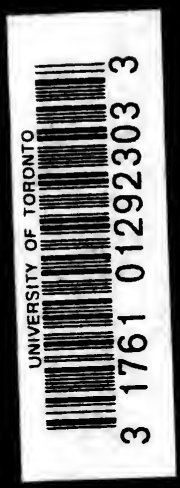



HANDBOUND AT THE TORONTO PRESS 
,

$$
\text { (⿻)丨 }
$$

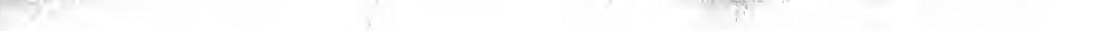


Digitized by the Internet Archive in 2007 with funding from Microsoft Corporation 

-

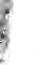

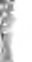

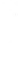

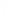





\section{A MANUAL}

OF

\section{VOLUMETRIC ANALYSIS}

FOR THE USE OF

PHARMACISTS, SANITARY AND FOOD CHEMISTS

AS WELL AS FOR

STUDENTS IN THESE BRANCHES

BY

HENRY W. SCHIMPF, PH.G., M.D.

Professor of Analytical Chemistry, Brooklyn College of Pharmacy; Member of the American Chemical Society; of the American Association for the Advancement of Science; of the American Pharmaceutical Association; of the New York State Pharmaceutical Association: of the King's County Pharmaceutical

Society; of the German Apothecaries' Society of New York City;

Honorary Member of the Alumni Association of the Brooklyn College of Pharmacy, etc., etc.

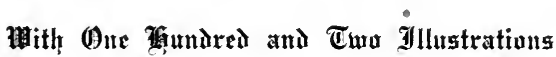

FIFTH EDITION, REVISED AND ENLARGED

FIRST THOUSAND

NEW YORK

JOHN WILEY \& SONS

LONDON: CHAPMAN \& HALL, LIMITED

$$
\text { Igog }
$$

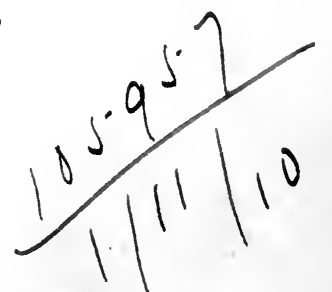


Authority to use for comment the Pharmacopoia of the United States of America, Eighth Decennial Revision, in this volume, has been granted by the Board of Trustees of the United States Pharmacopaial Convention, which Board of Trustees is in no way responsible for the accuracy of any translations of the official weights and measures or for any statements as to strength of official preparations.

Copyright, 1893, 1898, r909

BY

HENRY W. SCHIMPP 


\section{PREFACE TO THE FIFTH EDITION}

THE remarkable development of volumetric analysis during the past decade has necessitated a complete revision of my Text-Book of Volumetric Analysis. The present volume, which is called "A Manual of Volumetric Analysis," has been much enlarged and is almost entirely rewritten.

An effort has been made to bring it abreast of the times, in-so-far as methods of analysis are concerned. The book deals chiefly with pharmaceutical and food analysis, and it is hoped that chemists practising along these lines, as well as teachers and students, will find it of value.

The volumetric processes described are mostly such as have been tried and found of value.

The atomic weights used are those of the United States Pharmacopœia (based on $\mathrm{H} .=\mathrm{I}$ ); but for the convenience of chemists who prefer to work with the atomic weights based on $\mathrm{O}=\mathrm{r} 6$, a table of the International Atomic Weights is introduced at the front of the book, where it can easily be found. One hundred and fourteen pages are devoted to alkaloidal drug assaying.

Copious bibliographical references are given, and much care has been taken to make the index complete and comprehensive.

The author has given credit wherever it is due, and has in no case intentionally neglected this duty.

In the preparation of this edition the author has consulted, besides those works acknowledged in the former editions, the following: The Journal of the American Chemical Society, Chemical Abstracts, American Journal of Pharmacy, Proceedings of the American Phar- 
maceutical Association, Publications of the United States Department of Agriculture, The United States Pharmacopœia, The Pharmaceutical Review, Fresenius' Quantitative Chemical Analysis, Newth's Chemical Analysis, Lyons' Assay of Drugs, Holland's Medical Chemistry and Toxicology. The author also acknowledges his indebtedness to Dr. Joseph L. Mayer, for his assistance in revising the chapters on Water and Milk analyses.

H. W. S. 


\section{TABLE OF CONTENTS}

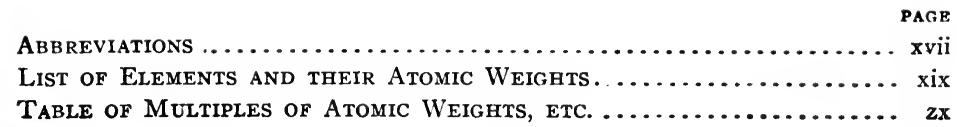

\section{PART I}

CHAPTER I

INTRODUCTION

CHAPTER II

General Principles

CHAPTER III

VolUMETRIC OR STANDARD SOLUTIONS $\ldots \ldots \ldots \ldots \ldots \ldots \ldots \ldots \ldots \ldots \ldots$

\section{CHAPTER IV}

INDiCATORS

Ionization Theory, 13. Chromophoric Theory, I4. Glaser's Classification of Indicators, 17. Requirements of a Good Indicator, 18. Alizarin, 19. Azolitmin, 19. Brazil-wood, 19. Cochineal, 19. Congored, I9. Corallin, 20. Eosin, 20. Gallein, 20. Hxmatoxylin, 20. Iodeosin, 21. Lacmoid, 2r. Litmus, 22. Luteol, 24. Methyl-orange, 24. Phenacetolin, 26. Phenolphthalein, 26. Poirrier blue, $\mathrm{C}_{4} \mathrm{~B}, 27$. Potassium Chromate, 27. Potassium Ferricyanid, 27. Resazurin, 28. Rosolic Acid, 28. Tropæolin O. O., 29. Turmeric, 29. Starch, 29.

A Guide for the Selection of Indicators, 30.

\section{CHAPTER V}




\section{CHAPTER VI}

ON THE USE, of Apparatus

The Reading of Instruments, 46 . The Calibration of Instruments, 49.

\section{CHAPTER VII}

Weights and Measures Used in Volumetric Analysis .............

\section{CHAPTER VIII}

Methods of Calculating Analyses . . . . . . . . . . . . . . . . .

Rules for Direct Percentage of Estimations, 53. Factors or Coefficients for Calculating the Analyses, 55. Table of Normal Factors, etc., for the Alkalies, Alkali Earths, and Acids, 57.

\section{CHAPTER IX}

SOME Vicarious Volumetric Methods

Volumetric Analysis without Weights and Standard Solutions, 58. Volumetric Analysis without a Burette, 59.

\section{CHAPTER X}

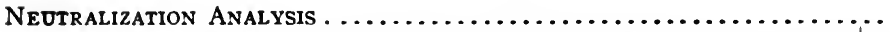

Alkalimetry, 64. Preparation of Standard Acid Solutions, 64. Estimation of Alkali Hydroxids, 74. Estimation of Alkali Carbonates, 77. Estimation of Mixed Alkali Hydroxid and Carbonate, 81. Estimation of Mixed Bicarbonates and Carbonates, 82. Estimation of Mixed Potassium and Sodium Hydroxids, 83. Estimation of Organic Salts of the Alkalies, 83. Table Showing Normal Factors, etc., of the Organic Salts of the Alkalies, 89. Estimation of Alkali Metals in their Salts, 89 . Estimation of Alkalies Combined with Volatile Acids, 9o. Estimation of Alkalies Combined with Non-volatile Acids, 9o. Estimation of Salts of the Alkali Earths, 91. Preparation of Normal Sodium Carbonate V.S., 92. Estimation of Mixed Hydroxids and Carbonates of the Alkali Earths, 93. Table Showing Normal Factors, etc., of the Alkali Earths, 94 .

Acidimetry, 94. Preparation of Standard Alkali Solutions, 96. Weighing Pipettes, 100. Estimation of Inorganic Acids, 99. Estimation of Organic Acids, ro4. Table of Acids which may be Estimated by Neutralization, 106. Estimation of Acids in Combination in Neutra! Salts, 108.

Table Showing Quantity of Substance to be Taken for Analysis in Direct Percentage Estimations, 107 
Precision in Determining End-reactions (Rakow), III. Preparation of Standard Silver Nitrate V. S., I I 2. Preparation of Standard Sodium Chlorid V. S., I I3. Preparation of Standard Potassium Sulphocyanate V. S., II4.

Estimation of Haloid Salts and Haloid Acids, I I 5. Titration without an Indicator (Gay-Lussac), I 20. Titration with Chromate Indicator (Mohr), I I5. Titration by Volhard's Method, I 22.

Estimation of Cyanogen, 125. Titration with Standard Silver V. S. to First Appearance of a Precipitate (Liebig), I25. Titration with Standard Silver V. S., using Iodid as an Indicator (Denigés), I 28. Titration with Standard Silver V. S., using Chromate as Indicator (Vielhaber), I 27.

Estimation of Silver Salts, I30. Titration with Standard Sodium Chlorid V. S., I30. Titration by Sulphocyanate Method, I3I. Estimation of Metallic Silver in Alloy, 132. Analysis of Certain Neutral Salts by Conversion into Chlorids, 132 .

Estimation of Alkali Iodids by Mercuric Chlorid V. S. (Personne), I34. Table of Substances Estimated by Precipitation, I35.

\section{CHAPTER XII}

Analysis by Oxidation and Reduction .................... 137

Analysis by Means of Potassium Permanganate V.S., I4I. Preparation of Standard Potassium Permanganate V.S., 142. On the Use of Empirical Potassium Permanganate V. S., 148. Typical Analyses with Potassium Permanganate V. S., 150. Direct Titrations, 150. Estimation of Ferrous Salts, I50. Estimation of Metallic Iron in Ferrum Reductum, 152. Estimation of Oxalic Acid and Oxalates, 153. Estimation of Hydrogen Dioxid, ${ }_{5} 6$. Estimation of Ferric Salts after Reduction, I62. Estimation of Nitrous Acid and Nitrites, 165. Residual Titrations, 167. Estimations in which the Excess of Permanganate is Found by Residual Titration with Oxalic Acid, 167. Estimation of Hypophosphites, 167. Methods Involving a Precipitation by Oxalic Acid and the Titration of the Excess by Permanganate, 170. Estimation of Calcium Salts, I 70. Methods Involving a Reduction by Means of Oxalic Acid and the Titration of the Excess of Permanganate, I7I. Estimation of Manganese Dioxid, I7I. Methods Involving a Reduction by Means of a Ferrous Salt, and Titration of the Excess by Permanganate, 172. Estimation of Nitrates (Pelouze), I72. Estimation of Chromic Acid and Chromates, 174. Methods Involving an Oxidation by Means of a Ferric Salt, and Titration of the Resultant Ferrous Salt by Means of Permanganate, 177. Estimation of Tin (Löwenthal), 177. Estimation of Copper (Fleitmann), I77.

Analyses by Means of Potassium Dichromate V.S., 178. Preparation of Standard Potassium Dichromate V. S., I79. Estimation of Ferrous 
Salts, 18r. Table of Substances which may be Estimated by Means of Potassium or Permanganate Dichromate, 185 .

Analysis by Indirect Oxidation, 185. Preparation of Standard Iodin V. S., 186. Preparation of Starch Indicator, 189. On the Use of Sodium Bicarbonate in Titrations with Iodin, 190. Estimation of Arsenous Compounds, 192. Estimation of Antimonous Compounds, 195. Estimation of Sulphurous Acids and Sulphites, 197. Table of Substances which may be Estimated by Means of Standard Iodin V. S., 201.

Estimation of Substances Readily Reduced, 202. Determinations Involving the Use of Sodium Thiosulphate (Iodometry), 202. Preparation of Standard Sodium Thiosulphate V. S., 203. Estimation of Free Iodin, 207. Estimation of Free Chlorin or Bromin, 209. Estimation of Hypochlorites, 2 Iо. Table Showing Degrees Gay-Lussac, 212. Estimation of Hydrogen Dioxid, 2 I 3

Distillation Methods, 214. Estimation of Manganese Dioxid, 218. Estimation of Chromic Acid and Chromates, 2rg. Estimation of Alkali Iodids, 220.

Digestion Methods, 222. Estimation of Chlorates, Bromates, and Iodates, 223. Estimation of Ferric Salts, 224.

Reduction Methods Involving the Use of Standard Arsenous Acid V.S., 226.

Preparation of Standard Arsenous Acid V S., 227. Estimation of Free Halogens, 228. Estimation of Hypochlorites, 230.

Reduction Methods Involving the Use of Stannous Chlorid Solution, 231. Estimation of Iron, 23r. Estimation of Mercuric Salts (Laborde), 233.

\section{PART II}

CHAPTER XIII

Acetic Acid and Acetates. Acetic Acid Table ............... 235

CHAPTER XIV

Boric ACID AND Borates

\section{CHAPTER XV}

Carbonic acid and Carbonates.

Carbonic Acid Gas in the Atmosphere, 246.

Table Showing Volume of $0.001 \mathrm{gm}$. of $\mathrm{CO}_{2}$ at Various Temperatures, 249. Carbonic Acid in Mineral Waters, 25r. Estimation of Small Quantities of Carbon Monoxid, $25^{3}$. 


\section{CHAPTER XVI}

Chlorin, Bromin, and Iodin 260

Chlorates, Bromates, and Iodates, 262 . Bromids or Iodids by Direct Titration with Chlorin Water, 265 .

\section{CHAPTER XVII}

Citric Acid and Citrates.

\section{CHAPTER XVIII}

Cyanogen and its Compounds.

Titration with Standard Silver Solution, 273. Titration with Standard Iodin V. S., 274. Titration with Standard Mercuric Chlorid V. S., 275. Titration with Standard Copper Solution, 275. By the Modified Kjeldahl Method, 276. The Iodometric Method for Ferricyanids, 280.

\section{CHAPTER XIX}

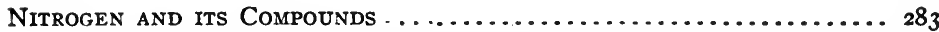

Method of Will and Varrentrapp, 283. The Ruffle Method, 286. The Kjeldahl Method (Modified), 287. The Jodlbauer-Kjeldahl Method, 290. Nitric Acid and Nitrates, 294. The Harcourt Method, 296. The Ulsch Method, 298. The Street-Ulsch Method, 298. The Pelouze Method, 299. The Schlösing Method, 299. The Iodometric Estimation (McGowan), 30r. Estimation of Nitrous Acid by $\mathrm{KMnO}_{4}, 303$. Estimation of Nitrous Acid Iodometric, 303.

\section{CHAPTER XX}

Oxalic Acid and Oxalates

\section{CHAPTER XXI}

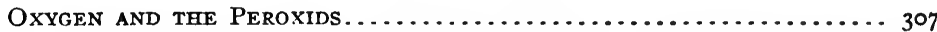

Estimation of Oxygen Dissolved in Water, 307. Hydrogen Dioxid, 309 .

\section{CHAPTER XXII}

Phosphoric Acid and Phosphates .

Stolba's Method, 3Ir. By Uranium Solution, 312. Segallé-Glückmann Method, 31 2. Pemberton's Molybdic Method, 316. Pemberton's New Method (McDonald), 3I7. Richardson's Method, 320. Estimation of Mixed Disodium and Trisodium Phosphate (Ahlum), 320. 
CHAPTER XXII

Salicylic Acid and Salicylates

\section{CHAPTER XXIV}

SUlPHUR AND ITS CoMpounds 326

Estimation of Sulphur in Alkali Sulphid, 327. Estimation of Hydrosulphuric Acid, 328. Estimation by Permanganate (Mohr), 328. Estimation by lodin, 329. Estimation by Arsenous Acid (Mohr), 329. Estimation by Silver Nitrate, 331. Estimation of Sulphurous Acid and Sulphites, 33r. Estimation of Sulphuric Acid and Sulphates, 33r.

\section{CHAPTER XXV}

Aluminum .

CHAPTER XXVI

Ammonium

CHAPTER XXVII

Antimony .

\section{CHAPTER XXVIII}

ARsenic

\section{CHAPTER XXIX}

BARIUM

\section{CHAPTER XXX}

Bismoth

CHAPTER XXXI

Calcium .

CHAPTER X.XXII

Correr. 362

CHAPTER XXXIII

Goln 


\section{CHAPTER XXXIV}

\section{CHAPTER XXXV}

LEAD 388

\section{CHAPTER XXXVI}

MAgNesium

\section{CHAPTER XXXVII}

MANGanese

\section{CHAPTER XXXVIII}

MERCURY 408

\section{CHAPTER XXXIX}

Silver .

CHAPTER XL

StRONTIUM

CHAPTER XLI

TiN 421

\section{CHAPTER XLII}

ZINC

\section{PART III}

\section{SANITARY ANALYSES AND VOLUMETRIC ANALYSES OF ORGANIC MEDICINAL SUBSTANCES}

\section{CHAPTER XLIII}

Sanitary Analysis of Water . . . . . . . . . . . . . . . . . 437

Collection of Sample, 437. Color, 437. Odor, 438. Reaction, 438. Suspended Matter, 438. Total Solids, 438. Organic and Volatile Matter or Loss on Ignition, 439. Chlorin, 439. Ammonia, 440. Nessler's Solution, 440. Albuminoid Ammonia, 443. Nitrates, 444. Nitrites, 445. Oxygen-consuming Power, 446. Phosphates, 447. Hardness, Temporary and Permanent, 448. Interpretation of Results, $45 \mathrm{r}$. 


\section{CHAPTER XLIV}

MILK.

Average Composition, 457. Colostrum, 458. Reaction, 458. Specific Gravity, 458. Lactometer, 459. Table for Correcting the Speciffc Gravity of Milk according to Temperature, 460. Adulterations of Milk, 459. Total Solids and Water, 459. Fat, Adam's Method, 461. Werner-Schmid Method, 462. Babcock Method, 462. Calculation Method, 463 . Calculation of Per Cent of Added Water, 464. Total Proteids, 465. Milk Sugar, 466.

\section{CHAPTER XLV}

ButTER .

Estimation of Volatile Acids (Reichert), 467. Estimation of Volatile Acids (A. O. A. C.), 467 .

\section{CHAPTER XLVI}

Some Technical Fxamination Methods for Fats, OILs, and Waxes... The Acid Value or Proportion of Free Fatty Acids, 472 . The Saponification Value (Kättstorfer's Number), 472. The Volatile Fatty Acid Value (Reichert's Number), 474. The Iodin Absorption Number (Hubl's Number), 474 The Hanus Method, 476. The Wijs Method, 477. Table Showing Iodin Absorption by the Three Methods, 477 . The Bromin Absorption Number, 478 .

\section{CHAPTER XLVII}

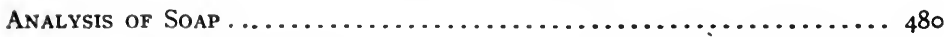

Free and Combined Alkali (Geisler), 480. Free Alkali (Devine), 482. Glycerin (Martin), 482.

\section{CHAPTER XLVIII}

Estimation of Stakch in Cereals, etc ...................... 484

Estimation after Conversion into Glucose by Heating with $\mathrm{HCl}, 486$. Lietz's Method, 486. Estimation after Inversion by Means of Diastase, 487 .

\section{CHAPTER XLIX}

Estimation of Sugars

By Fehling's Solution, 489. Various Methods of Determining Endreactions by Pavy's Solution, 493. By the Soxhlet-Fehling Method, 494. By the Permanganate Method, 495. Methods Depending upon the Reduction of Mercury, Knapp's Method, 495. Sachsse's Method, 496. 


\section{CHAPTER L}

PAGE

Volumetric Estimation of Alkaloids

498

Table Showing the Behavior of Some of the Alkaloids with Indicators, 503. Table Showing Factor for Various Alkaloids when Titrating with $\frac{\mathrm{N}}{10}$ Acid V. S., 504. Estimation by Mayer's Reagent, 505. Estimation of Alkaloids by Wagner's Reagent, 507. Estimation of Caffeine by Wagner's Reagent, 509. Gordin's Modified Alkalimetric Method, using Phenlophthalein as Indicator, $5 \mathrm{ro}$.

\section{CHAPTER LI}

Volumetric Assaying of Vegetable Drug:

Extraction of the Alkaloids, 5 I 2. Alkaloidal Assay by Immiscible Solvents, 51 2 General Methods of Assaying Drugs, 515. Keller Method, 515. Puckner Method, 5r6. Lyons Method, 5r6. Gordin Method, 517. Kebler-Keller Method, 517. Gordin and Prescott Method, 5 r8.

The Assaying of Crude Drugs, 520. Aconite Root (Keller), 520. Aconite Leaves: Keller, 52I; German Pharmacopoia, 52I. Aconite Root: U. S. P. VIII, 522; Gordin, 525; Kippenberger, 526; Fromme, 527 .

The Mydriatic Drugs, 527. Belladonna: Puckner (U. S. P. VIII), 527; Kebler, 528; Gordin and Prescott, 529. Hyoscyamus (Keller), 529. Scapola and Stramonium, 530. Cinchona: U.S. P. (1890), 531; Gordin, 532; Kippenberger, 533; German Pharmacopœia, 533; Florence, 534; Fromme, 535. Coca: U. S. P. VIII, 538; Kebler-Keller, 538; Lyons, 538; Squibbs, 539; Léger, 541. Colchicum: Gordin and Prescott, 542; Panchaud, 543. Conium: Lyons, 544; U. S. P. VIII, 544. Hydrastis Canadensis: U. S. P. VIII, 546; Puckner, 546; Gordin and Prescott, 547; Schreiber, 549. Ipecac: U.S. P. VIII, 552; Gordin, 552; Fromme, 553 .

Ipecac. Separate Estimation of Emetine and Cephaeline: Patterson, 553; Paul and Cownley, 554. Ipecac (Frerichs and Tapis), 555. Nux Vomica, 556.

Methods in which the "Total Alkaloids" is Determined: German Pharmacopœia, 556; Puckner, 557; Gordin, 558; Kippenberger, $55^{8}$.

Methods in which Strychnine is Determined: Keller, 559; U.S. P., 559; Webster and Pursel, 56ri Gordin, 56r; The Ferrocyanid Methods, 562. Opium: U. S. P. (1890), 564; U. S. P. VIII, 565; Lamar, 567; Mallinckrodt, 567; Parker, 568; Gordin and Prescott, 569; German Pharmacopœia, 570; Stevens, 571: Ascher, 572; Picard-Léger, 573; Tickle, 574. Physostigma: U. S. P. VIII, 576; Beckurts, 576. Pilocarpus, U. S. P. VIII, 578; Fromme, 578. Strophanthus (Barclay), 579. Tobacco (Keller), 580. Veratrum (Bredemann), 581. Wild Cherry Bark (Stevens), 583. 


\section{CHAPTER LII}

Assay of Galenical Preparations . . . . . . . . . . . . . . . 584

Lloyd's Methods, 584. Lýons' Method, 585. Steiglitz's Method, 586. Thompson's Method, 586. Katz's Method, 586. Kippenberger' Method, 587. Farr and Wright's Method, 590. Webster's Method, 590 Fluid Extract of Aconite, 592. Tincture of Aconite, 593. Extract of Belladonna, 593. Fluid Extract of Belladonna, 594. Tincture of Bella donna, 595. Puckner's Method, 595. Lyons' Method, 595. Thom's Bismuth-iodid Method, 598. Belladonna Plaster (U. S: P. VIII), 598. German Pharmacopeia Methods, 599. Fluid Extract of Cinchona, 600 . Fluid Extract of Coca, 6or. Fluid Extract of Gelsemium (Sayre), 60I. Fluid Extract of Hydrastis: U. S. P. VIII, 602; Heyl, 603; RustingSmeets, 603. Tincture of Hydrastis, 603. Fluid Extract of Ipecac, 604 . Extract of Nux Vomica: U. S. P (1890), 605; U. S. P. VIII, 606. Extract of Opium, 608. Tincture of Opium, 609. Extract of Physostigma, 6ro. Fluid Extract of Pilocarpus, 6ri. Alkaloidal Assay of Scale Salts, 612.

\section{CHAPTER LIII}

Phenol

Preparation of Standard Bromin V. S., 6r3. Lloyd's Hypobromite Method, 6r7. Waller's Method, 6r8. Crude Carbolic Acid, 6rg. Potassium Permanganate Method (Tocher), 620. Estimation of Phenol in Pharmaceutical Products, 621.

\section{CHAPTER LIV}

GlYcerin . . . . . . . . . . . . . . . . . . . . . . . . .

The Permanganate Method (Benedikt and Zsigmondy), 623. Estimation in Fats, 624. Herbig and Mangold's Method, 625. The Acetin Method (Benedikt and Cantor), 625. The Dichromate Method (Hehner), 627. The Iodic Acid Method (Chaumeil), 628. Estimation in Fluid Extracts, 628.

\section{CHAPTER LV}

TANNin :

Löwenthal's Method, 631. Fleury's Method, 634. Thompson's Method, using $\mathrm{H}_{2} \mathrm{O}_{2}, 634$. Lorenz's Method, using Safranin, 635 . Vignon's Method, using Silk, 635. Tannin in Wines, 636. Tannin in Tea, 636. Tannin in Cloves and Allspice, 637.

\section{CHAPTER LVI}

\section{FORMALDEHYDE}

The Ammonia Method: Legler, 639; Craig, 640. The Ammonium Chlorid Method (Schiff), 640. The Hydrogen Dioxid Method (Blank 
and Finkenbeiner), 641. The Iodiometric Method: Ramijn, 642; Reuter, 642; Taylor, 642. The Potassium Cyanid Method: Romijn, 643; Smith, 643. The Phloroglucin Method (Clowes and Cullens), 644.

\section{CHAPTER LVII}

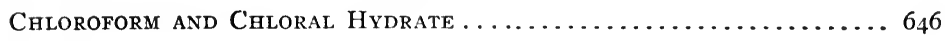

\section{CHAPTER LVIII}

Assaying Surgical Dressings . . ...................... 649

Carbolic Acid Dressings (Meissinger and Vortmann) 649. Salicylic Acid Dressings, 651. Boric Acid Dressings, 651. Sublimate Dressings: Denner, 65r; Link and Vaswinkel, 652; Beckurts, 653. Iodoform Dressings, 653. Styptic Cotton Dressings (Parker), 655.

\section{CHAPTER LIX}

Estimation of Compound Ethers...................... 656

Spirit of Nitrous Ether, 657 .

\section{CHAPTER LX}

URINE .

Reaction, 66o. Composition, 660. Specific Gravity, 66r. Total Solids, 662. Chlorids, 663. Phosphates, 663. Sulphates, 664. Total Acidity, 665. Joulie's Method, 665. Urea (Benedict and Gephart), 667. Uric Acid, 667. Uric Acid: Bartley's Method, 668; Iodic Acid Method (Merck), 670; Iodic Acid Method (Bouillet), 67r. Albumen, 672. Blood, 674. Pus, 674. Sugar, 674. Bile, 677.

\section{PART IV}

\section{A FEW GASOMETRIC METHODS}

\section{CHAPTER LXI}

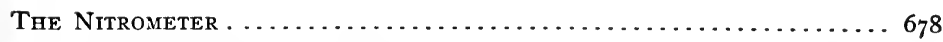

Charles' Law, 679. Boyle's Law, 680. Table of Factors for Temperature Corrections, 68r. Table of Factors for Barometric Corrections. $68 \mathrm{I}$. 


\section{CHAPTER LXII}

Spirit of Nitrous Ether, 683. Amyl Nitrite, 685. Sodium Nitrite, 685. Nitric Acid in Nitrates, 686.

\section{CHAPTER LXIII}

Assay of Hydrogen Dioxid 687

By Squibb's Urea Apparatus, 687. By Improvised Nitrometer, 687. The Hypochlorite Method, 688. The Hypobromite Method, 689.

\section{CHAPTER LXIV}

Estrmation of Soluble Carbonates . ....................... 69t Aromatic Spirits of Ammonia, 69r.

\section{CHAPTER LXV}

Estmation OF UREA AND URIC ACID 


\section{ABBREVIATIONS}

A. J. Ph............... American Journal of Pharmacy.

Am. Chem. J. . . ......... American Chemical Journal.

Am. Drug. ............. American Druggist.

A. O. A. C. . .......... Association of Official Agricultural Chemists.

Apoth. Ztg. . ............ Apotheker Zeitung.

Archiv. Ph. ............ Archives der Pharmacie.

At. wt. . ............. atomic weight.

Ber. Chem. Ges.......... Berichte der Deutschen Chemischen Gesellschaft.

Bull. Soc. Chim .......... Bulletin de la Société Chimique.

cc. .................. cubic centimeter.

Chem. News ............ Chemical News.

Chem. Ztg. ............ Chemiker Zeitung.

Compt. rend............ Comptes rendus des Seances de l'Académie des Sciences.

Chem. Centralbi......... Chemisches Centralblatt.

Cm.................. centimeter.

Drug. Cir............. Druggists' Circular.

Gaz. Ch. It............ Gazzetta chimica Italiana.

gm................. gram, I5.43235 grains.

gr. ................... grain.

J. A. C. S. . . . . . . J . Journal of the American Chemical Society.

J. S. C. I.............. Journal of the Society of Chemical Industry.

J. Chem. Soc. ........... Journal of the Chemical Society.

J. f. prakt. Ch. .......... Journal für praktische Chemie.

J. Anal. Chem........... Journal of Analytical Chemistry.

J. Pharm. et Chim. ....... Journal de Pharmacie et de Chimie.

Mg.................. milligrams.

Mm................. millimeter.

Monatsheft. ............ Monatshefte für Chemie.

Ph. Jour. and Trans ....... Pharmaceutical Journal and Transactions.

Ph. Centralh. ........... Pharmaceutische Centralhalle.

Ph. Rev.............. Pharmaceutical Review. 
Ph. Post.

Pharmaceutical Post.

Ph. Ger.

German Pharmacopœia.

Ph. Ztg. Pharmaceutische Zeitung.

T. S. test solution, according to U. S. P.

Trans. Brit. Ph. Conf. Transactions of the British Pharmaceutical Conference.

U. S. P. VIII. .......... United States Pharmacopœia, 8th Dec. Revision.

V. S. ............... volumetric solution.

Zeitschr. anal. Chem....... Zeitschrift für analytische Chemie.

Zeitschr. f. Chem......... Zeitschrift für Chemie.

Zeitschr. anorg. Chem. ..... Zeitschrift für anorganische Chemie.

Zeitschr. angew. Chem. ..... Zeitschrift für angewandte Chemie.

Zeitschr. physiol. Chem. .... Zeitschrift für physiologische Chemie. 
MULTIPLES OF NUMBERS REPRESENTING SOME ATOMIC WEIGHTS AND COMBINATIONS IN FREQUENT USE.

(Based on $\mathrm{H}=1$ ).

\begin{tabular}{|c|c|c|c|c|c|c|c|c|c|c|}
\hline $\mathrm{H}$ & 1 & 2 & 3 & 4 & 5 & 6. & 7 & 8 & 9 & $\mathrm{H}$ \\
\hline $\mathrm{O}$ & I 5.88 & 31.76 & 47.64 & $63 \cdot 5^{2}$ & 79.40 & 95.28 & III. I6 & 127.04 & 142.92 & $\mathrm{O}$ \\
\hline $\mathrm{OH}$ & 16.88 & $33 \cdot 76$ & 50.64 & 67.52 & 84.40 & IOI. 28 & 118.16 & 135.04 & 151.92 & $\mathrm{OH}$ \\
\hline $\mathrm{H}_{2} \mathrm{O}$ & 17.88 & $35 \cdot 76$ & 53.64 & $71 \cdot 5^{2}$ & 89.40 & 107.28 & 125.16 & 143.04 & 160.92 & $\mathrm{H}_{2} \mathrm{O}$ \\
\hline$N$ & 13.93 & 27.86 & $41 \cdot 79$ & 55.72 & 69.65 & $83 \cdot 58$ & $97 \cdot 5 \mathrm{I}$ & I I I. 44 & 125.37 & $\mathrm{~N}$ \\
\hline $\mathrm{NH}_{3}$ & 16.93 & 33.86 & 50.79 & $67 \cdot 72$ & 84.65 & $101 \cdot 58$ & I I $8.5 \mathrm{I}$ & 135.44 & 152.37 & $\mathrm{NH}_{3}$ \\
\hline $\mathrm{NH}_{4}$ & 17.93 & 35.86 & 53.79 & 71.72 & 89.65 & $107 \cdot 58$ & 125.51 & I 43.44 & 161.37 & $\mathrm{NH}_{4}$ \\
\hline $\mathrm{NO}_{3}$ & $61 \cdot 57$ & 123.14 & I 84.7 I & 246.28 & 307.85 & 369.42 & 430.99 & $492 \cdot 56$ & $554 \cdot 13$ & $\mathrm{INO}_{3}$ \\
\hline C. & I I.9I & 23.82 & $35 \cdot 73$ & 47.64 & 59.55 & $71 \cdot 46$ & $83 \cdot 37$ & $95 \cdot 28$ & 107.17 & C \\
\hline $\mathrm{CO}_{2}$ & $43 \cdot 67$ & $87 \cdot 34$ & 131.01 & 174.68 & 218.35 & 262.02 & 305.69 & $349 \cdot 36$ & 393.03 & $\mathrm{CO}_{2}$ \\
\hline $\mathrm{CO}_{3}$ & 59.55 & 119.10 & I 78.65 & $238: 20$ & $297 \cdot 75$ & $357 \cdot 30$ & 416.85 & 476.40 & 535.95 & $\mathrm{CO}_{3}$ \\
\hline $\mathrm{CN}$ & 25.84 & 51.68 & $77 \cdot 52$ & $103 \cdot 36$ & 129.20 & I 55.04 & 180.88 & 206.72 & $232 \cdot 56$ & $\mathrm{CN}$ \\
\hline $\mathrm{Cl}$ & $35 \cdot 18$ & 70.36 & $105 \cdot 54$ & 140.72 & 175.90 & 211.08 & 246.26 & $28 \mathrm{r} .44$ & 316.62 & $\mathrm{Cl}$ \\
\hline B & 79.36 & 158.72 & 238.08 & 317.44 & 396.80 & 476.16 & $555 \cdot 5^{2}$ & 634.88 & 714.24 & $\mathrm{Br}$ \\
\hline$\dot{I}$ & I 25.90 & 251.80 & $377 \cdot 70$ & 503.60 & 629.50 & $755 \cdot 40$ & $88 \mathrm{I} \cdot 30$ & 1007.2 & 1133.10 & $I$ \\
\hline $\mathrm{S}$ & 31.83 & 63.66 & $95 \cdot 49$ & I 27.32 & I 59. I 5 & 190.98 & $222.8 \mathrm{I}$ & 254.64 & 286.47 & $\mathrm{~S}$ \\
\hline $\mathrm{SO}_{4}$ & 95.35 & 190.70 & 286.05 & $381 \cdot 40$ & 476.75 & 572.10 & $667 \cdot 45$ & 762.80 & 858.15 & $\mathrm{SO}_{4}$ \\
\hline $\mathrm{PO}_{4}$ & 94.29 & I 88.58 & 282.87 & $377 \cdot 16$ & $471 \cdot 45$ & $565 \cdot 74$ & 660.03 & $754 \cdot 32$ & $848.6 \mathrm{I}$ & $\mathrm{PO}_{4}$ \\
\hline $\mathrm{Na}$ & 22.88 & $45 \cdot 76$ & 68.64 & $91 \cdot 52$ & I I 4.40 & 137.28 & 160.16 & I 83.04 & 205.92 & $\mathrm{Na}$ \\
\hline $\mathbf{K}$ & 38.86 & $77 \cdot 72$ & I I 6. 58 & I 55.44 & 194.30 & 233.16 & 272.02 & 310.88 & $394 \cdot 74$ & $\mathrm{~K}$ \\
\hline $\mathrm{Li}$ & 6.98 & 13.96 & 20.94 & 27.92 & $34 \cdot 90$ & 42.08 & 48.86 & 55.84 & 62.82 & $\mathrm{Li}$ \\
\hline $\mathrm{Ba}$ & 136.4 & 272.8 & 409.2 & $545 \cdot 6$ & 682.0 & 818.4 & 954.8 & 1091.2 & 1227.6 & $\mathrm{Ba}$ \\
\hline $\mathrm{Ca}$ & 39.8 & 79.6 & 128.4 & 159.2 & I99.0 & 238.8 & 278.6 & 318.4 & 358.2 & $\mathrm{Ca}$ \\
\hline $\mathrm{Ag}$ & 107.12 & 214.24 & $321 \cdot 36$ & 428.48 & 535.6 & $642 \cdot 72$ & 749.84 & 856.96 & 964.08 & $\mathrm{Ag}$ \\
\hline $\mathrm{Hg}$ & 198.5 & 397.0 & $595 \cdot 5$ & 794.0 & $992 \cdot 5$ & II 1 I. & 1389.5 & I 588.0 & I 786.5 & $\mathrm{Hg}$ \\
\hline \multirow[t]{2}{*}{$\mathrm{Fe}$} & $55 \cdot 5$ & I I I. O & 166.5 & 222.0 & $277 \cdot 5$ & 333.0 & 388.5 & 444.0 & $499 \cdot 5$ & $\mathrm{Fe}$ \\
\hline & 1 & 2 & 3 & 4 & 5 & 8 & 7 & 8 & 9 & \\
\hline
\end{tabular}




\section{A LIST OF ELEMENTS OCCURRING IN VOLUMETRIC METHODS, THEIR SYMBOLS, AND ATOMIC WEIGHTS}

\begin{tabular}{|c|c|c|c|c|}
\hline Name. & & $\begin{array}{l}\text { Atomic } \\
\text { Weights* } \\
\text { Based on } \\
\mathrm{H}=\mathrm{r} .000 .\end{array}$ & $\begin{array}{c}\text { Atomic } \\
\text { Weights } \dagger \\
\text { Based on } \\
\mathrm{O}=16\end{array}$ & $\begin{array}{c}\text { Approxi- } \\
\text { mate } \\
\text { Atomic } \\
\text { Weights. }\end{array}$ \\
\hline Aluminum $\ldots \ldots \ldots \ldots \ldots \ldots \ldots \ldots$ & $\mathrm{Al}$ & 26.9 & $27 \cdot I$ & 27.0 \\
\hline Antimony...$\ldots \ldots \ldots \ldots \ldots \ldots \ldots$ & $\mathrm{Sb}$ & II 9.3 & I 20.2 & 120.0 \\
\hline Arsenic...$\ldots \ldots \ldots \ldots \ldots \ldots \ldots \ldots$ & As & $74 \cdot 3$ & $75 \cdot 0$ & $75 \cdot 0$ \\
\hline 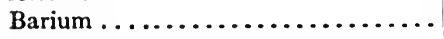 & $\mathrm{Ba}$ & 136.4 & $137 \cdot 4$ & 136.0 \\
\hline Bismuth $\ldots \ldots \ldots \ldots \ldots \ldots \ldots \ldots$ & $\mathrm{Bi}$ & 206.4 & 208.0 & 206.0 \\
\hline Boron...$\ldots \ldots \ldots \ldots \ldots \ldots \ldots \ldots$ & B & 10.9 & II.O & II.O \\
\hline Bromin...$\ldots \ldots \ldots \ldots \ldots \ldots \ldots \ldots$ & $\mathrm{Br}$ & $79 \cdot 36$ & $79 \cdot 96$ & 80.0 \\
\hline 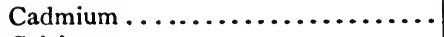 & $\mathrm{Cd}$ & III.6 & II 24 & III.O \\
\hline Calcium ...................... & $\mathrm{Ca}$ & 39.8 & 40.1 & 40.0 \\
\hline Carbon...$\ldots \ldots \ldots \ldots \ldots \ldots \ldots \ldots$ & C & II.9I & 12.0 & 12.0 \\
\hline 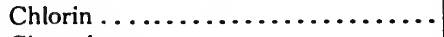 & $\mathrm{Cl}$ & $35 \cdot 18$ & $35 \cdot 45$ & 35.2 \\
\hline 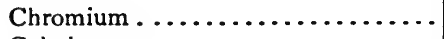 & $\mathrm{Cr}$ & $5 \mathrm{I} \cdot 7$ & 52.1 & 52.0 \\
\hline Cobalt $, \ldots \ldots \ldots, \ldots, \ldots, \ldots$, & Co & 58.56 & 59.0 & 58.0 \\
\hline Copper..$\ldots \ldots \ldots \ldots \ldots \ldots \ldots$ & $\mathrm{Cu}$ & $63 \cdot 1$ & 63.6 & 63.0 \\
\hline Fluorin $\ldots \ldots \ldots \ldots \ldots \ldots \ldots \ldots \ldots$ & $\mathrm{F}$ & 18.9 & 19.0 & 19.0 \\
\hline Gold . . . . . . . . . . . . . . . & $\mathrm{Au}$ & $195 \cdot 7$ & 197.2 & 196.0 \\
\hline Hydrogen...$\ldots \ldots \ldots \ldots \ldots \ldots$ & $\mathbf{H}$ & I. .000 & 1.008 & I.O \\
\hline lodin $\ldots \ldots \ldots \ldots \ldots \ldots \ldots \ldots \ldots$ & I & I $25 \cdot 9$ & I 26.97 & I 26.0 \\
\hline Iron $\ldots \ldots \ldots \ldots \ldots \ldots \ldots \ldots \ldots$ & $\mathrm{Fe}$ & $55 \cdot 5$ & $55 \cdot 9$ & 56.0 \\
\hline Lead $\ldots \ldots \ldots \ldots \ldots \ldots \ldots \ldots \ldots \ldots$ & $\mathrm{Pb}$ & 205.35 & 206.9 & 206.0 \\
\hline Lithium . . . . . . . . . . . . . . . & $\mathrm{Li}$ & 6.98 & 7.03 & 7.0 \\
\hline Magnesium .................. & $\mathrm{Mg}$ & $24 \cdot 18$ & $24 \cdot 3^{6}$ & 24.0 \\
\hline Manganese...$\ldots \ldots \ldots \ldots \ldots \ldots$ & $\mathrm{Mn}$ & 54.6 & 55.0 & 55.0 \\
\hline Mercury $\ldots . . . \ldots \ldots \ldots \ldots \ldots$ & $\mathrm{Hg}$ & 198.5 & 200.0 & 200.0 \\
\hline Molybdenum ................. & Mo & $95 \cdot 3$ & 96.0 & 95.0 \\
\hline Nickel ..................... & $\mathrm{Ni}$ & $5^{8} \cdot 3$ & 58.7 & 58.0 \\
\hline Nitrogen ....................... & $\mathrm{N}$ & 13.93 & 14.01 & 14.0 \\
\hline Oxygen...$\ldots \ldots \ldots \ldots \ldots \ldots$ & $\mathrm{O}$ & 15.88 & I6.0 & 16.0 \\
\hline Phosphorus . . . . . . . . . . . . . & $\mathrm{P}$ & 30.77 & 31.0 & 31.0 \\
\hline Platinum........................ & $\mathrm{Pt}$ & $193 \cdot 3$ & 194.8 & r94.0 \\
\hline Potassium..$\ldots \ldots \ldots \ldots \ldots \ldots \ldots$ & $\mathrm{K}$ & 38.86 & 39.15 & 39.0 \\
\hline Silver ...................... & $\mathrm{Ag}$ & 107.12 & 107.93 & 107.0 \\
\hline Sodium ...................... & $\mathrm{Na}$ & 22.88 & 23.05 & 23.0 \\
\hline 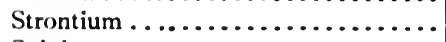 & $\mathrm{Sr}$ & 86.94 & 87.6 & 87.0 \\
\hline 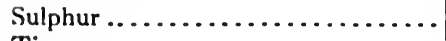 & $S$ & 31.83 & 32.06 & 32.0 \\
\hline $\operatorname{Tin} \ldots \ldots \ldots \ldots \ldots \ldots \ldots \ldots \ldots \ldots$ & $\mathrm{Sn}$ & 118.1 & II 9.0 & I 18.0 \\
\hline Zinc $\ldots \ldots \ldots \ldots \ldots \ldots \ldots \ldots \ldots \ldots$ & $\mathrm{Zn}$ & $64 \cdot 9$ & $65 \cdot 4$ & 65.0 \\
\hline
\end{tabular}

* Adopted in this book.

† International atomic weights, J. A. C. S., 1907, par. III. 


\section{A MANUAL}

OF

\section{VOLUMETRIC ANALYSIS}

\section{PART I}

\section{CHAPTER I \\ INTRODUCTION}

IN a chemical analysis the aim is to determine the nature of the chemical substances contained in a given compound or to ascertain their quantities. In the former case the analysis is a qualitative, in the latter a quantitative, one.

The quantitative analysis of a substance may be made either by the gravimetric or the volumetric method.

The Gravimetric Method consists in separating and weighing the constituents either in their natural states or in the form of new and definite compounds, the composition of which is known to the analyst. Fiom the weights of these new compounds the analyst can calculate the quantities of the original constituents.

Example. To determine the quantity of silver in a solution by the gravimetric method we proceed as follows:

Ten grams of a solution containing silver in the form of silver nitrate $\left(\mathrm{AgNO}_{3}\right)$ is placed into a beaker, and, after slightly acidulating with nitric acid, is treated with hydrochloric acid, drop by drop, until no further precipitation occurs. The precipitate which consists of silver chlorid $(\mathrm{AgCl})$ is then separated by filtration, thoroughly washed, dried, and weighed. Its weight is found to be $0.69 \mathrm{gm}$. The calculation is then made as follows: 142.3 gms. of silver chlorid represents I07.I 2 gms. of silver or I68.69 gms. of silver nitrate, as the equation shows:

$$
\underset{168.69}{\mathrm{AgNO}_{3}}+\mathrm{HCl}=\underset{\mathrm{I} 42.3}{\mathrm{AgCl}}+\mathrm{HNO}_{3} .
$$


Therefore, $0.69 \mathrm{gm}$. of silver chlorid will represent

or

$$
\begin{aligned}
& \frac{107.12}{142.3} \times 0.69=0.519 \mathrm{gm} . \text { of silver, } \\
& \frac{168.69}{142.3} \times 0.69=0.818 \mathrm{gm} . \text { of silver nitrate. }
\end{aligned}
$$

The Volumetric Method. This method depends upon the use of solutions (standard solutions) which are of known strength, and paying attention to the volume of such a solution which must be added to the substance under analysis to perform with it and complete a certain reaction. Thus, if we conduct an analysis by means of such a solution, and can express by chemical equation the reaction which takes place, we can readily and accurately calculate the quantity present of the substance under analysis.

Example.-If a silver solution is to be analyzed by this method it is treated with a standard solution of sodium chlorid, added slowly from a burette until no more silver chlorid is precipitated. Each cc. of this standard solution will precipitate a certain weight of silver as silver chlorid, and hence by noting the number of cc. used to complete the precipitation, the weight of the silver in the solution analyzed is easily ascertained.

The $\frac{\mathrm{N}}{\mathrm{IO}}$ sodium chlorid solution is generally used for this purpose. It is made by dissolving ${ }_{1}^{1} 0$ of the molecular weight of the salt (in grams) (5.806 gms.) in water sufficient to make $1000 \mathrm{cc}$. $1000 \mathrm{cc}$. of this solution will precipitate ${ }_{10}^{1}$ of the atomic weight of silver (in grams) (10.712 gms.), and hence each cc. of the sodium chlorid solution represents $0.0107 \mathrm{I} 2 \mathrm{gm}$. of metallic silver, and by multiplying this figure by the number of cc. used, the quantity of silver in the solution is found. If in the above analysis roo cc. of the $\frac{\mathrm{N}}{10}$ sodium chlorid solution were used, then $0.010712 \times 100=1.0712 \mathrm{gms}$. of metallic silver.

The reaction is illustrated by this equation:

$$
\begin{aligned}
& \mathrm{Ag} \quad \mathrm{NO}_{3}+\mathrm{NaCl}=\mathrm{AgCl}+\mathrm{NaNO}_{3} . \\
& \text { 10) } 107.12 \quad 10 \lcm{58.06} \\
& 1 0 0 0 \longdiv { 1 0 . 7 1 2 } \mathrm { gms } . \quad 1 0 0 0) \frac{5.806}{\mathrm{gms}} . \quad=1000 \mathrm{cc} . \frac{\mathrm{N}}{10} \mathrm{~V} . \mathrm{S} . \\
& 0.010712 \mathrm{gm} \text {. } 0.005806 \mathrm{gm} .=\text { I cc. } 10 \mathrm{~V} . \mathrm{S} \text {. }
\end{aligned}
$$


From the examples given it will be seen that the gravimetric operations consume no little time, and require the exercise of considerable skill. The washing of the precipitate must be thoroughly performed in order that it be freed from all adhering matter. The drying also is a matter of some consequence and must be performed in such a manner as to prevent the admixture of dust or the decomposition of the precipitate by excessive heat. A very accurate balance is also required.

The volumetric operations, on the other hand, do not require that the substance to be determined be separated in the form of a compound of known composition and weighed in the dry state; in fact, the substance may be accurately estimated when mixed 'with many others. It therefore obviates the necessity for the frequent separations and weighings which the gravimetric method demands, and enables the analyst to do the work in a very short time.

The instruments needed for volumetric work are few and simple, and comparatively little skill is required. Furthermore the results obtained are in most instances more accurate. 


\section{CHAPTER II}

GENERAL PRINCIPLES OF CHEMICAL COMBINATION UPON WHICH VOLUMETRIC ANALYSIS IS BASED

1. When substances unite chemically the union always takes place in definite and invariable proportions. Thus when silver nitrate and sodium chlorid are brought together, 168.69 parts (by weight) of silver nitrate and $5^{8.06}$ parts (by weight) of sodium chlorid will react with each other, producing 142.30 parts of a curdy white precipitate (silver chlorid).

These substances will react with each other in these proportions only.

If a greater proportion of silver nitrate than that above stated be added to the sodium chlorid, only the above proportion will react, the excess remaining unchanged.

The same is true if sodium chlorid be added in excess of the above proportions. For instance, if 200 parts of silver nitrate be mixed with 58.06 parts of sodium chlorid 168.69 parts only will react with the sodium chlorid, while $3 \mathrm{I} \cdot 3 \mathrm{I}$ parts of silver nitrate will remain unchanged. Again, when potassium hydroxid and sulphuric acid are mixed potassium sulphate is formed, III.48 parts of potassium hydroxid and 97.35 parts of sulphuric acid being required for complete neutralization. These two substances unite chemically in these proportions only.

The equation is

$$
\underset{111.48}{2 \mathrm{KOH}}+\underset{97.35}{\mathrm{H}_{2} \mathrm{SO}_{4}}=\mathrm{K}_{2} \mathrm{SO}_{4}+{ }_{2} \mathrm{H}_{2} \mathrm{O} .
$$

In other words, 111.48 parts of $\mathrm{KOH}$ will neutralize 97.35 parts of $\mathrm{H}_{2} \mathrm{SO}_{4}$, and consequently 97.35 parts of $\mathrm{H}_{2} \mathrm{SO}_{4}$ will neutralize III.48 parts of $\mathrm{KOH}$.

Oxalic acid and sodium carbonate react upon each other in the proportions shown in the equation

$$
\underbrace{\mathrm{H}_{2} \mathrm{C}_{2} \mathrm{O}_{4} \cdot{ }_{2} \mathrm{H}_{2} \mathrm{O}}_{125.10}+\underbrace{\mathrm{Na}_{2} \mathrm{CO}_{3}}_{105.3 \mathrm{I}}=\mathrm{Na}_{2} \mathrm{C}_{2} \mathrm{O}_{4}+\mathrm{CO}_{2}+{ }_{3} \mathrm{H}_{2} \mathrm{O}
$$


I25. I0 parts of crystallized oxalic acid are neutralized by I05.3 I parts of anhydrous sodium carbonate.

2. Definite chemical compounds always contain the same elements in exactly the same proportions, the proportions being those of their atomic weights, or some multiple of these weights.

Thus sodium chlorid $(\mathrm{NaCl})$ contains 22.88 parts of metallic sodium and 35.18 parts of chlorin, these being the atomic weights of sodium and chlorin respectively.

Potassium sulphate $\left(\mathrm{K}_{2} \mathrm{SO}_{4}\right)$ contains twice $38.86=77.72$ parts of potassium, 3 I .83 parts of sulphur, and four times $15.88=63.5^{2}$ parts of oxygen.

Potassium hydroxid $(\mathrm{KOH})$ contains 38.86 parts of potassium, I.5.88 parts of oxygen, and one part of hydrogen. Hydrochloric acid $(\mathrm{HCl})$ contains one part of hydrogen and 35.18 parts of chlorin.

Upon these facts the volumetric methods of analysis are based.

It has been shown that 97.35 gms. of sulphuric acid will neutralize III.48 gms. of potassium hydroxid; it is therefore evident that if a solution of sulphuric acid be made containing $48.675 \mathrm{gms}$. (half of the molecular weight) of the pure acid in rooo cc. that one cc. of this solution will neutralize $0.05574 \mathrm{gm}$. of potassium hydroxid. In estimating alkalies with this acid solution the latter is added from a burette, in small portions, until the alkali is neutralized, as shown by its reaction with some indicator.

Each cc. of the acid solution required before neutralization is complete indicates $0.05574 \mathrm{gm}$. of $\mathrm{KOH}$, and the number of cc. used multiplied by $0.05574 \mathrm{gm}$. gives the quantity of pure $\mathrm{KOH}$ in the sample analyzed.

One cc. of the same solution will neutralize $0.03976 \mathrm{gm}$. of sodium hydroxid $(\mathrm{NaOH}), 0.05^{26} 6 \mathrm{gm}$. of anhydrous sodium carbonate $\left(\mathrm{Na}_{2} \mathrm{CO}_{3}\right)$, etc.

If a solution of crystallized oxalic acid be made by dissolving (half of the molecular weight in grams) $62.55 \mathrm{gms}$. in sufficient water to make $1000 \mathrm{cc}$., we will have a normal solution, the neutralizing power of which is exactly equivalent to the above-mentioned normal sulphuricacid solution.

The strength of acids is estimated by alkali volumetric solutions. A normal solution of potassium hydroxid containing 55.74 gms. in the liter will neutralize exactly I liter of the normal acid solution; i cc. of this normal alkali will neutralize $0.036 \mathrm{~J} 8 \mathrm{gm}$. of $\mathrm{HCl}, 0.06255$ gm. of $\mathrm{H}_{2} \mathrm{C}_{2} \mathrm{O}_{4}+{ }_{2} \mathrm{H}_{2} \mathrm{O}$, or 0.048675 gm. of $\mathrm{H}_{2} \mathrm{SO}_{4}$, etc. 


\section{CHAPTER III}

\section{VOLLMETRIC OR STANDARD SOLUTIONS}

ANy solution employed in volumetric analysis for the purpose of estimating the strength of substances, that is, any solution the chemical power or titer of which has been determined, is designated a standard or volumetric solution. Such a solution is said to be "titrated" (French titre $=$ title or power), and is sometimes also called a set solution or a standardized solution. It may be normal, decinormal, empirical, or of any strength, so long as its strength is known.

When volumetric analysis first came into use the solutions were so made that each substance to be estimated had its own special volumetric solution, and this was usually of such strength as to give the result in percentages. Thus a certain strength of standard acid was employed for potash, another for soda, and a third for ammonia, and in testing the acids, each had its own special standard alkali. These solutions were known as normal solutions; they are still to some extent in use, and since solutions now designated as normal are of an entirely different character, it is important that no misconception should exist when a normal solution is spoken of. Furthermore, it is to be regretted that some authors still define normal solutions as those having the molecular weights in grams of the active reagent in a liter, whereas the normal solutions as now more generally accepted are those having the hydrogen equivalent, weighed in grams, in a liter; in other words:

Normal solutions are such as contain in one liter (I000 cc.) the molecular weight of the active reagent expressed in grams and reduced to the valency corresponding to one atom of replaceable hydrogen or its equivalent.

Thus in univalent or monobasic compounds the full molecular weight in grams is contained in a liter of the normal solution.

Example. Hydrochloric acid, $\mathrm{HCl}$, having one replaceable hydrogen atom, its normal solution would contain the full molecular weight, 36.18 grams in a liter. A normal solution of potassium hydroxid should contain 55.74 grams of $\mathrm{KOH}$ in a liter, while that of sodium hydroxid should contain 39.76 grams of absolute $\mathrm{NaOH}$.

Normal solutions of bivalent or dibasic compounds, contain in 
Iooo cc., one-half of the molecular weight in grams. Thus, oxalic acid $\mathrm{H}_{2} \mathrm{C}_{2} \mathrm{O}_{4}+{ }_{2} \mathrm{H}_{2} \mathrm{O}=125.10$, having two replaceable $\mathrm{H}$ atoms, one-half of its molecular weight in grams $=62.55$ is contained in a liter of its normal solution. For the same reason a liter of a normal solution of sulphuric acid contains $\frac{97.35}{2}=48.675$ gms., and a liter of a normal solution of sodium carbonate $\mathrm{Na}_{2} \mathrm{CO}_{3}$ contains $\frac{105.31}{2}=52.655$ gms., while in the case of trivalent or tribasic compounds one-third of the molecular weight in grams is contained in a liter of the normal solution.

Thus it will be seen that one cc. of any normal acid solution will neutralize one cc. of any normal alkali solution, because one molecule of a univalent acid will neutralize one molecule of a univalent alkali, or a half a molecule of a bivalent alkali. This is shown by the equations

$$
\begin{aligned}
& \mathrm{HCl}+\mathrm{NaOH}=\mathrm{NaCl}+\mathrm{H}_{2} \mathrm{O}, \\
& 36.18 \quad 39.76 \\
& { }_{2} \mathrm{HCl}+\mathrm{Na}_{2} \mathrm{CO}_{3}=2 \mathrm{NaCl}+\mathrm{H}_{2} \mathrm{O}+\mathrm{CO}_{2} \text {. }
\end{aligned}
$$

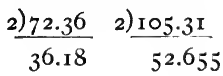

The value of a reagent as expressed by its hydrogen equivalent is readily seen in the case of acids and alkalies by reference to the chemical formula, but in such standard solutions as potassium dichromate, potassium permanganate, sodium thiosulphate, and others, the particular reaction in any given analysis must be taken into account in making a normal solution; for instance, when $\mathrm{K}_{2} \mathrm{Cr}_{2} \mathrm{O}_{7}$ is to be used as a precipitating agent its reaction is as follows:

$$
{ }_{2} \mathrm{Ba}\left(\mathrm{C}_{2} \mathrm{H}_{3} \mathrm{O}_{2}\right)_{2}+\mathrm{K}_{2} \mathrm{Cr}_{2} \mathrm{O}_{7}+\mathrm{H}_{2} \mathrm{O}={ }_{2} \mathrm{BaCrO}_{4}+{ }_{2} \mathrm{KC}_{2} \mathrm{H}_{3} \mathrm{O}_{2}+{ }_{2} \mathrm{HC}_{2} \mathrm{H}_{3} \mathrm{O}_{2}
$$

It is thus seen that one molecule of $\mathrm{K}_{2} \mathrm{Cr}_{2} \mathrm{O}_{7}$ will cause the precipitation of two atoms of barium in the form of chromate. Each atom of barium is chemically equivalent to two atoms of hydrogen; therefore one fourth of a molecule of $\mathrm{K}_{2} \mathrm{Cr}_{2} \mathrm{O}_{7}$ is equivalent to one atom of hydrogen. And therefore a normal solution of this salt, when used as a precipitating agent, must contain in one liter one fourth of its molecular weight in grams; $\frac{292.28}{4}=73.07 \mathrm{gms}$. 
If $\mathrm{K}_{2} \mathrm{Cr}_{2} \mathrm{O}_{7}$ is to be used as an oxidizing agent, the three atoms of oxygen which it yields for oxidizing purposes must be taken into account. When this salt oxidizes it splits up into $\mathrm{K}_{2} \mathrm{O}+\mathrm{Cr}_{2} \mathrm{O}_{3}+\mathrm{O}_{3}$. The three atoms of oxygen combine with and oxidize the salt acted upon, or they combine with an equivalent quantity of the hydrogen of an acid and liberate the acidulous part, which then combines with the salt. As the equations show,

$$
6 \mathrm{FeO}+\mathrm{K}_{2} \mathrm{Cr}_{2} \mathrm{O}_{7}=\mathrm{K}_{2} \mathrm{O}+\mathrm{Cr}_{2} \mathrm{O}_{3}+{ }_{3} \mathrm{Fe}_{2} \mathrm{O}_{3} \text { or }\left(\mathrm{Fe}_{6} \mathrm{O}_{9}\right) \text {; }
$$

$6 \mathrm{FeSO}_{4}+\mathrm{K}_{2} \mathrm{Cr}_{2} \mathrm{O}_{7}+{ }_{7} \mathrm{H}_{2} \mathrm{SO}_{4}=$

$$
\begin{aligned}
{ }_{7} \mathrm{H}_{2} \mathrm{O}+\mathrm{K}_{2} \mathrm{SO}_{4}+\mathrm{Cr}_{2}\left(\mathrm{SO}_{4}\right)_{3}+{ }_{3} \mathrm{Cr}_{2} \mathrm{O}_{7}\left(\mathrm{SO}_{4}\right)_{3} ; & { }_{3} \mathrm{SO}_{4}+{ }_{7} \mathrm{H}_{2} \mathrm{O}+\mathrm{K}_{2} \mathrm{SO}_{4}+\mathrm{Cr}_{2}\left(\mathrm{SO}_{4}\right)_{3} .
\end{aligned}
$$

Each of these atoms of oxygen are equivalent to two atoms of hydrogen. Thus $\mathrm{O}_{3}$ is equivalent to $\mathrm{H}_{6}$.

Hence a liter of a normal solution of $\mathrm{K}_{2} \mathrm{Cr}_{2} \mathrm{O}_{7}$, when used as an oxidizing agent, contains one sixth of its molecular weight in grams.

The same may be said of potassium permanganate when used as an oxidizing agent.

$2 \mathrm{KMnO}_{4}$ has five atoms of oxygen which are available for oxidizing purposes, and each of these is capable of taking two atoms of hydrogen from an acid and liberating the acidulous part. The hydrogen equivalent of this salt may therefore be said to be one tenth of the weight of $2 \mathrm{KMnO}_{4}$, and a normal solution of this salt contains $3 \mathrm{r} .396 \mathrm{gms}$. in a liter.

Sodium Thiosulphate (Hyposulphite), $\mathrm{Na}_{2} \mathrm{~S}_{2} \mathrm{O}_{3}$, is another instance. The molecule of this salt has two atoms of sodium, which have replaced two atoms of hydrogen of thiosulphuric acid. Thus it would seem that a normal solution should contain one half of the molecular weight in grams. But the particular reaction of this salt with iodin is taken into account.

One molecule reacts with one atom of iodin, as seen in the equation

$$
{ }_{2} \mathrm{Na}_{2} \mathrm{~S}_{2} \mathrm{O}_{3}+\mathrm{I}_{2}=2 \mathrm{NaI}+\mathrm{Na}_{2} \mathrm{~S}_{4} \mathrm{O}_{6}
$$

Since iodin is univalent, a molecule of the salt is equivalent to one atom of hydrogen.

A normal solution of this salt therefore contains the molecular weight in grams in a liter. 
Decinormal Solutions, $\frac{\mathrm{N}}{\mathrm{IO}}$, are one tenth the strength of normal solutions.

Centinormal Solutions, $\frac{\mathrm{N}}{\mathrm{IOO}}$, are one hundredth the strength of normal solutions.

Seminormal Solutions, $\frac{\mathrm{N}}{2}$, are one half the strength of normal solutions.

Double-normal Solutions, $\frac{N}{2}$, are twice the strength of the normal.

Empirical Solutions. A solution which does not contain an exact atomic proportion of the reagent may be employed as a volumetric solution after its strength or titer has been determined. Such a solution is said to be empirical, and solutions of this sort are very frequently used. To prepare solutions of exactly normal strength is a tedious process and often inconvenient. If the solution is approximately normal and its strength accurately determined, it may be used as it is. Again, in the case of standard solutions of the caustic alkalies, which, when not kept with all precautions, deteriorate readily through absorption of carbon dioxid from the air, as well as through their action upon the glass containers. To restore the titer of such solutions by the introduction of more of the alkali is an unnecessary waste of time, inasmuch as it is only necessary to determine its exact strength and then use it as it is. For instance, if an approximately normal solution of potassium hydroxid is on hand, its strength is determined as follows:

Ten cc. of an exactly normal oxalic or other acid solution are put into a beaker, and after diluting with a little water, and adding three or four drops of methyl orange, the "empirical potassium hydroxid solution is run in from a burette until the color of the solution changes from red to yellow; the number of cc. required is then noted.

Assuming that $10.4 \mathrm{cc}$. were required to neutralize the $10 \mathrm{cc}$. of normal acid. Hence its strength is $\frac{100}{104}$ or 0.9615 , that of a strictly normal solution, and the number of cc. used of it in any estimation must be multiplied by $\frac{100}{104}$ or 0.9615 and then by the normal factor for the substance analyzed.

It is a good plan to have the factor marked on the label of the 
bottle containing such an empirical solution. In this case it would be $\times 0.9615=$ normal.

Standard solutions for use in volumetric analysis are usually solutions of acids, bases, or salts, and in two cases elements, namely, iodin and bromin.

A standard solution of a base is usually used for the estimation of free acids.

A standard solution of an acid is usually used for the estimation of a free base, or the basic part of a salt, the acid of which can be completely expelled by the acid used in the standard solution. Example, carbonates.

A standard solution of a salt may be used as a precipitant, or it may be used as an oxidizing or reducing agent.

That part of the reagent in a standard solution which reacts with the substance under analysis is the active constituent of the solution. As $\mathrm{Ag}$ in $\mathrm{AgNO}_{3}$ is the active constituent of the standard solution of silver nitrate,

$$
\mathrm{AgNO}_{3}+\mathrm{NaCl}=\mathrm{AgCl}+\mathrm{NaNO}_{3},
$$

or $\mathrm{Cl}$ in $\mathrm{NaCl}$, is the active constituent of the standard solution of sodium chlorid.

If the reagent is a base, as $\mathrm{KOH}$, the basic part $\mathrm{K}$ is the active constituent. If the reagent is an acid, the active constituent is the acidulous part, as $\mathrm{SO}_{4}$ in $\mathrm{H}_{2} \mathrm{SO}_{4}$.

If the action of the reagent is oxidizing, then that part of the reagent which produces the oxidation is the active constituent.

The valence of an acid is shown by the number of replaceable hydrogen atoms it contains. Thus $\mathrm{HCl}$ is univalent, $\mathrm{H}_{2} \mathrm{SO}_{4}$ is bivalent, which means that a molecule of $\mathrm{HCl}$ is chemically equivalent to one atom of hydrogen, and a molecule of $\mathrm{H}_{2} \mathrm{SO}_{4}$ is chemically equivalent to two atoms of hydrogen.

The valence of a base is shown by the number of hydroxyls it is combined with. As, $\mathrm{KOH}$ is univalent, $\mathrm{Ca}(\mathrm{OH})_{2}$ is bivalent.

The valence of a salt is shown by the equivalent of base which has replaced the hydrogen of the corresponding acid.

Thus $\mathrm{NaCl}$, in which $\mathrm{Na}$ has replaced $\mathrm{H}$ of $\mathrm{HCl}$, is univalent.

$\mathrm{K}_{2} \mathrm{SO}_{4}$, in which $\mathrm{K}_{2}$ has replaced $\mathrm{H}_{2}$ of $\mathrm{H}_{2} \mathrm{SO}_{4}$, is bivalent.

In preparing standard solutions it must be remembered that most salts when dissolved in water reduce the temperature, while some, for instance sulphuric acid and alkaline hydroxids, cause a rise in tem- 
perature. Therefore the solutions should be allowed to stand a while so that they may attain the temperature of the air before being measured.

Furthermore, most salts cause a condensation in volume when dissolved in water; this must also be borne in mind.

It is always best to weigh out a little more of the salt than the amount required by theory; dissolve in water less than required for the finished solution, determine its strength, and then dilute to the proper strength. After dilution it should always be again carefully titrated, and proved normal.

To Titrate a substance means to test it volumetrically for the amount of pure substance it contains. The term is used in preference to "tested" or "analyzed," because these terms may relate to qualitative examinations as well as quantitative, whereas titration applies only to volumetric analysis.

Residual Titration, Re-titration, sometimes called Back Titration, consists in treating the substance under examination with standard solution in a quantity known to be in excess of that actually required; the excess (or residue) is then ascertained by residual titration with another standard solution.

Thus the quantity of the first solution which went into combination is found.

Example. Ammonium carbonate is treated first with $\frac{\mathrm{N}}{\mathrm{I}} \mathrm{H}_{2} \mathrm{SO}_{4}$ in excess, and the excess then found by titration with $\frac{\mathrm{N}}{\mathrm{I}} \mathrm{KOH}$.

The quantity of the $\frac{\mathrm{N}}{\mathrm{I}} \mathrm{KOH}$ used is then deducted from the quantity of $\frac{\mathrm{N}}{\mathrm{I}} \mathrm{H}_{2} \mathrm{SO}_{4}$ added, which gives the quantity of the latter which was neutralized by the ammonium carbonate. 


\section{CHAPTER IV}

\section{INDICATORS *}

IN volumetric analysis the substance to be analyzed in the state of solution is placed in a beaker and the standard solution is added from a burette until a certain reaction is produced. The exact moment when a sufficient quantity of the standard solution has been added is known by certain visible changes, which differ according to the substance analyzed and the standard solution used. When such a visible change occurs the "end reaction" is reached.

The end reaction manifests itself in various ways, as follows:

I. Cessation of precipitation.

2. First appearance of a precipitate.

3. Change of color.

In some cases, however, the addition of the standard solution to the substance under analysis does not produce either a precipitate or a change of color; in such cases we must resort to the use of an indicator.

An Indicator is a substance which is used in volumetric analysis, and which indicates by change of color, or some other visible effect, the exact point at which a given reaction is complete.

Generally the indicator is added to the substance under examination, but in a few cases it is used alongside, a drop of the substance being occasionally brought in contact with a drop of the indicator.

Thus in estimating an alkali with an acid-volumetric solution the alkali is shown to be completely neutralized when the litmus tincture which was added becomes faintly red or the phenolphthalein colorless. Again, when haloid salts are estimated with nitrate-of-silver solution, chromate of potassium is added as indicator. A white precipitate is produced as long as any halogen is present to combine with the silver, and when all is precipitated the chromate of potassium acts upon the silver nitrate, forming the red silver chromate, this color thus showing that all the halogen has been precipitated.

* For fuller information on indicators see A. I. Cohn's "Indicators and Test Papers," John Wiley \& Sons, New York. 


\section{THF IONIZATION OR DISSOCIATION THEORY}

When a soluble salt dissolves in water, its molecules split up or dissociate more or less completely into parts called ions. This behavior of substances, on going into solution, is known as electrolytic dissociation or ionization.

Ions are electrically charged atoms or groups of atoms and are of similar composition to the substances formed from the compound when an electric current is passed through the solution. The electropositive ions migrate to and collect around the negative pole (cathode) and hence are called cathions, while the electro-negative ions are called anions, because they concentrate around the positive pole or anode. The dissociation of a compound into its ions when an electric current is passed through its solution, although spoken of as electrolytic dissociation, is really not caused by the electric current, since the dissociation into ions occurs at once upon dissolving the substance in water and without the aid of an electric current, the action of the current being the transportation of the separated ions to the poles.

The dissociation of compounds into ions when dissolved in water is illustrated in the following list:

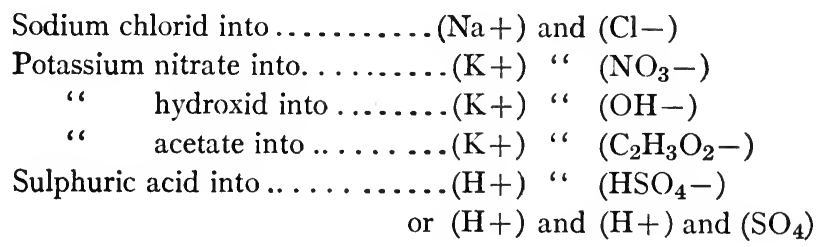

The extent of this dissociation depends upon the nature of the substance and the degree of dilution; the greater the dilution the more complete the dissociation. Furthermore, strong acids and bases dissociate readily, even in comparatively concentrated solutions, while the weaker acids and bases are more or less undissociated when dissolved, i.e., they are not readily split up into ions. Their salts, however, are immediately and completely ionzied. Therefore, upon neutralizing a weak acid or base an ionizable salt is formed. According to the theory of Arrhenius, the reactions of analytical chemistry are chiefly reactions between ions and not between atoms.

Strong acids, bases, and salts exist in solution not as molecules, but chiefly in the form of ions. The formation of silver chlorid by the 
reaction between silver nitrate and sodium chlorid takes place according to the following equation:

$$
\stackrel{+}{\mathrm{Ag}} / \overline{\mathrm{NO}} \mathrm{H}_{3} \mathrm{Aq} \cdot+\stackrel{+}{\mathrm{Na}} / \overrightarrow{\mathrm{Cl}} \mathrm{Aq} \cdot=\mathrm{AgCl}(\text { solid })+\stackrel{+}{\mathrm{Na}} / \overline{\mathrm{NO}} \mathrm{A}_{3} \mathrm{Aq} .
$$

The state of dissociation being denoted by the vertical line between the ions of the molecules.

This theory also explains why $\stackrel{+}{\mathrm{K}} / \mathrm{ClO}_{3}$ with $\stackrel{+}{\mathrm{Ag}} / \overline{\mathrm{NO}}$ dees not form $\mathrm{AgCl}$, in that the reaction involves the ion $\mathrm{ClO}_{3}$ and not the atom $\mathrm{Cl}$.

Theories of Indicators.-In connection with the use of indicators in neutralization analyses, the question as to the cause of the color changes is one of considerable interest.

Two distinct views are held. Of these the Ionization Theory of Ostwald has received almost universal acceptance. According to this theory the color changes are ascribed to a change in the indicator from a molecular to an ionic condition. As exemplified in the case of phenolphthalein the colorless molecule

$$
\mathrm{OCOC}_{6} \mathrm{H}_{4} \mathrm{C}-\left(\mathrm{C}_{6} \mathrm{H}_{4} \mathrm{OH}\right)_{2} \cdot . \cdot \cdot \cdot \cdot
$$

is dissociated into the red negative ion

$$
\mathrm{OCOC}_{6} \mathrm{H}_{4} \mathrm{C} \cdot\left(\mathrm{C}_{6} \mathrm{H}_{4} \mathrm{OH}\right) \mathrm{C}_{6} \mathrm{H}_{4} \overline{\mathrm{O}} \text {. . . . }
$$

In the other and less known view on this subject (the Chromophoric Theory),* the sensitiveness of the indicators and its color change is ascribed to a change in the constitution of the indicator, involving a chromophoric group, under the influence of hydrogen and hydroxyl ions. According to this view the color change is due (in the case of phenolphthalein), to a change of constitution from the colorless lactoid (I) with no chromophoric group, to the colored quinoid (III) with a quinone chromophore.

$$
\left(\mathrm{NaOOC} \cdot \mathrm{C}_{6} \mathrm{H}_{4}\right)\left(\mathrm{HOC}_{6} \mathrm{H}_{4}\right) \mathrm{C}: \mathrm{C}_{6} \mathrm{H}_{4}: \mathrm{O}, \text {. . . }
$$

and that the ionization of the sodium salt is merely a coincidence and not the cause of the color change.

Whicheres of these views is the correct one, remains for future investigit as to prove. That of Ostwald being most generally accepted at the present time is treated more fully below.

* See Julius Stieglitz, Jour. Am. Chem. Soc., XXV, I I 2 (1903). 
The Ionization Theory of Indicators.* The indicators used in alkalimetry and acidimetry are compounds of feeble acid or basic character, and hence not prone to dissociation in solution, but when neutralized, the salt formed ionizes the instant of its formation, the ions so liberated give rise to colors which differ from those of the original compounds.

Any feeble acid or base may be utilized as an indicator if its ions have a color different from that of the un-ionized compound. Strong acids or bases are not suited as indicators, because they ionize while in a free state on dilution, and thus give no color when neutralized.

A solution in which $\mathrm{H}$ ions predominate has an acid reaction, while one in which $\mathrm{OH}$ ions exist reàcts alkaline.

Phenolphthalcin is a feebly acid indicator, and in its undissociated state is colorless. It does not dissociate readily unless neutralized, but when sodium hydroxid is added to its solution, a sodium salt of phenolphthalein is formed which immediately ionizes and the ions liberated impart to the solution a brilliant red color. If now some acid is added the sodium salt is decomposed and the acid phenolphthalein again set free, and the solution becomes colorless.

If a few drops of phenolphthalein solution be added to an acid solution, ionization of the former is prevented by the presence of the stronger acid; if now some sodium hydroxid solution is added, the $\mathrm{OH}$ ions of the latter unite with the $\mathrm{H}$ ions of the acid, and when the acid is completely neutralized the first drop of excess of alkali unites with the phenolphthalein, forming a salt which immediately ionizes and produces the characteristic red color which shows the end of the reaction.

In the titration of a feeble acid the end point is often indistinct and is lacking in sharpness; this is because the indicator used has a greater tendency to ionize than the acid itself. In this case the $\mathrm{H}$ ions present just before the completion of the reaction are not in suffcient amount to fully retard the ionization of the indicator, and hence the latter dissociates partly before neutralization is complete and gives rise to an indefinite end-reaction. Therefore it is necessary when titrating a feeble acid that an indicator should be selected whose alkali salt ionizes with the production of a distinct color change, and whose tendency to ionize is less than that of the acid. Phenolphthalein is a

\footnotetext{
* See Ostwald's "Lehrbuch der Allgemeinen Chemie," r89r, and "Scientific Foundations of Analytical Chemistry," I900, also Walker's "Introduction to Physical Chemistry," 1899.
} 
suitable indicator in this case, provided a strong alkali be used for titrating.

Fixed alkalies readily yield ionizable salts with phenolphthalein, but ammonia does not. The latter being too weak a base to yield with so feeble an acid, a salt which can withstand the hydrolytic action of the water in dilute solutions, and as a consequence a larger excess of the ammonia must be used to overcome this. Thus is accounted for the imperfect color change of phenolphthalein when ammonia or its salts are present and why the color becomes visible only after a large excess of the alkali is added.

Paranitrophenol is also an acid indicator: it exists in solution in the form of undissociated colorless molecules, yet its electro-negative ion is intensely yellow in color. This compound has a slight tendency to dissociate in dilute solutions, but the presence of a trace of a stronger acid will overcome this tendency and the solution remains colorless. If an alkali is, however, added, a salt of paranitrophenol is formed which immediately ionizes and exhibits the intense yellow color of its liberated ion.

Other indicators exhibit a color in both the ionized and the nonionized state, but the colors in both conditions are different, as in the case of litmus lacmoid and methyl orange.

Methyl orange is both an acid and a base and will form ionizable salts with either acids or alkalies; its indicator characteristics are, however, due essentially to its basic character.

The salt which methyl orange forms with acids dissociates into red ions; this, upon the addition of an alkali, returns to its undissociated state, which is yellow. Because of its weak basic character its compound with acids is readily decomposed by alkalies, but it takes a strong acid or a relatively large quantity of a feeble acid to dissociate it in its non-ionized state, hence this indicator is very sensitive to alkalies, and much less so to acids.

With reference to the acid character of the indicator the explanation of its action, is that the non-ionized indicator is red, while its ion is yellow. Acids lessen its ionization and produce a red color, while alkalies produce a highly ionizable salt and hence a yellow color. When a weak but slightly ionizable acid is added to the methyl orange solution, the $\mathrm{H}^{\cdot}$ ions of the acid given up in excess of the amount required for neutralization are not sufficient to yield enough of a nonionizable salt to produce a decided red color, hence a large quantity of such a weak acid is required to give an acid indication. This would 
explain why methyl orange is not suitable as an indicator for weak acids, and why it is very sensitive to alkalies.

Litmus is an acid indicator which slightly dissociates in solution. Its non-ionized molecules are red, but its negative ions are blue. If it is added to an alkali, a salt is formed which at once ionizes and gives a blue color. If added to an acid, ionization is prevented and the red color of the non-ionized molecules appears.

From the above explanations it will be seen that indicators cannot be indiscriminately used, and that no one indicator will be suitable for every titration. Hence the indicator must be selected to suit each case. This selection is facilitated by reference to the classification of indicators, according to F. Glaser, 7tchr. f. a. Chem., I899, $273+$.

Group I. Indicators Forming Fairly Stable Salts. The members of this group comprise such indicators as are (I) of a strong acid character and which react readily with weak bases, or (2) of a feeble basic character and which require a strong acid to form a stable salt. Hence they will be found to be very sensitive to alkalies, and are useful in the titration of weak bases, as ammonia and the amine bases, as well as strong bases and acids. The indicators of this group are the following, arranged in the order of their sensitiveness towards alkalies:

(I) Iodeosin, Resazurin; (2) Tropæolin OO, Luteol; (3) Methyl and Ethyl Orange; (4) Congo Red; (5) Cochineal; (6) Lacmoid.

Group II. Indicators Possessing Faint Acid Properties and Yielding Salts whic hare Very Unstable. These are readily decomposed by relatively feeble acids, and are in consequence very sensitive towards acids, slightly so towards alkalies.

They are: (I) Rosolic acid; (2) Curcuma; (3) Phenolphthalein, Flavescin; (4) Alpha-naphtholbenzein.

Group III. Indicators Occupying a Place Midway between the Other Two Groups. They are somewhat stronger acids than those of Group II, but feebler than those of Group I.

They are fairly sensitive towards both acids and alkalies, but are more sensitive towards acids than those of Group I and less so towards alkalies. They are:

(I) Fluorescein, Phenacetolin; (2) Hæmatoxylin, Galleïn, Alizarin; (3) Litmus; (4) Paranitrophenol.

This division of indicators into groups, as above, facilitates the selection of an indicator suitable for the work in hand.

For instance, for titrating weak acids, a glance at the groups will show that the members of Group II are best adapted for this purpose. 
Again, weak bases will be best titrated by the indicators of Group I. Strong acids or bases may be titrated by means of any of the indicators.

The quantity of indicator taken in a titration is a matter of considerable moment. The smallest quantity which will produce a distinct color should be taken, but it is equally important that the quantity be not too small for the volume of liquid; for in high dilutions the hydrolytic action of water asserts itself, and intermediate tints will result, which interfere with the sharpness of the end color.

If too much of the indicator is used the sensitiveness is lessened, because acid or alkali must be added to convert the indicator into a salt, or when formed to decompose it; i.e., a minimum of excess of the titrating fluid would react with a small portion only of the indicator and intermediate tints would be produced, until sufficient of the titrating solution has been added to neutralize all of the indicator present. This is especially true when using centinormal solutions. 20 drops of litmus added to Io cc. of water require from Io to 14 drops of $\frac{\mathrm{N}}{\mathrm{IOO}}$ acid or alkali solution to produce a change of color. Thus the indicator itself takes up some of the standard solution, and hence the necessity for using as small a quantity of the indicator as possible; usually from 3 to 5 drops of the indicator may be taken to each 50 or 100 $\mathrm{cc}$. of the fluid titrated.

The degree of dilution of the substance titrated is also a matter of considerable moment. In very concentrated solutions ionization does not so readily occur, while too great a dilution diminishes the reactive ability of the ions because of their greater separation, and also because of the hydrolytic dissociation of water itself into $\mathrm{H}^{\cdot}$ and $\mathrm{OH}$ ions which react acid and alkaline respectively, and which brings about a premature dissociation of the indicator.

The Requirements of a Good Indicator, according to $\mathrm{H}$. A. Cripps, are:

I. The end reaction should be marked by a prominent change of color.

II. The smallest possible quantity of the reagent should be required to effect this change.

III. High tintorial power, which of itself assists in the fulfilment of the second requirement, less of the indicator being required.

IV. The change of color should not be affected by the impurities commonly present in the substance under examination, nor by the products of the reaction. 
In addition to these requirements it is a distinct advantage if the color reaction is equally decided in alcoholic as in aqueous liquids.

The following list includes the more reliable indicators in common use arranged alphabetically.

$$
\text { Alizarin: } \begin{aligned}
& \text { alkalies }=\text { Red } \\
& \text { acids }=\text { Yellow }
\end{aligned}
$$

This dye was first found in the roots of madder (Rubia tinctorium) but is now also obtained synthetically. A half per cent solution in alcohol is employed as an indicator.

\section{Azolitmin}

This is the color principle to which litmus owes its value as an indicator. Its extraction is explained under litmus. It is a highpriced article and is in consequence seldom used, the purified litmus tincture being preferred.

\section{Brazil Wood Test Solution: alkalies $=$ Purplish-red}

Boil 50 gms. of finely-cut Brazil-wood (the heart-wood of Peltophorum dubium (Sprengel) Briton, nat. ord. Leguminosa) with 250 (c. of water during half an hour, replacing from time to time. Allow the mixture to cool; strain; wash the contents of the strainer with water until roo cc. of strained liquid are obtained; add $25 \mathrm{cc}$. of alcohol and filter.

This indicator is especially serviceable for the titration of alkaloids, but it is useless in the presence of sulphurous acid, sulphites, or sulphids, as these substances decolorize the solution.

$$
\text { Cochineal Test Solution: } \begin{aligned}
& \text { alkalies }=\text { Violet } \\
& \text { acids }=\text { Yellowish-red }
\end{aligned}
$$

Cochineal is the dried female insect, Pseudococcus cacti Linné.

The test solution is made by macerating $3 \mathrm{gms}$. of unbroken cochineal for four days in $250 \mathrm{cc}$. of a mixture of I part of alcohol and 3 parts of water, by volume.

It is a very valuable indicator, especially for carbonates of the alkalies and alkaline earths, because it is not affected by $\mathrm{CO}_{2}$. It is also useful for titrating alkaloids, alkalies, alkali earths, ammonia, and inorganic acids, but is useless for most organic acids.

$$
\text { Congo Red: } \begin{aligned}
& \text { alkalies }=\text { Red } \\
& \text { acids }=\text { Blue }
\end{aligned}
$$

Congo red is a sodium tetrazo-diphenyl-naphtionate. It occurs in commerce in the form of lumps of a reddish-brown color. It is readily 
soluble in both water and alcohol. Its solution is exceedingly sensitive to free acids even in the presence of acid salts, and is likewise very sensitive to free carbonic and acetic acids. It may be employed for estimating free mineral acids, in the presence of most organic acids.

The test solution contains I per cent of the dye and ro per cent of alcohol.

\section{Corallin: $\begin{aligned} & \text { alkalies }=\text { Red } \\ & \text { acids }=\text { Yellow }\end{aligned}$}

This is a coloring-matter obtained from coal-tar and contains rosolic and para-rosolic acids. The test solution is made by dissolving one gram in ro cc. of alcohol and adding water to make roo cc. It is recommended for titrating free ammonia.

\section{Eosin : $\quad \begin{aligned} & \text { alkalies }=\text { Green furescence } \\ & \text { acids }=\text { Yellow }\end{aligned}$}

Tetra-bromo-resorcin-phthalein.-This is made by adding bromin to a solution of fluorescein in glacial acetic acid. Crystals gradualiy separate, which may be purified by conversion into a potassium salt and precipitated with an acid.

The composition of this substance is $\mathrm{K}_{2} \mathrm{C}_{20} \mathrm{H}_{6} \mathrm{Br}_{4} \mathrm{O}_{3}$.

\section{Gallein: alkalies $=$ Bright red}

Anthracene violet or pyrogallol-phthalein was proposed by $\mathbf{M}$. Dechan for use as an indicator.

It is prepared by heating a mixture of one part of phthalic anhydrid and two parts of pyrogallol, and finally recrystallizing in a similar way to phenolphthalein.

It is described as a dark reddish crystalline solid, possessing a greenish luster. It is nearly insoluble in water, but readily soluble in alcohol. In commerce it is frequently iound as a paste, mixed with water.

It forms a red coloration with alkalies, which is changed to yellowish brown on addition to an acid in excess.

It is said to be more delicate towards alkalies than phenolphthalein, and may be used in its stead for titrating many of the alkaloids. It may be used in the presence of ammonia or its salts. It indicates sharply with the organic acids. A solution in rectified spirit I-I000 is generally employed.

\section{Hamatoxylin: $\begin{aligned} & \text { alkalirr }=\text { V'iolet } \\ & \text { acids: Yellow ts Orange }\end{aligned}$}

A peculiar principle obtained from 1ngwood, having the composition $\mathrm{C}_{16} \mathrm{H}_{14} \mathrm{O}_{6}$, and crystallizing with one or three molecules of 
water. It is an efflorescent yellowish-rose colored substance, but when pure is said to be colorless, reddening on exposure to light.

It is soluble in hot water or alcohol. Its alcoholic solution is largely used as an indicator in the titration of alkaloids, for which it is considered the indicator par excellence.

The solution is prepared by dissolving one gram of the wellcrystallized material in $100 \mathrm{cc}$. of alcohol. In titrating use about three drops of this solution.

Its color reaction with carbonates and bicarbonates is interesting. When added to a solution of alkali-bicarbonate the reaction requires many seconds, and results in a gradually deepening carmine-red which is permanent, while in the case of soluble carbonates the reaction is instantaneous, a purple-red which changes rapidly through cherry, eosin-red to orange. The reaction with ammonium carbonate is similar to that with bicarbonate.

$$
\text { Iodeosin : } \begin{aligned}
& \text { alkalies }=\text { Rose-red } \\
& \text { acids }=\text { Yellow }
\end{aligned}
$$

Tetra-iodo-fluorescein Erythrosin B. This indicator is useful for minute quantities of alkali, as for instance, such as may be dissolved out from glass on contact with water. It is used in connection with highly dilute standard solutions only. The iodeosin solution is made by dissolving $.002 \mathrm{gm}$. of the indicator in $1000 \mathrm{cc}$. of pure ether. Titration with this indicator is carried out by introducing 50 to $100 \mathrm{cc}$. of the liquid to be titrated into a stoppered bottle and adding to to $20 \mathrm{cc}$. of the ethereal indicator solution and setting aside after shaking. The ethereal layer as well as the fluid will be colorless if the latter is neutral, but if traces of alkali are present the rose-red tint passes into the aqueous liquid leaving the ether colorless. If the fluid is acid, the ethereal layer is yellow. If preferred, 4 or 5 drops of a $1-10,000$ aqueous solution of the indicator may be added to the liquid, and ether then added. Iodeosin is particularly useful in titration of alkaloids, especially those of weak basicity, as emetine, atropine, morphine, etc.

\section{Lacmoid: $\quad \begin{aligned} & \text { alkalies }=\text { Blue } \\ & \text { acids }=\text { Red }\end{aligned}$}

Lacmoid is somewhat allied to litmus, but differs from it in many respects. It is a product of resorcin, and may be prepared by heating gradually to $110^{\circ} \mathrm{C}$. a mixture of 100 parts of resorcin, 5 parts of sodium nitrite, and 5 parts of water. After the violent reaction moderates it is heated to $\mathrm{I} 20^{\circ} \mathrm{C}$. until ammonia ceases to be evolved. 
The residue is then dissolved in warm water and the lacmoid precipitated therefrom by $\mathrm{HCl}$; the free acid is then removed by washing and the residue dried.

This constitutes the commercial lacmoid, which is not in a sufficiently pure state to be used as an indicator; its purification is effected according to Förster by treating the powder with boiling water, and acidulating the resulting blue solution with hydrochloric acid. After a few hours the precipitate is collected and washed with a little cold water and carefully dried, or it is dissolved in alcohol and the solution evaporated. Even after careful purification, lacmoid solution may still exhibit a violet tinge, which is a disturbing factor in accurate work. To remedy this defect, Förster suggests the addition of $5 \mathrm{gms}$. of beta-naphthol green to $3 \mathrm{gms}$. of purified lacmoid dissolved in $700 \mathrm{cc}$. of water and $300 \mathrm{cc}$. of alcohol. A sample of lacmoid which is only sparingly soluble in water should be rejected. The purer the article the more readily does it dissolve in water.

Lacmoid paper is prepared by dipping slips of calendered unsized paper into the blue or red solution and drying them.

Lacmoid is slightly affected by carbonic-acid gas. It may be used cold for the alkaline and earthy hydroxids, arsenites, and borates, and the mineral acids. The carbonates and bicarbonates of the alkalies and alkali earths are titrated hot with this indicator.

Many of the metallic salts, such as the sulphates and chlorids of iron, copper, and zinc, which are more or less acid to litmus, are neutral to lacmoid; therefore free acids in such solutions may be estimated by its aid.

Lacmoid paper reacts alkaline with the chromates of potassium or sodium, but neutral with the dichromates, so that a mixture of the two or of chromic acid and dichromate may be titrated by its aid.

\section{Litmus (Lacmus): $\begin{aligned} & \text { alkalies }=\text { Blue, } \\ & \text { acids }=\text { Red. }\end{aligned}$}

A pigment obtained by the fermentation of certain lichens, principally from Roccella tinctoria and $R$. fuciformis, but also from other species of lichen.

It occurs in commerce in small, friable, light cakes or cubes, of a violet color.

The coloring principles of litmus are azolitmin, erythrolitmin, and erythrolein. The first, which is the most important, is soluble in water, but insoluble in alcohol. The other two are readily soluble in alcohol, but only sparingly soluble in water. 
The U. S. P. process for making litmus test solution consists in exhausting coarsely powdered litmus with boiling alcohol.

The residue is then digested with about an equal weight of cold water so as to dissolve the excess of alkali present.

The blue solution thus obtained, after being acidulated, may be used to make red litmus-paper. Finally, the residue is extracted with abouit five times its weight of boiling water and the solution filtered.

The filtrate is preserved as test solution in wide-mouthed bottles, stoppered with loose plugs of cotton to exclude dust, but to admit air.

When kept in closed vessels litmus solution gradually loses color, but this returns upon exposure to air and consequent absorption of oxygen.

The fermentation to which the loss of color is due may be prevented by saturating the solution with $\mathrm{NaCl}$ or by the addition of thymol or phenol.

The British Pharmacopœia recommends to boil the litmus in powder with three successive portions of rectified spirit, and then to digest the residue in distilled water and filter, the object of these steps in the process being to get rid of the greater portion of erythrolitmin and erythrolein, which are soluble in alcohol. Then by treating the residue with water a larger proportion of azolitmin is dissolved, and the solution is contaminated with very little of the other two principles.

Litmus test solution should be of such strength that 3 drops added to $50 \mathrm{cc}$. of water will impart to the latter a distinct color. If one drop of $\frac{\mathrm{N}}{\mathrm{IO}}$ acid or alkali solution be added to this the color should change to red or blue.

Litmus may be used in a very large number of titrations. It is of value in the titration of most mineral acids and of a few organic acids, e.g., benzoic and oxalic. It is also useful in the titration of alkaline hydroxids when the latter are free from carbonates.

But for carbonates, bicarbonates, etc., a reliable end reaction can only be obtained by boiling the solution during the titration, in order to dispel the liberated $\mathrm{CO}_{2}$.

Free $\mathrm{CO}_{2}$ has an acid reaction with litmus, and interferes very much with the finding of the end reaction.

Litmus may be used for ammonia and for borax. It is of no use for phosphoric or arsenic acid, nor for sulphurous acid, phosphates, or arsenates, because the change of tint is too gradual. 
It is unsatisfactory in titrating many organic acids, e.g., tartaric and citric, but may be used for oxalic or benzoic, as before stated.

Sometimes it is required to perform a titration with litmus at night. Gas or lamp light is not adapted for showing the reaction satisfactorily, but by using a monochromatic light, such as the sodium flame, a very sharp line of demarcation may be found.

The operation should be conducted in a dark room, using a piéce of platinum foil sprinkled with salt or a piece of pumice-stone saturated with a solution of salt, heated in a Bunsen flame.

The red color then appears perfectly colorless, while the blue appears like a mixture of ink and water.

$$
\text { Luteol: } \begin{aligned}
& \text { alkalies }=\text { Yellow, } \\
& \text { acids }=\text { Colorless }
\end{aligned}
$$

Chemically it is an oxy-chlor-diphenyl-quinoxalin. It was suggested as an indicator by Autenrieth.

The solution for the purposes of an indicator is prepared by dissolving I part in roo parts of alcohol. Of this, four drops are sufficient for $50 \mathrm{cc}$. of fluid to be titrated.

In sensitiveness, luteol exceeds both litmus and phenolphthalein. It is more sensitive toward ammonia than Nessler's solution. Io cc. of a solution containing one drop of ammonia water per liter, is colored yellow immediately upon adding luteol, whereas with Nessler's solution it takes quite some time before a reaction is obtained.

\section{Methyl Orange: $\begin{aligned} & \text { alkalies }=Y \text { ellow, } \\ & \text { acids }=\text { Red. }\end{aligned}$}

Poirrier's Orange III, Tropaolin D, Helianthin, Mandarin-orange, Para-sulpho-benzeneazo-dimethylanilin.

This is prepared by the action of diazo-sulphanilic acid upon dimethylanilin; the acid so formed is converted into a sodium or ammonium salt, purified by reprecipitation with $\mathrm{HCl}$, and again converted into a sodium or ammonium salt. If prepared carefully and from the purest materials, it is a bright orange-red powder, perfectly soluble in water and slightly in alcohol; but it is often found in commerce as a dull orange-brown powder, often not completely soluble in water. Many conflicting statements have been made by operators as to the value of methyl orange as an indicator, which have tended to bring this indicator into disrepute.

Sutton has examined many specimens, but has not found any in which the impurities sensibly affected its delicate action. He claims 
that the common error is the use of too much indicator, and that some eyes are more sensitive to a change of tint than others.

Methyl orange is no doubt a very good indicator, but practice with it must be had in order to obtain good results. The author has found one sample which had a beautiful orange color, but which was absolutely useless as an indicator.

A. H. Allen describes as follows the characters and tests of a good article:

I. Aqueous solution, not precipitated by alkalies. (Orange I becomes red-brown; orange II brownish-red.)

2. Hot concentrated aqueous solution yields with $\mathrm{HCl}$ microscopic acicular crystals of the free sulphonic acid, soon changing to small lustous plates or prisms having a violet reflection. (Orange I gives yellow-brown color or flocculent precipitate; orange II brown-yellow precipitate.)

3. Dissolves in concentrated $\mathrm{H}_{2} \mathrm{SO}_{4}$ with a reddish or yellowishbrown color, which on dilution becomes fine red.

4. $\mathrm{BaCl}_{2}$ yields a precipitate.

5. $\mathrm{CaCl}_{2}$ yields no precipitate. (Orange I gives a red precipitate.)

6. $\mathrm{Pb}\left(\mathrm{C}_{2} \mathrm{H}_{3} \mathrm{O}_{2}\right)_{2}$ yields an orange-yellow precipitate.

7. $\mathrm{MgSO}_{4}$ in dilute solutions precipitates the coloring matter in microscopic crystals.

Methyl-orange T. S. is made by dissolving I gm. of methyl orange, in $1000 \mathrm{cc}$. of water. Add to it carefully diluted sulphuric acid in drops until the liquid turns red and just ceases to be transparent. Then filter.

The great value of this indicator consists in the fact that it is not affected by carbonic-acid gas, sulphureted hydrogen, or silicic, oleic, stearic, and many other acids.

It answers well for ammonia, but it is useless for most of the organic acids. Phosphoric and arsenic acids are rendered neutral to methyl orange when only one third of the acid has combined with the base, the end reaction being well defined. (Phenolphthalein indicates neutrality when two thirds of acid are combined.)

This indicator should not be employed when titrating with standard solutions which are weaker than decinormal, nor should it be used in any hot titrations, nor in excessive quantities. Two drops are sufficient for $50 \mathrm{cc}$. of the fluid to be titrated, or just enough to give it a faint tint. 


\section{Phenacetolin: alkali $\left\{\begin{aligned} \text { carbonates } & =\text { Red } \\ \text { hydroxids } & =\text { Yellow } \\ & =\text { Yellow }\end{aligned}\right.$}

This indicator is prepared by heating together for several hours equal molecular weights of phenol, glacial acetic acid, and sulphuric acid in a vessel provided with a reflux condenser. The product is then thoroughly washed with water to remove excess of acid and dried for use. It is only very slightly soluble in water, but dissolves readily in alcohol, forming a greenish-brown solution.

The solution yields with alkali hydroxids a scarcely perceptible pale yellow, but with normal carbonates of the alkalies, sulphids, and with ammonia it gives a decided pink color; with bicarbonates a more intense pink, while with acids a golden yellow.

This indicator is useful for estimating the amount of alkali or alkali earth hydroxids in the presence of carbonate, unless the hydroxid is present in too small quantity. Ammonia must not be present. The titration is carried out by adding the acid until a faint red color appears; this indicates that the alkali hydroxid or the lime has been neutralized. The further addition of the acid intensifies the red until the carbonate present in the mixture is neutralized, when a golden yellow color appears. The proportion of alkali hydroxid must be far in excess of the carbonate in order to obtain reliable results; furthermore, considerable practice is required in the use of this indicator in order to accustom the eve to the color changes.

A convenient strength of solution is $15: 00$.

$$
\text { - Phenolphthalein }\left(\mathrm{C}_{20} \mathrm{H}_{14} \mathrm{O}_{4}\right): \begin{aligned}
& \text { alkalies }=\text { acids }=\text { Rolorless } \\
& \text { acide }
\end{aligned}
$$

Preparation.-Five parts of phthalic anhydrid $\left(\mathrm{C}_{8} \mathrm{H}_{4} \mathrm{O}_{3}\right)$, ro parts of phenol $\left(\mathrm{C}_{6} \mathrm{H}_{6} \mathrm{OH}\right)$, and 4 parts of $\mathrm{H}_{2} \mathrm{SO}_{4}$ are heated together at $120^{\circ}$ to $130^{\circ} \mathrm{C}$. for several hours. The product is then boiled with water, and the residue, which consists of impure phenolphthalein, is dissolved in dilute soda solution and filtered. By neutralizing this solution the phenolphthalein is precipitated and may be purified by crystallization from alcohol; or the alcoholic solution may be boiled with animal charcoal, filtered, and the phenolphthalein reprecipitated by boiling water.

Uses.-Phenolphthalein is a very valuable indicator; it is extremely sensitive, and exhibits a well-marked and prompt change from colorless to pink, and vice versa. A few drops of the solution of the indicator show no color in neutral or acid liquids, but the faintest excess of alkali produces a sudden change to red. 
It may be employed in the titration of mineral and organic acids and most alkalies, but it is not suited for the titration of ammonia or its salts. It is very sensitive to $\mathrm{CO}_{2}$, and therefore in estimating carbonates the liquid must be boiled, as with litmus. It is inapplicable for borax, except in the presence of glycerin, because the color gradually fades away as the acid is added. One great advantage which phenolphthalein possesses is that its indications may be clearly read in many colored liquids; another is that it may be used in alcoholic liquids or in mixtures of alcohol and ether, and therefore many organic acids which are insoluble in water may be accurately titrated by its help.
of then ihalein T. S. is a one per cent solution in diluted alcohol.

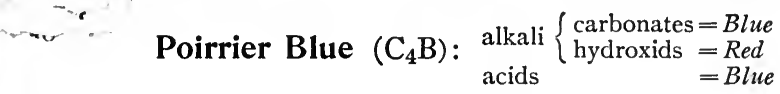

This indicator, which is closely allied to Gentian Blue in properties, is obtained by the action of sulphuric acid on triphenylrosanilin. It is a blue powder with a coppery luster. It dissolves in water and in alcohol, yielding blue solutions. $\mathrm{KOH}$ and $\mathrm{NaOH}$ change the color to red, but ammonia decolorizes it It is employed as an indicator in aqueous solution $\mathrm{r}: 500$. This indicator is exceedingly sensitive to acids. Borax and boric acid give a blue color; in the titration of boric acid the red color does not appear until the acid is completely neutralized. This indicator is recommended for the titration of hydrocyanic acid, toward which it is especially sensitive, the alkaline cyanids are alkaline in reaction to most indicators, but $\mathrm{C}_{4} \mathrm{~B}$ does not show an alkaline reaction until the $\mathrm{HCN}$ is completely neutralized, and a minute excess of the alkali hydroxid has been added. $\mathrm{C}_{4} \mathrm{~B}$ is of the character of a weak acid and its salts are very unstable; they are decomposed by water alone when in very great dilution, therefore the indicator must be used in sufficient quantity. The addition of a few drops of alcohol facilitates the color change, which is indeed a very sharp one.

Potassium-chromate Test-solution is used in the titration of haloid salts with silver-nitrate solution. It indicates that all the halogen has combined with the silver by producing a red-colored precipitate (silver chromate).

Potassium-ferricyanid Test-solution is used in the estimation of ferrous salts with potassium-dichromate solution. It gives a blue color to a drop of the solution on a white slab as long as any iron salt is present which has not been oxidized to ferric. 


\section{Resazurin: $\begin{aligned} & \text { alkalies }=\text { Blue } \\ & \text { acids }=\text { Red }\end{aligned}$}

This is a new indicator for alkalimetry, proposed by Crismer. It is prepared as follows: Dissolve $4 \mathrm{gms}$. of resorcin in $300 \mathrm{cc}$. of anhydrous ether and add 40 to 45 drops of nitric acid (sp. gr.1.25) saturated with nitrous anhydrid. Allow the mixture to stand in a cold place for two days, whereupon a deposit of blackish crystals, having a reddish-brown reflection, will be formed in the bottom of the vessel. The supernatant clear red liquid is decanted and the crystals washed with ether until the washings show a blue color with ammonia-water.

Resazurin $\left(\mathrm{C}_{12} \mathrm{H}_{7} \mathrm{NO}_{4}\right)$ is slightly soluble in water, mot, so in alcohol and freely soluble in acetic ether. It produces a bl. tion with water, alkalies, and alkali carbonates, which are $\ell$ alkali ol upon the addition of a slight excess of acid. To use this incularu' th alkalimetry, Crismer recommends the following solution: Resazurin $0.2 \mathrm{gm}$. dissolved in $40 \mathrm{cc}$. of $\frac{\mathrm{N}}{\mathrm{IO}}$ ammonia solution, and made up to $1000 \mathrm{cc}$. with distilled water.

This is deep blue in color and keeps well. Two or three drops are sufficient to color $200 \mathrm{cc}$. of liquid.

This indicator is not suited for the titration of nitric acid or monobasic organic acids, and it is not very sensitive to carbonic acid. It is, however, extremely sensitive to alkalies. If the solution is acidulated to a rose-red color and heated in a white glass flask, the solution will turn blue through the alkaline reaction of the dissolved glass before the boiling-point is reached.

This indicator is especially useful for borax.

$$
\text { Rosolic Acid }\left(\mathrm{C}_{20} \mathrm{H}_{14} \mathrm{O}_{3}\right): \begin{aligned}
& \text { alkalies }=\text { Red } \\
& \text { acids }=\text { Yellow }
\end{aligned}
$$

This compound is also called Aurin and Coralline, and is prepared as follows:

A mixture of phenol and sulphuric acid is placed upon a waterbath, and oxalic acid gradually added, waiting each time till the evolution of gas ceases, and using less oxalic acid than is required to attack all the phenol.

In this process the oxalic acid is decomposed into $\mathrm{CO}, \mathrm{CO}_{2}$, and $\mathrm{H}_{2} \mathrm{O}$. The CO immerliately reacts with the phenol and forms rosolic acid, as the following equation shows:

$$
{ }_{3} \mathrm{C}_{6} \mathrm{H}_{5} \mathrm{OH}+{ }_{2} \mathrm{CO}=\mathrm{C}_{20} \mathrm{H}_{14} \mathrm{O}_{3}+{ }_{2} \mathrm{H}_{2} \mathrm{O} .
$$


Commercial rosolic acid is a mixture of several derivatives, among them the above, methylaurin $\mathrm{C}_{20} \mathrm{H}_{16} \mathrm{O}_{3}$ and others. Commercial pœonin (also known as Aurin R.) [chiefly $\mathrm{C}_{19} \mathrm{H}_{14} \mathrm{O}_{3}$ ] may be used in place of rosolic acid.

Rosolic acid is soluble in diluted alcohol. Its color is pale yellow, not changed by acid, but turns violet red with alkalies.

It is an excellent indicator for the mineral acids and strong bases, weak ammoniacal solutions, oxalic acid, and other organic acids, except acetic.

The test solution is made by dissolving I gm. of the commercial rosoli - id in Io cc. of diluted alcohol, and then adding enough water to : User $100 \mathrm{cc}$.

Tropæolin (OO): $\begin{aligned} & \text { alkalies }=\text { Yellow } \\ & \text { acids }=\text { Yellowish-red }\end{aligned}$

This is used in the form of a solution containing $.5 \mathrm{gm}$. to rooo cc.

Turmeric Tincture. Digest any convenient quantity of ground curcuma-root (from Curcuma longa Linné, nat. ord. Scitaminea) repeatedly with small quantities of water, and throw this liquid away. Then digest the dried residue for several days with six times its weight of alcohol, and filter.

Turmeric Paper. Impregnate white, unsized paper with the tincture and dry it.

The color principle of turmeric is curcumin. It is seldom used in volumetric analysis, except in the form of turmeric paper. For highcolored solutions curcumin gives no reaction with acids, but becomes brown with alkalies. There is another color principle in turmeric besides curcumin, which is, however, useless in that it is indifferent to alkalies; it is soluble in water, and is extracted by digestion with water, after which the curcumin is dissolved out with alcohol.

Turmeric paper is especially useful, because of its peculiar reaction with boric acid, with which it develops a brown color after drying, and which color, when touched with caustic soda solution, is changed to dark green.

Starch Solution. This is useful as in indicator in iodometric estimations. It is sensitive to the extent of detecting iodin in solutions containing less than $\mathrm{I}$ in 2,000,000. It gives with iodin a darkblue color. Starch solution decomposes readily and should, therefore, be freshly prepared when needed. A decomposed solution gives a brownish-red or greenish color instead of a blue. If too much of the indicator is used the color produced is nearly black. About I cc. should 
be employed ordinarily in titrations, and the operation should be conducted at the ordinary temperature, as the application of heat destroys the color. It is further recommended that the starch indicator be introduced only toward the end of the reaction. The solution is prepared by mixing I gm. of potato starch with $10 \mathrm{cc}$. of cold water and then adding sufficient boiling water, with constant stirring to make $200 \mathrm{cc}$. of a thin jelly-like solution. The addition of two or three drops of mercuric chlorid T. S. will tend to prevent decomposition, though it is always best to prepare the solution when wanted. Arrowroot starch may be used instead of potato starch.

\section{A GUIDE FOR THE SELECTION OF INDICATORS.}

Hydroxids and Carbonates of the Alkalies and Alkali Earths. alkalthe titration of carbonates with standard acid solutions there is an evolution of $\mathrm{CO}_{2}$ which reacts acid with most indicators. Those indicators which are so affected cannot be employed in the presence of carbonates except in hot titrations, the $\mathrm{CO}_{2}$ being dispelled by boiling.

Those which are not so affected may be used in cold titrations and are therefore preferred, as heat affects many of the indicators. Alkali hydroxids almost invariably contain small amounts of carbonate, hence the selection of an indicator for either hydroxids or carbonates is to be made from the same list.

Indicators not affected by $\mathrm{CO}_{2}$.

(Cold Titrations).

\section{Methyl Orange:}

Useful for hydroxids of alkali and alkali earths and carbonates of alkalies. Large quantities of neutral salts must be absent; standard oxalic acid is useless.

Phenacetolin:

Large quantities of $\mathrm{NH}_{3}$ salts must not be present.

Congo Red:

Large quantities of salts of fixed alkalies $\mathrm{NH}_{3}, \mathrm{Ca}, \mathrm{Ba}$, and $\mathrm{Mg}$ must not be present.

Cochineal:

Not reliable in presence of salts of $\mathrm{Fe}, \mathrm{Al}$, or $\mathrm{Cu}$.

Iodeosin:

Useful, especially for minute quantities of alkali.

Galleïn.

\section{Indicators affected by $\mathrm{CO}_{2}$.} (Hot Titrations).

Litmus.

Phenolphthalein:

Useless if $\mathrm{NH}_{3}$ or its salts are present.

Lacmoid:

Used in residual titrations for car bonate.

Luteol.

Resazurin.

Rosolic Acid. 


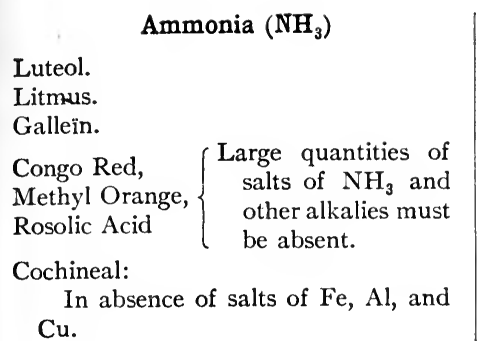

\section{Inorganic Acids}

$\left(\mathrm{H}_{2} \mathrm{SO}_{4}, \mathrm{HCl}, \mathrm{HNO}_{3}\right)$.

Phenolphthalein:

Useless in presence of $\mathrm{H}_{3} \mathrm{AsO}_{3}$.

Congo Red:

Not reliable for $\mathrm{HNO}_{2}$ or $\mathrm{H}_{2} \mathrm{SO}_{4}$.

Litmus.

\section{Lacmoid:}

Not reliable for $\mathrm{HNO}_{3}, \mathrm{HNO}_{2}$ or $\mathrm{H}_{2} \mathrm{~S}$.

Rosolic Acid.

Methyl Orange:

Not reliable for $\mathrm{HNO}_{2}$.

Cochineal.

Resazurin:

Not reliable for $\mathrm{HNO}_{3}$.

Luteol.

Galleïn.

\section{Ammonium Carbonate}

(Residual Titration).

Litmus.

Phenacetolin.

Methyl Orange.

Rosolic Acid

Phenolphthalein.

Galleïn.

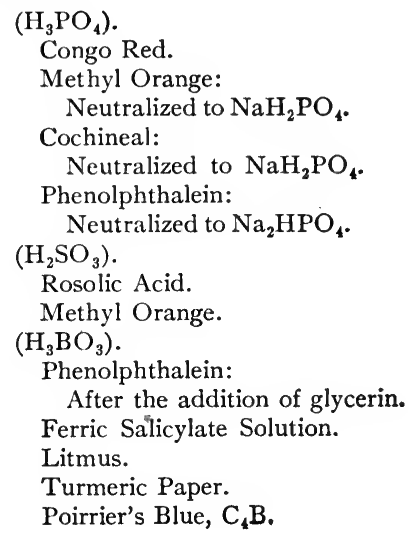

\section{Organic Acids}

Phenolphthalein (all).

Rosolic Acid (except Acetic, Citric and Tartaric).

Congo Red (Acetic Acid only).

Galleïn.

Litmus (Oxalic and Benzoic only). 


\section{CHAPTER V}

\section{APPARATUS USED IN VOLUMETRIC ANALYSES}

The Burette is a graduated glass tube which holds from 25 to roo cc. and is graduated in fifths or tenths of a cc., and provided at the lower end with a rubber tube and pinch-cock. The use of this instrument is to accurately measure quantities of standard solutions

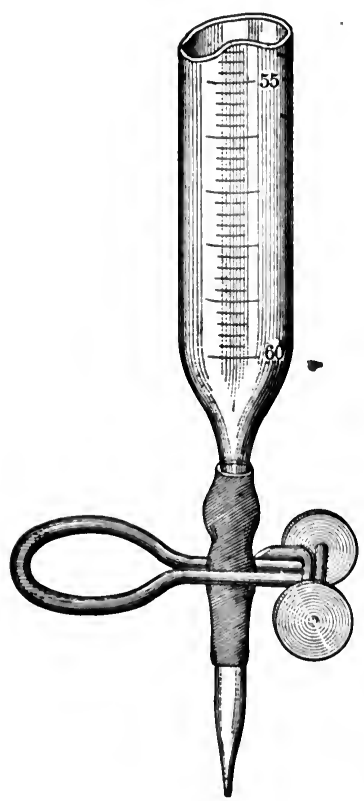

FIG. 1. used in an analysis. It is in an upright position when in use, and the flow of the solution can be regulated so as to run out in a stream or flow in drops by pressing the pinch-cock between the thumb and forefinger. The quantity of solution used can be read from the graduation on the outside of the tube. This is the simplest and most common form of burette, and is known as Mohr's (Fig. I).

The use of the pinch-cock in Mohr's burette may be dispensed with by introducing into the rubber tube a small piece of glass rod, which must not fit too tightly. By firmly squeezing the rubber tube surrounding the glass rod a small canal is opened, through which the liquid escapes. A very delicate action can in this way be obtained, and the flow of the liquid is completely under the control of the operator. (See Fig. 2.)

The greatest drawback to this burette is that it cannot be used for permanganate or other solutions that act upon the rubber.

This defect can be overcome by the use of a burette having a glass stop-cock in place of the rubber tubing and pinch-cock. This form has the additional advantage of being capable of delivering the solution in drops while both hands of the operator are disengaged (Fig. 3). 
Another good arrangement is that in which the tap is placed in an oblique position, so that it will not easily drop out of place (Fig. 4).

These glass stop-cock burettes should be emptied and washed immediately after use, especially if soda or potassa solution has been used; for these act upon the glass and often cause the stopper to stick so firmly that it cannot be turned or removed without danger of breaking the instrument.

The most satisfactory form of glass stop-cock is that shown in

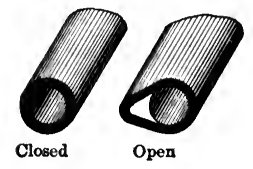

FIG. 2 .

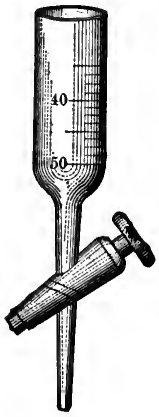

FIG. 4.

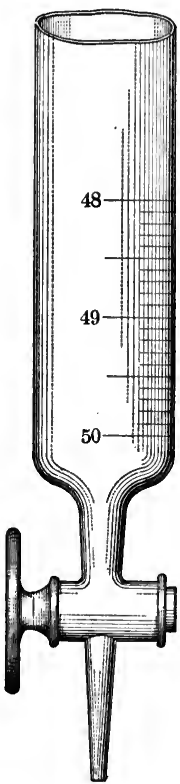

FIG. 3 .

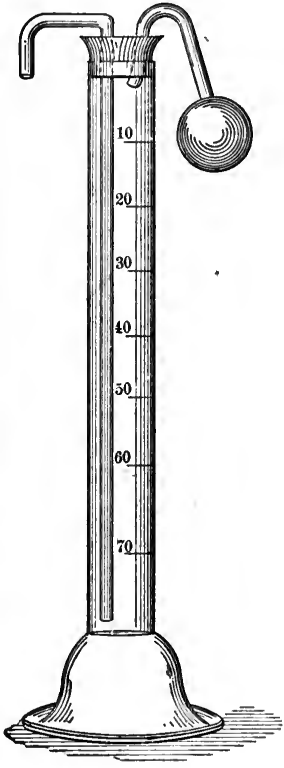

FIG. 5.

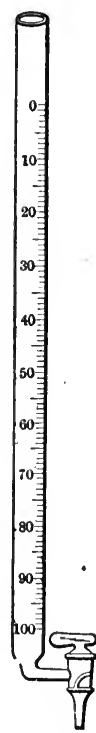

FIG. 6.

Fig. 6. Other forms of burettes are Mohr's Foot Burette, with rubber ball (Fig. 5). There is a hole in the rubber ball, and by placing the thumb over the hole and gently squeezing, the flow of the liquid may be regulated.

Bink's Burette (Fig. 7 is used by holding in the hand and inclining sufficiently to allow the quid to flow, then placing in an upright position, and reading when the surface of the liquid has settled.

Gay-Lussac's (Fig. 8) must also be inclined when used. A wooden foot is generally provided, into which this burette is placed to rest in 
an upright position. By inserting a tightly fitting cork into the open end and passing through this cork a small bent glass tube, the flow of the solution from the exit-tube can be nicely regulated by blowing through the small glass tube. The necessity for inclining the burette is thus obviated. (See Fig. 9).

The burette shown in Fig. Io with the spindle-shaped spout is used in the same manner as Bink's. It is claimed for the dilated spout

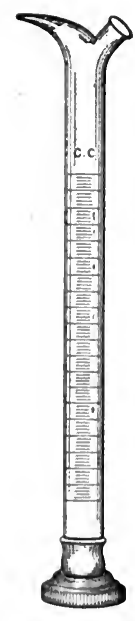

FIG. 7.

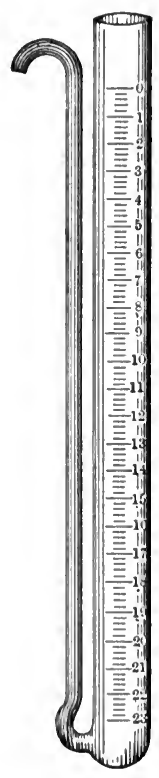

FIG. 8.

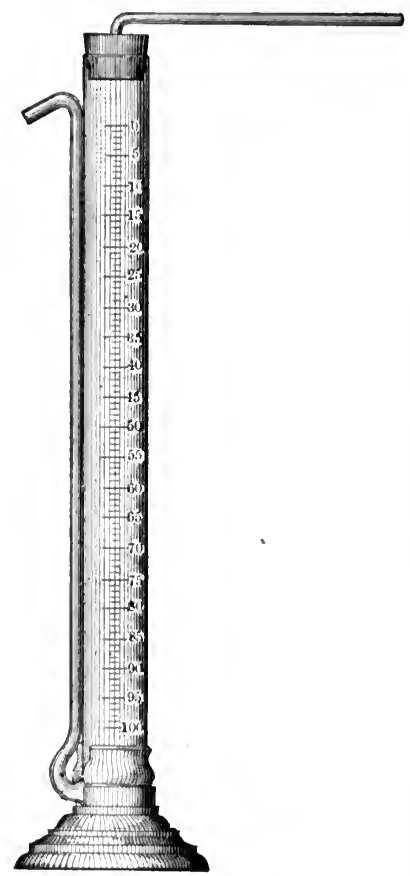

FIG. 9.

that it more readily admits of the delivery of single drops and prevents the too sudden back-dropping of the solution upon returning the burette to the upright position.

The three latter burettes being held in the hand when in use, there is a chance of increasing the bulk of the fluid by the heat of the hand, thus leading to errors in measurement.

Geissler's Foot Burette (Fig. I I) needs no further description.

A. Hesse has constructed the burette shown by Fig. I2, which 
facilitates the accurate standardization of normal test solution. The upper constricted part of the burette, having a capacity of $0.5 \mathrm{cc}$., is divided into $\frac{1}{10} \sigma \mathrm{cc}$., the next following main portion, as usual, into $\frac{1}{10}$ cc., and the lower constricted portion again in $\frac{1}{100} \mathrm{cc}$. for a distance of 2 cc. (from the 49 to $5 \mathrm{I}$ cc. mark). These graduations enable the practically absolute measurement of the solutions required when it is known that the total volume required for a titration reaches to within the limit of the constricted lower portion (to between 49 and $5 \mathrm{r}$ cc.), this having previously been ascertained experimentally. The upper

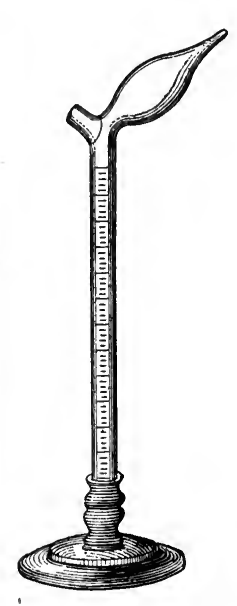

Fig. Io.

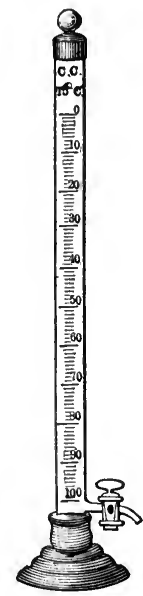

FIG. II.

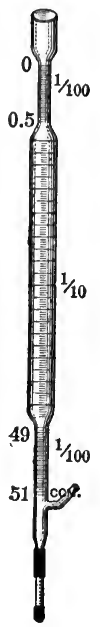

FIG. I2.

constricted portion is surmounted by a cup-shaped expansion, to prevent overflow in case the liquid is allowed to enter too zapidly.

When a number of estimations are to be made in which the same volumetric solution is employed, the arrangement shown in Figs. I3 and $\mathrm{I} 4$ is very serviceable.

A T-shaped glass tube is inserted between the lower end of the burette and the pinch-cock and connected by a rubber tube with a reservoir containing the volumetric solution. The tube which communicates with the reservoir is provided with a pinch-cock, which when open allows the solution to flow into and fill the burette in so gradual a manner that no bubbles are formed. The burette is emptied in the usual manner. 
F. Eo A. Automatic Burette (Fig. 15). This is used for the same purpose as the foregoing. It is provided with a side tube for connection with reservoir, and has an over-flow reservoir which prevents its being filled to above the zero mark. The three-way stop-cock is so arranged that if turned one way the inlet is opened and the

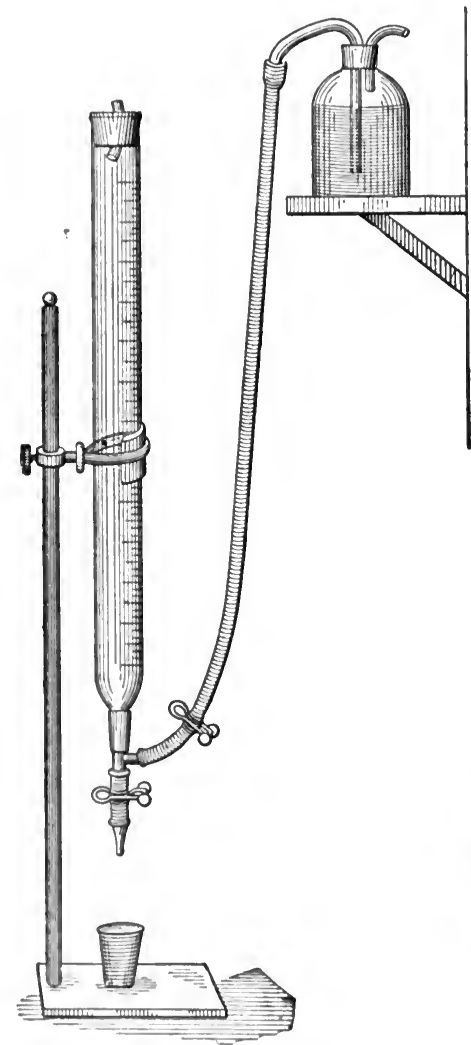

FIG. I3.

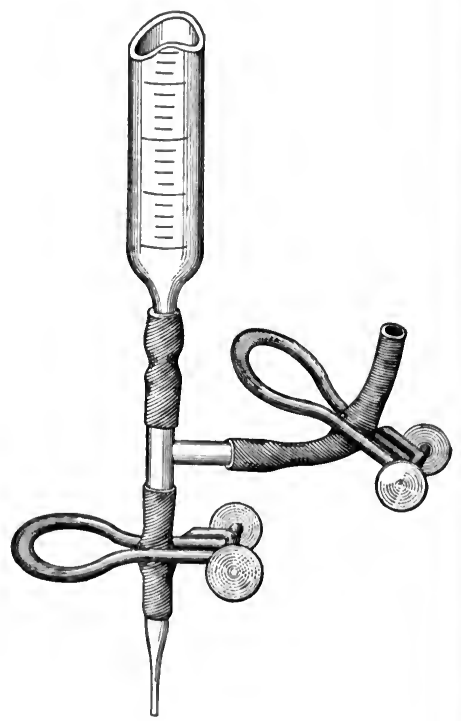

FIG. 14.

liquid from the reservoir flows into and fills the burette. If turned the other way the inlet is closed and the outlet is opened and the burette may be emptied. If the handle of the stop-cock is turned half-way round, both openings are closed.

There are many other forms of automatic burettes.

When working with solutions which are readily altered by contact 
with air, as for example, stannous chlorid, potassium, sodium, or barium hydroxid or ammonia, an arrangement like that depicted in Fig. 16 is very serviceable. In this the upper end of the burette is connected with the reservoir by means of a rubber tube, thus making an air-tight combination between the burette and the reservoir. Its

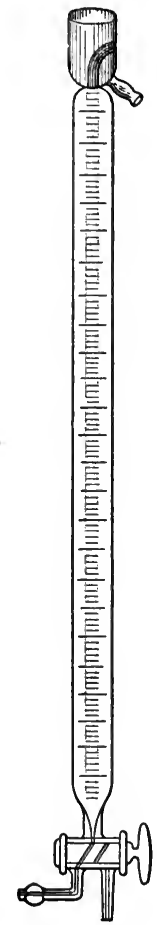

Fig. I5.

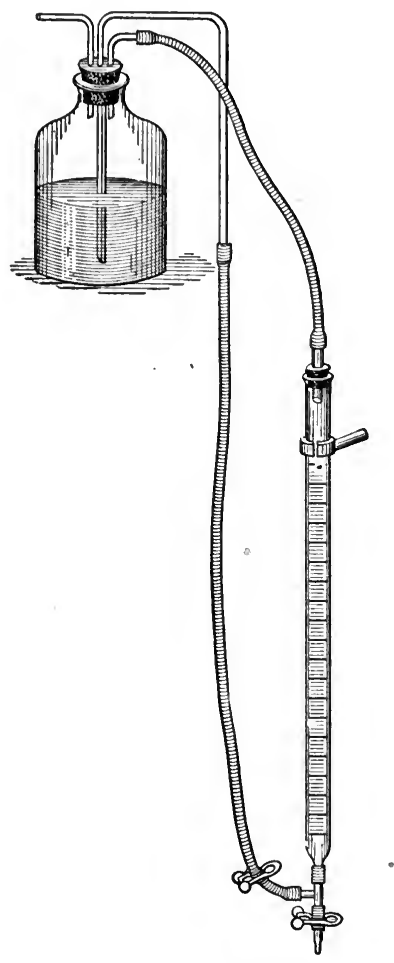

FIG. 16.

utility may be further enhanced by providing the reservoir with a sodalime tube or some other suitable absorption tube.

Another form of apparatus, and one which is better, is shown in Fig. 17. In this both the burette and the reservoir are provided with tubes containing soda-lime to insure a protection against the admission of $\mathrm{CO}_{2}$ and moisture from the air.

Fig. 18 depicts a form which answers the same purpose, as does also the instrument represented in Fig. I9. The latter having the 
advantage in being provided with an automatic zero-point burette, and in that the solution does not need to be passed through rubber tubes except at the outlet. It is furthermore provided, like the preced-

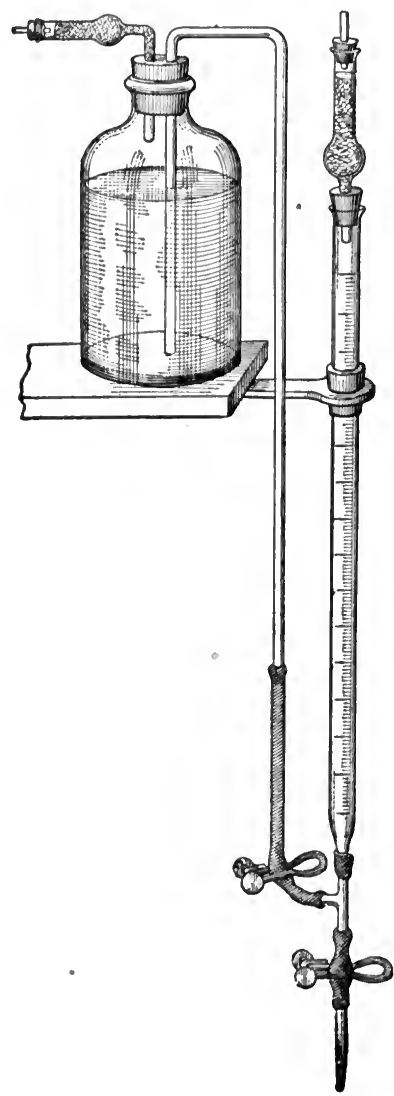

FIG. 17 .

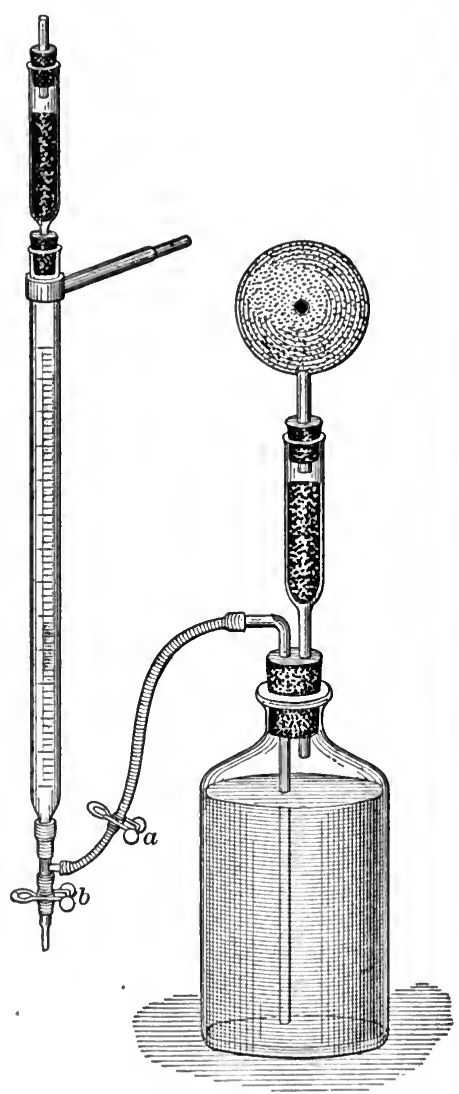

FIG. 18.

ing one, with an absorption tube placed between the rubber bulbs and the reservoir, as well as with one at the top of the burette.

Pinch-cocks used with Mohr's burettes are of various kinds. See Figs. 20, 21, and 22.

The screw-pinch cock,* Fig. 22 , is a very useful device; it mé be used like the ordinary pinch-cock by pressure with the finger 
upon $a-a$, when a rapid flow is desired, or the nut-screw (b) may be so adjusted as to allow a slower flow or to deliver the solution in drops, thus giving the operator the freedom of both hands for other work.

Burette-supports are of various forms. One of the best for one or two burettes is shown in Fig. 23. It is made of iron, can stand firmly upon an uneven surface, and does not easily tip over. The burettes are fastened to it br means of clamps, illustrated in Figs. 24 and 25 .

A revolving burette-holder for eight burettes is shown in Fig. 26. Burette-supports are also made with white porcelain base, which enables the operator more readily to see the change of color in the liquid titrated.

Pipettes are of two binds-those which are marked to deliver one quantity only, and those which are graduated on the stemlike burettes. Their use is to measure out portions of solutions with exactness.

Pipettes are filled by applying the mouth to the upper end and sucking the liquid up to the mark, then by closing the upper opening with the forefinger the liquid is presented from running out, but may be delivered in drops or allowed to run out to any mark by lessening the pressure of the finger orer the opening.

In using the pipettes of the first class (Fig. 27) the finger is raised and the instrument allowed to empty itself entirely. A drop or two, however, usually remains in the lower portion of the instrument, which may be blown out. By inclining the pipette and placing the point against the side of the ressel which is to receive the liquid, the instrument may be emptied more satisfactorily.

th Pipettes of the second class (Fig. 28) are never emptied completely of when in use. The flow of the liquid is regulated by the pressure of the finger over the upper opening, and stopped at the desired " point. 


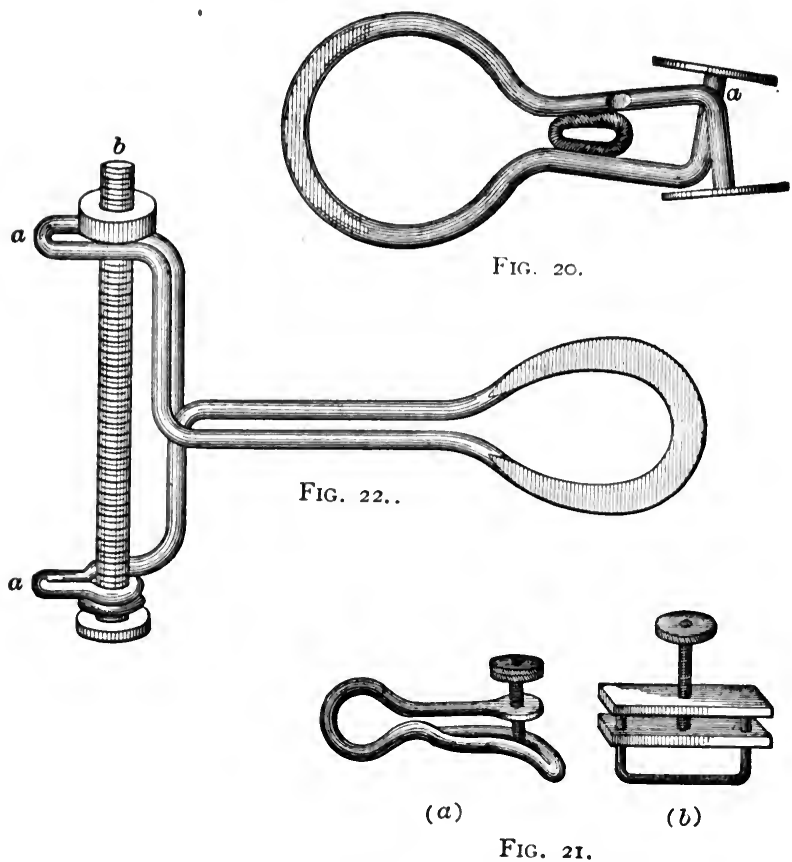

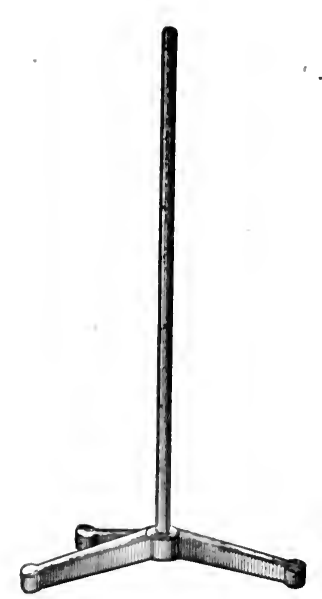

FIG. 23 .

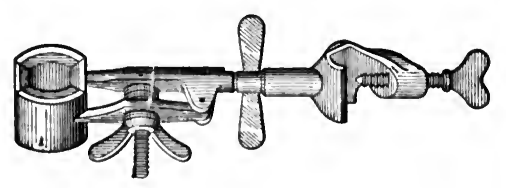

FIG. 24.

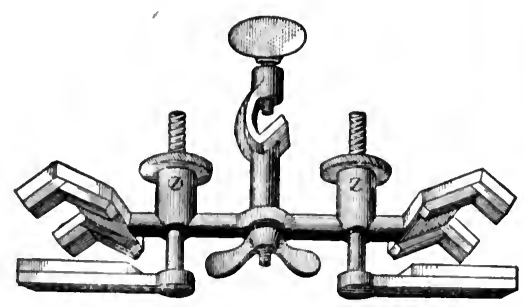

FIG. 25 . 
A very convenient form of pipette is one which has attached to its upper end a piece of rubber tubing, into which a short piece of glass rod has been inserted. By squeezing the rubber surrounding the glass rod firmly between the fingers, a canal is opened and the liquid can. be drawn up into the pipette by suction with the lips and run out again

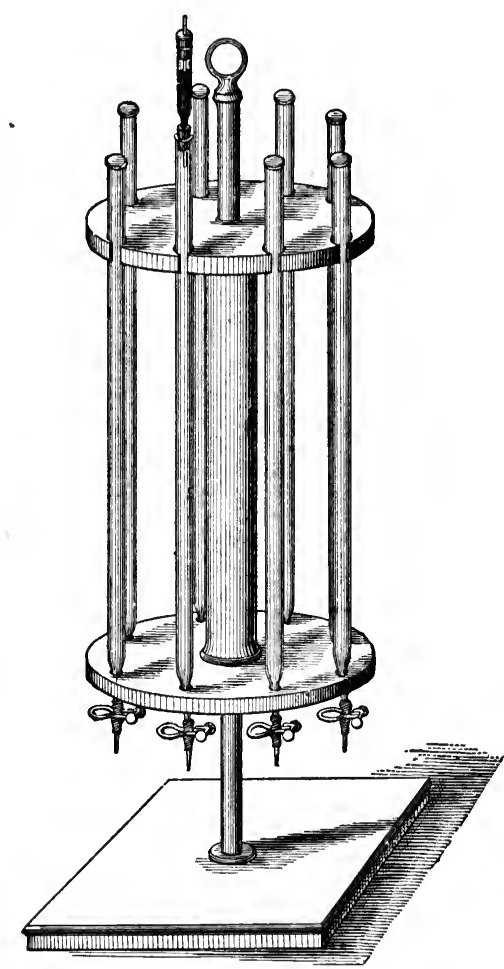

FIG. 26.

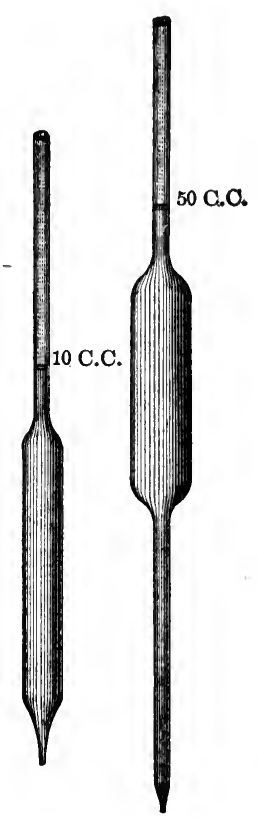

FIG. 27.

By removing the pressure the canal closes and the flow of the liquid is stopped at any point (Fig. 29).

The Nipple Pipette is very convenient for measuring small quantities of liquids, such as I or 2 cc. (Fig. 30 ).

When a volatile or highly poisonous solution is to be measured it is not advisable to suck it up with the mouth. The pipette in this case is filled by dipping it into the liquid contained in a long, narrow 
vessel, until the liquid reaches the proper mark on the pipette, then closing the upper opening and withdrawing. When this is done the liquid which adheres to the outside of the pipette should be dried off before the measured liquid is delivered.

A French firm has introduced pipettes provided with suction pumps, shown in various forms by Fig. $3 \mathrm{I}$, which possess the advantage over the ordinary forms provided with a compressible rubber bulb, that the liquid can with perfect ease be drawn up to the desired

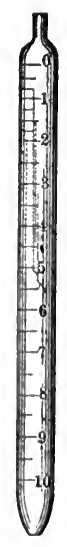

FIG. 28.

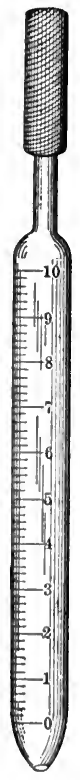

FIG. 29.

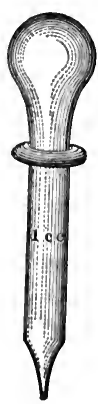

FIG. 30.
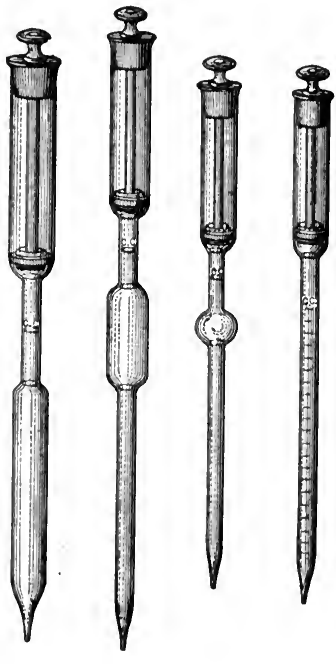

FIG. 31.

point on the scale, and with absolute accuracy maintained at the same height as long as may be desired.

The Measuring-flask is a vessel made of thin glass having a narrow neck, and so constructed as to hold a definite amount of liquid when filled up to the mark on the neck. These flasks are of various sizes, holding 100, 250, 500, 1000 cc., etc., but are generally called "Liter Flasks." (Fig. 32.)

Giles' Liter Flask, Fig. 33, is of 1100 cc. capacity, graduated at $1000 \mathrm{cc}$. and $1100 \mathrm{cc}$. Its value in making normal solutions is obvious, 
by taking one-tenth more of the reagent than would be used for making one liter of normal solution, and then filling up to the Iroo cc. mark, there is obtained roo.cc. of solution for ascertaining exact liter, leaving exactly one liter for correction.

Another very convenient liter flask is that shown in Fig. 34, which was designed by A. Gaske. The neck of the flask is somewhat prolonged and graduated from rooo cc. down to $920 \mathrm{cc}$. This arrangement permits any quantity of liquid to be removed for standardization, and the balance made up to the volume desired.

Liter flasks are used for making volumetric solutions.

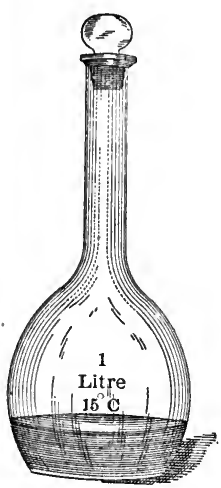

FIG. 32.

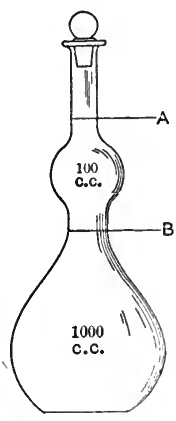

FIG. 33 .

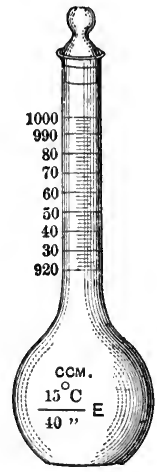

FIG. 34 .

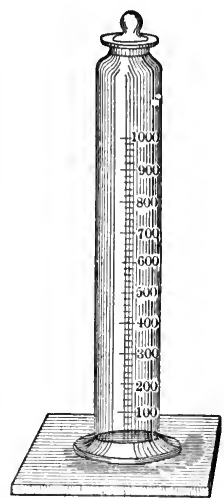

FIG. 35 .

Those which have the mark below the middle of the neck are to be preferred, because the contents can be more easily shaken.

Liter flasks are sometimes made with two marks on the neck very near together; the lower one is the liter mark. If the flask is required to deliver a liter, it must be filled to the upper mark; the difference between the two measures being the equivalent of the liquid which remains in the flask, adhering to the sides.

The Test Mixer, or Graduated Cylinder (Fig. 35), is for measuring and mixing smaller quantities of solutions. They are made of different sizes, holding 100, 250, 500, and 1000 cc., and graduated in fifths or tenths of a cc. 


\section{CHAPTER VI}

\section{ON THE USE OF APPARATUS}

IT is important that all apparatus used in volumetric analysis should be perfectly clean. Even new apparatus should be cleansed by passing dilute hydrochloric acid through them and then rinsing with distilled water.

If the burette, pipette, or other instrument is even slightly greasy, the liquid will not flow smoothly, and drops of the liquid will remain adhering to the sides, thus leading to inaccurate results.

Greasiness may be removed with dilute soda solution. If this fails the instrument should be allowed to remain for some little time in a solution containing sulphuric acid and potassium dichromate, which will radically remove all traces of grease.

The burette or other measuring instruments should never be filled with volumetric solution without first rinsing, even if the burette be perfectly dry.

It is well to wash the inside of the instrument with two or three small portions of the solution with which it is to be filled.

The burette may be filled with the aid of a funnel, the stem of which should be placed against the inner wall of the burette, so that the solution will flow down the side and thus prevent the formation of bubbles.

The burette should be filled to above the zero mark, and the airbubbles, if there are any, removed by gently tapping with the finger.

A portion of the liquid should then be allowed to run out in a stream so that no air-bubbles remain in the lower part of the burette. In the glass tap burette it can be easily seen if any air is present, but in the pinch-cock burette it is sometimes necessary to take hold of the rubber tube between the thumb and forefinger and gently stroke upward. Or the glass nip at the lower end of the burette may be pointed upward, and the pinch-cock opened wide so that a stream of the liquid will pass through and force out any air that may be inclosed.

If the titration is to be conducted at a high temperature, as in the estimation of carbonates, when litmus is used as the indicator, or 
in the estimation of sugar by copper solution, a long rubber tube should be attached to the lower end of the burette. The boiling can then be done at a little distance, and the expansion of the liquid in the burette avoided. The pinch-cock is fixed about midway on the tube.

Hart calls attention to the fact that if the fluid in a burette or pipette be run out rapidly at one time and slowly at another, different amounts of fluid are obtained.

This is due to the adhesion of the fluid to the inner sides of the instrument, and reading before it has settled down. It is therefore advisable always to deliver burettes slowly, as more constant results are then obtained.

Solutions which are measured by means of pipettes should be dilute, since concentrated solutions adhere to glass with different degrees of tenacity, and hence the amount of fluid delivered is slightly less than that measured.

The temperature of the solutions measured should be taken into account, since all liquids are affected by change of temperature, expanding and contracting as the temperature is increased or reduced.

This change of volume in the case of standard solutions does not exactly correspond to that in pure water; in fact some of them differ widely. The correction of the volume of a standard solution for the temperature by the expansion coefficient of water is not entirely satisfactory, but in the case of very dilute solutions this may be done.

Casamajor (C. N., Xxxv. I60) gives the following figures showing the relative contraction and expansion of water below and above $15^{\circ} \mathrm{C}$.:

$\begin{array}{cc}\text { Degree C. } & \text { Degree C. } \\ 8-.000590 & 17+.000305 \\ 9-.000550 . & 18+.000473 \\ \text { I0-.000492 } & 19+.000652 \\ \text { II }-.000420 & 20+.00084 \mathrm{I} \\ \mathrm{I} 2-.000334 & 2 \mathrm{I}+.001039 \\ \mathrm{I} 3-.000236 & 22+.001246 \\ \mathrm{I} 4-.000124 & 23+.001462 \\ \text { I5-normal } & 24+.001686 \\ \mathrm{I} 6+.000147 & 25+.001919\end{array}$

By means of these numbers it is easy to calculate the volume of liquid at $15^{\circ} \mathrm{C}$. corresponding to any volume observed at any temperature between $8^{\circ} \mathrm{C}$. and $25^{\circ} \mathrm{C}$. If $25 \mathrm{cc}$. of solution had been used at $20^{\circ} \mathrm{C}$., the table shows that I cc. of water passing from $15^{\circ}$ to $20^{\circ}$ 
is increased to $1.00084 \mathrm{I} \mathrm{cc}$. Therefore, by dividing $25 \mathrm{cc}$. by $\mathrm{I} .00084 \mathrm{I}$, the quotient, $24.97 \mathrm{cc}$. is obtained, which represents the volume at $15^{\circ} \mathrm{C}$. corresponding to $25 \mathrm{cc}$. at $20^{\circ} \mathrm{C}$.

These corrections are of value only for very dilute solutions and for water, but useless for concentrated solutions. Slight variations of atmospheric pressure may be disregarded.

\section{ON THE READING OF INSTRUMENTS}

In narrow vessels the surface of liquids is never level. This is owing to the capillary attraction exerted by the sides of the vessel upon the liquid, drawing the edge up and forming a saucerlike concavity

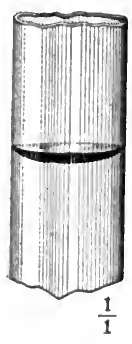

FIC. 36 .

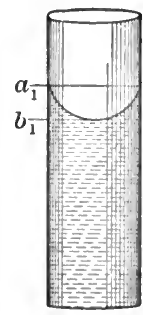

FIG. 37 .

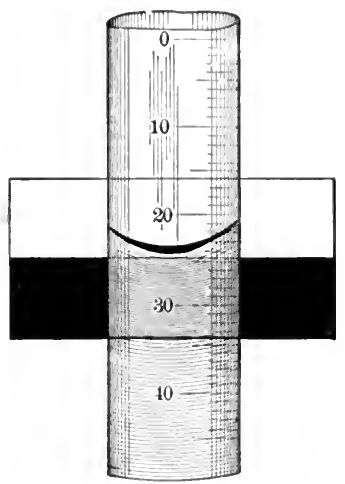

FIG. 38 .

(Fig. 36). All liquids present this concave surface except mercury, the surface of which is convex.

This behavior of liquids makes it difficult to find a distinct level, and in reading the measure either the upper meniscus (a) or the lower meniscus (b) may be used (Fig. 37).

The most satisfactory results are obtained if the lowest point of the curve $(b)$ is used, especially with light-colored solutions. But if dark-colored or opaque solutions are measured, it is necessary to use the upper meniscus (a) for reading.

In all cases the eye should be brought on a level with the surface of the liquid in reading the graduation.

The eye is very much assisted by using a small card, the lower half of which is black and the upper half white. This card is held behind the burette, the dividing line between white and black being 
about an eighth of an inch below the surface of the liquid. The eye is then brought on a level with it, and the lower meniscus can be distinctly seen as a sharply defined black line against the white background (Fig. $3^{8}$ ).

Erdman's Float, Fig. 39, is an elongated glass bulb, which is weighted at its lower end with mercury, to keep it in an upright posi-

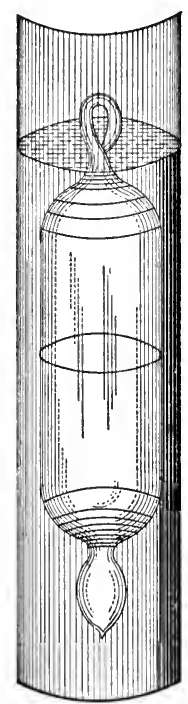

FIG. 39 .

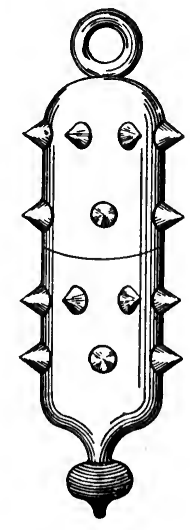

Fig. 40.

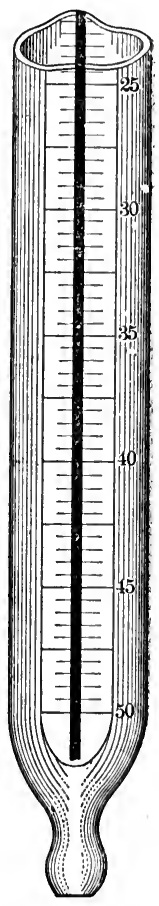

FIG. 4 I.

tion when floating. It is of such diameter that it will slide easily up and down inside of a burette. There is a ring at the top by which it can be lifted in or out by means of a bent wire. Around its center a line is marked. At this line instead of at the meniscus the reading is taken.

These floats are sometimes provided with a thermometer, and they then register the temperature as well as the volume.

Others are provided with projecting points along the sides, the 
object of which is to prevent it from adhering to the walls of the burette. See Fig. 40.

For the purpose of facilitating the reading, special forms of burettes are constructed which are provided with a dark longitudinal stripe on a white enameled background (Fig. 4I); the reflection of the dark stripe with the meniscus produces the peculiar appearance shown in Fig. 42. The narrowest point is at the middle of the meniscus, and

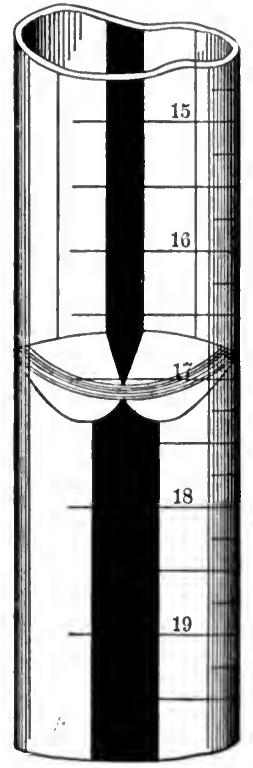

FIG. 42 。

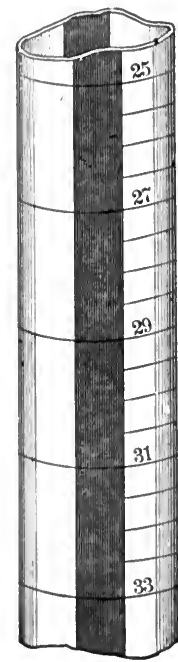

FIG. 43.

by reading from this point very accurate measurements are obtained. The same effect can be produced by holding behind an ordinary burette a white flexible card having a heavy black longitudinal stripe, about one-eighth inch in width.

Another form of burette designed for the purpose of facilitating reading is that provided with white enameled sides, leaving a strip of clear glass in front and back (Fig. 43). This form is especially adapted for use with dark-colored liquids such as iodin and permanganate. 


\section{CALIBRATION OF INSTRUMENTS}

Burettes are made from tubes of nearly uniform width. They are filled with distilled water at $15^{\circ} \mathrm{C} . * \quad\left(59^{\circ} \mathrm{F}\right.$.) to the $\circ$ mark, and then 25,50 , or $100 \mathrm{cc}$. run out, and another mark made at the surface of the liquid. The distance between these two marks is then divided into 25,50 , or roo equal parts, and the spaces again subdivided into fifths and tenths. Now it is very rarely possible to obtain tubes of exactly the same caliber throughout, and the divisions made as above do not always represent exactly what they are intended to.

If the tube is wider at one point the divisions at that point will contain more, and if it is narrower they will contain less, than they should.

Hence before using a new burette, or in fact any other measuring instrument, it is essential that the error, if any, should be determined. This is done as follows:

Fill the burette to the $\circ$ mark with distilled water at $15^{\circ} \mathrm{C}$. $\left(59^{\circ} \mathrm{F}\right.$.) and run out io cc. at a time into a small weighed flask, and weigh after each addition of ro cc.

Each ro cc. should weigh exactly ro gms., and every deviation found should be noted and taken into consideration in using the instrument.

\section{Example}

\begin{tabular}{|c|c|c|c|c|}
\hline Flask & & weig & | 20.0000 & rams \\
\hline “" & tro cc. & " & 30.1005 & 6 \\
\hline “ & $+20 \mathrm{cc}$. & “ & 40.0499 & \\
\hline “ & $+30 \mathrm{cc}$. & “ & 49.8000 & \\
\hline б & $+40 \mathrm{cc}$. & “" & 59.9700 & 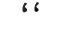 \\
\hline ، & $+50 \mathrm{cc}$. & “ & 70.0100 & \\
\hline
\end{tabular}

Thus the ist Io cc. weighed 10.1005 grams.

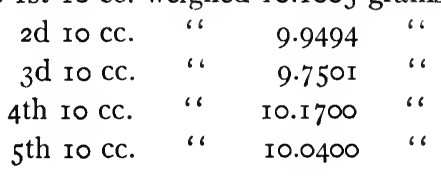

Having obtained these data, a table like the following may be constructed and kept in some convenient place where it can be readily

* Instead of $15^{\circ} \mathrm{C}$. $\left(59^{\circ} \mathrm{F}\right.$.) the temperature $25^{\circ} \mathrm{C}$. $\left(77^{\circ} \mathrm{F}\right.$.) is recommended in the U. S. P., because this more nearly approaches the ordinary temperature of the atmosphere in temperate climes. 
consulted whenever the burette it represents is being used. It is not necessary to carry the figure beyond the second decimal place.

\begin{tabular}{|c|c|c|c|c|c|}
\hline $\begin{array}{l}\text { No of cc. } \\
\text { as read on } \\
\text { Burette. }\end{array}$ & $\begin{array}{c}\text { No. of cc. } \\
\text { as } \\
\text { Corrected. }\end{array}$ & $\begin{array}{l}\text { No. of cc. } \\
\text { as read on } \\
\text { Burette. }\end{array}$ & $\begin{array}{c}\text { No. of cc. } \\
\text { as } \\
\text { Corrected. }\end{array}$ & $\begin{array}{l}\text { No. of ce } \\
\text { as read on } \\
\text { Burette. }\end{array}$ & $\begin{array}{l}\text { No. of ec. } \\
\text { as } \\
\text { Corrected. }\end{array}$ \\
\hline$I$ & I.OI & 14 & 14.06 & 27 & 26.79 \\
\hline 2 & 2.02 & I 5 & I 5.05 & 28 & $27 \cdot 7^{6}$ \\
\hline 3 & 3.03 & 16 & I6. 04 & 29 & 28.73 \\
\hline 4 & 4.04 & I 7 & 17.03 & 30 & 29.70 \\
\hline 5 & 5.05 & IS & I8.02 & 31 & 30.71 \\
\hline 6 & 6.06 & r 9 & I9.0I & $32^{\circ}$ & 31.72 \\
\hline 7 & 7.07 & 20 & 20.00 & 33 & 32.73 \\
\hline 8 & 8.08 & $2 \mathrm{I}$ & 20.97 & 34 & 33.74 \\
\hline 9 & 9.09 & 22 & 21.94 & 35 & $34 \cdot 75$ \\
\hline 10 & 10.10 & 23 & 22.91 & $3^{6}$ & $35 \cdot 76$ \\
\hline II & I I. O9 & 24 & 23.88 & 37 & 36.77 \\
\hline 12 & I 2.08 & 25 & 24.85 & $3^{8}$ & $37 \cdot 78$ \\
\hline 13 & 13.07 & 26 & 25.82 & 39 & $3^{8} \cdot 79$ \\
\hline
\end{tabular}

There should be no greater deviation than $0.15 \mathrm{cc}$. A burette which deviates more is best not used. In the foregoing table there is a deviation of $0.30 \mathrm{cc}$. at one point.

In order to test the accuracy of a pipette, fill to the mark with distilled water at $15^{\circ} \mathrm{C}$. $\left(59^{\circ} \mathrm{F}\right.$.); empty into a previously weighed flask, weigh again and thus determine the weight of the water measured. I gram is equal to I cc.

Liter flasks are tested as follows:

The flask, perfectly dry and clean, is counterpoised on a balance capable of turning with .005 when carrying about 2000 grams; it is then filled to the mark with distilled water at $15^{\circ} \mathrm{C}$. $\left(59^{\circ} \mathrm{F}\right.$.), and the increase in weight should be exactly the number of grams as the cc. indicated at the mark. 


\section{CHAPTER VII}

\section{WEIGHTS AND MEASURES USED IN VOLUMETRIC ANALYSIS}

THE metric or decimal system is used in this country and on the continent in Europe, but in England the grain system is sometimes used.

The unit of weight in the metric system is the gram (gm.).

A gram of distilled water at its maximum density, $4^{\circ} \mathrm{C} .\left(39^{\circ} \mathrm{F}\right.$.), measures one cubic centimeter (cc.).

A kilogram is 1000 gms.

A liter is rooo cubic centimeters.

Volumetric instruments are graduated in the metric system, but not at $4^{\circ} \mathrm{C}$. If they were, it would necessitate the carrying out of all volumetric operations at that temperature, and it would be impossible to do careful volumetric work, except for two or three months of the year, unless troublesome calculations for the correction of volume were made.

For this reason the temperature of $15^{\circ} \mathrm{C}$. $\left(59^{\circ} \mathrm{F}\right.$.) was taken as the standard, and at this temperature most volumetric instruments are graduated. In making very careful examinations the work should be done at this temperature.

It is, however, not imperative that this temperature or any other definite temperature be taken as the standard. The U. S. P., 8th Dec. Revis., recommends the temperature of $25^{\circ} \mathrm{C}$. $\left(77^{\circ} \mathrm{F}\right.$.) to be employed for graduating all instruments used in volumetric work, because this more nearly approaches the ordinary temperature of the atmosphere in chemical laboratories in this country. Whichever temperature is adapted the whole set of measuring instruments must be graduated at the same temperature, and the titrations must of course always be conducted with the standard solutions at the temperature at which the instruments are graduated or very near it.

One gram of distilled water at $15^{\circ} \mathrm{C}$. measures one cc. as used in volumetric analysis.

The true cc. weighs at $15^{\circ} \mathrm{C}$. only $0.999 \mathrm{gm}$. In the grain system used in England, I0,000 grains is taken as the standard of measure- 
ment. Sutton, in his "Handbook of Volumetric Analysis," proposes that ten-grain measures be called a decem, or for shortness dm.; this term corresponding to the cubic centimeter, and bearing the same relation to the ro,000-grain measure that the cubic centimeter does to the liter. A 10,000-grain measure contains 10,000 fluid grains, or 1000 decems. The flasks used in working by this system are graduated to hold 10,000, 5000, 2500, and 1000 grain measures. Burettes are graduated in 300-grain measures with I-grain divisions, 600 grains in 1 or 2 grain divisions, 1100 grains in 5 or 10 grain divisions, etc.

The system based upon the imperial-gallon measure of 70,000 grains is still to some extent in use. In this the decimillem ( 7 grains) bears the same relation to the pound (7000 grains) that the cubic centimeter does to the liter, or the decem to the 10,000-grain measure. 


\section{CHAPTER VIII}

\section{METHODS OF CALCULATING ANALYSES}

EACH cc. of a $\frac{\mathrm{N}}{\mathrm{I}}$ univalent volumetric solution contains $\frac{1}{1000}$ of the molecular weight in grams of its reagent, and will neutralize $\frac{1}{100} \overline{0}$ of the molecular weight of a univalent substance, or $\frac{1}{2000}$ of the molecular weight of a bivalent substance.

Each cc. of a $\frac{N}{I}$ bivalent volumetric solution contains $\frac{1}{20 \overline{0}}$ of the molecular weight in grams of its reagent, and will neutralize or combine with $\frac{1}{2000}$ of the molecular weight of a bivalent salt, or $\frac{1}{1000}$ of the molecular weight of a univalent salt.

A $\frac{\mathrm{N}}{\mathrm{IO}}$ is only $\frac{1}{10}$ the strength of a normal solution and will neutralize only ${ }_{10}^{1}$ the quantity of salt, etc.

Normal and decinormal solutions of acids should neutralize, respectively, normal and decinormal solutions of alkalies, volume for volume. Decinormal solution of silver nitrate and decinormal solution of hydrochloric acid or sodium chlorid should combine, volume for volume, etc.

\section{RULES FOR DIRECT PERCENTAGE ESTIMATIONS}

I. With normal solutions $1_{10}^{1}$ or $\frac{1}{20}$ (according to its atomicity) of the molecular weight in grams of the substance is weighed for titration, and the number of cc. of the V. S. required to produce the desired reaction is the percentage of the substance whose molecular weight has been used.

Thus, if sodium hydroxid $(\mathrm{NaOH}, 39.76)$ is to be examined by titration with normal acid solution $\frac{1}{10}$ of its molecular weight in grams ( 3.976 gms.) is weighed out, and each cc. of the normal acid solution required for its neutralization represents I per cent of pure sodium hydroxid. 
If sodium carbonate $\left(\mathrm{Na}_{2} \mathrm{CO}_{3}, 105.3 \mathrm{I}\right)$ is to be titrated $\frac{1}{20}$ of its molecular weight in grams ( 5.265 gms.) is taken.

2. With decinormal solutions $1 \frac{1}{100}$ or $\frac{1}{2} 0 \overline{0}$ of the molecular weight in grams of the substance to be analyzed is taken, and the number of cc. will, in like manner, give the percentage.

The following equations will serve to explain more fully:

Sodium hydroxid with $\frac{\mathrm{N}}{\mathrm{I}}$ sulphuric acid

$$
\begin{aligned}
& 2 \mathrm{NaOH}+\mathrm{H}_{2} \mathrm{SO}_{4}=\mathrm{Na}_{2} \mathrm{SO}_{4}+{ }_{2} \mathrm{H}_{2} \mathrm{O} \text {. } \\
& 2 \times 39.76=79.5^{2} 2 \underline{97.35} \\
& \text { 10) } 39.76 \quad 48.67=\text { to } 1000 \mathrm{cc} \text {. } \\
& 3.976 \text { gms. }=\text { to } 100 \mathrm{cc} \text {. }
\end{aligned}
$$

Thus the quantity taken, 3.976 gms., is the quantity of pure substance which is neutralizable by $100 \mathrm{cc}$. of the normal acid solution, and therefore each cc. of the latter represents $]_{0}^{1} \sigma 0$ of the quantity taken, or I per cent.

Sodium carbonate with $\frac{\mathrm{N}}{\mathrm{I}}$ sulphuric acid:

$$
\begin{aligned}
& \mathrm{Na}_{2} \mathrm{CO}_{3}+\mathrm{H}_{2} \mathrm{SO}_{4}=\mathrm{Na}_{2} \mathrm{SO}_{4}+\mathrm{H}_{2} \mathrm{O}+\mathrm{CO}_{2} \text {. } \\
& \text { 2) } 97.35 \\
& \text { 20) } 105.3 \mathrm{I} \quad 48.67=\text { to } 1000 \mathrm{cc} \text {. } \\
& 5.265 \mathrm{gms} \text {. }=\text { to } 100 \mathrm{cc} \text {. }
\end{aligned}
$$

With $\frac{\mathrm{N}}{10}$ sulphuric acid:

$$
\begin{aligned}
& 2 \mathrm{NaOH}+\mathrm{H}_{2} \mathrm{SO}_{4}=\mathrm{NaSO}_{4}+{ }_{2} \mathrm{H}_{2} \mathrm{O} . \\
& 2 \times 39.76=79.52 \quad \text { 2) } \underline{97.35} \\
& \text { I00) } 20.76 \quad 48.67=\text { to } 1000 \mathrm{cc} \text {. } \\
& .3976 \mathrm{gms} . \quad \quad \quad \text { to } 100 \mathrm{cc} \text {. }
\end{aligned}
$$

In the case of a trivalent substance as citric acid $\frac{1}{30}$ of the molecular weight in grams is taken for analysis when a normal solution is employed and $\frac{1}{3} \sigma 0$ when a decinormal solution is used.

In other words, when it is desired that each cc. of the standard solution should represent I per cent of the substance upon which it acts, the rule is to take for analysis as much of the substance as is represented by $100 \mathrm{cc}$. of the standard solution.

In the case of substances whose percentage of purity is high, it is advisable to take smaller quantities, in order to avoid the use of 
excessive quantities of standard solution. Thus sulphuric acid, which contains 92.5 per cent of absolute sulphuric acid, would require under the above conditions $92.5 \mathrm{cc}$. of normal alkali solution.

In the case of this acid, if $4.867 \mathrm{gms}$. are taken for analysis, each cc. of a normal alkali solution would represent I per cent of $\mathrm{H}_{2} \mathrm{SO}_{4}$.

If half of this quantity, i.e., 2.4335 gms. are taken for analysis, each cc. of the normal alkali will represent 2 per cent of $\mathrm{H}_{2} \mathrm{SO}_{4}$, and thus less of the standard solution will be required. Again, if 0.4867 $\mathrm{gm}$. be taken, each cc. of the standard alkali will represent ro per cent of $\mathrm{H}_{2} \mathrm{SO}_{4}$.

In the case of liquids where it is not always convenient to weigh off the exact quantity required for titration by the direct percentage method, the liquid is diluted to a convenient degree with water, and then a quantity of this diluted liquid (representing the weight required of the substance) is measured for analysis.

Example. A sulphuric acid solution of specific gravity 1.826 is to be analyzed. 2 cc. are accurately measured and diluted to roo cc. and then $\mathrm{r} 2.97 \mathrm{cc}$. of this solution (representing $0.4867 \mathrm{gm}$. of the acid) are taken for analysis.

Each cc. of $\frac{\mathrm{N}}{\mathrm{I}} \mathrm{NaOH}$ V. S. required in the titration represents Io per cent of absolute $\mathrm{H}_{2} \mathrm{SO}_{4}$. If $\frac{\mathrm{N}}{\mathrm{IO}} \mathrm{NaOH} \mathrm{V}$. S. is employed, each cc. will represent $\mathrm{I}$ per cent. To determine the amount of the diluted liquid to be taken we proceed as follows:

Two cc. of sulphuric acid specific gravity r .826 weigh $3.75^{2}$ gms., therefore the $100 \mathrm{cc}$. of diluted acid contain this weight, and I cc. of the same contains $0.0375^{2} \mathrm{gm}$.

If $0.0375^{2} \mathrm{gm}$. are contained in I cc., then $0.4867 \mathrm{gm}$. are contained in how many cc.?

$$
\begin{aligned}
& \text { gm. cc. gm. } \\
& .0375^{2}: \mathrm{r}:: .4867: x, \\
& \qquad x=\mathrm{r} 2.97 \mathrm{cc} .
\end{aligned}
$$

Factors or Coefficients for Calculating the Analyses. It frequently occurs that from the nature of the substance, or from its being in solution, this percentage method cannot be conveniently followed.

The best way to proceed in such a case is to find the factor. 
The first step in all cases is to write the equation for the reaction which takes place between the substance under analysis and the solution used.

For instance, a solution of caustic potash is to be examined, a $\frac{N}{\mathrm{I}}$ solution of sulphuric acid being used.

$$
\begin{aligned}
& { }_{2} \mathrm{KOH}+\mathrm{H}_{2} \mathrm{SO}_{4}=\mathrm{K}_{2} \mathrm{SO}_{4}+{ }_{2} \mathrm{H}_{2} \mathrm{O} \text {. } \\
& \text { 2) } \frac{\text { III.48 }}{55.74} \quad \frac{97.35}{48.67}=\text { to rooo cc. } \frac{N}{\mathrm{I}} \text { acid. } \\
& .05574 \mathrm{gm} . \quad .04867=\text { to } \quad \text { I cc. } \frac{\mathrm{N}}{\mathrm{I}} \text { acid. }
\end{aligned}
$$

The factor for $\mathrm{KOH}$ when $\frac{\mathrm{N}}{\mathrm{r}}$ solution is used is $.05574 \mathrm{gm}$., that being the quantity neutralized by each cc. of the $\frac{\mathrm{N}}{\mathrm{r}}$ acid. If $\frac{\mathrm{N}}{\mathrm{IO}}$ acid were used the factor would be $.005574 \mathrm{gm}$.

The number of cc. of the acid used to produce the desired result, when multiplied by the factor, gives the quantity in grams of $\mathrm{KOH}$ in the solution taken.

Example. If to grams of caustic-potash solution were taken, and $40 \mathrm{cc}$. of $\frac{\mathrm{N}}{\mathrm{I}}$ acid were required, the ro gms. of solution contained $.05574 \mathrm{gm} . \times 40=2.2296 \mathrm{gms}$. of pure $\mathrm{KOH}$.

To find the percentage, the following formula may be used.

$$
\frac{Q \times{ }_{\mathrm{I}} 00}{\mathrm{~W}}=\% \text {. }
$$

$Q=$ the quantity of pure substance found by calculation;

$W=$ weight of substance taken.

If the above example is taken, we have

$$
\frac{2.2296 \times 100}{10}=22.296 \% \text {. }
$$

Or the calculation may be made by proportion.

The quantity of the substance taken is always the first term, and the quantity of pure substance found, the second term.

The following rule is easily remembered: As the quantity taken is to the quantity found, so is $100: 0 \mathrm{x}$, the percentage of pure substance in the sample. 
Three terms of the equation being given, the fourth is found by multiplying the means and dividing the product by the given extreme. By applying this rule to the above case we have

$$
\text { 10: } 2.2296:: 100: x . \quad x=22.296 \% \text {. }
$$

Table Showing the Normal Factors, Etc., of the Alkalies, Alkaline EARTHS, AND ACIDS.

\begin{tabular}{|c|c|c|c|c|}
\hline Substance. & Formula. & $\begin{array}{l}\text { Molecular } \\
\text { Weight. }\end{array}$ & $\underset{\text { Normal }}{\text { Factor.* }}$ & $\begin{array}{c}\text { Quantity of } \\
\text { Substance } \\
\text { to be takent } \\
\text { so that each } \\
\text { cc. of } \frac{N}{I} \text { V.S. } \\
\text { will indicate } \\
\text { I per cent. }\end{array}$ \\
\hline 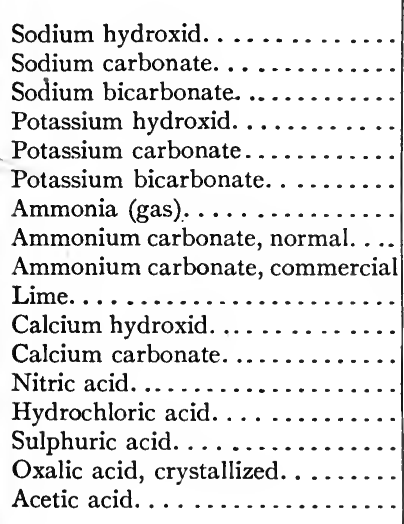 & $\begin{array}{c}\mathrm{NaOH} \\
\mathrm{Na}_{2} \mathrm{CO}_{3} \\
\mathrm{NaHCO}_{3} \\
\mathrm{KOH} \\
\mathrm{K}_{2} \mathrm{CO}_{3} \\
\mathrm{KHCO}_{3} \\
\mathrm{NH}_{3} \\
\left(\mathrm{NH}_{4}\right)_{2} \mathrm{CO}_{3} \\
\mathrm{~N}_{3} \mathrm{H}_{11} \mathrm{C}_{2} \mathrm{O}_{5} \\
\mathrm{CaO} \\
\mathrm{Ca}(\mathrm{OH})_{2} \\
\mathrm{CaCO} \\
\mathrm{HNO}_{3} \\
\mathrm{HCl} \\
\mathrm{H}_{2} \mathrm{SO}_{4} \\
\mathrm{H}_{2} \mathrm{C}_{2} \mathrm{O}_{4} \cdot \mathrm{H}_{2} \mathrm{O} \\
\mathrm{HC}_{2} \mathrm{H}_{3} \mathrm{O}_{2}\end{array}$ & $\begin{array}{r}39.76 \\
105 \cdot 3 \mathrm{I} \\
83.43 \\
55.74 \\
\mathrm{I} 37.27 \\
99.4 \mathrm{I} \\
\mathrm{I} 6.93 \\
95 \cdot 4 \mathrm{I} \\
\mathrm{I} 56.0 \mathrm{I} \\
55.68 \\
73 \cdot 56 \\
99 \cdot 35 \\
62.57 \\
36.18 \\
97 \cdot 35 \\
\mathrm{I} 25.10 \\
59.58\end{array}$ & $\begin{array}{l}0.03976 \\
0.05265 \\
0.08343 \\
0.05574 \\
0.06863 \\
0.09941 \\
0.05693 \\
0.0477 \\
0.052 \\
0.02784 \\
0.03678 \\
0.04967 \\
0.06257 \\
0.03618 \\
0.04867 \\
0.06255 \\
0.05958\end{array}$ & $\begin{array}{l}\text { gram. } \\
3 \cdot 976 \\
5 \cdot 265 \\
8.343 \\
5 \cdot 574 \\
6.863 \\
9.941 \\
1.693 \\
4 \cdot 77 \\
5 \cdot 2 \\
2.784 \\
3.678 \\
4 \cdot 967 \\
6.257 \\
3.618 \\
4.867 \\
6.255 \\
5 \cdot 95^{8}\end{array}$ \\
\hline
\end{tabular}

* This is the coefficient by which the number of cc. of normal solution used is to be multiplied in order to obtain the quantity of pure substance present in the material examined.

$\dagger$ This is the quantity of substance to be taken in direct percentage estimations. Each cc. of $\frac{N}{I}$ acid or alkali V. S. employed will then indicate I per cent: in the case of many of these substances it will, however, be better to take smaller quantities so that less of the standard solution be required. Thus if one half of the quantity be taken each cc. of the $\frac{N}{I}$ V.S will represent 2 per cent, if $\frac{1}{10}$ of the quantity be taken each cc. will represent Io per cent. If, however, $\frac{N}{10}$ solutions be used and $x_{1}^{x}$ of the quantity indicated in the tablc be taken each cc. will indicate I per cent. 


\section{CHAPTER IX}

\section{SOME VICARIOUS VOLUMETRIC METHODS}

VOLUMETRIC ANALYSIS WITHOUT WEIGHTS AND STANDARD SOLUTIONS

THIS is a matter of curiosity rather than of value, but under certain circumstances it might prove useful. The way in which this is carried out is best explained by an example.

Suppose we wish to determine the proportion of pure sodium chlorid in an impure specimen of salt. A portion of the latter is placed upon one pan of a balance and exactly counterpoised by placing on the other pan sufficient of the pure sodium chlorid. The samples are then dissolved in water and each titrated with a solution of silver nitrate of unknown strength and the calculation made as follows:

If the pure salt required $60 \mathrm{cc}$. of the silver solution, and the impure specimen $45 \mathrm{cc}$, then

$$
60: 45:: 100: x ; x=75 \text {, }
$$

the percentage of pure sodium chlorid in the salt analyzed.

This process it will be seen can be applied only to such substances of which pure specimens can be had, though in some instances a pure specimen of some other salt may be used as a substitute, and the result obtained by calculation.

For instance, suppose it is required to estimate sodium carbonate, and we have only pure calcium carbonate on hand to use as a weight. Equal weights are taken and each titrated with an acid solution. It is now necessary to find out how many cc. of acid solution would be required if pure sodium carbonate were used, instead of pure calcium carbonate, as a counterpoise The molecular weights of calcium carbonate and sodium carbonate are $100 *$ and $106 *$ respectively, and thus sodium carbonate would require $\frac{100}{106}$, the amount of acid selution as calcium carbonate.

\footnotetext{
* Approximate molecular weights.
} 
We will assume that the calcium carbonate required $60 \mathrm{cc}$. of the acid solution and the impure sal soda $40 \mathrm{cc} .60 \times \frac{100}{106}=56.6$, the number of cc. which an equal weight of sodium carbonate will require. Then

$$
56.6: 40:: 100: x ; x=70.67
$$

the percentage of pure sodium carbonate in the specimen analyzed.

With the exercise of a little ingenuity the method may be extended to a number of substances.

Koningh and Peacock have devised a method by which the same end is attained without the aid of a pure substance as a standard.

If impure sodium chlorid is to be examined, an equal weight of silver nitrate is taken and dissolved in sufficient water to make roo cc. of solution; this is placed in a burette, and the sodium chlorid titrated after the latter is dissolved.

Assuming that ro cc. of the silver solution were required, then

$$
\text { г69.7*: } 58.4^{*}:: \text { го : } x ; x=3.44 \% \text {. }
$$

In the estimation of sodium carbonate an equal weight of oxalic acid is taken and used in the same manner.

\section{VOLUMETRIC ANALYSIS WITHOUT A BURETTE}

The standard solutions are weighed instead of measured. This method is often resorted to where great accuracy is desired, for variations in temperature do not influence the result. It is, however, a slow process.

The standard solution is placed in a suitable flask (see Fig. 44), and the whole weighed on a delicate balance. The solutioin is then carefully run into the beaker containing the substance to be analyzed, and when the end reaction is obtained the flask is again weighec', and the difference in weight is the amount of solution used. The standard solution should of course be standardized by weight.

A weight burette is shown in Fig. 45, which is of a very serviceable kind. It was designed by $\mathrm{C}$. T. Heycock, $\uparrow$ and consists of

$A$, an ordinary glass cylinder of about $70 \mathrm{cc}$. capacity, very roughly divided into cc.'s. These divisions are not absolutely necessary, but

* Approximate molecular weights.

$\dagger$ Described at 1899 meeting of the Brit. Ph. Conf. 
they will be found useful in the case of a second titration of the same solution.

$B$, a hollow stopper with a small perforation opposite $C$, which is a glass tube used to admit the air when drawing out the solution without

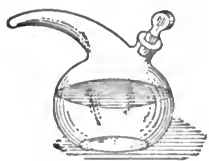

FIG. 44. taking out the stopper.

$E$, an ordinary glass stop-cock.

$D$, a wire loop for hanging the burette upon the hook above the balance pan.

A standard weight solution is made by taking a flask, carefully drying and weighing it, the standard substance being introduced into this from a stoppered weighing tube, water added to approximate volume desired and solution effected.

The whole is then weighed. The weight of dry flask being known, the weight of the solution is found.

The strength of this solution is then $x$ grams of standard substance in $x$ grams of the solution, which, therefore, becomes the standard solution.

The standard solution is placed in one of these burettes and another filled with the solution to be estimated.

Both are carefully weighed, a certain quantity from one, say 10.15 grams, is run into a suitable ressel, in which the titration can be made and again weighed. The titration is then carried out and the weight of the second burette found. It is seen that al definite weight of the solution of unknown strength requires a definite weight of standard solution, which in its turn contains a definite weight $\mathrm{ci}_{i}$ standard substance.

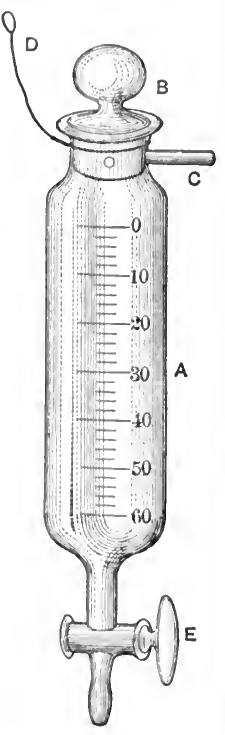

FIG. 45 .

From these data the strength of the unknown solution is obtained. It is essential when weighing both the empty flask and burettes that each is approximately counterpoised by a similar vessel upon the opposite scale-pan, so that the same amount of air is displaced on both sides, and thus corrections for variations of temperature and pressure are avoided.

As in ordinary volumetric work different substances will require slightly different manipulation; for instance, it will frequently be found convenient to weigh sufficient only of the standard substance for each titration. 
The advantages of this method of using weight burettes are:

I. Changes of temperature during the experiment, and differences of temperature of the two solutions exert no influence upon the result.

2. The errors arising from inaccurate calibration of the ordinary burette are entirely eliminated.

3. The question as to whether all pipettes are made to contain or deliver a certain volume, and, if the latter, whether the last drop should be blown out, is entirely avoided.

4. Supposing the weight of these burettes is carried to the third decimal place, it is equivalent to having a burette accurately graduated to one-thousandth part of a cubic centimeter.

5. The difficulty of reading correctly and uniformly the ordinary burettes owing to the unevenness of the meniscus, or from the fact of some of the solution adhering to the sides of the burette, particularly noticeable in dark solutions such as potassium permanganate, or from other causes, is obviously overcome. 


\section{CHAPTI:R X}

\section{ANALYSIS BY NELTRALIZATION}

THIs is based upon the fact that acids are neutralized by alkalies and alkalies by acids.

The strength of an acid is estimated by the quantity of alkali that is required to neutralize it. This process is called acidimetry.

The strength of an alkali is found by the quantity of an acid that is required to neutralize it. This process is called alkalimetry. The stronger the acid, the more alkali is required, and vice versa.

A substance is said to be alkaline when it turns red litmus blue; phenolphthalein, red; turmeric, brown, etc. Acid, when it turns blue litmus red; red phenolphthalein, colorless, etc.

The principal alkaline substances are the hydroxids and carbonates of sodium, potassium, and ammonium, and the hydroxids and oxids of calcium, barium, and strontium, and the alkaloids.

When an acid is brought in contact with an alkali combination takes place, and a neutral salt is formed. This combination takes place in definite and invariable proportions; thus: If III.48 parts of potassium hydroxid are mixed with 97.35 prarts of absolute sulphuric acid the alkali as well as the acid will be neutralized. If only 80 parts of the acid have been added the mixture would still be alkaline, for it requires 97.35 parts of the acid to neutralize. If more than 97.35 of the acid have been added, the mixture would consist of potassium sulphate and free sulphuric acid. The reaction is thus illustrated:

$$
\begin{array}{ccc}
2 \mathrm{KOH} & +\underset{\mathrm{H}_{2} \mathrm{SO}_{4}}{\mathrm{~S}_{2}}=\mathrm{K}_{2} \mathrm{SO}_{4}+{ }_{2} \mathrm{H}_{2} \mathrm{O} . \\
2 \mathrm{~K}=77.72 & \mathrm{H}_{2}=2.00 & \begin{array}{c}
\text { Potassium } \\
\text { sulphate }
\end{array} \\
2 \mathrm{O}=31.76 & \mathrm{~S}=31.83 & \\
2 \mathrm{H}=2.00 & \mathrm{O}_{4}=63.52 & \\
\text { III.45 } & 97.35 &
\end{array}
$$

Sodium hydroxid will unite with oxalic acid in the proportion 
of 79.52 parts by weight of the former and 25.10 parts by weight of the latter, as the equation shows.

$$
\begin{aligned}
& { }_{2} \mathrm{NaOH}+\mathrm{H}_{2} \mathrm{C}_{2} \mathrm{O}_{4} \cdot{ }_{2} \mathrm{H}_{2} \mathrm{O}=\mathrm{Na}_{2} \mathrm{C}_{2} \mathrm{O}_{4}+{ }_{4} \mathrm{H}_{2} \mathrm{O} \text {. } \\
& 2 \mathrm{Na}=45.76 \quad 6 \mathrm{H}^{\cdot}=6.00 \\
& { }_{2} \mathrm{O}=31.76 \quad \mathrm{C}_{2}=23.82 \\
& 2 \mathrm{H}^{\cdot}=2.00 \quad 6 \mathrm{O}=95.28 \\
& 79.52 \quad 125.10
\end{aligned}
$$

Ammonia water unites with hydrochloric acid as per the equation,

$$
\underset{34.8 \mathrm{I}}{\mathrm{NH}_{4} \mathrm{OH}}+\underset{36 . \mathrm{I} 8}{\mathrm{HCl}}=\mathrm{NH}_{4} \mathrm{Cl}+\mathrm{H}_{2} \mathrm{O} .
$$

Sodium carbonate with hydrochloric acid,

$$
\underset{\text { I05.3 }}{\mathrm{Na}_{2} \mathrm{CO}_{3}}+\underset{72.36}{2 \mathrm{HCl}}=2 \mathrm{NaCl}+\mathrm{H}_{2} \mathrm{O}+\mathrm{CO}_{2} .
$$

Upon a careful perusal of the foregoing equations it will be seen that since definite weights of acids neutralize definite weights of alkalies, the quantity of a certain alkali in solution can be easily determined by the quantity of an acid solution of known strength required to neutralize it, and vice versa.

Referring to the first equation we see that 97.35 gms. of $\mathrm{H}_{2} \mathrm{SO}_{4}$ neutralize III. $48 \mathrm{gms}$. of $\mathrm{KOH}$. If we prepare a normal solution of $\mathrm{H}_{2} \mathrm{SO}_{4}$ we take half the molecular weight, $97.35=48.675$ gms. to I000 cc. Half the molecular weight is taken because sulphuric acid is a bivalent acid. I000 cc. of this solution will neutralize $55.74 \mathrm{gms}$. of $\mathrm{KOH}$; hence I cc. will neutralize $0.05574 \mathrm{gm}$. of $\mathrm{KOH}$.

Thus if ro gms. of a solution of $\mathrm{KOH}$ be treated with the above normal solution of $\mathrm{H}_{2} \mathrm{SO}_{4}$, and it is found that $25 \mathrm{cc}$. of the acid solution are required to nutralize the alkali solution, the latter contains $25 \times 0.05574=\mathrm{r} .39+\mathrm{gm}$. of pure $\mathrm{KOH}$.

Since the acid and alkali as well as the neutral salt which is formed are colorless, and no visible change takes place during the reaction, it is necessary to add some substance which by change of color will show when the neutralization is complete. Such a substance is known as an indicator.

In the case of sodium hydroxid with oxalic acid (see the second equation), we find that $125.10 \mathrm{gm}$. of crystallized oxalic acid neu- 
tralizes $79.5^{2}$ gms. of $\mathrm{NaOH}$. Oxalic acid like sulphuric, is bivalent, therefore a normal solution of it contains half the molecular weight in grams, i.e., $62.55 \mathrm{gms}$. in $1000 \mathrm{cc}$.

rooo cc. will neutralize 39.76 gms. of $\mathrm{NaOH}$;

I cc. will neutralize $0.03976 \mathrm{gm}$. of $\mathrm{NaOH}$.

The neutralizing power of all normal acids is exactly the same, because they all contain in $1000 \mathrm{cc}$. the molecular weight in grams of the acid, in the case of univalent acids and half of the molecular weight in grams of bivalent acids.

Thus I cc. of any normal acid will neutralize $0.05574 \mathrm{gm}$. of $\mathrm{KOH}$ or $0.03976 \mathrm{gm}$. of $\mathrm{NaOH}$ or $\frac{1}{1000}$ of the molecular weight of any other univalent alkali, or $\overline{20} \overline{1}_{0}$ of the molecular weight of an alkali earth, the latter being bivalent. In like manner all decinormal solutions have a like neutralizing power, their neutralizing equivalence is one-tenth that of normal solutions.

Thus I cc. of a decinormal acid will neutralize $0.005574 \mathrm{gm}$. of $\mathrm{KOH}$ or $0.003976 \mathrm{gm}$. of $\mathrm{NaOH}$, etc.

\section{ALKALIMETRY}

Preparation of Standard Acid Solutions. It is possible to carry out the titration of most alkalies by means of one standard acid solution, but the same standard acid is not equally applicable in all cases; furthermore, the standard acids are frequently employed for other volumetric operations than neutralization, and therefore it is advisable to have a variety.

The standard oxalic acid solution is preferred by some, because of the ease with which it may be prepared, provided a pure oxalic acid is to hand. It does not, however, keep very long, is unreliable for use with methyl orange, and is inapplicable for the titration of alkali earths, because it forms insoluble compounds with these metals. Standard hydrochloric acid is the most desirable for alkali earths, because it forms soluble compounds with them; its disadvantage, however, is in its volatility and its consequent uselessness in hot titrations. Standard sulphuric acid is preferred by most analysts as heing the best general standard. A pure acid can be gotten without difficulty and the standard solution made from it is unaffected by boiling, and can therefore be used in hot as well as in cold titrations; it reacts sharply with the indicators and it keeps 
its titer indefinitely. It is, however, not suited for the titration of alkali earths, because it forms insoluble compounds with them which precipitate, and are very annoying to the operator. In the preparation of standard solutions the greatest care should be exercised in order that the product be absolutely accurate. The slightest inaccuracy in the strength of a standard solution will result in relative errors in the analysis. It is customary to prepare one standard solution, and then from this to adjust various others. For example, a normal oxalic acid may be made first, and by means of this a normal alkali solution, which in turn may be utilized for the adjusting of other standard acid solutions.

Normal Oxalic Acid $\left(\mathrm{H}_{2} \mathrm{C}_{2} \mathrm{O}_{4} \cdot 2 \mathrm{H}_{2} \mathrm{O}=125 . \mathrm{IO} ; \frac{\mathrm{N}}{\mathrm{I}} \mathrm{V} . \mathrm{S} .=62.55 \mathrm{gms}\right.$. in $1000 \mathrm{cc}$.)

Dissolve 62.55 gms. of pure oxalic acid (see below) in enough water to make at or near $15^{\circ} \mathrm{C}$. exactly $1000 \mathrm{cc}$.

Pure oxalic acid, crystallized, is in the form of colorless, transparent, clinorhombic crystals, which should leave no residue when ignited upon platinum foil. It is completely soluble in $\mathrm{I}_{4}$ parts of water at $15^{\circ} \mathrm{C}$. If the acid leaves a residue on ignition it should be purified by recrystallization, as directed in the U. S. P.

I cc. of $\frac{\mathrm{N}}{\mathrm{I}}$ oxalic acid V. S. is the equivalent of
Ammonia gas,
$\mathrm{NH}_{3} \ldots \ldots \ldots \ldots .0 .01693$
Potassium hydroxid,
KOH............. 0.05574
Sodium hydroxid,
$\mathrm{NaOH} \ldots \ldots . . . . .0 .03976$
Potassium permanganate, $\mathrm{KMnO}_{4} \ldots \ldots \ldots . .0 .031396$
Manganese dioxid,
$\mathrm{MnO}_{2} \ldots \ldots \ldots \ldots .0 .04318$
Calcium hydroxid,
$\mathrm{Ca}(\mathrm{OH})_{2} \ldots \ldots \cdots 0.03678$

Decinormal Oxalic Acid ( $\frac{\mathrm{N}}{\mathrm{IO}}$ V. S. $=6.255$ gms. in rooo cc.)

Dissolve 6.255 gms. of pure oxalic acid in enough water to make at or near $\dot{1} 5^{\circ} \mathrm{C}$. exactly $1000 \mathrm{cc}$.

Normal Hydrochloric Acid $\left(\mathrm{HCl}=36 . \mathrm{I} 8 ; \frac{\mathrm{N}}{\mathrm{I}} \mathrm{V} . \mathrm{S} .=36.18 \mathrm{gms}\right.$. in $1000 \mathrm{cc}$.)

Mix I30 cc. of hydrochloric acid of sp.gr. I.I 63 with enough water to measure, at or near $15^{\circ} \mathrm{C}$., $1000 \mathrm{cc}$.

Of this liquid (which is still too concentrated) measure carefully into a flask or beaker ro cc., add a few drops of phenolphthalein T. S., 
and gradually add from a burette $\frac{\mathrm{N}}{\mathrm{I}}$ potassium hydroxid V. S. until a permanent pale pink tint is produced. Note the number of cc. of $\frac{\mathrm{N}}{\mathrm{I}}$ potassium hydroxid solution consumed, and then dilute the acid

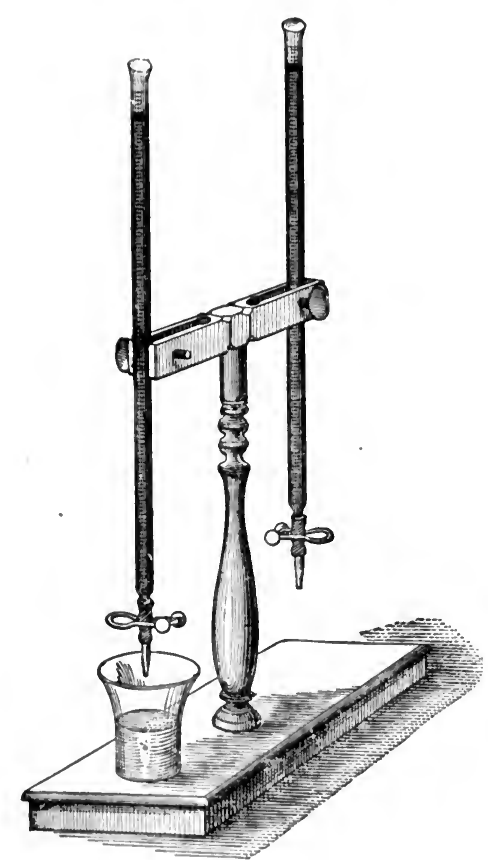

FIG. 46 . so that equal volumes of this and the $\frac{\mathrm{N}}{\mathrm{I}} \mathrm{KOH}$ V.S. neutralize each other.

Example: Assuming that the Io cc. of the acid solution re quired I2 cc. of the $\frac{\mathrm{N}}{\mathrm{I}} \mathrm{KOH}$, each Io cc. of the acid must be diluted to I2 cc., or the whole of the remaining acid in the same proportion.

After the dilution a new trial should be made. Io cc. of the acid V. S. should require exactly Io cc. of the alkali.

This method is fairly satisfactory if an accurately standardized normal potassium hydroxid solution is at hand; the latter, however, always contains a small quantity of carbonate, hence methyl orange would be more desirable as an indicator.

Other methods of standardizing acid solutions are:

Standardization by Means of the Specific Gravity. As the result of a careful experimental study of the various methods proposed for the standardization of acid volumetric solution, E. C. Worden and John Morton,* conclude that the most accurate and most easily performed method is that of taking the specific gravity in accurately calibrated pycnometers.

Standardization by Means of Borax. This is a ready and accurate method for the standardization of the strong mineral acids. The 
commercial borax is purified by recrystallization, the crystals are then finely pulverized and shaken with water at $25^{\circ}$ to $30^{\circ} \mathrm{C}$. The saturated solution is rapidly filtered and set aside to crystallize at a low temperature. The crystals so obtained are quite pure, and after draining are thoroughly dried between sheets of filtering paper, but without the use of heat or an exsiccator.

The neutralization takes place according to the following equation:

$$
\mathrm{Na}_{2} \mathrm{~B}_{4} \mathrm{O}_{7} \cdot 1 \circ \mathrm{H}_{2} \mathrm{O}+{ }_{2} \mathrm{HCl}={ }_{2} \mathrm{NaCl}+{ }_{4} \mathrm{H}_{3} \mathrm{BO}_{3} \text {. }
$$

Thus,

$$
379.40 \quad 72.36
$$

I89.7 gms. of borax neutralize 38.18 gms. of $\mathrm{HCl}$;

I89.7 gms. of borax neutralize rooo cc. $\frac{\mathrm{N}}{\mathrm{I}} \mathrm{HCl}$;

r.897 gms. of borax neutralize ro cc. $\frac{\mathrm{N}}{\mathrm{I}} \mathrm{HCl}$.

These figures form the basis for the standardization, which is conducted as follows:

r.897 $\mathrm{gm}$. of borax is accurately weighed, dissolved in $40 \mathrm{cc}$. of water, three drops of methyl orange * added, and then the hydrochloric acid solution to be standardized is run in from a burette until a red color appears. If for example $9 \mathrm{cc}$. are required, then each $9 \mathrm{cc}$. of the acid solution must be diluted to so cc., or the whole of the remaining solution in the same proportion. After the dilution a new trial should be made, and then the $1.897 \mathrm{gms}$. of borax should require exactly ro $\mathrm{cc}$. of the acid solution. If more than ro $\mathrm{cc}$. are required the acid solution is too weak.

Volumetric Standardization by Means of Silver Nitrate. The standardization of $\frac{N}{\mathrm{I}}$ hydrochloric acid may be effected also, by means of decinormal silver nitrate solution, as per the equation

$$
\underset{36.18}{\mathrm{HCl}}+\underset{168.69}{\mathrm{AgNO}_{3}}=\mathrm{AgCl}+\mathrm{HNO}_{3} .
$$

36.18 gms. of $\mathrm{HCl}=1000 \mathrm{cc}$. of $\frac{\mathrm{N}}{\mathrm{I}} \mathrm{V}$. S.

I6.869 gms. of $\mathrm{AgNO}_{3}=1000$ cc. of $\frac{\mathrm{N}}{\mathrm{IO}} \mathrm{V} . \mathrm{S}$.

* Phenolphthalein and litmus are inapplicable here. 
If Io cc. of the hydrochloric-acid solution are titrated with $\frac{\mathrm{N}}{10} \mathrm{AgNO}_{3}$ and $108 \mathrm{cc}$. of the latter are required, then each ro cc. must be diluted to $\left(\frac{108}{10}\right)=10.8 \mathrm{cc}$. If less than $100 \mathrm{cc}$. of the $\frac{\mathrm{N}}{10}$ silver nitrate were required, the acid solution is too dilute and a stronger solution should be prepared. After dilution, a second trial should be made, and the ro cc. of acid solution should then require for complete precipitation exactly roo cc. of $\frac{\mathrm{N}}{\mathrm{IO}} \mathrm{AgNO}_{3}$.

Another way is to take exactly 1.687 gms. of pure silver nitrate, dissolve it in $50 \mathrm{cc}$. of water, and then add the hydrochloric acid solution from a burette. Exactly ro cc. should be required. If less is required the solution must be diluted accordingly.

Gravimetric Standardization by Means of Silver Nitrate.* In this method the titer of the hydrochloric acid solution is determined by weighing the silver chlorid which a definite volume of it precipitates.

$20 \mathrm{cc}$. of the hydrochloric acid solution to be standardized are transferred to a $250 \mathrm{cc}$. Erlenmeyer flask, provided with a well-fitting rubber stopper. $50 \mathrm{cc}$. of a 5 per cent solution of silver nitrate are then added, the stopper inserted, and the flask shaken. Then sufficient of the silver solution is to be added to complete the precipitation. This should be added in portions of less than I cc. each and the flask stoppered and shaken after each addition. A large excess of silver nitrate must be avoided, because it possesses a slight solvent action upon the precipitate and necessitates much washing for its complete removal.

The precipitate is then allowed to settle, and the supernatant liquid when quite clear carefully decanted through a Gooch crucible. The precipitate is then twice or thrice shaken with pure water, faintly acidulated with nitric acid, and each time carefully decanted into the crucible as before, and finally the precipitate is transferred (by the use of a "spritz" bottle with pure water) to the crucible and dried in the air-bath at $130^{\circ}$ to $150^{\circ} \mathrm{C}$. to constant weight.

$$
\underset{36.18}{\mathrm{HCl}}+\mathrm{AgNO}_{3}=\underset{142.3}{\mathrm{AgCl}}+\mathrm{HNO}_{3} .
$$

*Hopkins, J. A. C. S., 1901, p. 72?. 
142.3 gms. of $\mathrm{AgCl}=36.18$ gms. of $\mathrm{HCl}=1000 \mathrm{cc}$. of $\frac{\mathrm{N}}{1} \mathrm{~V} . \mathrm{S}$;

r.423 gms. of $\mathrm{AgCl}=0.36 \mathrm{I} 8 \mathrm{gm}$. of $\mathrm{HCl}=$ ro cc. of $\frac{\mathrm{N}}{\mathrm{I}} \mathrm{V} . \mathrm{S}$.;

2.846 gms. of $\mathrm{AgCl}=0.7236 \mathrm{gm}$. of $\mathrm{HCl}=20 \mathrm{cc}$. of $\frac{\mathrm{N}}{\mathrm{I}} \mathrm{V} . \mathrm{S}$.

Supposing that the precipitate weighed 3.557 gms., then the solution is too strong and must be diluted thus:

If 2.846 gms. of $\mathrm{AgCl}=0.723 \mathrm{I}$ gm. of $\mathrm{HCl}$ or $20 \mathrm{cc}$. of its $\frac{\mathrm{N}}{\mathrm{I}} \mathrm{V}$. S., then 3.557 gms. of $\mathrm{AgCl}$ will represent

$$
\frac{3 \cdot 5.57}{2.846} \times: 0=25 \mathrm{cc}
$$

Therefore, each $20 \mathrm{cc}$. of the hydrochloric acid solution must be diluted to $25 \mathrm{cc}$. in order to make it a normal solution.

Standardization by Means of Calc-spar. Very accurate results may be attained by the use of pure crystallized calcium carbonate (Calc-spar, Iceland-spar), which, unlike an alkali, is not subject to hygroscopic variation.

$$
\begin{aligned}
& \mathrm{CaCO}_{3}+{ }_{2} \mathrm{HCl}=\mathrm{CaCl}+\mathrm{H}_{2} \mathrm{O}+\mathrm{CO}_{2} \text {. } \\
& \text { 2) } 99.35 \quad \text { 2) } \frac{72.36}{49.675} \text { gms. }=36.18 \text { gms. }=\text { rooo cc. of } \frac{\mathrm{N}}{\mathrm{I}} \mathrm{V} \text {. S. }
\end{aligned}
$$

Referring to the equation it will be seen that rooo cc. of $\frac{\mathrm{N}}{\mathrm{I}} \mathrm{HCl}$ will dissolve 49.675 gms. of pure $\mathrm{CaCO}_{3}$. Ioo cc. will therefore dissolve 4.9675 gms.

Weigh out 6 gms. of calc-spar in small pieces in a tared flask and add roo cc. of the acid solution to be standardized. When the reaction has ceased, pour off the solution, wash the residual in the beaker with water, pour off, and after drying the contents of the flask, weigh and deduct from original weight. The difference represents the $\mathrm{CaCO}_{3}$ which was dissolved.

Supposing that this difference be 5.65 gms., then the acid solution is too strong and must be diluted so that roo cc. will dissolve no more than 4.9675 gms. of $\mathrm{CaCO}_{3}$. 
The amount of dilution required in this case may be calculated thus:

$$
\frac{100}{4 ., 675} \times 5.65=113.74
$$

That is, each $100 \mathrm{cc}$. of the acid solution must be diluted to measure II $3.74 \mathrm{cc}$. This being the quantity of $\frac{\mathrm{N}}{\mathrm{I}} \mathrm{HCl} \mathrm{V.} \mathrm{S.} \mathrm{which}$ will dissolve 5.65 gms. of $\mathrm{CaCO}_{3}$. J. P. Catford (Chemist and Druggist), suggests the use of marble. The frequent presence of magnesium in this, the saturating power of whose carbonate is greater, weight for weight, than calcium carbonate, makes it necessary to determine the quantity present in the sample. If this quantity is small, the slight excess in saturating power is usually counterbalanced by the presence of traces of silica, and the sample may be weighed as $\mathrm{CaCO}_{3}$.

Half Normal Hydrochloric Acid $\left(\mathrm{HCl}=36.18 . \frac{\mathrm{N}}{2} \mathrm{~V} . \mathrm{S} .=\mathrm{I} 8.06\right.$ gms. in $1000 \mathrm{cc}$.).

Normal Sulphuric Acid $\left(\mathrm{H}_{2} \mathrm{SO}_{4}=97 \cdot 35 \cdot \frac{\mathrm{N}}{\mathrm{I}} \mathrm{V} . \mathrm{S} .=48.675 \mathrm{gms}\right.$. in $1000 \mathrm{cc}$.).

Mix carefully $30 \mathrm{cc}$. of pure concentrated sulphuric acid (sp.gr. r.835) with enough water to make about ro50 cc., and allow the liquid to cool to about $15^{\circ} \mathrm{C}$.

Titrate Io cc. of this liquid in the manner described under $\frac{\mathrm{N}}{\mathrm{I}}$ hydrochloric acid, and dilute it so that equal volumes of the acid and the alkali will neutralize each other.

The standardization of normal acid solutions may also be effected by the use of pure anhydrous sodium carbonate.

Standardization by Means of Sodium Carbonate. Pure anhydrous sodium carbonate may be obtained by heating to dull redness a few grams of pure sodium bicarbonate for about fifteen minutes. The resulting carbonate is practically free from impurity.

The sodium bicarbonate loses on ignition one half of its carbonic. acid gas:

$$
{ }_{2} \mathrm{NaHCO}_{3}+\text { Heat }=\mathrm{Na}_{2} \mathrm{CO}_{3}+\mathrm{CO}_{2}+\mathrm{H}_{2} \mathrm{O} .
$$

The bicarbonate should, however, be tested before igniting, and if more than traces of chlorid, sulphate, or thiosulphate are found, 
these may be removed by washing a few hundred grams, first with a saturated solution of sodium bicarbonate, and afterward with distilled water.

$0.5265 \mathrm{gm}$. of the pure anhydrous sodium carbonate is accurately weighed and dissolved in about $20 \mathrm{cc}$. of water in a flask and a few drops of methyl orange T. S. added as indicator. The acid to be "set" or "standardized" is then run into the sodium-carbonate solution until a permanent light-red color is produced. It should require exactly ro cc. of the $\frac{\mathrm{N}}{\mathrm{I}}$ acid solution.

If $8 \mathrm{cc}$. of the acid solution are consumed to bring about the required result, then every $8 \mathrm{cc}$. must be diluted to 10 cc., or the whole of the remaining solution must be diluted in this proportion:

$$
\begin{aligned}
& \mathrm{Na}_{2} \mathrm{CO}_{3}+\mathrm{H}_{2} \mathrm{SO}_{4}=\mathrm{Na}_{2} \mathrm{SO}_{4}+\mathrm{H}_{2} \mathrm{O}+\mathrm{CO}_{2} \text {. } \\
& \text { 2) } \frac{105.3 \mathrm{I}}{52.65} \text { gms. } \frac{2 \lcm{98}}{49}=\text { to } 1000 \mathrm{cc} . \frac{\mathrm{N}}{\mathrm{I}} \mathrm{V} \text {. S.; } \\
& 0.5265 \mathrm{gm} \text {. }=\text { to } 10 \mathrm{cc} \text {. }
\end{aligned}
$$

This method may be employed as well for the standardization of hydrochloric or of oxalic acid.

Standardization by the Iodometric Method.-This is a very accurate method and depends upon the fact that when diluted mineral acids (as $\mathrm{HCl}$ or $\mathrm{H}_{2} \mathrm{SO}_{4}$ ) are brought in contact with a mixture of potassium iodid and iodate, an equivalent amount of iodin is set free, as shown by the equation

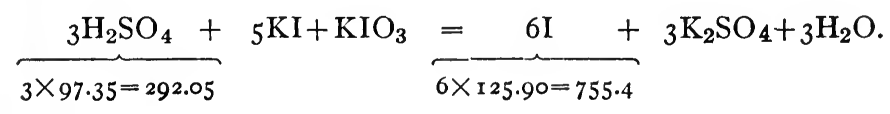

The liberated iodin is estimated by means of $\frac{\mathrm{N}}{\mathrm{I}}$ sodium thiosulphate solution.

The reaction between iodin and sodium thiosulphate is

$$
\underset{251.80}{\mathrm{I}_{2}+{ }_{25 \mathrm{Na}_{2}} \mathrm{~S}_{2} \mathrm{O}_{3} .5 \mathrm{H}_{2} \mathrm{O}=2 \mathrm{NaI}}+\mathrm{Na}_{2} \mathrm{~S}_{4} \mathrm{O}_{6}+\mathrm{IoH}_{2} \mathrm{O} .
$$

5 cc. (accurately measured) of the acid solution to be standardized are diluted with $50 \mathrm{cc}$. of water. 2 gms. of pure potassium iodid 
and $0.3 \mathrm{gm}$. of neutral potassium iodate are added, and the solution then carefully titrated with $\frac{\mathrm{N}}{\mathrm{IO}}$ sodium thiosulphate until the color of the iodin disappears, or if starch is used as indicator until the blue of starch iodid is destroyed. The quantity of $\frac{\mathrm{N}}{\mathrm{IO}}$ sodium thiosulphate used is divided by $10 *$ and the acid solution then diluted so that each $5 \mathrm{cc}$. will measure one tenth the quantity of $\frac{\mathrm{N}}{\text { IO }}$ thiosulphate used

Assuming that $60.6 \mathrm{cc}$. of the sodium thiosulphate solution were used, then each $5 \mathrm{cc}$. of the acid solution must be diluted to measure $6.06 \mathrm{cc}$.

Standardization by Ammonium Sulphate Method. $\dagger$ This consists in neutralizing the acid solution with pure ammonium hydroxid, evaporating and weighing the resulting ammonium sulphate. $25 \mathrm{cc}$. of the sulphuric acid solution to be standardized are carefully measured into a platinum evaporating dish. Ammonium hydroxid is then added in slight excess and the solution evaporated to dryness on a water-bath and the residue finally dried to constant weight at $120^{\circ} \mathrm{C}$.

$$
\underset{97.35}{\mathrm{H}_{2} \mathrm{SO}_{4}}+{ }_{2} \mathrm{NH}_{4} \mathrm{OH}=\underset{{ }_{1} \mathrm{I} \cdot 2 \mathrm{I}}{\left(\mathrm{NH}_{4}\right)_{2} \mathrm{SO}_{4}}+{ }_{2} \mathrm{H}_{2} \mathrm{O} .
$$

48.675 gms. or 1000 cc. $\frac{\mathrm{N}}{\mathrm{I}} \mathrm{H}_{2} \mathrm{SO}_{4}=65.605$ gms. $\left(\mathrm{NH}_{4}\right)_{2} \mathrm{SO}_{4}$;

4.8675 gms. or $100 \mathrm{cc} \cdot \frac{\mathrm{N}}{\mathrm{I}} \mathrm{H}_{2} \mathrm{SO}_{4}=6.5605$ gms. $\left(\mathrm{NH}_{4}\right)_{2} \mathrm{SO}_{4}$;

I.216875 gms. or 25 cc. $\frac{\mathrm{N}}{\mathrm{I}} \mathrm{H}_{2} \mathrm{SO}_{4}=\mathrm{I} .640+$ gms. $\left(\mathrm{NH}_{4}\right)_{2} \mathrm{SO}_{4}$.

Supposing the residue of ammonium sulphate, weighs I.84 gms., then

$$
\frac{1.84}{1.64} \times 25=28.04 \mathrm{cc} \text {. }
$$

*This converts the quantity of $\frac{\mathrm{N}}{10}$ thiosulphate into cubic centimeters of $\frac{\mathrm{N}}{\mathrm{I}}$ thiosulphate, which is equivalent to $\frac{\mathrm{N}}{\mathrm{I}}$ acid.

$\dagger$ M. Wenig, Ztschr. angew. Chem., IS92, p. 204. 
Therefore each $25 \mathrm{cc}$. of the acid solution must be diluted to $28.04 \mathrm{cc}$.

This method is simple, accurate, and rapid.

Standardization of Sulphuric Acid by Means of Barium Chlorid (Gravimetric). Iocc. of the acid solution to be standardized is diluted to about roo cc. and boiled in a covered beaker. To this boiling solution is slowly added a solution of pure barium chlorid until precipitation ceases. The precipitate is then thoroughly washed with hot water, and when all traces of barium chlorid have been removed it is dried and weighed. Suppose the precipitate of barium sulphate weighed $\mathrm{I} .24 \mathrm{gms}$. This corresponds to $0.5208 \mathrm{gm}$. of $\mathrm{H}_{2} \mathrm{SO}_{4}$ in the Io cc. As there should be only $0.48675 \mathrm{gm}$. present, we then measure off $4867.5 \mathrm{cc}$. and dilute it with water to the measure of $5208 \mathrm{cc}$. to make a normal solution.

Or we may calculate in this way, I.I 5875 gms. of barium sulphate corresponds to Iо cc. of the normal sulphuric acid, therefore $\mathrm{I} .24 \mathrm{gms}$. of barium sulphate will correspond to $10.7 \mathrm{cc}$. of the normal acid. Each Io cc. must therefore be dilute to $10.7 \mathrm{cc}$. or each IOO to $107 \mathrm{cc}$.

$$
\frac{10}{1.15^{875}} \times 1.24=10.7
$$

The reaction is thus illustrated

$$
\begin{aligned}
& \mathrm{H}_{2} \mathrm{SO}_{4}+\mathrm{BaCl}_{2}=\mathrm{BaSO}_{4}+{ }_{2} \mathrm{HCl} \text {. } \\
& \text { 2) } \frac{97.35}{48.675} \text { gms. }=1000 \mathrm{cc} . \frac{\mathrm{N}}{\mathrm{I}} \mathrm{V} . \mathrm{S} .=\frac{2)}{23 \mathrm{I} .75} \text { I } 5.875 \mathrm{gms} . \\
& 0.48675 \mathrm{gm} .=\quad \text { Iо cc. } \frac{\mathrm{N}}{\mathrm{I}} \text { V.S. }=\quad \text { 1.1 } 5875 \mathrm{gms} \text {. }
\end{aligned}
$$

Standardizatition of Sulphuric Acid may also be Affected by Means of the Specific Gravity. Besides normal sulphuric acid the U. S. P. also employs

and

$$
\begin{aligned}
& \text { Half normal } \frac{\mathrm{N}}{2} \text { sulphuric acid, } \\
& \text { Tenth normal } \frac{\mathrm{N}}{\mathrm{IO}} \text { sulphuric acid, } \\
& \text { Fiftieth normal } \frac{\mathrm{N}}{50} \text { sulphuric acid. }
\end{aligned}
$$




\section{ESTIMATION OF ALKALI HYDROXIDS}

Potassium and sodium hydroxids are usually titrated with $\frac{\mathrm{N}}{\mathrm{I}}$ sulphuric or hydrochloric acid; they are, however, so prone to absorb carbon dioxid out of the air that they are seldom free from carbonate, and hence the selection of an indicator is a matter of some importance. Phenolphthalein or litmus may be employed, but it is then advisable to boil the solution while titrating, in order to drive off the liberated carbon dioxid, because the latter gives an acid reaction with phenolphthalein and litmus and thus causes an end-reaction tint to appear before neutralization is complete. It is better, usually, to employ an indicator which is not affected by carbon dioxid. Methyl orange is mostly preferred; cochineal and Congo red are also useful. These indicators are especially serviceable in the presence of carbonates in that they are not affected by carbon dioxid, and can therefore be employed in direct titrations without the use of heat.

The quantity of carbonate in a recent sample of sodium or potassium hydroxid is so small usually that it is customary to disregard it and to report the total alkalinity as hydroxid.

A definite quantity of the sample (from $0.5 \mathrm{gm}$. to I gm. of the solid or an equivalent of a solution) is taken for analysis, dissolved in 30 to $50 \mathrm{cc}$. of water in a white porcelain dish or a beaker placed over a white surface, and a few drops of a suitable indicator added.

The vessel is then placed beneath a burette containing the standard acid solution and the latter run in, drop by drop, until the last drop just causes the color to change. The solution should be rotated or stirred after each addition of the standard acid.

The alkali hydroxids are so exceedingly hygroscopic that they take up water from the air while being weighed; it is therefore difficult to make a direct weighing with any degree of accuracy.

The best way is to take a small piece of the sample (about I gm.), place it immediately in a tared stoppered flask and take the weight accurately. It is then dissolved in water, transferred to the porcelain dish or beaker and titrated.

Potassium Hydroxid $(\mathrm{KOH}=55.74)$.

An accurately weighed portion (preferably less than I $\mathrm{gm}$.), is placed in a small beaker, dissolved in $50 \mathrm{cc}$. of water, three drops of methyl orange added, and the titration begun with $\frac{\mathrm{N}}{\mathrm{I}}$ sulphuric acid 
and continued until the yellow color of the solution is changed to red. Then the burette is carefully read to see how much of the acid solution was used. The number of cc. of the latter are multiplied by the normal factor for $\mathrm{KOH}(0.05574)$ and the result is the quantity of pure $\mathrm{KOH}$ in the sample taken for analysis.

The following equation illustrates the reaction:

$$
\begin{aligned}
& { }_{2} \mathrm{KOH}+\mathrm{H}_{2} \mathrm{SO}_{4}=\mathrm{K}_{2} \mathrm{SO}_{4}+{ }_{2} \mathrm{H}_{2} \mathrm{O} \\
& \text { 2) } \frac{\operatorname{III} .48}{55.74} \text { gms. } \frac{297.35}{48.675} \text { gms., quantity in } 1000 \text { cc. of } \frac{\mathrm{N}}{\mathrm{I}} \text { acid V. S.; } \\
& 0.05574 \mathrm{gm} \text {. (the factor for } \mathrm{KOH} \text { ), quantity neutralized by } \\
& \text { I cc. of } \frac{\mathrm{N}}{\mathrm{I}} \text { acid. }
\end{aligned}
$$

Thus rooo cc. of $\frac{\mathrm{N}}{\mathrm{I}} \mathrm{H}_{2} \mathrm{SO}_{4}$ V.S. containing 48.675 gms. of absolute $\mathrm{H}_{2} \mathrm{SO}_{4}$ will neutralize 55.74 gms. of $\mathrm{KOH}$. Therefore each cc. of $\frac{\mathrm{N}}{\mathrm{I}} \mathrm{H}_{2} \mathrm{SO}_{4}$ V.S. will neutralize $0.05574 \mathrm{gm}$. of pure $\mathrm{KOH}$.

Example. In the above analysis let it be assumed that $0.915 \mathrm{gm}$. of potassium hydroxid were taken and that $15.3 \mathrm{cc}$. of the standard acid were required to neutralize it, then $0.05574 \mathrm{gm} . \times_{15.3}=0.8528 \mathrm{gm}$. the quantity of pure $\mathrm{KOH}$ in the $0.915 \mathrm{gm}$. taken.

The percentage is then calculated in this way:

$$
\begin{aligned}
& 0.915: 0.8528:: \text { 100: } x ; \quad x=93.2+\text { per cent. } \\
& \frac{0.85^{28} \times 100}{0.915}=93.2 \text {. }
\end{aligned}
$$

Sodium Hydroxid $(\mathrm{NaOH}=39 \cdot 76)$.

This is estimated in exactly the same manner as described for potassium hydroxid, the following equation being applied:

$$
\begin{aligned}
& 2 \mathrm{NaOH}+\mathrm{H}_{2} \mathrm{SO}_{4}=\mathrm{Na}_{2} \mathrm{SO}_{4}+{ }_{2} \mathrm{H}_{2} \mathrm{O} \\
& \text { 2) } \underline{79.52} \quad \text { 2) } \\
& \frac{79.52}{39.76} \text { gms. } \frac{97.35}{48.675} \text { gms. }=\text { I } 000 \text { cc. } \frac{\mathrm{N}}{\mathrm{I}} \text { V.S. } \\
& \begin{array}{ll}
.03976 \mathrm{gm} . & \text { I cc. } \frac{\mathrm{N}}{\mathrm{I}} \text { V.S. } \\
\text { The factor. }
\end{array}
\end{aligned}
$$

The official solutions of potassium and of sodium hydroxid are estimated in this same manner, ro cc. may be taken for analysis, diluted with $20 \mathrm{cc}$. of water. 


\section{Ammonia Water $\left(\mathrm{NH}_{3} \cdot \mathrm{H}_{2} \mathrm{O}\right)$.}

3 cc. of ammonia water are put into a stoppered weighing bottle and the weight taken. $40 \mathrm{cc}$. of water are then added and the solution titrated with $\frac{\mathrm{N}}{\mathrm{I}}$ sulphuric acid. As indicator, litmus, methyl orange, or rosolic acid may be used. Phenolphthalein is useless for titrating ammonia and even methyl orange and rosolic acid are unsuitable in the presence of much salts of ammonium. Because of the volatile character of ammonia its solutions readily lose strength upon exposure. It is therefore best to measure a quantity into a weighing bottle and find its weight as directed for potassium hydroxids. If the specific gravity of the ammonia solution is known, the weight of a given volume is easily calculated, it being only necessary to multiply the volume in cc. by the sp.gr. Thus, if the sp.gr. of an ammonia solution is $0.95^{85}$ and the volume taken is $3 \mathrm{cc}$., the weight of the $3 \mathrm{cc}$. is $3 \times 0.9585=2.8755 \mathrm{gms}$.

In the titration with $\frac{\mathrm{N}}{\mathrm{I}}$ sulphuric acid each cc. of the latter represents $0.01693 \mathrm{gm}$. of $\mathrm{NH}_{3}$ as shown by the equation

$$
\begin{aligned}
& { }_{2} \mathrm{NH}_{3} \cdot \mathrm{H}_{2} \mathrm{O}+\mathrm{H}_{2} \mathrm{SO}_{4}=\left(\mathrm{NH}_{4}\right)_{2} \mathrm{SO}_{4}+{ }_{2} \mathrm{H}_{2} \mathrm{O} \\
& \frac{2) 33.86}{16.93} \text { gms. } \frac{2) 97.35}{48.675} \text { gms. }=1000 \text { cc. } \frac{\mathrm{N}}{\mathrm{I}} \mathrm{V} . \mathrm{S} \\
& \begin{array}{l}
.01693 \mathrm{gm} . \\
\text { Factor. }
\end{array} \quad \text { I cc. } \frac{\mathrm{N}}{\mathrm{I}} \text { V.S. }
\end{aligned}
$$

If 16.9 cc. of $\frac{\mathrm{N}}{\mathrm{I}}$ acid were required in the above assay, then $0.01693 \mathrm{gm} . \times 16.9=0.287 \mathrm{I}+\mathrm{gm}$., the quantity of pure $\mathrm{NH}_{3}$ in the 3 cc. (2.8755 gms.) of ammonia water taken.

The percentage is found as follows:

If $3 \mathrm{cc}$. of ammonia water weighing $2.8755 \mathrm{gms}$. contain $0.287 \mathrm{I}+\mathrm{gm}$. of $\mathrm{NH}_{3}$, Iо० gms. of ammonia water will contain $\times$ gm. of $\mathrm{NH}_{3}$

$$
\begin{gathered}
0.287 \mathrm{I} \times 100 \\
2.8755
\end{gathered}=9.98 \text { per cent. }
$$

Stronger ammonia water and spirit of ammonia may be estimated in the same manner.

The following are the alkalies which are official in the U.S. P.: 
Potassii hydroxidi. ............... 85 per cent.

Sodii hydroxidi. ................ 90

Liquor potassii hydroxidi. .......... 5

Liquor sodii hydroxidi............. 5

Aqua ammoniæ................. " 10 "

Aqua ammoniæ fortior. ............ 28

Spiritus ammoniæ.............. 10 ،

In the titration of all of these $\frac{\mathrm{N}}{\mathrm{I}}$ sulphuric acid is used and methyl orange is the indicator, except in the case of spiritus ammoniæ in which $\frac{\mathrm{N}}{2} \mathrm{H}_{2} \mathrm{SO}_{4}$ and litmus are recommended.

Estimation of Alkali Carbonates. When carbonates are treated with acids carbonic-acid gas is liberated. This gas shows an acid reaction with most indicators, and the reaction will seem to be completed before the alkali is entirely neutralized.

To avoid this, the titration may be conducted at the boiling temperature (hot way) in order to drive off the carbon dioxid. The standard acid being added until two minutes' boiling fails to restore the color indicating alkalinity. If the titration is conducted at a boiling temperature, it is advisable to attach to the lower end of the burette a long rubber tube with a pinch-cock fixed about midway on the tube.

The boiling can then be done at a little distance from the burette

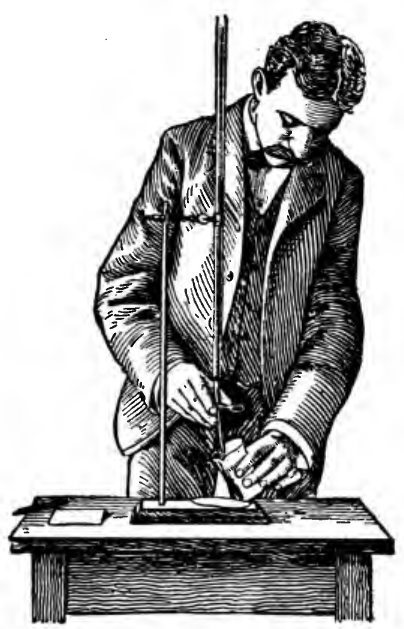

FIG. 47. and the expansion of the standard solution therein thus prevented.

Another method is to add to the carbonate a measured excess of the standard acid, and then after boiling to drive off the carbon dioxid, an indicator is added, and the excess of standard acid determined by titration with a standard alkali (residual titration way). The quantity of the latter deducted from the quantity of the standard acid taken, gives the quantity of the latter which reacted with the carbonate. 
Still another method is to titrate the carbonate direct, without heat (cold way), using an indicator which is not affected by carbon dioxid. The best of the indicators which are not so affected is methyl orange; others are cochineal and Congo red. When employing methyl orange, as in indicator, standard oxalic acid solution should not be used as the end-reaction is very indefinite and unreliable.

The end-reaction with this indicator, is at all events not a clearly marked one, and considerable practice and an eye for color is required to detect the point at which yellow changes to pale pink. It is a good plan to have on the bench two vials, one containing an acid and the other an alkali tinted with methyl orange, with which comparisons can be made.

Potassium Carbonate $\left(\mathrm{K}_{2} \mathrm{CO}_{3}=137.27\right)$.

Weigh carefully one gram of the salt, dissolve in a small quantity of water in a beaker or flask, add a few drops of methyl orange T. S., and titrate with normal sulphuric acid until a faint orange-red color appears.

$$
\begin{aligned}
& \mathrm{K}_{2} \mathrm{CO}_{3}+\mathrm{H}_{2} \mathrm{SO}_{4}=\mathrm{K}_{2} \mathrm{SO}_{4}+\mathrm{H}_{2} \mathrm{O}+\mathrm{CO}_{2} \\
& \text { 2) } \frac{\mathrm{I} 37.27}{68.635 \mathrm{gms} .} \frac{287.675}{\mathrm{gms} .}=1000 \mathrm{cc} . \frac{\mathrm{N}}{\mathrm{I}} \text { V.S. }
\end{aligned}
$$

Each cc. of $\frac{\mathrm{I}}{\mathrm{I}} \mathrm{H}_{2} \mathrm{SO}_{4}$, therefore, represents $0.068635 \mathrm{gm}$. of pure potassium carbonate.

If $\mathrm{r} 4.3 \mathrm{cc}$. of the normal acid are required the salt contains I $4.3 \times 0.068635 \mathrm{gm} .=0.98 \mathrm{I} 48 \mathrm{gm}$. of pure $\mathrm{K}_{2} \mathrm{CO}_{3}$ or $98 . \mathrm{I} 48$ per cent. If it is desired to use litmus or phenolphthalein, it will be necessary to boil the solution as described above.

Other alkali carbonates are estimated in exactly the same manner as this described for potassium carbonate.

Potassium Bicarbonate $\left(\mathrm{KHCO}_{3}=99.41\right)$.

$$
\begin{aligned}
& { }_{2} \mathrm{KHCO}_{3}+\mathrm{H}_{2} \mathrm{SO}_{4}=\mathrm{K}_{2} \mathrm{SO}_{4}+{ }_{2} \mathrm{H}_{2} \mathrm{O}+\mathrm{CO}_{2} \\
& \begin{array}{ll}
\text { 2) } 198.82 & \text { 2) } 97.35 \\
9
\end{array} \\
& 99.4 \text { I gms. } 48.675 \text { gms. }=1000 \text { cc. } \frac{N}{1} \text { V.S. }
\end{aligned}
$$

Each cc. of $\frac{\mathrm{N}}{\mathrm{I}}$ acid V. S. $=0.0994 \mathrm{I}$ gm. of $\mathrm{KHCO}_{3}$. 
Sodium Carbonate (crystallized) $\left(\mathrm{Na}_{2} \mathrm{CO}_{3} \cdot \mathrm{IoH}_{2} \mathrm{O}=284 . \mathrm{Ir}\right)$

$$
\begin{aligned}
& \mathrm{Na}_{2} \mathrm{CO}_{3} \cdot \mathrm{roH}_{2} \mathrm{O}+\mathrm{H}_{2} \mathrm{SO}_{4}=\mathrm{Na}_{2} \mathrm{SO}_{4}+\mathrm{Ir} \mathrm{H}_{2} \mathrm{O}+\mathrm{CO}_{2} \text {. } \\
& \text { 2) } \frac{284.1 \mathrm{Ir}}{\mathrm{I} 42.05} \text { gms. } \frac{97.35}{48.675} \text { gms. }=1000 \mathrm{cc} \cdot \frac{\mathrm{N}}{\mathrm{I}} \mathrm{V} . \mathrm{S} \text {. }
\end{aligned}
$$

Each cc. $\frac{\mathrm{N}}{\mathrm{I}}$ acid $=0.14^{2} \mathrm{gm}$. crystallized sodium carbonate.

Sodium Carbonate (anhydrous) $\left(\mathrm{Na}_{2} \mathrm{CO}_{3}=\mathrm{I05} \cdot 3 \mathrm{I}\right)$.

$\mathrm{Na}_{2} \mathrm{CO}_{3}+\mathrm{H}_{2} \mathrm{SO}_{4}=\mathrm{Na}_{2} \mathrm{SO}_{4}+\mathrm{H}_{2} \mathrm{O}+\mathrm{CO}_{2}$.
2) $105.3 \mathrm{I}$
52.65 gms. $\frac{97.35}{48.675}$ gms. $=1000$ cc. $\frac{N}{\mathrm{I}}$ V. S.

Each cc. of $\frac{\mathrm{N}}{\mathrm{I}}$ acid $=0.05^{2} 65 \mathrm{gm} . \mathrm{Na}_{2} \mathrm{CO}_{3}$.

Sodium Bicarbonate $\left(\mathrm{NaHCO}_{3}=83.43\right)$.

$$
\begin{aligned}
& { }_{2} \mathrm{NaHCO}_{3}+\mathrm{H}_{2} \mathrm{SO}_{4}=\mathrm{Na}_{2} \mathrm{SO}_{4}+{ }_{2} \mathrm{H}_{2} \mathrm{O}+{ }_{2} \mathrm{CO}_{2} . \\
& \frac{266.86}{83.43} \text { gms. } \frac{97.35}{48.675} \text { gms. }=1000 \text { cc. } \frac{\mathrm{N}}{\mathrm{I}} \text { V.S. }
\end{aligned}
$$

Each cc. of $\frac{\mathrm{N}}{\mathrm{I}}$ acid $=0.08343 \mathrm{gm} . \mathrm{NaHCO}_{3}$. ..

Lithium Carbonate $\left(\mathrm{Li}_{2} \mathrm{CO}_{3}=73.5 \mathrm{I}\right)$.

$$
\begin{aligned}
& \mathrm{Li}_{2} \mathrm{CO}_{3}+\mathrm{H}_{2} \mathrm{SO}_{4}=\mathrm{Li}_{2} \mathrm{SO}_{4}+\mathrm{H}_{2} \mathrm{O}+\mathrm{CO}_{2} \text {. } \\
& \text { 2) } \frac{73.5 \mathrm{I}}{36.75} \text { gms. } \frac{97.35}{48.675} \mathrm{gms} .=1000 \mathrm{cc} . \frac{\mathrm{N}}{\mathrm{I}} \text { V.S. }
\end{aligned}
$$

Each cc. of $\frac{\mathrm{N}}{\mathrm{I}}$ acid $=0.03675 \mathrm{gm} . \mathrm{Li}_{2} \mathrm{CO}_{3}$.

\section{Ammonium Carbonate $\left(\mathrm{N}_{3} \mathrm{H}_{11} \mathrm{C}_{2} \mathrm{O}_{5}=\mathrm{I}_{5} 6.0 \mathrm{I}\right)$.}

Normal ammonium carbonate has the formula $\left(\mathrm{NH}_{4}\right)_{2} \mathrm{CO}_{3}$, but the normal salt loses upon exposure $\mathrm{NH}_{3}$ and $\mathrm{H}_{2} \mathrm{O}$. The commercial salt, therefore, generally is a mixture of bicarbonate and carbamate.

$$
\begin{aligned}
& \left(\mathrm{NH}_{4}\right)_{2} \mathrm{CO}_{3}-\mathrm{NH}_{3}=\mathrm{NH}_{4} \mathrm{HCO}_{3} ; \\
& \left(\mathrm{NH}_{4}\right)_{2} \mathrm{CO}_{3}-\mathrm{H}_{2} \mathrm{O}=\mathrm{NH}_{4} \mathrm{NH}_{2} \mathrm{CO}_{2} .
\end{aligned}
$$

The commercial carbonate is therefore generally expressed thus:

$$
\text { . } \mathrm{NH}_{4} \mathrm{HCO}_{3} \cdot \mathrm{NH}_{4} \mathrm{NH}_{2} \mathrm{CO}_{2} \text { or } \mathrm{N}_{3} \mathrm{H}_{11} \mathrm{C}_{2} \mathrm{O}_{5} \text {. }
$$

This salt may be estimated by direct titration with normal or decinormal acid, using rosolic acid or methyl orange as an indicator. 
Two grams of the salt are taken dissolved in about $50 \mathrm{cc}$. of water and titrated with $\frac{\mathrm{N}}{\mathrm{I}} \mathrm{H}_{2} \mathrm{SO}_{4}$ V. S. The reaction is as follows:

$$
\begin{aligned}
& { }_{2} \mathrm{~N}_{3} \mathrm{H}_{11} \mathrm{C}_{2} \mathrm{O}_{5}+{ }_{3} \mathrm{H}_{2} \mathrm{SO}_{4}=3\left(\mathrm{NH}_{4}\right)_{2} \mathrm{SO}_{4}+{ }_{4} \mathrm{CO}_{2}+{ }_{2} \mathrm{H}_{2} \mathrm{O} \\
& \text { 6) } \frac{312.02}{52.003} \text { gms. } \frac{292.05}{48.675} \mathrm{gms} .=1000 \mathrm{cc} \cdot \frac{\mathrm{N}}{\mathrm{I}} \text { acid V. S. }
\end{aligned}
$$

Each cc. of $\frac{\mathrm{N}}{\mathrm{I}}$ acid V. S. represents $0.05^{2}$ gm. of $\mathrm{N}_{3} \mathrm{H}_{11} \mathrm{C}_{2} \mathrm{O}_{5}$ or $0.01693 \mathrm{gm}$. of $\mathrm{NH}_{3}$.

If in this titration $37.3 \mathrm{cc}$. of the standard acid are required then the two grams of ammonium carbonate contained $0.05^{2} \mathrm{gm} . \times 37.3$ $=\mathrm{I} .939 \mathrm{gms}$. of the salt.

$$
\frac{1.939 \times 100}{2}=96.9 \text { per cent. }
$$

If rosolic acid is used as indicator heat must be applied to expel carbon dioxid. The estimation of the carbonic acid may be effected by precipitating a definite weight of the salt with barium chlorid, collecting the precipitated barium carbonate, dissolving it in a measured excess of normal hydrochloric acid and retitrating with normal alkali, as described in Chapter XVII.

The method usually employed hy skilled analysts (the residual titration method), is to add a measured excess of the standard acid solution, and thus convert the ammonium carbonate into the less volatile ammonium sulphate; then gently boil to get rid of $\mathrm{CO}_{2}$, and titrate back with a standard alkali V.S. (using litmus as an indicator) until the excess of acid is neutralized. The quantity of free acid thus found, when deducted from the amount of acid first added, gives the quantity which was required to neutralize the ammonium carbonate.

Thus, 2 gms. in solution of ammonium carbonate are treated with 50 cc. of $\frac{\mathrm{N}}{\mathrm{I}} \mathrm{H}_{2} \mathrm{SO}_{4} \mathrm{~V}$. S., which is more than sufficient to neutralize it; the solution is then gently boiled to drive off $\mathrm{CO}_{2}$, a few drops of litmus tincture added, and then titrated with $\frac{\mathrm{N}}{\mathrm{I}} \mathrm{KOH}$ V. S. until the litmus no longer shows an acid reaction and the solution is neutral. 
Let us assume that 12.7 cc. of the $\frac{\mathrm{N}}{\mathrm{I}} \mathrm{KOH}$ V. S. were used. By deducting the $12.7 \mathrm{cc}$. from the $50 \mathrm{cc}$. of $\frac{\mathrm{N}}{\mathrm{I}}$ acid first added, we find $37.3 \mathrm{cc}$. of the acid went into combination with the ammonium salt, the calculation is then made as described above.

\section{MIXED ALKALI HYDROXID AND CARBONATE}

If it is desired to ascertain the proportion in which these exist in a mixture, we proceed as follows:

First determine the total alkalinity by means of normal hydrochloric acid, using methyl orange as indicator. Then dissolve a like quantity of the mixture in $15 \circ \mathrm{cc}$. of water and add sufficient barium chlorid to precipitate all of the carbonate as barium carbonate, and then add water to make $200 \mathrm{cc}$. and set aside to settle. When the supernatant liquid is clear take one fourth $(50 \mathrm{cc}$.) of it, and titrate with normal hydrochloric acid, using phenolphthalein as indicator.* The number of cc. multiplied by 4 will be the quantity of normal acid required by the caustic alkali. The difference between this and the number of cc. representing the total alkalinity is calculated as carbonate.

Example. Assuming that we are analyzing a mixture of sodium hydroxid and carbonate.

Two grams of the substance are dissolved in water and titrated with normal acid solution. $43.2 \mathrm{cc}$. of the latter are required. Another 2 grams is dissolved, treated with barium chlorid as directed, and one fourth of the clear solution titrated with normal acid. $5.6 \mathrm{cc}$. are required; then $5.6 \times_{4}=22.4$ cc., representing the sodium hydroxid.

$43.2 \mathrm{cc} .=$ total alkalinity;

$-22.4 \times 0.0397=0.889$ grams sodium hydroxid;

$20.8 \times 0.0526=1.094$ grams sodium carbonate.

Another way is to filter the mixture after barium chlorid has been added, titrate the filtrate with normal acid to find the quantity of

* The slight error which occurs in this method because the volume of the precipitate is included in the measured liquid, may be overcome by using the entire quantity of liquid, including the precipitate (instead of taking one-fourth of it), and titrating with oxalic acid V.S. in the presence of phenolphthalein. Oxalic acid in very dilute solutions does not react with alkali earth carbonates. 
hydroxid, then dissolve the precipitater barium carbonate in normal hydrochloric acid in excess, and retiterate with normal alkali, thus ascertaining the amount of carbonate.

When the alkaline carbonate is present in very small quantities the method of Lunge may be employed.

A few drops of phenacetolin solution are added to impart a scarcely perceptible yellow to the liquid. Normal acid solution is then run in until a pale rose tint appears, indicating that all the alkali hydroxid is neutralized; the volume of acid is noted, and the titration continued; the red color is intensified, and when the carbonate is entirely decomposed a golden-yellow color results.

Considerable practice is required with solutions of known composition to accustom the eye to the changes of color.

ESTIMATION OF ALKALI BICARBONATES WHEN MIXED WITH CARBONATES

Thompson's Method. Take 2 grams of the salt and dissolve in roo cc. of water. Divide the solution into two equal parts and titrate one portion with normal acid solution, using methyl orange as indicator, and note the quantity required. We will assume $13 \mathrm{cc}$.

Then treat the second portion with a measured excess (say $25 \mathrm{cc}$.) of normal sodium hydroxid solution free from $\mathrm{CO}_{2}$. This converts the bicarbonate into carbonate. Now add an excess of pure neutral barium chlorid solution in order to precipitate all the carbonate as barium carbonate, and then titrate with normal acid, using phenolphthalein as indicator, to determine the excess of sodium hydroxid. 15 cc. are required. Thus

and

$25-15=10 \mathrm{cc}$., the equivalent of bicarbonate,

$13-10=3 \mathrm{cc}$., the equivalent of carbonate;

10 $\times .0834=.834$ gm., sodium bicarbonate;

$3 \times .0526=.1578 \mathrm{gm}$., sodium carbonate.

\section{ESTIMATION OF ALKALIES IN THE PRESENCE OF SULPHITFS}

This is accomplished by adding hydrogen peroxid to the solution in order to convert the sulphite into sulphate, and then titrating in the usual way with normal acid. 
MIXED POTASSIUM AND SODIUM HYDROXIDS

These are estimated by treatment with tartaric acid solution, which converts them into bitartrates. The bitartrate of potassium is almost insoluble in solution of sodium bitartrate and hence may be separated by filtering. The sodium bitartrate is estimated in the filtrate by titration with normal sodium hydroxid solution. The potassium is found by difference.

Estimation of Organic Salts of the Alkalies. The tartrates, citrates, and acetates of the alkali metals are converted by ignition into carbonates, the whole of the base remaining in the form of carbonate.

Each molecular weight of a normal tartrate gives when ignited one molecular weight of carbonate:

$$
\underline{\mathrm{K}_{2}} \mathrm{C}_{4} \mathrm{H}_{4} \mathrm{O}_{6}=\underline{\mathrm{K}_{2}} \mathrm{CO}_{3} \text {. }
$$

Every two molecular weights of an acetate or an acid tartrate give one molecular weight of carbonate:

$$
\begin{aligned}
& { }_{2} \mathrm{KC}_{2} \mathrm{H}_{3} \mathrm{O}_{2}=\mathrm{K}_{2} \mathrm{CO}_{3} \text {; } \\
& { }_{2} \mathrm{KHC}_{4} \mathrm{H}_{4} \mathrm{O}_{6}=\overline{\mathrm{K}}_{2} \mathrm{CO}_{3} \text {. }
\end{aligned}
$$

Every two molecular weights of a normal citrate give three molecular weights of carbonate:

$$
\underline{{ }_{2} \mathrm{~K}_{3}} \mathrm{C}_{6} \mathrm{H}_{5} \mathrm{O}_{7}=\underline{{ }_{3} \mathrm{~K}_{2}} \mathrm{CO}_{3} .
$$

These reactions are taken advantage of in volumetric analysis, and the tartrates, citrates, and acetates of the alkalies are indirectly estimated by calculating upon the quantity of carbonate formed by burning them, the quantity of carbonate being found by titration in the usual manner.

The Process. Before igniting, the salt to be examined should be thoroughly dried in a desiccator over calcium chlorid or in a drying oven, the latter only for such salts as have no water of crystallization in their composition. If the weight is taken before and after, the amount of moisture present is determined. One or two grams of the dried salt is weighed accurately, placed in a porcelain crucible, and heat applied gradually, until dull redness is reached and white fumes cease to be given off. Upon applying heat to the salt, the latter swells, 
fuses, and then boils, and if the heat is applied too rapidly at this point, there is apt to be a considerable loss of material through sputtering. The completion of the ignition is known to be reached when the black contents of the crucible is dry and crisp. The crucible is then allowed to cool, and its contents treated with boiling water to dissolve out the alkali carbonate, and the solution filtered through a small, wetted filter into a flask or beaker. The filtrate should be perfectly colorless. If it has a yellow or brownish color it indicates incomplete ignition and should be rejected, and a fresh quantity of the salt subjected to ignition. The contents of the crucible and the filter should be washed with several small portions of water until the washings no longer show an alkaline reaction. The filtrate mixed with the wash water is now titrated with standard sulphuric or hydrochloric acid, using methyl orange as the indicator. From the quantity of carbonate found in the filtrate, the equivalent amount of the organic salt may be calculated. The quantity of standard acid employed is multiplied direct by the factor for the original salt.

In the case of salts of the alkali earths,* residual titration should be resorted to. The residue in the crucible being dissolved in standard hydrochloric acid, and retitrated with standard alkali.

Lithium salts, because of the sparing solubility of the carbonate in water, should also be titrated by the residual method.

Potassium Tartrate $\left({ }_{2} \mathrm{~K}_{2} \mathrm{C}_{4} \mathrm{H}_{4} \mathrm{O}_{6}+\mathrm{H}_{2} \mathrm{O}=476.16\right)$. Two grams of the salt are placed in a platinum or porcelain crucible and heated to redness in contact with the air until completely charred; that is to say, until nothing is left in the crucible but carbonate and free carbon.

The crucible is now cooled, and its contents treated with boiling water, which dissolves the potassium carbonate, the carbon being separated by filtration. In order to obtain every trace of carbonate it is well to wash the crucible with several small portions of hot water, and add the washings to the rest of the filtrate through the filter.

If the salt is completely carbonized the filtrate will be colorless, but if the carbonization is not complete the solution will be more or less colored and should be rejected, and a fresh quantity of the salt subjected to ignition.

* Organic salts of the alkali earths subjected to ignition as above are reduced partly to oxids. 
To the filtrate, which contains potassium carbonate, add a few drops of methyl-orange, and titrate with $\frac{\mathrm{N}}{\mathrm{I}}$ sulphuric acid V.S. until a light orange-red color appears ${ }^{\circ}$ and the carbonate is neutralized.

The following equations will explain the reactions:

$$
\underbrace{*_{2} \mathrm{~K}_{2} \mathrm{C}_{4} \mathrm{H}_{4} \mathrm{O}_{6}+\mathrm{H}_{2} \mathrm{O}}_{476.16}+{ }_{5} \mathrm{O}_{2}={ }_{2} \mathrm{~K}_{2} \mathrm{CO}_{3}+6 \mathrm{CO}_{2}+{ }_{54.54} \mathrm{H}_{2} \mathrm{O} \text {; }
$$

then

therefore

$$
\underset{274.54}{2 \mathrm{~K}_{2} \mathrm{CO}_{3}}+\underset{2}{2 \mathrm{H}_{2} \mathrm{SO}_{4}}=\underset{2}{\mathrm{I}_{2}} \mathrm{SO}_{4}+{ }_{2} \mathrm{H}_{2} \mathrm{O}+{ }_{2} \mathrm{CO}_{2} ;
$$

$$
\begin{aligned}
& { }_{2} \mathrm{~K}_{2} \mathrm{C}_{4} \mathrm{H}_{4} \mathrm{O}_{6}+\mathrm{H}_{2} \mathrm{O}={ }_{2} \mathrm{~K}_{2} \mathrm{CO}_{3}={ }_{2} \mathrm{H}_{2} \mathrm{SO}_{4} \text {, }
\end{aligned}
$$

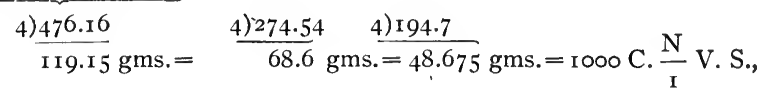

and each cc. of $\frac{\mathrm{N}}{\mathrm{I}} \mathrm{H}_{2} \mathrm{SO}_{4}$ represents $0.1 \mathrm{I} 9 \mathrm{gm}$. of potassium tartrate.

Example. Two gms. of potassium tartrate treated as described above require $16.3 \mathrm{cc}$. of $\frac{\mathrm{N}}{\mathrm{I}} \mathrm{H}_{2} \mathrm{SO}_{4} \mathrm{~V}$. S. It therefore contains $0.119 \times 16.3=1.9397 \mathrm{gms}$.

$$
\frac{\mathrm{I} .9397 \times 100}{2}=96.98 \text { per cent. }
$$

Potassium and Sodium Tartrate $\left(\mathrm{KNaC}_{4} \mathrm{H}_{4} \mathrm{O}_{6} \cdot 4 \mathrm{H}_{2} \mathrm{O}=280.18\right)$ (Rochelle Salt). This salt is treated in exactly the same way as described for potassium tartrate.

When ignited the double tartrate is converted into a double carbonate of potassium and sodium:

$$
\dagger \underbrace{2\left(\mathrm{KNaC}_{4} \mathrm{H}_{4} \mathrm{O}_{6} \cdot{ }_{4} \mathrm{H}_{2} \mathrm{O}\right)}_{560.36}+{ }_{5} \mathrm{O}_{2}={ }_{2} \mathrm{KNaCO}_{3}+6 \mathrm{CO}_{2}+\mathrm{I}_{2} \mathrm{H}_{2} \mathrm{O} ;
$$

* Since some carbon is always left behind, the reaction is probably more accurately written thus:

$$
{ }_{2} \mathrm{~K}_{2} \mathrm{C}_{4} \mathrm{H}_{4} \mathrm{O}_{6}+\mathrm{H}_{2} \mathrm{O}={ }_{2} \mathrm{~K}_{2} \mathrm{CO}_{3}+{ }_{5} \mathrm{C}+\mathrm{CO}_{2}+{ }_{5} \mathrm{H}_{2} \mathrm{O} .
$$

$\dagger$ The reaction is probably more accurately written thus:

$$
2\left(\mathrm{KNaC}_{4} \mathrm{H}_{4} \mathrm{O}_{6} \cdot{ }_{4} \mathrm{H}_{2} \mathrm{O}\right)=2 \mathrm{KNaCO}_{3}+{ }_{5} \mathrm{C}+\mathrm{CO}_{2}+\mathrm{I}_{2} \mathrm{H}_{2} \mathrm{O} \text {. }
$$


then

$$
\underset{24.258}{2 \mathrm{KNaCO}_{3}}+\underset{194.7}{\underset{2}{\mathrm{H}_{2} \mathrm{SO}_{4}}}=2 \underset{\mathrm{KNaSO}_{4}}{\mathrm{~K}_{2} \mathrm{CO}_{2}+{ }_{2} \mathrm{H}_{2} \mathrm{O}} ;
$$

therefore

$$
\begin{aligned}
& { }_{2} \mathrm{KNaC}_{4} \mathrm{H}_{4} \mathrm{O}_{6} \cdot \mathrm{H}_{2} \mathrm{O}={ }_{2} \mathrm{KNaCO}_{3}={ }_{2} \mathrm{H}_{2} \mathrm{SO}_{4} \text {, } \\
& \frac{4) 560.36}{140.04} \quad \frac{4) 242.56}{60.6} \quad \frac{4) 194.7}{48.675}=1000 \text { cc. } \frac{N}{I} \text { V.S. }
\end{aligned}
$$

and each cc. of $\frac{\mathrm{N}}{\mathrm{I}} \mathrm{H}_{2} \mathrm{SO}_{4}$ represents O.I $40 \mathrm{gm}$. of $\mathrm{KNaC}_{4} \mathrm{H}_{4} \mathrm{O}_{6} \cdot{ }_{4} \mathrm{H}_{2} \mathrm{O}$.

Example. If one gram of Rochelle salt treated as above described, requires 7 cc. of $\frac{\mathrm{N}}{\mathrm{I}}$ sulphuric acid, it contains $0 . \mathrm{I} 40 \times 7=0.980 \mathrm{gm} .=98$ per cent.

Potassium Bitartrate (Cream of Tartar) $\left(\mathrm{KHC}_{4} \mathrm{H}_{4} \mathrm{O}_{6}={ }_{\mathrm{I}} 86.78\right)$. The estimation of this salt is affected in the same way as the tartrate.

The bitartrate having but one atom of potassium in its molecule, it takes two molecules to form one molecule of carbonate.

$$
\underset{373.56}{*{ }_{2} \mathrm{KHC}_{4} \mathrm{H}_{4} \mathrm{O}_{6}}+{ }_{5} \mathrm{O}_{2}=\underset{137.27}{\mathrm{~K}_{2} \mathrm{CO}_{3}}+{ }_{7} \mathrm{CO}_{2}+{ }_{5} \mathrm{H}_{2} \mathrm{O} ;
$$

then

$$
\underset{{ }_{3} 7.27}{\mathrm{~K}_{2} \mathrm{CO}_{3}}+\underset{97.35}{\mathrm{H}_{2} \mathrm{SO}_{4}}=\mathrm{K}_{2} \mathrm{SO}_{4}+\mathrm{H}_{2} \mathrm{O}+\mathrm{CO}_{2} ;
$$

therefore

$$
\begin{aligned}
& { }_{2} \mathrm{KHC}_{4} \mathrm{H}_{4} \mathrm{O}_{6}=\mathrm{K}_{2} \mathrm{CO}_{3}=\mathrm{H}_{2} \mathrm{SO}_{4} \text {. }
\end{aligned}
$$

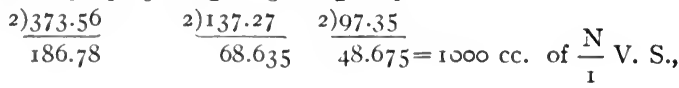

and each cc. of $\frac{\mathrm{N}}{\mathrm{I}} \mathrm{H}_{2} \mathrm{SO}_{4}$ V.S. $=0.18678$ gm. of $\mathrm{KHC}_{4} \mathrm{H}_{4} \mathrm{O}_{6}$.

Another way of estimating bitartrate is to dissolve a weighed quantity in hot water and titrate with $\frac{\mathrm{N}}{\mathrm{I}}$ potassium hydroxid until neutral, and thus the amount of tartaric acid existing as bitartrate is found. The bitartrate is acid in reaction. In detail the method is as follows:

* The reaction may also be written thus:

$$
{ }_{2} \mathrm{KHC}_{4} \mathrm{H}_{4} \mathrm{O}_{6}=\mathrm{K}_{2} \mathrm{CO}_{3}+{ }_{5} \mathrm{C}+{ }_{2} \mathrm{CO}_{2}+{ }_{5} \mathrm{H}_{2} \mathrm{O} \text {. }
$$


Two gms. of the bitartrate are dissolved in $100 \mathrm{cc}$. of hot water, a few drops of phenolphthalein T.S. added, and then titrated with $\frac{\mathrm{N}}{\mathrm{I}} \mathrm{KOH}$ V. S. until a faint pink color indicates that all of the acid has been neutralized. Not less than ro.6 cc. of the normal alkali should be required, corresponding to 98.9 per cent of pure salt.

The following equation will show the reaction:

$$
\begin{aligned}
\mathrm{KHC}_{4} \mathrm{H}_{4} \mathrm{O}_{6}+\mathrm{KOH} & =\mathrm{K}_{2} \mathrm{C}_{4} \mathrm{H}_{4} \mathrm{O}_{6}+\mathrm{H}_{2} \mathrm{O} . \\
\mathrm{I} 86.78 \quad 55.74 & =1000 \text { cc. of } \frac{\mathrm{N}}{\mathrm{I}} \mathrm{KOH} \text { V. S. }
\end{aligned}
$$

Each cc. of $\frac{\mathrm{N}}{\mathrm{I}} \mathrm{KOH}$ V.S. represents 0.18678 gm. of $\mathrm{KHC}_{4} \mathrm{H}_{4} \mathrm{O}_{6}$.

If 10.6 cc. are required for neutralization, then $10.6 \times 0.18678=$ I.979 gms.:

$$
\frac{\text { I. } 979 \times 100}{2}=98.9 \text { per cent } .
$$

Potassium Citrate $\left(\mathrm{K}_{3} \mathrm{C}_{6} \mathrm{H}_{5} \mathrm{O}_{7} \cdot \mathrm{H}_{2} \mathrm{O}=322.08\right)$.

then

$$
\underbrace{{ }_{2}\left(\mathrm{~K}_{3} \mathrm{C}_{6} \mathrm{H}_{5} \mathrm{O}_{7} \cdot \mathrm{H}_{2} \mathrm{O}\right)}_{644 . \mathrm{I} 6}+{ }_{9} \mathrm{O}_{2}={ }_{3} \mathrm{~K}_{2} \mathrm{CO}_{3}+{ }_{4} \mathrm{CO}_{2}+{ }_{7} \mathrm{H}_{2} \mathrm{O} \text {; }
$$

therefore

$$
\underset{4 \mathrm{II} .7 \mathrm{I}}{3 \mathrm{~K}_{2} \mathrm{CO}_{3}}+\underset{292.05}{\mathrm{H}_{2} \mathrm{SO}_{4}}={ }_{3} \mathrm{~K}_{2} \mathrm{SO}_{4}+{ }_{3} \mathrm{CO}_{2}+{ }_{3} \mathrm{H}_{2} \mathrm{O} ;
$$

$$
\begin{aligned}
& { }_{2} \mathrm{~K}_{3} \mathrm{C}_{6} \mathrm{H}_{5} \mathrm{O}_{7} \cdot \mathrm{H}_{2} \mathrm{O}={ }_{3} \mathrm{~K}_{2} \mathrm{CO}_{3}={ }_{3} \mathrm{H}_{2} \mathrm{SO}_{4} \text {. } \\
& \frac{6) 644 . \mathrm{I} 6}{\mathrm{I07.36}} \mathrm{gms} . \quad \frac{6)(\mathrm{Ir} .7 \mathrm{I}}{68.635} \frac{\text { 6) } 292.05}{48.675} \mathrm{gms} .=1000 \mathrm{cc} \cdot \frac{\mathrm{N}}{\mathrm{I}} \text { acid. }
\end{aligned}
$$

Thus each cc. of $\frac{\mathrm{N}}{\mathrm{I}}$ acid represents $0.10736 \mathrm{gm}$. of pure potassium citrate.

Potassium Acetate $\left(\mathrm{KC}_{2} \mathrm{H}_{3} \mathrm{O}_{2}=97.44\right)$. In estimating potassium acetate the salt is ignited and the residue treated in exactly the same manner as in the estimation of the citrates and tartrates before mentioned.

$$
\underset{194.88}{\mathrm{KC}_{2} \mathrm{H}_{3} \mathrm{O}_{2}}+{ }_{4} \mathrm{O}_{2}=\underset{137.27}{\mathrm{~K}_{2} \mathrm{CO}_{3}}+{ }_{3} \mathrm{H}_{2} \mathrm{O}+{ }_{3} \mathrm{CO}_{2} ;
$$

* The reaction may also be written thus:

$$
2\left(\mathrm{~K}_{3} \mathrm{C}_{6} \mathrm{H}_{5} \mathrm{O}_{7} \cdot \mathrm{H}_{2} \mathrm{O}\right)+{ }_{4} \mathrm{O}={ }_{3} \mathrm{~K}_{2} \mathrm{CO}_{3}+{ }_{7} \mathrm{C}+{ }_{2} \mathrm{CO}_{2}+{ }_{7} \mathrm{H}_{2} \mathrm{O} .
$$

† The reaction may also be written thus:

$$
{ }_{2} \mathrm{KC}_{2} \mathrm{H}_{3} \mathrm{O}_{2}+{ }_{4} \mathrm{O}=\mathrm{K}_{2} \mathrm{CO}_{3}+{ }_{2} \mathrm{C}+\mathrm{CO}_{2}+{ }_{3} \mathrm{H}_{2} \mathrm{O} \text {. }
$$


then

$$
\mathrm{K}_{2} \mathrm{CO}_{3}+\mathrm{H}_{2} \mathrm{SO}_{4}=\mathrm{K}_{2} \mathrm{SO}_{4}+\mathrm{H}_{2} \mathrm{O}+\mathrm{CO}_{2} \text {; }
$$

$137.27 \quad 97.35$

therefore

$$
\begin{aligned}
& { }_{2} \mathrm{KC}_{2} \mathrm{H}_{3} \mathrm{O}_{2}=\mathrm{K}_{2} \mathrm{CO}_{3}=\mathrm{H}_{2} \mathrm{SO}_{4} \text {. } \\
& \text { 2) } \frac{194.88}{97.44} \text { gms. } \frac{2) \frac{137.27}{68.635} \text { gms. }}{2)} \frac{97.35}{48.675} \text { gms. }=1000 \mathrm{cc} . \frac{\mathrm{N}}{\mathrm{I}} \mathrm{H}_{2} \mathrm{SO}_{4} \text {. }
\end{aligned}
$$

Each cc. therefore of $\frac{\mathrm{N}}{\mathrm{I}} \mathrm{H}_{2} \mathrm{SO}_{4}$ V. S. corresponds to $0.09744 \mathrm{gm}$. of potassium acetate.

If 10 cc. are required to neutralize the residue from I gm. of potassium acetate, the salt contains Io $\times 0.09744=0.9744$ gm., or 97.44 per cent.

Sodium Acetate $\left(\mathrm{NaC}_{2} \mathrm{H}_{3} \mathrm{O}_{2} \cdot 3 \mathrm{H}_{2} \mathrm{O}=\mathrm{I} 35.10\right)$.

$$
\underset{260.2}{\left(\mathrm{NaC}_{2} \mathrm{H}_{3} \mathrm{O}_{2} \cdot{ }_{3} \mathrm{H}_{2} \mathrm{O}\right)}+\underset{4}{\mathrm{O}_{2}}=\underset{105 \cdot 3}{\mathrm{Na}_{2} \mathrm{CO}_{3}} \text {, etc. }
$$

Each cc. of $\frac{\mathrm{N}}{\mathrm{I}} \mathrm{H}_{2} \mathrm{SO}_{4}$ V.S. represents $0.130 \mathrm{I}$ gm. of crystallized sodium acetate.

Sodium Benzoate $\left(\mathrm{NaC}_{7} \mathrm{H}_{5} \mathrm{O}_{2}=\mathrm{I} 43.0 \mathrm{I}\right)$.

$$
\underset{286.02}{2 \mathrm{NaC}_{7} \mathrm{H}_{5} \mathrm{O}_{2}}+\underset{15}{\mathrm{I}_{5} \mathrm{O}_{2}}=\underset{105.3}{\mathrm{Na}_{2} \mathrm{CO}_{3}} \text {, etc }
$$

Each cc. of $\frac{\mathrm{N}}{\mathrm{I}} \mathrm{H}_{2} \mathrm{SO}_{4}$ V. S. represents o.1430I gm. of sodium benzoate.

Sodium Salicylate $\left(\mathrm{NaC}_{7} \mathrm{H}_{5} \mathrm{O}_{3}={ }_{15} 8.89\right)$.

$$
\underset{317.78}{2 \mathrm{NaC}_{7} \mathrm{H}_{5} \mathrm{O}_{3}}+\mathrm{I}_{4} \mathrm{O}_{2}=\underset{\text { I05.3 }}{\mathrm{Na}_{2} \mathrm{CO}_{3}} \text {, etc. }
$$

Each cc. of $\frac{\mathrm{N}}{\mathrm{I}} \mathrm{H}_{2} \mathrm{SO}_{4} \dot{\mathrm{V}}$. S. represents o.r 5889 gm. of sodium salicylate.

Lithium Citrate $\left(\mathrm{Li}_{3} \mathrm{C}_{6} \mathrm{H}_{5} \mathrm{O}_{7}=208.56\right)$. As stated before, the organic salts of lithium and those of the alkali earth metals are best titrated by the residual method, after ignition, because the carbonates formed are insoluble in water. It is likewise best to use standard hydrochloric instead of standard sulphuric acid. The process for lithium citrate here given exemplifies the method. 
One gm. of the salt is thoroughly ignited in a porcelain crucible as described for potassium tartrate. The residue of lithium carbonate is then dissolved out of the crucible by adding 20 cc. of $\frac{\mathrm{N}}{\mathrm{I}}$ hydrochloric V. S. and filtering. The crucible and filter are washed with several small quantities of water and the washings added to the acid filtrate. Three drops of methyl orange are now added, and the solution titrated with $\frac{\mathrm{N}}{\mathrm{I}}$ sodium hydroxid V. S. until the yellow color appears. Assuming that $5.8 \mathrm{cc}$. of the standard alkali were required, then $20-5.8=\mathrm{I} 4.2 \mathrm{cc}$., the quantity of normal hydrochloric acid which reacted with the lithium carbonate. This quantity multiplied by the normal factor for lithium citrate $0.0695^{2}$ gives the weight of pure salt in the $\mathrm{r} \mathrm{gm}$. taken.

$$
0.06952 \times 14.2=0.987 \mathrm{gm} \text {. or } 98.7 \text { per cent. }
$$

The other lithium organic salts of the U. S. P. are assayed gravimetrically by conversion to sulphate.

Table Showing the Normal Factors, Etc., of the Organic Salts of the Alkali Metals

\begin{tabular}{|c|c|c|c|c|}
\hline Substance. & Formula. & $\begin{array}{l}\text { Molecular } \\
\text { Weight. }\end{array}$ & \begin{tabular}{|l|} 
Equivalent \\
Weight in \\
Carbonate.
\end{tabular} & $\begin{array}{l}\text { Normal } \\
\text { Factor. }\end{array}$ \\
\hline 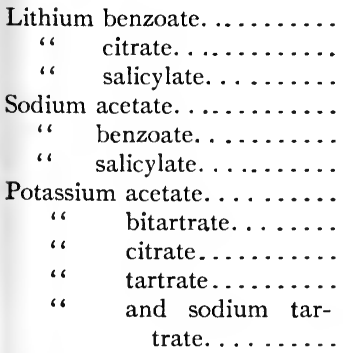 & $\begin{array}{c}\mathrm{LiC}_{7} \mathrm{H}_{5} \mathrm{O}_{2} \\
\mathrm{Li}_{3} \mathrm{C}_{6} \mathrm{H}_{5} \mathrm{O}_{7} \\
\mathrm{LiC}_{7} \mathrm{H}_{5} \mathrm{O}_{3} \\
\mathrm{NaC}_{2} \mathrm{H}_{3} \mathrm{O}_{2} \cdot 3 \mathrm{H}_{2} \mathrm{O} \\
\mathrm{NaC}_{7} \mathrm{H}_{5} \mathrm{O}_{2} \\
\mathrm{NaC}_{7} \mathrm{H}_{5} \mathrm{O}_{3} \\
\mathrm{KC}_{2} \mathrm{H}_{3} \mathrm{O}_{2} \\
\mathrm{KHC}_{4} \mathrm{H}_{4} \mathrm{O}_{6} \\
\mathrm{~K}_{3} \mathrm{C}_{6} \mathrm{H}_{5} \mathrm{O}_{7} \cdot \mathrm{H}_{2} \mathrm{O} \\
2 \mathrm{~K}_{2} \mathrm{C}_{4} \mathrm{H}_{4} \mathrm{O}_{6} \mathrm{H}_{2} \mathrm{O} \\
\\
\mathrm{KNaC}_{4} \mathrm{H}_{4} \mathrm{O}_{6} \cdot{ }_{4} \mathrm{H}_{2} \mathrm{O}\end{array}$ & $\begin{array}{r}\text { I } 27 . \text { I I } \\
208.56 \\
\text { I } 42.99 \\
\text { I } 35.10 \\
\text { I } 43.01 \\
\text { I } 58.89 \\
97.44 \\
\text { I86.78 } \\
322.08 \\
476.16 \\
\\
280.18\end{array}$ & $\begin{array}{c}3^{6.755} \\
\text { I } 10.265 \\
3^{6.755} \\
5^{2} .65 \\
5^{2} .65 \\
52.65 \\
68.685 \\
68.685 \\
205.95 \\
274.54 \\
\text { I } 21.29\end{array}$ & $\begin{array}{l}0.127 \text { II } \\
0.0695^{2} \\
0.14299 \\
0.1351 \\
0.14301 \\
0.15889 \\
0.09744 \\
0.18678 \\
0.10736 \\
0.11904 \\
0.14009\end{array}$ \\
\hline
\end{tabular}

Estimation of Alkali Metals in their Salts. This may be done by first converting the salt into a sulphate, and then by means of barium hydroxid, forming an alkali hydroxid which is finally converted into an alkali carbonate by means of carbon dioxid.

(a) $\mathrm{K}_{2} \mathrm{SO}_{4}+\mathrm{Ba}(\mathrm{OH})_{2}=\mathrm{BaSO}_{4}+2 \mathrm{KOH}$;

(b) ${ }_{2} \mathrm{KOH}+\mathrm{CO}_{2}=\mathrm{K}_{2} \mathrm{CO}_{3}+\mathrm{H}_{2} \mathrm{O}$. 
The conversion of the original salt into a sulphate may be done in several ways, depending upon whether the acid in combination is a volatile or a non-volatile one, as described below.

Alkalies Combined with Volatile Acids. A definite quantity of the salt in solution is treated with an excess of sulphuric acid and evaporated to dryness, and then further heated to drive off some of the excess of sulphuric acid. The residue which consists of the alkali as a sulphate is dissolved in water and treated with a slight excess of barium hydroxid solution. The mixture now contains the alkali in solution as hydroxid, and a precipitate of barium sulphate (see equation $(a)$ ), also the excess of barium hydroxid in solution. A stream of carbon dioxid $\left(\mathrm{CO}_{2}\right)$ is now passed through the mixture; this converts the alkali hydroxid into carbonate and at the same time, removes the barium hydroxid by precipitating it as barium carbonate (see equation $(b)$ ). When this conversion into carbonate is complete, the free carbon dioxid must be driven off by boiling, because barium carbonate is converted into the soluble barium bicarbonate, in the presence of free carbon dioxid. The mixture now contains the alkali in solution as a carbonate, and a sediment consisting of barium sulphate and barium carbonate. This mixture is now made up to a definite volume and the alkali carbonate titrated in the usual manner in an aliquot portion, which may be removed by filtration, or by means of a pipette, if the precipitate settles rapidly and leaves a clear supernatant liquid.

Alkalies Combined with Non-Volatile Acids. In the case of alkali salts of non-volatile acids, as phosphoric, boric, chromic, arsenic, molybdic, etc., the acid is removed by means of lead acetate, and the resulting alkali acetate converted into sulphate, by means of sulphuric acid.

(c) $\mathrm{K}_{2} \mathrm{CrO}_{4}+\mathrm{Pb}\left(\mathrm{C}_{2} \mathrm{H}_{3} \mathrm{O}_{2}\right)_{2}=\mathrm{PbCrO}_{4}+2 \mathrm{KC}_{2} \mathrm{H}_{3} \mathrm{O}_{2}$;

(d) ${ }_{2} \mathrm{KC}_{2} \mathrm{H}_{3} \mathrm{O}_{2}+\mathrm{H}_{2} \mathrm{SO}_{4}=\mathrm{K}_{2} \mathrm{SO}_{4}+{ }_{2} \mathrm{HC}_{2} \mathrm{H}_{3} \mathrm{O}_{2}$;

(e) $\mathrm{Pb}\left(\mathrm{C}_{2} \mathrm{H}_{3} \mathrm{O}_{2}\right)_{2}+\mathrm{H}_{2} \mathrm{SO}_{4}=\mathrm{PbSO}_{4}+{ }_{2} \mathrm{HC}_{2} \mathrm{H}_{3} \mathrm{O}_{2}$.

To the solution of the salt an excess of lead acetate solution is added; this causes a precipitation of the acid as a lead salt and converts the alkali into an acetate which remains in solution. (See equation $(c)$ ). The excess of lead acetate is also in solution. The mixture is filtered and the filtrate treated with a slight excess of sulphuric acid. This converts the alkali acetate into a sulphate (see equation $(d)$ ) and removes the lead acetate by precipitation in the form of lead sul- 
phate (see equation $(e)$ ) which is filtered out, and the solution of alkali sulphate treated as above discribed.

\section{ESTIMATION OF THE SALTS OF THE ALKALI EARTHS}

Standard sclution of hydrochloric or of nitric acid is preferred by many operators for the titration of hydroxids or carbonates of the alkali earths.

These acids possess the advantage over most other acids of forming soluble salts. The hydroxids may be estimated by any of the indicators, but as they readily absorb $\mathrm{CO}_{2}$ out of the air, they are generally contaminated with more or less carbonate, and the residual method should be used, i.e., a known excess of standard acid should be added, the mixture boiled to expel any trace of $\mathrm{CO}_{2}$, and retitrated with standard alkali.

The carbonates are of course estimated in the same way, as are also the organic salts of the alkali earths, after ignition. As an example:

One gram of calcium carbonate is mixed with $5 \mathrm{cc}$. of water. A measured excess of normal hydrochloric acid V. S. is now added, and the solution boiled to drive off the $\mathrm{CO}_{2}$. Then add a few drops of phenolphthalein, and titrate with $\frac{\mathrm{N}}{\mathrm{I}}$ alkali V.S. until a faint pink color is obtained.

Note the quantity of $\frac{\mathrm{N}}{\mathrm{I}}$ alkali used, and deduct this from the quantity of $\frac{N}{\mathrm{I}}$ acid first added, and the remainder will represent the amount of acid which combined with the calcium.

Each cc. of $\frac{\mathrm{N}}{\mathrm{I}}$ acid V. S. represents $0.049675 \mathrm{gm}$. of $\mathrm{CaCO}_{3}$.

$$
\begin{aligned}
& \mathrm{CaCO}_{3}+{ }_{2{ }_{2} \mathrm{HCl}}=\mathrm{CaCl}_{2}+\mathrm{H}_{2} \mathrm{O}+\mathrm{CO}_{2} . \\
& \frac{\left.{ }_{2}^{2}\right) \frac{72.35}{49.675}}{\text { gms. }}{ }_{36.18}^{6} \text { gms. or rooo cc. } \frac{\mathrm{N}}{\mathrm{I}} \text { acid V. S. }
\end{aligned}
$$

Assuming that $30 \mathrm{cc}$. of $\frac{\mathrm{N}}{\mathrm{I}} \mathrm{HCl} \mathrm{V.S.} \mathrm{were} \mathrm{added} \mathrm{to} \mathrm{the} \mathrm{I} \mathrm{gm}$. of $\mathrm{CaCO}_{3}$, and that II cc. of $\frac{\mathrm{N}}{\mathrm{I}} \mathrm{KOH}$ V.S. were required to bring the mixture 
back to neutrality, then $19 \mathrm{cc}$. of $\frac{\mathrm{N}}{\mathrm{I}} \mathrm{HCl}$ were actually required to saturate the $\mathrm{CaCO}_{3}$.

Therefore $0.049675 \times 19=0.9438$ or 94.38 per cent.

The hydroxids and carbonates may also be estimated by direct titration with standard hydrochloric acid (in the cold) using methyl orange as indicator. A better plan, however, would be to add the standard acid in slight excess, and then standard alkali until a distinct yellow color appears; the slight excess of alkali is then determined by adding standard hydrochloric acid until the red color reappears. A much more distinct color reaction is thereby obtained. The quantity of the standard alkali used is deducted from the total quantity of standard acid added.

Soluble salts of calcium, barium, and strontium, such as chlorids, nitrates, etc., may be readily estimated as follows:

A weighed quantity of the salt is dissolved in water, cautiously neutralized if it is acid or alkaline, phenolphthalein is added, the mixture beated to boiling, and standard solution of sodium carbonate delivered in from time to time, with constant boiling until the red color is permanent.

This process depends upon the fact that sodium carbonate forms with soluble salts of these bases insoluble neutral carbonates.

$$
\begin{aligned}
& \mathrm{CaCl}_{2}+\mathrm{Na}_{2} \mathrm{CO}_{3}=\mathrm{CaCO}_{3}+2 \mathrm{NaCl} . \\
& \mathrm{Ba}\left(\mathrm{NO}_{3}\right)_{2}+\mathrm{Na}_{2} \mathrm{CO}_{3}=\mathrm{BaCO}_{3}+2 \mathrm{NaNO}_{3} .
\end{aligned}
$$

Magnesium salts cannot be estimated in this way, as magnesium carbonate affects the indicator.

The alkali earth salts may also be estimated by dissolving them in water, precipitating the base as carbonate, with an excess of ammonium carbonate and some free ammonia. The mixture is then heated for a few minutes, and the carbonate separated by filtration, thoroughly

- washed with hot water till all soluble matters are removed, and then titrated with normal acdi V. S. as carbonate.

Normal Sodium Carbonate V. S. $\left(\mathrm{Na}_{2} \mathrm{CO}_{3}=105.3 \mathrm{I}\right)$ contains $5^{2} .65 \mathrm{gms}$. in I liter. This solution is made by dissolving $52.65 \mathrm{gms}$. of pure sodium carbonate (anhydrous) previously ignited and cooled, in distilled water, and diluting to $\mathrm{I}$ liter at $15^{\circ} \mathrm{C}$. $\left(59^{\circ} \mathrm{F}\right.$.).

If a pure salt is not at hand the solution may be made as follows: 
About 85 gms. of pure sodium bicarbonate, free from thiosulphate, chlorid, etc., are heated to dull redness (not to fusion) for about fifteen minutes to expel one half of the $\mathrm{CO}_{2}$; it is then cooled under a desiccator. When cool, weigh off $52.65 \mathrm{gms}$. and dissolve it in distilled water to make $\mathrm{I}$ liter at $15^{\circ} \mathrm{C}$. ( $59^{\circ} \mathrm{F}$.). This solution should neutralize $\frac{\mathrm{N}}{\mathrm{I}}$ acid V.S. volume for volume.

As an example of the process: Take of calcium chlorid one gm., dissolve it in a small quantity of water, neutralize the solution if it is acid or alkaline, heat to boiling, add a few drops of phenolphthalein, and titrate with $\frac{\mathrm{N}}{\mathrm{I}}$ sodium carbonate, delivered cautiously whilst boiling until the red color is permanent.

$$
\begin{aligned}
& \mathrm{CaCl}_{2}+\mathrm{Na}_{2} \mathrm{CO}_{3}=\mathrm{CaCO}_{3}+{ }_{2} \mathrm{NaCl}
\end{aligned}
$$

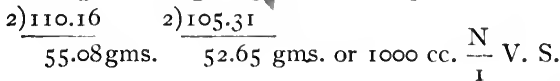

Each cc. of $\frac{\mathrm{N}}{\mathrm{I}} \mathrm{Na}_{2} \mathrm{CO}_{3}$ V.S. represents $0.05508 \mathrm{gm}$. of $\mathrm{CaCl}_{2}$. If $18 \mathrm{cc}$. are used the salt contains $0.05508 \mathrm{gm} . \times \mathrm{I} 8=0.99 \mathrm{I} \mathrm{gm}$. or 99.I per cent.

In the other method in which an excess of ammonium carbonate is added together with some free ammonia, the calcium is precipitated as carbonate, this is then separated by filtration, thoroughly washed with hot water to remove all soluble matters, and then titrated as directed for carbonate.

$$
\begin{aligned}
& \mathrm{CaBr}_{2}=\mathrm{CaCO}_{3}=\mathrm{H}_{2} \mathrm{SO}_{4} \text {. } \\
& \begin{array}{lll}
2) 198.5^{2} & \text { 2) } 99.35 & \text { 2) } 98
\end{array} \\
& 99.26 \text { gms. } 4.9 .675 \mathrm{gms} .49 \mathrm{gms} \text {. or } 1000 \mathrm{cc} . \frac{\mathrm{N}}{\mathrm{I}} \mathrm{V} . \mathrm{S} \text {. }
\end{aligned}
$$

Each cc. of $\frac{\mathrm{N}}{\mathrm{I}}$ acid thus represents $0.09926 \mathrm{gm}$. of $\mathrm{CaBr}_{2}$.

\section{The Estimation of Mixed Hydroxids and Carbonates of} Alkali Earths. This may be done as described under estimation of mixed alkali hydroxids and carbonates, page 8I, except that it is in this case unnecessary to precipitate the carbonate by barium chlorid in that the alkali earth carbonates are already insoluble. 
Table Showing Normal Factors, Etc., of the Alkali Earth Metals.

\begin{tabular}{|c|c|c|c|c|}
\hline Name of Salt. & Formula. & $\begin{array}{l}\text { Molecu. } \\
\text { lar } \\
\text { Weight. }\end{array}$ & $\begin{array}{c}\text { Standard Solution } \\
\text { Used }\end{array}$ & $\frac{\mathrm{N}}{\mathrm{r}}$ Factor. \\
\hline Barium carbonate.... & $\mathrm{BaCO}_{3}$ & 195.95 & $\mathrm{HCl}$ & 0.09797 \\
\hline " chlorid........ & $\mathrm{BaCl}_{2}$ & $206 \cdot 7^{6}$ & $\mathrm{Na}_{2} \mathrm{CO}_{3}$ & 0.10338 \\
\hline .. hỵdroxid..... & $\mathrm{Ba}(\mathrm{OH})_{2}$ & 170.16 & $\mathrm{HCl}$ & 0.08506 \\
\hline " nitrate...... & $\mathrm{Ba}\left(\mathrm{NO}_{3}\right)_{2}$ & $259 \cdot 54$ & $\mathrm{Na}_{2} \mathrm{CO}_{3}$ & 0.12977 \\
\hline Calcium bromid ..... & $\mathrm{CaBr}_{2}$ & $198.5^{2}$ & $\mathrm{Na}_{2} \mathrm{CO}_{3}$ & 0.09926 \\
\hline “ carbonate.... & $\mathrm{CaCO}_{3}$ & $99 \cdot 35$ & $\mathrm{HCl}+\mathrm{KOH}$ & 0.049675 \\
\hline " chlorid. ...... & $\mathrm{CaCl}_{2}$ & 110.16 & $\mathrm{NaCO}_{3}$ & 0.05508 \\
\hline hydroxid ..... & $\mathrm{Ca}(\mathrm{OH})_{2}$ & $73 \cdot 5^{6}$ & $\mathrm{HCl}$ & 0.03678 \\
\hline$" \quad$ oxid. $\ldots \ldots \ldots$ & $\mathrm{CaO}$ & 55.63 & $\mathrm{HCl}$ & 0.02784 \\
\hline $\begin{array}{l}\text { Magnesium carbonate } \\
\text { (true) } \ldots . . . . . . . .\end{array}$ & $\mathrm{MgCO}_{3}$ & $83 \cdot 73$ & $\begin{array}{c}\mathrm{HCl} \text { or } \mathrm{H}_{2} \mathrm{SO}_{4} \\
+\mathrm{KOH}\end{array}$ & $0.04186_{5}$ \\
\hline $\begin{array}{c}\text { Magnesium carbonate } \\
\quad(\text { U. S. P.)......... }\end{array}$ & $\begin{array}{c}\left(\mathrm{MgCO}_{3}\right)_{4} \mathrm{Mg}(\mathrm{OH})_{2} \\
+5 \mathrm{H}_{2} \mathrm{O}\end{array}$ & 482.26 & $\begin{array}{l}\mathrm{HCl} \text { or } \mathrm{H}_{2} \mathrm{SO}_{4} \\
+\mathrm{KOH}^{-}\end{array}$ & 0.048266 \\
\hline Magnesium oxid...... & $\operatorname{MgO}$ & 40.06 & $\begin{array}{l}\mathrm{HCl} \text { or } \mathrm{H}_{2} \mathrm{SO}_{4} \\
+\mathrm{KOH}\end{array}$ & 0.02003 \\
\hline Strontium bromid ..... & $\mathrm{SrBr}_{2}$ & 245.66 & $\mathrm{Na}_{2} \mathrm{CO}_{3}$ & 0.12283 \\
\hline " carbonate... & $\mathrm{SrCO}_{3}$ & I 46.49 & $\mathrm{HCl}+\mathrm{KOH}$ & 0.073245 \\
\hline iodid...... & $\mathrm{Srl}_{2}$ & 338.74 & $\mathrm{Na}_{2} \mathrm{CO}_{3}$ & 0.16937 \\
\hline lactate..... & $\mathrm{Sr}\left(\mathrm{C}_{3} \mathrm{H}_{5} \mathrm{O}_{3}\right)_{2}$ & 263.68 & $\mathrm{HCl}$ after ignition & 0.13184 \\
\hline oxid. ....... & $\mathrm{SrO}$ & 102.82 & $\mathrm{HCl}+\mathrm{KOH}$ & $0.0514 \mathrm{I}$ \\
\hline " salicylate... & $\mathrm{Sr}\left(\mathrm{C}_{7} \mathrm{H}_{5} \mathrm{O}_{3}\right)_{2}+2 \mathrm{H}_{2} \mathrm{O}$ & $394 \cdot 7^{2}$ & $\mathrm{HCl}$ after ignition & $0.1973^{6}$ \\
\hline
\end{tabular}

The haloid salts may be estimated by titration with $\frac{\mathrm{N}}{10} \mathrm{AgNO}_{3} \mathrm{~V} . \mathrm{S}$.

\section{ACIDIMETRY}

The Estimation of Acids by Neutralization.-In the previous pages it has been shown how alkalies are estimated by the use of acid solutions of known neutralizing power. In the estimation of acids, which will now be described, the order is reversed; alkaline solutions of known power being used in determining the strength of acids and of acid salts. Thus the procedure is analagous to that of the alkalimetric methods. The choice of the indicator, whether litmus, phenolphthalein or methyl orange, depends upon the particular acid to be estimated. Phenolphthalein is employed for the organic acids and boric acid and is preferred for phosphoric acid; while methyl orange and litmus are usually employed in the titration of the inorganic acids.

The standard alkali used may be either an hydroxid or a carbonate, the former is, however, usually preferred, because the car- 
bonate when brought in contact with an acid gives off carbonic acid gas $\left(\mathrm{CO}_{2}\right)$ which interferes to a great extent with most indicators. On the other hand, it must be remembered that the alkali hydroxids are very prone to absorb carbon dioxid from the atmosphere, therefore their solutions often contain some carbonate, the presence of which even in small quantities will occasion errors when used with most indicators, especially with litmus and phenolphthalein. It is therefore advisable, when using these indicators or others which are affected by carbon dioxid, to employ gentle heat

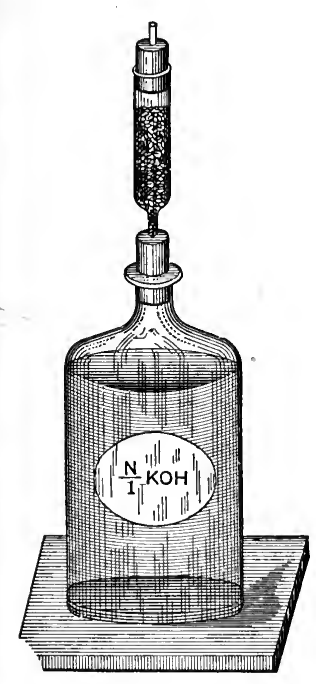

FIG. 48.

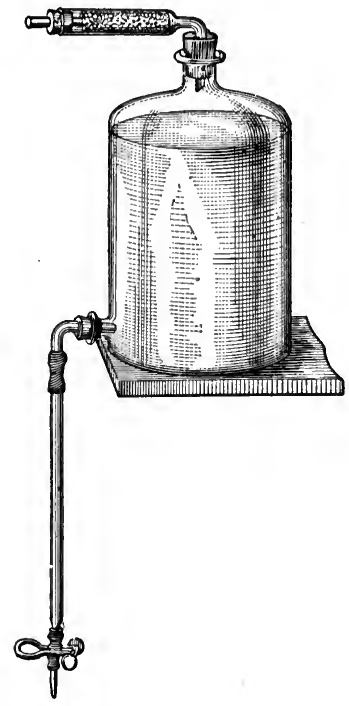

Fig. 49.

toward the close of each titration, in order to drive off the liberated gas. Methyl orange is not affected by this gas, and therefore heating is not necessary when this indicator is used. In fact, it is imperative that heat should not be employed with this indicator.

In acidimetrical operations when methyl orange is used as indicator, residual titrations may be advantageously done, because the change of color from yellow to red which is brought about by the acid is much more readily seen than that from red to yellow.

In the U. S. P. standard solutions of both potassium and sodium hydroxid are official. The former, however, is preferable, because it attacks glass more slowly, and less energetically, and also foams 
much less than does the sodium hydroxid solution. The neutralizing power of each is, however, the same. Standard solutions of alkali hydroxid should be preserved in small vials, provided with wellfitting rubber stoppers, or better still these should be provided with tubes filled with a mixture of soda and lime, which absorbs $\mathrm{CO}_{2}$ and prevents its access to the solution. A vessel of this description is pictured in Fig. 48.

An improvement upon this is shown in Fig. 49, since it allows of the burette being filled without removing the stopper, and consequently without any access of $\mathrm{CO}_{2}$ whatever,

Where a series of titrations of the same kind have to be made with the same alkali standard solution, the arrangement shown in Fig. I3 may be used, both the reservoir and the burette in this case being provided with soda-lime tubes.

\section{PREPARATION OF STANDARD ALKALI SOLUTIONS}

Normal Potassium Hydroxid $\left(\mathrm{KOH}=55.74 ; \frac{\mathrm{N}}{\mathrm{I}} \mathrm{V} . \mathrm{S} .=55.74\right.$ gms. in $1000 \mathrm{cc}$.).

Potassium hydroxid being prone to absorb carbon dioxid out of the air the pure article is not readily obtained in commerce. If pure potassium hydroxid were easily obtained it would only be necessary to dissolve $55.74 \mathrm{~g} n \mathrm{~s}$. in sufficient water to make $1000 \mathrm{cc}$. But since it always contains some $\mathrm{CO}_{2}$ and $\mathrm{H}_{2} \mathrm{O}$, it is necessary to take a slight excess and dilute the solution to the proper volume after havirg determined its strength.

The standardization may be effected by means of any of the standard acid solutions.

A satisfactory method for the preparation and standardization of this solution is as follows:

Dissolve $75 \mathrm{gms}$. of potassium hydroxid in sufficient water to make about $1050 \mathrm{cc}$ at $5^{\circ} \mathrm{C}$. $\left(59^{\circ} \mathrm{F}\right.$.), and fill a burette with a portion of this solution.

Dissolve $0.6255 \mathrm{gm}$. of pure oxalic acid in about ro cc. of water in a beaker or flask, add a few drops of phenolphthalein T. S., and then carefully add from the burette the potassium hydroxid solution, agitating frequently and regulating the flow to drops towards the end of the operation until a permanent pale pink color is obtained. Note the number of cc. of the alkali solution consumed, and then dilute the remainder so that exactly $10 \mathrm{cc}$. of the diluted liquid will be required to neutralize $0.6255 \mathrm{gm}$. of oxalic acid. Instead of weighing 
off $0.6255 \mathrm{gm}$. of the acid, ro cc. of its normal solution may be used.

Example. Assuming that $8 \mathrm{cc}$. of the stronger potassium hydroxid solution had been consumed in the trial, then each $8 \mathrm{cc}$. must be diluted to so cc., or the whole of the remaining solution in the same proportion. Thus if $8 \mathrm{cc}$. must be diluted to ro $\mathrm{cc}$., rooo cc. must be diluted to $1250 \mathrm{cc}$.

$$
\text { 8: 10:: 1000: } x \quad x=\mathrm{I} 250 \mathrm{cc} \text {. }
$$

It is always advisable to make another trial after diluting. Io cc. should then neutralize $0.6255 \mathrm{gm}$. of pure oxalic acid.

Standardization by Means of Potassium Bitartrate. The U. S. P., 8th Dec. Revis., recommends this method. It is based upon the reaction

$$
\begin{aligned}
\mathrm{KHC}_{4} \mathrm{H}_{4} \mathrm{O}_{6} & +\mathrm{KOH}=\mathrm{K}_{2} \mathrm{C}_{4} \mathrm{H}_{4} \mathrm{O}_{6}+\mathrm{H}_{2} \mathrm{O} . \\
\mathrm{I} 86.78 \mathrm{gms} . & =55.74 \mathrm{gms} .=\text { I000 cc. } \frac{\mathrm{N}}{\mathrm{I}} \mathrm{V} . \mathrm{S} . ; \\
\mathrm{I} 8.678 \mathrm{gms} . & =5.574 \mathrm{gms} .=\text { I00 cc. } \frac{\mathrm{N}}{\mathrm{I}} \mathrm{V} . \mathrm{S} . ; \\
\mathrm{I} .8678 \mathrm{gms} . & =0.5574 \mathrm{gm} .=\text { о० cc. } \frac{\mathrm{N}}{\mathrm{I}} \mathrm{V} . \mathrm{S} . ; \\
3.72 \mathrm{gms} . & =\text { I.I I } 48 \mathrm{gms} .=20 \mathrm{cc} \cdot \frac{\mathrm{N}}{\mathrm{I}} \mathrm{V} . \mathrm{S} .
\end{aligned}
$$

A solution of potassium hydroxid, 75 gms. in ro50 cc., is prepared and titrated against pure potassium bitartrate, using phenolphthalain as indicator.

$3.7^{2}$ gms. of purified dry potassium bitartrate $*$ are dissolved in

* Purified potassium bitartrate for standardizing caustic alkali volumetric solutions, is obtained according to the U. S. P. as follows: "To Ioo gms. of the salt contained in a beaker is added a mixture of $85 \mathrm{cc}$. of water and $25 \mathrm{cc}$. of diluted hydrochloric acid; the covered beaker is then placed upon a bath of boiling water and the mixture digested with occasional stirring for three hours. After quickly cooling, the solution is drained off from the precipitate, which is washed by effusion and decantation with two successive portions of $100 \mathrm{cc}$. each of water; after collecting the precipitate upon a plain filter, the washing with cold water is continued until the filtrate, after adding a few drops of nitric acid, ceases to become opalescent upon the addition of silver nitrate T.S. The precipitate of potassium bitartrate is then dissolved in the smallest possible volume of boiling water (about $5_{500} \mathrm{cc}$.), filtered, and the filtrate while being rapidly cooled is constantly stirred. When the mixture is cold, the crystalline precipitate is collected upon a plain filter, washed with 300 cc. of cold water, and, after thoroughly draining, dried at $120^{\circ} \mathrm{C}$. $\left(248^{\circ} \mathrm{F}\right.$.) until of constant weight. It should be kept in dry, securely stoppered bottles." 
60 cc. of boiling distilled water, a few drops (3) of phenolphthalein are added, and the solution of potassium hydroxid run into it (the solution being frequently boiled) until a pale pink color appears. Exactly $20 \mathrm{cc}$. will be required if the alkali solution is normal. If only $18 \mathrm{cc}$. are consumed, then each $18 \mathrm{cc}$. must be diluted to $20 \mathrm{cc}$. or the whole of the remaining solution in the same proportion.

Standardization by Means of Potassium Bi-iodate.* Potassium bi-iodate is an acid salt and may be directly titrated with potassium hydroxid using phenolphthalein as indicator.

One molecule of the bi-iodate is equivalent to one molecule of potassium hydroxid as shown by the equation

$$
\underset{386.94}{\mathrm{KH}\left(\mathrm{IO}_{3}\right)_{2}}+\underset{55.74}{\mathrm{KOH}}=2 \mathrm{KIO}_{3}+\mathrm{H}_{2} \mathrm{O} .
$$

To standardize a potassium-hydroxid solution, weigh accurately 3.8694 gms. of potassium bi-iodate, dissolve it in about $25 \mathrm{cc}$. of water, add a few drops of phenolphthalein, and then run into this, from a burette, the hydroxid solution which is to be standardized, until a pale pink color appears. Note the number of cc. used and dilute the soiution so that exactly ro cc. of it will neutralize $3.8694 \mathrm{gms}$. of the bi-iodate.

Example. Assuming that $8.2 \mathrm{cc}$. had been consumed, then each 8.2 cc. must be diluted to ro cc., or the whole of the remaining solution in the same proportion.

The advantages of this salt as an ultimate standard are (I) that it may be procured in the market in a state of absolute purity; $\dagger(2)$ that it is permanent, being neither deliquescent nor efflorescent; (3) that it can be dried at $110^{\circ} \mathrm{C}$. without decomposition; (4) that the results obtained with it are quite accurate, and (5) that it may be employed for standardizing most of the volumetric solutions commonly found in the laboratory. 389.

* See Meinecke, Chem. Ztg., XIX., 2. Also Caspari, Proc. A. Ph. A., I904,

+ According to Caspari, the salt may be readily prepared as follows: See A. Ph. A., 1904, 390. Potassium bicarbonate is mixed in solution with an equivalent amount of iorlic acid, and to the neutral solution is added an amount of iodic acid equal to the quantity first used. The solution is then evaporated until crystallization begins, and the first crop of crystals rejected. Those which separate after the solution has cooled to $50^{\circ} \mathrm{C}$. are almost pure and will be rendered absolutely pure if recrystallized. 
Normal Sodium Hydroxid $\left(\mathrm{NaOH}=39.76 ; \frac{\mathrm{N}}{1} \mathrm{~V} . \mathrm{S} .=39.76 \mathrm{gms}\right.$. in 1000 cc.).

Dissolve 54 gms. of sodium hydroxid in enough water to make about ro5० cc. of solution, fill a burette with a portion of this, and check it with normal acid, or a weighed quantity of oxalic acid or potassium bitartrate, in exactly the same manner as described for normal potassium hydroxid.

The standard alkali volumetric solutions which are official in the U. S. P. are:

Normal potassium hydroxid;

Tenth normal potassium hydroxid;

Fiftieth normal potassium hydroxid;

Hundredth normal potassium hydroxid;

Half normal potassium hydroxid (alcoholic);

Normal sodium hydroxid;

Double normal sodium hydroxid.

Other standard alkali solutions in frequent use are normal sodium carbonate, normal and other strengths of ammonia, and decinormal barium hydroxid.

Estimation of the Inorganic Acids. To weigh off directly a definite quantity of a fluid acid, is not a very easy matter. It is always a better plan to measure a small quantity of the acid and weigh it accurately in a tared and stoppered weighing flask (Fig. 50), then to add water and titrate with the standard alkali in the presence of a suitable indicator. If the specific gravity of the acid is known or can be easily taken, it is sufficient to measure a certain quantity of it by means of a pipette, and then determine its weight by multiplying the volume in cubic centimeters by the specific gravity. It must be remembered, however, that the liquid must be measured FIG. 50. at the same temperature at which the specific gravity was taken. This method is applicable to the diluted acids as well as to the concentrated acids of commerce, as hydrochloric, nitric, and sulphuric acids.

In the case of very volatile acids, i.e., such as evolve acid vapors at ordinary temperatures, the determination of the weight by means of the specific gravity is inadmissable. Such acids should be weighed 
in a Lunge pipette, Fig. $5^{1}$, or in a simple bulb pipette provided with a glass stop-cock, Fig. 52, or in a Grethan's pipette, Fig. 53.

The Lunge pipette is used by producing a vacuum in the bulb $(a)$, the air-tight glass mantle $(c)$ is then removed, and the tip of the tube $(d)$ sunk into the acid which is drawn up into the bulb, upon opening the cock $(b)$; when sufficient of the acid has been drawn into the apparatus the cock is closed, the tip of the pipette wiped, the glass mantle put in place, and the whole weighed. The weight of the empty pipette deducted gives the weight of the acid taken up. The pipettes shown in Figs. 52 and 53 are filled by applying direct

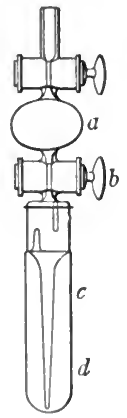

FIG. 51.

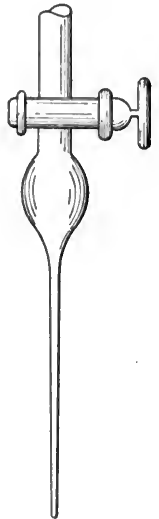

FIG. 52.

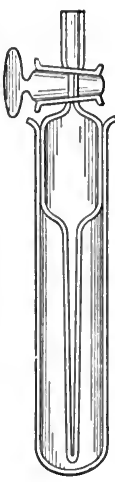

FIG. 53 .

suction with the lips, the operator protecting himself against inhalation of harmful vapors by attaching an absorption tube containing soda-lime, calustic soda, or similar substance.

The quantity of acid to be taken (in most cases) should be such as will require for neutralization from 20 to $50 \mathrm{cc}$. of the standard alkili. In the case of concentrated inorganic acids, 2 or 3 gms. may be taken, while in the case of the dilute acids, from 6 to $8 \mathrm{gms}$.

Any of the indicators may be employed for the inorganic acids, but because of the usual presence of carbonate in the standard alkali, methyl orange is preferred.

Hydrochloric Acid $(\mathrm{HCl}=36.18)$. About 2 cc. of hydrochloric acid (sp.gr. 1.048) are introduced into a tared weighing flask anc its weight accurately taken. (The weight is found to be 2.098 gms.) 
$20 \mathrm{cc}$. of water are now added, followed by two drops of methyl orange, and the solution carefully titrated with normal potassium hydroxid until the reddish color of the solution is changed to yellow.

Assuming that $\mathrm{I} 8.4 \mathrm{cc}$. were required, then $\mathrm{I} 8.4 \mathrm{cc}$. $\times 0.036 \mathrm{r} 8 \mathrm{gm}$. $=0.6657 \mathrm{gm}$. of absolute hydrochloric acid in the $2.098 \mathrm{gms}$. taken.

To find the per cent apply the proportion

$$
2.098 \text { gms. : } 0.6657 \text { gm. : : 100 : } x \quad x=3 \text { 1.68 per cent. }
$$

The equation is:

$$
\begin{aligned}
& \mathrm{HCl}+\mathrm{KOH}=\mathrm{KCl}+\mathrm{H}_{2} \mathrm{O} \\
& 36.18 \text { gms. }=55.74 \mathrm{gms} .=1000 \mathrm{cc} \cdot \frac{\mathrm{N}}{\mathrm{I}} \mathrm{V} . \mathrm{S} . \\
& .03618 \mathrm{gm} .=\quad .05574 \mathrm{gm} .=\text { I cc. } \frac{\mathrm{N}}{\mathrm{I}} \mathrm{V} . \mathrm{S} \text {. }
\end{aligned}
$$

Sulphuric Acid $\left(\mathrm{H}_{2} \mathrm{SO}_{4}=97.35\right)$. About I cc. of the concentrated. acid is weighed in a tared weighing flask and found to weigh $\mathrm{I} .8 \mathrm{gm}$. $20 \mathrm{cc}$. of water are added and then 2 drops of methyl orange, and the titration with normal potassium hydroxid begun, and cautiously continued until the yellowish color of the solution indicates the completion of the operation; note the number of cc. of alkali solution used and apply the equation

$$
\begin{aligned}
& \mathrm{H}_{2} \mathrm{SO}_{4}+{ }_{2} \mathrm{KOH}=\mathrm{K}_{2} \mathrm{SO}_{4}+{ }_{2} \mathrm{H}_{2} \mathrm{O} \\
& \text { 2) } \frac{97.35}{48.675} \text { gms. }=\frac{2) 11 \mathrm{I} .48}{55.74} \mathrm{gms} .=1000 \mathrm{cc} \cdot \frac{\mathrm{N}}{\mathrm{I}} \mathrm{V} . \mathrm{S} . \\
& 0.048675 \mathrm{gm} .=0.05574 \mathrm{gm} .=\mathrm{I} \text { cc. }{ }_{\mathrm{I}}^{\mathrm{N}} \text { V.S. }
\end{aligned}
$$

Thus each cc. of normal KOH V. S. represents $0.048675 \mathrm{gm}$. of pure $\mathrm{H}_{2} \mathrm{SO}_{4}$.

Assuming that in the above assay 34.I cc. were required, then $34 . \mathrm{I} \times 0.048675 \mathrm{gm} .=1.659 \mathrm{gm}$.

Then

$$
\frac{\mathrm{r} .659 \times 100}{1.8}=92.1 \text { per cent. }
$$

Phosphoric Acid $\left(\mathrm{H}_{3} \mathrm{PO}_{4}=97.29\right)$. In the assay of phosphoric acid by direct neutralization with standard alkali, the acid is converted into first $\mathrm{KH}_{2} \mathrm{PO}_{4}$ then $\mathrm{K}_{2} \mathrm{HPO}_{4}$, and finally into the normal 
$\mathrm{K}_{3} \mathrm{PO}_{4}$. We have no indicator which reliably shows the completion of the neutralization, i.e., the formation of the tribasic $\mathrm{K}_{3} \mathrm{PO}_{4}$. Litmus cannot be used as indicator here for the dipotassic or disodichydric phosphate $\left(\mathrm{K}_{2} \mathrm{HPO}_{4}\right.$ or $\left.\mathrm{Na}_{2} \mathrm{HPO}_{4}\right)$ which is formed is slightly alkaline towards litmus; the same is true of most other indicators.

It is recommended, therefore, in order to estimate phosphoric acid alkalimetrically, to prevent the formation of soluble phosphate of the alkali, and to bring the acid into a definite compound with an alkali earth, as follows:

The free acid in a diluted state is placed in a flask and a known volume of normal alkali in excess added in order to convert the whole of the acid in a basic salt. A few drops of rosolic acid are now added, and sufficient neutral $\mathrm{BaCl}_{2}$ solution poured in to combine with the phosphoric acid. The mixture is heated to boiling, and while hot the excess of alkali is titrated with $\frac{\mathrm{N}}{\mathrm{I}}$ acid.

The suspended basic phosphate, together with the liquid, possesses a rose-red color until the last drop or two of acid, after continuous heating and agitation, gives a permanent white or slightly yellowish milky appearance, when the process is ended.

The volume of normal alkali, less the volume of normal acid, represents the amount of alkali required to convert the phosphoric acid into a normal trisodic or tripotassic phosphate.

$$
\begin{aligned}
& \mathrm{H}_{3} \mathrm{PO}_{4}+3 \mathrm{KOH}=\mathrm{K}_{3} \mathrm{PO}_{4}+{ }_{3} \mathrm{H}_{2} \mathrm{O} . \\
& 3 \frac{97.29}{32.43} \text { gms. } \frac{167.22}{55.74} \text { gins. }=1000 \text { cc. of } \frac{\mathrm{N}}{\mathrm{I}} \text { KOH V. S. }
\end{aligned}
$$

Thus I cc. of $\frac{\mathrm{N}}{\mathrm{I}}$ alkali $=0.03243 \mathrm{gm}$. of $\mathrm{H}_{3} \mathrm{PO}_{4}$.

Thompson, however, has demonstrated that this acid may be accurately titrated by standard alkali when using either methyl orange or phenolphthalein, or both, successively.

If methyl orange is used the color changes upon the completion of the formation of monobasic phosphate, $\mathrm{KH}_{2} \mathrm{PO}_{4}$, as per the following equation:

$$
\mathrm{H}_{3} \mathrm{PO}_{4}+\mathrm{KOH}=\mathrm{KH}_{2} \mathrm{PO}_{4}+\mathrm{H}_{2} \mathrm{O} .
$$

If phenolphthalein is used, the color changes upon the completion of the formation of the dibasic phosphate $\mathrm{K}_{2} \mathrm{HPO}_{4}$.

$$
\mathrm{H}_{3} \mathrm{PO}_{4}+{ }_{2} \mathrm{KOH}=\mathrm{K}_{2} \mathrm{HPO}_{4}+{ }_{2} \mathrm{H}_{2} \mathrm{O} \text {. }
$$


In the method of Thompson, a weighed quantity of the acid, diluted with a little water, is titrated with normal potassium hydroxid, using methyl orange as an indicator until the red color of the solution changes to yellow, indicating the formation of the monobasic salt. The number of cc. of alkali solution required is multiplied by the factor 0.09729 . A few drops of phenolphthalein are now added and the titration continued until a pale red color appears, indicating the formation of the dibasic salt. The total number of cc. of alkali added is multiplied by 0.04865 . In either case, the quantity of absolute $\mathrm{H}_{3} \mathrm{PO}_{4}$ present in the sample is obtained. The U.S. P. recommends the use of phenolphthalein alone. For other methods for the assay of pnosphoric acid, see Part II.

\section{Hypophosphorous Acid $\left(\mathrm{HPH}_{2} \mathrm{O}_{2}=65.53\right)$.}

$$
\begin{aligned}
& \mathrm{HPH}_{2} \mathrm{O}_{2}+\mathrm{KOH}=\mathrm{KPH}_{2} \mathrm{O}_{2}+\mathrm{H}_{2} \mathrm{O} \\
& 65.53 \mathrm{gms} .=55.74 \mathrm{gms} .=1000 \text { cc. } \frac{\mathrm{N}}{\mathrm{I}} \mathrm{V} . \mathrm{S} .
\end{aligned}
$$

Thus each cc. of $\frac{\mathrm{N}}{\mathrm{I}}$ alkali represents $0.06553 \mathrm{gm}$. of $\mathrm{HPH}_{2} \mathrm{O}_{2}$.

Take about $5 \mathrm{gms}$. of the acid, dilute it with a small quantity of water, add a few drops of methyl orange, and titrate with $\frac{\mathrm{N}}{\mathrm{x}} \mathrm{KOH}$ until the solution assumes a yellow color.

Nitric Acid $\left(\mathrm{HNO}_{3}=62.57\right)$.

$$
\begin{aligned}
& \mathrm{HNO}_{3}+\mathrm{KOH}=\mathrm{KNO}_{3}+\mathrm{H}_{2} \mathrm{O} \\
& 62.57 \mathrm{gms} .=55.74 \text { gms. }=\text { roo0 cc. } \frac{\mathrm{N}}{\mathrm{I}} \text { V.S. }
\end{aligned}
$$

Each cc. of $\frac{\mathrm{N}}{\mathrm{I}} \mathrm{KOH}=0.06257 \mathrm{gm}$. of nitric acid. Take 3 cc. of the acid for analysis, weigh it accurately in a stoppered weighing bottle, dilute it with about $50 \mathrm{cc}$. of distilled water, and then titrate with $\frac{\mathrm{N}}{\mathrm{I}} \mathrm{KOH}$ V.S., using methyl orange as indicator. Multiply the number of cc. of standard alkali used by $0.06257 \mathrm{gm}$. then multiply this product by 100 and divide by the weight of the acid taken, the quotient will represent the percentage of absolute acid present in the sample. If the official nitric acid is being analyzed the specific gravity of which is $\mathrm{r} .403$, the weight of the $3 \mathrm{cc}$. will be $3 \times \mathrm{r} .403=4.209 \mathrm{gms}$.

Hydrobromic and hydriodic acids may be estimated in the same way as the foregoing, but it is usually preferred to estımate them by 
precipitation analysis, as described on pages I2O and I2I respectively. Sulphurous acid is best assayed by oxidation with iodin as described on page 198 .

Boric Acid $\left(\mathrm{H}_{3} \mathrm{BO}_{3}=61.54\right)$. This acid is estimated by neutralization with $\frac{\mathrm{N}}{\mathrm{I}} \mathrm{NaOH}$ in the presence of a large quantity of glycerin. (Thompson's Method, J. S. C. I., XII, 432). The addition of sufficient glycerin to a boric acid solution, so that no less than 30 per cent be present throughout the titration, develops the acidity of boric acid with regard to phenolphthalein to a great degree, and enables one to titrate direct with standard soda solution. I gm. of boric acid is dissolved in $50 \mathrm{cc}$. of water to this is added an equal volume of glycerin, then a few drops of phenolphthalein T. S., and the titration with normal sodium hydroxid begun and continued until a pink color appears.

Each cc. of $\frac{\mathrm{N}}{\mathrm{I}} \mathrm{NaOH}=0.06 \mathrm{I} 54$ gm. of $\mathrm{H}_{3} \mathrm{BO}_{3}$.

$$
\begin{aligned}
& \mathrm{H}_{3} \mathrm{BO}_{3}+\mathrm{NaOH}=\mathrm{NaH}_{2} \mathrm{BO}_{3}+\mathrm{H}_{2} \mathrm{O} \\
& 61.54 \quad 39.76 \text { gms. in } 1000 \text { cc. } \frac{\mathrm{N}}{\mathrm{I}} \text { V.S. } \\
& 0.06154 \mathrm{gm} . \quad \text { I cc. } \frac{\mathrm{N}}{\mathrm{I}} \text { V.S. }
\end{aligned}
$$

Organic Acids. As the individual organic acids require different indicators, the table on page $3 \mathrm{r}$ should be consulted in the selection of an indicator for a particular organic acid. Phenolphthalein is, however, the most suitable for organic acids generally.

Acetic Acid $\left(\mathrm{HC}_{2} \mathrm{H}_{3} \mathrm{O}_{2}=59.58\right)$. Mix 3 gms. of the acid with a small quantity of water, add a few drops of phenolphthalein T. S., and titrate with normal potassium hydroxid V. S. until a permanent pale-pink color is obtained, and apply the following equation:

$$
\underset{59 \cdot 53}{\mathrm{HC}_{2} \mathrm{H}_{3} \mathrm{O}_{2}}+\underset{55 \cdot 74}{\mathrm{KOH}}=\mathrm{KC}_{2} \mathrm{H}_{3} \mathrm{O}_{3}+\mathrm{H}_{2} \mathrm{O} .
$$

Thus 55.74 gms. or 1000 cc. of $\frac{\mathrm{N}}{\mathrm{I}} \mathrm{KOH} \mathrm{V.S.} \mathrm{will} \mathrm{neutralize} 59.58$ gms. of acetic acid; therefore each cc. of $\frac{\mathrm{N}}{\mathrm{I}} \mathrm{KOH}$ V. S. represents $0.0595^{8} \mathrm{gm}$. of acetic acid. 
If $18 \mathrm{cc}$. are required to neutralize $3 \mathrm{gms}$. of the acid, it contains $\mathrm{I} 8 \times 0.05958=\mathrm{I} .0724 \mathrm{gms}$. of absolute acetic acid.

$$
\frac{\mathrm{I} .0724 \times 100}{3}=35.74 \text { per cent. }
$$

Tartaric Acid $\left(\mathrm{H}_{2} \mathrm{C}_{4} \mathrm{H}_{4} \mathrm{O}_{6}=148.92\right)$. Dissolve 2 gms. of tartaric acid in sufficient water to make a solution, add a few drops of phenolphthalein and then pass into the solution from a burette $\frac{\mathrm{N}}{\mathrm{I}}$ potassium hydroxid V. S. until a faint pink tint is acquired by the solution, and apply the equation

$$
\begin{aligned}
& \mathrm{H}_{2} \mathrm{C}_{4} \mathrm{H}_{4} \mathrm{O}_{6}+{ }_{2} \mathrm{KOH}=\mathrm{K}_{2} \mathrm{C}_{4} \mathrm{H}_{4} \mathrm{O}_{6}+{ }_{2} \mathrm{H}_{2} \mathrm{O} \\
& \text { 2) } \lcm{48.92} \text { 2) II I. } 48 \\
& 74.46 \text { gms. } 55.74 \text { gms. }=1000 \mathrm{cc} . \frac{\mathrm{N}}{\mathrm{I}} \text { V.S. }
\end{aligned}
$$

Thus each cc. required for the neutralization of the acid represents $0.07446 \mathrm{gm}$. If $26.6 \mathrm{cc}$. are required then $26.6 \times 0.07446=\mathrm{x} .98 \mathrm{gms}$.

Then

$$
\frac{\mathrm{I} .98 \times 100}{2}=99 \text { per cent. }
$$

The other organic acids are assayed in exactly the same manner as that described for the foregoing.

Citric Acid $\left(\mathrm{H}_{3} \mathrm{C}_{6} \mathrm{H}_{5} \mathrm{O}_{7} \cdot \mathrm{H}_{2} \mathrm{O}=208 \cdot 50\right)$.

$$
\begin{aligned}
& \mathrm{H}_{3} \mathrm{C}_{6} \mathrm{H}_{5} \mathrm{O}_{7} \cdot \mathrm{H}_{2} \mathrm{O}+{ }_{3} \mathrm{KOH}=\mathrm{K}_{3} \mathrm{C}_{6} \mathrm{H}_{5} \mathrm{O}_{7}+{ }_{4} \mathrm{H}_{2} \mathrm{O} \text {. } \\
& \text { 3) } \frac{208.50}{69.5} \quad \frac{3) \mathrm{r} 67.22}{55.74 \mathrm{gms}}=1000 \mathrm{cc} . \frac{\mathrm{N}}{\mathrm{I}} \mathrm{V} \text {.S. }
\end{aligned}
$$

Each cc. of $\frac{\mathrm{N}}{\mathrm{I}} \mathrm{KOH}$ represents $0.0695 \mathrm{gm}$. of crystallized citric acid.

Oxalic Acid $\left(\mathrm{H}_{2} \mathrm{C}_{2} \mathrm{O}_{4} \cdot 2 \mathrm{H}_{2} \mathrm{O}=\mathrm{I} 25 . \mathrm{IO}\right)$.

$$
\begin{aligned}
& \mathrm{H}_{2} \mathrm{C}_{2} \mathrm{O}_{4} \cdot{ }_{2} \mathrm{H}_{2} \mathrm{O}+{ }_{2} \mathrm{KOH}=\mathrm{K}_{2} \mathrm{C}_{2} \mathrm{O}_{4}+{ }_{4} \mathrm{H}_{2} \mathrm{O} \text {. } \\
& \text { 2) } \frac{\mathrm{I} 25 . \mathrm{I}}{62.55} \quad \frac{\text { 2) } 11 \mathrm{I} .48}{55.74} \mathrm{gms} .=\text { I000 cc. } \frac{\mathrm{N}}{\mathrm{I}} \text { V.S. }
\end{aligned}
$$

Each cc. of $\frac{\mathrm{N}}{\mathrm{I}} \mathrm{KOH}$ represents $0.06255 \mathrm{gm}$. of crystallized oxalic acid. 
Lactic Acid $\left(\mathrm{HC}_{3} \mathrm{H}_{5} \mathrm{O}_{3}=89.37\right)$.

$$
\begin{gathered}
\mathrm{HC}_{3} \mathrm{H}_{5} \mathrm{O}_{3}+\mathrm{KOH}=\mathrm{KC}_{3} \mathrm{H}_{5} \mathrm{O}_{3}+\underset{\mathrm{H}_{2} \mathrm{O} .}{89.37} \quad 55.74 \text { gms. }=\text { rooo cc. } \frac{\mathrm{N}}{\mathrm{I}} \text { V.S. }
\end{gathered}
$$

Each cc. of $\frac{\mathrm{N}}{\mathrm{I}} \mathrm{KOH}$ represents $0.08937 \mathrm{gm}$. of lactic acid.

\begin{tabular}{|c|c|c|c|c|}
\hline & $\begin{array}{l}\text { Molec- } \\
\text { ular } \\
\text { Weight. }\end{array}$ & $\begin{array}{l}\text { Standard } \\
\text { Solution. }\end{array}$ & Indicator. & Factor.* \\
\hline Acetic, $\mathrm{HC}_{2} \mathrm{H}_{3} \mathrm{O}_{2} \ldots \ldots \ldots \ldots \ldots$ & $59 \cdot 5^{8}$ & $\frac{\mathrm{N}}{\mathrm{I}} \mathrm{KOH}$ & Phenolphthalein & $0.0595^{8}$ \\
\hline Boric, $\mathrm{H}_{2} \mathrm{BO}_{3} \ldots \ldots \ldots \ldots \ldots$ & $61 \cdot 54$ & $\frac{\mathrm{N}}{\mathrm{I}} \mathrm{NaOH}$ & “ with glycerin & 0.06154 \\
\hline Citric, $\mathrm{H}_{3} \mathrm{C}_{6} \mathrm{H}_{5} \mathrm{O}_{7} \cdot \mathrm{H}_{2} \mathrm{O} \ldots \ldots \ldots$ & 208.50 & $\frac{\mathrm{N}}{\mathrm{I}} \mathrm{KOH}$ & Phenolphthalein & 0.06950 \\
\hline Hydriodic, HI................ & I 26.90 & & Methyl orange & 0.12690 \\
\hline Hydrobromic, HBr............ & $80 \cdot 3^{6}$ & $\because$ & "6 & 0.08036 \\
\hline Hydrochloric, $\mathrm{HCl} . . . \ldots \ldots \ldots$. & $3^{6.18}$ & 16 & 16 & 0.03618 \\
\hline Hypophosphorous, $\mathrm{HPH}_{2} \mathrm{O}_{2} \ldots \ldots$ & $65 \cdot 53$ & "6 & Dh & 0.06553 \\
\hline Lactic, $\mathrm{HC}_{3} \mathrm{H}_{5} \mathrm{O}_{3} \ldots \ldots \ldots \ldots$ & $89 \cdot 37$ & 66 & Phenolphthalein & 0.08937 \\
\hline Nitric, $\mathrm{HNO}_{3} \ldots \ldots \ldots \ldots \ldots \ldots$ & $62 \cdot 57$ & "6 & Methyl orange & 0.06257 \\
\hline $\begin{array}{l}\text { Oxalic, } \mathrm{H}_{2} \mathrm{C}_{2} \mathrm{O}_{4} \cdot{ }_{2} \mathrm{H}_{2} \mathrm{O} \ldots \ldots \ldots \\
\text { Phosphoric, } \mathrm{H}_{3} \mathrm{PO}_{1} \ldots \ldots \ldots \ldots \ldots\end{array}$ & $\begin{array}{r}125.10 \\
97.29\end{array}$ & “" & Phenolphthalein & 0.06255 \\
\hline To formation of $\mathrm{KH}_{2} \mathrm{PO}_{1} \ldots \ldots \ldots$ & $\ldots \ldots$ & "6 & Methyl orange & 0.09729 \\
\hline ، $\lll \quad$ “ $\mathrm{K}_{2} \mathrm{HPO}_{4} \ldots \ldots \ldots$ & $\cdots \cdots \cdots$ & “6 & Phenolphthalein & 0.048645 \\
\hline " " " $\mathrm{K}_{3} \mathrm{PO}_{1} \ldots \ldots \ldots$ & $\cdots \cdots \cdots$ & $\cdots$ & Rosolic acid & 0.03243 \\
\hline $\left.\begin{array}{c}\text { Asammonio-magnesium phos- } \\
\text { phate. . . . . . . . . . }\end{array}\right\}$ & $\cdots \cdots \cdots$ & $\frac{\mathrm{N}}{10} \mathrm{HCl}$ & Methyl orange & 0.0048645 \\
\hline Sulphuric, $\mathrm{H}_{2} \mathrm{SO}_{4} \ldots \ldots \ldots \ldots$ & $97 \cdot 35$ & $\frac{\mathrm{N}}{\mathrm{I}} \mathrm{KOH}$ & "c & 0.048675 \\
\hline Tartaric, $\mathrm{HC}_{4} \mathrm{H}_{4} \mathrm{O}_{6} \ldots \ldots \ldots \ldots$ & 148.92 & ، & Phenolphthalein & $0.0^{74} 46$ \\
\hline Trichloracetic, $\mathrm{HC}_{2} \mathrm{Cl}_{3} \mathrm{O}_{2} \ldots \ldots$ & I62. I 2 & $\frac{\mathrm{N}}{\mathrm{I}} \mathrm{NaOH}$ & "6 & 0.016212 \\
\hline
\end{tabular}

Table of Acids which may be Estimated by Neutralization.

Certain of these acids are best assayed by other methods than neutralization. For example, hydriodic, hydrobromic, and hydrocyanic are usually assayed by precipitation with $\frac{\mathrm{N}}{\mathrm{IO}}$ silver nitrate; sulphurous acid is assayed by $\frac{\mathrm{N}}{\mathrm{IO}}$ iodin.

* This is the coefficient by which the number of cc. of standard solution is to be multiplied in order to obtain the quantity of pure acid in the sample analyzed. 
Table Showing Quantity of Substance to be Taken for Analysis in Direct Percentage Estimations

\begin{tabular}{|c|c|c|c|}
\hline Substance. & $\begin{array}{l}\text { Molecular } \\
\text { Weight. }\end{array}$ & $\begin{array}{c}\text { Quantity of } \\
\text { Substance to } \\
\text { be taken, so } \\
\text { that each cc. } \\
\text { of the V. S. } \\
\text { will Represent } \\
\text { r\%. } \\
\text { Grams. }\end{array}$ & $\begin{array}{c}\text { Percentage } \\
\text { Strength of } \\
\text { the Official } \\
\text { Substances. } \\
\text { Per Cent. }\end{array}$ \\
\hline Acetic acid. ${ }_{\|} \ldots \ldots \ldots \ldots$ & $59 \cdot 5^{8}$ & $5 \cdot 95^{8}$ & 36 \\
\hline $\begin{array}{r}\text { glacial } . \ldots \ldots \ldots \ldots \ldots \\
\text { Ammonium carbonate } \ldots \ldots \ldots \ldots \ldots\end{array}$ & $\begin{array}{r}59 \cdot 5^{8} \\
156.01\end{array}$ & $\begin{array}{l}5 \cdot 95^{8} \\
5 \cdot 2\end{array}$ & $\begin{array}{l}99 \\
97\end{array}$ \\
\hline 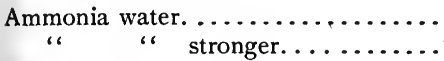 & $\begin{array}{l}\text { I6. } 93 \mathrm{NH}_{3} \\
\text { 16.93 } \mathrm{NH}_{3}\end{array}$ & $\begin{array}{l}\text { I. } 693 \\
\text { I. } 693\end{array}$ & $\begin{array}{l}\text { 10 } \\
28\end{array}$ \\
\hline Boric acid. ................... & 6 เ. 54 & 6.154 & 99.2 \\
\hline Citric acid $\left(+\mathrm{H}_{2} \mathrm{O}\right), \ldots \ldots \ldots \ldots \ldots \ldots$ & 208.50 & 6.95 & 99.5 \\
\hline Hydrochloric acid. . . . . . . . . . . . & 36.18 & 3.618 & 3 r.9 \\
\hline Hypophosphorous acid. ........... & $65 \cdot 5 \hat{3}$ & 6.553 & 30 \\
\hline Lactic acid.................... & 89.37 & 8.937 & 75 \\
\hline $\begin{array}{c}\text { Lithium } \\
" \quad \text { carbonate } \ldots \ldots \ldots \ldots \ldots \ldots \\
\text { citrate } \ldots \ldots \ldots \ldots \ldots \ldots\end{array}$ & $\begin{array}{r}73 \cdot 5^{1} \\
208 \cdot 5^{6}\end{array}$ & $\begin{array}{l}3.755 \\
6.952\end{array}$ & $\begin{array}{l}98.5 \\
98.5\end{array}$ \\
\hline Lime water $\left(\mathrm{Ca}(\mathrm{OH})_{2}\right) \ldots \ldots \ldots \ldots$ & $73 \cdot 56$ & 3.678 & 00.14 \\
\hline 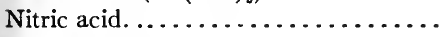 & 62.57 & 6.257 & 68 \\
\hline Oxalic acid. ................... & I 25. 10 & 6.255 & \\
\hline Phosphoric acid. ............... & 97.29 & & \\
\hline “ “ with methyl orange..... & 97.29 & 9.729 & 85 \\
\hline ، “ “ phenolphthalein... & 97.29 & 4.8645 & 85 \\
\hline Potassium acetate. ............... & $97 \cdot 44$ & 9.744 & 98 \\
\hline “ bicarbonate........... & I99.82 & $9.94 \mathrm{I}$ & 99 \\
\hline bitartrate. . . . . . . . . & 186.78 & 18.678 & 99 \\
\hline carbonate........... & 137.27 & 6.8635 & 98 \\
\hline citrate............ & 322.08 & 10.736 & 99 \\
\hline hydroxid........... & $55 \cdot 74$ & $5 \cdot 574$ & 85 \\
\hline " liquor.......... & $55 \cdot 74$ & $5 \cdot 574$ & 5 \\
\hline ، $\quad$ sodium tartrate. ......... & 280.08 & 14.009 & 99 \\
\hline Sodium acetate. ................ & I 35.10 & $13 \cdot 5^{1}$ & 99.5 \\
\hline “ benzoate.............. & I 43.01 & 14.301 & 99 \\
\hline " bicarbonate. . ........... & r66.86 & 8.343 & 99 \\
\hline “ carbonate $\left(+\mathrm{IoH}_{2} \mathrm{O}\right) \ldots \ldots$. & 284 . I I & 14.2 & \\
\hline Sulphuric acid. ................ & $97 \cdot 35$ & 4.8675 & 92.5 \\
\hline Tartaric acid. .................. & 148.92 & $7 \cdot 447$ & $99 \cdot 5$ \\
\hline
\end{tabular}


Referring to the table it will be seen that if the quantities indicated are taken for analysis, the amount of standard solution required for substances of high percentage strength will be very large (in some cases over $99 \mathrm{cc}$.), while for substances of low percentage strength, as for instance lime water, so small a volume of standard solution is required as to be unreadable (0.14 cc.). It is therefore advisable to take for analysis a smaller quantity of high percentage substances and a larger quantity of such substances as contain a low percentage. It is usually best to so adjust it, that no less than ro nor more than $30 \mathrm{cc}$. of the standard solution be required. . For example: In the case of citric acid, instead of taking for analysis 6.95 gms. it will be better to take one-fourth of this quantity, then each cc. of the standard solution used will represent 4 per cent, and only one fourth as much will be required, i.e., $24.9 \mathrm{cc}$. instead of $99.5 \mathrm{cc}$. Again in the case of lime water, if $36.78 \mathrm{gms}$. are taken instead of $3.768 \mathrm{gms}$., $\mathbf{1 . 4}$ cc. of the standard solution $\frac{\mathrm{N}}{\mathrm{I}}$ will be required, which is better than $0.14 \mathrm{cc}$., but in this case, it will be still better to use a decinormal $\left(\frac{N}{10}\right)$ solution, then 36.78 gms. of lime water would require for neutralization just I 4 cc. of the $\frac{N}{10}$ acid V.S. If half the quantity indicated in the table is taken, then each cc. of the standard solution will represent 2 per cent. If one-tenth the quantity is taken each cc. will represent 10 per cent. If double the quantity is taken each cc. will represent 0.5 per cent, etc.

\section{ESTIMATION OF ACIDS IN COMBINATION IN NEUTRAL SALTS}

This may be done in the case of a large number of salts, by adding to the solution of the salt a measured excess of alkali or alkali carbonate in the form of normal solution, and then ascertaining the excess by retitration with normal acid. Thus the amount of alkali which went into combination with the acid is obtained. Most bases are precipitated by the hydroxid; some, however, require the addition of carbonate to effect their precipitation.

The carbonate is required for alkali earth salts, magnesium salts, alum, zinc salts, bismuth salts, nickel, cobalt, and lead salts.

Example. 2 gms. of barium chlorid are dissolved in water and sufficient normal sodium carbonate added to make the liquid decidedly 
alkaline (say $20 \mathrm{cc}$.), and the whole diluted to $300 \mathrm{cc}$. and set aside to settle. Ioo cc. of the clear supernatant liquid are then removed with a pipette and titrated for excess of alkali with normal nitric acid or normal hydrochloric acid, of which say I. 2 cc. are required, making it $3.6 \mathrm{cc}$. for the whole quantity; therefore $20-3.6=\mathrm{I} 6.4 \mathrm{cc}$. is the measure of the alkali which combined with the acid of the original salt. This multiplied by $0.035^{1} 8$, the factor for chlorin, gives 0.57695 gm. of chlorin. 


\section{CHAPTER XI}

\section{ANALYSIS BY PRECIPITATION}

THE general principle of this method is that the determination of the quantity of a given substance is effected by the formation of a precipitate upon the addition of the standaid solution to the substance under examination. There are three ways of determining the end reaction in precipitation analyses.

I. By adding the standard solution until it ceases to produce any more precipitate, as in the estimation of silver by standard sodium chlorid, and the estimation of haloid salts and acids by means of standard silver nitrate. The application of this ending is almost limited to the above estimations, hecause in these only can accurate results be obtained. The silver halids formed are not only quite insoluble, but they have a tendency to curdle closely upon shaking (especially in acid solutions), and thus leave a clear supernatant liquid in which any further precipitation can be readily seen. Most of the other precipitates, such as barium sulphate, calcium oxalate, etc., although heavy and insoluble, are so finely divided and powdery that they do not readily subside.

2. By the use of an indicator, as in the estimation of haloid salts by means of standard silver nitrate solution, using neutral potassium chromate as the indicator. The latter is added to the haloid solution, and the silver nitrate V. S. delivered into the mixture until a. permanent red color (silver chromate) is produced. Silver nitrate reacts by preference with the halogen, and does not react with the chromate until the halogen has been entirely precipitated. Hence the production of a permanent red color marks the completion of the precipitation of the halogen.

Another illustration is in the estimation of silver by sulphocyanate solution, using ferric alum as indicator. The sulphocyanate produces with the silver a white precipitate of silver sulphocyanate, but when the precipitation of silver is complete the sulphocyanate reacts with the ferric alum present and a red ferric sulphocyanate appears and marks the cnd point. On the other hand the indicator may be used 
externally, i.e., alongside of the liquid being analyzed. A drop of the latter being brought in contact with a drop of the indicator at frequent intervals in the course of the titration. As in the estimation of phosphoric acid by means of uranium nitrate solution, in which potassium ferrocyanid is used as indicator.

3. By adding the standard solution until the first appearance of a precipitate, as in the estimation of cyanogen by silver nitrate solution, and the estimation of chlorin by mercuric nitrate V.S. In these estimations the standard solution is added to the solution of the substance under analysis until a precipitate appears.

\section{PRECISION IN DETERMINING END-REACTIONS}

In most volumetric precipitation processes no direct reading of the end-point is possible; filtration and trial with small quantities of the clear filtrate being usually necessary. P. N. Rakow (Chem. Zeit.) has found that many precipitates which remain obstinately suspended under ordinary conditions, and cause, in the liquid being titrated, an unmanageable turbidity, can be induced to collect and subside by the addition of some immiscible liquid heavier than water; for example, carbon disulphid or chloroform. Such liquids, although exerting no solvent action on the precipitate, mix intimately with it and carry it down, leaving the supernatant liquid sufficiently clear for the observation of any turbidity produced by the addition of a further quantity of the precipitating solution. Carbon disulphid and chloroform are usually but not invariably effective. Thus the former carries down silver chlorid rapidly and completely, but has no influence on the precipitation of barium sulphate. The author has found the method to work fairly well in the few cases he tried.

In the titration of chlorids by means of silver nitrate with neutral chromate as indicator, the end-reaction is more distinctly seen by gaslight than by daylight; and if very dilute solutions of chlorid are estimated the titration is best performed by gaslight, and even then the change of color from yellow to red is not easily perceived.

In order to overcome this difficulty Dupré suggests the following simple method:

The chlorid solution is placed in a white porcelain dish, a small quantity of neutral chromate added (sufficient to make the liquid yellow). Then the titration is begun and watched by looking through a flat glass cell containing some of the neutral chromate. 
If the solution in the cell corresponds fairly with the tint of the liquid in the porcelain dish, the latter will appear to be perfectly colorless, like pure water, and the first faint appearance of red becomes strikingly manifest, and no mistake can be made.

The same plan may be followed in other titrations, where the endreaction depends upon the perception of color changes.

Preparation of Decinormal $\left(\frac{\mathrm{N}}{\mathrm{IO}}\right)$ Silver Nitrate $\left(\mathrm{AgNO}_{3}=\mathrm{I} 68.69\right.$; $\frac{\mathrm{N}}{\mathrm{IO}}$ V. S. $=\mathrm{I} 6.869 \mathrm{gms}$. in $\left.1000 \mathrm{cc}\right)$. Dissolve $16.869 \mathrm{gms}$. of pure silver nitrate $*$ in sufficient water to make, at or near $15^{\circ} \mathrm{C} .\left(59^{\circ} \mathrm{F}\right.$.), exactly rooo cc. I liter of this solution thus contains ${ }_{1}^{1} 0$ of the molecular weight in grams of silver nitrate. It is therefore a decinormal solution.

If pure crystals of silver nitrate are not readily obtainable, and pure sodium chlorid is at hand, a solution of the silver nitrate may be made of approximate strength, a little stronger than necessary, and then standardized by means of the sodium chlorid, as follows: 0.1 6 I $2 \mathrm{gm}$. of sodium chlorid is dissolved in water, and a burette is filled with the solution of silver nitrate to be standardized. The silver solution is now slowly added from the burette to the sodiumchlorid solution contained in a beaker until no more precipitate of silver chlorid is produced.

If neutral potassium chromate is used as an indicator, the end of the reaction is shown by the appearance of yellowish-red silver chromate. This indication is extremely delicate. The silver nitrate does not act upon the chromate until all of the chlorid is converted into silver chlorid.

In the above reaction $20 \mathrm{cc}$. of silver nitrate should be required. But since the silver-nitrate solution is too strong, less of it will complete the reaction, and the solution must be diluted so that exactly $20 \mathrm{cc}$. will be required to precipitate the chlorin in $0.11612 \mathrm{gm}$. of $\mathrm{NaCl}$.

Thus if $\mathrm{I}_{7} \mathrm{cc}$. are used, each $17 \mathrm{cc}$. must be diluted to $20 \mathrm{cc}$., or each $170 \mathrm{cc}$. to $200 \mathrm{cc}$, , or the entire remaining solution in the same proportion.

After dilution a fresh trial should always be made.

\footnotetext{
* The U. S. P. directs that the silver nitrate should previous to weighing, be pulverized and dried in a covered porcelain crucible in an air-bath at $130^{\circ} \mathrm{C}$. $\left(206^{\circ} \mathrm{F}\right.$.) for one hour.
} 
Silver nitrate solution should be kept in dark amber-colored, glassstoppered bottles, carefully protected from dust.

Titration by decinormal silver nitrate V. S. may be managed in various ways, adapted to the special preparation to be tested.

I. In most cases it is directed by the U. S. P. to be used in the presence of a small quantity of potassium chromate $\mathrm{T}$. $\mathrm{S}$.

2. In some cases it is added until the first appearance of a permanent precipitate, as in potassium cyanid and hydrocyanic acid assays.

3. It may be used in all cases without an indicator by observing the exact point when no further precipitate occurs. But since this consumes too much time in waiting for the precipitate to subside, so as to render the supernatant liquid sufficiently clear to recognize whether a further precipitate is produced by the addition of the silver solution, it is impracticable.

4. It may be added in definite amount, known to be in excess of the quantity required, and the excess measured back by titration with decinormal potassium sulphocyanate V.S., or even with decinormal sodium chlorid V. S. (residual titration).

Decinormal $\frac{\mathrm{N}}{\mathrm{IO}}$ Sodium Chlorid $\left(\mathrm{NaCl}=58.06 ; \frac{\mathrm{N}}{\mathrm{IO}} \mathrm{V} . \mathrm{S} .=5.806\right.$ gms. in I000 cc). Dissolve 5.806 gms. of pure sodium chlorid in enough water to make exactly $1000 \mathrm{cc}$. at the ordinary temperature of the atmosphere.

Check this solution with decinormal silver nitrate. The two solutions should correspond, volume for volume:

Pure Sodium Chlorid may be prepared by passing into a saturated aqueous solution of the purest commercial sodium chlorid a current of dry hydrochloric acid gas. The crystalline precipitate is then separated and dried at a temperature sufficiently high to expel all traces of free acid.

The method of standardizing $\frac{\mathrm{N}}{\mathrm{IO}} \mathrm{NaCl}$ solution is as follows:

$0.3373^{8} \mathrm{gm}$. of silver nitrate is dissolved in ro cc. of distilled water, and the solution carefully titrated with $\frac{\mathrm{N}}{\mathrm{IO}} \mathrm{NaCl}$ V.S. until precipitation ceases. $20 \mathrm{cc}$. of the standard solution should be required.

$$
\begin{aligned}
& \mathrm{AgNO}_{3}+\mathrm{NaCl}=\mathrm{AgCl}+\mathrm{NaNO}_{3} . \\
& \begin{array}{lll}
\text { 10) } 168.69 & \text { 10) } 58.06
\end{array} \\
& \text { I6.869 gms. } 5.806 \text { gms., or } 1000 \text { cc. } \frac{\mathrm{N}}{\mathrm{IO}} \mathrm{NaCl} \text { V.S. }
\end{aligned}
$$


Each cc. of the standard solution represents $0.016869 \mathrm{gm}$. of pure $\mathrm{AgNO}_{3}$.

$$
\begin{aligned}
& 0.016869 \times 20=0.3373^{8} \mathrm{gm} . \\
& \frac{0.3373^{8} \times 100}{0.3373^{8}}=100 \text { per cent } .
\end{aligned}
$$

Decinormal $\frac{\text { N }}{\text { IO }}$ Potassium Sulphocyanate (Volhard's Solution) $\left(\mathrm{KSCN}=96.53 \frac{\mathrm{N}}{\mathrm{r}} \mathrm{V} . \mathrm{S} .=9.653\right.$ gms. in rooo cc.). Dissolve $10 \mathrm{gms}$. of pure crystallized potassium sulphocyanate (thiocyanate) in rooo cc. of water.

This solution, which is too concentrated, must be adjusted so as to correspond exactly in strength with decinormal silver nitrate V. S. For this purpose introduce into a flask ro cc. of $\frac{\mathrm{N}}{\mathrm{IO}} \mathrm{AgNO}_{3} \mathrm{~V}$. S., $5 \mathrm{cc}$. of ammonioferric sulphate T. S., and 5 cc. of diluted nitric acid.

Run into this mixture from a burette the sulphocyanate solution.

At first a white precipitate of silver sulphocyanate is produced, giving the fluid a milky appearance, and then as each drop of sulphocyanate falls in it is surrounded by a deep brownish-red cloud of ferric sulphocyanate, which quickly disappears on shaking, as long as any of the silver nitrate remains unchanged.

When the point of saturation is reached and the silver has all been precipitated, a single drop of the sulphocyanate solution produces a faint brownish-red color, which does not disappear on shaking.

Note the number of cc. of the sulphocyanate solution used, and dilute the whole of the remaining solution so that equal volumes of this and of the decinormal silver nitrate will be required to produce the permanent brownish-red tint. (The same tint of brown or red to which the volumetric solution is adjusted must be attained when the solution is used in volumetric testing.)

Assuming that $9.5 \mathrm{cc}$. of the sulphocyanate solution were required to produce the reaction, then each $0.5 \mathrm{cc}$. must be diluted to make Io cc., or the whole of the remaining solution in the same proportion.

Always make a new trial after the dilution to see if the solutions correspond, e.g., $50 \mathrm{cc}$. of $\frac{\mathrm{N}}{\mathrm{IO}}$ silver nitrate are taken, and $5 \mathrm{cc}$. of ammonioferric sulphate, $5 \mathrm{cc}$. of pure nitric acid and $200 \mathrm{cc}$. of water are added and there should be required exactly $50 \mathrm{cc}$. of the potassium sulphocyanate solution. The same depth of reddish-brown 
tint should be obtained in all assays by this method, as is obtained in standardizing the solution.

\section{ESTIMATION OF SOLUBLE HALOID SALTS}

The estimation of these salts is based upon the powerful affinity existing between the halogens and silver, and the ready precipitation of the resulting chlorid, bromid, and iodid. Standard solution of silver nitrate is used for this purpose, and for the sake of exactness and convenience is made of decinormal strength. In some cases it is advisable to use centinormal solutions.

Mohr's Method with Chromate Indicator. This method is the best to use, if the haloid salts are in neutral solution, and salts of lead, bismuth, barium, or iron are absent. If the solution is acid the indicator is inadmissable, in that acids have a solvent action upon silver chromate and thus prevent the end-reaction from being clearly and accurately observed. If the above-mentioned metals are present, the indicator is likewise useless as these bases form insoluble, highly colored compounds with the chromate. The neutral potassium chromate (yellow chromate) which is used as the indicator must be free from chlorid $*$ and should be used in the form of a 10 per cent solution.

In the volumetric analysis of soluble haloid salts (chlorids, bromids, and iodids) $0.5 \mathrm{gm}$. of the well-dried salt is dissolved in $40 \mathrm{cc}$. of water in a beaker. This is placed upon a white surface and a few drops of the chromate indicator (or sufficient to give the solution a pale yellow tint), added. The decinormal $\frac{\mathrm{N}}{\mathrm{IO}}$ silver nitrate solution is then added cautiously from a burette, stirring constantly until a permanent red tint is produced. The red tint is due to the formation of silver chromate, which does not appear permanent until the last trace of halogen has been precipitated.

The reactions are as follows:

and

$$
\mathrm{NaCl}+\mathrm{AgNO}_{3}=\mathrm{AgCl}+\mathrm{NaNO}_{3}
$$

$$
\mathrm{K}_{2} \mathrm{CrO}_{4}+{ }_{2} \mathrm{AgNO}_{3}=\mathrm{Ag}_{2} \mathrm{CrO}_{4}+{ }_{2} \mathrm{KNO}_{3} \text {. }
$$

* The presence of 'chlorid in the chromate solution may be determined by adding a small quantity of silver nitrate solution, and then some nitric acid. If the red precipitate dissolves completely and leaves a clear solution, chlorid is absent. If it is found to be present it may be removed by the addition of a few drops of silver nitrate solution, and filtering, without using any nitric acid. 
If the solution to be estimated is acid it should be accurately neutralized with ammonia, or sodium or calcium carbonate. If it is alkaline in reaction it should likewise be neutralized, using acetic acid for this purpose.

In the estimation of bromids and iodids it must not be forgotten to take into account the invariable presence of chlorids as an impurity.

The method in detail is exemplified in the following assays:

Estimation of Sodium Chlorid. I gm. of the well-dried sodium chlorid is dissolved in sufficient distilled water to measure roo cc. Of this solution Io cc. (representing o.I gm. of the salt) is taken, a few drops of neutral potassium chromate solution added, and then the $\frac{\mathrm{N}}{\mathrm{rO}}$ silver solution delivered from a burette with constant stirring or shaking until the chlorid is entirely precipitated as evidenced by the formation of a permanent red color (silver chromate). The equation is

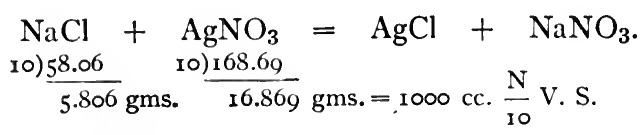

Tinus each cc. of the $\frac{\mathrm{N}}{10} \mathrm{~V}$. S. represents $0.005806 \mathrm{gm}$. of $\mathrm{NaCl}$.

If in the above assay $17 \mathrm{cc}$. of the silver solution were required, then i $7 \times 0.005803 \mathrm{gm} .=0.09865 \mathrm{I} \mathrm{gm}$. or $98.65 \mathrm{I}$ per cent.

$$
\frac{0.09865 \mathrm{I} \times 100}{0.1}=98.65 \mathrm{I} \text { per cent. }
$$

Estimation of Ammonium Bromid. $3 \mathrm{gms}$. of the salt are dried at $100^{\circ} \mathrm{C}$. ( $\left(2 \mathrm{I} 2^{\circ} \mathrm{F}\right.$ ) (to remove moisture, which the salt readily absorbs out of the air), and dissolved in sufficient water to measure $100 \mathrm{cc}$. ro cc. of this solution (representing $0.3 \mathrm{gm}$. of the salt) are placed in a beaker, a few drops of potassium chromate T.S. added, and then the $\frac{\mathrm{N}}{\mathrm{IO}}$ silver nitrate V.S. carefully delivered from a burette, until a permanent red coloration is produced. Apply the equation

$$
\begin{aligned}
& \mathrm{NH}_{4} \mathrm{Br}+\mathrm{AgNO}_{3}=\mathrm{AgBr}+\mathrm{NH}_{4} \mathrm{NO}_{3} \\
& \begin{array}{ll}
\text { 10) } 97.29 & \text { 10) } 168.69
\end{array} \\
& \text { 9.7 } 29 \text { gms. } 16.869 \text { gms. or } 1000 \mathrm{cc} \frac{\mathrm{N}}{10} \cdot \mathrm{AgNO}_{3} \mathrm{~V} . \mathrm{S} \text {. }
\end{aligned}
$$


Thus each cc. of the $\frac{\mathrm{N}}{\mathrm{IO}}$ V.S. represents $0.009729 \mathrm{gm}$. of $\mathrm{NH}_{4} \mathrm{Br}$.

The U. S. P. requirement is that not more than $31.6 \mathrm{cc}$. of $\frac{\mathrm{N}}{10} \mathrm{AgNO}_{3}$ be required for $0.3 \mathrm{gm}$. of ammonium bromid. If the salt is absolutely pure only $30.84 \mathrm{cc}$. would be required for $0.3 \mathrm{gm}$. The excess $\mathrm{s}$ due to the presence in the commercial salt of a certain amount of ammonium chlorid which is precipitated by the silver nitrate as well as the bromid, and which, having a lower molecular weight, requires proportionately more silver nitrate to precipitate it than the bromid does. The presence of chlorids must always be taken into account in the valuation of bromids, because the latter usually contain more or less of the former as an impurity.

The Determination of the Amount of Chlorid Present is Calculated as Follows: The amount of the salt examined equivalent to $1000 \mathrm{cc}$. of $\frac{\mathrm{N}}{\mathrm{IO}}$ silver nitrate solution is first found thus:

$$
\text { 31.6:0.3:: 1000 cc. : } x \quad x=9.493 \text { gms. }
$$

This is then deducted from the quantity of pure ammonium bromid ( $9.729 \mathrm{gm}$.) which is equivalent to rooo cc. of $\frac{\mathrm{N}}{\mathrm{IO}}$ silver nitrate solution.

$$
9.729-0.493=y . \quad y=0.236 \mathrm{gm} .
$$

$y$ represents the excess of $\frac{\mathrm{N}}{\mathrm{IO}}$ silver nitrate solution used up by' the ammonium chlorid, reckoned in terms of ammonium bromid, and since 5.3 II gms. of $\mathrm{NH}_{4} \mathrm{Cl}$ is equivalent to 9.729 gms. of $\mathrm{NH}_{4} \mathrm{Br}$, the excess which $\mathrm{NH}_{4} \mathrm{Cl}$ can consume is represented by

therefore,

$$
9.729-5.3 \mathrm{II}=4.4 \mathrm{I} 8 \mathrm{gms} .
$$

$$
4.418: 5.311:: 0.236: z . \quad z=0.283 \mathrm{gm} .
$$

$z$ represents the amount of $\mathrm{NH}_{4} \mathrm{Cl}$ present in 9.493 gms. of the sample. Lastly, calculate the percentage

$$
9.493: 0.283:: 100: p . \quad p=2.9^{8} \text { per cent of } \mathrm{NH}_{4} \mathrm{Cl} \text {. }
$$

Thus the salt examined contained 97.02 per cent of $\mathrm{NH}_{4} \mathrm{Br}$.

Estimation of Potassium Iodid. This is conducted in exactly the same manner as the preceding salts. The presence of chlorid 
$(\mathrm{KCl})$ as an impurity must likewise be taken into account, and the calculations made to determine its quantity, in the same manner as described under estimation of ammonium bromid.

$0.5 \mathrm{gm}$. of the well-dried salt is dissolved in ro cc. of water, 2 drops of neutral potassium chromate T.S. are added, and then the $\frac{\mathrm{N}}{\mathrm{IO}} \mathrm{AgNO}_{3}$ V.S. slowly added from a burette until a permanent red color of silver chromate is produced. Not more than $30.5 \mathrm{cc}$. nor less than $30 \mathrm{cc}$. of decinormal silver nitrate V.S. should be required. This quantity corresponds to roo per cent of the pure salt.

$$
\begin{aligned}
& \mathrm{KI}+\mathrm{AgNO}_{3}=\mathrm{AgI}+\mathrm{KNO}_{3} \text {. } \\
& \frac{10) \sqrt{164.76}}{16.476} \text { gms. } \frac{10) 168.69}{16.869} \text { gms. or } 1000 \text { cc. } \frac{\mathrm{N}}{\mathrm{IO}} \mathrm{AgNO}_{3} \mathrm{~V} \text {. S. }
\end{aligned}
$$

Each cc. of $\frac{\mathrm{N}}{\mathrm{IO}} \mathrm{AgNO}_{3}$ V.S. thus corresponds to $0.016476 \mathrm{gm}$. of potassium iodid.

Thus,

$$
0.016476 \times 30.5=0.5025 \mathrm{gm} .
$$

To determine the quantity of chlorid present as an impurity we calculate as follows:

The amount of the salt under examination equivalent to rooo cc. is first found.

$$
\text { 30.5 cc. : } 0.5 \text { gm. : : } 1000 \mathrm{cc} .=\dot{x} . \quad x=\mathrm{r} 6.393 \mathrm{gms} .
$$

This is deducted from 16.476 gms., the quantity of pure KI equivalent to $1000 \mathrm{cc}$. of $\frac{\mathrm{N}}{\mathrm{IO}} \mathrm{AgNO}_{3}$ V.S.

$$
\begin{aligned}
& \mathrm{r} 6.476 \\
& \mathrm{r} 6.393 \\
& .083 \mathrm{gm} .
\end{aligned}
$$

This represents the excess of standard silver solution used up by the $\mathrm{KCl}$, reckoned in terms of $\mathrm{KI}$.

Since 7.404 gms. of $\mathrm{KCl}$ is equivalent to $16.476 \mathrm{gms}$. of $\mathrm{KBr}$ the excess which $\mathrm{KCl}$ can consume is,

therefore,

$$
16.476-7.404=9.072 \text { gms.; }
$$

$$
9.072: 7.404:: 0.083: x . \quad x=0.615 \mathrm{gm} .
$$


$0.6 \mathrm{I} 5 \mathrm{gm}$. is the amount of $\mathrm{KCl}$ present in $16.393 \mathrm{gms}$. of the salt examined.

The percentage is now calculated

$$
\text { I6.393:0.615:: 100: } x . \quad x=3.75 \text { per cent of } \mathrm{KCl} \text {, }
$$

which leaves 96.25 per cent of pure KI.

The same method of assay is applied to the following salts:

Lithium Bromid.

$$
\begin{aligned}
& \mathrm{LiBr}+\mathrm{AgNO}_{3}=\mathrm{AgBr}+\mathrm{LiNO}_{3} . \\
& \text { 10) } 86.34 \quad \text { 10) } 168.69 \\
& 8.634 \text { gms. } 16.869 \text { gms. }=1000 \text { cc. } \frac{\mathrm{N}}{\text { IO }} \text { V.S. }
\end{aligned}
$$

Each cc. of the standard solution represents $0.008634 \mathrm{gm}$. of $\mathrm{LiBr}$. Potassium Bromid.

$$
\begin{aligned}
& \mathrm{KBr}+\mathrm{AgNO}_{3}=\mathrm{AgBr}+\mathrm{KNO}_{3} . \\
& \text { 10) } \underline{118.22} \text { 10) } 168.69 \\
& \text { Ir.822 gms. } 16.869 \text { gms. }=1000 \text { cc. } \frac{N}{10} \text { V.S. }
\end{aligned}
$$

Each cc. of the silver solution represents $0.011822 \mathrm{gm}$. of $\mathrm{KBr}$.

Sodium Bromid.

$$
\begin{aligned}
& \underset{\mathrm{NaBr}}{\mathrm{Na}}+\underset{\text { I0) } \mathrm{AgO}_{3}}{\mathrm{Ag}_{3}}=\mathrm{AgBr}+\mathrm{NaNO}_{3} . \\
& \underset{\text { I0.224 gms. } 16.869}{\text { gms. }}=\text { roo0 cc. } \frac{\mathrm{N}}{\text { Io }} \text { V. S. }
\end{aligned}
$$

Each cc. of the standard silver solution represents $0.010224 \mathrm{gm}$. of $\mathrm{NaBr}$.

\section{Strontium Bromid $\left(\mathrm{SrBr}_{2}\right)$.}

$$
\begin{aligned}
& \mathrm{SrBr}_{2}+{ }_{2} \mathrm{AgNO}_{3}=2 \mathrm{AgBr}+\mathrm{Sr}\left(\mathrm{NO}_{3}\right)_{2} \text {. } \\
& { }^{20)} \frac{245.66}{12.283} \text { gms. } 16.869 \text { gms. }=1000 \text { cc. } \frac{N}{10} \text { V.S. }
\end{aligned}
$$

Each cc. of the standard silver solution represents $0.0 \mathrm{I} 2283 \mathrm{gm}$. of $\mathrm{SrBr}_{2}$.

Zinc Bromid.

$$
\begin{aligned}
& \mathrm{ZnBr}_{2}+{ }_{2} \mathrm{AgNO}_{3}={ }_{2} \mathrm{AgBr}+\mathrm{Zn}\left(\mathrm{NO}_{3}\right)_{2} \text {. } \\
& \frac{2 0 \longdiv { 2 2 3 . 6 2 }}{\text { II.I8I gms. }} \frac{20)}{16.869} \text { gms. }=\text { rooo cc. } \frac{\mathrm{N}}{\text { IO }} \text { V.S. }
\end{aligned}
$$


Each cc. of the standard silver solution represents 0.0 II 8 I gm. of $\mathrm{ZnBr}_{2}$.

\section{Sodium Iodid.}

$$
\begin{aligned}
& \mathrm{NaI}+\mathrm{AgNO}_{3}=\mathrm{AgI}+\mathrm{NaNO}_{3} .
\end{aligned}
$$

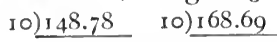

$$
\begin{aligned}
& \frac{148.78}{14.878} \text { gms. } \frac{168.69}{16.869} \mathrm{gms} .=1000 \text { cc. } \frac{\mathrm{N}}{10} \text { V.S. }
\end{aligned}
$$

Each cc. of the standard silver solution represents $0.014878 \mathrm{gm}$. of $\mathrm{NaI}$.

\section{Ammonium Chlorid.}

$$
\begin{aligned}
& \mathrm{NH}_{4} \mathrm{Cl}+\mathrm{AgNO}_{3}=\mathrm{AgCl}+\mathrm{NH}_{4} \mathrm{NO}_{3} \text {. } \\
& \begin{array}{ll}
\text { I0) } 53.11 & \text { 10) } 168.69
\end{array} \\
& 5.3 \text { I I gms. } 16.869 \text { gms. }=1000 \text { cc. } \frac{N}{10} \text { V.S. }
\end{aligned}
$$

Each cc. of the standard silver solution represents 0.0053 I I gm. of $\mathrm{NH}_{4} \mathrm{Cl}$.

Titration without an Indicator-Gay-Lussac's Method. In this method no indicator is used. The standard solution being added until it ceases to produce any further precipitation. This method is applicable to acid solution of the haloid salts, and to the haloid acids hydrochloric, hydrobromic, and hydriodic. Also to the estimation of silver by standard solution of sodium chlorid. The method is carried out in hot solutions, slightly acidulated with nitric acid, in order to facilitate the precipitation of the silver halid. The haloid acids are neutralized with an alkali and then slightly acidulated with nitric acid before the titration is begun. The calculations are precisely like those in the foregoing assays.

\section{ESTIMATION OF HALOID ACIDS}

These acids, namely, hydrochloric, hydrobromic, and hydriodic, may be estimated by Gay-Lussac's method above described, or they may be estimated by Mohr's Method, using neutral potassium chromate as an indicator. In this case it is necessary to carefully neutralize the acid with ammonia and then titrate with $\frac{\mathrm{N}}{\mathrm{IO}}$ silver nitrate solution, using a few drops of chromate as indicator in the manner described in the foregoing assays. They may also be estimated by Volhard's Method, in which an excess of the standard silver nitrate solution 
is used, in the presence of nitric acid, and the amount of the excess determined by residual titration with potassium sulphocyanate, using ferric alum as the indicator. This method is especially useful for iodids and hydriodic acid, in that the nitric acid need not be added until after an excess of silver nitrate solution is used, and thus liberation of iodin by the nitric acid avoided. This method is more fully described further on.

The estimation of the haloid acids may also be effected by neutralization with standard alkali, in the same way as other acids, but since hydrobromic and hydriodic acids are now frequently prepared by the method of Fothergill, in which potassium bromid or potassium iodid (according to the acid to be made) is brought in contact with tartaric acid (as shown in the equation), an excess of the latter acid is unavoidably present, and hence the neutralization method is inapplicable.

$$
\begin{gathered}
\underset{\begin{array}{c}
\text { Potassium } \\
\text { Iodid. }
\end{array}}{\mathrm{KI}}+\underset{\text { Tartaric acid. }}{\mathrm{H}_{2} \mathrm{C}_{4} \mathrm{H}_{4} \mathrm{O}_{6}}=\underset{\begin{array}{c}
\text { Potassium } \\
\text { Bitartrate. }
\end{array}}{\mathrm{KHC}_{4} \mathrm{H}_{4} \mathrm{O}_{6}} \underset{\begin{array}{c}
\text { Hydriodic } \\
\text { Acid. }
\end{array}}{\mathrm{HI} .} \\
\mathrm{KBr}
\end{gathered}
$$

Assay of Hydrobromic Acid, Using Chromate as Indicator. Io gms. of hydrobromic acid are diluted with sufficient distilled water to make roo cc. Io cc. of this solution, representing I gm. of the acid, is exactly neutralized with diluted ammonia water (using litmus solution as indicator); 3 drops of neutral potassium chromate are added, and then the $\frac{\mathrm{N}}{\mathrm{o}}$ silver nitrate run in from a burette until the solution acquires a permanent red tint. The following equation is then applied:

$$
\begin{aligned}
& \mathrm{HBr}+\mathrm{AgNO}_{3}=\mathrm{AgBr}+\mathrm{HNO}_{3} . \\
& \text { 10) } 80.36 \text { gms. } 10 \lcm{168.69} \text { gms. }
\end{aligned}
$$

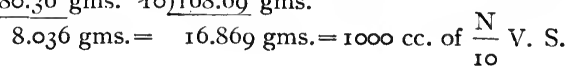

Thus each cc. of the $\frac{\mathrm{N}}{\mathrm{IO}}$ V. S. represents $0.008036 \mathrm{gm}$. of $\mathrm{HBr}$. then

Assuming that I2.44 cc. of the silver solution were consumed,

$$
0.008036 \times 12.44=0.0999 \mathrm{gm} .
$$

which is 9.99 per cent. 
If the assay is to be made by the direct percentage method, $8.036 \mathrm{cc}$. (8.04 cc.) of the solution (10 gms. in roo cc.) (representing 0.8036 gms. of the acid) should be taken, in which case each cc. of the standard silver solution consumed will at once indicate $I$ per cent.

Volhard's or Sulphocyanate Method. This method depends upon entirely precipitating the halogen in the presence of nitric acid, by a measured excess of standard silver nitrate solution, and then estimating the excess of silver by retitrating with standard sulphocyanate solution, using ferric alum as an indicator.

The sulphocyanate has a greater affinity for silver than it has for iron, and therefore, so long as any silver is in solution, the sulphocyanate will combine with it and form a precipitate of silver sulphocyanate.

As soon as the silver is all taken up, the sulphocyanate will combine with the ferric alum and strike a brownish-red color.

The sulphocyanate solution is to be made of such strength that it corresponds with the silver solution, volume for volume.

The difference between the volume of silver solution originally added and the volume of sulphocyanate solution used, will give the volume of silver solution equivalent to the haloid salt present.

This method has the advantage over the direct method for haloids with chromate indicator, in that it may be used in the presence of nitric acid. It thus enables one to estimate the haloids in the presence of phosphates or other salts which precipitate silver in neutral but not in acid solutions, and also in that the presence of barium, bismuth, lead, iron, and other metals do not interfere, as they do with the chromate in Mohr's method. The presence of mercury, however, exerts a disturbing influence upon the end reaction. The nitric acid acidulates the solution and thus facilitates the precipitation of silver by the halogens, and prevents its precipitation by other substances. The quantity of nitric acid employed is of no great importance, except in the case of iodids (because silver iodid is slightly soluble in nitric acid). Usually sufficient of the acid is added to just remove the color produced by the indicator. A very large excess of the acid would, however, interfere with the proper determination of the end reaction, in that it to a slight extent prevents the formation of ferric sulphocyanate. In the estimation of iodids by this method, the nitric acid should be added after the standard silver solution, while in the case of the other haloid salts the acid may be added before. 
The solutions required for this method are:

(I) Decinormal Silver Nitrate (Page I I2);

(II) Decinormal Potassium Sulphocyanate (Page II4);

(III) Ferric Alum Solution. (The indicator.)

This is a ro per cent aqueous solution of ferric-ammonium sulphate $\mathrm{Fe}_{2}\left(\mathrm{SO}_{4}\right)_{3} \cdot\left(\mathrm{NH}_{4}\right)_{2} \mathrm{SO}_{4}+{ }_{24} \mathrm{H}_{2} \mathrm{O}$.

(IV) Nitric Acid (C. P.). This must be free from nitrous acid. If it or any of the lower oxids of nitrogen are present they may be removed by diluting with one fourth part of water, and boiling until colorless.

The process is exemplified in the following assays:

Assay of Hydriodic Acid by the Sulphocyanate Method. Introduce into a $200 \mathrm{cc}$. stoppered flask $2 \mathrm{gms}$. of the acid, add 50 cc. of distilled water and $25 \mathrm{cc}$. (accurately measured) of decinormal silver nitrate, shake thoroughly, and then add $5 \mathrm{cc}$. of the ferric alum solution and 3 cc. of nitric acid C. P. The flask is stoppered and again thoroughly shaken, and finally, the decinormal potassium sulphocyanate run in slowly from a burette, until a permanent reddishbrown tint is produced. Note the number of cc. of sulphocyanate solution employed.

Deduct this from the $25 \mathrm{cc}$. of silver solution added, and multiply the remainder by the factor for HI, which is 0.01269 .

I.

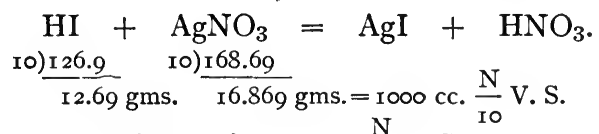

$$
\begin{aligned}
& 0.01269 \mathrm{gm} \text {. of } \mathrm{HI}=\mathrm{I} \text { cc. } \frac{\mathrm{N}}{\mathrm{IO}} \mathrm{V} \text {. S. }
\end{aligned}
$$

II.

$$
\begin{aligned}
& \mathrm{AgNO}_{3}+\mathrm{KSCN}=\mathrm{AgSCN}+\mathrm{KNO}_{3} . \\
& { }_{16.869} \text { gms. }=9.653 \text { gms. }=\text { roo० cc. } \frac{\mathrm{N}}{\mathrm{IO}} \text { V. S. }
\end{aligned}
$$

III. $\mathrm{Fe}_{2}\left(\mathrm{NH}_{4}\right)_{2}\left(\mathrm{SO}_{4}\right)_{4}+6 \mathrm{KSCN}=\mathrm{Fe}_{2}(\mathrm{SCN})_{6}+\left(\mathrm{NH}_{4}\right)_{2} \mathrm{SO}_{4}+{ }_{3} \mathrm{~K}_{2} \mathrm{SO}_{4}$.

The reddish-brown color which marks the end reaction is due to the formation of $\mathrm{Fe}_{2}(\mathrm{SCN})_{6}$ ferric sulphocyanate.

Assuming that in the above titration $9.3 \mathrm{cc}$. of decinormal sulphocyanate were employed, then $25 \mathrm{cc} .-9.3 \mathrm{cc} .=\mathrm{I} 5.7 \mathrm{cc}$.

$$
\begin{aligned}
& 0.01269 \times 15.7=0.199233 \mathrm{gm} . \\
& \frac{0.199233 \times 100}{2}=9.96+\% .
\end{aligned}
$$


The U.S.P. acidum hydriodicum dilutum is Io per cent in strength. Syrupus acidi hydriodici is I per cent.

Assay of Syrup of Hydriodic Acid. 6 gms. of the syrup are weighed off carefully in a $200 \mathrm{cc}$. stoppered flask, $20 \mathrm{cc}$. of distilled water are added, followed by ro cc. of decinormal silver nitrate and the mixture thoroughly shaken. $5 \mathrm{cc}$. of diluted nitric acid and 3 cc. of the ferric alum solution are now added, and after again shaking the mixture, it is titrated with decinormal potassium sulphocyanate until a permanent reddish-brown tint appears. If $5 . \mathrm{I} \mathrm{cc}$. of the sulphocyanate solution are used this quantity is deducted from the ro cc. of $\frac{\mathrm{N}}{\mathrm{IO}}$ silver nitrate solution added, which leaves $4.9 \mathrm{cc}$., the quantity of the latter which reacted with the syrup. Then

$$
\begin{aligned}
& 0.01269 \times 4.9=0.06218 \mathrm{Igm} . \\
& \frac{0.062181 \times 100}{6}=1.03+\% .
\end{aligned}
$$

Assay of Syrup of Ferrous Iodid by the Sulphocyanate Method. The U. S. P. directs to take Io gms. of the syrup, dilute it with distilled water to measure roo cc. Of this solution $15.36 \mathrm{cc}$. are mixed with $15 \mathrm{cc}$. of water, $6 \mathrm{cc}$. of decinormal silver nitrate and 2 cc. each of diluted nitric acid and ferric alum solution, and then after thoroughly shaking the mixture is titrated with decinormal sulphocyanate until a permanent reddish-brown tint appears. Not more than I cc. of the latter should be used. This I cc. deducted from the $6 \mathrm{cc}$. of decinormal silver nitrate leaves $5 \mathrm{cc}$., the quantity of the latter which reacted with the ferrous iodid. Each cc. represents I per cent. The equation is

$$
\begin{aligned}
& \mathrm{FeI}_{2}+{ }_{2} \mathrm{AgNO}_{3}=2 \mathrm{AgI}+\mathrm{Fe}\left(\mathrm{NO}_{3}\right)_{2} \text {. } \\
& \begin{array}{rr}
\text { 2) } 307.30 & 2 \lcm{337.38} \\
\text { 10) } 153.65 & \text { 10) } 168.69
\end{array} \\
& 15.365 \text { gms. } 16.869 \mathrm{gms} .=1000 \mathrm{cc} \cdot \frac{\mathrm{N}}{\mathrm{ro}} \mathrm{V} . \mathrm{S} . \\
& .015365 \mathrm{gm} \text {. of } \mathrm{FeI}_{2}=\text { I cc. } \frac{\mathrm{N}}{\mathrm{IO}} \mathrm{V} . \mathrm{S} \text {. }
\end{aligned}
$$

If each cc. of $\frac{\mathrm{N}}{\mathrm{IO}} \mathrm{AgNO}_{3}$ V.S. represent $0.015365 \mathrm{gm}$. of $\mathrm{FeI}_{2}$ then $5 \mathrm{cc} .=5 \times 0.015365 \mathrm{gm} .=0.076825$.

$$
\frac{0.0768_{25}}{1.536} \times 100=5 \text { per cent. }
$$


In this (direct percentage) method, a quantity of the syrup is taken which equals the weight of pure $\mathrm{FeI}_{2}$ represented by roo cc. of the decinormal silver nitrate solution.

Strontium Iodid $\left(\mathrm{SrI}_{2}\right)$ and $\mathrm{Zinc}$ Iodid $\left(\mathrm{ZnI}_{2}\right)$ are assayed in the U. S. P. by sulphocyanate method above described.

The sulphocyanate method may be used for the estimation of chlorids and bromids, as well as iodids.

When used for the estimation of chlorids, however, the precipitated silver chlorid must be removed by filtration, because of the action of ferric sulphocyanate upon silver chlorid which causes the results of the analysis to be too high. In the case of silver bromid no such reaction takes place, or if it does, the reaction is so slow as not to interfere in the least with the getting of accurate results. Therefore, when this method is used for the determination of bromids or iodids, there is no need for filtering to remove the precipitate. This matter is dealt with more fully on page $26 \mathrm{r}$.

\section{ESTIMATION OF CYANOGEN}

\section{Titration with Standard Silver Solution to First Appearance} of a Precipitate-Liebig's Method. This gives fairly accurate results. The cyanogen must be in the form of an alkali salt and in an alkaline solution. If hydrocyanic acid is to be estimated, it must be made alkaline by the addition of potassium or sodium hydroxid. The standard silver solution is then added cautiously and with constant stirring until a permanent precipitate of silver cyanid is produced. When silver nitrate is added to an alkaline solution of a cyanid, the precipitate which at first forms redissolves on stirring and a soluble double cyanid $(\mathrm{AgCN}, \mathrm{KCN}$ or $\mathrm{AgCN}, \mathrm{NaCN}$, depending upon the alkali used) is formed, and when all of the cyanid has been taken up, the further addition of silver nitrate causes a decomposition of this soluble double salt and the formation of a permanent precipitate of silver cyanid. Therefore, the first appearance of this precipitate affords a delicate proof of the completion of the reaction.

These equations illustrate the reactions.

$$
\begin{gathered}
2 \mathrm{NaCN}+\mathrm{AgNO}_{3}=\underset{\begin{array}{l}
\text { Double cyanid } \\
\text { of silver and sodium. }
\end{array}}{\mathrm{AgCN}}+\mathrm{NaNO}_{3} . \\
\mathrm{AgCN}, \mathrm{NaCN}+\mathrm{AgNO}_{3}=2 \mathrm{AgCN}+\mathrm{NaNO}_{3} .
\end{gathered}
$$


According to these equations it is seen that the end-reaction is reached when two molecules of the alkali cyanid have reacted with one molecule of silver nitrate. The slightest excess of silver nitrate above this quantity brings about a decomposition of the double salt and a precipitation of the silver cyanid, as above stated.

This double combination is so firm that if the estimation is done in the presence of a halogen, no permanent precipitate of silver halid is formed until after all of the cyanogen present has been converted into a double salt. This fact is taken advantage of in the U. S. P. processes for hydrocyanic acid and potassium cyanid in which potassium iodid is employed as indicator, in the presence of ammonia water. The latter prevents the precipitation of silver cyanids and thus allows the silver iodid to precipitate alone.

$$
\text { I cc. of } \begin{aligned}
\frac{\mathrm{N}}{\mathrm{IO}} \mathrm{AgNO}_{3} \text { V. S. }= & 0.005268 \mathrm{gm} . \mathrm{CN} ; \\
& 0.005368 \mathrm{gm} . \mathrm{HCN} ; \\
& 0.009744 \mathrm{gm} . \mathrm{NaCN} ; \\
& 0.01294 \mathrm{gm} . \mathrm{KCN} .
\end{aligned}
$$

Assay of Hydrocyanic Acid $(\mathrm{HCN}=26.84)$. Dilute hydrocyanic acid may be estimated by weighing out about 5 gms., and adding it without delay (to avoid evaporation) to sufficient sodium or potassium hydroxid solution to convert the acid into sodium or potassium cyanid $(\mathrm{NaCN}$ or $\mathrm{KCN})$ and leave the solution strongly alkaline. The mixture is then largely diluted with water (50 to $100 \mathrm{cc}$.); this is to enable one to more clearly observe the end-point.

The decinormal silver nitrate solution is then delivered in, until a permanent turbidity occurs.

The difficulty experienced in this process is in the conversion of the acid into the cyanid. Sodium cyanid has a strong alkaline reaction, turning litmus blue, when only a small proportion of the acid has been neutralized. If the titration is conducted before the acid is completely neutralized that which is free will not be acted upon. Indeed, cyanid of sodium may be estimated in the presence of hydrocyanic acid in this way.

According to Senier the following procedure will answer well :

To the dilute hydrocyanic acid add sodium hydroxid to strong alkaline reaction, determined by litmus tincture.*

* Poirrie- Bluc $C_{4} B$ is better, in that it is not affected by alkali cyanids, but gives a very sharp indication in the presence of the slightest excess of alkali 
Then titrate with $\frac{\mathrm{N}}{\mathrm{IO}}$ silver nitrate, drop by drop. If the liquid becomes acid, add a little more soda solution to bring it back to alkalinity, and continue the titration until the turbidity indicates the end of the reaction. The liquid must be kept alkaline throughout the process. It is not well to add too much alkali at the beginning as this will use up too much of the silver solution and make the reading a trifle too high. The following equations, etc., explain the reactions:

$$
\begin{aligned}
& { }_{2} \mathrm{HCN}+2 \mathrm{NaOH}=2 \mathrm{NaCN}+{ }_{2} \mathrm{H}_{2} \mathrm{O} \text {. }
\end{aligned}
$$

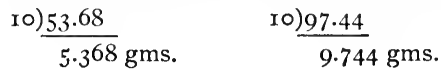

$$
\begin{aligned}
& { }_{2} \mathrm{NaCN}+\mathrm{AgNO}_{3}=\mathrm{AgCN}, \mathrm{NaCN}+\mathrm{NaNO}_{3} . \\
& \text { 10) } \frac{97.44}{9.744} \text { gms. } \frac{\text { I0) } 68.69}{16.869} \text { gms. or } 1000 \mathrm{cc} . \frac{\mathrm{N}}{\mathrm{IO}} \mathrm{V} . \mathrm{S} \text {. }
\end{aligned}
$$

It is seen that 5.368 gms. of real $\mathrm{HCN}$ are equivalent to $9.744 \mathrm{gms}$. of sodium cyanid, and represent 16.869 gms. of silver nitrate or 1000 cc. of the $\frac{\mathrm{N}}{\mathrm{IO}}$ V.S. That is, I000 cc. of the $\frac{\mathrm{N}}{\mathrm{IO}} \mathrm{AgNO}_{3} \mathrm{~V}$. S. may be added to a solution containing 9.744 gms. of sodium cyanid and no precipitate will be produced, but if one or two drops more of the standard solution be added, a precipitate is at once formed, the double salt being broken up and silver cyanid produced.

$$
\mathrm{AgCN}, \mathrm{NaCN}+\mathrm{AgNO}_{3}={ }_{2} \mathrm{AgCN}+\mathrm{NaNO}_{3} .
$$

Each cc. of the $\frac{\mathrm{N}}{\mathrm{IO}}$ silver solution which fails to produce a precipitate represents $0.009744 \mathrm{gm}$. of $\mathrm{NaCN}$, which is equivalent to $0.005368 \mathrm{gm}$. of HCN.

Titration with Standard Silver Solution, Using Chromate Indicator-Vielhaber's Method. This method is especially recommended for the assay of weak solutions containing hydrocyanic acid, as bitter almond oil, bitter almond water, cherry laurel water, etc., but it may also be employed for alkaline cyanids.

hydroxid. The amount of alkali used should be as near as possible that which is required to just convert the acid into the alkali cyanid, too much or too little alike affect the accuracy of the result. 
A sufficient quantity of an aqueous suspension of magnesium hydroxid $*$ to make the solution opaque and distinctly alkaline is added; this is followed by a few drops of potassium chromate indicator and then the $\frac{\mathrm{N}}{\mathrm{IO}}$ silver nitrate delivered into the mixture from a burette until a permanent red tint appears, as in the titration of haloid salts. The method is a very satisfactory one, if chlorids are absent.

The reactions in this method are the same as in the foregoing, but the end-reaction (the production of silver chromate) does not occur until the double cyanid is completely decomposed, at which point the addition of another drop of silver solution reacts with the chromate and produces the red precipitate (silver chromate).

The equations are as follows: Sodium is used in the equations instead of magnesium in order to make the explanation clearer.

\section{(a) ${ }_{2} \mathrm{NaCN}+\mathrm{AgNO}_{3}=\mathrm{AgCN}, \mathrm{NaCN}+\mathrm{NaNO}_{3}=\left({ }_{2} \mathrm{HCN}\right)$; \\ (b) $\mathrm{AgCN}, \mathrm{NaCN}+\mathrm{AgNO}_{3}=2 \mathrm{AgCN}+\mathrm{NaNO}_{3}$.}

These equations show that it requires two molecules of silver nitrate to completely precipitate two molecules of cyanid. 168.69 gms. of $\mathrm{AgNO}_{3}$ is equivalent to 26.84 gms. of $\mathrm{HCN}$, while by Liebig's method, I68.16 gms. of $\mathrm{AgNO}_{3}$ is equivalent to $53.68 \mathrm{gms}$. of $\mathrm{HCN}$.

$$
\begin{aligned}
\text { I cc. } \frac{\mathrm{N}}{\mathrm{IO}} \mathrm{AgNO}_{3} \text { V.S. }= & 0.002584 \mathrm{gm} . \mathrm{CN} ; \\
& 0.002684 \mathrm{gm} . \mathrm{HCN} ; \\
& 0.004872 \mathrm{gm} . \mathrm{NaCN} ; \\
& 0.006470 \mathrm{gm} . \mathrm{KCN}
\end{aligned}
$$

Example. I.35 gms. of the diluted acid is mixed with enough water and magnesia to make an opaque mixture of about ro cc. Add to this 2 or 3 drops of potassium chromate T. S., and then from a burette deliver the decinormal silver nitrate V. S. until a red tint is produced which does not disappear by shaking.

Titration with Standard Silver Solution, Using Potassium Iodid and Ammonia as Indicator. This method is recommended by W. J. Sharwood, J. A. C. S., $1897,400-434$, and is a modification 
of the method proposed by M. Georges Deniges, Ann. chim. phys., (7) $6.38 \mathrm{r}$.

In this method 5 gms. of hydrocyanic acid are diluted with distilled water to measure $50 \mathrm{cc}$. Then $25 \mathrm{cc}$. of this solution, after the addition of $5 \mathrm{cc}$. of ammonia water and 3 drops of a 20 per cent potassium iodid solution, are titrated with tenth-normal silver nitrate, until a slight permanent precipitate occurs. The ammonia water and potassium iodid in this process act as indicator.

The reactions may be expressed thus:

(I) $\mathrm{HCN}+\mathrm{NH}_{4} \mathrm{OH}=\mathrm{NH}_{4} \mathrm{CN}+\mathrm{H}_{2} \mathrm{O}$;

(2) ${ }_{2} \mathrm{NH}_{4} \mathrm{CN}+\mathrm{AgNO}_{3}=\mathrm{NH}_{4} \mathrm{Ag}(\mathrm{CN})_{2}+\mathrm{NH}_{4} \mathrm{NO}_{3}$;

(3) $\mathrm{NH}_{4} \mathrm{Ag}(\mathrm{CN})_{2}+\mathrm{AgNO}_{3}=\mathrm{NH}_{4} \mathrm{NO}_{3}+2 \mathrm{AgCN}$;

(4) $\mathrm{KI}+\mathrm{AgNO}_{3}=\mathrm{KNO}_{3}+\mathrm{AgI}$.

The silver nitrate forms with the cyanid a double salt which is soluble, no precipitate occurring until after all of the cyanid has entered into combination as the double salt; then the further addition of silver nitrate decomposes the double salt, and a precipitate of silver cyanid occurs. In the presence of ammonia water, however, as in the above assay, the precipitation of silver cyanid is prevented, but the iodid is now (not before) acted upon by the silver solution and a precipitate of silver iodid' occurs, which very delicately indicates the end reaction.

Each cc. of the standard silver nitrate solution used represent $0.005368 \mathrm{gm}$. of absolute $\mathrm{HCN}$. If $26.84 \mathrm{cc}$. of the above solution are taken instead of $25 \mathrm{cc}$., each cc. will represent at once 0.2 per cent. The U.S. P. dilute hydrocyanic acid should require when treated this way exactly ro cc. of silver solution, indicating 2 per cent of $\mathrm{HCN}$.

Potassium cyanid is assayed in the same way.

Estimation of Potassium Cyanid $(\mathrm{KCN}=64.70)$. I gm. of potassium cyanid is dissolved in sufficient distilled water to make Ioo cc., then $64.7 \mathrm{cc}$. of this solution mixed with $5 \mathrm{cc}$. of ammonia water and 3 drops of potassium iodid T. S. are titrated with $\frac{\mathrm{N}}{\mathrm{IO}} \mathrm{AgNO}_{3}$ V. S. until the appearance of a permanent precipitate. $47.5 \mathrm{cc}$. should be required. Each cc. indicates 2 per cent.

I cc. of $\frac{\mathrm{N}}{\mathrm{IO}} \mathrm{AgNO}_{3} \mathrm{~V} . \mathrm{S} .=0.01294 \mathrm{gm} . \mathrm{KCN}$. 


\section{ESTIMATION OF SILVER SALTS}

Soluble silver salts are estimated by direct titration with standard sodium chlorid, the process being exactly the converse of the precipitation methods for halogens. The standard sodium chlorid solution is added to the solution of the silver salt until precipitation ceases: Or the titration may be done in the presence of chromate indicator, the endpoint being then known to be reached when the red color of silver chromate disappears. The first of these methods is impracticable. Too much time being consumed in waiting for the precipitate to settle so as to render the supernatant liquid sufficiently clear to recognize whether a precipitate is produced in it by the further addition of the standard solution.

If chromate indicator is used, the end-point is easily over-stepped, because of the slow decomposition of the silver chromate by the chlorid. It is best to add an excess of sodium chlorid solution and then retitrate with standard silver nitrate solution until the red color appears.

Silver salts may also be titrated by means of standard sulphocyanate solution, using ferric alum as indicator.

Assay of a Solution of Silver Nitrate by Means of $\frac{\mathrm{N}}{\mathrm{IO}_{0}}$ Sodium

Chlorid Io gins. of the solution are introduced into a beaker and diluted with ro cc. of distilled water. Two drops of yellow potassium chromate solution are added as indicator, and then a measured excess of $\frac{\mathrm{N}}{\mathrm{IO}}$ sodium chlorid (sufficient of this must be added to completely destroy the red color), added slowly from a burette and with constant stirring. Assuming that $20 \mathrm{cc}$. were used, then the excess may be ascertained by titrating back with $\frac{\mathrm{N}}{\mathrm{IO}}$ silver nitrate V. S. until a permanent red tint is produced. Whatever number of cc. of $\frac{\mathrm{N}}{\mathrm{IO}}$ silver nitrate are used, that number represents the quantity of $\frac{\mathrm{N}}{\mathrm{IO}}$ sodium chlorid which was added in excess, and imust be deducted from the $20 \mathrm{cc}$. of the sodium chlorid solution emploved. Assuming this number to be $3 \mathrm{cc}$, then 3 from 20 leaves $17 \mathrm{cc}$., which is the exact quantity of $\frac{\mathrm{N}}{\mathrm{IO}} \mathrm{NaCl}$ V.S. which reacted with the silver in the solution examined.

The $\frac{\mathrm{N}}{\mathrm{IO}}$ factor for silver nitrate multiplied by $\mathrm{I} 7$ will then give 
the exact weight of silver nitrate in the ro gms. of solution taken. The following equation illustrates the reaction which occurs:

$$
\begin{aligned}
& \underset{\mathrm{AgNO}}{\mathrm{AgN}}+\underset{\mathrm{NaCl}}{\mathrm{N}}=\mathrm{AgCl}+\mathrm{NaNO}_{3} . \\
& \frac{168.69}{16.869} \text { gms. } \frac{58.06}{5.806} \text { gms. }=\text { rooo cc. } \frac{\mathrm{N}}{\text { Io }} \mathrm{V} . \mathrm{S} .
\end{aligned}
$$

$0.01689 \mathrm{gm}$. of silver nitrate is thus represented by each cc. of $\mathrm{N}$ sodium chlorid.

In the above assay $I 7$ cc. of the sodium chlorid solution were used, therefore the silver solution under analysis contains I7 Xo.or6869 gm. $=0.286773 \mathrm{gm}$. of pure $\mathrm{AgNO}_{3}$. The percentage of the solution is then found,

$$
\frac{0.286773 \times 100}{\text { I0 }}=2.86773 \text { per cent. }
$$

In the assay of silver nitrate crystals, $0.2 \mathrm{gm}$. is taken, dissolved in $10 \mathrm{cc}$. of distilled water, and then treated as in the foregoing assay. In the case of molded silver nitrate about the same quantity is taken for assay. Of mitigated silver nitrate, I gm. may be taken.

Silver oxid $\left(\mathrm{Ag}_{2} \mathrm{O}\right)$ may be converted into nitrate by solution in nitric acid and then tested as above. Free nitric acid is apt to be present in this case and therefore the snlution should be neutralized, before it is assayed, if the above described method is to be employed. The presence of free acid does not interfere, however, if the method of Gay-Lussac or the sulphocyanate method be employed.

\section{Assay of Silver Nitrate by Means of $\frac{N}{\text { Io }}$ Sulphocyanate.}

This method, as applied to the assay of halogen compounds, is described in the preceding pages. The great advantage which this method presents over the others, is that the presence of most other metals does not interfere. The only metal which does materially interfere with the determination of silver is mercury.

Example. A weighed quantity ( 0.2 to $0.5 \mathrm{gm}$.) of the silver salt is dissolved in water, some diluted nitric acid and ammonium ferric sulphate solution are added, and the mixture then titrated with $\frac{\mathrm{N}}{10}$ potassium sulphocyanate until a permanent reddish-brown color of ferric sulphocyanate is produced. 
The following equation explains the reactions:

$$
\begin{aligned}
& \mathrm{AgNO}_{3}+\mathrm{KSCN}=\mathrm{AgSCN}+\mathrm{KNO}_{3} \text {. } \\
& \text { 10) } \mathbf{6 8 . 6 9} \quad \text { 10) } 96.53 \\
& \text { I6.869 gms. } 9.653 \text { gms. or } 1000 \mathrm{cc} \text {. standard V.S. }
\end{aligned}
$$

Thus each cc. of the standard V. S. represents $0.016869 \mathrm{gm}$. of pure silver nitrate, or $0.010712 \mathrm{gm}$. of metallic silver.

Estimation of Metallic Silver and Silver Alloys. A quantity of the metal, weighing about $0.5 \mathrm{gm}$., is dissolved in ro cc. of nitric acid, and after complete solution is attained it is heated sufficiently to drive off all traces of nitrous acid. The solution is then diluted with about $100 \mathrm{cc}$. of distilled water and assayed by one of the methods described under the assay of solution of silver nitrate. The sulphocyanate method is the preferred one.

\section{THE ANALYSIS OF CERTAIN NEUTRAL SALTS BY CONVERSION INTO CHLORIDS.}

Many neutral salts may be indirectly estimated with great accuracy, by converting them into chlorids, salts of acids weaker than hydrochloric; and salts of volatile acids, of nearly the same chemical strength as hydrochloric acid, may be readily converted into chlorids by frequent evaporation to dryness with an excess of hydrochloric acid.

Sulphates are converted into chlorids by precipitation with barium chlorid, or better with barium hydroxid; the excess of the latter is then removed by passing $\mathrm{CO}_{2}$ through the solution, and then after the addition of an excess of hydrochloric acid evaporating to dryness.

Borates and Phosphates are precipitated by lead acetate solution, the excess of lead removed by means of sulphuric acid, and the resulting sulphate then treated as above described.

Carbonic Acid in Combination. The compound is slightly supersaturated with pure hydrochloric acid, the solution evaporated to dryness on a water-bath, and then heated for a short time in an air-bath. The residue, which consists of chlorid, is dissolved in water and titrated in the usual way with $\frac{\mathrm{N}}{\mathrm{IO}}$ silver nitrate, V. S., using neutral chromate as indicator.

Or the carbonate is treated with barium chlorid T. S., the precipitate well washed, and then dissolved on the filter with hydrochloric acid (covering it with a watch glass to prevent loss) the solution is then repeatedly evaporated to dryness, till all free hydrochloric acid is 
driven off. The barium is then precipitated by the addition of sodium sulphate solution, which is added in slight excess, and the mixture titrated with silver nitrate V. S. in the presence of chromate indicator. There is no necessity for filtering, as the precipitated barium sulphate does not interfere.

One molecule of sodium carbonate is equivalent to two molecules of sodium chlorid.

$$
\begin{aligned}
& \mathrm{Na}_{2} \mathrm{CO}_{3}={ }_{2} \mathrm{NaCl}+{ }_{2} \mathrm{AgNO}_{3}={ }_{2} \mathrm{AgCl}+2 \mathrm{NaNO}_{3} . \\
& \begin{array}{lll}
\text { 2) } 105.3 \mathrm{I} & \text { 2) } 1 \mathrm{r6.12} & \text { 2) } 337.38
\end{array} \\
& \text { 10) } 52.655 \mathrm{gms} \text {, ro) } 58.06 \mathrm{gms} \text {. 10) } 168.69 \mathrm{gms} \text {. }
\end{aligned}
$$

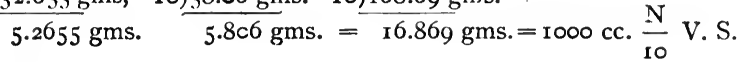

Thus I cc. of $\frac{\mathrm{N}}{\text { IO }} \mathrm{AgNO}_{3}$ V. S. represents 0.005806 gm. of $\mathrm{NaCl}$, which is equivalent to $0.0052655 \mathrm{gm}$. of $\mathrm{Na}_{2} \mathrm{CO}_{3}$.

Free Carbonic Acid Gas. The gas is collected by means of ammonia water and barium chlorid solution, and treated as described in the case of carbonic acid in combination.

$$
\text { I cc. of } \frac{\mathrm{N}}{\mathrm{IO}} \mathrm{AgNO}_{3}=0.0021835 \mathrm{gm} \text {. of } \mathrm{CO}_{2} \text {. }
$$

Organic Salts of the Alkalies and Alkali Earths. These salts are ignited to convert them into carbonates, then treated with hydrochloric acid, evaporated, and titrated as above described.

Chlorates are converted by ignition into chlorids.

Nitrates are converted into chlorids by evaporating with concentrated hydrochloric acid.

Ammonia, Ammonium Salts, and Nitrogen. Ammonia is conducted into diluted hydrochloric acid, the liquid then cautiously evaporated to dryness, the residue $\left(\mathrm{NH}_{4} \mathrm{Cl}\right)$ dissolved in water and titrated.

Ammonium salts and nitrogenous compounds, when burned with soda lime, evolve ammonia $\left(\mathrm{NH}_{3}\right)$ which is conducted into diluted hydrochloric acid, and treated as above.

$$
\text { I cc. } \frac{\mathrm{N}}{\mathrm{IO}} \mathrm{AgNO}_{3} \mathrm{~V} . \mathrm{S} .=0.00 \mathrm{I} 693 \mathrm{gm} . \mathrm{NH}_{3} \text {. }
$$

Besides the substances mentioned in the foregoing pages, many others may be accurately estimated by precipitation, as for instance, copper, manganese, lead, sulphocyanic acid, hydrosulphuric acid, 
phosphoric acid, glucose, etc., the methods for which are described under other headings.

Estimation of Alkali Iodids by Precipitation with Mercuric Chlorid Solution (Personne). Alkali iodids may also be estimated by titration with $\frac{\mathrm{N}}{\mathrm{IO}}$ mercuric chlorid V. S., the termination of the operation being indicated by the formation of a red precipitate.

$$
\begin{gathered}
{ }_{4} \mathrm{KI}+\mathrm{HgCl}_{2}={ }_{2} \mathrm{KCl}+\mathrm{HgI}_{2} \cdot{ }_{2} \mathrm{KI} \text { (soluble). . . . } \\
\mathrm{HgI}_{2} \cdot{ }_{2} \mathrm{KI}+\mathrm{HgCl}_{2}={ }_{2} \mathrm{KCl}+{ }_{2} \mathrm{HgI}_{2} . \quad . \quad . \quad .
\end{gathered}
$$

This process originated with M. Personne, and is founded on the fact that if a solution of mercuric chlorid be added to one of potassium iodid, in the proportion of one equivalent of mercuric chlorid to four of potassium iodid, red mercuric iodid is formed, which dissolves at once to a colorless solution. The slightest excess of mercuric chlorid will cause a brilliant red precipitate $\left(\mathrm{HgI}_{2}\right)$ to make its appearance.

$$
\begin{aligned}
& { }_{4} \mathrm{KI}+\mathrm{HgCl}_{2}={ }_{2} \mathrm{KCl}+\mathrm{HgI}_{2} \cdot 2 \mathrm{KI} \text { (soluble). } \\
& \text { 20)659.04 } \frac{20) 268.86}{32.952} \text { gms. } \frac{13.443}{\mathrm{Ims} .} \text { of rooo cc. of standard solution. }
\end{aligned}
$$

Thus each cc. of standard solution of the above strength represents $0.03295^{2} \mathrm{gm}$. of potassium iodid, which means that I cc. is the largest quantity of this standard solution which can be added to $0.03295^{2} \mathrm{gm}$. of potassium iodid without producing a permanent precipitate.

The above solution of mercuric chlorid is strictly a $\frac{N}{10}$ V. S. (Consult Estimation of Mercuric Salts on page 409.)

The author of this process states that neither chlorids, bromids, nor carbonates interfere with the reaction. 
Table of Substances Estimated by Precipitation

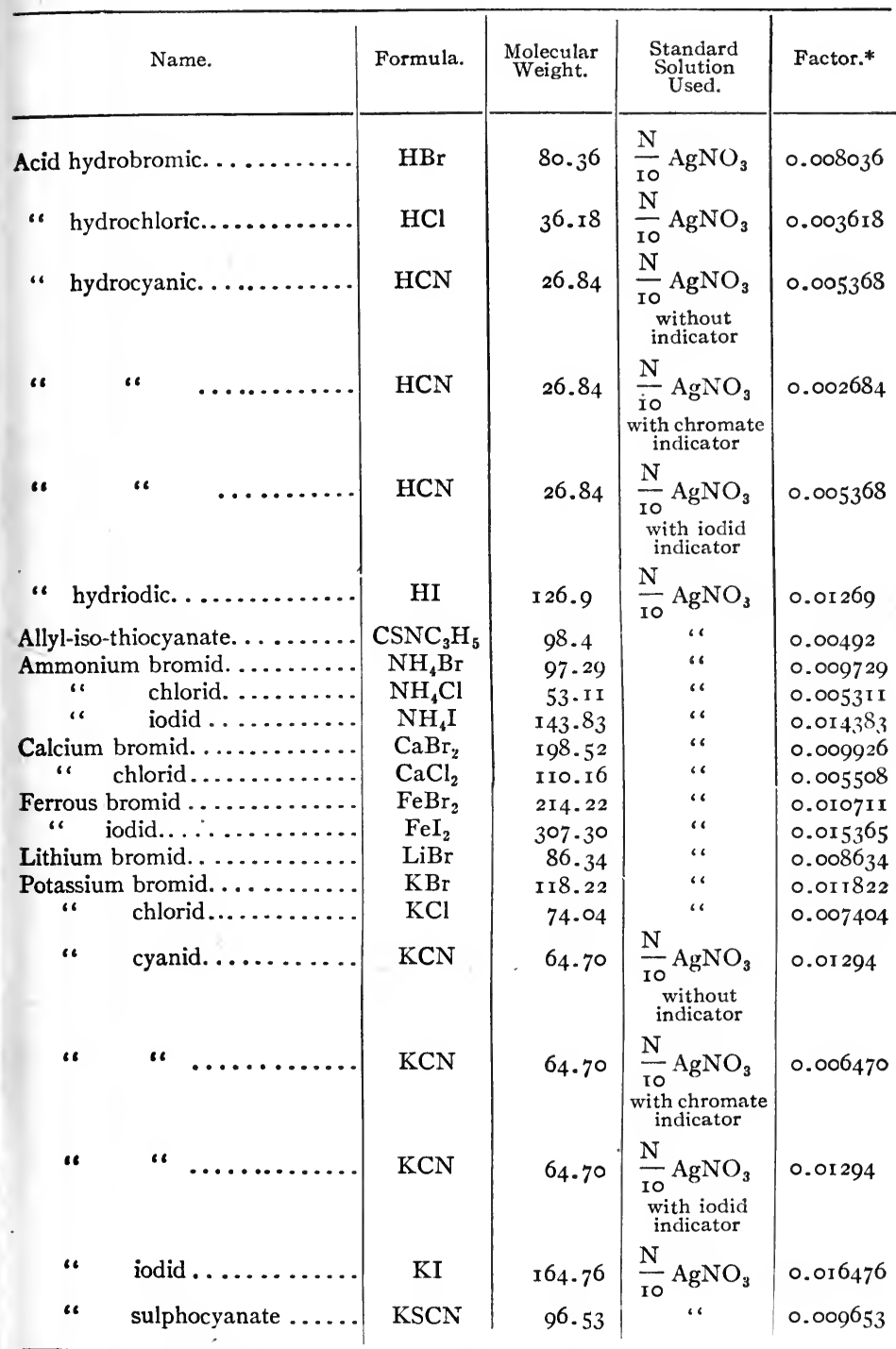

* This is the coefficient by which the number of cc. used of the decinormal solution is to be multiplied in order to obtain the quantity of pure substance in the sample analyzed. It represents the weight of the substance precipitated by $1 \mathrm{cc}$. of the decinormal solution. 
Table of Substances Estimated by Precipitation-Continued

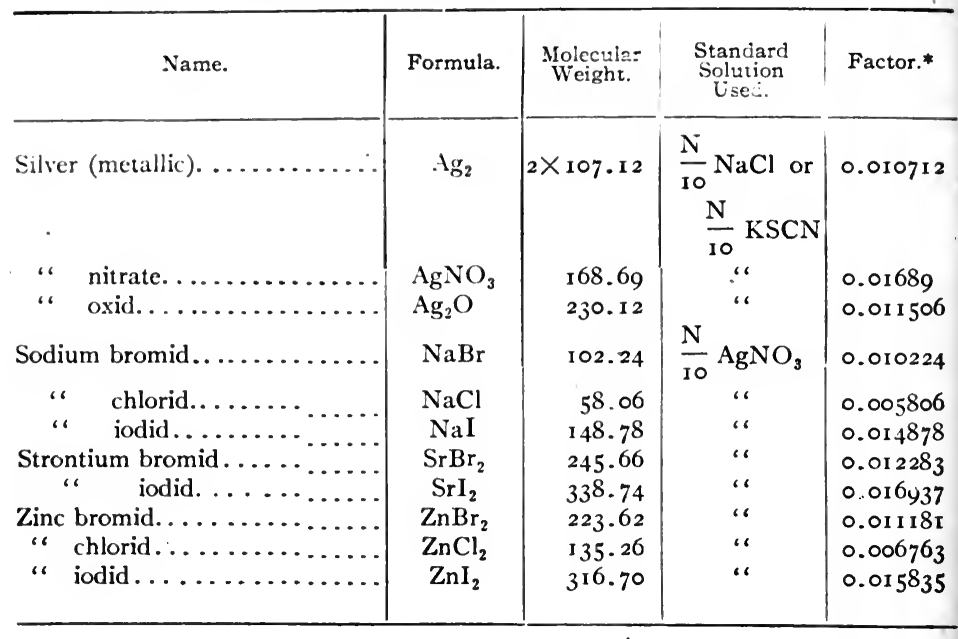




\section{CHAPTER XII}

\section{ANALYSIS BY OXIDATION AND REDUCTION}

An extensive series of analyses are made by these methods with extremely accurate results, in fact, the results are generally more accurate than those obtained by gravimetric methods.

The principle involved is exceedingly simple. An oxidizing agent is employed for the estimation of an oxidizable substance, and likewise a reducing agent is employed for the estimation of a reducible substance. Oxidizing agents are always reducible and reducing agents are always oxidizable. An oxidation and a reduction take place at the same time, i.e., the oxidizing agent is itself reduced in the operation and the reducing agent is at the same time oxidized.

Thus substances which are capable of absorbing oxygen or are susceptible of an equivalent action may be accurately estimated by subjecting them to the action of an oxidizing agent of known power, and from the quantity of the latter required for complete oxidation, the weight of the oxidizable substance is ascertained.

Example. Ferrous oxid ( $\mathrm{FeO})$, an oxidizable substance, is ever ready to take up oxygen, while potassium permanganate and potassium dichromate are always ready to give up some of their oxygen. When potassium permanganate gives up its oxygen in this way it is reduced and decolorized, while the ferrous oxid in taking up oxygen is oxidized to ferric oxid $\left(\mathrm{Fe}_{2} \mathrm{O}_{3}\right)$. The decolorization of the permanganate here spoken of is taken advantage of in volumetric analysis for the determination of the completion of the oxidation. The permanganate in the form of a standard solution being slowly delivered from a burette, until it is no longer decolorized, the iron salt is known to be completely oxidized, when the permanganate is no longer reduced. The reaction is as follows:

$$
\underset{\text { Ferrous oxid }}{\mathrm{IOFeO}}+2 \mathrm{KMnO}_{4}=\underset{\text { Ferric oxid }}{5 \mathrm{Fe}_{2} \mathrm{O}_{3}}+2 \mathrm{MnO}_{2}+\mathrm{K}_{2} \mathrm{O} .
$$


The oxidation of ferrous oxid by potassium dichromate is shown by the followng equation:

$$
6 \mathrm{FeO}+\mathrm{K}_{2} \mathrm{Cr}_{2} \mathrm{O}_{7}={ }_{3} \mathrm{Fe}_{2} \mathrm{O}_{3}+\mathrm{Cr}_{2} \mathrm{O}_{3}+\mathrm{K}_{2} \mathrm{O} .
$$

As before stated, an oxidation is always accompanied by a reduction, the oxidizing agent being itself reduced in the operation. As shown in the above equations, the manganic compound is reduced to a manganous, and the chromic to a chromous, while the ferrous salt is oxidized to a ferric condition.

In the same way any substance which readily yields oxygen in definite quantity or is susceptible of an equivalent action which involves its reduction to a lower quantivalence, may be estimated by ascertaining how much of a reducing agent of known power is required for its complete reduction.

Example. The available chlorin in bleaching powder may be accurately ascertained by treating it with a standard solution of arsenous oxid, and from the volume of the solution required for the complete reduction of the chlorin, the quantity of the latter present is found, or in other words, from the quantity of arsenous oxid $\left(\mathrm{As}_{2} \mathrm{O}_{3}\right)$, oxidized to arsenic oxid $\left(\mathrm{As}_{2} \mathrm{O}_{5}\right)$ the weight of the chlorin present is ascertained.

The principal substances which are used as oxidizing agents in volumetric analysis, are potassium permanganate, potassium dichromate, and iodin. The latter contains no oxygen, but it abstracts hydrogen from accompanying water and liberates the oxygen which does the oxidizing, hence iodin is known as an indirect oxidizing agent. The other two contain available oxygen which they readily give up when brought in contact with an oxidizable substance.

The principal reducing agents or deoxidizers which are used in volunetric analysis are, sodium thiosulphate, sulphurous acid, oxalic acid, arsenous oxid, stannous chlorid, ferrous oxid, hydriodic acid, hydrosulphuric acid, metallic zinc, and magnesium.

\section{VOLUMETRIC ANALYSES BY MEANS OF POTASSIUM PERMANGANATE}

When potassium permanganate solution is added to a solution of any readily oxidizable substance strongly acidulated with sulphuric acid, it undergoes reduction as shown in the equation below. The molecule $\left(2 \mathrm{KMnO}_{4}\right)$ has eight atoms of oxygen which it gives up in the process of oxidation. These eight atoms of oxygen unite with the replaceable hydrogen of an accompanying acid, liberating an 
equivalent amount of acidulous radical. Three of these atoms of oxygen liberate sufficient acidulous residual to combine with the potassium and manganese of the permanganate, while the other five atoms are available for direct oxidation.

$$
{ }_{2} \mathrm{KMnO}_{4}+{ }_{3} \mathrm{H}_{2} \mathrm{SO}_{4}=\mathrm{K}_{2} \mathrm{SO}_{4}+{ }_{2} \mathrm{MnSO}_{4}+{ }_{3} \mathrm{H}_{2} \mathrm{O}+{ }_{5} \mathrm{O},
$$

or, for combination with the hydrogen of more acid, more acidulous residual being set free, to combine with the salt acted upon.

$$
{ }_{2} \mathrm{KMnO}_{4} 8+\mathrm{H}_{2} \mathrm{SO}_{4}=\mathrm{K}_{2} \mathrm{SO}_{4}+{ }_{2} \mathrm{MnSO}_{4}+8 \mathrm{H}_{2} \mathrm{O}+{ }_{5}\left(\mathrm{SO}_{4}\right) .
$$

${ }_{5}\left(\mathrm{SO}_{4}\right)$ when combined with $\mathrm{IoFeSO}_{4}$ forms $\mathrm{Fe}_{10}\left(\mathrm{SO}_{4}\right)_{15}$ or ${ }_{5} \mathrm{Fe}_{2}\left(\mathrm{SO}_{4}\right)_{3}$, ferric sulphate. Thus it is seen that one molecule of potassium permanganate $\left(2 \mathrm{KMnO}_{4}\right)$ has the power of converting Io molecules of a ferrous salt to the ferric state.

The equation in full is

IoFeSO ${ }_{4}+2 \mathrm{KMnO}_{4}+8 \mathrm{H}_{2} \mathrm{SO}_{4}=\mathrm{K}_{2} \mathrm{SO}_{4}+2 \mathrm{MnSO}_{4}+8 \mathrm{H}_{2} \mathrm{O}+{ }_{5} \mathrm{Fe}_{2}\left(\mathrm{SO}_{4}\right)_{3}$.

We have seen that $2 \mathrm{KMnO}_{4}$ has 5 atoms of oxygen available for xidizing purposes, and that each of these will combine with 2 atoms of hydrogen. ${ }_{2} \mathrm{KMnO}_{4}$ is consequently chemically equivalent to ro atoms of hydrogen, and a normal solution of this salt when used is an oxidizing agent is one that contains in one liter one-tenth of the veight of $2 \mathrm{KMnO}_{4}$ expressed in grams, and a decinormal solution, ne which contains one-hundredth of this weight.

As before stated, .when potassium permanganate is brought in ontact with a ferrous salt or other oxidizable substance, it is decomosed and decolorized. Hence when titrating with a standard soluion of this salt it is decolorized so long as an oxidizable substance is resent; as soon, however, as the oxidation is completed the standard olution retains its color when added to the substance, and the first ppearance of a faint red color is the end-reaction, and the oxidation known to be completed.

In titrating with potassium permanganate it must be renembered hat excess of free acid (preferably sulphuric) should always be resent in the solution titrated, in order to keep the resulting mangaous and manganic oxids in solution; these, forming a dense brown recipitate, would make it difficult if not quite impossible to recogize the pinkish color of the end-reaction. Sulphuric acid alone if a large excess, has a reducing effect upon potassium permanganate. 
Nitric and hydrochloric acids are prejudicial and should be avoided; they are, however, frequently present in salts which are to be analyzed, and in such event should be removed by converting them into sulphate. By adding a small excess of sulphuric acid and applying heat, until hydrochloric acid or nitrous vapors are no longer evolved, the chlorid or the nitrate is converted into sulphate, and the deleterious effect of their presence overcome. Hydrochloric acid, unless present in very small quantities, and the titration conducted at a low temperature, will vitiate the analysis through its action upon the permanganate whereby chlorin is liberated $*$ thus:

$$
\mathrm{KMnO}_{4}+8 \mathrm{HCl}=\mathrm{KCl}+\mathrm{MnCl}_{2}+{ }_{4} \mathrm{H}_{2} \mathrm{O}+{ }_{5} \mathrm{Cl} \text {. }
$$

A very convenient way of obviating the irregularities due to the presence of hydrochloric acid is to add a few grams of manganous sulphatef to the solution before titrating it.

Mercuric sulphate $\ddagger$ and magnesium sulphate may also be used with satisfactory results.

Potassium permanganate being so readily decomposed by contact with organic matter, should be protected from such contact. I should never be filtered through paper (glass-wool or guncottor may be used), nor should it be used in a Mohr's burette or in an! other apparatus in which it is in contact with rubber or cork. Fur thermore, all substances of an oxidizing or reducing nature, asid

* This decomposition of the permanganate by hydrochloric acid is due to th presence of ferric salt, which latter seems to act catalytically, for oxalic acid ma be accurately titrated with permanganate even in the presence of hydrochlori acid, no chlorin being given off. Thus the decomposition of the permanganat is not due to the hydrochloric acid alone.

$\dagger$ Kessler and Zimmermann suggest using $20 \mathrm{cc}$. of a solution of manganor sulphate (200 gms. per liter.)

$\ddagger$ Cady and Ruediger (J. A. C. S., XIX-575) concluded from the followir general principles that it is possible to titrate iron with permanganate in the pre ence of hydrochloric acid if an excess of mercuric sulphate be added to the solutio Mercuric halids in solution ionize to an extremely slight extent, while the me curic salts of oxyacids are readily ionized, since compounds of slight ionizati always result when their constituent ions meet, mercuric halids are always $\mu \mathrm{r}$ duced when a mercuric salt of an oxyacid is added to a solution containing halogi ions. Therefore when mercuric sulphate solution and hydrochloric acid a mixed, ionization of both occurs, and the mercuric ions unite with the chlor ions and produce mercuric chlorid which is only very slightly ionized. In t presence of a large excess of mercuric sulphate, the mercuric ions resulting frc its dissociation diminish the ionization of the mercuric chlorid until it is pre tically nil. Thus no chlorin ions will be present in the solution to induce composition of the permanganate. The method is described in Chapter XXXI 
from that being analyzed, must be excluded from the solution. Among such substances may be mentioned hydriodic acid, sulphureted hydrogen, nitrous acid and the lower oxids of nitrogen, phosphorous and hypophosphorous acids, thiosulphuric, sulphurous, and all the other acids of sulphur except sulphuric, also ous salts and the metallic suboxids and peroxids.

Burettes and other apparatus which have been used for perman-1 ganate, should be emptied and rinsed immediately after use, and any manganic oxid which may be adhering to the glass should be removed by means of hydrochloric acid and boiled water.

Not only oxidizable substances but reducible substances may be estimated by means of potassium permanganate.

In the estimation of oxidizable substances the standard potassium permanganate is added directly to the acidulated solution of the substance being analyzed. The completion of the oxidation being then known by the appearance of a faint pinkish tint. This is the direct method.

In the estimation of reducible substances (i.e., oxidizing substances) he indirect or the residual method is employed.

In this an accurately weighed or measured quantity of the subitance is brought together with an excess of a third substance having educing power, and which is similarly effected by the permanganate ind by the substance analyzed. After completion of the reaction he excess of the reducing substance is found by titration with standard ermanganate. The difference between the quantity so found and hat originally added gives the quantity which reacted with the salt Inder analysis, and from this the calculation is made.

The Titration Methods in which permanganate is employed re classified in this ,Chapter under the following headings:

A. Direct Titrations.

$a$. Estimation of ferrous salts;

b. Estimation of iron in ferrum reductum;

c. Estimation of oxalic acid and oxalates;

$d$. Estimation of hydrogen dioxid and barium dioxid;

$e$. Estimation of ferric salts after reduction;

$f$. Estimation of nitrous acid and nitrites.

\section{B. Residual Titrations and Indirect Methods.}

a. Methods involving the addition of an excess of standard perpanganate, and retitration with standard oxalic acid, as estimation of ypophosphites. 
b. Methods involving a precipitation by oxalic acid and titration of the excess of the latter by standard permanganate, as estimation of calcium, gold, and lead.

c. Methods involving a reduction by means of oxalic acid and titration of the excess of the latter by standard permanganate, as estimation of manganese dioxid.

d. Methods involving a reduction by means of a ferrous salt and titration of the remaining unoxidized ferrous salt by standard permanganate, as estimation of nitrates (Pelouze) and chromates.

$e$. Methods involving the oxidation of the substance analyzed by means of a ferric salt and titration of the resultant ferrous salt, as estimation of $t i n$ and copper.

\section{Preparation of Decinormal $\left(\frac{N}{10}\right)$ Potassium Permanganate} $\left(2 \mathrm{KMnO}_{4}=313.96 ; \frac{\mathrm{N}}{\mathrm{IO}} \mathrm{V} . \mathrm{S} .=3.1396\right.$ gms. in I liter $)$. Absolutely pure poiassium permanganate cannot be obtained, therefore the preparation of a decinormal solution of this salt cannot be effected by simply dissolving the requisite proportion of the molecular weight in the water. The presence of oxidizable matter in the water used, the contact of dust and exposure to light, have a tendency to decompose the salt and hence weaken the standard solution. It is therefore advisatile to use boiling distilled water, to preserve the solution in amber glass bottles, provided with ground-glass stoppers. It will then retain its strength for several weeks, but should nevertheless be checked by titration immediately before using. It is not necessary, and it is usually undesirable, to make the solution an exact decinormal one. It is preferable to fix the titer of the solution and employ it as it is.

Place 3.5 gms. of pure crystallized potassium permanganate in a flask, ald $1000 \mathrm{cc}$. of distilled water, and boil until the crystals are dissolved; put a plug of absorbent cotton in the mouth of the flask and set it aside for two days so that any suspended matters may de posit. After the lapse of this time pour off the clear solution into glass-stoppered bottle, and when wanted for use standardize by either of the following methods:

Standardization by Means of Iron. Thin annealed binding wire, free from rust, is one of the purest forms of iron.*

\footnotetext{
* This contains 99.6 per cent of iron.
} 
O.I gm. of such iron is placed in a flask which is provided with a cork through which a piece of glass tubing passes, to the top of which a piece of rubber tubing is attached, which has a vertical slit about one inch long in its side, and which is closed at its upper end by a piece of glass rod (this arrangement is known as the "Bunsen Valve"). (See Fig. 54.) Diluted sulphuric acid is added and gentle heat applied. The iron dissolves and the steam and liberated hydrogen escape through the slit under slight pressure. The air is thus prevented from entering and the ferrous solution protected from oxidation.

A better form of apparatus in which to dissolve the iron and avoid

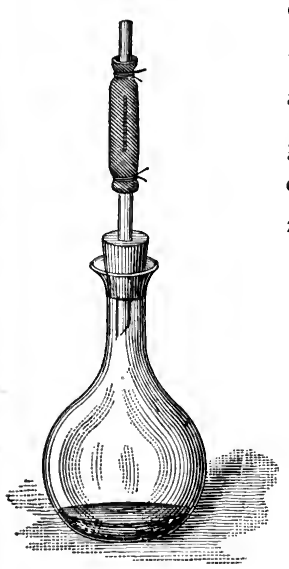

FIG. 54 . oxidation through admission of air is shown in Fig. 55. A roo cc. flask is fitted with a rubber stopper and a $\quad$ shaped glass tube; into this flask is placed $20 \mathrm{cc}$. of diluted sulphuric acid $(\mathrm{r}: 5)$ and then 2 or 3 crystals of pure sodium carbonate;

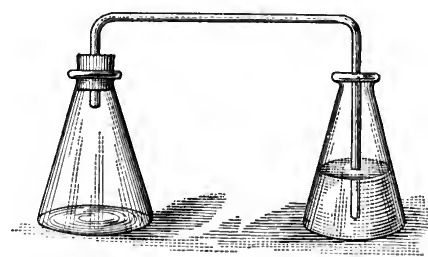

FIG. 55.

this causes an evolution of carbon dioxid which expels the air from flask. The o.I gm. of iron wire above described is now introduced, the stopper inserted, and a beaker containing a solution of pure sodium carbonate placed in position so that the tube will dip into the solution. Gentle heat is applied until the iron is wholly dissolved, and only a few minute particles of carbon remain (which must not be mistaken for iron). When the flame is withdrawn, the cooling of the flask and contents causes a drawing up of the sodium carbonate solution, but the first drops that enter the flask cause an effervescence with evolution of carbon dioxid, which drives the liquid back and at the same time fills the flask with the gas; this is repeated until the flask and contents are cold. Another useful form of apparatus for this purpose is depicted in Fig. $5^{6}$. 
When the iron is completely dissolved a small quantity of cold, recently boiled, distilled water should be used to rinse the lower end of the stopper and the neck of the

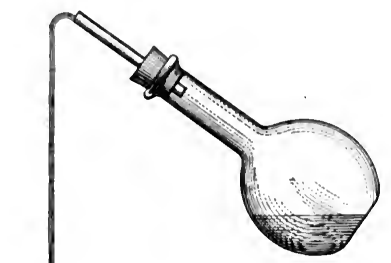
flask, and the titration with potassium permanganate at once begun and continued until a faint permanent pink color is produced. If the solution is decinormal, exactly $17.94 \mathrm{cc}$. will be required to produce this result.

The iron is converted by the sulphuric acid into ferrous sulphate, $\mathrm{Fe}_{2}+{ }_{2} \mathrm{H}_{2} \mathrm{SO}_{4}={ }_{2} \mathrm{FeSO}_{4}+{ }_{2} \mathrm{H}_{2}$. This ferrous sulphate is easily oxidized by the air, and therefore it is directed that access of air should be prevented, and the distilled water with which the solution is diluted previously boiled in order to drive off any Fig. 56 . dissolved free oxygen.

$$
\begin{aligned}
& \text { IoFeSO } 4+2 \mathrm{KMnO}_{4}+8 \mathrm{H}_{2} \mathrm{SO}_{4}
\end{aligned}
$$

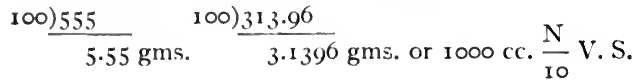

$$
\begin{aligned}
& ={ }_{5} \mathrm{Fe}_{2}\left(\mathrm{SO}_{4}\right)_{3}+\mathrm{K}_{2} \mathrm{SO}_{4}+2 \mathrm{MnSO}_{4}+8 \mathrm{H}_{2} \mathrm{O} \text {. }
\end{aligned}
$$

This equation, etc., shows that each cc. of $\frac{\mathrm{N}}{\mathrm{IO}}$ permanganate represents $0.00555 \mathrm{gm}$. of metallic iron.

Standardization by Means of Oxalic Acid. $0.06255 \mathrm{gm}$. of the pure crystallized acid is weighed (or ro cc. of decinormal oxalic acic carefully measured) and placed in a flask, with some dilute sulphuric acid and considerable water, the mixture warmed to about $60^{\circ} \mathrm{C}$ $\left(: 40^{\circ} \mathrm{F}\right.$ ), and the permanganate added from a burette.

The action is in this case less decisive and rapid than in the titra tion with iron, and more care should be used. The color disappear. slowly at first, but afterwards more rapidly.

Note the number of $\mathrm{cc}$. of the permanganate solution used, ani then dilute the remainder so that equal volumes of decinormal oxali acid and decinormal permanganate solution will exactly correspond. 
Example. Assuming that $9 \mathrm{cc}$. of the permanganate solution first prepared had been required to produce a permanent pink tint when titrated into ro cc. of $\frac{\mathrm{N}}{\mathrm{IO}}$ oxalic-acid solution, then the permanganate must be diluted in the proportion of 9 of permanganate and $\mathrm{I}$ of distilled water, or 900 and 100.

The U. S. P., 1890 , gives the following method for the preparation of this solution:

A stronger and a weaker solution is made and mixed in certain proportions to form a solution of the proper strength. It is said that when thus prepared the solution will keep its titer for months if properly preserved.

The Stronger Solution. 3.5 gms. of pure crystallized permanganate are dissolved in $1000 \mathrm{cc}$. of water by the aid of heat, and the solution then set aside in a closed flask for two days, so that any suspended matters may deposit.

The Weaker Solution. Dissolve 6.6 gms. of the salt in 2200 cc. of water in the same manner as above, and set this solution aside for two days.

These two solutions are then separately titrated in the following manner:

Introduce ro cc. of decinormal oxalic-acid solution into a flask, add I cc. of pure concentrated sulphuric acid, and before the mixture cools add the permanganate solution slowly from a burette, shaking the flask after each addition, and towards the end of the operation reducing the flow to drops. When the last drop is no longer decolorized, but imparts a pinkish tint to the liquid, the reaction is completed. Note the number of $\mathrm{cc}$. consumed. Finally, mix the two solutions in such proportions that equal volumes of the mixture and of $\frac{\mathrm{N}}{\mathrm{IO}}$ oxalic acid V. S. will exactly correspond.

To obtain the accurate proportions for mixing the two solutions, deduct Io from the number of cc. of the weaker solution consumed in the above titration; with this difference multiply the number of cc. of the stronger solution consumed: the product shows the number of cc. of the stronger solution needed for the mixture.

Then deduct the number of cc. of the stronger solution consumed in the titration from ro, and with the difference multiply the number of cc. of the weaker solution consumed: the product shows the number of cc. of the weaker solution needed for the mixture. 
Or, designating the number of cc. of the stronger solution by $S$, and the number of cc. of the weaker solution by $W$, and using the following formula, the proportions in which the solutions must be mixed are obtained:

$$
\begin{aligned}
& \text { Stronger Solution. Weaker Solution. } \\
& (W-\text { Io }) S+(\text { ro }-S) W .
\end{aligned}
$$

Example. Assuming that $9 \mathrm{cc}$. of the stronger and $10.5 \mathrm{cc}$. of weaker had been consumed in decomposing ro cc. of $\frac{\mathrm{N}}{10}$ oxalic acid V. S.; then, substituting these values in the above formula, we obtain

$$
(105-\text { io }) 9+(10-9) 10.5, \text { or } 4.5+10.5 \text {, }
$$

making ${ }_{5}$ cc. of final solution.

The bulk of the two solutions is now mixed in the same proportion: $450 \mathrm{cc}$. of the stronger and $1050 \mathrm{cc}$. of the weaker, or $900 \mathrm{cc}$. of the stronger and 2 roo cc. of the weaker.

After the solutions are thus mixed a new trial should be made, when so cc. of the solution should exactly decompose ro cc. of $\frac{\mathrm{N}}{\mathrm{ro}}$ oxalic acid $\mathrm{V}$. S.

The reaction between potassium permanganate and oxalic acid is illustrated by the following equation:

$$
\begin{aligned}
2 \mathrm{KMnO}_{4}+5\left(\mathrm{H}_{2} \mathrm{C}_{2} \mathrm{O}_{4} \cdot 2 \mathrm{H}_{2} \mathrm{O}\right)+{ }_{3} \mathrm{H}_{2} \mathrm{SO}_{4}= \\
\mathrm{K}_{2} \mathrm{SO}_{4}+2 \mathrm{MnSO}_{4}+\mathrm{IOCO}_{2}+\mathrm{r} 8 \mathrm{H}_{2} \mathrm{O} .
\end{aligned}
$$

Standardization by the Iodometric Method. This method, which was proposed by Volhard, is the most accurate and rapid for the standardization of permanganate. It is based upon the fact that potassium permanganate reacts with potassium iodid in solutions acidulated with either hydrochloric or sulphuric acid, and liberates an equivalent quantity of iodin, which may be estimated by standard solution of sodium thiosulphate. The reactions are illustrated oy the equations

(a) ${ }_{2} \mathrm{KMnO}_{4}+8 \mathrm{H}_{2} \mathrm{SO}_{4}+\mathrm{roKI}=2 \mathrm{MnSO}_{4}+6 \mathrm{~K}_{2} \mathrm{SO}_{4}+8 \mathrm{H}_{2} \mathrm{O}+{ }_{5} \mathrm{I}_{2}$; 313.96

$$
\mathrm{I}_{2}+2 \underbrace{\mathrm{Na}_{2} \mathrm{~S}_{2} \mathrm{O}_{3} \cdot 5 \mathrm{H}_{2} \mathrm{O}}_{49^{2.92}})=2 \mathrm{NaI}+\mathrm{Na}_{2} \mathrm{~S}_{4} \mathrm{O}_{6}+{ }_{10 H_{2}} \mathrm{O} .
$$


Thus it is seen that $2 \mathrm{KMnO}_{4}(3 \mathrm{r} 3.96$ gms.) containing five atoms of available oxygen, has the power of liberating its equivalent of iodin, i.e., Io atoms or I $259 \mathrm{gms}$. (see equation $a$ ) and that $492.92 \mathrm{gms}$. of sodium thiosulphate will reduce $25 \mathrm{I} .9 \mathrm{gms}$. of iodin (see equation $b$ ). Hence rooo cc. of $\frac{\mathrm{N}}{\mathrm{IO}}$ sodium thiosulphate (containing $24.646 \mathrm{gms}$.) will reduce, and therefore be equivalent to 12.59 gms. of iodin which in turn represents $3.1396 \mathrm{gms}$. of potassium permanganate. Therefore I cc. of the $\frac{\mathrm{N}}{\mathrm{IO}}$ thiosulphate represents $0.01259 \mathrm{gm}$. of iodin and $0.0031396 \mathrm{gm}$. of potassium permanganate, which latter is the quantity of potassium permanganate present in I cc. of its $\frac{\mathrm{N}}{\mathrm{IO}} \mathrm{V}$. S.

The process is conducted as follows: Into a $200 \mathrm{cc}$. flask place about $0.5 \mathrm{gm}$. of potassium iodid and ro cc. of diluted sulphuric acid, add to this (slowly from a burette) exactly ro cc. of the permanganate solution to be standardized and dilute the mixture (which is brown in color, because of the liberated iodin) with distilled water to about I50 cc. Then slowly titrate (with constant stirring) with an accurately standardized $\frac{\mathrm{N}}{\mathrm{s}}$ sodium thiosulphate until the color of the solution is a faint vellow, then add a few drops of starch solution and continue the titration until the color is discharged. Note the number of cc. consumed and diltue the permanganate with distilled water so that equal volumes of the two solutions correspond to each other.

Example. If I3 cc. of the thiosulphate solution were required, then each Io $\mathrm{cc}$. of the permanganate solution must be diluted to I3 cc.

Standardization with Ferrous Ammonium Sulphate (Mohr's salt $)\left(\mathrm{FeSO}_{4} \cdot\left(\mathrm{NH}_{4}\right)_{2} \mathrm{SO}_{4} \cdot 6 \mathrm{H}_{2} \mathrm{O}\right) . \quad 389.34$ gms. of this salt contains 55.5 gms. iron ( $3.5 \circ 7$ gms. contain $0.5 \mathrm{gm}$. of iron). $3.5 \circ 7 \mathrm{gms}$. of the salt are accurately weighed out and dissolved in sufficient recently boiled distilled water to make $250 \mathrm{cc}$. $50 \mathrm{cc}$. of this solution containing 0.I gm. of iron are transferred to a small flask, Io cc. of diluted sulphuric acid added, and then the permanganate solution to be standardized is run in slowly until a faint pinkish tint appears. Whatever number of cc. is consumed that number represents $0.1 \mathrm{gm}$. of iron, and must be diluted to $\mathrm{I} 8.02 \mathrm{cc}$. to make the solution exactly decinormal. 
Standardization by Means of Sodium Oxalate. Sodium oxalate may be used in the same manner as oxalic acid, the salt is anhydrous and may be accurately weighed, but it offers no advantage over oxalic acid.

Standardization with Hydrogen Peroxid in the Nitrometer is proposed by Lunge, J. S. C. I., IX, 2I. The volume of oxygen given off corrected for temperature and pressure, and calculated into weight, gives the weight of the permanganate present.

On the Use of Empirical Permanganate Solutions. $A$. If the standardization of the solution is done by means of iron as described on page $142,0.1 \mathrm{gm}$. of iron wire (representing $0.0996 \mathrm{gm}$. of pure iron) will require $17.94 \mathrm{cc}$. of the permanganate solution if the latter is exactly decinormal. If less than this quantity of solution is used (say 17.5 cc.) it indicates that the solution is stronger than decinormal, and may either be diluted so that each $17.5 \mathrm{cc}$. will measure 17.94, or it may be used as it is. This in most cases is preferable. The value of one cc. of the solution in iron is calculated thus:

$$
\text { i } 7.5 \text { cc. : I cc. : : } 0.0996 \text { gm. : } x . \quad x=0.00569 \mathrm{gm} \text {. }
$$

If a solution of this strength is to be used for the estimation of iron, simple multiplication of the number of cc. used by $0.00569 \mathrm{gm}$. gives the weight of $\mathrm{Fe}$ present. If, however, this solution is employed for the titration of other oxidizable substances, the number of $\mathrm{cc}$. consumed is multiplied by $0.00569 \mathrm{gm}$. and then by a fraction in which the numerator represents a quantity of the substance examined, equivalent in grams to an atom of iron in its reaction with permanganate, and the denominator is the atomic weight of iron.

Example. If in a titration, we use $40 \mathrm{cc}$. of a permanganate solution, the titer of which has been found to be I cc. $=0.00569 \mathrm{gm}$., the calculation would be:

in the case of ferrous sulphate $\left(\mathrm{FeSO}_{4}, \mathrm{I}_{50.85}\right)$,

$$
40 \times 0.00569 \mathrm{gm} . \times \frac{\mathrm{I} 50.85}{55.5}=0.6184 \mathrm{gm} . ;
$$

in the case of oxalic acid $\left(\mathrm{H}_{2} \mathrm{C}_{2} \mathrm{O}_{4} \cdot{ }_{2} \mathrm{H}_{2} \mathrm{O}=125.10\right)$,

$$
40 \times 0.00569 \mathrm{gm} . \times \frac{52.55}{55.5}=0.2564 \mathrm{gm} \text {; }
$$

in the case of hydrogen dioxid $\left(\mathrm{H}_{2} \mathrm{O}_{2}=33.76\right.$. $)$,

$$
40 \times 0.00569 \mathrm{gm} \cdot \times \frac{\mathrm{I} 6.88}{55.5}=0.0692 \mathrm{gm} .
$$


$B$. Another way. The solution just mentioned, of which $\mathrm{I} 7.50 \mathrm{cc}$. are consumed in titrating $0.1 \mathrm{gm}$. of iron wire, is compared with a true decinormal permanganate solution, of which $\mathrm{I} 7.94 \mathrm{cc}$. are consumed in the same reaction. The strength of the former solution is therefore $\frac{\mathrm{I} 794}{\mathrm{I} 75^{\circ}}$ as compared with a decinormal solution.

In titrating with this solution the number of $\mathrm{cc}$. consumed are to be multiplied by $\frac{\mathrm{I} 794}{\mathrm{I} 75^{\circ}}$ and then by the true decinormal factor for the substance being analyzed.

Example. $40 \mathrm{cc}$. of the solution are consumed.

In the case of ferrous sulphate $\left(\mathrm{FeSO}_{4}=\mathrm{I}_{50} 0.85\right)$, the decinormal factor (i.e., the weight of ferrous sulphate represented by I cc. of a true decinormal solution) is $0.015085 \mathrm{gm}$.

$$
40 \times \frac{\mathrm{I} 794}{\mathrm{I} 750} \times 0.015085 \mathrm{gm} .=0.6184 \mathrm{gm} .
$$

In the case of oxalic acid $\left(\mathrm{H}_{2} \mathrm{C}_{2} \mathrm{O}_{4}, 2 \mathrm{H}_{2} \mathrm{O}=\mathrm{I} 25 . \mathrm{IO}\right)$; the decinormal factor is $0.006255 \mathrm{gm}$.

$$
40 \times \frac{\mathrm{I} 794}{\mathrm{I} 750} \times 0.006255 \mathrm{gm} .=0.2564 \mathrm{gm} .
$$

In the case of hydrogen dioxid $\left(\mathrm{H}_{2} \mathrm{O}_{2}=33.76\right)$, the decinormal factor is $0.001688 \mathrm{gm}$.

$$
40 \times \frac{\mathrm{I} 794}{\mathrm{I} 75^{\circ}} \times 0.00 \mathrm{I} 688 \mathrm{gm} .=0.0692 \mathrm{gm} .
$$

C. If the standardization is done by means of oxalic acid, as described on page $\mathrm{I} 44$ in which Io cc. of a strictly decinormal oxalic acid solution are titrated with the permanganate solution which is being standardized, exactly Io cc. of the latter will be consumed if it is of decinormal strength. If in the trial, however, it is found that only $9.6 \mathrm{cc}$. are consumed it indicates that the solution is stronger than decinormal; its strength being expressed by $\frac{\text { IOO }}{96}$. If, on the other hand, more than ro cc. of the solution are consumed (say ro.4 cc.) the solution is below decinormal strength, namely, $\frac{100}{104}$.

In using a solution of the first strength the number of cc. of it consumed in any titration is to be multiplied by $\frac{100}{96}$ and then by the 
decinormal factor for the substance examined. In the case of the weaker solution the number of cc. consumed is multiplied by $\frac{\text { IOO }}{\text { IO.4 }}$ and then by the decinormal factor for the substance being analyzed.

Examples. Ferrous sulphate $\left(\mathrm{FeSO}_{4}=\mathrm{r}_{5} 0.85\right)$, is titrated with the stronger solution, $40 \mathrm{cc}$. of the latter being consumed.

Then,

$$
40 \times \frac{100}{96} \times 0.015086 \mathrm{gm} .=0.628 \mathrm{gm} .
$$

Oxalic acid $\left(\mathrm{H}_{2} \mathrm{C}_{2} \mathrm{O}_{4} \cdot 2 \mathrm{H}_{2} \mathrm{O}=125 \cdot 10\right), 40$.cc. are consumed.

Then,

$$
40 \times \frac{100}{96} \times 0.006255 \mathrm{gm} .=0.260 \mathrm{gm} .
$$

Hydrogen dioxid $\left(\mathrm{H}_{2} \mathrm{O}_{2}=33.76\right)$, 40 cc. are consumed.

Then,

$$
40 \times \frac{100}{96} \times 0.001688 \mathrm{gm} .=0.0703 \mathrm{gm} .
$$

$D$. If the checking of the permanganate solution is done by the iodometric method (page 146) and it is found that ro cc. of the permanganate requires the use of ${ }_{3} 3 \mathrm{cc}$. of decinormal thiosulphate solution; the titer of the solution is expressed with reference to decinormal as $\frac{13}{10}$. In using a solution of this strength, the number of cc. of it consumed in an analysis is multiplied by $\frac{13}{10}$ and then by the decinormal factor for the substance analyzed.

\section{TYPICAL ANALYSES WITI PERMANGANATE}

\section{A. Direct Ti'rations.}

a. Estimation of Ferrous Sulphate $\left(\mathrm{FeSO}_{4}+{ }_{7} \mathrm{H}_{2} \mathrm{O}=276.01\right)$. I gm. of ferrous sulphate is dissolved in $25 \mathrm{cc}$. of water and the solution strongly acidulated with sulphuric acid. Decinormal potassium permanganate is then delivered from a burette until a permanent pink tint is obtained, indicating the complete oxidation of the ferrous salt. 
The reaction is as follows:

$$
\begin{aligned}
& \left(\mathrm{roFeSO}{ }_{4}+{ }_{7} \mathrm{H}_{2} \mathrm{O}\right)+{ }_{2} \mathrm{KMnO}_{4}+8 \mathrm{H}_{2} \mathrm{SO}_{4} \\
& \text { 100) } 2760.1 \\
& 27.601 \text { gms. }=\frac{3.9}{3.139^{6}} \text { gm. }=1000 \text { cc. } \frac{\mathrm{N}}{\mathrm{IO}} \text { V.S. } \\
& 2.7601 \text { gms. = } \quad \text { 100 cc. } \frac{\mathrm{N}}{10} \text { V.S. } \\
& 0.02760 \text { r gm. }=\quad \text { I cc. } \frac{\mathrm{N}}{\text { ro }} \mathrm{V} \text {.S. } \\
& ={ }_{5} \mathrm{Fe}_{2}\left(\mathrm{SO}_{4}\right)_{3}+\mathrm{K}_{2} \mathrm{SO}_{4}+{ }_{2} \mathrm{MnSO}_{4}+{ }_{7} 8 \mathrm{H}_{2} \mathrm{O} \text {. }
\end{aligned}
$$

Thus 313.96 gms. of permanganate $=2760.1$ gms. of crystallized ferrous sulphate, which equals 555 gms. of metallic iron. I cc. of $\frac{\mathrm{N}}{\mathrm{IO}}$ permanganate solution therefore represents $0.02760 \mathrm{r} \mathrm{gm}$. of $\mathrm{FeSO}_{4}+{ }_{7} \mathrm{H}_{2} \mathrm{O}$ or 0.00555 gm. of Fe.

In the analysis $36 \mathrm{cc}$. of the $\frac{\mathrm{N}}{\mathrm{IO}}$ permanganate were consumed. The I gm. taken then contains $36 \times 0.02760 \mathrm{I}=0.993636 \mathrm{gm}$. or 99.36 per cent.

$$
\text { I : } 0.993636:: \text { 100: } x . \quad x=99.36+\text { per cent. }
$$

If it is desired that each cc. of the permanganate solution should represent a certain percentage of pure salt, a mo!ecular quantity of the salt should be taken for analysis instead of $\mathrm{I} \mathrm{gm}$. For example, if $2.760 \mathrm{I} \mathrm{gms.} \mathrm{be} \mathrm{taken,} \mathrm{each} \mathrm{cc.} \mathrm{of} \mathrm{the} \mathrm{decinormal} \mathrm{solution} \mathrm{con-}$ sumed will correspond to I per cent, because $2.760 \mathrm{I} \mathrm{gms}$. is the weight of crystallized ferrous sulphate which can be oxidized by Ioo cc. of the decinormal solution. If half of this weight be taken, i.e., I.38 gm. each cc. of the permanganate solution compound will represent 2 per cent of pure salt.

Granulated Ferrous Sulphate $\left(\mathrm{FeSO}_{4}+{ }_{7} \mathrm{H}_{2} \mathrm{O}\right)$ is estimated in the same way as the foregoing, and should correspond with it in strength.

Exsiccated (Dried) Ferrous Sulphate. This salt is tested in the same manner, as the other two sulphates. It contains a larger percentage of ferrous sulphate than the other two, having less 
water of crystallization. Its composition is approximately $\mathrm{FeSO}_{4}+{ }_{3} \mathrm{H}_{2} \mathrm{O}$.

$\mathrm{IoFeSO}_{4}+{ }_{2} \mathrm{KMnO}_{4}+8 \mathrm{H}_{2} \mathrm{SO}_{4}$

$\frac{100) 1508.5}{15.085}$ gms. $\frac{313.96}{3.1396}$ gms. or rooo cc. $\frac{N}{10}$ standard solution.

$$
={ }_{5} \mathrm{Fe}_{2}\left(\mathrm{SO}_{4}\right)_{3}+\mathrm{K}_{2} \mathrm{SO}_{4}+2 \mathrm{MnSO}_{4}+8 \mathrm{H}_{2} \mathrm{O} \text {. }
$$

Each cc. of the standard solution represents $0.015085 \mathrm{gm}$. of anhydrous (real) ferrous sulphate. If one gm. of the dried salt, treated as above described, requires $48 \mathrm{cc}$. of $\frac{\mathrm{N}}{\mathrm{IO}}$ permanganate solution, it contains

$$
0.015085 \times 48=0.72408 \mathrm{gm} \text {., }
$$

or 72.40 per cent of real ferrous sulphate, and $100.00-72.40=27.60$ per cent of water of crystallization.

Saccharated Ferrous Carbonate. The U. S. P. directs that I.I5 gms. of saccharated ferrous carbonate be dissolved in ro cc. of diluted sulphuric acid, then diluted with water to about roo cc., and then titrated with $\frac{\mathrm{N}}{\mathrm{IO}}$ potassium permanganate V. S. until a pink tint is produced in the liquid. $15 \mathrm{cc}$. should be required. This method is not an exact one, especially if heat is applied for solution of the powder, in that permanganate is reduced by the sugar present.

$b$. Estimation of Metallic Iron in Ferrum Reductum. Ferrum reductum (reduced iron) always contains besides metallic iron a varying quantity of oxid. Therefore, in assaying this preparation a method must be employed which will estimate the iron only, which is present as metallic iron. This may be done by means of a solution of mercuric chlorid which reacts with metallic iron only and not with the oxid.

The method is as follows:

$0.555 \mathrm{gm}$. of reduced iron is introduced into a glass-stoppered bottle, $50 \mathrm{cc}$. of mercuric chlorid solution ( $5 \mathrm{gms}$. in roo cc.) are added and the bottle heated on a water bath for one hour, agitating fiequently, but keeping the bottle well stoppered.

$$
{ }_{2} \mathrm{HgCl}_{2}+\mathrm{Fe}_{2}=2 \mathrm{FeCl}_{2}+{ }_{2} \mathrm{Hg},
$$

then allow it to cool, dilute the contents with water to roo cc., and filter. Take ro $\mathrm{cc}$. of the filtrate (representing $0.0555 \mathrm{gm}$. of 
reduced iron) add to it ro cc. of diluted sulphuric and io cc. of a solution of manganous sulphate $(\mathrm{I}: 5)$, introduce the mixture into a glassstoppered bottle (having a capacity of roo cc.), and titrate with decinormal permanganate until a permanent pink color is obtained. Each cc. of the permanganate solution represents $0.00555 \mathrm{gm}$. of metallic iron or to per cent.

$$
\begin{aligned}
& \text { Io } \mathrm{Fe} \mathrm{SO}_{4}+2 \mathrm{KMnO}_{4}+8 \mathrm{H}_{2} \mathrm{SO}_{4}={ }_{5} \mathrm{Fe}_{2}\left(\mathrm{SO}_{4}\right)_{3}+\mathrm{K}_{2} \mathrm{SO}_{4}+2 \mathrm{MnSO}_{4}+8 \mathrm{H}_{2} \mathrm{O} \\
& { }_{100)}^{5555} \text { gms. }{ }_{100)} \frac{313.96}{3.1396} \mathrm{gms} .=1000 \mathrm{cc} \cdot \frac{\mathrm{N}}{\mathrm{IO}} \mathrm{V} . \mathrm{S} . \\
& 0.00555 \mathrm{gms} .=0.0031396 \mathrm{gms} .=\mathrm{I} \mathrm{cc} \cdot \frac{\mathrm{N}}{\mathrm{IO}} \mathrm{V} . \mathrm{S} .
\end{aligned}
$$

If $9 \mathrm{cc}$. of the $\frac{\mathrm{N}}{\mathrm{ro}}$ permanganate are consumed, then $9 \times 0.00555$ $=0.04995 \mathrm{gm}$., and since the Io cc. of the iron solution taken for analysis represented $0.0555 \mathrm{gm}$., the per cent of metallic iron present is 90.

$$
0.0555: 100:: 0.04995: x . \quad x=90 .
$$

The use of manganous sulphate in this process is to prevent decomposition of permanganate by the hydrochloric acid. The quantity is, however, so small, that if the titration be conducted cold, its use is unnecessary.

Titration with an Empirical Permanganate Solution. A solution of permanganate which is found upon standardization to be of a strength in which I cc. is equivalent to $0.00512 \mathrm{gm}$. of $\mathrm{Fe}$ is to be used.

Each cc. of this solution is equivalent to the following quantities:

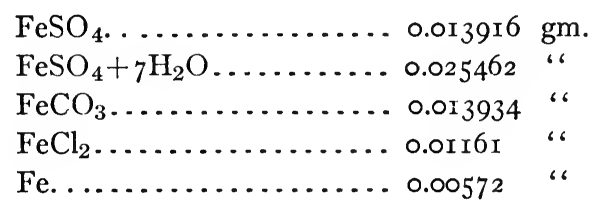

c. Estimation of Oxalic Acid and Oxalates with Potassium Permanganate Solution $\left(\mathrm{H}_{2} \mathrm{C}_{2} \mathrm{O}_{4}+{ }_{2} \mathrm{H}_{2} \mathrm{O}=\mathrm{I} 25.10 ; \mathrm{H}_{2} \mathrm{C}_{2} \mathrm{O}_{4}=89.34\right)$. The estimation of oxalic acid may be accurately made either by neutralization with a standard alkali or by oxidation with standard permanganate. The latter method is, however, the one to be employed in the case of oxalates. 
The oxidimetric estimation of oxalic acid is carried out as follows:

I gm. of the acid (accurately weighed) is dissolved in sufficient water to make $100 \mathrm{cc}$. Of this solution, Io cc. (representing 0.I gm. of the acid) is taken for analysis. 2 cc. of diluted sulphuric acid are added, the solution is heated to between $40^{\circ} \mathrm{C}$. and $60^{\circ} \mathrm{C}$., and keeping it at about this temperature is titrated with decinormal potassium permanganate, agitating constantly, until a faint rose tint marks the completion of the reaction.

Each cc. of the permanganate solution consumed represents $0.006255 \mathrm{gm}$. of crystallized oxalic acid.

The reaction is as follows:

$$
\begin{aligned}
& 5\left(\mathrm{H}_{2} \mathrm{C}_{2} \mathrm{O}_{4}+{ }_{2} \mathrm{H}_{2} \mathrm{O}\right)+{ }_{3} \mathrm{H}_{2} \mathrm{SO}_{4}+{ }_{2} \mathrm{KMnO}_{4} \\
& \text { 100)625.5 100) } 313.96 \\
& 6.255 \mathrm{gms} .=\frac{\underline{31} .96}{3.1396} \mathrm{gms} .=1000 \mathrm{cc} \cdot \frac{\mathrm{N}}{10} \mathrm{~V} . \mathrm{S} \text {. } \\
& 0.6255 \mathrm{gm} .=0.3^{1} 39^{6} \mathrm{gm} .=100 \mathrm{cc} \cdot \frac{\mathrm{N}}{\mathrm{IO}} \mathrm{V} . \mathrm{S} \text {. } \\
& 0.006255 \mathrm{gm} .=\quad 0.003^{1} 396 \mathrm{gm} .=\text { I cc. } \frac{\mathrm{N}}{\mathrm{IO}} \mathrm{V} . \mathrm{S} \text {. } \\
& =\mathrm{K}_{2} \mathrm{SO}_{4}+2 \mathrm{MnSO}_{4}+8 \mathrm{H}_{2} \mathrm{O}+5 \mathrm{O}_{2} \text {. }
\end{aligned}
$$

Direct Percentage Titration. $0.6255 \mathrm{gm}$. of crystallized oxalic acid . is oxidized by $100 \mathrm{cc}$. of $\frac{\mathrm{N}}{\mathrm{IO}}$ permanganate. Therefore if $0.6255 \mathrm{gm}$. of the acid is taken for analysis, each cc. of $\frac{\mathrm{N}}{10}$ permanganate will represent i per cent.

Titrating with an Empirical Solution. If the permanganate is checked with iron, we take into consideration that $2 \mathrm{KMnO}_{4}$ will oxidize ro atoms of iron ( 555 parts), and on the other hand 5 molecules of oxalic acid $(625.5$ parts). If the titer of the permanganate be found on experiment to be i cc. $=0.00569$, whatever number of cc. of this solution is consumed, is to be multiplied by 0.00569 and then $b y \frac{62.55}{55 \cdot 5}$.

Example. $0.3 \mathrm{gm}$. of oxalic acid require for oxidation $40 \mathrm{cc}$. of a permanganate solution whose titer is I cc. $=0.00569 \mathrm{gm}$. Fe, the calculation is made as follows:

$$
40 \times 0.00569 \mathrm{gm} . \times \frac{62.55}{55.5}=0.2564 \mathrm{gm} .
$$


$0.2564 \mathrm{gm}$. is the quantity of pure crystallized oxalic acid present in the $0.3 \mathrm{gm}$. taken for analysis. This is 85.46 per cent.

$$
\frac{0.2564 \times 100}{0.3}=85.46
$$

If the standardization of the permanganate is done by means of a decinormal oxalic acid, or by the iodometric method, the calculation is as described on pages 149 and 150 .

Oxalates are estimated in the same manner; a much larger quantity of sulphuric acid is, however, required. This serves to liberate the oxalic acid from its combination.

The presence of precipitates of sulphates of calcium, barium, or lead does not interfere with the recognition of the end-point.

Each cc. of $\frac{\mathrm{N}}{\mathrm{IO}}$ potassium permanganate represents

Oxalic acid anhydrous $\left(\mathrm{H}_{2} \mathrm{C}_{2} \mathrm{O}_{4}\right) \ldots \ldots \ldots \ldots . .004467 \mathrm{gm}$.

Oxalic acid crystallized $\left(\mathrm{H}_{2} \mathrm{C}_{2} \mathrm{O}_{4}+{ }_{2} \mathrm{H}_{2} \mathrm{O}\right) \ldots . .0 .006255$ "

This method may be applied to calcium salts which are soluble in water or in acetic acid, as well as to other metals which are precipitable as oxalates. It is, however, especially applicable to calcium, because of the readiness with which this metal may be separated from others as oxalate. The precipitation may be accomplished in either an ammoniacal or a weak acetic acid solution. If it is necessary to dissolve the calcium salt with the aid of hydrochloric acid, the solution must be rendered strongly alkaline with ammonia, and the precipitation effected with ammonium oxalate.

Example. $0.2 \mathrm{gm}$. of calcium carbonate is dissolved in the smallest necessary quantity of dilute acetic acid, then sufficient oxalic acid is added to completely precipitate the calcium as oxalate $\left(\mathrm{CaC}_{2} \mathrm{O}_{4}\right)$. This precipitate is then thoroughly washed on the filter. A hole is then made in the filter and the precipitate washed through the funnel into a flask (about $100 \mathrm{cc}$. of water being used) a small quantity, say about $2 \mathrm{cc}$. of dilute sulphuric acid are added, the mixture warmed to between $40^{\circ} \mathrm{C}$. and $60^{\circ} \mathrm{C}$. and titrated with decinormal potassium permanganate until a permanent rose tint appears.

Each cc. of the decinormal permanganate represents 0.004467 
gm. of oxalic acid (anhydrous) or 0.0049675 gm. of calcium carbonate.

If in the above assay, $39.5 \mathrm{cc}$. of the $\frac{\mathrm{N}}{\mathrm{IO}}$ permanganate were employed, then $0.0049675 \mathrm{gm} . \times 39.6=0.1957+\mathrm{gm}$.; this is the quanity of pure $\mathrm{CaCO}_{3}$ in the $0.2 \mathrm{gm}$. taken for analysis.

$$
\frac{0.1957 \times 100}{0.2}=97.85 \text { per cent. }
$$

If the substance to be examined contains iron, aluminum, manganese, etc., these metals must be removed before titrating.

Example. Dolomite (which contains besides calcium carbonate some iron, aluminum, and magnesium) is to be analyzed. A weighed quantity is dissolved in hydrochloric acid, and an excess of ammonia water is added. (If the ammonia water contains any carbonate, the precipitate must be again dissolved and reprecipitated). The strongly ammoniacal filtrate is treated with excess of oxalic acid, to induce complete precipitation. The precipitate is then collected on a filter, thoroughly washed, and oxalic acid estimated by titration with permanganate solution.

The calculation is based upon the fact that a molecular weight of oxalic acid (I25.I) will precipitate a molecule of $\mathrm{CaO}$ (55.68). The quantity of oxalic acid found is then multiplied by $\frac{55.68}{125 \cdot 10}$.

If an empirical permanganate solution is employed the titer of which is I cc. $=0.00569 \mathrm{gm}$. Fe, and $42 \mathrm{cc}$. of this be consumed, we calculate as follows:

$$
0.00569 \times 42 \times \frac{55.68}{125.10} \times \frac{62.55}{55.5}=0.139+\mathrm{gm} . \mathrm{CaO} .
$$

If, as in the case of dolomite a large quantity of magnesium is present, some of the difficultly soluble magnesium oxalate will be in the precipitate, mixed with the calcium oxalate; this may be remcred by dissolving the precipitate in diluted acetic acid and reprecipitating with pure ammonia.

d. Estimation of Hydrogen Dioxid and Barium Dioxid with Standard Potassium Permanganate. Hydrogen Dioxid (Hydrogen Peroxid; Aqua Hydrogenii Dioxidi, U. S. P.) $\quad\left(\mathrm{H}_{2} \mathrm{O}_{2}=33.76\right)$. 
This substance was official for the first time in the U. S. P., 1890, in which methods for its preparation, preservation, and assay were given. The official solution contained 3 per cent by weight of pure hydrogen dioxid, which corresponds to Io volumes of available oxygen.

Solution of hydrogen dioxid is an important commercial product, being used in the arts as well as in medicine.

It is sold as containing 5, Io, 15 , or 20 volumes of oxygen in solution. This should mean that a given volume of the solution yields from itself 5 , IO, I5, or 20 times its own volume of oxygen.

Thus, I cc. of a 5 -volume solution yields $5 \mathrm{cc}$. of oxygen; a Io-volume solution is one of which I cc. will yield Io cc. of oxygen, etc.

Many solutions of hydrogen dioxid are sent into the market under false pretences, being labeled as containing IO, I5, or 20 volumes of oxygen.

It is true a given volume of these solutions will yield the specified volume of oxygen when decomposed with potassium permanganate, but half of this oxygen comes from the permanganate itself. Therefore the hydrogen dioxid solution contains only half as much available oxygen as is given off in this decomposition.

Freshly bought samples of the five largest manufacturers, according to the analyses of Dr. Edward R. Squibb (Ephemeris, vol. Iv. No. 2), gave $9.2,8.7,8.4,10.9,9.7,8.6,8.5,7.3$, and 7.4 volumes. All of these were labeled as being of ${ }_{15}$ volumes strength. The author has had a similar experience.

In its purest and most concentrated form hydrogen dioxid is a syrupy colorless liquid, having an odor resembling that of chlorin or ozone.

One cc. of this concentrated hydrogen dioxid when decomposed at $0^{\circ} \mathrm{C}$. evolves 330.3 times its own volume of oxygen, at a pressure of $760 \mathrm{~mm}$. at $45^{\circ} \mathrm{N}$. latitude.

At a temperature of $100^{\circ}$ C. $-\left(22^{\circ}\right.$ F. $) \mathrm{H}_{2} \mathrm{O}_{2}$ decomposes rapidly into water and oxygen. This change also takes place at ordinary temperatures, but more slowly. In diluted solutions it is more stable, and may be concentrated by boiling without suffering much decomposition.

Dr. Squibb made a series of experiments in order to prove this, as well as the fact that solutions of hydrogen dioxid when kept in open vessels at the ordinary temperature become stronger instead of weaker, as was generally supposed. The water evaporates more rapidly than the dioxid decomposes. Part of the results of these 
experiments as published in the Ephemeris, vol. IV, No. 2, is as follows:

A freshly made solution that yielded 10.3 volumes of available oxygen was taken as the basis of the experiment. The evaporation was done on a water-bath, at temperatures varying from $55^{\circ}$ to $62^{\circ} \mathrm{C}$. $\left(13 \mathrm{I}^{\circ}\right.$ to $143.6^{\circ} \mathrm{F}$.), one $\mathrm{cc}$. of the concentrated solution being taken out for testing after each evaporation.

$200 \mathrm{cc}$. evaporated in 2 hours to $100 \mathrm{cc}$. tested 20.6 volumes: no apparent loss.

Ioo cc. of the 10.3-volume solution were added, and evaporated in 2 hours to $100 \mathrm{cc}$., tested 29.6 volumes: " 1.3 volumes loss.

Ioo cc. of the I0.3-volume solution were added, and evaporated in 2 hours to $100 \mathrm{cc}$., tested 36.5 volumes: 4.7 volumes loss.

roo cc. of the 10.3-volume solution were added, and evaporated in 2.5 hours to $23 \mathrm{cc}$., tested 146.8 volumes.

Another series of evaporations were made at higher temperatures, which also showed an increase in strength, but the loss was a little larger.

Hydrogen dioxid and potassium permanganate, though both oxidizing agents, will, when mixed in an acid solution, reduce each other. The reaction which occurs is probably primarily an oxidation of the $\mathrm{H}_{2} \mathrm{O}_{2}$ to a higher oxid $\left(\mathrm{H}_{2} \mathrm{O}_{4}(\right.$ ? $\left.)\right)$ which, however, immediately breaks up with the liberation of oxygen. The method of assaying hydrogen dioxid by means of permanganate is applicable not only to this substance but also to the estimation of barium dioxid and the soluble alkali peroxids. The method is usually carried out by adding the permanganate solution to the dioxid in a solution acidulated with sulphuric acid. Immediate decolorization of the permanganate occurs, as long as any hydrogen dioxid is present. When the latter has been entirely taken up the permanganate is no longer decolorized and a faint pink tint marks the end-point. In the estimation of the pharmacoporial or commercial dioxid solutions, containing 2 or 3 per cent of $\mathrm{H}_{2} \mathrm{O}_{2}$, a measured quantity is taken for analysis. The specific gravity of the solution being nearly that of water $\mathrm{I}$ cc. is taken to represent $\mathrm{I}$ gm. In the case of solutions of hydrogen dioxid of high percentage strength it is advisable to take a weighed quantity for analysis. If hydrochloric acid is present a small quantity of manganese sulphate should be introduced before titrating.

The assay is conducted as follows: 
Io cc. of the solution are accurately measured and diluted (in a graduated cylinder) with water to make $100 \mathrm{cc}$. Io cc. of this diluted liquid (containing I cc. of hydrogen dioxid solution) are transferred to a beaker, $5 \mathrm{cc}$. of diluted sulphuric acid ( $\mathrm{I}-8)$ are added and then the $\frac{\mathrm{N}}{\mathrm{IO}}$ permanganate solution run in from a burette, stirring after each addition until a permanent faint pink tint appears. The reaction is as follows:

$$
\begin{aligned}
& { }_{5} \mathrm{H}_{2} \mathrm{O}_{2}+{ }_{2} \mathrm{KMnO}_{4}+{ }_{3} \mathrm{H}_{2} \mathrm{SO}_{4}=\mathrm{K}_{2} \mathrm{SO}_{4}+2 \mathrm{MnSO}_{4}+8 \mathrm{H}_{2} \mathrm{O}+{ }_{5} \mathrm{O}_{2} \text {. } \\
& \frac{1.888}{\mathrm{gms} .} \frac{3^{\mathrm{I}} 3.96}{3.1396} \mathrm{gms} .=\text { rooo cc. } \frac{\mathrm{N}}{\mathrm{IO}} \text { permanganate V.S. } \\
& 0.001688 \mathrm{gm} .=0.003 \mathrm{r} 396 \mathrm{gm} .=\mathrm{I} \text { cc. } \frac{\mathrm{N}}{\mathrm{Io}} \text { permanganate V.S. }
\end{aligned}
$$

Thus each cc. of $\frac{\mathrm{N}}{\mathrm{IO}}$ permanganate represents $0.001688 \mathrm{gm}$. of absolute hydrogen dioxid. Assuming that in the above estimation I9 cc. of the permanganate solution were required, then the I cc. taken for analysis contained $0.001688 \mathrm{gm} . \times_{19}$, which is $0.032072 \mathrm{gm}$. of absolute $\mathrm{H}_{2} \mathrm{O}_{2}$. This corresponds to 3.2072 per cent.

The Direct Percentage Method. Io cc. of the solution are diluted with water to measure $100 \mathrm{cc}$. $\mathrm{I} 6.88 \mathrm{cc}$. of this diluted solution (containing r.688 cc. of hydrogen dioxid) are acidulated with sulphuric acid and titrated with decinormal permanganate as above described. Each cc. of the permanganate solution consumed will represent 0.1 per cent by weight of $\mathrm{H}_{2} \mathrm{O}_{2}$.

Titration with an Empirical Solution. A permanganate solution is on hand which is found upon standardization with iron to be I cc. $=0.00569 \mathrm{gm}$. Fe. To use this solution as it is, we take into consideration that $2 \mathrm{KMnO}_{4}=(3 \mathrm{I} 3.96)=\mathrm{IO}$ atoms of $\mathrm{Fe}(555)$ and also 5 molecules of $\mathrm{H}_{2} \mathrm{O}_{2}$ (168.8). $31.396 \mathrm{gm} . \mathrm{KMnO}_{4}=55.5 \mathrm{gm}$. $\mathrm{Fe}=\mathrm{I} 6.88$ gm. $\mathrm{H}_{2} \mathrm{O}_{2}$. Whatever number of cc. of this permanganate solution is used multiplied by $0.00569 \mathrm{gm}$. and then by $\frac{\mathrm{x} 6.88}{55.5}$ will give the weight of $\mathrm{H}_{2} \mathrm{O}_{2}$ present in the sample analyzed. See page $\mathrm{I} 48$.

Estimation of Volume Strength. Let us look at the above equation in a different light.

We there see that when potassium permanganate and hydrogen dioxid react, to atoms of oxygen are liberated.

The permanganate itself when decomposed liberates five atoms of 
oxygen. Therefore of the above ten atoms only five come from the hydrogen dioxid.

$$
\begin{aligned}
5 \mathrm{H}_{2} \mathrm{O}_{2} & ={ }_{5} \mathrm{H}_{2} \mathrm{O}+{ }_{5} \mathrm{O} \\
{ }_{2} \mathrm{KMnO}_{4}+{ }_{3} \mathrm{H}_{2} \mathrm{SO}_{4} & =\mathrm{K}_{2} \mathrm{SO}_{4}+{ }_{2} \mathrm{MnSO}_{4}+{ }_{3} \mathrm{H}_{2} \mathrm{O}+{ }_{5} \mathrm{O} .
\end{aligned}
$$

In order to find the factor for volume of available oxygen, see the following equation, etc.:

$$
\begin{aligned}
& { }_{5} \mathrm{H}_{2} \mathrm{O}_{2}+{ }_{2} \mathrm{KMnO}_{4}+{ }_{3} \mathrm{H}_{2} \mathrm{SO}_{4}=\mathrm{K}_{2} \mathrm{SO}_{4}+{ }_{2} \mathrm{MnSO}_{4}+8 \mathrm{H}_{2} \mathrm{O}+{ }_{5} \mathrm{O}+{ }_{5} \mathrm{O} \text {. }
\end{aligned}
$$

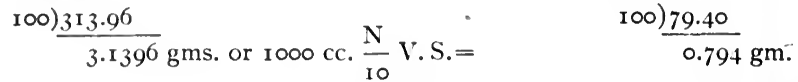

$$
\begin{aligned}
& \text { I cc. } \frac{\mathrm{N}}{\text { IO }} \text { V.S. }=\quad 0.000794 \mathrm{gm} \text {. }
\end{aligned}
$$

Thus it is seen that each cc. of $\frac{\mathrm{N}}{\mathrm{IO}}$ potassium permanganate V. S. represent $0.000794 \mathrm{gm}$. of oxygen. But we require to find the volume of oxygen, not the weight represented by I cc. of $\frac{\mathrm{N}}{\mathrm{IO}}$ permanganate.

I000 cc. of oxygen at $0^{\circ} \mathrm{C}$. and $760 \mathrm{~mm}$. pressure, weigh $\mathrm{I} .4276 \mathrm{I} 2$ gms. Therefore, if $1.4276 \mathrm{I} 2 \mathrm{gms}$. measure $1000 \mathrm{cc}$, $0.000794 \mathrm{gm}$. will measure $x . \quad x=0.556+\mathrm{cc}$.

$$
\frac{1000 \times 0.000794}{1.427612}=0.556 \mathrm{cc} .
$$

The factor, then, for volume of oxygen, liberated when hydrogen dioxid is titrated with $\frac{\mathrm{N}}{\mathrm{IO}}$ potassium permanganate is $c .556$, and the number of cc. of the $\frac{\mathrm{N}}{10}$ potassium permanganate consumed in the titration gives the volume of oxygen liberated by the quantity of hydrogen dioxid taken.

Thus if 19 cc. of the $\frac{N}{10}$ V. S. were required,

$$
0.55^{6} \times 19=10.564 \text { cc. of oxygen, }
$$

It is convenient to operate upon I cc. hydrogen dioxid solution. Then each cc. of potassium permanganate V.S. used will represent 
$0.55^{6} \mathrm{cc}$. of available oxygen, or $0.000794 \mathrm{gm}$. of oxygen, and it is only necessary to multiply the cc. by these numbers to obtain the volume or weight of available oxygen.

If any other quantity than I cc. of dioxid be taken for analysis, it will be necessary after multiplying by 0.556 to divide the result by the quantity of dioxid solution taken, in order to find volume strength.

Hydrogen dioxid solution may also be volumetrically assayed by Kingzett's method, which is described under Iodometry.

The gasometric estimation is also described further on.

Barium Dioxid (Barium Peroxid) $\left(\mathrm{BaO}_{2}=\mathrm{I} 68 . \mathrm{I} 6\right)$. This substance is assayed by treating it with an acid, and then estimating the liberated hydrogen dioxid, as follows:

Weigh ofi $2 \mathrm{gms}$. of the coarse powder, put it in a porcelain capsule, add about io cc. of ice-cold water, then $7.5 \mathrm{cc}$. of phosphoric acid, ( 85 per cent), and sufficient ice-cold water to make $25 \mathrm{cc}$. Stir and break up the particles with the end of the stirrer until a clear or nearly clear solution is obtained and all that is soluble is dissolved.

$5 \mathrm{cc}$. of this solution (which corresponds to $0.4 \mathrm{gm}$. of barium dioxid) is measured off for assay.

Drop into this from a burette, with constant stirring, decinormal potassium permanganate until a final drop gives the solution a permanent pink tint.

About $40 \mathrm{cc}$.of the decinormal permanganate should be required to produce this result.

In this process, the first step is the formation of hydrogen diroxid by treating the barium dioxid with phosphoric acid, as illustrated by the following equation:

$$
\underset{168.16}{\mathrm{BaO}_{2}}+\mathrm{H}_{3} \mathrm{PO}_{4}=\mathrm{BaHPO}_{4}+\underset{33.76}{\mathrm{H}_{2} \mathrm{O}_{2}} .
$$

The hydrogen dioxid is then estimated with decinormal permanganate as described above.

$$
\begin{aligned}
& 5\left(\mathrm{BaO}_{2}\right)={ }_{5} \mathrm{H}_{2} \mathrm{O}_{2}+{ }_{2} \mathrm{KMnO}_{4}+{ }_{3} \mathrm{H}_{2} \mathrm{SO}_{4} \\
& \text { 100) } 840.8 \quad \text { I00) } 168.8 \quad \text { I00) } 3 \mathrm{r} 3.96
\end{aligned}
$$

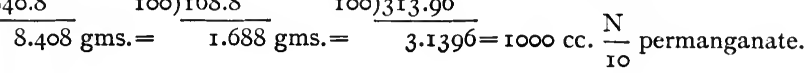

$$
\begin{aligned}
& =\mathrm{K}_{2} \mathrm{SO}_{4}+2 \mathrm{MnSO}_{4}+8 \mathrm{H}_{2} \mathrm{O}+{ }_{5} \mathrm{O}_{2} \text {. }
\end{aligned}
$$


Thus each cc. of $\frac{\mathrm{N}}{\mathrm{IO}}$ permanganate represents $0.001688 \mathrm{gm}$. of $\mathrm{H}_{2} \mathrm{O}_{2}$, which is equivalent to $0.008408 \mathrm{gm}$. of $\mathrm{BaO}_{2}$, as shown in above equation.

Assuming that $40 \mathrm{cc}$. of the decinormal solution of permanganate were required, then $0.008408 \times 10=0.33632 \mathrm{gm}$.

$$
\frac{0.33632 \times 100}{0.4}=84.08 \text { per cent of pure } \mathrm{BaO}_{2} \text {. }
$$

If an Empirical Solution is used, which was set with iron and whose titer is I cc. $=0.00569 \mathrm{gm}$. Fe, the quantity of the latter used, multiplied by $0.00569 \mathrm{gm}$. and then by $\frac{84.08}{55.5}$ will give the quantity of $\mathrm{BaO}_{2}$ present in the sample taken.

Example. $0.4 \mathrm{gm}$. were taken for analysis, and $38 \mathrm{cc}$. of the above ompirical solution were consumed, then

$$
38 \times 0.00569 \mathrm{gm} . \times \frac{84}{55.08}==0.3276
$$

If the empirical solution is checked with a decinormal oxalic acid, and $9.6 \mathrm{cc}$. are required to oxidize the ro cc. of standard oxalic acid, the permanganate solution is stronger than it should be. If this solution is used in the above-described assay and $38 \mathrm{cc}$. are employed, the calculation is made as follows:

Multiply 38 by $\frac{100}{9^{6}}$ and then by the true decinormal factor for $\mathrm{BaO}_{2}(0.008408$ gm.).

$$
38 \times \frac{100}{9^{6}} \times 0.008408=0.3328 \mathrm{gm} .
$$

e. Estimation of Ferric Salts by Means of Potassium Permanganate (After reduction). It is frequently necessary to estimate ferric salts by means of permanganate solution; this is particularly the case in compounds where ferric and ferrous salts are both present.

The ferric salts must of course be reduced to the ferrous state in order to estimate them with permanganate, or in fact with any oxidizing agent. There are many ways of affecting this reduction, but the best way (where permanganate is to be used) is no doubt by 
the use of metallic zinc or magnesium in sulphuric acid solutions. Hydrochloric acid may be used instead of sulphuric, but in that case the solution must be very dilute and of low temperature, in order to avoid the liberation of chlorin, which would spoil the analysis.

In concentrated or hot solutions hydrochloric acid acts upon permanganate as a reducing agent, as shown in the equation

$$
\mathrm{KMnO}_{4}+8 \mathrm{HCl}=\mathrm{KCl}+\mathrm{MnCl}_{2}+{ }_{4} \mathrm{H}_{2} \mathrm{O}+{ }_{5} \mathrm{Cl} \text {. }
$$

The irregularities due to the liberation of chlorin may be obviated by the addition of an excess of mercuric sulphate before titration, as suggested by Cady and Ruediger, or by the use of magnesium or manganous sulphate, as suggested by Kessler and Zimmermann (see page 140).

The reduction is effected by adding to the warm diluted solution of the ferric salt acidulated with sulphuric acid small pieces of pure metallic zinc or coarsely powdered magnesium, and setting aside in a covered vessel until the solution is colorless, or until it fails to produce a red color when a drop of it is brought in contact with a drop of sulphocyanate. The zinc used must be free from iron, or if the latter metal is present its quantity must be known. All of the zinc or magnesium must be dissolved before the titration is begun, otherwise the reduction would continue whilst the titration is being done. When the ferric salt is completely reduced the titration should be carried out without delay in order to avoid reoxidation through exposure to the air. Reoxidation takes place more readily in the presence of hydrochloric acid than when sulphuric acid is used. According to D. J. Carnegie, reduction takes place much more rapidly in neutral than in acid solutions. He suggests neutralization of the solution with ammonia, and after reduction the addition of sulphuric aciủ to keep the iron in solution. Other methods for the reduction of ferric salts are described further on.

The solution of the ferric salt to be estimated should not contain more than $0.15 \mathrm{gm}$. of metallic iron in $250 \mathrm{cc}$. To this quantity of solution about ro gms. of metallic zinc and $25 \mathrm{cc}$. of sulphuric are taken. The solution is kept at a temperature between $60^{\circ}$ and $80^{\circ} \mathrm{C}$. until the zinc is entirely dissolved, then the mixture is boiled in a flask provided with a valve stopper, as shown in Fig. 54 (in order to exclude air and prevent reoxidation). It is then rapidly cooled, and titrated with permanganate without delay. 
Example. Estimation of Ferric Chlorid. $0.35 \mathrm{gm}$. of the dried salt are dissolved in $250 \mathrm{cc}$. of water in a flask, $25 \mathrm{cc}$. of sulphuric acid are added, and then ro gms. of metallic zinc are introduced. The flask is then gently warmed until the zinc is entirely dissolved and the solution is colorless and fails to give a red color when a small portion of it removed with a glass rod, is brought in contact with a drop of potassium sulphocyanate. The solution is then brought to a boil, and after this rapidly cooled, avoiding entrance of air, and when cool titrated with $\frac{\mathrm{N}}{\mathrm{IO}}$ potassium permanganate, until a faint permanent pink color appears.

19.5 cc. of the permanganate were required. Each cc. of $\frac{\mathrm{N}}{\text { so }}$ permanganate represents $0.00555 \mathrm{gm}$. of metallic iron, which is equivalent to $0.016104 \mathrm{gm}$. of anhydrous ferric chlorid. Then if $19.5 \mathrm{cc}$. of the permanganate were employed, the quantity of real ferric chlorid present in the sample is $0.016104 \mathrm{gm} . \times_{19.5}$, which is $0.314028 \mathrm{gm}$., or 89.7 per cent, while the quantity of metallic iron is $0.00555 \mathrm{gm} . \times 19.5$ $=0.108225 \mathrm{gm}$. or 30.92 per cent.

The reactions are represented by the following equations:

$$
\begin{aligned}
& { }_{5} \mathrm{Fe}_{2} \mathrm{Cl}_{6}+{ }_{5} \mathrm{Zn}={ }_{10} \mathrm{FeCl}_{2}+{ }_{5} \mathrm{ZnCl}_{2} \text {. } \\
& \text { 100) } 1610.40 \quad 125^{8.6} \\
& \text { I6.104 }
\end{aligned}
$$

$\mathrm{roFeCl} 2+{ }_{2} \mathrm{KMnO}_{4}+{ }_{\mathrm{I}} 6 \mathrm{HCl}={ }_{5} \mathrm{Fe}_{2} \mathrm{Cl}_{6}+{ }_{2} \mathrm{KCl}+{ }_{2} \mathrm{MnCl}_{2}+8 \mathrm{H}_{2} \mathrm{O}$. 100) $1258.6 \quad$ 100) 313.96

$\mathrm{r} 2.586 \mathrm{gms} .=\frac{3.1396}{\mathrm{gms} .}=1000 \mathrm{cc} . \frac{\mathrm{N}}{10}$ permanganate.

Here it is shown that $1000 \mathrm{cc}$. of $\frac{\mathrm{N}}{\mathrm{IO}}$ permanganate containing $3.1396 \mathrm{gm}$. of the salt will oxidize 12.586 gms. of ferrous chlorid which represents 16.104 gms. of ferric chlorid or 5.55 gms. of metallic iron. Therefore each I cc. of the $\frac{\mathrm{N}}{10}$ permanganate will represent $\frac{1}{1000}$ part of each of these quantities, namely, $0.012586 \mathrm{gm}$. of $\mathrm{FeCl}_{2}, 0.016104$ $\mathrm{gm}$. of $\mathrm{Fe}_{2} \mathrm{Cl}_{6}$, and $0.00555 \mathrm{gm}$. of metallic iron.

Because of the frequent and almost invariable presence in zinc of carbon and iron, which have a reducing action upon permanganate, it is necessary to carry out a blank experiment, to determine the quantity of permanganate solution used up by these impurities in the zinc. 
This blank experiment must be conducted under the same conditions as the assay, and differs only in that the iron is left out.

Example. Io gms. of zinc from the same lot as used for the assay is treated with $250 \mathrm{cc}$. of water and $25 \mathrm{cc}$. of sulphuric acid, and when it is completely dissolved, the potassium permanganate solution is added, until a permanent pale pink tint results. The number of cc. consumed is deducted from the quantity employed in the assay; the difference is the quantity of the permanganate solution which was consumed by the ferrous chlorid. Another method for the reduction of ferric salts, previous to titration with permanganate, is that of $\mathrm{N}$. Matolcsy. In this the ferric salt is precipitated with ammonium sulphid and the precipitated ferrous sulphid dissolved in sulphuric acid, which converts it into ferrous sulphate. This is then titrated with permanganate after the $\mathrm{H}_{2} \mathrm{~S}$ has been driven off. The method is described in detail on page 382 .

If permanganate solution of the titer I $c c .=0.00569 \mathrm{gm}$. Fe be used, the calculation is

$$
0.00569 \times 19.5 \times \frac{\mathrm{I} 6 \mathrm{I} .04}{55.5}=0.3219 \mathrm{gm} . \mathrm{Fe}_{2} \mathrm{Cl}_{6},
$$

or 91.9 per cent.

f. Estimation of Nitrous Acid and Nitrites. Nitrous acid, when brought in contact with a potassium permanganate solution acidulated with sulphuric acid, is oxidized to nitric acid. Two molecules of $\mathrm{KMnO}_{4}$ reacting with 5 molecules of $\mathrm{HNO}_{2}$, as the equation shows,

$$
{ }_{5} \mathrm{HNO}_{2}+2 \mathrm{KMnO}_{4}+{ }_{3} \mathrm{H}_{2} \mathrm{SO}_{4}={ }_{5} \mathrm{HNO}_{3}+\mathrm{K}_{2} \mathrm{SO}_{4}+{ }_{2} \mathrm{MnSO}_{4}+{ }_{3} \mathrm{H}_{2} \mathrm{O} \text {. }
$$

In the case of nitrites, as for example sodium nitrite, the oxidation takes place in the same manner, and the process may be applied with equally good results to the salts, as well as to free $\mathrm{HNO}_{2}$. At ordinary temperatures the oxidation proceeds very slowly, but at a temperature of $40^{\circ} \mathrm{C}$. ( $104^{\circ} \mathrm{F}$ ) rapid reaction occurs. But because of the volatility of nitrous acid in acidulated solutions of its salts it is impossible to accurately estimate them by direct titration with permanganate at a raised temperature.

It is customary to add the nitrite solution to a measured volume of warm acidulated standard permanganate solution. The nitrite is 
then oxidized immediately as it comes in contact with the permanganate, and each drop added makes the permanganate lighter in color, and when complete decolorization of the permanganate is attained, the reaction is at an end.

The process in detail is as follows:

$100 \mathrm{cc}$. of $\frac{\mathrm{N}}{\mathrm{I} 0}$ potassium permanganate are measured into a flask, $5 \mathrm{cc}$. of diluted sulphuric acid ( $\mathrm{I}: 5)$ are added, and the solution warmed to $40^{\circ} \mathrm{C}$. $\left(104^{\circ} \mathrm{F}\right.$.). A solution of the nitrite (say sodium nitrite) is now prepared by dissolving I gm. of the salt in sufficient water to make $100 \mathrm{cc}$. This solution is placed in a burette and delivered slowly into the acidulated permanganate solution, with constant shaking, and reducing the flow to drops towards the end of the titration, until the permanganate is completely decolorized. We will assume that $38 \mathrm{cc}$. of the $\mathrm{NaNO}_{2}$ solution were used.

${ }_{5} \mathrm{NaNO}_{2}+{ }_{2} \mathrm{KMnO}_{4}+{ }_{3} \mathrm{H}_{2} \mathrm{SO}_{4}=5 \mathrm{NaNO}_{3}+\mathrm{K}_{2} \mathrm{SO}_{4}+2 \mathrm{MnSO}_{4}+{ }_{3} \mathrm{H}_{2} \mathrm{O}$ $\frac{100)}{3.4285} \mathrm{gms}$. $\frac{313.96}{3.1396} \mathrm{gms} .=1000 \mathrm{cc} \frac{\mathrm{N}}{10}$ permanganate.

Thus I00 cc. of $\frac{\mathrm{N}}{\mathrm{IO}}$ permanganate represent $0.34285 \mathrm{gm}$. of pure $\mathrm{NaNO}_{2}$. Therefore if $38 \mathrm{cc}$. of the sodium nitrite solution decolorized I00 cc. of $\frac{\mathrm{N}}{\mathrm{IO}}$ permanganate, the $38 \mathrm{cc}$. must contain $0.34285 \mathrm{gm}$. of $\mathrm{NaNO}_{2}$. If $38 \mathrm{cc}$. contains 0.34285 , 100 cc. contains $0.902 \mathrm{gm}$.

$$
38: 0.34285 .: 100: x ; \quad x=0.902 \text { gm., }
$$

and since I gm. of salt was dissolved in roo cc. of solution the percentage of pure $\mathrm{NaNO}_{2}$ is 90.2 per cent.

Nitrites may also be estimated by adding an excess of acidulated permanganate solution, warming the mixture, and retitrating with standard oxalic acid. This is the method which is directed in the U. S. P. for the assay of sodium nitrite.

The U. S. P. method is as follows:

To $30 \mathrm{cc}$. of $\frac{\mathrm{N}}{10}$ potassium permanganate add water to make about $150 \mathrm{cc}$. of solution, then add $5 \mathrm{cc}$. of sulphuric acid and ro cc. of a solution ( $\mathrm{rgm}$. in $100 \mathrm{cr}$.) of the sodium nitrite to be assayed, warm the mixture to $40^{\circ} \mathrm{C}$. $\left(104^{\circ} \mathrm{F}\right.$.) and allow to stand for five 
minutes, then titrate with $\frac{\mathrm{N}}{10}$ oxalic acid solution until complete decolorization is effected. Not more than $3.75 \mathrm{cc}$. of the latter should be required.

The volume o- $\frac{\mathrm{N}}{\mathrm{IO}}$ oxalic acid solution, deducted from the $30 \mathrm{cc}$. of $\frac{\mathrm{N}}{\mathrm{IO}}$ potassium permanganate solution used gives the quantity of the latter which reacted with the one gram of sodium nitrite, each cc. of $\frac{\mathrm{N}}{\mathrm{IO}}$ permanganate represents $0.0034285 \mathrm{gm}$. of $\mathrm{NaNO}_{2}$.

$$
30 \mathrm{cc} .-3.75 \mathrm{cc} .=26.25 \mathrm{cc} \text {. }
$$

then

$$
\begin{aligned}
& 0.0034285 \times 26.25=0.08999+. \\
& \frac{0.08999 \times 100}{0.1}=89.99+\text { per cent. }
\end{aligned}
$$

\section{B. Residual Titrations}

$a$ Methons in which an Excess of Standard Permanganate is Added, and the Excess Determined by Residual Titration with Standard Oxalic Acid.

Estimation of Hypophosphorous Acid and Hypophosphites.-An accurately weighed quantity of the acid or its salt is dissolved in water, the solution is strongly acidulated with sulphuric acid, and then a measured excess of $\frac{\mathrm{N}}{\mathrm{IO}}$ potassium permanganate solution added. The mixture is boiled for fifteen minutes to hasten and facilitate the oxidation, and then the excess of the permanganate solution estimated by residual titration, with $\frac{\mathrm{N}}{\mathrm{IO}}$ oxalic acid solution.

Hypophosphorous Acid $\left(\mathrm{HPH}_{2} \mathrm{O}_{2}=65.53\right) .3$ gms. of the acid accurately weighed are diluted with water to make $60 \mathrm{cc}$. Of this solution, $6 \mathrm{cc}$. (containing $0.3 \mathrm{gm}$. of the acid) are carefully removed with a pipette, and introduced into a flask. $7 \mathrm{cc}$. of sulphuric acid are 
added, and then $50 \mathrm{cc}$. of $\frac{\mathrm{N}}{10}$ potassium permanganate solution, and the mixture boiled for fifteen minutes.

The potassium permanganate, in the presence of sulphuric acid, oxidizes the hypophosphorous acid to phosphoric, as the equation shows:

$$
\begin{aligned}
& { }_{5} \mathrm{HPH}_{2} \mathrm{O}_{2}+6 \mathrm{H}_{2} \mathrm{SO}_{4}+2\left(2 \mathrm{KMnO}_{4}\right) \\
& \frac{2) \frac{327.65}{160.82}}{1.6382} \text { gms. } \quad \frac{206 \frac{627.92}{31.96}}{3.1396} \text { gms. or } 1000 \mathrm{cc} . \frac{\mathrm{N}}{\mathrm{IO}} \mathrm{V} . \mathrm{S} \text {. } \\
& ={ }_{5} \mathrm{H}_{3} \mathrm{PO}_{4}+6 \mathrm{H}_{2} \mathrm{O}+{ }_{2} \mathrm{~K}_{2} \mathrm{SO}_{4}+4 \mathrm{MnSO}_{4} \text {. }
\end{aligned}
$$

Each cc. of the decinormal V. S. represents $0.0016382 \mathrm{gm}$. of absolute hypophosphorous acid. The quantity of permanganate solution directed to be added is slightly in excess. The excess is then ascertained by retitration with decinormal oxalic acid. Each cc. of oxalic acid required corresponds to one cc. of decinormal permanganate which has been added in excess of the quantity actually required for the oxidation.

The excess of permanganate colors the solution red, and the oxalic acid V. S. is then added until the red color just disappears, which indicates that the excess of permanganate is decomposed.

$$
\begin{aligned}
{ }_{2} \mathrm{KMnO}_{4}+5\left(\mathrm{H}_{2} \mathrm{C}_{2} \mathrm{O}_{4} \cdot 2 \mathrm{H}_{2} \mathrm{O}\right)+{ }_{3} \mathrm{H}_{2} \mathrm{SO}_{4} \\
=\mathrm{K}_{2} \mathrm{SO}_{4}+2 \mathrm{MnSO}_{4}+18 \mathrm{H}_{2} \mathrm{O}+{ }_{10 \mathrm{CO}_{2}} .
\end{aligned}
$$

If $4.7 \mathrm{cc}$. of decinormal oxalic acid are required, it indicates that $50 \mathrm{cc} .-4.7 \mathrm{cc} .=45.3 \mathrm{cc}$. of decinormal permanganate were actually used up in oxidizing the hypophosphorous acid; therefore

$$
0.0016382 \mathrm{gm} . \times 45 \cdot 3=0.0742+\mathrm{gm} \text {. }
$$

of absolute hypophosphorous acid, $\mathrm{HPH}_{2} \mathrm{O}_{2}$, or

$$
\frac{0.0742 \times 100}{.3}=24.7 \text { per cent }
$$

In the above process, boiling facilitates the oxidation, but if the acid is boiled before sufficient permanganate has been added to completely oxidize it, decomposition will take place. Hence direct titra- 
tion with the permanganate is impossible, and the residual method must be resorted to.

Calcium Hypophosphite $\left(\mathrm{Ca}\left(\mathrm{PH}_{2} \mathrm{O}_{2}\right)_{2}=\mathrm{I} 68.86\right)$. O.I gm. of the salt is dissolved in ro cc. of water, then ro cc. of sulphuric acid and $50 \mathrm{cc}$. of decinormal potassium permanganate are added, and the mixture boiled for fifteen minutes.

The excess of permanganate is then found by retitrating with decinormal oxalic-acid solution.

The reactions which take place are expressed by the following equations:

$$
{ }_{5} \mathrm{Ca}\left(\mathrm{PH}_{2} \mathrm{O}_{2}\right)_{2}+{ }_{5} \mathrm{H}_{2} \mathrm{SO}_{4}={ }_{5} \mathrm{CaSO}_{4}+{ }_{10 H P H} \mathrm{O}_{2} ; \text {. . . }
$$

$$
\begin{aligned}
\mathrm{IoHPH}_{2} \mathrm{O}_{2}+\mathrm{I}_{2} \mathrm{H}_{2} \mathrm{SO}_{4}+4\left(2 \mathrm{KMnO}_{4}\right) \\
\quad=\mathrm{IOH}_{3} \mathrm{PO}_{4}+\mathrm{I}_{2} \mathrm{H}_{2} \mathrm{O}+{ }_{4} \mathrm{~K}_{2} \mathrm{SO}_{4}+8 \mathrm{MnSO}_{4} .
\end{aligned}
$$

These two reactions may be written together thus:

$$
\begin{aligned}
& { }_{5} \mathrm{Ca}\left(\mathrm{PH}_{2} \mathrm{O}_{2}\right)_{2}+\mathrm{I}_{7} \mathrm{H}_{2} \mathrm{SO}_{4}+4\left(2 \mathrm{KMnO}_{4}\right)
\end{aligned}
$$

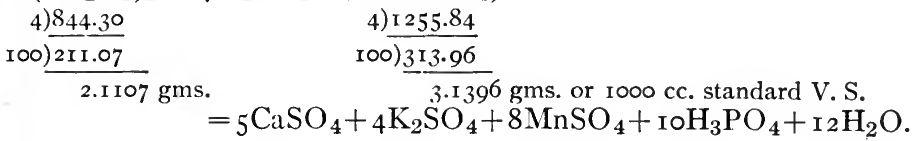

Thus each cc. of the standard permanganate represents 0.0021107 gm. of pure $\mathrm{Ca}\left(\mathrm{PH}_{2} \mathrm{O}_{2}\right)_{2}$. 50 cc. of decinormal potassium permanganate are about $3 \mathrm{cc}$. more than is necessary to oxidize 0.1 gm. of pure calcium hypophosphite. Therefore not more than $3 \mathrm{cc}$. of the standard oxalic acid solution should be required to decolorize the solution to which $50 \mathrm{cc}$. of permanganate has been added.

Then

$$
50 \mathrm{cc} .-3 \mathrm{cc} .=47 \mathrm{cc} \text {. }
$$

the quantity of $\frac{\mathrm{N}}{\mathrm{IO}}$ permanganate which was required for the oxidation of $0.1 \mathrm{gm}$. of the salt.

$$
0.0021107 \times 47=0.0992+\mathrm{gm} .
$$

the quantity of pure $\mathrm{Ca}\left(\mathrm{PH}_{2} \mathrm{O}_{2}\right)_{2}$ in the o.I gm. taken.

$$
\frac{0.099^{2} \times 100}{0.1}=99.2 \text { per cent pure. }
$$


The U. S. P. assay for sodium nitrite is a residual method coming under this head; it is described under $A, f$.

\section{b. Methons Involving a Precipitation by Oxalic Acid and the Titration of the Excess of the Latter with Standard Permanganate.}

Estimation of Soluble Calcium Salts. To a weighed quantity of the calcium salt dissolved in water, a measured excess of normal oxalic acid is added. The mixture is then made alkaline with ammonia and boiled, to facilitate the separation of the precipitate. The mixture is then cooled and diluted with water to an accurately measured volume, and after filtration an aliquot portion removed, acidulated with sulphuric acid, and carefully titrated with $\frac{\mathrm{N}}{\mathrm{IO}}$ potassium permanganate.

Example. $0.4 \mathrm{gm}$. of calcium chlorid is dissolved in water, Io cc. of normal oxalic acid added, the mixture made alkaline with ammonia water, and boiled for a few minutes. It is then filtered, the residue and filter washed with water, and after cooling, the combined filtrate and washings are diluted to make roo cc.

Of this solution $50 \mathrm{cc}$. are taken for analysis (representing $0.2 \mathrm{gm}$. of the salt), acidulated with sulphuric acid, and then titrated with $\frac{\mathrm{N}}{10}$ potassium permanganate to a faint rose tint.

The $50 \mathrm{cc}$. of solution represent $5 \mathrm{cc}$. of normal oxalic acid which is equivalent to $50 \mathrm{cc}$. of decinormal oxalic acid, so that whatever number of cc. of decinormal permanganate solution is required in the titration, that quantity is to be deducted from $50 \mathrm{cc}$. and the difference multiplied by the $\frac{N}{10}$ factor for calcium chlorid to find the quantity of pure $\mathrm{CaCl}_{2}$ present in $0.2 \mathrm{gm}$.

If $\mathrm{I}_{4} \mathrm{cc}$ of $\frac{\mathrm{N}}{\mathrm{IO}}$ permanganate are employed, then $\mathrm{I}_{4}$ from $50 \mathrm{cc}$. leaves $36 \mathrm{cc}$, the quantity of decinormal oxalic acid solution which combined with the $0.2 \mathrm{gm}$. of calcium chlorid. Then

$$
0.005508 \mathrm{gm} . \times 36=0.198288 \mathrm{gm} .,
$$

the quantity of pure $\mathrm{CaCl}_{2}$ present in the $0.2 \mathrm{gm}$. or 99.144 per cent. 
Calcium salts to be estimated by this method must be tolerably pure, and free at least from impurities which would react with oxalic acid or which would reduce permanganate.

Many of the less soluble calcium salts may be estimated by this method, but they must be subjected to longer treatment with the oxalic acid.

Gold and lead salts may also be estimated by the same method.

c. Methods Involving a Reduction by Means of Oxalic Acid, and Retitration of the Excess of the Latter with Potassium Permanganate.

Estimation of Manganese Dioxid $\left(\mathrm{MnO}_{2}\right)$. The estimation of manganese dioxid depends upon the fact that when it is boiled with oxalic acid in the presence of sulphuric acid a definite reaction takes place, as the equation shows:

$$
\mathrm{MnO}_{2}+\mathrm{H}_{2} \mathrm{C}_{2} \mathrm{O}_{4}+\mathrm{H}_{2} \mathrm{SO}_{4}=\mathrm{MnSO}_{4}+{ }_{2} \mathrm{CO}_{2}+{ }_{2} \mathrm{H}_{2} \mathrm{O} \text {. }
$$

In the estimation a measured excess of oxalic acid solution is added, together with some sulphuric acid, and the mixture heated until solution is complete.

The excess of oxalic acid is then found by retitration with standard permanganate solution. It is well to use a normal oxalic acid solution and a decinormal permanganate solution.

$0.5 \mathrm{gm}$. of the dioxid is a convenient quantity to operate upon. Each cc. of decinormal solution represents $0.0043 \mathrm{I} 8 \mathrm{gm}$. of $\mathrm{MnO}_{2}$.

Example. $0.5 \mathrm{gm}$. of $\mathrm{MnO}_{2}$ is treated with sulphuric acid and Io cc. of normal oxalic acid solution, which is equivalent to roo cc. of decinormal oxalic acid solution, the mixture treated, as described above, and upon retitrating $25 \mathrm{cc}$. of decinormal permanganate are required. Thus

$$
100 \mathrm{cc} .-25 \mathrm{cc} .=75 \mathrm{cc} \text {. }
$$

of $\frac{\mathrm{N}}{\mathrm{IO}}$ oxalic acid went into reaction with the $\mathrm{MnO}_{2}$.

Then

$$
\begin{aligned}
& 75 \times 0.004318=0.32385 \text { gm.; } \\
& \frac{0.32385 \times 100}{0.5}=64.77 \text { per cent. }
\end{aligned}
$$




\section{d. Methods Involving a Reduction by Means of a Standardized Solution of a Ferrous Salt, and Titration of the Remaining Unoxidized Ferrous Salt, by Permanganate.}

Estimation of Nitrates (Pelouze). This method consists in adding a weighed quantity of the nitrate to an acidulated solution of a ferrous salt of known strength, and, when reaction is complete, estimating the ferrous salt remaining, by titration with permanganate or in certain cases by means of dichromate V. S. The principle upon which the method is based is, that when nitric acid or a nitrate is brought in contact with a highly acidulated solution of a ferrous salt, the former gives off oxygen, which, passing over to the ferrous salt, oxidizes it to the ferric state, while at the same time NO is evolved. The reaction is

$$
\begin{aligned}
& \underset{\text { Nitric Acid. }}{2 \mathrm{HNO}_{3}}+\underset{\text { Ferrous Chlorid }}{6 \mathrm{HCl}}+\underset{3 \mathrm{~F}_{2} \mathrm{Cl}_{6}}{+6 \mathrm{FeCl}_{2} \mathrm{H}}+{ }_{2} \mathrm{NO} . \\
& 2 \times 62.57=125.14 \quad 6 \times 125.86=755.16 \\
& 6 \times 55.5=333.0
\end{aligned}
$$

Thus one molecular weight of nitric acid (62.57) will oxidize three molecular weights of ferrous salt, or three atoms of iron (I66.5).

Either hydrochloric or sulphuric acid may be employed. The former is preferred by most operators, and it is generally agreed that in order to attain results of sufficient precision the estimation should be done in the presence of hydrochloric acid only. In using hydrochloric acid, however, where the titrations are to be made with permanganate, certain precautions (previously mentioned) must be observed, because of the evolution of chlorin which will otherwise take place and spoil the analysis. This may be obviated by adding to the solution to be titrated an excess of manganese sulphate.

The NO which is produced during the reaction must be removed by boiling before titration with permanganate is begun. Air must be absolutely excluded during the entire process to prevent oxidation of ferrous salt by the atmospheric oxygen, as well as to prevent oxidation of $\mathrm{NO}$ to $\mathrm{HNO}_{3}$, which will oxidize more ferrous salt. The exclusion of air may be partially affected by the use on the flask of a Bunsen valve stopper (see Fig. 54), but the best method is to employ an apparatus so arranged that a constant stream of $\mathrm{CO}_{2}$ or $\mathrm{H}$ gas may be passed through it (see Fig. 57).

This method, although theoretically perfect, is in practice liable to great irregularities, and will give fairly good results only if the directions, 
especially those as to exclusion of air, are faithfully carried out. The method of Kjeldahl is to be preferred.

To conduct the process, weigh accurately $\mathrm{I} .5 \mathrm{gm}$. of flower wire * free from rust (the iron content of which is known), place it in an Erlenmeyer flask which is provided with a double perforated stopper fitted with two glass tubes, one of which should reach just to the surface of the liquid in the flask when in place, and the other, which is the outlet tube, should reach no lower than the bottom of the stopper. The first of these tubes is connected with an apparatus generating carbon dioxid or hydrogen, while the outlet tube serves to convey

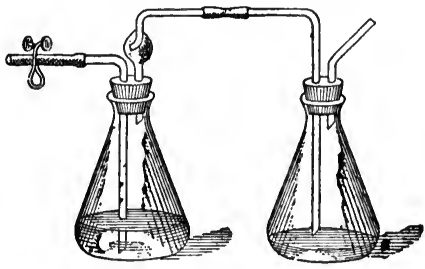

Fig. 57 . the gas into the air or into another flask containing water or an alkaline solution. 30 to $40 \mathrm{cc}$. of pure fuming hydrochloric acid are added to the iron wire in the flask, gentle heat is applied, and a stream of either $\mathrm{CO}_{2}$ or $\mathrm{H}$ passed through the flask and maintained throughout the entire process. When the iron is completely dissolved, the stopper is raised just long enough to introduce a small glass tube open at one end and containing the nitrate to be estimated. The quantity of nitrate taken must be equivalent to not more than $0.2 \mathrm{gm}$. of $\mathrm{HNO}_{3}$. The stopper is then reinserted, heat applied, and gradually increased until the reaction is complete. The free hydrochloric acid liberates nitric acid from the nitrate and oxidation of a portion of the iron is effected. The ferrous chlorid is oxidized to ferric chlorid, as the equation shows, and the solution becomes at first dark brown through the presence of NO. As the heat is increased, the dark-brown color of the solution is gradually changed to yellow, as ferric chlorid is formed, and increases in intensity until the reaction is complete, then the color remains stationary and indicates completion of oxidation. The solution is now allowed to cool, but the stream of $\mathrm{CO}_{2}$ or $\mathrm{H}$ gas is maintained. $40 \mathrm{cc}$. of a solution of manganese sulphate are now added (this is not necessary if sulphuric acid is used instead of hydrochloric), and titration with $\frac{\mathrm{N}}{\mathrm{IO}}$ potassium permanganate solution begun, in order to determine the quantity of unaltered ferrous salt

\footnotetext{
* Or fine piano-forte wire.
} 
remaining in the solution. Assuming that $89 \mathrm{cc}$. were required, the calculation is made as follows:

Since one molecule of $\mathrm{HNO}_{3}(62.57)$ reacts with three atoms of iron (166.5) the quantity of iron found to have been oxidized if multiplied by $\frac{62.57}{166.5}$ or by the factor $0.0375^{8}$ (obtained by dividing 62.57 by 166.5 ), will give the quantity of nitric acid present.

Example. I.5 gm. of iron wire, 99.6 per cent $\mathrm{Fe}=(\mathrm{I} .494 \mathrm{gm}$. of iron), is dissolved in hydrochloric acid as above described, and $0.6 \mathrm{gm}$. of potassium nitrate, $\mathrm{KNO}_{3}$ (100.43), added. After oxidation, $98 \mathrm{cc}$. of decinormal permanganate were required. Each cc. of $\frac{\mathrm{N}}{\mathrm{IO}} \mathrm{KMnO}_{4}=0.00555 \mathrm{gm}$. of $\mathrm{Fe}$.

$0.00555 \mathrm{gm} . \times 98=0.5439 \mathrm{gm}$. of oxidized iron. $\mathrm{r} .494 \mathrm{gm}$. of iron were originally taken.

Therefore,

$$
\begin{aligned}
& \mathrm{I} .4940 \\
& \frac{0.5439}{0.950 \mathrm{I}} \mathrm{gm} .=\text { the quantity of iron oxidized. }
\end{aligned}
$$

Then

$$
\frac{0.9501 \times 62.57}{166.5}=0.357 \text { gm. of } \mathrm{HNO}_{3} \text {, }
$$

which equals

$$
\frac{0.9501 \times 62.57 \times 100.43}{166.5 \times 62.57}=0.573 \mathrm{gm} . \text { of } \mathrm{KNO}_{3}
$$

or 95.5 per cent, pure.

It is usually advisable to use an $\frac{\mathrm{N}}{5}$ instead of an $\frac{\mathrm{N}}{10} \mathrm{KMnO}_{4}$ solution.

Chromic Acid and Chromates. Chromic acid oxidizes ferrous salts in the same manner as nitric acid does. The reaction is thus expressed:

$$
\underset{6 \times 55.5 \mathrm{Fe}=333}{\underset{5}{6} \mathrm{FeSO}_{4}+6 \mathrm{H}_{2} \mathrm{SO}_{4}+{ }_{2} \mathrm{CrO}_{3}=\mathrm{Cr}_{9} .68}=\mathrm{Cr}_{2}\left(\mathrm{SO}_{4}\right)_{3}+{ }_{3} \mathrm{Fe}_{2}\left(\mathrm{SO}_{4}\right)_{3}+6 \mathrm{H}_{2} \mathrm{O} .
$$

To an accurately weighed quantity of ferrous ammonium sulphate (Mohr's salt) $\mathrm{FeSO}_{4}+\left(\mathrm{NH}_{4}\right)_{2} \mathrm{SO}_{4}+6 \mathrm{H}_{2} \mathrm{O}$, (the permanganate titer 
of which is known) which is dissolved in a sufficient quantity of diluted sulphuric acid in an Erlenmeyer flask,* add a weighed quantity of the chromate or chromic acid in a concentrated aqueous solution. Warm the mixture on a water-bath, under a constant stream of carbon dioxid until the liquid assumes a clear green color. This occurs in a few minutes, and indicates complete reduction of the chromate.

Now allow the solution to cool, continuing the passage of carbon dioxid through the flask, and transfer the cold solution to a large beaker, and after diluting it to about $300 \mathrm{cc}$. and strongly acidifying it with sulphuric acid, titrate it for unoxidized ferrous salt by means of $\frac{\mathrm{N}}{\mathrm{IO}}$ potassium permanganate.

It is usually sufficient to mix the solutions cold, but it is better to employ heat after mixing. A large excess of ferrous salt is unneccessary. It is imperative to dilute the solution highly before titration, as then only can the end color point be accurately determined in the green solution. The use of an excess of sulphuric acid before titration is likewise demanded. A violet-red color marks the end-point, and unless too great a quantity of chromate be taken, or the solution be insufficiently diluted, it can be easily recognized. This method is applicable not only to free chromic acid and soluble chromates, but also to chromates which are insoluble in water. $\dagger$ It can therefore be employed for the indirect estimation of such bases as are precipitable by chromic acid, out of neutral, ammoniacal, or acetic acid solutions, as for instance lead, bismuth, and barium.

Finally, the method may be employed for the estimation of chromic oxids. The solution of the latter is treated with an excess of sodium carbonate, bromine water added, and heat applied until a clear solution results. This solution, which contains all of the chromium in the form of sodium chromate, is evaporated, the residue dissolved in dilute acetic acid and the chromium completely precipitated by means of lead acetate. The precipitated lead chromate is then treated as above.

* This flask should be provided with a stopper having two perforations through which glass tubes are passed, one of these, which serves to convey carbon dioxid gas, should reach close to the surface of the liquid, the other tube should end just below the stopper, and serve as the outlet tube. See Fig. 57.

$\dagger$ In the case of insoluble chromates the salt is shaken directly with the ferrous solution, and the mixture more highly diluted, and more strongly heated, than in the case of soluble salts. 
The calculation is made with reference to the equation, in which it is shown that one molecule 99.34 of chromic oxid $\left(\mathrm{CrO}_{3}\right)$ is equivalent to three molecules (166.5) of metallic iron. The quantity of iron oxidized, multiplied by $\frac{99.34}{166.5}$ gives the weight of chromic oxid present, and from this, its equivalent in potassium, sodium, lead, bismuth, or barium chromate is calculated.

In the case of potassium dichromate $\left(\mathrm{K}_{2} \mathrm{Cr}_{2} \mathrm{O}_{7}\right)$ one molecule (292.28) is equivalent to six atoms (333) of metallic iron. The quantity of iron oxidized is multiplied by $\frac{292.28}{333}$.

Example. To 1.5 gms. of ammonio-ferrous sulphate (containing $0.2 \mathrm{I}^{2} \mathrm{gm}$. Fe) add $0.124 \mathrm{I} \mathrm{gm}$. of $\mathrm{K}_{2} \mathrm{Cr}_{2} \mathrm{O}_{7}$ (molecular weight 292.28), and after complete oxidation, titrate the solution with $\frac{\mathrm{N}}{10} \mathrm{KMnO}_{4}$ to determine the quantity of unchanged ferrous salt. I $3 \mathrm{cc}$. are required. Each cc. represents 0.00555 gm. of Fe.

Thus, $13 \times 0.00555 \mathrm{gm} .=0.07215 \mathrm{gm}$., the quantity of iron which was not oxidized by the dichromate. This, deducted from the quantity of iron originally added $(0.2142-0.07215=0.14205 \mathrm{gm}$.), gives the quantity which was oxidized.

Then,

$$
\frac{0.14205 \times 99.34}{166.5}=0.08475+\text { gm. of } \mathrm{CrO}_{3}
$$

or

$$
\frac{0.14205 \times 292.28}{333}=0.1247 \text { gm. of } \mathrm{K}_{2} \mathrm{Cr}_{2} \mathrm{O}_{7} \text {. }
$$

Exalimple. To 1.5 gms. of ammonio-ferrous sulphate (containing $0.2142 \mathrm{gm}$. of $\mathrm{Fe}$ ) add the precipitate of barium chromate obtained from 0.249I gm. of $\mathrm{BaCl}_{2}+2 \mathrm{H}_{2} \mathrm{O}$ (molecular weight 242.52) and after complete oxidation, titrate with $\frac{\mathrm{N}}{\mathrm{JO}}$ permanganate. $7.8 \mathrm{cc}$. are consumed, thus $7.8 \times 0.00555=0.04329 \mathrm{gm}$. the quantity of unoxidized iron present. Then $0.2142-0.04329=0.17091 \mathrm{gm}$. of iron oxidized by the barium chromate.

$$
\frac{0.1709 \mathrm{r} \times 242.52}{166.5}=0.2489 \text { gm. } \mathrm{BaCl}_{2}+2 \mathrm{H}_{2} \mathrm{O} \text {. }
$$


Methods Involving the Oxidation of the Substance Analyzed by Means of a Ferric Salt, and Titration of the Resultant Ferrous Salt.

Estimation of Tin (Löwenthal). When stannous chlorid is brought in contact with ferric chlorid, it acts as a reducing agent, the ferric chlorid being reduced to ferrous, and the stannous chlorid oxidized to stannic. This reaction, which is an exact quantitative one, takes place according to the following equation:

$$
\underbrace{\mathrm{SnCl}_{2}}_{\frac{2) \text { I I } 8 . \mathrm{II}}{59.05}}+\underbrace{\mathrm{Fe}_{2} \mathrm{Cl}_{6}}_{\text {2) III }}=\mathrm{SnCl}_{4}+{ }_{25.5} \mathrm{FeCl}_{2} \text {. }
$$

Every 55.5 parts of iron reduced to the ferrous state represents 59.05 parts of tin. The quantity of ferrous iron produced is determined by titration. with $\frac{\mathrm{N}}{\mathrm{IO}}$ permanganate. Metallic tin, or any protosalt of tin, will dissolve in ferric chlorid solution, in the presence of a little hydrochloric acid and act in the manner described.

About $0.5 \mathrm{gm}$. of tin or an equivalent of stannous salt is introduced into a flask graduated at $250 \mathrm{cc}$. $5 \mathrm{cc}$. of tolerably concentrated ferric chlorid solution and a little hydrochloric acid are then added. It is well to drop into the flask a crystal of sodium carbonate, in order to produce carbon dioxid to replace the air in the flask and thus prevent oxidation by the oxygen of the air. The mixture is gently warmed until the tin is wholly dissolved, and then the solution diluted with cold, recently boiled water, to $250 \mathrm{cc}$. Of this solution $50 \mathrm{cc}$. is withdrawn by means of a pipette and titrated with $\frac{\mathrm{N}}{\mathrm{IO}}$ permanganate. Each cc. of $\frac{\mathrm{N}}{\mathrm{IO}}$ permanganate $=0.00555 \mathrm{gm} . \mathrm{Fe}=0.005905 \mathrm{gm}$. Sn.

It is always advisable when an exact assay is to be made, to make a blank experiment, using a like quantity of water and ferric chlorid solution, and deducting the quantity of permanganate solution used from the quantity required in the assay. The difference is calculated as tin.

Estimation of Copper (Fleitmann). The copper salt is first reduced either to cuprous oxid by means of glucose or to the metallic state by means of pure zinc. The reduced product is then dissolved 
in a mixture of ferric chlorid and hydrochloric acid, a little sodium carbonate added to expel the air, and the titration with $\frac{\mathrm{N}}{\mathrm{IO}}$ permanganate begun as in the preceding assay. The reaction is

or

$$
\mathrm{Cu}_{2} \mathrm{O}+\mathrm{Fe}_{2} \mathrm{Cl}_{6}+{ }_{2} \mathrm{HCl}={ }_{2} \mathrm{CuCl}_{2}+\mathrm{H}_{2} \mathrm{O}+{ }_{2} \mathrm{FeCl}_{2}
$$

$$
\underset{26_{3 . \mathrm{I}}}{\mathrm{Cu}}+\underbrace{\mathrm{Fe}_{2} \mathrm{Cl}_{6}}_{\frac{2 \mathrm{III}_{1}}{5.55}}=\mathrm{CuCl}_{2}+{ }_{2} \mathrm{FeCl}_{2}
$$

Each cc. of $\frac{\mathrm{N}}{\mathrm{jo}} \mathrm{KMnO}_{4}=0.00555 \mathrm{gm} . \dot{\mathrm{Fe}}=0.003 \mathrm{I} 55 \mathrm{gm} . \mathrm{Cu}$.

\section{VOLUMETRIC ANALYSIS BY MEANS OF POTASSIUM DICHROMATE}

In some respects the dichromate possesses advantages over permanganate:

I. It may be obtained in a pure state.

2. Its solution does not deteriorate upon standing as does that of permanganate.

3. It is not decomposed by contact with rubber as the permanganate is, and may therefore be used in Mohr's burette. Its great disdvantage, however, is that when used in the estimation of ferrous salts the end reaction can only be found by using an external indicator. The indicator which must be used is freshly prepared potassium ferricyanid T. S., a drop of which is brought in contact with a drop of the solution being tested, on a white slab, at intervals during the titration, the end of the reaction being the cessation of the production of the blue color, when the two liquids are brought together. Thus the estimation by potassium dichromate is cumbersome, and very exact results are not as easily obtained as with permaganate.

Besides ferrous salts, a great many other substances may be estimated by oxidation analysis with dichromate. Among them nitrates, sulphates, arsenous acid, barium, lead, ferric salts after reduction by stannous chlorid or an alkaline sulphite, but not after reduction by means of metallic zinc. The presence of the dissolved zinc salt interferes with the reaction of the ferricyanid indicator. Ferrous salts may be estimated in the presence of hydrochloric acid, by means of dichromate, without the precautions that apply in the case of permanganate. Chromium as chromate may be indirectly estimated; an excess of a solution of a ferrous salt being added and then the excess 
determined by dichromate. Iodids, thiosulphates, and alkalies may also be estimated by means of potassium dichromate.

\section{Preparation of Decinormal $\frac{N}{I 0}$ Potassium Dichromate} $\left(\mathrm{K}_{2} \mathrm{Cr}_{2} \mathrm{O}_{7}=292.28 ; \frac{\mathrm{N}}{\mathrm{IO}} \mathrm{V} . \mathrm{S} .=4.87 \mathrm{I} 3\right.$ gms. in $1000 \mathrm{cc}$. $)$.

$4.87 \mathrm{I} 3$ gms. of pure potassium dichromate $*$ which has been pulverized and dried at $120^{\circ} \mathrm{C}$. is dissolved in sufficient water to make I000 cc. of solution.

It will be noticed that $\frac{1}{60}$ of the molecular weight of the dichromate (expressed in grams) is taken in the preparation of $1000 \mathrm{cc}$. of this solution. The reason for this is that one molecule of potassium dichromate when treated with an acid yields three atoms of nascent oxygen which are available for oxidizing purposes, thus

$$
\mathrm{K}_{2} \mathrm{Cr}_{2} \mathrm{O}_{7}+{ }_{4} \mathrm{H}_{2} \mathrm{SO}_{4}=\mathrm{K}_{2} \mathrm{SO}_{4}+\mathrm{Cr}_{2}\left(\mathrm{SO}_{4}\right)_{3}+{ }_{4} \mathrm{H}_{2} \mathrm{O}+\mathrm{O}_{3} ;
$$

and since each atom of oxyen is equivalent to two atoms of hydrogen, one molecule of the dichromate must be equivalent to six atoms of hydrogen. Hence a normal solution of potassium dichromate, when used as an oxidizing agent, should contain one sixth of the molecular weight, expressed in grams, in rooo cc. (see definition for normal solution) and its decinormal solution $\frac{1}{60}$.

If a standard solution of potassium dichromate is to be made for use as a precipitant, as in the titration of barium, one fourth of the molecular weight is to be taken for $1000 \mathrm{cc}$. of the normal solution, as explained in Chapter III.

Standard solution of potassium dichromate is sometimes used as a neutralizing solution for estimating alkalies, phenolphthalein being used as indicator.

When used for this purpose the normal solution contains I46.14 gms. in I liter (one half the molecular weight in grams). It is then the exact equivalent of any normal acid V. S.

$$
\begin{aligned}
& { }_{2} \mathrm{KOH}+\mathrm{K}_{2} \mathrm{Cr}_{2} \mathrm{O}_{7}={ }_{2} \mathrm{~K}_{2} \mathrm{CrO}_{4}+\mathrm{H}_{2} \mathrm{O} \text {. } \\
& \text { 2) } \lcm{\mathrm{II} 2} \quad 2 \lcm{292.28} \\
& 5^{6} \text { gms. } 146.14 \text { gms., or } 1000 \text { cc. normal V.S. }
\end{aligned}
$$

Decinormal potassium dichromate may also be used in conjunction with potassium iodid and sulphuric acid for standardizing

* Potassium dichromate for use in volumetric analysis should respond to all the tests for purity given in the U.S. P., or it should be recrystallized several times and then dried. 
sodium thiosulphate. Iodin is liberated from potassium iodid in this reaction. The reaction is expressed by the equation

$$
\mathrm{K}_{2} \mathrm{Cr}_{2} \mathrm{O}_{7}+6 \mathrm{KI}+{ }_{7} \mathrm{H}_{2} \mathrm{SO}_{4}={ }_{4} \mathrm{~K}_{2} \mathrm{SO}_{4}+\mathrm{Cr}_{2}\left(\mathrm{SO}_{4}\right)_{3}+{ }_{7} \mathrm{H}_{2} \mathrm{O}+{ }_{3} \mathrm{I}_{2} \text {. }
$$

Thus one molecule of the dichromate will liberate six atoms of iodin, therefore, a normal solution should contain one sixth of the molecular weight, and a decinormal solution $\frac{1}{60}$ in $1000 \mathrm{cc}$. The solution is hence of the same strength as that which is used for oxidizing purposes. If the decinormal solution containing $\mathrm{I} 4.6 \mathrm{I} 4 \mathrm{gms}$. in I liter is used, it has the effect of a $\frac{3 \mathrm{~N}}{10}$ solution.

The decinormal solution which is used as an oxidizing agent is chemically equivalent to decinormal potassium permanganate. When used for the purpose of liberating iodin from potassium iodid, it is the equivalent of an equal volume of decinormal sodium thiosulphate.

For titrating ferrous salts the decinormal solution of dichromate is used in the following manner:

Make an aqueous solution of the ferrous salt, introduce it into a flask, and acidulate it with sulphuric or hydrochloric acid. Now add gradually from a burette the decinormal potassium dichromate until a drop taken out upon a white slab no longer shows a blue color with a drop of freshly prepared potassium ferricyanide T. S. Note the number of cc. of the standard solution used, multiply this number by the factor, and thus obtain the quantity of pure salt in the sample taken.

Ferrous salts strike a blue color with potassium ferricyanide T. S.; but as the quantity of ferrous salt gradually diminishes during the titration, the blue becomes somewhat turbid, acquiring first a green, then a gray, and lastly a brown shade. The process is finished when the greenish-blue tint has entirely disappeared.

The reaction of potassium dichromate with ferrous salts always takes place in the presence of free sulphuric or hydrochloric acid at ordinary temperatures. Nitric acid should not be used.

If it is desired to estimate ferric salts by this standard solution it is necessary to first reduce them.

This may be done by metallic magnesium, sulphurous acid, the alkali sulphites, or by stannous chlorid.

Standard potassium dichromate may be checked in the same way as standard permanganate, with pure metallic iron. 


\section{ESTIMATION OF FERROUS SALTS WITH POTASSIUM DICHROMATE.}

One molecule of potassium dichromate yields, under favorable circumstances, three atoms of 'oxygen. This is shown by the following equation:

$$
\mathrm{K}_{2} \mathrm{Cr}_{2} \mathrm{O}_{7}=\mathrm{Cr}_{2} \mathrm{O}_{3}+\mathrm{K}_{2} \mathrm{O}+\mathrm{O}_{3}
$$

Here it is seen that the three liberated atoms of oxygen combine at once with the ferrous oxid, converting it into ferric oxid:

$$
6 \mathrm{FeO}+\mathrm{O}_{3}=\mathrm{Fe}_{6} \mathrm{O}_{9} \text { or }{ }_{3} \mathrm{Fe}_{2} \mathrm{O}_{3} \text {. }
$$

In the oxidation of a ferrous salt, the reaction takes place only in the presence of an acid.

The dichromate then gives up its oxygen. Four of its oxygen atoms combine at once with the replaceable hydrogen of the accompanying acid, the other three being liberated. The three oxygen atoms thus set free are available either for direct oxidation or for combination with the hydrogen of more acid. In the latter case a corresponding quantity of acidulous radicals is set free.

$$
\mathrm{K}_{2} \mathrm{Cr}_{2} \mathrm{O}_{7}+{ }_{4} \mathrm{H}_{2} \mathrm{SO}_{4}=\mathrm{K}_{2} \mathrm{SO}_{4}+\mathrm{Cr}_{2}\left(\mathrm{SO}_{4}\right)_{3}+{ }_{4} \mathrm{H}_{2} \mathrm{O}+\mathrm{O}_{3} .
$$

In this case four of the liberated atoms of oxygen combine with eight of the atoms of hydrogen of sulphuric acid and liberate four $\mathrm{SO}_{4}$ radicals, which at once combine with the $\mathrm{K}_{2}$ and $\mathrm{Cr}_{2}$ of the dichromate. The other three atoms are set free. If seven sulphuric acid molecules are used instead of four molecules, the three free atoms of oxygen will liberate $3\left(\mathrm{SO}_{4}\right)$ :

$$
\mathrm{K}_{2} \mathrm{Cr}_{2} \mathrm{O}_{7}+{ }_{7} \mathrm{H}_{2} \mathrm{SO}_{4}=\mathrm{K}_{2} \mathrm{SO}_{4}+\mathrm{Cr}_{2}\left(\mathrm{SO}_{4}\right)_{3}+{ }_{7} \mathrm{H}_{2} \mathrm{O}+\left(\mathrm{SO}_{4}\right)_{3} .
$$

If this liberation of $3\left(\mathrm{SO}_{4}\right)$ takes place in the presence of a ferrous salt, the $3\left(\mathrm{SO}_{4}\right)$ will combine with six molecules of the ferrous salt, converting it into a ferric salt:

$$
6 \mathrm{FeSO}_{4}+{ }_{3} \mathrm{SO}_{4}=\mathrm{Fe}_{6}\left(\mathrm{SO}_{4}\right)_{9}={ }_{3} \mathrm{Fe}_{2}\left(\mathrm{SO}_{4}\right)_{3}
$$

$6 \mathrm{FeSO}_{4}+\mathrm{K}_{2} \mathrm{Cr}_{2} \mathrm{O}_{7}+{ }_{7} \mathrm{H}_{2} \mathrm{SO}_{4}=\mathrm{K}_{2} \mathrm{SO}_{4}+\mathrm{Cr}_{2}\left(\mathrm{SO}_{4}\right)_{3}+{ }_{7} \mathrm{H}_{2} \mathrm{O}+\left({ }_{3} \mathrm{Fe}_{2}\left(\mathrm{SO}_{4}\right)_{3}\right)$. 
If in the above case hydrochloric acid is used instead of sulphuric, fourteen molecules of the former must be taken to supply the necessary hydrogen.

The seven liberated atoms of oxygen must have fourteen atoms of hydrogen to combine with.

Three of these atoms of oxygen liberate six univalent or three bivalent acidulous radicals.

Therefore, since one molecule of $\mathrm{K}_{2} \mathrm{Cr}_{2} \mathrm{O}_{7}$ will give up for oxidizing purposes three atoms of oxygen, which are equivalent chemically to six atoms of hydrogen, one sixth of the molecular weight in grams of the dichromate, dissolved in sufficient water to make one liter, constitutes a normal solution, and one tenth of this quantity of $\mathrm{K}_{2} \mathrm{Cr}_{2} \mathrm{O}_{7}$ in a liter, a decinormal solution.

Thus the estimation of ferrous salts is effected by oxidizing them to ferric with an oxidizing agent of known power, the strength of the ferrous salt being determined by the quantity of the oxidizing agent required to convert it to ferric.

Saccharated Ferrous Carbonate $\left(\mathrm{FeCO}_{3}=115.05\right)$. I.1505 gms. of saccharated ferrous carbonate are dissolved in Io cc. of diluted sulphuric acid and the solution diluted with water to about I00 cc. The decinormal potassium dichromate is carefully added, until a drop of the solution taken out and brought in contact with a drop of freshly prepared solution of potassium ferricyanid ceases to give a blue color.

The number of cc. of the dichromate solution is read off and the following equations applied:

$$
\underset{690.3}{6 \mathrm{FeCO}_{3}}+6 \mathrm{H}_{2} \mathrm{SO}_{4}=\underset{905.10}{6 \mathrm{FeSO}_{4}}+6 \mathrm{H}_{2} \mathrm{O}+6 \mathrm{CO}_{2} ;
$$

then

$$
\begin{aligned}
& 6 \mathrm{FeCO}_{3} \text { or } 6 \mathrm{FeSO}_{4}+\mathrm{K}_{2} \mathrm{Cr}_{2} \mathrm{O}_{7}+{ }_{7} \mathrm{H}_{2} \mathrm{SO}_{4}= \\
& \begin{array}{lll}
6) 690.3 & 6 \lcm{905.10} & 6 \lcm{292.28} \\
1 0 \longdiv { 1 1 5 . 0 5 } & 1 0 \longdiv { 1 5 0 . 8 5 } & 10) 48.713
\end{array} \\
& \text { 11.505 gms. } 15.085 \text { gms. } 4.8713 \text { gms., or } 1000 \text { cc. } \frac{\mathrm{N}}{10} \mathrm{~K}_{2} \mathrm{Cr}_{2} \mathrm{O}_{7} \text { V. S. } \\
& \mathrm{K}_{2} \mathrm{SO}_{4}+\mathrm{Cr}_{2}\left(\mathrm{SO}_{4}\right)_{3}+{ }_{7} \mathrm{H}_{2} \mathrm{O}+{ }_{3} \mathrm{Fe}_{2}\left(\mathrm{SO}_{4}\right)_{3} \text {. }
\end{aligned}
$$

Thus each cc. of $\frac{\mathrm{N}}{\mathrm{IO}} \mathrm{K}_{2} \mathrm{Cr}_{2} \mathrm{O}_{7}$ represents 0.0 II $_{5} 05 \mathrm{gm}$. of pure ferrous carbonate or $0.00555 \mathrm{gm}$. of metallic iron. 
The U. S. P. saccharated ferrous carbonate requires about I $_{5} \mathrm{cc}$. of $\frac{\mathrm{N}}{10} \mathrm{~K}_{2} \mathrm{Cr}_{2} \mathrm{O}_{7} \mathrm{~V}$. S. for complete oxidation, corresponding to about I5 per cent.

$$
\begin{aligned}
& 0.011505 \times 15=0.172575 \mathrm{gm} . \\
& \frac{0.172575 \times 100}{\mathrm{I} . \mathrm{I} 505}=\mathrm{I} 5 \text { per cent. }
\end{aligned}
$$

If strong sulphuric acid is added to saccharated ferrous carbonate it will char the sugar, and a black mass of burnt sugar is obtained. This may be prevented by adding water first and then, slowly, the sulphuric acid.

Instead of sulphuric acid, hydrochloric acid may be used. This will not char the sugar; but the ferrous chlorid which is then formed is too readly oxidized by the air.

It has also been suggested that as hydrochloric acid so rapidly converts ordinary sugar into invert sugar as to render it easily attacked by the dichromate, it should be cautiously used, if at all. Phosphoric acid has none of these disadvantages, and may be employed with good results.

In making estimations of ferrous salts with potassium dichromate, care should be taken to avoid atmospheric oxidation. It is good practice to calculate approximately how much of the standard solution will probably be required to complete the oxidation,

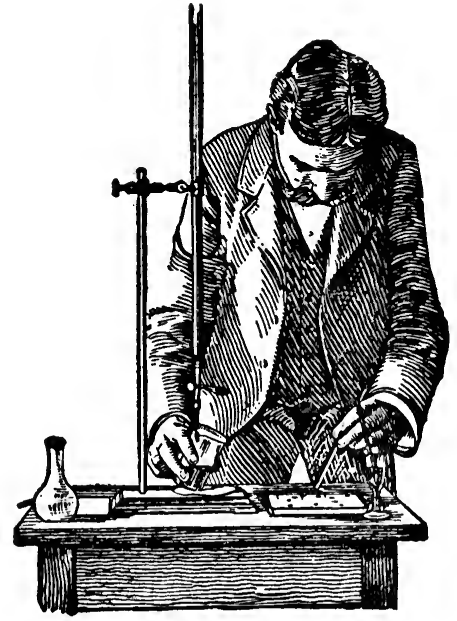

FIG. 58. and then add almost enough of the standard solution at once, instead of adding it slowly.

A white porcelain slab is then got ready, and placed alongside of the flask in which the titration is to be performed. Upon this slab is placed a number of drops of the freshly prepared solution of potassium ferricyanid, and at intervals during the titration a drop is taken from the flask on a glass rod and brought in contact with one of the drops 
on the slab. The glass rod should always be dipped in clean water after having been brought in contact with a drop of the indicator. See Fig. $5^{8}$.

When a drop of the solution ceases to give a blue color on contact with the indicator, the reaction is complete.

Ferrous Sulphate $\left(\mathrm{FeSO}_{4}+{ }_{7} \mathrm{H}_{2} \mathrm{O}=277.42\right)$. Dissolve about one gram of crystallized ferrous sulphate in a little water, add a good excess of sulphuric or hydrochloric acid, titrate with the decinormal potassium dichromate, as directed under Ferrous Carbonate, and apply the following equation:

$$
\begin{aligned}
& 6\left(\mathrm{FeSO}_{4} \cdot{ }_{7} \mathrm{H}_{2} \mathrm{O}\right)+\mathrm{K}_{2} \mathrm{Cr}_{2} \mathrm{O}_{7}+{ }_{7} \mathrm{H}_{2} \mathrm{SO}_{4}= \\
& \text { 6) } 1656.06 \quad \text { 6) } 292.28
\end{aligned}
$$

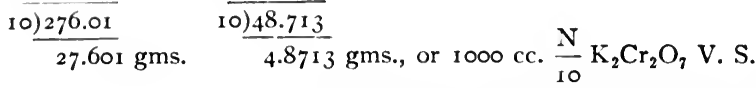

$$
\begin{aligned}
& { }_{3} \mathrm{Fe}_{2}\left(\mathrm{SO}_{4}\right)_{3}+\mathrm{K}_{2} \mathrm{SO}_{4}+\mathrm{Cr}_{2}\left(\mathrm{SO}_{4}\right)_{3}+{ }_{49} \mathrm{H}_{2} \mathrm{O} \text {. }
\end{aligned}
$$

Thus each cc. of the $\frac{\mathrm{N}}{10} \mathrm{~K}_{2} \mathrm{Cr}_{2} \mathrm{O}_{7} \mathrm{~V}$. S. represents $0.02760 \mathrm{Im}$. of crystallized ferrous sulphate or 0.015085 anhydrous. If I gm. of the salt is taken and dissolved as above, it should require about $37 \mathrm{cc}$. of the standard solution, equivalent to about 100 per cent.

\section{Anhydrous Ferrous Sulphate}

$6 \mathrm{FeSO}_{4}+\mathrm{K}_{2} \mathrm{Cr}_{2} \mathrm{O}_{7}+{ }_{7} \mathrm{H}_{2} \mathrm{SO}_{4}=3 \mathrm{Fe}_{2}\left(\mathrm{SO}_{4}\right)_{3}+\mathrm{K}_{2} \mathrm{SO}_{4}+\mathrm{Cr}_{2}\left(\mathrm{SO}_{4}\right)_{3}+{ }_{7} \mathrm{H}_{2} \mathrm{O}$. $6 \lcm{905.10}$ 6) 292.28 10) $1 5 0 . 8 5 \quad 1 0 \longdiv { 4 8 . 7 1 3 }$

${ }_{15} .085$ gms. 4.8713 gms., or 1000 cc. $\frac{\mathrm{N}}{\mathrm{IO}} \mathrm{K}_{2} \mathrm{Cr}_{2} \mathrm{O}_{7}$ V.S.

Each cc. of the standard solution represents $0.015085 \mathrm{gm}$. of real ferrous sulphate or $0.00555 \mathrm{gm}$. of metallic iron.

Dried (Exsiccated) Ferrous Sulphate of the U. S. P. has the approximate composition $\mathrm{FeSO}_{4}+{ }_{3} \mathrm{H}_{2} \mathrm{O}$.

It is tested in the same manner as the anhydrous ferrous sulphate.

Granulated Ferrous Sulphate $\left(\mathrm{FeSO}_{4}+{ }_{7} \mathrm{H}_{2} \mathrm{O}\right)$ is tested in the same manner as crystallized ferrous sulphate, with which it should correspond in strength. 
Table of Substances which may be Estimated by Means of Potassitm Permanganate or Potassium Dichromate

\begin{tabular}{|c|c|c|c|}
\hline Name. & Formula. & $\begin{array}{l}\text { Molecular } \\
\text { Weight. }\end{array}$ & $\frac{N}{10}$ Factor. \\
\hline Acid chromic .... & $\mathrm{CrO}_{3}$ & $99 \cdot 34$ & 0.0033 .113 \\
\hline “ hypophosphorous ......... & $\mathrm{HPH}_{2} \mathrm{O}_{2}$ & $65 \cdot 53$ & 0.0016382 \\
\hline " nitric $\ldots \ldots \ldots \ldots \ldots \ldots \ldots \ldots$ & $\mathrm{HNO}_{3}$ & 62.57 & 0.002085 \\
\hline “ nitrous.$\ldots \ldots \ldots \ldots \ldots \ldots$ & $\mathrm{HNO}_{2}$ & 46.69 & 0.0023345 \\
\hline " oxalic (anhydrous)..$\ldots \ldots \ldots \ldots$ & $\mathrm{H}_{2} \mathrm{C}_{2} \mathrm{O}_{4}$ & $89 \cdot 34$ & 0.004467 \\
\hline " " $\quad$ (crystallized) $\ldots \ldots \ldots \ldots \ldots$ & $\mathrm{H}_{2} \mathrm{C}_{2} \mathrm{O}_{4}+2 \mathrm{H}_{2} \mathrm{O}$ & I25. I & 0.006255 \\
\hline Barium dioxid ..... & $\mathrm{BaO}_{2}$ & I68. 16 & 0.008408 \\
\hline Calcium chlorid ...... & $\mathrm{CaCl}_{2}$ & 110.16 & 0.005508 \\
\hline 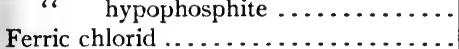 & $\mathrm{Ca}\left(\mathrm{PH}_{2} \mathrm{O}_{2}\right)_{2}$ & 168.86 & 0.0021107 \\
\hline $\begin{array}{l}\text { Ferric chlorid ........ } \\
\text { " hypophosphite. }\end{array}$ & $\mathrm{FeCl}_{3}$ & I6I.04 & 0.016104 \\
\hline $\begin{array}{l}\text { “ } \text { hypophosphite . } \\
\text { “ } \text { sulphate ...... }\end{array}$ & $\mathrm{Fe}\left(\mathrm{PH}_{2} \mathrm{O}_{2}\right)_{3}$ & 249.09 & 0.024909 \\
\hline $\begin{array}{l}\text { " sulphate ....... } \\
\text { Ferrous carbonate.... }\end{array}$ & $\mathrm{Fe}_{2}\left(\mathrm{SO}_{4}\right)_{3}$ & $397 \cdot 05$ & 0.0198525 \\
\hline $\begin{array}{c}\text { Ferrous carbonate } \ldots . . \\
\text { " oxid } \ldots . . . .\end{array}$ & $\begin{array}{l}\mathrm{FeCO}_{3} \\
\mathrm{FeO}\end{array}$ & II 5.05 & 0.011505 \\
\hline “ $\quad$ oxid ...... & $\begin{array}{l}\mathrm{FeO} \\
\mathrm{FeSO}_{4}\end{array}$ & $71 \cdot 38$ & 0.007138 \\
\hline $\begin{array}{l}\text { “ sulphate (anh } \\
\text { “، (crys }\end{array}$ & $\mathrm{FeSO}_{4}$ & 150.85 & 0.015085 \\
\hline Ferrum (metallic) $\ldots \ldots$ & $\mathrm{FeSO}_{4}+{ }_{7} \mathrm{H}_{2} \mathrm{O}$ & 276.01 & 0.027601 \\
\hline $\begin{array}{l}\text { Ferrum (metallic) } \ldots \ldots \\
\text { Hydrogen dioxid ...... }\end{array}$ & $\begin{array}{c}\mathrm{Fe}_{2} \\
\mathrm{H}_{2} \mathrm{O}_{2}\end{array}$ & III.0 & 0.00555 \\
\hline $\begin{array}{l}\text { Hydrogen dioxid .... } \\
\text { Manganese dioxid ... }\end{array}$ & $\mathrm{H}_{2} \mathrm{O}_{2}$ & $33 \cdot 76$ & 0.001688 \\
\hline 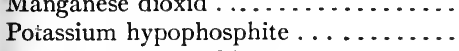 & $\mathrm{MnO}_{2}$ & $86 \cdot 3^{6}$ & 0.004318 \\
\hline $\begin{array}{l}\text { Poiassium hypophospnite .. } \\
\text { Sodium hypophosphite .... }\end{array}$ & $\mathrm{KPH}_{2} \mathrm{O}_{2}$ & I03.39 & 0.00258475 \\
\hline $\begin{array}{l}\text { Sodium hypophosphite } \\
\text { “، nitrite }\end{array}$ & $\mathrm{NaPH}_{2} \mathrm{O}_{2}$ & $87 \cdot 4 \mathrm{I}$ & 0.00218525 \\
\hline & $\mathrm{NaNO}_{2}$ & 68.57 & 0.0034285 \\
\hline
\end{tabular}

\section{ANALYSIS BY INDIRECT OXIDATION}

This method of analysis is based upon the oxidizing power of iodin.

Iodin acts upon the elements of water, forming hydriodic acid with the hydrogen, and liberating oxygen in a nascent state.

Nascent oxygen is a very active agent, and readily combines with and oxidizes many substances, such as arsenous oxid, sulphurous acid, sulphites, thiosulphates, hydrosulphuric acid, the lower oxids of antimony, and their salts.

$$
\begin{gathered}
\mathrm{As}_{2} \mathrm{O}_{3}+2 \mathrm{H}_{2} \mathrm{O}+2 \mathrm{I}_{2}=4 \mathrm{HI}+\mathrm{As}_{2} \mathrm{O}_{5} \\
\mathrm{H}_{2} \mathrm{SO}_{3}+\mathrm{H}_{2} \mathrm{O}+\mathrm{I}_{2}=2 \mathrm{HI}+\mathrm{H}_{2} \mathrm{SO}_{4} .
\end{gathered}
$$

Therefore iodin is said to be an indirect oxidizer, and may be used for the estimation of a great variety of substances with extreme accuracy. 
When iodin is brought in contact with certain oxidizable substances it is decolorized. This decolorization occurs as long as some of the oxidizable substance is present, and ceases when oxidation is complete. Hence when the yellow color of iodin shows itself in the solution being analyzed the reaction is known to be at an end. In most cases a more delicate end-reaction is obtained by using starch solution as an indicator. This gives a distinct and unmistakable blue color with the slightest excess of iodin.

In making an analysis with standard iodin solution, the substance under examination is brought into dilute solution (usually alkaline), the starch solution added, and then the iodin, in the form of a standard solution, is delivered in from a burette, stirring or shaking constantly, until a final drop colors the solution blue; a sign that a slight excess of iodin has been added.

Preparation of Decinormal Iodin $\left(\mathrm{I}=125.9 ; \frac{\mathrm{N}}{10} \quad\right.$ V. S. $=$ I2.59 gms. per liter). Dissolve I 2.59 gms. of pure * iodin in $300 \mathrm{cc}$. of distilled water containing $\mathrm{I} 8 \mathrm{gms}$. of pure potassium iodid. Then add enough water to make the solution measure at $15^{\circ} \mathrm{C}$. $\left(59^{\circ} \mathrm{F}\right.$.) exactly $1000 \mathrm{cc}$.

The solution should be kept in small glass-stoppered vials, in a dark place.

The potassium iodid used in this solution acts merely as a solvent for the iodin.

If pure iodin is used in making this solution, there is no necessity for checking (standardizing) it.

But if desired, the solution may be checked against pure arsenous acid or sodium thiosulphate. If there is any doubt as to the purity of the iodin, it is best to take a larger quantity, say 14 gms. instead of the $12.59 \mathrm{gms}$. directed above, and then dilute the resulting solution to the proper strength after standardizing.

* If pure iodin be not at hand, it may be prepared from the commercial article as follows:

Powder the iodin and heat it in a porcelain dish placed over a water-bath, stirring constantly with a glass rod for 20 minutes. Any adhering moisture, together with any cyanogen iodid, and most of the iodin bromid and iodin chlorid, is thus vaporized.

Then triturate the iodin with about 5 per cent of its weight of pure, dry potassium iodid. The iodin bromid and chlorid are thereby decomposed, potassium bromid and chlorid being formed and iodin liberated from the potassium iodid.

The mixture is then returned to the porcelain clish, covered with a clean glass funnel, and heated on a sand-bath. A pure resublimed iodin is then obtained. 


\section{Standardization of Iodin V. S. by Means of a Decinormal} Sodium Thiosulphate Solution. $25 \mathrm{cc}$. of the iodin solution are accurately measured off into a beaker, and then from a burette the $\frac{\mathrm{N}}{10}$ thiosulphate is delivered until the solution is of a pale yellow color, two or three drops of starch solution are then added, and the titration with the thiosulphate solution continued until the blue color of starch iodid is discharged.

If the iodin solution is exactly decinormal, the $25 \mathrm{cc}$. will require 25 cc. of decinormal sodium thiosulphate to exactly complete the reaction.

If on the other hand more than $25 \mathrm{cc}$. of thiosulphate solution is required, it indicates that the iodin solution is too concentrated, and must be diluted so as to correspond with the decinormal thiosulphate solution, volume for volume.

Example. Assuming that in the above titration $27 \mathrm{cc}$. of the thiosulphate solution were used, then each $25 \mathrm{cc}$. of the iodin solution must be diluted with water to make $27 \mathrm{cc}$. in order to convert the iodin solution into a strictly decinormal solution. If, however, the iodin solution is found to be weaker, as evidenced by its using up less than its own volume of $\frac{\mathrm{N}}{\mathrm{IO}}$ thiosulphate, its relative strength should be noted on the label of the container.

Thus if only $24.8 \mathrm{cc}$. of the thiosulphate solution are used up, then I cc. of the latter equals $\frac{25}{24.8}$ cc. or $1.008 \mathrm{cc}$. of the iodin solution.

I cc. of this iodin solution is equivalent to 0.992 cc. of $\frac{\mathrm{N}}{\text { IO }}$ thiosulphate, which is the same as saying I cc. $=0.992 \mathrm{cc}$. of $\frac{\mathrm{N}}{\mathrm{IO}}$ iodin, or expressed in another way, I cc. of this iodin solution contains $0.01249+$ gm. of iodin.

Such an rodin solution may be used as an empirical solution, and in any assay the quantity of it (in cubic centimeters) which is consumed is divided by 1.008 or multiplied by $\frac{24.8}{25}$ or by $0.99^{2}$, and then multiplied by the decinormal factor for the substance analyzed. Another way is to multiply the cc. of this iodin solution used by the weight of ipdin contained in each cc. and then by a fraction in which the numerator represents the quantity of the substance analyzed equal to an atom of iodin and the denominator is the atomic weight of iodin. 
Example. O.I gm. of arsenous acid consumes $20 \mathrm{cc}$. of this empirical solution. How much absolute $\mathrm{As}_{2} \mathrm{O}_{3}$ does it contain? The $\frac{\mathrm{N}}{10}$ factor for $\mathrm{As}_{2} \mathrm{O}_{3}$ is $0.0049 \mathrm{II} \mathrm{gm}$.

$$
\begin{aligned}
& \text { Method (a) } \frac{20 \times 0.0049 \mathrm{II}}{\mathrm{I} .008}=0.974 \mathrm{gm} \text {. } \\
& \text { ، } \quad \text { (b) } \frac{24.8}{25} \times 0.0049 \mathrm{II}=0.974 \mathrm{gm} \text {. } \\
& \text { “ } \quad(c) \quad 20 \times 0.992 \times 0.0049 \mathrm{II}=0.974 \mathrm{gm} . \\
& \text { ، (d) } 20 \times 0.01249 \times \frac{49.11}{125.9}=0.974 \mathrm{gm} \text {. }
\end{aligned}
$$

It is a good plan to have the factors marked on the labels. In the above case the label may be marked

$$
\times \frac{24.8}{25} \text { or } \times 0.992 \text { or } \quad \text { I cc. }=0.01249 \text { gm. iodin. }
$$

\section{Standardization of Iodin V..S. by Means of Arsenous Oxid.} $0.2 \mathrm{gm}$. of pure resublimed vitreous arsenous oxid is weighed off very carefully into a flask.

$50 \mathrm{cc}$. of water are added and then after the addition of 2 gms. or more of sodium bicarbonate, the mixture is gently warmed and shaken until the arsenous oxid is completely dissolved.*

To this solution a few drops of starch indicator are added, and then the iodin solution delivered carefully from a burette until a blue color marks the end of the reaction.

$$
\begin{aligned}
& \mathrm{As}_{2} \mathrm{O}_{3}+\mathrm{I}_{4}+{ }_{2} \mathrm{H}_{2} \mathrm{O}=\mathrm{As}_{2} \mathrm{O}_{5}+{ }_{4} \mathrm{HI} \text {. } \\
& 196.44 \quad 4 \times 125.9
\end{aligned}
$$

49.I I gms. of $\mathrm{As}_{2} \mathrm{O}_{3}=125.9$ gms. of iodin;

$$
4.9 \mathrm{I} 1 \text { “ “ } \mathrm{As}_{2} \mathrm{O}_{3}=\mathrm{I} 2.59 \text { “" “" “ } 1000 \mathrm{cc} \cdot \frac{\mathrm{N}}{\mathrm{IO}} \text { V.S. }
$$

$0.2 \mathrm{gm}$. of $\mathrm{As}_{2} \mathrm{O}_{3}$ will require

$$
\frac{1000 \times 0.2}{4.91 \mathrm{I}}=40.72 \mathrm{cc} \text {. of a true } \frac{\mathrm{N}}{\mathrm{IO}} \text { iodin V.S. }
$$

* Arsenous oxid is much more readily soluble in alkali hydroxid, than in carbonated alkalies, therefore the following method of making the solution is preferred: $0.2 \mathrm{gm}$. of arsenous oxid are dissolved in a small quantity of boiling water with the aid of potassium hydroxid (free from sulphur), the solution is th ien acidified with hydrochloric acid, and then again made alkaline by the addition of sodium bicarbonate. The latter must be added in considerable excess byeing careful, however, to avoid loss of solution during effervescence. 
Assuming that in the above titration $37.4 \mathrm{cc}$. of the iodin solution were used, then the iodin solution is too concentrated and must be diluted so that each $37.4 \mathrm{cc}$. will be made up to $40.72 \mathrm{cc}$.

After diluting in this way a new trial should be made.

It is a good plan to make a decinormal solution of the arsenous oxid by dissolving 4.9 I I gms. of the pure oxid and $30 \mathrm{gms}$. of sodium bicarbonate in sufficient water to make $1000 \mathrm{cc}$. at $15^{\circ} \mathrm{C}$. and to titrate this with the iodin solution. $25 \mathrm{cc}$. of this solution should require for complete oxidation, exactly $25 \mathrm{cc}$. of the iodin solution, if the latter is strictly of decinormal strength.

The Starch Solution. This solution, which is used as an indicator in iodometric determinations, is made as follows:

I gm. of starch (potato, arrowroot, or corn starch), is triturated with Io cc. of cold water, until a smooth mixture is obtained, then sufficient boiling water is added, with constant stirring, to make $200 \mathrm{cc}$. of a thin, translucent fluid. If the solution is not translucent it should be boiled for about three minutes, then allowed to cool, and filtered. This solution does not keep very long, in fact, it becomes useless after standing one day, therefore it should be freshly prepared when required.

This indicator is very sensitive to iodin-it will detect one part of iodin in 3,500,000. If the solution is not clear, or contains flocks of insoluble starch, and the characteristic leautiful blue color is not obtained with iodin; instead, a greenish or brownish color is produced, and the insoluble particles are even colored black and are decolorized with difficulty.

The blue color which starch gives with iodin constitutes a very delicate indication of the slightest excess of iodin. This color is usually regarded as being due to the formation of a compound of starch and iodin, called iodid of starch. It is a compound of very unstable character and of doubtful composition.

Sodium thiosulphate behaves towards iodid of starch exactly as it does towards free iodin-it takes up the iodin and thus discharges the blue color.

Iodid of starch dissociates upon heating, but reunites upon cooling, hence it is advisable to avoid heat in estimations where starch is used as an indicator.

In order to prevent the deterioration of this solution a few drops of chloroform may be added; this will preserve it for a long time. Oil of cassia is also recommended as a preservative. Moerk adds 2 cc. 
of the oil to a liter of the cooled starch solution. Zinc chlorid or iodid added to the boiling starch solution will prevent its decomposition for a long time. A starch solution so made, however, should not be used in titrations of sulphids, because zinc reacts with sulphids.

In the case of solutions containing carbonates, the precipitate of zinc carbonate is so small in amount that it does not interfere in the least with the recognition of the end-reaction tint. Mercuric iodid is also a very valuable preservative.

o.or gm. of mercuric iodid in a liter of the starch solution is quite sufficient. A very satisfactory indicator is the commercial soluble starch which is made by heating potato starch with glycerin and precipitating the starch by repeated treatment with alcohol. This starch dissolves readily in hot water, forming a clear solution, which gives a very delicate reaction with iodin. It is best preserved under alcohol, the latter being removed by filtration and evaporation, when the starch is wanted for making a solution.

In making starch solution for use as an indicator, long continued boiling should be avoided, as this converts some of the starch into dextrin.

On the Use of Sodium Bicarbonate in Titrations with Iodin. In these titrations an excess of alkali is necessary in order to neutralize the hydriodic acid formed. See equations on page 185 .

Alkali hydroxids or carbonates cannot be used for this purpose, because they react with free iodin or even with starch iodid. Bicarbonates ordinarily have no such action, and therefore sodium bicarbonate is usually directed to be added in excess to the solution to be titrated with iodin.

It is well known that sodium hydroxid solution reacts with free iodin, with formation of hypoiodite and iodid.

$$
{ }_{2} \mathrm{NaOH}+\mathrm{I}_{2}=\mathrm{NaIO}+\mathrm{NaI}+\mathrm{H}_{2} \mathrm{O},
$$

the hypoiodite quickly forming iodate.

$$
3 \mathrm{NaIO}=2 \mathrm{NaI}+\mathrm{NaIO}_{3} .
$$

It is also now a recognized fact that sodium carbonate is partly hydrolyzed when in solution, with formation of some sodium hydroxid as per equation,

$$
\mathrm{Na}_{2} \mathrm{CO}_{3}+\mathrm{H}_{2} \mathrm{O}=\mathrm{NaOH}+\mathrm{NaHCO}_{3} .
$$


It therefore reacts in much the same way with iodin as the hydroxid, though to a less extent.

On the other hand, it is generally supposed that bicarbonate of soda is without effect on iodin, and when, in iodometric estimations, addition of sodium bicarbonate is indicated, little attention is given to amount added as long as it be in excess.

The experiments of W. A. Puckner, Proc. A. Ph. A., I904, 408, prove that we are entirely wrong in the supposition that sodium bicarbonate has no effect upon iodin. He showed that when using I to 2 gms. of the bicarbonate, an error of $\mathrm{r} .5$ to $4.5 \mathrm{cc}$. of decinormal iodin may be introduced even when the sodium bicarbonate used is of exceptional purity, and especially proven to be free from carbonate, sulphite or thiosulphate. He shows that when sodium bicarbonate is added to a decinormal iodin solution, residual titration with sodium thiosulphate will show a considerable loss of free iodin, which went into combination in some form or other (probably iodid) and that the quantity so lost is proportional to (I) the mass of sodium bicarbonate; (2) the time of the interaction (the reaction is slow); (3) the concentration of the solution; (4) the temperature, and (5) the size of the flask in which reaction occurs. These phenomena are due to the fact that sodium bicarbonate when dissolved in water undergoes hydrolysis, thus

$$
2 \mathrm{NaHCO}_{3}=\mathrm{Na}_{2} \mathrm{CO}_{3}+\mathrm{H}_{2} \mathrm{CO}_{3} \text { or }\left(\mathrm{H}_{2} \mathrm{O}+\mathrm{CO}_{2}\right) \text {. }
$$

This breaking up of the $\mathrm{NaHCO}_{3}$ into $\mathrm{Na}_{2} \mathrm{CO}_{3}$ and $\mathrm{H}_{2} \mathrm{CO}_{3}$, and the latter into $\mathrm{H}_{2} \mathrm{O}$ and $\mathrm{CO}_{2}$, continues until the pressure of the $\mathrm{CO}_{2}$ above is equal to the pressure of the gas in the solution, i.e., until equilibrium has been reached. In concentrated solutions of $\mathrm{NaHCO}_{3}$ the amount hydrolyzed is much greater than in dilute solutions. An elevation of temperature materially increases the absorption of iodin.

Less iodin is lost when smaller flasks are used, provided the glass stopper completely shuts off communication with the atmosphere. The $\mathrm{CO}_{2}$ will escape from the solution until its pressure in the solution is equal to that of the gas above. Thus, since a larger volume of air is contained in a larger flask, more $\mathrm{CO}_{2}$ passes from the liquid before equilibrium is established, hence more $\mathrm{NaHCO}_{3}$ is decomposed, and more iodin in consequence absorbed.*

* For further study of equilibrium, see the work of Dr. H. N. McCoy, Am. Ch. J., vol. XXIV, 437 . 
Reasoning from the above observations it may be said that: 1. Though sufficient sodium bicarbonate be used to more than neutralize the hydriodic acid formed, the solution titrated should be well diluted. 2. That the titration should be done cold. 3. That the titration should be done in small stoppered flasks, and 4. It should be done quickly.

\section{ESTIMATION OF ARSENOUS COMPOUNDS}

These compounds are estimated by means of iodin in a manner similar to that described under standardization of iodin solution by means of arsenous oxid. The method is as follows:

Arsenous Oxid, Arsenous Acid, Arsenous Anhydrid, Arsenic Trioxid $\left(\mathrm{As}_{2} \mathrm{O}_{3}=196.44\right)$. When arsenous acid is brought in contact with iodin in the presence of water and an alkali, it is oxidized into arsenic acid and the iodin is decolorized. The reaction is:

$$
\begin{aligned}
\mathrm{As}_{2} \mathrm{O}_{3}+{ }_{2} \mathrm{I}_{2}+{ }_{2} \mathrm{H}_{2} \mathrm{O} & =\mathrm{As}_{2} \mathrm{O}_{5}+4 \mathrm{HI} \\
\mathrm{NaHCO}_{3}+\mathrm{HI} & =\mathrm{NaI}+\mathrm{H}_{2} \mathrm{O}+\mathrm{CO}_{2} .
\end{aligned}
$$

The alkali should be in sufficient quantity to combine with the hydriodic acid formed, and must be in the form of potassium or sodium bicarbonate.

The hydroxids or carbonates should not be used. Starch solution is used as the indicator, a blue color being formed as soon as the arsenous acid is entirely oxidized into arsenic acid.

o.1 gm. of arsenous acid is accurately weighed and dissolved, together with about I gm. of sodium bicarbonate, in $20 \mathrm{cc}$. of water heated to boiling. Allow the liquid to cool, add a few drops of starch T. S., and allow the decinormal iodin to flow in, shaking or stirring the mixture constantly, until a permanent blue color is produced. The following equation illustrates the reaction:

$$
\begin{aligned}
& \mathrm{As}_{2} \mathrm{O}_{3}+{ }_{2} \mathrm{H}_{2} \mathrm{O}+{ }_{2} \mathrm{I}_{2}=4 \mathrm{HI}+\mathrm{As}_{2} \mathrm{O}_{5} \text {. }
\end{aligned}
$$

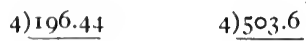

$$
\begin{aligned}
& \text { 10) }+9.11 \quad \text { 10) } \lcm{125.9} \\
& 4.91 \mathrm{I} \text { gms. } \frac{125.9}{12.59} \text { gms. or } 1000 \mathrm{cc} \frac{\mathrm{N}}{\mathrm{IO}} \text { I V.S. }
\end{aligned}
$$

Thus each cc. of $\frac{\mathrm{N}}{10}$ I V.S. represents 0.0049 I gm. of pure $\mathrm{As}_{2} \mathrm{O}_{3}$. 
If $20 \mathrm{cc}$. are consumed in the test, then

$$
\begin{aligned}
& 0.0049 \text { I } \times 20=0.09822 \mathrm{gm} . \\
& \frac{0.09822 \times 100}{0.1}=98.22 \text { per cent. }
\end{aligned}
$$

The U. S. P. requirement is 99.8 of $\mathrm{As}_{2} \mathrm{O}_{3}$. The starch T. S. is not used in the U. S. P. process, and the end of the reaction is known by the iodin being no longer decolorized. But with starch the indication is exceedingly delicate, and it should always be used.

Liquor Acidi Arsenosi, U. S. P. Measure accurately ro cc. of the solution, add to it I gm. of sodium bicarbonate, and boil for a few minutes. Then allow the liquid to cool, and dilute it to $50 \mathrm{cc}$. with water. A little starch T. S. is then added and the decinormal iodin run in from a burette, until a final drop produces the blue color of starch iodid.

Each cc. of $\frac{\mathrm{N}}{\mathrm{IO}}$ I V. S. represents 0.0049 I I gm. of $\mathrm{As}_{2} \mathrm{O}_{3}$. (See Estimation of Arsenous Acid.)

The U.S.P. requirement is that $24.6 \mathrm{gms}$. of the liquor acidi arsenosi, when treated as above, will consume $50 \mathrm{cc}$. of decinormal iodin. Two grams of the bicarbonate are used.

$$
\begin{aligned}
& 0.0049 \mathrm{II} \times 50=0.24555 \mathrm{gm} . \\
& \frac{0.24555 \times 100}{24.6}=\mathrm{I} \text { per cent. }
\end{aligned}
$$

Liquor Potassii Arsenitis, U. S. P. (Fowler's Solution). The process is similar to the foregoing.

24.6 gms. of the solution are diluted with water to roo cc.; the mixture is very slightly acidified with diluted hydrochloric acid, and then made alkaline with 2 gms. of sodium bicarbonate. It should require not less than $50 \mathrm{cc}$. of $\frac{\mathrm{N}}{\mathrm{IO}} \mathrm{I}$ V. S., corresponding to I per cent of $\mathrm{As}_{2} \mathrm{O}_{3}$. The use of hydrochloric acid, in this process, is to neutralize any potassium hydroxid which may have been formed through hydrolysis of the potassium bicarbonate contained in the preparation, and at the same time to decolorize the solution in order to better see the yellow color of the end reaction No starch is used in the official process. 


\section{The Direct Percentage Assay of Arsenous Compounds.} A quantity of arsenous acid is taken, which is equal to the weight of pure $\mathrm{As}_{2} \mathrm{O}_{3}$, oxidized by 100 cc. of decinormal iodin, i.e., 0.49 I I gm.

If 0.491 I $\mathrm{gm}$. of the sample be taken then each cc. of $\frac{\mathrm{N}}{\mathrm{IO}} \mathrm{I} \mathrm{V} . \mathrm{S}$. will represent $\frac{1}{100}$ of this quantity or I per cent of pure $\mathrm{As}_{2} \mathrm{O}_{3}$. In the case of weak solutions of arsenic, as liquor acidi arsenosi, liquor potassi arsenitis, etc., which contain only I per cent of arsenous acid. A much larger quantity should be taken for analysis, otherwise the quantity of standard iodin solution used will be so small as to diminish the accuracy of the test.

Thus, if only 0.49 I I gm. of either of the above solutions be taken, no more than I cc. of the standard solution would be required. It is better to take enough of the preparation to use up 30 to $50 \mathrm{cc}$. of standard solution. As above seen the U. S. P. directs 24.6 gms. to be taken; this should use up about $50 \mathrm{cc}$. of $\frac{\mathrm{N}}{\mathrm{IO}}$ iodin V. S.

Arsenous Iodid $\left(\mathrm{AsI}_{3}=45^{2.10}\right)$.

$$
\begin{aligned}
& { }_{2} \mathrm{AsI}_{3}+{ }_{5} \mathrm{H}_{2} \mathrm{O}+{ }_{2} \mathrm{I}_{2}=\mathrm{IOHI}+\mathrm{As}_{2} \mathrm{O}_{5} \text {. } \\
& \text { 4) } 904.20 \quad 4 \lcm{503.6} \\
& \text { I0 } \lcm{226.05} \quad \text { 10 } \lcm{125.9}
\end{aligned}
$$

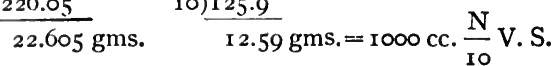

Thus each cc. of $\frac{\mathrm{N}}{\mathrm{IO}}$ iodin V. S. $=0.022605 \mathrm{gm}$. of arsenous iodid or $0.00372 \mathrm{gm}$. of metallic arsenic.

The U. S. P. directs to take $0.5 \mathrm{gm}$. of arsenous iodid and $2 \mathrm{gms}$. of sodium bicarbonate and dissolve them in $50 \mathrm{cc}$. of water. The titration is conducted without the use of starch, the end point being the production of a slight yellow tint. $2 \mathrm{I} .9 \mathrm{cc}$. of $\frac{\mathrm{N}}{\mathrm{IO}}$ iodin should be consumed.

$$
\begin{aligned}
& 0.022605 \times 2 \mathrm{I} .9=0.4950495 \mathrm{gm} . \\
& \frac{0.4950495 \times 100}{0.5}=99 \text { per cent }
\end{aligned}
$$

of which 82.7 per cent should be iodin and 16.3 per cent metallic arsenic. 


\section{ESTIMATION OF ANTIMONY COMPOUNDS}

Antimonous oxid $\left(\mathrm{Sb}_{2} \mathrm{O}_{3}\right)$ or any of its compounds may be accurately estimated by means of iodin, in a manner similar to that described for the estimation of arsenous oxid. The antimonous oxid being oxidized to antimonic oxid, as per equation.

$$
\mathrm{Sb}_{2} \mathrm{O}_{3}+{ }_{2} \mathrm{H}_{2} \mathrm{O}+{ }_{2} \mathrm{I}_{2}={ }_{4} \mathrm{HI}+\mathrm{Sb}_{2} \mathrm{O}_{5} \text {. }
$$

The antimonous oxid is dissolved and kept in solution by the aid of tartaric acid, and then after the addition of an excess of sodiumbicarbonate, the solution is titrated with $\frac{\mathrm{N}}{\mathrm{IO}}$ iodin, using starch as an indicator. Accurate results can only be obtained if the solution is sufficiently alkaline to neutralize the hydriodic acid formed during the reaction. The titration should be conducted without delay after the addition of the bicarbonate, otherwise a precipitate of antimonous hydrate will be formed, upon which iodin has little effect. The antimony must be in solution to be properly attacked by the iodin.

To o.r gm. of antimonous oxid $20 \mathrm{cc}$. of water are added and the mixture heated to boiling; to this tartaric acid is added in small portions at a time until the oxid is completely dissolved. The solution is then neutralized by means of sodium carbonate, and sufficient of a saturated solution of sodium bicarbonate is added to make the solution distinctly alkaline (about Io cc. is required for o.r gm. of the antimonous oxid). The mixture is now ready for titration with standard iodin solution. This should be done immediately. The appearance of a permanent blue color marks the end point, starch being used as indicator.

$$
\begin{aligned}
& \mathrm{Sb}_{2} \mathrm{O}_{3}+2 \mathrm{H}_{2} \mathrm{O}+2 \mathrm{I}_{2}=4 \mathrm{HI}+\mathrm{Sb}_{2} \mathrm{O}_{5} . \\
& \begin{array}{l}
\text { 4) } 286.24 \\
\text { I0) } \frac{7 \mathrm{I} .548}{7.1548} \text { gms. }
\end{array} \frac{4) \frac{503.6}{\mathrm{I} 25.9}}{\mathrm{I} 2.59} \text { gms. or } 1000 \text { cc. } \frac{\mathrm{N}}{\text { IO }} \mathrm{V} . \mathrm{S} \text {. }
\end{aligned}
$$

I cc. of $\frac{\mathrm{N}}{\mathrm{IO}}$ iodin represent $0.007 \times 548 \mathrm{gm}$. of $\mathrm{Sb}_{2} \mathrm{O}_{3}$.

The solution of the oxid may be made by means of hydrochloric acid, and after adding a portion of tartaric and diluting with water, sodium bicarbonate is added and the titration conducted as above. 
Other compounds of antimony may be estimated in the same way. Antimonic compounds are reduced to antimonous sulphid $\left(\mathrm{Sb}_{2} \mathrm{~S}_{3}\right)$ by precipitating with hydrogen sulphid, and after thoroughly washing the precipitate, dissolving it in hydrochloric acid; thus a solution of antimonous chlorid is obtained from which all traces of hydrogen sulphid are expelled by boiling. This solution is diluted with water, tartaric acid added, and finally, after making alkaline with sodium bicarbonate, titrated with the standard iodin solution as above described.

Antimony and Potassium Tartrate (Tartar Emetic) $\left(2\left(\mathrm{~K}[\mathrm{SbO}] \mathrm{C}_{4} \mathrm{H}_{4} \mathrm{O}_{6}\right)+\mathrm{H}_{2} \mathrm{O}=659.80\right)$. This is the only antimonial compound for which the U. S. P. gives direction for a volumetric assay.

The U. S. P. directions are: I gm. of the salt is dissolved in sufficient water to make Ioo cc. $32.99 \mathrm{cc}$. of this solution, representing $0.3299 \mathrm{gm}$. of the salt, are taken for assay. $20 \mathrm{cc}$. of a cold saturated solution of sodium bicarbonate are added, then a little starch T. S., and the mixture titrated with $\frac{\mathrm{N}}{\mathrm{IO}}$ iodin until a permanent blue color appears. Not less than $19.9 \mathrm{cc}$. of the iodin solution should be required.

The calculation is as follows:

$$
\begin{aligned}
& { }_{2} \mathrm{~K}(\mathrm{SbO}) \mathrm{C}_{4} \mathrm{H}_{4} \mathrm{O}_{6}+\mathrm{H}_{2} \mathrm{O}+{ }_{2} \mathrm{I}_{2}+{ }_{3} \mathrm{H}_{2} \mathrm{O}={ }_{4} \mathrm{HI}+{ }_{2} \mathrm{KHC}_{4} \mathrm{H}_{4} \mathrm{O}_{6}+{ }_{2} \mathrm{HSbO}_{3} \\
& \text { 4) } 659.80 \\
& \text { 10) } \lcm{164.95} \\
& \text { 4) } 503.6 \\
& 16.495 \mathrm{gms} \text {. } \\
& \text { 10) } \lcm{125.9} \\
& \frac{125.9}{\mathrm{I} 2.59} \text { gms. }=1000 \mathrm{cc} . \frac{\mathrm{N}}{\mathrm{IO}} \text { V.S. }
\end{aligned}
$$

I cc. of $\frac{\mathrm{N}}{\mathrm{IO}}$ iodin represents $0.0 \mathrm{I} 6495 \mathrm{gm}$. of ${ }_{2} \mathrm{~K}(\mathrm{SbO}) \mathrm{C}_{4} \mathrm{H}_{4} \mathrm{O}_{6}$ $+\mathrm{H}_{2} \mathrm{O}$ (crystallized tartar emetic).

$\mathrm{K}(\mathrm{SbO}) \mathrm{C}_{4} \mathrm{H}_{4} \mathrm{O}_{6}$ (anhydrous tartar emetic $)=320.96$.

$$
\frac{2) \frac{320.9^{6}}{10)}}{160.4^{8}}
$$

Thus I cc. of $\frac{\mathrm{N}}{\mathrm{IO}}$ iodin represents $0.016048 \mathrm{gm}$. of anhydrous tartar emetic. 
If $19.9 \mathrm{cc}$. of $\frac{\mathrm{N}}{10}$ iodin are consumed, then

$$
0.016495 \mathrm{gm} . \times 19.9=0.3282405 \mathrm{gm} .
$$

$$
\frac{0.3282405 \times 100}{0.3299}=99.5 \text { per cent of the crystallized salt; }
$$

$0.016048 \times 19.9=0.315355^{2} \mathrm{gm}$. or 95.6 per cent of the anhydrous salt.

\section{ESTIMATION OF SULPHUROUS ACID AND SULPHITES}

These substances may be accurately estimated by means of a standard solution of iodin. When sulphurous acid or one of its salts is brought in contact with iodin, a complete oxidation takes place. The sulphurous acid is oxidized to sulphuric acid and the sulphite to a sulphate, as the equations show.

$$
\begin{gathered}
\mathrm{H}_{2} \mathrm{SO}_{3}+\mathrm{H}_{2} \mathrm{O}+\mathrm{I}_{2}={ }_{2} \mathrm{HI}+\mathrm{H}_{2} \mathrm{SO}_{4}, \\
\mathrm{Na}_{2} \mathrm{SO}_{3}+\mathrm{H}_{2} \mathrm{O}+\mathrm{I}_{2}=2 \mathrm{HI}+\mathrm{Na}_{2} \mathrm{SO}_{4}, \\
\mathrm{NaHSO}_{3}+\mathrm{H}_{2} \mathrm{O}+\mathrm{I}_{2}={ }_{2} \mathrm{HI}+\mathrm{NaHSO}_{4} .
\end{gathered}
$$

There are two methods which may be employed. In one method the substance is brought into solution in water, an excess of sodium bicarbonate is added, and then the standard iodin solution is run in until a faint yellow color of free iodin marks the end-reaction. If starch solution is used as indicator the end-point is the production of a blue color. The other method is that of Giles and Shearer, who, in a very valuable series of experiments detailed in the J. S. C. I., III, I97, and IV, 303, suggest the following modification:

The weighed sulphurous acid or the sulphite (in fine powder) is added to an accurately measured excess of $\frac{\mathrm{N}}{\text { ro }}$ iodin, without diluting with water. After the mixture has been allowed to stand for about one hour, with frequent shaking, the oxidation is complete, and the excess of iodin is ascertained by titrating back with $\frac{\mathrm{N}}{\mathrm{FO}}$ sodium thiosulphate. 
The quantity of the latter deducted from the quantity of $\frac{\mathrm{N}}{\mathrm{IO}}$ iodin solution added, will give the quantity of the latter, which reacted with the sulphite.

The neutral and acid sulphites of the alkalies, alkali earths, and even zinc and aluminum, may be accurately estimated in this manner, The less soluble salts requiring, of course, more time and shaking, to insure their complete oxidation. The latter is the U. S. P. method.

Sulphurous Acid (Acidum Sulphurosum, U. S. P.) - This is an aqueous solution of sulphur dioxid $\left(\mathrm{SO}_{2}=63.59\right)$ containing not less than 6 per cent, by weight, of the gas.

Sulphurous acid when brought in contact with Iodin is oxidized into sulphuric, the iodin being decolorized because of its union with the hydrogen of the accompanying water, forming hydriodic acid.

Two grams of sulphurous acid are taken and diluted with distilled water (recently boiled and cooled $*$ ) to about $25 \mathrm{cc}$. Two grams of sodium bicarbonate are added, and then the decinormal iodin V. S. is delivered into the solution (to which a little starch T. S. had been previously added) until a permanent blue color is produced. . At least $40 \mathrm{cc}$. of the standard iodin solution should be consumed before this color appears.

The following equations, etc., show the reaction that takes place:

$$
\begin{aligned}
& \mathrm{H}_{2} \mathrm{SO}_{3}+\mathrm{H}_{2} \mathrm{O}+\mathrm{I}_{2}={ }_{2} \mathrm{HI}+\mathrm{H}_{2} \mathrm{SO}_{4} \text {. } \\
& \begin{array}{rr}
\text { 2) } 81.47 & 2 \lcm{251.8} \\
\text { 10) } 40.735 & \text { 10 } \lcm{125.9}
\end{array}
\end{aligned}
$$

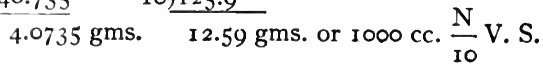
Thus each cc. of the $\frac{\mathrm{N}}{10} \mathrm{~V}$. S. represents $0.0040735 \mathrm{gm}$. of pure
$\mathrm{H}_{2} \mathrm{SO}_{3}$.

Sulphurous acid being, however, looked upon as a solution of $\mathrm{SO}_{2}$ in water, the quantity of this gas is generally estimated in analyses.

$$
\begin{aligned}
& \mathrm{H}_{2} \mathrm{O}, \mathrm{SO}_{2}+\mathrm{H}_{2} \mathrm{O}+\mathrm{I}_{2}={ }_{2} \mathrm{HI}+\mathrm{H}_{2} \mathrm{SO}_{4} \text {. } \\
& \begin{array}{ll}
\text { 2) } 63.59 & \text { 2) } 251.8
\end{array}
\end{aligned}
$$

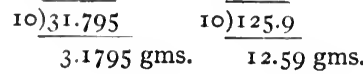

* "Recently boiled" insures absence of air, the oxygen of which would partially oxidize the sulphurous acid, and "cooled" is directed to avoid loss of $\mathrm{SO}_{2}$, which would occur if hot water were used. 
Thus each cc. of $\frac{\mathrm{N}}{\mathrm{IO}} \mathrm{V}$. S. consumed before the blue color appears represents $0.003 \mathrm{I} 795 \mathrm{gm}$. of $\mathrm{SO}_{2}$.

If $40 \mathrm{cc}$. are consumed in the above analysis, the 2 gms. contain

$$
0.0031795 \times 40=0.12718
$$

then

$$
\frac{0.12718 \times 100}{2}=6.35 \text { per cent of } \mathrm{SO}_{2}
$$

$A$ better method, however, is to measure 2 cc. into a stoppered weighing bottle, and weigh accurately. Then pour this into a solu, tion of sodium bicarbonate, and after the addition of starch solutiontitrate with $\frac{\mathrm{N}}{\mathrm{IO}}$ iodin.

The U. S. P. directs to introduce into a stoppered weighing bottle, 2 cc. of sulphurous acid and weigh accurately. To this add $50 \mathrm{cc}$. of $\frac{\mathrm{N}}{\mathrm{IO}}$ I V. S., and allow it to stand for five minutes, then titrate with $\frac{\mathrm{N}}{\mathrm{IO}}$ sodium thiosulphate until the mixture is just decolorized. Subtract the number of cc. of thiosulphate used from the $50 \mathrm{cc}$. of $\frac{\mathrm{N}}{\mathrm{IO}}$ I V.S. added, and multiply the difference by 0.318 , and divide this product by the weight of the acid taken. The quotient represents the percentage of absolute $\mathrm{SO}_{2}$ in the acid.

When a solution containing sulphur dioxid is to be measured by means of a pipette, it is never advisable to fill the instrument by suction in the usual manner, as this would cause a loss of the gas. A better plan is to fill the pipette by pressure, by the use of an arrangement similar to that shown in Fig. 59 .

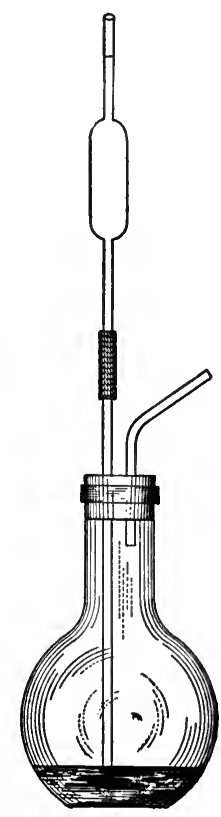

FIG. 59 .

The solution containing sulphur dioxid or other volatile substance is poured into a flask which is provided with a stopper through which two glass tubes pass; one of these tubes reaches to near the bottom of the flask and the other projects about one half an inch below the 
stopper and is bent outward above. To the upper end of the former the pipette is attached by means of a piece of rubber tubing. By blowing into the flask through the shorter tube the liquid is caused to rise and fill the pipette, which may then be easily pulled out of the rubber tube connection.

Sodium Sulphite $\left(\mathrm{Na}_{2} \mathrm{SO}_{3}+{ }_{7} \mathrm{H}_{2} \mathrm{O}=250.39\right)$. I gm. of the salt is dissolved in $25 \mathrm{cc}$. of distilled water recently boiled to expel air, and after the addition of an excess of sodium bicarbonate a little starch T. S. is added, and then the decinormal iodin V. S. delivered in from a burette, until the blue color of starch iodid appears, which does not disappear upon shaking or stirring.

The reaction is expressed as follows:

$$
\begin{aligned}
& \underbrace{\mathrm{Na}_{2} \mathrm{SO}_{3}+{ }_{7} \mathrm{H}_{2} \mathrm{O}}+\mathrm{I}_{2}={ }_{2} \mathrm{HI}+\mathrm{Na}_{2} \mathrm{SO}_{4}+6 \mathrm{H}_{2} \mathrm{O} \text {. } \\
& \begin{array}{ll}
\text { 2) } 250.39 & \text { 2) } 251.8
\end{array} \\
& 1 0 \longdiv { 1 2 5 . 1 9 } \quad 1 0 \longdiv { 1 2 5 . 9 } \\
& \text { 12.519 gms. } 12.59 \text { gms. or } 1000 \text { cc. } \frac{N}{10} \text { iodin V. S. }
\end{aligned}
$$

Thus each cc. of the standard solution represents $0.012519 \mathrm{gm}$. of crystallized sodium sulphite.

If I $\mathrm{gm}$. of the salt is taken, to find the percentage multiply the factor by the number of cc. of standard solution consumed, and the result by 100 .

The U.S. P. directs that $0.5 \mathrm{gm}$. of the finely powdered crystals be added to $50 \mathrm{cc}$. of $\frac{\mathrm{N}}{\mathrm{IO}}$ iodin, contained in a $100 \mathrm{cc}$. glass-stoppered flask, and allowed to stand for one hour (shaking frequently); the solution is then titrated with $\frac{\mathrm{N}}{\mathrm{IO}}$ sodium thiosulphate until the color is discharged.

Potassium Sulphite $\left(\mathrm{K}_{2} \mathrm{SO}_{3}+{ }_{2} \mathrm{H}_{2} \mathrm{O}=192.95\right)$. Operate upon $0.5 \mathrm{gm}$. in the same manner as for sodium sulphite.

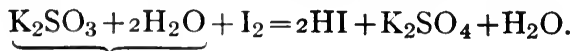

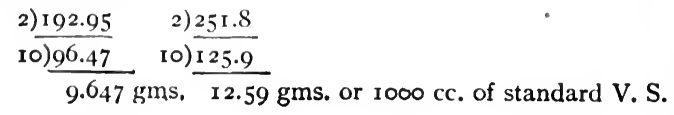

Each cc. of the $\frac{\mathrm{N}}{\mathrm{IO}}$ iodin represents $0.009647 \mathrm{gm}$. of crystallized potassium sulphite. 
Sodium Bisulphite $\left(\mathrm{NaHSO}_{4}=103.35\right)$. Operate upon about $0.25 \mathrm{gm}$. in the same manner as for sodium sulphite, and apply the following equation:

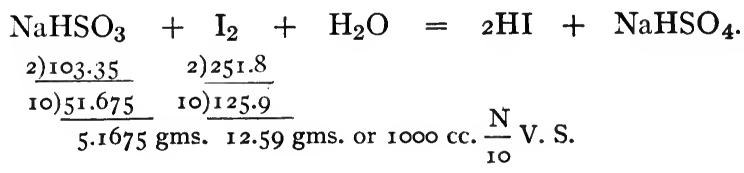

Thus each cc. of $\frac{\mathrm{N}}{\mathrm{IO}}$ iodin represents $0.005 \mathrm{I} 675 \mathrm{gm}$. of sodium bisulphite.

Sodium Thiosulphate (Sodium Hyposulphite) $\left(\mathrm{Na}_{2} \mathrm{~S}_{2} \mathrm{O}_{3}+{ }_{5} \mathrm{H}_{2} \mathrm{O}\right.$ $=246.46$ ). This salt, when brought in contact with iodin, is converted into sodium iodid and sodium tetrathionate. The reaction is expressed by the equation

$$
{ }_{2} \mathrm{Na}_{2} \mathrm{~S}_{2} \mathrm{O}_{3}+\mathrm{I}_{2}=2 \mathrm{NaI}+\mathrm{Na}_{2} \mathrm{~S}_{4} \mathrm{O}_{6} .
$$

Table of Substances which may be Estrmated by Means of Standard IODIN SOLUTION

\begin{tabular}{|c|c|c|c|}
\hline Name. & Formula. & $\begin{array}{l}\text { Molecular } \\
\text { Weight. }\end{array}$ & $\frac{\mathrm{N}}{10}$ Factor. \\
\hline 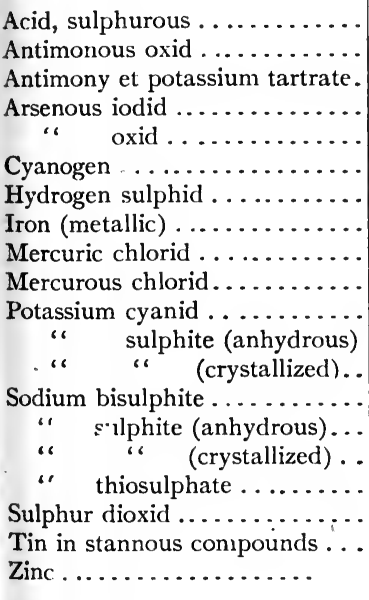 & $\begin{array}{c}\mathrm{H}_{2} \mathrm{SO}_{4} \\
\mathrm{Sb}_{2} \mathrm{O}_{3} \\
2\left(\mathrm{~K}(\mathrm{SbO}) \mathrm{C}_{4} \mathrm{H}_{4} \mathrm{O}_{6}\right)+\mathrm{H}_{2} \mathrm{O} \\
\mathrm{AsI}_{3} \\
\mathrm{As}_{2} \mathrm{O}_{3} \\
\mathrm{CN} \\
\mathrm{H}_{2} \mathrm{~S} \\
\mathrm{Fe}_{2} \\
\mathrm{HgCl}_{2} \\
\mathrm{HgCl} \mathrm{KCN} \\
\mathrm{K}_{2} \mathrm{SO}_{3} \\
\mathrm{~K}_{2} \mathrm{SO}_{3}+{ }_{2} \mathrm{H}_{2} \mathrm{O} \\
\mathrm{NaHSO}_{3} \\
\mathrm{Na}_{2} \mathrm{SO}_{3} \\
\mathrm{Na}_{2} \mathrm{SO}_{3}+{ }_{7} \mathrm{H}_{2} \mathrm{O} \\
\mathrm{Na}_{2} \mathrm{~S}_{2} \mathrm{O}_{3}+{ }_{5} \mathrm{H}_{2} \mathrm{O} \\
\mathrm{SO}_{2} \\
\mathrm{Sn}_{2} \\
\mathrm{Zn}_{2}\end{array}$ & $\begin{array}{l}81.47 \\
286.24 \\
659.80 \\
451.1 \\
\text { I96.44 } \\
25.84 \\
33.83 \\
111.0 \\
268.86 \\
233.68 \\
64.70 \\
157.19 \\
192.95 \\
103.35 \\
125.23 \\
250.39 \\
246.46 \\
63.59 \\
236.2 \\
\text { I } 29.8\end{array}$ & $\begin{array}{l}0.0040735 \\
0.0071548 \\
0.016495 \\
0.022605 \\
0.004911 \\
0.001292 \\
0.0016915 \\
0.002775 \\
0.026886 \\
0.023368 \\
0.003235 \\
0.0078595 \\
0.009648 \\
0.005168 \\
0.0062615 \\
0.012519 \\
0.024646 \\
0.003180 \\
0.005905 \\
0.0032450\end{array}$ \\
\hline
\end{tabular}


It is estimated as follows: I gm. of the salt is dissolved in $20 \mathrm{cc}$. of water, a few drops of starch T. S. are added, and then the $\frac{\mathrm{N}}{\mathrm{IO}}$ iodin is delivered in from a burette, until the appearance of blue starch iodid indicates an excess of iodin.

Each cc. of $\frac{\mathrm{N}}{\mathrm{IO}}$ iodin represents $0.015706 \mathrm{gm}$. of the anhydrous salt, or $0.024646 \mathrm{gm}$. of the crystalline salt.

Many other substances besides those mentioned in the foregoing pages may be estimated by titration with standard iodin solution. Among them are cyanids, hydrogen sulphid, stannous compounds, mercurous compounds, metallic zinc, and aluminum.

\section{ESTIMATION OF SUBSTANCES READILY REDUCED}

Any substance which readily yields oxygen in a definite quantity, or is susceptible of an equivalent action, which involves its reduction to a lower quantivalence, may be quantitatively tested by ascertaining how much of a reducing agent of known power is required by a given quantity of the substance for its complete reduction.

The principal reducing agents which may be employed in volumetric analysis are sodium thiosulphate, sulphurous acid, arsenous acid, oxalic acid, metallic zinc, and magnesium.

The sodium thiosulphate is the only one which is employed officially in the U. S. P. in the form of a volumetric solution. It is used in the estimation of free iodin, and indirectly of other free halogens, or compounds in which the halogen is easily liberated, as in the hypochlorites, etc.

ESTIMATIONS INVOLVING THE USE OF SODIUM THIOSULPHATE V. S. (Iodometry)

When sodium thiosulphate acts upon iodin, sodium tetrathionate and sodium iodid are formed, and the solution is decolorized.

This reaction takes place in definite proportions: one molecular weight of the thiosulphate absorbs one atomic weight of iodin.

$$
{ }_{2} \mathrm{Na}_{2} \mathrm{~S}_{2} \mathrm{O}_{3}+\mathrm{I}_{2}=2 \mathrm{NaI}+\mathrm{Na}_{2} \mathrm{~S}_{4} \mathrm{O}_{6} \text {. }
$$

Chlorin cannot be directly titrated with the thiosulphate, but by adding to the solution containing free chlorin an excess of potassium 
iodid, the iodin is liberated in exact proportion to the quantity of chlorin present, atom for atom.

$$
\mathrm{Cl}_{2}+{ }_{2} \mathrm{KI}=2 \mathrm{KCl}+\mathrm{I}_{2} \text {. }
$$

Then by estimating the iodin, the quantity of chlorin is ascertained. All bodies which contain available chlorin, or which when treated with hydrochloric acid evolve chlorin, may be estimated by this method.

Also, bodies which contain available oxygen, and which when boiled with hydrochloric acid evolve chlorin, such as manganates, chromates, peroxids, etc., may be estimated in this way.

Solutions of ferric salts, when acidulated and boiled with an excess of potassium iodid, liberate iodin in exact proportion to the quantity of ferric iron present.

Thus sodium thiosulphate may be used in the estimation of a great variety of substances with extreme accuracy.

Preparation of Decinormal Sodium Thiosulphate (Hyposulphite) $\left(\mathrm{Na}_{2} \mathrm{~S}_{2} \mathrm{O}_{3}+{ }_{5} \mathrm{H}_{2} \mathrm{O}=246.46\right.$ contains 24.646 gms. in I liter). Sodium thiosulphate is a salt of thiosulphuric acid in which two atoms of hydrogen have been replaced by sodium; it therefore seems that a normal solution of this salt should contain one half the molecular weight in grams in one liter.

But this salt is used chiefly for the estimation of iodin, and, as stated before, one full molecular weight reacts with and decolorizes one atomic weight of iodin, and since one atom of iodin is chemically equivalent to one atom of hydrogen, a full molecular weight of sodium thiosulphate must be contained in a liter of its normal solution.

Sodium thiosulphate is easily obtained in a pure state, and therefore the proper weight of the salt, reduced to powder and dried between heets of blotting-paper, may be dissolved directly in water, and made up to one liter.

The U. S. P. directs that a stronger solution than necessary be made, its tier found, and then the solution diluted to the proper measure.

30 gms. of selected crystals of the salt are dissolved in enough vater to meke, at or near $15^{\circ} \mathrm{C} .\left(59^{\circ} \mathrm{C}\right.$. $)$, $1000 \mathrm{cc}$.

This concentrated solution is then standardized by one of the followng methods:

a. Standardization by Means of $\frac{\mathrm{N}}{\mathrm{IO}}$ Iodin. Transfer ro cc. of this olution into a flask or beaker, add a few drops of starch T.S., 
and then gradually deliver into it from a burette decinormal iodin solution, in small portions at a time, shaking the flask after each addition, and regulating the flow to drops toward the end of the operation. As soon as a blue color is produced which does not disappear upon shaking, but is not deeper than pale blue, the reaction is completed. Note the number of cc. of iodin solution used, and then dilute the thiosulphate solution so that equal volumes of it and the decinormal iodin will exactly correspond to each other, under the above-mentioned conditions.

Example. The ro cc. of sodium thiosulphate, we will assume, require 10.7 cc. of decinormal iodin.

The sodium-thiosulphate solution must then be diluted in the proportion of $10 \mathrm{cc}$. to $10.7 \mathrm{cc}$., or $1000 \mathrm{cc}$. to 1070 cc.

After the solution is thus diluted a new trial should be made, in the manner above described, in which $50 \mathrm{cc}$. of the thiosulphate solution should require exactly $50 \mathrm{cc}$. of the decinormal iodin to produce a faint blue color.

The solution should be kept in small dark amber-colored, glass-stoppered bottles, carefully protected from dust, air, and light.

One cc. of this solution is the equivalent of:

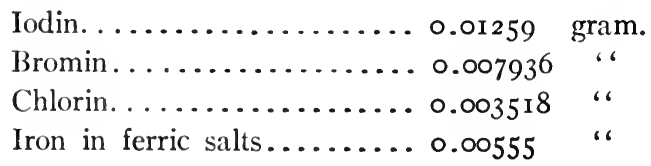

b. Standardization by Means of Potassium Dichromate. The potassium dichromate should be pure, if not it should be purified by triple recrystallization and then heating in a porcelain crucible until the entire mass is just fused: Then setting aside to cool in a desiccator over calcium chlorid or sulphuric acid. The mass falls to a crystalline powder. Of this purified potassium dichromate, weigh off a definite quantity, say 0.2 gm., dissolve it in a small quantity of water, and pour the solution into a beaker containing 2 gmis. of pure potassium iodid (free from iodate) and $100 \mathrm{cc}$. of water. Acidulate the solution with $5 \mathrm{cc}$. of concentrated hydrochloric or sulphuric acid, cover the beaker, and let stand for about five minutes, then titrate with the thiosulphate solution to be standardized (using starch as an 
indicator) until the blue color is just discharged. The calculation is then made as follows:

$$
\underset{292.28}{\mathrm{~K}_{2} \mathrm{Cr}_{2} \mathrm{O}_{7}} \underset{6 \times 164.76}{+6 \mathrm{KI}}+{ }_{14} \mathrm{HCl}={ }_{2} \mathrm{CrCl}_{3}+8 \mathrm{KCl}+{ }_{7} \mathrm{H}_{2} \mathrm{O}+{ }_{3} \mathrm{I}_{2} .
$$

Thus $292.28 \mathrm{gms}$. of potassium dichromate oxidizes $6 \times 164.76$ $=988.56 \mathrm{gms}$. of potassium iodid and liberates therefrom $6 \times 125.9$ $=755.4$ gms. of iodin.

The potassium iodid must be in excess, in fact for each atom of iodin liberated one molecule of potassium iodid must be present at the completion of the reaction in order to keep the iodin in solution, and thus prevent loss by volatilization.

If $292.28 \mathrm{gms}$. of potassium dichromate liberate $755.4 \mathrm{gms}$. of iodin, 0.2 gms. will liberate

$$
\frac{755.4 \times 0.2}{292.28}=0.5168 \mathrm{gm} . \text { of iodin }
$$

$0.01259 \mathrm{gm}$. of iodin $=$ I cc. of $\frac{\mathrm{N}}{10}$ thiosulphate.

$$
0.5168 \text { ، “ “ }=41.05 \text { cc. of } \frac{\mathrm{N}}{10} \text { thiosulphate. }
$$

Therefore, if in the above assay $37.60 \mathrm{cc}$. of the thiosulphate V. S. were consumed, it must be diluted so that each $37.60 \mathrm{cc}$. will measure ir.o5 cc. in order to convert the thiosulphate solution into a true decihormal solution. A new trial should then be made with the diluted olution to see if its strength is correct.

The pharmacopœial method is a modification of this; it is as follows: To a solution of about I gm. of potassium iodid in Io cc. of diluted ulphuric acid contained in a $500 \mathrm{cc}$. flask, add slowly from a burette o cc. of $\frac{\mathrm{N}}{\text { IoO }}$ potassium dichromate, shaking after each addition. Cover he mouth of the flask with a watch-glass and let stand for five minutes, hen dilute to about $250 \mathrm{cc}$. with distilled water, add some starch T. S., nd then from a burette the trial solution of sodium thiosulphate, in mall portions at a time, shaking after each addition, and towards the nd of the operation reducing the flow to drops, until the blue color $f$ the mixture changes to green. Note the number of cc. of the thio- 
sulphate solution consumed. Then dilute it with distilled water, so that equal volumes of it and $\frac{\mathrm{N}}{\mathrm{I} 0}$ dichromate will exactly correspond to each other under the above conditions at $25^{\circ} \mathrm{C} .\left(77^{\circ} \mathrm{F}\right.$.)

Example. Assuming that $16 \mathrm{cc}$. of the thiosulphate solution were consumed, then each $16 \mathrm{cc}$. must be diluted to measure exactly $20 \mathrm{cc}$.

c. Standardization by Means of Potassium Bi-iodate. This method depends upon the fact that when potassium iodid and biiodate are brought together in the presence of a small quantity of an acid, an equivalent amount of iodin is set free. The reaction is illustrated by the equation:

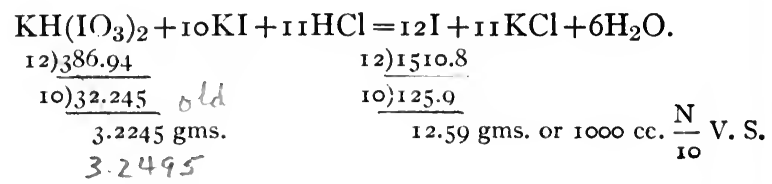

Thus it is seen that one molecule of the bi-iodate causes the liberation of 12 atoms of iodin, against which the thiosulphate solution is standardized.

In order not to use up too large a quantity of the thiosulphate solution in the titration, a very small quantity of the bi-iodate must be taken, and since a small error in weighing this would entail a relatively large error in. the results it is best to use the bi-iodate in the form of a solution of known strength and thus obviate the difficulty.

A decinormal solution of the bi-iodate, i.e., one containing in $1000 \mathrm{cc} .3 .2245 \mathrm{gms}$. of the salt may be used to advantage. Such a solution will keep unchanged for years, and may be employed as follows for the standardization of sodium thiosulphate solution:

Into a glass-stoppered flask of $250 \mathrm{cc}$. capacity introduce ro cc. of a 5 per cent solution of potassium iodid,* one cc. of diluted hydrochloric acid and exactly $25 \mathrm{cc}$. of the above potassium bi-iodate solution.

This brownish-yellow solution is now titrated with the sodium thiosulphate solution which is slowly delivered from a burette until the solution becomes pale yellow in color; a few drops of starch solution are now added, and the titration continued (the flow being reduced

* The potassium iodid must be in sufficient quantity not only to react with the bi-iodate quantitatively, as shown in the equation, but also to dissolve the iodin which is liberated. 
to drops) until the blue color is just discharged. During the titration, the flask should be frequently stoppered and vigorously shaken.

When the blue color is discharged, note the number of cc.'s used. If an exactly decinormal thiosulphate solution is taken, $25 \mathrm{cc}$. will be required to react with the $25 \mathrm{cc}$. of potassium bi-iodate solution, under the above conditions. If, on the other hand, only $22 \mathrm{cc}$. of the thiosulphate solution are used in the titration, then the latter is too strong, and must be diluted so that each $22 \mathrm{cc}$. will measure $25 \mathrm{cc}$. in order to make it strictly decinormal.

d. Standardization by Means of Potassium Permanganate.Sodium thiosulphate solution may be accurately standardized by means of potassium permanganate, if the other substances used for this purpose are not at hand. The method is easily understood by referring to the iodometric standardization of potassium permanganate solution, see page 146 .

Estimation of Free Iodin $(I=I 25.9)$. Iodin in the dry state or in the form of a tincture is brought into aqueous solution by means of pure potassium iodid and then titrated with standard sodium thiosulphate. The potassium iodid is used here to dissolve the iodin; it must be free from iodate $\left(\mathrm{KIO}_{3}\right)$ because the presence of this salt would cause a liberation of iodin from the potassium iodid.

Dry iodin is assayed as follows:

About $0.5 \mathrm{gm}$. of iodin are placed in a tightly stoppered weighing bottle and accurately weighed. One gram of potassium iodid and $50 \mathrm{cc}$. of water are added, and when the iodin is dissolved, $\frac{\mathrm{N}}{\mathrm{IO}}$ sodium thiosulphate is delivered from a burette in small portions at a time, shaking after each addition until the reaction is nearly completed, and the solution is of a faint yellow color. - A few drops of starch indicator are now added, and the titration with the thiosulphate, continued drop by drop until a final drop just discharges the blue color. The number of cc. of the thiosulphate solution used is noted. This number multiplied by the decinormal factor for iodin gives the weight of the latter present in the sample assayed.

$$
\begin{aligned}
& \mathrm{I}_{2}+{ }_{2}\left(\mathrm{NaS}_{2} \mathrm{O}_{3}+{ }_{5} \mathrm{H}_{2} \mathrm{O}\right)=\mathrm{Na}_{2} \mathrm{~S}_{4} \mathrm{O}_{6}+2 \mathrm{NaI}+\mathrm{IOH}_{2} \mathrm{O} . \\
& \text { 2) } 251.8 \quad \text { 2) } 492.92
\end{aligned}
$$

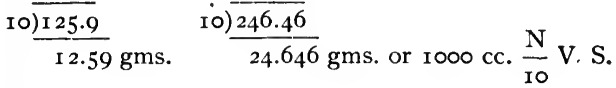


Each cc. of $\frac{N}{10}$ thiosulphate represents $0.01259 \mathrm{gm}$. of iodin.

If in the above assay $39 \mathrm{cc}$. of $\frac{\mathrm{N}}{\mathrm{IO}}$ sodium thiosulphate were consumed, then

$$
\begin{aligned}
& 0.01259 \times 39=0.49 \mathrm{Igm} . \\
& \frac{0.49 \mathrm{I}+100}{0.5}=98.2 \text { per cent. }
\end{aligned}
$$

Liquor Iodi Compositus (I.ugol's Solution). This is an aqueous solution of iodin and potassium iodid.

It is estimated for iodine in the same way as the foregoing. The potassium iodid acts merely as a solvent for free iodin, and does not enter into the reaction.

Ten or twelve grams of the solution is a convenient quantity to operate upon. Starch T. S. is the indicitor.

Tinctura Iodi (Tincture of Iodin). This is an alcoholic solution of free iodin, and must be diluted with a solution of potassium iodid, before titration, in order to provide sufficient liquid to keep the resulting salts in solution and to prevent the precipitation of iodin, which would result upon the addition of the aqueous standard solution.

Indirect Iodometric Estimations. The titration methods previously described, in which iodin is used as a standard solution and the estimation of free iodin by means of sodium thiosulphate, are classed as direct iodometric methods. The following methods, in which the strength of the substance under analysis is determined by the quantity of iodin which it liberates from an iodid, are known as indirect iodometric methods. Potassium iodid is added in excess * to an acidulated solution of the substance, and the liberated iodin estimated by means of standard thiosulphate. These methods are among the most accurate of all volumetric analyses, and take in a very large class of substances. Among the substances which may be analyzed by this method are chlorin and bromin, and all substances which readily liberate these elements: ferric salts, manganates, chromates, metallic peroxids, and other substances from which oxygen can be easily liberated.

* The iodid should be in sufficient excess to keep the liberated iodin in solution as KI.I. 
Free Chlorin or Bromin. Free chlorin acts upon potassium iodid, liberating iodin as per the equation

$$
\mathrm{Cl}_{2}+{ }_{2} \mathrm{KI}={ }_{2} \mathrm{KCl}+\mathrm{I}_{2} \text {. }
$$

Thus it is seen that each atom of chlorin will liberate one atom of iodine, hence by determining the quantity of iodin by means of a standard thiosulphate solution the quantity of chlorin present is easily ascertained. (I $25.9 \mathrm{gms}$. of $\mathrm{I}=35.18 \mathrm{gms}$. of $\mathrm{Cl}$ ). The same applies to free bromin, one atom of bromin (79.36) will liberate one atom of iodin (I25.9) $\mathrm{Br}_{2}+2 \mathrm{KI}=2 \mathrm{KBr}+\mathrm{I}_{2}$. 1000 cc. of $\frac{\mathrm{N}}{\mathrm{IO}}$ sodium thiosulphate is equivalent to 12.59 gms. of iodin, and hence to $3.5 \mathrm{I} 8$ gms. of chlorin or $7.936 \mathrm{gms}$. of bromin.

Thus I cc. of $\frac{\mathrm{N}}{\mathrm{IO}}$ sodium thiosulphate is equivalent to $0.01259 \mathrm{gm}$. of iodin; $0.0035^{18} \mathrm{gm}$. of chlorin; $0.007936 \mathrm{gm}$. of bromin.

Chlorin cannot be directly titrated with sodium thiosulphate because instead of the tetrathionate being formed as with iodin, sulphuric acid is produced, furthermore there is no readily observable end point as there is with iodin.

Aqua Chlori (Chlorin Water). This is an aqueous solution of chlorin, $\mathrm{Cl}=35.18$, containing at least 0.4 per cent of the gas.

The estimation of chlorin is effected in an indirect way, namely, by determining the quantity of iodin which it liberates from potassium iodid.

A definite quantity of chlorin will liberate a definite quantity of iodin from an iodid; these quantities are in exact proportion to their atomic weights, as the equation shows :

$$
\begin{aligned}
& \mathrm{Cl}_{2}+{ }_{2} \mathrm{KI}={ }_{2} \mathrm{KCl}+\mathrm{I}_{2} \text {. } \\
& \begin{array}{l}
\text { 2) } \frac{70.36}{35.18} \\
3.518 \text { gms. }
\end{array}
\end{aligned}
$$

Thus it is seen that by estimating the liberated iodin the quantity of chlorin may be determined with accuracy.

Ten grams is a convenient quantity to operate upon. To this about half a gram of potassium iodid is added. A little starch T. S. is then introduced, and the titration is begun with decinormal sodium thiosulphate. 
When the blue color of starch iodid has entirely disappeared the reaction is finished.

The reaction between iodin and sodium thiosulphate is illustrated by the following equation:

$$
\begin{aligned}
& \mathrm{I}_{2}+2\left(\mathrm{Na}_{2} \mathrm{~S}_{2} \mathrm{O}_{3}+{ }_{5} \mathrm{H}_{2} \mathrm{O}\right)=2 \mathrm{NaI}+\mathrm{Na}_{2} \mathrm{~S}_{4} \mathrm{O}_{6}+\mathrm{I}_{2} \mathrm{H}_{2} \mathrm{O} . \\
& \frac{2 \longdiv { 2 5 1 . 8 }}{1 0 \longdiv { 1 2 5 . 9 }} \quad \frac{2 \longdiv { 4 9 2 . 9 2 }}{12.59} \mathrm{gms} \text {. } \frac{246.46}{24.646} \mathrm{gms} \text { or I000 cc. } \frac{\mathrm{N}}{\mathrm{IO}} \mathrm{V} . \mathrm{S} \text {. }
\end{aligned}
$$

Thus we see that Iooo cc. of $\frac{\mathrm{N}}{10} \mathrm{Na}_{2} \mathrm{~S}_{2} \mathrm{O}_{3.5} \mathrm{H}_{2} \mathrm{O}$ represent I 2.59 gms. of iodin, which are equivalent to 3.518 gms. of chlorin.

Each cc. therefore is equivalent to $0.003518 \mathrm{gm}$. of chlorin. This number is the factor which, when multiplied by the number of cc. of $\frac{\mathrm{N}}{\mathrm{IO}}$ thiosulphate used, gives the weight in grams of chlorin contained in the quantity of chlorin water acted upon.

Chlorinated Lime (Calx Chlorinata, Chlorid of Lime, Bleachingpowder). This substance was formerly supposed to be a compound of lime and chlorin, $\mathrm{CaOCl}_{2}$, and hence the name chlorid of lime. It is now generally considered to be a mixture principally of calcium chlorid and calcium hypochlorite, $\mathrm{CaCl}_{2}+\mathrm{Ca}(\mathrm{ClO})_{2}$ or $\mathrm{Ca}(\mathrm{OCl}) \mathrm{Cl}$. The hypochlorite is the active constituent. This - is a very unstable salt, and is readily decomposed even by carbonic acid. When treated with hydrochloric acid it gives off chlorin.

The value of chlorinated lime as a bleaching or disinfecting agent depends upon its available chlorin; that is, the chlorin which the hypochlorite yields when treated with an acid.

In estimating the available chlorin, the latter is liberated with hydrochloric acid. This liberated gas, then, acting upon potassium iodid, sets free an equivalent amount of iodin. The quantity of iodin is then determined, and thus the amount of available chlorin found. 0.2 to $0.4 \mathrm{gms}$. are convenient quantities to operate upon.

The U.S. P. directs to introduce into a stoppered weighing bottle between 3 and 4 gms. of chlorinated lime and weigh accurately. (In order to make the description simpler we will assume that $3.5 \mathrm{gms}$. is the weight taken). This is then triturated thoroughly with $50 \mathrm{cc}$. of water, and the mixture transferred to a graduated vessel, together with the rinsings and made up to $1000 \mathrm{cc}$. with water. This is thor- 
oughly shaken, Ioo cc. of it (representing $0.35 \mathrm{gm}$. of the sample) are removed by means of a pipette and treated with I gm. of potassium iodid $*$ and $5 \mathrm{cc}$. of diluted hydrochloric acid, and into the resulting reddish-brown liquid, the $\frac{\mathrm{N}}{\mathrm{IO}}$ sodium thiosulphate is delivered from a burette. Towards the end of the titration, when the brownish color of the liquid is very faint, a few drops of starch T. S. are added and the titration continued until the bluish or greenish color produced by the starch has entirely disappeared. Not less than $30 \mathrm{cc}$. of the volumetric solution should be required to produce this result.

The reactions which take place in this process are illustrated by the following equations:

$$
\mathrm{Ca}(\mathrm{OCl}) \mathrm{Cl}+{ }_{2} \mathrm{HCl}=\mathrm{CaCl}_{2}+\mathrm{H}_{2} \mathrm{O}+\mathrm{Cl}_{2}
$$

or

$$
\mathrm{Ca}(\mathrm{OCl}) \mathrm{Cl}+\mathrm{H}_{2} \mathrm{SO}_{4}=\mathrm{CaSO}_{4}+\mathrm{H}_{2} \mathrm{O}+\mathrm{Cl}_{2} \text {, }
$$

$$
\begin{aligned}
& \mathrm{Cl}_{2}+{ }_{2} \mathrm{KI}={ }_{2} \mathrm{KCl}+\mathrm{I}_{2} \text {. } \\
& \text { 2) } 70.36 \text { 2 } 25 \mathrm{I} .8
\end{aligned}
$$

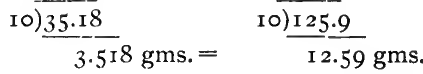

$\mathrm{I}_{2}+{ }_{2}\left(\mathrm{Na}_{2} \mathrm{~S}_{2} \mathrm{O}_{3}+{ }_{5} \mathrm{H}_{2} \mathrm{O}\right)=2 \mathrm{NaI}+\mathrm{Na}_{2} \mathrm{~S}_{4} \mathrm{O}_{6}+\mathrm{IoH} \mathrm{H}_{2} \mathrm{O}$.
2) 251.8
2) 492.92
10) $\lcm{125.9}$
I $2.59 \mathrm{gms}$.
10) $\frac{246.46}{24.646}$
24.646 gms. $=1000$ cc. $\frac{\mathrm{N}}{\mathrm{IO}} \mathrm{V} . \mathrm{S}$

It is thus seen that I cc. of the decinormal sodium thiosulphate represents $0.01259 \mathrm{gm}$. of iodin, which in turn is equivalent to $0.0035 \mathrm{I} 8$ gm. of chlorin.

Then

$$
0.0035 \mathrm{I} 8 \times 3 \circ=0.10554 \mathrm{gm} .
$$

$\frac{0.10554 \times 100}{0.35}=30.15$ per cent available chlorin.

This is a very rapid method for estimating chlorin; but when calcium chlorate is present in the bleaching-powder (and it often is, through imperfect manufacture) the chlorin from it is recorded, as

* In order to assume a sufficient excess of potassium iodid, take twice as much of it as of the bleaching powder. 
well as that from the hypochlorite, the chlorate being decomposed into chlorin, etc., by hydrochloric acid. The chlorate, however, is of no value in bleaching; its chlorin is not available. Hence, unless the powyder is known to be free from chlorate, the analysis should be made by means of arsenous-acid solution, or by using acetic acid instead of hydrochloric, and thus avoid liberating chlorin from chlorate which may be present.

The strength of bleaching powder is expressed in per cent of available chlorin or in degrees (Gay-Lussac). The latter represents the number of liters of chlorine, at $0^{\circ} \mathrm{C}$. and $760 \mathrm{~mm}$. pressure, available from one kilogram of the bleaching powder. The relation between these two ways of expressing the value is shown in the table following:

\begin{tabular}{c|c||c|c||c|c}
\cline { 1 - 2 } $\begin{array}{c}\text { Degrees. } \\
\text { Gay-Lussac. }\end{array}$ & $\begin{array}{c}\text { Per Cent } \\
\text { Chlorin. }\end{array}$ & $\begin{array}{c}\text { Degrees. } \\
\text { Gay-Lussac. }\end{array}$ & $\begin{array}{c}\text { Per Cent } \\
\text { Chlorin. }\end{array}$ & $\begin{array}{c}\text { Degrees. } \\
\text { Gay-Lussac. }\end{array}$ & $\begin{array}{c}\text { Per Cent } \\
\text { Chlorin. }\end{array}$ \\
\cline { 1 - 3 } \cline { 1 - 2 } 65 & 20.65 & 80 & 25.42 & 95 & 30.19 \\
66 & 20.97 & 81 & 25.74 & 96 & 30.51 \\
67 & 21.29 & 82 & 26.06 & 97 & 30.82 \\
68 & 21.61 & 83 & 26.37 & 98 & 31.14 \\
69 & 21.93 & 84 & 26.69 & 99 & 31.46 \\
70 & 22.24 & 85 & 27.01 & 100 & 31.78 \\
71 & 22.56 & 86 & 27.33 & 101 & 32.09 \\
72 & 22.88 & 87 & 27.65 & 102 & 32.41 \\
73 & 23.20 & 88 & 27.96 & 103 & 32.73 \\
74 & 23.51 & 89 & 28.28 & 104 & 33.05 \\
75 & 23.83 & 90 & 28.60 & 105 & 33.36 \\
76 & 24.15 & 91 & 28.92 & 106 & 33.68 \\
77 & 24.47 & 92 & 29.33 & 107 & 34.00 \\
78 & 24.79 & 93 & 29.55 & 108 & 34.32 \\
79 & 25.10 & 94 & 29.87 & 109 & 34.64 \\
\hline
\end{tabular}

The various bleaching preparations of the market which depend upon their available chlorin are all salts of hypochlorous acid $(\mathrm{HClO})$ or solutions of such salts.

Eau de Javelle (Javelle's Water) is a solution of potassium hypochlorite and potassium chlorid. A solution of magnesium hypochlorite is known in commerce as Ramsay's or Grouvelle's Bleaching Fluid. The solution known as W'ilson's Bleaching Fluid contains aluminum hypochlorite.

Liquor Sodæ Chlorinatæ (Solution of Chlorinated Soda; Labarraque's Solution). This is an aqueous solution of several chlorin compounds of sodium, principally sodium chlorid and hypochlorite, containing at least 2.4 per cent by weight of available chlorin. 
In this solution, as in chlorinated lime, it is the available chlorin which is estimated. The chlorin is first liberated with hydrochloric or sulphuric acid; this then liberates iodin from potassium iodid, and the free iodin is then determined by standard sodium thiosulphate.

Seven grams of chlorinated soda solution are mixed with $50 \mathrm{cc}$. of water, 2 gms. of potassium iodid, and ro cc. of hydrochloric acid are then added, together with a few drops of starch T. S. Into this mixture the decinormal sodium thiosulphate is delivered from a burette until the blue or greenish tint of the liquid is just discharged. Each cc. of $\frac{\mathrm{N}}{\mathrm{IO}}$ thiosulphate used up represents $0.0035^{18} \mathrm{gm}$. of available chlorin. The potassium iodid should always be added before the hydrochloric acid, so that the chlorin has potassium iodid to act upon as it is liberated, and thus loss of chlorin is obviated.

Bromin Water, or any substance containing free bromin, may be assayed in exactly the same manner as that described for chlorin : water. Free chlorin must, however, be absent.

Each cc. of $\frac{\mathrm{N}}{\mathrm{IO}}$ thiosulphate solution represents $0.007936 \mathrm{gm}$. of bromin.

Assay of Hydrogen Dioxid $\left(\mathrm{H}_{2} \mathrm{O}_{2}=33.76\right)$. The iodometric method, which originated with Kingzett, $*$ is based upon the fact that iodin is liberated from potassium iodid by hydrogen dioxid, in the presence of sulphuric acid, and that this liberation of iodin is in direct proportion to the available oxygen contained in the dioxid.

Then by determining the amount of iodin liberated, the available oxygen is readily found.

$$
\begin{aligned}
& \mathrm{H}_{2} \mathrm{O}_{2}+\mathrm{H}_{2} \mathrm{SO}_{4}+2 \mathrm{KI}=\mathrm{K}_{2} \mathrm{SO}_{4}+{ }_{2} \mathrm{H}_{2} \mathrm{O}+\mathrm{I}_{2} \text {. } \\
& \text { 2) } \frac{33.76}{\mathrm{I} 6.88}=\mathrm{r} \text { available } \mathrm{O}=\frac{2) \mathrm{r} 5.88}{7.94} \frac{\text { 2) } 25 \mathrm{I} .8}{\mathrm{I} 25.9}
\end{aligned}
$$

This shows that 125.9 gms. of iodin are liberated by 16.88 gms. of absolute dioxid, which are equivalent to $7.94 \mathrm{gms}$. of available oxygen.

Thus rooo cc. of $\frac{\mathrm{N}}{\mathrm{IO}}$ sodium thiosulphate V. S., which absorb and 
consequently represent $12.59 \mathrm{gms}$. of iodin, are equivalent to I.688 gms. of $\mathrm{H}_{2} \mathrm{O}_{2}$ or $0.794 \mathrm{gm}$. of available oxygen.

Each cc. of this $\frac{\mathrm{N}}{\mathrm{IO}}$ V. S., then, represents, $0.00 \mathrm{I} 688 \mathrm{gm}$. of $\mathrm{H}_{2} \mathrm{O}_{2}$, and $0.000794 \mathrm{gm}$. of available oxygen.

The coefficients for weight of $\mathrm{H}_{2} \mathrm{O}_{2}$ and of oxygen, it is seen, are identical with those used in the permanganate process. Therefore the coefficient for volume is also the same in this method as in the other if $\mathrm{r}$ cc. be taken for assay.

The process is carried out as follows: Take 2 or 3 cc. of sulphuric acid, dilute it with about $30 \mathrm{cc}$. of water, add an excess of potassium iodid (about I gm.), and then I cc. of hydrogen oxid. After the mixture has been allowed to stand five minutes starch T. S.* is added, and the titration with $\frac{\mathrm{N}}{\mathrm{IO}}$ sodium thiosulphate begun.

Note the number of cc. required to discharge the blue color, and multiply this number: by $0.001688 \mathrm{gm}$. to find the quantity, by weight, of $\mathrm{H}_{2} \mathrm{O}_{2}$; by $0.000794 \mathrm{gm}$. to find the weight of available oxygen; by $0.556 \mathrm{cc}$. to find the volume of available oxygen.

If $18 \mathrm{cc}$. are required, the solution is of $0.556 \times 18=10.008$ volume strength.

$$
\begin{aligned}
& 0.001688 \times 18=0.030384 \text { or } 3.0384 \text { per cent } \mathrm{H}_{2} \mathrm{O}_{2} \text {. } \\
& 0.000794 \times 18=0.014292 \text { or } 1.4292 \text { per cent of oxygen. }
\end{aligned}
$$

With this method the author has always obtained satisfactory results. The lack of uniformity in the reaction, which is frequently reported, is doubtless due to the use of insufficient acid or to taking a too concentrated solution of the dioxid.

The best results are obtained if the solution is not more than two volumes strength.

The sulphuric acid used in this assay must be free from sulphurous acid, arsenous acid, and nitric acid, and the potassium iodid must contain no iodate.

Distillation Methods. Manganates, chromates, metallic peroxids, and a great variety of substances containing oxygen, will, when heated

* Starch T. S. may he omitted, as the decolorization of the iodin is distinctly seen if the beaker is placed upon a white surface. 
with concentrated hydrochloric, liberate an equivalent amount of chlorin. This is illustrated by the following equation:

$$
\mathrm{MnO}_{2}+{ }_{4} \mathrm{HCl}=\mathrm{MnCl}_{2}+{ }_{2} \mathrm{H}_{2} \mathrm{O}+\mathrm{Cl}_{2} .
$$

The chlorin which is evolved, is passed into a solution of potassium iodid and liberates an equivalent of iodin, which latter substance is then estimated by titration with sodium thiosulphate solution. The quantity so found is therefore a measure of the original substance and of its oxygen content. The process may be carried out by means of the apparatus devised by Bunsen, Fig. 60, or by that of Fresenius, Fig. 6I, or Mohr, Fig. 62.

An accurately weighed quantity of the substance to be analyzed is introduced into the round-bottomed flask $a$, Fig. 6o. The flask is

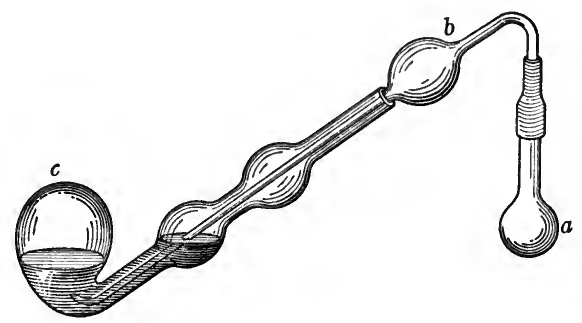

FIG. 60.

then filled to about two-thirds its capacity with concentrated hydrochloric acid, and quickly connected by means of a short rubber tube with a long-bulbed delivery tube, $b$, which is introduced into and extends to the bottom of an inverted bulbed retort, $c$. The larger bulb of the retort is filled to two thirds of its capacity with a Io per cent solution of potassium iodid. Heat is applied to the flask, and the chlorin distils over into the potassium iodid solution, which becomes brownish-red through liberation of iodin. The distillation is continued until about one third of the acid fluid has passed over or until a peculiar cracking sound indicates the absorption of hot hydrochloric acid vapor.

The flask, together with its delivery tube, is then slowly removed, the heating being, however, continued until the tube is entirely withdrawn, in order to prevent the iodid solution being drawn over into the flask. The retort is then shaken so that any traces of chlorin 
which may have escaped absorption, are taken up and the contents of the retort poured into a beaker; the retort and the delivery tube are then rinsed with water and the rinsings added to the fluid in the beaker, and titration with standard sodium thiosulphate begun immediately.

It is important that the quantity of potassium iodid be sufficient to keep the liberated iodin in solution, and that the potassium iodid be free from iodate, also that the titration be started without delay to avoid liberation of iodin through action of the air upon the strongly acid potassium iodid solution. When all of the chlorin has passed

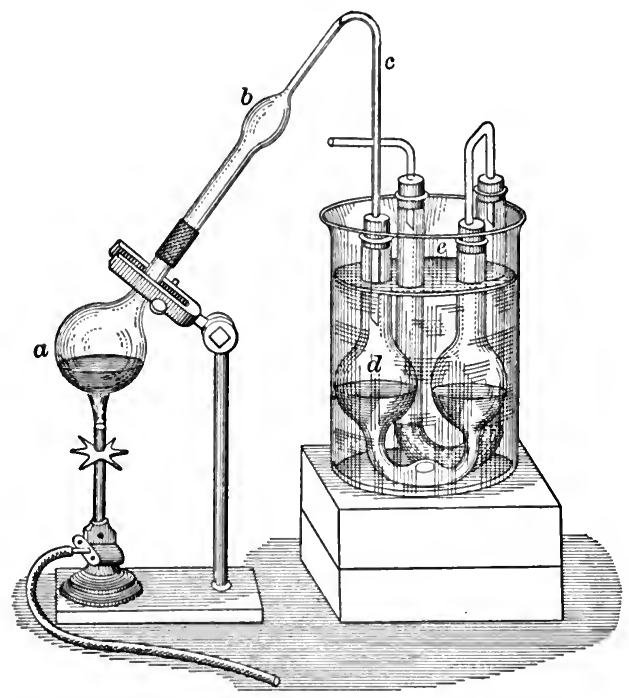

FIG. 61.

over and hydrochloric acid gas begins to distil, the liquid in the retort is apt to be drawn back into the flask because of the great affinity which hydrochloric acid gas has for water, and the resultant condensation in the flask. This regurgitation may be avoided by introducing into the generating flask a small piece of magnesite, which slowly dissolves in the acid solution and so keeps up a constant flow of carbon dioxid, which by its pressure prevents back flow of the fluid. The bulbs in the retort and delivery tube are also calculated to prevent this regurgitation.

The Fresenius apparatus is illustrated in Fig. 6r. In this the potassium iodid solution is contained in two joined $\mathrm{U}$-shaped tubes. 
The delivery tube from the distilling flask enters one of the U-tubes through a paraffin-soaked cork (which fits tightly), and terminates just above the potassium iodid solution. In operation, the U-tubes should be kept in ice water, and all the fittings should be air-tight. Paraffincovered cork stoppers only should be used.

After all the chlorin has passed over or when about one-third of the acid has distilled over, the apparatus is allowed to stand for a few minutes, to permit all traces of chlorin to become absorbed; the

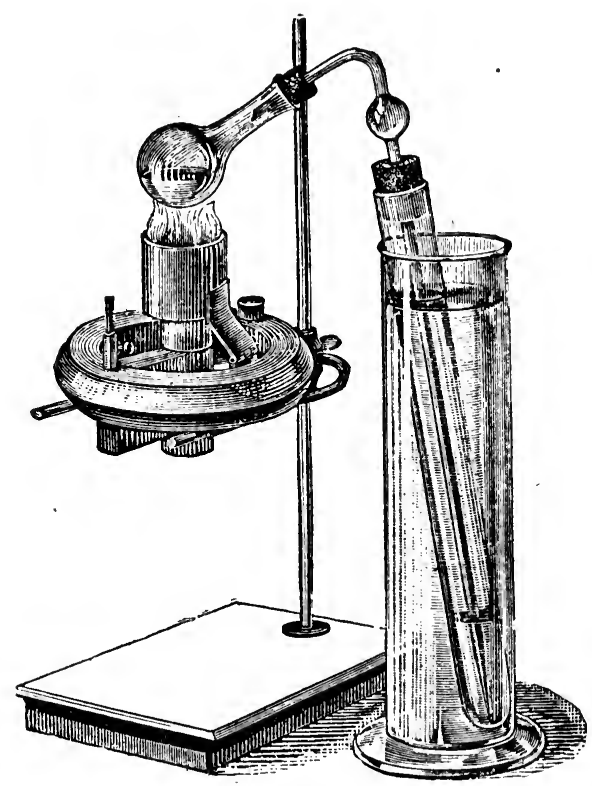

FIG. 62.

application of a suction pump to the rear outlet tube will help to bring about this result.

Mohr's apparatus, shown in Fig. 62, is of very simple construction and easy to use.

The distilling flask is fitted with a paraffin-soaked cork, through - which a delivery tube containing one bulb passes; this delivery tube again passes through a common cork which loosely fits a stout, large test tube, containing the potassium iodid solution. The delivery tube is drawn out to a fine point and reaches to near the bottom of the test 
tube. The latter is placed, when in operation in a hydrometer jar containing cold water.

Estimation of Manganese Dioxid $\left(\mathrm{MnO}_{2}=86.36\right)$. $0.4 \mathrm{gm}$. of pulverized manganese dioxid is placed into the distilling flask ( $a$, Fig. 60 or Fig. 6I) and the latter filled to two-thirds of its capacity with concentrated hydrochloric acid and connected without delay with the vessel containing the potassium iodid solution. The flask is then gradually heated so that a steady current of chlorin passes over into the potassium iodid solution. When the evolution of chlorin gas begins to diminish, the heat is slowly raised to boiling, and continued at this point until about one third of the acid liquid has distilled over. The delivery tube is then removed and rinsed as previously described, and the liberated iodin titrated by means of $\frac{\mathrm{N}}{\mathrm{IO}}$ sodium thiosulphate solution, of which we will assume $60 \mathrm{cc}$. were consumed.

The reactions are as follows:

$$
\begin{aligned}
& \mathrm{MnO}_{2}+2 \mathrm{KI}=\mathrm{MnCl}_{2}+{ }_{2} \mathrm{H}_{2} \mathrm{O}+\mathrm{Cl}_{2} \text {. } \\
& 86.36 \quad 70.36 \\
& \mathrm{Cl}_{2}+2 \mathrm{KI}=2 \mathrm{KCl}+\mathrm{I}_{2} \text {. } \\
& 70.36 \quad 25 \mathrm{I} .8 \\
& \mathrm{I}_{2}+{ }_{2} \mathrm{Na}_{2} \mathrm{~S}_{2} \mathrm{O}_{3} \cdot{ }_{5} \mathrm{H}_{2} \mathrm{O}=2 \mathrm{NaI}+\mathrm{Na}_{2} \mathrm{~S}_{4} \mathrm{O}_{6}+\mathrm{roH}_{2} \mathrm{O} \text {. } \\
& \frac{12) 251.8}{1 2 \longdiv { 1 2 5 . 9 }} \quad \begin{array}{l}
\text { 10) } \frac{492.92}{246.46} \\
24.59
\end{array} \\
& 0.01259 \mathrm{gm} \text {. } \\
& =\quad \text { I cc. } \frac{N}{10} \text { V.S. }
\end{aligned}
$$

These equations show that $492.9^{2}$ gms. of sodium thiosulphate will decolorize $25 \mathrm{r} .9 \mathrm{gms}$. of iodin, which quantity is liberated by $70.36 \mathrm{gms}$. of chlorin, which is itself liberated from hydrochloric acid by 86.36 gms. of manganese dioxid.

Therefore I cc. of a decinormal solution of sodium thiosulphate (containing $24.646 \mathrm{gms}$. in $1000 \mathrm{cc}$.) is equivalent to $0.0 \mathrm{I} 259 \mathrm{gm}$. of I; $0.0035 \mathrm{I} 8 \mathrm{gm}$. of $\mathrm{Cl} ; 0.0043 \mathrm{I} 8 \mathrm{gm}$. of $\mathrm{MnO}_{2} ; 0.000794 \mathrm{gm}$. of $\mathrm{O}$ (available).

The $60 \mathrm{cc}$. of the thiosulphate solution used in this assay will therefore represent $0.004318 \times 60=0.25908 \mathrm{gm}$. of pure $\mathrm{MnO}_{2}$, or 64.77 per cent.

$$
\frac{0.25908 \times 100}{0.4}=64.77 \text { per cent. }
$$


This is the method which should be used for the assay of native manganese dioxid. The freshly precipitated manganese dioxid of the Pharmacopœia may be assayed by the more easily performed digestion method described on page 222 .

Estimation of Chromic Acid and Chromates. Chromic Anhydrid, Chromium Trioxid $\left(\mathrm{CrO}_{3}=99.34\right)$, when heated with concentrated hydrochloric acid, liberates chlorin as per the equation,

$$
\underset{99 \cdot 34}{\mathrm{CrO}_{3}+6 \mathrm{HCl}}=\mathrm{CrCl}_{3}+{ }_{3} \mathrm{H}_{2} \mathrm{O}+\underset{3 \times 35.18}{\mathrm{Cl}_{3} .}
$$

99.34 parts of $\mathrm{CrO}_{3}$ liberates $3 \times 35.18$ parts of $\mathrm{Cl}$, hence one atomic weight of chlorin, 35.18 parts, represents $33.1 \mathrm{I}_{3}+$ parts of $\mathrm{CrO}_{3}$. Or I cc. of $\frac{\mathrm{N}}{\mathrm{IO}}$ sodium thiosulphate represents $0.0033 \mathrm{II} 3 \mathrm{gm}$. of $\mathrm{CrO}_{3}$.

Potassium Dichromate $\left(\mathrm{K}_{2} \mathrm{Cr}_{2} \mathrm{O}_{7}=292.28\right)$. This salt, as explained in a previous chapter, has three atoms of exygen available for oxidation. A molecule of this salt is therefore equivalent to six atoms of chlorin, and when boiled with hydrochloric acid will liberate six atoms of chlorin, as the equation shows.

$$
\begin{aligned}
& \mathrm{K}_{2} \mathrm{Cr}_{2} \mathrm{O}_{7}+{ }_{14} \mathrm{HCl}=2 \mathrm{KCl}+{ }_{2} \mathrm{CrCl}_{3}+{ }_{7} \mathrm{H}_{2} \mathrm{O}+{ }_{3} \mathrm{Cl}_{2} \text {. } \\
& 292.28 \\
& 6 \times 35.18
\end{aligned}
$$

Thus one atom of liberated chlorin will represent one sixth of 292.28 , which is $48.7 \mathrm{I} 3+$ parts of potassium dichromate. Then I cc. of $\frac{\mathrm{N}}{\mathrm{IO}}$ sodium thiosulphate will represent $0.00487 \mathrm{I} 3 \mathrm{gm}$. of $\mathrm{K}_{2} \mathrm{Cr}_{2} \mathrm{O}_{7}$. In the same way all other chromates may be treated, but these compounds will, when treated with hydrochloric acid, liberate chlorin at once and without the application of much heat, hence some chlorin is apt to be lost before the distillation flask can be connected with the apparatus, and therefore it is more convenient to employ the digestion method later described.

The reaction in the case of neutral potassium chromate is as follows:

$$
\underset{\text { I } 92.94}{\mathrm{~K}_{2} \mathrm{CrO}_{4}}+8 \mathrm{HCl}=2 \mathrm{KCl}+{ }_{4} \mathrm{H}_{2} \mathrm{O}+\mathrm{CrCl}_{3}+\mathrm{Cl}_{3} .
$$

Lead peroxid, $\mathrm{PbO}_{2}$; cobalic oxid, $\mathrm{Co}_{2} \mathrm{O}_{3}$; nickel oxid, $\mathrm{Ni}_{2} \mathrm{O}_{3}$, as well as many other substances, may he assayed by this distillation method. 
Estimation of Alkali Iodids by the Distillation Method. This method is based upon the fact that metallic iodids when treated with ferric salts in acidulated solucion yield up all of their iodin. As shown in the equation

$$
\mathrm{Fe}_{2}\left(\mathrm{SO}_{4}\right)_{3}+2 \mathrm{KI}=\mathrm{K}_{2} \mathrm{SO}_{4}+{ }_{2} \mathrm{FeSO}_{4}+\mathrm{I}_{2} .
$$

The iodin thus set free is distilled into a solution of potassium iodid and its quantity determined by titration with sodium thiosulphate in the usual manner.

For the reaction, ferric sulphate or ammonio-ferric alum may be used; the latter is, however, preferred because of its paler color. Ferric sulphate and ferric chlorid are so dark in color that the determina.

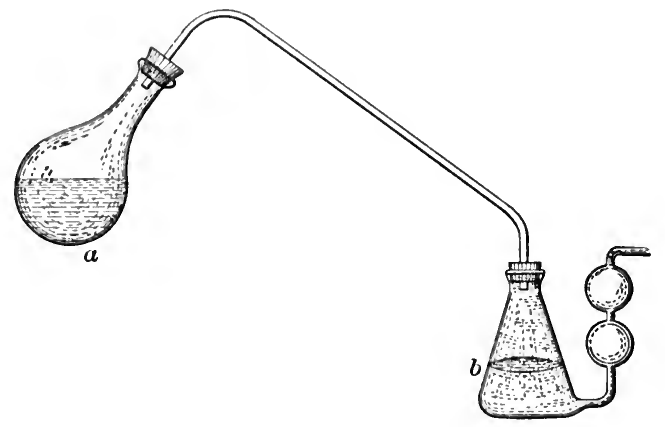

Fig. 63.

tion of the end-reaction is quite a difficult matter; furthermore, these salts frequently contain traces of nitrates which if present liberate chlorin from the chlorids or distil over and liberate iodin from the potassium iodid solution in the receiving vessel. Ferric chlorid is particularly objectionable because of the tenacity with which it holds the last portions of iodin.

The distillation may be done in the Fresenius apparatus, Fig. 6r, or hetter in that shown in Fig. 63. The latter consists of a $100 \mathrm{cc}$. distilling flask (a), connected by means of a glass tube with a nitrogen flask $(b)$, which contains a 10 per cent potassium iodid solution, and which is kept in a vessel of ice water when in use. The stoppers used are cork, well soaked in paraffin. The construction of the flask is particularly suitable, because it presents a large surface to the vapor of iodin which distils over, and because the titration can be done 
directly in it, thus avoiding the necessity of transferring its contents to a beaker or other vessel.

The glass tube which conveys the iodin vapor must not be carried into the solution of potassium iodid and must not be drawn to a fine point. The reason for this is that the iodin condensing at the point would soon choke up the tube and so prevent the further passage of iodin vapor. Any iodin which condenses in the tube is washed down into the potassium iodid solution by the steam during the distillation.

The Process. Into the flask $(a)$ is introduced about $5 \mathrm{gm}$. of ammonio-ferric alum, $50 \mathrm{cc}$. of water, $20 \mathrm{cc}$. of diluted sulphuric acid ( $\mathrm{I}: \mathrm{IO}$ ), and the iodid to be examined, accurately weighed. Take about $0.5 \mathrm{gm}$. The flask is then connected with the receiving vessel (b), which is about half filled with a ro per cent potassium iodid solution, and after connections are made tight, heat is gradually applied to the distilling flask. After most of the iodin has passed over, the heat is raised to boiling, and continued at this temperature until about one fourth of the liquid has passed over, and the solution in the distilling flask is no longer of a brown color.

When the receiving vessel has sufficiently cooled, it is disconnected and its contents titrated with decinormal sodium thiosulphate, using starch as indicator. Before beginning titration, however, it is necessary to rinse the lower extremity of the tube and the stopper into the solution in the receiving vessel, in order that every trace of iodin be collected.

The calculation is then made as follows:

(a) $\mathrm{Fe}_{2}\left(\mathrm{SO}_{4}\right)_{3} \cdot\left(\mathrm{NH}_{4}\right)_{2} \mathrm{SO}_{4}+2 \mathrm{KI}=\mathrm{K}_{2} \mathrm{SO}_{4}+\left(\mathrm{NH}_{4}\right)_{2} \mathrm{SO}_{4}+2 \mathrm{FeSO}_{4}+\mathrm{I}_{2}$. $329 \cdot 5^{2}$ 251.8

(b) $\mathrm{I}_{2}+{ }_{2} \mathrm{Na}_{2} \mathrm{~S}_{2} \mathrm{O}_{3}+{ }_{5} \mathrm{H}_{2} \mathrm{O}=2 \mathrm{NaI}+\mathrm{Na}_{2} \mathrm{~S}_{4} \mathrm{O}_{6}+10 \mathrm{H}_{2} \mathrm{O}$.

$$
\begin{aligned}
\frac{251.8}{10)} & \frac{25.9}{12.59} \mathrm{gms} . \\
0.01259 \mathrm{gm} . & =\quad \text { I cc. } \frac{\mathrm{N}}{24.62} \mathrm{~V} . \mathrm{S} .
\end{aligned}
$$

Referring to the above equations we see that $251.8 \mathrm{gms}$. of iodin are liberated from $329.5^{2} \mathrm{gms}$. of potassium iodid, thus $125.9 \mathrm{gms}$. of iodin represents $164.76 \mathrm{gms}$. of potassium iodid, and therefore I cc. 
of decinormal sodium thiosulphate solution representing $0.01259 \mathrm{gm}$. of iodin will at the same time represent $0.016476 \mathrm{gm}$. of KI.

If in the above assay $29 \mathrm{cc}$. of $\frac{\mathrm{N}}{\mathrm{O}}$ sodium thiosulphate are consumed, we multiply the factor for $\mathrm{KI}=0.016476 \mathrm{gm}$. by 29 , this gives $0.477604 \mathrm{gm}$., the quantity of pure KI in the $.5 \mathrm{gms}$. taken, which is about 95 per cent.

Digestion Methods. The distillation methods above described may be avoided in many cases and the more easily performed digestion process used. For instance, freshly precipitated manganese dioxid,

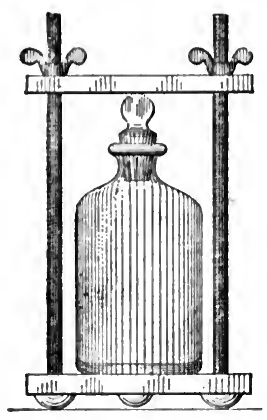

Fig. 64. lead peroxid, chromic acid, chlorates, bromates, iodates, ferric salts, and a great many other substances, may be assayed by mere digestion with hydrochloric acid at a slightly elevated temperature.

The digestion is performed in a strong glass bottle, provided with an accurately fitting groundglass stopper which is tied down by means of wire or secured by a clamp. See Fig. 64 .

Before using the bottle for this operation it should be tested by securely tying down the stopper and immersing the bottle entirely in hot water to see if the stopper fits sufficiently tight. If it does not, bublles of air will escape from inside, and the bottle is useless for the purpose intended. In that event the stopper must be reground into the neck of the bottle with a little very fine emery and water. The capacity of the bottle may vary from 50 to $150 \mathrm{cc}$.

The Process. The substance is accurately weighed and introduced into the bottle together with a small quantity of coarsely powdered glass or small pure flint pebbles (to prevent caking, especially in the case of insoluble powders). A sufficient excess of potassium iodid solution is then added, followed by some pure concentrated hydrochloric acid. The stopper is then quickly inserted, firmly secured by wire or a clamp, and the bottle placed in a water bath, and the water gradually heated to boiling; this temperature being continued until decomposition is complete, which is usually in about half an hour. The hottle is then allowed to cool slowly and its contents emptied into a beaker. Then after washing the bottle and adding the washings to the contents of the beaker the liberated iodin is estimated by titration with sodium thiosulphate. 
The potassium iodid used in this process must be absolutely free from iodate.

I cc. of $\frac{\mathrm{N}}{10}$ sodium thiosulphate is equivalent to

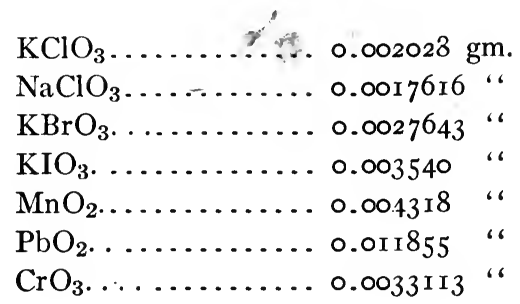

Estimation of Chlorates, Bromates, and Iodates. The estimation of these salts is based upon the fact that in each case one equivalent of the acid or its monobasic salt liberates six equivalents of chlorin and consequently six equivalents of iodin when decomposed by the digestion method.

This is illustrated by the equations:

$$
\begin{gathered}
\mathrm{KClO}_{3}+6 \mathrm{HCl}={ }_{3} \mathrm{H}_{2} \mathrm{O}+\mathrm{KCl}+\mathrm{Cl}_{6} ; \\
\mathrm{KBrO}_{3}+6 \mathrm{HCl}={ }_{3} \mathrm{H}_{2} \mathrm{O}+\mathrm{KBr}+\mathrm{Cl}_{6} ; \\
\mathrm{KIO}_{3}+6 \mathrm{HCl}={ }_{3} \mathrm{H}_{2} \mathrm{O}+\mathrm{KI}+\mathrm{Cl}_{6} .
\end{gathered}
$$

and

$$
\mathrm{Cl}_{6}+6 \mathrm{KI}=6 \mathrm{KCl}+\mathrm{I}_{6} \text {. }
$$

In the distillation process, however, bromates and iodates liberate only four equivalents of iodin, while bromous chlorid and iodous chlorid remain in the retort, therefore in these cases the digestion is preferable to the distillation method.

If the bromate or iodate to be assayed contains any bromid or iodid, bromin or iodin respectively will be liberated upon the addition of the acid, according to the equations

$$
\begin{aligned}
5 \mathrm{KBr}+\mathrm{KBrO}_{3}+6 \mathrm{HCl} & =6 \mathrm{KaCl}+{ }_{3} \mathrm{H}_{2} \mathrm{O}+\mathrm{Br}_{6} \\
5 \mathrm{KI}+\mathrm{KIO}_{3}+6 \mathrm{HCl} & =6 \mathrm{KaCl}+{ }_{3} \mathrm{H}_{2} \mathrm{O}+\mathrm{I}_{6}
\end{aligned}
$$

therefore the method is not applicable for the assay of such mixtures. 
The presence of either of these salts may be ascertained by moistening a small quantity of the salt with dilute sulphuric acid, when if a yellow or brown coloration results, either a bromid or an iodid respectively is present.

Example. Estimation of Potassizm Chlorate. $0.2 \mathrm{gm}$. of the salt is introduced into the digestion bo about $100 \mathrm{cc}$. capacity, Io cc. of water added, and about 4 gms. of potassium iodid (or sufficient of its saturated solution). This is followed by Io cc. of concentrated hydrochloric acid, the stopper quickly inserted, firmly secured by wiring or a clamp, and the flask placed, stopper downward, in a water-bath. The water is gradually raised to boiling and kept at this temperature for about half an hour. It is then allowed to cool slowly, and the contents of the bottle washed into a beaker and titrated with decinormal sodium thiosulphate, using starch as indicator. The number of cubic centimeters of $\frac{\mathrm{N}}{\mathrm{IO}}$ thiosulphate solution used, multiplied by $0.002028 \mathrm{gm}$., gives the weight of pure $\mathrm{KClO}_{3}$ present in the sample.

In the assay of bromates and iodates a smaller quantity of hydrochloric acid may be used and a lower temperature, say $50^{\circ} \mathrm{C}$., is suffcient for decomposition.

Example. Estimation of Potassium Bromate. $0.2 \mathrm{gm}$ of the salt are dissolved in $15 \mathrm{cc}$. of water, $4 \mathrm{gms}$. of potassium iodid are added, followed by $4 \mathrm{cc}$. of concentrated hydrochloric acid. The bottle is securely closed, as in the foregoing assay, and heated for half an hour at $50^{\circ} \mathrm{C}$. Then after decomposition, titration with $\frac{\mathrm{N}}{\mathrm{IO}}$ sodium thiosulphate solution is begun, each cc. of which represents $0.0027643 \mathrm{gm}$. of pure $\mathrm{KBrO}_{3}$.

If To $^{1} 00$ of one sixth of the molecular weight of either salt be taken for assay, each cc. of the $\frac{\mathrm{N}}{\mathrm{IO}}$ thiosulphate solution used will indicate I per cent purity.

Estimation of Ferric Salts. When a ferric salt in an acidulated solution is digested with an excess of potassium iodid the salt is reduced to the ferrous state, and iodin is set free.

$$
\mathrm{Fe}_{2} \mathrm{Cl}_{6}+2 \mathrm{KI}=2 \mathrm{FeCl}_{2}+2 \mathrm{KCl}+\mathrm{I}_{2} .
$$

One atom of iodin is liberated for each atom of iron in the ferric state. The liberated iodin is then determined by sodium thiosul- 
phate in the usual way. I25.9 gms. of iodin $=55.5$ gms. of metallic iron.

This is the method of the U. S. P. It is given in detail here.

$0.555 \mathrm{gm}$. of the salt is dissolved in Io or $15 \mathrm{cc}$. of water and $2 \mathrm{cc}$. of hydrochloric acid in a glass-stoppered bottle having a capacity of about roo cc. I gm. of potassium iodid is then added, and the mixture digested for half an hour at a temperature of $40^{\circ} \mathrm{C}$. (IO4 ${ }^{\circ} \mathrm{F}$.). During the digestion the stopper should be left in the bottle, and the heat not allowed to rise too high, otherwise the liberated iodin will be volatilized. When cool a few drops of starch T. S. are added. It is now ready for titrating with $\frac{\mathrm{N}}{\mathrm{IO}}$ sodium thiosulphate. Each cc. corresponds to I per cent of metallic iron.

When the quantity of metallic iron and the chemical formula for the ferric salt under estimation are known, the quantity of pure salt is easily found by calculation.

In all the estimations of ferric iron it is convenient to take $0.555 \mathrm{gm}$. of the salt. Each cc. of the volumetric solution used will then represent I per cent of metallic iron, assuming the atomic weight of iron to be 55.5 .

Ferric salts may be tested in many other ways. For instance:

A ferric salt in solution may be filtered through a column of zinc dust, which reduces it to the ferrous state. This is then estimated with potassium permanganate $\mathrm{V}$. S. in the usual manner, or the ferric solution is treated with a few small pieces of zinc or magnesium coarsely powdered, until complete reduction is effected. When a red color is no longer produced by sulphocyanate of potassium the ferric salt is completely reduced, and may be estimated with potassium permanganate V. S.

Stannous chlorid, ammonium bisulphite, and other substances may also be used as reducing agents.

Ferric Chlorid $\left(\mathrm{Fe}_{2} \mathrm{Cl}_{6}\right.$ or $\mathrm{FeCl}_{3}$. $) \quad 0.555 \mathrm{gm}$. of the dry salt is dissolved in a glass-stoppered bottle (having a capacity of about roo cc.) in Io cc. of water and 2 cc. of hydrochloric acid, and after the addition of I gm. of potassium iodid is kept for half an hour at a temperature of $40^{\circ} \mathrm{C}$. ( $104^{\circ} \mathrm{F}$.), then cooled and titrated with decinormal sodium thiosulphate until the color of the liquid is discharged. Each cc. of the decinormal thiosulphate solution used represents 0.00555 gm., or I per cent of metallic iron, or $0.016104 \mathrm{gm}$. of pure ferric chlorid. 
The following equations illustrate the reactions:

$$
\begin{aligned}
& \mathrm{Fe}_{2} \mathrm{Cl}_{6}+{ }_{222.08} \mathrm{KI}={ }_{2} \mathrm{FeCl}_{2}+{ }_{2} \mathrm{KCl} \\
& \frac{{ }_{16 \mathrm{I} .04}}{2 \frac{\mathrm{I}_{25} \mathrm{r} .8}{\mathrm{I} 25.9}}
\end{aligned}
$$

Then

$$
\begin{aligned}
& \mathrm{I}_{2}+2 \mathrm{Na}_{2} \mathrm{~S}_{2} \mathrm{O}_{3} \cdot{ }_{5} \mathrm{H}_{2} \mathrm{O}=2 \mathrm{NaI}+\mathrm{Na}_{2} \mathrm{~S}_{4} \mathrm{O}_{6}+10 \mathrm{H}_{2} \mathrm{O} \text {. } \\
& \text { 2) } 2 5 1 . 8 \quad 2 \longdiv { 2 . 4 6 . 4 6 }
\end{aligned}
$$

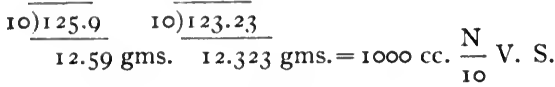

Thus each cc. of $\frac{\mathrm{N}}{\mathrm{IO}}$ thiosulphate represents $0.0 \mathrm{r} 259 \mathrm{gm}$. of iodin and consequently $0.016104 \mathrm{gm}$. of $\mathrm{Fe}_{2} \mathrm{Cl}_{6}$.

The assay of other official ferric salts is practically the same as that described above.

Each cc. of $\frac{\mathrm{N}}{\mathrm{IO}}$ sodium thiosulphate is equivalent to

Ferrum, Fe.

$0.00555 \mathrm{gm}$.

Ferric ammonium sulphate $\mathrm{Fe}_{2}\left(\mathrm{SO}_{4}\right)_{3} \cdot\left(\mathrm{NH}_{4}\right)_{2} \mathrm{SO}_{4} \ldots 0.026413$

" chlorid, $\mathrm{Fe}_{2} \mathrm{Cl}_{6} \ldots \ldots \ldots \ldots \ldots \ldots \ldots \ldots \ldots . . .016104$

“، " (cryst.), $\mathrm{Fe}_{2} \mathrm{Cl}_{6}+\mathrm{I}_{2} \mathrm{H}_{2} \mathrm{O} \ldots \ldots \ldots \ldots \ldots .0 .026832$

" nitrate, $\mathrm{Fe}_{2}\left(\mathrm{NO}_{3}\right)_{6} \ldots \ldots \ldots \ldots \ldots \ldots \ldots \ldots . .0 .02402 \mathrm{I}$

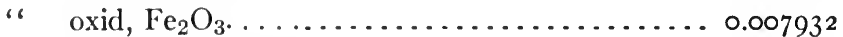

“ sulphate, $\mathrm{Fe}_{2}\left(\mathrm{SO}_{4}\right)_{3} \ldots \ldots \ldots \ldots \ldots \ldots \ldots \ldots . .0 .0198525$

In the case of the official scale salts of iron, which are mostly of indefinite and variable composition, it is the quantity of metallic iron present which is determined.

The official solutions of ferric salts are estimated in the same manner. It is the rule to take I.I I gms. of the solution for assay; then each cc. of the thiosulphate will represent 0.5 per cent of metallic iron.

REDUCTION METHODS INVOLVING THE USE OF STANDARD ARSENOUS ACID SOLUTION (Chlorometry)

As previously described, arsenous oxid when brought in contact with iodin in an alkaline solution results in an oxidation of the former to arsenic oxid, and a conversion of the iodin to hydriodic acid, as shown in the equation

$$
\mathrm{As}_{2} \mathrm{O}_{3}+{ }_{2} \mathrm{H}_{2} \mathrm{O}+\mathrm{I}_{4}=\mathrm{As}_{2} \mathrm{O}_{5}+{ }_{4} \mathrm{HI} \text {. }
$$


Advantage is taken of this reaction for the estimation, not only of arsenous and antimonous compounds, but also of iodin and the other halogens, chlorin and bromin, as well as of all those bodies which when heated with hydrochloric acid evolve chlorin, as for instance, the peroxids.

The reaction with chlorin is as follows:

$$
\mathrm{Cl}_{4}+{ }_{2} \mathrm{H}_{2} \mathrm{O}+\mathrm{As}_{2} \mathrm{O}_{3}={ }_{4} \mathrm{HCl}+\mathrm{As}_{2} \mathrm{O}_{5}
$$

This reaction is really an oxidation, so far as the formation of arsenic oxid $\left(\mathrm{As}_{2} \mathrm{O}_{5}\right)$ is concerned, but there is no accompanying reduction. The conversion of the halogen to an haloid acid is not strictly a reduction in the accepted sense of the word. Nevertheless, for obvious reasons, we speak of analyses done by means of arsenous acid as reduction methods.

The chief value of this method is found in the estimation of free chlorin, as in chlorin water, and the available chlorin existing in hypochlorites or that evolved from hydrochloric acid by heating with peroxids. Hence the designation "chlorometry."

In carrying out this method, free alkali must be present to combine with the haloid acid which is formed. The alkali must be in the form of bicarbonate. Normal carbonates or hydroxids are not suitable, see page 190.

The solutions required are:

Decinormal iodin, see page 186 ;

Decinormal arsenous acid;

Starch solution, see page 189 ; or iodized starch test paper.

Preparation of Decinormal $\frac{\mathrm{N}}{\mathrm{IO}}$ Arsenous Acid $\left(\mathrm{As}_{2} \mathrm{O}_{3}=\mathrm{I} 96.44 ;\right)$
$\left(\frac{\mathrm{N}}{\mathrm{IO}}\right.$ V. S. $\left.=4.9 \mathrm{I} \mathrm{I} \mathrm{gms.} \mathrm{in} \mathrm{I} \mathrm{liter.}\right)$

4.95 gms. of the purest sublimed arsenous anhydrid $\left(\mathrm{As}_{2} \mathrm{O}_{3}\right)$ are dissolved in about $250 \mathrm{cc}$. of distilled water with the aid of about 20 gms. of pure sodium bicarbonate. The anhydrid should be in fine powder, and the mixture heated to effect complete solution. This is then diluted with some water, cooled, and made up to rooo cc. It is then standardized with decinormal iodin, using starch as indicator. Decinormal arsenous acid solution should correspond, volume for volume, with decinormal iodin solution.

If this solution is made from pure arsenous acid it will hold its 
titer for years, but if any sulphur is present there will be an absorption of oxygen from the air and a consequent oxidation to arsenic oxid. If the presence of sulphur is suspected, the solution should be tested with silver nitrate, when its presence will be indicated by the formation of a reddish precipitate.

Iodized Starch Test Paper. A portion of starch solution is mixed with a few drops of potassium iodid solution and in this are soaked strips of pure white filtering paper. This test paper is used in the damp state; it is then far more sensitive.

Estimation of Free Halogens. The estimation of chlorin, bromin, or iodin by the chlorometric method depends, as before stated, upon their power of oxidizing arsenous acid. When a free halogen is brought in contact in alkaline solution with arsenous acid, the latter is oxidized to arsenic acid, while the halogen is transformed into a haloid acid, as per equations

$$
\left.\begin{array}{r}
\mathrm{Cl}_{4} \\
\mathrm{Br}_{4} \\
\mathrm{I}_{4}
\end{array}\right\}+{ }_{2} \mathrm{H}_{2} \mathrm{O}+\mathrm{As}_{2} \mathrm{O}_{3}=\mathrm{As}_{2} \mathrm{O}_{5}+\left\{\begin{array}{l}
{ }_{4} \mathrm{HCl} \\
4 \mathrm{HBr} . \\
{ }_{4} \mathrm{HI}
\end{array}\right.
$$

The estimation may be carried out in two ways: ist, by direct titration with a standard arsenous oxid solution, using iodized starch test paper as indicator; $2 \mathrm{~d}$, by residual titration, an excess of the standard arsenous oxid being taken, and then retitrating with standard iodin solution, using starch as indicator. The residual titration method need not be employed for free iodin, as this can be titrated direct with the arsenous oxid solution, using starch as indicator. Furthermore, iodin need not be brought into solution to be titrated by this method.

The estimation of free halogens by the direct chlorometric method is as follows:

An accurately weighed quantity of substance made alkaline by the addition of sodium bicarbonate is titrated with decinormal arsenous acid solution, and from time to time during the titration a drop of the solution is removed on the end of a pointed glass rod and brought in contact with a piece of iodized starch test paper. So long as free chlorin or bromin is present the liquid will cause a blue stain on the test paper, but when the halogen is all taken up no blue color is produced.

If the exact point is overstepped the residual method must be used. A little additional excess of the arsenous acid solution may be added, 
together with a few drops of starch solution, and the excess then titrated by means of decinormal iodin solution until the blue color is produced. The volume of decinormal iodin solution so used, deducted from the total volume of arsenous acid solution taken, gives the exact quantity which was oxidized by the halogen and from this the percentage of chlorin or bromin may be calculated.

Example r. Estimation, of Chlorin in Chlorin Water. $20 \mathrm{cc}$. of chlorin water (sp.gr. 1.o) titrated by the direct method required $22 \mathrm{cc}$. of decinormal arsenous acid solution before the iodized starch test paper indicated the completion of the reaction.

By referring to the equation we see that each cc. of the arsenous acid solution represents $0.0035 \mathrm{I} 8 \mathrm{gm}$. of chlorin. Therefore, if $22 \mathrm{cc}$. were used, the $20 \mathrm{cc}$. of chlorin water must have contained $22 \times 0.0035 \mathrm{I} 8$ gm. of chlorin, which is $0.077396 \mathrm{gm}$.

The $20 \mathrm{cc}$. of chlorin water (sp.gr. r.o) weigh 20 gms. Hence

$$
\frac{0.077396 \times 100}{20}=0.38+\text { per cent. }
$$

Example 2. The $20 \mathrm{cc}$. of chlorin water weighing $20 \mathrm{gms}$. were treated with $26 \mathrm{cc}$. of the arsenous acid solution, starch solution was then added, and the excess of arsenous acid solution titrated by means of decinormatiodin. $4 \mathrm{cc}$. of the latter were required, then 4 from $26 \mathrm{cc}$. leaves $22 \mathrm{cc}$, the quantity of the $\frac{\mathrm{N}}{\mathrm{IO}}$ arsenous acid solution which reacted with the chlorin. The calculation is the same as in Example I.

The reaction is as follows:

$$
\begin{aligned}
& \mathrm{Cl}_{4}+{ }_{2} \mathrm{H}_{2} \mathrm{O}+\mathrm{As}_{2} \mathrm{O}_{3}={ }_{4} \mathrm{HCl}+\mathrm{As}_{2} \mathrm{O}_{5} \text {. } \\
& \text { 4) } 1 4 0 . 7 2 \quad 4 \longdiv { 1 9 6 . 4 4 } \\
& \text { 10) } 35.18 \text { IO) } 49.11 \\
& 3.5^{18} \text { gms. }=\frac{1 0 \longdiv { 4 9 . 1 1 }}{4.91 \mathrm{I}} \text { gms. or roo0 cc. } \frac{\mathrm{N}}{\text { IO }} \mathrm{V} . \mathrm{S} \text {. } \\
& 0.0035^{18} \text { gms. } \\
& \text { I cc. } \frac{\mathrm{N}}{\mathrm{IO}} \text { V.S. }
\end{aligned}
$$

Estimation of Available Chlorin in Bleaching Powder. $3.5 \mathrm{gms}$. of the bleaching powder (chlorinated lime) are triturated thoroughly with $50 \mathrm{cc}$. of water, and the mixture transferred to a. graduated vessel, together with the rinsings, and made up to rooo cc. with water. This is thoroughly shaken. roo cc. of it (representing $0.35 \mathrm{gm}$. of 
the sample) is removed by means of a pipette and titrated with decinormal arsenous acid solution, as described in the foregoing assay, using either the iodized starch test paper as indicator or retitrating the excess of $\frac{\mathrm{N}}{\mathrm{IO}}$ arsenous acid solution, added by means of $\frac{\mathrm{N}}{\mathrm{IO}}$ iodin solution. chlorin.

Each cc. of $\frac{\mathrm{N}}{\mathrm{IO}} \mathrm{As}_{2} \mathrm{O}_{3}$ V. S. represents $0.0035 \mathrm{I} 8 \mathrm{gm}$. of available

$$
\begin{aligned}
& { }_{2} \mathrm{Ca}(\mathrm{OCl}) \mathrm{Cl}+\mathrm{As}_{2} \mathrm{O}_{3}=\mathrm{As}_{2} \mathrm{O}_{5}+{ }_{2} \mathrm{CaCl}_{2} \text {. } \\
& \text { 4) } 140.72 \quad 4) 196.44 \\
& \text { 10) } 35.18 \quad \text { 10) } 49.11 \\
& 3.518 \text { gms. }=4.911 \text { gms. or } 1000 \text { cc. } \frac{N}{10} \text { V.S. }
\end{aligned}
$$

As seen by referring to the above equation this process determines the value of the chlorinated lime by measuring the amount of arsenous acid which the oxygen present in the active constituent $(\mathrm{Ca}(\mathrm{OCl}) \mathrm{Cl})$ is capable of oxidizing. In the formula of this compound there are two atoms of chlorin and one atom of oxygen. Therefore the quantity of bleaching powder which yields 35.18 parts of available chlorin will also supply 8 parts of oxygen; this may therefore be taken as the measure of the chlorin. The same method may be employed for the assay of all other solutions containing available chlorin.

Assay of Manganese Dioxid (Chlorometric). The chlorometric assay of manganese dioxid, as well as that of all other bodies which liberate chlorin when heated with hydrochloric acid, may be made in similar manner to that described for the iodometric assays of these substances, the same apparatus, etc., being used. solution.

The liberated chlorin is, however, titrated with $\frac{\mathrm{N}}{\mathrm{IO}}$ arsenous acid

The chlorin may be distilled into a solution of sodium carbonate and this solution then titrated with $\frac{\mathrm{N}}{\mathrm{IO}}$ arsenous acid or the chlorin may be distilled directly into a measured volume of $\frac{\mathrm{N}}{\mathrm{IO}}$ arsenous acid solution and the latter then titrated with $\frac{\mathrm{N}}{\mathrm{IO}}$ iodin solution, using starch as indicator, the difference between the volume of iodin solution used and that of the arsenous acid solution taken is the measure of the latter which reacted with the chlorin. 
It is a good plan in each case to divide the solution into two or three equal parts and to titrate each separately.

\section{REDUCTION METHODS INVOLVING THE USE OF STANNOUS CHLORID}

Stannous chlorid $\left(\mathrm{SnCl}_{2}\right)$ is a very powerful reducing agent. Its action in this respect depends upon its affinity for chlorin which it readily abstracts from most other chlorids. In its action upon mercuric chlorid, a portion of the latter is always reduced to the metallic state.

This reducing action of stannous chlorid is utilized in certain volumetric processes, especially in the estimation of iron. In this case it possesses an advantage over permanganate, in that the iron must be in the ferric state, in which condition it is most usually found, while if permanganate is used, a preliminary reduction to the ferrous state is necessary before titrating. The great disadvantage, however, is in the fact that even short contact with air will quickly oxidize it, and thus spoil its titer. In consequence of this it must be frequently tested, and can be used only in the form of empirical solutions.

It is particularly useful in the titration of ferric salts, which salts can be accurately estimated by direct titration with it, the end-point being recognized by the disappearance of the yellow color of the ferric solution. These salts may also be estimated residually by adding an excess of stannous chlorid solution of known strength and retitrating the excess by means of standard iodin, using starch as indicator.

The reactions are:

and

$$
\mathrm{Fe}_{2} \mathrm{Cl}_{6}+\mathrm{SnCl}_{2}={ }_{2} \mathrm{FeCl}_{2}+\mathrm{SnCl}_{4}
$$

$$
\mathrm{SnCl}_{2}+{ }_{2} \mathrm{HCl}+\mathrm{I}_{2}=\mathrm{SnCl}_{4}+{ }_{2} \mathrm{HI} \text {. }
$$

The Estimation of Iron by Means of Stannous Chlorid Solutions may be accurately affected by the following procedure, as suggested by Fresenius. The solutions necessary are:

(a) A solution of ferric chlorid containing Io gms. of pure iron in a liter.

This is made by dissolving 10.04 gms. of thin annealed binding wire (which contains 99.6 per cent of pure iron) in a sufficient quantity of pure hydrochloric acid. A small quantity of potassium chlorate is then added to effect complete oxidation of the iron, and the excess 
of chlorin expelled by boiling. This solution is then cooled and diluted to one liter.

(b) A solution of stannous chlorid made by dissolving about ro gms. of pure tin in $200 \mathrm{cc}$. of strong, pure hydrochloric acid. This may be done by heating the tin in small pieces with the acid in a flask, and introducing a few pieces of platinum foil to excite galvanic action. The solution so obtained is diluted to about one liter with distilled

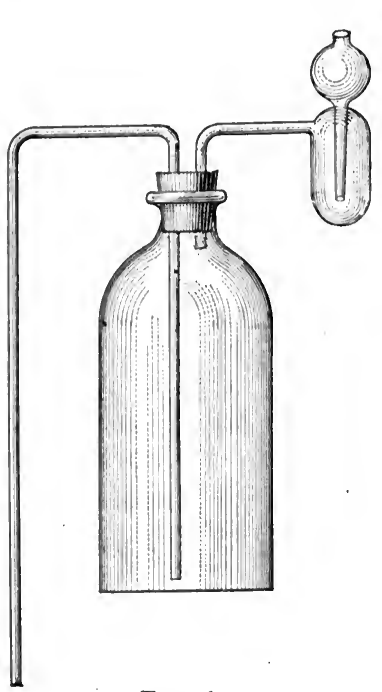

Fig. 65. water and should be preserved in a bottle, such as shown in Fig. 65 , to which air can only gain access through a strongly alkaline solution of pyrogallic acid. When so kept the strength of the solution can be preserved for several weeks.

(c) A solution of iodin in potassium iodid. This may be approximately or exactly decinormal.

The procedure is as follows:

Ist. The relation between the tin solution and the iodin solution is found.

$2 \mathrm{~d}$. The relation between the tin solution and the iron solution is determined.

3d. The assay.

The relation between the tin solution and the iodin solution is found as follows:

Two cubic centimeters of the tin solution are put into a beaker, a little starch solution added, and the iodin solution then delivered in from a burette until the blue color occurs. If $4 \mathrm{cc}$. are used then each 2 cc. of iodin solution represents I cc. of tin solution.

The relation between the tin solution and the iron solution is found as follows:

Fifty cubic centimeters of the iron solution (representing $0.5 \mathrm{gm}$. of iron) are put into a small flask together with a little hydrochloric acid and heated to gentle boiling. The tin solution is then delivered from a burette until the yellow color of the iron solution is nearly discharged. It is then added continuously, drop by drop, until the color is entirely gone. Assuming that $35 \mathrm{cc}$. were required, then each $35 \mathrm{cc}$. of tin solution are equivalent to $0.5 \mathrm{gm}$. of pure iron. If the end-point is not clearly recognized and an excess of the tin solution 
was added, the solution should be quickly cooled, a few drops of starch solution added, and the excess estimated by titrating with the iodin solution, each cc. of which represents $0.5 \mathrm{cc}$. of the tin solution. The excess so found, deducted from the total quantity of tin solution added, gives the quantity of the latter, which corresponds to $0.5 \mathrm{gm}$. of iron.

Having determined these data, the analyst can readily estimate any unknown quantity of iron in solution in the ferric state.

If the iron is partly or wholly in the ferrous state it may be oxidized by adding some potassium chlorate and boiling to expel excess of chlorin.

The Assay. A solution of iron taken for analysis, required $24 \mathrm{cc}$. of the tin solution. The quantity of iron present is calculated by proportion as follows:

$$
35 \text { cc. : } 0.5 \text { gm. :: } 24 \text { cc. : } x ; \quad x=0.34 \text { gm. }
$$

To secure accurate results the iron solution assayed must be fairly concentrated, because then the end-reaction is more readily seen, and also because the greater the dilution the larger the amount of tin solution will be required. It is good policy to use very little excess of the tin solution, so that only a very small quantity of iodin solution is required.

Estimation of Mercuric Salts (Laborde). This depends upon the fact that stannous chlorid solution added to a solution of a mercuric salt reduces the latter first to mercurous chlorid (calomel) and finally the calomel to metallic mercury. The reduction to calomel results in the formation of a white precipitate, and when the mercuric salt is completely reduced the stannous chlorid acts upon and reduces the calomel to metallic mercury, which results in the production of a characteristic brownish color.

The reactions are as follows:

$$
\begin{aligned}
\mathrm{SnCl}_{2}+{ }_{2} \mathrm{HgCl}_{2} & =\mathrm{SnCl}_{4}+{ }_{2} \mathrm{HgCl} ; \\
\mathrm{SnCl}_{2}+{ }_{2} \mathrm{HgCl} & =\mathrm{SnCl}_{4}+\mathrm{Hg}_{2} .
\end{aligned}
$$

According to Laborde the tin solution is made by dissolving $8 \mathrm{gms}$. of pure tinfoil by means of heat in $100 \mathrm{cc}$. of pure hydrochloric acid, and diluting to 2 liters.

This tin solution is checked against a solution of mercuric chlorid containing ro gms. per liter. To counteract the hindering effect of 
the hydrochloric acid the solution under analysis, containing $0.1 \mathrm{gm}$. of mercuric chlorid, is mixed with $5 \mathrm{cc}$. of a solution containing $100 \mathrm{gms}$. of anmonium acetate and $100 \mathrm{cc}$. of acetic acid to the liter. The acetic acid promotes the disappearance of the brown color which occurs at the point where the tin solution is in excess, but before reduction is complete. The titration with the tin solution is continued until a permanent brown color occurs.

If the brown color is too dark from overstepping of the end-point, the addition of I cc. of the mercuric chlorid solution will render the solution white again, and the titration can then be carried further.

This method, which is convenient, rapid, and very accurate, can be employed in many cases. If the mercuric solution contains any free mineral'acids, the latter must be neutralized with ammonia (in the presence of ammonium acetate, to prevent formation of ammoniated mercury.)

The presence of alkali and alkali earth salts or most salts of other metals (except iron, gold and platinum), do not in the least interfere with the accuracy of the results. The same is true of organic acids, either free or in combination with alkali. 


\section{PART II}

\section{CHAPTER XIII}

\section{ACETIC ACID AND ACETATES}

Vinegar. Vinegar is impure diluted acetic acid. Its strength may be estimated in the same manner as acetic acid.* Phenolphthalein must be used as an indicator. Litmus will give only approximate results, because potassium and sodium acetate both have a slightly alkaline reaction with litmus, but show no reaction with phenolphthalein. $\dagger$ The absence of mineral acids must be assured before the volumetric test is applied.

The strength of vinegar may also be estimated by distilling i $10 \mathrm{cc}$. until roo cc. come over. The roo cc. will contain 80 per cent of the whole acetic acid present in the rIo cc., and may be titrated; or the specific gravity of the distillate may be taken, and, by consulting the table on the next page, the per cent strength of the distillate found. By adding 20 per cent to this the strength of the original vinegar is obtained.

Vinegar usually contains from three per cent to six per cent of acetic acid.

\section{ESTIMATION OF FREE MINERAL ACIDS IN VINEGAR}

Mr. Hehner has devised the method given below, which has the merit of being speedy, scientific, and accurate.

The method is based upon the fact that acetates of the alkalies are always present in commercial vinegar, and when vinegar is evaporated to dryness, and the ash ignited, the acetates of the alkalies are converted into carbonates. If the ash has an alkaline reaction no

* See page ro4.

† Even dark-colored vinegar may be titrated in this way when diluted. If the color, however, is too dark, litmus-paper or phenolphthalein paper may be used by bringing a drop of the liquid in contact with the paper from time to time during the titration. 
ACETIC ACID TABLE

\begin{tabular}{|c|c|c|c|c|c|}
\hline $\begin{array}{l}\text { Per Cent } \\
\text { of } \\
\text { Absolute } \\
\text { Acetic } \\
\text { Acid. }\end{array}$ & $\begin{array}{l}\text { Specific Gravity } \\
\text { at }\left\{\begin{array}{l}15^{\circ} \mathrm{C} . \\
59^{\circ} \mathrm{F} .\end{array}\right.\end{array}$ & $\begin{array}{l}\text { Per Cent } \\
\text { of } \\
\text { Absolute } \\
\text { Acetic } \\
\text { Acid. }\end{array}$ & $\begin{array}{l}\text { Specific Gravity } \\
\text { at }\left\{\begin{array}{l}15^{\circ} \mathrm{C} . \\
59^{\circ} \mathrm{F} .\end{array}\right.\end{array}$ & $\begin{array}{l}\text { Per Cent } \\
\text { of } \\
\text { Absolute } \\
\text { Acetic } \\
\text { Acid. }\end{array}$ & $\begin{array}{l}\text { Specific Gravit } \\
\text { at }\left\{\begin{array}{l}15^{\circ} \mathrm{C} . \\
59^{\circ} \mathrm{F} .\end{array}\right.\end{array}$ \\
\hline I & I. .0007 & 26 & I. $0_{3}{ }^{6} 3$ & $5^{I}$ & 1.0623 \\
\hline 2 & 1.0022 & 27 & I. .0375 & $5^{2}$ & I. $.063 I$ \\
\hline 3 & I. . 0037 & 28 & I. 0388 & 53 & I. 06,38 \\
\hline 4 & 1.0052 & 29 & 1.0400 & 54 & I. .0646 \\
\hline 5 & I. . 0067 & 30 & I. 0412 & 55 & I. .653 \\
\hline 6 & 1.0083 & 31 & I. 0424 & $5^{6}$ & 1.0660 \\
\hline 7 & I. . 0098 & $3^{2}$ & I. $043^{6}$ & 57 & I. .0666 \\
\hline 8 & I.OII 3 & 33 & I. .0447 & $5^{8}$ & I. 0673 \\
\hline 9 & I.OI 27 & 34 & I. 0459 & 59 & I. 0679 \\
\hline IO & 1.0142 & 35 & I. 0470 & 60 & I. .0685 \\
\hline II & I.OI 57 & $3^{6}$ & I. 048 I & $6 I$ & I. $069 \mathrm{r}$ \\
\hline 12 & I.OI 71 & 37 & I. $049^{2}$ & 62 & I. .0697 \\
\hline 13 & I.0185 & $3^{8}$ & I. 0502 & 63 & 1.0702 \\
\hline 14 & 1.0200 & 39 & 1.0513 & 64 & I. 0707 \\
\hline I 5 & 1.0214 & 40 & 1.0523 & 65 & 1.0712 \\
\hline 16 & I. .0228 & $4 I$ & I. 0533 & 66 & I.0717 \\
\hline 17 & 1.0242 & 42 & 1.0543 & 67 & I. 0721 \\
\hline 18 & I. 0256 & 43 & $1.055^{2}$ & 68 & I. 0725 \\
\hline 19 & 1.0270 & 44 & I. 0562 & 69 & I. 0729 \\
\hline 20 & I. 0284 & 45 & I. 0571 & 70 & I. .0733 \\
\hline $2 I$ & 1.0298 & 46 & I. 0580 & 71 & I. .0737 \\
\hline 22 & I. O3 II & 47 & I. $05^{8} 9$ & 72 & I. 0740 \\
\hline 23 & 1.0324 & 48 & I. 0598 & 73 & I. .0742 \\
\hline 24 & I. 0337 & 49 & I. .0607 & 74 & I. 0744 \\
\hline 25 & $1.035^{\circ}$ & 50 & I. 06 I 5 & 75 & 1.0746 \\
\hline
\end{tabular}

free mineral acid is present. If, however, the ash is neutral or acid some free mineral acid must be present.

The quantitative process in detail is as follows: $50 \mathrm{cc}$. of vinegar are mixed with $25 \mathrm{cc}$. of $\frac{\mathrm{N}}{\mathrm{I}}$ soda or potash. The liquid is evaporated to dryness on a water-bath, and the residue carefully incinerated at the lowest possible temperature, to convert the acetates into carbonates. When cooled, $25 \mathrm{cc}$. of $\frac{\mathrm{N}}{\mathrm{IO}}$ sulphuric acid are added, the mixture heated to expel $\mathrm{CO}_{2}$ and filtered. The filter is washed with hot water, phenolphthalein T. S. added, and the filtrate and washings carefully titrated with $\frac{\mathrm{N}}{\mathrm{IO}}$ alkali. Each cc. of $\frac{\mathrm{N}}{\mathrm{IO}}$ alkali used represents $0.0046875 \mathrm{gm} . \mathrm{H}_{2} \mathrm{SO}_{4}$ or $0.0036 \mathrm{I} 8 \mathrm{gm}$. $\mathrm{HCl}$. 
Mohr's Method for Estimating Acetic Acid in Vinegar. Take 20 gms. of the vinegar, add an excess of pure precipitated calcium carbonate (say 3 gms.), set aside until reaction is complete, shaking occasionally, and then boil to drive off the $\mathrm{CO}_{2}$.

Now separate the residual calcium carbonate by filtration, wash it thoroughly with boiling water, and dissolve in a measured excess of $\frac{\mathrm{N}}{\mathrm{I}}$ hydrochloric acid, say $35 \mathrm{cc}$, and titrate back with $\frac{\mathrm{N}}{\mathrm{I}}$ sodium hydroxid, using phenolphthalein as indicator. Assuming that $4 \mathrm{cc}$. were used, then $35-4=3 \mathrm{I}$, the number of cc. of $\frac{\mathrm{N}}{\mathrm{I}}$ hydrochloric acid which reacted with the residual calcium carbonate. Thus we have $3 \mathrm{I} X$ $0.049675 \mathrm{gm} .=\mathrm{r} .54 \mathrm{gms}$. of residual calcium carbonate. Deduct this from the 3 gms. taken, and we arrive at the quantity which was taken up by the acetic acid, namely, I.46 gms.

Therefore the 20 gms. of vinegar contain

$$
\frac{\mathrm{I} .46 \times \mathrm{II} 9.16}{99.35}=\mathrm{I} .75 \text { gms. or } 8.75 \text { per cent. }
$$

The reactions are as follows:

$$
\mathrm{CaCO}_{3}+{ }_{2} \mathrm{HC}_{2} \mathrm{H}_{3} \mathrm{O}_{2}=\mathrm{Ca}\left(\mathrm{C}_{2} \mathrm{H}_{3} \mathrm{O}_{2}\right)_{2}+\mathrm{H}_{2} \mathrm{O}+\mathrm{CO}_{2} \text {. }
$$

$$
99.35 \quad \text { I19.16 }
$$

Hence I gm. of calcium carbonate represents I.2 gms. of acetic acid.

$$
\begin{aligned}
& \mathrm{CaCO}_{3}+{ }_{2} \mathrm{HCl}=\mathrm{CaCl}_{2}+\mathrm{H}_{2} \mathrm{O}+\mathrm{CO}_{2} \text {. }
\end{aligned}
$$

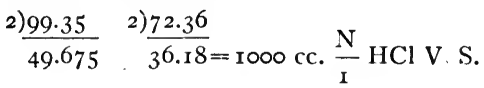

$$
\begin{aligned}
& 0.049675=\text { I сс." “" “ }
\end{aligned}
$$

This process answers well for dark-colored liquids and is especially seful for impure brown pyroligneous acid.

Pettenkofer's Method. A measured excess of standard barium ydroxid solution is added to the acetic acid or the vinegar, and titrated ack with decinormal acid, using turmeric paper as indicator. This pethod is the best for high-colored vinegars. 


\section{ESTIMATION OF METALliC ACETATES}

Acetates of lead, iron, etc., are treated with an excess of normal alkali carbonate, which precipitates the metal as carbonate while an alkali acetate is formed in the solution. The mixture is boiled, filtered, and the precipitate thoroughly washed on the filter with hot water. The filtrate and washings are mixed and made up to a definite volume. An aliquot portion is taken out and titrated with $\frac{N}{I}$ acid solution. The difference between the quantity of acid used and that of the alkali carbonate originally added is calculated into acetate by multiplying by $0.06 \mathrm{gm}$. If other salts than acetate are present, proceed as follows: Add excess of alkali carbonate solution to precipitate the metal, exactly neutralize the filtrate with hydrochloric acid, evaporate to dryness, ignite the residue to convert the acetate into carbonate, and then titrate with normal acid solution in the usual way.

Any other organic acid present will of course be recorded as acetic.

Acetates of Sodium and Potassium. These acetates may be estimated by ignition, which reduces them to carbonates, when they can be titrated with standard acid, as described under Analysis of Organic Salts of the Alkalies, page 83 .

Acetate of Lime. The presence of tarry matters and other impurities in the commercial acetate of lime makes it necessary to adopt special methods for its valuation. In the case of very impure, darkcolored samples the acetic acid can only be estimated by distillation with phosphoric acid and water to nearly dryness, and then estimating the acetic acid direct with $\frac{\mathrm{N}}{\mathrm{IO}}$ alkali. The distillation should be conducted in a retort, so arranged that there can be no vitiation of the distillate through sputtering of the acid liquid in the retort. I gm. of the sample is placed in a retort and 10 cc. of a 40 per cent phosphoric acid added, together with about $40 \mathrm{cc}$. of water, and the distillation commenced over a naked flame and continued to near dryness. The retort is then cooled, $50 \mathrm{cc}$. of water introduced, and the distillation agrain performed, and the same repeated a third time. This will be sufficient to carry over all of the acetic acid. The combined distillates are then titrated with $\frac{\mathrm{N}}{\mathrm{IO}}$ alkali, using phenolphthalein as indicator.

II eber's Method (Z. A. C., Xxiv 6r4) is based on the insolubility of silver acetate in alcohol. Io gms. of the sample are powdered and placed in a $250 \mathrm{cc}$. flask, a little water added and heated to extract all soluble matter; then after cooling, the mixture is diluted to $250 \mathrm{cc}$; the solution filtered and $25 \mathrm{cc}$. of the filtrate mixed in a beaker with $50 \mathrm{cc}$. of absolute alcohol, and the acetic acid precipitated by adding 
alcoholic solution of silver nitrate. The precipitate, which consitss of silver acetate, together with any chlorid, sulphate, etc., is then washed on a filter with 60 per cent alcohol, till all free silver nitrate is removed. The precipitate is then dissolved in weak $\mathrm{HNO}_{3}$ and the solution titrated with $\frac{\mathrm{N}}{\mathrm{IO}} \mathrm{NaCl}$. This method gives very satisfactory results.

K. R. Haberland (Zeitsch f. Anal. Chem., I899, 217), submits the following method for the estimation of commercial acetates:

Ten grams of the finely powdered acetate of lime are introduced into a $300 \mathrm{cc}$. flask, together with $50 \mathrm{cc}$. of water and I $\mathrm{cc}$. of hydrochloric acid (sp.gr. I.I24), a condenser is attached and the mixture distilled until the fluid is of a syrupy consistency, then 50 or $60 \mathrm{cc}$. of distilled water are added and the distillation continued to dryness. The distillate is received in a $250 \mathrm{cc}$. flask and water added to the mark.

The distillate which contains hydrochloric-acid as well as acetic acid is titrated for total acidity in one portion, and for hydrochloric acid in another, as follows:

Fifty cubic centimeters of the distillate are titrated with normal sodium hydroxid, using phenolphthalein as indicator. The quantity of normal alkali used multiplied by 5 gives total acidity.

Then $25 \mathrm{cc}$. of the distillate are placed into a $100 \mathrm{cc}$. flask, $15 \mathrm{cc}$. of pure nitric acid are added, followed by an excess of decinormal silver nitrate (the quantity of which is known), and finally distilled water to make $100 \mathrm{cc}$. After complete subsidence of the precipitate, $50 \mathrm{cc}$. of the clear supernatant liquid are removed and titrated with decinormal ammonium sulphocyanate, using ammonio-ferric sulphate as indicator. The amount of sulphocyanate solution used, multiplied by 2 , is deducted from the quantity of decinormal silver nitrate solution added. This gives the quantity of the latter, which represents the hydrochloric acid present in $25 \mathrm{cc}$. of the distillate. 


\section{CHAPTER XIV}

\section{BORIC ACID AND BORATES}

Free Boric Acid may be estimated by means of barium hydroxid, as suggested by Will. The method is said to be fairly accurate.

The boric acid solution is titrated with a barium hydroxid solution of known strength, until the turbidity appearing at first is completely and exactly removed.

The equation is as follows:

$$
{ }_{4} \mathrm{H}_{3} \mathrm{BO}_{3}+\mathrm{Ba}(\mathrm{OH})_{2}=\mathrm{BaB}_{4} \mathrm{O}_{7}+{ }_{7} \mathrm{H}_{2} \mathrm{O} .
$$

Thompson's Method (Jour. Soc. Chem. Ind., XII, 432). The addition of glycerin to a boric acid solution to the extent of 30 per cent develops the acidity of the acid to a great degree. It may then be titrated with standard sodium hydroxid solution, using phenolphthalein as indicator. (See page 104.)

Boric Acid in Borax may be estimated as follows:

Add methyl orange solution (on which $\mathrm{H}_{3} \mathrm{BO}_{3}$ has no effect) to the solution of borax, and then just sufficient standard sulphuric acid solution to acidulate. Boil and exactly neutralize with standard sodium hydroxid. All the boric acid is now in a free state; sufficient glycerin is now added so that the solution contains at least 30 per cent, and the titration with standard sodium hydroxid is begun, in the presence of phenolphthalein.

$$
\begin{aligned}
& \text { I cc. } \frac{\mathrm{N}}{\mathrm{I}} \mathrm{NaOH}=0.06 \mathrm{I}_{54} \mathrm{gm} . \mathrm{H}_{3} \mathrm{BO}_{3} ; \\
& \text { I cc. } \frac{\mathrm{N}}{\mathrm{I}} \mathrm{NaOH}=0.05013 \mathrm{gm} . \mathrm{Na}_{2} \mathrm{~B}_{4} \mathrm{O}_{7} .
\end{aligned}
$$

E. F. Smith's Process (Am. Chem. Jour., 1882). Take ro cc. of borax solution containing $0.1 \mathrm{gm}$.; add ro cc. of solution of manganese sulphate, containing $0.06 \mathrm{gm}$. of $\mathrm{MnSO}_{4}$, and finally $20 \mathrm{cc}$. of strong alcohol. A white flocculent precipitate of $\mathrm{MnB}_{4} \mathrm{O}_{7}$ separates. Set aside for half an hour to settle; filtcr, wash the precipitate with alcohol, and evaporate the filtrate and washings to dryness. Then dissolve the 
residual manganese salt in water, add some strong solution of zinc sulphate, heat to near the boiling point, and titrate with potassium permanganate until a permanent pink is produced. Each cc. of the permanganate represents $0.0044985 \mathrm{gm}$. of $\mathrm{MnSO}_{4}$.

In the above titration $6.4 \mathrm{cc}$. were required $=0.02879 \mathrm{gm}$.

This deducted from the $0.06 \mathrm{gm}$. added gives us $0.031209 \mathrm{gm}$., the amount which combined with the borax.

I 49.95 gms. $\mathrm{MnSO}_{4}=200.5^{2}$ gms. $\mathrm{Na}_{2} \mathrm{~B}_{4} \mathrm{O}_{7}$

I 49.95 gms. $\mathrm{MnSO}_{4}=\mathrm{I}_{3} 8.88$ gms. $\mathrm{B}_{2} \mathrm{O}_{3}$.

Thus the O.I gm. of borax analyzed contained

$0.04 \mathrm{I} 7 \mathrm{gm}$. of pure $\mathrm{Na}_{2} \mathrm{~B}_{4} \mathrm{O}_{7}=4 \mathrm{I} .7$ per cent

or

$$
0.0289 \text { gm. of } \mathrm{B}_{2} \mathrm{O}_{3} \quad=28.9 \text { per cent. }
$$

The reactions are as follows:

$$
\begin{gathered}
\mathrm{Na}_{2} \mathrm{~B}_{4} \mathrm{O}_{7}+\mathrm{MnSO}_{4}=\mathrm{MnB}_{4} \mathrm{O}_{7}+\mathrm{Na}_{2} \mathrm{SO}_{4} . \\
{ }_{3} 00.5^{2} \quad{ }^{149.95} \\
\mathrm{MnSO}_{4}+{ }_{449.85} \mathrm{KMnO}_{4}+{ }_{2} \mathrm{H}_{2} \mathrm{O}=\mathrm{K}_{2} \mathrm{SO}_{4}+{ }_{3} \mathrm{MnSO}_{4}+{ }_{2} \mathrm{H}_{2} \mathrm{SO}_{4} .
\end{gathered}
$$

With Ferric Salicylate as Indicator. Jules Wollf (Compt. rend., I30-II28) suggests the use of a solution of ferric salicylate in sodium salicylate as an indicator for the titration of boric acid and borates.

In the case of borax, for instance, the solution is treated with a known volume of standard acid in excess. The ferric salicylate indicator is then added, and the free acid is titrated until the violet tint is replaced by a clear madder-red color. The end-reaction is very sharp. If ammonia salts are present an excess of soda is added and the mixture boiled to drive off the ammonia before adding the acid.

The indicator is prepared as follows: 5 to 6 gms. of sodium salicylate are dissolved in $25 \mathrm{cc}$. of distilled water, ferric chlorid solution added drop by drop until a slight permanent turbidity results, the solution is filtered and divided into two equal parts; to one a sufficient quantity of soda solution is added to give a deep orange tint, the other is treated with acid to the development of a red tint. The two are then mixed and ro gm. of sodium salicylate dissolved in the mixture. 
Gladding's Process (J. A. C. S., I898, 288). I gm. of the substance to be examined is placed in a flask resting on a wire screen over a Bunsen burner, together with a little 95-per-cent methyl alcohol and 5 cc. of syrupy phosphoric acid. Flask A (Fig. 66), two-thirds filled with methyl alcohol, is placed on the water-bath E. Flask B is connected with condenser $\mathrm{D}$, and flask $\mathrm{C}$ connected to receive the distillate. Heat is now applied to the water-bath $\mathrm{E}$, and when the methyl alcohol boils, flask $\mathrm{A}$ is connected to the tube which passes to the bottom of flask B. A current of methyl-alcohol vapor is thus continually passing through the liquid in flask $\mathrm{B}$, and carries over the boric acid. Heat is applied under flask $\mathrm{B}$ and so regulated that the liquid remains between 15 and $25^{\circ} \mathrm{C}$.

The distillation is carried on in this way for about half an hour, or until about $100 \mathrm{cc}$. of distillate are obtained. 'A mixture of $40 \mathrm{cc}$.

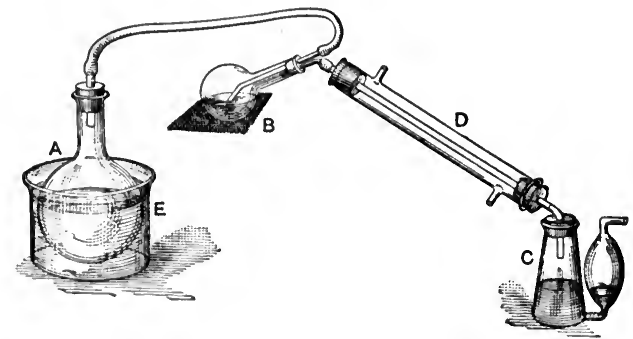

FIG. 66.

glycerin and roo cc. water is now carefully neutralized, using phenolphthalein as indicator, and then added to the distillate, and the mixture titrated with standard soda solution. A blank assay should be made, using all the reagents, and any acidity found deducted from the final results. Gladding found 36.57 per cent of boric acid in borax, the theoretical per cent being 36.65 , while boric acid itself gave 99.9 per cent.

In this process the distillation must be continued until all of the boric acid has passed over. This usually occupies about half an hour.

The method depends upon the fact that boric acid in alcoholic solution is remarkably volatile. If a vessel containing such a solution be covered with a glass plate and allowed to stand for about twentyfour hours at the ordinary temperature of the atmosphere, a distinct white coating of boric acid will be deposited upon the glass. The presence of boric acid in a substance may be determined by treating it with sulphuric acid and alcohol in a test-tube closed with a stopper bearing a bent glass tube, boric acid being then deposited in the tube. 
The quantitative analysis of boric acid in mixtures or compounds may be made in this way. The acid is first extracted with absolute alcohol in an extraction apparatus provided with a reflux condenser and the solution so obtained distilled.

Gladding found that a distillation of crystallized boric acid made without the addition of phosphoric or other acid yielded all of the boric acid present. A similar distillation of borax he found to yield 19.50 per cent of boric acid out of a total of 36.65 per cent present, or slightly more than 50 per cent of the whole amount. 


\section{CHAPTER XV}

\section{CARBONIC ACID AND CARBONATES}

ALKALI carbonates may be accurately estimated by titration with standard acid solutions, as described on page 177 .

They may also be estimated by precipitation with calcium or barium chlorid and the precipitated carbonate then treated with an excess of standard acid, and retitrated with standard alkali.

Calcium chlorid is preferred where it can be used, because the physical characters of the calcium carbonate are such as to render it more rapidly and thoroughly washed than is the case with barum carbonate. If caustic alkalies are present, however, barium chlorid must be used, as calcium hydroxid is very insoluble, and is in consequence precipitated with the carbonate.

If ammonia is present, the precipitation of calcium carbonate or barium carbonate is not complete. In this case it is necessary to heat the mixture for several hours.

Example. The carbonate is dissolved in water, heated and treated with calcium or barium chlorid in excess, and the mixture boiled for a few minutes, filtered, and the precipitate rapidly washed with several portions of hot water. The precipitate together with the filter is placed in a flask and a measured excess of normal acid added, and the mixture boiled until the precipitate is dissolved and the $\mathrm{CO}_{2}$ expelled.

Phenolphthalein is then added, and lastly normal alkali from a burette until a faint pink appears.

The quantity of normal alkali used is deducted from the acid added, and the quantity of the latter which went into combination with the precipitate found. The reactions are written thus:

$$
\mathrm{Na}_{2} \mathrm{CO}_{3}+\mathrm{BaCl}_{2}=\mathrm{BaCO}_{3}+2 \mathrm{NaCl} ;
$$

then

$$
\mathrm{BaCO}_{3}+{ }_{2} \mathrm{HCl}=\mathrm{BaCl}_{2}+\mathrm{H}_{2} \mathrm{O}+\mathrm{CO}_{2} .
$$

The factors for the alkali carbonates are the same as when estimated by direct titration with acid, which see. See also the Gasometric Method, page 69r. 


\section{CARBONIC ACID IN INSOLUBLE CARBONATES}

This may be estimated by. decomposition with an acid, and conducting the $\mathrm{CO}_{2}$ into strong ammonia-water which absorbs it completely. The $\mathrm{CO}_{2}$ is then precipitated by calcium chlorid and estimated as explained under alkali carbonates. The ammonia-water must be free from $\mathrm{CO}_{2}$. If any be present, it must be removed by means of calcium chlorid. The decomposition is effected in the apparatus shown in Fig. 67. The carbonate and some water is put in $A$, hydrochloric acid in $b$, ammonia water in $B$, and some pieces of broken glass in $c$, through which the ammonia is poured into the flask.

The flask containing the ammonia-water is heated until it is filled with its fumes. Then the hydrochloric acid is run into the carbonate by opening the pinch-cock, and when decomposition of the carbonate is complete the liquid is boiled, and finally a slow current of air free from $\mathrm{CO}_{2}$ is drawn through the apparatus to carry over the last traces of the gas.

The apparatus is then disconnected, $c$ is rinsed into $B$, calcium chlorid added and the solution boiled for some time, and the prerifitated carbonate treated as explained in the foregoing process.

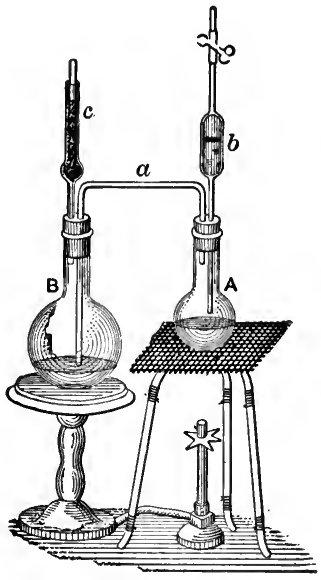

FIG. 67 . In either of the foregcing processes the precipitated barium or calcium carbonate may be dissolved in hydrochloric acid, evaporated to dryness and the amount of chlorin, as chlorid, found by means of $\frac{\mathrm{N}}{10}$ silver nitrate solution in presence of chromate.

I cc. $\frac{\mathrm{N}}{10}$ acid or silver $=0.0021835 \mathrm{gm} . \mathrm{CO}_{2}$;

I cc. $\frac{\mathrm{N}}{\mathrm{IO}}$ acid or silver $=0.00_{52}^{26} \mathrm{gm} . \mathrm{Na}_{2} \mathrm{CO}_{3}$.

Certain insoluble carbonates may also be estimated, by adding to a weighed quantity of the salt a measured excess of $\frac{\mathrm{N}}{\mathrm{I}}$ sulphuric acid, then boiling to drive off $\mathrm{CO}_{2}$ and titrating the excess of acid 
by means of $\frac{\mathrm{N}}{\mathrm{I}}$ or $\frac{\mathrm{N}}{10}$ alkali, as described under Estimation of Calcium Carbonate, page $9 \mathrm{I}$.

In alkali carbonates containing bicarbonate, the excess of carbonic acid over that necessary to form neutral carbonate may be found by adding barium chlorid in excess to the somewhat dilute solution, then standard baryta-water in excess, and estimating the excess by $\frac{\mathrm{N}}{\mathrm{IO}}$ oxalic acid, as above. In this case, all the carbonic acid contained as neutral carbonate is precipitated by the barium chlorid first added, but the remainder stays in solution, and is estimated by addition of the baryta-water. See page 82 .

Carbonic acid in solution in water may be measured by adding an excess of standard baryta-water, and estimating the excess by $\frac{\mathrm{N}}{\mathrm{IO}}$ oxalic acid. The whole of the carbonic acid is precipitated as carbonate immediately upon addition of the baryta-water, and the excess is found by adding $\frac{\mathrm{N}}{\mathrm{I}}$ oxalic or hydrochloric acid, until a glass rod dipped in the fluid no longer produces a brown-red spot upon turmeric paper. The end of the reaction is very sharply shown. When carbon dioxid is contained in a gaseous mixture, it may be estimated by passing through a measured volume of standard baryta-water, and, without filtering, estimating the excess of baryta.

$$
\text { cc. } \frac{\mathrm{N}}{10} \operatorname{acid} \times 0.002 \mathrm{1} 83=\mathrm{CO}_{2} \text {. }
$$

\section{CARBONIC-ACID GAS IN THE ATMOSPHERE}

This is done by the modified Pettenkofer's method as follows: A glass globe or bottle holding from 5 to ro liters is filled with the air to be tested, by means of a bellows; baryta-water of known strength is then introduced in convenient quantity.

The bottle is then securely closed and set aside for about one hour, rotating it at intervals, so that the liquid is spread over the entire inner wall of the bottle.

When the time is up the baryta-water is emptied out quickly into a beaker, covered carefully with a watch-glass, and when the barium carbonate has subsided a portion of the clear liquid is withdrawn and titrated with $\frac{\mathrm{N}}{10}$ oxalic acid solution.* The difference between the

\footnotetext{
* $\frac{\mathrm{N}}{\mathrm{IO}}$ hydrochloric acid may be used with equally good results.
} 
quantity of oxalic acid solution required to neutralize the barium hydroxid solution, before and after contact with the air, is the quantity equivalent to the carbonic acid gas absorbed.

The Baryta-water is made by dissolving about. 7 gms. of pure crystallized barium hydroxid in I000 cc. of distilled water.

This solution, being prone to absorb $\mathrm{CO}_{2}$ out of the air, must be kept in a special bottle, such as is illustrated in Fig. 49, which prevents access of $\mathrm{CO}_{2}$ and admits of the withdrawal of any quantity of solution without inverting the bottle.

The Bottle which is used to collect the air should hold from 5 to Io liters, and its exact capacity must be known. This may be found by filling the bottle to the bottom of the cork with water and then accurately measuring the water. Before using the bottle it must be absolutely dry.

The Analysis. Into the bottle, the capacity of which is exactly known-we will assume it to be $7100 \mathrm{cc}$.-is blown the air to be tested, by means of a bellows.

I00 cc. of the baryta-water are then introduced, thus leaving 7000 cc. of air in the bottle.

The bottle is now securely closed and set aside for about half an hour, rotating it occasionally so as to spread the liquid over the entire inner wall of the bottle. While waiting for the half hour to expire, a convenient quantity of baryta-water is taken and its strength compared with decinormal oxalic acid solution by titrating with the latter, using phenolphthalein as indicator.

50 cc. of baryta water is a convenient quantity. This is placed in a beaker, a few drops of phenolphthalein added, and then titrated with the $\frac{\mathrm{N}}{\mathrm{IO}}$ acid solution until the color just disappears.

. Let us assume that $40 \mathrm{cc}$. of the latter were consumed; $80 \mathrm{cc}$. will then be consumed by roo cc. of baryta-water.

$$
\begin{aligned}
& \mathrm{Ba}(\mathrm{OH})_{2}+\mathrm{H}_{2} \mathrm{C}_{2} \mathrm{O}_{4}+{ }_{2} \mathrm{H}_{2} \mathrm{O}=\mathrm{BaC}_{2} \mathrm{O}_{4}+{ }_{4} \mathrm{H}_{2} \mathrm{O} \text {; }
\end{aligned}
$$

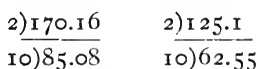

$$
\begin{aligned}
& \frac{85.08}{8.508} \text { gms. } \frac{102.55}{6.255} \text { gms. or } 1000 \text { cc. } \frac{N}{\text { IO }} \text { V.S. } \\
& \mathrm{Ba}(\mathrm{OH})_{2}+\mathrm{CO}_{2}=\mathrm{BaCO}_{3}+\mathrm{H}_{2} \mathrm{O} \text {. }
\end{aligned}
$$

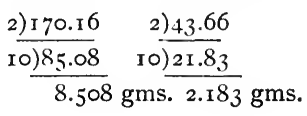


These equations show that 2.183 gms. of carbon dioxid will neutralize as much barium hydroxid as $1000 \mathrm{cc}$. of $\frac{\mathrm{N}}{\mathrm{IO}}$ oxalic acid solution. And thus each cc. of the $\frac{\mathrm{N}}{10}$ oxalic acid solution is chemically equivalent to $0.002183 \mathrm{gm}$. of carbon dioxid; therefore $100 \mathrm{cc}$. of the barytawater is capable of absorbing $80 \times 0.002183 \mathrm{gm} .=0.17464 \mathrm{gm}$. of $\mathrm{CO}_{2}$.

The next step is to determine the quantity of $\mathrm{CO}_{2}$ that was absorbed by the $100 \mathrm{cc}$. of baryta-water which was introduced into the bottle of air.

The liquid is poured out of the bottle into a small beaker, carefully covered with a watch-glass, and the barium carbonate allowed to settle. Then $50 \mathrm{cc}$. of the clear supernatant liquid are drawn out of the beaker by means of a pipette, treated with a few drops of phenolphthalein T. S., and titrated with the $\frac{\mathrm{N}}{10}$ oxalic acid until the red color is just discharged. Note the number of cc. consumed, double it, and deduct this number from 80 , the quantity which roo cc. of barytawater consumed before being brought in contact with $\mathrm{CO}_{2}$.

Example. Assuming that $3.0 \mathrm{cc}$. of the oxalic acid solution were required by the $50 \mathrm{cc}$. of the baryta-water after exposure, the roo cc. then would require $60 \mathrm{cc}$. There is thus a loss of alkalinity equivalent to $20 \mathrm{cc}$. of $\frac{\mathrm{N}}{\mathrm{IO}}$ oxalic acid. This is due to the absorption of carbon dioxide, which neutralizes the hydroxid by forming a carbonate.

Now since each cc. of $\frac{\mathrm{N}}{10}$ oxalic acid is chemically equivalent to $0.002183 \mathrm{gm}$. of $\mathrm{CO}_{2}$, the baryta-water must have absorbed

$$
20 \times 0.002183 \mathrm{gm} .=0.02366 \mathrm{gm} \text {. of } \mathrm{CO}_{2} .
$$

Therefore the $7000 \mathrm{cc}$. of air which the bottle held, contained 0.04366 gm. of $\mathrm{CO}_{2}$.

In stating the result of an analysis the quantity of $\mathrm{CO}_{2}$ by volume in $10,000 \mathrm{cc}$. of air is generally given.

In the above case $7000 \mathrm{cc}$. of air contained $0.04366 \mathrm{gm}$. of $\mathrm{CO}_{2}$; I0,000 cc. of this same air, then, contains

$$
\frac{0.04366 \times 10,000}{7000} \text { or } \frac{0.04366 \times \text { i0 }}{7}=0.06237 \mathrm{gm} .
$$


If several bottles are in use it is convenient to mark upon them the multiplier and divisor, thus:

$$
\frac{10,000}{7000} \text { or } \frac{\text { 10 }}{7} \text {. }
$$

In calculating the volume of a gas, the temperature and pressure must be taken into acount.

By referring to the following table the volume occupied by $0.00 \mathrm{Igm}$. of $\mathrm{CO}_{2}$ at different temperatures can be seen.

The volume of $0.06237 \mathrm{gm}$. of $\mathrm{CO}_{2}$ at $16^{\circ} \mathrm{C}$. is

$$
\frac{0.06237 \times 0.53843}{0.001}=33.518 \mathrm{cc} \text {. }
$$

Table showing Volume of .oor Gm. of Carbon Dioxid at Various

\begin{tabular}{|c|c|c|c|c|c|}
\hline $\mathrm{C}^{\circ}$ & $\mathrm{F} .{ }^{\circ}$ & Cc. & C. ${ }^{\circ}$ & $\mathrm{F}^{\circ}$ & Cc. \\
\hline$\circ$ & $3^{2}$ & $0.5086_{3}$ & I3 & 55.4 & $0.533^{\mathrm{I} 4}$ \\
\hline I & 33.8 & $0.5^{1049}$ & I4 & 57.2 & $0.5347 \mathrm{I}$ \\
\hline 2 & 35.6 & 0.51235 & I 5 & 59 & $0.53^{6} 57$ \\
\hline 3 & $37 \cdot 4$ & $0.5^{\mathrm{I}} 45^{\mathrm{I}}$ & I 6 & 60.8 & 0.53843 \\
\hline 4 & 39.2 & $0.5^{1608}$ & I 7 & 62.6 & 0.54030 \\
\hline 5 & $4 \mathrm{I}$ & $0.5^{1} 794$ & I 8 & 64.4 & 0.54216 \\
\hline 6 & 42.8 & 0.51980 & I9 & 66.2 & 0.54402 \\
\hline 7 & 44.6 & 0.52167 & 20 & 68 & $0.545^{89}$ \\
\hline 8 & 46.4 & $0.5^{2353}$ & $2 I$ & 69.8 & 0.54775 \\
\hline 9 & 48.2 & 0.52539 & 22 & 71.6 & 0.54961 \\
\hline 10 & 50 & 0.52726 & 23 & 73.4 & $0.55^{1} 77$ \\
\hline I I & 51.8 & 0.529 I 2 & 24 & 75.2 & 0.55334 \\
\hline 12 & 53.6 & 0.53098 & & & \\
\hline
\end{tabular}
TEMPERATURES

If the pressure remains constant, the volume of a gas increases regularly as the temperature increases, and decreases as the temperature decreases. (Charles' Law.)

This expansion or contraction amounts to $\frac{1}{2} \frac{1}{3}$ of the volume of the gas for each degree centigrade.

Thus by calculation the volume of $0.00 \mathrm{I} \mathrm{gm} . \mathrm{CO}_{2}$ (0.50863 cc.) at any temperature may te found.

$$
\frac{1}{2} \frac{1}{3} \text { of } 0.50863=0.001863 \text {. }
$$

Then to find the volume at any given $\mathrm{C}$. temperature multiply the degree of temperature by 0.001863 , and add the answer to $0: 50863$. 
The Pettenkofer method has long been the favorite, but it contains certain inherent sources of error, which, however, can be obviated by skilful manipulation and with the use of improved apparatus. The principal errors of this method and its usual modifications are due to absorption of carbon dioxid from the outer air or the breath of the operator, and the action of the caustic alkali on the glass of the container, as well as the presence of small amounts of the precipitated barium carbonate in the fluid taken out for titration.

These errors can be obviated by employing Walker's method,* as modified by Woodman, $\uparrow$ which is outlined briefly, as follows:

To a definite volume of air, usually $\mathrm{I}$ to 2 liters, is added a measured amount of standard barium hydroxid, care being taken to avoid contact of the solution with the air. After the absorption of the carbon dioxid, the solution is filtered under reduced pressure, through asbestos, and the clear barium hydroxid received in to a known excess of standard hydrochloric. The absorption vessel is rinsed out with water, free from carbon dioxid. The excess of acid is then determined by titration with barium hydroxid.

The standard solutions required are $\frac{\mathrm{N}}{50}$ hydrochloric acid, and approximately $\frac{\mathrm{N}}{\mathrm{I} 00}$ barium hydroxid, its exact strength relative to acid being found by titration when required.

The bottles in which the air is collected for analysis should be of about 2 liters capacity (the exact capacity must be known), and coated on the inside with paraffin; this prevents action of the alkali on the glass during the absorption.

It also admits of more complete draining and washing. The bottles should be provided with rubber stoppers, through which pass a long tube reaching to near the bottom of the bottle, and a short tube which reaches just below the stopper. Both tubes may be provided with stop-cocks or with rubber tubing and pinch-cocks. The bottles are best filled with the air to be analyzed, by suction applied at the short tube, the air entering through the longer tube; this reduces the danger of contamination by the breath of the opeartor.

The absorption is carried on for about thirty minutes and the solution then filtered through an asbestos filter in a Soxhlet filtering tube under diminished pressure, all air being excluded. The filtrate being received in a measured quantity of the standard hydrochloric acid, the absorption bottle must be thoroughly rinsed with 100 to I $50 \mathrm{cc}$. of water, free from carbon dioxid, or with a prepared "wash water" made as suggested by Walker by adding I cc. of a ro per cent solu- 
tion of barium chlorid and 3 drops of phenolphthalein, then titrating with barium hydroxid to a faint permanent pink tint.

The barium hydroxid solution must be kept in a bottle coated on the inside with either paraffin or barium carbonate (to prevent action of the alkali on the glass), and provided with a device for protecting the-solution against contact with carbon dioxid of the air (see Fig. 49).

All rubber stoppers and connections with which the solution may come in contact should be cleaned. with a five per cent potash solution, washed, and boiled with a dilute dichromate and sulphuric acid mixture, and then rubbed and washed until free from acid.

ESTIMATION OF CARBONIC ACID IN NATURAL WATERS

Carbonic acid exists in natural waters presumably in three different forms:

(I) Free carbonic acid $\left(\mathrm{H}_{2} \mathrm{CO}_{3}\right)$. It is generally considered that the carbon dioxid $\left(\mathrm{CO}_{2}\right)$ which is dissolved in water exists as a true acid having the formula $\mathrm{H}_{2} \mathrm{CO}_{3}$.

(2) Normal carbonates of calcium and magnesium $\left(\mathrm{CaCO}_{3}\right.$ and $\left.\mathrm{MgCO}_{3}\right)$ and of the alkalies.

(3) Bicarbonates of the same $\mathrm{CaH}_{2}\left(\mathrm{CO}_{3}\right)_{2}$ and $\mathrm{MgH}_{2}\left(\mathrm{CO}_{3}\right)_{2}$.

The carbonic acid existing free may be completely driven off by heating the water. Carbonic acid existing in the half-bound state, i.e., bicarbonate, may also be driven off (though less completely) on boiling. Hence these two forms are distinguished as volatile, while that existing as normal carbonate is fixed.

The estimation of carbonic acid in natural waters consists in determining the amount of free and half-bound carbonic acid which may be present.

The Pettenhofer Method. This method is based upon the action which calcium or barium hydroxid has upon free and half-bound carbonic acid, whereby insoluble calcium and barium carbonates are formed and precipitate out of solution. A measured excess of the hydroxid is used, and the portion unacted upon is determined volumetrically with a standard acid solution.

The reaction is as follows:

$$
\mathrm{CaH}_{2}\left(\mathrm{CO}_{3}\right)_{2}+\mathrm{CO}_{2}+2 \mathrm{Ba}(\mathrm{OH})_{2}=2 \mathrm{BaCO}_{3}+\mathrm{CaCO}_{3}+{ }_{2} \mathrm{H}_{2} \mathrm{O} \text {. }
$$

If magnesium bicarbonate is present in the water the reaction between it and the barium or calcium hydroxid is the same as shown in above equation; but magnesium carbonate $\left(\mathrm{MgCO}_{3}\right)$, being more soluble than the carbonates of calcium and barium, does not precipitate as readily, and instead reacts with the hydroxid, forming magnesium hydroxid which precipitates. The presence therefore of magnesium carbonate, or in fact any magnesium salt, causes the 
calcium or barium hydroxid to be used up. This precipitation of magnesium is prevented by the introduction of ammonium chlorid, which, by forming a soluble salt of ammonium and magnesium chlorid, prevents any loss of calcium or barium hydroxid.

The reaction is as follows:

$\mathrm{MgCO}_{3}+{ }_{4} \mathrm{NH}_{4} \mathrm{Cl}+\mathrm{Ba}(\mathrm{OH})_{2}=\mathrm{MgCl}_{2}\left(\mathrm{NH}_{4} \mathrm{Cl}\right)_{2}+\mathrm{BaCO}_{3}+2 \mathrm{NH}_{4} \mathrm{OH}$.

As here shown an equivalent amount of ammonium hydroxid is formed and hence the alkalinity is not changed. Heat must not be applied in this case, as this would volatilize the ammonia.

If alkali carbonate, sulphate, or any other alkali salt is present, the acid of which would be precipitated by the calcium or barium hydroxid, the addition of a small quantity of a neutral solution of barium or calcium chlorid will remove it.

This addition, besides removing carbonates and other interfering salts, also prevents any irregularities which might arisc from the presence of free alkali in the calcium or barium hydroxid or of magnesium carbonate in the water itself. This irregularity is due to a double decomposition which occurs between magnesium or alkali oxalate and calcium carbonate (which is seldom entirely absent from the titrated fluid), forming calcium oxalate and alkali or magnesium carbonate, which two latter will of course take up oxalic acid.

The Process. $100 \mathrm{cc}$. of the water are put into a flask of about $200 \mathrm{cc}$. capacity, $3 \mathrm{cc}$. of strong calcium or barium chlorid solution, and $2 \mathrm{cc}$. of a saturated solution of ammonium chlorid are added, followed by $45 \mathrm{cc}$. of calcium or barium hydroxid solution, the strength of which was previously ascertained by means of $\frac{\mathrm{N}}{10}$ acid. The flask is then closed with a well-fitting rubber stopper, shaken and set aside for about twelve hours or until the precipitate has fully settled. The fluid contents of the flask measure $150 \mathrm{cc}$. $50 \mathrm{cc}$. of this clear liquid are removed by means of a pipette and titrated with $\frac{\mathrm{N}}{\mathrm{IO}}$ oxalic acid, using turmeric paper as the indicator. The quantity used multiplied by 3 gives the total quantity of calcium or barium hydroxid solution left. This deducted from the original $45 \mathrm{cc}$. added, gives the quantity which reacted with the carbon dioxid present. This quantity multiplied by $0.002 \mathrm{I} 83 \mathrm{gm}$. will give the weight of carbon dioxid existing free or in the form of bicarbonate in the $100 \mathrm{cc}$. of water taken for analysis. In making such an analysis the first step is to determine the relation between the calcium or barium hydroxid solution and the $\frac{\mathrm{N}}{10}$ oxalic acid. This is done by titrating $45 \mathrm{cc}$., with the 
acid, shaking thoroughly during the operation until the alkaline reaction has just vanished. This end-reaction is known to be reached when a drop taken out on a pointed glass rod and applied to turmeric paper produces no brown spot. Two trials should be made, the first a rough one, the second should be exact.

If the water contains only free carbonic acid it is best to use barium instead of calcium hydroxid, because the calcium carbonate first formed is amorphous and perceptibly soluble in water, to which it imparts an alkaline reaction. Hence the unprecipitated lime cannot be estimated until the calcium carbonate has separated in crystalline form, which is insoluble and takes from eight to ten hours.

Instead of $\frac{\mathrm{N}}{\mathrm{IO}}$ oxalic acid, standard sulphuric or hydrochloric acid may be used, and the turmeric paper may be replaced as indicator by rosolic acid or any other indicator which is not affected by ammonium compounds.

This method is simple, expeditious, and fairly satisfactory, but there are a great many precautions which must be observed in order to obtain reliable results. They are as follows:*

(I) The avoidance of exposure to air of the barium hydroxid solution and of the sample of water to be analyzed. The former should be kept in a bottle whose outlet to the air is provided with a tube containing fused calcium chlorid and stick potash. (See Fig. 48.) Undue exposure of the water should likewise be avoided, to prevent its absorbing carbon dioxid, which it does readily.

(2) The standardization of the barium hydroxid should be carried out in a manner similar to the method employed in the titration of the sample. If the titration is done in the presence of rosolic acid, the standardization should be done with the same indicator and in the presence of ammonium chlorid.

(3) The titer of the oxalic acid solution (if this is used), should be found just before using, as it deteriorates rapidly.

(4) The use of a syphon to introduce the water into the bottle in which the precipitation is done.

(5) The reagents should be added to the sample in the order given in the description of the process. The barium hydroxid solution being added last, and in order to avoid any exposure it should be introduced by means of a long delivery tip on the burette, the lower end of which dips below the surface of the sample in the bottle.

(6) The allowance of sufficient time for the separation of the carbonate in crystalline form before withdrawing the supernatant liquid for titration.

* Ellms and Beneker, J. A. C. S., XXIII, 4I 2. 
(7) The bottle in which the precipitation is done should be provided with a tightly fitting stopper, either rubber or, better still, ground glass stoppers, well vaselined.

(8) The removal of the $50 \mathrm{cc}$. of liquid for titration should be carefully done in order to avoid stirring up the precipitate in the bottom.

(9) The titration of the withdrawn portion of the liquid should take place immediately, and the acid should be run in quickly.

Ellms and Beneker, J. A. C. S., xxIII, 405, detail numerous carefully made experiments appertaining to this subject.

Even with strict observance of all of these precautions, there are several sources of error, as pointed out by Forbes and Pratt, J. A. C. S., $\mathrm{xxv}$, 744: "They are due to the fact that working on such a small quantity of water as $100 \mathrm{cc}$. and employing $\frac{\mathrm{N}}{\mathrm{IO}}$ acid, very slight errors in the titration of the aliquot portion of $50 \mathrm{cc}$., in measuring out the sample for titration and in measuring the original sample, make a considerable difference in the final results."

In order to lessen the effect of these errors Forbes and Pratt have modified the method as follows:

"Ground-gliss stoppered bottles, holding approximately $480 \mathrm{cc}$., are accurately calibrated by weighing completely filled with water. The bottle is filled with the water to be analyzed by means of a syphon, the glass stopper inserted, leaving no air bubbles, and the neck of the bottle wiped dry. The glass stopper is then carefully removed and $57 \mathrm{cc}$. of the water withdrawn by means of an accurately calibrated pipette, in order to make room for the reagents. $3 \mathrm{cc}$. of strong barium chlorid solution (8 gms. per liter), 2 cc. of saturated ammonium chlorid solution, and $50 \mathrm{cc}$. of standard barium hydroxid are then introduced, the bottle quickly stoppered, well shaken, and set aside to settle."

"There is now in the bottle an air-space of $2 \mathrm{cc}$. which is left to avoid the possibility of loss of liquid when the stopper is inserted. After the precipitated carbonate have completely settled out, several portions of roo cc. are syphoned off and titrated with $\frac{\mathrm{N}}{50}$ sulphuric acil. The barium hydroxid used is approximately $\frac{N}{15}$. The figure obtained by averaging several results of titration of portions of $100 \mathrm{cc}$. is taken as the true value."

The use of this large quantity of water and the titration of roo cc. portions reduce considerably the errors due to the difficulty of obtaining the end-point and those due to inaccuracies of measurement.

'The decrease in the concentration of the barium hydroxid solution due to the use of the larger quantity of water makes it necessary to 
allow a longer time for the precipitation of the carbonates. Twelve to sixteen hours, or over night, is usually sufficient.

Trillich * describes a radical modification of the Pettenkoffer method, in which the precipitation of magnesium hydroxid is allowed to take place, instead of being prevented by the addition of ammonium chlorid. Then from a direct gravimetric determination of the amount of magnesium present in another portion of the sample, the proper correction is applied to the result obtained volumetrically. Since 40 parts of $\mathrm{MgO}$ will react with as much barium hydroxid as 44 parts of $\mathrm{CO}_{2}$, the correction is attained by multiplying each part of $\mathrm{MgO}$ present by $\mathrm{I} . \mathrm{I}$ and subtracting the product from the apparent amount of carbonic acid found by the volumetric determination. This method differs from the Pettenkoffer method in that $5 \mathrm{cc}$. of barium chlorid solution are used instead of $3 \mathrm{cc}$., omitting ammonium chlorid and using phenolphthalein as indicator for the determination of free and half-bound carbonic acid.

It is recommended to use a barium hydroxid solution in which, to each 9 gms. of barium hydroxid $0.5 \mathrm{gm}$. of barium chlorid is added, in order to convert the sodium or potassium hydroxids which are common impurities of barium hydroxid into chlorids and thus obviate the disturbing effect which these impurities would occasion.

In using this method it is quite evident as pointed out by Ellms and Beneker, that unless the precipitation of the magnesium by barium hydroxid is complete, an error is introduced into the correction. A large excess of barium hydroxid is necessary in order to effect complete precipitation.

In order to differentiate between free, half-bound, and fixed carbonic acid, Trillich uses that portion of his solution which contains the precipitated carbonates and titrates it with standard hydrochloric acid, using cochineal as indicator. This gives the total carbonic acid.

By subtracting the free and half-bound from this he obtains the fixed carbonic acid, and by finding the difference between the free and half-bound and the fixed, he estimates the free carbonic acid (assuming the half-bound to be equivalent to the fixed).

Lunge-Trillich or Seyler Method. $\dagger$ This method, which is looked upon as the best volumetric method for the determination of free and half-bound carbonic acid, is based upon the assumption that in the bicarbonates of the alkali earth bases, there is one molecule of half-bound carbonic acid for each molecule of fixed, and that these bicarbonates are neutral to phenolphthalein.

When a solution containing free carbonic acid is titrated with

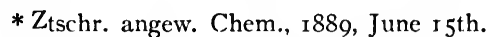

† Chem. News, 70-104 (1894), Analyst, 22-31 2 (I897). 
sodium carbonate, using phenolphthalein as indicator, sodium bicarbonate is formed,

$$
\mathrm{H}_{2} \mathrm{CO}_{3}+\mathrm{Na}_{2} \mathrm{CO}_{3}=2 \mathrm{NaHCO}_{3},
$$

which is neutral to phenolphthalein. As soon as the free acid is all taken up, the further addition of sodium carbonate produces a pink color. Thus the free carbonic acid is obtained directly. The fixed carbonic acid may then be determined by Hehner's method, and from this the half-bound is obtained, assuming this to be equal to the fixed, in waters which are acid to phenolphthalein. With waters that are alkaline to phenolphthalein the determination of the "phenolphthalein alkalinity" is first made, and then the total alkalinity with lacmoid or methyl orange by the Hehner process. Twice the phenolphthalein alkalinity subtracted from the total alkalinity gives the half-bound carbonic acid, no free acid being present in this case, and the halfbound being less than the fixed, i.e., some of the normal carbonates are held in solution without the aid of any bicarbonate.

If no free carbonic acid is present the half-bound may equal the fixed, and the water will be neutral to phenolphthalein.

Thus it is assumed that if the water be acid or neutral to phenolphthalein there is present for each molecule of fixed carbonic acid, one molecule of half-bound acid, and that if a water is alkaline to phenolphthalein only, one half of the carbonic acid in the form of normal carbonate is found by titrating with acid in the presence of phenolphthalein, as per equation:

$$
{ }_{2} \mathrm{Na}_{2} \mathrm{CO}_{3}+\mathrm{H}_{2} \mathrm{SO}_{4}=2 \mathrm{NaHCO}_{3}+\mathrm{Na}_{2} \mathrm{SO}_{4}
$$

The pink color produced by phenolphthalein with sodium carbonate is destroyed when one half of the latter becomes saturated with the carbonic acid liberated by the mineral acid.

The carbonates of alkaline earth bases act in a similar manner.

$$
{ }_{2} \mathrm{CaCO}_{3}+\mathrm{H}_{2} \mathrm{SO}_{4}=\mathrm{CaH}_{2}\left(\mathrm{CO}_{3}\right)_{2}+\mathrm{CaSO}_{4} \text {. }
$$

The details of the Seyler process are as follows:

For the determination of free carbonic acid, roo cc. of the water are introduced into a tall glass cylinder by means of a siphon, several drops of a neutral alcoholic solution of phenolphthalein are added, and the solution titrated with $\frac{\mathrm{N}}{50}$ sodium carbonate, stirring carefully and thoroughly until a faint permanent pink color is obtained.

For the determination of the fixed carbonic acid from which the half-bound is estimated, Hehner's method is used. 
The same or a fresh portion of the water is titrated with $\frac{\mathrm{N}}{50}$ sulphuric acid, using methyl orange or lacmoid as indicator. Seyler gives a series of formulas for calculating the results which greatly simplifies the work.

If $100 \mathrm{cc}$. of the sample are operated upon and the standard solutions are $\frac{\mathrm{N}}{50}$, the following formulæ give the results in parts per million:

\section{For Waters which are Acid or Neutral to Phenolphthalein.}

Free carbonic acid............... $=4.4 p$

Fixed or half-bound carbonic acid..... $=4.4 \mathrm{~m}$

Volatile carbonic acid. ............ $=4.4(m+p)$

Total carbonic acid. ............. $=4.4(2 m+p)$

$p=c c$. of $\frac{\mathrm{N}}{50}$ sodium carbonate required,

$m=$ cc. of $\frac{\mathrm{N}}{50}$ sulphuric acid required in the presence of methyl orange or lacmoid.

II. For Waters which are Alkaline to Phenolphthalein.

Fixed carbonic acid............. $=4.4 \mathrm{~m}$

Half-bound or volatile carbonic acid.. $=4.4\left(m-2 p^{\prime}\right)$

Total carbonic acid. ............. $=4.4\left(2 m-2 p^{\prime}\right)$

$m=$ cc. $\frac{\mathrm{N}}{50}$ sulphuric acid required in the presence of methyl orange or lacmoid,

$p^{\prime}=$ cc. $\frac{\mathrm{N}}{50}$ sulphuric acid required in the presence of phenolphthalein.

Examples. Waters which are Acid to Phenolphthalein.

For Free Carbonic Acid. Ioo cc. of the sample titrated with $\frac{\mathrm{N}}{50}$ sodium carbonate in the presence of phenolphthalein require $4.5 \mathrm{cc}$. of the standard solution, therefore $4.4 \times 4.5=19.85$ parts per million.

For Fixed and Half-bound Carbonic Acid. roo cc. titrated with $\frac{\mathrm{N}}{50}$ sulphuric acid in the presence of methyl orange required $\mathrm{I} .34 \mathrm{cc}$. of standard acid, therefore $4.4 \times 1.34=5.896$ parts per million.

The Volatile Carbonic Acid is the sum of the above two results, i.e., 25.746 parts per million.

The Total Carbonic Acid present is the sum of the fixed, half-bound, and free, i.e., I $9.85+5.896+5.896=3 \mathrm{r} .64 .2$ parts per million. 


\section{For Waters which are Alkaline to Phenolphthalein.}

Fixed Carbonic Acid. Ioo cc. of the sample titrated with $\frac{\mathrm{N}}{50}$ sulphuric acid, using methyl orange as indicator, required $3.6 \mathrm{cc}$. Therefore $4.4 \times 3.6=15.84$ parts per million.

Half-bound or Volatile Carbonic Acid. Ioo cc. of the sample titrated with $\frac{\mathrm{N}}{50}$ acid in the presence of phenolphthalein required $\mathrm{I} .2 \mathrm{cc}$.

Twice this amount subtracted from $3.6 \mathrm{cc}$. and then multiplied by 4.4 will give 5.28 parts per million.

[For further details of this method see Journal of the American Chemical Society, J. W. Ellms, XXI, 359; Ellms and Beneker, XXIII, 405; and Forbes and Pratt, XXV, 742.]

\section{Precautions:}

I. If the water contains much free carbonic acid it is better to take less than $100 \mathrm{cc}$.

2. The stirring should not be too vigorous, otherwise there will be a loss of $\mathrm{CO}_{2}$.

3. The titration should be quickly completed in order to avoid absorption of $\mathrm{CO}_{2}$ from the air.

4. The sodium carbonate solution should be made with water which has been thoroughly boiled and cooled out of contact with the air, and the finished solution should not be exposed any more than absolutely necessary.

Estimation of Small Quantities of Carbon Monoxid in the Air; Iodometrically.* This method depends upon the reaction between carbon monoxid and iodin pentoxid at $150^{\circ} \mathrm{C}$. or over, attention to which was first called by Ditte in $1870, \uparrow$

$$
\mathrm{I}_{2} \mathrm{O}_{5}+{ }_{5} \mathrm{CO}=\mathrm{I}_{2}+{ }_{5} \mathrm{CO}_{2} .
$$

The process is conducted by Kinnicut and Sanford as follows:

Twenty-five grams of iodin pentoxid are placed in a small U-tube, which is suspended in an oil or glycerol bath and connected with a Wolff blood-absorption tube containing $0.5 \mathrm{gm}$. potasium iodid dissolved in $5 \mathrm{cc}$. of water. The tube containing the iodin pentoxid is also connected with two U-tubes, one containing sulphuric acid, the other small pieces of potassium hydroxid, so as to remove from the air to be analyzed (before it comes in contact with the iodin pentoxid) all unsaturated hydrocarbons, hydrogen sulphid, sulphur dioxid, and other reducing gases which would react with the iodin pentoxid.

* L. P. Kinnicut and G. R. Sanford. J.A.C.S., XXI1, I4.

† Bull. Suc. Chim., I3, 318. 
The oil bath in which the U-tube containing the iodin pentoxid is suspended is heated to $150^{\circ} \mathrm{C}$. (The reaction is not quantitative at lower temperatures.) A measured quantity of air ( $\mathrm{I}$ liter is a convenient quantity) is passed through the apparatus at the rate of about I liter in two hours, the rate being controlled by forcing the air out of the container by means of mercury, the flow of which is regulated by means of a stop-cock. The temperature and barometric pressure must of course be noted, and the volume of air analyzed reduced to $0^{\circ} \mathrm{C}$. and $760 \mathrm{~mm}$. pressure.

The titration of the liberated iodin is made in the Wolff bloodabsorption apparatus employing $\frac{\mathrm{N}}{\mathrm{I} 000}$ sodium thiosulphate. Nicloux, Compt. rend., I26, 746, determined the iodin set free by the depth of color formed in a chloroform solution. Gautier, Compt. rend., I 26, 93 I , determined the quantity of carbon dioxid formed.

A modification of this process is described by Morgan and McWhorter, J. A. C. S., XxIx, I 589. 


\section{CHAPTER XVI}

\section{CHLORIN, BROMIN, AND IODIN}

FrEe chlorin may be estimated by the addition of potassium iodid, and titration of the liberated iodin by means of sodium thiosulphate, as described on page 209.

The same method may be employed for the estimation of free bromin. Free iodin is estimated by direct titration with sodium thiosulphate. See page 207.

The available chlorin, as for instance that existing in bleaching powder, Javelle water, etc., is estimated by liberating the chlorin with an acid, and then, after the addition of potassium iodid, estimating the liberated iodin. See page 2 ro.

The estimation of free halogens may also be made by titration with standard arsenous acid solution, as described on page 228, and by means of the same standard solution the available chlorin in bleaching powder and other hypochlorites may be estimated. By the same methods the chlorin which is liberated by heating certain substances, as for instance the metallic peroxids, with hydrochloric acid may be accurately estimated. See page 214 .

Gross, J. A. C. S., xxv, 989 , estimates free iodin by converting it into zinc iodid and titrating with $\frac{\mathrm{N}}{\mathrm{IO}}$ silver nitrate, using potassium chromate as indicator. 2 gms. of the iodin are placed in a flask, $40 \mathrm{cc}$. of water, and about $4 \mathrm{gms}$. of shot-zinc are added. The flask is then shaken and allowed to stand with stopper inserted until fluid is colorless. When all the iodin is taken up by the zinc, the solution is filtered into a half liter flask, the residue well washed with hot water until free from iodid, and the fluid then made up to $500 \mathrm{cc}$. $50 \mathrm{cc}$. of the solution are placed in a porcelain capsule and titrated with $\frac{\mathrm{N}}{\mathrm{IO}}$ silver nitrate, using potassium chromate as indicator, until endpoint, a slight brownish color, is reached.

Chlorids, Bromids, and Iodids. These salts are most readily estimated by titration with standard silver solution, as described under analysis by precipitation (page I IO). A distillation method for alkali iodid is described on page 220 . 
The modified Personne method in which alkali iodids are titrated with standard mercuric chlorid solution, is described on page I 34 .

These salts may also be estimated by means of Volhard's sulphocyanate method. See page i 22 .

In the case of chlorids, however, as pointed out by M. A. Rosanoff and A. E. Hill,* it is necessary to remove the precipitated silver chlorid by filtration before beginning titration with the sulphocyanate solution; this is because ferric sulphocyanate is decomposed by the silver chlorid according to the following equation:

$$
\mathrm{Fe}(\mathrm{CNS})_{3}+3 \mathrm{AgCl}=\mathrm{FeCl}_{3}+3 \mathrm{AgCNS} \text {. }
$$

In the experiments of Rosanoff and Hill, it was furthermore demonstrated that this decomposition is a rapid one. Forty-three per cent of sulphocyanate is destroyed by only an equivalent of silver chlorid in two minutes. It must therefore be expected that a large excess of silver chlorid (such as is present during a titration by Volhard's method) will destroy practically all of the sulphocyanate in a few seconds. This in fact is the case. In the estimation of bromids and iodids it is unnecessary to filter out the silver precipitate.

Chlorids, Bromids, and Iodids may be estimated in presence of each other by the method of Benedict and Snell (J. A. C. S., Xxv, I I38).

This method is based upon the fact that potassium iodate $\left(\mathrm{KIO}_{3}\right)$ will liberate iodin from iodids upon acidification with acetic acid, and bromin from bromids on acidification with dilute nitric acid.

The process which is subjoined includes:

(I) A determination of total halogens by titration with silver nitrate or any of the usual methods.

(2) A determination of iodin.

(3) A determination of chlorin; the bromin being estimated by difference.

For the determination of iodin a quantity of the substance containing not over $0.5 \mathrm{gm}$. of iodin or $0.15 \mathrm{gm}$. of chlorin, is dissolved in water and made up to $50 \mathrm{cc}$. in a $100 \mathrm{cc}$. cylinder with a close fitting glass stopper. Neutral potassium iodate is added in about twice the quantity necessary to react with all the bromin and iodin believed to be present. The mixed solution is acidified with 4 or $5 \mathrm{cc}$. of 30 per cent acetic acid and shaken with 30 to $40 \mathrm{cc}$. of carbon disulphid until all the liberated iodin has been taken up by the latter. 'The aqueous solution is now separated from the carbon disulphid layer by filtration through a wet filter and the carbon disulphid is thoroughly washed with cold water on the filter. The filtrate and washings are reserved for the chlorin determination. The carbon disulphid solu- 
tion is transferred to another beaker by puncturing the filter, and is covered with 20 to $25 \mathrm{cc}$. of 75 per cent alcohol; any carbon disulphid left adhering to the filter is rinsed into the beaker with a portion of the 75 per cent alcohol.

The iodin is now titrated with sodium thiosulphate with constant stirring. No starch indicator is necessary.

For the determination of the chlorin, the aqueous filtrate from the carbon disulphid is treated with $5 \mathrm{cc}$. nitric acid (sp.gr. I.I8) to liberate the bromin, and is boiled in a covered beaker until colorless. The excess of iodate is next destroyed by adding a quantity of potassium iodid slightly in excess of the amount necessary to react with it. The solution is again boiled until colorless, 2 or $3 \mathrm{cc}$. more of dilute nitric acid being added if the color is not completely discharged after ten or fifteen minutes' boiling. A minute or two after the color has completely disappeared the solution is taken from the flame, cooled, and neutralized with sodium carbonate. To secure exact neutralization, a little calcium carbonate may be added at first and then sodium carbonate solution, until a precipitate just forms. The chlorin is then determined by titration with $\frac{\mathrm{N}}{\mathrm{IO}}$ silver nitrate, üsing potassium chromate as indicator.

Chlorates, Bromates, and Iodates. These may be estimated by titrating with $\frac{\mathrm{N}}{\mathrm{IO}}$ silver nitrate solution after ignition. They are reduced by heat to chlorids, bromids, and iodids respectively.

$$
\begin{aligned}
& \text { (a) } \mathrm{KClO}_{3} \text { theat }=\mathrm{KCl}+\mathrm{O}_{3} \text {. } \\
& \text { (b) } \mathrm{KCl}+\mathrm{AgNO}_{3}=\mathrm{AgCl}+\mathrm{KNO}_{3} \text {. } \\
& \text { 10) } 74.04 \text { 10) } 168.69 \\
& 7.404 \text { gms. } 16.869=1000 \mathrm{cc} \frac{\mathrm{N}}{\mathrm{IO}} \mathrm{AgNO}_{3} \mathrm{~V} \text {. S. }
\end{aligned}
$$

Thus each cc. of the $\frac{\mathrm{N}}{\mathrm{IO}} \mathrm{AgNO}_{3}$ V. S. represents $0.007404 \mathrm{gm}$. of $\mathrm{KCl}=0.012168 \mathrm{gm} . \mathrm{KClO}_{3}$. The factor is $10 \frac{1}{000}$ the molecular weight in grams of any univalent chlorate, bromate, or iodate and 20000 that of bivalent salts.

Chlorates, Bromates, and Iodates may also be estimated by digestion with excess of hydrochloric acid in the presence of potassium iodid. In each case the liberated halogen acts upon the potassium iodid and sets free an equivalent of iodin, the amount of which is then estimated by means of $\frac{\mathrm{N}}{\mathrm{IO}}$ sodium thiosulphate.

(a) $\mathrm{KClO}_{3}+6 \mathrm{KI}+6 \mathrm{HCl}={ }_{7} \mathrm{KCl}+{ }_{3} \mathrm{H}_{2} \mathrm{O}+\mathrm{I}_{6}$. I 21.68 
(b) $\mathrm{I}_{2}+2\left(\mathrm{Na}_{2} \mathrm{~S}_{2} \mathrm{O}_{3} \cdot{ }_{5} \mathrm{H}_{2} \mathrm{O}\right)={ }_{2} \mathrm{Na}_{2}+\mathrm{Na}_{2} \mathrm{~S}_{4} \mathrm{O}_{6}+\mathrm{IoH}_{2} \mathrm{O}$,

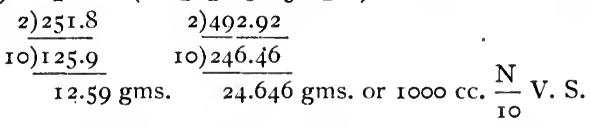

Each cc. of $\frac{\mathrm{N}}{\mathrm{IO}} \cdot \mathrm{Na}_{2} \mathrm{~S}_{2} \mathrm{O}_{3}=0.01259 \mathrm{gm}$. of iodin $=0.0020428 \mathrm{gm}$. of $\mathrm{KClO}_{3}$.

This method is more fully described on page 222 .

By Reduction with Hydroxylamin Sulphate. Fritz Weber, in Pharm. Ztg., I906, 364, employs hydroxylamin sulphate as a reducing agent for the quantitative estimation of chlorates, bromates, and iodates, the resulting chlorid, bromid, and iodid being titrated with standard silver nitrate. For the estimation of chlorate:

Dissolve I $\mathrm{gm}$. of the chlorate in $200 \mathrm{cc}$. of water, add $20 \mathrm{gms}$. of hydroxylamin sulphate, acidulate with nitric acid, warm, and titrate with standard silver nitrate.

For the estimation of bromate:

Dissolve I gm. of the bromate in $200 \mathrm{cc}$. of water, supersaturate with ammonia water, add 20 gms. of hydroxylamin sulphate, acidulate as above with nitric acid, and titrate with standard silver nitrate.

For the estimation of iodate.

Dissolve I gm. of the iodate in roo cc. of water, supersaturate with ammonia water, add to gms. of hydroxylamin sulphate, and proceed as above described for bromate. If any iodin is liberated, a little sulphurous acid is added, and the solution again acidulated with nitric acid.

Titrations with Standard Potassium Iodate $\left(\mathrm{KIO}_{3}\right)$. According to L. W. Andrews, J A. C. S., Xxv, 756, potassium iodate may be employed as a standard for titrating iodids, chlorates, and free iodin, as well as arsenous, antimonous, and ferrous compounds, in fact for the estimation "of almost all the substances to which Bunsen's process of distillation with potassium iodid and hydrochloric acid is applicable, with at least equal precision and far simpler apparatus."

When potassium iodate is added to a solution of an iodid, in the presence of a small quantity of acid, iodin is set free, as per equation:

$$
5 \mathrm{KI}+\mathrm{KIO}_{3}+6 \mathrm{HCl}=6 \mathrm{KCl}+{ }_{3} \mathrm{I}_{2}+{ }_{3} \mathrm{H}_{2} \mathrm{O} \text {. }
$$

If, however, a larger quantity of acid is present, iodin chlorid (ICl) forms as

$$
2 \mathrm{KI}+\mathrm{KIO}_{3}+6 \mathrm{HCl}=3 \mathrm{KCl}+3 \mathrm{ICl}+{ }_{3} \mathrm{H}_{2} \mathrm{O} .
$$

If chloroform or carbon tetrachlorid is used as an indicator, the immiscible solvent remains violet in the first case, but in the latter 
becomes colorless, while the supernatant solution becomes bright yellow from the presence of the iodin chlorid.

This behavior is explained as follows:

Iodin chlorid being the salt of a very weak base, undergoes hydrolysis in a neutral or feebly acid solution with the production of hypoiodous acid (HIO) and hydrochloric acid.

$$
\mathrm{ICl}+\mathrm{H}_{2} \mathrm{O}=\mathrm{HIO}+\mathrm{HCl} \text {. }
$$

The hypoiodous acid undergoes spontaneous conversion into iodic acid, whereas in the presence of a great excess of acid this hydrolysis is prevented.

The Process. Titration of Iodid. $0.2 \mathrm{gm}$. of potassium iodid are dissolved in $20 \mathrm{cc}$. of water in a glass-stoppered bottle of $250 \mathrm{cc}$. capacity, $5 \mathrm{cc}$. of chloroform, and $30 \mathrm{cc}$. of concentrated hydrochloric (sp.gr. I.2 I) are added and the mixture titrated with standard potassium iodate (10.62 gms. in the liter) shaking briskly until the chloroform loses its color. The end-reaction is exceedingly sharp. Each cc. of the standard iodate $=0.016476 \mathrm{gm}$. of potassium iodid.

$$
\begin{aligned}
& { }_{2} \mathrm{KI}+\mathrm{KIO}_{3}+6 \mathrm{HCl}={ }_{3} \mathrm{KCl}+{ }_{3} \mathrm{ICl}+{ }_{3} \mathrm{H}_{2} \mathrm{O} .
\end{aligned}
$$

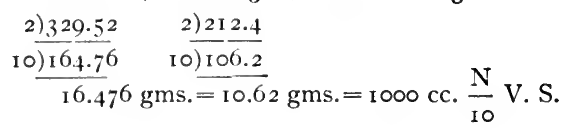

If a standard solution of acid potassium iodate is used under the above conditions, that is, with a great excess of acid, the reaction is probably as follows:

$$
\begin{aligned}
& { }_{4} \mathrm{KI}+\mathrm{KH}\left(\mathrm{IO}_{3}\right)_{2}+\mathrm{I}_{2} \mathrm{HCl}=6 \mathrm{ICl}+{ }_{5} \mathrm{KCl}+\mathrm{HCl}+6 \mathrm{H}_{2} \mathrm{O} .
\end{aligned}
$$

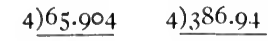

$$
\begin{aligned}
& \text { 10) } \frac{164.76}{16.476} \text { gms. } \frac{10) 96.735}{9.6735} \text { gms. or } 1000 \mathrm{cc} \text {. standard solution. }
\end{aligned}
$$

Titration of Free Iodin $0.3 \mathrm{gm}$. of the iodin is dissolved in ro cc. of a solution of potassium iodid which is checked against the standard iodate solution. Io cc. of hydrochloric acid and $5 \mathrm{cc}$. of chloroform are added and the mixture titrated with the standard iodate, as above described. If the potassium iodid solution used contains 16.476 gms. per liter, then Io cc. will take up ro cc. of the standard iodate solution. Therefore ro cc. deducted from the quantity of the latter required, gives the quantity which reacted with the free iodin. Each cc. represents $0.02518 \mathrm{gm}$. of free iodin.

Titration of Chlorates. To the solution of the chlorate add an exactly known amount of pure potassium iodid in a glass-stoppered 
bottle and an amount of fuming, pure hydrochloric acid at least onethird greater than the volume of the solution. Close the bottle tightly and allow it to stand fifteen minutes after shaking, then add $5 \mathrm{cc}$. of chloroform. On now shaking, the chloroform must become deep violet. (If the color is pale an insufficiency of iodid has been arded, and it is advisable to begin again.) Then add the decinomal iodate with intermittent shaking until the chloroform becomes colorless, which point may be estimated with the utmost precision. Each cc. of $\frac{\mathrm{N}}{\mathrm{I}}$ iodate solution is equivalent to $0.00268 \mathrm{gm}$. of $\mathrm{ClO}_{3}$.

The estimation of iodates, $*$ bromates, $\dagger$ and hypochlorites, $\$$ may be made by the use of hydrazin (diamid) which is a very powerful reducing agent. The halogen in each case is reduced to the halid form, while the nitrogen of the hydrazin is set free. The halid may then be titrated with standard silver nitrate, or if preferred the nitrogen may be measured.

Iodids may also be estimated by means of standard potassium $b i$-iodate, in faintly acid solutions with very satisfactory results (see standardization of sodium thiosulphate solution), or by means of potassium dichromate (also see standardization of sodium thiosulphate solution), or by means of potassium permanganate (see iodometric standardization of permanganate).

Estimation of Bromids or Iodids by Direct Titration with Chlorin Water. If for any reason the determination of these salts cannot conveniently or satisfactorily be made by titration with standard silver nitrate, the much less accurate method of titration with chlorin water may be employed.

This method depends upon determining the quantity of chlorin water of known strength required to convert the bromin into $\mathrm{BrCl}$. If to an aqueous solution of a bromid a small quantity of chlorin water is added, bromin is set free.

$$
{ }_{2} \mathrm{KBr}+\mathrm{Cl}_{2}={ }_{2} \mathrm{KCl}+\mathrm{Br}_{2} \text {. }
$$

If a larger quantity of chlorin water is added the bromin is converted into bromin monochlorid, $\mathrm{BrCl}$.

$$
\mathrm{KBr}+\mathrm{Cl}_{2}=\mathrm{KCl}+\mathrm{BrCl} \text {. }
$$

The addition of a still larger quantity of chlorin water will form $\mathrm{BrCl}_{5}$.

$$
\mathrm{Br}_{2}+\mathrm{Cl}_{10}={ }_{2} \mathrm{BrCl}_{5}
$$

* Riegler, Z. anal. Chem., 42, 677.

† Schlotter, Z. anorg. Chem., 37, 164.

$\ddagger$ Roberts and Rancoli, Chem. Centrlbl., I904, I, I 294 . 
which is finally converted into bromic acid.

$$
\mathrm{BrCl}_{5}+{ }_{3} \mathrm{H}_{2} \mathrm{O}=\mathrm{HBrO}_{3}+{ }_{5} \mathrm{HCl} \text {. }
$$

The bromid in solution is treated with chlorin water in the presence of chloroform (carbon disulphid or carbon tetrachlorid may also be used). The chloroform takes up the liberated bromin and becomes yellow or dark brown in color according to the quantity of bromin dissolved, but as more chlorin water is added the color of the chloroform solution becomes paler through conversion of the bromin into bromin chlorid $(\mathrm{BrCl})$, and finally, when a sufficient excess has been added, the solvent becomes quite colorless. The point at which all of the bromin is converted into $\mathrm{BrCl}$ is easily recognized after a little practice by the solvent becoming yellowish white. This indication is fairly sharp if the vessel in which the titration is performed is placed upon a white surface. The complete decolorization of the solvent cannot be utilized as the end-reaction, as this decolorization does not occur until the chlorin water has been added in decided excess.

The method is carried out according to Reimann, as follows:

The titer of the chlorin water as compared to $\frac{\mathrm{N}}{100}$ sodium thiosulphate is first found. Then knowing how many cubic centimeters of the sodium thiosulphate solution correspond to a given volume of the chlorin water it is easy to calculate how much bromin the same volume of chlorin water will convert into $\mathrm{BrCl}$.

Into a flask provided with a well-fitting ground-glass stopper a weighed quantity of the bromid to be analyzed is introduced, dissolved in water, a small quantity of chloroform added, and the mixture titrated slowly with the chlorin water, closing the flask and shaking briskly after each addition, and observing the color of the chloroform layer; as soon as this is pale yellowish-white, the delivery of the chlorin water is stopped, and the quantity used noted, and the calculation made.

By reference to the equation it is seen that one molecule of $\mathrm{KBr}$ requires two atoms of chlorin to transform the bromin into $\mathrm{BrCl}$, hence one cc. of $\frac{\mathrm{N}}{100}$ sodium thiosulphate represents in this case, not $0.0007936 \mathrm{gm}$. of bromin, but rather one half of this quantity, which is $0.0003968 \mathrm{gm}$. and hence likewise $0.00059 \mathrm{I} \mathrm{gm}$. of $\mathrm{KBr}$, or $0.0005 \mathrm{II2}$ gm. of $\mathrm{NaBr}$.

Example. To ro cc. of chlorin water, I gm. of potassium iodid is added, and the solution titrated.with $\frac{\mathrm{N}}{10}$ thiosulphate in the usual manner. I 2 cc. are required. Thus the ro cc. of chlorin water are 
equivalent to $120 \mathrm{cc}$. of $\frac{\mathrm{N}}{\mathrm{IOO}}$ sodium thiosulphate. $0.21 \mathrm{gm}$. of the potassium bromid to be analyzed is now dissolved in ro cc. of water, 2 cc. of chloroform added, and the solution titrated with the above chlorin water. $28.6 \mathrm{cc}$. are required. If Io $\mathrm{cc}$. of chlorin water are equivalent to $\mathrm{I} 2 \mathrm{cc}$. of $\frac{\mathrm{N}}{\mathrm{IO}}$ thiosulphate, $28.6 \mathrm{cc}$. are equivalent to $34.3 \mathrm{cc}$, or $343 \mathrm{cc}$. of $\frac{\mathrm{N}}{\mathrm{I} O 0}$ sodium thiosulphate, and since each cc. of the latter represent $0.00059 \mathrm{II} \mathrm{gm}$. of pure $\mathrm{KBr}$ the $0.2 \mathrm{I} \mathrm{gm}$. taken contain $0.00059 \mathrm{II}$ gm. $\times 343=0.202747 \mathrm{gm}$. of pure $\mathrm{KBr}$, or 96.54 per cent.

The estimation of iodids * by this method is similar to that of bromids, the reaction with chlorin being the same.

The first addition of chlorin water liberates iodin, then it reacts with iodin to form $\mathrm{ICl}$, then $\mathrm{ICl}_{5}$ and finally, $\mathrm{HIO}_{3}$.

Free iodin and iodin monochlorid (ICl) dissolve in chloroform or carbon disulphid, forming a violet to blue solution, according to the quantity of the substance present. While iodin pentachlorid $\left(\mathrm{ICl}_{5}\right)$ or $\mathrm{HIO}_{3}$ on the other hand form colorless solutions. Therefore in titrating with chlorin the disappearance of the violet color may be taken as the end-reaction and the quantities of chlorin used as the measure of the iodin which was converted into $\mathrm{ICl}_{5}$. The reaction is

$$
\mathrm{KI}+6 \mathrm{Cl}=\mathrm{ICl}_{5}+\mathrm{KCl} \text {. }
$$

Thus it is seen that one atom of iodin or one molecule of potassium iodid is the equivalent of six atoms of chlorin.

Therefore if the quantity of chlorin water used in the titration is calculated into cubic centimeters of $\frac{\mathrm{N}}{\mathrm{s} 00}$ sodium thiosulphate, as directed under Estimation of Bromids, by this method, each cc. of $\frac{\mathrm{N}}{\mathrm{I} 00}$ sodium thiosulphate used will represent in this case

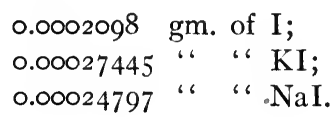

The presence of hydrochloric, or rather of a large excess of hydrochloric acid, will materially alter the reaction in this case. A much smaller quantity of chlorin water will be required. Andrews, J. A. C. S., $\mathrm{Xxv}, 757$.

*Dupré, Ann. Chem. (Liebig), 94, 365, I855. 
The Determination of Chlorids or Bromids in the Presence of Sulphocyanate. The method proposed by Rosanoff and Hill, J. A. C. S., Xxix, 1467 , depends upon oxidizing the sulphocyanate to hydrocyanic acid by means of nitric acid, expelling nearly all of the hydrocyanic acid, and determining the residual chlorid or bromid, in the presence of the traces of hydrocyanic acid by the Volhard method. The sulphocyanate originally present is found by difference. The sum of chlorid and sulphocyanate, or of bromid and sulphocyanate, is established by precipitation with excess of standard silver nitrate V. S. and determination of the excess by titration with standard ammonium sulphocyanate V. S. Precipitates containing silver chlorid must be filtered off before the excess of silver nitrate is determined, while in the case of the bromid filtration is unnecessary.

The method in detail is as follows:

"Determination of Chlorids. The following solutions are required: (a) a fifteenth-normal solution of silver nitrate, standardized gravimetrically or by weight of silver nitrate used; $(b)$ a fifteenth-normal solution of ammonium sulphocyanate, standardized against the silver nitrate by Volhard's method; $(c)$ a saturated solution of iron-ammonium alum, free from chlorids and treated with nitric acid to lighten the color.

"A measured volume of the chlorid-sulphocyanate mixture is diluted so that the normality of the chlorid is about one-fiftieth. The solution is treated with I cc. of the iron-ammonium alum and brought to a boil in a large Erlenmeyer flask. To the gently boiling solution concentrated nitric acid is added at the rate of about three drops a minute, during which operation the liquid should be frequently stirred to prevent superebullition. The addition of the acid in the above described manner is continued until the color of the solution has faded to a pale orange, and then without further addition of acid the solution is allowed to boil for a few minutes longer, by which treatment the last traces of sulphocyanate are oxidized. The complete oxidation requires about twenty minutes time; a change of color to pure yellow indicates the completion of the operation. The odor of hydrocyanic acid is now practically gone.

"Cool the solution thoroughly under the tap and add to it one third its volume of concentrated nitric acid. Add a measured amount of $\frac{\mathrm{N}}{\mathrm{I}_{5}}$ silver nitrate in moderate excess above the quantity required to precipitate the chlorid and shake the mixture until the precipitate is well coagulated, which requires but a few minutes' agitation when the solution is cool, but a much longer time if the solution is heated. When the precipitate is well coagulated bring the solution again to a 
gentle boil, maintaining it at this temperature for about five minutes. The liquid above the precipitate must become perfectly clear.

"Filter the solution while still hot through a rapid-running double filter, paying no attention to the opalescence which forms in the filtrate upon cooling. Wash the precipitate with hot water containing a few drops of nitric acid. Allow the liquid to cool thoroughly, dilute until the volume is about three times that of the solution before filtration, add 2 cc. of the iron indicator for every $100 \mathrm{cc}$. of solution and run in the standardized sulphocyanate to a strong red color. Stir the solution for five minutes and then add the standardized silver nitrate drop by drop, with stirring, until the color just disappears. 'A single drop of the sulphocyanate solution should then give a permanent pink color; should it fail to do so, titrate back and forth with the silver nitrate and ammonium sulphocyanate until the color changes on addition of a single drop. The titration is best conducted in a porcelain dish, against the white ground of which the end-point is most accurately determined; in glassware transmitted light effects may be misleading. The total silver nitrate added, minus the total ammonium sulphocyanate added, is the silver nitrate equivalent of the chlorid present.

"Determination of Bromids. The analysis of bromid-sulphocyanate mixtures is conducted precisely as that of the chlorid mixtures, with three modifications: (a) the solution to be treated with nitric acid for the destruction of the sulphocyanates should be diluted to roughly one-hundredth normality with respect to bromids; $(b)$ after the sulphocyanate has been decomposed at the boiling temperature and the liquid thoroughly cooled, only one sixth its volume of concentrated itric acid is added; $(c)$ the precipitated silver salts should not be iltered off before the excess silver nitrate is determined."

This method depends upon the following assumptions: (I) that ulphocyanates may be decomposed at a temperature of about $100^{\circ} \mathrm{C}$. y such concentrations of nitric acid as are insufficient either to oxidize hlorids or bromids or to cause the volatilization of hydrochloric or yydrobromic acid; (2) that all but traces of the hydrocyanic acid ormed by this decomposition can be volatilized during the time required or the oxidation itself; (3) that the silver cyanid formed is sufficiently oluble in hot nitric acid to be separated quantitatively from the silver hlorid by filtration; (4) that small amounts of silver cyanid do not terfere with the determination of the excess of silver by means of a alphocyanate titration since it acts as a soluble silver salt. 


\section{CHAP'TER XVII}

\section{CITRIC ACID AND CITRATES}

FREE citric acid may be estimated by titration with standard sodium hydroxid solution in the presence of phenlophthalein as indicator. Citrates of potassium sodium and lithium may be estimated as directed under Estimation of Organic Salts of the Alkalies, page 83. The citrate being converted into a carbonate, and then titrated with standard acid, using methyl orange as indicator.

Citrates of the Alkalies and Earths may be estimated by treating with a solution of lead nitrate or acetate. The resulting precipitate of lead citrate is then washed with a mixture of equal parts of alcohol and water, and then suspended in water and treated with $\mathrm{H}_{2} \mathrm{~S}$ gas until all the lead is precipitated as sulphid. The lead sulphid is separated and the clear solution boiled to expel $\mathrm{H}_{2} \mathrm{~S}$, and then titrated with normal alkali solution.

Each cc. of the latter used $=0.070 \mathrm{gm}$. of crystallized citric acid.

This method may be employed for estimating solutions of citrate of magnesia.

Lime-juice or Lemon-juice, the chief constituent of which is citric acid, may be estimated by titrating with $\frac{\mathrm{N}}{10}$ potassium hydroxid in the same manner as other acid solutions.

Lime-juice contains on an average 7.84 per cent, rarely as much as to per cent, and very seldom as little as 7 per cent, of citric acid.

Commercial lime-juice frequently contains sulphuric, hydrochloric, or tartaric acid. Therefore, before applying this test, the absence of notable quantities of these acids must be insured by qualitative tests.

Warrington's Method (Jour. Chem. Soc., 1875, 934). $20 \mathrm{cc}$. ordinary juice or $4 \mathrm{cc}$. of concentrated juice are neutralized with norma sodium hydroxid solution and diluted to about $50 \mathrm{cc}$. The mixture i: heated to boiling and a small excess of calcium chlorid solution added The boiling is continued for about half an hour, the precipitate col lected on a filter and washed with hot water.

The filtrate and washings are mixed and concentrated to abou I5 cc.; a few drops of ammonia-water are added and the precipitat produced, collected separately and washed. 
Both filters and their precipitates are then dried and incinerated at a low red heat and the residue titrated with $\frac{\mathrm{N}}{\mathrm{I}}$ hydrochloric acid.

The process depends upon the formation of sodium citrate, which is precipitated as calcium citrate. This is converted by ignition into carbonate, which is finally titrated with the normal acid.

Each cc. represents 0.0695 gm. of $\mathrm{H}_{3} \mathrm{C}_{6} \mathrm{H}_{5} \mathrm{O}_{7}, \mathrm{H}_{2} \mathrm{O}$. 


\section{CHAPTER XVIII}

\section{CYANOGEN AND ITS COMPOUNDS}

Tht estimation of cyanogen in the form of alkali cyanids or hydrocyanic acid may be effected in a number of ways. The most satisfactory and simplest are those depending upon the reaction with silver nitrate. The method of Liebig (see page 125), in which standard silver nitrate solution is added, until the first appearance of a permanent precipitate of silver cyanid, is one of the oldest and best known, and the results obtained with it are quire accurate in the absence of certain impurities. The cyanogen in mercuric cyanid in cyanogen bromid, and in the double cyanids of silver, gold, nickel, cobalt, iron, copper, zinc, and a few other metals cannot be estimated by this method.

The method of Vielhaber (see page 127), using chromate as an indicator, and titrating with silver nitrate, is very popular, has a welldefined end-reaction, and is quite accurate, but like the foregoing is useless in the presence of certain metallic impurities.

The Denigé-Sharwood method in which potassium iodid and ammonia are used as indicators (see page 128 ) is equally if not more accurate than the before-mentioned methods, and has the advantage that it can be employed in the presence of many of the impurities which interfere with the working of the others. Sharwood (J. A. C. S., $\mathrm{I} 897,400)$, in an extensive series of well-conducted experiments, comparing this method with Liebig's, shows the special applicability of the method, for spent cyanid solutions, etc., in the cyanid method of gold extraction, and proves the superiority of the method over Liebig's where the cyanid solutions tested are impuie. With pure solutions the results are practically identical. The standard solution, he says, should not be over twentieth normal. See also Engineering and Mining Journal, I898, 2 I 6.

In Volhard's method, an excess of standard silver nitrate solution is used, and the excess determined by residual titration with sulphocyanate solution, using ferric alum as an indicator. This method can be made to give reliable results. Its disadvantage over the others is that the precipitated silver cyanid and the sulphocyanate react upon one another during the titration, and hence make it necessary to remove the former by filtration before titrating back with the sulphocyanate 
solution. This is a decided inconvenience, and hence the foregoing methods are usually preferred. The irregularities caused by the reaction between silver cyanid and sulphocyanate may to some extent be avoided by using a very weak sulphocyanate solution, which less readily dissolves the silver cyanid.

A weighed quantity of the cyanid is treated with a measured excess of decinormal silver nitrate solution in order to unite the cyanogen entirely with the silver as silver cyanid; the mixture is then made up to a certain volume by the addition of distilled water, thoroughly shaken and a portion filtered through a dry filter. An aliquot portion of the whole solution is then removed by means of a pipette, some ammonio-ferric sulphate solution added, then strongly acidulated with nitric acid, and titrated for the excess of silver nitrate with $\frac{N}{10}$ potassium sulphocyanate solution. The quantity of silver being found in the aliquot portion, it is multiplied by the proper figure, and the quantity of silver in the entire solution is ascertained. This deducted from the quantity originally added gives the quantity which went into combination with the cyanogen.

$$
\begin{aligned}
\text { Each cc. of } \frac{\mathrm{N}}{\mathrm{IO}} \mathrm{AgNO}_{3} \text { V. S. } & =0.002584 \text { gm. CN. } \\
& =0.002684 \text { " } \mathrm{HCN} . \\
& =0.006470 “ \mathrm{KCN} . \\
& =0.004872 \text { “ } \mathrm{NaCN} . \\
& =0.012509 \text { " } \mathrm{Hg}(\mathrm{CN})_{3} .
\end{aligned}
$$

This is essentially the method of Volhard, described on page I22. The end-reaction is known by the appearance of a red color. In estimating cyanids by this method the above-described procedure must be carefully followed.

The following precautions must be taken, which are not necessary when the halogens are estimated by this method, namely:

The cyanogen must be completely combined with the silver and under no circumstances must the solution be acidulated before the silver solution is added, otherwise the cyanid will be converted into hydrocyanic acid, as the equation shows:

$$
\mathrm{KCN}+\mathrm{HNO}_{3}=\mathrm{KNO}_{3}+\mathrm{HCN} .
$$

This would not only cause a loss by volatilization, but would seriously endanger the health or even the life of the analyst. 
Furthermore, the sulphocyanate solution must not be added to the solution which contains the silver cyanid in suspension, as is done in the case of the halogens, because the sulphocyanate will react with silver cyanid, which is not the case with the haloid salts of silver. Hence it is directed to filter the liquid and operate upon an aliquot portion.

In the estimation of solutions containing free $\mathrm{HCN}$, such as bitteralmond water, a weighed quantity of the latter is poured directly into the silver nitrate solution in order to lose as little as possible of the hydrocyanic acid.

Cyanids insoluble in water are treated with an acid in order to set free the hydrocyanic acid, which is distilled over and received in a solution of potassa.

Titration with Standard Iodin Solution (Fordos and Gelis.*) This method is based upon the reaction of free iodin on potassium cyanid, which is as follows:

$$
\mathrm{KCN}+2 \mathrm{I}=\mathrm{KI}+\mathrm{ICN} .
$$

Two equivalents of iodin correspond to one equivalent of cyanogen.

The standard iodin solution is added to the cyanid soiution, until it ceases to lose its color. The end-reaction is known by the solution becoming yellow. If free hydrocyanic acid is to be determined, the latter should first be made alkaline by the cautious addition of sodium or potassium hydroxid, any excess of alkali hydroxid is then converted into bicarbonate by the addition of carbonic acid water. Hydroxid, and even normal carbonate, will decolorize iodin. Pure alkali cyanids do not need this preparation for analysis-they are simply dissolved in water and titrated with standard iodin. Commercial cyanids are, however, frequently contaminated with alkali hydroxid or carbonate, and must therefore be treated with carbonic acid water as described.

Sulphids must be absent, as this will vitiate the results.

The Process. Five grams of potassium cyanid accurately weighed are dissolved in water to make $500 \mathrm{cc}$. Of this solution, ro cc. (representing $0.1 \mathrm{gm}$. of the cyanid) are removed for analysis, about $250 \mathrm{cc}$. of water are added and then $100 \mathrm{cc}$. of carbonic acid water, the solution shaken and then titrated with $\frac{\mathrm{N}}{\mathrm{IO}}$ iodin to a slight but permanent yellow color. Guérin suggests the employment of borax, instead of alkali hydroxid, before titration of hydrocyanic acid by this method

* Jour. de chim. et de Pharm., XXIII, 48; Jour. f. prakt. Chem., LIX, 255. 
or by that of Liebig. Io cc. of the dilute acid are treated with Io cc. of 3 per cent borax solution.

$$
{ }_{2} \mathrm{HCN}+\mathrm{Na}_{2} \mathrm{~B}_{4} \mathrm{O}_{7}=\mathrm{H}_{2} \mathrm{~B}_{4} \mathrm{O}_{7}+2 \mathrm{NaCN} .
$$

Each cc. of $\frac{\mathrm{N}}{\mathrm{IO}}$ iodin V. S. $=0.001342 \mathrm{gm}$. of $\mathrm{HCN}$;

$$
=0.003235 \text { “ “ } \mathrm{KCN} \text {. }
$$

Titration with Standard Mercuric Chlorid Solution (Hannay).* In this method the cyanid in solution, made alkaline by the addition of ammonia water, is titrated with a standard solution of mercuric chlorid (13.443 gm. per Iooo cc.), stirring constantly until a bluish-white opalescence is produced (mercurammonium chlorid $\left(\mathrm{NH}_{2} \mathrm{Hg}\right) \mathrm{Cl}$ ). With pure cyanid the reaction is very delicate, but it is not so accurate with impure commercial cyanids, though good results may be obtained in the presence of some impurities, such as alkaline salts, sulphocyanate, and cyanates. The end-reaction is best observed if the beaker is placed on a black surface.

Titration with Standard Copper Solution (Flajolot). This method depends upon the reaction between copper sulphate and an alkali cyanid, a double cyanid of copper and the alkali being formed. The reaction is illustrated by the following equation:

$$
{ }_{4} \mathrm{KCN}+\mathrm{CuSO}_{4}=(\mathrm{KCN})_{2} \cdot \mathrm{Cu}(\mathrm{CN})_{2}+\mathrm{K}_{2} \mathrm{SO}_{4} .
$$

The end-reaction is recognized by the decolorization of the alkaline copper sulphate solution.

According to the equation, 247.85 gms. of crystallized copper sulphate, $\mathrm{CuSO}_{4}+{ }_{5} \mathrm{H}_{2} \mathrm{O}$, are decolorized by 258.80 gms. of potassium cyanid.

To carry out this method a standard solution is made, containing in rooo cc. 24.785 gms. of pure crystallized copper sulphate. Twenty cubic centimeters of this solution are introduced into a small flask and ammonia water added until the precipitate which at first forms is just redissolved, and the solution assumes a deep-blue color. The cyanid solution to be tested (and which should contain about 5 or 6 gms. per liter), is delivered from a burette, drop by drop, until the last drop added just decolorizes the copper solution. In order to see this change clearly it is well to place the flask on a white surface.

The $20 \mathrm{cc}$. of the copper solution made as above described represent $0.5177 \mathrm{gm}$. of potassium cyanid. Therefore whatever quantity of the 
cyanid solution is taken to bring about the discoloration, that quantity contains $0.5177 \mathrm{gm}$. of potassium cyanid, $\mathrm{KCN}$, or its equivalent of $\mathrm{HCN}, \mathrm{NaCN}$, or $\mathrm{CN}$.

Example. Assuming that $160 \mathrm{cc}$. of the potassium cyanid solution were used for the decolorization of the $20 \mathrm{cc}$. of copper solution, then the $160 \mathrm{cc}$. contains $0.5177 \mathrm{gm}$. of pure $\mathrm{KCN}$. We may now calculate the per cent of pure $\mathrm{KCN}$ in the sample of commercial salt taken for analysis If $5 \mathrm{gms}$. of the sample were dissolved in sufficient water to make $1000 \mathrm{cc}$., $\mathrm{r} 60 \mathrm{cc}$. will contain $0.8 \mathrm{gm}$. of the sample. And since the assay showed that $160 \mathrm{cc}$. of the solution contained $0.5177 \mathrm{gm}$. of pure $\mathrm{KCN}$, therefore $0.8 \mathrm{gm}$. of the sample contains $0.5177 \mathrm{gm}$. of $\mathrm{KCN}$.

$$
\frac{0.5177 \times 100}{0.8}=64.7 \text { I per cent. }
$$

By the Modified Kjeldahl Process. To $0.2 \mathrm{gm}$. of the cyanid add 2 or 3 gms. of phenolsulphuric acid,* Io to $20 \mathrm{cc}$. of highly concentrated sulphuric, and 0.1 to $0.2 \mathrm{gm}$. of yellow mercuric oxid. $\dagger$

These substances are to be introduced into a round-bottomed flask of 200 to $250 \mathrm{cc}$. capacity, which is placed in an oblique position on a square of wire gauze or of asbestos and heated to just below the boiling point of the liquid. This digestion should be continued until the solution is clear, and all of the nitrogen is converted into ammonium sulphate. Several hours will be consumed before this end is attained.

In order to condense any acid vapors and to prevent loss by spurting, a glass bulb tube may be inserted into the neck of the flask. At the end of the operation the heat is withdrawn and the solution, which should be colorless, is transferred (after cooling) to the distillation flask. See Fig. 57 .

An excess of sodium hydroxid is added to it after the apparatus is set up, and the distillation begun, the liberated.ammonia gas being conducted into a receiving flask containing a measured quantity of $\frac{\mathrm{N}}{\mathrm{IO}}$ sulphuric acid. This absorbs the ammonia, forming mmonium sulphate. When about half of the liquid has distilled over, the process is completed, and the contents of the receiver are titrated in the pres-

* Dissolve $5 \circ$ gms. of phenol in a small quantity of concentrated sulphuric acid, and then make up the solution to $100 \mathrm{cc}$. with more concentrated sulphuric acid.

† The mercuric oxid made from mercuric nitrate is not suitable-that made in the wet way is to be used. 
ence of methyl orange with $\frac{\mathrm{N}}{\mathrm{IO}}$ sodium hydroxid solution. The volume of the latter required, deducted from the quantity of $\frac{\mathrm{N}}{\text { Io }}$ sulphuric acid originally taken, gives the quantity of the latter which was neutralized by ammonia, and hence represents the nitrogen of the cyanid tested.

Example. 50 cc. of $\frac{\mathrm{N}}{\mathrm{IO}}$ culphuric acid were in the receiver. After distillation the contents of the receiver required for complete neutralization, just 2 I cc. of $\frac{\mathrm{N}}{\mathrm{IO}}$ sodium hydroxid solution. Hence $50 \mathrm{cc} .-2 \mathrm{I} \mathrm{cc}$. $=29 \mathrm{cc}$. is the quantity of $\frac{\mathrm{N}}{\mathrm{IO}}$ sulphuric acid which was neutralized by the ammonia which distilled over.

I6.93 gms. of ammonia represents $64.70 \mathrm{gms}$. of potassium cyanid.

$$
\begin{aligned}
& { }_{2} \mathrm{NH}_{3}+\mathrm{H}_{2} \mathrm{SO}_{4}=\left(\mathrm{NH}_{4}\right)_{2} \mathrm{SO}_{4}
\end{aligned}
$$

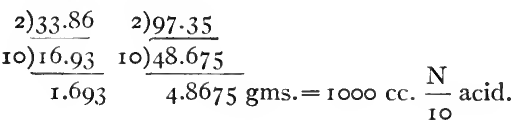

Thus each cc. of $\frac{\mathrm{N}}{\mathrm{IO}}$ sulphuric acid represents $0.001693 \mathrm{gm}$. of $\mathrm{NH}_{3}$, which is equivalent to $0.006470 \mathrm{gm}$. of $\mathrm{KCN}$.

If $29 \mathrm{cc}$. were neutralized by the evolved ammonia, then $29 \times 0.006470$ gm. $=0.18763 \mathrm{gm} . \quad$ Therefore the sample tested is 93.8I per cent pure.

Assay of Insoluble Cyanids. Cyanids insoluble in water are treated with an acid in order to set free the hydrocyanic acid which is distilled over and received in a solution of potassa.

Care must be taken in distilling hydrocyanic acid, insamuch as it is partially decomposed into formic acid and ammonia in the presence of much free acid. To overcome this, the distilling apparatus shown in Fig. 68 is used. The hydrochloric acid is contained in the pipette. The cyanid together with some water is placed in the flask. The water in the flask is heated to boiling, and the hydrochloric acid allowed to flow in slowly, drop by drop. In this way the hydrocyanic acid formed by each drop of hydrochloric acid distils over immediately with the vapor of water, and is condensed by means of the Liebig's condenser. The reaction is as follows:

$$
\mathrm{Hg}(\mathrm{CN})_{2}+{ }_{2} \mathrm{HCl}={ }_{2} \mathrm{HCN}+\mathrm{HgCl}_{2} \text {. }
$$


If any hydrochloric acid distills over with the hydrocyanic acid, the analysis is spoiled, or at least cannot be concluded by titration with silver nitrate, because hydrochloric acid reacts with silver. This may be avoided by the use of nitric, sulphuric, or phosphoric acid.

If potassium ferrocyanid is to be estimated in this way it is treated with sulphuric acid; but it must be remembered that only half of the contained cyanogen distils over, the rest remains in combination with potassium and iron.

$$
{ }_{2} \mathrm{~K}_{4} \mathrm{Fe}(\mathrm{CN})_{6}+{ }_{3} \mathrm{H}_{2} \mathrm{SO}_{4}=6 \mathrm{HCN}+\mathrm{K}_{2} \mathrm{Fe}_{2}(\mathrm{CN})_{6}+{ }_{3} \mathrm{~K}_{2} \mathrm{SO}_{4} \text {. }
$$

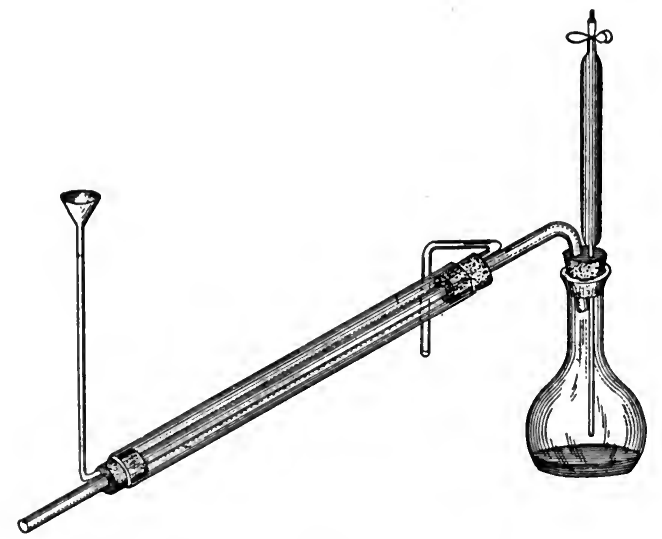

FIG. 68.

Ferrocyanids. Alkali ferrocyanids may be estimated by potassium permanganate or dichromate. The reaction is as follows:

$$
\begin{aligned}
{ }_{5} \mathrm{~K}_{4} \mathrm{Fe}(\mathrm{CN})_{6}+\mathrm{KMnO}_{4}+{ }_{4} \mathrm{H}_{2} \mathrm{SO}_{4} \\
={ }_{5} \mathrm{~K}_{3} \mathrm{Fe}(\mathrm{CN})_{6}+\mathrm{MnSO}_{4}+{ }_{3} \mathrm{~K}_{2} \mathrm{SO}_{4}+{ }_{4} \mathrm{H}_{2} \mathrm{O} .
\end{aligned}
$$

The ferrocyanid is thus oxidized to ferricyanid. The former is yellow in color, the latter red. Therefore the end-reaction is the appearance of a red color, but much practice is required in order to recognize the first appearance of a red, which is very difficult in the greenish-yellow solution. The end-reaction may also be found by bringing a drop of the solution in contact, on a white slab, with a drop of ferric chlorid solution; when a blue color is no longer produced by this contact the end-point is reached.

The process is conducted as follows: 2 gms. of the ferrocyanid are dissolved in sufficient water to make I liter of solution. roo cc. of this, 
representing $0.2 \mathrm{gm}$. of the salt, are acidulated with sulphuric acid, placed in a white porcelain dish, and titrated with the permanganate. I cc. of the permanganate represents

or

$$
\begin{aligned}
& 0.036598 \mathrm{gm} \text {. of } \mathrm{K}_{4} \mathrm{Fe}(\mathrm{CN})_{6}, \\
& 0.04 \mathrm{I} 962 \mathrm{gm} \text {. of } \mathrm{K}_{4} \mathrm{Fe}(\mathrm{CN})_{6}+{ }_{3} \mathrm{H}_{2} \mathrm{O} \text {. }
\end{aligned}
$$

Ferricyanids. These salts may be estimated after reduction to ferrocyanids by titrating with permanganate, as described in the preceding.

The ferricyanid is treated with an excess of potassa or soda and boiled, while small quantities of strong solution of ferrous sulphate are added from time to time, until the precipitate produced is black in color. The solution is then diluted to a convenient quantity, say $300 \mathrm{cc}$.

Ioo cc. of this solution are then taken out, acidified strongly with sulphuric acid, and titrated with $\frac{\mathrm{N}}{\mathrm{IO}}$ permanganate, as directed for the estimation of ferrocyanids.

The process is based upon the fact that ferrous sulphate reduces the ferricyanid to ferrocyanid in the form of a blue precipitate, Turnbull's blue $\left(\mathrm{Fe}_{3} \mathrm{Fe}_{2}(\mathrm{CN})_{12}\right)$, as the equation shows:

$$
\mathrm{K}_{6} \mathrm{Fe}_{2}(\mathrm{CN})_{12}+{ }_{3} \mathrm{FeSO}_{4}=\mathrm{Fe}_{3} \mathrm{Fe}_{2}(\mathrm{CN})_{12}+{ }_{3} \mathrm{~K}_{2} \mathrm{SO}_{4}
$$

This blue precipitate when boiled with an alkali is immediately reduced to magnetic oxid $\left(\mathrm{Fe}_{3} \mathrm{O}_{4}\right)$, and the alkali ferrocyanid goes in solution, as shown by equation:

$$
\mathrm{Fe}_{3} \mathrm{Fe}_{2}(\mathrm{CN})_{12}+8 \mathrm{KOH}=\mathrm{Fe}_{3} \mathrm{O}_{4}+2 \mathrm{~K}_{4} \mathrm{Fe}(\mathrm{CN})_{6}+{ }_{4} \mathrm{H}_{2} \mathrm{O} \text {. }
$$

In the analysis, whatever quantity of permanganate is used must be multiplied by three, because only one third of the entire solution is titrated.

Each cc. of permanganate represents

$$
0.036598 \mathrm{gm} \text {. of } \mathrm{K}_{4} \mathrm{Fe}(\mathrm{CN})_{6} \text {, }
$$

which equals

$$
0.032712 \mathrm{gm} \text {. of } \mathrm{K}_{3} \mathrm{Fe}(\mathrm{CN})_{6} \text {. }
$$

Most of the insoluble ferricyanids are converted into potassium ferricyanid by boiling, with $\mathrm{KOH}$.

The reduction of ferricyanid may also be effected by means of nascent hydrogen developed from zinc and potassa, or by means of sodium amalgam. De Haen employs lead oxid for this purpose in alkaline solution, the reaction being expressed thus:

$$
{ }_{2} \mathrm{~K}_{3} \mathrm{Fe}(\mathrm{CN})_{6}+\mathrm{PbO}+2 \mathrm{KOH}=2 \mathrm{~K}_{4} \mathrm{Fe}(\mathrm{CN})_{6}+\mathrm{PbO}_{2}+\mathrm{H}_{2} \mathrm{O} \text {. }
$$


Sodium peroxid has also been suggested as an agent for completely and rapidly reducing ferri to ferrocyanid. About $0.06 \mathrm{gm}$. of the peroxid is required for $0.5 \mathrm{gm}$. of ferricyanid in $100 \mathrm{cc}$. of water. The mixture is heated until all effervescence is over, acidified with sulphuric acid, cooled, and titrated with permanganate, as above described.

Another method consists in boiling with an excess of potassium hydroxid, then cooling, and adding sufficient $\mathrm{H}_{2} \mathrm{O}_{2}$ to make the solution yellow. The excess of $\mathrm{H}_{2} \mathrm{O}_{2}$ is then gotten rid of by boiling, and the solution acidified with sulphuric acid and titrated with permanganate. Whatever method of reduction is used one molecule of ferricyanid becomes one molecule of ferrocyanid.

The Iodometric Method (Lenssen). Hydroferricyanic acid reacts with potassium iodid in the same manner as ferric salts do, hence the estimation of ferricyanids may be readily effected by the following process, in which the ferricyanid in strongly acidulated solution is digested with potassium iodid. Sufficient acid must be added to liberate the hydroferricyanic acid. The reaction may be expressed by the equation below:

$$
{ }_{2} \mathrm{~K}_{3} \mathrm{Fe}(\mathrm{CN})_{6}+2 \mathrm{KI}+{ }_{2} \mathrm{HCl}={ }_{2} \mathrm{~K}_{4} \mathrm{Fe}(\mathrm{CN})_{6}+\mathrm{I}_{2}+{ }_{2} \mathrm{HCl} .
$$

According to this equation potassium ferricyanid is reduced to potassium ferrocyanid and an equivalent of iodin is set free. In this reaction there is always produced more or less hydroferrocyanic acid, the presence of which has a disturbing influence upon the accuracy of the result. This disturbing effect can be entirely avoided, according to C. Mohr, by the addition of a quantity of iron-free zinc sulphate, which forms zinc ferrocyanid.

The process is as follows:

An accurately weighed quantity of the ferricyanid is dissolved in a convenient quantity of water, some dry potassium iodid is added, together with a tolerable quantity of pure hydrochloric and an excess of iron-free zinc sulphate solution. After a few minutes standing the excess of acid is partially neutralized with sodium bicarbonate, so that the solution is only very faintly acid. At this stage the zinc ferricyanid first formed is converted into zinc ferrocyanid and an equivalent of iodin is set free, which may be titrated with $\frac{\mathrm{N}}{\mathrm{IO}}$ sodium thiosulphate. I cc. of $\frac{\mathrm{N}}{\mathrm{IO}}$ sodium thiosulphate represents

$0.012 .59 \mathrm{gm}$. of iodin;

$0.032712 \mathrm{gm}$. of potassium ferricyanid. 
The estimation of mixed potassium ferro- and ferricyanids may be done by first estimating the former by titration with permanganate, as described, and then estimating in another portion the quantity of the combined salts, after reduction to ferrocyanid.

Sulphocyanates. These salts, when not containing too much impurity, may be readily estimated by means of standard silver nitrate solution, with which they form silver sulphocyanate. Ammonioferric alum serves as indicator. The reaction is fully explained under Estimation of Silver, Volhard's method, page r $3 \mathrm{r}$.

To carry out the process, 7 to ro gms. of the sample are dissolved in sufficient water to make rooo cc. This solution, which should be clear, is filled into a burette and carefully delivered into a white porcelain dish containing $20 \mathrm{cc}$. of $\frac{\mathrm{N}}{\mathrm{IO}}$ silver nitrate (or some other measured quantity), and $5 \mathrm{cc}$. of ammonio-ferric sulphate T. S. together with enough nitric acid to make the solution colorless. The titration is continued until the solution assumes a permanent reddish color.

Each cc. of $\frac{\mathrm{N}}{\mathrm{IO}}$ silver nitrate solution represents

or

$$
\begin{aligned}
& 0.009653 \text { gm. of } \mathrm{KCNS} \text {, } \\
& 0.00756 \text { " " } \mathrm{NH}_{4} \mathrm{CNS} \text {. }
\end{aligned}
$$

Example. Io gms. of potassium sulphocyanate are dissolved in sufficient water to make $1000 \mathrm{cc}$. $20 \mathrm{cc}$. of $\frac{\mathrm{N}}{\mathrm{TO}}$ silver nitrate solution were taken and $24 \mathrm{cc}$. of the sulphocyanate solution were used up.

The 20 cc. of $\frac{\mathrm{N}}{\mathrm{IO}}$ silver nitrate represents $20 \times 0.009653 \mathrm{gm} .=$ $0.19306 \mathrm{gm}$. of KCNS. Then the $24 \mathrm{cc}$. of the sulphocyanate solution analyzed contain $0.19306 \mathrm{gm}$. of pure KCNS.

I000 cc. of the solution contain ro gms. of the sample, therefore $24 \mathrm{cc}$. will represent $0.24 \mathrm{gm}$. of the sample. Then if $0.24 \mathrm{gm}$. of the sample contain $0.19306 \mathrm{gm}$. of $\mathrm{KCN}$, 100 gms. will contain $80.44 \mathrm{gm}$.; thus the salt is 80.44 per cent pure.

Estimation by Means of Cupric Sulphate in Presence of a Reducing Agent (Barnes and Liddle).* This method, which is easily carried out and gives good technical results, depends upon the fact that when a solution of cupric sulphate is added to a solution of a sulphocyanate, in the presence of sodium bisulphite, the insoluble cuprous sulphocyanate is precipitated. The end-reaction is found by bringing a drop of the solution in contact with a drop of ferrocyanid 
solution, when a brown color will be produced. The reaction is as follows:

$$
{ }_{2} \mathrm{KCNS}+{ }_{2} \mathrm{CuSO}_{4}+\mathrm{Na}_{2} \mathrm{SO}_{3}+\mathrm{H}_{2} \mathrm{O}=\mathrm{Cu}_{2}(\mathrm{CNS})_{2}+\mathrm{K}_{2} \mathrm{SO}_{4}+2 \mathrm{NaHSO}_{4} \text {. }
$$

The standard solution of cupric sulphate used contains $6.196 \mathrm{gms}$. per liter, I cc. of which represents $0.00144 \mathrm{I} 7 \mathrm{gm}$. of CNS.

The Process. $3 \mathrm{gms}$. of the sample are dissolved in sufficient water to make $1000 \mathrm{cc} .25 \mathrm{cc}$. of this solution are introduced into a flask, together with $3 \mathrm{cc}$. of a concentrated solution of sodium bisulphite, and the mixture boiled.

The copper sulphate solution is filled into a burette.

When the liquid in the flask has reached the boiling point, about $20 \mathrm{cc}$. of the copper solution are run in, the flask shaken, and the precipitate allowed to settle. A drop of the solution is then taken out on a glass rod anc brought in contact with a drop of five per cent potassium ferrocyanid solution on a white porcelain tile. If no brown color appears, more copper solution must be added; this addition (I cc. at a time, or less) should be continued, with frequent testing, as just described, until the brown color is produced immediately upon contact. A brown color which develops in the mixed drops after a short time (say one minute or a little less), is no indication of the completion of the reaction.

Two or more trials should be made; the first gives approximately the quantity of copper solution required. In the subsequent trials the amount of copper solution first added should be as near as possible the quantity which is required to complete the reaction. 


\section{CHAPTER XIX}

\section{NITROGEN AND ITS COMPOUNDS}

SEVERAL of the better methods for the estimation of nitrogen in organic substances depend upon converting the nitrogen into ammonia, which is then estimated by the usual methods. The older method of Will and Varrentrapp possesses merely an historic interest, in that it is now almost entirely replaced by the equally exact, but more rapid, and readily performed method of Kjeldahl and its modifications. The former method depends upon the fact that the $\mathrm{N}$ of most nitrogenous organic bodies may be converted into ammonia by heating to a dull red heat with soda-lime (a mixture of caustic lime and caustic soda). The ammonia thus evolved is passed into a measured quantity of standard acid, and its quantity determined by retitration with standard alkali.

The substance to be analyzed, intimately mixed with soda lime,

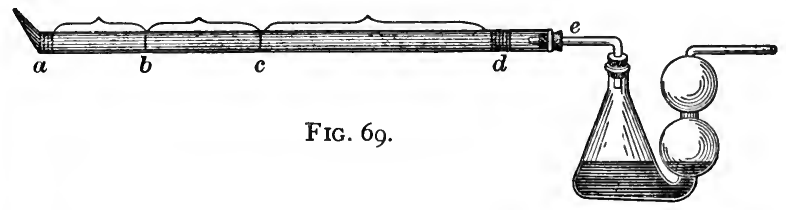

is introduced into a combustion tube made of hard glass, which is open at one end and drawn to a closed point at the other. See Fig. 69. The tube should be about $15 \mathrm{~mm}$. in diameter and $50 \mathrm{~cm}$. in length. The open end is fitted with cork, through which a glass tube passes, and which conveys the evolved ammonia into an absorption flask containing a measured quantity of standard sulphuric acid.

In filling the tube an asbestos plug is first introduced into the far end to prevent plugging of the point $(a)$ by the soda lime; tha soda lime in coarse pieces, is then introduced to $(b)$ and from $(b)$ to $(c)$, the weighed quantity of the substance to be analyzed mixed intimately with powdered soda lime; then from $(c)$ to $(d)$ the tube is filled with more coarsely powdered soda lime, and finally, a small asbestos plug, to prevent any of the soda lime from being passed over into the flask. The tube is now placed in a combustion furnace, the flask containing the standard acid connected, and heat applied, first to that portion 
of the tube from $(c)$ to $(\dot{d})$ until the soda lime in this portion is dull red, then the other end of the tube is heated in the 'same way, the middle portion being kept cool until the soda lime at both ends has been heated to the proper point. The middle portion is then heated, and the heat throughout the tube continued until no more ammonia passes over, and the acid solution begins to rise toward the tube; an aspirator is now applied to the outer end of the absorption flask, the distal end of the combustion tube is broken off and a current of air is drawn through the whole apparatus. Finally, the acid in the absorption flask is retitrated with standard alkali solution, and thus the quantity of liberated $\mathrm{NH}_{3}$ found, and from this the $\mathrm{N}$ is readily calculated.

This method is not applicable in the presence of nitrates.

For Example. $25 \mathrm{cc}$. of $\frac{\mathrm{N}}{10} \mathrm{H}_{2} \mathrm{SO}_{4}$ were originally placed in the absorption flask, and after the completion of the combustion $5 \mathrm{cc}$. of $\frac{\mathrm{N}}{\mathrm{s} O} \mathrm{NaOH}$ were required for neutralization. Therefore, $20 \mathrm{cc}$. of $\frac{\mathrm{N}}{10}$ $\mathrm{H}_{2} \mathrm{SO}_{4}$ were neutralized by the evolved $\mathrm{NH}_{3}$.

Each cc. of $\frac{\mathrm{N}}{10} \mathrm{H}_{2} \mathrm{SO}_{4}$ represents $0.001693 \mathrm{gm}$. of $\mathrm{NH}_{3}$ which is equivalent to $0.001393 \mathrm{gm}$. of $\mathrm{N}$.

Precaution. The soda lime must not be heated above a dark red, otherwise the liberated $\mathrm{NH}_{3}$ will be decomposed into $\mathrm{N}$ and $\mathrm{H}$.

The method of carrying out this process as adopted by the Association of Official Agricultural Chemists $*$ is as follows:

(I) Preparation of Reagents.

(a) Standard solution of hydrochloric acid, standard alkali solution, and cochineal indicator, the preparation of which are described under the Kjeldahl method, as carried out by the A. O. A. C. See page 290.

(b) Soda Lime. Excellent soda lime may be easily and quickly prepared by adding 2.5 parts of quicklime, to I part by weight, of commercial caustic soda (such soda as is used in the Kjeldahl method) dissolved in a sufficient amount of water to slake the lime. The mixture is then dried and heated in an iron pot to incipient fusion, and when cold is ground and sifted. Two sizes of granules are required in this method:

$\left(b_{1}\right)$ Fine enough to pass through a $2.5 \mathrm{~mm}$. sieve.

$\left(b_{2}\right)$ Fine enough to pass through a $1.25 \mathrm{~mm}$. sieve. 
(c) Sodium Carbonate and Lime or Slaked Lime. Instead of soda lime, Johnson's mixture of sodium carbonate and lime or slaked lime may be used.

Slaked lime may be granulated by mixing it with a little water to form a thick mass, which is dried in the water oven until hard and brittle. It is then ground and sifted as above. Slaked lime is much easier to work with than soda lime and gives excellent results, though it is probable that more of it should be used in proportion to the substance to be analyzed than is the case with soda iime.

(2) Apparatus.

(a) Asbestos. The asbestos used should be ignited and kept in a glass-stoppered bottle.

(b) Combustion Tubes. These are about $40 \mathrm{~cm}$. long and of $\mathrm{I} 2$ $\mathrm{mm}$. internal diameter, drawn out to a point and closed at one end.

(c) U-tubes. Large-bulb U-tubes with glass stopcocks, or Will's tubes with four bulbs.

(3) Determination.

The substance to be analyzed should be powdered finely enough to pass through a sieve of I mm. mesh. 0.7 or I.4 gms., according to the amount of nitrogen present, are used for the determination. Into the closed end of the combustion tube put a small loose plug of asbestos, and upon it about $4 \mathrm{~cm}$. of fine soda lime. In a porcelain dish or mortar mix the substance to be analyzed, thoroughly but quickly, with enough fine soda lime to fill about $16 \mathrm{~cm}$. of the tube, or about forty times as much soda lime as substance, and put the mixture into the combustion tube as quickly as possible by means of a wide-neck funnel, rinsing out the dish and funnel with a little more fine soda lime, which is to be put in on top of the mixture. Fill the rest of the tube to about $5 \mathrm{~cm}$. from the. end with granulated soda lime, making it as compact as possible by tapping the tube gently while held in a nearly upright position during the filling. The layer of granulated soda lime should not be less than $12 \mathrm{~cm}$. long. Lastly, put in a plug of asbestos about $2 \mathrm{~cm}$. long, pressed rather tightly, and wipe out the end of the tube to free it from adhering particles.

Connect the tube by means of a well-fitting rubber stopper or cork with the U-tube, or Will's bulbs, containing ro cc. of standard acid, and adjust it in the combustion furnace so that the end projects about $4 \mathrm{~cm}$. from the furnace, supporting the U-tube or Will's bulbs suitably. Heat the portion of the tube containing the granulated soda lime to a moderate redness, and when this is attained extend the heat gradually through the portion containing the substance, so as to keep up a moderate and regular flow of gases through the bulbs, maintaining the heat of the first part until the whole tube is heated uniformly to 
the same degree. Keep up the heat until gases have ceased bubbling through the acids in the bulbs, and the mixture of substance and' soda lime has become white, or nearly so, which shows that the combustion is finished. The combustion should occupy about three-quarters of an hour, or not more than one hour. Remove the heat, and when the tube has cooled below redness break off the closed tip and aspirate air slowly through the apparatus for two or three minutes, to bring all the ammonia into the acid. Disconnect, wash the acid into a beaker or flask, and titrate with the standard alkali.

During the combustion the end of the tube projecting from the furnace must be kept heated sufficiently to prevent the condensation of moisture, yet not enough to char the stopper. The heat may be regulated by a shield of tin slipped over the projecting end of the combustion tube.

It is found very advantageous to attach a Bunsen valve to the exit tube, allowing the evolved gases to pass out freely, but preventing a violent "sucking back" in case of a sudden condensation of steam in the bulbs.

The Ruffle Method (as adopted by A. O. A. C.).*

(i) Preparation of Reagents.

(a) Standard solutions and indicator, the same as in foregoing method.

(b) A mixture of equal parts by weight of fine slaked lime and finely powdered sodium thiosulphate, dried at $100^{\circ}$.

(c) A mixture of equal parts of finely powdered granulated sugar and flowers of sulphur.

(d) Granulated soda lime.

(2) Apparatus.

(a) Combustion tubes of hard, Bohemian glass, $70 \mathrm{~cm}$. long and $\mathrm{I} .3 \mathrm{~cm}$. in diameter.

(b) Bulbed U-tubes or Will's bulbs.

(3) The Determination.

Clean the U-tube and introduce ro cc. of standard acid.

Fill the combustion tube as follows:

(a) A loosely fitting plug of asbestos, previously ignited, and then from 2.5 to $3.5 \mathrm{~cm}$. of the thiosulphate mixture.

(b) The weighed portion of the substance to be analyzed is intimately mixed with from 5 to ro gms. of the sugar and sulphur mixture.

(c) Pour on a piece of glazed paper or in a porcelain mortar a sufficient quantity of the thiosulphate mixture to fill $25 \mathrm{~cm}$. of tube, then add the substance to be analyzed, as previously prepared, 
mix carefully, and pour into the tube; shake down the contents of the tube; rinse off the paper or mortar with a small quantity of the thiosulphate mixture, then fill up with soda lime to within $5 \mathrm{~cm}$. of the end.

(d) Place another plug of ignited asbestos at the end of the tube and close with a cork.

(e) Hold the tube in a horizontal position and tap on the tube until there is a gas channel all along the top. Make connections with the U-tube containing the acid; aspirate and see that the apparatus is tight.

The Combustion.

Place the prepared combustion tube in the furnace, letting the open end project a little, so as not to burn the cork. Commence by heating the soda lime portion until it is brought to a full red heat. Then turn on slowly jet after jet toward the farther end of the tube, so that the bubbles come off two or three a second. When the whole tube is red hot the evolution of the gas has ceased and the liquid in the U-tube begins to recede toward the furnace. Attach the aspirator to the other limb of the U-tube, break off the end of the combustion tube, and draw a current of air through for a few minutes. Detach the U-tube and wash its contents into a beaker or porcelain dish, add a few drops of cochineal solution, and titrate.

The Kjeldahl Method (modified). In this method the nitrogenous organic substance is digested with concentrated sulphuric acid in the presence of an oxidizing agent. The nitrogen is thus converted into ammonia, which in the presence of the large excess of sulphuric acid, forms ammonium sulphate. The solution is then cooled, diluted with water, an excess of caustic soda added, and the evolved ammonia $\left(\mathrm{NH}_{3}\right)$ distilled over into a measured volume of standard acid, and the amount found by titration in the usual way. As oxidizing agent, either of the following substances may be employed: Metallic mercury, yellow mercuric oxid, copper oxid, platinic chlorid, potassium permanganate, or acid potassium sulphate.

The flask in which the digestion is done should be well annealed, should have a rather long neck, and a rounded bottom, and should hold I 50 to $250 \mathrm{cc}$.

The distillation flask should be of the Erlenmeyer pattern, made of hard Bohemian glass, and of about 500 cc. capacity. It should be fitted with a rubber stopper, and a bulbed delivery tube, to prevent the spray of the boiling alkaline liquid from being carried over into the acid solution in the receiver, with which it is connected. A condensing apparatus need not be connected unless the temperature of the laboratory in which the distillation is conducted be high, though usually a condenser is used. Fig. 57 shows the apparatus without 
condenser, while Fig. 70 shows same with condenser attached, and arranged for making a number of distillations simultaneously.

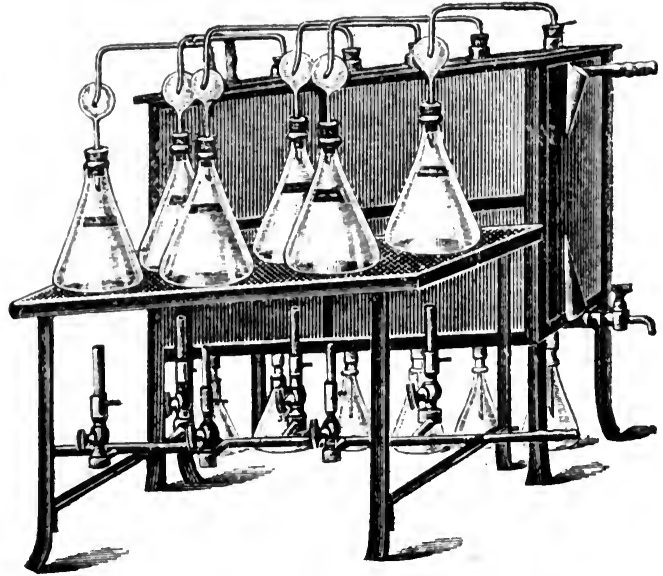

FIG. 70.

The Process. 0.2 to $0.5 \mathrm{gm}$. or a larger quantity of the nitrogenous substance (the amount taken depending upon the nature of the substance and the amount of nitrogen present in it;, is introduced into the digestion flask, ro to $20 \mathrm{cc}$. of pure concentrated sulphuric acid are added, followed by $0 . x$ to $0.2 \mathrm{gm}$. of yellow mercuric oxid, and the flask placed in an inclined position on wire gauze or asbestos, and

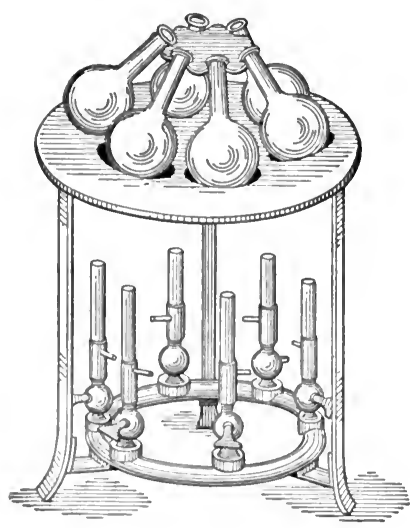

FIG. 7 I. cautiously heated for ten to fifteen minutes or until frothing has ceased. The arrangement shown in Fig. $7 \mathrm{I}$ is suitable for this purpose; as many as six flasks may be heated at once on this apparatus.

The heat is now raised so that the acid liquid boils briskly, and is continued at this point for about fifteen minutes. 5 to $10 \mathrm{gms}$. of potassium sulphate are then added and the heating continued until the black color of the liquid is destroyed, and it is clear and colorless.

The flask is now removed and cooled and its contents transferred with the aid of several portions of water to the distilling flask. 
About Io cc. of 4 per cent potassium sulphid solution are added, followed by a decided excess of saturated sodium hydroxid solution. A few small pieces of granulated zinc are now added, and the flask at once connected with the rest of the apparatus. The distillation is now begun by applying heat, and continued until all of the ammonia has passed over into the standard acid, and the concentrated solution can no longer be safely boiled. This usually requires half an hour. The contents of the receiving flask are then titrated with standard alkali solution in the usual manner.

The use of mercuric oxid hastens the oxidation. 'The use of potassium sulphid is to remove the mercury, as mercurous sulphid, and thus prevent the formation of mercur-ammonium compounds which

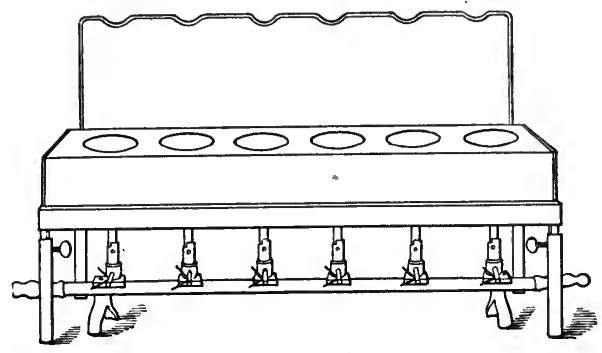

FIG. 72.*

the soda solution cannot completely decompose. The use of zinc is to prevent violent bumping by evolving hydrogen gas.

The sulphuric acid used must be free nitrates and ammonium sulphate.

The solution of sodium hydroxid used must be free from nitrates and nitrites. The indicator may be methyl orange or litmus, but not phenolphthalein.

Example. $0.5 \mathrm{gm}$. of the nitrogenous substance are digested with 20 cc. of $\mathrm{H}_{2} \mathrm{SO}_{4}$ and $0.2 \mathrm{gm}$. of $\mathrm{HgO}$. The evolved $\mathrm{NH}_{3}$ is received in 100 cc. of $\frac{\mathrm{N}}{\mathrm{IO}} \mathrm{H}_{2} \mathrm{SO}_{4}$. Methyl orange is added as indicator, and the solution titrated with $\frac{\mathrm{N}}{\mathrm{IO}} \mathrm{NaOH}, 60 \mathrm{cc}$. of which is required for neutralization. Hence $40 \mathrm{cc}$. of $\frac{\mathrm{N}}{\mathrm{IO}}$ sulphuric acid were neutralized by the evolved $\mathrm{NH}_{3}$.

* Fig. 72 shows a shelf upon which the flasks can be placed in a reclining position for digestion, and which may likewise be employed for heating the distillation flasks. 
Each cc. of $\frac{\mathrm{N}}{\mathrm{rO}} \mathrm{H}_{2} \mathrm{SO}_{4}$ is neutralized by $0.00 \mathrm{r} 693 \mathrm{gm}$. of $\mathrm{NH}_{3}$, and hence represents $0.001393 \mathrm{gm}$. of $\mathrm{N}$. Therefore the $0.5 \mathrm{gm}$. of the substance analyzed contained $0.001393 \times 40=0.0557^{2} \mathrm{gm}$. of $\mathrm{N}$ or II.I 4 per cent.

The above method must be somewhat modified, if the substance examined contains any nitrates.

The Kjeldahl Method (modified by Jodlbauer) gives in such cases very accurate results. This is conducted as follows:

The substance to be analyzed (in requisite amount) is put into the digestion flask, together with I or 2 gms. of zinc dust. 20 to $30 \mathrm{cc}$. of concentrated sulphuric acid containing 2 gms. of salicylic acid are then poured over the mixture so as to cover it at once. Heat is then applied gently until frothing is over, and after the addition of potassium sulphate, the process is completed as described in the foregoing. Instead of salicylic acid, phenol may be taken (see Estimation of Cyanids by Modified Kjeldahl Process). A globule of mercury or a small quantity of the yellow mercuric oxid may be added as well. This method may be used for nitrates alone.

Small quantities of nitrates or nitrites may be estimated by mere distillation with zinc, or still better, with aluminum and caustic soda. Large quantities of nitrates or nitrites cannot, however, be accurately estimated by this means, because too much time is consumed.

The estimation of potassium nitrate by the Jodlbauer modification of Kjeldahl's process, is illustrated by the following example:

$0.2 \mathrm{gm}$. of $\mathrm{KNO}_{3}$ are put into the digestion flask together with 2 cc. of phenol-sulphuric acid, ro to 20 cc. of concentrated $\mathrm{H}_{2} \mathrm{SO}_{4}$ and $0.2 \mathrm{gm}$. of $\mathrm{HgO}$, and heated until colorless. The evolved ammonia is received in 50 cc. $\frac{\mathrm{N}}{10} \mathrm{H}_{2} \mathrm{SO}_{4}$, and for the neutralization $30 \mathrm{cc}$. of $\frac{\mathrm{N}}{\mathrm{IO}} \mathrm{NaOH}$ were required.

Thus 20 cc. of $\frac{\mathrm{N}}{10} \mathrm{H}_{2} \mathrm{SO}_{4}$ were neutralized by the evolved $\mathrm{NH}_{3}$. Each cc. of $\frac{\mathrm{N}}{10} \mathrm{H}_{2} \mathrm{SO}_{4}=0.001393 \mathrm{gm}$. of nitrogen or $0.010043 \mathrm{gm}$. of
$\mathrm{KNO}_{2}$.

The Kjeldahl Method (as adopted by the Association of Official Agricultural Chemists).* (Not applicable in the presence of nitrates). (i) Preparation of Reagents.

(a) Standard Hydrochloric Acid, the absolute strength of which has been determined by precipitating with silver nitrate and weighing the silver chlorid, as follows:

* Bulletin No. 107, U. S. Dept. of Agriculture. 
By means of a preliminary test, with silver nitrate solution, to be measured from a burette, with excess of calcium carbonate to neutralize free acid, and potassium chromate as indicator, determine exactly the amount of silver nitrate required to precipitate all the hydrochloric acid.

To a measured and also weighed portion of the standard acid, add from a burette one drop more of the silver nitrate solution than is required to precipitate the hydrochloric acid. Heat to boiling, cover from light, and allow to stand until the precipitate is granular. Then wash with hot water through a Gooch crucible, testing the filtrate to prove excess of silver nitrate. Dry the silver chlorid at $140^{\circ}$ to $15^{\circ} \mathrm{C}$.

(b) Standard Sulphuric Acid. For ordinary work, half normal acid is recommended. For works determining very small quantities of nitrogen, one tenth normal is recommended.

(c) Standard Alkali Solution. The strength of this solution, relative to the acid, must be accurately determined; one tenth normal ammonia solution is recommended.

(d) Sulphuric Acid, specific gravity $\mathbf{1} .84$, free from nitrates and also from ammonium sulphate.

(e) Metallic Mercury or Mercuric Oxid. The latter should be prepared in the wet way, but not from mercuric nitrate.

(f) Potassium permanganate in fine powder.

(g) Granulated Zinc, Pumice Stone, or Zinc Dust. Used to prevent bumping. When zinc dust is used, 0.5 will be sufficient.

(h) Potassium Sulphid Solution, 40 gms. to one liter.

(i) Sodium Hydroxid Solution. A saturated solution free from nitrates.

(j) Indicator. A solution of cochineal is prepared by digesting and frequently agitating 3 gms. of pulverized cochineal in a mixture of $50 \mathrm{cc}$. of strong alcohol and $200 \mathrm{cc}$. of distilled water for a day or two at ordinary temperatures. The filtered solution is employed as indicator.

(2) Apparatus.

(a) Kjeldahl Digestion Flasks. These are pear-shaped, round-bottom flasks, made of hard, moderately thick, well-annealed glass, having a total capacity of about $250 \mathrm{cc}$. They are $22 \mathrm{~cm}$. long and have a maximum diameter of $6 \mathrm{~cm}$., tapering gradually to a long neck, which is $2 \mathrm{~cm}$. in diameter at the narrowest part and flared a little at the edge.

(b) Distillation Flasks. A flask of ordinary shape, of about $55^{\circ} \mathrm{cc}$. capacity. It is fitted with a rubber stopper, and with a bulb tube above to prevent the possibility of sodium hydroxid being carried over mechanically during distillation. The bulbs may 
be about $3 \mathrm{~cm}$. in diameter, the tubes being of the same diameter as the condenser and cut off obliquely at the lower end, which is fastened to the condenser by a rubber tube.

(c) Kjeldahl Flasks for both Digestion and Distillation. These are pear-shaped, round-bottom flasks, having a total capacity of about $55^{\circ} \mathrm{cc}$. , made of hard, moderately thick, and well-annealed glass. When used for distillation, the flasks are fitted with rubber stoppers and bulb tubes, as given under Distillation Flasks.

(3) Determination.

(a) The Digestion. From ${ }^{2} 0.7$ to 3.5 gms. of the substance to be analyzed, according to its proportion of nitrogen, are brought into a digestion flask with approximately $0.7 \mathrm{gm}$. of mercuric oxid, or its equivalent in metallic mercury, and $20 \mathrm{cc}$. of sulphuric acid. The flask is placed in an inclined position, and heated below the boiling point of the acid for from five to fifteen minutes, or until frothing has ceased. If the mixture froth badly, a small piece of paraffin may be added to prevent it. The heat is then raised until the acid boils briskly. No further attention is required till the contents of the flask have become a clear liquid, which is colorless, or at least has only a very pale straw color. The flask is then removed from the flame, held upright, and while still hot, potassium permanganate is dropped in carefully and in small quantities at a time, till, after shaking, the liquid remains of a green or purple color.

(b) The Distillation. After cooling, the contents of the flask are transferred to the distilling flask with about $200 \mathrm{cc}$. of water, a few pieces of granulated zinc, pumice stone, or $0.5 \mathrm{gms}$. of zinc dust when found necessary to keep the contents of the flask from bumping, and $25 \mathrm{cc}$. of potassium sulphid solution are added, with shaking. Next add $50 \mathrm{cc}$. of the soda solution, or sufficient to make the reaction strongly alkaline, pouring it down the side of the flask so that it does not mix at once with the acid solution. Connect the flask with the condenser, mix the contents by shaking, and distil until all ammonia has passed over into the standard acid. The first $\mathrm{r} 50 \mathrm{cc}$. of the distillate will generally contain all the ammonia This operation usually requires from forty minutes to one hour and a half. The distillate is then titrated with standard alkali.

The use of mercuric oxid in this operation greatly shortens the time necessary for digestion, which is rarely over an hour and a half in case of substances most difficult ot oxidize, and is more commonly less than an hour. In most instances the use of potassium permangal'nate is quite unnecessary, but it is believed that in exceptional cases it is required for complete oxidation, and in view of the unrertainty it is 
always used. The potassium sulphid removes all the mercury from the solution, and so prevents the formation of mercur-ammonium compounds which are not completely decomposed by the sodium hydroxid. The addition of zinc gives rise to an evolution of hydrogen and prevents violent bumping. Previous to use the reagents should be tested by a blank experiment with sugar, which will partially reduce any nitrates that are present, which might otherwise escape notice.

\section{Gunning Method.* (Not applicable in the presence of nitrates.)}

(i) Preparation of Reagents.

(a) Potassium Sulphate. This reagent should be pulverized before using.

The other standard solutions and reagents used are the same as those described under Kjeldhal method.

(2) Apparatus.

The apparatus used is the same as that described in the Kjeldahl method.

(3) Determination. In a digestion flask holding from 250 to 500 cc. place from 0.7 to 3.5 gms. of the substance to be analyzed, according to its proportion of nitrogen. Then add זo gms. of powdered potassium sulphate and from 15 to $25 \mathrm{cc}$. (ordinarily about $20 \mathrm{cc}$.) of concentrated sulphuric acid. Conduct the digestion as in the Kjeldahl process, starting with a temperature below boiling point and increasing the heat gradually until frothing ceases. Digest until the mixture is colorless or nearly so. Do not add either potassium permanganate or potassium sulphid. Dilute, neutralize, and distil as in the Kjeldahl method. In neutralizing it is convenient to add a few dops of phenolphthalein indicator, by which one can tell when the acid is completely neutralized, remembering that the pink color, which indicates an alkaline reaction, is destroyed by a considerable excess of strong fixed alkali. The distillation and titration are conducted as in the Kjeldahl method.

Kjeldahl Method (modified to include the nitrogen of nitrates). $\dagger$

(i) Preparation of Reagents.

Besides the reagents given under the Kjeldahl method, there will be needed-

(a) Zinc Dust. This should be an impalpable powder; granulated zinc or zinc filings will not answer.

(b) Sodium Thiosulphate.

(c) Commercial Salicylic Acid. 
(2) Apparatus.

The apparatus used is the same as in the Kjeldahl method.

(3) Determination. Place from 0.7 to 3.5 gms. of the substance to be analyzed into a Kjeldahl digestion flask, add $30 \mathrm{cc}$. of sulphuric acid containing I gm. of salicylic acid and shake until thoroughly mixed, then add 5 gms. of crystallized sodium thiosulphate; or add to the substance $30 \mathrm{cc}$. of sulphuric acid containing 2 gms. of salicylic acid, then add gradually 2 gms. of zinc dust, shaking the contents of the flask at the same time. Finally, place the flask on the stand for holding the digestion flasks, where it is heated over a low flame until all danger from frothing has passed. The heat is then raised until the acid boils briskly and the boiling continued until white fumes no longer escape from the flask. This requires about five or ten minutes. Add approximately $0.7 \mathrm{gm}$. of mercuric oxid or its equivalent in metallic mercury, and continue the boiling until the liquid in the flask is colorless or nearly so. In case the contents of the flask are likely to become solid before this point is reached, add Io cc. more of sulphuric acid. Complete the oxidation with a little potassium permanganate in the usual way, and proceed with the distillation as described in the Kjeldahl method. The reagents should be tested by blank experiments.

Gunning Method (modified to include the nitrogen of nitrates).*

(I) Preparation of Reagents.

Besides the reagents given under the Gunning method, there will be needed-

(a) Sodium Thiosulphate.

(b) Commercial Salicylic Acid.

(2) Apparatus.

The apparatus used is the same as that given under the K jeldahl method.

(3) Determination. In a digestion flask, holding from 250 to 500 cc., place from 0.7 to $3.5 \mathrm{gms}$. of the substance to be analyzed, according to the amount of nitrogen present. Add from 30 to $35 \mathrm{cc}$. of salicylic acid mixture, namely, $30 \mathrm{cc}$. sulphuric acid to I gm. of salicylic acid, shake until thoroughly mixed, and allow to stand from five to ten minutes, with frequent shaking; then add 5 gms. of sodium thiosulphate and to gms. of potassium sulphate. Heat very gently until frothing ceases, then heat strongly until nearly colorless. Dilute, neutralize, and distil, as in the Gunning method.

Nitric Acid Nitrates. The assaying of nitric acid, in a solution containing no other acid may be accomplished most simply by neu-

* A. O. A. C. Bulletin No. I07. 
tralization, as described on page I03. The strength of a solution of pure nitric acid may also be readily found by taking its specific gravity and referring to a table such as that in the U. S. P.

The determination of combined nitric acid is, on the other hand, a much more difficult problem, though one of greatest importance. A great many methods have been prepared, all of them more or less complicated. Only, the simplest and best will be described here. Whichever method is selected for use it is advised that it be tried several times upon weighed quantities of pure potassium nitrate, in order to become familiar with the method and to acquire sufficient skill in its use to attain accurate results.

I. Conversion with Chlorids. Nitrates may be evaporated with concentrated hydrochloric acid, and the resulting chlorid dissolved in water and titrated with decinormal silver nitrate.

2. Distillation with Sulphuric Acid. Nitrates are decomposed by distillation with moderately dilute sulphuric acid. 'The nitric acid which passes over is received in a measured quantity of standard alkali.

The Process. I gm. of nitrate is introduced into a tubulated retort, together with a cooled mixture of 5 cc. of sulphuric acid and ro cc. of water. The distillation is performed at a temperature of $160^{\circ}$ to $I 70^{\circ} \mathrm{C}$. on a paraffin or sand-bath, and occupies

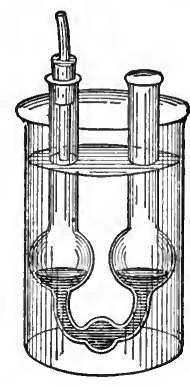

FIG. 73 . about three or four hours. The retort is connected with a bulbed U-tube (see Fig. 73) containing a measured quantity of standard soda solution.

After the distillation is complete, the U-tube is washed out and the excess of alkali found by titration with standard acid.

The distillation may be performed in a partial vacuum, * by connecting the retort air tight with a receiver having two tubulures. Place the normal alkali, diluted with water, in the receiver, and the dilute sulphuric acid in the retort. Heat the contents of both vessels to boiling, with the tubulures open. When the air is expelled from both vessels, drop the nitrate contained in a small glass tube through the tubulure of the retort, quickly insert the stopper, and then insert the stopper of the receiver and take away both lamps. The retort is placed upon a water-bath, and, as the nitric acid will all go over at this temperature in the vacuum, the distillation may be left to take care of itself. The greatest advantage obtained by this process is that there is far less danger of sulphuric acid going over with the nitric acid than when the temperature is raised to $170^{\circ} \mathrm{C}$.

\footnotetext{
* Finkener, Zeit. Anal. Chem., I, 309.
} 
3. Decomposition of Nitrates by Alkali Hydroxids or Carbonates. Nitric acid, combined with bases which are precipitable by alkali hydroxid or carbonate, may be estimated by simply boiling with an excess of standard alkali or carbonate. After cooling, the solution is diluted to about $200 \mathrm{cc}$, the precipitate allowed to settle, and a portion of the clear liquid drawn off, and the free alkali remaining in it determined by titration with standard acid.

In many nitrates, the bases of which are precipitable by hydrogen sulphid, the nitric acid may be determined by adding to the salt in solution about its own weight of some neutral organic salt, e.g., Rochelle salt, and precipitating the metal by hydrogen sulphid. The filtrate and washings are brought to a definite bulk, and free acid is determined in aliquot portions.

4. Conversion of Nitric Acid into Ammonia. If a nitrate is heated in an alkaline solution in which nascent hydrogen is being evolved in

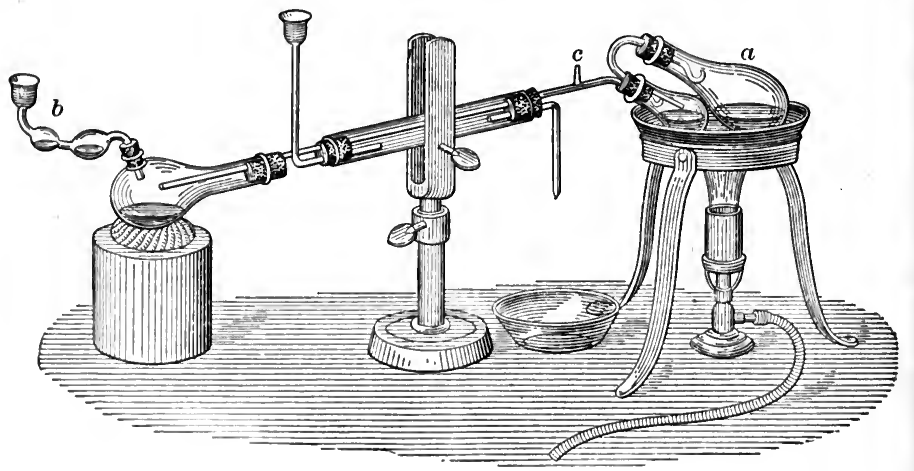

FIG. 74 .

sufficient quantity, the nitric acid is converted into ammonia. From the quantity of ammonia so produced, the quantity of the nitric acid present is readily determined.

Fr. Schultze was the first to utilize this reaction for the quantitative estimation of nitric acid. He was soon followed by W. Wolf, Harcourt, and Siewert. Schultze employed platinized zinc, Harcourt and Siewert used zinc and iron filings. The combination of zinc and iron affords most satisfactory results.

Harcourt employs the apparatus shown in Fig. 74.

The distilling flask (a) holds about $200 \mathrm{cc}$., and is connected with a smaller flask in such a way that both may be placed obliquely upon a sand-bath, as shown in the figure; this arrangement is to prevent the spurting of the boiling liquids into the exit tubes-the latter as an extra 
precaution, are bent into the form of hooks. From the small flask a long tube passes through a Liebig's condenser into a tubulated receiver containing normal sulphuric acid colored with litmus and provided with a bulb-tube $(b)$ of peculiar form. This also contains some of the colored sulphuric acid. The tube which passes through the condenser has a small tubulure $(c)$ closed with a rubber stopper; through this tubulure water may be passed when the distillation is over, in order to wash any traces of ammonia into the receiver. All the corks of the apparatus should be soaked in paraffin.

The bulb-tube $(b)$ on the receiver is turned half way round so as to bring it into a vertical position, and a measured quantity (more than sufficient to fix the ammonia) of normal sulphuric acid is passed into it, to the receiver, a small quantity of litmus tincture having been added. The tube is then turned back to the position shown in the figure, and a little more normal sulphuric acid measured into it. The flasks are now removed, and into the smaller tube, some water is introduced, and the flask placed back into position. Into the larger flask $(a)$ is put 50 gms. of finely-granulated zinc and $25 \mathrm{gms}$. of pure iron filings (purified by sifting, and then heating in a current of hydrogen, or igniting in a covered crucible). The weighed quantity of the nitrate is then added (say $0.5 \mathrm{gm}$.), followed by $25 \mathrm{cc}$. of water and $25 \mathrm{cc}$. of potassium hydroxid solution, of sp.gr. I.3. The flask is then quickly connected, and after standing for about one hour, heat is applied directly under the distillation flask $(a)$, until its contents boil. When distillation begins, the heat is so applied that the water in the smaller flask boils gently. In this manner the fluid is twice distilled and any potassium hydroxid which may have been carried over from $(a)$ is completely retained in the smaller flask.

From one to two hours are required for the distillation. It may be stopped when the hydrogen, which is evolved more fully as the potash solution becomes more concentrated, has passed through the bulb tube $(b)$ for five or ten minutes. When the apparatus has cooled, and the fluid in the bulb tube has receded, the rubber stopper is removed from the tubulure $(c)$ and the tube rinsed into the receiver. The bulb tube is also rinsed with water. The receiver is then disconnected, the lower end of the condenser tube which has projected to the middle of the receiver is also rinsed off into the acid solution, and the latter then titrated with normal alkali.

The Zinc-Iron Method. Under this name the Association of Official Agricultural Chemists adopted the following simplied procedure.*

"Ten grams of the sample are dissolved in $500 \mathrm{cc}$. of water. Of this solution $25 \mathrm{cc}$., corresponding to one-half gram, are placed in a

* Bulletin No. Io7, U. S. Dept. of Agriculture. 
distillation flask of about 400 cc. capacity, I 20 cc. of water added, also about 5 gms. of well washed and dried zinc dust and an equal weight of reduced iron. To the solution are added $80 \mathrm{cc}$. of sodium hydroxid of $32^{\circ} \mathrm{B}$. The flask is then connected with the condensing apparatus and the distillation carried on synchronously with the reduction, the ammonia being collected in carefully standardized acid. The distillation is continued for one or two hours, or until roo cc. have been distilled. The resulting distillate is titrated as in the Kjeldahl method."

This method is applicable to the determination of nitric and ammoniacal nitrogen.

The Ulsch Method. This method may be used for the estimation of alkali nitrates when no ammonium salt or other form of nitrogen is present. It depends upon the fact that when a solution of an alkali nitrate is heated with dilute sulphuric acid, together with some reduced iron, the nitrogen is converted into ammonium sulphate, and the ammonia is then distilled off by boiling with sodium hydroxid, as in Kjeldahl's method.

The Process. $0.5 \mathrm{gm}$. of the alkali nitrate is dissolved in $25 \mathrm{cc}$. of water (or an equivalent quantity of manure solution, though not more than $30 \mathrm{cc}$.), and put into a $150 \mathrm{cc}$. flask, together with $5 \mathrm{gms}$. of reduced iron and $20 \mathrm{cc}$. of dilute sulphuric acid (I.3). The reaction is allowed to go on in the cold until effervesence ceases, the flask being placed in an oblique position. Then the contents of the flask are boiled for six or seven minutes and allowed to cool. The liquid is then transferred to a Kjeldahl distilling flask, an excess of soduim hydroxid and a few pieces of zinc are added. The distillation of the ammonia is then done as in the Kjeldahl process.

If the iron is suspected of containing some form of nitrogen or ammonia, a blank experiment should be made with the iron.

The following modification is that adopted by the Association of Official Agricultural Chemists, Bullet in No. Io7, U. S. Dept. Agriculture.

Ulsch Method (Modified by Street). (Applicable to all nitric and ammoniacal nitrogen determinations.)

Place I gm. of the sample in a half liter flat-bottom flask. Add about $30 \mathrm{cc}$. of water, 2 to 3 gms. of reduced iron, and ro cc. of a mixture of strong sulphuric acid with an equal volume of water; shake well and allow to stand for a short time. This will remove the danger of an explosion caused by the otherwise violent action which takes place. Close the neck of the flask with a rubber stopper through which passes a dropping bulb filled with water. The flask having thus been stoppered, place it on a slab to which a moderate heat is applied. Heat the solution slowly, boil it for five minutes, and cool. Add about roo cc. of water, a little paraffin, and from 7 to ro gms. of magnesium oxid. The flask is then connected with a condenser, 
such as is used in the Kjeldahl method, and the mixture boiled for forty minutes, the ammonia being collected in a known amount of standard acid. When magnesia is used, assurance must be had that it is strongly in excess, and forty minutes are necessary for the complete distillation of the ammonia. The contents of the receiver are titrated, as in the Kjeldahl method.

In the analysis of nitrate salts, proceed as above, except that $25 \mathrm{cc}$. of the nitrate solution equivalent to $0.25 \mathrm{gm}$. of the sample are employed with 5 gms. of reduced iron. After boiling add $75 \mathrm{cc}$. of water, and an excess of sodium hydroxid, and complete the determination as above.

The Kjeldahl process, as modified by Jodlbauer, may be used in the presence of non-nitrogenous organic matter, with very satisfactory results. This method is described on page 290 .

The Kjeldahl and Gunning methods, modified to include nitrogen of nitrates, as adopted by the Association of Official Agricultural Chemists, may also be employed with good results.

The Pelouze method depends upon the oxidation of ferrous salts by the nitrate in the presence of hydrochloric acid. This method, which is described on page 172 , is theoretically perfect, but in practice serious errors are liable to creep in. It is not available in the presence of organic matter. This method has been variously modified, and with care good results may be obtained. Among the modifications may be mentioned, $(a)$ the use of potassium dichromate instead of permanganate in the titration of the residual ferrous salt; $(b)$ the direct titration of the resulting ferric salt by means of standard stannous chlorid, after the manner described on page $23 \mathrm{I} ;(c)$ the iodometric estimation of the resulting ferric salt, after the manner described on page 224. An excess of potassium iodid is added, and the liberated iodin estimated with sodium thiosulphate.

Schlösing's Method. This method was devised for the estimation of nitric acid in tobacco. It affords the very important advantage that it may be used in the presence of organic matter, and has successfully passed through the ordeal of numerous and searching experiments. It is conducted in the apparatus shown in Fig. 75 .

In this method the solution of nitrate is boiled in a flask until all air is expelled, then an acid solution of ferrous chlorid is drawn in, the mixture boiled, and the resulting nitric oxid collected over mercury in a bell jar filled with mercury and milk of lime; the gas is finally brought without loss in contact with oxygen and water, so as to convert it into nitric acid, which latter is then titrated with standard alkali in the usual manner. The reaction is:

$$
6 \mathrm{FeCl}_{2}+2 \mathrm{KNO}_{3}+8 \mathrm{HCl}={ }_{3} \mathrm{Fe}_{2} \mathrm{Cl}_{6}+2 \mathrm{KCl}+{ }_{4} \mathrm{H}_{2} \mathrm{O}+\mathrm{N}_{2} \mathrm{O}_{2} .
$$


The nitrate is dissolved and introduced into the flask $A$, the drawnout neck of which is connected by means of a rubber tube $a$ with the glass tube $b$, at the lower end of which is another rubber tube $c$ six inches in length. The solution of the salt which must be neutral or alkaline is boiled down to a small volume, so that the aqueous vapor will completely expel all of the air from the flask and tubes. The end of the tube $c$ is then placed into a solution of ferrous chlorid in hydrochloric acid contained in a small glass vessel. The flame is now removed, and the condensation of the aqueous vapor in the flask will cause the ferrous chlorid solution to rise and enter the flask $A$. This may be regulated by compressing the tube $c$ with the fingers. When sufficient of the iron solution is absorbed, a little hydrochloric

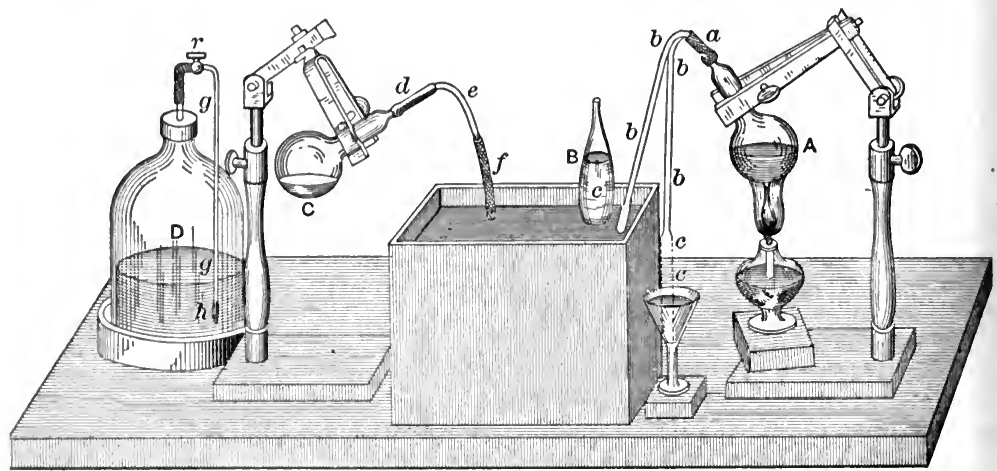

FIG. 75.

acid is allowed to pass in, in the same manner, in three or four portions in order to completely wash the tubes of any ferrous chlorids. In these operations, as well as in all subsequent transfers, it is important to exclude all traces of air. This is easy, provided the drop of liquid which hangs to $c$ when it is carried from one liquid to another is not allowed to fall off. Finally, the tube is rinsed by allowing a little boiled water to recede, and then the tube $c$ closed by means of an iron compressioncock while still filled with water, and its end immersed into the mercury in the trough and brought up under the bell jar $B$. Heat is again applied under the flask $A$, and its contents will boil violently and become black. As soon as pressure is evident within the tube $c$ the compression-cock is removed and the nitric oxid gas allowed to pass into the bell jar receiver $B$. The reaction is usually complete in about eight minutes, when the tube may be removed from under $B$. The receiver $B$ is a small bell jar made from an adapter, and should have a capacity of three or four times the volume of the gas to be received. 
In cases where the evolution of gas is too rapid it may be necessary to immerse the entire receiver, in order to facilitate the condensation of the vapors. The upper part of the receiver is drawn out so as to admit of its insertion into the rubber tube from $C$ and the breaking off of its point. The receiver is first filled with water in order to expel all the air, and then with mercury.

Then by means of a curved pipette a sufficient quantity of thick and well-boiled milk-of-lime is introduced. This is to absorb the hydrochloric acid which boils over from the flask, and thus the nitric oxid is obtained free from traces of acid vapor. Should the nitric oxid be evolved in quantities greater than the receiver will hold at once, the cock is closed, and the flame removed from under the flask. The receiver is then emptied, as below described, charged anew with water, mercury, and milk-of-lime, reconnected and the boiling resumed. When the nitric oxid has been completely collected in the receiver it is transferred to the flask $C$, there to be converted into nitric acid by means of oxygen. The flask $C$ contains a small quantity of water (about roo cc.); it is provided with a rubber tube $d$ connected with a glass tube $e$, the other end of which carries a rubber tube $f$ five inches long. The water in $C$ is boiled to expel all atmospheric air, and while still vigorously boiling, $f$ is connected with the tip of the belljar $B$ which has previously been slightly scratched with a diamond and the tip then broken off. At first the aqueous vapor condenses in the bell jar and expels at the same time the small quantity of milkof-lime remaining in the tip. On now removing the flame a return current is soon established, which draws the nitric oxid into $C$. If this proceeds too rapidly $f$ is compressed by the fingers; as soon as the milk-of-lime in the bell jar has reached the $\operatorname{rim}$ of $f$, the latter is closed by a compression-cock; 20 to $30 \mathrm{cc}$. of pure hydrogen (washed by passing through sulphuric acid and through milk-of-lime) are now introduced into the bell jar in order to complete the transferral of the nitric oxid into $C$. The tube $f$ is now removed from the tip of the bell jar and connected with the glass tube $h$ of the jar $D$, which contains oxygen under pressure; the cock $r$ is now opened and the oxygen allowed to enter $C$. It is absorbed with the appearance of red fumes and the formation of nitric acid. The cock $r$ is closed, the jar $D$ separated, and after half an hour, the flask being occasionally shaken, the nitric acld is dissolved in the water and may be estimated by a standard alkali solution.

The Iodometric Estimation of Nitric and Chloric Acids. McGowan, J. C. S., LXIX, 530 and LXI, 87, describes methods for the iodometric estimation of these acids.

The method is based upon the principle that when a concentrated solution of a nitrate or chlorate is warmed with an excess of pure, 
strong hydrochloric acid, the salts are completely decomposed, the nitrate being resolved into nitrosyl chlorid and chlorin. The reaction is as follows:

$$
\mathrm{HNO}_{3}+{ }_{3} \mathrm{HCl}=\mathrm{NOCl}+\mathrm{Cl}_{2}+{ }_{2} \mathrm{H}_{2} \mathrm{O} .
$$

With chlorates only chlorin is evolved. The operation is to be conducted in an atmosphere of carbon dioxid, and the escaping gases passed through a solution of potassium iodid. The amount of iodin liberated is exactly equivalent to the whole of the chlorin present (free and combined), nitric oxid escaping in the operation upon nitric acid. One molecule of nitric acid thus liberates three atoms of iodin which can be titrated in the usual manner with standard thiosulphate

The details of the methods are given in Sutton's "Volumetric Analysis," and in Z. f. angew. Chem., Aug 15, 1890, Details of a process depending upon the same principle are given by De Koninck and Nihoul.

Nitrous Acid. Estimation by Means of Standard Permanganate. The direct titration of nitrites by means of potassium permanganate is not usually employed, because nitrites in acidulated solution evolve nitrous acid, which being very volatile will occasion a considerable loss. This especially, since the complete oxidation of the nitrous acid requires a slightly raised temperature. This loss may be to some extent obviated by taking very dilute solutions of nitrite for analysis, say not stronger than one part of nitrite in one liter of water. The most satisfactory ways of estimating nitrites by means of potassium permanganate are described on page 165 . The permanganate may be added in excess to the nitrite solution and the excess then found by retitrating with oxalic acid, or the nitrite in solution may be delivered from a burette into a measured quantity of stand- ard permanganate until the latter is just decolorized.

The standardization of the permanganate solution used in this estimation is best accomplished by titration against a weighed amount of pure silver nitrite, which is prepared as follows: Pure potassium nitrate is fused at a strong red heat for some time, allowed to cool, dissolved in hot water, and the greater part of the undecomposed nitrate crystallized out; the potassium nitrite, being very soluble, remains in the mother liquor. A small portion of the mother liquor is then treated with a few drops of silver nitrate. If the precipitate is brown or yellowish, the heat has been too intense, and a portion of the nitrite been decomposed. Add nitric acid to the rest of the mother liquor until a portion tested with silver nitrate gives a white precipitate, and then add silver nitrate until a precipitate ceases to form. The precipitate is washed with a little cold water, dissolved in boiling water, 
and recrystallized once or twice. The crystals obtained have the composition $\mathrm{AgNO}_{2}$.

In the absence of nitrates, nitrous acid and nitrites may be estimated by converting the nitrogen into ammonia, and estimating the latter, as described under nitric acid, or by determining the oxidizing action on ferrous salt. In the presence of nitric acid the permanganate method may be used.

To estimate the quantity of nitrogen tetroxid in red fuming nitric acid, place a few cubic centimeters in about $500 \mathrm{cc}$. of cold, distilled water, and determine the quantity of nitrous acid produced-one molecule of nitrous anhydrid found corresponds to two molecules of nitrogen tetroxid. The latter, when mixed with a large quantity of water as above, is decomposed into nitric and nitrous acid.

$$
\mathrm{N}_{2} \mathrm{O}_{4}+\mathrm{H}_{2} \mathrm{O}=\mathrm{HNO}_{3}+\mathrm{HNO}_{2} \text {. }
$$

The Estimation of Nitrous Acid in the Chamber Acid of Sulphuric Acid Works. The specific gravity is first determined, then the acid is delivered out of a small burette into a measured quantity of standard permanganate. The latter must be largely diluted, preferably with hot water. The addition of sulphuric acid is not absolutely necessary, but it is a good plan to add a small quantity before titrating, in order to avoid any possibility of a precipitation of manganese dioxid. The calculation is then made as follows:

The weight of the acid taken is determined by multiplying the volume consumed in the titration by the specific gravity. Then, knowing the titer of the permanganate solution, we can readily calculate the quantity of nitrous acid.

Each cc. of $\frac{\mathrm{N}}{\text { IO }}$ potassium permanganate $=0.0023345 \mathrm{gm}$. of $\mathrm{HNO}_{2}$.

The Iodometric Method.* This method is based upon the reaction between nitrous acid and an iodid in which an equivalent of iodin is liberated, as shown in the following equation:

$$
{ }_{2} \mathrm{HNO}_{2}+{ }_{2} \mathrm{HI}={ }_{2} \mathrm{H}_{2} \mathrm{O}+{ }_{2} \mathrm{NO}+\mathrm{I}_{2} .
$$

The liberated iodin is then estimated by means of standard thiosulphate in the usual manner.

The process must be conducted with complete exclusion of air, in order to avoid oxidation of the NO, and a consequent excessive liberation of iodin. If the operation is conducted in an atmosphere of carbon dioxid or hydrogen, very good results may be obtained.

* Legler, Ph. Centralh. March 9, I905. 
The simple apparatus described below will answer very well for this purpose.

A small, wide-mouthed flask is fitted with a rubber stopper having four perforations; through one of these an inlet tube is passed which reaches below the surface of the liquid in the flask; into another, an outlet tube is fitted, the inner end of which is flush with the lower surface of the stopper, and the other end dips into water to prevent entrance of atmospheric air. Into the other holes the tips of two burettes are fitted. One of these burettes contains dilute sulphuric acid and the other $\frac{\mathrm{N}}{10}$ thiosulphate.

The Process. Into the flask is introduced a quantity of the nitrite solution to be examined, containing about o.I gm. of nitrous acid, and the solution boiled to drive out air. $5 \mathrm{cc}$. of a ro per cent solution of potassium iodid, or a few small crystals of the dry salt, together with a few drops of starch solution are added, and the flask immediately closed with the stopper and attached apparatus. A good stream of $\mathrm{CO}_{2}$ or $\mathrm{H}_{2}$ is then passed through the apparatus in order to completely drive out all the air. 5 or Io cc. of diluted sulphuric acid are then introduced, and after standing a short time the liberated iodin is estimated in the flask by means of the $\frac{\mathrm{N}}{\mathrm{IO}}$ thiosulphate. The tips of the two burettes must of course be completely filled with the reagents, so that no air will be introduced when their contents are delivered into the flask. I cc. of $\frac{\mathrm{N}}{10}$ thiosulphate $=0.01259 \mathrm{gm}$. of iodin, which in turn represents $0.004669 \mathrm{gm}$. of $\mathrm{HNO}_{2}$. 


\section{CHAPTER XX}

\section{OXALIC ACID AND OX̣ALATES}

FREE oxalic acid may be estimated by direct titration with standard alkali. See page ro5.

It may likewise be estimated by adding an excess of calcium acetate to the solution, which must be neutral or very faintly acidified with acetic acid. Alumina, chromium sesqui oxid, copper, and ferric salts must be absent. The precipitation is made in a hot solution. The precipitate of calcium oxalate is washed with hot water, heated to redness in a crucible, whereby it is converted into a mixture of calcium carbonate and oxid, and is then dissolved in an excess of normal acid, and the excess found by retitration with normal alkali.

The best method, however, for oxalic acid and oxalates as well, is the permanganate method, described on page 153 .

If the titration is conducted with an empirical permanganate solution, standardized by means of iron, we must take into consideration that $2 \mathrm{KMnO}_{4}$ is equivalent to five molecules of $\mathrm{H}_{2} \mathrm{C}_{2} \mathrm{O}_{4}(=446.70)$ on the one hand and ten atoms of iron $(=555)$ on the other.

Hence if in the estimation of a weighed quantity of oxalic acid, $40 \mathrm{cc}$. of a permanganate solution (I cc. of which $=0.0057 \mathrm{gm}$. Fe) are consumed. The quantity of iron represented by $40 \times 0.0057$ is calculated into oxalic acid by multiplying it by $\frac{446.7}{555}$ for anhydrous oxalic acid.

Thus

$$
40 \times 0.0057 \times \frac{446.7}{555}=0.183+\mathrm{gm} .
$$

For crystallized oxalic acid multiply by

$$
\frac{5 \times 125.1}{555} \text { or } \frac{625.5}{555} \text {. }
$$

For oxalic acid anhydrid $\left(\mathrm{C}_{2} \mathrm{O}_{3}\right)$ multiply by

$$
5 \times \frac{71.46}{555} \text { or } \frac{357 \cdot 3}{555} \text {. }
$$


In the titration of salts of oxalic acid the acidulation with sulphuric acid serves not only to prevent the precipitation of manganese dioxid, but also to liberate the oxalic acid, a sulphate of the metal being formed as a by-product. The titration of oxalates by this method can be carried out not only with such salts as form clear solutions with the excess of sulphuric acid, but also with those which form insoluble or scantily soluble sulphates, as calcium, strontium, barium, and lead. With the raised temperature and frequent shaking, the reaction is a quantitative one, and the presence of the white precipitate of sulphate in no way interferes with the recognition of the end-reaction. 


\section{CHAPTER XXI}

\section{OXYGEN AND PEROXIDS}

Oxygen Dissolved in Water. Mohr's method, which depends upon the oxidation of ferrous salts and subsequent titration with potassium permanganate, is briefly as follows:

When a solution of ferrous sulphate made strongly alkaline is poured into water containing dissolved oxygen, the latter is completely absorbed, and upon then acidifying the solution, an amount of ferric sulphate is produced equivalent to the dissolved oxygen of the water.

The water to be tested is placed in a bottle provided with a carefully ground stopper, and a weighed amount of iron wire dissolved in sulphuric acid, or of ferrous-ammonium sulphate, dissolved in water, is added. The bottle is then filled with pure carbon dioxid, an excess of sodium hydroxid solution added, the bottle quickly closed, shaken, and allowed to stand for ten or fifteen minutes. The stopper is then removed, an excess of sulphuric acid introduced, and finally, the mixture titrated with $\frac{\mathrm{N}}{\mathrm{IO}}$ potassium permanganate for excess of ferrous salt. In filling the bottle with carbon dioxid, the gas must not be allowed to run into the bottle longer than is necessary to fill it, as otherwise a part of the oxygen dissolved in the water will diffuse into it, and the results obtained will be too low.

Each cc. of $\frac{\mathrm{N}}{\text { IO }}$ permanganate represents $0.038934 \mathrm{gm}$. of

$$
\mathrm{FeSO}_{4}+\left(\mathrm{NH}_{4}\right)_{2} \mathrm{SO}_{4}+6 \mathrm{H}_{2} \mathrm{O} \text { and } 0.000794 \mathrm{gm} \text {. of oxygen. }
$$

Therefore by multiplying the quantity of ferrous ammonium sulphate which was oxidized by $0.0204 \mathrm{gm}$. we arrive at the quantity of oxygen which was contained, dissolved in the water. Of course the quantity of the iron salt found by titration must be deducted from the quantity originally added to the water.

The reaction may be expressed by this equation:

$$
{ }_{2} \mathrm{FeSO}_{4}+\mathrm{O}+\mathrm{H}_{2} \mathrm{SO}_{4}=\mathrm{Fe}_{2}\left(\mathrm{SO}_{4}\right)_{3}+\mathrm{H}_{2} \mathrm{O} .
$$


Example. $0.5 \mathrm{gm}$. of the ferrous ammonium sulphate were employed, and in the titration $10.4 \mathrm{cc}$. of $\frac{\mathrm{N}}{\mathrm{IO}}$ permanganate were required. $0.5 \mathrm{gm}$. of ferrous ammonium sulphate are represented by $\mathrm{r} 2.84 \mathrm{cc}$. of $\frac{\mathrm{N}}{\mathrm{IO}}$ permanganate, therefore $\mathrm{I} 2.84-\mathrm{IO} .40=2.44$, the quantity of $\frac{\mathrm{N}}{\mathrm{IO}}$ permanganate which represents the dissolved oxygen in the water. $2.44 \times 0.000794=0.00193736 \mathrm{gm}$. oxygen.

The calculation may also be made as follows:

I 5.88 parts by weight of oxygen, will oxidize $2 \times 55.5$ parts of iron, as the above equation shows.

One cubic centimeter of $\frac{\mathrm{N}}{\mathrm{IO}}$ permanganate $=0.00555 \mathrm{gm}$. of iron. $0.5 \mathrm{gm}$. of ferrous ammonium sulphate $=0.07 \mathrm{Ig} \mathrm{gm}$. of iron. If $10.4 \mathrm{cc}$. of the permanganate solution are required, then $10.4 \times 0.00555$ deducted from $0.0719=0.01418 \mathrm{gm}$. iron oxidized.

Then

$$
\frac{0.01418 \times 15.88}{2 \times 55.5}=0.00202+\text { gm. oxygen }
$$

Schützenberger's Method. This method depends upon the use of sodium hyposulphite $\left(\mathrm{Na}_{2} \mathrm{SO}_{2}\right)$ as a reducing agent. 20 to $30 \mathrm{cc}$. of water are introduced into a large Woulff's bottle of about $2000 \mathrm{cc}$. capacity, filled with pure hydrogen. Indigo, carmine solution is added in sufficient quantity to color the solution slightly blue. Standard hyposulphite solution is then cautiously added so as to just discharge the color. To this decolorized liquid, $250 \mathrm{cc}$. of the water to be examined are added. The dissolved oxygen present restores the blue color by oxidation, and the amount of hyposulphite solution now required to again decolorize the liquid is the measure of the dissolved oxygen present. The hyposulphite solution is prepared by the action of zinc dust on a saturated solution of sodium bisulphite, containing an excess of sulphurous acid. For more detailed descriptions of this method and its modifications, see Sutton's "Volumetric Analysis," $9^{\text {th }}$ edition.

See also International Scientific Series, "Fermentation," by $\mathbf{P}$. Schützenberger; Schützenberger and Risler, J. Chem. Soc., I873, p. 936; Roscoe and Lunt, J. Chem. Soc., I889, p. 552; Ramsay and Williams. J. Chem. Soc., I886, p. 75I; Dupré, Analyst., x, I56; M. A. Adams, J. Chem. Soc., LXI, 3 Io. 
Other methods are.

The Iodometric Method of Thresh, J. Chem. Soc., LVII, I85.

The Manganous Hydrate Method of Winkler, Berichte, I888-285 I. Seyler's Modification of Foregoing, C. N., Lxx, I5I.

Hydrogen Dioxid (Peroxid of Hydrogen). The assay of hyen dioxid solution may be conducted titrimetrically by either the permanganate method or the iodometric method of Kingzett; the former is described on page 156 , the latter on page 213 . Gasometric methods are described in Part IV. Of all of these methods the Kingzett method is decidedly the best, in that it is very easily carried out, and that its accuracy is practically unaffected by the presence of any of the substances usually added as preservatives. It is the best method for ethereal peroxid solutions.

The accuracy of the permanganate methods is slightly affected by the presence of moderate quantities of glycerin or boroglycerin, but in the presence of the quantities that would be added to the solution by manufacturers (which would hardly exceed I per cent) the difference in results would scarcely be noticeable. In the presence of salicylic acid, however, this method is worthless. Ether also has a marked disturbing influence upon the accuracy of the results. In fact, generally speaking, in the presence of organic matter the permanganate methods are not to be depended upon. As regards the gasometric methods in general, they require more time, attention, and apparatus than titration methods, and the results obtained by them do not approach the latter in accuracy, unless suitable corrections, requiring tedious calculation, are made for variation in temperature and atmospheric pressure.

Among the other methods for the assay of hydrogen dioxid may be mentioned

(a) Decomposition by Means of Silver Oxid and Measurement of the Oxygen Liberated. The reaction, according to E. Rieger, is represented by the following equation:

$$
{ }_{3} \mathrm{H}_{2} \mathrm{O}_{2}+{ }_{2} \mathrm{Ag}_{2} \mathrm{O}={ }_{3} \mathrm{H}_{2} \mathrm{O}+\mathrm{Ag}_{4} \mathrm{O}+{ }_{2} \mathrm{O}_{2} .
$$

The $\mathrm{Ag}_{4} \mathrm{O}$ being further decomposed into $\mathrm{Ag}_{2} \mathrm{O}$ and $\mathrm{Ag}_{2}$.

(b) Decomposition by Means of Potassium Ferricyanid in Alkaline Solution. Quincke, Zeitschr. f. anorg. Chem., I3, I. The volume of oxygen liberated is noted, and the strength of the hydrogen dioxid solution calculated therefrom.

The equation is

$$
{ }_{2} \mathrm{~K}_{3} \mathrm{Fe}(\mathrm{CN})_{6}+{ }_{2} \mathrm{KOH}+\mathrm{H}_{2} \mathrm{O}_{2}={ }_{2} \mathrm{~K}_{4} \mathrm{Fe}(\mathrm{CN})_{6}+{ }_{2} \mathrm{H}_{2} \mathrm{O}+\mathrm{O}_{2} \text {. }
$$

(c) By Oxidation of Arsenous Acid. According to B. Grützner, Arch. d. Ph., $237-507$, the assay of hydrogen dioxid can be car- 
ried out with exactness by oxidation of arsenous acid $\left(\mathrm{As}_{2} \mathrm{O}_{3}\right)$ in an alkaline solution, and then, by means of standard iodin V. S., the excess of $\mathrm{As}_{2} \mathrm{O}_{3}$ determined.

$$
\mathrm{As}_{2} \stackrel{\bullet}{\mathrm{O}}_{3}+{ }_{2} \mathrm{H}_{2} \mathrm{O}_{2}=\mathrm{As}_{2} \mathrm{O}_{5}+{ }_{2} \mathrm{H}_{2} \mathrm{O} \text {. }
$$

Barium Dioxid may be assayed by the method described on page $\mathrm{I} 6 \mathrm{I}$.

Sodium Peroxid may be assayed by titration with standard permanganate. The peroxid being added to cold water acidulated with sulphuric acid and titrated with the permanganate, or a known weight of the peroxid may be added to an excess of the standard permanganate, acidulated with sulphuric acid, and the excess determined by residual titration with standard oxalic acid. Archbutt (Analyst, xx, 5), recommends a gasometric method in which precipitated cobalt sesquioxid is employed for the decomposition of the peroxid.

The Estimation of the Active Oxygen in Inorganic Persulphates may be carried out by heating a weighed amount of the persulphate with a solution of ferrous ammonium sulphate (Mohr's salt) and titrating the unoxidized portion with standard permanganate. This method cannot be used for organic persulphates. 


\section{CHAPTER XXII}

\section{PHOSPHORIC ACID AND PHOSPHATES}

FREE phosphoric acid is usually estimated by neutralization in the manner described on page IoI.

Phosphoric Acid may also be estimated by Stolba's* method, as Ammonio-magnesium Phosphate.

$0.2 \mathrm{gm}$. of phosphoric acid is supersaturated with ammonia water, so as to convert all of the acid into ammonium phosphate and leave an excess of the alkali.

$$
\underset{97.29}{\mathrm{H}_{3} \mathrm{PO}_{4}}+{ }_{2} \mathrm{NH}_{4} \mathrm{OH}=\underset{{ }_{3} \mathrm{~J}_{2} \mathrm{I}_{5}}{\left(\mathrm{NH}_{4}\right)_{2} \mathrm{HPO}_{4}}+{ }_{2} \mathrm{H}_{2} \mathrm{O} .
$$

An excess of magnesia mixture $\dagger$ is now added in order to precipitate all of the phosphoric acid in the form of ammonio-magnesium phosphate.

$$
\underset{\mathrm{I}_{3} \mathrm{I} . \mathrm{I} 5}{\left(\mathrm{NH}_{4}\right)_{2} \mathrm{HPO}_{4}}+\mathrm{MgSO}_{4}=\underset{\mathrm{I}_{3} 6.40}{\mathrm{Mg}\left(\mathrm{NH}_{4}\right) \mathrm{PO}_{4}}+\mathrm{NH}_{4} \mathrm{HSO}_{4}
$$

The precipitate is washed, first with ammonia water, and then the ammonia is entirely removed by washing with alcohol of 50 per cent or 60 per cent strength.

The precipitate is now dissolved in a measured excess of $\frac{\mathrm{N}}{\mathrm{IO}}$ hydrochloric acid, a few drops of methyl-orange T. S. added, and the excess of acid found by titrating back with $\frac{\mathrm{N}}{\text { IO }}$ potassium hydroxid. The difference between the number of cc. of $\frac{\mathrm{N}}{\mathrm{IO}} \mathrm{HCl}$ added and the

* Fres. Zeitschrift., 16, 100.

† Magnesia Mixture. Dissolve ro gms. of magnesium sulphate or chlorid and $20 \mathrm{gms}$. of ammonium chlorid in $80 \mathrm{cc}$. of water, add $42 \mathrm{cc}$. of ammonia water, set aside for a few days in a well-stoppered bottle, and filter. It should never be used freshly made. 
quantity of $\frac{\mathrm{N}}{10} \mathrm{KOH}$ used gives the quantity of the former which went into combination with the ammonio-magnesium phosphate.

$$
\underset{136.40}{\mathrm{Mg}\left(\mathrm{NH}_{4}\right)} \mathrm{PO}_{4}+\underset{72.36}{2 \mathrm{HCl}}=\mathrm{NH}_{4} \mathrm{H}_{2} \mathrm{PO}_{4}^{\circ}+\mathrm{MgCl}_{2} \text {. }
$$

By consulting the equations given, it will be seen that 72.36 gms. of $\mathrm{HCl}$ are equivalent to ${ }_{3} 6.4$ gms. of $\mathrm{Mg}\left(\mathrm{NH}_{4}\right) \mathrm{PO}_{4}$, or ${ }_{3} 3 \mathrm{I} .15 \mathrm{gms}$. of $\left(\mathrm{NH}_{4}\right)_{2} \mathrm{HPO}_{4}$, or 97.29 gms. of $\mathrm{H}_{3} \mathrm{PO}_{4}$.

This means that $1000 \mathrm{cc}$. of a decinormal $\left(\frac{\mathrm{N}}{\mathrm{ro}}\right)$ solution of $\mathrm{HCl}$, containing 3.618 gms. of the acid, represents $\frac{1}{2} \frac{1}{0}$ of each of these quantities; and one cc. of $\frac{\mathrm{N}}{\mathrm{IO}} \mathrm{HCl}$ thus represents $0.004864 \mathrm{gm}$. of phosphoric acid.

In this estimation care must be taken that all free ammonia is removed from the precipitate, and that the whole of the ammoniomagnesium phosphate is decomposed by the acid before titration with the $\frac{\mathrm{N}}{\mathrm{IO}}$ alkali. This may be insured by using a rather large excess of the acid and warming.

Example. To the precipitate of ammonio-magnesium phosphate obtained from $0.2 \mathrm{gm}$. of phosphoric acid, $50 \mathrm{cc}$. of $\frac{\mathrm{N}}{\mathrm{rO}} \mathrm{HCl}$ are added. In titrating back $15.3 \mathrm{cc}$. of $\frac{\mathrm{N}}{\mathrm{IO}} \mathrm{KOH}$ are required. Hence $34.7 \mathrm{cc}$. of the acid went into combination with the double salt.

Then

and

$$
34.7 \times 0.004864=0.16878+\text { gm. }
$$

$\frac{0.16878 \times 100}{.2}=84.39$ per cent of absolute phosphoric acid.

This method is said to give good results.

By Precipitation as Uranium Phosphate. One of the best methods for the estimation of phosphates is by means of a standard solution of uranium. The process is conducted in hot solutions containing acetic acid. The uranium solution is run into the solution under examination, until a drop of the latter gives a reddish brown color when brought in contact on a white porcelain slab with a drop of potassium ferrocyanid solution.

The Standard Uranium Solution is prepared by dissolving $38.5 \mathrm{gms}$. of uranium acetate or an equivalent quantity of yellow uranium oxid 
and about $50 \mathrm{cc}$. of glacial acetic acid in about $900 \mathrm{cc}$. of water and then so adjusting the solution that it and the standard phosphate solution will correspond, volume for volume.

The Standard Phosphate Solution is prepared by dissolving 35.56 gms. of crytallized sodium phosphate $\left(\mathrm{Na}_{2} \mathrm{HPO}_{4}+\mathrm{I}_{2} \mathrm{H}_{2} \mathrm{O}\right)$ in sufficient water to make $1000 \mathrm{cc}$. Each cc. of this solution represents $0.007047 \mathrm{gm}$. of $\mathrm{P}_{2} \mathrm{O}_{5}$.

Instead of using the sodium phosphate we may prepare the standard phosphate by using microcosmic salt $\left(\mathrm{Na}\left[\mathrm{NH}_{4}\right] \mathrm{HPO}_{4}+{ }_{4} \mathrm{H}_{2} \mathrm{O}\right)$, 20.762 gms. of which are dissolved in sufficient water to make $1000 \mathrm{cc}$.

Standardization of the Uranium Solution. $20 \mathrm{cc}$. of the standard phosphate solution are mixed with a quantity of very dilute acetic acid, the liquid is heated to boiling, then after removing the flame, the uranium solution is run in from a burette, about five drops at a time, until a drop of the solution gives the required reaction with potassium ferrocyanid; 2 cc. of the standard phosphate solution are then added, the liquid again boiled, and again titrated with the uranium solution. The total quantity of uranium solution employed corresponds to $22 \mathrm{cc}$. of the standard phosphate solution, and whatever that quantity is it must be diluted to $32 \mathrm{cc}$. so that it will correspond, volume for volume, with the standard phosphate solution. One cubic centimeter of it will then represent 0.007047 gm. of $\mathrm{P}_{2} \mathrm{O}_{5}$.

The Process for the Estimation of Phosphoric Acid is carried out exactly in the manner just described for the standardization of the uranium solution. It is a good plan to add $2 \mathrm{cc}$. of standard phosphate solution to facilitate the determination of the exact close of the titration; the phosphoric acid in these $2 \mathrm{cc}$. must of course be deducted from the total phosphoric acid found. In determining phosphoric acid by this process, the absence of all bases except the alkalies and alkaline earths and manganese must be assured, likewise all nonvolatile or reducing organic acids, such as citric, tartaric, oxalic, and, formic acids, must be absent. The presence of hydrogen sulphid, sulphurous oxid, hydriodic acid, or the acids of arsen ic likewise interfere.

Instead of titrating direct with the uranium solution as above described, it is usually a better plan to add an excess of the standard uranium solution, and then retitrate with standard phosphate.

If tricalcic phosphate is to be estimated, the uranium solution should be standardized with a solution of tricalcic phosphate, and in the process of titration it is necessary to add nearly the full amount of uranium solution before boiling the mixture, so as to prevent the precipitation of calcium phosphate * which is apt to occur in acetic acid solution when heated, or the inverted process following, may be used.

*Sutton's "Volumetric Analysis," gth edition. 
The Inverted Uranium Method, which is described as follows by Muir, in his translation of Fleischer's "Volumetric Analysis,"* affords exceedingly accurate results. The alkali or alkali earth phosphate is dissolved in acetic acid, the liquid made up to a fixed volume (say $200 \mathrm{cc}$.), and a portion of it placed in a burette. $20 \mathrm{cc}$. of standard uranium solution are heated almost to boiling, with the addition of a few drops of acetic acid. The liquid should remain perfectly clear; if a turbidity occurs, more acetic acid must be added. The phosphate solution is then run in until a drop of the hot liquid ceases to give a reddish-brown color with potassium ferrocyanid, and the number of cc. used is noted. The liquid is again heated nearly to boiling, and the standard uranium solution is cautiously added from another burette until the brown color is again obtained with ferrocyanid. The number of cc. of uranium solution used is added to the $20 \mathrm{cc}$. originally taken; the sum represents the amount corresponding to the quantity of phosphate solution run in from the burette. Each cc. of the standard uranium solution represents $0.007047 \mathrm{gm}$. of $\mathrm{P}_{2} \mathrm{O}_{5}$.

Estimation by Uranic Nitrate. $\dagger$ The solutions required are:

I. A standard uranium solution.

2. A standard phosphate solution.

3. A solution of sodium acetate in dilute acetic acid.

4. A freshly prepared solution of potassium ferrocyanid.

Standard Uranium Solution. Either the acetate $\ddagger$ or nitrate of uranium may be employed.

Thirty-five grams of the salt are dissolved in about rooo cc. of water. The solution keeps better if about $50 \mathrm{cc}$. of glacial acetic acid are included.

Standard Phosphate Solution. 5.886 gms. of crystallized noneffloresced microcosmic salt (ammonio-sodic phosphate) are dissolved in water and diluted to one liter.

Fifty cubic centimeters of this solution will represent $0.1 \mathrm{gm}$. of $\mathrm{P}_{2} \mathrm{O}_{5}$.

The Sodium Acetate Solution is made by dissolving 100 gms. of sodium acetate in water, adding $50 \mathrm{cc}$. of glacial acetic acid, and diluting to one liter.

The standard uranium solution is titrated against the above standard phosphate solution and diluted so that $20 \mathrm{cc}$. of the uranium solution will be equivalent to $50 \mathrm{cc}$. of the phosphate solution. $50 \mathrm{cc}$. of the phosphate solution are placed in a beaker, $5 \mathrm{cc}$. of sodium acetate

* Second edition, 1877 , p. I 6 .

† For fuller details, see Sutton's "Volumetric Analysis," 9th ed.

$\ddagger$ If the acetate is used, the addition of sodium acetate mav be omitted, since the sodium acetate is added to prevent the possible occurrence of free nitric acid. 
solution are added, and the mixture heated to nearly boiling. The uranium solution is then delivered in from a burette, until a drop of the hot solution brought in contact on a white porcelain plate with a drop of the freshly prepared ferrocyanid solution produces a brown color (uranic ferrocyanid). A second and third titration should always be made so as to ascertain the exact strength of the uranium solution, which is then diluted so that $20 \mathrm{cc}$. correspond to 50 of the phosphate solution. If $18.7 \mathrm{cc}$. were required, then each $\mathrm{I} 8.7 \mathrm{cc}$. must be diluted to 20 , or 935 to 1000 .

In estimating phosphoric acid by standard uranium solution it is absolutely essential that all the above conditions should be present. That is, the bulk of fluid should be the same, the quantity of phosphate acted upon should be nearly the same (o.I gm. in $50 \mathrm{cc}$.), the same relative amount of sodium acetate, and the same depth of color in testing.

In the analysis, the phosphate is dissolved in water, if no ammonia is present, I cc. of ro per cent solution is added and neutralized with the least possible quantity of acetic acid; then $5 \mathrm{cc}$. of sodium acetate, and water to make $50 \mathrm{cc}$. The solution is then heated to near boiling, and the uranium solution run in as described.

Several titrations should be made; the first will give roughly the amount required, and that may be taken as a guide.

Each cc. of uranium solution $=0.005 \mathrm{gm}$. of $\mathrm{P}_{2} \mathrm{O}_{5}=0069 \mathrm{H}_{3} \mathrm{PO}_{4}$. This method depends upon the fact that when uranic nitrate is added to a solution of an orthophosphate the whole of the $\mathrm{P}_{2} \mathrm{O}_{5}$ is precipitated as yellow uranyl phosphate. If ammonia is present, the $\mathrm{P}_{2} \mathrm{O}_{5}$ is precipitated as uranyl-ammonium phosphate.

If a mineral acid is present, as when phosphate is dissolved by the use of hydrochloric or nitric acid, a corresponding amount of ammonium acetate and ammonia-water in excess must be added, followed by acetic acid to neutralize. The reactions may be expressed as follows:

$$
\mathrm{Na}_{2} \mathrm{HPO}_{4}+\mathrm{UO}_{2}\left(\mathrm{NO}_{3}\right)_{2}=\mathrm{UO}_{2} \mathrm{HPO}_{4}+{ }_{2} \mathrm{NaNO}_{3},
$$

or

$\mathrm{Na}\left(\mathrm{NH}_{4}\right) \mathrm{HPO}_{4}+\mathrm{UO}_{2}\left(\mathrm{NO}_{3}\right)_{2}=\left(\mathrm{UO}_{2}\right)\left(\mathrm{NH}_{4}\right) \mathrm{PO}_{4}+\mathrm{NaNO}_{3}+\mathrm{HNO}_{3}$.

Gluickmann's Method (R. Segalle's Modification) (Z. f. anal. Chem., 1895). To the solution of phosphoric acid add a measured excess of normal ammonia solution; then sufficient of a neural solution of magnesium sulphate to cause the precipitation of all of the phosphoric acid as ammonio-magnesium phosphate. The mixture is then made up to a definite volume, shaken vigorously, and immediately filtered. 
The excess of ammonia is then determined by titration with normal acid solution. The number of cc. of acid solution used is deducted from the quantity of normal ammonia added, and the difference represents the phosphoric. The reactions which take place are represented by the following equations:

$$
\begin{gathered}
\mathrm{H}_{3} \mathrm{PO}_{4}+{ }_{3} \mathrm{NH}_{3}=\left(\mathrm{NH}_{4}\right)_{3} \mathrm{PO}_{4} ; \\
\left(\mathrm{NH}_{4}\right)_{3} \mathrm{PO}_{4}+\mathrm{MgSO}_{4}=\mathrm{NH}_{4} \mathrm{MgPO}_{4}+\left(\mathrm{NH}_{4}\right)_{2} \mathrm{SO}_{4} .
\end{gathered}
$$

Thus it is seen that one molecule of phosphoric acid is neutralized by three molecules of ammonia.

$$
\begin{aligned}
& \mathrm{H}_{3} \mathrm{PO}_{4}+{ }_{3} \mathrm{NH}_{3} \text {. } \\
& \text { 3) } 97.29 \quad 3 \lcm{50.79} \\
& 32.43 \text { gms. }=\frac{30.79}{16.93} \text { gms. or } 1000 \text { cc. } \frac{\mathrm{N}}{10} \text { V.S. } \\
& 0.03243 \mathrm{gm} .=\quad \text { I cc. } \frac{\mathrm{N}}{10} \text { V.S. }
\end{aligned}
$$

The presence of the magnesium sulphate does not interfere in the least with the titration of the ammonia.

Pemberton's Molybdic Method (Ch. News, Xlvi, 4). This process is based upon the fact that if an aqueous solution of ammonium molybdate be added to a hot solution of a phosphate in the presence of a large quantity of ammonium nitrate and a small excess of nitric acid, the phosphoric acid will be completely precipitated in the form of ammonium phospho-molybdate.

The standard molybdate solution is made by dissolving $89.543 \mathrm{gms}$. of the crystallized salt in about $900 \mathrm{cc}$. of water, and if the solution is not quite clear a few drops of ammonia-water are added, and it is then diluted with water to rooo cc.

The solution should be standardized with a solution of phosphate of known strength.

The Analysis. Take a quantity of the phosphate not containing more than o.I gm. of $\mathrm{P}_{2} \mathrm{O}_{5}$, add a small quantity of water, then 2 cc. of nitric acid (sp.gr. I.4) and Io gms. of granular ammonium nitrate, and heat the solution to $140^{\circ} \mathrm{F}$. or over. Then run in some of the standard molybdate solution, stirring constantly; set aside in a warm place for a few minutes in order to allow the yellow precipitate to settle and leave the supernatant liquid, not clear, but containing widely disseminated particles, in which the yellow cloud produced by the further addition of molybdate solution may be readily seen.

When the precipitation is thought to be nearly complete, the titration is continued carefully, with the aid of a Beale's filter (Fig. 87). By means of the Beale's filter a small portion is taken out of the solution 
at intervals, and tested with a drop or two of the molybdate solution. If a precipitate is produced the solution is washed back into the beaker with a little hot water, and the titration continued until a portion of the filtered solution tested as above no longer yields a precipitate.

If the end-point has been overstepped, a measured quantity of phosphate solution of known strength is added, and the titration with molybdate resumed, the quantity of phosphate thus added being deducted from the amount found.

Each cc. of the molybdate solution represents 0.003 gm. of $\mathrm{P}_{2} \mathrm{O}_{5}$ or $0.004 \mathrm{gm} . \mathrm{H}_{3} \mathrm{PO}_{4}$.

About three titrations should be made: the first shows about how much of the molybdate solution is required, the second gives approximate results, the third will give exact results.

The process is not reliable in the presence of silicates, organic matter, or organic acids.

Pemberton's New Molybdic Method Modified (Bulletin No. 107, U. S. Dept. Agriculture). This method depends upon the precipitation of ammonium phospho-molybdate, and then titrating the precipitate alkalimetrically. The process requires great delicacy of manipulation, but gives excellent results. It is especially suitable for fertilizers.

The solutions required are:

Molybdate Solution. Dissolve Ioo gms. of molybdic acid in $144 \mathrm{cc}$. of ammonium hydroxid, specific gravity 0.90 , and $27 \mathrm{I} \mathrm{cc}$. of water; slowly, and with constant stirring, pour the solution thus obtained into $489 \mathrm{cc}$. of nitric acid (sp.gr. I.42), and II 48 cc. of water. Keep the mixture in a warm place for several days, or until a portion heated to $40^{\circ} \mathrm{C}$. deposits no yellow precipitate of ammonium phosphomolybdate. Decant the solution from any sediment and preserve in glassstoppered vessels.

For use add to roo cc. of this solution $5 \mathrm{cc}$. of nitric acid, sp.gr. I.42. Filter each time before using.

Standard Potassium Hydroxid Solution. This solution contains 18.17106 gms. of potassium hydroxid to the liter. It is prepared by diluting $323.8 \mathrm{I}$ cc. of normal potassium hydroxid (which, has been freed from carbonates by barium hydroxid) to one liter. Ioo cc. of the solution should neutralize $32.38 \mathrm{cc}$. of normal acid. One cc. of this is equal to o.0oI of $\mathrm{P}_{2} \mathrm{O}_{5}$, or I per cent if O.I gm. of the substance is taken for analysis.

Normal sodium hydroxid may be used instead of potassium. The dilution is made in exactly the same way.

Standard Nitric Acid Solution. This solution should correspond in strength to the standard alkali solution, or may be one half that strength. It is standardized by titrating against that solution, using phenolphthalein as indicator. Any mineral acid may be used. The 
indicator is made by dissolving I $\mathrm{gm}$. of phenolphthalein in roo cc. of alcohol.

If a soluble phosphate is to be analyzed, dissolve I $\mathrm{gm}$. in sufficient water to make $250 \mathrm{cc}$. $25 \mathrm{cc}$. of this solution, representing 0.I gm. of the substance, is taken for analysis. If the phosphate is in an insoluble compound or organic substance, 2. gms. are treated by one of the methods given below. After solution, cool, dilute to 200 or 250 cc., mix and pour on a dry filter.

Total Phosphoric Acid. (a) Dissolve in $30 \mathrm{cc}$. of concentrated nitric acid and a small quantity of hydrochloric acid and boil until organic matter is destroyed.

(b) Evaporate with 5 cc. of magnesium nitrate, ignite, and dissolve in hydrochloric acid.

(c) Add $30 \mathrm{cc}$. of concentrated hydrochloric acid, heat, and add cautiously, in small quantities at a time, about $0.5 \mathrm{gm}$. of finely pulverized potassium chlorate to destroy organic matter.

(d) Dissolve in from I 5 to $30 \mathrm{cc}$. of strong hydrochloric acid and from 3 to ro cc. of nitric acid. This method is recommended for fertilizers containing much iron or aluminum phosphate.

Determination. (I) For percentages of 5 or below use an aliquot corresponding to $0.4 \mathrm{gm}$. of substance; for percentages between 5 and 20 use an aliquot corresponding to $0.2 \mathrm{gm}$. of substance, and for percentages above 20 use an aliquot corresponding to $0 . \mathrm{I} \mathrm{gm}$. of substance. Add from 5 to ro cc. of nitric acid, depending on the method of solution (or the equivalent in ammonium nitrate), nearly neutralize with ammonium hydroxid, dilute to from 75 to roo cc., heat in water bath to from $60^{\circ}$ to $65^{\circ} \mathrm{C}$., and for percentages below 5 add from 20 to $25 \mathrm{cc}$. of freshly filtered molylodate solution. For percentages between 5 and 20 add from 30 to $35 \mathrm{cc}$. of molybdate solution; stir, let stand about fifteen minutes, filter at once, wash once or twice with water by decantation, using from 25 to $30 \mathrm{cc}$. each time, agitating the precipitate thoroughly and allowing to settle; transfer to filter and wash with cold water until two fillings of the filter do not greatly diminish the color produced with phenolphthalein by one drop of the standard alkali. Transfer precipitate and filter to beaker or precipitating vessel, dissolve in small excess of standard alkali, add a few drops of phenolphthalein solution, and titrate with standard acid.

(2) Proceed as directed in (I), with this exception: Heat in a water bath at $45^{\circ}$ to $50^{\circ} \mathrm{C}$, add the molybdate solution, and allow to remain in the bath with occasional stirring for thirty minutes.

(3) Proceed as in (I) to the point where the solution is ready to place in the water bath. Then cool solution to room temperature, add molybdate solution at the rate of $75 \mathrm{cc}$. for each decigram of phosphoric acid present, place the stoppered flask containing the solu- 
tion in a shaking apparatus and shake for thirty minutes at room temperature, filter at once, wash, and titrate as in preceding method.

Water-soluble Phosphoric Acid. Place 2 gms. of the sample on a $9-\mathrm{cm}$. filter, wash with successive small portions of water, allowing each portion to pass through before adding more, until the filtrate measures about $250 \mathrm{cc}$. If the filtrate be turbid, add a little nitric acid. Make up to any convenient definite volume, mix well, use an aliquot portion of the solution corresponding to 0.2 or 0.4 gms., add Io $\mathrm{cc}$. of concentrated nitric acid and ammonium hydroxid until a slight permanent precipitate is formed, dilute to $60 \mathrm{cc}$., and proceed as under the preceding method (I).

Citrate-insoluble Phosphoric Acid. Make the solution according to the directions given before and determine the phosphoric acid in an aliquot corresponding to 0.4 gms., as directed for total phosphites.

Determination in Acidulated Samples. Heat roo cc. of strictly neutral ammonium citrate solution of $\mathrm{r} .09 \mathrm{sp} . \mathrm{gr}$. to $65^{\circ} \mathrm{C}$. in a flask placed in a warm-water bath, keeping the flask loosely stoppered to prevent evaporation. When the citrate solution in the flask has reached $.65^{\circ} \mathrm{C}$. drop into it the filter containing the washed residue from the water-soluble phosphate determination, close tightly with a smooth rubber stopper, and shake violently until the filter paper is reduced to a pulp. Place the flask in the bath and maintain it at such a temperature that the contents of the flask will stand at exactly $65^{\circ} \mathrm{C}$. Shake the flask every five minutes.

At the expiration of exactly thirty minutes from the time the filter and residue are introduced, remove the flask from the bath and immediately filter the contents as rapidly as possible; wash thoroughly with water at $65^{\circ} \mathrm{C}$. Then proceed as under $(a)$ or $(b)$.

(a) Transfer the filter and its contents to a crucible, ignite until all organic matter is destroyed, add from Io to $\mathrm{r}_{5} \mathrm{cc}$. of strong hydrochloric acid, and digest until all phosphate is dissolved, or $(b)$ return the filter with contents to digestion flask, add from 30 to 35 , cc. of strong nitric acid, and from 5 to ro cc. of strong hydrochloric acid, and boil until all phosphate is dissolved. Dilute to $200 \mathrm{cc}$., mix well, and pass through a dry filter. Take a definite portion of the filtrate and proceed as under total phosphoric acid.

Determination of Non-acidulated Samples. Treat 2 gms. of the phosphatic material without previous washing with water, precisely in the way above described, except that in case the substance contains much animal matter (bone, fish, etc.), the residue, insoluble in ammonium citrate, is to be dissolved by the treatment described under (b), or by digestion with concentrated sulphuric acid in the presence of a small quantity of sodium or potassium nitrate. 
Citrate-soluble Phosphoric Acid. The sum of the water-soluble and citrate-insoluble subtracted from the total phosphoric acid, gives the citrate-soluble phosphoric acid.

When phosphoric acid is determined in acid phosphate by the Pemberton volumetric method or its usual modifications, the results do not agree with those obtained gravimetrically by the A. O. A. C. method, asserts W. D. Richardson,* and the error frequently amounts to $+\mathrm{I}$ per cent $\mathrm{P}_{2} \mathrm{O}_{5}$.

The disturbing substance is probably sulphuric acid, and if this be removed by barium chlorid, the volumetric method may be applied and accurate results obtained.

Richardson recommends the following procedure:

Weigh 2 gms. into a $250 \mathrm{cc}$. flask, digest by boiling with $30 \mathrm{cc}$. of concentrated nitric acid and $5 \mathrm{cc}$. concentrated hydrochloric acid, then add ro cc. water and boil for five minutes. Add 25 to $30 \mathrm{cc}$. of Io per cent barium chlorid, cool, and make up to volume. Filter through a dry filter, rejecting the first portion of the filtrate, and take $25 \mathrm{cc}$. for the determination. From this point on follow the A. O. A. C. modification of the Pemberton Method.

Estimation of Mixed Disodium and Trisodium Phosphates (Ahlum). + Both of these phosphates may be titrated with standard acid, both are alkaline to methyl orange, while sodium-dihydrogen phosphate is neutral. The end reaction therefore occurs when either or both are converted into the acid phosphate. The equations are:

$$
\begin{aligned}
\mathrm{HCl}+\mathrm{Na}_{2} \mathrm{HPO}_{4} & =\mathrm{NaH}_{2} \mathrm{PO}_{4}+\mathrm{NaCl} ; \\
2 \mathrm{HCl}+\mathrm{Na}_{3} \mathrm{PO}_{4} & =\mathrm{NaH}_{2} \mathrm{PO}_{4}+2 \mathrm{NaCl} .
\end{aligned}
$$

Thus trisodium phosphate requires two molecules of acid, while the disodium hydrogen phosphate requires but one, therefore I cc. of $\frac{\mathrm{N}}{\mathrm{IO}}$ hydrochloric acid will be equivalent to $0.008 \mathrm{I} 465 \mathrm{gm}$. of $\mathrm{Na}_{3} \mathrm{PO}_{4}$ or 0.014105 gm. of $\mathrm{Na}_{2} \mathrm{HPO}_{4}$.

If carbon dioxid is passed into a solution of trisodium phosphate, the latter will be converted into disodium hydrogen phosphate, with the formation of a definite quantity of sodium carbonate.

$$
\mathrm{CO}_{2}+2 \mathrm{Na}_{3} \mathrm{PO}_{4}+\mathrm{H}_{2} \mathrm{O}=2 \mathrm{Na}_{2} \mathrm{HPO}_{4}+\mathrm{Na}_{2} \mathrm{CO}_{3} \text {. }
$$

As the sodium carbonate is directly proportional to the trisodium phosphate, a determination of the latter salt, when in mixture with

\footnotetext{
* J. A. C. S., XXIX, r3I4.

$\dagger$ C. Chester Ahlum, J. A. C. S., XXVIII, 533.
} 
disodium hydrogen phosphate can be easily accomplished, i.e., by passing carbon dioxid into a solution of the mixed salts, and determining the sodium carbonate formed.

The Process. Two grams of the mixed salt are dissolved in water and carbon dioxid passed through the solution until the reaction is complete (about ten or fifteen minutes). The solution is then evaporated to dryness and the sodium carbonate estimated by the Schrötter apparatus. The amount of carbon dioxid eliminated, multiplied by 7.46 , gives the amount of trisodium phosphate present in the mixture.

One gram of the mixed salts is dissolved in water and titrated with $\frac{\mathrm{N}}{\mathrm{IO}}$ hydrochloric acid. The number of cubic centimeters required by the trisodium phosphate is obtained by dividing the amount (in grams) of trisodium phosphate found in the above determination by $0.016292(2 \times 0.00846)$. The number of cubic centimeters required by the disodium hydrogen phosphate is the difference between the number obtained in the titration and the number required by the trisodium phosphate. The number of cubic centimeters required by the disodium hydrogen phosphate, multiplied by 0.01405 , gives the amount of disodium hydrogen phosphate in the mixture.

If the original mixture contains sodium carbonate, a determination of this ingredient will be necessary in order to estimate the amount of sodium carbonate formed from the trisodium phosphate and also to make a correction in the titration.

Example.

(a) 2 gms. of mixed salts gave 0.16 gms. $\mathrm{CO}_{2}$, .

$$
0.16 \text { gm. } \times 7.46=\mathrm{I} .1936 \text { gms. } \mathrm{Na}_{3} \mathrm{PO}_{4} .
$$

(b) I gm. of mixed salts required ror. $83 \mathrm{cc} . \frac{\mathrm{N}}{\mathrm{IO}} \mathrm{HCl}$.

(c) I.1936 gms. $\mathrm{Na}_{3} \mathrm{PO}_{4}$ required 73.25 cc. $\frac{\mathrm{N}}{\text { IO }} \mathrm{HCl}$.

$$
\left(\frac{1.1936}{0.016292}=73.25\right) \text {. }
$$

(d) The $\mathrm{Na}_{2} \mathrm{HPO}_{4}$ required гоr.83-73.25 cc. $=28.58$ cc. of $\frac{\mathrm{N}}{\mathrm{IO}} \mathrm{HCl}$.

Therefore I gm. of the sample contained

and

$$
0.014105 \times 28.58=0.4037+\text { gm. of } \mathrm{Na}_{2} \mathrm{HPO}_{4}
$$

$$
\frac{\text { I.19.36 }}{2}=0.5968+\text { gm. of } \mathrm{Na}_{3} \mathrm{PO}_{4} \text {. }
$$


Ahlum's Alternative Method (J. A. C. S., xxvIII, 535). Ahlum also proposes the following:

Alternative Method. If a solution containing disodium hydrogen phosphate and trisodium phosphate is acidified and then neutralized with sodium carbonate, adding an excess of the latter, we have as a result a solution containing disodium hydrogen phosphate and sodium carbonate.

If a titration were to be made of the mixed salts before treatment and a titration made, after making allowance for the amount of sodium carbonate found in excess, we find the amount of acid required by the phosphates after treatment is less than that required before, the difference heing directly proportional to the amount of trisodium phosphate present.

This is made clear by referring to the reactions above, noting that disodium hydrogen phosphate requires but one molecule of acid while the trisodium phosphate requires two.

This method in detail is as follows: $0.5 \mathrm{gm}$. is dissolved in $50 \mathrm{cc}$. of water and titrated with $\frac{\mathrm{N}}{\mathrm{IO}}$ hydrochloric acid. I.O gm. is dissolved in $50 \mathrm{cc}$. of water containing a drop of methyl orange. Hydrochloric acid is added in slight excess and the solution boiled for ten minutes. Sodium carbonate is added in excess and the solution concentrated by boiling as far as possible. It is then transferred to a weighed platinum dish, evaporated to dryness on a steam-bath, dried in an oven and weighed.

The mass in the dish is broken up and pulverized with a procelain pestle, guarding against loss. One-half of the amount of solid matter found is weighed off and the carbon dioxid determined by the Schrötter apparatus. The remaining half of the solids is dissolved in $50 \mathrm{cc}$. of water and titrated with $\frac{\mathrm{N}}{\mathrm{IO}}$ hydrochloric acid.

The following example will be explanatory: $0.5 \mathrm{gm}$. was titrated with $\frac{\mathrm{N}}{\mathrm{IO}}$ hydrochloric acid, requiring 23.I cc. One gram was treated in the manner described above, titrating a solution of one-half of the solids and estimating the carbon dioxid in the other half. The amount of carbon dioxid found was $0.0149 \mathrm{gm}$. The amount of sodium carbonate equivalent to the carbon dioxid eliminated $=0.0149 \times 2.4 \mathrm{II} 5$ $=0.0359 \mathrm{gm}$.

The number of cubic centimeters of $\frac{\mathrm{N}}{\mathrm{IO}}$ hydrochloric acid equivalent to the sodium carbonate present $=0.0359 \div 0.0053=6.7$.

The number of cubic centimeters of $\frac{\mathrm{N}}{10}$ hydrochloric acid required after treatment was found to be 20.1 . 
cc.

Total $\frac{\mathrm{N}}{\mathrm{IO}}$ acid required................... 20.I

$\frac{\mathrm{N}}{\mathrm{IO}}$ acid required by $\mathrm{Na}_{2} \mathrm{CO}_{3} \ldots \ldots \ldots \ldots \ldots \ldots, 6.7$

$\frac{\mathrm{N}}{\mathrm{IO}}$ acid required by $\mathrm{Na}_{2} \mathrm{HPO}_{4} \ldots \ldots \ldots \ldots \ldots \ldots+\mathrm{I}_{3.4}$

$\frac{\mathrm{N}}{\mathrm{IO}} \mathrm{HCl}$ required by the $\mathrm{Na}_{3} \mathrm{PO}_{4}+\mathrm{Na}_{2} \mathrm{HPO}_{4} \ldots . .23 . \mathrm{I}$

$\frac{\mathrm{N}}{\text { IO }} \mathrm{HCl}$ required by the $\mathrm{Na}_{2} \mathrm{HPO}_{4}$ (the original

with that formed from the trisodium phosphate).. 13.4

Difference...................... 9.7

The difference in the amount of acid required is due to the loss in alkalinity of the trisodium phosphate, caused by the conversion of this salt into disodium hydrogen phosphate, thereby requiring just one half of the acid as when in the tribasic state.

In the above example the amount of $\frac{\mathrm{N}}{\mathrm{IO}}$ hydrochloric acid necessary to completely act upon the trisodium phosphate would be $9.7 \times 2$ $=\mathrm{I} 9.4 \mathrm{cc}$. As each cubic centimeter is equivalent to $0.0082 \mathrm{gm}$. of trisodium phosphate, the amount of this salt in the mixture will be I $9.4 \times 0.0082 \times 200=31.9$ per cent.

The number of cubic centimeters required by the disodium hydrogen phosphate is obtained by subtracting the number of cubic centimeters required by the trisodium phosphate from the number of cubic centimeters obtained in the original titration.

In the above example this would be $23.1 \mathrm{cc} .-\mathrm{I} 9.4 \mathrm{cc} .=3.7 \mathrm{cc}$. As each cubic centimeter of $\frac{\mathrm{N}}{\mathrm{IO}}$ hydrochloric acid is equivalent to $0.0142 \mathrm{gm}$. of disodium hydrogen phosphate, the amount of this salt present in the mixture would be $3.7 \times 0.0142 \times 200=10.5$ per cent.

The accuracy of the determinations depends upon the determination of the sodium carbonate. As all sources of error may be traced to this determination, this should be performed with care and results checked. 


\section{CHAPTER XXIII}

\section{SALICYLIC ACID AND SALICYLATES}

FREE salicylic acid may be readily estimated by titrating its aqueous solution with decinormal sodium hydroxid, using phenolphthalein as indicator.

$$
\begin{aligned}
& \mathrm{HC}_{7} \mathrm{H}_{5} \mathrm{O}_{3}+\mathrm{NaOH}=\mathrm{NaC}_{7} \mathrm{H}_{5} \mathrm{O}_{3}+\mathrm{H}_{2} \mathrm{O} \text {. }
\end{aligned}
$$

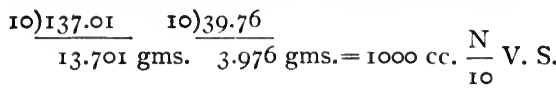

Thus each cc. of $\frac{\mathrm{N}}{\mathrm{IO}} \mathrm{NaOH}$ represents $0.01370 \mathrm{Igm}$. of salicylic acid.

Salicylates of the alkalies and alkali earths may be estimated by incineration of the salt (thereby converting it into carbonate), and titrating the resulting carbonate with standard acid, using methyl orange as indicator See page 88 .

W. Fresenius and L. Grünhut (Zeitschrift für analytische Chemie, I899, 292), submitted the several methods which have been proposed for the estimation of salicylic acid to a critical study.

Separation by Immiscible Solvents. From a solution of sodium salicylate acidulated with sulphuric acid all the salicylic acid could readily be abstracted with chloroform. This chloroformic solution could, however, not be brought to.dryness without loss of acid, and this method can therefore only be depended upon for approximate results.

The Iodometric Method. This method depends upon the formation of iodin compounds of salicylic acid when an alkaline solution of the latter is treated with an excess of iodin solution and then rendered acid. From the amount of iodin rendered insoluble, the salicylic acid is calculated. While Vortmann (Anleitung zur chemischen Analyse organischer Stoffe, Wien, I89I, pp. 320 and 40I) calculates one molecule of acid for every six atoms of iodin combined, the authors demonstrate that the amount of iodin combined depends largely upon the conditions of the experiment; thus in their work they find in sodium salicylate from 64.7 I per cent to 92.70 per cent of acid, while theory requires 86.23 per cent. 
Titration with Bromin Solution. This method of Fr. Freyer, I896 (Chemiker-Zeitung, 20, p. 820) is based upon the same principle as the estimation of phenol according to Koppeschaar. Excellent results were obtained when of the volumetric bromin solution (sodium bromate 3 gms., sodium bromid 20 gms., water, $1000 \mathrm{cc}$.)* roo cc. were diluted with $300 \mathrm{cc}$. of water, to this $20 \mathrm{cc}$. of hydrochloric acid added, and to this mixture $20 \mathrm{cc}$. of an approximately I per cent solution of substance under examination was gradually added. After standing for five minutes, occasionally stirring, 30 to $40 \mathrm{cc}$. of a ro per cent potassium iodid solution is added and the residual iodin titrated in the usual way.

The reaction in the process is as follows:

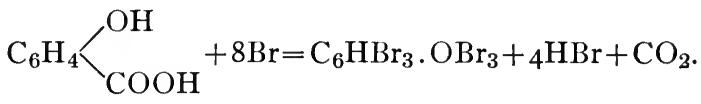

On adding the solution of potassium iodid, not only does the excess of bromin liberate an equivalent of iodin, but the tribromphenol bromid also reacts thus,

$$
\mathrm{C}_{6} \mathrm{HBr}_{3} \cdot \mathrm{OBr}+{ }_{2} \mathrm{KI}=\mathrm{C}_{6} \mathrm{HBr}_{3} \cdot \mathrm{OK}+\mathrm{KBr}+\mathrm{I}_{2} .
$$

Hence 6 atoms of bromin correspond to one molecule of salicylic acid.

* See Estimation of Phenol. 


\section{CHAPTER XXIV}

\section{SULPHUR AND ITS COMPOUNDS}

SULPHUR may be estimated by converting it into sulphuric acid. This is accomplished by heating the finely-powdered substance with strong nitric acid to which some crystals of potassium chlorate have been added. The solution is covered with a watch-glass until all spurting has ceased, and then evaporated to dryness on the sand-bath with an excess of pure hydrochloric acid. The residue is dissolved in hydrochloric acid, and again evaporated to dryness, redissolved in water, a few drops of hydrochloric acid added, and the sulphuric acid estimated by $\frac{\mathrm{N}}{\mathrm{IO}}$ barium chlorid. (See Sulphuric Acid.) The sulphur of most insoluble sulphids may be oxidized to sulphuric acid and determined in the same way. The object of the evaporations is to drive off the nitric acid, which interferes with the estimation of the sulphuric acid.

Another way for estimating sulphur in insoluble sulphids, as in iron or copper pyrites, consists in igniting the sulphid with potassium chlorate and sodium carbonate. The sulphur is converted entirely into sulphuric acid which reacts with an equivalent of sodium carbonate forming sodium sulphate. An accurately weighed quantity of the substance is fused with a known weight of pure sodium carbonate in excess, and in the presence of potassium chlorate. And the resulting mass titrated with normal acid to find the quantity of unaltered sodium carbonate. The quantity of normal acid required, subtracted from the quantity required to saturate the sodium carbonate originally added, is the quantity of normal acid representing the carbonate which reacted with the sulphuric acid produced, and from which the proportion of sulphur is easily calculated.

Each cc. of $\frac{\mathrm{N}}{\mathrm{r}}$ sulphuric acid $=0.015915 \mathrm{gm}$. of sulphur.

It is advisable to take I $\mathrm{gm}$. of the substance and $5.2655 \mathrm{gms}$. of pure anhydrous sodium carbonate for the assay. 5.2655 gms. of $\mathrm{Na}_{2} \mathrm{CO}_{3}$ represent $100 \mathrm{cc}$. of $\frac{\mathrm{N}}{10} \mathrm{H}_{2} \mathrm{SO}_{4}$, therefore, it is only necessary to subtract the number of cc. of $\frac{\mathrm{N}}{\mathrm{IO}} \mathrm{H}_{2} \mathrm{SO}_{4}$ used after ignition from 
I00, and multiply the remainder by the factor for sulphur $0.015915 \mathrm{gm}$. in order to arrive at the weight of sulphur in I gm. of the substance. This weight multiplied by roo gives the percentage.

Example. I gm. of finely powdered $\mathrm{FeS}_{2}$ is mixed thoroughly with 5.2655 gms. of anhydrous sodium carbonate, and $8 \mathrm{gms}$. each of potassium chlorate and sodium chlorid, and gradually exposed in a platinum crucible to a dull red heat for ten minutes. The crucible is allowed to cool, its contents treated with warm water, and the solution so obtained filtered. The insoluble residue is then boiled with water, the water passed through a filter, and the residue washed on the filter until all soluble matter is dissolved.

The mixed filtrate is then titrated with normal sulphuric acid, using methyl orange as the indicator.

If $66.8 \mathrm{cc}$. of normal sulphuric acid are required, this deducted from $100=33.2 \mathrm{cc}$. This multiplied by $0.0159 \mathrm{I} 5=0.5283+\mathrm{gm}$. or 52.83 per cent of sulphur.

The results by this method are by no means exact, and vary at times as much as I.5 per cent. It is, however, suitable for rough technical purposes. The inaccuracy of this process is caused by the volatilization of some of the sulphur in the form of chlorid, and also the formation of ferric sulphate from which the acid is expelled at a red heat.

The reaction involved is as follows:

$$
{ }_{2} \mathrm{FeS}_{2}+{ }_{5} \mathrm{KClO}_{3}+{ }_{4} \mathrm{Na}_{2} \mathrm{CO}_{3}=\mathrm{Fe}_{2} \mathrm{O}_{3}+4 \mathrm{Na}_{2} \mathrm{SO}_{4}+{ }_{5} \mathrm{KCl}+{ }_{4} \mathrm{CO}_{2} \text {. }
$$

The sodium chlorid is used to moderate the violence of the reaction.

Caution. Great caution must be exercised in using potassium chlorate in this method, because many sulphids, especially antimony sulphid, afford violent explosions.

Estimation of Sulphur in Alkali Sulphid. The estimation is the exact reverse of the process for estimating zinc by means of sodium sulphid.

The reaction is as follows:

$$
\mathrm{Na}_{2} \mathrm{~S}+\mathrm{ZnSO}_{4}=\mathrm{ZnS}+\mathrm{Na}_{2} \mathrm{SO}_{4} \text {. }
$$

77.59

The $\frac{\mathrm{N}}{\mathrm{IO}}$ zinc solution may be made by dissolving $3.245 \mathrm{gms}$. of pure metallic zinc in hydrochloric acid, supersaturating with ammonia, and diluting to I liter. Or by dissolving I4.275 gms. of pure crystallized zinc sulphate $\left(\mathrm{ZnSO}_{4}+{ }_{7} \mathrm{H}_{2} \mathrm{O}=285.4 \mathrm{I}\right)$ in water, making strongly alkaline with ammonia water and diluting to I liter. The indicator is nickel protochlorid or alkaline lead solution. 
Each cc. of this zinc solution will indicate respectively,

$$
\begin{aligned}
& 0.0015915 \text { gm. sulphur; } \\
& 0.0038795 \text { “ } \text { sodium sulphid; } \\
& 0.0054777 \text { “" } \text { potassium sulphid; } \\
& 0.0033845 \text { “ ammonium sulphid. }
\end{aligned}
$$

The sulphid is dissolved in water and the zinc solution added from a burette until no dark color is shown, when a drop of the solution tested is brought in contact with a drop of nickel sulphate on a white porcelain tile. Instead of the nickel sulphate, an alkaline lead solution may be used as indicator.

Sulphids may also be estimated by adding an excess of zinc solution, washing the precipitated sulphid rapidly, out of contact of air, digesting with an excess of ferric sulphate, and estimating the ferrous salt with permanganate (see Zinc). $\frac{\mathrm{N}}{10}$ permanganate $\times 0.0015915=\mathrm{S}$, etc.

$$
\mathrm{ZnS}+\mathrm{Fe}_{2}\left(\mathrm{SO}_{4}\right)_{3}=\mathrm{ZnSO}_{4}+\mathrm{S}+{ }_{2} \mathrm{FeSO}_{4} .
$$

Estimation of Hydrosulphuric Acid. By Permanganate (Mohr). When ferric sulphate is added to a solution of $\mathrm{H}_{2} \mathrm{~S}$ in water the following reaction takes place:

$$
\mathrm{Fe}_{2}\left(\mathrm{SO}_{4}\right)_{3}+\mathrm{H}_{2} \mathrm{~S}={ }_{2} \mathrm{FeSO}_{4}+\mathrm{H}_{2} \mathrm{SO}_{4}+\mathrm{S} .
$$

The sulphur separates as a white powder, making the fluid milky. The $\mathrm{FeSO}_{4}$ formed is a measure of the $\mathrm{H}_{2} \mathrm{~S}$, and may be estimated by permanganate. The precipitated sulphur does not interfere.

An acid solution of ferric sulphate which must be free from ferrous sulphate is poured into a flask and the solution of hydrogen sulphid measured in with a pipette whose point just touches the surface of the solution. The flask is closed and allowed to stand for an hour, shaking frequently. At the end of this time the liquid must still possess a yellow color, from excess of ferric sulphate, and upon opening the flask no odor of $\mathrm{H}_{2} \mathrm{~S}$ should be present. The solution is considerably diluted and titrated with permanganate until the rose color appears. If the solution of hydrogen sulphid is very dilute, it should stand three or four hours before being titrated.

When the $\mathrm{H}_{2} \mathrm{~S}$ solution also contains thiosulphates, or other substances which reduce permanganate, the $\mathrm{H}_{2} \mathrm{~S}$ must be precipitated by alkaline zinc solution added in excess. The precipitate is rapidly washed, transferred to a flask containing an acid solution of ferric sulphate, and after standing half an hour in a warm place, the solution is diluted and titrated with permanganate. 
Each cc. of $\frac{\mathrm{N}}{10}$ permanganate $=0.0016915 \mathrm{gm}$. of $\mathrm{H}_{2} \mathrm{~S}$.

In like manner metallic sulphids which are soluble in dilute sulphuric acid may be estimated by adding the dry sulphids in known quantity to an acid solution of ferric sulphate, and after standing in a warm place as above stated may be titrated with permanganate.

Alkali sulphids may be estimated in the same manner as hydrogen sulphid.

By Iodin. When iodin and hydrogen sulphid are brought together $i^{n}$ solution the following reaction occurs:

$$
\mathrm{H}_{2} \mathrm{~S}+2 \mathrm{I}={ }_{2} \mathrm{HI}+\mathrm{S} \text {. }
$$

The reaction is not regular, however, when performed in an acid solution, but in the presence of alkali bicarbonates the results are constant. The method may be employed for the estimation of alkali sulphates.

The process may be conducted as follows, according to Sutton:

ro cc. or any other necessary volume of $\frac{\mathrm{N}}{\mathrm{I} 00}$ iodin solution are measured into a $500 \mathrm{cc}$. flask and the $\mathrm{H}_{2} \mathrm{~S}$ solution to be examined added until the color disappears. $5 \mathrm{cc}$. of starch solution are then added, and $\frac{\mathrm{N}}{\mathrm{I00}}$ iodin until the blue color appears. The flask is then filled to the $500 \mathrm{cc}$. mark with distilled water. The respective volumes of iodin and starch solution, together with the added water, deducted from $500 \mathrm{cc}$. will show the volume of water actually titrated by the iodin. A correction should be made for the excess of iodin necessary to produce the blue color.

Mohr's Procedure. The $\mathrm{H}_{2} \mathrm{~S}$ solution is made alkaline with ammonium carbonate or sodium bicarbonate, starch solution is added, and then the standard iodin solution until the blue color appears.

Each cc. of $\frac{\mathrm{N}}{100}$ iodin $=0.00016915 \mathrm{gm}$. of $\mathrm{H}_{2} \mathrm{~S}$.

The estimation may likewise be made by adding the $\mathrm{H}_{2} \mathrm{~S}$ solution to a solution of copper sulphate, boiling for a few minutes, filtering off the precipitated sulphid, dissolving it in nitric acid, evaporating the solution to dryness with excess of sulphuric acid, and estimating the copper by means of potassium iodid and sodium thiosulphate. See Copper.

Each cc. of $\frac{\mathrm{N}}{\mathrm{IO}}$ sodium thiosulphate $=0.00 \mathrm{I} 69 \mathrm{I} 5 \mathrm{gm}$. of $\mathrm{H}_{2} \mathrm{~S}$. 
The estimation of $\mathrm{H}_{2} \mathrm{~S}$ in sulphids which are insoluble in water, may be carried out as follows:

A weighed quantity of the sulphid is introduced into a flask, provided with a double perforated stopper; through one of the perforations the stem of a separatory funnel is passed, through the other a glass delivery tube (see Fig. 76). The funnel tube extends to near the

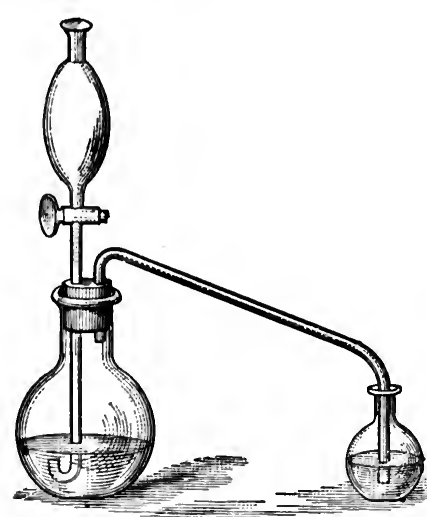

FiG. 76 . bottom of the flask and is bent to form a hook, the opening of which is under water. The delivery tube begins at the lower end of the stopper and ends in another flask containing sodium bicarbonate solution. The funnel contains diluted sulphuric acid, which, upon opening the glass stopcock, is allowed to flow into the flask, upon the contained sulphid, the $\mathrm{H}_{2} \mathrm{~S}$ liberated is conducted into the solution of sodium bicarbonate which absorbs it completely. A current of air aspirated through the apparatus insures absorption of the entire $\mathrm{H}_{2} \mathrm{~S}$ developed. The sodium bicarbonate solution of $\mathrm{H}_{2} \mathrm{~S}$ is then titrated with the standard iodin, in the presence of starch.

By Arsenous Acid (Mohr). When $\mathrm{H}_{2} \mathrm{~S}$ is added to a solution of arsenous acid, the following reaction occurs:

$$
{ }_{3} \mathrm{H}_{2} \mathrm{~S}+\mathrm{As}_{2} \mathrm{O}_{3}=\mathrm{As}_{2} \mathrm{~S}_{3}+{ }_{3} \mathrm{H}_{2} \mathrm{O} \text {. }
$$

The arsenous acid is added in excess, and the excess then found by titration with $\frac{\mathrm{N}}{\mathrm{IO}}$ iodin and starch.

A measured quantity, say 10 cc. of $\frac{\mathrm{N}}{\mathrm{IO}}$ arsenous acid solution is put into a flask together with a measured quantity, say $20 \mathrm{cc}$. of the $\mathrm{H}_{2} \mathrm{~S}$ solution, sufficient hydrochloric acid is then added to make the solution distinctly acid and diluted to $300 \mathrm{cc}$. The precipitated arsenic sulphid is separated by filtration, and $100 \mathrm{cc}$. of the clear colorless filtrate taken out, neutralized with sodium bicarbonate, and then titrated with $\frac{\mathrm{N}}{\mathrm{IO}}$ iodin, using starch as indicator. The quantity of arsenous acid V. S. so found multiplied by 3 is deducted from the original ro cc. and the remainder multiplied by the factor for $\mathrm{H}_{2} \mathrm{~S}$, which is 0.002537 gm. 
By Silver Nitrate. Hydrogen sulphid water may also be estimated by adding an accurately measured quantity of the water to be analyzed, to an accurately measured quantity of standard silver nitrate solution, and after thoroughly shaking, diluting to a definite volume. After the precipitate of silver sulphid has subsided, an aliquot portion of the clear supernatant liquid is removed by means of a pipette, and in this the unchanged silver nitrate determined by means of standard sulphocyanate solution. The difference between the quantity of unchanged silver nitrate (in the whole) found by titration and the quantity originally added, is calculated into $\mathrm{H}_{2} \mathrm{~S}$.

The reaction is as follows:

$$
\begin{aligned}
& \mathrm{H}_{2} \mathrm{~S}+2 \mathrm{AgNO}_{3}=\mathrm{Ag}_{2} \mathrm{~S}+{ }_{2} \mathrm{HNO}_{3} . \\
& 3.83
\end{aligned}
$$

Each cc. of $\frac{\mathrm{N}}{\mathrm{IO}} \mathrm{AgNO}_{3}=0.0016915 \mathrm{gm} . \mathrm{H}_{2} \mathrm{~S}$.

Sulphurous Acid and Sulphites. Sulphurous acid and sulphites may be accurately estimated by titration with standard iodin, as described on page 197 .

Sulphurous acid may also be estimated by neutralization with standard alkali. It acts in about the same way as does phosphoric acid, i.e., with methyl orange as indicator, the yellow color appears upon the formation of $\mathrm{KHSO}_{3}$.

$$
\mathrm{H}_{2} \mathrm{SO}_{3}+\mathrm{KOH}=\mathrm{KHSO}_{3}+\mathrm{H}_{2} \mathrm{O},
$$

while if phenolphthalein is used as indicator, the end-point does not appear until normal potassium sulphite $\mathrm{K}_{2} \mathrm{SO}_{3}$ is produced.

$$
\mathrm{H}_{2} \mathrm{SO}_{3}+2 \mathrm{KOH}=\mathrm{K}_{2} \mathrm{SO}_{3}+{ }_{2} \mathrm{H}_{2} \mathrm{O} \text {. }
$$

In the first instance one molecule of sulphurous acid is neutralized by one equivalent of $\mathrm{KOH}$; in the second instance two equivalents of $\mathrm{KOH}$ are required.

Sulphuric Acid and Sulphates. The free acid is estimated by neutralization with normal alkali.

Sulphuric acid in sulphates may be estimated by various volumetric methods, though the gravimetric method is undoubtedly the most satisfactory. The principal volumetric methods are as follows:

With Barium Chlorid. The sulphate is dissolved in water, acidified with hydrochloric acid, heated to boiling, and decinormal barium chlorid * carefully added until no further precipitation occurs.

* The decinormal barium chlorid solution is made by dissolving I $2.126 \mathrm{gms}$. of pure crystallized barium chlorid $\left(\mathrm{BaCl}_{2}+2 \mathrm{H}_{2} \mathrm{O}\right)$ in water to make one liter. 
The end of the reaction may be determined by the use of Beale's filter, Fig. 87 , or by placing a drop of the clear solution on a plate of black glass or a mirror, and bringing in contact with it a drop of barium chlorid solution.

Wildenstein first proposed this method of estimating sulphuric acid in acid solution by means of barium chlorid. Barium sulphate is deposited rapidly from a hot solution containing excess of sulphuric acid, leaving a clear solution, but as the excess of acid grows smaller it settles more slowly, until near and beyond the neutral point we must either wait a long time for the precipitate to settle or by some means filter a small portion of the solution and test the filtrate to see whether enough barium chlorid has been added. For the filtration, Wildenstein employs the following special apparatus, shown in Fig. 77. $A$ is a bottle of white glass whose bottom has been

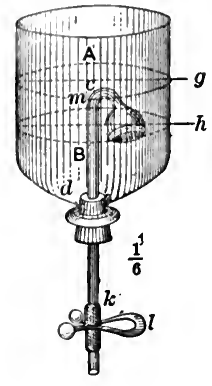

FIG. 77 . removed, and which will contain about $900 \mathrm{cc}$. An ordinary quart bottle answers the purpose. The bent funnel-tube $B$ is covered in the following way: A piece of muslin is first laid over the aperture, then two pieces of fine Swedish filterpaper, and finally another piece of muslin. The whole is then fastened tightly over the mouth of the tube by means of waxed thread, and the projecting edges neatly trimmed. In binding over the aperture care must be taken not to break the filter-paper. Fill the vessel with hot water above the funnel-tube, and by opening the pinch-cock the funnel-tube is completely filled. Add the solution of the sulphate, which is to be estimated, and acidify with a few drops of hydrochloric acid, and then add the barium chlorid from the burette gradually, after each addition opening the pinch-cock and allowing a volume of fluid corresponding to the contents of the tube to flow into a small beaker; this is then returned to the beaker, another portion run out into the same beaker without rinsing it, and this tested by a drop of barium chlorid from the burette. If a precipitate forms, the contents of the beaker are returned to the bottle, a little more barium chlorid added, the contents of the tube emptied and returned to the beaker, and then a second portion run into the beaker and tested, and so on until the solution fails to give a perceptible precipitate with a drop of barium chlorid after a lapse of two minutes. It is necessary to empty the contents of the tube each time before testing, since the barium chlorid which is added to the solution in the bottle does not enter the funnel tube until the pinch-cock is opened. In the precipitation of sulphuric acid by barium chlorid a point occurs where both barium chlorid and sulphuric acid produce a precipitate. This 
point marks the time when barium chlorid and sulphuric acid are present in solution in precisely equivalent quantities and therefore this point should be taken as the end of an analysis. After a little practice it is easy to hit this point and great accuracy may be attained.

With Barium Chlorid and Potassium Dichromate.* Add an excess of $\frac{\mathrm{N}}{\mathrm{IO}}$ barium chlorid solution and heat to boiling, then add some ammonia water and titrate the excess of barium chlorid with decinormal potassium dichromate. The latter is added in small portions, boiling after each addition until the fluid above the precipitate is of a faint yellow color. The decinormal potassium dichromate solution is made by dissolving 7.307 gms. of the salt in sufficient water to make rooo cc. The reactions are as follows:

(a) $\mathrm{K}_{2} \mathrm{SO}_{4}+\mathrm{BaCl}_{2}=\mathrm{BaSO}_{4}+2 \mathrm{KCl}$;

(b) ${ }_{2} \mathrm{BaCl}_{2}+\mathrm{K}_{2} \mathrm{Cr}_{2} \mathrm{O}_{7}+\mathrm{H}_{2} \mathrm{O}=2 \mathrm{BaCrO}_{4}+2 \mathrm{KCl}+{ }_{2} \mathrm{HCl}$.

Each cc. of $\frac{\mathrm{N}}{\mathrm{IO}}$ barium chlorid solution represents

$$
\begin{aligned}
& 0.0086535 \text { gm. of } \mathrm{K}_{2} \mathrm{SO}_{4} \text {; } \\
& 0.0048675 \text { “" " } \mathrm{H}_{2} \mathrm{SO}_{4} \text {. }
\end{aligned}
$$

In order to obtain satisfactory results by this method the solution must be neutral or slightly alkaline and must contain no carbonate or other acid besides sulphuric, capable of precipitating barium from a neutral solution.

If the solution under analysis contains carbonates, a slight excess of hydrochloric acid is added, and the solution boiled until all carbon dioxid is driven off, and then ammonia (which must be free from carbonate) is added to alkaline reaction. The solution is heated to boiling and then barium chlorid solution added in excess, more ammonia is added and then potassium dichromate by small portions, boiling after each addition until the supernatant liquid is faintly yellow.

By Precipitation as Lead Sulphate. A decinormal solution of lead nitrate is prepared by dissolving 16.4245 gms. of pure dry lead nitrate in sufficient water to make $1000 \mathrm{cc}$. The sulphate is dissolved in water and titrated with the lead nitrate solution until precipitation is complete. A solution of potassium iodid may be used as indicator. The reaction is known to be completed when a drop of the solution

* Wildenstein, Fres. Zeit., I, 323. 
brought in contact with a drop of the indicator on a porcelain slab gives a yellow color, due to the formation of lead iodid. The reaction is:

$$
\begin{aligned}
& \mathrm{K}_{2} \mathrm{SO}_{4}+\mathrm{Pb}\left(\mathrm{NO}_{3}\right)_{2}=\mathrm{PbSO}_{4}+{ }_{2} \mathrm{KNO}_{3} \\
& \text { 2) } 173.07 \quad \text { 2) } 328.49
\end{aligned}
$$

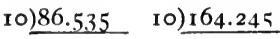

$$
\begin{aligned}
& 8.6535 \quad \frac{\mathrm{N}}{\mathrm{I} 6.4245}=1000 \mathrm{cc} \frac{\mathrm{N}}{\mathrm{IO}} \mathrm{V} \text {.S. }
\end{aligned}
$$

Fach cc. $=0.0086535 \mathrm{gm}$. of $\mathrm{K}_{2} \mathrm{SO}_{4}$;

$$
=0.0048675 \text { " " } \mathrm{H}_{2} \mathrm{SO}_{4} \text {. }
$$




\section{CHAPTER XXV}

\section{ALUMINUM}

Alum and Aluminum Salts. The salt is dissolved in water, phenolphthalein added, and then a measured excess of $\frac{\mathrm{N}}{\mathrm{I}}$. sodium hydroxid. This makes the solution red.

$$
\mathrm{Al}_{2}\left(\mathrm{SO}_{4}\right)_{3}+6 \mathrm{NaOH}=\mathrm{Al}_{2}(\mathrm{OH})_{6}+{ }_{3} \mathrm{Na}_{2} \mathrm{SO}_{4} \text {. }
$$

The $\mathrm{Al}_{2}(\mathrm{OH})_{6}$ dissolves in the excess of $\mathrm{NaOH}$.

Normal acid solution is now added until the red color disappears, the quantity of the acid solution used is deducted from the alkali added, and the remainder multiplied by the factor.

$$
\begin{aligned}
\text { Each cc. } & \text { ×0.0I69 gm. }=\mathrm{Al}_{2} \mathrm{O}_{3} ; \\
\times 0.05664 \mathrm{gm} . & =\mathrm{Al}_{2}\left(\mathrm{SO}_{4}\right)_{3} .
\end{aligned}
$$

The $\mathrm{Ph}$. Germ. directs the following procedure for estimating aluminum sulphate:

One gram of the salt is dissolved in $10 \mathrm{cc}$. of water and $1.2 \mathrm{gm}$. of barium chlorid added. Then a few drops of phenolphthalein T. S. are added and the mixture titrated with $\frac{\mathrm{N}}{\mathrm{I}}$ potassium hydroxid until red color appears.

The process depends upon the fact that the acid combined with aluminum behaves toward the indicator as though it were in a free state. The red color does not appear until the aluminum is completely precipitated. In the case of sulphate of aluminum, however, the addition of alkali hydroxid solution is apt to cause the precipitation of basic sulphate of aluminum.

Hence in this process barium chlorid is added in order to convert the sulphate into chlorid of aluminum, which can be accurately titrated with the alkali solution. 
The reactions are:

$$
\mathrm{Al}_{2}\left(\mathrm{SO}_{4}\right)_{3}+{ }_{3} \mathrm{BaCl}_{2}=\mathrm{Al}_{2} \mathrm{Cl}_{6}+{ }_{3} \mathrm{BaSO}_{4} ;
$$

then

$$
\mathrm{Al}_{2} \mathrm{Cl}_{6}+6 \mathrm{KOH}=\mathrm{Al}_{2}(\mathrm{OH})_{6}+6 \mathrm{KCl} \text {. }
$$

Aluminum sulphate is apt to have some free acid, and this is of course included in the calculation together with the combiried acid.

Hence not more than $8.7 \mathrm{cc}$. of $\frac{\mathrm{N}}{\mathrm{I}} \mathrm{KOH}$ V. S. should be used; any quantity above that would indicate free acid.

Each cc. of $\frac{\mathrm{N}}{\mathrm{I}}$ alkali $=0.05664 \mathrm{gm}$. of $\mathrm{Al}_{2}\left(\mathrm{SO}_{4}\right)_{3}$. The free acid may be estimated by the use of tropæolin O. O., which reacts only with free acid.

Titration with Standard Barium Hydroxid (A. H. White).* This method depends upon precipitating the free and combined sulphuric acid of alum, by titrating with standard barium hydroxid in the presence of Rochelle salt; and then titrating another portion of the sample with barium hydroxid in the presence of sodium citrate. The second titration gives the free acid plus two thirds of the acid combined with alumina. There is no precipitation of aluminum hydroxid in either case, and hence the end-reaction with phenolphthalein is very sharply defined. The difference between the two titrations upon the same quantities of material represents one third of the sulphuric acid combined with alumina, and hence one third of the alumina.

The Process. Dissolve 3 gms. of alum in sufficient water to make 100 cc. Take $25 \mathrm{cc}$. sample, add $50 \mathrm{cc}$. strictly neutral ro per cent potassium sodium tartrate, and titrate with fifth-normal barium hydroxid, using phenolphthalein as indicator. This is equivalent to the sulphuric acid combined with alumina, plus the free acid. Evaporate a duplicate $25 \mathrm{cc}$. sample to dryness on the water-bath, dissolve in $50 \mathrm{cc}$. strictly neutral to per cent sodium citrate solution, allow to stand ten minutes and titrate with barium hydroxid using phenolphthalein indicator as before. The difference between these results is equivalent to one third of the sulphuric acid combined with the alumina, and hence to one third of the alumina. The barium hydroxid solution should be standardized by a blank determination upon a solution of sulphuric acid in which approximately enough precipitated aluminum hydroxid has been dissolved to correspond to aluminum sulphate. The aluminum hydroxid may be best made by precipitation of the 
chlcrid to insure absence of sulphate. Caustic soda, even when freed from carbon dioxid by barium hydroxid, does not give such satisfactory results as the barium hydroxid.

Iodometric Method (Stock).* When a mixture of potassium iodid and iodate acts upon an aluminum salt in solution, a precipitate of aluminum hydroxid is formed, and a corresponding quantity of iodin is liberated, according to the equation:

$$
\mathrm{Al}_{2}\left(\mathrm{SO}_{4}\right)_{3}+{ }_{5} \mathrm{KI}+\mathrm{KIO}_{3}+{ }_{3} \mathrm{H}_{2} \mathrm{O}=2 \mathrm{Al}(\mathrm{OH})_{3}+{ }_{3} \mathrm{~K}_{2} \mathrm{SO}_{4}+6 \mathrm{I}
$$

This is a reaction which begins very rapidly in the cold, but which is not complete for several days. The velocity of the reaction can, however be accelerated if the iodin liberated during the reaction is removed by means of sodium thiosulphate, or by allowing the precipitation to take place when hot. By working on a water-bath, the reaction is complete in a few minutes, and by removing the iodin at the same time by means of standard thiosulphate solution; an accurate method of estimating alumina can be devised. The precipitation is complete even in dilute solutions.

* Alfred Stock, Chem. News, Feb. I6, I900, 83, from Compt. rend., I30, Jan. 22, I900, No. 4 . 


\section{CHAPTER XXVI}

\section{AMMONIA AND AMMONIUM SALTS}

Ammonium Salts may be estimated by distilling them with potassium or sodium hydroxid, and receiving the ammonia $\left(\mathrm{NH}_{3}\right)$ which distils over, in a known volume or normal or decinormal acid. After the distillation is completed, the quantity of the ammonia is found by titrating back with normal or decinormal alkali. The apparatus illustrated in Fig. 67 may be used for this purpose.

The ammonium salt in solution is put into flask $A ; b$ contains strong solution of sodium hydroxid. The receiving-flask $B$ contains a measured quantity of normal hydrochloric acid, which is poured in through the tube $c$, containing fragments of glass.

The pinch-cock upon $b$ is opened, which allows the sodium hydroxid solution to run into the flask, and the solution is then gently boiled until all the ammonia is driven over and absorbed by the normal acid.

Care must be taken not to heat too strongly, or some of the fixed alkali may be projected up into the connecting-tube and carried over into the acid in flask $B$.

After the distillation is completed the acid adhering to the broken glass in $c$ is washed into the flask, phenolphthalein added, and the excess of acid found by titrating with normal alkali. The amount of normal alkali used is deducted from the quantity of normal acid added, and the remainder is the acid which combined with the ammonia.

$$
\begin{aligned}
& \mathrm{NH}_{3}+\mathrm{HCl}=\mathrm{NH}_{4} \mathrm{Cl} . \\
& 16.93 \quad 36.18=\text { I00c cc. } \frac{\mathrm{N}}{\mathrm{I}} \text { V. S. } \\
& .01693 .03618=\quad \text { I cc. } \frac{\mathrm{N}}{\mathrm{I}} \text { V. S. }
\end{aligned}
$$

Thus

$$
\text { I cc. } \begin{aligned}
\frac{\mathrm{N}}{\mathrm{I}} \mathrm{HCl} & =0.01393 \mathrm{gm} . \mathrm{N} ; \\
& =0.01693 \mathrm{gm} . \mathrm{NH}_{3} ; \\
& =0.01793 \mathrm{gm} . \mathrm{NH}_{4} ; \\
& =0.0531 \mathrm{gm} . \mathrm{NH}_{4} \mathrm{Cl} .
\end{aligned}
$$

This method may be employed for ammonia in all of its salts and compounds; either sodium or potassium hydroxid or lime may be 
used for liberating, except in the case of substances containing nitrogenous organic compounds, in which case the use of freshly ignited magnesia is preferred, because the alkali and alkali earth hydroxids convert organic nitrogen into ammonia.

Indirect Method. In the case of pure ammoniacal salts or solutions free from acid, the following method may be employed.

To a weighed quantity of the salt a measured quantity of normal sodium hydroxid is added, and the mixture boiled in an open vessel until all the ammonia is expelled.

The residual alkali in the flask is then titrated with normal acid, and the difference between the normal acid used and the normal soda added, gives the quantity of the latter which reacted with the ammonium salt.

The reaction is thus expressed:

$$
\begin{aligned}
\mathrm{NH}_{4} \mathrm{Cl}+\mathrm{NaOH} & =\mathrm{NaCl}+\mathrm{H}_{2} \mathrm{O}+\mathrm{NH}_{3} ; \\
\text { each cc. of } \frac{\mathrm{N}}{\mathrm{I}} \mathrm{NaOH} & =0.053 \mathrm{I} \mathrm{I} \mathrm{gm} . \mathrm{NH}_{4} \mathrm{Cl} ; \\
& =0.0656 \text { " }\left(\mathrm{NH}_{4}\right)_{2} \mathrm{SO}_{4} .
\end{aligned}
$$

The estimation of ammonium carbonate is described on page 79 . The carbonic acid in this may be determined by adding to the hot solution of the salt sufficient barium chlorid to precipitate the carbonate as barium carbonate. The precipitate is then well washed, and dissolved in excess of normal acid and retitrated with normal alkali, the number of cc. of normal acid taken up, multiplied by 0.021835 , gives the weight of carbon dioxid in the sample. See page 244 .

By Means of Formaldhyde (Ronchése). This method depends upon the fact that a neutral solution of formaldehyde added in excess to a neutral solution of any ammonium salt, will react with formation of hexamethylenamin and liberation of the corresponding acid. The liberated acid is titrated with $\frac{\mathrm{N}}{\mathrm{IO}}$ sodium hydroxid, using phenolphthalein as indicator. Cochineal or methyl orange cannot be employed, as hexamethylenamin reacts alkaline with these indicators. The solution of ammonium salt is diluted to $100 \mathrm{cc}$. with recently boiled distilled water, a few drops of phenolphthalein added, and then a large excess of a neutral 20 per cent solution of formaldehyde. The solution is then carefully titrated with decinormal alkali. In case the sample originally contained free acid, it is divided into two equal portions, in one the acidity is determined as above, in the other the total acidity, using an indicator not effected by ammonium salts, as rosolic acid or fluorescein. 


\title{
CHAPTER XXVII
}

\author{
ANTIMONY
}

Oxidation by Iodin in Alkaline Solution (Mohr). Antimonous Oxid, or any of its compounds, is estimated in the manner described on page 195 .

Solution of the oxid is first effected by means of tartaric acid, and any excess of the latter neutralized by sodium carbonate. Then for every $0.1 \mathrm{gm}$. of $\mathrm{Sb}_{2} \mathrm{O}_{3}$, Iо cc. of a cold saturated solution of sodium bicarbonate are added, then starch solution, and finally titrated with $\frac{\mathrm{N}}{\mathrm{IO}}$ iodin.

Each cc. of $\frac{\mathrm{N}}{10}$ iodin V. S. $=0.005965 \mathrm{gm} . \mathrm{Sb}$;

$$
=0.007{ }^{6} 6 \mathrm{gm} . \mathrm{Sb}_{2} \mathrm{O}_{3} \text {. }
$$

One gram of the antimonous oxid is weighed out into a $250 \mathrm{cc}$. flask. About 2 gms. of tartaric acid and 25 cc. of water are added and the mixture shaken until the antimonous oxid is dissolved. The solution is then just neutralized with sodium carbonate and diluted with water to make $250 \mathrm{cc}$. $50 \mathrm{cc}$. of this solution are removed by means of a pipette, transferred to a beaker, and $30 \mathrm{cc}$. of a cold, saturated solution of sodium bicarbonate added, and after the addition of a few drops of starch solution, the titration with decinormal iodin is begun at once.

Example. In the above titration 26.8 cc. of decinormal iodin solution were consumed. Therefore the $\mathbf{I}$ gm. taken for analysis would require $26.8 \times 5=\mathrm{I} 34 \mathrm{cc}$.

Antimonous sulphid may be dissolved in hydrochloric acid by the aid of heat, tartaric acid added, and then the solution made alkaline by the addition of sufficient sodium bicarbonate and titrated with decinormal iodin as above.

Metallic antimony in solution (free from arsenic and tin), in fact all antimony compounds, may be converted into sulphid by means of hydrogen sulphid. The precipitated sulphid is thoroughly washed and then dissolved in hydrochloric acid, the solution is boiled until all traces of hydrogen sulphid have been removed, and after diluting 
with water and adding tartaric acid and then sodium bicarbonate to alkalinity, the titration is conducted with decinormal iodin in the presence of starch, as before described.

When assaying antimonous compounds by this method, the titration with standard iodin must be begun, and completed without delay, as otherwise a portion of the antimony will be precipitated as antimonous hydrate, upon which iodin has no effect. F. H. Alcock ( $\mathrm{Ph}$. Jour., 1900, 362), recommends the following modification to avoid this precipitation, and thus permit of working with less haste.

Weigh off I gm. of tartar emetic, add $50 \mathrm{cc}$. of water and ro gms. of Rochelle salt, and after solution is effected, add 3 gms. or more of sodium bicarbonate and make up to a suitable volume. Of this solution an aliquot is titrated with the iodin solution.

If solution of antimonous chlorid is to be assayed, $5 \mathrm{cc}$. of the solution are taken and the other ingredients added in the same quantities. If antimonous oxid is to be assayed, I gm. is converted into tartar emetic by the aid of 2 gms. of potassium bitartrate, ro gms. of Rochelle salt are added, followed by an excess of sodium bicarbonate and water to make $200 \mathrm{cc}$; an aliquot portion of the solution is then titrated with iodin.

Type Metal (containing lead and antimony). $0.5 \mathrm{gm}$. are dissolved in the smallest possible quantity of aqua regia, and then an excess of aqua ammonia and yellow ammonium sulphid are added and the mixture allowed to digest for several hours. The precipitated lead sulphid is then separated by filtration and thoroughly washed. The filtrate and washings are then made slightly acid with diluted sulphuric acid, and heated to drive off all hydrogen sulphid. The resulting precipitate of antimony sulphid is then dissolved in hydrochloric acid, and after the addition of tartaric acid and sodium bicarbonate, as above described, titration with $\frac{\mathrm{N}}{\mathrm{TO}}$ iodin is begun, using starch as indicator. If greater accuracy is desired, the precipitate, containing lead sulphid, is dissolved in hydrochloric acid and again treated with ammonia and ammonium sulphid.

Titration with Standard Potassium Bromate in Acid Solution (Györy).* The method which may be used for arsenic as well as antimony, is based upon the oxidation of arsenous or antimonous oxid by means of a standard solution of potassium bromate, in the presence of hydrochloric acid. For a I per cent solution of arsenous acid an equal volume of diluted hydrochloric acid should be taken. In the case of antimony, however, a larger quantity of the acid is used; suff-

* Zeitschr. f. analyt. Chem., 32, 416 (189.3). 
cient must be added to prevent precipitation of the antimony during titration as a result of the increasing dilution of the solution; thus for $0.33 \mathrm{gm}$. of tartar emetic, $25 \mathrm{cc}$. or more of diluted hydrochloric acid should be employed. The reactions with arsenic and with antimony are as follows:

and

$$
3 \mathrm{As}_{2} \mathrm{O}_{3}+{ }_{2} \mathrm{HCl}+2 \mathrm{KBrO}_{3}=2 \mathrm{KCl}+{ }_{2} \mathrm{HBr}+3 \mathrm{As}_{2} \mathrm{O}_{5}
$$

$$
{ }_{3} \mathrm{Sb}_{2} \mathrm{O}_{3}+{ }_{2} \mathrm{HCl}+{ }_{2} \mathrm{KBrO}_{3}=2 \mathrm{KCl}+{ }_{2} \mathrm{HBr}+{ }_{3} \mathrm{Sb}_{2} \mathrm{O}_{5} \text {. }
$$

Methyl-orange solution (0.I gm. in roo cc. water) is added to the acid solution as indicator. The slightest excess of the standard bromate solution completely decolorizes the red solution, through liberation of bromin.

The decinormal potassium bromate is made by dissolving $\frac{1}{60}$ of the molecular weight in grams of pure crystallized potassium bromate $\left(\mathrm{KBrO}_{3}\right)$ dried at $110^{\circ} \mathrm{C}$. in sufficient distilled water to make $1000 \mathrm{cc}$.

Each cc. of this solution represents

$$
\begin{aligned}
& 0.0049 \text { I gm. of } \mathrm{As}_{2} \mathrm{O}_{3} ; \\
& 0.007 \mathrm{I} 56 \text { "“ " } \mathrm{Sb}_{2} \mathrm{O}_{3} ; \\
& 0.016495 \text { " “ }{ }_{2} \mathrm{~K}(\mathrm{SbO}) \mathrm{C}_{4} \mathrm{H}_{4} \mathrm{O}_{6} \mathrm{H}_{2} \mathrm{O} \text {. }
\end{aligned}
$$

Titration with Standard Iodate (Andrews). The determination of antimony by this method is precisely like that of arsenic, which see.

Oxidation by Dichromate or Permanganate in Presence of Hydrochloric Acid (Kessler).* The solutions required are:

(a) Standard Arsenous Oxid. 5 gms. of pure arsenous oxid are dissolved with the aid of sodium hydroxid solution, hydrochloric acid is added until the solution is slightly acid, and then roo cc. of pure hydrochloric acid (sp.gr. I.I2) are added and the solution diluted with water to $1000 \mathrm{cc}$.

Each cc. of this solution $=0.005 \mathrm{gm}$. of arsenous oxid, which corresponds to $0.007287 \mathrm{gm}$. of antimonous oxid $\left(\mathrm{Sb}_{2} \mathrm{O}_{3}\right)$.

(b) Solution of Potassium Dichromate. $2.5 \mathrm{gms}$. of the salt in $1000 \mathrm{cc}$.

(c) Solution of Ferrous Sulphate. Made by dissolving I.I gms. of pure iron wire in $20 \mathrm{cc}$. of diluted sulphuric acid (I:4) and adding water to make $1000 \mathrm{cc}$.

(d) Solution of Potassium Ferricyanid, freshly prepared (Indicator).

The Relation betueen the. Dichromate Solution and the Iron solution is found as follows: 
From a burette to $\mathrm{cc}$. of the dichromate solution are run into a beaker, $5 \mathrm{cc}$. of hydrochloric acid and $50 \mathrm{cc}$. of water are added, and then the iron solution delivered into the mixture from another burette, until the fluid is green. Then continue adding the iron solution, I cc. at a time, testing a drop of the fluid after each addition by bringing it in contact with a drop of the ferricyanid solution on a white slab, until a blue color is obtained. Then add $0.5 \mathrm{cc}$. more of the dichromate solution, and again the iron solution in drops until the blue color just appears. Now read off both burettes and calculate how much dichromate solution corresponds to ro cc. of the iron solution.

If the solutions are made as above described ro cc. of the iron solution will correspond to about $3.9 \mathrm{cc}$. of the dichromate solution, i.e., each cubic centimeter of the former will correspond to $0.3^{\circ} \mathrm{cc}$. of the dichromate solution.

\section{The Relation between the Dichromate Solution and the} Arsenous Oxid Solution is now to be ascertained.

Ten cubic centimeters of the arsenous oxid solution are introduced into a beaker, together with $20 \mathrm{cc}$. of hydrochloric acid (sp.gr. I.I2) and from 80 to roo cc. of water.*

The dichromate solution is then run in until the yellow color of the fluid indicates it to be in excess. The mixture is allowed to react a few minutes and then the ferrous sulphate solution added until a drop of the solution from the beaker gives, with a drop of the ferricyanid solution, a blue color. The end-point is more accurately determined by adding $0.5 \mathrm{cc}$. of the dichromate solution and again titrating with the iron solution in drops until the precise end-point is obtained. Then deduct from the total quantity of dichromate solution used the amount corresponding to the iron solution employed, ie., about $0.39 \mathrm{cc}$. for each cc. of iron solution. This will give the quantity of dichromate solution required for the oxidation of Io $\mathrm{cc}$. of the arsenous oxid solution; about $20.2 \mathrm{cc}$. will be required.

From these data, the quantity of antimony corresponding to roo cc. of the dichromate solution is easily calculated.

If each cubic centimeter of the arsenous oxid solution corresponds to $0.007287 \mathrm{gm}$. of $\mathrm{Sb}_{2} \mathrm{O}_{3}$ and $\mathrm{roO} \mathrm{cc}$. of the dichromate solution represents $49 \mathrm{cc}$. of the arsenous oxid solution, roo cc. of the dichromate solution will represent $0.357663 \mathrm{gm}$. of $\mathrm{Sb}_{2} \mathrm{O}_{3}$, or each cubic centimeter 0.00357663 gm. $\mathrm{Sb}_{2} \mathrm{O}_{3}$.

The Actual Analysis. If organic matter, heavy metallic oxids, or other oxidizable bodies are absent, the antimonous compound is dis-

* The water must be measured, because uniformity of action is insured only if the volume of hydrochloric acid present is not less than $\frac{1}{6}$ nor more than $\frac{1}{3}$ of the entire volume of solution. 
solved at once in hydrochloric acid not less than one sixth nor more than one third of the volume of the solution should consist of hydrochloric acid (sp.gr. I.12). The dichromate solution is now run in, and the titration carried out precisely as directed for the determination of the relationship between the arsenous oxid solution and the dichromate solution.

The use of more than one third of hydrochloric acid will interfere with a nice and precise determination of the end-reaction with potassium ferricyanid. Tartaric acid is inadmissible here as a solvent, because it interferes with the action of chromic acid on the ferrous salt. If the direct determination of antimony in the hydrochloric acid solution is not practicable, precipitate it with hydrogen sulphid, and after washing the precipitate, dissolve it in hydrochloric acid on a water-bath, and after removing the hydrogen sulphid by the addition of a saturated solution of mercuric chlorid in hydrochloric acid, proceed as directed.

The Titration uith Potassium Permanganate. The same proportion of hydrochloric acid solution is necessary as in the foregoing. The permanganate solution which may contain about $\mathrm{r} .5 \mathrm{gms}$. of the pure crystallized salt in the liter is added, till the rose color is permanent. The addition of a small quantity of magnesium sulphate prevents the decomposition of permanganate by the hydrochloric acid. Tartaric acid, at least in the proportion in which it exists in tartar emetic, does not interfere. Hence since tartar emetic can easily be obtained in a pure state, it may be employed for standardizing the permanganate solution.

Estimation of Antimonous Sulphid by Oxidation with Ferric Sulphate, and Titration of the Resultant Ferrous Sulphate with Permanganate (J. Hanus).* The reaction is as follows:

$$
\mathrm{Sb}_{2} \mathrm{~S}_{3}+{ }_{5} \mathrm{Fe}_{2}\left(\mathrm{SO}_{4}\right)_{3}+8 \mathrm{H}_{2} \mathrm{O}={ }_{2} \mathrm{H}_{3} \mathrm{SbO}_{4}+{ }_{1} \circ \mathrm{FeSO}_{4}+{ }_{5} \mathrm{H}_{2} \mathrm{SO}_{4}+\mathrm{S}_{3} \text {. }
$$

The sulphid is boiled for fifteen minutes in a beaker with an excess of ferric sulphate, allowed to cool, and after adding an excess of sulphuric acid, titrated with potassium permanganate. $\mathrm{Sb}_{2} \mathrm{~S}_{3}$ corresponds to IoFe.

Antimonic Acid and its Salts $†$ are dissolved and strongly acidified with hydrochloric acid, a strong solution of sodium sulphite is then gradually added, the mixture boiled to drive off the $\mathrm{SO}_{2}$, a drop of phenolphthalein solution added, and then $\mathrm{KOH}$ until slightly alkaline, as shown by red color. Then a small excess of tartaric acid is added and the process completed as for antimonous acid.

$$
\text { I cc. } \frac{\mathrm{N}}{\mathrm{IO}} \text { iodin }=0.005965 \mathrm{gm} . \mathrm{Sb} \text {. }
$$

* Apoth. Ztg. Ang. 31, 1898, 6r 3 .

$\dagger$ According to Von Knorre, Zeitsch. angew. Chem. (I888), 155. 
The reduction to antimonous oxid may also be made by means of $\mathrm{H}_{2} \mathrm{~S}$. See page 196 .

Other articles on the titrimetric estimation of antimony which may be referred to with profit are:

"Estimation of Antimony in Alloys, such as Babbitt and Type Metals." H. Yockey. J. A. C. S. (I906), page I 435 .

"Estimation of Antimony in the Presence of Organic Matter." Norton and Koch. J. A. C. S. (I905), page I247.

"Estimation of Antimony and Arsenic in Ores," etc. A. H. Low. J. A. C. S. (I906), page I7I5. 


\section{CHAPTER XXVIII}

\section{ARSENICUM}

Oxidation by Iodin in Alkaline Solution (Mohr). The estimation of arsenous compounds by this method is fully described on page 192. See also pages i 88 and 190 .

Oxidation by Potassium Dichromate (Kessler). This method is exactly the same as is minutely described for antimony. See page 342 . In its simpler form it is as follows:

o.I gm. of the substance is dissolved in about ro cc. of water with the aid of hydrochloric acid. Then $20 \mathrm{cc}$. of hydrochloric acid (sp.gr. I.I2) and $80 \mathrm{cc}$. of water are added. An excess of $\frac{\mathrm{N}}{\mathrm{IO}}$ potassium dichromate (say $30 \mathrm{cc}$.) is now introduced, the mixture allowed to react for a few minutes and then retitrated with a ferrous sulphate solution which corresponds in strength with the dichromate. A freshly prepared solution of potassium ferricyanid is used as the indicator. The difference between the quantities of the ferrous sulphate and dichromate solutions used gives the quantity of the latter which reacted with the arsenous oxid. In order to find the end-reaction more accurately, it is advised to add another one half or I cc. of the dichromate and again retitrate with the ferrous sulphate solution. The reaction is as follows:

$$
\begin{aligned}
& 3 \mathrm{As}_{2} \mathrm{O}_{3}+{ }_{2} \mathrm{~K}_{2} \mathrm{Cr}_{2} \mathrm{O}_{7}+{ }_{\mathrm{I}} 6 \mathrm{HCl}={ }_{4} \mathrm{KCl}+{ }_{4} \mathrm{CrCl}_{3}+3 \mathrm{As}_{2} \mathrm{O}_{5}+8 \mathrm{H}_{2} \mathrm{O} ; \\
& \text { 2) } 589.32 \quad 2) 587.56 \\
& \text { 6) } \\
& \text { 10) } 4 9 . 1 1 \quad 1 0 \longdiv { 4 8 . 7 1 3 }
\end{aligned}
$$

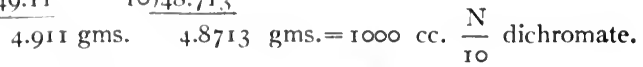

I cc. $\frac{\mathrm{N}}{\mathrm{IO}}$ dichromate $=0.0049 \mathrm{II} \mathrm{gm.} \mathrm{As}_{2} \mathrm{O}_{3}$.

The reaction between the ferrous sulphate and the dichromate is shown on page 184 .

In the above estimation the volume of hydrochloric acid must not be less than one sixth nor more than one third that of the solution.

If the direct titration of the arsenous salt in hydrochloric acid solution is not practicable, it is precipitated by $\mathrm{H}_{2} \mathrm{~S}$; the precipitate is 
washed and placed in a stoppered bottle together with a saturated solution of mercuric chlorid in hydrochloric acid (sp.gr. I.I2) and gently heated until the precipitate is white, then water is added in such amount that the hydrochloric acid present in the liquid be not less than one sixth of its volume. The titration with the $\frac{\mathrm{N}}{\mathrm{IO}}$ dichromate is then carried out as above described.

By Titration with Standard Potassium Iodate. Andrews (J. A. C. S., 25, p. 759) suggests a method for the estimation of arsenous oxid or chlorid based upon the following equation:

$$
{ }_{2} \mathrm{AsCl}_{3}+\mathrm{KIO}_{3}+{ }_{5} \mathrm{H}_{2} \mathrm{O}={ }_{2} \mathrm{H}_{3} \mathrm{AsO}_{4}+\mathrm{KCl}+\mathrm{ICl}+{ }_{4} \mathrm{HCl} \text {. }
$$

The process is conducted in the same manner as that for the estimation of iodids by means of standard potassium iodate solution (see page 263 ), with the exception that too great a concentration of hydrochloric acid must be avoided. The amount of the latter added to the arsenous solution should be sufficient to make the hydrochloric acid equal to about 20 per cent of the entire mixture at the end of the titration. Chloroform is used as the indicator, $5 \mathrm{cc}$. being usually taken, and the standard iodate solution run in from a burette, at first nearly the quantity which in the judgment of the operator the arsenous solution will require, then the liquid is shaken and the titration continued cautiously until the chloroform is decolorized.

Example. 0.I gm. of arsenous oxid is dissolved in Io cc. of water, $5 \mathrm{cc}$. of fuming hydrochloric acid added, and then, after introducing $5 \mathrm{cc}$. of chloroform, the titration with the iodate solution is begun. Nine cubic centimeters are added at once, and then more is added, drop by drop until the end-point is reached.

Each cc. of $\frac{\mathrm{N}}{10} \mathrm{KIO}_{3}=0.009822 \mathrm{gm}$. of $\mathrm{As}_{2} \mathrm{O}_{3}$.

Since copper does not interfere in the least with the application of this method, it is possible, for example, to titrate the arsenic in Paris green directly without preliminary separation.

$0.5 \mathrm{gm}$. of Paris green are dissolved in $15 \mathrm{cc}$. of water and $25 \mathrm{cc}$. of fuming hydrochloric acid and directly titrated with the decinormal iodate solution, in the presence of chloroform as indicator.

Arsenic Oxid by Precipitation with Uranium Solution. Arsenic acid forms with uranic nitrate or acetate a precipitate which is analogous in composition to that produced by phosphoric acid. The estimation is conducted in exactly the same way as that of phosphoric acid and under precisely similar conditions as to quantity of fluid, the amount of acetate and acetic acid added, and depth of color obtained, with indicator. 
The arsenic must be in the state of $\mathrm{As}_{2} \mathrm{O}_{5}$. If it is in the form of $\mathrm{As}_{2} \mathrm{O}_{3}$ it may be oxidized to $\mathrm{As}_{2} \mathrm{O}_{5}$ by evaporation with strong nitric acid, neutralizing with an alkali, and then dissolving in acetic acid.

The uranium solution may be standardized by means of pure sodium arsenate or by a weighed quantity of pure arsenous oxid converted into arsenic oxid by evaporation with strong nitric acid, neutralizing with alkali, and then dissolving in acetic acid.

Arsenic Oxid $\left(\mathrm{As}_{2} \mathrm{O}_{5}\right)$. This may be estimated by iodin as directed for $\mathrm{As}_{2} \mathrm{O}_{3}$, if it be first reduced to the latter form by boiling with potassium iodid in the presence of hydrochloric acid in large excess until the iodin vapors are entirely dissipated. It is then cooled, neutralized with sodium carbonate, then bicarbonate added in excess and titrated with $\frac{\mathrm{N}}{10}$ iodin as directed for $\mathrm{As}_{2} \mathrm{O}_{3}$.

$$
\text { I } \mathrm{cc} .=0.005705 \mathrm{gm} . \mathrm{As}_{2} \mathrm{O}_{5} \text {. }
$$

Arsenic Oxid and Arsenates may also be estimated by means of magnesia mixture in exactly the same way as described for phosphoric acid. See page 3 II

Each ,cc. of $\frac{\mathrm{N}}{\mathrm{IO}}$ hydrochloric acid V. S. represents $0.005705 \mathrm{gm}$. $\mathrm{As}_{2} \mathrm{O}_{5}$.

By Distillation with Chromic and Hydrochloric Acids (Bunsen). When potassium dichromate is boiled with hydrochloric acid, chlorin is given off in accordance with the following reaction:

$$
\mathrm{K}_{2} \mathrm{Cr}_{2} \mathrm{O}_{7}+{ }_{14} \mathrm{HCl}={ }_{2} \mathrm{CrCl}_{3}+2 \mathrm{KCl}+{ }_{7} \mathrm{H}_{2} \mathrm{O}+6 \mathrm{Cl} \text {. }
$$

One molecule $\mathrm{CrO}_{3}$ gives 3 atoms chlorin. If arsenous oxid be present, it is oxidized to arsenic oxid at the expense of a part of the chlorin, and less is therefore given off. $\mathrm{As}_{2} \mathrm{O}_{3}+{ }_{4} \mathrm{Cl}+{ }_{2} \mathrm{H}_{2} \mathrm{O}=\mathrm{As}_{2} \mathrm{O}_{5}+$ ${ }_{4} \mathrm{HCl}$. The excess of chlorin evolved from a measured quantity of dichromate over that required to oxidize the arsenous to arsenic oxid is received in a solution of potassium iodid, and the iodin titrated with $\frac{\mathrm{N}}{\mathrm{IO}}$ thiosulphate. If we let $a$ represent the cc. of $\frac{\mathrm{N}}{10}$ dichromate taken, and $b$ the cc. of $\frac{\mathrm{N}}{\mathrm{IO}}$ thiosulphate used,

$$
\begin{aligned}
& a-b \times 0.0019644=\mathrm{As} ; \\
& a-b \times 0.0049 \mathrm{II}=\mathrm{As}_{2} \mathrm{O}_{3} .
\end{aligned}
$$

The distillation may be conducted in one of the apparatus described under distillation methods, page 214 and shown in Figs. 60, 61, and 62. 
By' Titration with Standard Bromate (Györy). This method is described under antimony.

\section{ESTIMATION OF ARSENIC IN SMALL QUANTITIES, AS IN CASES OF POISONING}

Houzean's Method (Comp. rend., LXxv). The substance containing the arsenic is placed in a Marsh's apparatus, and the arseniureted hydrogen given off is passed into a measured amount of $\frac{\mathrm{N}}{\mathrm{IO}}$ silier nitrate solution. A part of the silver nitrate is reduced to metallic silver, which may be separated by filtration and the filtrate tirrated with $\frac{\mathrm{N}}{\mathrm{IO}}$ sodium chlorid. The loss of silver corresponds to the arsenic.

$$
\begin{aligned}
& \mathrm{AsH}_{3}+6 \mathrm{AgNO}_{3}+{ }_{3} \mathrm{H}_{2} \mathrm{O}=6 \mathrm{Ag}+\mathrm{H}_{3} \mathrm{AsO}_{3}+6 \mathrm{HNO}_{3} \text {. } \\
& \text { 6) } 75 \quad 6)(1018.2 \\
& \text { I0) } \longdiv { 1 2 . 5 } 5 \longdiv { 5 0 \longdiv { 6 0 . 7 } } \\
& \frac{1 0 0 0 \longdiv { 1 . 2 5 }}{.00125} \frac{1 0 0 0 \longdiv { 1 6 . 9 7 }}{.01697}=1000 \mathrm{cc} . \frac{\mathrm{N}}{10} \text { V.S. }
\end{aligned}
$$

The number of $\mathrm{cc}$. of sodium chlorid deducted from the number of cc. of $\frac{\mathrm{N}}{\mathrm{IO}} \mathrm{AgNO}_{3}$ solution first taken gives the number of cc. of the latter which was reduced by the $\mathrm{AsH}_{3}$.

Each cc. thus reduced represents $0.00125 \mathrm{gm}$. of As, or $0.00408 \mathrm{gm}$. $\mathrm{As}_{2} \mathrm{O}_{3}$.

R. C. Cowley and J. P. Catford,* suggest the utilization of Reinsch's test for the quantitative estimation of arsenic in small quantities! The authors assert that by this method it is possible to definitely measure to the $\frac{1}{30}$ of a milligram or even to $\frac{1}{5000}$ of a grain.

The method is as follows:

A few inches of fine copper wire coiled into a helix by twisting it around a glass tube, is immersed in ro cc. of the liquid to be tested, to which one fifth of its volume of hydrochloric acid has been added. The liquid and acid are contained in a test tube, which is supported upright in a salt water-bath by means of a loop of wire resting on the edges of the bath. The coil of copper wire is arranged so that it shall reach from the bottom of the arsenical liquid to above its surface. The test tube must be immersed in the salt water-bath so that the liquid it contains shall be below the level of the liquid in the bath; the bath is to be kept simmering, without, however, reaching the boiling point,

\footnotetext{
* Pharm. Jour, 1904, 897.
} 
for about an hour. The projecting extremity of the copper is now to be pressed down below the surface of the liquid, and if it remains bright, after continuing the application of heat for another fifteen minutes, the arsenic will be all removed from the liquid, and the wire may be removed to a small dish, rinsed without touching it with the fingers, and the deposit then dissolved off by a cubic centimeter of bromin water containing a little hydrobromic acid. The clean wire is lifted out, rinsed with water, and if thought necessary may be returned to the acid liquid to make sure that all the arsenic has been deposited from it. The bromin solution now contains the arsenic as arsenous acid. To it, I cc. of solution of potassium hydroxid is added, and the liquid is boiled until the light green copper compounds are broken up. During this treatment the cupric oxid formed as an intermediate compound oxidizes the arsenic, and a solution, the alkali arsenate results, which is filtered from the copper oxids. An aliquot part of the filtrate may be reserved and tested for arsenic acid by the molybdate reagent after evaporation; the remainder is reduced again to arsenite and titrated with $\frac{\mathrm{N}}{100}$ or other suitably weak solution of iodin.

For a burette the authors use a pipette graduated in hundredths of a cubic centimeter. The flow is controlled by slipping over the top of the pipette a piece of rubber tubing compressed by a screw clamp.

\section{ESTIMATION OF ARSENIC IN PARIS GREEN}

Smith's Method.* This method, which is generally considered the most accurate, depends upon precipitating the copper as cuprous oxid by boiling with sodium hydroxid. The arsenic being present as arsenite, acts as a reducing agent upon the copper. The filtrate, which contains some arsenate, is concentrated by boiling, and after acidulation with hydrochloric acid the arsenate is wholly reduced to arsenite by means of an excess of potassium iodid. The liberated iodin is then taken up by sodium thiosulphate, the solution neutralized with sodium carbonate, and after the addition of an excess of sodium bicarbonate the arsenite is titrated with $\frac{\mathrm{N}}{\mathrm{IO}}$ iodin solution in the presence of starch as indicator.

The Process. $2 \mathrm{gms}$. of the Paris green are weighed out, and about $100 \mathrm{cc}$. of water and 2 gms. of sodium hydroxid added. The solution is brought to a boil, and boiling continued for a few minutes. It is then allowed to cool to room temperature and the solution made

*J. A. C. S., XXXI (1899), 769 
up to $250 \mathrm{cc}$. The well-shaken liquid is filtered through a dry filter and $50 \mathrm{cc}$. taken for the analysis. This portion, equal to $0.4 \mathrm{gm}$. is concentrated to about one half its volume and allowed to cool to $80^{\circ} \mathrm{C}$. An equal volume of strong hydrochloric acid is then added, accompanied by $3 \mathrm{gms}$. of potassium iodid, and the whole allowed to stand for ten minutes (longer is not necessary). The deep red solution is slightly diluted with water to dissolve the precipitate caused by the potassium iodid, and a dilute solution of thiosulphate added until the color just disappears. This solution is then made neutral by addition of dry sodium carbonate and finally an excess of sodium bicarbonate is added. Decinormal iodin solution is then delivered from a burette and the end-reaction noted by starch solution.

The Avery and Beans' Method.* This method, which has the advantage of consuming very little time is as follows: Sample the Paris green by quartering (as one would an ore for assaying) down to about I gm. Pulverize this small sample in an agate mortar and weigh out 0.2 to $0.3 \mathrm{gm}$. into a beaker of about $300 \mathrm{cc}$. capacity. Add about $25 \mathrm{cc}$. of water, and to the Paris green, suspended in water, add, with constant stirring, concentrated hydrochloric acid until solution is just effected; from. 6 to Io drops are usually sufficient. Now add to the acid solution sodium carbonate solution till a slight permanent precipitate is formed, and at this point add 2 or 3 gms. of Rochelle salt in solution. The tartrate will at once dissolve the precipitated copper and prevent further precipitation during the subsequent titration. Dilute to about $200 \mathrm{cc}$.; add solid sodium bicarbonate and starch solution, and titrate with $\frac{\mathrm{N}}{\mathrm{IO}}$ iodin solution in the usual way.

This method, it will be seen, rests on the principle that arsenous acid may be titrated with iodin in the presence of cupric salts, provided an alkali tartrate be present.

By this method most excellent results are obtained if the Paris green examined is pure, but as Haywood pointed out, $\dagger$ if the samples of Paris green contain considerable free arsenous oxid the results are always low. This is due to the fact that the free arsenous oxid is not readily soluble in cold hydrochloric acid. Avery also calls attention to this, $\ddagger$ and suggests gentle boiling, and if solution of the free arsenous oxid is not thereby effected, add a cold saturated solution of sodium acetate, using about $3 \mathrm{gms}$. of the salt for each $0.1 \mathrm{gm}$. of the green. After solution is effected, an alkali tartrate is added,

* J A. C. S., XXIII (IgoI), 485 .

$\dagger$ J. A. C. S., XXV (1903), 963 .

$\ddagger$ J. A. C. S., XXV (1903), гоg6. 
and solid bicarbonate, and the diluted solution titrated in the usual manner.

Haywood's Modification of the Avery and Beans' Method.* Sample the Paris green (as one would an ore for assaying) down to 2 gms. Pulverize this small sample in a mortar and place from 0.3 to $0.4 \mathrm{gm}$. in a beaker. Add about $25 \mathrm{cc}$. of water and, while constantly stirring, add concentrated hydrochloric acid, a drop at a time, until all the Paris green is in solution and the free arsenous oxid remains as a residue. Filter, and wash the residue. The arsenous oxid in the filtrate is determined in exactly the same manner as in the Avery-Bean's method. The filter and contents are dropped back into the beaker, which also receives the water used in washing the funnel. Five grammes of sodium bicarbonate are added and the solution boiled until the arsenous oxid is completely dissolved (this takes from 5 to ro minutes). The resulting solution is cooled and acidified, using a drop of methyl orange to read the change. It is then made alkaline again with bicarbonate, starch added, and titrated with iodin as usual.

Other articles on the volumetric estimation of arsenic are:

Pierce's Method, by Standard Silver. Sutton's "Volumetric Analysis."

J. F. Bennett's "Modification of Pierce's Method." J. A. C. S., Xxi (I 899), 43 r.

Pattinson's "Estimation of Arsenous Sulphid by Iodin." J. S. C. I., 1898 , 2 II.

A. H. Low, "Estimation of Antimony and Arsenic in Ores," etc. J. A. C. S. (1906), I7 I5.

F. A. Norton and A. E. Koch, "A Method for the Estimation of Arsenic and Antimony in the Presence of Organic Matter." J. A. C. S., XXvir (1905), 1247 .

$$
\text { * J. A. C. S., } \operatorname{xxV}\left(\operatorname{rg}_{3}\right), 967 .
$$




\section{CHAPTER XXIX}

\section{BARIUM}

Barium Oxid or Hydroxid may be titrated with standard acid, using phenolphthalein as indicator. But since the hydroxid frequently contains carbonate through absorption of carbonic acid gas from the atmosphere, it is usually a better plan to add an excess of standard acid, boil to expel any carbonic acid, and then retitrate with standard alkali.

Barium Carbonate and Organic Salts of Barium (the latter after ignition) may of course be assayed in the same way. See Estimation of the Salts of the Alkali Earths, page 9r. Under the same heading will also be found a method for the estimation of soluble salts of barium, such as chlorid and nitrate, by titration with a standard solution of sodium carbonate. Barium chlorid in the absence of other chlorids may also be estimated by the use of tenth-normal silver nitrate solution, as in the case of alkali chlorids, after precipitating the barium by means of potassium sulphate. The precipitated barium sulphate may be separated from the potassium chlorid formed and the - filtrate titrated with the tenth-normal silver nitrate, using potassium chromate as indicator, or the titration may be done without first filtering. The precipitate of barium sulphate has no disturbing effect.

$$
\text { Each cc. of } \begin{aligned}
\frac{\mathrm{N}}{\mathrm{IO}} \text { silver solution } & =0.00682 \mathrm{gm} \text {. of } \mathrm{Ba}, \\
& =0.010338 \mathrm{gm} \text {. of } \mathrm{BaCl}_{2} .
\end{aligned}
$$

Soluble barium salts may also be estimated by precipitation with sulphuric acid. The process" is the converse of that for sulphuric acid and sulphate by means of barium chlorid (see Chapter XXIV).

By Titration with Potassium Dichromate. The barium salt in alkaline solution is titrated with standard potassium dichromate until precipitation is complete and the supernatant liquid shows a slight yellow tint.

The dichromate solution used for this purpose is a decinormal solution, but it differs in strength from that used as an oxidizing solution.

It is made by dissolving 7.307 gms. of pure potassium dichromate in water, and diluting to make a liter. Each cc. of this decinormal potassium dichromate represents $.00682 \mathrm{gm}$. of $\mathrm{Ba}$. 
In the analysis the barium compound is dissolved in water, ammonia water free from carbonate is added to alkaline reaction, the mixture heated to $70^{\circ} \mathrm{C}$., and titrated with the dichromate solution, allowing to settle after each addition until the supernatant fluid shows a slight yellow tint. Lead and all other metals which form chromate insoluble in ammoniacal solution, must of course be absent. Calcium and small quantities of strontium exercise no influence upon the result.

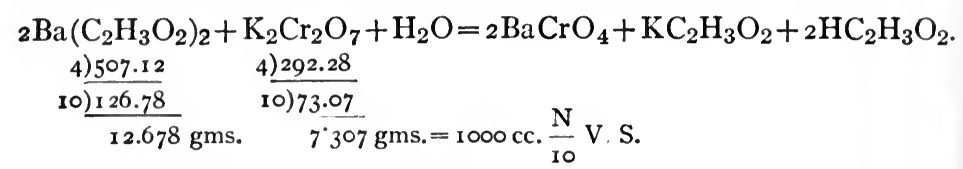

The Indirect Iodometric Method. This mehod depends upon precipitaing the barium by means of potassium chromate, and then subjecting the precipitated barium chromate to the digestion process described on page 222 .

The neutral or slightly ammoniacal solution of the barium salt is treated with potassium chromate in slight excess at a boiling temperature, by which the barium is completely precipitated as chromate. The solution is filtered, the precipitate washed with hot water until the filtrate is entirely colorless, and while still moist the precipitate, together with the filter, is pushed into the stoppered digestion bottle (Fig. 64), some concentrated hydrochloric acid is added, and then the air in the bottle is displaced by carbon dioxid. (This may be accomplished by throwing in some sodium bicarbonate and waiting until effervescence ceases). An excess of potassium iodid is then introduced, the stopper inserted and securely fastened in the frame; the bottle is then placed in a water-bath and heated for about half an hour. After the reaction is completed, the bottle is allowed to cool, opened, and the free iodin found by titrating with decinormal sodium thiosulphate, using starch as indicator.

The reaction between the barium chromate and potassium iodid and hydrochloric acid is as follows:

$$
{ }_{2} \mathrm{BaCrO}_{4}+6 \mathrm{KI}+\mathrm{I} 6 \mathrm{HCl}={ }_{2} \mathrm{BaCl}_{2}+{ }_{2} \mathrm{CrCl}_{3}+6 \mathrm{KCl}+6 \mathrm{I}+8 \mathrm{H}_{2} \mathrm{O} .
$$

Thus it is seen that one molecule of barium chromate is the equivalent of three atoms of iodin, i.e., one third of an atom of barium corresponds to one atom of iodin, or $4.546 \mathrm{gms}$. of barium corresponds to I $2.59 \mathrm{gms}$. of iodin, which in turn corresponds to I liter of $\frac{\mathrm{N}}{\text { IO }}$ sodium thiosulphate. Therefore each cc. of $\frac{\mathrm{N}}{\mathrm{IO}}$ sodium thiosulphate $=0.004546 \mathrm{gm}$. of Ba. 


\section{CHAPTER XXX}

\section{BISMUTH}

By Precipitation as Oxalate (Muir). The bismuth in nitric acid solution is treated with a strong solution of oxalic acid in considerable excess, and the mixture shaken up and then set aside to settle.

The supernatant liquid is then poured off and the precipitated oxalate boiled for five or ten minutes with successive quantities of about $50 \mathrm{cc}$. of water, which converts it into the basic oxalate.

So soon as the supernatant liquid ceases to show an acid reaction the transformation is complete.

The precipitate is then dissolved in dilute sulphuric acid and titrated with $\frac{\mathrm{N}}{20}$ potassium permanganate.

The original bismuth solution must be free from hydrochloric acid and must contain just sufficient nitric acid to prevent the precipitation of basic nitrate before the oxalic acid solution is added.

One molecule of oxalic acid corresponds to one atom of bismuth, or I25.I $=206.9$. The reaction may be represented as follows:

$$
\mathrm{Bi}\left(\mathrm{NO}_{3}\right)_{3}+\mathrm{H}_{2} \mathrm{C}_{2} \mathrm{O}_{4}+\mathrm{H}_{2} \mathrm{O}=\mathrm{Bi}(\mathrm{OH}) \mathrm{C}_{2} \mathrm{O}_{4}+{ }_{3} \mathrm{HNO}_{3} \text {. }
$$

Each cc. of the $\frac{\mathrm{N}}{20}$ permanganate solution represents $0.005^{1} 7_{2} \mathrm{gm}$. of

The results by this method are fairly accurate, though usually a -little too high. This is doubtless due to incomplete conversion to the basic oxalate.

By Precipitation as Chromate (Mohr). The metal must be in in the form of nitrate, and must be free from chlorin. If not in solution it is dissolved in nitric acid, then ammonia water is added until a slight precipitate forms, then some dilute nitric acid to dissolve the precipitate. An excess of potassium dichromate is now added and the solution heated until the precipitate subsides.

The clear liquid should be tested with ammonia water and potassium dichromate, and if neither of these produce a precipitate the entire solution is poured upon an asbestos filter, and the precipitate on the filter washed with hot water. The precipitate, together with 
the filter, is then placed in a flask, a weighed amount of ferrous ammonium sulphate (Mohr's salt) added, followed by some sulphuric acid, and whilst a stream of carbonic acid gas or hydrogen is passed through the flask, it is heated gently until reaction is complete.

The unchanged ferrous salt is then found by titration with decinormal potassium permanganate $\mathrm{V}$. S.

This method is for obvious reasons not a very exact one, the following method gives much better results. See also page 174 .

Iodometric Estimation of the Chromate* Rupp and Shaumann suggest the following procedure:

The solution of bismuth containing the smallest possible quantity of free acid, is poured into a known volume of standard potassium dichromate. The solution is then diluted, shaken energetically, and after ten minutes filtered. After making certain that the whole of the bismuth is precipitated, the excess of dichromate is found in an aliquot portion of the filtrate, by adding a slight excess of potassium iodid, acidulating with sulphuric acid, and titrating the liberated iodin by means of standard sndium thiosulphate. The reactions are explained on page 204 .

By Precipitation as Phosphate (Muir). This method depends upon the complete precipitation of bismuth from its solution, by means of sodium phosphate, in the presence of free acetic acid, and then determining the excess of sodium phosphate by means of a standard uranium solution.

The standard sodium phosphate solution used is prepared by dissolving $35.56 \mathrm{gms}$. of crystallized sodium phosphate $\left(\mathrm{Na}_{2} \mathrm{HPO}_{4}+\right.$ $\mathrm{I}_{2} \mathrm{H}_{2} \mathrm{O}$ ) in water to make $1000 \mathrm{cc}$. Each cc. of this solution represents $0.007047 \mathrm{gm}$. of $\mathrm{P}_{2} \mathrm{O}_{5}$. The standard uranium solution is made by dissolving $38.5 \mathrm{gms}$. of uranium acetate, or an equivalent quantity of yellow uranium oxid, and about $50 \mathrm{cc}$. of glacial acetic acid in about I000 cc. of water, and then so adjusting the solution that it and the standard phosphate will correspond, volume for volume.

The titration should be conducted in the presence of an approximately equal amount of sodium acetate and free acetic acid.

The solution containing bismuth, which must be free from hydrochloric or sulphuric acid, is mixed with a considerable excess of sodium acetate; the liquid is heated to boiling, and a measured volume (excess) of standard sodium phosphate solution run in. After boiling a few minutes the liquid is filtered into a measuring flask, the precipitate thoroughly washed with hot water, and the mixed filtrate and washings made up to the mark. An aliquot portion of the solution is then titrated for excess of the phosphate, by the inverted uranium process

* Zeitsch. anorg. Chem., XXXII, 359. 
(see p. 314.) The precipitate of bismuth phosphate has the composition $\mathrm{BiPO}_{4}$. The reaction may be expressed as follows:

$$
\mathrm{Bi}\left(\mathrm{NO}_{3}\right)_{3}+\mathrm{Na}_{2} \mathrm{HPO}_{4}={ }_{2} \mathrm{NaNO}_{3}+\mathrm{HNO}_{3}+\mathrm{BiPO}_{4} \text {. }
$$

Thus each cc. of the phosphate solution represents $0.02069 \mathrm{gm}$. of Bi.

By Precipitation as Molybdate** To $20 \mathrm{cc}$. of a solution of bismuth nitrate (containing about o.r gm. of $\mathrm{Bi}$ ) in a beaker add one cc. of nitric acid and an excess of ammonium molybdate reagent (U. S. P.), about three or four times the theoretical amount-in this case $25 \mathrm{cc}$. The whole is then carefully neutralized with ammonia water, using methyl orange to determine the neutral point, and 2 or 3 drops of 30 per cent nitric acid introduced.

The bismuth is then completely precipitated as molybdate. The composition of this precipitate is probably $\mathrm{BiNH}_{4}\left(\mathrm{MoO}_{4}\right)_{2}$. The mixture is then warmed, without boiling, by placing on a thick asbestos pad over a low flame, until the fine flocculent precipitate has collected. The precipitate is then stirred with a glass rod until it is broken up, and finally allowed to settle, which it does rapidly, forming a compact sediment which is easily washed.

The supernatant liquid, which must be perfectly clear, is decanted through a plain filter paper, and the precipitate washed twice by decantation with a three per cent solution of ammonium sulphate, after which it is washed on the filter with the ammonium sulphate solution. It is then dissolved in diluted sulphuric acid and run through a Jones' reductor with suction; $†$ after this it is strongly acidified with sulphuric acid and immediately titrated with standard potassium permanganate. The degree of reduction of the molybdenum is believed to correspond to $\mathrm{Mo}_{24} \mathrm{O}_{37}$ and the ratio of bismuth to molybdenum is close to $I: 2 .+$

The color of the molybdate precipitate is pure white, but if the conditions are not followed exactly, a slightly yellowish compound results which gives varying but always lower results. This yellow molybdate precipitate can, however, be easily changed to the white, by first making the solution alkaline with ammonia, to throw down bismuth oxyhydrate, and then dissolving the latter in nitric acid, all this being done in the whole mixture of precipitate and solution. The

* Herman S. Riederer, J. A. C. S., XXV (1903), 907.

$\dagger$ A reductor with a column of zinc 40 to $50 \mathrm{~cm}$. in length, in a tube $1.25 \mathrm{~cm}$. in diameter is recommended, in order to avoid the necessity of more than one passage through the zinc. The suction flank should be large enough to admit the titration being made in it directly, thus air has less chance of reoxidizing the molybdous oxid which is to be determined by the permanganate.

$\ddagger$ See Miller and Frank, J. A. C. S., (1903), 9rg. 
clear solution is now re-treated as a new solution, except that it is not always necessary to add more ammonium molybdate.

The important conditions in this work are, that the temperature of the solution passed through the reductor should be 70 to $75^{\circ} \mathrm{C}$. the time of passage seven to ten minutes, volume of solution $200 \mathrm{cc}$. and the acidity Io cc. concentrated sulphuric acid. After the passage of the molybdate solution, I00 cc. of hot water should be passed through the reductor, and the titration begun at once. The reduction is theoretically as follows:

$$
{ }_{5} \mathrm{Mo}_{24} \mathrm{O}_{37}+70 \mathrm{KMnO}_{4}=\mathrm{I}_{2} 0 \mathrm{MoO}_{3}+35 \mathrm{~K}_{2} \mathrm{O}+70 \mathrm{MnO} .
$$

Each cc. of $\frac{\mathrm{N}}{\text { IO }}$ permanganate solution will represent $0.003544 \mathrm{gm}$. of Bi. 


\section{CHAPTER XXXI}

\section{CALCIUM}

THE best methods for the quantitative estimation of calcium depend upon its precipitation as oxalate. Any calcium salt soluble in water or acetic acid may be estimated as follows:

Dissolve in water and add ammonia water until the solution is alkaline, and then ammonium chlorid and oxalate. Heat gently until the calcium oxalate has completely separated, collect on a filter, wash thoroughly, dissolve in warm dilute sulphuric acid, and estimate the oxalic acid liberated by means of $\frac{\mathrm{N}}{\mathrm{IO}}$ permanganate. If the calcium salt is insoluble in water but soluble in dilute acetic acid, it is to be dissolved in the smallest possible quantity of the latter, and the calcium precipitated as oxalate with sulphuric acid; the excess of oxalate is determined by titration with $\frac{\mathrm{N}}{\mathrm{IO}}$ permanganate.

Rupp and Bergdoldt * give the following directions for estimating calcium and other alkali earths:

Ten to twenty centimeters of the solution of a calcium salt are placed in a flask together with $\mathrm{I}$ or 2 gms. of ammonium chlorid, and heated to boiling; ammonia water is then added in slight excess followed by a known volume of $\frac{\mathrm{N}}{\mathrm{I}}$ oxalic acid or of ammonium oxalate of known titer, and the heating continued for several minutes. The flask with contents is then placed in cold water, and when cool brought to a specific volume with water and filtered. The first few cc. of filtrate are rejected and then 50 or $100 \mathrm{cc}$. collected, diluted to I 50 or $200 \mathrm{cc}$. with hot water, and Io cc. of dilute sulphuric acid, without previous addition of ammonia. The excess of oxalic acid is then found by titration with permanganate.

Each cc. of $\frac{\mathrm{N}}{\mathrm{IO}}$ permanganate $=0.00 \mathrm{I} 99 \mathrm{gm} . \mathrm{Ca}$;

$$
\begin{aligned}
& =0.004968 \text { “ } \mathrm{CaCO}_{3} ; \\
& =0.002784 \text { “ } \mathrm{CaO} ;
\end{aligned}
$$

* Arch. d. Ph., 242, VI, Aug. I904. 
Even difficultly soluble calcium salts, such as the sulphate, phosphate, turtrate, and citrate, may be converted into oxalates by longer treatment with oxalic acid, or ammonium oxalate.

The following method may be employed if the solution of a calcium salt, to be estimated, contains no other substance capable of reducing permanganate. To the solution of the calcium salt a measured volume in excess of a solution of ammonium oxalate is added, the mixture warmed, and then diluted to $200 \mathrm{cc}$. The precipitate is allowed to settle, and 50 or $100 \mathrm{cc}$. of the clear supernatant liquid removed by means of a pipette, and after acidulating, 2 to $3 \mathrm{gms}$. of manganous sulphate are added, and the oxalic acid titrated with $\frac{\mathrm{N}}{\text { ro }}$ permanganate.

$$
\text { Each cc. } \begin{aligned}
\frac{N}{\text { IO }} \text { oxalic acid } & =0.00199 \text { gm. Ca; } \\
& =0.002784 \text { " CaO; } \\
& =0.005508 \text { " } \mathrm{CaCl}_{2} .
\end{aligned}
$$

(See also page i 70.)

The alkalimetric methods for the estimation of calcium salts and other alkali earths are described on page 9r.

The above described oxalic acid method may be employed in the estimation of calcium even in the presence of iron, aluminum, or magnesium. As for instance, in dolomite or limestone, as follows:

Ten grams of the pulverized limestone are dissolved in dilute hydrochloric acid, the insoluble residue separated by filtration, and the solution diluted to $1000 \mathrm{cc}$. $50 \mathrm{cc}$. of the clear solution are introduced into a beaker, ammonia added to slight alkalinity, and then a sufficient quantity of acetic acid is added to redissolve the precipitated ferric hydroxid. The solution is then heated to boiling, and sufficient ammonium oxalate added to precipitate all the calcium as oxalate. If the precipitation is done in a boiling solution, and an excess of ammonium oxalate is used the precipitate settles rapidly, and is of a crystalline character, in which state filtration and washing are easily accomplished. The mixture is then filtered through a plain filter, the entire precipitate being transferred to the filter by using a spritz bottle and a glass rod tipped with a piece of rubber tubing, and washed with hot water until the washings no longer react acid. The filter is then placed over a porcelain capsule, and the precipitate dissolved by moistening it with dilute sulphuric acid, and washed into the capsule with hot water, any occurrence of calcium sulphate will not in the least interfere.

The solution in the capsule is then titrated with $\frac{\mathrm{N}}{10}$ permanganate. 
Instead of dissolving the precipitate on the filter, it may be introduced directly into a beaker, by making a hole in the bottom of the filter and washing the precipitate through it, then treating with dilute sulphuric acid and titrating as above under constant agitation.

Another method is: Take a sample of dolomite $(0.5 \mathrm{gm}$.), dissolve in hydrochloric acid, and dilute to $250 \mathrm{cc}$. with water; to this add about five drops of nitric acid and boil for a few minutes. Then add an excess of ammonia, and filter to remove the precipitate of aluminum and ferric hydroxids, wash the precipitate thoroughly with hot water, and to the mixed filtrate and washings add oxalic acid solution until the calcium is completely precipitated as oxalate. The precipitate is collected, washed, and redissolved in water by aid of hydrochloric acid, and after making alkaline with ammonia, the calcium is again precipitated by oxalic acid. This precipitate is then thoroughly washed, mixed with about roo cc. of water and sufficient dilute sulphuric acid, and finally titrated with $\frac{\mathrm{N}}{\mathrm{IO}}$ permanganate. 


\section{CHAPTER XXXII}

\section{COPPER}

By Precipitation as Cuprous Oxid. Copper may be precipitated as cuprous oxid $\left(\mathrm{Cu}_{2} \mathrm{O}\right)$ by means of grape-sugar, from an alkaline solution of the metal containing tartaric acid.

To the copper solution tartaric acid or an alkali tartrate is added, and then an excess of sodium or potassium hydroxid. A clear, deep, blue colored liquid should be obtained, if not, more tartaric acid must be added. A considerable quantity of grape-sugar is now added, and the liquid heated on a water-bath (though not above $90^{\circ} \mathrm{C}$.) until the precipitate becomes bright red in color. This precipitate is separated by filtration through a plug of asbestos, and washed with hot water until the washings are perfectly clear and colorless. The precipitate, together with the asbestos, is then transferred to a flask containing an acid solution of a ferric salt and heated until the cuprous oxid is completely dissolved. The reaction is as follows:

$$
\mathrm{Cu}_{2} \mathrm{O}+\mathrm{Fe}_{2} \mathrm{Cl}_{6}+{ }_{2} \mathrm{HCl}={ }_{2} \mathrm{CuCl}_{2}+{ }_{2} \mathrm{FeCl}_{2}+\mathrm{H}_{2} \mathrm{O} \text {. }
$$

The resulting ferrous salt is then titrated with permanganate. It is advisable to pass a stream of carbon dioxid or hydrogen through the flask whilst the cuprous oxid is dissolving, to prevent oxidation of ferrous salt.

As shown by the above equation, for every two equivalents of copper, two equivalents of iron are reduced, i.e., 63.I gins. of copper represent $55.5 \mathrm{gms}$. of iron. Therefore each cc. of $\frac{\mathrm{N}}{\mathrm{IO}}$ potassium permanganate represents $0.00555 \mathrm{gm}$. of $\mathrm{Fe}$, which is equivalent to $0.0063 \mathrm{I} \mathrm{gm}$. of $\mathrm{Cu}$.

Estimation as Sulphid. This method depends upon precipitating copper out of alkaline solutions by means of sodium sulphid. The composition of the precipitate so obtained varies, according to the temperature at which the precipitation is done. If the precipitation is carried out at the ordinary temperature of the atmosphere the precipitate will consist of simple copper sulphid $(\mathrm{CuS})$, which, however, settles so slowly in the alkaline solution that the determination of the end-point becomes a very difficult matter, and hence the titration at this temperature is quite impracticable. Working at higher tem- 
peratures the composition of the precipitate is quite different, being of the nature of an oxysulphid, but its advantage lies in the fact that the precipitate settles rapidly and hence admits of a rapid and accurate determination of the end-point. The method of Pelouze depends upon working at a temperature of from $60^{\circ}$ to $80^{\circ} \mathrm{C}$. The precipitate is then an oxysulphid of the composition $\mathrm{CuO} . \mathrm{CuS}$.

The end-point is indicated by the discharge of the blue color of the alkaline copper solution, and by noting the point at which the further addition of the standard sulphid solution fails to produce a precipitate.

The sodium sulphid is standardized against a solution of pure copper sulphate of known strength, which is best made by dissolving 39.279 gms. of pure copper sulphate $\left(\mathrm{CuSO}_{4} \cdot{ }_{5} \mathrm{H}_{2} \mathrm{O}\right)$ in sufficient water to make I liter. Each cc. of this solution will contain o.cr gm. of copper.

Precipitation in Acid Solution. The estimation of copper by means of sodium sulphid is comparatively simpler if the precipitation is carried out in acid solution instead of in an ammoniacal solution. The precipitate in this case does not vary in composition with the temperature at which the operation is conducted. Its composition is invariably $\mathrm{CuS}$.

The copper solution is diluted to about $200 \mathrm{cc}$. with hot water in a stoppered flask, acidulated with hydrochloric acid, and the standard sulphid solution added little by little, replacing the stopper and shaking after each addition until the end-point is reached. The CuS formed here subsides quickly. The reaction is thus illustrated:

$$
\mathrm{CuSO}_{4}+\mathrm{Na}_{2} \mathrm{~S}=\mathrm{CuS}+\mathrm{Na}_{2} \mathrm{SO}_{4} .
$$

\section{By Precipitation as Metallic Copper, and Subsequently Titration} with Ferric Chlorid and Permanganate. Into the copper solution, which must contain no nitric acid or other metal precipitable by zinc (as bismuth or lead), is placed a rod of clean pure zinc. The copper deposits in the metallic state. As soon as the deposition of the copper is complete, which is known by the solution being colorless, the zinc is removed, and the precipitated spongy copper collected carefully on a filter, upon which it is thoroughly washed. The precipitate, together with the filter, is then transferred to a flask containing a mixture of ferric chlorid and hydrochloric acid, and gentle heat applied. The copper is thus dissolved according to the equation

$$
\mathrm{Cu}+\mathrm{Fe}_{2} \mathrm{Cl}_{6}=\mathrm{CuCl}_{2}+{ }_{2} \mathrm{FeCl}_{2} \text {. }
$$

In order to prevent oxidation of the resulting ferrous chlorid a stream of hydrogen is passed through the flask whilst the copper is dissolving, or a small quantity of sodium carbonate may be added 
to the contents of the flask, which, by disengaging carbon dioxid, expels the atmospheric air present and thus prevents oxidation. When the copper is completely dissolved the solution is titrated with permanganate, $55.5 \mathrm{Fe}=3 \mathrm{I} .55 \mathrm{Cu}$. Each cc. of $\frac{\mathrm{N}}{\mathrm{IO}}$ permanganate represents $0.00555 \mathrm{gm}$. of $\mathrm{Fe}$, which is the equivalent of $0.003 \mathrm{I} 55 \mathrm{gm}$. of $\mathrm{Cu}$.

By Means of Potassium Cyanid. This method originated with Parkes.* When potassium cyanid is added to the blue ammoniacal solution of a copper salt, the solution loses its blue color, and copper and ammonium cyanid is formed. Cyanogen is also liberated, which reacts on the free ammonia, producing urea, urea oxalate, ammonium cyanid, and formate (Liebig). The reaction varies with the amount of free ammonia, ammoniacal salts, the concentration of the solution, and the temperature. The process therefore is worthless, unless all analyses made by it are done under the same conditions. Manganese, nickel cobalt, mercury, silver, and larger quantities of zinc (over 3 per cent) must first be removed. Cadmium materially increases the quantity of potassium cyanid used. Arsenous, antimonous and stannous salts have a deleterious effect, but in the oxidized state have no influence upon the result.

The titration should be conducted at or near the boiling temperature. The lower the temperature the greater the quantity of potassium cyanid solution used. The results also appear to vary with the speed at which the titration is conducted.

In order to avoid the irregularities occasioned by the presence of varying quantities of ammonia or its salts, J. L. Davies † substituted sodium carbonate for the ammonia, the value of which modification is confirmed by Fessenden $\ddagger$ and by Fernekes and Koch, $\S$ as well as by Sutton and others.

The acid copper solution is neutralized with sodium carbonate, and then an excess of the latter is added, to nearly redissolve the precipitate. It is not necessary to add sufficient sodium carbonate to completely dissolve the precipitate, as the latter dissolves readily upon the addition of the standard cyanid solution.

The cyanid solution is then run in until the blue color of the solution is just discharged. Sutton || neutralizes the copper solution with sodium carbonate, and adds a trifling excess, and then I cc. of ammonia, sp.gr. 0.960 . This addition of ammonia is, howeve:, entirely unnecessary.

* Mining Journal, $185 \mathrm{I}$.

$\S$ J. A. C. S., XXVII, I 225.

$\dagger$ Chem. News, 58, I $3 \mathrm{I}$.

II Handbook of Volumetric Analysis.

$\ddagger$ Chem. News, 61, 253-283. 
Sutton further states that the presence of iron in the solution does not interfere, in fact it is rather an advantage, acting as an indicator. Lead and aluminum have no influence upon the accuracy of the titration.

The reaction between potassium cyanid and the copper salt may be expressed by the equation

$$
\mathrm{CuSO}_{4}+{ }_{4} \mathrm{KCN}=\mathrm{K}_{2} \mathrm{Cu}(\mathrm{CN})_{4}+\mathrm{K}_{2} \mathrm{SO}_{4} .
$$

In standardizing the cyanid solution it is advisable to do so under the same conditions, as in the actual assay. Commercial potassium cyanid is never entirely pure, and therefore the requisite quantity required for making a strictly normal solution varies greatly. It is the custom to weigh off 7 gms. of commercial potassium cyanid, dissolve it in $1000 \mathrm{cc}$. of water, and then standardize it against pure metallic copper dissolved in nitric acid, and transferred into sulphate by evaporating with sulphuric acid. Or against pure recrystallized copper sulphate.

As seen in the above equation four molecules of $\mathrm{KCN}$ are the equivalent of one atom of $\mathrm{Cu}$. This standard solution does not keep its titer very long, hence freshly prepared solutions only should be used. This process reversed serves for the estimation of cyanids (see page 275).

Steinbeck's Process for Ores.* 5 gms. of finely powdered ore are put into a flask with 40 to $50 \mathrm{cc}$. crude hydrochloric acid (sp.gr. I.16) and $6 \mathrm{cc}$. nitric acid (prepared by mixing nitric acid (sp.gr. I.I2), with its own bulk of water). Ores containing much sulphur or bitumen must be roasted before being treated with acid. The flask is heated on a sand-bath for half an hour, and then boiled for fifteen minutes; then filtered into a large beaker and the residue washed with water. A rod of zinc, weighing about 50 gms. and as free from lead as possible, is surrounded with a stout piece of platinum foil and immersed in the copper solution. The precipitation is usually complete in about half an hour. The precipitate of metallic copper is collected together with the platinum foil upon which a part has deposited, and, if it amounts to 6 per cent of the ore (known approximately from its bulk), dissolved in $8 \mathrm{cc}$. of the nitric acid before mentioned. If more than 6 per cent, it is dissolved in $\mathrm{r} 6 \mathrm{cc}$. nitric acid, diluted to roo cc. and one half taken. To the solution of copper ro cc. ammonia water (prepared by mixing one volume sp.gr. 0.93 with two volumes of water) is added, and the fluid titrated with potassium cyanid until the blue color has disappeared.

* Zeitschr. f. Analyt. Chem., VIII, I, and Chem. News, XIX, I8I. 
Dulin's Process for Ores.* The copper ore is treated in the usual manner so as to obtain a solution of copper partially free from lead and silver. (See Low's process.) This solution is boiled with strips of aluminum foil, which results in the complete precipitation of the copper, together with any silver which may be present, and which is always so small as to be negligible. If cadmium is present it is only partially precipitated, beginning after the copper is thrown down. If the boiling be stopped immediately after the copper is precipitated, which a practiced eye will readily detect, the amount of cadmium precipitated is so small as not to cause a sensible error. The liquid is then decanted off from the aluminum foil and copper quickly washed several times with hot water, care being taken not to wash away any particles of copper. Three cc. of nitric acid are then added to the flask and boiled until the copper is dissolved. The solution is then treated with ammonia or with sodium carbonate, as described under Estimation by Means of Potassium Cyanid, and titrated with the cyanid solution in the usual way.

\section{ESTIMATIONS WITH POTASSIUM IODID AND SODIUM THIOSULPHATE}

Iodometric Method. This method $\dagger$ is based upon the reaction between potassium iodid and copper sulphate, in acid solution. The reaction is expressed as follows:

$$
{ }_{2} \mathrm{CuSO}_{4}+4 \mathrm{KI}=\mathrm{Cu}_{2} \mathrm{I}_{2}+{ }_{2} \mathrm{~K}_{2} \mathrm{SO}_{4}+2 \mathrm{I} .
$$

Cuprous iodid is precipitated as a dirty white powder, while iodin is set free. The latter is then immediately titrated with sodium thiosulphate and starch, and thus the corresponding quantity of copper found.

The copper should be in the form of sulphate, and the solution must contain no nitric or hydrochloric acid, though acetic acid may be present.

If nitric acid is present, the solution must be evaporated with sulphuric acid until the former is expelled. Other salts of copper may be converted into sulphate by precipitating with $\mathrm{H}_{2} \mathrm{~S}$, dissolving the resulting sulphid in nitric acid, and then evaporating the solution to dryness after adding some sulphuric acid and sodium sulphate.

Each cc. of $\frac{\mathrm{N}}{\mathrm{IO}}$ thiosulphate $=0.0063 \mathrm{Igm}$. Cu. In titrating copper by this method it is of course necessary to remove all other substances

\footnotetext{
* Dulin, J. A. C. S., XVII, 346.

† De Haen, Annal. d. Chem. u. Pharm., XCI, 237, and Brown, Quart. Jour. of Chem. Soc., $\mathrm{X}, 65$.
} 
which cause a liberation of iodin from potassium iodid, such as ferric salts, free chlorin, or nitrites. This can readily be accomplished by placing into the solution a rod of clean pure zinc. Metallic copper then quickly and completely separates as a spongy precipitate, which may be collected upon a filter, and after thoroughly washing, dissolved in nitric acid. This nitric acid solution is evaporated to dryness after the addition of sulphuric acid, and the resulting copper sulphate dissolved in water and treated with potassium iodid, as above described.

In alloys containing besides copper, zinc, tin, or lead, I or 2 gms. of the alloy in coarse powder are dissolved in concentrated nitric acid, and the solution diluted with water to Ioo cc. After the metastannic acid has settled to the bottom, the clear solution is poured off, and a measured or weighed quantity of the latter transferred to a porcelain capsule. The excess of nitric acid is neutralized by means of calcium carbonate, and any excess of the latter removed by the addition of a few drops of diluted hydrochloric acid. The now weakly acid solution is then treated with potassium iodid and the liberated iodin titrated in the usual manner.

In copper-nickel alloys the separation of the nickel must be brought about. For this purpose the method of Dewilde may be employed. The alloy is dissolved in hydrochloric acid containing some nitric acid. The excess of acid is removed by evaporating or by neutralizing with sodium hydroxid. The resulting chlorid is dissolved in about $50 \mathrm{cc}$. of water, and to this is added twice as much potassium bitartrate as was taken of the alloy for analysis, and the mixture warmed. An alcoholic solution of potassium hydroxid is then added in small portions at a time until the precipitate which at first forms is redissolved. The solution is then allowed to cool and some pure grape-sugar in solution added and the liquid boiled for one or two minutes.

The copper is then completely precipitated as $\mathrm{Cu}_{2} \mathrm{O}$. This precipitate is collected on a filter, washed thoroughly, dissolved in dilute nitric acid, and the resulting copper nitrate solution titrated as above described.

\section{Iodometric Methods for the Technical Assay of Copper Ores.} J. W. Westmoreland $*$ recommends the following for copper in its various ores:

The ore is dissolved in nitric acid, to which hydrochloric acid is also added later on. The solution is then evaporated to dryness, with excess of sulphuric acid. Thus the bases are converted into sulphates. The residue is then treated with warm water and any lead sulphate or other insoluble matters, separated by filtration. The filtrate is heated to boiling and precipitated with sodium thiosulphate; the copper is 
thereby completely precipitated as sulsulphid mixed with sulphur. This can be readily filtered off and washed. Arsenic and an imony, if present, are also precipitated, but tin, zinc, iron, nickel, cobalt, and manganese are not precipitated.

The precipitate is washed thoroughly with hot water, dried and roasted,* and the resulting copper oxid and sulphid dissolved in nitric acid. The excess of acid is then removed by evaporation and sodium carbonate added to precipitate part of the copper and to neutralize any remaining mineral acid. Sufficient acetic acid is now added to produce a clear solution, and then about ten parts of potassium iodid to one of copper, supposed to be present, are added, and the titration of the liberated iodin carried out by means of sodium thiosulphate, in the usual manner.

About 5 gms. of the ore should be taken for the assay; for high percentage ores relatively smaller quantities are to be taken."

Estimation of Copper in Ores Containing Iron. (Andrew M. Fairlie. $\dagger$ ) For the precipitation of copper and its separation from iron, zinc or aluminum, thiocyanate is used in preference to sodium thiosulphate. In the following process the thiocyanate is used.

The following solutions are required:

(a) Sodium thiosulphate, 19.5 gms. per liter.

(b) Ammonium thiocyanate, roo gms. per liter.

(c) Potassium iodid, 50 per cent.

(d) Starch solution.

To Standardize the Solutions. $0.2 \mathrm{gm}$. of copper are dissolved in $5 \mathrm{cc}$. of diluted nitric acid ( $\mathrm{I}: \mathrm{I})$ and after the addition of $50 \mathrm{cc}$. of water boiled for five or ten minutes. A slight excess of ammonia is then added and the liquid acidified with acetic acid and cooled. $6 \mathrm{cc}$. of the potassium iodid solution are then added, and the liberated iodin titrated with the standard sodium thiosulphate.

The Process. Of the ore to be examined a sufficient quantity is taken to make a solution containing 0.2 to $0.24 \mathrm{gm}$. of copper., 2 gms. of potassium chlorate are added together with Io cc. of nitric acid (sp.gr. 1.42), and the vessel covered and shaken for a short time. Heat is then applied and the chlorin and nitrous vapors driven off. The solution is then cooled quickly, ro cc. of hydrochloric acid (sp.gr. r.2) are added, and again boiled for one or two minutes. By this procedure all sulphur present is oxidized, while the copper dissolves and a white sand-like residue remains. The solution is then diluted (with out filtering) with $50 \mathrm{cc}$. of water, an excess of ammonia is added, and after acidifying the liquid with sulphuric acid and heating nearly to

* The roasting causes most of the arsenic and the sulphur to be expelled.

$\dagger$ Chem. Ztg., 1904, Rep. 385 . 
boiling, Io cc. of sulphurous acid are added to reduce the iron present. The copper is then precipitated by adding 5 cc. of the thiocyanate solution, filtered, and washed with hot water.

The precipitate, together with the filter, is then introduced into a suitable flask and dissolved in concentrated nitric acid, driving off the acid vapors by boiling; ammonia water is then added, and finally acetic acid to faint acid reaction, and when the liquid is cool, $6 \mathrm{cc}$. of the potassium iodid solution are added, and the titration with thiosulphate begun. Upon the addition of the sulphurous acid the solution should be neutral, and during the titration large quantities of ammonium acetate must not be present, since this hinders to some extent the reaction between copper and the iodid.

Low's Process. The following modification by A. H. Low * gives excellent results:

The sodium thiosulphate solution is standardized as follows: I9 gms. of the salt are dissolved in I liter of water. $0.2 \mathrm{gm}$. of pure copper foil are placed in a $250 \mathrm{cc}$. flask, and $5 \mathrm{cc}$. of a mixture of equal parts of nitric acid (sp.gr. I.42) and water are added and the mixture boiled until red fumes cease to be given off. Now remove the flame and add 6 or 7 gms. of crystallized zinc acetate and about I5 cc. of water, or about $20 \mathrm{cc}$. of a cold saturated solution of the zinc salt. Heat to boiling, cool, and dilute to about $50 \mathrm{cc}$. Now add $3 \mathrm{gms}$. of potassium iodid and shake gently until dissolved. Then titrate at once with the thiosulphate solution, until the brown tinge has become weak, and then add sufficient starch solution to produce a blue color, and continue the titration cautiously until the blue color vanishes. I cc. of the thiosulphate solution should correspond to about $0.005 \mathrm{gm}$. of copper. In assaying ores, etc., when half a gram is taken, I cc. of the standard solution will be equal to one per cent of copper. The use of the zinc acetate is to counteract the nitric acid used in dissolving the copper. $\dagger$

The Process. Treat half a gram of the ore in a flask of $250 \mathrm{cc}$. capacity with 5 or $6 \mathrm{cc}$. of strong nitric acid, and boil gently until nearly dry. Then add 5 cc. of strong hydrochloric acid and boil again.

* J. A. C. S., XVIII, 458, and XXIV, I082.

$\dagger$ In a later modification of this process, Low uses instead of zinc acetate, $5 \mathrm{cc}$. of strong bromin water. The mixture is boiled until the bromin is expelled; this is to insure complete destruction and removal of the red fumes. It is then removed from the heat, and a slight excess of strong ammonia water added. ordinarily $7 \mathrm{cc}$. of ammonia water $0.90 \mathrm{sp}$.gr.; then boiled to expel excess of ammonia, which is shown by a change of color of the liquid and a partial precipitation of the copper. Now strong acetic acid is added ( 3 or 4 cc. of the 80 per cent) and boil if necessary to redissolve the copper; cool, add the potassium iodid, and proceed as above described. 
As soon as the incrusted matter has dissolved, add $6 \mathrm{cc}$. of strong sulphuric acid and heat strongly over a naked flame, until more volatile acids are expelled and the fumes of sulphuric acid are coming off freely. Allow to cool and then add $25 \mathrm{cc}$. of cold water, and heat to boiling, to dissolve any anhydrous sulphates of iron, etc. Now filter, to remove more especially any lead sulphate, and receive the filtrate in a beaker about $2 \frac{1}{2}$ inches in diameter. Wash the flask and filter with hot water, and endeavor to keep the volume of the filtrate down to 50 or $60 \mathrm{cc}$.

Place in the beaker two pieces of sheet aluminum, which, for the sake of convenience in subsequent washing, may be prepared as follows: Stout sheet aluminum, say about one sixteenth of an inch in thickness, is cut into two pieces an inch and a half square, and then the four corners are bent for about a quarter of an inch, alternately up and down at right angles. This scheme prevents the pieces from lying flat against each other or upon the bottom of the beaker, and their washing is thus facilitated. Add one drop of strong hydrochloric acid, cover the beaker and heat to boiling for about seven minutes.*

Transfer the solution back to the original flask, and by means of a wash-bottle of hot water, rinse in also as much of the copper as possible, leaving the aluminum behind. Drain the beaker as completely as possible, and temporarily set it aside with the aluminum, which may still retain a little copper. Allow the copper in the flask to settle and then decant the liquid through a filter. Again wash the copper similarly two or three times with hot water, retaining it as completely as possible in the flask. Now pour upon the aluminum in the beaker $5 \mathrm{cc}$. of a mixture of equal volumes of strong sulphuric acid and water, and warm the beaker gently but do not boil. See that any copper

* In Low's later modification of this method the directions from here on are as follows: There is danger of the finely divided copper being slightly oxidized in the subsequent washing. To prevent this, and at the same time, precipitate any traces of copper still remaining in solution, add about $\mathrm{i} 5 \mathrm{cc}$. of strong hydrogen sulphid water. If the copper in the ore does not exceed much over 20 per cent, proceed as follows: Decant the supernatant liquid through a $9-\mathrm{cm}$. filter, add Io cc. of strong hydrogen suplhid water to the residue in the beaker, and then transfer the liquid and precipitate to the filter. Wash thoroughly with cold water without delay, to prevent oxidation. Now place the clean original flask under the funnei. Pour over the aluminum in the beaker $5 \mathrm{cc}$. of a mixture of equal parts of strong nitric acid (sp.gr. 1.42) and water. This dissolves any adhering particles of copper. Heat to boiling and pour over the precipitate on the filter so as to dissolve all the copper Now without washing, pour $5 \mathrm{cc}$. of a cold saturated aqueous solution of bromin into the filter, and then wash the beaker and filter with hot water. Boil the filtrate, which usually does not exceed $75 \mathrm{cc}$. in bulk, to thoroughly expel the excess of bromin. Then remove from the heat and add ammonia water in slight excess (about $7 \mathrm{cc}$. of strong ammonia water). Boil off the excess of ammonia, acidify with acetic acid, and finish the process as described under the Standardization of Sodium Thiosulphate Solution. 
present is dissolved and pour the warm solution through the filter last used, thus dissolving any retained particles of copper, and receive the filtrate in the flask containing the main portion of the copper. At this stage do not wash either the aluminum or the filter, but simply remove the flask and set the beaker in its place. Heat the mixture in the flask to boiling and see that all the copper is dissolved. Then add about half a gram of potassium chlorate and again boil for a moment. This is to oxidize any arsenic present to arsenic acid and is a very important point. Remove the flask from the lamp and again place it under the funnel and wash the beaker, aluminum and filter with as little hot water as possible, again boil sufficiently to remove every trace of red fumes. All the copper is now in the flask as nitrate. Add the zinc acetate and proceed from this point precisely as described with the original nitrate of copper solution in the standardization of the thiosulphate; finally calculate the precentage of copper present from the amount of standard thiosulphate required.

A sufficient excess of potassium iodid should be taken, not less than 3 gms. - an excess does no harm. Silver does not interfere. Lead and bismuth are without effect, except that by forming yellow iodids, they may mark the end-point before the starch is added. Arsenic, when oxidized as described, has no effect. The return of the blue tinge in the liquid by long standing after titration, is of no significance, but a quick return of the color, which an additional drop or two of the thiosulphate does not permanently destroy, may indicate either incomplete combination of all the nitric acid with the zinc, or a failure in completely boiling off the red fumes.

Note. $-0.5 \mathrm{gm}$. of copper requires theoretically $-2.65 \mathrm{gms}$. of potassium iodid, yet when only this proportion is used the reaction is slow. It is therefore better in practice to use an excess, the quantity used being governed by the amount of copper present, which can always be approximately estimated. Low suggests to use about $\mathrm{r}$ gm. of potassium iodid for every $\mathrm{r} 5$ per cent of copper.

By Preciptiation with Thiocyanate (Volhard). The estimation of copper by means of thiocyanate solution depends upon the fact that in the presence of a reducing agent thiocyanates completely precipitate copper out of its hot solution, as cuprous thiocyanate:

$$
{ }_{2} \mathrm{CuSO}_{4}+{ }_{2} \mathrm{KSCN}+\mathrm{SO}_{2}+{ }_{2} \mathrm{H}_{2} \mathrm{O}={ }_{2} \mathrm{CuSCN}+{ }_{2} \mathrm{H}_{2} \mathrm{SO}_{4}+\mathrm{K}_{2} \mathrm{SO}_{4} \text {. }
$$

The reducing agent best suited for this purpose is sulphurous acid. The precipitate of cuprous thiocyanate is white in color and quite insoluble. The estimation is best carried out by residual titration; an accurately measured quantity (excess) of $\frac{\mathrm{N}}{\mathrm{IO}}$ potassium thioeyanate is added to the copper solution, and the uncombined excess deter- 
mined by titrating with $\frac{\mathrm{N}}{\mathrm{IO}}$ silver nitrate. Ferric alum is used as indicator. This process is not available in the presence of silver, mercury cyanids, halogens, or arsenic in the oxidized state.

The Process. A sample of metallic copper or an alloy, say $0.5 \mathrm{gm}$., is dissolved in a smail quantity of concentrated nitric acid (sp.gr. 1.42), and the solution heated until excess of acid and nitrous fumes are expelled. About roo cc. of water are added and the solution neutralized carefully with sodium carbonate, until a slight precipitate begins to form. A sufficient quantity of sulphurous acid is now added to give the solution a strong odor of $\mathrm{SO}_{2}$. The solution is then heated to boiling and an excess of $\frac{\mathrm{N}}{\mathrm{IO}}$ potassium thiocyanate slowly added. The greenish coloration which is produced upon each addition of the thiocyanate is due to the formation of cupric thiocyanate; this upon agitation is reduced to the white cuprous thiocyanate which settles rapidly. The addition of the standard solution is continued until no further coloration is produced and the copper is completely precipitated.

The quantity of thiocyanate solution used is noted, and after the solution has cooled down it is diluted with distilled water to exactly $500 \mathrm{cc}$. This solut ion is then thoroughly shaken and filtered, and roo cc. (representing $0.1 \mathrm{gm}$. of the metal) is removed to a beaker, ro cc. of ammonio-ferric sulphate solution added as indicator, then 10 cc. of nitric acid, and the titration with $\frac{\mathrm{N}}{\mathrm{IO}}$ silver nitrate begun and continued until the solution is colorless. The quantity of silver solution consumed, deducted from the quantity of thiocyanate solution, gives the quantity of the latter which reacted with and hence represents the copper. A more accurate determination of the end-point may be obtained by retitrating with the thiocyanate, after the silver nitrate, in which case the quantity of silver used is deducted from the total quantity of thiocyanate.

Precipitation as Cuprous Thiocyanate and the Acidimetric Determination of the Combined Thiocyanic Acid (W. E. Garrigues).* This method depends upon precipitating the copper as cuprous thiocyanate $(\mathrm{CuSCN})$ in the presence of sulphurous acid, according to the method of Rivot, and then acidimetrically determining the combined thiocyanic acid in the precipitate.

The solution of copper sulphate, slightly acidulated with sulphuric acid, is warmed and treated with a sufficient excess of suiphurous acid 
to impart a distinct odor to the solution. An excess of ammonium thiocyanate solution is now added, and the precipitate allowed to settle (the settling is facilitated by warming). The fluid is then filtered, preferably through a Gooch crucible, and the precipitate well washed with water. The filter and contained precipitate are then transferred to a beaker and boiled with a measured excess of standard caustic alkali, for a few minutes, then cooled and diluted to a convenient volume (say $200 \mathrm{cc}$.). The reaction which occurs is as follows:

$$
{ }_{2} \mathrm{CuSCN}+2 \mathrm{NaOH}=\mathrm{Cu}_{2}(\mathrm{OH})_{2}+2 \mathrm{NaSCN} .
$$

The cuprous hydroxid is then filtered off and the filtrate titrated to neutrality with normal hydrochloric acid, using methyl orange as indicator. One cc. of $\frac{\mathrm{N}}{\mathrm{I}} \mathrm{NaOH}=0.06_{3 \mathrm{I}} \mathrm{gm}$. Cu.

The results obtained by this process compare very favorably with those obtained by the iodometric method. Garrigues claims even greater accuracy than the iodometric method is capable of. It is especially useful in alloy assaying.

Precipitation as Cuprous Thiocyanate and Subsequent Titration of the Thiocyanic Acid by Means of Potassium Permanganate (H. A. Guess)** The first steps of this method are the same as in the foregoing. The precipitated cuprous thiocyanate is washed by decantation with hot water, and then transferred by washing onto a filter.

The funnel with its contents is then placed in the original precipitation flask and a boiling ten per cent solution of caustic soda poured through. The operation is repeated with a further portion of the caustic soda solution, the filter being filled full each time. The cuprous thiocyanate is decomposed, and cuprous hydroxid remains on the filter, while sodium thiocyanate passes through. The filter and contents are well washed with hot water, the alkaline filtrate is made decidedly acid with dilute sulphuric acid, and the liberated thiocyanic acid titrated with standard potassium permanganate while still warm. According to the equation

$$
\mathrm{IoHCNS}+\mathrm{I}_{2} \mathrm{KMnO}_{4}+8 \mathrm{H}_{2} \mathrm{SO}_{4}=6 \mathrm{~K}_{2} \mathrm{SO}_{4}+\mathrm{I}_{2} \mathrm{MnSO}_{4}+\mathrm{s} . \mathrm{HCN}+8 \mathrm{H}_{2} \mathrm{O} \text {, }
$$

the iron factor multiplied by 0.1892 should give the theoretical amount of copper. Guess found that by using 0.192 as the factor greater accuracy was obtained.

This factor overcomes the loss occasioned by the slight solubility

*J. A. C. S., XXIr, zo8. 
of the cuprous thiocyanates and the consequent incomplete precipitation.

The permanganate solution used for this purpose is best standardized against pure copper, under the same conditions as the regular assays.

\section{TITRATION OF THE IRON EQUTVALENT OF COPPER BY MEANS OF POTASSIUM PERMANGANATE}

Meade's Process.* The copper is brought into solution as a sulphate, either by dissolving it in sulphuric acid, or by evaporation of its solution with sulphuric acid. The greater part of the free acid is neutralized by ammonia, the solution warmed, sulphurous acid added until the solution smells strongly of the reagent, and then a slight excess of ammonium or potassium thiocyanate added. The copper is immediately precipitated as cuprous thiocyanate. Stirring and warming renders the precipitate heavy and easily handled. The solution is filtered through asbestos, using the pump, and well washed. The precipitate and filter are thrown into the beaker in which the precipitation was made, and heated with a solution of caustic soda or potash. The resulting cuprous hydroxid is filtered on asbestos and washed well with hot water.

The precipitate and filter are again placed in the same beaker, and an excess of ferric chlorid or ferric sulphate (free from nitric acid, free chlorin or ferrous salts), together with a little dilute sulphuric acid added. The cuprous oxid reduces a corresponding amount of iron from the ferric to the ferrous state.

$$
\mathrm{Cu}_{2} \mathrm{O}+\mathrm{Fe}_{2} \mathrm{Cl}_{6}+{ }_{2} \mathrm{HCl}={ }_{2} \mathrm{CuCl}_{2}+{ }_{2} \mathrm{FeCl}_{2}+\mathrm{H}_{2} \mathrm{O} .
$$

The beaker is warmed and stirred until all the copper oxid is dissolved. The solution is then poured through a perforated platinum disk and the asbestos, which stays behind, washed with water to which has been added a little sulphuric acid and a little ferric chlorid or sulphate. The solution is then titrated with permanganate. The iron equivalent to the permanganate used, multiplied by 1.125 , gives the weight of copper in the sample. Instead of sulphurous acid, ammonium or sodium bisulphate may be used. Copper is the only metal precipitated by an alkaline thiocyanate out of an acid solution. Therefore the presence of arsenic, antimony, bismuth, and zinc will not interfere with the accuracy of the results. The filtration may be done through a Gooch crucible.

* J. A. C. S., XX, 6 ro. 
S. W. Parr's Process.* This method depends upon precipitating the copper as cuprous thiocyanate, as in the preceding processes; then decomposing the precipitate by heating with sodium hydroxid, and titrating the alkaline solution at once with potassium permanganate until a green tint is imparted to the liquid portion of the mixture. The reaction to this point is as follows:

$$
8 \mathrm{CuOH}+{ }_{2} \mathrm{KMnO}_{4}+8 \mathrm{H}_{2} \mathrm{O}=2 \mathrm{KOH}+8 \mathrm{Cu}(\mathrm{OH})_{2}+\mathrm{Mn}_{2}(\mathrm{OH})_{6} .
$$

The appearance of the green tint indicates that the copper has been completely oxidized, and that the permanganate is acting upon the sodium thiocyanate with formation of the green manganates, as per the reaction:

$$
\begin{aligned}
& 8 \mathrm{KMnO}_{4}+\mathrm{NaCNS}+\mathrm{I} \circ \mathrm{NaOH} \\
& \qquad={ }_{4} \mathrm{~K}_{2} \mathrm{MnO}_{4}+{ }_{4} \mathrm{Na}_{2} \mathrm{MnO}_{4}+\mathrm{NaCNO}+\mathrm{Na}_{2} \mathrm{SO}_{4}+{ }_{5} \mathrm{H}_{2} \mathrm{O} .
\end{aligned}
$$

The alkaline solution is then treated with diluted sulphuric acid $(\mathrm{I}: 5)$ slowly added with constant stirring until the solution becomes clear; a divided excess of the diluted acid is then added, and the whole warmed to $60^{\circ}$ or $70^{\circ} \mathrm{C}$., and the titration with the permanganate continued to the usual pink-end reaction. This is really a titration of cuprous thiocyanate by permanganate. For further details, the articles themselves are to be read.

By Precipitation with Potassium Ferrocyanid (Spica). When a solution of a copper salt is treated with potassium ferrocyanid a brown precipitate of copper ferrocyanid forms:

$$
{ }_{2} \mathrm{CuSO}_{4}+\mathrm{K}_{4} \mathrm{Fe}(\mathrm{CN})_{6}=\mathrm{Cu}_{2} \mathrm{Fe}(\mathrm{CN})_{6}+{ }_{2} \mathrm{~K}_{2} \mathrm{SO}_{4} .
$$

This reaction is the basis of a method proposed by M. Spica $\dagger$ for the estimation of copper. The end-point, i.e., the point at which a slight excess of the ferrocyanid is present, is determined by means of ferric chlorid. A drop of the solution being titrated is removed on a glass rod and brought in contact with a piece of bibulous paper, which has been previously impregnated with a solution of ferric chlorid and dried. The production of a blue color on this test paper indicates that an excess of the ferrocyanid has been added, and hence that the copper is completely precipitated.

* J A. C. S., XXII, 685, and XXIV, 580.

$\dagger$ Matteo Spica, Chem. Centrabl., 65, 8I 5 (I894). 
The standard solution used is made by dissolving $0.84 \mathrm{gm}$. of pure crystallized potassium ferrocyanid in water to make rooo cc. Each cc. of this solution represents $0.00 \mathrm{Igm}$. of $\mathrm{CuSO}_{4}+{ }_{5} \mathrm{H}_{2} \mathrm{O}$.

The copper solution to be titrated and which should be a 0.5 to I per cent solution is slightly acidified with hydrochloric acid before titrating. The titration should be conducted hot.

This method can be used only for pure copper solutions, since under similar conditions many other metals are precipitated. For instance, lead, silver, mercury, cadmium, bismuth, zinc, iron, manganese, cobalt, and nickel. It must be admitted, however, that with pure copper solutions very satisfactory results are obtained.

Estimation by Means of Stannous Chlorid (Fr. Weil).* This process depends upon the fact that a hot solution of a cupric salt in the presence of considerable excess of hydrochloric acid, shows a distinct greenish yellow color. The addition of the minutest excess of stannous chlorid solution decolorizes the copper solution instantly, and thus affords a sharp easily distinguishable end-reaction. The reaction is as follows:

$$
{ }_{2} \mathrm{CuCl}_{2}+\mathrm{SnCl}_{2}=\mathrm{Cu}_{2} \mathrm{Cl}_{2}+\mathrm{SnCl}_{4} \text {. }
$$

The further certainty that the reaction is complete and that the stannous chlorid is in slight excess, is afforded on adding a few drops of mercuric chlorid solution to a small portion of the cooled liquid; any precipitate of calomel indicates the presence of stannous chlorid.

The stannous chlorid may be prepared as described on page 232 . It should be standardized against a solution of copper sulphate as follows:

Twenty-five cc. of a copper sulphate solution (containing 0.I gm. $\mathrm{Cu}$ ) are introduced into a roo-cc. flask. $5 \mathrm{cc}$. of pure concentrated hydrochloric acid are added, and the liquid heated to boiling. The stannous chlorid solution is added to the boiling liquid, rapidly at first, but towards the end by drops, until the liquid is colorless. $5 \mathrm{cc}$. more of hydrochloric acid are added, if a slight color develops more stannous chlorid solution is added until the color is again discharged, and the end-point affirmed by testing with mercuric chlorid solution, as above described. In titrating any cupric salt, the procedure is the same; nitric acid and ferric salts must be absent, as must also mercuric salts, and all other substances which react with stannous chlorid.

*Zeitschr. f. analyt. Chem., IX, 297. 


\section{BIBLIOGRAPHY}

Steinbeck, Chem. News, XIX, I8I.

Davies, Chem. News, LVIII, I3I.

Fessenden, Chem. News, LXI, 253-283.

J. J. and C. Beringer, Chem. News, XuIX, 3.

Spica, Chem. Centrbl., LXv, 8I5 (I894).

Parkes, Mining Jour., (I85I).

Dulin, J. A. C. S., XvII, 346.

Low, J. A. C. S., XviII, 458, and XxIv, 708.

Garrigues, J. A. C. S., XIX, 940.

Meade, J. A. C. S., xx, 6 ro.

Shengel and Smith, J. A. C. S., XXI, 932.

Parr, J. A. C. S., XxII, 685, and Xxiv, 580.

Guess, J. A. C. S., xxiv, 708.

Fernekes and Koch, J. A. C. S., XXVII, I224.

Griggi, Boll. Chim. Farm., XLII, 392.

Westmoreland, J. S. C. I., v, 51 .

Liebig, Ann. Chem., 95, I18.

Mohr, Ann. Chem., 94, 148.

Weil, Zeitschr. f. analyt. Chem., IX, 297. 


\section{CHAPTER XXXIII}

\section{GOLD}

THE gold must be in the form of chlorid $\left(\mathrm{AuCl}_{3}\right)$.

To the solution of gold chlorid a measured excess of $\frac{\mathrm{N}}{\mathrm{I}}$ oxalic acid solution is added and the mixture set aside for twenty-four hours.

The solution is then made up to a definite volume (say $300 \mathrm{cc}$.). Then by means of a pipette roo cc. are removed, and the excess of oxalic acid found by titrating with $\frac{\mathrm{N}}{10}$ permanganate in the presence of sulphuric acid. The reaction is:

$$
{ }_{2} \mathrm{AuCl}_{3}+{ }_{3} \mathrm{H}_{2} \mathrm{C}_{2} \mathrm{O}_{4}=2 \mathrm{Au}+6 \mathrm{HCl}+6 \mathrm{CO}_{2} .
$$

Each cc. of $\frac{\mathrm{N}}{\mathrm{I}}$ oxalic acid solution $=0.06523 \mathrm{gm}$. of $\mathrm{Au}$, or 0.1004 gm. of $\mathrm{AuCl}_{3}$. 


\section{CHAPTER XXXIV}

\section{IRON}

Ferrous Salts are estimated by direct titration with potassium permanganate or dichromate, as described in Chapter XII.

Ferric Salts may be estimated by means of potassium permanganate or dichromate, but they must first be reduced to the ferrous state. The reduction may be accomplished in various ways, as follows:

The ferric salt in solution may be percolated through a column of zinc dust, which reduces it to the ferrous state, and the iron may then be estimated in the usual way with permanganate.

Another way for reducing ferric salts is to treat the solution with small pieces of metallic zinc or magnesium and a little sulphuric acid. The reduction is known to be complete when the solution ceases to give a red color with potassium sulphocyanate. The change of color of the ferric solution from dark to light is also an indication of reduction.

When zinc or magnesium are used the metal must be entirely dissolved before titration is begun.

For full details of the method for estimating ferric salts after reduction, see page 162 .

The zinc used must be free from iron, or if any is present its exact quantity should be known.

The iron solution to be reduced by zinc should not contain more than 0.12 in $200 \mathrm{cc}$., and for this quantity about Io gms. of zinc and $20 \mathrm{cc}$. of sulphuric acid should be employed.

Stannous chlorid solution is also employed for reducing ferric salts. This solution is made by dissolving ro gms. of pure tin in $200 \mathrm{cc}$. of strong hydrochloric acid and diluting to rooo cc. It should be freshly made when needed.

After reduction by stannous chlorid the titration may be performed with dichromate.

When stannous chlorid is used, sufficient must be added to completely reduce the iron, but an excess, even the slightest, must be avoided. A practiced eye can very closely determine the point of complete reduction by the discharge of the color in the ferric solution, provided the solution is tolerably concentrated. A surer way is to use 
a concentrated aqueous solution of mercuric chlorid; this converts any excess of stannous chlorid present to stannic chlorid, while a turbidity due to the precipitation of mercurous chlorid results.

If the excess of stannous chlorid were not thus destroyed, it would consume the dichromate, and thus lead to inaccurate results. The presence of the mercurous chlorid precipitate does not in the least interfere with the titration, which should be begun with potassium dichromate at once.

Other reducing agents for ferric salts which are used in technical analyses, are sodium sulphite, ammonium bisulphite, and sulphurous acid. When these are used, the ferric solution should be dilute and not too acid. The sulphite is added to the ferric solution and heated in a flask until the color is discharged. It is then boiled until all sulphur dioxid is driven off, quickly cooled and titrated with the standard dichromate.

After reduction by any method, the titration should be started without delay, because the iron is rapidly re-oxidized upon exposure.

Ferric salts may also be estimated direct, i.e., without previous reduction, but the first mentioned methods are by far the best.

The Direct Titration by Stannous Chlorid is explained on page 23I.

The Direct Titration by Sodium Thiosulphate. When a ferric salt is treated under suitable conditions with a solution of sodium thiosulphate, the following reaction occurs and a fairly accurate estimation of the ferric salt may be obtained:

$$
\mathrm{Fe}_{2} \mathrm{Cl}_{6}+{ }_{2} \mathrm{Na}_{2} \mathrm{~S}_{2} \mathrm{O}_{3}+{ }_{2} \mathrm{HCl}=\mathrm{H}_{2} \mathrm{~S}_{4} \mathrm{O}_{6}+{ }_{4} \mathrm{NaCl}+{ }_{2} \mathrm{FeCl}_{2} \text {. }
$$

Sodium thiosulphate was first suggested for use for this purpose by Scherer in 1859 , afterwards by Kremer and Landolt (Zeitschr. f. analyt Chem., I, 214). Later Oudemans in the same journal (VI, 129) described the method briefly given below.

Oudemans' Process. A small quantity of a solution of pure copper sulphate ( $\mathrm{I}$ cc. of a I per cent solution) is added to the ferric salt, and then a few drops of a solution of potassium sulphocyanate, which colors the fluid intensely red. Sodium thiosulphate is then run in slowly from the burette. A few minutes should elapse after each addition of thiosulphate. When the color communicated by the sulphocyanate has disappeared, a milkiness makes its appearance, due to cuprous sulphocyanate, which is formed. This marks the completion of the reaction. The operator is very apt to overstep the mark, and the best plan is to add thiosulphate in very slight excess, and find the amount in excess by adding starch, and titrating back with iodin. 
The method is tolerably good and sufficiently accurate for technical estimations, but not entirely satisfactory where great exactness is required. The copper sulphate is supposed by Oudemans, who first used it, to act by transferring oxygen from the ferric salt to the thiosulphate. The estimation may be made without using copper sulphate, if the solution be heated to $40^{\circ} \mathrm{C}$.

$$
\text { I cc. } \frac{\mathrm{N}}{\text { IO }} \text { thiosulphate }=0.00555 \mathrm{gm} . \text { Fe. }
$$

Kerner gives the following as being a satisfactory procedure:

The iron solution, in which the metal must be in complete oxidation (best obtained by using hydrogen dioxid in excess and boiling off the excess), is acidified with hydrochloric acid. Sodium acetate is added until the mixture is red, and then dilute hydrochloric acid until the red color is discharged; the solution is then diluted until the iron amounts to 0.25 per cent. An excess of $\frac{\mathrm{N}}{\mathrm{IO}}$ sodium thiosulphate solution is then added, and the excess determined by titration with $\frac{\mathrm{N}}{\mathrm{IO}}$ iodin solution and starch.

J. T. Norton (Am. J. Sci., CLviII, 25-30) made careful experiments with this thiosulphate method, and found that the best results are obtained under the following conditions: The concentration of the iron solution must not be greater than $0.1 \mathrm{gm}$. ferric oxid in $400 \mathrm{cc}$; the amount of acid present should not exceed I cc. of strong hydrochloric acid in the same volume; the time of reaction must be as short as possible to prevent progressive oxidation by air, and the excess of thiosulphate used should, for the same weight of iron, be not less than I5 cc. of a decinormal solution.

Moreau * describes the following modification for the titration of solution of ferric chlorid:

Five gms. of the solution is treated with $2 \mathrm{cc}$. of pure hydrochloric acid and diluted with water to $80 \mathrm{cc}$. Io cc. of this is measured off off and diluted with 20 to $30 \mathrm{cc}$. of water, about $0.1 \mathrm{gm}$. of sodium salicylate is then added as an indicator, followed by $10 \mathrm{cc}$. of a 10 per cent solution of cupric sulphate. The mixture is then titrated with $\frac{N}{\text { IO }}$ sodium thiosulphate, added, drop by drop, until the liquid has lost its violet color, or shows only the blue color of the copper. The interval between drops should be, near the end, five seconds. The reaction between the ferric salt and the thiosulphate, which is shown on

* Bull. de Sciences Pharm., from Pharm. Jour., I904, 744. 
page 380 , takes place only in warm solutions, but in the presence of copper it proceeds in the cold.

The best method, however, for simple ferric solutions is the iodometric method, in which the ferric salt is reduced by digestion with potassium iodid, and the liberated iodin which is set free in this reaction, titrated with standard sodium thiosulphate solution (see page 224).

Titration by Permanganate after Precipitation as Ferrous Sulphid (N. Matolcsy, Ph. Post., 1903, 4I). This method depends upon the fact that ferric salts, as well as ferrous salts, are precipitated by ammonium sulphid in the form of ferrous sulphid:

$$
\begin{aligned}
\mathrm{Fe}_{2} \mathrm{Cl}_{6}+3\left(\mathrm{NH}_{4}\right)_{2} \mathrm{~S} & =2 \mathrm{FeS}+6 \mathrm{NH}_{4} \mathrm{Cl}+\mathrm{S}, \\
\mathrm{FeCl}_{2}+\left(\mathrm{NH}_{4}\right)_{2} \mathrm{~S} & =\mathrm{FeS}+2 \mathrm{NH}_{4} \mathrm{Cl},
\end{aligned}
$$

and that the precipitate dissolves in sulphuric acid, forming ferrous sulphate, which may be titrated with permanganate solution in the usual manner, after the expulsion of hydrogen sulphid.

The Process. To the aqueous solution of the iron salt, acidulated with hydrochloric acid, an excess of $\mathrm{H}_{2} \mathrm{~S}$ solution is added and then ammonia water sufficient to make the solution alkaline; this causes the precipitation of the iron as ferrous sulphid.

In order to bring about the agglutination of the precipitate, some ammonium chlorid is added and the solution slightly warmed. The precipitate is then collected, washed with ammonium sulphid solution, and then dissolved in dilute sulphuric acid. The resulting solution of $\mathrm{FeSO}_{4}$ is then boiled until the $\mathrm{H}_{2} \mathrm{~S}$ is completely driven off, i.e., until the steam evolved no longer blackens silver nitrate paper. The addition to the boiling solution of a small piece of calcite, or pure marble, facilitates the dispersion of the $\mathrm{H}_{2} \mathrm{~S}$ and insures an even distribution of the heat. The solution is then quickly cooled and titrated with decinormal potassium permanganate solution, in the usual manner.

Titration of Ferric Salts by Permanganate after Reduction with Stannous Chlorid.* The following solutions are required:

(a) A Five Per Cent Solution of Stannous Chlorid. This solution need not be freshly prepared, but it must be clear and should give no precipitate when diluted with twenty times its volume of water and boiled. It may be kept in good condition by acidifying with hydrochloric acid and putting a few fragments of tin into the bottle.

(b) A Mercuric Sulphate Solution. This is made by adding to $200 \mathrm{gms}$. of mercuric sulphate, $80 \mathrm{cc}$. of sulphuric acid (conc.), and

* Cady and Ruediger, J. A. C. S., XIX, 577, describe this method for titration with permanganate, in the presence of hydrochloric acid. 
adding to this paste, $800 \mathrm{cc}$. of water. If a yellow precipitate of basic mercuric sulphate is formed, add more sulphuric acid. Add to this Ioo gms. of orthophosphoric acid and dilute to one liter.

The Process. $\mathrm{I}$ gm. of the substance is dissolved in $15 \mathrm{cc}$. of hydrochloric acid (sp.gr. I.IO) in a small Erlenmeyer flask, 2 cc. of the mercuric sulphate solution $(b)$ is added, and the whole heated to boiling and the stannous chlorid solution added in small quantities at a time. The precipitate that may be temporarily produced as each drop of stannous chlorid is added, will dissolve after boiling a few seconds, until all the iron is reduced, when the solution becomes colorless, and the addition of a single drop of stannous chlorid produces a perceptible turbidity or a précipitate which does not redissolve on boiling. This shows the end of the reduction. The contents of the flask is rinsed into a beaker and diluted to about $300 \mathrm{cc}$. $50 \mathrm{cc}$. of dilute sulphuric acid are added, and then $45 \mathrm{cc}$. of the mercuric sulphate solution, and the titration begun at once with standard permanganate.

The other method proposed by the same authors is as follows:

Dissolve in water with a varying quantity of hydrochloric acid, dilute to roo cc., and heat to boiling. Reduce with stannous chlorid, adding small portions at a time until the solution becomes colorless, and a droplet gives no red color with potassium thiocyanate. Any excess of stannous chlorid that may have been used is oxidized by adding potassium permanganate, drop by drop, until a droplet of the iron solution gives a faint red color with thiocyanate. One or two drops of stannous chlorid solution are now added, the whole process being carried on at a boiling heat. The solution is then cooled. $50 \mathrm{cc}$. of dilute sulphuric acid are added, and for every ro cc. of hydrochloric acid that has been used add $35 \mathrm{cc}$. of the mercuric sulphate solution; dilute to $400 \mathrm{cc}$. and titrate with permanganate.

\section{ESTIMATION OF METALLIC IRON IN REDUCED IRON}

Professor E. Schmidt, of Marburg, recommends * the following process:

Weigh accurately $0.4 \mathrm{gm}$. of reduced iron, and place in a roo-cc. flask with ro cc. of water, and add 2 gms. of pure dry iodin.

The iodin combines with metallic iron, but does not react with any ferric oxid which may be present.

$$
\mathrm{Fe}_{2}+2 \mathrm{I}_{2}={ }_{2} \mathrm{FeI}_{2} \text {. }
$$

Now rinse down the iodin left in the neck of the flask with some water, and add I gm. of potassium iodid; when all of the iodin has

* Proc. Soc. German Naturalists and Physicians, Sept., I897 
dissolved, add sufficient water to make roo cc. Shake the flask and allow to stand for several hours.

Then measure off $50 \mathrm{cc}$. of the clear liquid and titrate the free iodin with decinormal sodium thiosulphate, using starch as an indicator. The reaction is thus expressed:

$$
\begin{aligned}
& \mathrm{I}_{2}+{ }_{2} \mathrm{Na}_{2} \mathrm{~S}_{2} \mathrm{O}_{3},{ }_{5} \mathrm{H}_{2} \mathrm{O}=\mathrm{Na}_{2} \mathrm{~S}_{4} \mathrm{O}_{6}+2 \mathrm{NaI}+\mathrm{IoH}_{2} \mathrm{O} \text {. } \\
& \text { 2) } 251.8 \quad 2 \lcm{492.92} \\
& \text { 10) } \lcm { 1 2 5 . 9 } \quad 1 0 \longdiv { 2 4 6 . 4 6 } \\
& \text { I2.59 gms. } \frac{246.46}{24.646} \text { gms. } \\
& .01259 \mathrm{gm} \text {. } .024646 \mathrm{gm} \text {. “ I cc. “" “ }
\end{aligned}
$$

Example. Assuming that $9 \mathrm{cc}$. of the decinormal solution were employed in titrating the $50 \mathrm{cc}$., then $\mathrm{I} 8 \mathrm{cc}$. would be required by the entire quantity.

As seen in the above equation, each cc. of the decinormal solution represents $0.01259 \mathrm{gm}$. of iodin; hence if $\mathrm{I} 8 \mathrm{cc}$. are employed we have $18 \times 0.01259 \mathrm{gm} .=0.22662 \mathrm{gm}$., the quantity of free iodin.

Then by subtracting this amount from the quantity of iodin taken (2 gms.) we ascertain the quantity which went into combination with the iron, namely, I.7733 gms. All that is now necessary is to ascertain by calculation the quantity of metallic iron represented by the above weight of iodin.

$$
\begin{gathered}
\mathrm{Fe}+\mathrm{I}_{2}=\mathrm{FeI}_{2} ; \\
55.5 \quad{ }_{25} \mathrm{I} .8 \\
\frac{\mathrm{I} .7733 \times 55.5}{25 \mathrm{I} .8}=0.35 \mathrm{II}+\mathrm{gm} .
\end{gathered}
$$

Thus the $0.40 \mathrm{gm}$. of reduced iron taken contained $0.35 \mathrm{II}+\mathrm{gm}$. of metallic iron, or 87 per cent.

This process, very slightly modified, is official in the U.S.P. and the Pharm. G. In both of these standards the iron is dissolved in a solution of iodin in potassium iodid.

The Estimation of Metallic Iron in Reduced Iron may also be made by the process of digestion with mercuric chlorid. This converts all the iron present in a free state to ferrous chlorid, which is then titrated by potassium permanganate (see page 152 ).

To confirm the assay, add a few drops of alcohol to decolorize (or decompose) the excess of permangante, then add I gm. of potassium iodid, and digest for half an hour at a temperature of $40^{\circ} \mathrm{C}$. (I04 ${ }^{\circ} \mathrm{F}$.).

$$
\begin{aligned}
& \mathrm{Fe}_{2}\left(\mathrm{SO}_{4}\right)_{3}+{ }_{2} \mathrm{KI}={ }_{2} \mathrm{FeSO}_{4}+\mathrm{I}_{2}+\mathrm{K}_{2} \mathrm{SO}_{4} . \\
& \begin{array}{ll}
\text { 2) } \mathrm{III} & 25 \mathrm{I} .80 \\
\frac{\mathrm{IO}}{\frac{55.5}{5.55}} & \frac{125.9}{\mathrm{I2.59}}
\end{array}
\end{aligned}
$$


The cooled solution is mixed with a few drops of starch test solution, which gives it a dark blue color, because of the formation of iodid of starch. Then add carefully, from a burette, decinormal sodium thiosulphate until the blue color is discharged.

$$
\begin{aligned}
& \mathrm{I}_{2}+2\left(\mathrm{Na}_{2} \mathrm{~S}_{2} \mathrm{O}_{3} \cdot{ }_{5} \mathrm{H}_{2} \mathrm{O}\right)=2 \mathrm{NaI}+\mathrm{Na}_{2} \mathrm{~S}_{4} \mathrm{O}_{6}+\mathrm{IoH}_{2} \mathrm{O} \text {. } \\
& \begin{array}{rr}
\text { 2) } 25 \text { I. } & \text { 2) } 492.92 \\
\text { 10) } 125.9 & \text { 10 } \lcm{246.46}
\end{array} \\
& \text { I } 2.59 \text { gms. } \frac{\mathrm{N}}{24.646} \text { gms. or I000 cc. } \frac{\mathrm{N}}{10} \mathrm{Na}_{2} \mathrm{~S}_{2} \mathrm{O}_{3} \text { V. S. }
\end{aligned}
$$

Thus each cc. of the standard thiosulphate represents $0.01259 \mathrm{gm}$. of iodin, or $0.00555 \mathrm{gm}$. of metallic iron.

In both of these estimations the quantity of standard solution used should be the same.

Total Iron Content of Ferrum Reductum. Reduced iron should contain at least 90 per cent of metallic iron. But there is always more or less oxidation of the iron upon exposure and long standing, so that for this reason and through imperfect reduction in its manufacture there is always present some ferric oxid and more or less sulphid, arsenic, and copper, as well as insoluble matter.

The total iron content, i.e., free and combined, may be estimated as follows: * o.r gm. of the sample is digested upon a water-bath with hydrochloric acid to complete solution. Io cc of sulphuric acid are now added, and the hydrochloric acid evaporated off. The whole is then rinsed into an Erlenmeyer flask, diluted to roo cc., 2 grns. of pure zinc added and the flask set aside in a warm place for about one hour, after which time the contents are filtered through a glass plug and immediately titrated with standard permanganate in the usual way.

Another 0.I gm. of the same sample is then assayed for free iron by either of the foregoing methods. The quantity of free iron found subtracted from the total iron gives the amount of iron present in a combined state, principally as ferric oxid.

Estimation of the Iron in Liquor Ferri Albuminata. $20 \mathrm{cc}$. of the solution are mixed in a roo-cc. flask with $20 \mathrm{cc}$. of water and $3 \mathrm{cc}$. of diluted sulphuric acid $(\mathrm{I}: 5)$ the mixture is heated on a waterbath until the brown precipitate at first produced becomes whitish.

The contents of the flask are then allowed to cool, diluted with water to roo cc. and filtered. $20 \mathrm{cc}$. of this solution are then measured off, and potassium permanganate solution added drop by drop until the whole quantity of solution is faintly red, the color being, however,

* G. C. Steventon, Proc. A. Ph. A., 1894, 172. 
transient, and when it disappears and leaves the solution colorless (the addition of a few drops of alcohol will facilitate the discharge of the color), 2.5 to $3 \mathrm{gms}$. of potassium iodid are added, the flask stoppered securely, and set aside for one hour at the ordinary temperature. At the end of this the liberated iodin is titrated with $\frac{\mathrm{N}}{\mathrm{IO}}$ sodium thiosulphate and the calculation made in the usual manner.

Estimation of Iron in Iron Ores. It is usually required in estimating the quantity of iron in iron ores to determine the ferrous and the ferric oxid. This may be done as follows:

$0.5 \mathrm{gm}$. of the ore in fine powder is dissolved with careful exclusion of air * in $20 \mathrm{cc}$. of a mixture of equal parts of strong hydrochloric acid and water, and the resulting solution titrated with potassium dichromate or permanganate, for the quantity of ferrous oxid.

$$
\text { Each cc. of } \begin{aligned}
\frac{\mathrm{N}}{\mathrm{IO}} \text { dichromate } & =0.00555 \mathrm{gm} . \mathrm{Fe} ; \\
& =0.007 \mathrm{I} 38 \text { “ } \mathrm{FeO} ; \\
& =0.007932 \text { " } \mathrm{Fe}_{2} \mathrm{O}_{3} .
\end{aligned}
$$

Another $0.5 \mathrm{gm}$. of the finely powdered ore is then dissolved as above, and when complete solution is effected, the liquid is diluted with water, $15 \mathrm{cc}$. of strong sulphuric acid are added, a few fragments of pure zinc are introduced, and the ferric oxid present thus reduced to the ferrous state; air is excluded during the reduction to prevent reoxidation, and the titration with permanganate immediately begun. The total iron content is thus obtained, and by subtracting the ferrous oxid found from the total iron in the ore, the amount of iron in the ferric state is ascertained.

If any doubt exists as to the complete reduction of the iron, a drop of the solution is withdrawn on a glass rod and tested with potassium thiocyanate. A red color will indicate the presence of unreduced iron. In this event it will be necessary to add more inc and possibly more acid in order to completely reduce the remaining ferric oxid.

Note. If permanganate is used as the titrating reagent, the amount of hydrochloric acid must be small, and the solution cold and very dilute, otherwise a vitiating reaction will take place between the hydrochloric acid and the permanganate (see page I 40).

In estimating toial iron, the reduction to the ferrous state may be made either by means of sodium sulphite or stannous chlorid. If these reducing agents are used, the titration should be done by means of potassium dichromate.

* The apparatus shown in Fig. 57 may be used for this purpose. 
If the ore under examination is not readily soluble in hydrochloric acid, solution may be effected by the addition of some potassium chlorate, and the application of heat. The solution is then evaporated to dryness, and the residue dissolved in hydrochloric acid. 'Any residue which may be left after treatment with hydrochloric acid, should be separated by filtration and fused with sodium carbonate; this will render all the iron soluble. Ores which contain organic matter or pyrites should first be roasted in an open platinum crucible. 


\title{
CHAPTER XXXV
}

\author{
LEAD
}

Lead Oxid or Carbonate is estimated by dissolving in a measured excess of normal nitric acid solution, and then titrating back with normal sodium carbonate or hydroxid solution until a faint milkiness appears, or phenolphthalein which has been added turns red. The quantity of normal alkali solution used is deducted from the quantity of normal acid added, and the remainder multiplied by the factor for the lead salt examined.

$$
\begin{aligned}
& \mathrm{Pb}=0.102675 \mathrm{gm} . ; \\
& \mathrm{PbO}=0.11061 \mathrm{gm} . ; \\
& \mathrm{PbCO}_{3}=0.13245 \mathrm{gm} .
\end{aligned}
$$

Soluble lead salts may be converted into carbonates by adding an excess of ammonium carbonate. The precipitated lead carbonate is then washed with hot water and estimated as above.

Before titrating the nitric acid solution with the standard alkali, Mohr adds enough neutral solution of sodium sulphate to precipitate the lead as sulphate. The excess of nitric acid is then determined by means of the standard alkali, without previous filtration.

By Titration with Standard Dichromate. Lead salts may also be estimated by precipitation with a standard solution of potassium dichromate. The end-point is reached when a further addition of a drop of the dichromate solution fails to produce a precipitate. Beale's filter may here be employed, or a neutral solution of silver nitrate may be used as an indicator, by contact on a porcelain slab. The dichromate solution used for precipitating lead is the same as that used for the estimation of barium containing $7.307 \mathrm{gms}$. in a liter. The reaction is as follows:

$$
{ }_{2} \mathrm{~Pb}\left(\mathrm{C}_{2} \mathrm{H}_{3} \mathrm{O}_{2}\right)_{2}+\mathrm{H}_{2} \mathrm{O}+\mathrm{K}_{2} \mathrm{Cr}_{2} \mathrm{O}_{7}=2 \mathrm{PbCrO}_{4}+{ }_{2} \mathrm{KC}_{2} \mathrm{H}_{3} \mathrm{O}_{2}+{ }_{2} \mathrm{HC}_{2} \mathrm{H}_{3} \mathrm{O}_{2} \text {. }
$$

The lead solution must not contain much free acid. Sufficient sodium acetate is added to saturate the mineral acid and set free an equivalent of acetic acid. 
Each cc. of $\frac{\mathrm{N}}{\text { ro }}$ dichromate $=0.0102675 \mathrm{gm} . \mathrm{Pb}$; $=0.01106 \mathrm{I} \mathrm{gm} . \mathrm{PbO}$.

J. H. Wainwright (J. A. C. S., I897, 389) modifies this method as follows:

One gm. of litharge, for example, is dissolved in to to I 5 cc. of nitric acid (sp.gr. 1.20), the solution is neutralized with ammonia in excess, and a considerable excess of acetic acid is added. The solution is then boiled and the standard potassium dichromate solution run in. When nearly enough of the latter has been used to completely precipitate the lead, the mixture is again boiled until the precipitate of lead chromate has become orange colored. The titration is now continued, one-half cc. or less at a time, stirring after each addition, until the reaction is nearly complete, which is known by the sudden clearing up of the solution, the lead chromate settling promptly to the bottom of the beaker. This will occur if the solution is hot, usually within one cc. of the end of the reaction.

The dichromate should now be added, drop by drop, allowing settling after each addition, and testing by bringing a drop or two of the solution in contact with a drop of silver nitrate solution on a white porcelain tile, until a distinct red color is produced.

The dichromate solution should be made of such strength that one cc. will equal about $0.0 \mathrm{Igm}$. of lead, and should be standardized either by means of pure metallic lead or pure "white lead," in which the metal has been accurately determined gravimetrically.

This method is particularly adapted to the assay of such substances as litharge, white lead, red lead, pig lead, etc., and for lead ores in which the lead exists as carbonate. "White lead" may be dissolved directly in dilute acetic acid and the resulting solution titrated without filtration. In the case of "red lead" solution should be effected by means of nitric acid, boiling, and then adding, drop by drop, a dilute solution of oxalic acid until the lead oxid formed is completely dissolved. If organic matter is present, the solution should be filtered.

The essential points to be observed in order to attain success are:

First. The lead solution should be concentrated and decidedly acid with acetic acid.

Second. Other metals should be absent, especially such as may exist in the lower forms of oxidation; antimony and tin are especially to be avoided, unless they are thoroughly oxidized by repeated evaporation with fuming nitric acid. Bismuth also should be absent.

Third. The solution should be kept as near the boiling-point as possible during the titration. 
Precipitation as Chromate and Digestion of the Precipitate with a Ferrous Salt. If the lead salt is acid, sodium acetate is added in sufficient quantity to saturate the mineral acid and set free an equivalent of acetic acid. The solution is warmed and potassium dichromate is added in sufficient quantity to precipitate all of the lead.

The precipitate is then collected on an asbestos filter and washed thoroughly with warm water; and then together with the asbestos is placed in a flask containing a weighed quantity of ferrous ammonium sulphate and the solution warmed until the chromate is completely decomposed. A current of hydrogen or carbon dioxid is passed through the flask during the reaction (see Fig. 57). The resulting solution contains ferric and chromic sulphate, together with lead sulphate and some unoxidized ferrous salt.

The quantity of the latter is found by $\frac{\mathrm{N}}{\mathrm{IO}}$ permanganate, after acidulation with $\mathrm{H}_{2} \mathrm{SO}_{4}$ (see p. I74), and deducted from the original weight, the remainder multiplied by $0.1757 \mathrm{gm}$. will give the weight of lead. The reactions which occur in this process are:

(a) ${ }_{2} \mathrm{~Pb}\left(\mathrm{NO}_{3}\right)_{2}+\mathrm{K}_{2} \mathrm{Cr}_{2} \mathrm{O}_{7}+\mathrm{H}_{2} \mathrm{O}={ }_{2} \mathrm{PbCrO}_{4}+2 \mathrm{KNO}_{3}+{ }_{2} \mathrm{HNO}_{3}$;

(b) ${ }_{2} \mathrm{PbCrO}_{4}+6 \mathrm{FeSO}_{4} \cdot\left(\mathrm{NH}_{4}\right)_{2} \mathrm{SO}_{4}+6 \mathrm{H}_{2} \mathrm{O}+8 \mathrm{H}_{2} \mathrm{SO}_{4}=\mathrm{Cr}_{2}\left(\mathrm{SO}_{4}\right)_{3}$

$$
+2 \mathrm{PbSO}_{4}+{ }_{3} \mathrm{Fe}_{2}\left(\mathrm{SO}_{4}\right)_{3}+6\left(\mathrm{NH}_{4}\right)_{2} \mathrm{SO}_{4}+6 \mathrm{H}_{2} \mathrm{O} \text {. }
$$

I $168.02 \mathrm{gms}$. of the ferrous-ammonium sulphate $=205.35 \mathrm{gms}$. of $\mathrm{Pb}$.

Thus I $\mathrm{gm}$. of ferrous ammonium sulphate $=0.1757 \mathrm{gm}$. Pb.

I gm. of $\mathrm{Fe}=1.2313 \mathrm{gms}$. of $\mathrm{Pb}$.

By Titrating with Excess of Dichromate and Determining the Excess of the latter by Titration with Standard Solution of Ammonio-ferrous Sulphate. This modification of the foregoing was suggested by A. S. Cushman and J. Hayes-Campbell,* and consists in adding a measured quantity (excess) of standard potassium dichromate solution to the lead solution containing some sodium acetate. The precipitated lead chromate is separated by filtration, the precipitate thoroughly washed with hot water. The mixed filtrate and washings are then titrated with a standardized solution of ammonioferrous sulphate, using a freshly prepared solution of potassium ferricyanid as an outside indicator. The standardization of the iron solution is effected by titrating against a decinormal solution of potassium dichromate. The standard ferrous solution should be preserved under a layer of paraffin oil, in a stock bottle provided with a siphon tube and pinch-cock; this arrangement being used to prevent too rapid oxidation of the standard solution.

* J. A. C. S., XVII, gor. 
The Assay of a Lead Ore by this method is carried out as follows:

"One gm. of finely pulverized ore is digested in a casserole, or evaporating-dish, with ${ }_{5} 5 \mathrm{cc}$. of a mixture of two parts of nitric acid and one part of sulphuric acid until decomposition is complete. Io cc. or more of sulphuric acid are now added, and the liquid evaporated until it fumes freely. Cool, dilute with ro cc. of dilute sulphuric acid (I: IO), and then add gradually $40 \mathrm{cc}$. of water. Heat to boiling, filter, and wash by decantation with dilute sulphuric acid ( $\mathrm{I}: \mathrm{IO}$ ), getting as little of the lead sulphate on the filter as possible. To the residue in the dish add $20 \mathrm{cc}$. of strong ammonia, then make slightly acid with acetic acid. Boil until the lead sulphate is dissolved, then pour the liquid through the filter, having first moistened the paper with ammonia. Wash the filter with water containing ammonium acetate, and finally once or twice with hot water. Cool the filtrate and run in from a burette an excess of standard dichromate solution, stirring until the precipitate settles rapidly and the supernatant liquid has a yellow color. Allow to settle for a few minutes, then filter, under pressure if possible, wash a few times and titrate the filtrate with the standard ammonioferrous sulphate solution."

"In case the ore is known to be free from bismuth and antimony, it may be dissolved in nitric acid alone, and the solution neutralized with an excess of ammonia and then made acid with acetic acid." This solution is then immediately titrated as above. The results by this method are a trifle low.

The Technical Assay of Lead Ores by the Conversion of the Precipitated Chromate into Lead Oxalate, and Titration of the latter by Means of Permanganate. The following method is described by A. H. Low, $*$ who considers it the best for technical work:

Take $0.5 \mathrm{gm}$. of the ore and treat it in a 6-ounce flask by the usual methods to obtain the washed lead sulphate, etc., on a 9-cm. filter.

The lead sulphate on the filter is dissolved by treatment with hot sodium acetate solution. The sodium acetate solution is made by diluting a cold saturated solution of sodium acetate with an equal bulk of water and adding $40 \mathrm{cc}$. of 80 per cent glacial acetic acid per liter.

The completeness of the extraction of the lead sulphate is ascertained by passing a small quantity of the sodium acetate solution through the filter and testing with potassium dichromate. A yellow precipitate indicates incomplete extraction of the lead sulphate. The solution of lead sulphate so obtained is shaken, and heated, if necessary, to redissolve any separated precipitate; and ıo cç of a 5 per cent solution of commercial potassium dichromate are added and the mixture heated

* J. A. C. S., XXX, $5^{87}$ 
to boiling, until the precipitate is basic, which is known by its becoming reddish yellow. Then filter hot, and wash the precipitate only once, simply to clear the upper edge of the filter. The precipitate is then washed off the filter into a flask by means of a jet of hot oxalic acid solution, using 25 to $40 \mathrm{cc}$., and subsequently with hot water. The oxalic acid solution consists of a cold saturated solution of oxalic acid one part and water three parts.

To the mixture in the flask add grain alcohol and boil until all the chromic acid is reduced and the lead converted into oxalate. Then add $30 \mathrm{cc}$. of cold water and cool the solution; filter, wash out the flask, and then wash the precipitate ten times with cold water.

Place $5 \mathrm{cc}$. of strong sulphuric acid in a flask, dilute with hot water to about 125 cc.; add the filter and precipitate, and titrate with standard permanganate to the usual pink tinge.

Neither calcium nor antimony will interfere with this method, but the presence of a large quantity of bismuth will materially raise the result. Most of the bismuth is, however, removed as sulphate and chromate. This method is a good technical one, though an error of 0.01 to 0.05 may be expected.

By Precipitating with Excess of Potassium Chromate and Determining the Excess Iodometrically. The reactions are:

(a) $\mathrm{Pb}\left(\mathrm{NO}_{3}\right)_{2}+\mathrm{K}_{2} \mathrm{CrO}_{4}=\mathrm{PbCrO}_{4}+2 \mathrm{KNO}_{3}$;

(b) ${ }_{2} \mathrm{~K}_{2} \mathrm{CrO}_{4}+6 \mathrm{KI}+8 \mathrm{H}_{2} \mathrm{SO}_{4}=\mathrm{Cr}_{2}\left(\mathrm{SO}_{4}\right)_{3}+{ }_{5} \mathrm{~K}_{2} \mathrm{SO}_{4}+\mathrm{I}_{6}+8 \mathrm{H}_{2} \mathrm{O}$;

(c) $\mathrm{I}_{6}+6\left(\mathrm{Na}_{2} \mathrm{~S}_{2} \mathrm{O}_{3}+{ }_{5} \mathrm{H}_{2} \mathrm{O}\right)={ }_{3} \mathrm{Na}_{2} \mathrm{~S}_{4} \mathrm{O}_{6}+6 \mathrm{NaI}+30 \mathrm{H}_{2} \mathrm{O}$.

The potassium chromate is added in excess, the mixture heated, filtered, the precipitate washed, and then the potassium chromate estimated in the filtrate by adding potassium iodid in excess and titrating for free iodin with sodium thiosulphate in the usual way.

Titration with Standard Sulphate Solution. A better method is the converse of that described for sulphates (p. 333). In this the lead salt in solution is titrated with a decinormal solution of potassium sulphate until precipitation is complete or until a drop of the solution ceases to produce a yellow spot upon paper impregnated with potassium iodid and sodium thiosulphate.

The lead should be in the form of nitrate. If any free nitric acid is present this should be neutralized by means of ammonia water. Before applying a drop of the solution to the test paper it is important to allow the suspended lead sulphate to settle, because this will also react with the potassium iodid. The decinormal factors are the same as those given above. The reaction is as follows:

$$
\mathrm{Pb}\left(\mathrm{NO}_{3}\right)_{2}+\mathrm{K}_{2} \mathrm{SO}_{4}=\mathrm{PbSO}_{4}+2 \mathrm{KNO}_{3} \text {. }
$$


The $\frac{\mathrm{N}}{\mathrm{IO}}$ solution of potassium sulphate is made by dissolving 8.6535 gms. of pure anhydrous potassium sulphate in sufficient distilled water to make one liter.

By Precipitating as Oxalate. Lead may also be estimated by precipitating as oxalate, and then titrating the oxalate with permanganate, or by adding an excess of oxalic acid, and then retitrating with permanganate in an aliquot portion of the supernatant liquid. The lead should be in the form of a soluble salt, such as acetate or nitrate.

The Distillation Method. Lead in the form of peroxid $\left(\mathrm{PbO}_{2}\right)$ may be assayed by the distillation method described on page 2 I4.

A weighed quantity of the lead peroxid is boiled with concentrated hydrochloric acid, and the chlorin set free is conveyed into a solution of potassium iodid, from which it liberates iodin. The iodin is then titrated with sodium thiosulphate in the usual way. 


\section{CHAPTER XXXVI}

\section{MAGNESIUM}

Most magnesium compounds may be converted into the sulphate by evaporating, treating with concentrated sulphuric acid, evaporating to dryness and heating to dull redness to drive off the excess of acid. The heat must not be raised higher than dull redness, otherwise some of the sulphate is apt to be decomposed. Dissolve the residue in water, add a few drops of hydrochloric acid, and determine the sulphuric acid by means of $\frac{\mathrm{N}}{10}$ barium chlorid.

Each cc. of $\frac{\mathrm{N}}{\mathrm{IO}}$ barium chlorid $=0.001209 \mathrm{gm} . \mathrm{Mg}$;

$$
\begin{array}{lll}
=0.0020 & \text { “ } & \mathrm{MgO} ; \\
=0.004 \mathrm{I} 86 & \text { “ } & \mathrm{MgCO}_{3} ; \\
=0.005967 & \text { “ } & \mathrm{MgSO}_{4} .
\end{array}
$$

Estimation as Phosphate (Stolba). Magnesium salts may be precipitated as ammonio-magnesium phosphate and the precipitate then titrated with $\frac{\mathrm{N}}{10}$ hydrochloric acid, or with uranium solution as directed under estimation of phosphoric acid.

The magnesium in the form of a soluble salt is dissolved in a small quantity of water, at least twice the quantity of ammonium chlorid is added, and then ammonia water to make strongly alkaline. Then sodium phosphate solution is added in excess, and the mixture allowed to stand twelve hours. The magnesium is thus completely precipitated as ammonio-magnesium phosphate.

This precipitate is separated by filtration and washed, first with a mixture of water, three parts, and ammonia water, one part, and then with 50 or 60 per cent alcohol to remove the ammonia.

The precipitate is then dissolved in a measured excess of $\frac{\mathrm{N}}{10}$ hydrochloric acid, a few drops of methyl orange added, and the excess of acid found by retitrating with $\frac{\mathrm{N}}{10}$ potassium hydroxid. The difference 
between the quantities of acid and alkali solutions used is the quantity of the fcrmer which reacted with the ammonio-magnesium phosphate.

Each cc. of $\frac{\mathrm{N}}{\mathrm{IO}}$ hydrochloric acid $=0.001209 \mathrm{gm} . \mathrm{Mg}$;

$$
=0.0020 \text { " " } \mathrm{MgO} \text {. }
$$

Or, the precipitate of ammonio-magnesium phosphate may be dissolved in acetic acid and estimated by uranium solution, as directed under Phosphoric Acid.

Each cc. of uranium solution $=0.001695 \mathrm{Mg}$;

$$
=0,0028 \mathrm{I} 7 \mathrm{MgO} \text {. }
$$

Stolba's method gives fairly accurate results in the assaying of cements, limestone, and with soluble magnesium salts generally. Its objectionable feature lies in the difficulty encountered and the time consumed in the removal of the ammonia by alcohol.

Meade's Method (J. A. C. S., I899, 746) has the great advantage over the foregoing in that it can be worked much more rapidly.

It consists essentially in using sodium arsenate instead of sodium phosphate. The precipitate obtained is then magnesium-ammonium arsenate $\left(\mathrm{Mg}_{2}\left(\mathrm{NH}_{4}\right)_{2} \mathrm{As}_{2} \mathrm{O}_{8}+\mathrm{H}_{2} \mathrm{O}\right)$ instead of magnesium-ammonium phosphate. The arsenic in the precipitate is then estimated by Williamson's process (J. Soc. Dyers and Colorists, May, I896), which depends upon the reaction between arsenic oxid and potassium iodid in the presence of hydrochloric or sulphuric acid. The arsenic is reduced as per the equation, and an equivalent of iodin is set free.

$$
\mathrm{As}_{2} \mathrm{O}_{5}+{ }_{4} \mathrm{KI}+{ }_{4} \mathrm{HCl}=\mathrm{As}_{2} \mathrm{O}_{3}+{ }_{4} \mathrm{KCl}+{ }_{2} \mathrm{H}_{2} \mathrm{O}+{ }_{2} \mathrm{I}_{2} \text {. }
$$

For every molecule of arsenic oxid reduced, corresponding to two atoms of magnesium, four atoms of iodin are liberated. The latter is then titrated with standard sodium thiosulphate.

The Standard Sodium Arsenate solution is made by dissolving 12.29 gms. of pure arsenous oxid in nitric acid, evaporating to dryness on a water-bath, neutralizing with sodium carbonate, and making up to $1000 \mathrm{cc}$. with water. Each cc. of this solution is equivalent to $0.005 \mathrm{gm}$. of Mg.

The Standard Sodium Thiosulphate solution is made to correspond with the above by direct titration, or made to correspond with a standard iodin solution containing 52.24 gms. of pure resublimed iodin and $75 \mathrm{gms}$. of potassium iodid in a liter. Each cc. $=0.005 \mathrm{gm}$. $\mathrm{Mg}$.

The Process. Pour the magnesium solution, which should not contain too great an excess of ammonium chlorid or oxalate, into a large Erlenmeyer flask or gas-bottle of sufficient size. A'dd one third the volume of the solution of strong ammonia and $50 \mathrm{cc}$. of sodium 
arsenate. Cork up tightly and shake vigorously for ten minutes. Allow the precipitate to settle somewhat, and wash with a mixture of three parts of water and one part of strong ammonia until the washings cease to react for arsenic. Avoid using an excess of the washing fluid, however. Dissolve the precipitate in dilute hydrochloric acid (I:I), allowing the acid solution to run into the flask in which the precipitation was made, and wash the filter-paper with the dilute acid until the washings and solution measure 75 or $100 \mathrm{cc}$. Cool, if not already so, and add from 3 to $5 \mathrm{gms}$. of potassium iodid free from iodate. Allow the solution to stand a few minutes, and then run in the standard sodium thiosulphate until the color of the liberated iodin fades to a pale straw color. Add starch solution and titrate until the blue is discharged. If preferred, an excess of the thiosulphate may be added, then starch and standard iodin until the blue color is produced. On adding the iodid of potassium to the acid solution, a brown precipitate forms, which, however, dissolves when the thiosulphate is added.

G. B. Frankforter and Lillian Cohn (J. A. C. S., I907, p. I464) avoid the difficulty usually experienced with the starch indicator in the presence of strong hydrochloric acid by omitting its employment altngether. They assert that a much sharper end-point can be obtained if the starch indicator is not used. They employ the following modification for the determination of magnesium in water.

Measure out $500 \mathrm{cc}$. of the water to be examined, precipitate out the iron group and the calcium by the ordinary methods. Acidify and evaporate until the salts start to crystallize out. Make up to Ioo cc., transfer to an Erlenmeyer flask, add one third of the volume of concentrated ammonia and $25 \mathrm{cc}$. of a Io per cent solution of sodium arsenate, cork, and shake vigorously for ten minutes. After the precipitate has settled, filter and wash with dilute ammonia until the washings are free from arsenic, using as small a quantity of ammonia as possible. Now add ro cc. of sulphuric acid ( $1: 4)$, and allow it to run into the flask in which the precipitation was made. Wash the filter-paper with hot water until the total solution measures about roo cc. Now add ro cc. of sulphuric acid_ ( I: I), cool, and add $3.5 \mathrm{gms}$. of pure potassium iodid. Allow the solution to stand for five minutes and titrate with thiosulphate to a pale straw color. Then add the thiosulphate cautiously, drop by drop, until the yellow solution becomes colorless.

The commercial magnesium carbonate and magnesium oxid may be examined readily, by dissolving a weighed quantity (recently ignited and cooled) in a measured excess of normal sulphuric acid, and then retitrating with normal alkali in the presence of methyl orange as indicator. 


\section{CHAPTER XXXVII}

\section{MANGANESE}

\section{Estimation of the Available Oxygen in Manganese Ores.} The oxids of manganese are as follows: $\mathrm{MnO}, \mathrm{Mn}_{3} \mathrm{O}_{4}, \mathrm{Mn}_{2} \mathrm{O}_{3}, \mathrm{MnO}_{2}$; $\mathrm{MnO}_{3}$ (anhydrid of $\mathrm{H}_{2} \mathrm{MnO}_{4}$ ); and $\mathrm{Mn}_{2} \mathrm{O}_{7}$ (anhydrid of $\mathrm{H}_{2} \mathrm{Mn}_{2} \mathrm{O}_{8}$ or ${ }_{2} \mathrm{HMnO}_{4}$ ).

With the exception of the first or protoxid all the others will, when distilled with hydrochloric acid, cause a liberation of chlorin. The amount of chlorin liberated is proportional to the available oxygen, i.e., the excess of oxygen over that necessary to form protoxid. This is shown in the following equations:

$$
\begin{aligned}
\mathrm{Mn}_{3} \mathrm{O}_{4}+8 \mathrm{HCl} & ={ }_{3} \mathrm{MnCl}_{2}+{ }_{4} \mathrm{H}_{2} \mathrm{O}+{ }_{2} \mathrm{Cl} \\
\mathrm{Mn}_{2} \mathrm{O}_{3}+6 \mathrm{HCl} & =2 \mathrm{MnCl}_{2}+{ }_{3} \mathrm{H}_{2} \mathrm{O}+{ }_{2} \mathrm{Cl} \\
\mathrm{MnO}_{2}+{ }_{4} \mathrm{HCl} & =\mathrm{MnCl}_{2}+{ }_{2} \mathrm{H}_{2} \mathrm{O}+{ }_{2} \mathrm{Cl} ; \\
\mathrm{K}_{2} \mathrm{MnO}_{4}+8 \mathrm{HCl} & =2 \mathrm{KCl}+\mathrm{MnCl}_{2}+{ }_{4} \mathrm{H}_{2} \mathrm{O}+{ }_{4} \mathrm{Cl} ; \\
2 \mathrm{KMnO}_{4}+{ }_{1} 6 \mathrm{HCl} & =2 \mathrm{KCl}+2 \mathrm{MnCl}_{2}+8 \mathrm{H}_{2} \mathrm{O}+\mathrm{IOCl} .
\end{aligned}
$$

The assay may be carried out in several ways: $(a)$ The chlorin evolved may be conducted by means of a suitable apparatus into a solution of potassium iodid from which an equivalent of iodin is set free. The amount of the latter is then found by titration with sodium thiosulphate. (See page 2 18 .)

(b) The chlorin may be allowed to react with a known weight of ferrous salt, and the amount of iron salt which at the completion of the reaction is unchanged is found by titration with standard permanganate. Thus the amount of manganese oxid, of known composition or of available oxygen, is calculated from the quantity of ferrous salt oxidized.

(c) The chlorin may be passed into a solution of sodium carbonate and this solution titrated with standard arsenous acid solution, or the chlorin may be distilled directly into a measured volume of $\frac{\mathrm{N}}{\mathrm{IO}}$ arsenous acid, and the latter then retitrated with $\frac{\mathrm{N}}{\mathrm{IO}}$ iodin solution. (See page 230.) 
If sulphuric acid is used instead of hydrochloric acid, the available oxygen of the manganese oxid will be evolved instead of its equivalent of chlorin. See equations:

$$
\begin{aligned}
\mathrm{Mn}_{3} \mathrm{O}_{4}+{ }_{3} \mathrm{H}_{2} \mathrm{SO}_{4} & =3 \mathrm{MnSO}_{4}+{ }_{3} \mathrm{H}_{2} \mathrm{O}+\mathrm{O} ; \\
\mathrm{Mn}_{2} \mathrm{O}_{3}+{ }_{2} \mathrm{H}_{2} \mathrm{SO}_{4} & =2 \mathrm{MnSO}_{4}+{ }_{2} \mathrm{H}_{2} \mathrm{O}+\mathrm{O} ; \\
\mathrm{MnO}_{2}+\mathrm{H}_{2} \mathrm{SO}_{4} & =\mathrm{MnSO}_{4}+\mathrm{H}_{2} \mathrm{O}+\mathrm{O} .
\end{aligned}
$$

This reaction may be made use of for determining the available oxıgen of manganese ores in two ways:

First. By causing the evolved oxygen to oxidize a ferrous salt, and from the quantity of ferrous salt so oxidized, the amount of available oxygen is obtained. The reactions between the higher oxids of manganese and ferrous salts are illustrated by the following equations:

$$
\begin{aligned}
\mathrm{Mn}_{3} \mathrm{O}_{4}+{ }_{2} \mathrm{FeSO}_{4}+{ }_{4} \mathrm{H}_{2} \mathrm{SO}_{4} & =3 \mathrm{MnSO}_{4}+\mathrm{Fe}_{2}\left(\mathrm{SO}_{4}\right)_{3}+{ }_{4} \mathrm{H}_{2} \mathrm{O} \\
\mathrm{Mn}_{2} \mathrm{O}_{3}+{ }_{2} \mathrm{FeSO}_{4}+{ }_{3} \mathrm{H}_{2} \mathrm{SO}_{4} & =2 \mathrm{MnSO}_{4}+\mathrm{Fe}_{2}\left(\mathrm{SO}_{4}\right)_{3}+{ }_{3} \mathrm{H}_{2} \mathrm{O} \\
\mathrm{MnO}_{2}+{ }_{2} \mathrm{FeSO}_{4}+{ }_{2} \mathrm{H}_{2} \mathrm{SO}_{4} & =\mathrm{MnSO}_{4}+\mathrm{Fe}_{2}\left(\mathrm{SO}_{4}\right)_{3}+{ }_{2} \mathrm{H}_{2} \mathrm{O} \\
\mathrm{K}_{2} \mathrm{MnO}_{4}+{ }_{4} \mathrm{FeSO}_{4}+{ }_{4} \mathrm{H}_{2} \mathrm{SO}_{4} & =\mathrm{K}_{2} \mathrm{SO}_{4}+\mathrm{MnSO}_{4}+{ }_{2} \mathrm{Fe}_{2}\left(\mathrm{SO}_{4}\right)_{3}+{ }_{4} \mathrm{H}_{2} \mathrm{O}
\end{aligned}
$$

Second. The oxids of manganese (with the exception of $\mathrm{MnO}$ ), when digested with oxalic acid in the presence of sulphuric acid, convert the oxalic acid into carbon dioxid and water. If a known weight of oxalic acid (in excess) be taken, the amount of the latter remaining unchanged after the reaction is found by titration with permanganate, and the difference between this amount and that originally added is the measure of the available oxygen in the manganese ore. The reactions are:

$$
\begin{aligned}
\mathrm{Mn}_{3} \mathrm{O}_{4}+\mathrm{H}_{2} \mathrm{C}_{2} \mathrm{O}_{4}+{ }_{3} \mathrm{H}_{2} \mathrm{SO}_{4} & =3 \mathrm{MnSO}_{4}+{ }_{2} \mathrm{CO}_{2}+{ }_{4} \mathrm{H}_{2} \mathrm{O} \\
\mathrm{Mn}_{2} \mathrm{O}_{3}+\mathrm{H}_{2} \mathrm{C}_{2} \mathrm{O}_{4}+{ }_{2} \mathrm{H}_{2} \mathrm{SO}_{4} & =2 \mathrm{MnSO}_{4}+{ }_{2} \mathrm{CO}_{2}+{ }_{3} \mathrm{H}_{2} \mathrm{O} \\
\mathrm{MnO}_{2}+\mathrm{H}_{2} \mathrm{C}_{2} \mathrm{O}_{4}+\mathrm{H}_{2} \mathrm{SO}_{4} & =\mathrm{MnSO}_{4}+{ }_{2} \mathrm{CO}_{2}+{ }_{2} \mathrm{H}_{2} \mathrm{O} \\
\mathrm{K}_{2} \mathrm{MnO}_{4}+\mathrm{H}_{2} \mathrm{C}_{2} \mathrm{O}_{4}+{ }_{2} \mathrm{H}_{2} \mathrm{SO}_{4} & =\mathrm{MnSO}_{4}+\mathrm{K}_{2} \mathrm{SO}_{4}+{ }_{4} \mathrm{CO}_{2}+{ }_{4} \mathrm{H}_{2} \mathrm{O} .
\end{aligned}
$$

Example of First Method. By Oxidation of Ferrous Salt. I gin. of soft iron wire is weighed out and dissolved in about $40 \mathrm{cc}$. of dilute sulphuric acid $(\mathrm{r}: 4)$ in the apparatus, shown in Fig. 55, or that shown in Fig. 56. When the iron is completely dissolved $0.8 \mathrm{gm}$. of finely powdered manganese ore (previously dried at $100^{\circ} \mathrm{C}$.) is introduced into the flask and the cork with its tube replaced. The mixture is then gently warmed until the ore is completely dissolved. The solu- 
tion is then cooled, diluted with recently boiled water, and the excess of ferrous sulphate found by titration with permanganate. The excess of unoxidized iron thus found deducted from the weight of iron taken gives the quantity of iron which reacted with and was oxidized by the manganese dioxid, and from this the quantity of the latter, or of available oxygen, can easily be calculated. Thus $14.2 \mathrm{cc}$. of $\frac{\mathrm{N}}{\mathrm{IO}}$ permanganate (I cc. $=0.00555 \mathrm{gm}$. Fe) are consumed.

$$
0.00555 \times 14.2=0.0788 \mathrm{r}^{*} \mathrm{gm} .=\text { excess of iron } .
$$

I gm. of iron (99.6 per cent of $\mathrm{Fe})=0.996 \mathrm{gm}$., the quantity of iron origirally taken.

Therefore $0.996-0.0788 \mathrm{I}=0.9 \mathrm{I} 7 \mathrm{I} 9 \mathrm{gm}$. of iron oxidized by the $0.8 \mathrm{gm}$. of manganese oxid.

Then $55 \cdot 5: 0.91719:: 43.18: x ; x=0.7 \mathrm{I} 2+$ gm. of $\mathrm{MnO}_{2}$ or 89 per cent.

Example of Second Method. By the Oxidation of Oxalic Acid (Mohr). $0.5 \mathrm{gm}$. of the finely pulverized and dried manganese ore is weighed into a flask and ro cc. of normal oxalic acid solution added. $25 \mathrm{cc}$. of dilute sulphuric acid $(\mathrm{r}: 4)$ are then added and the mixture gently heated until solution is complete. The solution is then cooled and made up to $100 \mathrm{cc}$. with cold water, and the excess of oxalic acid found by titration with decinormal potassium permanganate.

Assuming that 6.I cc. of the permanganate were employed in titrating $25 \mathrm{cc}$. of the above solution, then the whole will require $24.4 \mathrm{cc}$. of the permanganate solution. The ro cc. of normal oxalic acid originally used are equivalent to $100 \mathrm{cc}$. of decinormal oxalic acid and the same amount of decinormal permanganate. Therefore by deducting $24.4 \mathrm{cc}$. from roo cc. we obtain the quantity of decinormal acid which was oxidized, i.e., $75.6 \mathrm{cc}$.

Each cc. of decinormal oxalic acid $=0.004318 \mathrm{gm}$. of $\mathrm{MnO}_{2}$;

$$
=0.000794 \text { " of available } \mathrm{O} \text {. }
$$

Thus 75.6×0.004318 gm. $=0.3264408 \mathrm{gm}$. of $\mathrm{MnO}_{2}$;

$75.6 \times 0.000794 \mathrm{gm} .=0.0600264 \mathrm{gm}$. of $\mathrm{O}$ (available).

The Distillation Method, described on page 2I4, is undoubtedly the quickest and most accurate method for determining the quantity of available oxygen present in the ore, which to manufacturers of bleaching powder, is a matter of considerable moment, inasmuch as this method directly expresses the quantity of chlorin which is evolved by 
treating the ore with hydrochloric acid. It furthermore enables them to cstimate the quantity of hydrochloric acid which any particular sample of ore may require for its complete decomposition. (See Scherer and Rumpf, Chem. News, xx, 302, Am. Reprint, vI, I870, page 82).

With regard to the method of Mohr, involving the oxidation of oxalic acid, it is generally conceded that this method has advantages over the iron method. There is no fear of false results occurring from the presence of air, and it requires only one weighing for each test.

Furthermore, the results are very uniform, according to $\mathrm{B}$. $\mathrm{H}$. Paul (Chem. News, XxI, I6, Am. Reprint, vi, I45 (I870)). The method has also the advantage of giving results which fairly represent the amount of available oxygen in manganese ores; for any iron that may be present as metal or protoxid will consume an equivalent quantity of permanganate solution, and thus apparently reduce the quantity of oxalic acid decomposed by the dioxid to an extent proportionate to the quantity of iron existing in the ore. Thus, for instance, if the quantity of oxalic acid decomposed by 100 grains of manganese ore free from iron or protoxid of iron were 109.53 grains, the ore would contain 76.5 per cent dioxid, and the whole of that would be available. But, if the roo grains of ore also contain 5.6 grains of metallic iron, or an equivalent of protoxid, the permanganate required for peroxidizing that iron would represent 6.3 grains of oxalic acid, and the quantity of oxalic acid decomposed by the dioxid would appear so much less than it really was, or 103.23 grains instead of 109.53 grains. Accordingly, the amount of dioxid would be represented as 72.1 per cent instead of 76.5 per cent, and the latter would in fact be the amount of dioxid available for generating chlorin.

Manganese Dioxid may also be cstimated by the following method:

$0.2 \mathrm{gm}$. of the substance is placed in a $250-\mathrm{cc}$. flask, an excess of potassium iodid solution added, and then strongly acidulated with hydrochloric acid. The flask is stoppered, and allowed to stand until the manganese dioxid is completely dissolved. The solution is then diluted with water to the $250-\mathrm{cc}$. mark, and $25 \mathrm{cc}$. of it taken out and titrated with centinormal sodium thiosulphate, using starch solution as the indicator. The method depends upon the fact that $\mathrm{MnO}_{2}$, when treated with $\mathrm{HCl}$, gives off two atoms of chlorin for each molecule of $\mathrm{MnO}_{2}$, and in the presence of an iodid the chlorin liberates an equivalent of iodin. Hence two atoms of iodin represent one molecule of $\mathrm{MnO}_{2}$. The reaction is thus expressed:

$$
\mathrm{MnO}_{2}+{ }_{4} \mathrm{HCl}+{ }_{2} \mathrm{KI}=\mathrm{MnCl}_{2}+2 \mathrm{KCl}+\mathrm{I}_{2}+2 \mathrm{H}_{2} \mathrm{O} \text {. }
$$


Thus each cc. of $\frac{\mathrm{N}}{\mathrm{I00}}$ sodium thiosulphate represents $0.0012590 \mathrm{gm}$. of iodin, which is equivalent to $0.004318 \mathrm{gm}$. of manganese dioxid.

Direct Titration with Permanganate (Guyard).* A dilute, neutral, or faintly acid solution of manganese salt is heated to $80^{\circ} \mathrm{C}$., and $\frac{\mathrm{N}}{\mathrm{IO}}$ potassium permanganate solution added, so long as a brownish red precipitate of hydrated $\mathrm{MnO}_{2}$ forms and until the occurrence of the characteristic rose color of permanganate. In neutral solution the reaction is exact; but a large excess of hydrochloric or sulphuric acid causes irregularity. Iron and chromium must also be absent. The manganese compound may be dissolved in nitro-muriatic acid, boiling if necessary, and the excess of acid neutralized. The reaction is written thus:

$$
3 \mathrm{MnSO}_{4}+{ }_{2} \mathrm{KMnO}_{4}+{ }_{2} \mathrm{H}_{2} \mathrm{O}=\mathrm{K}_{2} \mathrm{SO}_{4}+{ }_{5} \mathrm{MnO}_{2}+{ }_{2} \mathrm{H}_{2} \mathrm{SO}_{4} ;
$$

I cc. of $\frac{\mathrm{N}}{10}$ permanganate $=0.001638 \mathrm{gm}$. of $\mathrm{Mn}$.

Example. One or two grams of the substance to be examined is dissolved in nitro-hydrochloric acid and the mixture boiled for some time in order to convert all of the manganese into chlorid. The solution is nearly neutralized with sodium hydroxid, diluted with boiling water to I or 2 liters, and maintained at a temperature of $80^{\circ} \mathrm{C}$., whilst titrating with standard potassium permanganate.

Volhard's Permanganate Method. This depends upon the fact that when a faintly acid, hot solution of a manganous salt is treated with potassium permanganate, a precipitation of hydrated manganese dioxid results, the permanganate solution being decolorized so long as any manganous salt is present. The reaction is as in the foregoing method, and takes place according to Volhard only in the presence of other non-oxidizable, highly basic metals, as for instance zinc oxid or a zinc salt.

The end-reaction is most distinctly observed when working in a solution slightly acidified with sulphuric acid, together with a few drops of nitric acid. Organic matter must be absent, as must also quantities of chlorid over $0.5 \mathrm{gm}$. per liter. Large quantities of iron, except in the ferric state, must be likewise absent. Excessive quantities of iron, if present, are best removed by the addition of zinc oxid, whereby the former is precipitated as hydroxid, the manganese remaining in solution.

The permanganate solution used in this titration is standardized 
against a solution of manganic sulphate. The zinc oxid or zinc sulphate used should not have a reducing action upon the permanganate. The method of carrying out this process is determined by the presence or absence of iron.

If iron is absent (or present in very small amounts only), the manganese solution in the form of manganese sulphate is introduced into a flask, together with $10 \mathrm{cc}$. of a zinc sulphate solution (100 gms. crystallized zinc sulphate to a liter), and the mixture diluted so that it will contain not more than $0.25 \mathrm{gm}$. of manganese in roo cc. This, if neutral, is acidified by the addition of a few drops of concentrated nitric acid and heated to boiling. The heat is then withdrawn and the solution titrated at first rapidly, then slowly and carefully, with the permanganate until the characteristic pinkish tint is obtained. The brownish precipitate of manganese dioxid will to some extent interfere with an accurate observation of the end-point, but if the flask in which the titration is performed is rotated throughout the process, the precipitate settles as a rule quickly enough to enable the operator to distinguish clearly the end color.

If larger quantities of iron are present, as, for instance, in spiegeleisen ferro-manganese, and other ores. A quantity of the substance (= to about $0.3 \mathrm{gm}$. of $\mathrm{Mn}$ ) is dissolved in nitric acid, the solution evaporated to dryness in a porcelain dish, the residue heated until the nitrate is completely decomposed, thereby destroying any organic matter present. The residual oxids are then digested with hydrochloric acid, adding a little strong sulphuric acid and evaporating to dryness, The resulting sulphate is then slightly acidified with nitric acid, dissolved in warm water, then washed into a liter flask, and nearly neutralized with sodium carbonate or hydroxid. Sufficiont pure zinc oxid, made into a cream, is now added to precipitate all of the iron. The liquid above the precipitate should be milky white. The flask is then filled to the mark with water, shaken, and $200 \mathrm{cc}$. filtered off into a boiling flask, acidified with two drops of nitric acid (sp.gr. I.2), boiled, and titrated with the permanganate whilst still hot.

The permanganate method is rapid and simple, nevertheless it is $v$ sry generally ignored, because of the fact that under certain condiditions inconsistent results are obtained. The sources of error are: (a) Incomplete destruction of the organic matter present; $(b)$ excessive addition of zinc oxid and in hot solution; $(c)$ standardizing the permanganate with iron instead of with manganese.

By Reduction of Potassium Ferricyanid (Lenssen).* This method is based upon the fact that when a manganous salt in solu-

* Journ. f. prakt. Chem., LXXX, 408. 
tion, mixed with one equivalent of a ferric salt for every equivalent of $\mathrm{MnO}$, is acted upon at a boiling temperature by excess of an alkaline solution of potassium ferricyanid; all of the manganese is precipitated as $\mathrm{MnO}_{2}$ and a corresponding quantity of potassium ferrocyanid is formed, as per the equation:

$$
{ }_{2} \mathrm{~K}_{3} \mathrm{Fe}(\mathrm{CN})_{6}+4 \mathrm{KOH}+\mathrm{MnSO}_{4}={ }_{2} \mathrm{~K}_{4} \mathrm{Fe}(\mathrm{CN})_{6}+\mathrm{K}_{2} \mathrm{SO}_{4}+\mathrm{MnO}_{2}+{ }_{2} \mathrm{H}_{2} \mathrm{O} \text {. }
$$

The ferrocyanid formed is then measured by means of standard potassium permanganate, as explained on page 278 , and the amount of manganese calculated from this.

According to the above equation, one atomic weight of manganese gives rise to two molecular weights of potassium ferrocyanid. The manganese must be present in the form of a manganous salt, and all other reducing agents must of course be absent. If no ferric salt is present in the solution the precipitate will consist of $\mathrm{MnO}_{2}$ and varying proportions of $\mathrm{MnO}$, and the results will hence be inaccurate.

The Process. The manganous salt is dissolved in water or acid, and enough ferric chlorid solution added to make certain of having at least one molecule of $\mathrm{Fe}_{2} \mathrm{Cl}_{6}$ to one atom of $\mathrm{Mn}$. This is added gradually to a boiling strongly alkaline solution of potassium ferricyanid contained in a flask. After boiling the mixture for a few minutes the brownish-black precipitate becomes granular and less bulky. It is then allowed to cool completely, and is poured into a half-liter flask, diluted to the mark with water, shaken, and the precipitate allowed to settle (or it may be filtered before diluting and the precipitate washed with water, and the filtrate after acidulation with sulphuric or hydrochloric acid titrated with permanganate). An aliquot portion of the clear solution is removed by means of a pipette, acidified with sulphuric acid, and the amount of ferrocyanid contained in it determined by titration with standard permanganate. A small error is introduced here from neglecting the volume of the precipitate. It is therefore a better plan to filter, wash the precipitate, and titrate an aliquot portion of the mixed filtrate and washings. The filtration should, however, be done only when the solution is completely cooled, for if the liquid is filtered hot, the filter will have a reducing action, and too high results will be obtained. Fresenius suggests certain precautions which must be observed:

(a) The solution of potassium ferricyanid and potassium hydroxid must not be boiled too long, as this causes a small amount of ferricyanid to be reduced to ferrocyanid.

(b) The potassium hydroxid used must be quite free from organic matter, and if there be any doubt upon this point, it should be fused in a silver dish before being used. 
(c) The precipitate of $\mathrm{MnO}_{2}$ must be thoroughly washed.

(d) The ferric salt should not be in large excess, otherwise inaccuracies result.

$$
\text { I cc. of } \begin{aligned}
\frac{\mathrm{N}}{10} \text { permanganate } & =0.00273 \mathrm{gm} \text {. of } \mathrm{Mn} \text {; } \\
& =0.0035^{24} \text { “" “" } \mathrm{MnO} ; \\
& =0.0043 \mathrm{I} 8 \text { “" } \mathrm{MnO}_{2} .
\end{aligned}
$$

The Bismuthate Method (Blair).* This method, as originally proposed by Schneider, depends upon oxidizing a manganous salt to permanganic acid by means of bismuth tetroxid in the presence of an excess of nitric acid. The permanganic acid formed is very stable in nitric acid of sp.gr. 1.r35, when the solution is cold. But in hot solutions the excess of bismuth tetroxid is rapidly decomposed and nitric then acts upon and decomposes the permanganic acid.

In the cold, however, the excess of bismuth salt may be filtered off, and to the clear liquid an excess of ferrous salt added and the amount of the latter necessary to oxidize the permanganic acid, determined by titration with potassium permanganate.

The method is accurate, except in the presence of even traces of hydrochloric acid. Reddrop and Ramage first suggested the use of sodium bismuthate instead of bismuth tetroxid, because the latter is difficult to obtain free from chlorids. Sodium bismuthate may be prepared as follows: Heat twenty parts of caustic soda nearly to redness in an iron or nickel crucible and add, in small quantities at a time, ten parts of pure, dried basic bismuth nitrate. Then add two parts of sodium peroxid, and pour the brownish-yellow fused mass on an iron plate to cool; then break up in a mortar, extract with water, and collect on an asbestos filter. The residue, after thorough washing by decantation, is dried in a water-oven, then broken up, and passed through a fine sieve.

The Method for Steels. Dissolve I gm. of drillings in $50 \mathrm{cc}$. of nitric acid (sp.gr. 1.135) in an Erlenmeyer flask of 200-cc. capacity. Cool, and add about $0.5 \mathrm{gm}$. of bismuthate. Heat for a few minutes, or until the pink color has disappeared, with or without the precipitation of manganese dioxid. Add sulphurous acid, ferrous sulphate solution or sodium thiosulphate in sufficient amount to clear the solution and heat until all nitrous oxid has been driven off. Cool to about $15^{\circ} \mathrm{C}$., add an excess of bismuthate and agitate for a few minutes; add $50 \mathrm{cc}$. of water containing $30 \mathrm{cc}$. nitric acid to the liter, and filter through an asbestos felt on a platinum cone into a 300-cc. Erlenmeyer flask, and wash with $50 \mathrm{cc}$. to $100 \mathrm{cc}$. of the same acid. The arrangement 
shown in Fig. 78 has proved very satisfactory. Run into the flask from the pipette, shown in Fig. 79, a measured volume of ferrous sulphate solution and titrate to a faint pink color with permanganate. The number of $\mathrm{cc}$. of the permanganate solut ion obtained, subtracted from the number corresponding to the volume of ferrous sulphate used, will give the volume of permanganate equivalent to the manganese in the sample, which, multiplied by the value of the permanganate in manganese, gives the amount of manganese in the steel.

Pig Iron. Dissolve I gm. in 25 cc. of nitric acid (sp.gr. I.I35) in a small beaker, and as soon as the action has ceased filter into a $200-\mathrm{cc}$. Erlenmeyer flask, wash with $30 \mathrm{cc}$. of the same acid, and proceed as for steel.

Iron Ores Containing Less than 2 Per Cent Manganese. Treat I gm. in a platinum dish with 4 cc. of strong sulphuric acid, io cc.

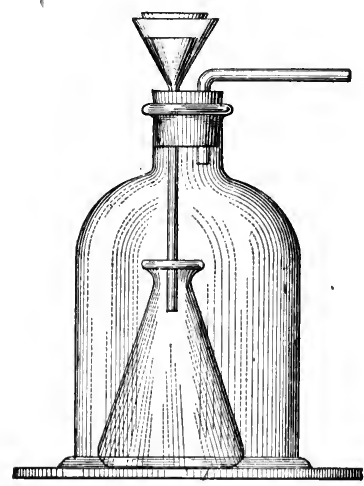

FIG. 78 .

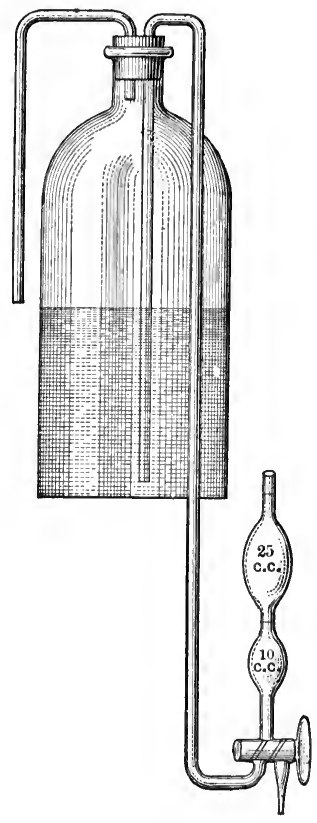

FIG. 79.

of water and ro to $20 \mathrm{cc}$. of hydrofluoric acid. Evaporate until the sulphuric acid fumes freely. Cool, and dissolve in 25 cc. of I.I 35 nitric acid. If no appreciable residue remains, transfer to a $200-\mathrm{cc}$. flask, using 25 cc. of I.I 35 nitric acid to rinse the dish and proceed as usual. If there is an appreciable residue, filter on a small filter into a beaker, wash with water, burn the filter and residue, and fuse with a small amount of potassium bisulphate; dissolve in water with the addition of a little nitric acid, add to main filtrate, evaporate nearly to dryness, take up in I.I35 nitric acid and transfer to the flask as before. 
Manganese Ores and Iron Ores High in Manganese. Treat I $\mathrm{gm}$. as in the case of iron ores, using a little sulphurous acid if necessary. Transfer the solution to a 500-cc. flask, dilute to the mark, mix thoroughly, and measure into a flask from a pipette such a volume of the solution as will give from $I$ to 2 per cent of manganese and enough strong nitric acid (sp.gr. 1.4) to yield a mixture of I.I35 acid in a volume of $5^{\circ}$ to $60 \mathrm{cc}$. For example, in a 50 per cent ore use io cc. of the solution and add $30 \mathrm{cc}$. of water and Io cc. of nitric acid (I.4). In this case the manganese must be calculated on $\delta_{50}^{1}$ of a gram, or $20 \mathrm{mg}$. of ore. When the ore contains a much smaller amount of manganese, say 5 or ro per cent, it is better to make up the solution to say $100 \mathrm{cc}$. instead of $500 \mathrm{cc}$.

Reagents. Nitric Acid (sp.gr. I.I35). A mixture of three parts of water and one part of strong nitric acid will answer.

Nitric Acid (3 per cent). 30 cc. of strong nitric acid to the liter.

Permanganate Solution and Ferrous Sulphate Solution. I gm. of potassium permanganate to the liter gives a solution of convenient strength, and $12.4 \mathrm{gms}$. of ferrous ammonium sulphate and $50 \mathrm{cc}$. of sulphuric acid made up to the liter, gives a solution which is almost equal to the permanganate solution. The strength of the ferrous sulphate solution changes quite rapidly, while the permanganate remains unaltered for months.

By using a constant volume of the ferrous sulphate solution and testing it against the permanganate solution every day the calculation of the result is very simple. It is necessary that the conditions should be the same in testing the ferrous sulphate solution as in titrating a solution of manganese. The following method of procedure may be adopted: Measure into a 200-cc. flask 50 cc. of nitric acid (r.135); cool, and add a small amount of bismuthate, dilute with $50 \mathrm{cc}$. of 3 per cent nitric acid; filter into a $300-\mathrm{cc}$. flask, and wash with $50 \mathrm{cc}$. of 3 per cent nitric acid. If the felt is well coated with bismuthate, it is unnecessary to add any to the nitric acid in the flask, as filtration through the mass of bismuthate on the felt will answer the purpose. Run in from the pipette (Fig. 79) 25 cc. of ferrous sulphate solution and titrate with the permanganate to a faint pink. This gives the value in permanganate of the ferrous sulphate solution. With this method of procedure any number of determinations may be made with a variation of less than $0.1 \mathrm{cc}$.

The permanganate solution may be standardized with iron in the usual way and calculating its value in manganese. The proportion is $55.5: 54.6$. Or it may be standardized by titrating a steel containing a known amount of manganese and getting the value of the solution by dividing the percentage of manganese by the number of cc. of the permanganate used. Or, thirdly, by making a solution of 
pure manganese sulphate and evaporating a weighed amount of it to dryness, heating it to redness and weighing as manganese sulphate, which, multiplied by 0.36424 , gives the amount of manganese. 5 gms. of "C. P." manganese sulphate dissolved in $500 \mathrm{cc}$. of water and filtered will give a solution containing about $0.0035 \mathrm{gm}$. of manganese to the gram of solution. Weigh I to $3 \mathrm{gms}$. of the solution in a crucible, transfer to a $200-\mathrm{cc}$. flask, using $50 \mathrm{cc}$. of nitric acid (I.I35); cool, add 0.5 to I gm. of bismuthate, and allow it to stand for three or four minutes, shaking at intervals. Add $50 \mathrm{cc}$. of 3 per cent nitric acid, filter through the asbestos filter and wash with 50 or $60 \mathrm{cc}$. of the same acid. Run $25 \mathrm{cc}$. of the ferrous sulphate solution into the flask from the pipette and titrate with the permanganate solution to a faint pink.

Subtract the number of cc. of the permanganate solution obtained from the value of the $25 \mathrm{cc}$. of ferrous sulphate solution in permanganate, and the result is the number of cc. of permanganate corresponding to the manganese in the manganese sulphate solution used. Divide the weight of the manganese in the manganese sulphate used by the number of cc. of permanganate, and the result is the value of $0.006354 \mathrm{gm}$., or the sample contains 0.635 per cent of manganese.

Example. I gm. manganese sulphate solution contains $0.0035^{62} \mathrm{gm}$. manganese; 2.0372 gms. manganese sulphate solution equals 0.0072565 gm. manganese; $25 \mathrm{cc}$. ferrous sulphate solution equals $24.5 \mathrm{cc}$. permanganate solution; 2.0372 gms. manganese sulphate, after oxidation and addition of $25 \mathrm{cc}$. of ferrous sulphate solution, require $3.6 \mathrm{cc}$. of permanganate; $24.5 \mathrm{cc} .-3.6 \mathrm{cc} .=20.9 \mathrm{cc}$; 0.0072565 divided by $20.90=.0003472$, or I gm. permanganate equals $0.0003472 \mathrm{gm}$. manganese. If then $\mathrm{I} \mathrm{gm}$. of steel, after oxidation and addition of $25 \mathrm{cc}$. of ferrous sulphate solution, requires $6.2 \mathrm{cc}$. of permanganate to give the pink color, then $24.5-6.2 \mathrm{cc} .=\mathrm{I} 8.3 \times 0.0003472=0.006354^{\circ} \mathrm{gm}$. or the sample contains 0.635 per cent manganese.

When the proper precautions are observed, this method gives very accurate results, especially for material containing up to two per cent of manganese. The reaction is very delicate. 


\section{CHAPTER XXXVIII}

\section{MERCURY}

By Precipitation as Mercurous Chlorid. The metal must be present in the form of mercurous salt only. A weighed quantity of the solution is treated with a measured excess of $\frac{\mathrm{N}}{\mathrm{IO}}$ sodium chlorid, which precipitates all the mercury as mercurous chlorid. The mixture is filtered, the precipitate well washed, and the mixed filtrates titrated with $\frac{\mathrm{N}}{\mathrm{IO}}$ silver nitrate solution, using potassium chromate as indicator. The solution must be neutral.

The quantity of silver solution used is deducted from the quantity of sodium chlorid solution added, and the difference is the quantity of the latter which reacted with the mercurous salt.

I cc. of $\frac{\mathrm{N}}{\mathrm{IO}}$ sodium chlorid $=0.026007 \mathrm{gm} . \mathrm{HgNO}_{3}$,

$$
=0.01985 \text { " } \mathrm{Hg} \text {. }
$$

Estimation of Mercurous Chlorid by Iodin and Thiosulphate (Hempel). The mercurous chlorid is treated with potassium iodid and $\frac{\mathrm{N}}{\mathrm{IO}}$ iodin solution until it is completely dissolved. The reaction is as follows:

$$
\mathrm{Hg}_{2} \mathrm{Cl}_{2}+6 \mathrm{KI}+\mathrm{I}_{2}={ }_{2} \mathrm{HgK}_{2} \mathrm{I}_{4}+2 \mathrm{KCl} \text {. }
$$

Starch solution is then added, which gives a blue color with the excess of iodin. The mixture is then titrated with $\frac{\mathrm{N}}{\mathrm{IO}}$ thiosulphate until the blue color disappears, and lastly with $\frac{\mathrm{N}}{\mathrm{IO}}$ iodin until the blue color returns. Subtract the cc. of thiosulphate from the total quantity of iodin added, and the remaining cc. will be the measure of the mercury.

$$
\text { Each cc. } \begin{aligned}
\frac{\mathrm{N}}{\mathrm{IO}} \text { iodin } & =0.01985 \mathrm{gm} . \mathrm{Hg} ; \\
& =0.023368 \text { “ } \mathrm{Hg}_{2} \mathrm{Cl}_{2}
\end{aligned}
$$


In the analysis, to I gm. of calomel take about $2.5 \mathrm{gms}$. of potassium iodid and roo cc. of $\frac{\mathrm{N}}{\mathrm{ro}}$ iodin.

Mercurous Salts other than the chlorid may be converted into the chlorid by precipitation with sodium chlorid, the precipitate well washed, and treated as directed for mercurous chlorid.

Mercuric Salts may be converted into mercurous chlorid by adding sodium chlorid, an excess of ferrous sulphate, and sodium hy. droxid to alkaline reaction. The mixture is allowed to stand for a short time, shaking frequently. Then hydrochloric acid is added until the solution becomes clear, and the mercurous chlorid white, and free from iron. The precipitate is then separated, washed, and treated as directed under Mercurous Chlorid. The reaction is as follows:

${ }_{2} \mathrm{HgSO}_{4}+{ }_{2} \mathrm{NaCl}+{ }_{2} \mathrm{FeSO}_{4}+6 \mathrm{NaOH}=\mathrm{Hg}_{2} \mathrm{Cl}_{2}+\mathrm{Fe}_{2}(\mathrm{OH})_{6}+{ }_{4} \mathrm{Na}_{2} \mathrm{SO}_{4}$.

Estimation of Mercuric Salts by Precipitation as Iodid (Personne's).* This method is described under the estimation of iodids (page r 34 ).

The mercuric solution must be added to the potassium iodid solution; a reversal of the process is not reliable.

The mercuric salt must be in the form of a neutral solution of mercuric chlorid, and it should be considerably diluted, say to 300 or $500 \mathrm{cc}$., and as a preliminary trial $20 \mathrm{cc}$. of decinormal potassium iodid solution should be taken, and titrated with the mercuric solution. Two or more titrations should be made, the first will give the approximate figure.

This method depends upon the fact that when a neutral solution of mercuric chlorid is added to a solution of potassium iodid, a colorless solution is formed, until the proportion of the mercuric chlorid is to that of potassium iodid as I to 4 . As soon as this proportion is exceeded, and more mercuric chlorid added, the solution is colored red through precipitation of mercuric iodid, which marks the endreaction.

The reagents required are:

Decinormal Potassium Iodid, made by dissolving 32.952 gms. of the pure salt and diluting to a liter;

Decinormal Mercuric Chlorid, made by dissolving 13.443 gms. of pure mercuric chlorid, together with about 30 gms. of sodium chlorid (to assist solution) in water to make one liter. 
The mercuric chlorid to be estimated, which must be neutral, is dissolved in water and added to an excess of the decinormal potassium iodid. The excess of potassium iodid is then formed by titrating from a burette, with decinormal mercuric chlorid, until the red color makes its appearance.

The reaction is expressed by the following equation:

$$
{ }_{4} \mathrm{KI}+\mathrm{HgCl}_{2}=2 \mathrm{KCl}+\mathrm{HgI}_{2} 2 \mathrm{KI} .
$$

Each cc. of $\frac{\mathrm{N}}{\mathrm{IO}}$ potassium iodid represents $0.009975 \mathrm{gm}$. of $\mathrm{Hg}$;

$$
0.013443 \text { “" “ } \mathrm{HgCl}_{2} \text {. }
$$

Estimation of Mercuric Salts by Oxidation of Ferrous Salts (Mohr). The mercury in the form of mercuric chlorid is dissolved in water in a flask, a weighed amount of ferrous ammonium sulphate dissolved in water added, and then sodium hydroxid, until the ferrous oxid is precipitated and the solution is decidedly alkaline. The mixture is then allowed to stand for a few minutes, shaking frequently. The mercuric chlorid is thus reduced to mercurous chlorid, and the ferrous salt to ferric hydroxid. The mixture is muddy and dark in color because of the presence of ferric and ferrous hydroxid and mercurous chlorid.

The reaction may be expressed by the following equation:

$$
\mathrm{HgCl}_{2}+\mathrm{FeCl}_{2}=\mathrm{HgCl}+\mathrm{FeCl}_{3} .
$$

Sufficient hydrochloric or sulphuric acid is now added to completely dissolve the iron hydroxids, leaving the mercurous chlorid perfectly white. The solution is then diluted to the mark, shaken, filtered through a dry filter, and a portion measured out and titrated for excess of ferrous salt, using either dichromate or permanganate. If permanganate is to be used, it is advisable to use sulphuric instead of hydrochloric acid for dissolving the precipitated iron hydroxids.

Example. I gm. of mercuric chlorid in solution was treated with $3 \mathrm{gms}$. of the double iron salt, and the solution then made freely alkaline with sodium hydroxid. The precipitated iron hydroxids are dissolved, through the addition of sulphuric acid, and the solution diluted to $300 \mathrm{cc}$., filtered, and $100 \mathrm{cc}$. titrated with $\frac{\mathrm{N}}{\mathrm{IO}}$ permanganate. $\quad 13.4 \mathrm{cc}$. were required. Therefore the entire quantity would require three times $\mathrm{I} 3.4 \mathrm{cc} .=40.2 \mathrm{cc}$, which, deducted from $77 \mathrm{cc}$. (the quantity required for $3 \mathrm{gms}$. of double iron salt), left $36.8 \mathrm{cc}$. which $=1.43152 \mathrm{gms}$. of undecomposed iron salt, which, multiplied by the factor $0.6905 \mathrm{gm}$. 
$=0.9884+$ gm. of pure mercuric chlorid; or the $36.8 \mathrm{cc}$. may be multiplied by the $\frac{\mathrm{N}}{\mathrm{IO}}$ factor for mercuric chlorid.

Mercurous Salts may be converted into mercuric chlorid by precipitating them as mercurous chlorid by means of sodium chlorid, washing the precipitate, adding sodium hydroxid, and passing chlorin through the solution. The solution is then acidified with hydrochloric acid, and evaporated on a water-bath to expel the excess of chlorin.

Mercuric Salts may be converted into mercuric chlorid by evaporating with hydrochloric acid, being careful, however, not to boil the solution or some of the chlorid will be lost.

By Potassium Cyanid (Hannay)** This method depends upon the fact that ammonia produces in mercurial solutions a precipitate or an opalescence (according to the degree of concentration), which is removed by a definite amount of potassium cyanid. To the mercury in the form of mercuric chlorid, ammonia is added until a considerable amount of a white precipitate has formed (an excess of ammonia does no harm). A solution of potassium cyanid is then added until the precipitate redissolves and leaves the liquid perfectly clear. The strength of the standard potassium cyanid is best determined by titration in the same manner against a weighed amount of pure mercuric chlorid. Since it decomposes slowly by keeping, the strength must be redetermined before each series of analyses. The temperature at which the titration is conducted should not be below $8^{\circ}$ nor above $20^{\circ} \mathrm{C}$.

This method is not interfered with by the presence of organic matter, and in its modifications by Tuson and Neison (J. Chem. S., 1877, 679), Chapman Jones (same journal, I.xI, 364), and Sutton ("Manual of Volumetric Analysis"), it is useful for a great variety of salts.

See Fstimation of Cyanogen, page 275 .

By Direct Titration with Sodium Thiosulphate (Scherer). $\dagger$ This method depends upon the fact that when sodium thiosulphate is added to a mercurial solution, a precipitate is produced so long as any mercury remains in solution. The standard sodium thiosulphate is added until the last drop fails to produce a precipitate. The precipitate settles rapidly and the proper end-point is easily found.

The thiosulphate used is of twentieth-normal strength. It is made by dissolving $12.323 \mathrm{gms}$. of the pure crystallized salt in sufficient

* J. Chem. S., 1873,565 .

† Lehrbuch der chemie, I, 5 I3. 
water to make one liter. The reaction which takes place with this solution and mercurous nitrate is

$$
\mathrm{Hg}_{2}\left(\mathrm{NO}_{3}\right)_{2}+\mathrm{Na}_{2} \mathrm{~S}_{2} \mathrm{O}_{3}+\mathrm{H}_{2} \mathrm{O}=\mathrm{Hg}_{2} \mathrm{~S}+\mathrm{Na}_{2} \mathrm{SO}_{4}+{ }_{2} \mathrm{HNO}_{3} .
$$

The Estimation of Mercurous Nitrate. The solution, which must be free from mercuric salt, is diluted, heated, and the standard thiosulphate run in from a burette until no further precipitate is produced. The sulphid settles rapidly and the end-point is easily found.

Each cc. of the thiosulphate $=0.01985 \mathrm{gm} . \mathrm{Hg}$;

$$
\begin{aligned}
& =0.026007 " \text { " } \mathrm{Hg}_{2}\left(\mathrm{NO}_{3}\right)_{2} ; \\
& =0.020644 " \mathrm{Hg}_{2} \mathrm{O} .
\end{aligned}
$$

Estimation of Mercuric Nitrate. Sodium thiosulphate precipitates the mercury from mercuric nitrate, according to the following reaction:

$$
{ }_{3} \mathrm{Hg}\left(\mathrm{NO}_{3}\right)_{2} \quad{ }_{2} \mathrm{Na}_{2} \mathrm{~S}_{2} \mathrm{O}_{3}+2 \mathrm{H}_{2} \mathrm{O}=2 \mathrm{HgS} . \mathrm{Hg}\left(\mathrm{NO}_{3}\right)_{2}+{ }_{2} \mathrm{Na}_{2} \mathrm{SO}_{4}+{ }_{4} \mathrm{HNO}_{3} \text {. }
$$

The mercurial solution is highly diluted, put into a stoppered flask, a little nitric acid added, and then the thiosulphate solution delivered from a burette, the flask being vigorously shaken meanwhile until the last drop produces no further yellow precipitate. In order to better distinguish the end-reaction Scherer recommends that the solution be diluted to a definite volume, when the greater part of the metal is precipitated, and a measured volume of the clear fluid taken out with a pipette, and the titration finished upon this portion. A second like quantity may then be taken out and titrated in the same way to check the analysis.

Each cc. of the thiosulphate $=0.01488 \mathrm{gm} . \mathrm{Hg}$,

$$
=0.1607 \text { "HgO. }
$$

Estimation of Mercuric Chlorid. The reaction in this case is as follows:

$$
{ }_{3} \mathrm{HgCl}_{2}+{ }_{2} \mathrm{Na}_{2} \mathrm{~S}_{2} \mathrm{O}_{3}+{ }_{2} \mathrm{H}_{2} \mathrm{O}={ }_{2} \mathrm{HgS} . \mathrm{HgCl}_{2}+{ }_{2} \mathrm{Na}_{2} \mathrm{SO}_{4}+{ }_{4} \mathrm{HCl} \text {. }
$$

The solution is acidified with hydrochloric acid, considerably diluted, and heated nearly to boiling; and then the thiosulphate carefully added as long as a white precipitate is formed. Care must be taken not to add an excess, otherwise a dirty looking gray, or even black, color will be produced. The end-reaction in this analysis is 
not nearly so easily seen as in the foregoing, and filtracion by means of a Beale's filter is necessary in order to distinguish the exact endpoint.

Each cc. of the thiosulphate $=0.01488 \mathrm{gm} . \mathrm{Hg}$,

$$
=0.10607 \text { " } \mathrm{HgO} \text {. }
$$

This method gives good results with pure mercurous nitrate. But it is practically inaccurate, if any mercuric nitrate is present, and this is usually the case.

Mercuric Chlorid may also be estimated by reduction with stannous chlorid, as described on page 233 .

Estimation of Mercuric Chlorid in Colored Tablets or Solutions.* Aniline colors, now frequently used in solutions and tablets of mercuric chlorid for antiseptic use, interfere with the volumetric estimation of the mercury contained in them. This may be overcome by precipitating the mercury with metallic iron, oxidizing the ferrous chlorid formed with potassium permanganate, which destroys the dye, and then estimating the ferric chlorid with potassium iodid and decinormal sodium thiosulphate. From $20 \mathrm{cc}$. of a four per cent solution of mercuric chlorid all the mercury is precipitated when a few grams of reduced iron are added and the mixture agitated frequently during one hour. The reactions are:

(a) $\mathrm{HgCl}_{2}+\mathrm{Fe}=\mathrm{FeCl}_{2}+\mathrm{Hg}$;

(b) $\mathrm{I}_{\mathrm{CFeCl}}+2 \mathrm{KMnO}_{4}+\mathrm{I} 6 \mathrm{HCl}=2 \mathrm{KCl}+2 \mathrm{MnCl}_{2}+{ }_{5} \mathrm{Fe}_{2} \mathrm{Cl}_{6}+8 \mathrm{H}_{2} \mathrm{O}$;

(c) $\mathrm{Fe}_{2} \mathrm{Cl}_{6}+2 \mathrm{KI}=\mathrm{FeCl}_{2}+\mathrm{I}_{2}+2 \mathrm{KCl}$;

(d) $\mathrm{I}_{2}+2 \mathrm{Na}_{2} \mathrm{~S}_{2} \mathrm{O}_{3}=2 \mathrm{NaI}+\mathrm{Na}_{2} \mathrm{~S}_{4} \mathrm{O}_{6}$.

The Process. I gm. of the mercuric chlorid tablets is dissolved in $50 \mathrm{cc}$. of water. $25 \mathrm{cc}$. of this solution containing $0.5 \mathrm{gm}$. of substance is agitated frequently during one hour with a few grams of reduced iron. $20 \mathrm{cc}$. of the solution are then filtered into a flask, $5 \mathrm{cc}$. of dilute sulphuric acid and $5 \mathrm{cc}$. of a ro per cent manganese sulphate solution are added. The addition of the latter prevents the liberation of chlorin, and thus obviates the necessity for heating to drive it off. A sufficient quantity of a one per cent solution of potassium permanganate is now added to produce a permanent red-colored solution, and then a few drops of alcohol or tartaric acid solution are added to decompose the excess of potassium permanganate. This accomplished, I to 2 gms. of potassium iodid are added, the solution digested for half an hour, and then titrated with sodium thiosulphate in the presence of starch.

* E. Rupp, Arch. d. Pharm., 238, 98. 
Further work on this subject by E. Rupp (Arch. d. Pharm., 1905, 300 ) resulted in the following method:

Several cubic centimeters of a 35 per cent formaldehyde solution are made alkaline by the addition of some weak sodium hydroxid solution, and the mercurial solution added whilst agitating the flask. This mixture is warmed for ten or fifteen minutes on a water-bath, and then after cooling, considerably acidified with acetic acid. A sufficient excess of $\frac{\mathrm{N}}{\mathrm{IO}}$ iodin is then added, the flask securely stoppered and shaken for five minutes; and when the precipitated mercuric potassium iodid is dissolved, the solution is titrated with $\frac{\mathrm{N}}{\mathrm{IO}}$ thiosulphate, using starch as indicator.

$$
\text { Each cc. of } \begin{aligned}
\frac{\mathrm{N}}{\mathrm{IO}} \text { iodin solution } & =0.01002 \mathrm{gm} . \mathrm{Hg}, \\
& =0.01355 " \mathrm{HgCl}_{2} .
\end{aligned}
$$

The Process. Five sublimate tablets are dissolved in water to make $500 \mathrm{cc}$. of solution. Of this $20 \mathrm{cc}$. are taken, $3 \mathrm{cc}$. of formaldehrde solution are added, followed by ıо cc. of caustic soda solution and $20 \mathrm{cc}$. of water. This mixture is warmed, as described above, and allowed to cool, when $30 \mathrm{cc}$. of 30 per cent acetic acid are added, followed by $25 \mathrm{cc}$. of $\frac{\mathrm{N}}{\mathrm{IO}}$ iodin solution, and the mixture titrated with $\frac{\mathrm{N}}{\mathrm{IO}}$ thiosulphate. The quantity of the latter used, deducted from $25 \mathrm{cc}$, gives the quantity of $\frac{\mathrm{N}}{\mathrm{IO}}$ iodin which represents the mercuric
chlorid.

The Personne Method, described on page 409, may be employed with good results for the estimation of mercuric chlorid in sublimate tablets. The presence of anilin colors; or sodium chlorid, does not in the least interfere.

Titration by Means of Potassium Dichromate. This method, which is recommended by F. M. Litterscheid,* is based upon the observation that when a solution of mercuric chlorid is treated with an excess of solution of potassium dichromate in the cold, and then with ammonia to distinctly neutral reaction, the mercury is completely precipitated, forming a lemon-yellow precipitate, separating rapidly after figorous shaking and soon assuming a granular character. After twenty minutes standing, the filtrate gives no reaction for mercury with either sulphureted hydrogen or stannous chlorid. The resulting

* Arch. d. Pharm., 1903, 306-313. 
compound, dimercur-ammonium chromate, is practically insoluble in water.

The dichromate solution used is the same as that used for standardizing $\frac{\mathrm{N}}{\text { 10 }}$ sodium thiosulphate $(4.8713 \mathrm{gms}$. in rooo cc). See page 179. The method is carried out as follows:

Mercuric chlorid solution $(\mathrm{r}: 40)$ is mixed in a roo-cc. flask with an excess of $\frac{\mathrm{N}}{\text { Io }}$ potassium dichromate. Ten per cent ammonia-water is then added, drop by drop, with rotation of the flask until the liquid is distinctly alkaline. The mixture is then shaken frequently during ten minutes and diluted to roo cc.; again shaken and allowed to stand for six hours. An aliquot part is then filtered off (about one half, rejecting the first portion of the filtrate) and in this determining the excess of dichromate. This is done after acidulation with sulphuric acid $(\mathrm{r}: 5)$, and the addition of an excess of potassium iodid, by titrating with $\frac{\mathrm{N}}{\mathrm{IO}}$ sodium thiosulphate. The quantity of the latter used, deducted from the quantity of $\frac{\mathrm{N}}{\mathrm{IO}}$ dichromate solution added, gives the quantity of the latter which reacted with and hence represents the mercuric chlorid. The potassium iodid used must of course be free from iodate.

Each cc. of the dichromate solution $=0.276 \mathrm{gm}$. Hg.

Estimation of Mercury in its Organic Compounds. E. Rupp and Th. Nöll * recommend the following procedure for hydrargyrum salicylicum: $0.3 \mathrm{gm}$. of the salicylate is heated with $4 \mathrm{gms}$. of potassium sulphate and $5 \mathrm{cc}$. of concentrated sulphuric acid in a $150-\mathrm{cc}$. flask, resting on wire gauze and provided with an oblique reflux tube about $50 \mathrm{~cm}$. long. As soon as the mixture is clear and colorless, the reflux tube is rinsed with 5 to ro cc. of sulphuric acid, and O.I to $0.2 \mathrm{gm}$. of potassium permanganate added. The heating is renewed until the solution is colorless. The whole is now diluted to roo cc. and titrated with decinormal sulphocyanate, using iron alum as indicator, until a reddish-brown color appears.

Each cc. of the decinormal solution $=0.010015 \mathrm{gm}$. of $\mathrm{Hg}$.

Analysis of Mercuric Cyanid. $\dagger$ One gram is dissolved in water to make roo cc. of solution. In this a determination of mercury and of cyanogen may be made as follows:

Determination of Mercury. $20 \mathrm{cc}$. of the solution is introduced into a glass-stoppered bottle, I-2 gms. of potassium iodid and 3-5 cc.

* Arch. d. Pharm., 243, No. I (Feb. 27, r 905), I-5.

†E. Rupp and F. Lehmann, Pharm. Ztg., 52, 1020. 
of a 15 per cent potassium hydroxid solution. To this is added a mixture of $2-3 \mathrm{cc}$. of formaldehyde solution (35 per cent) and $20 \mathrm{cc}$. of water, and the solution shaken for two minutes, or until complete reduction ensues. Sufficient acetic acid is then added to acidify the solution, and this is followed by $25 \mathrm{cc}$. of $\frac{\mathrm{N}}{10}$ iodin and shaken until the mercury is entirely dissolved. Then there are added io cc. of diluted sulphuric acid, and the excess of iodin is found by titration with $\frac{\mathrm{N}}{\mathrm{IO}}$ sodium thiosulphate.

Each cc. of $\frac{\mathrm{N}}{\mathrm{IO}}$ iodin $=0.012509 \mathrm{gm} . \mathrm{Hg}(\mathrm{CN})_{2}$.

Determination of Cyanid Radicle. Place into a glass-stoppered bottle ro cc. of the mercuric cyanid solution; add a little water and 5 -10 cc. of potassium hydroxid solution (15 per cent), and $25 \mathrm{cc}$. of $\frac{\mathrm{N}}{10}$ iodin, mix, warm thirty minutes on a water-bath, then dilute with water to make roo cc. Acidify with about $25 \mathrm{cc}$. of diluted hydrochloric acid, and after two minutes, titrate the excess of iodin with $\frac{\mathrm{N}}{\mathrm{IO}}$ sodium thiosulphate.

Each cc. of $\frac{\mathrm{N}}{\mathrm{IO}}$ iodin $=0.0062545 \mathrm{gm}$.

The following equations illustrate the reactions:

(I) ${ }_{4} \mathrm{KOH}+{ }_{4} \mathrm{I}=2 \mathrm{KIO}+2 \mathrm{KI}+{ }_{2} \mathrm{H}_{2} \mathrm{O}$;

(2) $\mathrm{Hg}(\mathrm{CN})_{2}+2 \mathrm{KIO}+2 \mathrm{KI}=\mathrm{K}_{2} \mathrm{HgI}_{4}+2 \mathrm{KCNO}$;

(3) $\mathrm{KIO}+\mathrm{KI}+2 \mathrm{HCl}=2 \mathrm{I}+2 \mathrm{KCl}+\mathrm{H}_{2} \mathrm{O} \cdot \mathrm{Hg}(\mathrm{CN})_{2}$.

Assay of Ammoniated Mercury.* Triturate $0.3 \mathrm{gm}$. of the substance, and rinse into a stoppered bottle with $50 \mathrm{cc}$. of water and add 3 gms. of potassium iodid. Shake until solution is effected; add two drops of a 0.2 per cent alcoholic solution of methyl orange, and titrate with $\frac{\mathrm{N}}{\mathrm{IO}}$ hydrochloric acid. The reaction is as follows:

$$
{ }_{2} \mathrm{HgNH}_{2} \mathrm{Cl}+{ }_{4} \mathrm{KI}+{ }_{2} \mathrm{H}_{2} \mathrm{O}={ }_{2} \mathrm{HgI}_{2}+2 \mathrm{KCl}+2 \mathrm{NH}_{3}+2 \mathrm{KOH} \text {. }
$$

Each cc. of $\frac{\mathrm{N}}{\mathrm{IO}}$ hydrochloric acid $=0.0 \mathrm{I} 248 \mathrm{gm}$. of $\mathrm{HgNH}_{2} \mathrm{Cl}$.

* Rupp and Lehmann, Pharm. Ztg., 52, ror4. 


\section{CHAPTER XXXIX}

\section{SILVER}

Estimation by Precipitation as Chlorid. Silver in solution (slightly acidified with nitric acid) when treated with sodium chlorid is completely precipitated as silver chlorid. See page Izo.

The end-reaction may be determined in three ways:

First. By shaking the solution violently after each addition of the sodium chlorid solution, and allowing the silver chlorid to subside before adding more of the reagent. The end-point is then reached when the further addition of sodium chlorid fails to produce a precipitate in the clear supernatant liquid.

Second. By filtering a small portion by means of a Beale's filter and testing the clear liquid.

Third. By the use of neutral potassium chromate as indicator. In this case it is always better to add the standard solution of sodium chlorid (usually decinormal) in slight excess; then add a few drops of the indicator and titrate the excess of sodium chlorid solution by means of decinormal silver nitrate. The end-point is known by the solution becoming red, through formation of silver chromate. Accuracy cannot so easily be obtained if the chromate is added to a silver solution, and then sodium chlorid until the red color changes to white:

First. Because the precipitated silver chromate, if allowed to stand any length of time, is not easily decomposed by the sodium chlorid solution and the operator is apt to overstep the proper point.

Second. Because the change from red to white is less easy to observe than that from white to red.

These methods and the preparation of the standard solutions are fully described in the chapter on Precipitation Analyses.

The Sulphocyanate Method is described on page I3I.

The Starch Iodid Method (Pisani). This method is especially applicable to the analysis of ores containing lead and copper, but not mercury, tin, iron, manganese, antimony, arsenic, or gold.

The method is based upon the fact that when starch iodid is added to a neutral solution of a silver salt, the silver is precipitated as iodid, and the blue color of the starch iodid is destroyed as long as any silver remains in solution. When, however, the silver is entirely precipitated, the starch iodid colors the solution blue. 
Preparation of the Starch Iodid Solution. Weigh out 2 gms. of iodin into a mortar, add 15 gms. of starch, and rub it up well with 6 or 8 drops of water. This mass is transferred to a well-stoppered flask and heated on a water-bath for about one hour or until it has assumed a dark bluish-gray color. Sufficient water is then added to dissolve it. The strength of this solution is then ascertained by means of a solution of silver. About o.or gm. of silver is weighed out, dissolved in nitric acid, evaporated to dryness, dissolved again in about $80 \mathrm{cc}$. of water; some precipitated calcium carbonate added, and the starch iodid solution run in from a burette until the solution takes a greenish-blue tint. The $0.0 \mathrm{Igm}$. of silver should require about $50 \mathrm{cc}$. of the starch iodid solution.

The amount of silver taken for an analysis should not contain more than 0.01 to $0.02 \mathrm{gm}$. of silver, and in the presence of copper must be greatly diluted, in order to weaken the color of the copper, say from 60 to $100 \mathrm{cc}$. If more than $0.02 \mathrm{gm}$. of silver be present, the larger portion may be precipitated by means of $\frac{\mathrm{N}}{\mathrm{IO}}$ sodium chlorid, the precipitate filtered off, and the remaining silver determined as above. The amount so found is then added to that which has been precipitated by the sodium chlorid. If lead is present in the solution it may be removed by the addition of sulphuric acid, filtering, and then neutralizing the excess of acid by means of calcium carbonate, and filtering again before titrating. Field (Chem. News, II, I7) uses a solution of iodin in potassium iodid with starch. 


\section{CHAPTER XL}

\section{STRONTIUM}

Strontium Oxid and Hydroxid may be estimated by dissolving in water and titrating with decinormal hydrochloric acid in the presence of an indicator. A better way is by residual titration, i.e., adding a measured excess of $\frac{\mathrm{N}}{\mathrm{IO}}$ hydrochloric acid, and retitrating with $\frac{\mathrm{N}}{\mathrm{IO}} \mathrm{NaOH}$ in the presence of phenolphthalein, after boiling the acid solution.

I cc. $\frac{\mathrm{N}}{\text { IO }}$ hydrochloric acid $=0.004347 \mathrm{gm} . \mathrm{Sr}$;

$$
=0.006035 \text { " } \mathrm{Sr}(\mathrm{OH})_{2} \text {. }
$$

Strontium Carbonate may be estimated by residual titration as above described.

I cc. $\frac{\mathrm{N}}{\text { IO }}$ hydrochloric acid $=0.007322 \mathrm{gm} . \mathrm{SrCO}_{3}$.

Strontium Chlorid may be estimated by precipitating the metal as sulphate by means of potassium sulphate, and then titrating the filtrate containing potassium chlorid, with decinormal silver nitrate solution, and chromate as indicator.

$$
\begin{aligned}
& \mathrm{SrCl}_{2}+\mathrm{K}_{2} \mathrm{SO}_{4}=\mathrm{SrSO}_{4}+{ }_{2} \mathrm{KCl} ; \\
& \mathrm{KCl}+\mathrm{AgNO}_{3}=\mathrm{AgCl}+\mathrm{KNO}_{3} .
\end{aligned}
$$

I cc. $\frac{\mathrm{N}}{\text { IO }}$ silver nitrate solution $=0.007865 \mathrm{gm}$. $\mathrm{SrCl}_{2}$.

The same method may be employed for strontium iodid and bromid. These haloid salts may also be titrated direct with silver nitrate, as described on page II 5 .

Strontium Nitrate may be estimated by adding to its solution an excess of sodium carbonate, thoroughly washing the resulting precipitate of strontium carbonate with hot water, and then estimating the carbonate as described above.

All soluble strontium salts may be estimated by precipitation as oxalate, in the absence of calcium, barium, and other metals precipitable by oxalic acid. 
A strong solution of oxalic acid is added in excess, then an equal volume of alcohol. Let stand over night, filter through sand, dry the precipitate thoroughly to expel the alcohol, wash it into a beaker, add dilute sulphuric acid to decompose the oxalate, and titrate with $\mathrm{N}$ $\frac{\mathrm{N}}{10}$ permanganate.

I cc. $\frac{\mathrm{N}}{\mathrm{IO}}$ permanganate $=0.004347 \mathrm{gm}$. Sr. 


\section{CHAPTER XLI}

TIN

Titration with Iodin in Alkaline Solution (Lenssen).* In this method tin is estimated in the form of stannous chlorid. To a solution of this salt acidulated with hydrochloric acid, or a solution of metallic tin in hydrohloric acid, a tolerable quantity of Rochelle salt is added, and then a sirong solution of sodium bicarbonate, until the solution is alkaline. If the solution becomes cloudy upon addition of the sodium bicarbonate, more Rochelle salt must be added. Starch solution is then added and $\frac{\mathrm{N}}{\mathrm{IO}}$ iodin solution from a burette until the blue color appears. See equation below.

Stannous Chlorid of Commerce is estimated according to Dietze (Ph. Ztg., I897, r9I) as follows:

One gram is dissolved in water, a few drops of hydrochloric acid added, and the solution diluted to roo cc. $25 \mathrm{cc}$. of this solution are mixed with I gm. of tartaric acid, 2 or 3 gms. of sodium bicarbonate, and a few drops of starch solution, and the mixture titrated with $\frac{\mathrm{N}}{\mathrm{IO}}$ iodin solution until a blue color appears. 2 I.9 cc. to $22 . \mathrm{I} \mathrm{cc}$. of the latter should be employed.

Each cc. of $\frac{\mathrm{N}}{\text { IO }}$ iodin $=0.01 \mathrm{I} 2 \mathrm{II}$ gm. of $\mathrm{SnCl}_{2}+{ }_{2} \mathrm{H}_{2} \mathrm{O}$.

The cc. used multiplied by 4 and then by the factor gives the quantity in the I gm. taken. This multiplied by roo gives the per cent. The reaction is as follows:

$$
\mathrm{SnCl}_{2}+{ }_{2} \mathrm{H}_{2} \mathrm{O}+{ }_{2} \mathrm{HCl}+\mathrm{I}_{2}=\mathrm{SnCl}_{4}+{ }_{2} \mathrm{H}_{2} \mathrm{O}+{ }_{2} \mathrm{HI} \text {. }
$$

If a salt of tin, other than stannous chlorid, is to be estimated, it may easily be brought into this condition by precipitating the metal from its solution by means of pure metallic zinc, washing the finely divided tin, and dissolving in strong hydrochloric acid in contact with pieces of metallic platinum in an atmosphere of hydrogen or carbon dioxid.

* Jour. f. prak. Chem., LXXVIII, 200. 
Estimation in Acid Solution. As pointed out by S. W. Young (J. A. C. S., XIx, 809), the estimation of stannous chlorid may be accurately made in an acid solution by means of iodin.

The method of operation is very simple, and consists in bringing the stannous salt into solution, preferably with dilute hydrochloric acid, adding starch paste, and then titrating with standard iodin to a blue color. Neither oxidizing nor reducing substances must be present.

Great care must be exercised to prevent oxidation of the stannous salt solution by undue exposure to air. It should be prepared rapidly and immediately titrated.

The potassium iodid must be free from iodate.

Since thiosulphate cannot be satisfactorily used in acid solution to titrate against iodin, a dilute stannous chlorid solution may be employed for this purpose. This solution may be preserved under coal-oil, and even then should be checked against the iodin very frequently because it changes its strength very rapidly.

The results obtained by the author with this method are a trifle high, which is attributed by him to the standardization of the iodin by thiosulphate in neutral solution, whereas the assay is done in an acid solution.

Young obtained better results by standardizing in the following way:

A fresh solution of stannous chlorid was prepared of such strength as to be approximately equivalent to the iodin solution. Portions of C. P. potassium dichromate were then weighed out (about $0.2 \mathrm{gm}$.). These were titrated with the stannous chlorid solution until the dichromate was completely reduced, and then titrated back with iodin solution until the starch-iodid blue was produced. The reaction is as follows :

$$
\mathrm{K}_{2} \mathrm{Cr}_{2} \mathrm{O}_{7}+{ }_{3} \mathrm{SnCl}_{2}+{ }_{1} \mathrm{HCl}_{-}=2 \mathrm{KCl}+{ }_{2} \mathrm{CrCl}_{3}+{ }_{7} \mathrm{H}_{2} \mathrm{O}+{ }_{3} \mathrm{SnCl}_{4}
$$

the relationship between the iodin and the stannous chlorid being thus accurately determined.

See also Young and Adams, J. A. C. S., XIX, 515; "Action of Iodin on Solutions of Stannous Chlorid;" and Wilson H. Low, "Determination of Antimony and Tin in Babbitt, Type Metal, or Other Alloys," J. A. C. S., xxix, 66.

Indirect Titration by Ferric Chlorid and Permanganate (Löwenthal; * also Stromeyer $\dagger$ ). This method is based upon the 
fact that when stannous chlorid is brought in contact with ferric chlorid the latter is reduced to the ferrous state, whilst the stannous chlorid is oxidized to the stannic condition. The quantity of ferrous salt then found by means of potassium permanganate is the measure of the stannous salt present originally. This method is more fully described on page r 77. Cupric chlorid may be used instead of ferric chlorid..

- Stannic Salts, also tin compounds containing iron, are dissolved in water, hydrochloric acid added, and some pieces of pure metallic zinc introduced, and left in for ten or twelve hours. The tin which is so precipitated is (after being thoroughly washed) dissolved in hydrochloric acid and titrated as above described, or it may at once be mixed with the ferric chlorid solution, a little hydrochloric acid added, and when solution is complete titrated with permanganate.

Stannic salts may also be reduced to stannous sulphid by treatment with $\mathrm{H}_{2} \mathrm{~S}$. The precipitated sulphid is then well washed mixed with ferric chlorid, gently warmed, the sulphur separated by filtration, and the filtrate titrated with permanganate as above. The reaction is:

$$
\mathrm{SnS}_{2}+{ }_{2} \mathrm{Fe}_{2} \mathrm{Cl}_{6}=\mathrm{SnCl}_{4}+{ }_{4} \mathrm{FeCl}_{2}+\mathrm{S}_{2} \text {. }
$$

By Means of Potassium Dichromate (Reynolds).* This is a modification of Streng's original method. The oxidation of stannous chlorid by air is prevented by carbon dioxid, and a new and delicate indicator is used. The indicator is prepared by heating azobenzene with concentrated sulphuric acid till the beginning of a violent reaction; the whole is then poured into a large volume of water, forming a deep red solution. Stannous chlorid will decolorize this solution, while potassium dichromate will restore the color. It is not affected by ferrous salts nor by sodium thiosulphate.

The Process. Place $20 \mathrm{cc}$. of concentrated hydrochloric acid in a 200-cc. Erlenmeyer flask, fitted with a cork through which passes a tube reaching to within $1.5 \mathrm{~cm}$. of the bottom and a short tube about $6 \mathrm{~mm}$. wide at the top and 2 at the bottom, cut slantwise.

The tube has a side hole just below the cork to allow the gas to escape when adding the standard dichromate.

Fill the flask with $\mathrm{CO}_{2}$ from a cylinder, the gas passing first through titanous sulphate and then through sulphuric acid. Heat the hydrochloric acid nearly to boiling and allow to cool in a current of $\mathrm{CO}_{2}$.

Weigh out from 0.6 to I gm. of the metal to be analyzed, in a platinum gauze basket, and lower into the acid. When dissolved, 
heat nearly to boiling to drive out hydrogen, cool, and run in the standard dichromate through the short tube to within one cc. of the correct amount. Then add enough indicator to insure masking the green of the chromic salt when the color of the indicator is restored. Remove the basket and finish the titration by delivering the remainder of the dichromate directly into the solution.

The method gives good results in the case of pure tin, ferro-tin, and britannia metal, but is not reliable for pewter, phosphor-tin, or manganese-tin. 


\section{CHAPTER XLII}

\section{.. ZINC}

Zinc Oxid and Carbonate. Benedikt and Cantor (Zeit. angew. Chem., I888, 236, 237) show that the above compounds can be accurately titrated by adding a measured excess of normal acid solution and titrating back with normal alkali, using methyl-orange as indicator.

$$
\begin{aligned}
& \mathrm{ZnO}+\mathrm{H}_{2} \mathrm{SO}_{4}=\mathrm{ZnSO}_{4}+\mathrm{H}_{2} \mathrm{O} \text {; } \\
& \text { 2) } \frac{80.78}{40.39} \quad \frac{98}{49}=1000 \text { cc. } \frac{N}{I} \text { V.S. } \\
& \mathrm{ZnCO}_{3}+\mathrm{H}_{2} \mathrm{SO}_{4}=\mathrm{ZnSO}_{4}+\mathrm{H}_{2} \mathrm{O}+\mathrm{CO}_{2} \text {. } \\
& \frac{2) \mathrm{r} 24.45}{62.225} \quad \frac{98}{49}=1000 \mathrm{cc} \cdot \frac{\mathrm{N}}{\mathrm{I}} \text { V.S. }
\end{aligned}
$$$$
\text { I cc. } \begin{aligned}
\frac{\mathrm{N}}{\mathrm{I}} \mathrm{H}_{2} \mathrm{SO}_{4} & =0.03245 \text { gm. } \mathrm{Zn} ; \\
& =0.04039 \text { “ } \mathrm{ZnO} ; \\
& =0.06222 \text { “ } \mathrm{ZnCO}_{3} .
\end{aligned}
$$

Other salts of zinc may be treated by the same method. The zinc salt is dissolved in water ( $50 \mathrm{cc} .=$ about $0.1 \mathrm{gm}$. of $\mathrm{ZnO}$ ), phenophthalein is added and the solution titrated with standard sodium hydroxid to an intense red color, a few cc. more of the standard alkali then added, and after boiling the mixture titrating the excess of sodium hydroxid with standard acid.

Estimation as Oxalate (Leison). The zinc salt in solution preferably as sulphate and neutral in reaction, is treated with an excess of a strong solution of oxalic acid, and then a volume of strong alcohol equal in bulk to the zinc solution, is added. This is allowed to stand twelve hours, and then the precipitated zinc oxalate separated by filtration through a plug of ashestos, and thoroughly washed with alcohol, and dried in an air-bath. The asbestos plug and precipitate are placed in hot dilute sulphuric acid, by which the oxalate is dis- 
solved, and the mixture titrated with $\frac{\mathrm{N}}{10}$ potassium permanganate solution.

Each cc. of $\frac{\mathrm{N}}{10}$ permanganate $=0.003245 \mathrm{gm}$. of $\mathrm{Zn}$;

$$
\begin{aligned}
& =0.004039 \text { ، “ " } \mathrm{ZnO} ; \\
& =0.0080125 \text { " " } \mathrm{ZnSO}_{4} \text {. }
\end{aligned}
$$

Zinc Dust. Zinc dust is generally a mixture of metallic zinc, zinc oxid, and often some zinc carbonate. It is largely used as a reducing agent, and its value in this respect is proportionate to the metallic zinc it contains. Hence it is important to be able to estimate the quantity of free metal in a sample. This may be done as follows:

A weighed portion of the zinc dust, free from lumps, is introduced into a flask provided with a ground-glass stopper, and a measured excess of a centinormal solution of iodin added and the mixture digested for some time. The metallic zinc is acted upon by the iodin, and the zinc iodid is formed; the oxid is not affected.

When the reaction is completed, the excess of iodin solution is determined by retitration with centinormal sodium thiosulphate solution, and the quantity of the latter employed is deducted from the quantity of iodin solution added.

Each cc. of $\frac{\mathrm{N}}{\mathrm{IOO}}$ iodin V. S. $=0.00032,45 \mathrm{gm}$. of metallic zinc.

Indirect Method. Precipitation as Sulphid and Conversion of the Latter into Chlorid, with Subsequent Titration of the Chlorid by Volhard's Method (Mann). The reagents required are:

Silver Chlorid. Well washed, preserved under water, and protected from light.

Decinormal Silver Nitrate.

Decinormal Ammonium Sulphocyanate.

Ferric Alum Indicator.

Pure Nitric Acid.

The Process. 0.5 to $\mathrm{I} \mathrm{gm}$. of the zinc ore is dissolved in nitric acid. $\mathrm{H}_{2} \mathrm{~S}$ is passed through the solution to remove heavy metals. Iron and aluminum are separated by double precipitation with ammonia. The mixed filtrates are acidified with acetic acid and the zinc precipitated as $\mathrm{ZnS}$ by means of $\mathrm{H}_{2} \mathrm{~S}$. The liquid is then boiled in order to drive off excess of $\mathrm{H}_{2} \mathrm{~S}$, which is known to be removed completely when a drop of the filtered liquid fails to stain lead paper.

The precipitate is then allowed to settle, the supernatant liquid decanted, and the precipitate transferred to a filter with a little hot water without further washing. The filter and contents are then placed into a beaker; $50 \mathrm{cc}$. of hot water added, and a sufficient quan- 
tity of moist silver chlorid added to decompose the zinc sulphid and be present in slight excess. The mixture is now boiled until it begins to settle clear; several drops of dilute sulphuric acid are added, and the whole of the zinc sulphid will soon be converted into zinc chlorid. The insoluble matter, consisting of silver chlorid and free sulphur, is removed by filtration and washed. The chlorid in the mixed filtrate and washings is then estimated by the following method:

To 200 or $300 \mathrm{cc}$. of the cooled filtrate are added $5 \mathrm{cc}$. of the ferric alum indicator and enough pure nitric acid to remove the yellow color of the iron. A measured quantity (excess) of the standard silver nitrate is then added, and finally the titration with standard sulphocyanate begun. The volume of the latter required to produce the red end-color is deducted from the quantity of the silver nitrate added, and the difference is the quantity of silver nitrate which represents the zinc chlorid present. The reactions are:

$$
\begin{gathered}
\mathrm{ZnS}+2 \mathrm{AgCl}=\mathrm{ZnCl}_{2}+\mathrm{Ag}_{2} \mathrm{~S}, \\
\mathrm{ZnCl}_{2}+2 \mathrm{AgNO}_{3}=2 \mathrm{AgCl}+\mathrm{Zn}\left(\mathrm{NO}_{3}\right)_{2} .
\end{gathered}
$$

This method gives exceedingly good results.

Precipitation with Standard Sodium Sulphid. This process, which gives very good results, is the reverse of the method for the estimation of sulphids described on page 327 . The materials required are:

Standard Sodium Sulphid. This is prepared by passing $\mathrm{H}_{2} \mathrm{~S}$ into a solution of sodium hydroxid until saturated, adding sodium hydroxid until the solution no longer smells of $\mathrm{H}_{2} \mathrm{~S}$, and then diluting it until it nearly corresponds to the standard zinc solution.

Standard Zinc Solution. Made by dissolving $43.976 \mathrm{gms}$. of pure zinc sulphate $\left(\mathrm{ZnSO}_{4}+{ }_{7} \mathrm{H}_{2} \mathrm{O}\right)$ in water to make rooo cc. Such a solution contains o.or gm. of zinc per cc.

Alkaline Lead Indicator. Made by heating together lead acetate, tartaric acid, and sodium hydroxid solution in excess, until a clear solution is produced. Sodium nitro-prussid, nickelous, and cobaltous chlorids are among the substitutes suggested as indicator.

Determination of the Relative Strength of the Standard Solutions. $50 \mathrm{cc}$. of the zinc solution $(=0.5 \mathrm{gm} . \mathrm{Zn})$ are placed into a beaker and sufficient of a solution containing three parts of ammonia and one part of ammonium carbonate are added until the precipitate which, at first, forms is redissolved. The sodium sulphid solution is then added from a burette, stirring after each addition. From time to time a drop of the alkaline lead solution is taken out from a bottle containing it, by means of a glass rod, and placed upon a piece of white filtering paper, and near it upon the same piece of paper, a drop 
of the turbid zinc solution. The liquids spread over the paper and run into each other, and as soon as an excess of sodium sulphid solution is present, show a dark line where the drops meet. It is not always easy to hit the exact end-point on the first trial, but a second or a third trial will give accurate results. In the analysis itself the zinc solution must be made alkaline with ammonia, as above described. Two burettes are to be used, one containing the standard sodium sulphid solution and the other the standard zinc solution. The sulphid is added until the lead indicator shows it to be in excess. If this point is overstepped in the first trial, as may easily happen, a few cc. of the standard zinc solution may be added, and the titration again proceeded with.

If zinc ores are to be examined, an ammoniacal zinc solution is prepared for analysis as follows:

One or two grams of the ore, according to the quantity of zinc present, are finely pulverized, introduced into a wide-mouthed flask, and treated with hydrochloric acid, to which a little nitric acid is added. This mixture is warmed in order to facilitate solution, and then the excess of acid removed by heat. Lead, if present, is removed by the addition of a few drops of $\mathrm{H}_{2} \mathrm{SO}_{4}$ previous to complete dryness. The residue is then extracted with water and filtered. Should heavy metals be present, they are to be removed by $\mathrm{H}_{2} \mathrm{~S}$; the solution will still contain iron and possibly also manganese. Boiling with a little nitric acid is necessary, if the iron is not fully oxidized. If only traces of manganese are present, a few drops of bromized $\mathrm{HCl}$ should be added. When cold the solution is supersaturated with ammonia to precipitate the iron, which is separated by filtration and washed with ammoniacal warm water. If the quantity of iron is large, the precipitate will hold much zinc (about one-fifth of its weight). It should then be dissolved in $\mathrm{HCl}$ and reprecipitated as basic acetate. The filtrate from this second precipitate is then added to the original zinc filtrate, and the whole made up to one liter.

Precipitation as Sulphid and then Treatment with Ferric Salt and Titration of the Resulting Ferrous Salt with Permanganate (Schwartz). This method is based upon the fact that when zinc sulphid is mixed with ferric sulphate and sulphuric acid, the following reaction takes place:

$$
\mathrm{ZnS}+\mathrm{Fe}_{2}\left(\mathrm{SO}_{4}\right)_{3}={ }_{2} \mathrm{FeSO}_{4}+\mathrm{ZnSO}_{4}+\mathrm{S} \text {. }
$$

The ferrous sulphate so produced is estimated with permanganate, and the proportional quantity of zinc present ascertained.

Ferric chlorid and hydrochloric acid may be used instead of the 
sulphate and sulphuric acid, and dichromate may be employed instead of permanganate.

The Process. The zinc solution to be examined, in a tall cylinder, is made alkaline by the addition of sodium or ammonium hydroxid (or in the assay of zinc ores the ammoniacal zinc solution, described in the foregoing method, is taken). A slight excess of sodium or ammonium sulphid is then added, the cylinder filled with warm distilled water, covered closely, and set aside in a warm place for several hours. A tube drawn out at one end and containing a loosely fitting plug of asbestos, is then arranged, as in Fig. 8o. The bent tube is connected with a suction-pump. After the liquid above the precipitate in the cylinder has become perfectly clear, and the precipitate settled, decant the clear fluid through the filter. As soon as the clear fluid has run through, close the tube with a tight cork, fill the cylinder with warm, recently-boiled, water containing a little ammonium hydrate, cork tightly, and allow the flask to stand until the precipitate again subsides; then pour the clear fluid upon the filter, and repeat this three or four times; finally, wash most of the precipitate upon the asbestos filter, and wash rapidly with warm water containing ammonia until a drop of the filtrate produces no black precipitate in an alkaline lead solution. Then remove the tube containing the asbestos and zinc sulphid from the Woulfe's bottle, and, by means of a stiff wire inserted at the small end, force the asbestos and zinc sulphid into the cylinder

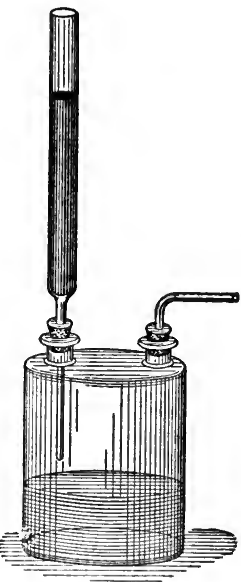

FIG. 80. in which the precipitation was made, wash any zinc sulphid still adhering to the tube into the cylinder, and add a quantity of an acid solution of ferric sulphate more than sufficient to decompose the zinc sulphid. Stopper the cylinder securely, and allow to stand in a warm place, shaking occasionally. The reaction which takes place may be represented thus:

$$
\mathrm{Fe}_{2}\left(\mathrm{SO}_{4}\right)_{3}+\mathrm{ZnS}={ }_{2} \mathrm{FeSO}_{4}+\mathrm{ZnSO}_{4}+\mathrm{S} \text {. }
$$

After the reaction is completed, the solution should possess a yellow color due to undecomposed ferric salt. The stopper is now removed, no odor of $\mathrm{H}_{2} \mathrm{~S}$ should be perceptible, and the solution should be diluted and titrated with $\frac{\mathrm{N}}{\mathrm{IO}}$ permanganate. If the solution is very dilute, the finely divided precipitate of sulphur does not interfere. 
Each cc. of $\frac{\mathrm{N}}{\mathrm{IO}}$ permanganate $=0.003245 \mathrm{gm} . \mathrm{Zn}$;

$$
\begin{aligned}
& =0.004039 \text { “ } \mathrm{ZnO} ; \\
& =0.0080125 \text { “ } \mathrm{ZnSO}_{4} .
\end{aligned}
$$

\section{Precipitation as Sulphid with Iron Indicator (Schaffner).*}

Reagents Required. Sodium Sulphid Solution and Zinc Solution are prepared as directed on page 427. I cc. of the former should precipitate $0.01 \mathrm{gm}$. of zinc, and I cc. of the latter should contain just $0.01 \mathrm{gm}$. of zinc.

Ferric Hydroxid Indicator. 3 gms. of iron wire are dissolved in $\mathrm{HCl}$ with the aid of heat; a little $\mathrm{HNO}_{3}$ is added, and the solution boiled so as to convert the ferrous into ferric chlorid, and the solution then diluted to $100 \mathrm{cc}$.

Just before using, add one or two drops (always taking the same number of drops) to I cc. of undiluted ammonia-water. Each drop produces a ring of ferric hydroxid, which requires but a few moments to impart the desired opacity to the liquid. In about one minute, the suspended ferric hydroxid is ready for use. $\dagger$

The Process. Prepare the ammoniacal zinc solution as described on page 428 . To $500 \mathrm{cc}$. of this solution add ferric hydroxid indicator suspended in ammonia (see above), and then run in the standard sodium sulphid solution until the greater part of the ferric hydroxid, collected on the sides and bottom of the beaker, acquires a brown or a black tint (the same tint should be used in all the titrations). It is advisable to keep the solution for titration at from $40^{\circ}$ to $60^{\circ} \mathrm{C}$. A check titration carried out under exactly equal conditions, with a known quantity of zinc, gives comparative data for calculation.

Estimation as Ferrocyanid. The use of potassium ferrocyanid for the volumetric estimation of zinc was first suggested by Galletti. The precipitation is effected in acetic acid solution at $40^{\circ} \mathrm{C}$., and the milky appearance which the liquid assumes when the potassium ferrocyanid is in excess serves as an indication of the end-reaction.

The Standard Potassium Ferrocyanid solution contains 43.104 gms. per liter. I cc. of this $=0.01 \mathrm{gm}$. of zinc, but its actual working power must be found by titration against a standard zinc solution, made by dissolving to gms. pure metallic zinc in hydrochloric acid, and making up to I liter. The ferrocyanid solution should be freshly prepared

* Jour. f. prakt. Chem., LXXIII, 4 Io.

$\dagger$ Ferric chlorid solution may be used instead and dropped directly into the ammoniacal zinc solution.

$\ddagger$ Zeitschr. f. analyt. Chem., IV, 213, and the method later modified by him in VIII, 135 and XIV, 190 
frequently, or should be adjusted when needed. The adjustment should be made in precisely the same way as the actual analysis of the ores, which is as follows:

$25 \mathrm{cc}$. of the zinc solution are treated in a beaker with $15 \mathrm{cc}$. of ammonia-water (sp.gr. 0.900). Acetic acid is then very cautiously added to acidify the solution. $50 \mathrm{cc}$. of acid ammonium acetate solution (made by adding together $20 \mathrm{cc}$. of ammonia-water, sp.gr. 0.900 , 15 cc. of acetic acid, and $65 \mathrm{cc}$. of distilled water) are then added to liquid, and the whole diluted to $250 \mathrm{cc}$., and warmed to about $50^{\circ} \mathrm{C}$. The ferrocyanid solution is then delivered from a burette, until the whole of the zinc is precipitated. The first change of color, from white to ash gray, is taken as the end-point. A better endreaction is, however, attainable if a drop of the solution is brought in contact on a white porcelain slab with a drop of uranium acetate solution. The ferrocyanid solution should correspond, volume for volume, with the standard zinc solution.

This method is available in the absence of copper, manganese, nickel, and cobalt, but moderate quantities of iron and lead may be present.

Precipitation in Hydrochloric Acid Solution (Fahlberg).* This method is somewhat simpler than the foregoing. The same ferrocyanid solution is used., The zinc solution is prepared by dissolving Io gms. of pure zinc in hydrochloric acid, adding $50 \mathrm{gms}$. of ammonium chlorid, and diluting to one liter. The ammonium chlorid causes the precipitate to assume a more fine flocculent character, which is quite an advantage.

The working value of the ferrocyanid solution is ascertained as follows:

Fill two burettes, one with the ferrocyanid solution and the other with the standard zinc solution. Then place in a beaker $50 \mathrm{cc}$. of the zinc solution, add Io to $15 \mathrm{cc}$. of hydrochloric acid (sp.gr. I.I2), and $45^{\circ} \mathrm{cc}$. of water, and while gently stirring, run in the ferrocyanid solution in quantities of $\mathrm{I}$ cc. at a time until a drop of the liquid, brought in contact with a drop of uranium nitrate solution on a white slab, gives a permanent brownish-red color.

Now carefully run in the zinc solution until the reaction disappears, then again add the ferrocyanid solution, drop by drop, until the endreaction is again attained. The ferrocyanid solution is then diluted, so that equal volumes of it and the zinc solution react. Ferric chlorid may also be used as indicator.

Miller (J. A. C. S., XVIII, I IO2) suggests the use of a strong solution

* Zeitschr. f. analyt. Chem., XIII, 379. 
of hydrochloroplatinic acid $\left(\mathrm{H}_{2} \mathrm{PtCl}_{6}\right)$, acidified with $\mathrm{HCl}$, as an indicator for the titration of zinc by $\mathrm{K}_{4} \mathrm{Fe}(\mathrm{CN})_{6}$ when performed in a hot solution. The end-reaction is a bright emerald green, which develops in a few seconds but does not work in a cold solution. This indicator is used in the same way as uranium solution, and is less affected by varying amounts of $\mathrm{HCl}$.

The nature of the precipitate produced in zinc solutions upon the addition of potassium ferrocyanid has been the subject of considerable discussion. The prevailing idea that a normal ferrocyanid is formed and that the reaction occurs as per the equation,

$$
\mathrm{K}_{4} \mathrm{FeCy}_{6}+{ }_{2} \mathrm{ZnCl}_{2}=\mathrm{Zn}_{2} \mathrm{FeCy}_{6}+{ }_{4} \mathrm{KCl} \text {, }
$$

is criticised by Miller,* who asserts that the precipitate has the composition, $\mathrm{Zn}_{3} \mathrm{~K}_{2}\left[\mathrm{Fe}(\mathrm{CN})_{6}\right]_{2}$. In proof of which a solution of potassium ferrocyanid ( $\mathrm{I} \mathrm{cc}$. of which $=0.010 \mathrm{gm}$. of $\mathrm{Zn}$ ) would contain $32.32 \mathrm{gms}$. of $\mathrm{K}_{4} \mathrm{Fe}(\mathrm{CN})_{6}+{ }_{3} \mathrm{H}_{2} \mathrm{O}$ to the liter, if the reaction proceeds, as per the above equation; whereas it has been proven by experiment, that I cc. of a solution containing 43.104 gms. of $\mathrm{K}_{4} \mathrm{Fe}(\mathrm{CN})_{6}+{ }_{3} \mathrm{H}_{2} \mathrm{O}$ to the liter, equals $0.010 \mathrm{gm}$. of zinc. This would indicate that two molecular weights of potassium ferrocyanid are equivalent to three atoms of zinc, and hence the composition of the precipitate formed is probably as asserted by Miller, $\mathrm{Zn}_{3} \mathrm{~K}_{2}\left[\mathrm{Fe}(\mathrm{CN})_{6}\right]_{2}$, and the equation may be written:

$$
{ }_{2} \mathrm{~K}_{4} \mathrm{FeCy}_{6}+{ }_{3} \mathrm{ZnCl}_{2}=\mathrm{Zn}_{3} \mathrm{~K}_{2}\left[\mathrm{Fe}(\mathrm{CN})_{6}\right]_{2}+6 \mathrm{KCl} \text {. }
$$

In a series of researches Wyronboff comes to the conclusion that the precipitate produced by the action of potassium ferrocyanid upon a solution of a zinc salt has the composition, $3 \mathrm{Zn}_{2} \mathrm{Fe}(\mathrm{CN})_{6} \cdot \mathrm{K}_{4} \mathrm{Fe}(\mathrm{CN})_{6}$ $+{ }_{12} \mathrm{H}_{2} \mathrm{O}$, which to an extent agrees with the findings of Miller.

The fact is that the composition of the precipitate differs with varying conditions in an analysis, such as quantity and temperature of the solution and quantity of acid present. The indicator used also has an influence.

According to Stone and Van Ingen (J. A. C. S., XIX, 545), in a cold solution containing one seventh of a cc. of $\mathrm{HCl}$ in Ioo, uranium showed the end-reaction when the quantities of zinc and ferrocyanid were sufficient to form $7 \mathrm{n}_{2} \mathrm{Fe}(\mathrm{CN})_{6}$. In a hot solution containing to cc. of $\mathrm{HCl}$, uranium gave the reaction with $\mathrm{Zn}_{3} \mathrm{~K}_{2}\left[\mathrm{Fe}(\mathrm{CN})_{6}\right]_{2}$. In both of these cases a drop of the indicator was mixed with a drop of the solution on a porcelain plate. In a cold solution containing one seventh of a cc. of the acid, uranium gave a reaction with $\mathrm{Zn}_{4} \mathrm{~K}_{4}\left[\mathrm{Fe}(\mathrm{CN})_{6}\right]_{3}$ 
when drops of it and the solution were placed side by side on a filterpaper, so that the uranium did not touch the precipitate, but only the clear solution that filtered from it. Under the same conditions copper and ferric chlorid, as indicators, gave the same end-point.

A strong solution of cobalt placed on a porcelain plate and a drop of the solution by it, so that the two touch but do not mix, showed the reaction for $\mathrm{Zn}_{4} \mathrm{~K}_{4}\left[\mathrm{Fe}(\mathrm{CN})_{6}\right]_{3}$ when the solution was mixed with very dilute cobalt the reaction was for $\mathrm{Zn}_{3} \mathrm{~K}_{2}\left[\mathrm{Fe}(\mathrm{CN})_{6}\right]_{3}$. In both cases one-seventh cc. of hydrochloric acid was present and the solution was cold. Platinum indicator, when used in a hot solution, gives the reaction with $\mathrm{Zn}_{3} \mathrm{~K}_{2}\left[\mathrm{Fe}(\mathrm{CN})_{6}\right]_{2}$. For fuller discussion of this subject see Miller and Mathews; also Stone and Van Ingen (J. A. C. S., XIX, 542 and 547). Similar variations occur in the composition of the precipitate, produced with manganese and potassium ferrocyanid.

Determination of $Z$ inc in the Presence of Manganese, using Cobalt Nitrate as Indicator.* The methods commonly used for the separation of manganese, before titrating with ferrocyanid, take too long and are, moreover, unsatisfactory. Stone therefore suggests the following procedure, in which the two metals (after separation of other metals of the iron group) are titrated together; the manganese determined in a separate portion by titration with permanganate, and the zinc found by difference. The usual indicators for ferrocyanid, namely, uranium salts, ferric chlorid, and copper sulphate, are not suited here, because they react with the precipitated manganese ferrocyanid. Cobalt nitrate, however, gives a very delicate reaction, and is not affected by as much as one part of hydrochloric acid in fourteen of water, nor does it react on the precipitates.

The indicator should be quite dilute and should be used on a porcelain slab, a drop of it is brougnt in contact with a drop of the solution to be tested. The drops should touch, but not mix. A faint green line appearing immediately, marks the end-point. The reagents required are:

Standard Potassium Ferrocyanid, 30 gms. to I liter. This is standardized by titrating against solutions containing known amounts of zinc or manganese in slightly acidulated solutions, using cobalt nitrate solution as an outside indicator. The solution should be about the volume used in an actual analysis. The amount of ferrocyanid solution required to five a reaction with cobalt in this volume of acidulated solution riust be noted and deducted for each titration. It is about $0.7 \mathrm{cc}$. for a volume of $140 \mathrm{cc}$.

Standard Potassium Permanganale, $\mathrm{r} .99$ gms. per liter. I cc. $=$ o.00I gm. Mn.

* G. C. Stone, J. A. C. S., XVII, 473. 
The Process. The ore is dissolved in $\mathrm{HCl}$, using $\mathrm{KClO}_{3}$ as an oxidizer, taking care to have sufficient acid to keep the manganese in solution.

Lead alone need not be separated; copper can be precipitated by lead, or lead and copper can both be precipitated by aluminum; cadmium may be precipitated by $\mathrm{H}_{2} \mathrm{~S}$ and the filtrate oxidized. Iron and alumina are best separated by barium carbonate, which must be free from alkali carbonates and hydroxids, barium hydroxid, and ammonium salts. A sufficiently pure salt for this purpose can be obtained by suspending the commercial pure barium carbonate (free from ammonium salts) in water and warming for several hours with two or three per cent of its weight of barium chlorid.

The thoroughly oxidized solution of the ore is washed into a 500-cc. flask, cooled, and barium carbonate suspended in water added until the precipitate curdles. Then pour into a beaker, mix thoroughly, let it settle, decant the clear liquid through a dry filter, dilute to $500 \mathrm{cc}$., and take portions of 50,100 , or $200 \mathrm{cc}$. for each titration. The titration should be started at once, or some zinc will precipitate.

One portion, which should contain between 0.01 and $0.04 \mathrm{gm}$. of manganese, is diluted to about $200 \mathrm{cc}$., heated nearly to boiling in a porcelain dish, and titrated rapidly with permanganate, stirring vigorously.

A second portion, made slightly acid with $\mathrm{HCl}$, is now titrated for the zinc and manganese together in the cold.

A large excess of $\mathrm{HCl}$ must be avoided because of its solvent action upon manganese ferrocyanid, $5 \mathrm{cc}$. of $\mathrm{HCl}$ to $100 \mathrm{cc}$. of solution containing about $0.03 \mathrm{gm}$. of $\mathrm{Mn}$ is about right.

If the manganese is present in appreciable quantity, the color of the precipitate will darken as the ferrocyanid is run in, and quite suddenly change to light greenish yellow shortly before the end is reached. It is not necessary to test with the cobalt solution, until I or $2 \mathrm{cc}$. of the ferrocyanid have been added after the lightening of the precipitate.

Example. I cc. of the ferrocyanid solution used equaled 0.00606 gm. of zinc, or $0.00384 \mathrm{gm}$. of manganese; I cc. of the permanganate solution equaled $0.00 \mathrm{Igm}$. of manganese. $2.5 \mathrm{gms}$. of the ore were treated as above described.

$50 \mathrm{cc}$. of the solution was diluted, heated, and titrated with permanganate, requiring $\mathrm{I} 8.45 \mathrm{cc} .=$ to 7.38 per cent of manganese. I $00 \mathrm{cc}$. titrated with ferrocyanid required $27.85 \mathrm{cc}$.

The previous titration had shown that there was $0.0369 \mathrm{gm}$. of manganese present, which would require 9.6I cc. of ferrocyanid; deducting this from 27.85 left $18.24 \mathrm{cc}$. for the $z$ inc $=$ to $0.11053 \mathrm{gm}$. or 22.1 I per cent. 
By Precipitation as Arsenate, and Titration of the Arsenate with Standard Sodium Thiosulphate (Meade).* This method consists in precipitating zinc by means of sodium arsenate, and then after dissolving the precipitated zinc ammonium arsenate in dilute hydrochloric acid, adding potassium iodid and titrating the liberated iodin by means of standard thiosulphate. The reactions may be expressed as follows:

$$
\begin{gathered}
\mathrm{ZnCl}_{2}+\mathrm{Na}_{2} \mathrm{HAsO}_{4}+\mathrm{NH}_{4} \mathrm{OH}=\mathrm{ZnNH}_{4} \mathrm{AsO}_{4}+{ }_{2} \mathrm{NaCl}+\mathrm{H}_{2} \mathrm{O}, \\
\mathrm{As}_{2} \mathrm{O}_{5}+{ }_{4} \mathrm{KI}+{ }_{4} \mathrm{HCl}=\mathrm{As}_{2} \mathrm{O}_{3}+{ }_{4} \mathrm{KCl}+{ }_{2} \mathrm{H}_{2} \mathrm{O}+{ }_{2} \mathrm{I}_{2} .
\end{gathered}
$$

Each cc. of $\frac{\mathrm{N}}{\text { IO }}$ thiosulphate solution represents $0.003245 \mathrm{gm}$. of $\mathrm{Zn}$.

The Process. A definite weight of, say I gm., of zinc oxid is dissolved in dilute hydrochloric acid; an excess of ammonia is added, and then $50 \mathrm{cc}$. of a ro per cent solution of sodium arsenate. This mixture is then diluted to about $750 \mathrm{cc}$., warmed, and nitric acid added until a slight turbidity appears; then acetic acid is added instead, I cc. at a time, until the solution reacts acid to test paper.

The precipitate has now changed its character from curdy and flocculent to heavy and granular; this change being facilitated by heating and stirring. The precipitate after settling is filtered off and washed. The filter is then punctured and the precipitate washed through into a beaker; 50 to $60 \mathrm{cc}$. of dilute hydrochloric acid are added. The paper and the beaker in which the precipitate was formed are washed with dilute acid until the solution and washings measure 85 to $100 \mathrm{cc}$. 3 gms. of potassium iodid are then added, and after a few minutes standing, the titration with thiosulphate is begun. The use of starch as indicator is unnecessary, as the discharge of the iodin color gives a sufficiently delicate end-reaction.

The separation of zinc from calcium and magnesium may be made by taking advantage of the fact that calcium and magnesium are precipitated from alkaline solutions by sodium arsenate, while zinc separates from an acid solution.

The solution is made strongly ammoniacal, sodium arsenate added, and the calcium and magnesium arsenates filtered off.. The solution is then made acid with nitric and acetic acids and filtering off the precipitated zinc arsenate, and determining as above described.

Precipitation as Phosphate (Walker). $\dagger$ This method is an adaptation of the principles of Stolba's $\ddagger$ method for magnesium. It gives better results with zinc than with magnesium.

* J. A. C. S., XXII, 353 .

$\dagger$ J. A. C. S., XXIII, 468.

$\ddagger$ Chen. Centrabl., r866. 727 ; see also pages $3^{11}$ and 394 , this book. 
The Process. "To the zinc solution, which should contain ammonium chlorid, a large excess of ammonia is added; then a large excess of sodium phosphate. The solution remains clear, but if the excess of ammonia is cautiously neutralized, a white cloud is formed, as each drop of the acid falls into the strong ammoniacal liquid. On stirring, this cloud dissolves until nearly all the ammonia is neutralized, when the whole solution becomes milky.

"It should now be heated to about $75^{\circ} \mathrm{C}$. and stirred constantly, at the same time continuing the addition of dilute acid, drop by drop. In a very few minutes the precipitate becomes crystalline, and with care the liquid may be almost perfectly neutralized. It is a, good plan to add a small piece of litmus-paper to the liquid; this should not turn red but should remain blue or violet, while the hot liquid should have no odor or only a very faint odor of ammonia. When the precipitation is made as above, the zinc ammonium phosphate is easily filtered, which may be safely done after five minutes standing. The precipitate should be washed with cold water until the washings show only a faint trace of chlorids; then the paper with the precipitate is returned to the beaker in which the precipitation was made, an excess of standard acid added, a few drops of methyl orange, and the exact point of neutrality determined with standard alkali. According to the equation

$$
\mathrm{ZnNH}_{4} \mathrm{PO}_{4}+\mathrm{H}_{2} \mathrm{SO}_{4}=\mathrm{ZnSO}_{4}+\mathrm{NH}_{4} \mathrm{H}_{2} \mathrm{PO}_{4} \text {, }
$$

we see that I cc. of the normal acid corresponds to $0.03245 \mathrm{gm}$. $\mathrm{Zn}$."

Since the zinc ammonium phosphate is not precipitated in the presence of a large excess of ammonia, the process may be used in the presence of magnesium, which is precipitated in the strongly alkaline liquid, and the filtrate neutralized to precipitate the zinc.

\section{REFERENCES}

"Assay of Zinc Ores." A. H. Low. J. A. C. S., xxir, I98.

"Assay of Zinc Ores." W. G. Waring. J. A. C. S., XxvI, 4.

"Comparison of Methods of Zinc Analyses." Nissenson and Kettembeil. Chem. Ztg., xxix, 95I.

\section{"A Stzy of the Ferrocyanid Method." W. H. Seaman. J. A. C. S., Xxix, 205 \\ "Repon of Committee on Zinc Ore Analyses." J. A. C. S., Xxix, 262. \\ "Tables for Use with the Ferrocyanid Method for. Zinc Analyses." Chem. Eug., I, I 90.}

"Volumetric Method for Zinc." W. H. Keen. J. A. C. S., xxx, 225. Note on same method, Ibid. 904 .

"Assay of Zinc." E. W. Buskett. Mines and MineraIs, xxiıII, I83.

"Estimation of Zinc by Schaffner's Method." $\dot{V}$. Hassreidter. Z. angew. Chem., xxr (2), 66. 


\section{- PART III \\ CHAPTER XLIII}

\section{SANITARI ANALYSIS OF WATER}

IN collecting samples of water great care must be exercised in order to secure a fair representation of the water and to avoid the introduction of foreign matters.

The samples should be collected in clean, stoppered bottles having a capacity of about one gallon.

It is well to completely fill the bottle with water, then empty it, and again fill with the water to be analyzed.

In taking samples from lakes, reseryoirs, or slow streams the bottle should be submerged, so as to avoid collecting any water that has been in direct contact with the air.

In collecting from pump-wells a few gallons should be pumped out before taking the sample, in order to remove that which has been standing in the pump.

If the public water-supply is to be analyzed, take the water from a hydrant communicating directly with the street main, and not from a cistern.

At the time of collecting, a record should be made of those surroundings and conditions which might influence the character of the water, such as proximity of cesspools, sewers, stables, and factories.

It should also be noted whether the sample is from a deep or shallow well, a river, spring, or artesian well.

The nature of the soil and the different strata of the locality must also be taken into account.

The sample should be kept in the dark and analyzed with as little delay as possible. .

Color. This may be taken by looking down through $\mathrm{d}$ column of water in a colorless glass tube about two feet long, standing upon a piece of white paper.

A comparison is made with a second tube containing distilled water. 
Another way of determining the color is by the use of a colorless glass tube two feet long and two inches in diameter, closed at each end, with disks of colorless glass cemented on, but having a small opening at one end for filling and emptying the tube.

To use this tube, it is half filled with the water to be examined and placed in a horizontal position. A piece of white paper is held at one end of the tube, and then by looking through from the other end the color of the liquid is observed, and a comparison of tint made between the lower half of the tube containing the water and the upper half containing air.

Odor. Three or four ounces of water are placed in a small flask fitted with a cork through which is passed a thermometer; the flask is placed in a water-bath and heated to $100^{\circ} \mathrm{F}$. The flask is then shaken, the cork withdrawn, and the odor immediately observed.

In this way, satisfactory and uniform tests are obtained, and a practised nose can frequently detect the pollution.

Reaction. This may be determined by the use of a neutral solution of litmus. If an acid reaction is obtained, the water should be boiled in order to determine if it is due to carbonic acid; if the red color disappears upon boiling, the acid reaction is due to carbonic acid.

Phenolphthalein or lacmoid may also be used for this purpose.

Suspended Matter. A liter of the turbid water is passed through . a dried and weighed filter. The filter is then again dried and weighed, and the increase in weight represents the suspended matter in one liter of the water.

\section{TOTAL SOLIDS}

A platinum dish having a capacity of about $\mathrm{r} 20 \mathrm{cc}$. is heated to redness, cooled under a desiccator, and weighed. roo cc. of the water is then introduced and evaporated on a water-bath (the dish being placed on a sheet of filter-paper to prevent any deposition from the water on the bottom) until the residue appears dry. The dish and residue are then placed in an air oven (kept at a temperature of about $105^{\circ} \mathrm{C}$.) for thirty minutes, cooled in a desiccator, and weighed. In waters of exceptional purity it may be advisable to use a larger quantity, such as $250 \mathrm{cc}$.

The increase in weight of the dish represents approximately the total solids contained in the water taken.

It has been found that the figure for total solids obtained thus does not truly represent the sum of the organic and mineral matters in all cases.

'Experiments have been made with urea dissolved in varying 
quantities of water. Where the solution contained $\mathbf{I}$ gm. of urea the residue after evaporation varied from 0.98 to $0.007 \mathrm{gm}$.

Besides the possible loss of organic matter during the evaporation, some of the mineral constituents may retain with great obstinacy large quantities of water in the form of water of crystallization, which would cause an error in the opposite direction.

Thus the determination of total solids is only an approximation.

\section{ORGANIC AND VOLATILE MATTER-LOSS ON IGNITION}

Though the mineral matter in a water must to some extent be taken into account in judging of a water, the organic matter is of far greater importance. The really injurious matters are more probably the organic.

It is therefore important to determine as near as possible their quantity and nature.

It was naturally supposed that by igniting the residue obtained from evaporation of the water, the organic matter would be burned out, and that the loss of weight would then represent the organic matter.

Ignition, however, decomposes other salts which may be contained in water, and may even volatilize some wholly; therefore the loss on ignition cannot be truly called the organic matter. Hence the expressions "Organic and Volatile Matter," and "Loss on Ignition." "The determination is made as follows:

Heat the dish and residue from the "total solids" estimation to redness, cool in a desiccator, and weigh. The difference in the weight is reported as "Organic and Volatile Matter," or "Loss on Ignition." The degree of blackening which takes place, gives some idea of the probable amount of organic matter present.

\section{CHLORIN}

may be estimated by the use of decinormal or centinormal silver nitrate solution; but analysts generally use a solution of such strength that I cc. will represent $0.00 \mathrm{I} \mathrm{gm}$. of chlorin.

Standard Silver Nitrate Solution. Dissolve 4.8022 gms. of pure recrystallized silver nitrate in sufficient water to make rooo cc.

Potassium Chromate Solution. Five grams of neutral potassium chromate are dissolved in roo cc. of water and a weak solution of silver nitrate added, drop by drop, until a slight permanent red precipitate is produced, which is allowed to settle in the bottle, or separated by filtration.

The Process. Measure out roo cc. of the water to be analyzed into a beaker or white basin; add a few drops of the potassium chromate 
solution; then run in slowly from a burette the silver nitrate solution until a slight red tint appears. Note the number of cc. of silver solution used. Each cc. represents $0.00 \mathrm{I}$ gm. (I milligram) of chlorin. If the chlorin is present in small quantity, about $250 \mathrm{cc}$. of the water should be evaporated to about one fifth before titrating with the silver nitrate solution. If the basin which is being employed in the titration is placed next to a similar basin containing the same amount of potassium chromate and a volume of distilled water equal to the sample, the reading of the end-reaction is very much simplified.

Example. Ioo cc. of water taken, 4 cc. of silver solution consumed; thus showing that the $100 \mathrm{cc}$. of water contained $0.004 \mathrm{gm}$. of chlorin.

For method of stating results see Calculation of Results.

The water must be perfectly neutral before titration. If acid, it must be shaken with a little pure precipitated calcium carbonate.

\section{AMMONIA}

When organic matter decomposes spontaneously, it first forms ammonia, then nitrites, and finally nitrates. Thus the presence of ammonia in water is generally conceded to indicate decomposing organic matter, and hence its determination is an important part of the sanitary examination of water.

The ammonia is generally spoken of as free ammonia and albuminoid ammonia, or, more properly, as ammonium salts and ammonia from organic nitrogen.

The sanitary examination of a water should always include a quantitative determination of nitrogen in both compounds.

The process requires several solutions and considerable care in manipulation. The solutions required are:

I. Nessler's Solution, made by dissolving $35 \mathrm{gms}$. of potassium iodid in $100 \mathrm{cc}$. of water and $17 \mathrm{gms}$. of mercuric chlorid in $300 \mathrm{cc}$. of water. The liquids may be heated to aid solution, but if so, must be again cooled. When solution is complete, add the latter to the former until a permanent precipitate is produced; then dilute with a 20 per cent solution of sodium hydroxid to I000 cc. Now add mercuric chlorid solution again until a permanent precipitate is formed. Let the mixture stand until settled, then decant off the clear solution for use. The bulk of the solution should be kept in a well-stoppered bottle, and a small quantity transferred from time to time to a small bottle, from which it should be used. This solution improves on keeping, and reacts with extremely minute quantities of ammonia.

2. Sodium Carbonate Solution. A 20 per cent solution of pure freshly-ignited sodium carbonate in water free from ammonia. 
3. Standard Ammonium Chlorid Solution. Dissolve $0.3 \mathrm{I} 38 \mathrm{gm}$. of pure ammonium chlorid in water to roo cc. For use dilute I cc. of this solution with $99 \mathrm{cc}$. of distilled water free from ammonia. Each cc. of this solution contains $0.0000 \mathrm{Igm}$. of ammonia.

4. Alkaline Potassium Permanganate Solution. Dissolve $200 \mathrm{gms}$. of pure-potassium hydroxid and 8 gms. of pure potassium permanganate in sufficient ammonia-free water to make $1000 \mathrm{cc}$.

5. Ammonia-free Water. If the distilled water of the laboratory gives a reaction with Nessler's solution, it should be treated with sodium carbonate, about I gm. to the liter, and boiled until one fourth has been evaporated.

A good clear hydrant water when treated with sodium carbonate and distilled yields ammonia-free water. The first portion which comes over has of course some ammonia in it, and small portions of the distillate should be tested with Nessler's reagent until no more reaction is obtained; the remainder, except the very last portion, should be collected.

Ammonia-free water may also be obtained by distilling water acidulated with sulphuric acid. In the first two processes the ammonia is converted into a volatile salt and is easily dissipated, or appears in the first distillate; in the last process it is converted into a nonvolatile salt, which does not distil over.

Apparatus Required. A round bottom flask having a capacity of about $1000 \mathrm{cc}$., fitted with a one-holed rubber stopper (which has been boiled in a solution of potassium hydroxid, until free from ammonia and then boiled in distilled water), through which passes a tube projecting for a considerable distance into the tube of a Liebig condenser. The tube of the flask and the Liebig condenser are connected by a rubber connection, thus making the apparatus airtight at this point. To obtain the most satisfactory results, the tube from the flask should be of block tin and should lead to an upright condenser (which is preferably of copper). Instead of a flask a retort may be used, as shown in Fig. 8I.

The heat is applied by means of a low-temperature burner, the iron ring of which is removed, so that the retort rests directly upon the gauze.

Cylinders for Comparative-color Tests. These cylinders are made of pure colorless glass, about one inch in diameter, having a capacity of about roo cc. and graduated at $50 \mathrm{cc}$. These should either have a milk-glass foot, or should stand upon white paper. Six or more of thése are required.

The Process. The flask and condenser are thoroughly rinsed with ammonia-free water. Then about $200 \mathrm{cc}$. of ammonia-free water are introduced and about ro cc. of sodium carbonate solution 
added to make the water alkaline. The whole is then boiled by placing the flask on a piece of gauze over a Bunsen burner, until $50 \mathrm{cc}$. of distillate are obtained. This distillate is transferred to one of the colorcomparison cylinders, and $2 \mathrm{cc}$. of Nessler's reagent added. If a yellow color is produced, which develops more fully in three or five minutes, ammonia is present, either from imperfect cleansing of the apparatus of from the sodium carbonate. In this event the distillation must be carried on until the distillate no longer reacts with Nessler's reagent. The apparatus is then known to be ammonia-

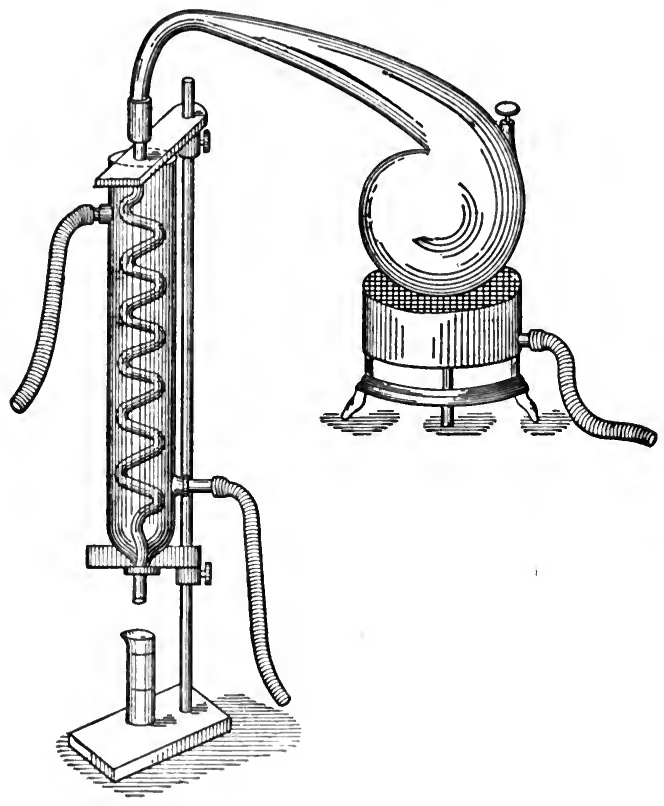

FIG 8I.

free. $500 \mathrm{cc}$. of the water under analysis are then introduced, made alkaline with Io cc. of sodium carbonate solution, and distilled. Each 50 cc. of distillate is then Nesslerized as follows, until the distillates cease to give a reaction, thus indicating that all the free ammonia has been expelled.

Prepare several standard ammonia tubes by adding to tube No. I, $\frac{1}{2}$ cc. ammonium chlorid solution; to tube No. 2, I cc. of ammonium chlorid solution; to tube No. $3, \mathrm{I} \frac{1}{2} \mathrm{cc}$; to tube No. $4,2 \mathrm{cc}$; and to tube No. $5,2 \frac{1}{2}$ cc. The tubes then contain respectively $0.000005 \mathrm{gm}$., $0.0000 \mathrm{~g} g \mathrm{gm} ., 0.000015 \mathrm{gm} ., 0.00002 \mathrm{gm}$., and $0.000025 \mathrm{gm}$. of ammonia, 
and are filled to the 50-cc. mark with ammonia-free water. 2 cc. of Nessler's reagent is then added to each tube. The amount of ammonia is then determined in each $50 \mathrm{cc}$. of distillate by adding 2 cc. of Nessler's reagent and matching the color so produced with the standard ammonia tubes, until one is found which exactly matches the color of the distillate. Knowing the strength of the standard it is easy to calculate the amount of ammonia in the distillate. The amount in each distillate is noted; the total being the amount of ammonia in the $500 \mathrm{cc}$. of water under analysis. Example:

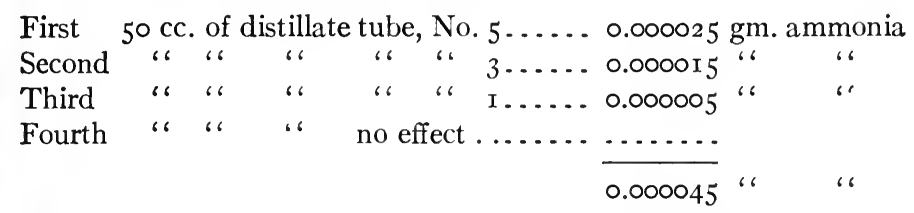

The residue in the retort serves for the determination of the nitrogen of the organic matter (albuminoid ammonia), which is converted by the alkaline permanganate into ammonia.

$50 \mathrm{cc}$. of the alkaline permanganate are placed in a porcelain dish of about $150 \mathrm{cc}$. capacity, the dish nearly filled with distilled water, and then the liquid boiled down to $50 \mathrm{cc}$.

This is added to the residue in the retort, the distillation resumed, and the ammonia estimated in each $50 \mathrm{cc}$. as described. Another way is to continue the distillation after the addition of the alkaline permanganate until the distillate no longer gives a reaction for ammonia. This insures absence of ammonia which might be accidently introduced with the reagents or otherwise. The apparatus being ammoniafree, now introduce $500 \mathrm{cc}$. of the water under analysis and distil; Nesslerize each 50 cc., as described before. Deduct the amount of free ammonia beforc found from the total found in this determination and the difference will be the "albuminoid ammonia."

It is the practice of some analysts to mix the distillates of each of the above operations, and thus make determinations merely of the total quantity of ammonia and albuminoid ammonia. By so doing valuable information may be lost, since it has been pointed out that the ammonia may be differently distributed in the distillates, according to the state, decomposing or otherwise, in which the ammonia exists in the water. If the ammonia distils over very rapidly, it indicates that the organic matter is in a putrescent or decomposing condition.

If, on the other hand, it distils gradually, it indicates the presence of organic matter in a comparatively stable or fresh condition. It is best, therefore, to keep the record of each distillate, so that the rapidity with which the ammonia is set free, as well as the actual amount, may be known. 
The greatest care should be exercised in order to avoid the introduction of ammonia in any way during the course of the analysis, since small quantities of ammonia compounds and nitrogeneous matters are everywhere present. All measuring vessels, cylinders, etc., should be thoroughly rinsed with ammonia-free water before using.

Determinations of ammonia to be accurate should be performed in a room removed from the main laboratory, where ammoniacal fumes from reagents will by no means contaminate the atmosphere.

\section{NITROGEN AS NITRATES}

Solutions Required. Acid Phenyl Sulphate. 370 gms. of strong sulphuric acid are added to 30 gms. of pure phenol and placed in a flask, which is submerged in boiling water for six hours. This reagent should be preserved in a glass-stoppered bottle.

Standard Potassium Nitrate. $0.722 \mathrm{gm}$. of pure potassium nitrate, previously heated to a temperature just sufficient to fuse it, is dissolved in water, and the solution made up to $1000 \mathrm{cc}$. I cc. of this solution will contain $0.000 \mathrm{Igm}$. of nitrogen.

The Process. A measured volume of water is evaporated just to dryness in a platinum or porcelain dish, having previously added ${ }_{10}^{10} \mathrm{cc}$. of a saturated solution of sodium carbonate. 2 cc. of the acid phenyl sulphate are added and thoroughly mixed with the residue by means of a glass rod, and the dish gently warmed. The liquid is then diluted with about $25 \mathrm{cc}$. of water, a slight excess of ammonium hydroxid (about $5 \mathrm{cc}$.) added, and the solution made up to roo cc.

The color produced is compared with that of prepared tubes of standard nitrate solution as follows: ro cc. of standard potassium nitrate solution are now similarly evaporated in a platinum or porcelain basin on a water-bath, just to dryness, and the residue moistened with 2 cc. of the acid phenyl sulphate, and mixed thoroughly with a glass rod. A small amount of water is added and the mixture made up to $1000 \mathrm{cc}$. with distilled water. Each cc. of this solution= $0.00000 \mathrm{gm}$. of nitrogen from nitrates. Definite but varying amounts of this solution are then measured into Nessler tubes of roo cc. capacity. An excess of ammonia water (about $5 \mathrm{cc}$.) is added to each tube and then distilled water to the mark. The matching of the colors is then done in a similar manner to that described for ammonia.

If $100 \mathrm{cc}$. of water under analysis are taken the amount of nitrate found will be that contained in $100 \mathrm{cc}$. The reactions are:

$$
\begin{aligned}
& \mathrm{C}_{6} \mathrm{H}_{4}(\mathrm{OH}) \mathrm{SO}_{3} \mathrm{H}+{ }_{3} \mathrm{HNO}_{3}=\mathrm{C}_{6} \mathrm{H}_{2}(\mathrm{OH})\left(\mathrm{NO}_{2}\right)_{3}+\mathrm{H}_{2} \mathrm{SO}_{4}+{ }_{2} \mathrm{H}_{2} \mathrm{O} \text {. } \\
& \text { Acid phenyl sulphate. } \quad \text { Trinitrophenal (picric acid). }
\end{aligned}
$$

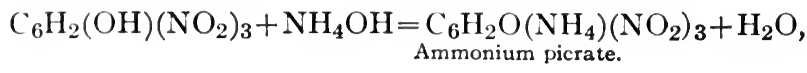


The nitric acid used in the above equation is derived from the potassium nitrate by the action of sulphuric acid.

The ammonium picrate imparts a yellow color to the solution, the intensity of which is proportional to the amount present.

Care should be taken that the same quantity of acid phenyl sulphate is used for the water and for the comparison liquid, otherwise different tints instead of depths of tints are produced.

With river or spring waters 25 to roo cc. should be evaporated for the test, but with subsoil and other waters which probably contain much nitrates to cc. will be sufficient.

\section{NITROGEN AS NITRITES}

Solutions Required. I. Naphthylammonium Chlorid (Naphthylamin Hydrochlorate). Saturated solution in water free from nitrites. It should be colorless $(0.5 \mathrm{gm}$. dissolved in roo cc. of boiling water). This solution should be kept in a glass-stoppered bottle with a little animal charcoal, which will keep the solution colorless.

2. Sulphanilic Acid (Para-amido-benzene-Sulphonic Acid). A saturated solution in water free from nitrites ( $\mathrm{I} \mathrm{gm}$. in $100 \mathrm{cc}$. of hot water).

Hydrochloric Acid. $25 \mathrm{cc}$. of concentrated pure hydrochloric acid mixed with $75 \mathrm{cc}$. of water free from nitrites.

Standard Sodium Nitrite. $0.220 \mathrm{gm}$. pure silver nitrite is dissolved in pure water, and a dilute solution of pure sodium chlorid added until a precipitate ceases to form. It is then diluted with pure water to I000 cc. and allowed to stand until clear. For use $5 \mathrm{cc}$. of this solution are diluted to I000 cc. It must be kept dark. I cc. of the dilute solution is equivalent to $0.000000 \mathrm{rgm}$. of nitrogen.

A standard solution of silver nitrite is used by some chemists, but the above is said to give better results.

The Process. $100 \mathrm{cc}$. of the water are placed in one of the colorcomparison cylinders, the measuring vessels and cylinder having previously been rinsed with the water to be tested. By means of a pipette introduce into the water I cc. each of the solutions of sulphanilic acid, dilute hydrochloric acid, and naphthylammoniùm chlorid in the order named. It is convenient to have three pipettes-one for each of these solutions, and to use them for no other purpose. In all cases the pipettes should be rinsed with ammonia-free water before using them. Into a series of roo cc. Nessler tubes, introduce varying but definite amounts of the standard nitrite solution and make up to roo cc. with pure water; then add the same reagents as were added to the water in the other cylinder.

A pink color is produced in the presence of nitrites, which requires in dilute solutions half an hour for complete development. 
At the end of that time the sample under analysis is matched, and the calculation made as explained under nitrates.

The reactions are explained by the following equations:

$$
\begin{aligned}
& \mathrm{C}_{2} \mathrm{H}_{4} \mathrm{NH}_{2} \mathrm{HSO}_{3}+\mathrm{HNO}_{2}=\mathrm{C}_{6} \mathrm{H}_{4} \mathrm{~N}_{2} \mathrm{SO}_{3}+{ }_{2} \mathrm{H}_{2} \mathrm{O} \text {; } \\
& \text { Sulphanilic acid. Para-diazo-benzene-sulphonic acid. } \\
& \underset{\substack{\text { aphthammonium } \\
\text { chlorid. }}}{\mathrm{C}_{6} \mathrm{H}_{4} \mathrm{~N}_{2} \mathrm{SO}_{3}}+\underset{\substack{\text { Azo-alpha-amido-naphthalene- } \\
\text { parazo-benzene-sulphonic acid. }}}{\mathrm{C}_{10} \mathrm{H}_{7} \mathrm{NH}_{3} \mathrm{Cl}}=\underset{\mathrm{C}}{\mathrm{C}_{10} \mathrm{H}_{6}\left(\mathrm{NH}_{2}\right) \mathrm{NCN}_{6} \mathrm{H}_{4} \mathrm{HSO}_{3}}+\mathrm{HCl} .
\end{aligned}
$$

The last-named body gives the color to the liquid.

\section{OXYGEN-CONSUMING POWER}

Potassium permanganate readily yields up its oxygen, especially in the presence of a strong mineral acid, as sulphuric. It oxidizes many salts and organic matter.

This property lead to the idea that this salt may be used for burning up (chemically speaking) the organic matter in water, and that the quantity of permanganate used could be relied upon as a means of measuring the organic matter in water.

This method does not distinguish between animal and vegetable matter, nor does the quantity of permanganate consumed represent only the organic matter.

The organic matters in water are very variable in character and condition, and their oxidability is subject to much difference.

Nevertheless, as a high oxygen-consuming power certainly indicates pollution by organic matter, the process is of considerable value.

The following is a convenient method for approximating the oxygen-consuming power of a water:

Solutions Required. Potassium Permanganate. $0.395^{2} \mathrm{gm}$. of pure potassium permanganate is dissolved in distilled water, and the solution made up to $1000 \mathrm{cc}$. I cc. of this solution will yield under favorable circumstances $0.000 \mathrm{Igm}$. of oxygen.

Diluted Sulphuric Acid. $50 \mathrm{cc}$. of pure sulphuric acid are mixed with roo cc. of water, and then just sufficient of the permanganate solution added to give the mixture a faint pink color, which remains after standing in a warm place four hours.

The Process. Five stoppered bottles having a capacity of $500 \mathrm{cc}$. are thoroughly cleansed with strong sulphuric acid and then carefully rinsed with pure water, and $250 \mathrm{cc}$. of the water to be tested put into each one. Io cc. of the dilute sulphuric acid is then added to each, together with regularly increasing quantities of the standard permanganate, say 2, 4, 6, 8, and 10 cc. respectively. 
At the end of an hour they should be examined, to see which, if any, are decolorized. At the end of the fourth hour they should again be examined, and again at the expiration of twenty-four hours.

If all of the bottles are decolorized at or before the fourth hour an additional ro cc. of the permanganate solution should be added to each bottle.

With ordinary waters the first and probably the second bottle will be decolorized, while a little color will remain in the third, and the color in the fourth and fifth will be but little diminished. In this way an approximate figure for the oxygen-consuming power of the water may be obtained, which in most cases is all that is necessary. If a closer figure is desired, the experiment may be repeated, using quantities of permanganate intermediate between those marking the limits of the reaction.

Thus if the second bottle is decolorized and a faint color still remains in the third, repeat the experiment with $5 \mathrm{cc}$. of the permanganate.

This method of procedure has an advantage over some of the other processes, because the rate of oxidation can easily be seen. This is considered by some to be of more importance than the actual amount of oxygen consumed.

It must be remembered that nitrites, ferrous salts, sulphids, etc., consume oxygen as well as organic matter. It is therefore important to boil water containing hydrogen sulphid in order to drive the latter off. Nitrites may be removed by treating the water with sulphuric acid, and boiling. The nitrite is thus converted into nitrous acid, which is driven off by the heat. Or the oxygen required to convert the nitrites present into nitrates may be deducted from the total amount of oxygen consumed. Fourteen parts of nitrogen as nitrite require sixteen parts of oxygen for oxidation into nitrate.

\section{PHOSPHATES}

Solutions Required. Ammonium Molybdate. Made by dissolving ro gms. of molybdic anhydrid in a mixture of $15 \mathrm{cc}$. of concentrated ammonia (sp.gr. 0.900) and $25 \mathrm{cc}$. of water. This solution is poured slowly, and with constant stirring, into a mixture of $65 \mathrm{cc}$. of concentrated nitric acid (sp.gr. I.4) and $65 \mathrm{cc}$. of water, and allowed to stand until clear. It should be kept dark.

The Process. One liter of the water is evaporated to about $50 \mathrm{cc}$; a few drops of a dilute solution of ferric chlorid are added, followed by a slight excess of ammonia. Ferric hydroxid is thus precipitated, which carries down with it all the phosphate. This precipitate is separated by filtration, dissolved on the filter in the smallest possible 
quantity of hot dilute nitric acid, and a little water passed through the filter. The filtrate and washings should not exceed $5 \mathrm{cc}$., and should if more, be evaporated to this bulk.

The solution is now heated nearly to boiling and $2 \mathrm{cc}$. of the ammonium molybdate solution added. If after half an hour an appreciable precipitate is formed, it is collected on a small weighed filter and its weight found after thorough drying. This weight, multiplied by 0.05 , gives the amount of $\mathrm{PO}_{4}$. If the quantity is too small to be collected and weighed in this manner, it is usually reported as "traces," "heavy traces," or "very heavy traces."

\section{HARDNESS}

The hardness of water, that is, its soap-destroying power, is due principally to the presence of calcium salts; but salts of magnesium, iron, and other metals may also contribute to this effect.

Two kinds of hardness are recognized:

I. "Temporary," which is due to the presence in water of the bicarbonates of calcium, magnesium, etc. By boiling, these salts are decomposed, the carbonic acid gas being driven off, and the neutral carbonate formed, which is precipitated. Thus the water loses its hardness upon boiling.

$$
\mathrm{CaH}_{2}\left(\mathrm{CO}_{3}\right)_{2}=\mathrm{CaCO}_{3}+\mathrm{H}_{2} \mathrm{O}+\mathrm{CO}_{2} \text {. }
$$

2. "Permanenţ" hardness is due to the presence in water of salts of the above-mentioned metals which are not removed by boiling, such as the sulphates.

Hardness is estimated by means of a standard soap solution.

Many samples of water possess both temporary and permanent hardness, and it is sometimes desirable to estimate them separately.

The total hardness is estimated in one sample, and the hardness in another sample is determined after boiling and filtering off the precipitated calcium carbonate.

The hardness found after boiling is the permanent hardness, and is the most objectionable form. The difference between the total and permanent hardness is the temporary hardness. To express the hardness in some tangible form, the usual custom in this country and in England is to give results in the corresponding amounts of calcium carbonate, i.e., practically to determine the amount of soap destroyed by a measured quantity of water, and then to state the results as the amount of calcium carbonate which would destroy that quantity of soap. 
The reaction which takes place when soap is added to a hard water, is illutsrated in the following equations:

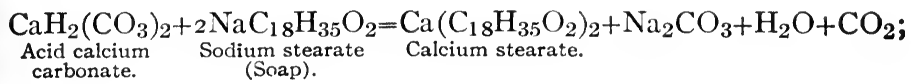

or,

$$
\underset{\text { Calcium sulphate. }}{\mathrm{CaSO}_{4}+\mathrm{NaC}_{18} \mathrm{H}_{35} \mathrm{O}_{2}=\mathrm{Ca}\left(\mathrm{C}_{18} \mathrm{H}_{35} \mathrm{O}_{2}\right)_{2}+\mathrm{Na}_{2} \mathrm{SO}_{4}} \text {. }
$$

The calcium stearate, which is an insoluble calcium soap, is precipitated in both cases as a white curd-like mass.

The method for estimating hardness in water by the use of soap solution is known as Clark's method.

Solutions Required. Standard Soap Solution. Dissolve to gms. of shavings of air-dried Castile soap in a liter of dilute alcohol. Filter the solution if it is not clear, and keep it in a tightly-stoppered bottle.

Standard Calcium Chlorid Solution. Dissolve I gm. of pure calcium carbonate in the smallest excess of hydrochloric acid, then carefully neutralize with ammonia water, and add sufficient water to make up to one liter.

One cc. of this solution will contain the equivalent of $0.00 \mathrm{Im}$. of calcium carbonate. This solution is used for determining the strength of the soap solution, which is done as follows:

Measure ro cc. of this solution into a glass-stoppered bottle capable of holding about $250 \mathrm{cc}$.; add $90 \mathrm{cc}$. of distilled water, and run in the soap solution, drop by drop, from a burette until a lather is formed, which remains for five minutes, and extends over the entire surface of the liquid when the bottle is placed in a horizontal position. Note the number of cc. of soap solution used.

We now repeat the experiment with roo $\mathrm{cc}$. of distilled water. The amount of the soap solution required to produce a permanent lather with the distilled water must be deducted from the amount used in the first test. Usually it will be about one half or one cc.

The ro cc. of the calcium chlorid solution contained the equivalent of o.oro gm. of $\mathrm{CaCO}_{3}$. Suppose in the above-mentioned test $8.5 \mathrm{cc}$. of the soap solution were used to produce a permanent lather, and $0.5 \mathrm{cc}$. were used by the distilled water. Then $8 \mathrm{cc}$. were used to precipitate $0.010 \mathrm{gm}$. of $\mathrm{CaCO}_{3}$. Thus each cc. of this soap solution will represent $\frac{1}{8}$ of $0.010 \mathrm{gm} .=0.00125$ of calcium carbonate.

The soap solution may either be used as it is, or it may be diluted with dilute alcohol so that about 10.5 or I I $\mathrm{cc}$. of it will be required to produce a permanent lather with Io cc. of the standard calcium 
chlorid solution. If so diluted each cc. will represent $0.00 \mathrm{Igm}$. of $\mathrm{CaCO}_{3}$.

This is a convenient strength, because if $100 \mathrm{cc}$. of water are operated upon, each cc. of the soap solution used will represent I part of $\mathrm{CaCO}_{3}$ in 100,000 parts of water.

Measure roo cc. of the water under analysis into the well-stoppered bottle. Add the soap solution gradually from a burette in fractions of a cc. at a time, shaking well after each addition until a soft lather is obtained, which, if the bottle is placed at rest on its side, remains continuous over the whole surface for five minutes.

The soap should not be added in large quantities at a time, even if the volume required is approximately known.

If magnesium salts are present, a kind of scum (simulating a lather) will be seen before the reaction is completed. The character of this scum must be carefully watched, and the soap solution added very carefully, with an increased amount of shaking after each addition. The point when the false lather due to the magnesium salt ceases and the true persistent lather is produced is comparatively easy to distinguish.

If more than $23 \mathrm{cc}$. of the soap solution are consumed by the roo cc. of water, a smaller quantity of water should be taken (say 50 or $25 \mathrm{cc}$.) and made up to roo cc. with distilled water, recently boiled. In such case the quantity of soap solution used must be multiplied by 2 or 4 .

If the first-mentioned soap solution is used each cc. represents $0.00125 \mathrm{gm}$. If the second solution is used each cc. represents $0.00 \mathrm{Igm}$. of $\mathrm{CaCO}_{3}$, and if $100 \mathrm{cc}$. of water are acted upon each cc. represents I part of $\mathrm{CaCO}_{3}$ in 100,000 .

If $70 \mathrm{cc}$. of water are acted upon, instead of Ioo cc., each cc. of soap solution used represents I gm. per $70,000 \mathrm{cc}$., which corresponds to I gr. per imperial gallon ( 70,000 grs.) or I degree of hardness.

These estimations are, however, only approximate, for the lather does not form until the reaction between the soap and the calcium in the water is completed, and then the quantity of soap solution required to produce the lather depends upon its strength.

Dr. Clark, the originator of this method, has shown that 1000 grains of distilled water (free from hardness) require I.4 measures of soap solution, each measure being the volume of Io grains of distilled water at $\mathrm{I}^{\circ} \mathrm{C}$.

For Permanent Hardness. To determine the hardness after boiling, measure roo cc. of the water under analysis into a flask, and with a file make a mark on the flask to indicate this volume; then add $100 \mathrm{cc}$. of distilled water, and boil until the amount is reduced to the roo-cc. mark; filter rapidly, and test in the same manner as 
described above. One half or one cc. is deducted from the soap solution used for the calculation.

Among German chemists it is customary to designate the soapdestroying power equivalent to one part of $\mathrm{CaCO}$ in 100,000 , as one degree of hardness.

Among French chemists each degree of hardness represents one part of $\mathrm{CaCO}_{3}$ in 100,000 .

\section{INTERPRETATION OF RESULTS}

Statement of Analysis. The composition of water is generally expressed in terms of a unit of weight in a definite volume of liquid, but no fixed standard is used. The proportions are, however, most generally expressed in parts per million, this being the most satisfactory and convenient, since there are $1,000,000$ milligrams in a liter, it is readily seen that the number of milligrams of a constituent present in a liter is at once the parts per million.

Example. If in the determination of chlorin it is found that $100 \mathrm{cc}$. of water contain $0.004 \mathrm{gm}$. (4 milligrams), then one liter will contain $0.040 \mathrm{gm}$. (40 milligrams) or 40 parts per million.

Many chemists prefer to express the results in parts per hundred thousand. Sometimes, generally by English chemists, the figures are given in grains per imperial gallon of 70,000 grains; less frequently, in grains per U. S. gallon of 58,328 grains.

To calculate the grains in one U. S. gallon, multiply the milligrams in a liter by the factor 0.058328 . If it is desired to report the parts per 100,000, the figure is obtained from the number of milligrams in $100 \mathrm{cc}$. For example, roo cc. of water contain 4 milligrams ( $0.004 \mathrm{gm}$.$) , then there are 4$ parts per I00,000.

Passing Judgment. In order to pass judgment upon the analytical results from a sample of water, the analyst must know to which class of water it belongs-whether river water, well water, or artesianwell water. He must know something of the soil and geological character of the locality from which the water is obtained, as well as other conditions of the locality which might affect the quality of the water, such as proximity of stables, cesspools, sewers, factories, etc.

Color. Water of the highest purity is clear, colorless, odorless, and nearly tasteless. But the color of water is no indication of its quality. A turbid or colored water is not necessarily a dangerous one, neither is a clear, colorless water always a safe water.

Odor. For comfort, if for nothing else, potable water should be free from odor. Water sometimes has an unpleasant odor and taste, yet it may be used with perfect safety for domestic purposes. At other times the odor may give rise to suspicions which a subsequent 
examination may confirm. Thus by the odor alone the safety of the water cannot be told.

Total Solids. This is intended to represent the total solid matters dissolved in the water; but since much of the organic matter as well as some of the inorganic matter is volatilized by evaporation, the total solids obtained by this method are only the total non-volatile solids. The indication is thus lower than it should be. On the other hand, certain salts, especially calcium sulphate, retain water of crystallization, thus producing an effect in the opposite direction.

The total solids so obtained contain both organic and inorganic matters, either of which may be injurious or not. Mineral waters contain large quantities of inorganic salts. Much smaller quantities of total solids in other waters might indicate pollution.

Large quantities of mineral solids, especially of marked physiological action, are known to render water non-potable; but no absolute maximum or minimum can be assigned as the limit of safety. An arbitrary limit has, however, been fixed by sanitary authorities of 60 parts per 100,000; and if the solid residue does not exceed 57 parts per 100,000 , there is no reason for rejecting a water. Many waters, especially artesian waters, which are in constant use, contain much larger quantities.

The loss on ignition should never reach 50 per cent of the total residue.

Chlorin in potable waters is very largely derived from sodium and potassium chlorids of urine and sewage.

Food contains considerable amounts of chlorids, and still more is added by way of condiment in the shape of salt. The chlorin thus taken into the system is again thrown off in the excreta, and thus appears in the sewage; hence the presence of large quantities of chlorin in water is taken as an indication of pollution. Urine contains about 500 parts of chlorin per 100,000 . The average quantity found in sewage is about II.5 parts per 100,000. Over 5 parts per 100,000 of chlorin in a water may be considered, in most cases, to be due to pollution of the water by sewage or animal excretions. The chlorin itself is not a dangerous constituent of water, but its presence in large quantities is an unfavorable indication. Nevertheless too much dependence must not be put upon the amount of chlorin in water as a means of judging of its purity, for dangerous vegetable matter may exist in it without its presence being indicated by chlorin. The maximum amount of chlorin per 100,000, given by the Rivers Pollution Commission, is 21.5 parts, the minimum 6.5 parts. Various conditions, however, which affect the proportion of chlorin, such as the nature of the strata through which the water passes, proximity to the sea, etc., must be taken into account. 
Nitrogen in Ammonia. Ammonium compounds are usually the result of spontaneous putrefactive fermentation of nitrogeneous organic matter; nitrites are then formed, and finally nitrates. Ammonium compounds may also result from the reduction of nitrites and nitrates in the presence of excess of organic matter. Therefore in either case the presence of ammonia suggests contamination.

This fact is so generally conceded that the estimation of ammonia in water is a very important part of the sanitary examination.

In the water from deep wells an excess of ammonia is nearly always found, but its presence here cannot always be considered an adverse condition, since it is derived largely from the decomposition of nitrates, and shows previous contamination; but the water having undergone extensive filtration and oxidation, its organic matter is presumably converted into harmless bodies.

Rain-water often contains large proportions of ammonium compounds, which it dissolves out of the air in its descent; but here also, this fact cannot condemn the water, since it does not indicate contamination with dangerous organic matter.

An average of $7 \mathrm{I}$ samples of rain-water collected in England contained 0.05 parts per 100,000, including an exceptional maximum of $0.2 \mathrm{I}$ parts.

Fischer (Chemische Technologie des Wassers) gives two analyses of typically good wells, containing respectively 0.048 and 0.044 parts per 100,000, and of two typically bad shallow wells, containing respectively 0.084 and 2.227 parts per 100,000.

Albuminoid Ammonia. If water yields no albuminoid it is free from recently added organic contamination. If it contain more than 0.01 part per I00,000 it is looked upon as suspicious, and when it reaches 0.015 parts per 100,000 it is to be condemned. When free ammonia is present in considerable quantity, then the albuminoid ammonia becomes suspicious when it reaches 0.005 parts per 100,000. An opinion should not, however, be formulated without a knowledge of the source cf the water; for, as has been said before, free ammonia may exist in large quantities in deep wells without indicating contamination. Wanklyn gives the following standards:

High purity .... 0.000 to 0.004 I of albuminoid ammonia per 100,000

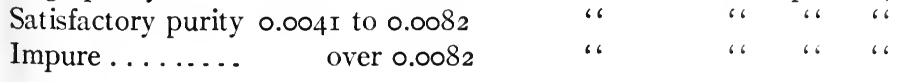

In the absence of free ammonia he does not condemn a water unless the albuminoid ammonia exceeds 0.0082 parts per 100,000; but he condemns a water yielding 0.0123 parts of albuminoid ammonia, under all circumstances. 
Nitrogen as Nitrates. Nitrates are normally present in all natural waters, and are derived chiefly from the oxidation of animal matters. The nitrogen of organic matters liberated by putrefaction, is first converted into ammonia; then this is oxidized into nitrous and finally into nitric acid. These changes are due partially to direct oxidation and partially to certain micro-organisms which have the power of converting nitrogeneous organic matter into nitrites and nitrates. Nitrates and nitrites in themselves, in the quantity in which they exist in water, are perfectly harmless. They are, however, an indication of previous contamination; and many analysts believe that a water which has once been contaminated is always open to suspicion. Others consider them of little importance in determining the impurity of a water. Water which is laden with organic matter is purified by percolating through the ground, the nitrogeneous matter being converted into nitrates; therefore deep wells may contain large quantities of nitrates without being essentially impure, while the water from shallow wells should be condemned if the nitrates are excessive.

Certain strata, as the chalk formation, yield large amounts of nitrates to water. If the nitrogen as nitrates exceeds 0.6 parts per 100,000 the water is suspected.

Nitrogen as Nitrites. Some chemists regard the presence of nitrites as an indication that the oxidation of the dangerous compounds has probably been incomplete, and accordingly condemn water in which nitrites are found. Leeds places the nitrites in American rivers at 0.03 per 100,000 . The average in good waters is placed at about 0.0014 per 100,000. When the quantity exceeds 0.02 parts per 100,000 it is considered an indication of previous contamination.

Oxygen-consuming Power. This is intended to represent the oxidizable organic matter in the water. But there are other substances in water besides organic matters which absorb oxygen, namely, nitrites, which are thus oxidized to nitrates; ferrous salts, which are oxidized into ferric salts, etc. Thus the oxygen-consuming power does not represent the organic matter alone. However, a water having a high oxygen-consuming power may be considered as polluted.

The following basis for interpreting results of this method are given by Frankland and Tidy:

Oxygen Absorbed in 3 Hours.

High organic purity . . . . . . . .

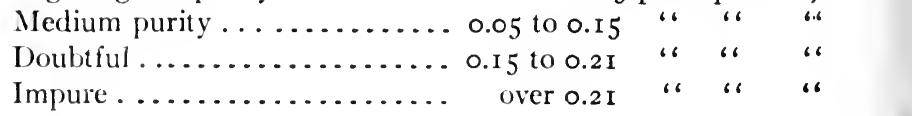


Phosphates. Sewage contains large amounts of phosphates, but water usually contains alkali or earthy carbonates, which precipitate the phosphates; therefore the absence of phosphates does not indicate purity. But their presence may indicate sewage contamination. 0.06 parts per 100,000 is regarded with suspicion (calculated as $\mathrm{PO}_{4}$ ).

Hardness. On account of the presence of considerable amounts of calcium compounds in our food, sewage is lsually very hard, containing especially calcium sulphate. The hardness of water, therefore, has some bearing upon the question as to whether the water is probably polluted with sewage or not. But water may be hard, yet otherwise perfectly pure. The test for the degree of hardness is therefore of little importance in determining sewage, as the figures below show that water uncontaminated by sewage may be very hard.

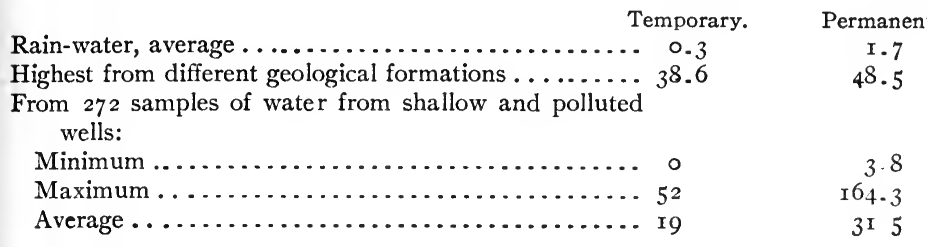

The above figures are parts in Io०,000. The hardness has, however, much significance from an economic point of view. Hard water is objectionable for domestic purposes in washing, because of its soapdestroying power, and for manufacturing purposes in boilers. It has no bad effect upon the health, but is by some considered wholesome.

Standards. Certain standards have been fixed by some chemists for determining the purity or impurity of water, according to which, if certain figures are exceeded, the water is to be condemned.

Dr. Tidy's classification depends upon the amount of oxygen consumed, from potassium permanganate, after standing three hours:

I. Great organic purity ............. o to 0.05

2. Medium purity ..................... 0.05 to 0.15

3. Doubtful ....................... 0.15 to $0.2 \mathrm{I}$

4. Impure ....................... over $0.2 \mathrm{I}$

These standards are applied to waters other than upland surfacewaters, in which larger quantities of oxygen may be absorbed.

Wanklyn's standard is based upon the indications of the amounts of free and albuminoid ammonia, as follows:

I. Extraordinary purity....... $\circ$ to 0.005 part albuminoid $\mathrm{NH}_{3}$.

2. Satisfactory purity ......... 0.005 to 0.010 " " " "

3. Dirty ................. " over 0.010 " " 
If the albuminoid ammonia exceeds 0.005 parts per 100,000 the free ammonia must be taken into account. If the free ammonia is in large quantity it is a suspicious sign. If it is in small quantity or altogether absent, the water should not be condemned, unless the albuminoid ammonia is something like 0.010 parts per 100,000; while over 0.015 should condemn the water absolutely.

The following is a list of analyses of waters which were pronounced good. The results are given in parts per 100,000:

\begin{tabular}{|c|c|c|c|c|}
\hline & I. & II. & III. & IV. \\
\hline Chlorin & 0.877 & 1.333 & 9.294 & I. 578 \\
\hline Free ammonia ....... & 0.0004 & none & 0.002 & 0.0002 \\
\hline Albuminoid ammonia ....... & none & 0.0006 & 0.005 & 0.0022 \\
\hline Oxygen absorbed (in 3 hours). & 0.0054 & 0.0016 & 0.0255 & 0.0008 \\
\hline $\mathrm{N}$ in nitrates and nitrites ...... & $0.25^{25}$ & 0.3376 & 0.0107 & 0.2633 \\
\hline Total hardness.......... & 1923 & 14.0000 & I3.33 & 2.079 \\
\hline Permanent hardness... & 3.715 & 3.934 & 3.060 & I.980 \\
\hline Organic and volatile matters .... & I 5 & 1.7 & trace & 2.100 \\
\hline Total solids (dried at $230^{\circ} \mathrm{F}$.) .. & 24.4 & 27 & $37 \cdot 40$ & $9 \cdot 40$ \\
\hline
\end{tabular}

The following were pronounced bad:

\begin{tabular}{|c|c|c|c|c|}
\hline & I. & II. & III. & IV. \\
\hline Chlorin . & 0.316 & 62.43 & 4.208 & 28.230 \\
\hline Free ammonia .... & 0.0196 & 0.278 & none & 00105 \\
\hline Albuminoid ammonia ......... & 0.0678 & 0.0030 & 0.0105 & 0.0395 \\
\hline Oxygen absorbed (in 3 hours) ..... & 0.2912 & 0.133 & 0.0165 & 0.2110 \\
\hline $\mathrm{N}$ in nitrates and nitrites ......... & 0.0283 & none & 0.247 & 0.6210 \\
\hline Total hardncss................. & 6.940 & $27 \cdot 72$ & 13.068 & $50 . \infty 0$ \\
\hline Permanent hardness ............. & $3 \cdot 5$ & $23 \cdot 76$ & 2.574 & 32.670 \\
\hline Organic and volatile matters.... & 0.5 & 19.5 & trace & 8.00 \\
\hline Total solids (dried at $230^{\circ} \mathrm{F}$.). . & 15.60 & 156.20 & 30.50 & 146.50 \\
\hline
\end{tabular}

I, Back of slaughter-house; II, Drive-well on beach; III, Well; IV, Well 30 feet deep. 


\section{CHAPTER XLIV}

\section{MILK}

MILK is the nutritive secretion of glands (the mammary glands) which are characteristic of the mammalia.

This secretion takes place as a result of pregnancy and delivery, and continues for a variable period, constituting the entire food of the young animal until it is able to live upon other foods.

The milk of different animals contains qualitatively identical or analagous ingredients to that of the cow, namely, fat (which is held in suspension), nitrogeneous matters, such as casein and albumin, milk sugar, inorganic salts, and water.

The average composition of cow's milk is as follows:

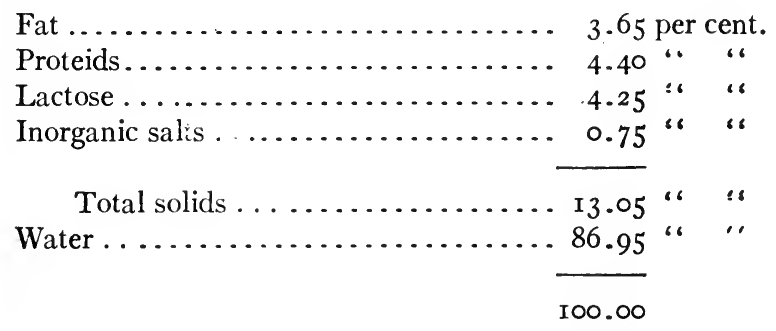

In the milk of different animals, however, these ingredients are in different proportions, as the following table shows:

\begin{tabular}{|c|c|c|c|c|}
\hline & Human. & Goat. & Mare. & Ass. \\
\hline 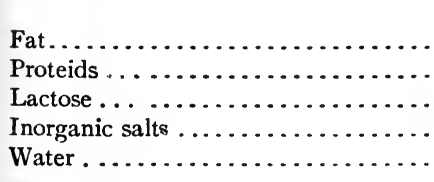 & $\begin{array}{r}\text { Per cent. } \\
3.40 \\
2.45 \\
5.75 \\
0.35 \\
88.05\end{array}$ & $\begin{array}{c}\text { Per cent. } \\
5.2 \\
3.8 \\
4.3 \\
0.7 \\
86.0\end{array}$ & $\begin{array}{rl}\text { Per cent. } & \\
\text { I } . ~ & \text { I } \\
2 & 2 \\
5 & 8 \\
0.3 \\
90 & 6\end{array}$ & $\begin{array}{rl}\text { Per cent. } & \text { cr } \\
1 & \circ \\
2 & 7 \\
5 & 3 \\
\circ & 4 \\
90 & 6\end{array}$ \\
\hline ? & $100 . \infty$ & 1000 & 100.0 & 100.0 \\
\hline Total solids . . . . . . . . . . . . . & II. 95 & 14.0 & 94 & 94 \\
\hline
\end{tabular}


Milk is a perfect natural emulsion. The casein appears to be the emulsifying agent, a film of which envelops each globule of fat, thus preventing cohesion.

The inorganic salts are chiefly the phosphates of sodium and calcium, and the chlorids of sodium and potassium, but magnesium and iron are also generally present.

The proteids consist mainly of casein with some albumen, the proportion being about as 6 to $\mathrm{I}$.

Besides the above-mentioned constituents milk also contains a very small quantity of peptone, kreatin, leucin, etc. Also gases, such as $\mathrm{CO}_{2}, \mathrm{O}$, and $\mathrm{N}$.

Colostrum is the milk secreted in the early stages of lactation; it is rich in proteids, often containing as much as 20 per cent, and contains a few corpuscles of a peculiar character, which look like epithelium cells, called colostrum corpuscles.

Reaction. The reaction of the milk of herbivorous animals is generally alkaline, that of carnivora is generally acid. The reaction of cow's milk is generally neutral, sometimes slightly acid, rarely alkaline.

Specific Gravity. This varies in normal cow's milk from 1.029 to I.035. It should not be below 1.029.

An excess of fat lowers the specific gravity and the removal of fat raises it. Thus skimmed milk has a higher specific gravity than normal milk.

These facts are made use of for the detection of the ordinary adulterations.

Determinations of the specific gravity of milk should always be made at the temperature of $60^{\circ} \mathrm{F}$, and may be made by any of the ordinary methods. See table of corrections for temperatures other than $60^{\circ} \mathrm{F}$., page 460 . A special hydrometer known as the lactometer is, however, generally used. The lactometer is graded from $\circ$ at the top to $\mathrm{I} 20$ at the bottom. In taking the specific gravity with this instrument the temperature of the milk must be $60^{\circ} \mathrm{F}$.

For every $2 \frac{1}{2}^{\circ}$ of temperature above the $60^{\circ}$ standard, one degree is to be added to the reading of the lactometer; below $60^{\circ} \mathrm{F}$. a similar subtraction is to be made.

On the lactometer scale $0=1.000$, the specific gravity of pure water; at $60^{\circ} \mathrm{F} .100=\mathrm{I} .029$, the specific gravity of the poorest allowable milk at the same temperature.

If in a sample of milk the lactometer stands at 80 the sample contains about 80 per cent of standard milk and 20 per cent of water. If the lactometer stands at 90 , the sample contains about ro per cent of water. 


\begin{tabular}{|c|c|c|c|}
\hline Lactometer Reading. & Specific Gravity. & Lactometer Reading. & Specific Gravity. \\
\hline 0 & I. 0000 & 70 & 1.0203 \\
\hline Io & I,, 0029 & 80 & I.0232 \\
\hline 20 & $1.005^{8}$ & 90 & I. 0261 \\
\hline 30 & I. .0087 & 100 & I. 0290 \\
\hline 40 & I. OI I 6 & I IO & I. 0319 \\
\hline 50 & I. OI 45 & I 20 & I. .0348 \\
\hline 60 & I. OI 74 & $\cdot$ & \\
\hline
\end{tabular}

The Adulterations of Milk. The adulterations usually practised are the abstraction of cream (skimming) or the addition of water, or both. Occasionally the addition of some foreign substance, as sodium carbonate, borax, common salt, or sugar, is met with; or preservatives, as formaldehyde, boric or salicylic acids.

The detection of adulterations usually depends upon the deternation of the specific gravity, the fat, total solids, and the ash.

These ingredients are, however, present in milk in varying proportions, and hence certain limits of allowable variations have been determined upon from time to time.

The standard adopted in many States in this country is, for specific gravity, not less than I.029, for total solids, not less than 12 per cent, for fat 3 per cent. The total solids may vary legally from I 2 to I3.I3 per cent, and the solids not fat, from 8.5 to 9.5 per cent.

Estimation of Total Solids and Water. A small, shallow platinum or porcelain dish about $\mathrm{I}_{2}^{\frac{1}{2}}$ inches in diameter is heated to redness, allowed to cool, and weighed. $5 \mathrm{cc}$. of milk (the specific gravity of which has been accurately determined) are then put in, and the dish and contents placed on a water-bath and kept there until there is no further loss in weight. In conducting the evaporation on the water-bath, it is a good plan to place below the dish a piece of filter paper in order to prevent depositions from the water on the bottom of the dish. The weight of the dry residue, minus the tare of the dish, equals the total solids.

Then by multiplying this by roo, and dividing by the weight of milk taken, the percentage of total solids is found. Thus

$$
\frac{\text { Total solids } \times \text { roo }}{\text { weight of milk }}=\text { per cent of total solids. }
$$

This deducted from roo gives the per cent of water. The weight of the milk taken is the volume multiplied by the specific gravity.

Fat. Where great accuracy is unnecessary the fat may be determined by treating the total solid residue with successive portions of warm ether until the fat is completely dissolved out. The ethereal 


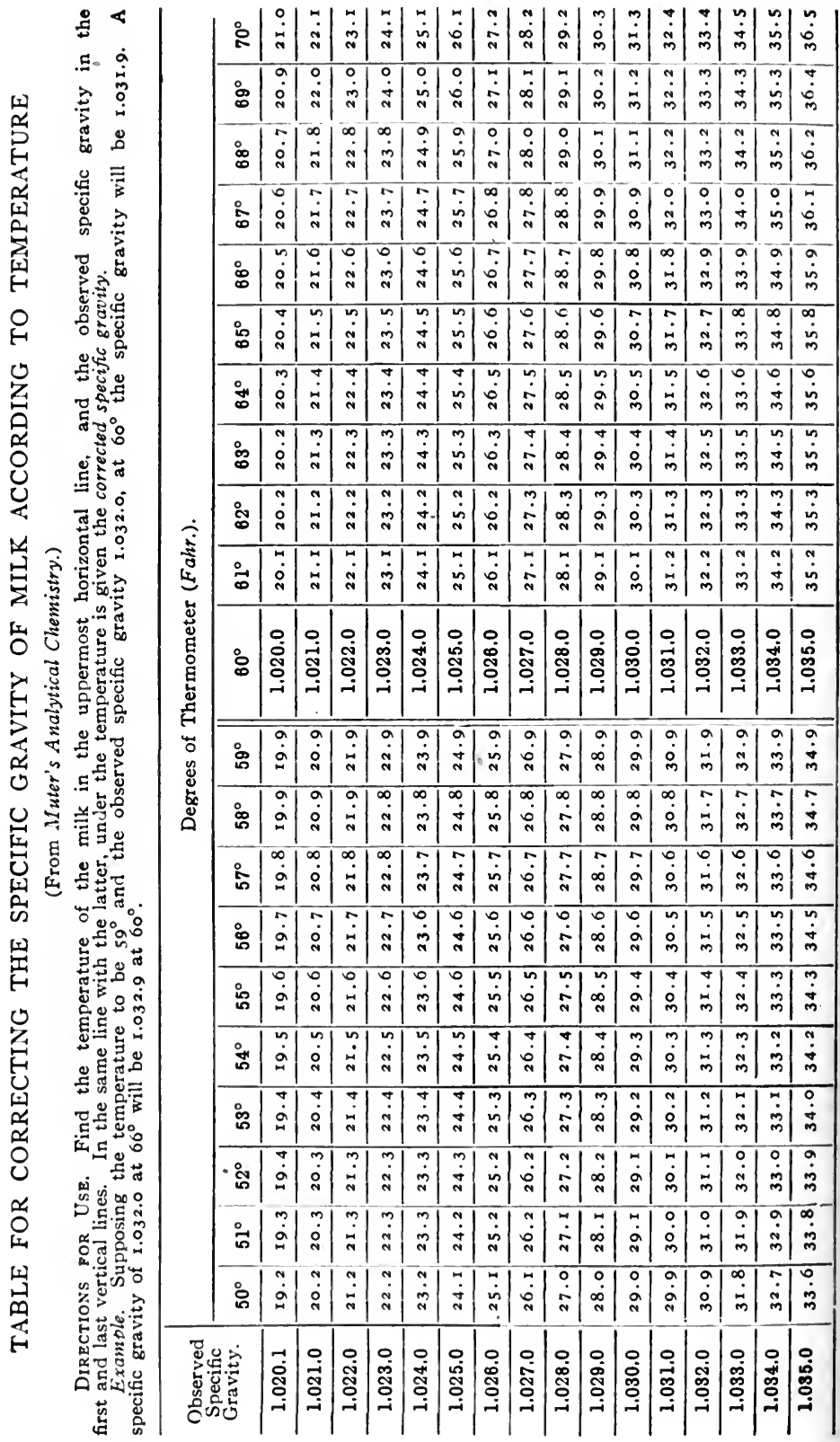


solution is then evaporated and the fat which remains behind is weighed, or the residue in the dish may be again weighed. The loss of weight then represents the fat. The results so obtained are 0.3 to 0.5 per cent too low.

Adams' Method is the officially recognized method for the accurate estimation of fat in milk.

This consists essentially in spreading the milk over absorbent paper, drying, and extracting the fat. The paper used for this purpose must previously have been thoroughly exhausted with alcohol and ether, and should be in long narrow strips.

The procedure is as follows: $5 \mathrm{cc}$. of the milk are put into a small beaker and weighed. A strip of the absorbent paper which has been rolled into a coil is thrust into the beaker containing the milk. In a few minutes nearly the whole of the milk will be absorbed; the coil is then withdrawn, and stood dry end down upon a sheet of glass.

It is important to take up the whole of the milk from the beaker, as the paper has a selective action, removing the watery constituents by preference over the fat. The beaker is again weighed, and the milk taken found by difference.

The paper charged with the milk is now dried in a water over and placed into the tube $C$ of a Soxhlet extraction apparatus (Fig. 82). About $75 \mathrm{cc}$. of ether are introduced into the tared flask $A$ of the apparatus, the reflux condenser $D$ attached and heat applied to $A$ by means of a water-bath and continued until exhaustion is complete. The flask is then detached, the ether removed by distillation, and the flask and fat which remains is weighed.

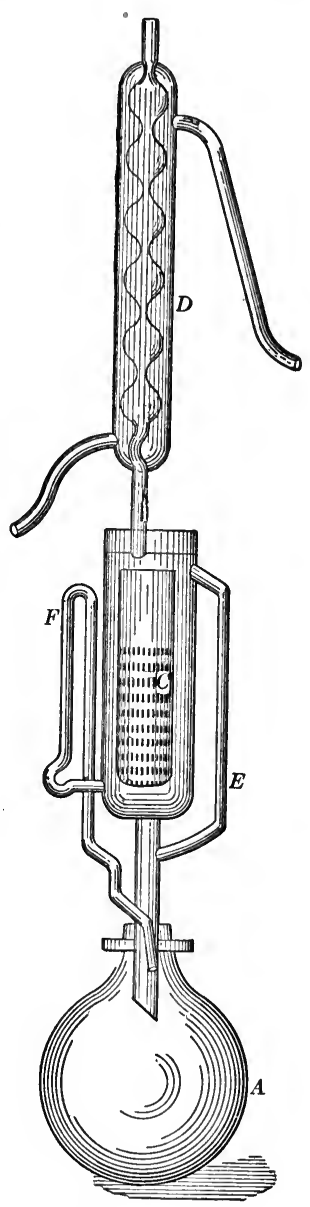

FIG. 82.

The vapor of the ether rises through $E$, condenses in $D$, and drops into the tube $C$ containing the paper charged with the milk. When the instrument has become filled with ether to the level of the top of $F$, it is automatically syphoned back into the flask charged with part of the fat. This process repeats itself until the whole of the fat is extracted, when the flask is detached and the ether removed 
by distillation as above stated. The weight of the tared flask, deducted from the weight of the flask and fat, gives the weight of the fat.

The paper may be charged with the milk by spreading the latter over the surface of the paper by means of a pipette.

The Werner-Schmid Method. This is a satisfactory and at the same time a rapid method for the determination of fat, and is especially suitable for sour milk.

ro cc. of the milk are put into a long tube having a capacity of about $50 \mathrm{cc}$, and ro cc. of strong hydrochloric acid are added; or the milk may be weighed in a small beaker and

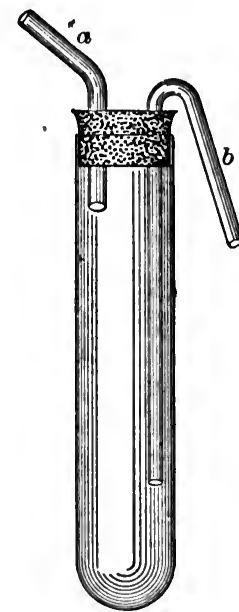

FIG. 83. washed into the tube with the acid. The liquids are mixed and boiled for $1 \frac{1}{2}$ minutes, or until the liquid turns dark brown, but not black. The tube and contents are then cooled, and 30 cc. of ether are added, shaken, and allowed to stand until the acid liquid and ether have separated. The cork is now taken out and the wash-bottle arrangement inserted (see Fig. 83). The stopper of this should be of cork, not of rubber, since the ether has a solvent action upon the latter. The lower end of the exit tube, $b$, is adjusted so as to rest immediately above the junction of the two liquids. The ethereal solution of fat is then drawn off, by gently blowing into tube $a$, and received in a weighed beaker. Two more portions of ro cc. each are shaken successively with the acid liquid, blown out, and added to the first. The ethereal solution is then heated on a water-bath, and the residue of fat weighed. The results agree quite closely with the Adams method.

The Babcock Centrifugal Method. This method is easily and quickly manipulated, and gives very accurate results, which compare very well with those obtained by the Adams method. It is carried out as follows: Into the long-neck graduated bottle which accompanies the apparatus, add from the graduated pipette exactly I $7.6 \mathrm{cc}$. of the well mixed milk, and to this add i $7.5 \mathrm{cc}$. (about) of commercial sulphuric acid. The acid is carefully poured down the side of the bottle to allow the air to escape, and when all has been added, the contents of the bottle are mixed by giving it a rotary motion. The bottle is then placed in one of the sockets of the machine. Another bottle is then treated in exactly the same way and placed in the opposite socket, to maintain the equilibrium, the cover put on the machine, and the bottle whirled at about 700 to 1200 revolutions per minute 
for seven minutes. Hot water is then added to the base of the neck, the bottles put back in the machine and whirled for about three minutes. After this the bottles are removed and hot water added to about the 7 per cent mark on the stem of the bottle. After which they are again whirled for about two minutes and taken out of the machine. The per cent of fat is then read off direct from the stem of the bottle. Each time the bottles are whirled the cover should be on the machine. Immediately after using, the bottles should be washed out with hot water containing some alkali.

The readings should be taken at a temperature between $130^{\circ}$ and $150^{\circ} \mathrm{F}$. when the fat is wholly liquid. The apparatus is pictured in Fig. 84 .

Calculation Method. This rests upon the assumption that every per cent of solids not fat, raises the specific gravity by a definite
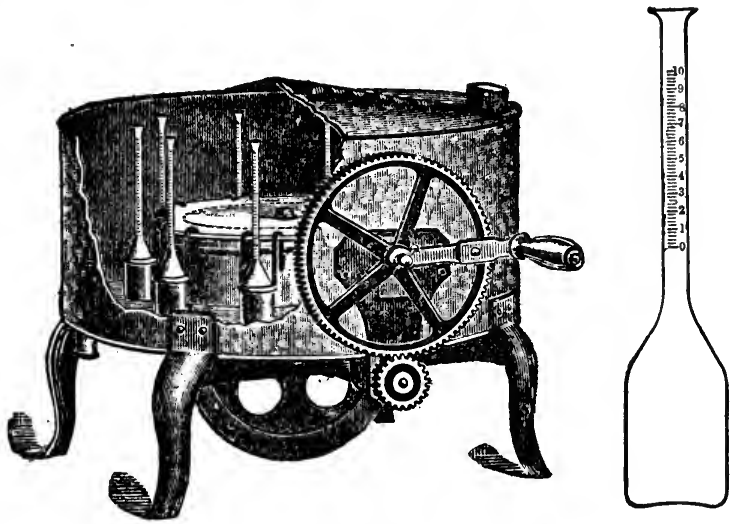

FIG. 84 .

amount, while every per cent of fat lowers it by a definite amount. An accurate determination of the per cent of total solids and of the specific gravity therefore furnish the necessary data for calculating the amount of fat.

Hehner and Richmond have devised the following formula:

$$
F=0.859 T-0.2186 G ;
$$

in which $F=$ fat, $T$ =total solids, and $G=$ the last two units of the specific gravity and any decimal. Thus if the specific gravity is 1029, $G$ will be 29; if I029.5, $G$ will be 29.5 . 
Example. Let us assume in the examination of a certain milk that the specific gravity was 1030 , and that it contained 12 per cent of total solids. We then have

$$
\text { Fat }=0.859 \times 12-0.2186 \times 30=3.75 \text { per cent. }
$$

When the per cent of fat is known, the formula may be transposed so as to calculate the total solids, as follows:

$$
T=\frac{F+0.2186 G}{0.859} .
$$

Example. A sample of milk is found to contain 3.75 per cent of fat, and its specific gravity is 1030 ; then

$$
\text { Total solids }=\frac{3.75+0.2186 \times 30}{0.859}=\mathrm{I} 2 \text { per cent. }
$$

Ash. The ash may be determined by evaporating $20 \mathrm{gms}$. of milk to dryness in the presence of $6 \mathrm{cc}$. of nitric acid, and then heating at a dull red heat until the ash is white. The organic matter is thus all burned off, and the residue is weighed and calculated as ash. The ash should be about 0.75 per cent, never below 0.67 .

To Calculate the Per-cent of Pure Milk and of Added Water, the following formula may be adopted, which is based upon the legal standard of the State of New York, viz., 3 per cent of fat, I 2 per cent of total solids, and 9 per cent of "solids not fat."

If, however, a milk has 3 per cent of fat and only 8.5 per cent of "solids not fat," it need not be considered as definitely proved to be adulterated.

The quantity of added water should, however, always be calculated upon the average standard of 9 per cent "solids not fat," provided the milk is certainly well below the limit of 8.5 per cent.

The "solids not fat" are used as a basis for the calculation because they are a fairly constant quantity, the fat being variable. The calculation is made thus:

$$
\frac{\text { "Solids not fat } " \times \text { roo }}{9}=\text { per cent of pure milk present; }
$$

and the difference between this result and roo will of course give the added water. 
Example. A sample of milk upon analysis was found to contain 8. I per cent of solids not fat; then

$$
\frac{8.1 \times 100}{9}=\frac{810.0}{9}=90 \text { per cent }
$$

of pure standard milk and ro per cent of water.

Total Proteids. The method most generally employed is the Kjeldahl Method. The following reagents and apparatus are required: Decinormal hydrochloric acid, decinormal ammonia or other alkali, saturated solution of sodium hydrate, free from nitrates (sulphuric acid, sp.gr. I.84), pulverized potassium sulphate, cochineal or methylorange indicator, Kjeldahl flasks, any convenient form of distillation flask, those described on page 287 , with bulbed outlet tube, are very satisfactory.

The process is conducted as follows: To $5 \mathrm{cc}$. of milk the specific gravity of which is known, contained in the Kjeldahl flask, add Io gms. of pulverized potassium sulphate and $20 \mathrm{cc}$. of sulphuric acid. The flask is placed in an inclined position and heated over a free flame or on a gauze; at first at a low heat until frothing ceases; then the heat is raised and continued until the mixture is either colorless or at most has a faint straw color. Then cool and transfer to a distillation flask with about $200 \mathrm{cc}$. of water, add sodium hydroxid solution to alkaline reaction, using phenolphthalein as indicator. The flask is now connected with a condenser, the joint being made tight and the distillation carried on until about $150 \mathrm{cc}$. have been distilled over into a known quantity of standard acid. To avoid bumping during the distillation, a small piece of pumice stone or a small amount of granulated zinc may be employed. Now add cochineal or methyl-orange indicator, and titrate the unneutralized acid by means of decinormal alkali.

This process depends upon the destruction of the nitrogeneous organic matter by the sulphuric acid and the formation of ammonium sulphate. This in turn is broken up by the sodium hydroxid into ammonia, which is distilled over into a measured quantity of standard acid. The loss of acidity of the acid solution is the measure of the ammonia which distilled over. Each cc. of decinormal acid $=.001393$ gm. of nitrogen, assuming that the percentage of nitrogen in the proteids of milk is 16 , the quantity of nitrogen found multiplied by 6.25 gives the proteids. Some chemists base their calculation upon the assumption that the proteids of milk contain 15.88 per cent of nitrogen, in which case the nitrogen is multiplied by 6.38 in order to obtain the quantity of proteids. 
Rittenhausen's Copper Process. The solutions required are: (I) Copper-sulphate solution, 34.64 gms. in 500 cc.; (2) Sodium-hydroxid solution, I 2 gms. to $500 \mathrm{cc}$.

Ten grams of the mllk are diluted to Ioo cc. with distilled water and placed in a beaker; $5 \mathrm{cc}$. of the copper-sulphate solution are now added and thoroughly mixed.

The sodium-hydroxid solution is now added, drop by drop, stirring constantly until the precipitate settles quickly, and the liquid is neutral or feebly acid. It should never be alkaline, as an excess of alkali prevents the precipitation of some of the proteids.

The precipitate which includes the fat carries down all of the copper. It is washed by decantation and collected upon a weighed dry filter, the contents of the filter being washed until the total filtrate measures about $250 \mathrm{cc}$. This filtrate, which contains no copper, is reserved for the determination of the sugar by Fehling's Solution.

The precipitate is washed once by strong alcohol to remove adhering water; it is then washed several times with ether to remove the fat. The residue on the filter, which consists of the proteids and copper hydroxid, is dried at $265^{\circ} \mathrm{F}$. in the air-bath and weighed. It is then transferred to a porcelain crucible and incinerated, and the residue weighed.

The weight of the filter and contents less the weight of the filter and residue after ignition, gives the weight of the proteids.

The Milk-sugar is estimated in the mixed filtrate from the precipitated proteids by the use of Fehling's Solution in the usualway. (See Estimation of Sugar.)

Detection of Formaldehyde (Hehner).* To the milk contained in a test tube add strong sulphuric acid containing a few drops of ferric chlorid solution. The acid should be carefully added so that it does not mix with the milk. At the junction of the liquids a violet or blue color will appear if the milk contains one or more parts of formaldehyde per 10,000 . If a large quantity of formaldehyde is present, the test does not work so satisfactorily, and it is best to dilute the milk.

Detection of Borax or Boric Acid. Take a small quantity of milk, make it alkaline with lime-water, and evaporate it to dryness. Ignite the residue to destroy organic matter and dissolve in a small quantity of water with the aid of hydrochloric acid, adding about I cc. of the latter in excess. Dip a piece of turmeric paper in this solution and dry it. If borax or boric acid is present the paper will acquire a peculiar red color, which is changed to dark blue-green upon moistening with ammonia water, but is restored by acid.

* Analyst, I895, XX, I55. 


\title{
CHAPTER XLV
}

\author{
BUTTER
}

AN analysis of butter comprises the estimation of water, fat, casein, ash, sodium chlorid, and also volatile fatty acids.

The water is determined by drying to a constant weight; the fat, by extraction with ether; the casein, by heating the residue after extraction of fat, to just below redness, until the former becomes white. The loss of weight represents casein and the residue ash. In the ash the chlorin may be determined by dissolving in water and titrating with standard silver nitrate solution. Salt is estimated by washing the butter with several portions of hot water and titrating the aqueous solution with standard silver nitrate solution.

The estimation of salt is conducted according to the official method of the Association of Official Agricultural Chemists, as follows:

Weigh in a counterpoised beaker from 5 to ro gms. of butter, using portions of about I gm. from different parts of the sample. Add about $20 \mathrm{cc}$. of hot water, and after the butter is melted transfer the whole to a separatory funnel. Insert the stopper and shake for a few moments. Let stand until the fat has all collected on the top of the water, then draw off the latter into a flask, being careful to let none of the fat globules pass. Again add hot water to the beaker and repeat the extraction from ten to fifteen times, using each time from Io to $20 \mathrm{cc}$. of water. The washings will contain all but a mere trace of the sodium chlorid originally present in the butter. Determine its amount in the whole or an aliquot of the liquid by the volumetric silver nitrate method, with potassium chromate as indicator.

The Estimation of Volatile Acids. This is undoubtedly the best process for detecting the admixture of foreign fats with butter. This process depends upon the fact that butter contains certain constituents which, when appropriately treated, yield volatile acids in much larger quantity than is obtained from any of the practicable substitutes for butter. These acids are principally butyric and caproic. The process consists in saponifying the fat with an alkali, then separating the fatty acids by neutralization, and distilling off 
the volatile acids for titration with standard alkali. "Reichert's number" is the number of cc. of decinormal alkali solution required to neutralize the acids distilled from $2.5 \mathrm{gms}$. of fat. The results are, however, often specified for 5 or 10 gms. of the fat.

The operations involved in this process do not admit of any arbitrary variation, and reliable and comparable results can only be obtained by strictly adhering to the prescribed details.

The following process is adopted by the Association of Official Agricultural Chemists. The solutions required are:

Sodium Hydroxid Solution. Ioo gms. of sodium hydroxid are dissolved in roo cc. of distilled water. The alkali should be as free as possible from the carbonates, and be preserved out of contact with the air.

Potassium Hydroxid Solution. Ioo gms. of the purest potassium hydroxid are dissolved in $58 \mathrm{cc}$. of hot distilled water, cooled in a stoppered vessel, and the clear liquid decanted, and preserved out of contact with the air.

Sulphuric Acid. Mix $200 \mathrm{cc}$. of the strongest acid with $1000 \mathrm{cc}$. of water.

Alcohol of about 95 per cent, redistilled from caustic soda.

Standard Barium Hydroxid Solution. Accurately standardized, approximately decinormal.

Indicator. Dissolve I $\mathrm{gm}$. of phenolphthalein in го० cc. of 95 per cent alcohol.

The process:

Weighing the Butter. The butter to be examined should be melted and kept in a dry, warm place at about $60^{\circ} \mathrm{C}$. for two or three hours, until the water and curd have entirely settled out. The clear supernatant fat is poured off and filtered through a dry filter-paper in a jacketed funnel containing boiling water. Should the filtered fat in a fused state not be perfectly clear, it must be filtered a second time. This is to remove all foreign matter and any trace of moisture. The saponification flasks are prepared by thoroughly washing with water, alcohol, and ether, wiping perfectly dry on the outside, and heating for one hour at the temperature of boiling water. The flasks should then be placed in a tray by the side of the balance and covered with a silk handkerchief until they are perfectly cool. They must not be wiped with a silk handkerchief within fifteen or twenty minutes of the time they are weighed. The weight of the flasks having been accurately determined, they are charged with the melted fat in the following way:

A pipette with a long stem, marked to deliver $5.75 \mathrm{cc}$., is warmed to a temperature of about $50^{\circ} \mathrm{C}$. The fat having been poured back 
and forth once or twice into a dry beaker in order to thoroughly mix it, is then taken up in the pipette and the nozzle of the pipette carried to near the bottom of the flask, having been previously wiped to remove any adhering fat, and $5.75 \mathrm{cc}$. of fat are allowed to flow into the flask. After the flasks have been charged in this way they should be recovered with the silk handkerchief and allowed to stand fifteen or twenty minutes, when they are again weighed.

The Saponification. Three methods may be employed:

I. Under Pressure with Alcohol. Io cc. of 95 per cent alcohol are added to the fat in the flask, and then $2 \mathrm{cc}$. of the caustic soda solution. A soft cork stopper is now inserted in the flask and tied down with a piece of twine. The saponification is then completed by placing the flask upon the water or steam bath (see Fig. 85). The flask during the saponification, which should last one hour, should be gently rotated from time to time, being careful not to project the soap for any distance up its sides. At the end of an hour the flask, after having been cooled to near the room temperature, is opened.

2. Under Pressure without the Use of Alcohol. Place 2 cc. of the potassium hydroxid in the flask containing the fat, which must be round bottomed and made of wellannealed glass to resist the pressure; cork, and heat as in the previous method. Rotate the flask very gently during the saponification, taking great care that none of

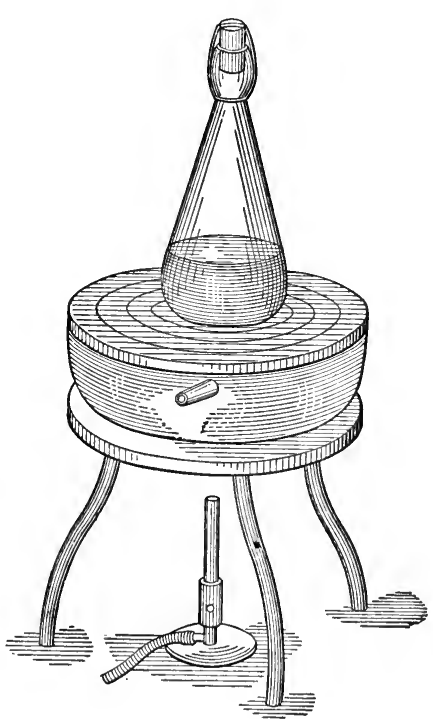

FIG. 85 . the fat rises on the sides of the flask out of reach of the alkali. Potash makes a softer soap than soda and thus allows a complete saponification without the use of alcohol. This method avoids the danger of formation of esters and the trouble of removing the alcohol after saponification.

3. With a Reflux Condenser and the Use of Alcohol. Place ro cc. of the 95 per cent alcohol in the flask containing the fat, add $2 \mathrm{cc}$. of the sodium hydroxid solution with a reflux condenser (a glass tube not less than I meter in length is allowable), and heat on the steam bath until the sapónification is complete. 
Removal of the Alcohol. The stoppers having been laid loosely in the mouth of the flask, the alcohol is removed by dipping the flask into a steam bath. The steam should cover the whole of the flask except the neck. After the alcohol is nearly removed, frothing may be noticed in the soap, and to avoid any loss from this cause or any creeping of the soap up the sides of the flask, it should be removed from the bath and shaken to and fro until the frothing disappears. The last traces of alcohol vapor may be removed from the flask by waving it briskly, mouth down, to and fro, or better, by a current of carbon dioxid free air.

Dissolving the Soap. After the removal of the alcohol the soap should be dissolved by adding $135 \mathrm{cc}$. of recently boiled distilled water

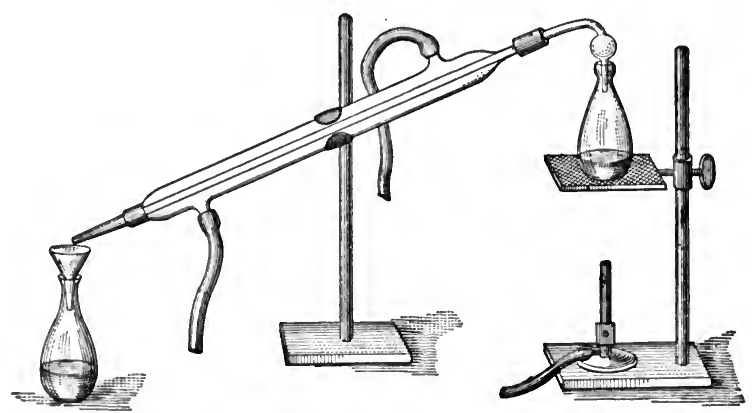

FIG. \&6.

(or I $_{2}$ cc. if potassium hydroxid was used in the saponification), warming on the steam bath, with occasional shaking until solution of the soap is complete.

Setting Free the Fatty Acids and Distilling. Cool to from $60^{\circ}$ to $70^{\circ} \mathrm{C}$., throw in a few pieces of pumice stone, add $5 \mathrm{cc}$. of the dilute sulphuric acid (or $8 \mathrm{cc}$. if potassium hydroxid was used in the saponification), stopper as in the method of saponification, and heat on the water-bath until the fatty acids form a clear, transparent layer on top of the water. This may take several hours. Cool to room temperature, add a few pieces of pumice stone, and connect with a glass condenser, as in Fig. 86.

Heat slowly with a naked flame until ebullition begins, and distil, regulating the flame in such a way as to collect I Io cc. of distillate in as nearly thirty minutes as possible.

Mix this distillate, filter through a dry filter, and titrate roo cc. with the standard barium hydroxid solution, using $0.5 \mathrm{cc}$. of phenol- 
phthalein as indicator. The red color should remain unchanged for two or three minutes.

Increase the number of cubic centimeters of tenth-normal alkali used by one-tenth, divide by the weight of fat taken, and multiply by five to obtain the Reichert-Meissl number. Correct the result by the figure obtained in a blank experiment.

When treated as above described, 5 gms. of genuine butter never yields less acidity than is represented by $24 \mathrm{cc}$. of $\frac{\mathrm{N}}{\mathrm{IO}}$ alkali. It is true, however, that the butter made from the milk of a single cow, especially towards the end of her period of lactation, has been known to fall slightly below this figure, but the average butter, as produced from the mixed milk of a herd, usually requires $27 \mathrm{cc}$. or more. Oleomargarin requires about I cc. beef-fat, and lard about the same. Cacao butter requires about $7 \mathrm{cc}$.

The percentage of butter-fat in a mixture of fats, 5 gms. being taken: $(n-0.6) \times 3.65=$ percentage of true butter-fat.

A rapid method for detecting oleomargarin or an admixture of it with butter is to heat the suspected substance in a small tin dish directly over a gas flame. If it melts quietly, foams, and runs over the dish, it is butter; if it sputters noisily as soon as heated and foams but little, it is oleomargarin.

Another way is to heat the butter for a moment with an alcoholic solution of sodium hydroxid and then empty into cold water. It gives a distinct odor of pineapples (due to ethyl butyrate), while oleomargarin gives only an alcoholic odor. 


\section{CHAPTER XLVI}

SOME TECHNICAL EXAMINATION METHODS FOR FATS, OILS, AND WAXES

The Acid Value or Proportion of Free Fatty Acids. This indicates the number of milligrams of $\mathrm{KOH}$ required to neutralize the free fatty acids in I gm. of oil, fat or wax. The standard alkali used is in alcoholic solution and may be $\frac{N}{2}, \frac{N}{5}$, or weaker, depending upon the nature of the fat. Phenolphthalein is the indicator. The fat is dissolved, according to Geissler, in ether, but alcohol or purified methylated spirit, chloroform or a mixture of alcohol and ether may be used. The solvent, whichever is used, must be free from acidity, and should be neutralized with $\frac{\mathrm{N}}{\mathrm{IO}}$ alkali if necessary.

The Process. Io gms. of the oil are accurately weighed into a flask and about $50 \mathrm{cc}$. of solvent added. A few drops of phenolphthalein are then added and the titration with alcoholic $\frac{\mathrm{N}}{2}$ potassium hydroxid solution begun, shaking constantly until the first appearance of a pink color. Care must be taken not to add too great an excess of the alkali, otherwise saponification will occur. A small excess may, however, be added and retitrated with standard acid, a more distinct end point is then obtained. In the case of waxes or solid fats, the solvent is added, heat applied until it boils, and the titration at once started.

I cc. of $\frac{\mathrm{N}}{2} \mathrm{KOH}=0.02787 \mathrm{gm}$. of $\mathrm{KOH}$ or $27.87 \mathrm{mgms}$.

The number of cc. used, multiplied by 27.87 and then divided by the weight of oil taken, gives the milligrams of $\mathrm{KOH}$ neutralized by the free fatty acids of the oil, i.e., the acid value.

The Saponification Value (Köttstorfer Number)* This indicates the number of milligrams of $\mathrm{KOH}$ required for the complete saponification of one gram of fat or oil. Reagents required are:

Alcoholic Potassium Hydroxid Solution, made by dissolving 40 gms. of potassium hydroxid in I liter of 95 per cent alcohol.

* J. Köttstorfer, I879, Zeitschr. anal. Chem.; XVIII, I99. 
Half-Normal Hydrochloric Acid Solution. Each'cc. of 'which $=27.87 \mathrm{mg}$. of $\mathrm{KOH}$.

Indicator. Phenolphthalein I gm. in I00 cc. of 95 per cent alcohol.

The Process. Into an Erlenmeyer flask capable of holding $200 \mathrm{cc}$, accurately weigh about I.5 gms. of the fat (previously purified and filtered). Run into this from a burette $25 \mathrm{cc}$. of the alcoholic potassium hydroxid solution. Then place a small funnel in the mouth of the flask or cover it loosely with a watch crystal, and set it in a steam bath for half an hour or until the fat is entirely saponified. The operation is facilitated by occasional agitation. The flask is then removed, its contents cooled, and titrated with the half-normal hydrochloric acid, using phenolphthalein as indicator. The alcoholic potassium hydroxid solution is standardized by conducting a blank experiment similar in every detail to the above with the exception that the fat is omitted.

The Köttstorfer number is then ascertained by subtracting the number of cc. of the standard hydrochloric acid used in the analysis from the number necessary to neutralize $25 \mathrm{cc}$. of the alcoholic potassium hydroxid solution in the blank experiment, multiplying the difference by 27.87 and dividing by the weight of fat taken.

Example. I.5 gms. of the fat was treated with $25 \mathrm{cc}$. of alcoholic potassium hydroxid solution, under the above described conditions, and in titrating the excess, $2 \mathrm{I} .5 \mathrm{cc}$. of $\frac{\mathrm{N}}{2}$ hydrochloric acid was required. In the blank experiment $25 \mathrm{cc}$. of the alcoholic potassium hydroxid required 32 cc. of the $\frac{\mathrm{N}}{2}$ acid. Then $32-21.5=10.5$.

$$
\frac{10.5 \times 27.87}{1.5}=195
$$

I95 is the milligrams of $\mathrm{KOH}$ neutralized by I gm. of the oil, or the Köttstorfer saponification number.

TABLE SHOWING U. S. P. REQUIREMENT AS TO SAPONIFICATION NUMBER

Lard oil ............ I95 to 197

Almond oil (expressed) ... I I I to 200

Cottonseed oil ........... I91 to 196

Linseed oil ............ I87 to 195

Cod-liver oil.......... I 75 to 185
Olive oil ........... I9 to 195

Castor oil ............ I 79 to $\mathrm{I} 80$

Theobroma oil ......... I88 to I95

Croton oil ............. 21 2 to 218

Yellow wax ........... 90 to 96 
The Reichert Number Volatile Fatty Acid Value.* This indicates the number of cc. of decinormal $\mathrm{KOH}$ required to neutralize the volatile fatty acids distilled from 2.5 gms. of fat. This method is conducted as follows: 2.5 gms. of the clear filtered fat are taken in an Erlenmeyer flask of about I50 cc. capacity with I gm. of potassium hydroxid and $20 \mathrm{cc}$. of 80 per cent alcohol, and the whole digested on a water-bath (rotating the flask frequently) until saponification is complete and the alcohol all removed. $50 \mathrm{cc}$. of water are then added, and $20 \mathrm{cc}$. of dilute sulphuric acid ( $\mathrm{r}: \mathrm{IO})$, and the mixture distilled. The distillate is collected in a $50 \mathrm{cc}$. flask into which is set a funnel with a wetted filter to collect any insoluble fat acid. The first ro or $20 \mathrm{cc}$. of distillate are returned to the flask, and the $50 \mathrm{cc}$. distilled. This is treated with a few drops of phenolphthalein and then titrated with decinormal potassium hydroxid. The number of $\mathrm{cc}$. consumed is the Reichert Number.

In the Meissl modification (the Reichert-Meissl method), 5 gms. of the fat are taken and a more complete distillation of the volatile acids effected. The method is particularly useful in determining the genuineness and purity of butter. It is described in detail in Chapter XLV.

Iodin Absorption Number of Fats and Oils (Hübl's Number). $\dagger$ This is the percentage of iodin absorbed by a fat or an oil under certain conditions. In other words it is the number of parts of iodin absorbed by Ioo parts of an oil.

Reagents required:

(a) Hübl's Iodin Solution. Dissolve 25 gms. of pure iodin in 500 cc. of alcohol, and mix this solution with $500 \mathrm{cc}$. of alcohol containing 30 gms. of pure mercuric chlorid, and set aside for twenty-four hours. The mercuric chlorid solution should be filtered if necessary before it is mixed with the alcoholic iodin solution. This solution loses its strength rapidly, and should therefore be tested before using.

(b) Decinormal sodium thiosulphate.

(c) Potassium iodid solution, 20 gms. in $100 \mathrm{cc}$.

(d) Starch paste indicator.

The Process. To about 0.3 gm. $\ddagger$ of the fat or oil, accurately weighed and dissolved in ro cc. of chloroform, contained in a glassstoppered bottle of $250 \mathrm{cc}$. capacity, add $25 \mathrm{cc}$. of the iodin solution. Stopper the bottle securely and place in a cool, dark place for four

* E. Reichert, Zeitschr. anal. Chem., XVIII, 68.

† Dingler's Polyt. Jour., 1884, 281; Am. Ch. Jour., VI, 285.

¥ In the case of drying oils which have a very high absorbent power, as linseed oil, use from 0.15 to $0.20 \mathrm{gm}$.; for oil of theobroma and similar fats, use $0.80 \mathrm{gm}$. 
hours.* At the expiration of this time, the mixture must still possess a brown color; if it does not, a further measured quantity of the iodin solution must be added and the mixture again set aside. Finally $20 \mathrm{cc}$. of the potassium iodid solution are added together with $50 \mathrm{cc}$. of water, and the mixture titrated with the decinormal sodium thiosulphate until the color is almost discharged, when a few drops of starch indicator are added and the titration continued until the solution is colorless.

The Standardization of the Iodin Solution is effected by subjecting it to the same treatment as in the assay, but with the oil omitted, and at the end of four hours, titrating with the decinormal thiosulphate.

The difference between the number of cc. of thiosulphate solution used in the blank test and the number used in the actual assay, is multiplied by 12.59 , and this divided by 3 , gives the iodin value of the oil under analysis.

When the quantity of the oil or fat taken is not $0.3 \mathrm{gm}$., then the product is not divided by 3 , but by the figure corresponding to the quantity taken; thus if $0.15 \mathrm{gm}$. are taken the product is divided by I.5.

Another way of making the calculation is as follows:

The difference between the cc. of thiosulphate used in the blank test, and the cc. used in the analysis, is multiplied by 0.01259 , then by Ioo, and the product divided by the weight in grams of the oil taken for analysis.

\section{Example:}

Number of cc. of thiosulphate used in blank test. . . . . . . $45 \cdot 4$

"، " " " " " " analysis............. $26 . \mathrm{r}$

، ، ، “ representing iodin absorbed. ........... 19.3

Iodin number is $\frac{19.3 \times 0.01259 \times 100}{0.3}=80.9$.

This method, as is well known, is based upon the fact that the unsaturated glycerids in the oils form addition products with the iodin. The mercuric chlorid and iodin contained in the alcoholic solution interact with a formation of mercuric chloriodid and iodin chlorid; the latter is supposed to be the active agent.

$$
\mathrm{HgCl}_{2}+\mathrm{I}_{2}=\mathrm{HgClI}+\mathrm{ICl}
$$

* The time allowed does not give the complete iodin absorption power of an oil or fat and cannot be compared with determinations in which six to twelve hours have been used. It gives, however, very satisfactory comparative results, but the time factor must be very closely observed. 
The mercuric chlorid also acts the part of a carrier of halogen similar to that played by mercury in the Kjeldahl process when dissolving the substance in sulphuric acid.

Gill and Adams, J. A. C. S., XxiI, I2, call attention to the fact that not only addition products, but also substitution products, are formed in this process, which vary in amount with the time of action and the strength of the solution. This is a feature which interferes with the accurate determination of the iodin number, giving as it does a higher figure. In order to prevent this formation of substitution products, and thus overcome the discrepancy in results, these authors suggest the use of mercuric iodid instead of mercuric chlorid, making the solution with methyl alcohol (free from acetone and anhydrous). They claim that by the use of a solution so made a truer iodin absorption number is obtained. A great disadvantage of the Hübl method is that the solution quickly loses its strength; another is, the length of time required for the absorption. As above described, four hours are required, but this is not sufficient time to allow of complete absorption of the iodin. It is, however, a good practice to have a fixed time limit; the process then gives very satisfactory comparative results.

The Hanus Method* for the determination of the iodin absorption number is conducted in a manner similar to the above. It differs in the composition of the iodin solution used. This is prepared as follows:

Dissolve 13.2 gms. of iodin in rooo cc. of glacial acetic acid $(99.5$ per cent), showing no reduction with potassium dichromate and sulphuric acid; add enough bromin to double the halogen content determined by titration (about $3 \mathrm{cc}$. is the proper amount). Heat may be employed for the solution of the iodin, but the liquid must be cold when the bromin is added.

The Absorption of Iodin. Add $25 \mathrm{cc}$. of the iodin solution to the chloroformic solution of the fat, allow to stand, with occasional shaking, for thirty minutes. The excess of iodin should be at least 60 per cent of the amount added.

The titration of the unabsorbed iodin is conducted as in the Hübl's method, except that only ro cc. of the potassium iodid solution are taken, and $100 \mathrm{cc}$. of water added.

The concensus of opinion among analysts is that the Hanus method is the most satisfactory. Its principal advantage over the Hübl method lies in the facts, $(a)$ that the solution (which consists of iodin bromid in acetic acid), will keep its strength, or at least change very

* Zeitschr. Nahr. u. Genus. (rgor), gr3. 
little in three months; and $(b)$ that the time required for the reaction with the oil is comparatively short, thirty minutes being usually allowed. Deiter has found, however, that ten to fifteen minutes is quite suffcient.

The Wijs Method. Wijs (in Berichte [1898] 750) uses an iodin solution which holds its titer much longer than Hübl's, and acts much more rapidly. The same results are, however, obtained. The disadvantage of Wijs's method lies in the difficulty encountered in preparing the solution, which consists of iodin monochlorid in strong acetic acid. It is prepared by dissolving 13 gms. of pure iodin in a liter of 99 per cent acetic acid, and then taking the titer by thiosulphate. Chlorin gas (free from $\mathrm{HCl}$ ), is then passed into the solution until the titer is doubled. The proper ending is known when the color changes from brown to light yellow. The process of titrating the fat is carried out exactly as in Hübl's method, except that the time required for absorption is much less. For fats of low iodin value, less than five minutes is required; for others, from five to ten minutes.

Usually fifteen to thirty minutes are given. Like the Hanus method its advantage lies in the shortness of the time required for reaction with the oil, and also in the fact that the solution remains constant for a very long time. The results obtained with it and the Hanus method compare closely with those of the Hübl method. In using the Wijs solution an excess of about forty per cent should be added to the oil.

\section{TABLE SHOWING IODIN ABSORPTION BY THE THREE METHODS}

\begin{tabular}{|c|c|c|c|}
\hline & $\begin{array}{l}\text { Hübl's No. } \\
4 \text { Hours. }\end{array}$ & $\begin{array}{l}\text { Wijs' No. } \\
\text { 30 Minutes. }\end{array}$ & $\begin{array}{l}\text { Hanus' No. } \\
\text { 30 Minutes. }\end{array}$ \\
\hline Almond oil (expressed) $\ldots \ldots \ldots \ldots \ldots \ldots \ldots$ & 98.2 & 99. I & 98.7 \\
\hline 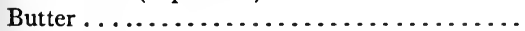 & $35 \cdot 4$ & 35.9 & 35.4 \\
\hline Castor oil $\ldots \ldots \ldots \ldots \ldots \ldots \ldots \ldots \ldots$ & $87 \cdot 3$ & 88. & 87.5 \\
\hline Cocoanut oil $\ldots \ldots \ldots \ldots \ldots \ldots \ldots \ldots \ldots$ & 8.4 & 9.I & 8.6 \\
\hline Cod-liver oil $\ldots \ldots \ldots \ldots \ldots \ldots \ldots \ldots \ldots \ldots$ & $144 \cdot 3$ & 145.8 & I 43.9 \\
\hline Cottonseed oil $\ldots \ldots \ldots \ldots \ldots \ldots \ldots \ldots$ & $104 \cdot 3$ & 106.5 & 106.6 \\
\hline Lard oil $\ldots \ldots \ldots \ldots \ldots \ldots \ldots \ldots$ & $69 \cdot 5$ & 70.5 & 69.7 \\
\hline Linseed oil $\ldots \ldots \ldots \ldots \ldots \ldots \ldots \ldots \ldots \ldots$ & I 79.6 & I 88.3 & I86.2 \\
\hline Mustard oil $\ldots \ldots \ldots \ldots \ldots \ldots \ldots \ldots \ldots \ldots$ & II 3 . I & II 8.3 & II $5 \cdot 3$ \\
\hline Olive oil $\ldots \ldots \ldots \ldots \ldots \ldots \ldots \ldots \ldots \ldots \ldots \ldots$ & 86. I & 86.7 & 86.8 \\
\hline Oleomargarin..$\ldots \ldots \ldots \ldots \ldots \ldots \ldots \ldots \ldots$ & $5^{2 . I}$ & $53 \cdot 7$ & $5^{2} \cdot 3$ \\
\hline Peanut oil...$\ldots \ldots \ldots \ldots \ldots \ldots \ldots \ldots \ldots \ldots$ & 96.3 & 99- & $97 \cdot 3$ \\
\hline Rape oil $\ldots \ldots \ldots \ldots \ldots \ldots \ldots \ldots \ldots$ & 102.4 & 105.6 & 105.2 \\
\hline Sesame oil $\ldots \ldots \ldots \ldots \ldots \ldots \ldots \ldots \ldots \ldots$ & 106.4 & 106.8 & 106.5 \\
\hline Theobroma oil $\ldots \ldots \ldots \ldots \ldots \ldots \ldots \ldots \ldots \ldots \ldots$ & $35 \cdot 4$ & 34.2 & $35 \cdot 5$ \\
\hline
\end{tabular}




\section{REFERENCES}

Volte and Logan. J. A. C. S., xxIII, 156. "A Comparison between the Bromin and Iodin Absorption Figures of Various Oils." They allow twentyfour hours for the absorption, hence their figures differ widely from those of other experimenters.

Wijs. Chem. Rev., Fett u. Hartz Ind., vi, 6 (I899).

Hanus. Zeitschr. f. Nahrung u. Genus., Iv, 913 (I90I).

Wijs. Zeitschr. f. Nahrung u. Genus., v, 499 (I902).

Tolman. J. A. C. S., XxVI, 826.

Frank and Kaminetzky. Chem. Centr., I, 696 (1905).

Tolman and Munson. J. A. C. S., Xxv, 244.

Marshall. J. S. C. I., 213 (1900).

The Bromin Value (The Bromin Absorption Number). This indicates the percentage of bromin absorbed by oils, fats or resins. The solvent used for both the fat and the bromin is either carbon disulphid or carbon tetrachlorid. The former is, however, seldom used because of its offensive odor; the latter is very satisfactory. The excess of bromin after absorption is found by titration with standard sodium thiosulphate, using potassium iodid and starch as the indicator, or by retitration with a standard solution of beta-naphthol.

This process has been much improved by Mcllhiney, J.A.C.S., I899, I084, who determines both "the bromin addition" and "the bromin substitution." The process consists briefly in adding to a weighed portion of the fat or resin in a stoppered bottle, an excess of standard solution of bromin in carbon tetrachlorid, and after the reaction has taken place, determining the excess of bromin by adding an aqueous solution of potassium iodid and titrating with standard sodium thiosulphate. Any $\mathrm{HBr}$ which may have been formed is determined in the aqueous solution. The per cent of bromin found as $\mathrm{HBr}$ is called the substitution figure, and total per cent of bromin absorbed, less twice the bromin substitution figure, gives the bromin addition figure.

The process in more detail is as follows:

A blank test is first made to determine the strength of the bromin solution as follows: $10 \mathrm{cc}$. of carbon tetrachlorid are mixed with 20 cc. of the standard bromin solution $\left(\frac{\mathrm{N}}{3}\right)$ and titrated with the sodium
thiosulphate.

In the analysis itself 0.2 of oil to be analyzed is weighed into a glass-stoppered bottle and dissolved in Io cc. of carbon tetrachlorid. $20 \mathrm{cc}$. of $\frac{\mathrm{N}}{3}$ bromin in carbon tetrachlorid are then added from a pipette, and after a minute's standing 20 to $30 \mathrm{cc}$. of ro per cent solution of 
KI are introduced, the amount necessary depending upon the excess of bromin present.

In order to prevent any loss of bromin or $\mathrm{HBr}$ on removal of the stopper, a short piece of wide rubber tubing is slipped over the lip of the bottle so as to form a well around the stopper. It is advisable also to cool the bottle by placing it in cracked ice. Into the well so formed the potassium iodid solution may be poured and the stopper opened slightly. If the bottle has been well cooled the iodid solution will be sucked into the bottle. No loss of bromin or of $\mathrm{HBr}$ is sustained by this procedure. The bottle is now shaken and the liberated iodin titrated with $\frac{\mathrm{N}}{\mathrm{IO}}$ thiosulphate. When the titration is finished, 5 cc. of a neutral 2 per cent solution of potassium iodate is added; this liberates a quantity of iodin equivalent to the $\mathrm{HBr}$ formed, and on titrating this with $\frac{\mathrm{N}}{\mathrm{IO}}$ thiosulphate, the bromin substitution figure is calculated.

The principal advantage which this process has over others "is that if the oil or fat contains substances with which bromin reacts to form substitution products, the fact will be made known by the production of $\mathrm{HBr}$, which is determined in one part of the process. The ordinary animal and vegetable fats, such as olive oil, lard, cottonseed oil, linseed oil, etc., which are practically pure glycerids, absorb almost the whole of the bromin which disappears by direct addition, that is to say, the bromin unites with pairs of carbon atoms which had previously been connected by double or triple bonds, and consequently but little $\mathrm{HBr}$ is produced by the reaction." "The most frequent adulterants of fatty oils are petroleum and rosin, both relatively cheap. Both of these react with bromin to produce $\mathrm{HBr}$, and thereby give very distinct indication of their presence." (See the original papers by McIlhiney, J. A. C. S., XVI, 275; XXI, 1084, and XXIV, II09.) 


\section{CHAPTER XLVII}

\section{ANALYSIS OF SOAP}

(a) Estimation of Water and Volatile Matters. 10 gms. of the soap are dried to a constant weight at $100^{\circ} \mathrm{C}$. and carefully weighed; the loss of weight $=$ water.

(b) Free Fats. The dried soap contained as above, is exhausted with petroleum ether of low boiling point. The petroleum ether is then evaporated off and the residue weighed; this is the weight of the fat contained in ro gms. of the soap.

(c) Fatty Acids. The residue from (b) which is free from fat and which represents Io gms. of the soap, is weighed and half of it dissolved in water. Normal nitric acid is then added in excess to liberate the fatty acids. These are collected on a tared filter, dried and weighed. This weight when doubled gives the amount of fatty acids in Io gms. of the soap. The reaction is illustrated by this equation:

$$
\underset{\text { Sodium oleate. }}{\mathrm{NaC}_{18} \mathrm{H}_{33} \mathrm{O}_{2}}+\mathrm{HNO}_{3}=\underset{\text { Oleic acid. }}{\mathrm{HC}_{18} \mathrm{H}_{33} \mathrm{O}_{2}}+\mathrm{NaNO}_{2} \text {. }
$$

The acid filtrate is now titrated with normal soda or potash, using phenolphthalein as an indicator. The difference between the volumes of acid and alkali solutions used gives roughly the quantity of total alkali.

(d) Chlorids and Sulphates. The residual neutral liquid from the above is divided into two equal parts, in one of which chlorin is estimated by $\frac{\mathrm{N}}{10} \mathrm{AgNO}_{3}$, using potassium chromate.

In the other sulphuric acid is estimated with barium chlorid.

(e) Free Alkali (i.e., the alkali which does not exist as soap). Ten gms. of the soap are dissolved in hot alcohol, and one drop of phenolphthalein T.S. added; then carbonic-acid gas is passed through the solution until the color disappears. The free alkali is thus converted into sodium carbonate, which is insoluble in alcohol and may be separated by filtration. The residue on the filter is washed with hot alcohol, and then dissolved in a little water and titrated with $\frac{\mathrm{N}}{\mathrm{IO}}$ 
acid in the presence of methyl-orange. The number of cc. used multiplied by $0.003 \mathrm{I}$ gives the grams of free alkali, as $\mathrm{Na}_{2} \mathrm{O}$, in the ro gms. of soap.

(f) Combined Alkali. The alcoholic solution from the above, which contains the combined alkali and the fatty acids, is diluted with a little water, methyl-orange added, and the mixture titrated with decinormal acid. The quantity of combined alkali is thus found. The number of cc. of acid consumed multiplied by $0.003 \mathrm{I}$ gives the quantity as $\mathrm{Na}_{2} \mathrm{O}$.

Another way is to evaporate the alcoholic solution to dryness, the residue then ignited, and the soap thus converted into alkali carbonate. This is dissolved in water and titrated with normal or decinormal acid in the presence of methyl-orange.

The fatty acids are found by using the factor 0.028014 or 0.28014 . The number of cc. of decinormal acid used in the above titration, when multiplied by 0.028014 , or of normal acid when multiplied by 0.28014 , gives the quantity of fatty acid as oleic. Soaps, however, contain various fatty acids, the molecular weights of which differ.

Therefore in estimating the fatty acids volumetrically, the neutralizing power of the acids liberated from soap, expressed in cc. of standard alkali, and called the saponification equivalent, is employed.

Geissler determines the free and combined alkali in soap as follows:

Ten grams of the soap are dissolved in roo cc. of water, phenolhpthalein T.S. added, and the solution titrated with $\frac{\mathrm{N}}{\mathrm{I}}$ hydrochloric acid until the color is just discharged. The quantity of $\frac{N}{I}$ hydrochloric acid used represents the free alkali and is calculated as carbonate.

Each cc. of $\frac{\mathrm{N}}{\mathrm{I}}$ acid $=0.05^{26} 6$ gm. of $\mathrm{Na}_{2} \mathrm{CO}_{3}$ or $0.06863 \mathrm{gm}$. of $\mathrm{K}_{2} \mathrm{CO}_{3}$.

The $\frac{\mathrm{N}}{\mathrm{r}}$ acid is now added in excess in order to liberate the fatty acids, and the mixture is heated to melt the fatty acids and cause them to form a clear oily layer on the surface. After the mixture has cooled off, the watery layer is separated and the fatty acids washed with water. The washings are added to the aqueous liquid and titrated with $\frac{\mathrm{N}}{\mathrm{I}}$ potassium hydroxid until the red color reappears; this gives the excess of acid, and when deducted from the quantity of acid added 
after decolorization of the phenolphthalein, gives the quantity of the acid which combined with and hence represents the combined alkali of the soap. This is also calculated as carbonate, using the same factors as given above.

Divine $*$ recommends the following method for determining the free alkali in soaps: To a solution 2 gms. of the soap in $50 \mathrm{cc}$. of alcohol, contained in a flask provided with a reflux condenser, an excess of $\frac{\mathrm{N}}{\mathrm{IO}}$ stearic acid is added, and the flask heated on a water-bath until a clear solution is obtained. The excess of stearic acid is then determined with $\frac{\mathrm{N}}{\mathrm{IO}}$ soda solution, the difference giving the data for total free alkali, both hydroxid and carbonate. In a second experiment with the same quantity of soap, the carbonate is removed by means of Io per cent solution of barium chlorid, and the remaining free alkali estimated as before. The difference between the total free alkali as previously ascertained and the caustic alkali as ascertained in the second experiment, gives the amount of free alkali present as carbonate in the sample.

Determination of Glycerin. E. Martin $\dagger$ recommends the following method for determining the glycerin in soaps: ro gms. of the sample is dissolved in $50 \mathrm{cc}$. of hot water. The fatty acids are liberated by the addition of a slight excess of sulphuric acid, the heat being continued until the fat is completely melted. The solution is then filtered through a wetted filter, and the fatty acids left on the filter are washed with boiling water. The filtrate is treated with excess of basic lead acetate solution, and, after standing for half an hour, the liquid is filtered into a graduated $250 \mathrm{cc}$. flask. The precipitate is washed, excess of lead removed by the addition of sulphuric acid, and the whole made up to $250 \mathrm{cc}$. After subsidence $25 \mathrm{cc}$. of the liquid is filtered or pipetted off, introduced into a conical flask with $25 \mathrm{cc}$. of potassium dichromate solution ( 84.565 gms. $\mathrm{K}_{2} \mathrm{Cr}_{2} \mathrm{O}_{7}$ per liter), $20 \mathrm{cc}$. of sulphuric acid ( $5 \circ$ per cent), and heated on the waterbath for half an hour. After cooling, the excess of dichromate is titrated back by means of ferrous ammonium sulphate (I60 gms. $\mathrm{FeSO}_{4} \mathrm{Am}_{2} \mathrm{SO}_{4} 6 \mathrm{H}_{2} \mathrm{O}$ and 20 gms. $\mathrm{H}_{2} \mathrm{SO}_{4}$ per liter) in the usual manner by spotting out on a white tile, and using potassium ferricyanid as an indicator. These solutions should first be "set" against each other. When $\mathrm{V}=$ the number of cubic centimeters of the solution of ferrous ammonium sulphate, equivalent to $25 \mathrm{cc}$. of dichromate solution, and

* Pharm. Centralh., Jan. 3, r9or. 7; from Chem. Ztg. (Rep.), r900, 330.

† Pharm. Jour., Dec. 31, 1904, 958; from Moniteur Scient. (4), 17, 797 . 
$\mathrm{v}=$ the number of cc. of this solution actually used to titrate back the unused dichromate, then $\frac{\mathrm{V}-\mathrm{v}}{\mathrm{V}} \times 25=$ the percentage of giycerin in the soap. If the combined glycerin also be required, as in the case of superfatted soap, another determination after saponification must be performed. The difference of the total and free glycerin will be the amount of the combined glycerin. 


\section{CHAPTER XLVIII}

ESTIMATION OF STARCH IN CFREALS, ETC.

By Precipitation with Barium Hydroxid.-The method about to be described depends upon the fact that when barium hydroxid is brought in contact with starch, an insoluble compound is formed, the formula of which is $\mathrm{C}_{24} \mathrm{H}_{40} \mathrm{O}_{20} \mathrm{BaO}$. This combination takes place in definite proportions, so that if an excess of barium hydroxid solution is added to the starchy substance, and then the excess estimated, the quantity which combined with and which consequently represents the amount of starch present, is found. Solutions required:

I. Decinormal Hydrochloric Acid. (3.6I8 gms. to I liter.) Each cc. represents $0.0076 \mathrm{I} 4 \mathrm{gm}$. of $\mathrm{BaO}$.

2. Baryta-water (barium hydroxid solution), made by dissolving about 7 gms. of pure crystallized barium hydroxid in rooo cc. of water. This should be kept in a special vessel, such as is illustrated in Figs. 48 or 49 .

The Process. The sample is finely powdered, and I gm. weighed out for analysis. This is rubbed up with successive portions of water (using not more than $50 \mathrm{cc}$.) and transferred to a flask having a capacity of about I5o cc. The flask and contents are now heated upon a waterbath for half an hour to thoroughly gelatinize the starch. If the substance analyzed contains oil, this must first be extracted in a "Soxhlet" apparatus before the water is added.

If free starch is to be experimented with, 0.2 or $0.3 \mathrm{gm}$. instead of I gm. should be taken.

When the starch is gelatinized, the solution is cooled, and $25 \mathrm{cc}$. of the baryta-water are added. The flask is corked, and well shaken for two minutes; proof spirit is then added to make $125 \mathrm{cc}$, the flask again corked, thoroughly shaken, and set aside to settle. While settling a check is made upon Io cc. of the baryta-water mixed with $50 \mathrm{cc}$. of recently boiled distilled water, by titrating with decinormal hydrochloric acid, using phenolphthalein as indicator. The number of cc. of $\frac{\mathrm{N}}{\mathrm{IO}}$ hydrochloric acid used, is noted, and when multiplied by $2 \frac{1}{2}$, the total strength of the 25 cc. of the baryta-water employed in the analysis is obtained. 
When the settling of the insoluble compound is completed, $25 \mathrm{cc}$. of the clear liquid is drawn off (this is $\frac{1}{5}$ of the entire quantity) with a pipette and rapidly titrated with the $\frac{\mathrm{N}}{\mathrm{IO}}$ acid V. S. in the presence of a few drops of phenolphthalein T.S. The number of cc. consumed is noted, multiplied by 5 , and then deducted from the number representing the total strength of $25 \mathrm{cc}$. baryta-water. The difference is the quantity which went into combination, with the starch.

Each cc. of the $\frac{\mathrm{N}}{\mathrm{IO}}$ hydrochloric acid represents $0.007614 \mathrm{gm}$. of $\mathrm{BaO}$, which is equivalent to $0.0324 \mathrm{gm}$. of starch.

Therefore by multiplying the number of cc. representing the quantity of baryta-water which combined with the starch by 0.0324 gm., the quantity of starch present in the sample is obtained.

Example. I gm. of substance was taken, mixed with $50 \mathrm{cc}$. of water, $25 \mathrm{cc}$. of baryta-water, and sufficient proof spirit to make I $25 \mathrm{cc}$. This is set aside and allowed to settle.

The reaction which takes place is as follows:

$$
\begin{aligned}
& { }_{2} \mathrm{C}_{12} \mathrm{H}_{20} \mathrm{O}_{10}+\mathrm{BaO}, \mathrm{H}_{2} \mathrm{O}=\mathrm{C}_{24} \mathrm{H}_{40} \mathrm{O}_{20} \cdot \mathrm{BaO}+\mathrm{H}_{2} \mathrm{O} \text {. } \\
& \text { Starch. } \\
& \text { 2) } \frac{648}{324} \quad \frac{2 \lcm{52.28}}{76.14}
\end{aligned}
$$

While settling, the strength of the baryta-water is determined by titrating with decinormal hydrochloric acid the following equation being applied:

$$
\begin{aligned}
& \mathrm{BaO}, \mathrm{H}_{2} \mathrm{O}+{ }_{2} \mathrm{HCl}=\mathrm{BaCl}_{2}+2 \mathrm{H}_{2} \mathrm{O} \text {. }
\end{aligned}
$$

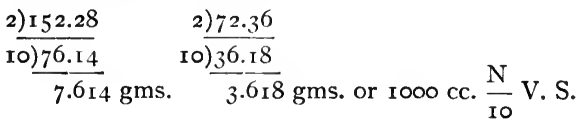

Thus each cc. represents $0.0076 \mathrm{I} 4 \mathrm{gm}$. of $\mathrm{BaO}$.

Ten cc. of the baryta-water are taken, and 8 cc. of the $\frac{\mathrm{N}}{\mathrm{IO}}$ acid solution are required to neutralize this. Therefore $25 \mathrm{cc}$. of barytawater will require $2 \frac{1}{2} \times 8 \mathrm{cc} .=20 \mathrm{cc}$. of $\frac{\mathrm{N}}{\mathrm{IO}}$ acid V.S.

When the settling is completed, $25 \mathrm{cc}$. of the clear solution is drawn off and titrated with $\frac{\mathrm{N}}{10}$ acid V. S. We will assume that $2.5 \mathrm{cc}$. of the $\frac{\mathrm{N}}{\mathrm{IO}}$ acid V. S. are required; therefore the entire quantity of solution will neutralize $5 \times 2.5 \mathrm{cc} .=\mathrm{I} 2.5 \mathrm{cc}$. 
The difference between $12.5 \mathrm{cc}$. and $20 \mathrm{cc} .=7.5 \mathrm{cc}$, which is the loss of alkalinity expressed in cc. of $\frac{\mathrm{N}}{\mathrm{IO}}$ acid V. S. Each cc. of alkalinity lost, expressed as $\frac{\mathrm{N}}{\mathrm{IO}}$ acid V. S., indicates that $0.0076 \mathrm{I} 4 \mathrm{gm}$. of $\mathrm{BaO}$ went into combination with starch; and since $0.0076 \mathrm{r} 4 \mathrm{gm}$. of $\mathrm{BaO}$ represents $0.0324 \mathrm{gm}$. of starch, the substance analyzed contains $7.5 \times 0.0324 \mathrm{gm}$. or $0.243 \mathrm{gm}$. of starch.

$$
\frac{0.243 \times 100}{I}=24.3 \text { per cent. }
$$

Estimation of Starch after Inversion. This method of estimating starch consists in converting it into glucose and then estimating the glucose with Fehling's solution.

The starch is weighed and boiled in a flask with water containing hydrochloric acid for several hours; the solution is then cooled, neutralized with potassium hydroxid, and diluted so that one part of starch, or rather sugar, shall be contained in 200 parts of water. This is put into a burette and titrated into Io cc. of Fehling's solution, as described under Sugar.

In estimating the starch in baking powder, 2 to $5 \mathrm{gms}$. of the powder are introduced into an Erlenmeyer flask, 150 to $200 \mathrm{cc}$. of a 4 per cent solution of hydrochloric acid are added and the solution gently boiled for four hours, after which the flask and contents are cooled, neutralized by adding sodium hydroxid, and made up to a definite volume. It is then ready for testing with Fehling's solution.

According to O. Lietz * the estimation of starch in substances containing but small quantities of cellulose, may be made as follows: into a flask of about $500 \mathrm{cc}$. capacity put 2 to ro gms. of the substance, according to the quantity of starch present. Add $75 \mathrm{cc}$. of alcoholic potassium hydroxid solution (containing 5 per cent of $\mathrm{KOH}$ and prepared with 90 per cent alcohol); connect with an upright condenser and warm on asbestos over a naked flame or on a waterbath, so that the alcohol boils gently for twenty minutes. Then cool somewhat and filter by suction through a plug of asbestos. Wash with hot alcohol and return the residue, together with the asbestos, to the flask, rinsing into the flask any particles which may be adhering to the funnel. Add $200 \mathrm{cc}$. of water and $20 \mathrm{cc}$. of hydrochloric acid, and invert the starch by heating on a water-bath for two and a half hours. Nearly neutralize with potassium hydroxid, leaving the liquid faintly acid, and dilute to $300 \mathrm{cc}$. Of this fluid remove $25 \mathrm{cc}$., esti-

* Berichte D. Pharm. Gesellschaft, 1902, I 53. 
mate the dextrose present (by Allihn's or Fehling:s method), and calculate it into starch; roo parts of glucose $=90.85$ parts of starch.

If a larger proportion of cellulose is present, the residue which is left after treating with alcoholic potassium hydroxid is returned to the orignial flask and 30 to $60 \mathrm{cc}$. of a 3 to 5 per cent aqueous potassium hydroxid solution added. Warm until the mass is almost entirely dissolved. Dilute to $400 \mathrm{cc}$., filter off $200 \mathrm{cc}$., neutralize with hydrochloric acid, add $20 \mathrm{cc}$. more of the acid, invert by heating, and proceed as above.

\section{Estimation of Starch after Inversion by Means of Diastase.}

The treatment of starch with malt infusion or pure diastase at a temperature not above $70^{\circ} \mathrm{C}$., readily converts the starch into maltose, but the solution also contains, besides maltose, various dextrins in proportions varying with the temperature at which the diastase acts. The digestion may vary from fifteen minutes to fifteen hours. Complete conversion of the starch may be determined by testing occasionally with iodin. A blank experiment should be made, especially if the digestion is carried on beyond half an hour. A like quantity of the same diastase solution thould be digested at the same temperature and for the same time, and the amount of sugar found deducted from the total quantity found in the analysis. Faulenbach $*$ makes use of the following solution of diastase: Crush 3.5 kilos of fresh green malt, treat with a mixture of two liters of water and four liters of glycerin and let stand for one week, stirring occasionally; then express and filter. This solution is very stable. Five drops of it will dissolve I gm. of starch; 15 drops of it contain a quantity of carbohydrate $=$ to $0.00 \mathrm{Igm}$. of glucose.

A quanticy of the substance to be tested (containing about 2 gms. of starch) is boiled to gelatinize the starch. Fifteen drops of the diastase solution are then added, and the mixture digested at $63^{\circ} \mathrm{C}$. It is then filtered to separate the undissolved cellulose, etc., and heated with $20 \mathrm{cc}$. of hydrochloric acid in a water-bath for three hours. The acidity is then just destroyed by means of caustic soda, the glucose determined, o.0oI $\mathrm{gm}$. deducted, and the starch calculated from the glucose.

O'Sullivan $\dagger$ employs pure diastase, prepared as follows: Pour sufficient water over 2 or 3 kilos of finely crushed, pale malt, to just cover it. Let stand for three or four hours, then express and filter the solution. Add alcohol (sp.gr. o.83) until the liquid above the flocculent precipitate becomes opalescent. Collect the precipitate,

* Zeitschr. f. physiol. Chem., VII, 5 10; and Chem. Centralh., I883, 632.

† Jour. Chem. Soc., XLV (1884 
wash it first with alcohol (sp.gr. 0.86 to 0.88 ) then with absolute alcohol, and press it between linen. Finally dry it in a vacuum over sulphuric.

The Determination of Starch in Cereals is effected as follows: 5 gms. of the finely-ground substance is treated successively with ether, with alcohol at $35^{\circ}$ to $40^{\circ} \mathrm{C}$., and with water at the same temperature, so as to remove the fat, sugar, soluble albuminates and soluble carbohydrates. The residue is then boiled at $63^{\circ} \mathrm{C}$. to gelatinize the starch, and allowed to cool. 0.025 to $0.035 \mathrm{gm}$. of the diastase dissolved in a small quantity of water are now added and the mixture kept at $62^{\circ}$ to $63^{\circ} \mathrm{C}$. for an hour. It is then heated to boiling, filtered, the insoluble residue washed with hot water, and the filtrate diluted to I00 cc. In this solution are determined on the one hand the maltose by Fehling's solution, and on the other hand the dextrin by polarization, deducting from the total polarization that due to maltose. Both maltose and dextrin are then calculated into starch and the results added. 


\section{CHAPTER XLIX}

\section{ESTIMATION OF SUGARS}

By Fehling's Solution. If a solution of grape sugar is warmed with caustic potash or soda, together with a cupric salt, the grape sugar is oxidized and the cupric oxid is reduced to cuprous oxid, which separates as a yellow or reddish precipitate. If there is more cupric oxid present than the grape sugar can reduce, a black precipitate of cupric oxid falls, which hides the red cuprous oxid, and hence the presence of grape and other reducing sugars may be overlooked.

In order to overcome this difficulty certain substances may be added to the alkaline solution which will prevent the precipitation of cupric oxid, but not cuprous oxid; among such substances may be mentioned glycerin and the neutral tartrates. The former is used in Haines' solution, while the double tartrate of sodium and potassium is used for this purpose in Fehling's solution.

If to a portion of hot Fehling's solution some grape sugar or other reducing sugar is added, a quantity of the cupric oxid is reduced corresponding to the quantity of sugar present. The unreduced cupric oxid remaining in solution while a distinct yellow or reddish precipitate of cuprous oxid is produced. The solution above this precipitate is a clear blue. If, however, the quantity of grape sugar added is sufficient to completely reduce the copper present, the supernatant liquid will be colorless.

Preparation of Fehling's Solution. (a) The Copper Solution. 34.67 gms. of carefully selected small crystals of pure cupric sulphate are dissolved in sufficient water to make, at or near $15^{\circ} \mathrm{C} .\left(59^{\circ} \mathrm{F}\right.$.), exactly $500 \mathrm{cc}$. Keep in small well-stoppered bottles.

(b) The Alkaline-tartrate Solution. I73 gms. of potassium and sodium tartrate (Rochelle salt) and 75 gms. of potassium hydroxid, U. S. P., are dissolved in sufficient water to make, at or near $15^{\circ} \mathrm{C}$. $\left(59^{\circ} \mathrm{F}\right.$.), exactly $500 \mathrm{cc}$. Keep in small rubber-stoppered bottles.

For use, equal quantities of the two solutions should be mixed at the time required.

One molecular weight of water-free glucose will reduce five molecular weights of cupric oxid, i.e., $\mathbf{I} 78.74$ gms. of glucose will reduce I239.25 gms. of crystallized copper sulphate $\left(\mathrm{CuSO}_{4}+{ }_{5} \mathrm{H}_{2} \mathrm{O}\right)$. If 
pure chemicals are used in the preparation of this solution there will be no need of standardizing it, but if the solution is old and its titer doubtful, the following method of standardization may be employed.

Dissolve $0.95 \mathrm{gm}$. of pure cane sugar in $50 \mathrm{cc}$. of water, add $2 \mathrm{cc}$. of hydrochloric acid, and heat to $70^{\circ} \mathrm{C}$. for ten minutes. Then neutralize with sodium carbonate and dilute to I liter.

Fifty cc. of this solution should exactly reduce ro cc. of Fehling's solution. Io cc. of the mixed Fehling's solution is equivalent to

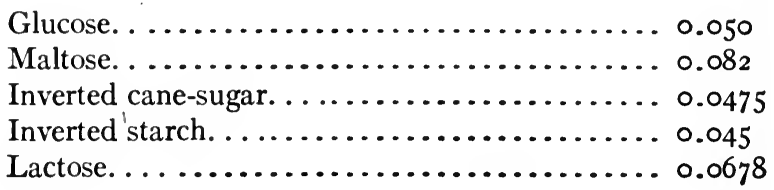

The Process. $0.5 \mathrm{gm}$. or less of the sugar is dissolved in Ioo cc. of water. This liquid is placed in a burette. Io cc. of the Fehling's solution are mixed with $40 \mathrm{cc}$. of water and placed in a porcelain dish over a Bunsen burner and heated to boiling. The sugar solution is then run in from the burette, until all blue color is destroyed.

The Calculation. Io cc. of Fehling's solution are always taken; and whatever the quantity of glucose or sugar solution is required to effect reduction, that quantity contains the equivalent of ro cc. of Fehling's solution. Thus if $\mathrm{I} 2 \mathrm{cc}$. of the sugar solution were required to reduce Io $\mathrm{cc}$. of Fehling's solution, the $12 \mathrm{cc}$. contain $0.05 \mathrm{gm}$. of glucose or $0.082 \mathrm{gm}$. of maltose, etc. I00 $\mathrm{cc}$. of the solution therefore contain $x \mathrm{gm}$. of glucose.

$$
\frac{0.05 \times 100}{12}=0.416 \mathrm{gm} . \text { glucose }
$$

In order to obtain reliable results it is important that the process be carried out exactly as laid down in the above directions, and that the quantity of sugar present in solution be no greater than one per cent. The degree of heat and the time occupied in the process, as well as the concentration of the Fehling's solution, have a very important bearing upon the accuracy of the results. The complete reduction of the copper (using undiluted Fehling's), after the addition of the requisite quantity of sugar, does not take place instantly. The time required varies somewhat with the different sugars. For instance, with glucose, invert sugar and levulose, the reduction is not complete until after heating two minutes; with maltose, four minutes, and with lactose six minutes are required. 
It is always advisable to complete the titration in as short a time as possible. A preliminary test should always be made, in which the approximate quantity of the solution required is found; then a second and more accurate titration can be done in which the sugar solution may be added more boldly, and the time of boiling and exposure of the copper solution to the air much lessened.

Determination of the End-point. It is always somewhat difficult to determine the exact point at which the blue color disappears, owing to the presence of the precipitated suboxid of copper. This difficulty may be overcome by the addition of some substance which will prevent the precipitation of the cuprous oxid, such as ammonium hydroxid or potassium ferrocyanid. When the latter is used the disappearance of the blue color can then be readily seen, as the solution remains clear to the end, turning from blue to green, and finally brown, which indicates the end of the reaction.

Professor Bartley reports this method as accurate, reliable, and rapid, provided the solution be not boiled during the reduction. $\mathrm{He}$ recommends to add to the Fehling's solution in the porcelain basin Io cc. of a io per cent freshly prepared solution of potassium ferrocyanid and $30 \mathrm{cc}$. of water. The ferrocyanid does not precipitate the copper in alkaline solution.

L. Beulaygue (Compt. rend., I38, 5I) suggests the following method for determining the end-reaction when titrating sugar solution in the usual manner with Fehling's reagent, using solution of sodium monosulphid as the indicator. When the end of the reaction is near, a little of the hot solution is applied, by means of a glass rod, to two superimposed white filter papers. The upper one acts as a filter, retaining the particles of cuprous oxid. The lower paper is withdrawn, and the moist spot touched with a drop of the sodium monosulphid reagent, when an immediate black stain of cupric sulphid is formed if the reaction is not complete. By successive spotting out and testing in this manner, a very accurate reading of the endreaction may be obtained. It is important, when standardizing the Fehling's solution, that the same indicator should be employed. Potassium ferrocyanid in a solution, acidified by either hydrochloric or acetic acid, may be employed in a similar manner. The end-reaction is then indicated by the disappearance of the red color from the last spot.

Ley and Dichgens (Pharm. Ztg., 48, No. 68 P., 689-670) employ Fehling's solution in excess, heating as usual, filtering off the reduced cuprous oxid, and treating an aliquot part of the filtrate with a standardized solution of potassium ferricyanid, also in excess. This latter excess is then titrated in the well-known manner with potassium iodid and sodium thiosulphate (see page 280 ), in a medium acidulated with $\mathrm{HCl}$, using starch paste as indicator-the reaction here being sharp 
and easily recognized. The figures thus obtained permit the accurate calculation of the quantity of Fehling's solution originally consumed, and thus of the percentage of glucose in the substance examined.

S. A. Vasey * finds that the addition of a quantity of precipitated calcium carbonate or finely powdered barium sulphate facilitates the recognition of end-point in sugar determinations with Fehling's solution. The mixture of a measured quantity of this solution with about two teaspoonfuls of either of the compounds named, is heated to the boiling point and the sugar solution is run in, the mixture being constantly stirred until the supernatant fluid becomes colorless-the exact point being easily recognized by the complete and rapid precipitation of the cuprous oxid, which is carried down by the chalk or barium sulphate, leaving the liquid limpid and transparent.

E. F. Harrison, $\nmid$ who has employed Fehling's solution somewhat extensively in quantitative sugar estimations, has found the indicators usually recommended to determine the end-point of reaction to be unsatisfactory. It was suggested by him that the action of cupric salts in liberating iodin from iodid might be utilized for this purpose with advantage, and his experiments determine the superiority of this over the other indicators heretofore proposed. The indicator is prepared by boiling $0.05 \mathrm{gm}$. of starch with a few cc. of water, adding ro $\mathrm{gm}$. of potassium iodid and diluting to roo cc. This indicator should be prepared as required. In use 0.5 to $1.0 \mathrm{cc}$. of this solution is acidified with about 5 or 1o drops of acetic acid, and one drop or more of the liquid in process of titration added. As long as unreduced copper is present, a color is produced, varying from red to blue, and of greater or less intensity, according to the nearness of the end-point. The production of no color marks the end of the reaction. The indicator is available with one drop of a solution containing one part of cupric sulphate in twenty thousand parts.

If the sugar to be cxamined be either glucose, maltose, or lactose, it may be titrated directly; but if it be cane-sugar, it must first be inverted. This is done by dissolving the sugar $(0.475 \mathrm{gm}$.) in about Ioo cc. of water, adding 3 or 4 drops of strong hydrochloric acid, and boiling briskly for ten or fifteen minutes. This is then allowed to cool, neutralized with potassium hydroxid, and made up to $100 \mathrm{cc}$. with water.

The sugar in urine may be estimated by this process. The urine is placed in the burette and run into the boiling Fehling's solution in

* Lancet, I84 (1903), I, I37. † Trans. Brit. Pharm. Conf., 1903 568, 569. 
the usual manner. If it contain a large quantity of sugar, it must be diluted two or three times.

In estimating with Fehling's solution it is well to attach a rubber tube eight to twelve inches in length to the lower end of the burette, so that the boiling need not be done directly under the burette, and thus cause incorrect readings through the expansion of the liquid therein.

Pavy's Method. This consists in adding ammonia water to the ordinary Fehling's solution, in order to prevent the precipitation of cuprous oxid, which has a tendency to hide the end-reaction. Thus the disappearance of the blue color which constitutes the end-reaction is distinctly seen.

Pavy's solution is made by dissolving 170 gms. of Rochelle salt and I70 gms. of potassium hydroxid in sufficient water. Then 34.65 gms. of copper sulphate are separately dissolved in water with the aid of heat, and the two solutions are mixed and diluted to I liter.

I20 cc. of this solution are now taken and mixed with $400 \mathrm{cc}$. of ammonia water (sp.gr. o.88) and diluted with water to I liter. This constitutes Pavy's solution, or rather Pavy-Fehling solution, of which ro cc. $=$ I cc. of Fehling's solution, i.e., ro cc. of Pavy's solution $=0.005$ gm. of glucose.

The process is conducted as follows: Io cc. of the Pavy's solution are diluted with $20 \mathrm{cc}$. of water and placed in a small flask, and heated to and kept at the boiling point, while the glucose solution properly diluted is added from a burette. The glucose solution should be added at about the rate of roo drops per minute until the blue color is just destroyed. The sugar solution should be so diluted that not less than 4 nor more than $7 \mathrm{cc}$. are required to produce the decoloration.

In order to avoid the nuisance of filling the laboratory with ammoniacal vapors, the titration may be performed in a small flask provided with a well-fitting cork, having two holes, through one of which the spit of the burette is passed, and through the other an escapetube which conducts the vapors into a vessel containing water or diluted hydrochloric acid.

Several titrations should always be made in order to obtain exact results, and it is advisable to check the solution against a sugar solution of known strength, since the ratio of reduction is seriously influenced by the amount of potassium hydroxid present and the strength of the ammonia water.

The calculation is exactly the same as that in the use of Fehling's solution, except that Io cc. of Pavy $=0.005$ gm. glucose. 
The Soxhlet-Fehling Method. The solutions required are: (a) Copper Sulphate Solution. Dissolve 34.639 gms. of $\mathrm{CuSO}_{4} \cdot{ }_{5} \mathrm{H}_{2} \mathrm{O}$ in water and dilute to $500 \mathrm{cc}$.

(b) Alkaline Tartrate Solution. Dissolve 175 gms. of Rochelle salts and $50 \mathrm{gms}$. of sodium hydroxid in water and dilute to $500 \mathrm{cc}$.

(c) Mixed Solution. Mix equal volumes of solutions $(a)$ and $(b)$ immediately before use.

A preliminary titration is made to determine the approximate per cent of reducing sugar in the material as follows: roo cc. of the mixed solution are placed in a porcelain dish, heated to boiling, and the sugar solution added in small portions at a time until the liquid, after sufficient boiling (the duration of which must depend upon the kind of sugar, see page 490), no longer appears blue. From this preliminary test calculate the approximate quantity of sugar corresponding with $100 \mathrm{cc}$. of Soxhlet-Fehling solution, as per the following table.

On boiling one per cent solutions of the different sugars with undiluted Soxhlet-Fehling solution, the following results are obtained:

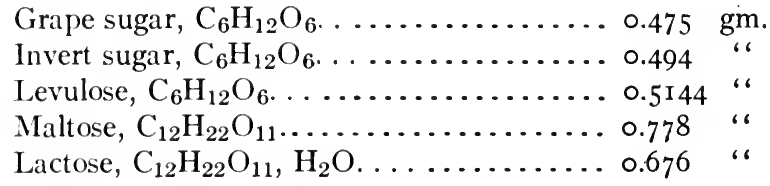

Then a solution of the sugar to be examined is made, which contains approximately one per cent. Place into a beaker $100 \mathrm{cc}$. of the mixed copper solution and add approximately the amount of the sugar solution for its complete reduction. Boil for two minutes. Filter through a folded filter and test a portion of the filtrate for copper by the use of acetic acid and potassium ferrocyanid. Repeat the test, varying the volume of sugar solution, until two successive amounts are found which differ by $0.1 \mathrm{cc}$., one giving complete reduction and the other leaving a small amount of copper in solution. The mean of these two readings is taken as the volume of the solution, required for the complete precipitation of $100 \mathrm{cc}$. of the copper reagent.

Uncler these conditions $100 \mathrm{cc}$. of the mixed copper reagent require $0.475 \mathrm{gm}$. of anhydrous dextrose or $0.494 \mathrm{gm}$. of invert sugar for compleie reduction. Calculate the percentage by the following formula:

$\mathrm{V}=$ the volume of the sugar solution required for the complete reduction of $100 \mathrm{cc}$. of the copper reagent.

$\mathrm{W}=$ the weight of the sample in I $\mathrm{cc}$. of the sugar solution. 
Then

or $\frac{100 \times 0.475}{V W}=$ per cent of dextrose, $\frac{\mathrm{roo} \times 0.494}{\mathrm{VW}}=$ per cent of invert sugar.

The Permanganate Method.* Place $60 \mathrm{cc}$. of the mixed Fehling's alkaline copper solution in a beaker, add $60 \mathrm{cc}$. of water, and heat to boiling. Add $25 \mathrm{cc}$. of the sugar solution, which must not contain more than $0.250 \mathrm{gm}$. of dextrose, and boil for two minutes. Filter immediately through asbestos without diluting, then filter through a Gooch crucible, wash the beaker and precipitate thoroughly with hot water, without any effort to transfer the precipitate to the filter. Wash the asbestos film and adhering cuprous oxid into a beaker, add about $30 \mathrm{cc}$. of hot water, and heat the precipitate and asbestos thoroughly. Rinse the crucible with $50 \mathrm{cc}$. of hot saturated solution of ferric sulphate in 20 per cent sulphuric acid, receiving the rinsings in the beaker containing the precipitate. After the cuprous oxid is dissolved, wash the solution into a large Erlenmeyer flask and immediately titrate with standard solution of potassium permanganate. One cc. of the permanganate solution should equal o.oro gm. of copper. In order to determine the strength of this solution, make six or more determinations with the same sugar solution, titrating one half of the precipitates obtained, and determining the copper in the others by electrolysis. The average weight of copper obtained by electrolysis, divided by the average number of cubic centimeters of permanganate solution required for the titration, is equal to the weight of copper equivalent to one cc. of the standard permanganate solution. A solution standardized with iron or oxalic acid will give too low results.

METHODS DEPENDING UPON THE REDUCTION OF MERCURY COMPOUNDS

Knapp's Method. The Standard Mercuric Cyanid Solution. Io gms. of pure dry mercuric cyanid are dissolved in water, roo cc. of caustic soda solution (sp.gr. r.145) are added, and the liquid diluted to measure I liter.

The method as originally used by Knapp consisted in adding the sugar solution gradually to the mercurial solution, was found by Soxhlet and others to be inaccurate. Brumme, and also Soxhlet, assert that the reducing effect of sugar is greater when the requisite quantity

\footnotetext{
* One of the methods of the Assoc. of Official Agricultural Chemists, Bulletin No. 107 .
} 
of the sugar solution is added all at once, in a manner analogous to that of the Soxhlet-Fehling method. Ioo cc. of the mercury solution are taken, and as near as can be judged the entire requisite quantity of the 0.5 or I per cent sugar solution added all at once, the liquid boiled for two or three minutes and then tested to see if it still contains mercury. Several tests are made with larger and smaller quantities of sugar solutions until two tests are made in which the quantity of sugar differs but very little, one of which containing, however, a slight quantity of mercury and the other being free from it.

The end of reaction is found by placing a drop of the clear liquid above the precipitate on a piece of white filter paper and holding this over an open bottle of fuming hydrochloric acid, and then over strong sulphureted hydrogen water. A light brown stain so produced indicates mercury.

According to Soxhlet, the following relations exist between Knapp's solution and the various sugars. Ioo cc. of Knapp's solution are reduced by the following quantities of sugar, using a 0.5 per cent sugar solution:

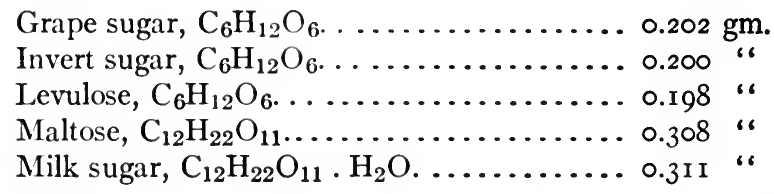

Sachsse's Method. The Standard Mercuric Iodid Solution. I $8 \mathrm{gms}$. of pure dry mercuric iodid and $25 \mathrm{gms}$. of pure potassium iodid are dissolved in water, and to this solution is added $80 \mathrm{gms}$. of caustic potash dissolved in water, and then the whole diluted to $\mathrm{I}$ liter.

In using this solution more mercury seems to be reduced if the sugar is added gradually than if added all at once. The reverse is true in the case of Knapp's solution. It is, however, necessary, in order to obtain comparable results, to proceed in the manner prescribed by Soxhlet for Knapp's solution. It is also to be remembered that the reducing action of sugar upon this solution differs according as a $I$ per cent or a 0.5 per cent solution is used. It is therefore important to have the sugar solution as nearly 0.5 per cent as possible.

The operation is carried out by using roo cc. of Sachsse's solution and proceeding in the same manner as described under Knapp's method. An alkaline stannous oxid solution is used as indicator. The end-reaction is found by removing a few drops of the clear supernatant liquid and mixing it with the alkaline stannous oxid solution on a porcelain plate. A black precipitate indicates a large quantity of mercury, a brown precipitate indicates a very small quantity. The 
alkaline stannous oxid solution may be prepared by simply supersaturing a stannous chlorid solution with caustic soda. Ioo cc. of Sachsse's solution are reduced by the following quantities of sugar, when in 0.5 per cent solution, according to Soxhlet, under the above conditions.

Grape sugar. . . . . . . .

Invert sugar. . . . . . . . . . . . . . . 0.269

Levulose......................... 0.213

Maltose............................ 0.491

Milk sugar.......................... 0.387

\section{REFERENCES}

Munson and Walker, "Unification of Reducing Sugar Methods." J. A. C. S., XxvirI, $66_{3}$.

Oerum, Zeitschr. anal. Chem., xuIII, 365 .

Rosenthal, Zeitschr. anal. Chem., XLIII, 252.

Craven and Hill. J. S. C. I., XVI, 98I; and XVII, I24.

Knapp, Zeitschr. anal. Chem., IX, 395.

Sachsse, Zeitschr. anal. Chem., xvı, I2 r. 


\section{CHAPTER L}

\section{ESTIMATION OF ALKALOIDS (VOLUMETRICALLY)}

IN making alkaloidal assays of drugs it has long been the custom to evaporate the final ethereal or chloroformic extract, and to weigh the residue as alkaloid. This residue seldom, if ever, consists of the pure alkaloid, and the impurities-i.e., non-alkaloidal matter-is variable in amount and difficult to entirely remove, consequently gravi- metric results are in many cases very wide of the truth, and hence unreliable.

The volumetric methods are in most cases much more satisfactory.

While the results of the titration of the total alkaloids of drugs cannot be called absolutely accurate, nevertheless experience has shown that they are nearer the truth than those obtained by the gravimetric method.

In estimating an alkaloid by titration it is essential to know the formula and molecular weight of the alkaloid, as well as the equivalent of acid with which it will combine.

The quantity of alkaloid present is easily calculated when we know that a molecular weight of a monobasic acid or half a molecular weight of a dibasic acid will combine with and neutralize a molecular weight of an alkaloid, provided the alkaloid is a monacid base. If the alkaloid is a diacid base, one molecular weight will combine with two molecules of a monobasic acid or one molecular weight of a dibasic acid.

Sparteine and emetine (?) are diacid alkaloids; most of the others are monacid bases.

Examples. Monacid alkaloids:

$$
\begin{aligned}
& \underset{\text { Quinine. }}{\mathrm{C}_{20} \mathrm{H}_{24} \mathrm{~N}_{2} \mathrm{O}_{2}}+\mathrm{HCl}=\mathrm{C}_{20} \mathrm{H}_{24} \mathrm{~N}_{2} \mathrm{O}_{2} \cdot \mathrm{HCl} ;
\end{aligned}
$$

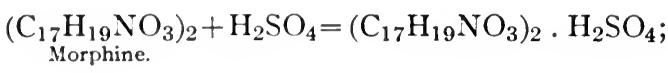

$$
\begin{aligned}
& \underset{\substack{\text { Strychnine. } \\
\left(\mathrm{C}_{21} \mathrm{H}_{22} \mathrm{~N}_{2} \mathrm{O}_{2}\right)_{2}}}{ }+\mathrm{H}_{2} \mathrm{SO}_{4}=\left(\mathrm{C}_{21} \mathrm{H}_{22} \mathrm{~N}_{2} \mathrm{O}_{2}\right)_{2} \cdot \mathrm{H}_{2} \mathrm{SO}_{4} \text {; }
\end{aligned}
$$


Diacid alkaloids:

$$
\begin{gathered}
\underset{\text { Sparteine. }}{\mathrm{C}_{15} \mathrm{H}_{26} \mathrm{~N}_{2}}+{ }_{2} \mathrm{HCl}=\mathrm{C}_{15} \mathrm{H}_{26} \mathrm{~N}_{2}(\mathrm{HCl})_{2} . \\
\underset{\text { Emetine (Kunz). }}{\mathrm{C}_{30} \mathrm{H}_{40} \mathrm{~N}_{2} \mathrm{O}_{5}}+\mathrm{H}_{2} \mathrm{SO}_{4}=\mathrm{C}_{30} \mathrm{H}_{40} \mathrm{~N}_{2} \mathrm{O}_{5} \cdot \mathrm{H}_{2} \mathrm{SO}_{4} .
\end{gathered}
$$

The quantity of alkaloid present in the substance is easily calculated, as illustrated by this equation:

$$
\begin{aligned}
& \mathrm{C}_{20} \mathrm{H}_{24} \mathrm{~N}_{2} \mathrm{O}_{2} \\
& \text { Quinine. }
\end{aligned}
$$

Thus I cc. of $\frac{\mathrm{N}}{\mathrm{ro}} \mathrm{V} . \mathrm{S} .=0.032 \mathrm{1} 82 \mathrm{gm}$. of quinine.

$$
\begin{aligned}
& \underset{\text { Sparteine. }}{\mathrm{C}_{15} \mathrm{H}_{26} \mathrm{~N}_{2}}+{ }_{2} \mathrm{HCl}=\mathrm{C}_{15} \mathrm{H}_{26} \mathrm{~N}_{2}(\mathrm{HCl})_{2} .
\end{aligned}
$$

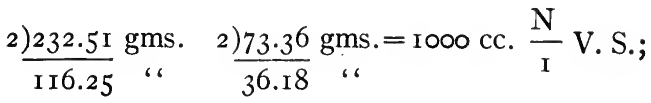

$$
\begin{aligned}
& \text { I } 1.625 \text { “ } \quad 3.618 ،=1000 \mathrm{cc} . \frac{\mathrm{N}}{\mathrm{IO}} \text { V.S.; }
\end{aligned}
$$

I cc. of $\frac{\mathrm{N}}{10} \mathrm{~V} . \mathrm{S}$. hence $=0.011625 \mathrm{gm}$. of sparteine.

Thus I000 cc. of $\frac{\mathrm{N}}{\mathrm{IO}}$ hydrochloric acid will combine with $\frac{1}{10}$ of the molecular weight in grams of a monacid alkaloid, or $\frac{1}{2} \overline{0}$ of the molecular weight of a diacid alkaloid.

In the case of drugs where two or more alkaloids are present, accurate results can only be obtained by determining how much of each alkaloid is present by a separate assay. But often it is assumed that the alkaloids are present in equal quantities, and the mean of their molecular weights is taken as the basis for the calculation.

It must be borne in mind, however, that in titrating alkaloids the greatest care must be exercised and all precautions closely observed. in order to attain any degree of accuracy. The volumetric solutions must be prepared with the greatest care and must be absolutely accu- 
rate. The eye must be trained (as it can only be through practice), to distinguish the end-colors of the indicators employed, a matter of some difficulty. Furthermore, all measuring instruments used must be accurate, or they should be carefully calibrated in order to find the necessary factor for correction.

The Volumetric Solutions usually employed in titrating alkaloids are $\frac{\mathrm{N}}{10}, \frac{\mathrm{N}}{20}, \frac{\mathrm{N}}{25}, \frac{\mathrm{N}}{50}$, and $\frac{\mathrm{N}}{100}$. The weaker solutions give more accurate results, as will be understood if we remember that $a \frac{N}{I}$ is Io $\times$ stronger than a $\frac{N}{10}$ and $100 \times$ stronger than $\frac{N}{100}$ V. S. Then if $\frac{N}{1}$ solution be used, one drop may overstep the neutral point, while if the same solution were treated with $\frac{\mathrm{N}}{10}$ solution 5 drops would be required to neutralize, which is equivalent to using one half a drop of $\frac{N}{I}$ solution. A $\frac{N}{100}$ solution will of course be capable of even more delicate work. In the case just mentioned 45 drops of the $\frac{\mathrm{N}}{100}$ may exactly neutralize the solution, hence less than half a drop of $\frac{N}{I}$ and between 4 and 5 drops of $\frac{N}{10}$ are represented. Therefore in all delicate alkaloidal titrations weak standard solutions should be usedin fact, in all titrations where great accuracy is required.

If the alkaloid be from a recent extraction and is in the form of a free alkaloid, it is dissolved in a measured excess of $\frac{\mathrm{N}}{\mathrm{IO}}$ acid solution and the excess of acid solution then determined by residual titration with $\frac{\mathrm{N}}{100}$ alkali solution.

In this the $\frac{\mathrm{N}}{\mathrm{IO}}$ sulphuric acid solution is preferred, except in the case of quinine or cinchonine, in which $\frac{\mathrm{N}}{\mathrm{I}}$ hydrochloric acid gives
better results.

The process in detail is as follows: Place 2 gms. of the alkaloid into a beaker, add $75 \mathrm{cc}$. of $\frac{\mathrm{N}}{\mathrm{IO}}$ sulphuric acid solution, and warm on a water-bath until the alkaloid is completely dissolved. The solution is then allowed to cool and diluted to roo cc.

Io $\mathrm{cc}$. of the solution (containing $0.2 \mathrm{gm}$. of the alkaloid and $7.5 \mathrm{cc}$. of $\frac{\mathrm{N}}{\mathrm{IO}}$ sulphuric acid solution) are removed by means of a pipette and 
retitrated with $\frac{\mathrm{N}}{\mathrm{I} O 0}$ potassium hydroxid solution. One tenth of the quantity of the $\frac{\mathrm{N}}{\mathrm{IOO}}$ alkali used is deducted from the $7.5 \mathrm{cc}$. of the $\frac{\mathrm{N}}{\mathrm{IO}}$ acid solution, and the remainder is the quantity of the latter which combined with and hence represents the alkaloid present. This, if multiplied by the factor, gives the weight of the alkaloid.

Either hæmatoxylin solution or Brazil-wood T. S. may be employed as the indicator.

If the alkaloid is soluble in alcohol, as are quinine and codeine, it may be treated as follows:

Place 2 gms. of the alkaloid in a graduated cylinder, dissolve in alcohol, and dilute the solution up to roo cc. with alcohol. Remove Io cc. of this solution (containing $0.2 \mathrm{gm}$. of the alkaloid) and place in a beaker, add the indicator and run in the decinormal acid solution to slight excess. Rotate the beaker several times, let stand for a few minutes, wash down the sides of the beaker with distilled water, using about $40 \mathrm{cc}$., and retitrate the excess of acid with $\frac{\mathrm{N}}{\mathrm{I} O 0}$ potassium hydroxid solution, until end-color is given by the indicator. Deduct one tenth of the quantity of the $\frac{\mathrm{N}}{\mathrm{IOO}}$ solution used from the quantity of $\frac{N}{10}$ acid solution added, and the remainder is the quantity of the latter, which combined with and hence represents the alkaloid present.

The indicators best suited for most alkaloids are Hamatoxylin, Brazil-wood, Cochineal, Iodeosin, and Litmus.

A. H. Allen states: "In titrating an alkaloid with methyl-orange as indicator it is rarely convenient to employ an aqueous solution of the base:

"A solution in proof-spirit can be employed, but the indicator is much less sensitive under such conditions.

"I have found it preferable, especially when an alkaloid is much colored, as is frequently the case in assaying bases directly extracted from their sources, to dissolve the alkaloid in a little chloroform, ether, amylic-alcohol, or other suitable immiscible solvent.

"The solution is placed in a small stoppered cylinder, together with a few cc. of water colored with a drop or two of methyl-orange. Then on gradually running in the standard acid from a burette, and agitating thoroughly after each addition, it is easy to observe the end of the reaction, as the coloring matter remains in the immiscible layer and presents a marked contrast to the red color of the aqueous liquid."

Allen has obtained satisfactory results with aconitine and its allies, 
even when working on as little as $0.030 \mathrm{gm}$., by using ether as a solvent and titrating with $\frac{\mathrm{N}}{50}$ hydrochloric acid.

In the titration of cinchona alkaloids such anomalous results are obtained that there is some doubt as to whether the relation of these alkaloids to acids is thoroughly understood. When quinine is titrated with an acid, almost twice as much of the latter is used, when methylorange is the indicator, as when Brazil-wood is employed as indicator. This is probably due to the fact that ordinary quinine sulphate is slightly alkaline to methyl-orange, and the end-reaction with this indicator is not reached until the acid sulphate is formed, while with Brazil-wood as indicator the end-reaction is reached sooner, that is, when the normal sulphate is formed, which is practically neutral to this indicator.

Quinine sulphate is also neutral to cochineal, but distinctly alkaline to litmus; hence the latter, like methyl-orange, is inapplicable in the titration of quinine. These anomalies should be had in mind when working upon the cinchona bases.

In titrating alkaloids the personal equation plays an important part. It is generally correct to titrate to the point where a change of color is developed, though there is no agreement among authorities as to the proper end-reaction tints, and each operator relies upon his own judgment.

Lyman F. Kebler says: "In order to obtain standard end-reaction tints for alkaloids it will be necessary to prepare some absolutely pure alkaloid. Treat a molecular quantity of the alkaloid with an equivalent of the acid in question to form a neutral salt, then add one drop. more of the decinormal acid for an acid color-reaction.

"For alkaline tints add one drop of the centinormal alkali solution to a solution of neutral alkaloidal salt theoretically prepared."

The color changes produced by the principal indicators used in alkaloidal titrations are as follows:

\begin{tabular}{|c|c|c|}
\hline & Acid. & Alkali. \\
\hline 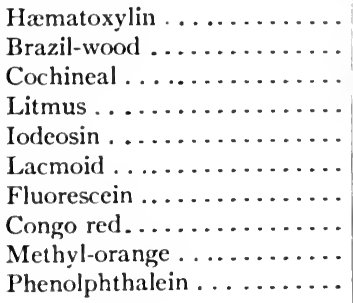 & $\begin{array}{c}\text { Yellow } \\
\text { “ } \\
\text { Yellowish-red } \\
\text { Red } \\
\text { Yellow } \\
\text { Red } \\
\text { Green fluorescence } \\
\text { Blue } \\
\text { Red } \\
\text { Colorless }\end{array}$ & $\begin{array}{c}\text { Blue } \\
\text { Purplish-red } \\
\text { Purplish } \\
\text { Blue } \\
\text { Rose-red } \\
\text { Blue } \\
\text { No Hluoresc., yellowish } \\
\text { Red } \\
\text { Straw-yellow } \\
\text { Red }\end{array}$ \\
\hline
\end{tabular}


TABLE SHOWING BEHAVIOR OF SOME OF THE ALKALOIDS WITH INDICATORS

\begin{tabular}{|c|c|c|c|c|}
\hline Name. & Formula. & $\begin{array}{l}\text { Methyl- } \\
\text { orange. }\end{array}$ & $\begin{array}{l}\text { Phenolph- } \\
\text { thalein. }\end{array}$ & Litmus. \\
\hline 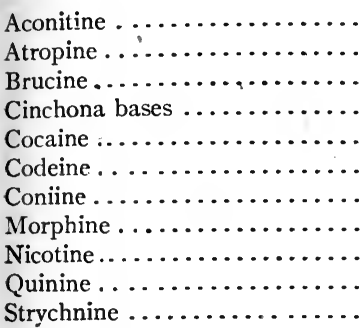 & $\begin{array}{l}\mathrm{C}_{34} \mathrm{H}_{47} \mathrm{NO}_{11} \\
\mathrm{C}_{17} \mathrm{H}_{23} \mathrm{NO}_{3} \\
\mathrm{C}_{23} \mathrm{H}_{28} \mathrm{~N}_{2} \mathrm{O}_{4} \\
\ldots \ldots . . . \\
\mathrm{C}_{17} \mathrm{H}_{21} \mathrm{NO}_{4} \\
\mathrm{C}_{81} \mathrm{H}_{21} \mathrm{NO}_{3} \\
\mathrm{C}_{8} \mathrm{H}_{17} \mathrm{~N} \\
\mathrm{C}_{17} \mathrm{H}_{19} \mathrm{NO}_{3} \\
\mathrm{C}_{5} \mathrm{H}_{7} \mathrm{~N} \\
\mathrm{C}_{20} \mathrm{H}_{24} \mathrm{~N}_{2} \mathrm{O}_{2} \\
\mathrm{C}_{21} \mathrm{H}_{22} \mathrm{~N}_{2} \mathrm{O}_{2}\end{array}$ & 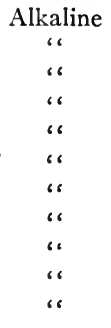 & $\begin{array}{c}\text { Neutral } \\
\text { Alkaline } \\
\text { Neutral } \\
\text { ‘، } \\
\text { Alkaline } \\
\text { “ } \\
\text { Faintly acid } \\
\text { Alkaline } \\
\text { Neutral } \\
\text { “، }\end{array}$ & 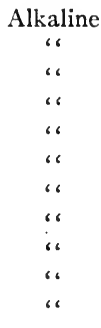 \\
\hline
\end{tabular}

\section{TITRATION OF ALKALOIDAL SALTS}

Prof. Plugge made a number of experiments with a view to determine the possibility of estimating volumetrically the amount of acid contained in alkaloidal salts, and from this determining the amount of alkaloid. He found-

(I) That in the salts of the weak opium bases narcotine, papaverine, and narceine the amount of acid can be volumetrically estimated with either litmus or phenolphthalein, the reaction being as precise and well defined as if no aikaloid were present.

(2) That in the salts of alkaloids in general, the acid can be readily determined by the use of phenolphthalein the volatile alkaloids coniine and nicotine being exceptions; and that in the case of morphine, brucine, codeine, and thebaine, phenolphthalein may be used with certain restrictions.

(3) That the free acid in solutions of alkaloidal salts can be determined by the use of litmus, but in solutions of weak opium bases litmus cannot be used. The entire quantity of acid, both free and combined, may be determined by the use of phenolphthalein. The difference between the two titrations gives the quantity of acid united to the base.

Thus he estimates the alkaloid by titrating the acid of the salt of the alkaloid with standard alkali, and from the result calculates the quantity of alkaloid present. He first determines the uncombined (free) acid by titrating with standard alkali in the presence of litmus.

He then titrates another portion of the solution in the presence of phenolphthalein to determine the total quantity of acid (both free and combined) present, and from this, indirectly, the quantity of alkaloid is calculated.

For the estimation of the alkaloid in a commercial salt, such as quinine sulphate, strychnine sulphate, etc.: 
Dissolve the salt in hot water and titrate with $\frac{\mathrm{N}}{\mathrm{IO}}$ sodium hydroxid solution, using phenolphthalein, methyl-orange, or some other suitable indicator.

The acid in combination with the alkaloid acts as though it were a free acid, and may be readily estimated by this method.

Phenolphthalein should be used with caution, as an indicator, in titrating morphine salts, as this alkaloid has a faint acid reaction with it.

It is generally preferable to titrate the solution of the salt of an alkaloid with $\frac{\mathrm{N}}{\mathrm{IO}}$ potassium hydroxid to exact neutrality, using phenolphthalein as indicator. The alkaloid which is thus set free and in a neutral liquid may be titrated in the same by means of $\frac{\mathrm{N}}{10}$ hydrochloric acid, using Brazil-wood T. S. as indicator. This gives very good results, and the two titrations are a check upon each other.

TABLE SHOWING THE FACTOR FOR VARIOUS ALKALOIDS IVHEN TITRATING WITH $\frac{\mathrm{N}}{\mathrm{rO}}$ ACID OR ALKALI

\begin{tabular}{|c|c|c|c|}
\hline Name. & Formula. & $\begin{array}{l}\text { Molecular } \\
\text { Weight. }\end{array}$ & Factor. \\
\hline 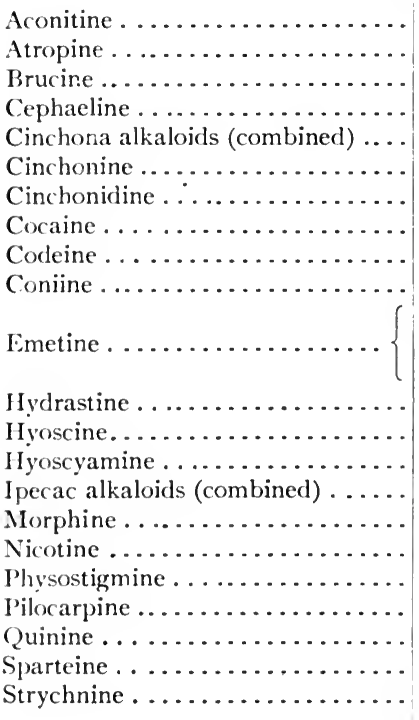 & 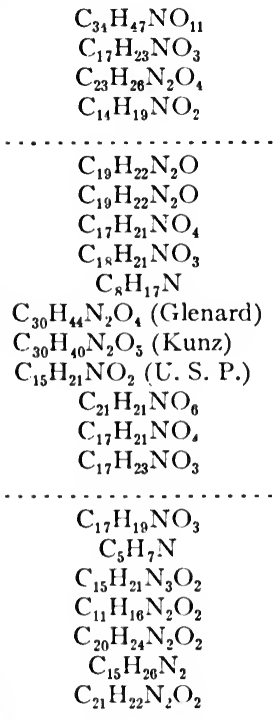 & $\begin{array}{l}640.55 \\
287.04 \\
391 \cdot 31 \\
231.43 \\
\cdots \cdots \cdots \\
292.03 \\
292.03 \\
300.92 \\
296.95 \\
126.21 \\
492.68 \\
504.56 \\
245 \cdot 34 \\
380.32 \\
300.92 \\
287.04 \\
\cdots \cdots \cdots \\
283.04 \\
80.48 \\
273.20 \\
206.63 \\
321.82 \\
232.51 \\
331.73\end{array}$ & $\begin{array}{l}0.06406 \\
0.02870 \\
0.03913 \\
0.02314 \\
0.03069 \\
0.02920 \\
0.02920 \\
0.03009 \\
0.02969 \\
0.01262 \\
0.02463 \\
0.02522 \\
0.02453 \\
0.0380 .3 \\
0.03009 \\
0.02870 \\
0.02384 \\
0.02830 \\
0.008048 \\
0.02732 \\
0.020663 \\
0.03218 \\
0.001625 \\
0.03317\end{array}$ \\
\hline
\end{tabular}


New Acidimetric Method for Alkaloidal Assay. Elie Falieries (Compt. rend., I29, IIO) proposes to avoid the difficulty which surrounds the determination of the end-reaction when titrating colored solutions of alkaloids by titrating back the excess of acid with ammoniacal cupric oxid solution. The end of the reaction is sharply defined by the precipitation of cupric oxid, which produces a very clearly marked precipitate. The cupric oxid solution is obtained by dissolving Io gms. of cupric sulphate in one half liter of water and adding ammonia until the precipitate formed at first is almost entirely redissolved, filling up to rooo cc. with distilled water, filtering and determining the amount of alkali present by titration with decinormal sulphuric acid. The estimation itself is carried out as follows: 0.10 gm. of the alkaloid is placed in a small, narrow cylinder, $20 \mathrm{cc}$. of decinormal sulphuric acid added, and when solution has been effected the excess of sulphuric acid titrated back by means of the cupric oxid solution. The sulphuric acid combined with the alkaloid takes no part in the reaction.

\section{ESTIMATION OF ALKALOIDS BY MAYER'S REAGENT}

The results of titrating with Mayer's solution have only an approximate value, being influenced to a large extent by various conditions, such as degree of dilution, mode of conducting the operation, and the length of time allowed for precipitation after each addition of the reagent.

The Mayer's solution is added from a burette, and the precipitate allowed to subside after each addition until no further precipitation takes place, which can be seen by bringing a drop of the clear supernatant liquid in contact on a watch-glass, with two or three drops of the reagent.

A more common practice is to filter the solution after each addition of the reagent, using the same filter. When ro cc. of the filtered liquid are no longer affected by two drops of the reagent, the titration is complete.

If a considerable length of time is allowed to elapse after each addition of reagent, it is found that the results of a titration will coincide more nearly with what theory requires; but the principal advantage which volumetric analysis has over gravimetric, namely, rapidity of execution, is thereby forfeited.

The presence-of alcohol, free acetic acid, or ammonia vitiates the result; but gum, albumen, glucose, or extractives in moderate quantities have no effect upon the reaction.

In all comparative titrations with this reagent the dilution of the 
alkaloidal solution should be the same. The solution should be slightly acid, and its strength about $\mathrm{I}: 200$.

In titrations where the end-reaction can only be ascertained by the cessation of the formation of a precipitate, it is often necessary to filter a portion of the turbid solution at intervals

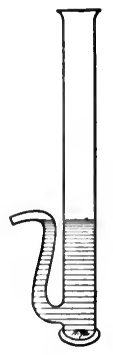
during the titration, and test it to see whether the process is completed. In such cases Beale's filter, Fig. 87 , may be used. Over the lower end of this instrument a piece of filter-paper is tied, and over that a piece of thin muslin to keep the paper from being broken. When dipped into a turbid mixture the clear liquid rises, and may be poured out of the little spout for testing. If the process is shown to be unfinished, the contents are washed back to the bulk of the liquid, and small portions filtered out at intervals until the process is found to be completed.

FIG. 87 .

The Decinormal Mayer's Solution, $\frac{\mathrm{N}}{10}$ Mercuric Potassium Iodid $\left(\mathrm{HgI}_{2}+2 \mathrm{KI}=783.98 .39 .2\right.$ gms. in a liter), is made as follows:

Dissolve $\mathrm{I} 3.44$ gms. of pure mercuric chlorid in $600 \mathrm{cc}$. of water, and $49.8 \mathrm{gms}$. of potassium iodid in $100 \mathrm{cc}$. of water.

Mix the two solutions, and then add enough water to make a mixture measure at or near $15^{\circ} \mathrm{C} .\left(59^{\circ} \mathrm{F}\right.$.) exactly rooo cc.

The reaction which takes place when these two solutions are mixed is

$$
\mathrm{HgCl}_{2}+{ }_{4} \mathrm{KI}=\mathrm{HgI}_{2}+2 \mathrm{KI}+2 \mathrm{KCl}
$$

A. B. Lyons and many others prefer to use a solution of half the above strength.

Each cc. of the decinormal solution, according to Dr. Mayer, precipitates of-

\begin{tabular}{|c|c|c|}
\hline $\begin{array}{cc}\mathrm{gm} . \\
\ldots .0267\end{array}$ & Coniine $\ldots \ldots 0_{0.00416}^{g m}$ & Quinidine ..... $0.01 \mathrm{gm} 20$ \\
\hline .0 .0145 & ine ..... 0.0200 & Quinine ....... 0.0108 \\
\hline 0.0233 & 0.0213 & 0.0167 \\
\hline .0 .0102 & Nicotine ...... 0.00405 & Veratrine ...... \\
\hline
\end{tabular}

The precipitates are hydriodates of the alkaloids, respectively, with iodid of mercury; but Lyons finds that they are not of definite composition, though the variation is very slight. This reagent will give similar precipitates with all of the alkaloids, except perhaps colchicine, caffeine, and the glucoside digitalin. 
ESTIMATION OF ALKALOIDS BY WAGNER'S REAGENT

Wagner's reagent has long been known to have the power of completely precipitating most alkaloids, even in dilute solutions. It is a very delicate reagent, and is said to precipitate ${ }_{500}^{1} \frac{1}{0} 00$ of a grain of the alkaloid in I grain of water.

The use of a potassium iodid solution of iodin as an alkaloidal precipitant was first recommended by Bouchardat as early as 1839 , but it was not until I86I that Wagner proposed the decinormal solution for use in volumetric analysis.

Because of the many difficulties that attended its use, through imperfect knowledge of the composition of the precipitate formed and the variations noticed with different alkaloids, it dropped into disuse for a time.

Recently Wagner's reagent has been again brought into notice as a volumetric reagent for alkaloids. This reagent is a solution of iodin in potassium iodid (see Decinormal Iodin V. S., page I86). Its use in volumetric analysis depends upon precipitating the alkaloids in the form of definite periodids, and its advantages over other methods are its sharp end-reactions and accurate results. The operations being performed in acid solutions, the presence of ammonia or other alkalies does not interfere.

The alkaloid in acidulated solution is treated with Wagner's reagent, added in excess. The precipitate is allowed to settle, and an aliquot portion of the clear liquid decanted, and titrated with decinormal thiosulphate solution, to determine the excess of iodin. This, deducted from the quantity of iodin added, gives the quantity of the latter which combined with the alkaloid.

The difficulty that presents itself is that different alkaloids when treated under apparently the same conditions give periodids of entirely different composition. Thus morphine is said to give with Wagner's reagent Alkaloid $\mathrm{HI}$. $\mathrm{I}_{3}$; codeine gives Alkaloid $\mathrm{HI}$. $\mathrm{I}_{4}$; caffeine gives Alkaloid $\mathrm{HI} . \mathrm{I}_{4}$.

Thus in the first case one equivalent of morphine equals three of iodin, in the case of codeine and caffeine one equivalent of the alkaloid equals in each case four of iodin; therefore we must ascertain exactly the composition of the precipitate in a particular case before we can make use of the reagent for volumetric analysis. When the composition of the different periodids, as produced under the conditions of titrations, is exactly known, this method may be placed upon a sound asis.

The General Method of Procedure in the estimation of the trength of an aqueous solution of an alkaloidal salt is as follows Prescott and Gordin, J. A. C. S., xx, 722): To about ro cc. of the 
decinormal iodin solution, diluted with a little water, $\mathrm{I}$ cc. of the acidulated alkaloidal solution is added, and the mixture well shaken for a few minutes. Should the precipitate separate out very quickly and the supernatant liquid be clear and of a light yellowish or greenish color, or altogether colorless, the alkaloidal solution is too strong and must be diluted, till after a few trials the clear supernatant liquid retains a very dark-red iodin color. The acidulated alkaloidal solution is then made up to a given volume, and 10 or $15 \mathrm{cc}$. of it are run from a burette into a graduated cylinder, into which has been previously put 25 or $30 \mathrm{cc}$. of the decinormal iodin solution diluted with a little water. The mixture is then made up to a given volume and shaken till the supernatant liquid is perfectly transparent and is of a very dark-red iodin color. This point is very important, and should the clear liquid not have this dark-red color, the experiment with most alkaloids (except morphine and possibly some others) should be repeated, putting more decinormal iodin solution into the graduated vessel, or decreasing the number of cc. of the alkaloidal solution. It would not do to add more decinormal iodin solution to the same mixture, as the iodin must be in large excess during the whole operation in order to prevent the formation of lower periodids, which, once formed, might not take up fresh iodin and form higher periodids. Only with morphine, which in such solutions forms but one periodid, these precautions are not necessary. When the liquid has become perfectly clear, an aliquot portion of it is filtered off and the excess of iodin determined by standard sodium thiosulphate. From these data is obtained the quantity of iodin consumed. This quantity of iodin multiplied by the "ratio" of alkaloid to the one of iodin gives the quantity of alkaloid sought. If preferred, but generally with less convenience to the chemist, the number of $\mathrm{cc}$. of decinormal solution of iodin consumed may be multiplied by the "alkaloidal factor of I cc. $\frac{\mathrm{N}}{10}$ iodin V. S."

IODIN-FACTORS OF THE ALKALOIDS SO FAR DETERMINED

\begin{tabular}{|c|c|c|c|}
\hline & $\begin{array}{l}\text { Higher Periodid } \\
\text { Formed. }\end{array}$ & $\begin{array}{l}\text { Ratio of Alka- } \\
\text { loid to One } \\
\text { of Iodin. }\end{array}$ & $\begin{array}{l}\text { Alkaloidal } \\
\text { Factor } \\
\text { of I cc. } \frac{\mathrm{N}}{10} \\
\text { Iodin. }\end{array}$ \\
\hline 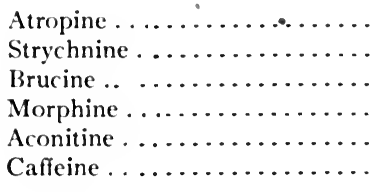 & $\begin{array}{l}\mathrm{C}_{17} \mathrm{H}_{23} \mathrm{NO}_{3} \mathrm{HI} . \mathrm{I}_{8} \\
\mathrm{C}_{21} \mathrm{H}_{22} \mathrm{~N}_{2} \mathrm{O}_{2} \mathrm{HI} . \mathrm{I}_{6} \\
\mathrm{C}_{23} \mathrm{H}_{28} \mathrm{~N}_{2} \mathrm{O}_{4} \mathrm{HI} . \mathrm{I}_{6} \\
\mathrm{C}_{17} \mathrm{H}_{19} \mathrm{NO}_{3} \mathrm{HI} . \mathrm{I}_{3} \\
\mathrm{C}_{33} \mathrm{H}_{45} \mathrm{NO}_{2} \mathrm{HI} . \mathrm{I}_{6} \\
\mathrm{C}_{8} \mathrm{H}_{10} \mathrm{~N}_{4} \mathrm{O}_{2} \mathrm{HI} . \mathrm{I}_{4}\end{array}$ & $\begin{array}{l}0.2849 \\
0.4390 \\
0.5179 \\
0.74918 \\
0.3834\end{array}$ & $\begin{array}{l}0.0036048 \\
0.00555467 \\
0.00655299 \\
0.00947937 \\
0.00485\end{array}$ \\
\hline
\end{tabular}




\section{ESTIMATION OF CAFFEINE BY WAGNER'S REAGENT}

Caffeine may be titrated as follows: $0.1 \mathrm{gm}$. of caffeine is dissolved in $30 \mathrm{cc}$. of water and acidulated with 5 or 6 drops of hydrochloric acid. $\frac{\mathrm{N}}{\mathrm{IO}}$ iodin solution is then run in from a burette, a few $\mathrm{cc}$. at a time, until $30 \mathrm{cc}$. have been used; this is a little over one and one third the quantity required theoretically to precipitate o.I gm. of caffeine. The precipitated caffeine periodid is separated by filtration through a dry asbestos filter after five minutes' standing, and an aliquot portion of the filtrate then titrated with $\frac{\mathrm{N}}{\mathrm{IO}}$ sodium thiosulphate solution, in order to determine the excess of iodin. The difference between the quantity of thiosulphate used for the whole filtrate and the quantity of iodin solution originally added, gives the quantity of the latter which reacted with the caffeine.

Each cc. of the $\frac{\mathrm{N}}{\mathrm{IO}}$ iodin solution $=0.00485 \mathrm{gm}$. of caffeine.

The calculation in detail is as follows:

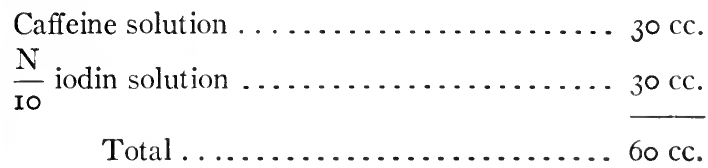

$30 \mathrm{cc}$. of filtrate required $5.8 \mathrm{cc} . \frac{\mathrm{N}}{\mathrm{IO}}$ thiosulphate;

whole " " "

$$
\begin{aligned}
& \text { 30 cc. } \frac{\mathrm{N}}{\text { IO }} \operatorname{iodin}-\text { r r.6 cc. " } \\
& =\text { I } 8.4 \text { cc. of } \frac{\mathrm{N}}{\text { IO }} \text { iodin consumed by caffeine, }
\end{aligned}
$$

$\mathrm{I} 8.4 \times 0.00485=0.08924 \mathrm{gm}$. caffeine 
GORDIN'S MODIFIED ALKALIMETRIC METHOD, USING PHENOLPHTHALEIN AS INDICATOR

The alkaloidal residue obtained by any of the extraction meihods in use is dissolved in a measured excess of $\frac{\mathrm{N}}{20}$ hydrochloric acid solution. Wagner's or Mayer's reagent is then added, a little at a time, with frequent shaking, until the alkaloids are completely precipitated. The mixture is then diluted with water to $100 \mathrm{cc}$. and shaken until the double salt of the alkaloid and reagent completely separate. When allowed to stand a few minutes, the supernatant liquid should be clear, and if Wagner's reagent has been used, this will be of a dark-red color. The liquid is now filtered, and $50 \mathrm{cc}$ of the filtrate (representing one half of the alkaloid) is treated with a Io per cent solution of sodium thiosulphate added, drop by drop, until the color of the free iodin disappears. This discolorization is not needed if Mayer's reagent has been used. A few drops of the indicator phenolphthalein are now introduced, and the excess of acid estimated by retitration with $\frac{\mathrm{N}}{20}$ potassium hydroxid solution. This, deducted from one half of the volume of the standard acid solution employed, indicates the number of cc. of $\frac{\mathrm{N}}{20}$ hydrochloric acid solution, which combined with the alkaloid in $50 \mathrm{cc}$. of the solution. This number, multiplied by two and then by the factor for the alkaloid present, gives the total quantity of alkaloid.

Example. An alkaloidal residue consisting of morphine was dis. solved in $30 \mathrm{cc}$. of $\frac{\mathrm{N}}{20} \mathrm{HCl} \mathrm{V.S.} \mathrm{Then} \mathrm{Wagner's} \mathrm{reagent} \mathrm{was} \mathrm{added} \mathrm{in}$ excess as described, and the mixture made up to roo cc. with water. $50 \mathrm{cc}$. of this were filtered off, decolorized as directed, and titrated with $\mathrm{x}$ 20

$\mathrm{KOH} \mathrm{V.S.} 10 \mathrm{cc}$. were required, which corresponds to $20 \mathrm{cc}$. for the entire quantity. Then $20 \mathrm{cc}$. deducted from the $30 \mathrm{cc}$. of $\frac{N}{20} \mathrm{HCl}$ V. S. added, leaves $10 \mathrm{cc}$, the quantity required to neutralize the morphine. The $\frac{N}{20}$ factor for morphine (0.0137 gm., Gordin), multiplied by $10=0.137 \mathrm{gm}$., the quantity of alkaloid in the residue examined.

The $\frac{\mathrm{N}}{\mathrm{IO}}$ factor for morphine here given is somewhat lower than the 
theoretical equivalent. It was ascertained by experiment with a sample of the anhydrous alkaloid.

The following factors (by Gordin) were obtained by comparing their molecular weights with that of morphine, which factor was determined by experiment:

Morphine......... $0.0137 \mathrm{gm} .=$ I cc. $\frac{\mathrm{N}}{20} \mathrm{HCl} \mathrm{V.} \mathrm{S.}$

Hydrastine ......... 0.0184 " =

Strychnine.......... 0.0160" " =

Caffeine, cryst . ...... $0.0102 "$ " =

Cocaine ............ 0.0146" $"=$

Atropine .......... 0.0139 " =

66
66
66
66 


\section{CHAPTER LI}

\section{VOLUMETRIC ASSAYING OF VEGETABLE DRUGS}

\section{EXTRACTION OF THE ALKALOIDS}

Selection of the Sample. Care must be taken to secure a fairly representative sample.

If the drug is in small pieces or consists of seeds or leaves, mix it well, take a portion, pulverize it, and of the powder take a sufficient quantity for the assay. If the drug be in large lumps, which vary in quality, select a few representative lumps and cut from each a fairly representative section; pulverize these, mix well, and weigh off a sufficient quantity for the assay.

If drying is necessary, the loss of weight in drying must be made note of.

The Exhaustion of the Drug is usually effected by maceration in a suitable menstruum, although percolation, boiling, and hot repercolation must be employed in some cases.

The Choice of Solvent depends upon the nature of the drug. Water dissolves, besides the alkaloids, so much inert matter that the subsequent steps in the assay are liable to be interfered with. Alcohol dissolves too much of the resinous matter, and besides does not penetrate the drug very well. Acidulated water has been much used, but chloroform and ether, separately and in various combinations, are now most generally employed for exhausting drugs in conjunction with alcohol and ammonia. Petroleum benzin has of late been recommended.

Prollius' fluid, or some modification of it, is very satisfactory.

Prollius' Fluid consists of ether $325 \mathrm{cc}$., alcohol $25 \mathrm{cc}$., and concentrated ammonia water ro cc.

IIodified Prollius' Fluid consists of ether $250 \mathrm{cc}$, chloroform 80 to roo cc., alcohol $25 \mathrm{cc}$, concentrated ammonia water ro cc.

Alkaloidal Assay by Immiscible Solvents. The following is the U. S. P. description of this procedure:

"Nearly all alkaloids are practically insoluble in water, but they are soluble in alcohol, chloroform, ether, amyl-alcohol, benzene, petroleum benzin, or mixtures of several of these. The salts of these alka- 
loids, however, are soluble in water, but practically insoluble in the above-mentioned solvents. The process of assay by immiscible solvents, which is generally known as the "shaking-out" process, is based on this property of alkaloids, and it is carried out by treating liquid extracts that have been freed from alcohol with an immiscible solvent in the presence of an excess of alkali. This liberates the alkaloid, and, on becoming free, if not so previously, it is dissolved by the immiscible solvent. This solution is then separated, transferred to another container, shaken with an excess of acid largely diluted with water. The acid combining with the free alkaloid forms a salt, which now leaves the immiscible solvent and is found in the aqueous solution. This process is sometimes repeated, in case the alkaloidal solution is still colored. The apparatus used in this operation of shaking-out is termed a "separator," (see Fig. 88) and consists of an oval or pear-shaped glass vessel, with an opening at the top supplied with a well-ground glass stopper, and an outlet tube at the bottom, provided with an accurately fitting glass stop-cock. The solvents directed to be used in this Pharmacopœia are alcohol, chloroform, ether, and various mixtures of both containing at least 75 parts of ether in

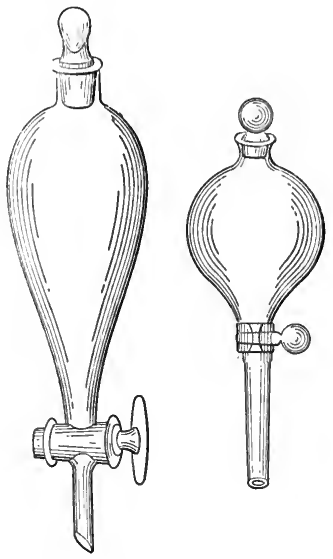

Squibb's Pattern

FIG. 88 . Ioo parts of solvent by volume. In the case of chloroform, the solvent will collect at the bottom of the separator, and can be drawn off, but the ethereal or ether-chloroform mixture will form the upper portion of the liquid in the separator, and the aqueous layer must first be drawn off into a suitable vessel, and the ethereal layer then transferred to another vessel. It is not necessary or desirable to shake the mixture of immiscible solvent and water violently, for a rotation of the separator or a gentle shaking for about a minute will answer all purposes. At times, an emulsion of the water and the solvent is formed, especially if the shaking is too violent, and in order to separate this, it is advisable to proceed as follows: If the solvent is heavier than the water, add more of the former, a little water, and a slight amount of alcohol; if the solvent is lighter than the water, add sufficient saturated sodium chlorid solution or crystals of sodium chlorid. A safe procedure to avoid the forming of emulsions is to invert the separator several times, and then to at once begin rotating to keep the solvents well mixed. To insure a complete extraction of the alkaloid, it is desirable to treat 
the liquid three times with the immiscible solvent, and this is to be followed by a rinsing of the empty separator with repeated small portions of the same solvent. The separator should not be filled to more than two thirds of its capacity at any time, and if its contents should become heated by the neutralization of acid by alkali, or vice versa, it should be cooled to the temperature of the room, before opening the stopper, by immersing it in running water. The final operation must always be the collection of the free alkaloid by the use of a portion of the immiscible solvent, drawing this off into a beaker, rinsing with small portions of the solvent to prevent possible loss. The beaker is then placed on a water-bath and gently heated, to remove the solvent by evaporation, leaving the alkaloids in the beaker in the dry form, and usually in the condition of a resinous or varnish-like mass. It is then either weighed as such or dissolved in volumetric acid solution, delivered in measured quantity from a burette, and the excess of the acid titrated with volumetric alkali solution with the use of an indicator. Should the final residual alkaloids still be slightly colored, it is preferable to employ iodeosin as the indicator, as the alkaloidal solution contains ether and the ethereal layer retains in solution coloring matter or impurity which may be present. If the alkaloids are not colored, hæmatoxylin or cochineal may safely be used.

"The quantity of alkaloid is found by multiplying the number of cc. of volumetric acid consumed by a constant factor, depending upon the molecular weight of the individual alkaloid."

"The factor in each case represents the weight in grams of the alkaloid required to neutralize I cc. of volumetric acid.

In connection with this, attention is called to the fact that some of the most important methods of isolating alkaloids in drug assays and in toxicological investigations depend upon the solubility of the free alkaloids in ether, chloroform, benzene, etc., and the relative insolubility of alkaloidal salts in the same solvents. There are, however, a number of exceptions to this rule, and special attention is called to the fact, that in many cases alkaloids pass from decidedly acid aqueous solutions (in which they certainly occur as alkaloidal salts) into chloroform and ether, in the shaking-out methods. While the amount of alkaloid so dissolved by these solvents is never very great, still the quantity is an appreciable one. This behavior occurs in the case of caffeine, colchicine, and narcotine; and under certain conditions also in the case of strychnine, atropine, veratrine, and other bases. This transfer of alkaloid to chloroform occurs more particularly in the case of alkaloids of weak basic character and when the solution is neutral or only feebly acid, or when the alkaloid is in combination with a comparatively weak acid, as citric, tartaric, etc. Furthermore. 
some alkaloidal salts, notably those of weak bases, are transferred as such to chloroform, especially the salts of hydrochloric acid, hydrobromic acid, and nitric acid. In the case, however, of the sulphates, phosphates, tartrates, and citrates of strongly basic alkaloids, no transfer occurs, or at most, only minute quantities of the alkaloids pass over. In order to prevent a transfer of alkaloid or alkaloidal salt out of an aqueous solution to an immiscible solvent, the use of sulphuric acid is to be recommended, and in all toxicological investigations due regard should be paid to the above named conditions. For more details, see the paper by Edward Schaer, Proc. A. Ph. A., Igo6, 425 .

\section{GENERAL METHODS OF ASSAYING DRUGS}

No rule can be formulated as to the method of extraction, or the solvent to be employed, which can be applied to all drugs; each must be dealt with in accordance with the properties of the contained alkaloids and their state of combination. Several methods are, however, in use which may be applied to a large number of different drugs, and with slight special modifications to many more.

The Keller Method * has been widely accepted, and in its various modifications is certainly the most practical.

In brief it is as follows: A convenient quantity of the drug in fine powder is introduced into a flask with about ten times its weight of menstruum, usually a mixture of one part of chloroform and about eight parts of ether. This is allowed to stand for about ten minutes, a small quantity of ammonia water added, the flask stoppered and shaken frequently during several hours. A sufficient quantity of water is then added to cause the powder to cake together and leave the ethereal fluid quite clear. Of this ethereal fluid an aliquot portion is removed by decantation and the alkaloid extracted by shaking out with dilute acid. By this procedure the alkaloids are almost wholly dissolved out of the drug, even when the latter is in a coarse powder. A more detailed description of this method is given in the assay of aconite root.

A serious objection to this method lies in the taking of a so-called aliquot part: First, because of the well-known solubility of ether in water, and conversely of water in ether, as a result of which the volume of the ethereal stratum is materially changed. Furthermore, commercial ether contains variable quantities of alcohol, hence the change in volume will not be always the same.

* C. C. Keller, Schweitz. Wochenschr. f. Chem. u. Pharm., XXX, 50I-509; A. J. Ph., LXV, 78, and LXVI, 42. 
Another source of error in the aliquot part is found in the volatile nature of the solvents used. In warm weather it is impossible to avoid loss by volatilization, hence the aliquot part taken is too large.

W. A. Puckner * has described a modification of the Keller method which avoids the use of the aliquot part. He uses only one half of the ethereal solvent for the maceration, and after the usual maceration transfers the drug to a small percolator in which, after the ethereal solution has been well drained off, the marc is percolated with the same menstruum to complete exhaustion. The quantity of ethereal solvent required is not materially greater than in the Keller method, while the quantity of alkaloid obtained for weighing or titrating is larger because it represents the whole of the sample taken for the assay. In the case of drugs containing a very small proportion of alkaloid this is an important advantage.

The objection to this plan is that the transfer of the mass from the flask in which the maceration has been conducted to a suitable percolator, which should not be more than $3 \mathrm{~cm}$. in diameter, requires very dextrous manipulation, or it will be attended with loss of alkaloid.

A. B. Lyons $\dagger$ recommends the following procedure: Provide a cylindrical percolator about $20 \mathrm{~cm}$. in length and 2 to $2.5 \mathrm{~cm}$. in internal diameter, ending in a tube $5 \mathrm{~cm}$. long and about $3 \mathrm{~mm}$. in internal diameter. A glass stop-cock in the tube would be a very desirable improvement. In absence of this, the rate of flow of the percolate must be controlled by packing the tube more or less firmly with absorbent cotton. Since the solvent is to be a very mobile fluid, the packing should generally be quite firm.

Having prepared the percolator, moisten the drug (5, I0, I 5, 20 gms. or more, according to richness in alkaloid-the finer the powder the better) with the mixture of ammonia, alcohol, and ether-chloroform, the proportions of which will be somewhat varied to suit different drugs. If 10 gms. of such a drug as belladonna leaf are to be used for the assay, the mixture may consist of: Stronger water of ammonia, I cc.; alcohol, 4 cc.; ether-chloroform (6:I vol.), 5 cc. Moisten in a small evaporating-dish, transfer quickly to the percolator, pressing the powder down firmly with a glass rod. The small amount of powder that remains adhering to the dish, spatula, and glass rod can be easily transferred to the percolator by aid of a little absorbent cotton, which is firmly pressed down upon the powder. The percolator is then to be covered and allowed to stand five to ten minutes, so that the ammonia may thoroughly permeate the drug. A mixture of

* Ph. Rev., XVI, I80, and XX, 457.

$\dagger$ Ph. Rev., Nov., 1903, and Proc. A. Ph. A., I903, 254. 
ether and chloroform, or whatever solvent is best suited to the extraction of the alkaloid present, is next added and the powder percolated with it to exhaustion. It is easy generally to secure a rate of flow of one drop per second, which will insure thorough exhaustion by the time that 50 to $75 \mathrm{cc}$. of percolate has passed. When it is believed that the exhaustion is complete, test this by collecting ${ }_{15}$ or 20 drops, stirring this with a drop of normal sulphuric acid, evaporating off the ethereal solvent and testing the acid solution with Meyer's or Wagner's reagent.

From this point the assay is to be carried on in the usual manner.

H. M. Gordin* proposes a new method in which special forms of apparatus are used, including a combination percolator and shaking tube in which the distillation of the ethereal solution to dryness is avoided; another feature of which is the use of fixed alkali hydroxid or carbonate instead of ammonia for the liberation of the alkaloids. By this method the loss occasioned through the transferring of the material from one vessel to another is avoided, and the injury to certain alkaloids by heat prevented. The process and apparatus are described under Aconite, page 523 .

Kebler's Modification of the Keller Method. $\dagger$ Treat Io gms. of the dry powdered drug in a 250 -cc. flask with 25 gms. of chloroform and 75 gms. of ether; stopper the flask securely, agitate well for a few minutes and add Io gms: of ammonia-water U.S. P., and shake frequently during one hour. Then on adding $5 \mathrm{gms}$. more of the ammonia-water and shaking, the suspended powder agglutinates into a lump and leaves the solution clear after a few minutes' standing. Then proceed by $\mathrm{A}$ or $\mathrm{B}$.

A. When the mixture has completely separated, 50 gms. (representing $5 \mathrm{gms}$. of the drug) are poured off into a beaker and heated on a water-bath until the solvent is evaporated. Io cc. of ether are then added and again evaporated. The varnish-like residue is then dissolved in $15 \mathrm{cc}$. of warm alcohol and water added to slight permanent turbidity, then the indicator is addesd, followed by an excess of standard acid solution and the mixture retitrated with standard alkali.

B. When the mixture has completely separated pour off 50 gms. into a separatory funnel, and add $20 \mathrm{cc}$. of acidulated water, agitate, and when the liquids have separated draw off the aqueous solution into a second separatory funnel. Repeat this operation with two more portions of $15 \mathrm{cc}$. of acidulated water. Now render the contents of the separatory funnel alkaline by adding ammonia-water. This liberates the alkaloids, which are then separated by treatment with a mixture of 
chloroform three parts (by volume) and ether one part, using three successive portions, first $20 \mathrm{cc}$., then twice $15 \mathrm{cc}$.

The chloroform-ether solution is heated on a water-bath until the solvent is evaporated, and then the varnish-like residue treated twice with $8 \mathrm{cc}$. of ether and again evaporated.

The residue is then dissolved in $15 \mathrm{cc}$. of alcohol, water added to slight permanent turbidity, and then the indicator. Titrate in usual way with decinormal acid and centinormal alkali.

Gordin and Prescott's Method (J. A. C. S., XxI, 232). This may be used when the alkaloid is to be estimated by Wagner's reagent, or by any other method. I to 4 gms. of the finely powdered drug is weighed into a low wide-mouthed vessel with a round bottom, holding 8 to ro ounces and having a well-fitted cork, such as a screw-top ointment jar. The powder is rubbed up with a small pestle to a fine paste by adding a little of a solvent mixture composed of stronger ammonia-water and alcohol each $5 \mathrm{cc}$., chloroform ro cc., and ether $20 \mathrm{cc}$. Then a few more cc. of this mixture are added, so as to have the drug well covered with the liquid, using in all about five times the amount of the drug taken. The vessel is corked with the pestle inside and is set aside for about four or five hours, taking care to agitate by rotating very frequently during that interval. After that time the cover is removed and the vessel kept in a current of air, stirring frequently till all odor of ammonia has disappeared. With a good draught and frequent stirring the powder will be almost perfectly dry in about two hours. The vessel is then put in a vacuum desiccator over sulphuric acid for about four or five hours. An amount of powdered sodium chlorid equal to about five or six times the amount of drug employed is then carefully mixed in, with the use of the pestle and the whole thrown into a small percolator having a plug of cotton at the bottom. The vessel is then cleaned out several times with small quantities of sodium chlorid and the cleanings added to the percolator. The mixture in the percolator is then covered with a piece of cotton, which is pressed down with a piece of glass, and a suitable menstruum, usually chloroform, is poured slowly into the percolator till the menstruum begins to drop from the bottom of the percolator; the flow is stopped, the percolator covered and set aside for five or six hours. After that time the stop-cock is opened and the drug exhausted with the menstruum percolating until ten drops of the percolate evaporated on a watch-glass and the residue taken up with a few drops of acidulated water, the solution shows no turbidity whatever on adding a few drops of Wagner's reagent. When finished, the percolate which is received in an evaporating-dish, is placed in a good draught at a temperature of about $30^{\circ} \mathrm{C}$. When the liquid is reduced to a very small volume Io cc. of acidulated water are added, and then a few cc. 
of ether, so as to have the ethereal liquid cover the aqueous solution. (If an alkalimetric assay is intended the acidulated water should be $\frac{\mathrm{N}}{\mathrm{IO}}$ sulphuric acid, and taken in definite quantity.) Then the whole is stirred with a glass rod till all the ethereal liquid is evaporated off. The liquid is then filtered and the evaporating-dish and filter washed several times with the acidulated water. In this way a colorless solution of the alkaloid is obtained, which can be worked up for any method of assay.

\section{LITERATURE ON ALKALOIDAL ASSAYING}

I8go. O. Schweissinger and G. Sarnow. A. J. Ph., LXIII, 96.

I89I. O. Schweissinger. Ph. Centrh., xxxII, 583 .

189r. A. H. Allen. Analyst, XVII, I86 and 215.

189. H. Beckurts. A. J. Ph., LXIV, 25.

1892. L. Barthe. A. J. Ph., LXv, 638 .

1892. A. H. Allen. Ch. News, LXVI, 259.

I892. C. C. Keller. Schweiz. Wochenschr. f. Chem. u. Ph., xxx, 501-509; A. J. Ph., Lxv, 78 .

I893. C. C. Keller. A. J. Ph., LXVI, 42.

I893. C. C. Keller. Schweiz. Wochenschr. f. Chem. u. Ph., xxxi, 473.

1893. C. Caspari and A. R. L. Dohme. A. J. Ph., LXvi, 473.

I894. H. Beckurts. Zeitschr. Oesterreich. Apoth. Ver., XLVIII, 624; and Am. Druggist, xxv, 328.

I894. K. Schwickerath. Bull. Ph., vIII, 56 and 246 .

I894. L. F. Kebler. Proc. A. Ph. A., XLII, I93.

I895. L. F. Kebler. A. J. Ph., Lxvir, 398,

1895. R. A. Cripps. Abstr. Chem. and Drugg., XLVII, I98.

1898. C. Kippenberger. Apoth. Ztg., page 664.

I898. N. Rusting. Pharm. Centrh., Aug. I8, page 603 .

1898. Prescott and Gordin. J. A. C. S., Xx, 722.

I898. W. Lenz. Pharm. Ztg., Sept. 24, page 683.

1899. Gordin and Prescott. Proc. A. Ph. A., 26r and 27I.

I899. Gordin and Prescott. A. J. Ph., Jan., pages I4-I3.

1899. Gordin and Prescott. Ph. Rev., Nov., page 495.

I899. E. Failieres. Ph. Jour., Sept., page 295.

I goo. E. Schmidt. Apoth. Ztg., Jan. 6, page i 3.

1900. C. Kippenberger. Ph. Rev., Aug., page 373 .

I9or. H. Proelss. Apoth. Ztg., No. 88.

1903. A. B. Stevens. Pharm. Arch., vI, 49.

1903. A: B. Lyons. Ph. Rev., Nov.

I905. G. Fromme. Geschäftsbericht, Cæsar and Loretz, page 5 I.

I905. H. M. Gordin. A. J. Ph., page 464.

I906. H. M. Gordin. Proc. A. Ph. A., page 377.

I906. E. Schaer. Proc. A. Ph. A., page 425.

1908. W. A. Puckner. A. J. Ph., Lxxx, page 66. 


\section{THE ASSAYING OF CRLDE DRUGS}

The Extraction of the Alkaloids. This may be effected by numerous methods. The following are those most frequently recommended at the present time. Though these methods are of general application, yet owing to slight differences in the solvent action of the fluids used, and for other reasons, one method is better adapted for a particular drug than another.

The Keller method, as modified by Puckner,* in which the drug is exhausted by percolation after maceration, and the use of an aliquot part avoided, is one of the best general methods. The percolation method of Lyons, $\uparrow$ also gives very excellent results. Gordin's method, in which a special combination percolator and shaking tube is employed, and fixed alkali used instead of ammonia, obviates the necessity for evaporation of the ethereal solution to dryness, thus preventing injury to certain alkaloids. The methods suggested by Gordin and Prescott, $\S$ and by Kebler, $\|$ are also useful in some cases.

\section{ASSAY OF ACONITE}

Assay of Aconite Root (C. C. Keller). Place I 2 gms. of the root (in No. 80 powder) in a 250 -cc. flask, add 30 gms. of chloroform and 90 gms. of ether; stopper securely and shake the flask for five or ten minutes; then add ro gms. of ammonia-water U. S. P. and shake frequently during half an hour, and introduce $20 \mathrm{gms}$. of water and again shake; this causes the drug to gather in lumps and permits the chloroform-ether mixture to separate so that it can be easily poured off.

When the mixture has completely separated, pour off ivo gms. of the chloroform-ether solution (representing ro gms. of the drug) into a separatory funnel and treat at once with $25 \mathrm{cc}$. of a I per cent solution of hydrochloric acid. Agitate, and when the liquids have separated, draw off the aqueous solution into a second separatory funnel, and repeat the operation with two more portions of the hydrochloric acid solution, using first 15 and then ro cc.

The acidulated solution is then rendered alkaline by adding ammonia-water and the reprecipitated alkaloid removed by treatment with successive portions of a mixture of chloroform 3 parts and ether 2 parts (by weight), using in all about 100 gms. of the mixed solvent. It is best to add a portion of the solvent before the ammonia.

The chloroform-ether solution is collected in a beaker or flask and the solvent distilled off. The residuum is treated with two or three small portions of ether and the latter removed by heating on a waterbath. The alkaloids are then dissolved in ro cc. of absolute alcohol,

\footnotetext{
* Page 516. †Page 516. ‡Page 523. § Page 518. \|Page 517.
} 
and water added to slight permanent turbidity. The solution is then titrated with $\frac{\mathrm{N}}{\mathrm{IO}}$ hydrochloric acid in the presence of hæmatoxylin as
indicator.

I cc. of $\frac{\mathrm{N}}{\mathrm{IO}}$ acid equals $0.0640 \mathrm{gm}$. of aconitine, the principal alkaloid.

Assay of Aconite Leaves. This assay is the same as that of the root. Use the following quantities: aconite leaves (No. 80 powder) 25 gms., ether Ioo gms., chloroform 25 gms., ammonia-water U. S. P. Io gms. Pour off I05 cc. of the chloroform-ether solution, and after it has stood for a few minutes to settle, transfer roo gms. of it into a separatory funnel and proceed as above. In this way a clearer solution is obtained.

The German Pharmacopœia Method. This is a modification of the foregoing. In it the precipitation of the alkaloid is effected by means of sodium hydroxid, and iodeosin is the indicator. The method is as follows:

(a) Place 12 gms. of the drug (in fine powder, and dried at $100^{\circ} \mathrm{C}$.) into a 250-cc. bottle, add 30 gms. of chloroform and $90 \mathrm{gms}$. of ether, stopper the bottle, and shake it briskly.

(b) Add ro cc. of a mixture of two parts (by weight) of soda solution (Pharm. G. I5 per cent) and one part of water, and shake frequently during three hours; then add Io cc. of water, or as much more as may be necessary to cause the powder to aggultinate into lumps upon shaking. The liquid becomes clear after a few minutes' standing and car be decanted almost completely from the sediment.

(c) After standing for one hour, decant Ioo gms. of the clear chloroform-ether solution (representing Io gms. of the drug), and pass it through a small, dry, well-covered filter into a flask and distil off about half of it. Introduce the rest into a separating funnel, rinsing the flask three times with a mixture of chloroform and ether $(\mathrm{r}: 3)$, using $5 \mathrm{cc}$. each time and adding the rinsings to the contents of the separator.

(d) Then add $25 \mathrm{cc}$. of $\frac{\mathrm{N}}{100}$ hydrochloric acid; shake thoroughly, and set aside until the liquids have separated. If necessary, add a little more ether to insure more complete separation.

(e) Draw off the aqueous layer, pass it through a filter moistened with water into a Ioo-cc. flask, wash the chloroform-ether mixture in the separator with three portions of water (Io cc. each), passing the washings through the same filter into the flask and further dilute with water to roo cc. 
(f) Place $50 \mathrm{cc}$. of this solution into a white glass flask, add $50 \mathrm{cc}$. of water and sufficient ether to form a layer $\mathrm{I} \mathrm{cm}$. in depth; then five drops of iodeosin solution and titrate with $\frac{\mathrm{N}}{100}$ potassium hydroxid, shaking the flask after each addition of the standard solution until the lower aqueous layer assumes a pale red color. Not more than $8.5 \mathrm{cc}$. should be required. The $\frac{\mathrm{N}}{100}$ factor for aconitine is $0.0064 \mathrm{gm}$.

(g) The Calculation. The $25 \mathrm{cc}$. of $\frac{\mathrm{N}}{100}$ hydrochloric acid take up the alkaloids representing Io gms. of the drug. One half of this when titrated with $\frac{\mathrm{N}}{\mathrm{IOO}} \mathrm{KOH}$ requires, we will assume, $8.5 \mathrm{cc}$. which represents the quantity of free $\frac{\mathrm{N}}{100}$ acid solution in the quantity titrated. Twice this quantity, i.e., I 7 cc. of the $\frac{\mathrm{N}}{\mathrm{IOO}}$ alkali then represents $\mathrm{I} 7 \mathrm{cc}$. of $\frac{\mathrm{N}}{\mathrm{IOO}}$ acid solution in a free state. This subtracted from the $25 \mathrm{cc}$. originally added leaves $8 \mathrm{cc}$., which latter is the quantity of $\frac{\mathrm{N}}{100}$ acid which was taken up by the alkaloid present. This, multiplied by $\frac{\mathrm{N}}{\mathrm{IOO}}$-factor for aconitine (0.0064 gm.), gives us $0.05^{\mathrm{I} 2}$ gm., the quantity of aconitine in Io gms. of "the drug (0.512 per cent).

The Method of the U. S. P. VIII.* Introduce Io gms. of the aconite root (in No. 40 powder) into a 200-cc. Erlenmeyer flask, add $75 \mathrm{cc}$. of a mixture of alcohol, seven parts, and distilled water, three parts (by volume), stopper the flask securely, and agitate it at intervals during four hours. After placing a pledget of cotton in the bottom of a small cylindrical glass percolator $(25 \mathrm{~mm}$. in diameter), carefully transfer the contents of the flask to the percolator. When the liquid has all passed through, continue the percolation with more of the same mixture until $150 \mathrm{cc}$. of percolate have been obtained. Pour the percolate into a shallow porcelain evaporating-dish, and evaporate it to dryness at a temperature not exceeding $60^{\circ} \mathrm{C}$. ( $140^{\circ} \mathrm{F}$.). Add $5 \mathrm{cc}$. of tenth-normal sulphuric acid V. S. and ro cc. of distilled water. When the extract is dissolved, filter the liquid into a separator, washing the dish, and filter with about $40 \mathrm{cc}$. of distilled water, and add the washings to the separator. Add $25 \mathrm{cc}$. of ether and $2 \mathrm{cc}$. of ammonia-water to the separator and agitate it for one minute. Draw

* Proposed by A. B. Stevens, Pharm. Arch., 1903, VI, 49. 
off the lower layer into a flask and filter the ether solution into a beaker. Return the contents of the flask into the separator, add I 5 cc. of ether, and again agitate for one minute. Draw off the lower layer into the flask and filter the ether solution into the beaker. Repeat the shaking out, with two other portions of Io cc. each of ether. Evaporate the combined ether solutions to dryness and dissolve the residue in $3 \mathrm{cc}$. of tenth-normal sulphuric acid V. S. Add to the solution five drops of hæmatoxylin T.S., and then carefully run in fiftiethnormal $\mathrm{KOH} \mathrm{V}$. S. until a violet color is produced, the transition stages being as follows: First, yellow, then green, finally passing into violet. Divide the number of cc. of fiftieth-normal KOH V. S. used by 5 ; subtract this number from 3 (the $3 \mathrm{cc}$. of tenth-normal sulphuric acid V. S. taken), multiply the remainder by 0.06406 , and this product by Io, which will give the percentage of aconitine in the aconite.

Each cc. of $\frac{\mathrm{N}}{\mathrm{IO}}$ sulphuric acid represents $0.06406 \mathrm{gm}$. of aconitine $\left(\mathrm{C}_{34} \mathrm{H}_{47} \mathrm{NO}_{11}\right)$.

H. M. Gordin,* in commenting upon the U. S. P. alkaloidal assay processes, points out that in this process, as well as in several others, the filtration of the first acid liquid, as directed, is attended with some difficulty in that the liquid being thicker and slimy soon clogs up the filter. He proposes a new method in which special forms of apparatus are used, and in which the distillation of the ethereal solution to dryness is avoided, and furthermore the liberation of the alkaloid effected by means of fixed alkali hydroxid or carbonate. In the presence of the impurities which always accompany the alkaloid obtained in an assay, the distillation to dryness of the ethereal or chloroformic solution frequently causes, a partial resinification of the alkaloid, which renders it difficultly soluble in dilute acids without heating. Heat in the presence of free acid is liable to injure the alkaloid.

If ammonia is employed to liberate the alkaloid, it is necessary to distil the ethereal solution to dryness, or at least to a small bulk, in order to drive off the ammonia which is dissolved in the ether and which would vitiate the results of the titration. If, on the other hand, sodium carbonate or hydroxid is used for liberating the alkaloid, there will be no need for distillation to dryness inasmuch as the fixed alkalies are not taken up by the immiscible solvents. The latter will therefore contain; besides the alkaloid, no other basic substance except minute traces of ammonia formed by the action of the fixed

* Proc. A. Ph. A., I906, 377. 
alkali upon the albuminous matter of the drug. Such traces of ammonia can be easily and quickly removed either by drawing air over the surface of the ethereal liquid or more quickly by concentrating the ethereal solution upon a warm water-bath to about one half of its original volume.

Ether alone cannot be used as an immiscible solvent in these methods, because, since it dissolves water, it takes up some fixed alkali when shaken with an alkaline solution. But if instead of ether alone, chloroform alone, or a mixture of three volumes of ether and one volume of chloroform, or a mixture of two or three volumes of ether and one volume of petroleum ether be shaken with a solution of a fixed alkali, no trace of alkali goes into the immiscible solvent even if the alkaline solution contains 50 to 60 per cent of alcohol. This can be shown by filtering the immiscible solvent, after shaking it with the alkaline solution, through a plain filter of ordinary filter-paper, having four folds on each side and previously moistened with ether, and then shaking up the ethereal liquid with a little water. Neither phenolphthalein nor any other delicate indicator will show the presence of alkali in the aqueous liquid.

In order to eliminate the sources of error involved in transferring ethereal liquids * from one vessel to another, Gordin suggests the use of special apparatus. These are described and illustrated below.

The combination percolator and shaking tube (Fig. 89) is designed to avoid the necessity of transferring the mixture of drug and menstruum from one vessel to another. Both the shaking and the percolating of the drug may be carried out in it.

This apparatus is described by Gordin in A. J. Ph., I905, 464, as follows: "The apparatus consists of a cylindrical tube drawn out at both ends so that it has the shape of an ordinary percolator, but a neck like an ordinary bottle. The main body of the tube has an inner diameter of $25 \mathrm{~mm}$. and is $200 \mathrm{~mm}$. in length. The lower, longer but narrower, drawn-out part has an inner diameter of $14 \mathrm{~mm}$., and is $30 \mathrm{~mm}$. long. At the juncture of this narrow tube to the main body of the tube there are three rather deep indentations in the narrow tube. The upper bottle-neck shaped end of the tube has an inner diameter of $17 \mathrm{~mm}$. and is $10 \mathrm{~mm}$. long. The whole apparatus is made of sirong glass of about I mm. in thickness.

The tube is used in the following way: A piece of cotton is placed in a piece of cheese cloth and then pushed up from below, with the cloth upwards, into the tube so that the plug reaches the indentations and closes the tube rather tightly. The plug is then followed by more

* Ethereal liquids have a tendency to "creep" to the outer sides of the vessels. 
cotton, so as to nearly fill the narrow tube, and the ratter is closed by a good perforated cork through which passes a thin glass stopcock. After closing the stop-cock, the weighed out drug is introduced through the open end of the tube, and after adding the menstruum the apparatus is closed with a good cork.

The tube can now be shaken without the plug becoming dislodged. After shaking the tube the prescribed length of time, it is set aside with the stop-cock downwards till the drug has well settled, and the percolation then finished in the usual way.

By means of separating funnel (Fig. 90), aqueous and ethereal liquids can be drawn off through separate outlets and the contamination of the immiscible liquids by each other completely avoided. By means

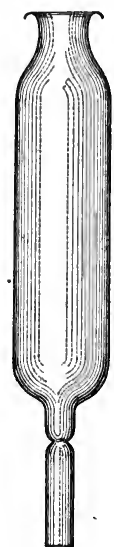

Fig. 89 .

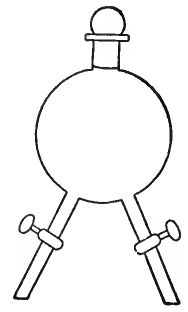

FIG. 90.

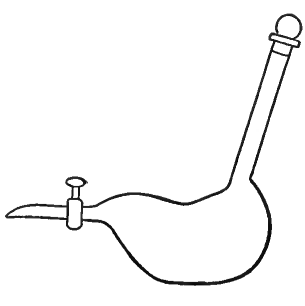

FIG. 9I.

of separator (Fig. 9r) the concentration of an ethereal liquid can be accomplished by placing the funnel in warm water and connecting the long goose neck with a condenser, and the necessity for transferring the liquid from a distilling-flask to a separatory funnel thus avoided.

Gordin's Modified Assay for Aconite Root. Put Io gms. of aconite root in No. 60 powder into the percolator shaking tube, add $50 \mathrm{cc}$. of a mixture of three volumes ether and one volume chloroform and $5 \mathrm{cc}$ of a Io per cent solution of sodium carbonate. After closing the tube shake the whole thoroughly during one hour, then percolate with the same immiscible solvent to exhaustion. In order to obtain a perfectly clear liquid free from any trace of fixed alkali pass the stop-cock of the percolator through a very wide cork placed over a small funnel which contains a small plain double 
filter of ordinary filter-paper having four folds on each side. The cork serves as a cover to prevent evaporation. The percolate can be received into any vessel, or better into special separatory funnel (Fig. 9r). The percolate is then concentrated, by placing the funnel in warm water, to about one half of the original volume to remove traces of ammonia and, when cold, diluted again with ether to approximately the original volume. The ethereal liquid is now shaken out once with excess of standard acid and then washed twice with water. The excess of acid is then titrated in the usual way. As it is always advisable to control the acidimetric estimation by a gravimetric one the acid liquid can be received into a separating funnel of suitable capacity and the titration carried out directly in this funnel. After the titration the liquid is made strongly alkaline with sodium hydroxid and shaken out three times with chloroform. The indicator added all remains in the alkaline liquid and the colorless chloroformic solution, after evaporation, leaves the alkaloid to be weighed and identified by special reactions. Assayed by this method a sample of good aconite root gave (volumetrically) I.02 per cent alkaloids.

Method of C. Kippenberger (Apoth. Ztg., I898, 664). 18 gms. of the root in fine powder are extracted with $180 \mathrm{cc}$. of alcohol containing $3 \mathrm{gms}$. of tartaric acid. I $20 \mathrm{cc}$. of the filtrate are evaporated to dryness, avoiding high temperatures. The residue is dissolved in $60 \mathrm{cc}$. of water containing 2 cc. of dilute hydrochloric acid (G. Ph.), and $50 \mathrm{cc}$. of the filtrate (representing Io gms. of the drug) are precipitated with an excess of iodin solution, 20 to $40 \mathrm{cc}$. being used.

The iodin solution is made as follows: Iodin 20 gms., potassium iodid $60 \mathrm{gms}$., water to make $1000 \mathrm{cc}$. The mixture is let stand about half an hour in order to insure complete precipitation of the periodid. The precipitate is then collected upon a small plaited filter, washed twice with water containing a few drops of the iodin solution, and dissolved in pure acetone (of which 15 to $20 \mathrm{cc}$. are required), observing that it be completely dissolved, and that there be no waste. The acetone solution is then treated with alkali hydroxid solution in excess, to precipitate the pure alkaloid, then diluted hydrochloric acid is added in excess to take up the alkaloid, and the mixture diluted with water and shaken up with petroleum-ether to remove the acetone, the last traces of which are removed by warming on a water-bath. The liquid is now supersaturated with alkali hydroxid, and shaken out with chloroform. The chloroformic solution of the pure alkaloid so olstained is evaporated and the amount of alkaloid determined either by direct weighing or by titration. This method may be applied to most other drugs, but the amount of solvents and precipitant must, of course, vary with the nature of the drug assayed. 
G. Fromme's Method.* 7 gms. of aconite of medium fineness, 70 gms. ether, and 5 gms. 15 per cent sodium hydroxid solution are shaken frequently and vigorously during half an hour and then as much as possible poured through a pledget of cotton into a flask; I cc. of water is added to the ethereal liquid, the mixture well shaken, put aside until perfectly clear, and then $50^{\circ} \mathrm{gms}$. or as much as possible ( $10 \mathrm{gms} .=\mathrm{I} \mathrm{gm}$. of drug) poured off. This is extracted with 15 , Io, and Io gms. of I per cent hydrochloric acid. Then the acid extractions are just neutralized with ammonium hydroxid, and the alkaloid abstracted with I5, Io, and Io gms. of chloroform, and successively passed through a 3 to $4 \mathrm{~cm}$. plain filter into a roo-cc. tared Erlenmeyer flask. The chloroform is distilled from a waterbath, the residue twice dissolved in ether, 5 gms. each time, brought to dryness, the residue dried to constant weight in a desiccator and weighed. As a check the residue is dissolved in a few cc. of absolute alcohol, about $20 \mathrm{cc}$. of water, and a few drops of hæmatoxylin solution added and its alkalinity determined with $\frac{\mathrm{N}}{\mathrm{IO}}$ hydrochloric acid.

THE MYDRIATIC DRUGS

These drugs, namely, belladonna, hyoscyamus, scopola, and stramonium, are assayed according to the U. S. P. by the Keller method, as modified by W. A. Puckner (Ph. Rev., xvI, I80; and xx, 457). (See page 516).

Assay of Belladonna (U. S. P.). Place Io gms. of belladonna (leaves or root in No. 60 powder) in an Erlenmeyer flask, and add $50 \mathrm{cc}$. of a mixture of chloroform one part and ether four parts (both by volume). After inserting the stopper securely, allow the flask to stand ten minutes, then add $2 \mathrm{cc}$. of ammonia water mixed with $3 \mathrm{cc}$. of distilled water, and shake the flask well at frequent intervals during one hour. Then transfer as much as possible of the contents of the flask to a small percolator which has been provided with a small pledget of cotton packed firmly in the neck and inserted in a separator containing $6 \mathrm{cc}$. of normal sulphuric acid, diluted with $20 \mathrm{cc}$. of distilled water. When the liquid has passed through the cotton, pack the drug firmly in the percolator with the aid of a glass rod, and having rinsed the flask with Io cc. of the chloroform-ether mixture, transfer the remaining contents of the flask to the percolator, by the aid of several small portions ( $5 \mathrm{cc}$.) of the chloroform-ether mixture, and continue the percolation with successive small portions of the

\footnotetext{
* Geschäftsbericht, Cæsar and Loretz, I905, 5r.
} 
same liquid (using in all $50 \mathrm{cc}$.). Next shake the separator well for one minute after securely inserting the stopper, and when the liquids have completely separated, draw off the acid solution into another separator. Add to the chloroform-ether mixture ro cc. of sulphuric acid mixture of the same strength as that previously used, agitate well, and again draw off the acid solution into the second separator; repeat this operation once more, drawing off the acid solution as before. Introduce into the acid solution contained in the second separator a small piece of red litmus paper, then add ammonia-water until the liquid is distinctly alkaline, and shake out with three successive portions of chloroform I5, I5, and $5 \mathrm{cc}$; collect the chloroform solutions in a beaker, place it on a water-bath containing warm water, and allow the chloroform to entirely evaporate. Dissolve the residue in $3 \mathrm{cc}$. of ether, and let this also entirely evaporate. To the alkaloidal residue add $3 \mathrm{cc}$. of $\frac{\mathrm{N}}{\mathrm{IO}}$ sulphuric acid and 5 drops of hæmatoxylin T. S. (or iodeosin T. S.); then titrate the excess of acid with $\frac{\mathrm{N}}{50} \mathrm{KOH}$. Divide the number of cc. of $\frac{\mathrm{N}}{50} \mathrm{KOH}$ used by 5 , subtract the quotient from 3 (the $3 \mathrm{cc}$. of $\frac{\mathrm{N}}{\mathrm{IO}}$ sulphuric acid taken), and multiply the remainder by 0.0287 , and this product by Io; the result will be the percentage of total mydriatic alkaloids contained in the belladonna. The pharmacopoial requirement is, not less than 0.35 per cent for the leaves and not less than 0.5 per cent for the root of mydriatic alkaloids.

It is suggested that in carrying out this process, the percolation be continued to exhaustion, as indicated by Wagner's reagent, and that double the quantity of drug be taken for the assay.

Assay of Belladonna Leaves (Lyman F. Kebler, J. A. C. S., Xvir, 828). Place 10 gms. of the powder in a 250 -cc. flask, add 25 gms. of chloroform and $75 \mathrm{gms}$. of ether; stopper the flask securely, agitate well for several minutes, add Io gms. of ro per tent ammonia-water, then agitate frequently and during one hour. On adding 5 gms. more of the ammonia-water and shaking well, the suspended powder agglutinates into a lump, and the liquid becomes clear after standing a few minutes and can be poured off almost completely.

When the mixture has completely separated, pour $50 \mathrm{gms}$. into a separatory funnel, and treat it at once with $20 \mathrm{cc}$. of acidulated water. After thorough agitation and complete separation remove the aqueous solution into a second separatory funnel. Repeat the above operation twice more successively with I $_{5} \mathrm{cc}$. of slightly acidulated water.

The acidulated aqueous solution in the second separatory funnel 
is then rendered alkaline with ammonia-water and the reprecipitated alkaloid removed by adding successively $20 \mathrm{cc}, 15 \mathrm{cc}$., and $15 \mathrm{cc}$. of a mixture of 3 parts (by volume) of chloroform and I part of ether. Collect the chloroform-ether mixture in a beaker and distil off the solvent. The varnish-like residue is next dissolved in $\mathrm{I}_{5} \mathrm{cc}$. of alcohol with heat, water is added to slight permanent turbidity, a few drops of hæmatoxylin solution added, then a slight excess of $\frac{\mathrm{N}}{20}$ sulphuric acid, and retitrate with $\frac{\mathrm{N}}{20}$ alkali solution.

Each cc. of $\frac{\mathrm{N}}{20}$ acid $=0.01445 \mathrm{gm}$. of alkaloid as atropin.

The use of decinormal sulphuric acid and of centinormal alkali solution is preferred by many; one tenth the number of cc.'s of the alkali used is then deducted from the quantity of decinormal acid added and the remainder multiplied by $0.0289 \mathrm{gm}$.

The Periodid Method of Gordin and Prescott may be employed for the assay of belladonna (see page 5I8).

Other methods recommended are:

Frank X. Moerk (A. J. Ph., I899, I05-I20 and 320-326).

E. R. Squibb (A. J. Ph., I900, 4-6).

La Wall and Pursel (A. J. Ph., I899, 394).

E. Schmidt (Apoth. Ztg., I900, I3, I4).

W.A . Puckner (Ph. Review, I898, I80-183 and 303-308; also I902, 457-463).

Fromme, (Geschäftsbericht, Cæsar and Loretz, 1905, 87).

Farr and Wright (Ph. Jour., Xx, 546).

Beckurts (Apoth. Ztg., XviII, 67).

Thoms (Bericht. d. Ph. Gesell., xv, 85).

Pearson and Roberts (A. J. Ph. (I908), Lxxx, 368).

Assay of Hyoscyamus Leaves. Hyoscyamus leaves may be assayed by the method of Keller, or by some modification of it.

Keller's Method is as follows: To 20 gms. of the leaves dried over sulphuric acid, reduced to No. 40 powder and contained in a shortnecked flask, are added roo cc. of chloroform-ether mixture (chloroform 20 cc., ether U.S. P. 80 cc.), and after standing for a few minutes, Io cc. of ammonia-water (Io per cent), then the flask is well corked and shaken thoroughly and frequently for one hour. At the end of this time the mixture is transferred to a small narrow percolator (improvised by drawing out a piece of glass tubing or a large test-tube about $25 \mathrm{~mm}$. in diameter) provided with a plug of cotton at the outlet and the percolate received in a separator. When nearly all the liquid has passed through, the drug is packed down rather firmly by means of a glass rod. When the liquid no longer drops from the 
percolator, $25 \mathrm{cc}$. of the same chloroform-ether mixture is poured upon the drug remaining in the flask, and with it the emainder of the drug transferred to the percolator.

When this liquid also has passed, a further $25 \mathrm{cc}$. of the same menstruum is used to complete the extraction. The united chloroform-ether solution, containing the alkaloid from 20 gms. of the drug, is now extracted successively with IO, IO, IO, and Io cc. of I per cent hydrochloric acid. This acid solution, which has been received in a second separator, is rendered alkaline with $5 \mathrm{cc}$. of ammonia-water (Io per cent) and shaken with 20, I0, and Io cc. of chloroform. The chloroform as it is drawn off is passed through a small pellet of cotton (to retain any water accidentally carried over) into a shallow beaker and evaporated at a temperature of $25^{\circ}$ to $35^{\circ} \mathrm{C}$. When all has evaporated the residue is dissolved in $5 \mathrm{cc}$. of ether and again brought to dryness. In the residue the alkaloid is determined by adding ether 2 or 3 cc., cochineal test solution 3 to 5 drops, and decinormal acid in slight but distinct excess, and after complete solution has taken place, and the ether which was added to assist in the solution of the alkaloid has evaporated, determining the excess of acid with decinormal alkali.

The number of cc. of decinormal acid combined with the alkaloid, multiplied by the factor $0.02868 \mathrm{gm}$., indicates the weight of alkaloid calculated as hyoscyamine in $20 \mathrm{gms}$. of the drug.

If $\frac{\mathrm{N}}{2}$ acid is used the factor is $0.01434 \mathrm{gm}$. Hæmatoxylin may be employed as indicator.

The U. S. P. recommends the employment of the same method as that used for belladonna, with the exception that $25 \mathrm{gms}$. of hyoscyamus are to be taken, the quantity of chloroform-ether mixture, which is added at first, increased from 50 to $100 \mathrm{cc}$., and the product at the end of the assay multiplied by 4 instead of ro. Not less than 0.08 per cent of mydriatic alkaloids should be present.

\section{LITERATURE}

W. A. Puckner. Ph. Rev., I 898 (Vol. xvi), I80 and (Vol. xxmI).

H. Beckurts. Apoth. Ztg., XVIII, 67.

W. A. Puckner. Proc. A. Ph. A., I899, 297.

H. Beckurts. Ph. Centralh., I 894,566 .

K. Schwickerath. Ph. Rundschau, I893 (Vol. XI), 282

E. Schmidt. Apoth. Ztg., I900, I3, I4.

Scopola and Stramonium are both assayed by the same methods recommended for belladonna and hyoscyamus. The former should 
contain not less than 0.5 per cent and the latter not less than 0.35 per cent of mydriatic alkaloids.

\section{ASSAY OF CINCHONA}

The U. S. P. (1890) Method. (a) For Total Alkaloids. 20 gms. of the cinchona in very fine powder (No. 80 or finer and completely dried at $100^{\circ} \mathrm{C}$.) are introduced into a bottle provided with an accurately fitting glass stopper, and to this is added $200 \mathrm{cc}$. of a previously prepared mixture of I9 volumes of alcohol, 5 volumes of chloroform, and I volume of ammonia-water; the bottle is stoppered, and thoroughly and frequently shaken during four hours. The liquid is then passed through a plug of cotton in a funnel into another bottle, being careful that there occurs no loss by evaporation.

I00 cc. of the clear filtrate (representing Io gms. of cinchona) are transferred to a beaker and evaporated to dryness. The crude alkaloids thus obtained are dissolved in ro cc. of water and $4 \mathrm{cc}$. of normal sulphuric acid with the aid of gentle heat. The cooled solution is then filtered into a separator, and the beaker and filter washed with water until the washings no longer have an alkaline reaction, using as little water as possible.

Now add $5 \mathrm{cc}$. of potassium hydroxid V. S., or sufficient to render the liquid alkaline. The alkaloids are thereby reliberated, and may be shaken out by chloroform. $20 \mathrm{cc}$. of chloroform are first added, and the extraction repeated, using Io cc. at a time, until a drop of the last chloroform extraction leaves no residue when evaporated on a watch-glass.

The chloroformic extracts are then mixed, evaporated in a tared beaker, the residue dried at $100^{\circ} \mathrm{C} .\left(2 \mathrm{I} 2^{\circ} \mathrm{F}\right.$.) and weighed.

The weight multiplied by io will give the precentage of total alkaloids in the specimen tested.

The volumetric method cannot very well be employed here, as the alkaloids exist in varying proportions and are very numerous, thus making it difficult to find a factor which will answer for all cases.

However, some experimenters dissolve the weighed alkaloids in Io or I5 gms. of alcohol, adding water until slight permanent turbidity appears, and then titrate with $\frac{\mathrm{N}}{10}$ hydrochloric acid, using hæmatoxylin as indicator. I cc. of the $\frac{\mathrm{N}}{\mathrm{TO}}$ acid is assumed to be equivalent to $0.0315 \mathrm{gm}$. of calisaya alkaloids and of $0.0304 \mathrm{gm}$. of succirubra alkaloids.

(b) For Quinine. Transfer $50 \mathrm{cc}$. of the clear filtrate remaining over from the preceding process (and representing 5 gms. of cinchona) 
to a beaker, evaporate it to dryness, and proceed as directed in the assay for total alkaloids, using, however, only half the amounts of volumetric acid and alkali there directed.

Add the united chloroformic extracts containing the alkaloids in solution, gradually and in small portions at a time, to about 5 gms. of powdered glass contained in a porcelain capsule placed over a xater-bath, so that when the contents of the capsule are dry, all or nearly all of the dry alkaloids shall be in intimate admixture with the powdered glass, and the chloroform completely expelled. Now moisten the residue with ether, and having placed a funnel containing a filier $(7 \mathrm{~cm}$. in diameter) and well wetted with ether over a small graduated tube $A$, transfer to the filter the ether-moistened residue from the capsule. Rinse the latter, several times if necessary, with fresh ether, so as to transfer the whole of the residue to the filter; then percolate with ether, drop by drop, until exactly Io cc. of percolate are obtained. Then collect another Io cc. by similar slow percolation with ether in a second graduated tube $B$. Transfer the contents of the tubes to two small tared capsules, properly marked, $A$ and $B$, and evaporate to a constant weight at $100^{\circ} \mathrm{C}$. $\left(212^{\circ} \mathrm{F}\right.$.) and weigh them. (The residue in $A$ will contain practically all of the quinine, together with a portion of the alkaloids less soluble in ether; the residue in $B$ will consist almost entirely of these alkaloids.)

From the amount of residue obtained in $A$ deduct that contained in $B$. This will give approximately the amount of quinine present in the 5 gms. of sample. Multiply this by 20 , and the percentage of quinine containing one molecule of water is obtained.

Cinchona calisaya should contain not less than 5 per cent of total alkaloids, and at least 2.5 per cent of quinine.

The assay method of the 1900 U. S. P. is a gravimetric one, and is based on Keller's method. It is directed to macerate $15 \mathrm{gms}$. of the drug in No. 80 powder or finer, with I $25 \mathrm{cc}$. of ether, $25 \mathrm{cc}$. of chloroform, and ro cc. of ammonia-water during five hours. From I00 cc. of the decanted liquid the alkaloids are abstracted with dilute acid. In one half of the acid extraction the total alkaloids are determined, in the other haif the ether-soluble alkaloids are determined. This method is criticized by A. B. Lyons (Proc. A. Ph. A., 1906, 440) and by J. M. Francis (same vol., page 453). The ether-soluble alkaloids include quinine, quinidine, and cinchonidine.

Gordin's Modified Alkalimetric Method.* 4 gms. of the very finely powdered bark are digested with $100 \mathrm{cc}$. of modified Prollius fluid, shaking frequently during four hours. $50 \mathrm{cc}$. of the liquid

* Proc. A. Ph. A., 1900, 125. 
(representing 2 gms. of the bark) are then drawn off, evaporated, and the residue taken up with strongly acidulated water and filtered. The filtered liquid is then made strongly alkaline with potassium hydroxid, and shaken out three times with a mixture of 3 parts of ether and $\mathrm{I}$ of chloroform, using $25 \mathrm{cc}$. each time. The united ethereal liquids are then shaken up with about half a gram of calcined magnesia. This completely removes the small quantity of water together with traces of the alkali present.

The ethereal liquid is filtered into a flask, 40 cc. of $\frac{\mathrm{N}}{40} \mathrm{H}_{2} \mathrm{SO}_{4} \mathrm{~V}$. S. added, and after shaking well the ethereal liquid is completely distilled off. The acid liquid is then poured into a roo-cc. measuring flask, the distilling flask washed twice with $5 \mathrm{cc}$. of water, and Mayer's reagent added in small quantities at a time till the reagent is in considerable excess. The flask is then filled up to the roo-cc. mark with water and shaken until the supernatant liquid is clear, the liquid is filtered and $50 \mathrm{cc}$. of the clear filtrate, titrated with $\frac{\mathrm{N}}{40} \mathrm{KOH}$, using phenolphthalein as the indicator.

For total alkaloids, the mean factor of quinine and cinchonidine was taken, which for $\frac{\mathrm{N}}{40}$ acid V. S. is $0.0077 \mathrm{gm}$.

C. Kippenberger's Method. 9 gms. of the finely-powdered bark are extracted with $90 \mathrm{cc}$. of alcohol containing $9 \mathrm{cc}$. of diluted hydrochloric (G. Ph.) and 6o cc. of the filtrate are evaporated to dryness. The residue is dissolved in $60 \mathrm{cc}$. of water containing $6 \mathrm{cc}$. diluted hydrochloric acid (G. Ph.), and $50 \mathrm{cc}$. of this filtrate (= 5 gms. of the bark) are treated with 30 to $40 \mathrm{cc}$. of the iodin solution, modified as under Alcoholic Extract of Cinchona, and then further treated as under Assay of Aconite Leaves.

In the alkalimetric estimation of cinchona, hæmatoxylin, Brazilwood, or cochineal may be used as indicator. To these indicators, compounds like the normal quinine sulphate react neutral. To methylorange, on the other hand, the acid sulphate of quinine reacts neutral and the normal sulphate alkaline. Hence if this latter indicator is used, the titration equivalent for quinine is one-half what it is with the other indicators.

The German Pharmacopœia Method. Place I2 gms. of the drug (in fine powder, and dried at $100^{\circ} \mathrm{C}$.) into a 250 -cc. flask, add 30 gms. of chloroform and 90 gms. of ether, stopper the bottle and shake it briskly.

Add Io cc. of soda solution (G. Ph. I5 per cent) and shake frequently for three hours; then add Io cc. of water, or as much more as may be necessary to cause the powder to agglutinate into lumps 
upon shaking and the supernatant ethereal liquid to become quite clear. After standing for one hour, decant $100 \mathrm{gms}$. of the clear chloroform-ether solution (representing Io gms. of the drug), and pass it through a small, dry, well covered filter into a flask and distil off about half of it. Introduce the rest into a separating funnel, rinsing the flask three times with a mixture of chloroform and ether $(\mathrm{r}: 3)$, using $5 \mathrm{cc}$. each time and adding the rinsings to the contents of the separator.

Then add 25 cc. of $\frac{\mathrm{N}}{\mathrm{IO}}$ hydrochloric acid, shake thoroughly, and set aside until the liquids have separated. If necessary, add a little more ether to insure more complete separation. Draw off the aqueous layer, pass it through a filter moistened with water, into a roo-cc. Hlask, wash the chloroform-ether mixture in the separator with three portions of water (Io cc. each), passing the washings through the same filter into the flask, and further dilute with water to $100 \mathrm{cc}$. Remove $50 \mathrm{cc}$. of this solution, add a few drops of a freshly prepared alcoholic solution of hæmatoxylin, and titrate with $\frac{\mathrm{N}}{\mathrm{IO}} \mathrm{KOH}$ until, upon shaking, a violet-blue color is obtained. Not more than $4.3 \mathrm{cc}$. should be required.

Florence's Method.* (I) The Simple Rapid Method. This method is recommended where extreme accuracy is not required. I 2 gms. of the finely-powdered bark are shaken with I 20 gms. of the pure alcohol-free ether, Io cc. of Io per cent sodium hydroxid are added, and the mixture is frequently shaken during one hour. Then Io gms. of water are added, so that the powdered bark may separate as a coherent mass, whereupon the ether extraction is decanted and shaken with 20 to $30 \mathrm{cc}$. of lime water to remove resinous constituents. Now decant roo gms. (or an aliquot portion) of the ether extraction into a glass-stoppered vessel, add $30 \mathrm{cc}$. of water, allow $\frac{\mathrm{N}}{\mathrm{IO}}$ ethereal solution of oxalic acid (freshly prepared by dissolving $0.63 \mathrm{gm}$. crystallized oxalic acid in $100 . \mathrm{cc}$. of pure ether) to flow into the ethereal solution of alkaloid from a burette, as long as a precipitate is produced or until a drop of the aqueous layer reacts neutral with litmus. The number of cc. of oxalic acid solution consumed, multiplied by 0.035 , gives the amount of total alkaloid in $10 \mathrm{gms}$. of bark (= 100 gms. of ether extraction).

Under the conditions of this experiment, all of the cinchona alkaloids in the ether extraction form white precipitates which, with the

* Bull. des scienc. pharmacolog., I906, $3^{6} 5$ (Pharm. Centralh., I907, 48, 405 . 
exception of the quinine oxalate formed, are dissolved in the water provided for this purpose. The

Determination of Quinine is therefore simply effected by collecting the precipitate on a filter, washing it with water until lime water ceases to produce turbidity, then drying and weighing it. The quantity of quinine oxalate so ascertained, multiplied by 0.878 , gives the amount of quinine in Io gms. of the sample ( $\mathrm{r}$ gm. quinine oxalate $=0.878 \mathrm{gm}$. quinine).

(II) The Exact Method. In the exact method the drug is treated in an extraction apparatus with a mixture of ether, four parts, and chloroform, one part, until a portion of the percolate is not rendered turbid by addition of ethereal oxalic acid solution. The ethereal liquid is then extracted in a separatory funnel with three portions of lime water, the latter extracted twice with a little ether, the ethereal liquids united, brought to dryness and the residue weighed as total alkaloids. To determine the quinine the total alkaloids are dissolved in ether, or in ether to which one fifth its volume of chloroform has been added, and $30 \mathrm{cc}$. of an aqueous saturated solution of quinine oxalate and then the quinine precipitated with the ethereal oxalic acid solution, as in the short method. The ether is decanted to a tared filter, then the precipitate transferred to the filter and washed with a saturated solution of quinine oxalate until the washings are rendered no more turbid on the addition of lime water than a saturated solution of quinine oxalate when treated in the same way. The precipitate is allowed to drain, the filter thd precipitate pressed between filter-paper to absorb most of the retained wash fluid and weighed. It is then dried, finally at $100^{\circ} \mathrm{C}$. and again weighed. Since I cc. water dissolved $0.00069 \mathrm{gm}$. quinine oxalate there is subtracted from the last weight $0.00069 \mathrm{gm}$. for every gram difference between the first and second weight and also the weight of the filter-the remainder is the weight of quinine oxalate. The quinine oxalate solution is prepared by treating quinine sulphate with sodium hydroxid and ether, precipitating the ether solution of quinine with an ether solution of oxalic acid, collecting the precipitate, washing it with ether and drying it.

Fromme's Method. G. Fromme* recommends the following method for assaying cinchona bark: 2.5 gms. of the air-dry powder (fine or coarse) are heated for ten minutes on a steam bath with a mixture of $2 \mathrm{cc}$. of pure 25 per cent hydrochloric acid and $20 \mathrm{cc}$. of distilled water. After cooling, 50 gms. of ether and $25 \mathrm{gms}$. of chloroform are added, the mixture is vigorously shaken, then supersaturated with $5 \mathrm{cc}$. of 15 per cent sodium hydroxid solution, vigorously and continiously shaken

* Ph. Ztg., I, No. 73 (1905), 770; from Ber. of Cæsar and Loretz. 
during ten minutes, and $\mathrm{I} .5 \mathrm{gms}$. of powdered tragacanth having been added, the mixture is once more well shaken. The now clear chloroform-ether solution is filtered into a thoroughly cleansed 200-gm. flask until $60 \mathrm{gms}$. of filtrate (corresponding to 2 gms. of the bark) are collected, and in this the alkaloid is determined either gravimetrically or volumetrically as follows:

a. Gravimetrically. The filtrate ( 60 gms., 2 gms. bark) is shaken out with 20 , IO, Iо cc. of I per cent hydrochloric acid-if necessary, with a fourth portion, consisting of ro cc. of $\frac{\mathrm{N}}{\mathrm{IO}}$ hydrochloric acid if a reaction is obtained with Mayer's reagent in the last portion of acid liquid. The united acid liquids are mixed with ${ }_{5} 5 \mathrm{cc}$. of chloroform, ammonia-water is added in moderate excess, and the mixture is vigorously shaken. The chloroform solution is filtered through a double filter into a small, tared Erlenmeyer flask, and the aqueous liquid shaken out twice more than Io and Io cc. of chloroform, the solution being filtered through the same double filter into the flask. The chloroform is distilled off and the residue heated to $100^{\circ} \mathrm{C}$. to constant weight; the ascertained weight, multiplied by $5^{\circ}$, giving the percentage of alkaloid.

b. Volumetrically. The filtrate $(60$ gms. $=2$ gms. bark) is subjected to evaporation (or distillation); the residual alkaloid is dissolved in $10 \mathrm{cc}$, of alcohol, ro cc. of ether and $30 \mathrm{cc}$. of water are added, followed by a few drops of hæmatoxylin solution, and titrated with $\frac{\mathrm{N}}{10}$ hydrochloric acid, shaking after each addition, and adding towards the end of the titration 10 and $30 \mathrm{cc}$. more of water. The titration is ended as soon as the liquid assumes a lemon-yellow color. Each cc. of the $\frac{\mathrm{N}}{\mathrm{I} 0}$ acid combines with $0.0309 \mathrm{gm}$. of cinchona alkaloids; by multiplying the number of cc. of $\frac{\mathrm{N}}{10}$ acid consumed by 0.0309 , the amount of alkaloid in 2 gms. of bark is ascertained, and this, multiplied by $5 \circ$, gives the percentage. Of the two methods the gravimetric method is regarded as the more reliable.

A. Panchaud $*$ has determined that cinchona alkaloids readily decompose chloroform according to $\mathrm{CHCl}_{3}+\mathrm{O}=\mathrm{COCl}_{2}+\mathrm{HCl}$. If cinchona alkaloids are dissolved in chloroform in the evening and titrated the next morning, from 20 to roo per cent of the alkaloid will be found to have been neutralized by the hydrochloric acid produced in the decomposition of the chloroform. Since the decomposition of

* Schweiz. Wochenschr. f. Pharm., 44, 580 (Chem. Centralbl., 1906, 2, 1212. 
$0.0229 \mathrm{gm}$. chloroform will produce sufficient hydrochloric acid to neutralize $0.120 \mathrm{gm}$. alkaloids, the error liable to be introduced thereby in the volumetric estimation of cinchona alkaloids is obvious. Panchaud therefore cautions that any solution of cinchona alkaloids which contains chloroform must be evaporated at once.

A. Limmer, at the University of Strasburg, has studied this question and has not confirmed Panchaud's results. While he found that a number of alkaloids do decompose chloroform with formation of chlorid, the amount so decomposed is slight. There is, nevertheless, some danger of error from this decomposition in the estimation of alkaloids, and it should be borne in mind.

\section{LITERATURE}

De Vrij. Ph. Jour. and Trans., I875, 46r.

De Vrij. The Hague, July 5, r880.

J. Muter. Analyst, I880, 223.

A. Petit. Chem. and Drug., I884.

G. Shimoyama. Arch. d. Ph. (1885), 8I-20).

E. Landrin. Compt. rend., ro8, 750.

W. Haubensak. Schweiz. Wochenschr. (I89I), I47.

C. Kursteiner. Schweiz. Wochenschr. (I892), xxx, 473.

W. Lenz. Ph. Ztg., Sept. 24 (1898), 683, in which chloral hydrate is usèd as a solvent.

E. R. Squibb. A. J. Ph. (1899), $3^{12}$, in which acetic acid is used as a menstruum.

Raymond High. Am. Drug. (I899), 354.

Sidney C. Gadd. Ph. Jour. (4), 2 I, I34.

J. M. Francis. Bull. Ph. (1905), 364 .

Vigeron. J. Ph. Chim. (6), 2I, I80.

P. W. Roberts. Proc. Chem. Soc., 21, 242.

W. Duncan. Ph. Jour. (4), 20, 437.

\section{ASSAY OF COCA LEAVES}

The principal active alkaloid of this drug is cocaine, which may be readily extracted by means of the ordinary solvents, especially ether, petroleum-ether (benzin), and also kerosene. This alkaloid is very unstable and is accompanied by other kindred alkaloids which may, however, be converted into ordinary cocaine. In assaying coca and its preparations, it is not absolutely necessary to determine the quantity of cocaine. It is usually considered sufficiently accurate to estimate the ether-soluble (or benzin-soluble) alkaloids.

For the extraction Keller's process may be employed with very satisfactory results. Quite as good results may be obtained by the 
use of petroleum-benzin, with a boiling-point of about $80^{\circ} \mathrm{C}$. The kerosene process of Dr. Squibb is also a good one.

The U.S. P. VIII Process. Place ro gms. of the coca leaves in an Erlenmeyer flask, add $50 \mathrm{cc}$. of a mixture of chloroform I volume, and ether four volumes, and insert the stopper securely. Allow the flask to stand ten minutes, then add $2 \mathrm{cc}$. of ammonia-water mixed with $3 \mathrm{cc}$. of distilled water, and shake the flask well, at frequent intervals, during one hour. Then transfer as much as possible of the contents of the flask to a small percolator which has been provided with a pledget of cotton packed firmly in the neck, and inserted in a separator containing $6 \mathrm{cc}$. of normal sulphuric acid V. S., diluted with $20 \mathrm{cc}$. of distilled water. When the liquid has passed through the cotton, pack the coca firmly in the percolator with the aid of a glass rod, and, having rinsed the flask with ro cc. of chloroform-ether mixture, transfer the remaining contents of the flask to the percolator by the aid of several small portions $(5 \mathrm{cc}$.) of a chloroform-ether mixture, using the same proportions as before, and continue the percolation with successive small portions of the same liquid (in all $50 \mathrm{cc}$.). Next, shake the separator well for one minute, after securely inserting the stopper, and when the liquids have completely separated, draw off the acid liquid into another separator. Add to the chloroform-ether mixture Io cc. of a sulphuric acid mixture, using the same proportions as before, agitate well, and again draw off the acid liquid. Repeat this operation once more, drawing off the acid solution as before into the second separator, introduce a small piece of red litmus paper, add ammoniawater until the liquid is distinctly alkaline, and shake out with three successive portions of ether $\left(25,20\right.$, and $\mathrm{I}_{5} \mathrm{cc}$.): Collect the ether solutions in a beaker, place it on a water-bath filled with warm water, and allow the ether to evaporate entirely. Dissolve the residue in $3 \mathrm{cc}$. of ether, and let this also evaporate completely. To the alkaloidal residue add $4 \mathrm{cc}$. of tenth-normal sulphuric acid V. S. and five drops of hamatoxylin or iodeosin T. S., then titrate the excess of acid with fiftieth-normal potassium hydroxid V. S. Divide the number of cc. of fiftieth-normal potassium hydroxid V. S. used by 5, subtract this number from 4 (the $4 \mathrm{cc}$. of tenth-normal sulphuric acid V.S. taken), and multiply the remainder by 0.03 and this product by ro, to obtain the percentage of ether-soluble alkaloids contained in the coca.

Among other methods are Kebler's modification of Keller's method. (See page 5 I 7.)

Lyons' Process No. I (Lyons' "Assay of Drugs "). Into a fourounce prescription vial put ro gms. of the drug in moderately fine powder. Pour in Ioo cc. of Prollius fluid, cork securely and shake very frequently during four or five hours. Then draw off $50 \mathrm{cc}$. of 
the clear liquid (representing 5 gms. of the drug) and evaporate it to dryness. The residue is taken up with acid-free ether, drained off, and again evaporated to dryness. Once more ro cc. of pure ether are added together with $5 \mathrm{cc}$. of $\frac{\mathrm{N}}{20}$ acid V. S., the ether evaporated off, and after adding an indicator, the excess of acid is retitrated with $\frac{\mathrm{N}}{20}$ alkali. The quantity of alkali used deducted from the $5 \mathrm{cc}$. gives the quantity of $\frac{\mathrm{N}}{20}$ acid V. S. which combined with the alkaloid. This, multiplied by the factor and then by 2, gives the quantity of alkaloid present in the Io gms. taken. The factor for cocaine when $\frac{\mathrm{N}}{20} \mathrm{~V}$. S. is used is $0.01565 \mathrm{gm}$.

The Squibb Kerosene Process as Modified by Lamar.* This process, which, according to several experimenters, is quite satisfactory, is as follows:

Place 25 gms. of the powdered leaf into a covered jar of about $450 \mathrm{cc}$. capacity, add $25 \mathrm{cc}$. of an approximatelv 2 per cent ammonia solution (Squibb uses 7 per cent solution of crystallized sodium carbonate), and mix well together by means of a stout glass rod of such a length that, while in the jar, will allow the cover to rest in its normal position. Permit this to macerate for half an hour, covered, stirring from time to time. At the end of this time, the odor of ammonia being still perceptible, add gradually, with stirring, $75 \mathrm{cc}$. of kerosene oil. Then cover the jar and set aside for an hour or more, stirring frequently, after which transfer to a cylindrical percolator of $500 \mathrm{cc}$. capacity and percolate with the oil at the rate of six or eight drops per minute, collecting about $450 \mathrm{cc}$. of percolate. Transfer this percolate to a separatory funnel of 700 to $75^{\circ} \mathrm{cc}$. capacity (Squibb's pattern) and add $25 \mathrm{cc}$. of $\frac{\mathrm{N}}{\mathrm{IO}}$ hydrochloric acid and shake continually for ten minutes. Allow the separator to rest, and when the liquids have separated draw off the acid liquid into another separator of 265 to $285 \mathrm{cc}$. capacity. Then shake the oil in the separator with two further portions of $25 \mathrm{cc}$. each of $\frac{\mathrm{N}}{\mathrm{jO}}$ hydrochloric acid, mixing the three acid solutions so collected. To the united acid solutions, now add $20 \mathrm{cc}$. of ether and shake well. When the liquids have separated, the acid solution is drawn off into another separator and ${ }_{5} \mathrm{cc}$. of ether added

* See Squibbs' Ephemeris, III, 1104; and William R. Lamar, A. J. Ph. (IgOr), I 25 . 
to it, the mixture shaken, and the separated acid solution again drawn off into a third separator, thus removing the last traces of kerosene oil and coloring matter. The ether remaining in the first separator is shaken successively with two portions of water, of $5 \mathrm{cc}$. each, after separation has taken place; these in their turn are added to the second ether washing, and after separating, drawn off into the third separator, containing the major portion of the acid solution. To this is then added a sufficient quantity of ro per cent ammonia-water diluted with four times its volume of water to render the liquid slightly alkaline. Now extract the alkaloids with three successive portions of ether, using 40,30 , and $30 \mathrm{cc}$., taking care in each instance to allow the ether to separate completely, drawing off the aqueuus liquid carefully into another separator and pouring the ethereal solution of the alkaloids out through the upper opening of the separator into a tared beaker of 160 cc. capacity.

Rinse the separator with ro cc. of ether, pouring it out at the top, into the separator containing the aqueous portion. Now rinse the rim of the separator and its cork stopper with ether in such a manner as to cause the ether to flow into the separator containing the aqueous portion, and finally add more ether to make in all $30 \mathrm{cc}$. for the second extraction. Shake the separator with its contents actively for a few minutes, then allow the liquids to separate, drawing off the aqueous portion into the separator previously emptied, the ethereal layer being added to that already in the beaker. This operation is repeated a third time. The beaker containing the ethereal solution of the alkaloids is set in a warm place $\left(3 \circ-35^{\circ} \mathrm{C}\right.$.), and as soon as the ether has evaporated, it is dried at a temperature of $60^{\circ} \mathrm{C}$. until of a constant weight, this usually requiring about three hours. The weight obtained, multiplied by 4 , expresses the percentage of alkaloids in the leaf.

The alkaloids so obtained are almost colorless, possessing only a faint cream tint, and are beautifully crystalline in appearance. If it is desirable, as a check upon the weight, they may be titrated, using an excess of $\frac{\mathrm{N}}{20}$ sulphuric acid (about $25 \mathrm{cc}$.) and a few cc. of ether to facilitate the solution, and after the ether has been entirely dissipated, the excess of acid is determined by means of $\frac{\mathrm{N}}{20}$ potassium hydroxid, using cochineal tincture as indicator.

The factor for the pure alkaloids as determined by numerous assays is $0.01514 \mathrm{gm}$. as the equivalent of I cc. of $\frac{\mathrm{N}}{20}$ sulphuric acid. The extremes are 0.01493 and $0.0155 \mathrm{gm}$. 
Léger's Process.* Having determined the amount of moisture in a small amount of the powdered leaves, a quantity of the same powder equivalent to $25 \mathrm{gms}$. of the dried leaves is intimately mixed in a mortar with $5 \mathrm{gms}$. of magnesia and $15 \mathrm{cc}$. of distilled water. The mixture is introduced into a $\mathrm{I}$-liter wide-mouthed, glass-stoppered flask, and treated with $625 \mathrm{cc}$. of ether (sp.gr. 0.72I), saturated with water. The flask is then stoppered, tied down with a piece of cloth, well shaken up, and set aside for twelve hours, with frequent agitation. The whole is then shaken up, transferred to a filter, the filtrate collected in a 500-cc. graduated flask, the funnel being covered with a glass plate during filtration. The $500 \mathrm{cc}$. of filtrate thus collected, equivalent to 20 gms. of dry powder, is distilled in several portions from a dry 250-cc. flask by plunging the latter in warm water. The green residue is dissolved in $20 \mathrm{cc}$. of neutral ether, Io cc. of $\frac{\mathrm{N}}{30}$ hydrochloric acid, and $20 \mathrm{cc}$. of water are added, the flask closed with a rubber stopper, and agitated. The whole contents are then trans. ferred to a separator, and the acid liquid, after separation, withdrawn into a conical flask. The ether layer is then twice shaken out with $25 \mathrm{cc}$. of distilled water, these washings being added to the acid liquid in the flask. This acid solution is filtered through a moistened double filter into a wide-mouthed glass-stoppered 500-cc. flask, and the filter thoroughly washed through into the same. Sufficient distilled water is added to make up the volume to $150 \mathrm{cc}$., when sufficient neutral ether to give a layer $\mathrm{I} \mathrm{cm}$. deep is added. Five or six drops of 0.2 per cent iodeosin solution are then added, and the amount of free acid titrated back in the usual manner with $\frac{\mathrm{N}}{\mathrm{IO}}$ potassium hydroxid solution. The number of cc. of acid thus found to be combined with the coca alkaloids, multiplied by 0.1535 , gives the percentage of these in the powder.

\section{LITERATURE ON THE ASSAY OF COCA}

Koehler. A. J. Ph., I888, 238.

Van der Marck. Ph. Ztg. (I889), 39, 282.

Schwickerath. Ph. Runds., I893, 282.
A. Gunn. J. Ph. u. Chem., I893, 99, I52.
K. De Jong. Ph. Ztg., No. 37 (I905), 991.
E. R. Squibb. Ephemeris, Vol. III, I IоI.
Bloementhal. Ph. Ztg., No. 47 (1905), 497.
H. Matthes and O. Ramstedt. Ph. Ztg. (I906), LI, го3г. 


\section{ASSAY OF COLCHICUM}

Owing to the ready decomposition of the alkaloid colchicine (the active principle of the root and seeds of Colcinicum autumnale) by both acids and alkalies, its extraction by immiscible solvents, such as Prollius' fluid, etc., is not advisable. This, together with the presence of oily matter in the drug, makes the assay of colchicum a matter of considerable difficulty. The drug is best exhausted by hot extraction with alcohol, and the following method, which depends upon the saponification of the alkatoid and retitration with acid, may be used.

Method of Gordin and Prescott (Proc. A. Ph. A., I900, I33). $25 \mathrm{gms}$. of the powdered drug are exhausted in a Soxhlet extraction apparatus with strong alcohol for two hours. The alcohol is then distilled off completely, the oily residue taken up with about ro cc. of hot water, and the mixture poured into a separating funnel. The extraction apparatus is washed several times with hot water and the washings added to the mixture in the separating funnel; $203 \mathrm{cc}$. of petroleum ether are now poured carefully into the separating funnel and the latter inclined in different directions without shaking. The petroleum ether then takes up the oily drops adhering to the sides of the vessel. In fifteen or twenty minutes the oil will be found floating on the surface of the aqueous liquid. The lower aqueous liquid is run ofi into a roo-cc. measuring-tiask.

In order to completely remove any colchicine which may still be in the oil, about ro cc. of water are added to the contents of the separator, the latter shaken vigorously, and its contents drawn off into an evaporatingdish. The petroleum ether is then evaporated off by heating on a waterbath. This will leave a clear aqueous liquid with an oily layer on its surface. This is returned to the separator and treated as before with 2 or $3 \mathrm{cc}$. of petroleum ether, and the aqueous liquid drawn off into the measuringflask. The washing of the oil with water may be repeated until no trace of colchicine is leit in the oil, as shown by acidulating the aqueous liquid drawn off and treating with Mayer's or Wagner's reagent.

The turbid liquid in the flask is now diluted to roo cc., I or 2 gms. of purified talcum added, the flask thoroughly shaken, and the liquid filtered through a dry filter.

$80 \mathrm{cc}$. of the clear filtrate (representing $20 \mathrm{gms}$. of the drug) are measured off into a separator and shaken out three times with chloroform, using about $30 \mathrm{cc}$. each time. The chloroformic solutions of the alkaloid are filtered into a flask and the chloroform distilled off. The residue of colchicine left in the flask is treated with Io cc. of water, and while the flask is kept on a boiling water-bath air is drawn over its surface by means of a pump for about half an hour. The colchicine 
can now be estimated either gravimetrically or by saponification or by both methods in succession, using one method as a check upon the other. For the saponification method $40 \mathrm{cc} . \frac{\mathrm{N}}{40} \mathrm{KOH} \mathrm{V.S.} \mathrm{are}$ added to the solution of the colchicine, the flask connected with a reversed condenser, and the liquid boiled briskly on a wire gauze for two hours, then cooled, the liquid diluted to about $100 \mathrm{cc}$,, and the excess of $\mathrm{KOH}$ titrated back with $\frac{\mathrm{N}}{40} \mathrm{HCl}$ V. S., using phenolphthalein as indicator. The number of cc. of $\frac{\mathrm{N}}{40} \mathrm{HCl}$ deducted from 40 gives the quantity of $\frac{\mathrm{N}}{40} \mathrm{KOH}$, which was consumed in the saponification. This divided by 20 gives the percentage of colchicine in the drug.

Assuming that $12.2 \mathrm{cc}$. of the $\frac{\mathrm{N}}{40} \mathrm{KOH}$ were consumed, then the 20 gms. contained $0.6 \mathrm{I}$ per cent of colchicine.

The $\frac{\mathrm{N}}{40}$ factor is about $0.0099275 \mathrm{gm}$.

Panchaud's Method.* Panchaud has determined the solubility of colchicine in mixtures of chloroform, ether, and petroleum-ether. $\mathrm{He}$ finds that petroleum-ether having a boiling-point of $50^{\circ}$ to $60^{\circ} \mathrm{C}$. must be used in the assay and the ether must be completely dehydrated over metallic sodium. For the estimation of colchicine he directs that I 5 gms. coarsely powdered colchicum seeds be treated in a flask with I50 gms. chloroform, the mixture shaken frequently during thirty minutes, then $6 \mathrm{cc}$. Io per cent ammonia-water added and the mixture shaken thoroughly. After occasional shaking during one half hour, Io̊ gms. are to be filtered off through a plain filter of $20 \mathrm{~cm}$. diameter into a 200-cc. Erlenmeyer flask, the funnel being kept covered. The solution is distilled to complete dryness and the residue dissolved in I gm. dry chloroform, I gm. dry ether added, and then 30 gms. dry petroleum-ether. The liquid and precipitate is transferred to a plain filter of $8 \mathrm{~cm}$. diameter, using further petroleum-ether to complete the transfer. The funnel containing the precipitate is placed on an empty flask and the precipitate dissolved with warm chloroform, care being taken that it is completely dissolved by washing the edge of the filter with chloroform. The chloroformic solution is distilled and the residue dissolved in 15 drops of chloroform, 2 gms. absolute ether added and, after solution, $30 \mathrm{cc}$. dry petroleum-ether. The liquid

*Schweiz. Wochenschr. f. Ch. u. Ph., 1906, 564; Ph. Centrh., I907 (48), 75 . 
and precipitate is poured on a tared, plain filter of $8 \mathrm{~cm}$. diameter. Floccules adhering to the flask are dissolved in five drops of chloroform, I gm. ether added and then Io gms. dry petroleum-ether; the mixture transferred to the first filter and the precipitate washed with a little petroleum-ether. The weight of the precipitate, plus $0.0022 \mathrm{gm}$. (correction for solubility of colchicine in the quantity of solvent used), multiplied by ro, furnishes the colchicine content of the drug.

\section{ASSAY OF CONIUM}

The active principle of this drug is the volatile alkaloid coniine. Because of the volatile nature of this alkaloid, specimens of the drug are frequently deficient in alkaloid and sometimes entirely devoid of coniine. The volatile character of this alkaloid makes it furthermore necessary to exercise great care in its estimation. The following method of A. B. Lyons, and which is also the pharmacopoial method, is a practicable one:

Place the conium in a $200-\mathrm{cc}$. Erlenmeyer flask, add $100 \mathrm{cc}$. of a mixture of ether 98 parts, alcohol 8 parts, and ammonia-water 3 parts (by volume), insert the stopper securely, and shake the flask at intervals during four hours. After the powder has settled, decant $50 \mathrm{cc}$. of the clear liquid into a beaker (representing 5 gms. of conium), and add sufficient normal sulphuric acid V.S. to produce a distinctly acid reaction. Evaporate the ether at a gentle heat by the aid of a waterbath; then add ${ }_{15} \mathrm{cc}$. of alcohol, and set the beaker aside in a cool place for two hours to allow the ammonium sulphate to deposit. Filter the liquid; wash the residue and filter with a little alcohol, and add the washings to the filtrate; neutralize any excessive amount of acid with sodium carbonate T.S., being careful to retain a slight acidity. Concentrate the liquid to $3 \mathrm{cc}$. by the aid of a gentle heat on a water-bath, add $3 \mathrm{cc}$. of distilled water and two drops of normal sulphuric acid V.S. Add $\mathrm{I} 5 \mathrm{cc}$. of ether to remove traces of fatty matter, pour off the ether solution and repeat the washing with 15 cc. of ether. Then transfer the acid liquid to a separator, introduce a small piece of red litmus paper, and add sufficient sodium carbonate 'T. S. to render the liquid slightly alkaline; then shake out with successive portions of $15,{ }_{5}$, and $10 \mathrm{cc}$. of ether. The assay is then finished gravimetrically, according to the U. S. P., by following (I) or, volumetrically, by following directions under (II).

(I) To the combined ether solutions, in a tared beaker, add, drop by drop, sufficient hydrochloric acid solution ( 5 per cent) to insure an excess of acid, and then evaporate the ether by a gentle heat on a. water-bath. Remove the excess of hydrochloric acid by adding to 
the residue $3 \mathrm{cc}$. of alcohol and heating gently to evaporate the liquid, repeat this operation once, and dry the residue (coniine hydrochlorid) at a temperature not exceeding $60^{\circ} \mathrm{C}$. ( $\left(140^{\circ} \mathrm{F}\right.$.) until the weight, after cooling in a desiccator, remains constant. The weight of the residue multiplied by 0.777 , and this product by 20 , gives the percentage of coniine contained in the conium.

(II) Treat the ethereal solution with exsiccated calcium sulphate (neutral) to remove minute droplets of alkaline water, and then titrate with standard acid $\frac{\mathrm{N}}{20}$ or $\frac{\mathrm{N}}{10}$ sulphuric acid. Iodeosin is the indicator. Each cc. of $\frac{\mathrm{N}}{20}$ acid represents $0.01262 \mathrm{gm}$. of coniine.

A. B. Lyons, in his "Handbook of Assaying of Drugs and Galenicals," gives an alternative process in which the drug is moistened with an equal weight of 5 per cent crystallized sodium carbonate solution, and then macerated for four hours with petroleum-benzin, or percolated with this menstruum. Then, in an aliquot portion of this percolate, the alkaloid is extracted by shaking out with acidulated water, the assay being then completed as directed in the preceding process. Other methods are proposed by Schwickerath $(\mathrm{Ph}$. Record, I893, 282) and R. A. Cripps (Ph. Jour. Trans. (3), I8, pp. 12, 54, 820 and 888); J. v. Braun (Berichte d. Chem. Ges., 38, 3108); W. A. H. Naylor (Brit. Col. Drug., 48, 77); T. Maben (Brit. Col. Drug., 48, 83).

\section{ASSAY OF HYDRASTIS CANADENSIS}

This drug contains two principle alkaloids, namely, hydrastine $\left(\mathrm{C}_{21} \mathrm{H}_{21} \mathrm{NO}_{6}\right)$ and berberine $\left(\mathrm{C}_{20} \mathrm{H}_{17} \mathrm{NO}_{4}\right)$. The former, a colorless crystalline alkaloid, is the characteristic alkaloid of the drug and is recognized as the true active principle of hydrastis canadensis. The other is a deep yellow alkaloid and is the one to which the drug owes its bitterness and its color. This alkaloid is found in many other plants and is therefore not characteristic of hydrastis. Hydrastine is freely soluble in ether and is also soluble in petroleum-benzin and the usual alkaloidal solvents. It is very feebly alkaline. Berberine, on the other hand, is practically insoluble in ether, but is soluble in chloroform and forms crystallizable salts which are mostly insoluble in water, particularly in the presence of acids. In all assays of this drug its therapeutic value is based upon the quantity of hydrastine present. The most satisfactory assay methods for this drug are gravimetric. Hydrastine being so feebly alkaline, alkalimetric methods are not satisfactory as a rule. Lyons, in his "Assaying of Drugs and Galenicals," 
I899, page 177 , asserts that he finds no difficulty in the titration, using hæmatoxylin, cochineal, or methyl-orange. A residual titration may be made, indeed, in a sufficiently concentrated aqueous solution without using any indicator, owing to the sparing solubility of the alkaloid.

The U. S. P. Method. Introduce $15 \mathrm{gms}$. of the hydrastis (in No. 60 powder) into an Erlenmeyer flask of $250 \mathrm{cc}$. capacity, add I50 cc. of ether, shake the flask during ten minutes, and add $5 \mathrm{cc}$. of ammonia-water, again shaking the flask at intervals during half an hour. Then add I5 cc. of distilled water to the mixture in the flask and shake it until the drug collects in masses, and at once pour off, into a measuring-cylinder, $100 \mathrm{cc}$. of the supernatant ether solution and transfer it to a separator. Add I 5 cc. of normal sulphuric acid V. S. to the separator, and shake it moderately during one minute. Allow the liquids to separate, and draw off the lower acid liquid into a second separator. Again shake out the ether solution with $5 \mathrm{cc}$. of normal sulphuric acid V.S. and $5 \mathrm{cc}$. of distilled water, and shake the separator for one minute. After the liquids have separated, draw off the acid solution as before into the second separator. Repeat the same process with $5 \mathrm{cc}$. of distilled water, drawing this also into the second separator. Introduce a small piece of red litmus paper into the second separator, add enough ammonia-water to render the liquid alkaline, and then $25 \mathrm{cc}$. of ether, and shake the separator moderately during one minute, and when the liquids have separated draw off the lower alkaline liquid into another separator, and the ether solution into a tared beaker. Again shake out the alkaline liquid, using $20 \mathrm{cc}$. of ether, shake the separator for one minute, and when the liquids have separated, draw off the alkaline liquid into the other separator, and the ether solution into the tared beaker. Finally, again shake out the alkaline liquid, using $\mathrm{I}_{5} \mathrm{cc}$. of ether, proceeding as before, and adding the ether solution to the liquid in the tared beaker. Evaporate the ether carefully with the aid of a water-bath, and dry the alkaloidal residue in the beaker to a constant weight at $100^{\circ} \mathrm{C}$. $\left(2 \mathrm{I} 2^{\circ} \mathrm{F}\right.$.). The weight found, multiplied by Io, will give the percentage of hydrastine in the hydrastis.

Puckner* gives the following modification of the foregoing:

The modified assay process is carried out as follows: To $5 \mathrm{gms}$. drug in No. 60 powder and contained in an Erlenmeyer flask, 50 cc. ether is added, and the mixture rotated occasionally during ten minutes. Then ammonia-water, 2 cc., is added, the mixture shaken thoroughly and frequently during one-half hour, and then transferred to a percolator. The percolate is received in a separator and the

* Ph. Rev., I908, I36. 
drug extracted with a further quantity of ether, $50 \mathrm{cc}$. The ethereal liquid is extracted successively with a mixture of dilute hydrochloric acid, 2 cc., and water, 18 cc. with a mixture of dilute hydrochloric acid, 5 drops, and water, Io cc., and finally with water, 10 cc. The united extractions are rendered alkaline with ammonia-water, using litmus test solution as an indicator and extracted with three portions of ether, 20 cc. each, using three separators. The united ethereal solutions are evaporated at a moderate temperature, and the residue dried at $98^{\circ}$ to $100^{\circ}$ until it ceases to lose in weight.

The Method of Gordin and Prescott.* This method, which estimates both hydrastine and berberine, is based upon the following plan: The alkaloids of the powdered root, are first set free by the action of a mixture of ammonia and ether (stronger ammonia-water $5 \mathrm{cc}$., alcohol $5 \mathrm{cc}$., ether $30 \mathrm{cc}$.). After drying the powder is extracted with absolute ether, and the ethereal extract, after evaporation of the ether and taking up of the residue with acidulated water, is used for the estimation of hydrastine. Through the powdered root left in the extraction apparatus, air is passed till it is dry, and then the powder is extracted with alcohol to exhaustion. The alcoholic extract, after dilution with water, evaporation of the alcohol and taking up the residue with dilute acetic acid, is used for the estimation of berberine. The berberine is first precipitated as berberine acetone, the latter washed, decomposed by the aid of acid, and the purified berberine estimated by standard solution of potassium iodid, silver nitrate, and ammonium thiocyanate.

The Assay. Io gms. of the finely powdered hydrastis are rubbed up to a paste with a few cc. of the above-mentioned ammonia-ether mixture in an eight-ounce ointment jar, and a few cc. more of the same mixture are then added so as to have the powder well covered with the liquid. The small pestle is then left inside, and the jar well covered and set aside over night. The jar is then opened, put into a good current of air till the odor of ammonia has disappeared, and then in a vacuum over sulphuric acid for about five or six hours; the powder is then inclosed in filter-paper, placed in a Soxhlet extraction apparatus, the jar rinsed out several times with powdered glass or with coarsely powdered barium nitrate, the rinsings added to the Soxhlet, the latter connected with an Erlenmeyer flask containing about 40 or 50 cc. of absolute ether, and the extraction conducted in the usual way, till a few drops, after evaporation of the ether and acidulation, give no reaction with Mayer's or with Wagner's solution.

The Erlenmeyer is then detached from the Soxhlet, the ether

* J. A. C. S., I899. 735 . 
poured out into a flat evaporating-dish, the Erlenmeyer washed out several times with water containing about 2 per cent sulphuric acid, the washings added to the contents of the evaporating-dish, and the latter put into a draught at about $30^{\circ} \mathrm{C}$. till the ether has disappeared.

The contents of the dish are poured into a roo-cc. flask, the dish washed, the washings added into the flask and the latter filled up to the Ioo-cc. mark. The solution containing hydrastine sulphate, of which every Io cc. represent I gm. of the root, is used for the estimation of hydrastine.

For the iodometric estimation $20 \mathrm{cc}$. of the filtered solution (representing 2 gms. of the drug) are run from a burette into a I00-cc. flask containing 20 or $30 \mathrm{cc}$. of a standardized solution of iodin of any known strength (that in the neighborhood of one per cent is the best) and the analysis carried out exactly as described on page 507. From the amount of iodin consumed the amount of hydrastine is deduced by using the factor of the hydrastine hexaiodid, i.e., $0.60403 \mathrm{gm}$. of hydrastine for one of iodin consumed.

For the estimation of berberine a current of dry air is passed through the Soxhlet till all the ether is removed, the Soxhlet connected with an Erlenmeyer containing 40 or $50 \mathrm{cc}$. of alcohol, and the extraction continued until the alcohol comes out colorless. The alcoholic extract containing the berberine, and considerable quantities of extractive matter, is poured out into an evaporating-dish, the Erlenmever washed out with hot water and a little dilute acetic acid, the washings added to the evaporating-dish and the latter kept on a water-bath, adding water from time to time till all the alcohol has disappeared. A little more diluted acetic acid is now added, the dish covered, and when completely cold its contents are filtered into an Erlenmeyer having the capacity of about 300 or $400 \mathrm{cc}$.*

Six to eight cc. of acetone are added to the contents of the Erlenmeyer, to which the washings of the dish and the filter have been added, and then a io per cent solution of sodium hydroxid is added, drop by drop, till the precipitate first formed ceases to disappear on shaking, and the liquid acquires a strongly alkaline reaction. The Erlenmeyer is then stoppered and shaken in a circular direction for about ten or fifteen minutes, and then set aside in a cool place for

* In the remaining procedure, the simplest way would be to precipitate the berberine with hydrochloric or nitric acid, but in this case a considerable amount of extractive matter contaminates the precipitate and too high a yield would result though the error in this respect might be compensated to some extent by the solubility of the hydrochlorid or nitrate in water. But the best way is to purify the berberine by converting it into berberine acetone, regenerate the alkaloid by means of sulphuric acid and then estimate it volumetrically by standart potassium iodid. 
two or three hours. The berberine acetone separates out in crystals, some of which adhere to the sides of the vessel. The supernatant liquid is then poured on a small filter, the precipitate washed once or twice by decantation and then on the filter till the washings are colorless. The filter is then pierced and, by means of the wash-bottle, the precipitate is returned to the same Erlenmeyer in which the precipitation took place. In this way all loss is avoided. To the precipitate about 4 or $5 \mathrm{cc}$. of a 5 per cent solution of sulphuric acid is now added, and then water enough to make about 100 or $200 \mathrm{cc}$. The Erlenmeyer is now put into hot water when the precipitate will completely dissolve in the course of a few minutes. The solution is now poured out into a long-necked flask, washing the Erlenmeyer several times, the flask put on an asbestos plate and kept very gently boiling for about an hour an a half or two hours, adding hot water from time to time if necessary. The flask is now cooled and its contents poured out into a liter flask containing roo cc. of $\frac{\mathrm{N}}{20}$ potassium iodid solution. The flask is washed several times, the washings added to the measuring-flask and the latter filled up to one liter and set aside over night. $500 \mathrm{cc}$. are now filtered off into another liter flask, $50 \mathrm{cc}$. of $\frac{\mathrm{N}}{20}$ silver nitrate and nitric acid added to the flask, which is filled up to one liter, well shaken, filtered, and $500 \mathrm{cc}$. of the liquid titrated back with $\frac{\mathrm{N}}{40}$ ammonium sulphocyanate, using ferric alum as indicator. Twice the number of cc. of sulphocyanate solution used is equal to the number of cc. of the potassium iodid solution consumed by the berberine, representing Io gms. of the hydrastis root. By multiplying the number of cc. of $\frac{\mathrm{N}}{20}$ potassium iodid consumed by 0.167 I 25 , the per cent of anhydrous berberine in the root is obtained, as I cc. of the potassium iodid solution is equal to $0.0167125 \mathrm{gm}$. of berberine.

O. Schreiber* has subjected ten samples of hydrastis root, as found in the European markets, to alkaloidal assay by the following method: The amount of moisture having been determined in Io gms. of the powdered sample by drying to constant weight, the dried powder was moistened with a mixture of ammonia, 5 cc., alcohol, 5 cc., and ether, $30 \mathrm{cc}$., and dried. It was then extracted in a Soxhlet with ether; the ether extract shaken out with 15 gms. of 5 per cent hydrochloric acid in a graduated cylinder. The ethereal layer was decanted, the acid extract washed with more ether to remove resinous

* Ph. Post., I901, 36, 321. 
matter, and the ether decanted. The volume of ether over the acid liquor was then adjusted to exactly $50 \mathrm{cc}$., Io cc. of ammonia added, and the whole well shaken until all of the precipitated alkaloid was dissolved in the ethereal layer. After separation, $40 \mathrm{cc}$. of this was decanted $\left(=\frac{4}{5}\right.$ of the whole), into a tared capsule, about half the ether evaporated off with a gentle heat, the rest allowed to evaporate spontaneously. In this manner almost colorless crystals of hydrastine were obtained, which were finally dried to constant weight on the water-bath. The poorest sample examined was thus shown to contain 2.85 per cent, and the best 4.16 per cent of alkaloid.

\section{ASSAY OF IPECAC}

In the U. S. P. VIII ipecac is defined as "The dried root, to which may be attached a portion of the stem not exceeding $7 \mathrm{~cm}$. in length, of Cephaelis Ipecacuanha (Brotero), A. Richard (Fam. Rubiacce), known commercially as Rio, Brazilian, or Para ipecac, or the corresponding portion of Cephaelis Acuminata, Karsten, known commercially as Carthagena ipecac, yielding when assayed by the process given below, * not less than 2 per cent of 'ipecac alkaloids.' " Ipecac contains, according to Paul and Cownley, three distinct alkaloids, namely, emetine, cephæline, and psychotrine. There is a marked difference between Brazilian and Carthagena ipecac in the relative proportions of the contained alkaloids. In the former the proportion of emetine in the total alkaloidal content is about 70 per cent, while in the latter it is about 40 per cent. The table by Paul and Cownley on page 55I shows the relative proportion in the two varieties of root.

The two first alkaloids differ considerably in their physiological and therapeutic action. Emetine being regarded as the expectorant and cephaline as the emetic principle of the drug. The two varieties of the drug cannot therefore be considered as therapeutically identical. Because of this difference in the proportion of the contained alkaloids it is always advisable to specify the variety of ipecac assayed. The third alkáloid (psychotrine) is present in such small quantity that it may be ignored in the assay of the root. It is desirable not only to insure complete extraction of the alkaloids but their separation. This latter is, however, not absolutely necessary since it is considered sufficiently accurate for the purpose of the pharmacist to express the result as mixed alkaloids, as in the U. S. P. assay.

* The U.S. P. process. 


\begin{tabular}{|c|c|c|c|}
\hline \multirow{2}{*}{. } & \multicolumn{2}{|c|}{ Brazilian. } & \multirow{2}{*}{ Columbian. } \\
\hline & Root. & Stems. & \\
\hline 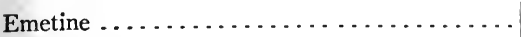 & I. 45 & I . I 8 & 0.89 \\
\hline 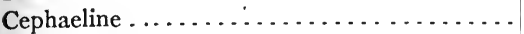 & $0.5^{2}$ & 0.59 & I. 25 \\
\hline Psychotrine...$\ldots \ldots \ldots \ldots \ldots \ldots \ldots$ & 0.04 & 0.03 & 0.06 \\
\hline
\end{tabular}

TABLE SHOWING PER CENT OF EACH ALKALOID IN THE TOTAL ALKALOIDAL CONTENT

\begin{tabular}{|c|c|c|c|}
\hline & \multicolumn{2}{|c|}{ Brazilian. } & \multirow{2}{*}{ Columbian. } \\
\hline & Root. & Stems. & \\
\hline 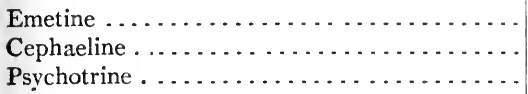 & $\begin{array}{r}72.14 \\
25.87 \\
\text { I. } 99\end{array}$ & $\begin{array}{r}65.6 \\
32.8 \\
\text { I. } 6\end{array}$ & $\begin{array}{r}40.5 \\
56.8 \\
27\end{array}$ \\
\hline
\end{tabular}

In this assay, which is a typical Keller method, the two principal alkaloids are assumed to be present in almost equal proportions, and the factor for "ipecac alkaloids" is found by taking the mean of their molecular weights. Thus

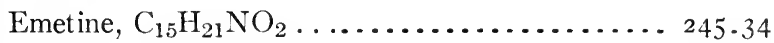

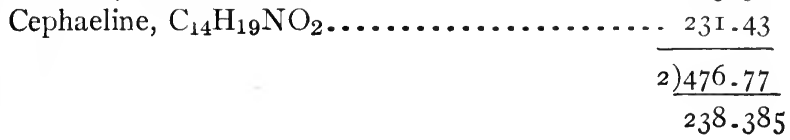

The formulæ here given tor emetine and cephaeline depend upon the assumption that these alkaloids are non-acid bases. The formula of $\mathrm{Kunz}, \mathrm{C}_{30} \mathrm{H}_{40} \mathrm{~N}_{2} \mathrm{O}_{5}$, for emetin is obviously incorrect, because he operated upon a mixture of the alkaloids. Kunz considered emetin to be a diacid base, witk a molecular weight of 504.5. Therefore in assaying ipecac, if the alkaloidal value is expressed in terms of emetinKunz, it will not differ very greatly from an assay in which the result is expressed as "ipecac alkaloids." The $\frac{\mathrm{N}}{10}$ factor being in the former case $0.025^{2} \mathrm{gm}$. and in the latter $0.0238 \mathrm{gm}$. However, an assay 'calculated in either of these ways, although it is sufficiently accurate for the purpose of the pharmacist, does not give the relative proportions of the two principal alkaloids; and since these alkaloids 
differ decidedly in their therapeutic action, it is a matter of some importance to know which is present in the greater quantity. The advisability of specifying the variety of ipecac assayed is therefore apparent.

The U. S. P. Method. "Introduce 15 gms. of the ipecac (in No. 80 powder) into an Erlenmeyer flask of 250 cc. capacity, add I $5 \mathrm{cc}$. of ether and $35 \mathrm{cc}$. of chloroform, shake the flask during five minutc:, and then add 3 cc. of ammonia-water and again shake the flask at intervals during half an hour. Now arld ro cc. of distilled water, shake the liquid until the powder collects in masses, and pour off roo cc. of the clear ethereal solution into a measuring-cylinder. Transfer the latter to a separator, add Io cc. of normal sulphuric acid and Io cc. of distilled water. Shake the separator moderately during two minutes. and when the liquids have separated, draw off the lower acid solution into a second separator. Repeat the shaking out of the ether solution with $3 \mathrm{cc}$. of normal sulphuric acid and $5 \mathrm{cc}$. of distilled water, drawing the acid solution into the second separator. Repeat the shaking out again, using io cc. of distilled water, and add the aqueous solution to the second separator. Reject the ether in the first separator, introduce a small piece of red litmus paper into the second separator, add enough ammonia-water to render the liquid alkaline, and $25 \mathrm{cc}$. of ether, and then shake the separator vigorously during one minute; draw off the alkaline aqueous liquid into another separator, and transfer the ether solution to a flask. Add $20 \mathrm{cc}$. of ether to the alkaline liquid in the separator, shake it for one minute, and, having allowed the liquids to separate, draw off the alkaline liquid into the other separator, and transfer the ether solution to the flask. Again shake out the alkaline liquid with Io cc. of ether, and, when the fluids have separated, reject the alkaline liquid and add the ether solution to the liquid in the flask. Distil the ether from the flask with the aid of a water-bath, and dissolve the alkaloidal residue in $\mathrm{I} 2 \mathrm{cc}$. of tenth-normal sulphuric acid, warming it gently on a water-bath if necessary. Then add five drops of hæmatoxylin T. S. and titrate with fifticth-normal potassium hydroxid. Divide the number of cc. of fiftieth-normal potassium hydroxid used by 5 , subtract the quotient from I2 (the I2 cc. of tenth-normal sulphuric acid taken), and multiply the remainder by 0.0238 , and this product by 10, which will give the percentage of alkaloids in the ipecac."

The German Pharmacopœa employs the same method for ipecac as for aconite.

Gordin's Method (A. J. Ph., I906, 461). Put 5 gms. of ipecac (No. 60 powder) into the shaking tube (Fig. 89); add 2.5 cc. of a Io per cent solution of sodium carbonate and $25 \mathrm{cc}$. of a mixture of three volumes of ether and one volume of chloroform. After shaking 
for one hour, percolate to exhaustion. Shake out the percolate three times with small quantities of very dilute sulphuric acid, add excess of sodium hydroxid, and shake out three times with ether-chloroform. Distil the ethereal solution to about one-half, dilute with ether to about the original volume, and finish as directed for aconite root (page 525 ).

G. Fromme's Method.* This simple method was designed to overcome the difficulty in titrating the ipecac alkaloids, occasioned by their discoloring the solutions.

Six grams of the drug in fine powder, 120 gms. ether, and $5 \mathrm{cc}$. ammonia-water to per cent, are shaken during half an hour, put aside to settle, and then Ioo gms. decanted through a pledget of cotton. The ether is distilled off, the residue dissolved in $5 \mathrm{cc}$. of absolute alcohol, $20 \mathrm{cc}$. of ether, and Io cc. of water. Three drops of hæmatoxylin solution are then added and the $\frac{\mathrm{N}}{\mathrm{IO}}$ acid run in; toward the end of the titration $30 \mathrm{cc}$. of water are added gradually and the addition of the standard acid continued until the color change is complete.

Separate Estimation of Emetine and Cephaeline (Paterson). $\dagger$ The following method is recommended as being accurate, quick, and easy and well adapted to small quantities (say ro gms.) of the root. Agitate ro gms. of the powdered drug with Io cc. of ammonia water (or Io cc. of sodium carbonate solution $\mathrm{I}: 3$ ) and $\mathrm{I} 20 \mathrm{cc}$. of a menstruum, composed of I part of chloroform, I part of amyl alcohol, and 3 parts of ether, in a stoppered bottle during one hour. Then add from Io to $15 \mathrm{cc}$. of water in order to aggregate the powder; decant Ioo cc. of the ethereal liquid, evaporate it to one half, and shake it out with $\mathrm{I}_{5}$ cc. (or an excess) of $\frac{\mathrm{N}}{\mathrm{IO}}$ hydrochloric acid, followed by three portions of $5 \mathrm{cc}$. each of water. To the aqueous solution of the alkaloids so obtained now add an excess (about 2 cc.) of normal potassium hydroxid, and shake it out with ether in four portions of 15 , IO, Io, and 5 cc. respectively, reserving both the ethereal and aqueous portions. Having mixed the ethereal solution, shake out three times with Io, 5 , and $5 \mathrm{cc}$. of $\frac{\mathrm{N}}{2 \mathrm{c}}$ potassium hydroxid, mix the latter and shake out with Io cc. of ether; then evaporate the ethereal solution, and weigh the residue as emetine (or titrate it; I cc. $\frac{\mathrm{N}}{\mathrm{jO}}$ acid $=0.0245 .3 \mathrm{gm}$. emetine). Finally, mix all the aqueous solutions, acidify with hydro-

* Ph. Ztg., XLIX (Sept. I 7, I904), 79г.

$\dagger$ Ph. Jour., July I 8 and 25, I903, 73-75 and ror, I02. 
chloric acid. Make alkaline with ammonia, and shake out with four portions of $20,10,10$, and $5 \mathrm{cc}$. of ether-chloroform $(1: 6)$; evaporate and weigh as cephaeline. Instead of weighing the cephaeline it may also be titrated-the factor being 0.02314-using methyl-orange as indicator.

Paul and Cownley's Method.* Mix $50 \mathrm{gms}$. of the powdered ipecac with Io gms. of freshly-slaked lime, moistened with water, and extract by percolation with amylic alcohol. Extract the alkaloids from the percolate by shaking out with dilute sulphuric acid, make the solution alkaline with ammonia-water and shake out with ether. The psychotrine will remain in the aqueous solution from which it can be removed by chloroform. The ethereal solution is evaporated and the residue titrated with seminormal hydrochloric acid. The hydrochloric acid solution is mixed with sodium hydroxid solution in excess, and shaken out with ether to remove emetine. Some cephaeline, however, accompanies it, so that it is necessary to redissolve the emetine in dilute acid and repeat the treatment with soda and ether until the residual alkaline solution is no longer rendered cloudy upon the addition of ammonium chlorid. The purified emetine is finally determined by titration with standard acid. The cephaeline is obtained from the alkaline residues containing it, by acidifying, adding ammonia and shaking out with ether. It is finally titrated with standard acid. The total number of cc. of seminormal hydrochloric acid used in titrating the separated bases should be equal to the number required before their separation. When the separation has been satisfacotrily made, the emetine hydrochlorid should be readily obtained in crystalline form on evaporation of the solution; and the solution of cephaeline hydrochlorid should give the characteristic crystals of cephaeline when shaken out with ether and ammonia.

The following table is interesting in that it demonstrates that the two standard methods, Keller's and Paul and Cownley's, give very closely agreeing results:

\begin{tabular}{|c|c|c|c|}
\hline & \multirow{2}{*}{$\begin{array}{c}\text { Keller. } \\
\text { Total } \\
\text { Alkaloids. }\end{array}$} & \multicolumn{2}{|c|}{ Paul and Cownley. } \\
\hline & & Emetine. & Cephaeline. \\
\hline Rio root $\ldots \ldots \ldots \ldots \ldots \ldots$ & 2.8 .46 & 2.026 & 0.842 \\
\hline Rio root . ............ & 2.297 & $\mathrm{I} \cdot 355$ & 0.984 \\
\hline Johore root $\ldots \ldots \ldots \ldots \ldots \ldots \ldots$ & $25 \mathrm{II}$ & 1.539 & 0.820 \\
\hline Carthagena root...$\ldots \ldots \ldots \ldots$ & 2.875 & I. 544 & 1389 \\
\hline
\end{tabular}

* Ph. Jour., ז896 (April 25), 32 I. 
G. Frerichs and N. de Fuentes Tapis* propose the following method, in which the small quantity of psychotrine (believed to be medicinally inert) is ignored:

Agitate 6 gms. of the finely powdered root for one hour with a mixture of 60 gms. ether and $5 \mathrm{cc}$. ammonia solution, or in place of the latter, $5 \mathrm{cc}$. of sodium carbonate solution $(\mathrm{I}: 3)$, then adding $\mathrm{Io} \mathrm{cc}$. water and evaporating $50 \mathrm{gms}$. of the ether solution $(=5 \mathrm{gms}$. of the drug) to one-half, after filtering, shaking that liquid out with ro cc. decinormal hydrochloric acid and washing it twice with Io cc. water. The acid liquor diluted to roo cc. is then titrated with decinormal potassium hydroxid in the presence of a layer of ether, using iodeosin as an indicator. I cc. of decinormal hydrochloric acid is taken as being equal to $0.024 \mathrm{I} \mathrm{gm}$. emetine and cephaeline, corresponding to the mean of 248 and 234. Instead of titrating the alkaloid as described, the acid solution may be shaken out with ether and ammonia, the ether residue being dried at $100^{\circ} \mathrm{C}$. and weighed; or the dried residue may be titrated; but as a slight decomposition always occurs on drying these sensitive bases, giving rise to a strongly colored solution, the titration is not in that case very distinct.

\section{LITERATURE}

1885. A. B. Lyons. A. J. Ph., page 53I.

r889. Cripps and Whitley. Ph. Jour. Trans., page 721 .

I89o. Arndt. Apoth. Ztg., page 78r.

I89r. Kottmeyer. Ph. Post., pages 9I 3-933.

1895. R. A. Cripps. Ph. Jour., page 159.

1896. Paul and Cownley. Ph. Jour. (Apr. 25), page 32ז.

I896. Lyman F. Kebler. A. J. Ph., page rg6.

1899. Geschäftsbericht, Cæsar and Loretz (Sept.).

I900. J. V. S. Stanislaus. Proc. Indiana Ph. A., pages 6I-64.

I900. Geschäfetsbericht, Cæsar and Loretz (Sept.).

I9o0. La Wall and Pursel. Proc. Penn. Ph. A., page I60.

I900. F. C. Bird. Ph. Jour., pages I 75-1 78, 334-335, 4I4-4I6.

rgor. Paul and Cownley. A. J. Ph., pages 57-66 and 107-1 I6.

1902. Paul and Cownley. Ph. Jour., pages 256 and 317 .

1902. Frerichs and N. de Fuentes Tapis. Arch. d. Ph., 240 (July 25), page 390; (Sept. ro), page 40 r.

1903. Hammond and Sayre. Drug. Circular, No. 47, page 227.

I903. Paterson. Ph. Jour., pages $73-75$ and ror-Io2.

I904. G. Fromme. Ph. Ztg. (Sept. I 7), page 79r.

rgo6. H. M. Gordin. A. J. Ph., page $46 \mathrm{r}$.

* Arch. d. Pharm., 240 (Sept. Io, I9§2), 40r. 


\section{ASSAY OF NUX VOMICA}

The alkaloids of nux vomica are strychnine and brucine. The proportion of strychnine in the total alkaloidal content is from 40 to upward of 50 per cent. The same alkaloids are present in ignatia, but in this the proportion of strychnine is somewhat larger. These two alkaloids are somewhat similar in their medicinal properties, but the toxicity of strychnine is much greater than that of brucine.

In the assay methods of nux vomica, the object is either to determine the total alkaloidal content, or to separate and determine the quantity of strychnine. A determination of the total alkaloids gives a fairly good estimate of the value of a sample of the drug, but it must be admitted that the most satisfactory method is one which includes a determination of the quantity of strychnine.

Methods in which the "Total Alkaloids" is Determined. Nux vomica may be assayed by the Keller method, page 5r5, and by the various modifications of it, among them, Puckner's, page 516; Lyons', page 516; and Kebler's, page 517, besides those described below.

The $\frac{\mathrm{N}}{\mathrm{ro}}$ factor for total alkaloids is $0.03615^{2} \mathrm{gm}$. This factor is obtained by assuming that the two alkaloids strychnine and brucine are present in equal proportions, and taking the mean of their molecular weights.

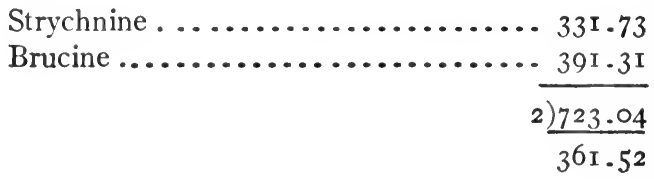

Hæmatoxylin or Brazil-wood solutions may be used as indicators. The German Pharmacopœia Method. I5 gms. of nux vomica (in medium fine powder, dried at $100^{\circ} \mathrm{C}$.) are introduced into a flask, Ioo gms. of ether and $50 \mathrm{gms}$. of chloroform are added and the flask thoroughly shaken. Then Io cc. of a mixture of two parts of sodium hydroxid solution (Ph. G. I5 per cent) and one part of water are added, and the mixture shaken frequently during three hours. Then 15 cc. or a sufficient quantity of water are added, to cause the powder to agglutinate into a lump upon shaking, and the supernatant chloroform-ether solution to separate clear. After standing one hour Ioo gms. of the chloroform-ether solution (representing Io gms. of the drug) are filtered through a dry, well covered filter into a flask.

About one half of this is distilled off, and the rest introduced into 
a separating funnel, rinsing the flask three times with a mixture of chloroform and ether $(\mathrm{I}: 3)$, using $5 \mathrm{cc}$. eack time, and adding the rinsings to the contents of the separator. The mixed solutions are then shaken with Io cc. of $\frac{\mathrm{N}}{\mathrm{IO}}$ hydrochloric acid, and set aside until the liquids have separated. If necessary, add a little more ether to insure the complete separation of the chloroform-ether solution. The lower aqueous solution is now drawn off and filtered through a small filter, moistened with water, into a roo-cc. flask. Wash the chloroformether solution by shaking out with three portions of water (Io cc. each), passing the washings through the same filter, and further wash with water also passed through the same filter to make roo cc. Measure off $50 \mathrm{cc}$. of this solution into a white glass flask of $200 \mathrm{cc}$. capacity and add $50 \mathrm{cc}$. of water, and sufficient ether to form a layer $\mathrm{I} \mathrm{cm}$. in depth. Then add five drops of iodeosin solution and titrate with $\frac{\mathrm{N}}{100} \mathrm{KOH}$, shaking after each addition of the standard alkali solution until the lower layer assumes a pale red color. Not more than $15.6 \mathrm{cc}$. should be required.

$$
\text { Each cc. of } \frac{\mathrm{N}}{100} \mathrm{HCl} 0.003615^{2} \mathrm{gm} \text {. of total alkaloids. }
$$

Puckner's Method (Proc. A. Ph. A., I903, I97). In this method the taking of an aliquot part is avoided, the drug being exhausted by maceration and percolation. By eliminating the aliquot part the use of dry-measuring vessels and their subsequent cleansing is avoided, the loss of volatile solvent by evaporation is of no consequence, and the time of maceration may be reduced to an hour or half an hour, while in the methods where aliquot parts are taken three, six, or even twelve hours' maceration, with frequent, or even continuous, agitation is directed.

To 5 gms. of powdered nux vomica, add $2 \mathrm{cc}$. of ammonia-water and $50 \mathrm{cc}$. of a mixture, made by mixing $7 \mathrm{cc}$. of alcohol, $23 \mathrm{cc}$. of chloroform and $70 \mathrm{cc}$. of ether. Shake frequently during one hour. Then transfer the whole to a small percolator, and receive the percolate in a separator. When the menstruum has all passed through, the drug is packed down and exhausted by percolating with $80 \mathrm{cc}$. of the same mixture. The alkaloids are then extracted from the percolate by shaking out with ro, Io, and ro cc. of normal sulphuric acid. To the combined acid extractions, a drop of cochineal T. S. is added, then an excess of ammonia-water, and the alkaloids abstracted with Io, Io, and Io cc. of chloroform. After the evaporation of the chloroform, the alkaloidal residue is titrated with $\frac{\mathrm{N}}{\mathrm{IO}}$ acid, cochineal being
used as indicator. 
The Modified Alkalimetric Method (Gordin's). 8 gms. of the drug in No. 50 powder are extracted for about two and a half hours in a Soxhlet apparatus with alcohol, the alcoholic extract reduced by evaporation to about Io cc., and then diluted with acidulated water to $50 \mathrm{cc}$. This liquid is then filtered through a little talcum powder and $25 \mathrm{cc}$. of the clear filtrate (representing 4 gms. of the drug) made strongly alkaline with potassium hydroxid solution, shaken out three times with a mixture of ether and chloroform ( 3 and I), using $25 \mathrm{cc}$. each time. The united ethereal liquids are then shaken up with $0.5 \mathrm{gm}$. of calcined magnesia. This completely removes the small quantity of water together with traces of alkali contained in the ethereal liquid. The ether-chloroform is filtered into a flask, $40 \mathrm{cc}$. of $\frac{\mathrm{N}}{40}$ sulphuric acid added, the flask shaken well, and the ethereal liquid removed by distillation.

The acid liquid is then poured into a roo-cc. measuring-flask. The distilling flask is washed two or three times with $5 \mathrm{cc}$. of water and the washings added to the contents of the measuring-flask. Mayer's reagent is then added in small quantities at a time, until the reagent is in considerable excess. The flask is then filled up to the roo-cc. mark, shaken till the supernatant liquid is clear, the liquid filtered, and $50 \mathrm{cc}$. of the clear filtrate removed and carefully titrated with $\frac{\mathrm{N}}{10}$ potassium hydroxid solution, using phenlophthalein as the indicator and the $\frac{\mathrm{N}}{40}$ factor for total alkaloids $0.009 \mathrm{I} \mathrm{gm}$.

Kippenberger's Method (Apoth. Ztg., I898, 664-674). I5 gms. of the drug in fine powder (No. 50) are extracted with a mixture of $140 \mathrm{cc}$. alcohol, $7 \mathrm{cc}$. water, and 3 cc. diluted hydrochloric acid. $100 \mathrm{cc}$. of the filtered extract are evaporated to dryness on a waterbath, the residue dissolved in $60 \mathrm{cc}$. of water containing $2 \mathrm{cc}$. of dilute hydrochloric acid, the solution filtered, and $45 \mathrm{cc}$. of the filtrate (representing $7.5 \mathrm{gms}$. of the drug) are introduced into a flask, and treated with $20 \mathrm{cc}$. of iodin solution (iodin 20 gms., potassium iodid 60 gms., water to make Iooo cc.).

The mixture is allowed to stand about ten minutes or until the alkaloids are completely precipitated as periodid. The precipitate is collected on a small plaited filter and washed two or three times with water to which a few drops of the iodin solution have been added.

The precipitate is then dissolved in 'acetone, about $20 \mathrm{cc}$. being required for complete solution, an excess of potassium hydroxid solution is added, and then an excess of hydrochloric acid. The mixture is diluted with water and shaken out with petroleum ether to remove 
the acetone, the last traces of which are removed by heating on a waterbath.

The liquid is now supersaturated with potassium hydroxid solution and shaken out with chloroform. The chloroformic solution of the pure alkaloid so obtained is cautiously evaporated and the amount of alkaloid determined either by direct weighing or by titration.

Methods in which Strychnin is Determined. The determination of strychnine in admixture with brucine, as in nux vomica and ignatia, is not a difficult matter. This may be made (I) By oxidizing the brucine sulphate or picrate by means of dilute nitric acid into compounds having no basic character, while the strychnine remains unchanged, or at least only slightly altered under the same conditionsGerock,* Keller, $\dagger$ Nagelvoort, $\ddagger$ and Gordin $\$$ have elaborated processes lased upon this reaction and a modification of Gordin's procedure is now official in the U.S. P. VIII. (2) By precipitating the strychnine as ferrocyanid, as designed by Beckurts and Holst $\|$ and Dunstan and Short.

In Gerock's method, the alkaloids are precipitated by picric acid, and after treating the combined picrates with dilute nitric acid (sp.gr. I.056), the unchanged strychnine is collected and weighed.

Keller's Method. The alkaloidal residue from an extraction (about $0.3 \mathrm{gm}$.) is dissolved by the aid of water-bath heat, in Io cc. of dilute sulphuric acid (Io per cent). To this liquid, when cold, I cc. of nitric acid is added (sp.gr. I.42), the mixture well mixed and set aside for one and a half to two hours. The liquid is then treated with Io cc. of ammonia-water, and the strychnine shaken out with a mixture consisting of equal parts of chloroform and ether, successive portions being taken, using about $80 \mathrm{cc}$. in all. Then put into a flask $40 \mathrm{cc}$. of the filtered chloroform-ether solution, distil off the solvent, dry at $95^{\circ}$ to $100^{\circ} \mathrm{C}$. and weigh. The distillation should be discontinued when crystallization of the strychnine begins and the remainder of the solvent driven off by a current of air.

The U. S. P. Method. In this method the brucine is destroyed according to the conditions worked out by H. M. Gordin, and the strychnine isolated and titrated. The directions are, however, somewhat at variance with those of Gordin, which, according to several critics, spoils the method.

* Arch. d. Ph., I889, I 58 and A. J. Ph., I889, I80.

$\dagger$ Zeit. Oest. Apoth. Ver., r893, 587; Proc. A. Ph. A., r894, 53r.

† Proc. A. Ph. A., I893 I64.

\$ Proc. A. Ph. A., 1902, 336 .

il Arch. d. Ph. (3), XXV, 3 I3.

I Brit. Pharmacopoia. 
The Process. Introduce 20 gms. of the nux vomica into a $250-\mathrm{cc}$. Erlenmeyer flask and add to it $200 \mathrm{cc}$. of a mixture of $137.5 \mathrm{cc}$. of ether, $44 \mathrm{cc}$. of chloroform, $13.5 \mathrm{cc}$. of alcohol, and $5 \mathrm{cc}$. of ammoniawater; insert the stopper securely and macerate with frequent shaking during one hour and allow it to stand in a cool place for twelve hours. Decant into a measuring cylinder roo cc. of the liquid (representing Io gms. of nux vomica), and pour this into a separator, preferably of a globular shape. Rinse the cylinder with a little chloroform, add this to the separator, and then add $15 \mathrm{cc}$. of normal sulphuric acid V. S.; shake the mixture moderately during one minute, being careful to avoid emulsification; when the liquids have separated completely, craw off the acid liquid into a beaker. Repeat the shaking out with successive portions of 5 and $3 \mathrm{cc}$. of normal sulphuric acid V.S.; collect the acid solutions and pour them into a separator. If a drop of the last acid solution yields a precipitate with mercuric potassium rodid T. S., repeat the shaking out of the ether solution with $5 \mathrm{cc}$. of normal sulphuric acid V.S. To the combined acid solutions in the separator add a small piece of red litmus paper, $25 \mathrm{cc}$. of chloroform and then sufficient ammonia-water to render the liquid alkaline, and shake the separator thoroughly. When the liquids have separated draw off the chloroform into a flask of $100 \mathrm{cc}$. capacity, and repeat the shaking out of the alkaline liquid with two successive portions of I5 cc each of chloroform, adding the latter to that already in the flask. Evaporate the combined chloroformic solutions in the flask until the alkaloidal residue is dry, then dissolve it in $15 \mathrm{cc}$. of sulphuric acid ( 3 per cent) warming it on a water-bath. When the solution has cooled, add $3 \mathrm{cc}$. of a cooled mixture of equal volumes of nitric acid (sp.gr I.40) and distilled water, and after rotating the liquid a few times, set it aside for exactly ten minutes, shaking it gently three times during this interval. Transfer the resulting red liquid to a separator containing $25 \mathrm{cc}$. of an aqueous solution of sodium hydroxid (I : IO) and wash the flask three times with very small amounts of distilled water, and add the washings to the separator. If the liquid is not turbid add $2 \mathrm{cc}$. more of the solution of sodium hydroxid. Now add $20 \mathrm{cc}$. of chloroform to the separator, and shake it well by a rotating motion for a few minutes; allow the liquids to separate, and draw off the chloroform through a small filter wetted with chloroform, into a flask. Repeat this twice, using ro cc. of chloroform each time, and draw off both portions into the flask, using the same filter. Finally, wash the filter and funnel with $5 \mathrm{cc}$. of chloroform, and then evaporate all the chloroform by means of a water-bath very carefully, to avoid decrepitation. To the alkaloidal residue add $6 \mathrm{cc}$. of tenth-normal sulphuric acid V.S., five drops of iodeosin T. S., about $80 \mathrm{cc}$. of distilled water, and $20 \mathrm{cc}$. of ether. When all the 
alkaloid is dissolved, titrate the excess of acid with fiftieth-normal potassium hydroxid V. S. until the aqueous liquid just turns pink. Divide the number of cc. of fiftieth-normal potassium hydroxid V. S. used by 5 , subtract this number from 6 (the $6 \mathrm{cc}$. of tenth-normal sulphuric acid V.S. taken), multiply the remainder by $0.033^{2}$, and this product by Io, which will give the percentage of strychnine in the nux vomica.

This method, although essentially that of Gordin, differs in some important respects; for instance, nitric acid (sp.gr. I.40) is used, whereas Gordin directs to use a nitric acid of sp.gr. I.42. This means a difference of 4.5 per cent in the strength of the acid, which, according to Gordin, Smith, and Webster and Pursel, is sufficient a difference to destroy the value of the method. Another change from the original method is the omission of the amyl alcohol at the end of the evaporation 'of the alkaloidal solution. The use of this is intended to obviate the necessity of evaporation by heating and thus prevent loss of strychnine.

Webster and Pursel point out that the lack of uniformity in results with this method is due to the absence or varying proportions, if present, of the lower oxids of nitrogen in the nitric acid, and conclude that the most suitable reagent for nitrating the brucine would be nitric acid containing a fair proportion of the lower oxids of nitrogen in solution. This may be secured either by substituting fuming nitric acid (commercial nitrous acid sp.gr. I.42) for the U. S. P. nitric acid, or better, by adding to the latter a certain quantity of sodium nitrite.

These modifications, added to the text of the U.S. P., are as follows: Dissolve the alkaloidal residue in $15 \mathrm{cc}$. of 3 per cent sulphuric acid. To this solution add $3 \mathrm{cc}$. of a mixture of equal parts of nitric acid (sp.gr. I.40) and distilled water; then add I cc. of a 5 per cent solution of sodium nitrite in water, and after rotating the liquid a few times, set aside exactly 30 minutes, stirring it gently three times during the interval. The solution is then made alkaline and shaken out in the usual way. This modification they affirm is accurate over a wide range of temperature.

Gordin's Method.* The following description in the author's own words is from his paper read before the A. Ph. A. (1902): The mixed alkaloids, for example, the residue of total alkaloids obtained in the assay of nux vomica from 8 to ro gms. of the drug, are dissolved in 15 cc. of 3 per cent sulphuric acid by the aid of water-bath heat, the solution is cooled to ordinary temperature, and $3 \mathrm{cc}$. of a previously prepared and cooled mixture of equal parts of strong nitric

* Proc. A. Ph. A. (I902), $33^{6}$. 
acid (sp.gr. I.42) and water added to the alkaloidal solution. The liquid is set aside for exactly ten minutes, shaking it gently three or four times during this time. The red liquid is now transferred to a separator containing 20 or $25 \mathrm{cc}$. of to per cent sodium hydroxid solution,* and the vessel in which the digestion of the alkaloids had taken place is washed three or four times with very small amounts of water. The liquid in the separator will now be very turbid, from separation of strychnine. If this is not the case, there is not enough alkali, and a further addition of $\mathrm{I}$ or $2 \mathrm{cc}$. alkali must be made. After the addition of sufficient alkali, the liquid is shaken out three times with chloroform, using $20 \mathrm{cc}$. for the first shaking out, and ro cc. each time for the two subsequent ones. The chloroformic solution is filtered through a small plain double filter, arranged so that there are four folds of paper on each side, into a light, tared flask, taking care to wash the stem of the separator with a little chloroform; the filter and stem of the funnel are also washed a few times with small amounts of chloroform, and to the perfectly colorless solution of strychnine thus obtained, are added 2 or $3 \mathrm{cc}$. of pure amyl alcohol which distils between $128^{\circ}$ and $132^{\circ} \mathrm{C}$, and leaves no residue on evaporation.

The chloroform is now distilled off completely and the small amount of amyl alcohol left behind removed by keeping the vessel on the water-bath and blowing air over its opening, but so as not to blow out some alkaloid by the air current. The strychnin obtained in this method is very pure and may be weighed, or titrated with standard acid, using hæmatoxylin as indicator.

The Methods Depending upon the Precipitation of Strychnine by Means of Potassium Ferrocyanid are those of Beckurts and Holst and Dunstan and Short. The former consists in dissolving the alkaloidal residue in water strongly acidulated with hydrochloric acid, and titrating with standard solution of potassium ferrocyanid which precipitates the strychnine but leaves the brucine in solution. For determining the end-point a drop of the solution is taken out on a glass rod and brought in contact with paper moistened with a weak solution of ferric chlorid. This is repeated frequently until a blue color is produced, indicating that all of the strychnine has been precipitated. Because of the tediousness and inconvenience of the method of finding the end-point, this method is not in great favor; it is, however, capable of very exact results. The method of Dunstan and Short is the

\footnotetext{
* It is best to place the alkali in the separator while the alkaloids are being digested with the acids, so that after the lapse of ten minutes, when the acid liquor is poured into the separator, the action of nitric acid upon strychnine is quickly arrested.
} 
official method of the British pharmacopœia. In this, the precipitated strychnine ferrocyanid is collected on a filter and washed with acidulated water. It is then decomposed by ammonia and the strychnine shaken out with chloroform.

\section{LITERATURE}

Schweissinger. Am. Drug., I885, 230.

Gerock. Arch. d. Ph., I889, I58, and A. J. Ph. (ז889), s So.

Nagelvoort. Proc. A. Ph. A., I893, I65.

Keller. Zeit. Oest. Ap. Ver., I893, 587, and Schweitz. Wochenschr., I893, 33, 452; also Proc. A. Ph. A., I894, 531.

Gomberg. J. A. C. S., I896, 339.

Prescott and Gordin. J. A. C. S., I898, 722; and Proc. A. Ph. A., I899, 278.

Lenton. Ph. Jour. (Vol. 2I), 864.

Reynolds and Sutcliffe. Ph. Jour. (76), 555.

Beckurts and Holst. Arch. d. Ph. (3), xxv, 313.

C. E. Smith. A. J. Ph., I896, 189.

Bird. Ph. Jour., I900, Sept. 8th, 286.

Farr and Wright. Proc. A. Ph. A., I901, 883 .

Gordin. A. J. Ph., I901, 211; and Proc. A. Ph. A., I902, 336.

F. J. Smith. A. J. Ph., 1903, 253.

Puckner. Proc. A. Ph. A., 1903, 197.

Howard. Analyst, 1905 (xxx), 26r.

Farr and Wright. Trans. Brit. Ph. Conf. (Yearbook), 226-238; and Chem. and Drug., July 28 , I906.

Webster and Pursel. A. J Ph., I907, I-7.

Gordin. A. J. Ph., r907, 6r.

\section{ASSAY OF OPIUM}

Opium contains numerous alkaloids of which morphine is the most abundant and by far the most important. It exists in the drug in the form of a salt of meconic acid, in which form is is readily soluble in water. Narcotine and most of the other alkaloids being relatively insoluble in water are left behind in the aqueous extraction. Any narcotine which dissolves may be separated by precipitating the morphine with ammonia in the presence of ether. The latter holds narcotine in solution. The separation may be also effected by treatment with lime; this dissolves morphine but renders narcotine insoluble. Lead acetate $*$ and lead subacetate $\dagger$ are also employed for the purpose of separating morphine from its accompanying impurities. 
Morphine (the alkaloid) is nearly insoluble in cold water. In its crystalline form it is very sparingly soluble in ether, but if ether is present at the moment that the alkaloid is set free by an alkali, it dissolves much more readily but most of it will in a short time crystallize out of the ethereal solution.

The U. S. P. Method 1890. This is the method proposed by Dr. Squibb.* It is described in the U. S. P. as follows: Opium, in any condition to be valued, Io gms.; ammonia-water, $3.5 \mathrm{cc}$; alcohol, ether, water, each a sufficient quantity. Introduce the opium (which, if fresh, should be in very small pieces, and if dry, in very fine powder) into a bottle having a capacity of $300 \mathrm{cc}$.; add $100 \mathrm{cc}$. of water; cork well. Agitate the bottle frequently during twelve hours; then pour the whole as evenly as possible upon a wetted filter having a diameter of $12 \mathrm{~cm}$., and when the liquid has drained off wash the residue with water carefully dropped upon the edges of the filter and contents , until I50 cc. of filtrate are obtained. Then carefully transfer the moist opium back to the bottle by means of a spatula, add $50 \mathrm{cc}$. of water, agitate thoroughly and repeatedly during fifteen minutes, and return the whole to the filter.

When the liquid has drained off, wash the residue as before until the second filtrate measures $150 \mathrm{cc}$., and finally collect about $20 \mathrm{cc}$. more of a third filtrate.

Evaporate in a tared capsule; first, the second filtrate to a small volume, then add the first filtrate, rinsing the vessel with the third filtrate, and continue the evaporation until the residue weighs I $_{4} \mathrm{gms}$. Pour the liquid into a tared flask, rinse the capsule, and add the rinsings until the entire solution weighs 20 gms. Then add $12.2 \mathrm{cc}$. of alcohol; shake well; add $25 \mathrm{cc}$. of ether; shake again. Now add the ammonia-water, cork well, shake for ten minutes, and set aside for at least six hours or overnight, so that the crystals may form.

At the expiration of this time decant the ethereal layer upon a double, plain, rapidly acting filter previously wet with ether; add ro cc. of ether to the contents of the flask, rotate, and again decant. Repeat this operation with another Io cc. of ether. Then pour the liquid in the bottle upon the filter, in small portions at a time, so as to transfer the greater portion of the crystals to the filter, and wash the remaining crystals on to the filter with the aid of a small quantity of water, using not more than ro cc. Then wash the crystals, first with a few drops of water, then with an alcoholic solution of morphine, and finally with ether to displace the alcohol. Dry the crystals to a constant weight and weigh on a tared watch-glass.

* See Ephemeris III, I $50,116 \dot{r}$. 
The weight of the crystals obtained, when multiplied by ro, represents the percentage of crystallized morphine present in the sample of gum. Opium should contain 9 per cent; the powdered not less than I2 per cent nor more than $\mathrm{T} 2.5$ per cent.

Instead of weighing, the crystals may be dissolved in $\frac{\mathrm{N}}{\mathrm{IO}}$ sulphuric acid, and the solution retitrated with $\frac{\mathrm{N}}{50}$ potassium hydroxid solution, using hæmatoxylin as the indicator and the $\frac{\mathrm{N}}{50}$ factor for morphine
$0.006015 \mathrm{gm}$.

The result of this titration is not entirely satisfactory in that the impurity mixed with the morphine crystals containing as it does calcium compounds (meconate and sulphate) is capable of neutralizing strong acids. These impurities are, however, in a great part insoluble in limewater, whereas morphine is soluble, hence, in the 8th Revision of the U. S. P., an attempt is made to separate the alkaloid by treating the weighed precipitate with lime water. The insoluble residue containing the impurities is then separated by filtration, dried and weighed; the latter weight deducted from the former gives the soluble portion, which is computed as morphine.

The lime water filtrate so obtained, is however, colored, and hence indicates that some of the impurity is soluble. This soluble portion may amount to 4 or 5 per cent.

It is also possible, as pointed out by Parker and others, that the lime water reacts with a portion of the impurities (calcium-ammoniummeconate), changing it to calcium meconate, so that the separated residue has neither the same weight nor composition as the impurities originally weighed with the morphine; therefore this method, though an improvement over the old, is, nevertheless, far from perfect.

Other solvents, such as potassium hydroxid, ethyl, and methyl alcohol, which have been tried, with a view to a separation of the morphine from its impurities, are without any better results.

The U. S. P. Method (8th Decennial Revision). Opium, in any condition to be valued, Io gms., ammonia-water, $3.5 \mathrm{cc}$., alcohol, ether, distilled water, limewater, each a sufficient quantity. Introduce the opium (which, if fresh, should be in very small pieces, and if dry in very fine powder) into an Erlenmeyer flask having a capacity of about $300 \mathrm{cc}$., add roo cc. of distilled water, stopper the flask, and agitate it every ten minutes (or continuously in a mechanical shaker) during three hours. Then pour the contents as evenly as possible upon a wetted filter having a diameter of $12 \mathrm{~cm}$., and, when the liquid has drained off, wash the residue with distilled water, carefully dropped upon the edges of the filter and its contents, until I $_{5} \mathrm{O}$ cc. of filtrate 
have been obtained. Then carefully transfer the moist opium back to the flask by means of a spatula, add $50 \mathrm{cc}$. of distilled water, agitate it thoroughly and repeatedly during fifteen minutes, and return the whole to the filter. When the liquid has drained off, wash the residue as before, until the second filtrate measures $150 \mathrm{cc}$., and finally collect about $20 \mathrm{cc}$. more of a third filtrate. Evaporate carefully in a tared dish; first, the second filtrate to a small volume, then add the first filtrate, rinsing the vessels with the third filtrate, and continue the evaporation until the residue weighs 14 gms. Rotate the concentrated solution about in the dish until the rings of extract are redissolved, pour the liquid into a tared Erlenmeyer flask having a capacity of about roo cc., and rinse the dish with a few drops of water at a time until the entire solution, after the rinsings have been added to the flask, weighs $20 \mathrm{gms}$. Then add Io gms. (or I $2.2 \mathrm{cc}$.) of alcohol, shake the flask well, add $25 \mathrm{cc}$. of ether, and repeat the shaking. Now add the ammonia-water from a graduated pipette or burette, stopper the flask with a sound cork, shake it thoroughly during ten minutes, and then set it aside, in a moderately cool place, for at least six hours, or over night.

Remove the stopper carefully, and should any crystals adhere to it, brush them into the flask. Place in a small funnel two rapidly acting filters, of a diameter of $7 \mathrm{~cm}$., plainly folded, one within the other (the triple fold of the inner filter being laid against the single side of the outer filter), wet them well with ether, and decant the ethereal solution as completely as possible upon the inner filter. Add Io cc. of ether to the contents of the flask, rotate it, and again decant the ethereal layer upon the inner filter. Repeat this operation with another portion of Io cc. of ether. Then pour the liquid in the flask into the filter, in portions, in such a way as to transfer the greater portion of the crystals to the filter and, when the liquid has passed through, transfer the remaining crystals to the filter by washing the flask with several portions of water, using not more than $15 \mathrm{cc}$. in all. Use a feather or rubbed-tipped glass rod to remove the crystals that adhere to the flask. Allow the double filter to drain, then apply water to the crystals, drop by drop, until they are practically free from mother-liquor, and afterwards wash them, drop by drop, from a pipette, with alcohol previously saturated with powdered morphine. When this has passed through, displace the remaining alcohol by ether, using about $10 \mathrm{cc}$. or more, if necessary. Allow the filter to dry in a moderately warm place, at a temperaure not exceeding $60^{\circ} \mathrm{C}$. $\left(140^{\circ} \mathrm{F}\right.$.) until its weight remains constant, then carefully transfer the crystals to a tared watch-glass and weigh them.

Place the crystals (which are not quite pure) in an Erlenmeyer flask, add lime water (Io cc. for each o.I gm. of morphine) and shake 
the flask at intervals during half an hour. Pass the liquid through two counterpoised rapidly acting, plainly folded filters, one within the other (the triple fold of the inner filter being laid against the single fold of the outer filter), rinse the flask with more limewater and pass the washings through the filter until the filtrate, after acidulating, no longer yields a precipitate with mercuric potassium iodid T. S. Press the filters until nearly dry between bibulous paper and dry them to a constant weight, then weigh the contents, using the outer filter as a counterpoise. Deduct the weight of the insoluble matter on the filter from the weight of the impure morphine previously found. The difference, multiplied by ro, represents the precentage of crystallized morphine contained in the opium.

Lyons objects to the direction that the precipitate be washed with morphinated alcohol, as moderate changes in temperature affect the solubility, causing the solution to deposit or dissolve morphine, and also morphine is apt to be deposited by evaporation. He prefers liberal washing with morphinated water, and drying the filter between folds of absorbent paper.

Lamar's Modification of the U. S. P. VIII Method. This is given in Bulletin No. 105, A. O. A. C., U. S. Dept. of Agriculture, in the following words:

Proceed as directed by the Pharmacopœia to precipitation of morphine. To the 20 gms. of aqueous extract add 60 gms. of alcohol, stopple flask, shake well for one minute and set aside for thirty minutes, during which time the precipitated material should have completely subsided. Decant the clear supernatant liquid into a tared 250-cc. evaporating-dish, transfer the precipitate to a $7-\mathrm{cm}$. filter previously moistened with a mixture of alcohol (three parts) and water (one part.) The last portions of the residue are transferred to the filter by using small portions of the above hydro-alcoholic solution. The filtrate is to be collected in the tared evaporating-dish. Continue washing the residue and filter by dropping the alcoholic solution on the filter and the residue until the filtrate is no longer bitter. Add $35 \mathrm{cc}$. of water to the contents of the evaporating-dish and evaporate on water-bath to I4 gms., then proceed as directed by the Pharmacopœia.

Mallinckrodt suggests a reassay of the precipitated impure morphine crystals by a modification of the old lime method as follows:

Place 1.2 gms. mixed crude morphine in an 8o-cc. Erlenmeyer flask, add $0.5 \mathrm{gm}$. freshly slacked lime and $20 \mathrm{cc}$. water, cork, and shake occasionally for one half hour. Filter into a similar tared flask with gentle suction (reinforcing the point of the filter with a platinum or hardened paper cone), wash the flask and residue with lime water until the total filtrate and washings amount to 35 gms. Add $3 \mathrm{cc}$. 
alcohol, $20 \mathrm{cc}$. ether, rotate, add $0.5 \mathrm{gm}$. ammonium chlorid, cork, and shake vigorously.

Let stand two hours, then filter, dry, and weigh the precipitated morphin according to the directions of the Pharmacopœia.

This method is not expeditious, and its results need correction by factors not yet well determined. Mallinckrodt thinks that a correction of 20 to $30 \mathrm{mg}$. should be added to the weight of the reassay morphine for solubility in the mother-liquor, which is equivalent to raising all results about $0.2 \mathrm{I}$ per cent.

An aqueous extraction of opium contains sulphate and meconate of morphine and other alkaloids, meconic acid, calcium salts, extractive, resinous matters, etc. Barium chlorid has been used for removing the sulphuric acid, ammonium oxalate for removing the calcium salts, and alcohol for removing the extractives and other impurities. Lead acetate has also been suggested for removing impurities, and recently Parker has devised a method in which lead subacetate is employed for this purpose and oxalic acid to remove the excess of lead. Any small a mount of lead still remaining in solution is finally removed by treatment with hydric sulphid. The method is as follows: *

Parker's Method. Introduce ro gms. of the opium into a 300 -cc. flask, add Ioo cc. of water, cork and shake for two and one half hours; add $25 \mathrm{cc}$. of lead subacetate solution; cork and shake for one half hour. Filter through a wetted filter $12 \mathrm{~cm}$. in diameter, and wash the residue carefully with water until the total filtrate amounts to about $\mathrm{I} 75 \mathrm{cc}$. Return the residue to the flask, add $50 \mathrm{cc}$. of water, cork, shake about ten minutes and return the whole to the filter, washing the residue until the second filtrate amounts to about $\mathrm{r} 50 \mathrm{cc}$. Combine the two filtrates in a beaker, and from a burette add normal oxalic acid solution, at first in portions of about $5 \mathrm{cc}$. at a time, stirring and allowing to settle after each addition, and then more slowly, until the point where precipitation just ceases is reached (about $26 \mathrm{cc}$.); then add $5 \mathrm{cc}$. more, or $\mathrm{I}$ cc. for each 3 per cent of morphine if the approximate amount is known. Filter the solution, wash the precipitate with water, and evaporate the filtrate in flat-bottomed dishes to a volume of about $20 \mathrm{cc}$., uniting the whole in one dish when the volume is sufficiently reduced, and rinsing carefully after with water. Treat the concentrated solution in the dish (facilitated by slightly tilting the latter) with hydrogen sulphid, and filter through a $5-\mathrm{cm}$. paper, washing the dish and filter after into a small evaporator, with a minimum amount of hot water. Evaporate to small volume (somewhat less than that finally required), transfer to a tared roo-cc. flask,

* From Proc. A. Ph. A., 1907, 495. 
rinsing after with a minimum amount of hot water, and add water to bring the weight to 10 , 15 , or 20 gms. as may be desired. Add $5,7.5$ or 10 gms. of alcohol, as the case may be, rotate, add $25 \mathrm{cc}$. of ether, rotate again and add 2 cc. of ammonia-water (Io per cent), or a moderate excess. (Cork the flask, shake, and suspend a strip of dry, neutral litmus paper under the cork; it should turn blue in about one minute. Cork, shake vigorously for ten minutes and set aside for twelve hours or over night in a cool place.)

Filter through double counterpoised filters and wash, dry, and weigh as directed by the Pharmacopœia, except that a saturated solution of morphine in water is used instead of pure water for washing the precipitate. The precipitate, or a weighed portion of it, is dissolved in a known amount of decinormal acid, and after addition of cochineal indicator, is titrated back with fiftieth-normal potassium hydroxid solution.

Method of Gordin and Prescott (Proc. A. Ph. A., I900, I26). Materials and Utensils for the Assay. Opium in very fine powder, powdered sodium chlorid, an ethereo-ammoniacal mixture composed of stronger ammonia-water and alcohol, each $5 \mathrm{cc}$., chloroform 1o cc., and ether $20 \mathrm{cc}$; benzene boiling at about $80^{\circ} \mathrm{C}$; $\frac{\mathrm{N}}{40}$ sulphuric acid and $\frac{\mathrm{N}}{40}$ potassium hydroxid solution; phenolphthalein solution; $\frac{\mathrm{N}}{10}$ iodin solution (Wagner's reagent); $\frac{\mathrm{N}}{\mathrm{IO}}$ sodium thiosulphate solution; a small Dunstan and Short extraction apparatus (Proc. A. Ph. A., xxI, 33); a screw-top ointment jar having a concave bottom within, and a small pestle, just long enough to rest half upright within the jar when it is closed.

Directions for the Assay. Weigh out 2 gms. of the opium into the ointment jar, rub it by means of the pestle with a few cc. of the ethereoammoniacal solution to a smooth paste, taking care not to smear the sides of the jar unnecessarily, then add about $2 \mathrm{cc}$. more of the same mixture, so as to have the opium well covered with the liquid; screw down the top, leaving the pestle inside, and set the jar aside for five or six hours. After that time the jar is opened, about Io gms. of sodium chlorid well mixed with the opium, and the open jar placed in a good current of air, stirring frequently with the pestle to prevent lumping. In about an hour, when the powder is nearly dry, the jar is placed in a vacuum desiccator containing besides sulphuric acid a vessel of paraffin and left there over night. The jar is then taken out, any lumps in the powder carefully crushed, and the mixture transferred to glazed paper and then to the inner tube of the extraction apparatus, in the bottom of which a plug of cotton has been placed. 
The jar is rubbed out several times with small quantities of sodium chlorid, the rinsings added to the tube, and having placed a plug of cotton and a piece of glass on top of the powder, the tube is connected with the glass stop-cock by means of rubber tubing, and regulating the flow with the stop-cock, the powder is extracted with benzene by percolating very slowly until, upon evaporating four or five drops of the percolate on a watch-glass and dissolving the residue in ten or twelve drops of slightly acidulated water, no turbidity appears upon the addition of a few drops of Wagner's reagent. A current of dry air is then passed through the tube until the powder is dry, the tube placed into the outer jacket of the apparatus, and the latter connected with a small round-bottomed flask containing 40 to $50 \mathrm{cc}$. of chloroform, and the powder extracted until exhaustion is complete. This will take about two and one-half hours. Care should be taken that only a small portion of the bottom of the flask be heated, and that a layer of the solvent be constantly on top of the powder. The chloroform is then distilled off and the residue dissolved in $60 \mathrm{cc} . \frac{\mathrm{N}}{40}$ sulphuric acid by the aid of gentle heat.

The solution is poured into a Ioo-cc. graduated cylinder, the latter filled up to the mark of roo cc., and the liquid filtered, using a little talcum powder if necessary. $50 \mathrm{cc}$. of the clear filtrate (representing I gm. of the opium) are put into a roo-cc. measuring-flask and Wagner's reagent added in small quantities at a time, shaking well after each addition till the supernatant liquid is very dark red. The flask is then filled up to roo cc. and shaken till the supernatant liquid is perfectly transparent and dark red. The liquid is filtered and in $50 \mathrm{cc}$. of the clear filtrate, after decolorizing it with a few drops of the thiosulphate solution, titrate the free acid with $\frac{\mathrm{N}}{40}$ potassium hydroxid, using phenolphthalein as indicator. Figure out the number of cc. of the $\frac{\mathrm{N}}{40}$ acid consumed by I gm. of the opium and multiply it by $0.7 \mathrm{I}$; the result is the percentage of morphine in the drug.

In order to obtain very exact results the acid should be standardized with a small quantity of anhydrous morphine.

The German Pharmacopœial Method. 6 gms. of medium fine powdered opium are mixed with $6 \mathrm{gms}$. of water, the mixture is rinsed with water into a dry, weighed flask; then by the addition of more water, the contents of the flask is made to weigh $54 \mathrm{gms}$. After the mixture has been allowed to stand (with frequent shaking) for several hours, the mass is pressed through a dry piece of linen. Of the liquid so obtained, 42 gms. are filtered through a dry folded filter $(10 \mathrm{~cm}$. in 
diameter) into a dry flask. Add to this filtrate 2 gms. of sodium salicylate solution ( $\mathrm{r}: 2)$ and shake well. Then filter off 36 gms. of the clear solution (representing 4 gms. of opium) through a dry folded filter ( $10 \mathrm{~cm}$. in diameter) into a flask. Mix the filtrate (rotating the flask) with Io gms. of ether and add 5 gms. of a mixture consisting of 17 gms. of ammonia-water and 83 gms. of water. The flask is then stoppered and strongly shaken for ten minutes and set aside for twentyfour hours. At the end of this time the ether layer is poured off as completely as possible onto a plain filter $(8 \mathrm{~cm}$. in diameter). To the aqueous solution remaining in the flask is added another Io gms. of ether, the mixture is rotated a few moments and the ether again poured onto the filter. The aqueous solution is then poured onto the filter, paying no attention to the crystals adhering to the walls of the flask, and the filter as well as the flask rinsed three times with ether-saturated water, using 5 gms. each time. After the flask is thoroughly drained and the filtrate entirely passed through, the morphine crystals are dried, and dissolved in $25 \mathrm{cc}$. of $\frac{\mathrm{N}}{10}$ hydrochloric acid. The solution is poured into a roo-cc. flask, the filter and flask are then carefully washed and the solution diluted to roo cc. $50 \mathrm{cc}$. of this solution are then introduced into a 200-cc. bottle of white glass. $50 \mathrm{cc}$. of water are added, and enough ether to make a layer $\mathrm{I} \mathrm{cm}$. in depth. Then, after the addition of five drops of iodeosin solution, there is delivered from a burette $\frac{\mathrm{N}}{\mathrm{IO}}$ potassium hydroxid, shaking thoroughly after each addition until the lower aqueous layer assumes a pale red color. To obtain this color not more than 5.4 nor less than 4.I cc. of the alkali should be required. This method, compared with others, has been found to give results which are I to I.2 per cent low.

A. B. Stevens' Method.* Take 4 gms. of opium in fine powder and triturate in a mortar with 2 gms. of fresh burnt lime (not airslaked) and ro cc. of water until a uniform mixture results. Add I9 cc. of water and stir frequently for half an hour. Filter through a dry filter, about $\mathrm{Io} \mathrm{cm}$. in diameter. Transfer exactly $\mathrm{I} 5 \mathrm{cc}$. to a 6o-cc. bottle. To this add 4 cc. of alcohol and ro cc. of ether and shake the mixture. Then add $0.5 \mathrm{gm}$. of ammonium chlorid. Shake well and frequently during half an hour. Set aside in a cool place for twelve hours. Remove the stopper carefully and preserve, with any adhering crystals, for further use. Pour the ethereal layer into a small funnel, the neck of which has been previously closed with a

\footnotetext{
* Pharm. Archiv., March, I902, 4r.
} 
piece of absorbent cotton. Rinse the bottle with ro cc. of ether, and when this has passed through, pour the contents of the bottle into the funnel. Without trying to remove all the crystals from the bottle, wash the bottle and contents of the funnel with morphinated water until the washings are colorless. When the crystals have drained, place the funnel in the bottle containing adhering crystals, and with a small glass rod drawn out to a curved point, lift the cotton and rinse the crystals into the bottle with $\mathrm{I} 2 \mathrm{cc}$. of decinormal sulphuric acid, using the cotton on the end of the rod to detach any adhering crystals. Place the cotton in the bottle, replace the cork and agitate until the crystals are all dissolved. Rinse the cork and funnel with water and titrate the excess of acid with $\frac{\mathrm{N}}{40}$ potassium hydroxid. The number of cc. of decinormal acid consumed by the morphine, multiplied by 1.5038, will give the percentage of morphine obtained. To this add I.I2 for the morphine remaining in solution.

Prof. L. E. Sayre, who has had considerable experience with this method, states that it leaves little chance for error, is easy of manipulation, economical in point of time, and will give trustworthy and concordant results. He believes that the process may be much shortened by allowing the mixture of opium solution and ammonium chlorid to stand four hours, instead of twelve, in a cool place.

Dr. Ph. Ascher* gives the following modification of Stevens' method, which he found to give results within a few milligrams of the quantity of morphine originally taken. The full modified process, showing the modifications in italics, is as follows:

Place 4 gms. of dried or powdered opium in a roo-cc. tared porcelain evaporating-dish, add $5 \mathrm{cc}$. of $\mathrm{KOH}$ solution, 5 per cent or its equivalent of a stronger solution, mix thoroughly with a rubber-tipped glass rod and ev'aporate on water-bath or drying closet, until of constant weight, then add 2 gms. of dry, freshly-slaked lime and Io cc. of water and triturate continually for fifteen minutes until a perfectly smooth mixture results. Finally, add I9 cc. of water, triturating frequently during half an hour and filter through a dry filter about Io $\mathrm{cm}$. in diameter. Transfer exactly $15 \mathrm{cc}$. to a Ioo-cc. Erlenmeyer flask and add to this $4 \mathrm{cc}$. of alcohol and ro cc. of concentrated ether and shake the mixture. Then add $0.5 \mathrm{gm}$. ammonium chlorid. Shake well and frequently during half an hour. Set aside in a cool place for twelve hours.

Remove the stopper carefully and preserve with any adhering crystals for future use. Pour the ethereal layer into a small funnel, 
the neck of which has been previously closed with a piece of absorbent cotton. Rinse the flask with ro cc. of ether, shake continually for five minutes and pour as before into the funnel, and when this has passed through, pour the contents of the flask into the funnel. Add $5 \mathrm{cc}$. of ether to the flask, rotate gently and pour into funnel, repeating with $5 \mathrm{cc}$. more of ether. Without trying to remove all the crystals from the bottle, wash the flask and contents of the funnel with saturated solution of morphin, small portions at a time, using $15 \mathrm{cc}$. in all. When the crystals have drained, place the funnel in the bottle containing adhering crystals, and with a small rod drawn out to a curved point, lift the cotton and rinse the crystals into the bottle with $\mathrm{I} 2 \mathrm{cc}$. of decinormal sulphuric acid, using the cotton on the end of the rod to detach any adhering crystals. Place the cotton carefully into the flask, replace the stopper and agitate until the crystals are all dissolved. Rinse the cork and funnel with water, and titrate the excess of acid with fortieth-normal potassium hydroxid solution, using hæmatoxylin as indicator.

Divide the number of cc. of potassium hydroxid solution used by 4 and subtract the product from I 2 cc. of acid used; the remainder will be the amount of acid consumed by the morphine, which number, multiplied by 1.5046 , and the addition of 0.070 as the corrective factor for loss of morphine during estimation, gives the percentage of morphine in the sample under examination. The corrective factor 0.II2, given by Stevens, the author considers too high; he finds 0.070 to be nearer the difference between the results obtained and the amount of substance originally taken.

The use of potassium hydroxid in this process, with subsequent evaporation to dryness, is for the purpose of expelling ammonia, the presence of salts of which in opium, Ascher concludes, interferes with the estimation of morphine in that complete solution of this alkaloid by treatment with lime is prevented.

I. Picard * suggests the following modification of Leger's method for the assay of opium: 6 gms. of opium in No. I 20 powder, dried at $60^{\circ} \mathrm{C}$., are rubbed down with a very little lime water to make a soft mass, which is thoroughly worked. The remainder of $48 \mathrm{cc}$. of lime water is then added so as to form a homogeneous mixture, which is carefully covered and set aside for two hours. $50 \mathrm{cc}$. of 5 per cent solution of sodium salicylate is then mixed in, and after ten minutes' contact the mixture is thrown on a cloth and strained with expression. The strained liquid is then filtered through a small filter into a small flask graduated at $36 \mathrm{cc}$. and to that volume of filtrate $4 \mathrm{cc}$. of pure

* Bull. Soc. Pharm. de Bordeaux, I906 (XLVI), 250; and Ph. Jour., I907, 59. 
ether is added. The liquid is then neutralized with dilute solution of ammonia, added drop by drop, and tested after each addition with litmus paper. When neutral, six drops in excess are added, the flask is corked, shaken for ten minutes and set aside for twenty-four hours. The liquid is then passed through two counterpoised filters, any adhering crystals of morphin being washed off the flask on to the filter with $8 \mathrm{cc}$. of water. The beak of the funnel is then closed with a piece of India-rubber tubing carrying a pinch-cock. The filter and funnel are then filled with distilled water to which a few drops of pure ether have been added. After five minutes' contact this water is run off and the washing repeated a second time in the same manner. The precipitate is then drained, dried at $100^{\circ} \mathrm{C}$, , and weighed. If desired the dry precipitate may be washed with $20 \mathrm{cc}$. of benzene and again dried and weighed. This may remove a trace of narcotine, but, in the author's opinion, it is not necessary.

Thomas Tickle * describes a new process for the morphiometric assay of opium, the essential point of which consists in the employment of metacresol as a solvent of the alkaloid. In contact with a concentrated solution of morphine, cresol readily dissolves the liberated alkaloid up to a strength of 40 per cent, whilst amyl alcohol dissolves only $5 \mathrm{mg}$. per cc. In very weak solutions, however, the cresol only takes up about twice as much as amyl aclohol, a fact due to the aqueous layer being saturated with cresol, and, therefore, a stronger solvent than water alone. But this intermiscibility of solvent and water is diminished by the admixture of some other solvent, notably amyl alcohol. The general process outlined by the author for the isolation of morphine consists in liberating the alkaloid contained in $100 \mathrm{cc}$. of solution with sodium bicarbonate, agitating with a mixture of pure or recently distilled cresol (two parts) and amyl alcohol (one part) in four separate fractions. The mixed fractions, totaling $30 \mathrm{cc}$, are next treated with ${ }_{15} \mathrm{cc}$. of ether, which has the curious property of annulling all tendency of the solvent to retain alkaloid; then $30 \mathrm{cc}$. of petroleum ether is added to further facilitate the extraction by diluted acetic acid. Io cc. of I per cent acetic acid are used for the first shaking out of the morphine, and $5 \mathrm{cc}$. for succeeding extractions until exhausted. The solution of morphine acetate thus obtained is cvaporated to dryness, taken up with water and placed in a covered vessel side by side with an open beaker containing very dilute ammonia. The morphine solution rapidly absorbs ammonia vapor and deposits the alkaloid in a crystalline state. By this ingenious procedure there is no danger of introducing excess of ammonia in which the alkaloid

*Ph. Jour., Feb. 16, 1907, 162-164. 
is more soluble than in water. The crystals thus obtained are dried at $110^{\circ} \mathrm{C}$. and weighed.

\section{LITERATURE ON OPIUM ASSAYING}

J. Perger. J. Chem. Soc., I884, 70 I.

J. H. Wainwright. J. A. C. S., I 885 (Vol. 7), 48.

P. C. Plugge. Archiv. de Ph., I887, 343.

E. Dieterich. Helfenberger Annalen, I887, 54.

D. B. Dott. Ph. Jour., I888, 701 .

E. R. Squibb. Ephemeris, I889 (Vol. III), II50-I I6I.

Flückiger. A. J. Ph., I890, I4.

J B. Nagelvoort. A. J. Ph., I890, 407.

Beckurts. Apoth. Ztg., I891, 526.

D. B. Dott. Britt. and Col. Drug., I894, 372.

D. B. Dott. Ph. Jour., I894, 847 .

D. B. Dott. Ph. Jour., I895, 497.

Farr and Wright. Ph. Jour., I897, 202.

Montemartini and Trasciatti. J. Chem. Soc., I898, 27I (Vol. II).

Gordin and Prescott. J. A. C. S., I898, 725 .

Lyons. "Assaying of Drugs and Galenicals." Nelson Baker \& Co. I899.

Lamar. A. J. Ph., 1900, 36 .

Gordin and Prescott. Proc. A. Ph. A., I900, I 26.

L. E. Sayre. Drug. Cir., Sept., I90I, I80.

A. B. Stevens. Pharm. Arch., March, 1902, 4I.

H. E. Matthews. Trans. Britt. Ph. Conf., I903, 570.

P. L. Aslanoglon. Chem. News., Dec., 1903, 286.

C. E. Caspari. Proc. A. Ph. A., I904, 386.

$\mathrm{Ph}$. Schidrowitz. Analyst, March, I904.

L. F. Kebler. Proc. A. Ph. A., I904, 369-375.

Leo Eliel. Proc. Ind. Ph. A., I9o6.

Ph. Ascher. A. J. Ph., 1906, 262.

I. Picard. Ph. Jour., 1907, 59 .

D. B. Dott. Ph. Jour., 1907,78 .

Thomas Tickle. Ph. Jour., 1907, 162.

C. E. Parker. Proc. A. Ph. A., $1907,490$.

Bulletin No. 107, Bureau of Chem. U. S. Dept. of Agric.

Allen's "Commercial Organic Analysis.

\section{ASSAY OF PHYSOSTIGMa (Calabar bean)}

This drug contains three alkaloids, namely, physostigmine (also called eserine), eseridine, and calabarine. The first of these is the active principle, which together with eseridine is readily removed from an aqueous solution by treatment with ammonia or alkali bicarbonate, and shaking out with ether. Calabarine being insoluble in ether is left behind. All three alkaloids are soluble in chloroform. 
The U. S. P. VIII Method. Introduce $20 \mathrm{gms}$. of physostigma (in No. 6o powder) into an Erlenmeyer flask of about $250 \mathrm{cc}$. capacity, add $200 \mathrm{cc}$. of ether, and shake the flask well during ten minutes. Then add ro cc. of an aqueous solution of sodium bicarbonate ( $(: 20)$, and shake the mixture vigorously at intervals during four hours. Allow the powder to settle, and decant roo cc. of the ether solution (representing Io gms. of physostigma) into a measuring cylinder; then transfer it to a separator, introduce a small piece of blue litmus paper, and add sufficient normal sulphuric acid V. S. to render the liquid acid, and then Io cc. of distilled water. Shake the liquid well for several minutes, and draw off the aqueous layer into another separator. Repeat the extraction, using 2 cc. of normal sulphuric acid V. S. and $8 \mathrm{cc}$. of distilled water, add the acid aqueous layer to the second separator, and finally again shake out the ether solution, using I cc. of normal sulphuric acid V. S. and 9 cc. of distilled water, adding this also to the second separator. To the combined acid liquids in the second separator, add $25 \mathrm{cc}$. of ether, a small piece of red litmus paper, and sufficient sodium bicarbonate solution $(\mathrm{I}: 20)$ to render it alkaline. Shake the separator for one minute, allow the liquids to separate, and draw off the ether into a beaker. Repeat the shaking out process with $20 \mathrm{cc}$. and again with $15 \mathrm{cc}$. of ether added to the separator, shake each time for one minute, allow the liquids to separate, and draw off the ether into the beaker. Carefully evaporate the ether from the combined solutions by means of a water-bath, and when dry, dissolve the residue in $5 \mathrm{cc}$. of tenth-normal sulphuric acid V. S. and $20 \mathrm{cc}$. of ether, which must be strictly neutral, and transfer this solution to a bottle, rinsing with $80 \mathrm{cc}$. of water; add five drops of iodeosin T. S., and titrate the excess of acid with fiftieth-normal potassium hydroxid V.S., until, after shaking, the aqueous liquid just acquires a pink color. Divide the number of cc. of fiftieth-normal potassium hydroxid V.S. used by 5, subtract the quotient from 5 (the $5 \mathrm{cc}$. of tenth-normal sulphuric acid V. S. taken), and multiply the remainder by 0.0273 , and this product by 10 ; the result will be the percentage of alkaloids soluble in ether contained in the physostigma. The figure 0.0273 represents-the weight in grams of alkaloids (mainly physostigmine) required to neutralize I cc. of tenth-normal sulphuric acid V.S.

H. Beckurts* recommends the following method for assaying calabar beans: 20 gms. of the powdered drug (sieve $\mathrm{v}$ of the G. P., IV) are shaken frequently during three hours with $\mathrm{I} 20$ gms. of ether and ro $\mathrm{cc}$. of a ro per cent solution of potassium bicarbonate; then

* Apoth. Ztg., XX, No. 67 (1905), 670. 
$90 \mathrm{gms}$. of the ether solution are filtered, one half of the ether is distilled off, and the residue transferred to a separator by the aid of some of the recovered ether. After adding Io cc. of petroleum benzene to prevent subsequent emulsification, the ether solution is shaken out with Io cc., and then thrice successively with $5 \mathrm{cc}$. of $\frac{\mathrm{N}}{\mathrm{IO}} \mathrm{HCl}$; the acid liquids are united, $45 \mathrm{gms}$. of ether and ro cc. of a 10 per cent solution of potassium bicarbonate are added, and the mixture, after several vigorous shakings, is allowed to separate. Then $30 \mathrm{gms}$. of the ether solution (corresponding to Io gms. of the drug) are mixed with Io cc. $\frac{\mathrm{N}}{\mathrm{I} O \mathrm{O}} \mathrm{HCl}, 20 \mathrm{cc}$. of water, and five drops of alcoholic iodeosin solution, and the excess of acid is determined by titration with $\frac{\mathrm{N}}{\mathrm{IOO}} \mathrm{NaOH}$. Fach cc. of $\frac{\mathrm{N}}{\mathrm{I} 00} \mathrm{HCl}$ corresponds to $0.00273 \mathrm{gm}$. of physostigmine. Under the conditions of this assay method, calabarine, being insoluble in ether, is excluded, while the alkaloids extracted by the ether-physostigmine and eseridine-do not undergo unfavorable change during twenty-four hours. Caustic alkalies, as well as carbonates, cannot be used for the shaking out of these alkaloids from their acid solutions because they decompose the bases, the solution assuming a red color. If ether and then sodium or potassium bicarbonate is added to the alkaloidal solution no decomposition occurs. There is no troublesome emulsification produced during the direct assay of the beans, but when applying the method to the assay of the extract there is considerable annoyance from this source, while the color reaction of the indicator, which is quite sharp when operating with the drug, is less distinct when assaying the extract, this being probably due to a partial decomposition of alkaloid during its preparation.

\section{LITERATURE}

W. A. H. Naylor. Britt. and Col. Drugg., XLviri, 77.

H. M. Gordin. Proc. A. Ph. A., 1906, 380.

Lyons. "Assaying of Drugs and Galenicals."

\section{ASSAY OF PILOCARPUS LEAVES (Jaborandi)}

The leaves of several species of pilocarpus are found in the market and sold as jaborandi. The alkaloidal content of these is variable and often below the official requirement. Two species of pilocarpus, namely, P. Jaborandi and P. Microphyllis, are official in the U. S. P., in which the leaves, when assayed by the official process, should yield not less than 0.5 per cent of the alkaloids. 
Several alkaloids are present in this drug. Of these, pilocarpine is the most important and, in fact, the one upon which alone the therapeutic value of the drug depends. The others are isopilocarpine and pilocarpidine.

The U. S. P. and other methods recommended for the assay of this drug all depend upon a determination of the total alkaloidal content. This information is not considered of great value, since it gives no indication of the amount of the principal alkaloid (pilocarpine) present, but until a more satisfactory method is found we must be content with such as we have.

The U. S. P. Method. This is based upon the method proposed by Lyons and is as follows: Moisten ro gms. of pilocarpus (in No. 60 powder) with $2 \mathrm{cc}$. of ammonia-water and $3 \mathrm{cc}$. of chloroform, and at once pack it firmly in a small cylindrical percolator, which has been provided with a pledget of cotton packed firmly in the neck. Percolate the powder slowly with chloroform containing about 2 per cent of ammonia-water, until it is exhausted, about roo cc. of menstruum usually being sufficient. Pour into a separator the percolate, and shake it out with I $_{5} \mathrm{cc}$. of normal sulphuric acid V. S., transferring the acid aqueous layer to another separator, and repeating the shaking out of the chloroform solution with $2 \mathrm{cc}$. of normal sulphuric acid V.S., mixed with $8 \mathrm{cc}$. of distilled water. Add the acid layer to the second separator, and again repeat the shaking out with ro cc. of distilled water, and add the aqueous liquid to the second separator. Introduce into the second separator a small piece of red litmus paper, add enough ammonia-water to render the liquid alkaline and shake out the liquid with $20 \mathrm{cc}$. of chloroform, drawing off the chloroformic solution into a beaker. Repeat the shaking out with two portions of 15 and Io cc. each of chloroform, and add the chloroformic solutions to the beaker. Evaporate the chloroform by means of a water-bath, and dissolve the alkaloidal residue in $7 \mathrm{cc}$. of tenthnormal sulphuric acid V.S. Add five drops of cochineal T.S. or iodeosin T.S., and titrate the excess of acid with fiftieth-normal potassium hydroxid V. S. Divide the number of cc. of fiftieth-normal potassium hydroxid V. S. used by 5, subtract the quotient from 7 (the $7 \mathrm{cc}$. of tenth-normal sulphuric acid V.S. taken), and multiply the remaindet by 0.02 , and this product by Io; the result will be the percentage of alkaloids contained in the pilocarpus. The figure 0.02 represents the weight in grams of alkaloids (mainly pilocarpine) required to neutralize I cc. of tenth-normal sulphuric acid V. S.

G. Fromme * recommends the following method for the assay of

* Pharm. Rev., Nov., I90I, 504; from Cæsar and Loretz, Geschäfts-Bericht for 1901 
jaborandi leaves: 15 gms. of the leaves, in moderately fine powder, are macerated in $150 \mathrm{gms}$. of chloroform and $15 \mathrm{gms}$. of ammoniawater for half an hour, under frequent agitation; the whole is then transferred to a plain filter and covered with a glass plate, the filtration towards the end being hastened by pouring a little water on the dregs. When a little more than roo gms. of filtrate has been obtained, this is shaken with I cc. of water and set aside until any fine powder which may have passed through the filter has been taken up by the water and the chloroformic solution is perfectly clear. Then roo gms. of this solution, representing Io gms. of the drug, are extracted succes sively with 30,20 , and Io cc. of I per cent hydrochloric acid; the united acid extraction is shaken with $20 \mathrm{cc}$. of ether to remove chlorophyl, fat, and resin, and, the acid layer being drawn off, it is rendered alkaline by ammonia and shaken out successively with 30,20 , and Io cc. of chloroform. The chloroform is evaporated and the residue weighed or titrated as may be most expedient-I cc. of $\frac{\mathrm{N}}{\text { I00 }}$ hydrochloric acid being eaual to $0.00208 \mathrm{gm}$. of pilocarpine.

\section{LITERATURE}

H. A. D. Jowett. Britt. Pharm. Conf., I899.

Farr and Wright. Britt. Pharm. Conf., I899.

Dohme and Engelhardt. Drug. Cir., Feb., I900, 28.

H. M. Gordin. Proc. A. Ph. A., I906, 380.

E. W. Mann. Britt. and Col. Drug., xL, 493 .

W. A. H. Naylor. Britt. and Col. Drug., XLVIII, 78.

\section{ASSAY OF STROPHANTHUS}

Strophanthus contains the glucosid strophanthin, which is its active principle. This glucosid is soluble in water, less soluble in alcohol, and almost insoluble in chloroform or ether. It is decomposed by mineral acids, strophanthidin and glucose resulting. Strophanthidin dissolves in alcohol and in chloroform, but is almost insoluble in water.

Barclay's Method (Ph. Jour. and Trans., Nov. 28, I896). According to Barclay, if an alcoholic extract of the drug be exhausted with water and the aqueous extract thus obtained is in turn exhausted with absolute alcohol, the product consists almost wholly of strophanthin. The method of assay which he proposes depends upon the conversion of the whole of the strophanthin into strophanthidin, thereby avoiding the possible loss occasioned by partial splitting up of the glucosid during the process of extraction. From the strophanthidin obtained it is easy to determine the amount of strophanthin in the drug. The seeds are extracted with alcohol, most of the alcohol 
recovered by distillation, water is added, and the remainder of the alcohol driven off by gentle heat, and to the aqueous mixture chloroform is added to extract the oils and fats present. The chloroform layer is then removed and the aqueous liquid acidified with dilute sulphuric acid and heated for one hour on a water-bath; the resulting turbid solution is shaken out several times in a separator with portions of chloroform until the chloroform removes nothing further from the solution. The combined chlcroform solutions are evaporated carefully and the residue, consisting of strophanthidin, carefully dried at a temperature not exceeding $150^{\circ} \mathrm{F} .\left(65.5^{\circ} \mathrm{C}\right.$ ) and weighed. The weight multiplied by the factor 2.74 gives the amount of strophanthin originally present in the drug. In the above assay operate on $12 \mathrm{gms}$. of the drug finely powdered. Macerate about twelve hours, with frequent shaking, with $180 \mathrm{cc}$. of alcohol. U. S. P. Pour off I50 cc. of the tincture, representing ro gms. of the drug, and remove the alcohol by evaporation on a water-bath or by distillation. To the residue add $30 \mathrm{cc}$. of water, transfer the aqueous mixture to a separator, shake out with two successive portions of chloroform (Io cc. each time) to remove fatty substances. Transfer the aqueous liquid to a flask, add I cc. of dilute sulphuric U. S. P., and heat on a water-bath for one hour, having connected the flask with a reflux condenser. The resulting turbid liquid is then shaken out in a separator with three successive portions of chloroform (ro cc. each). The combined chloroform solution is evaporated at $150^{\circ} \mathrm{F} .\left(65.5^{\circ} \mathrm{C}\right.$.), the residue weighed and multiplied by 2.74 to find amount of strophanthin in Io gms. of the drug.

\section{LITERATURE}

G. Fromme. Report of Cæsar and Loretz, and Pharm. Ztg. (Ig05), LXXIII, 772 .

A. R. L. Dohme. Drug. Cir., 1900, 132.

\section{ASSAY OF TOBACCO}

According to the method of C. C. Keller (Schweiz. Wochenschr. f. Pharm., I899, 309), 6 gms. of the dry tobacco are shaken vigorously in a $200-\mathrm{cc}$. flask with $60 \mathrm{cc}$. each of ether and petroleum ether, Io cc. solution of potassa (2o per cent), and the shaking repeated during half an hour. After the mixture is allowed to stand during three or four hours $100 \mathrm{cc}$. of the solution are filtered through a small plaited filter into a $100-\mathrm{cc}$. flask and a strong current of air is passed through the filtrate in order to drive off the ammonia (a small quantity of which is liberated during the process). After ammonia has been completely driven off (as shown by litmus paper, held in the air, which has passed through 
the solution no longer turning blue) Io cc. of alcohol and one drop of iodeosin in ro cc. of water are added, the flask is stoppered, and the contents are vigorously shaken. The nicotine and iodeosin are taken up by the water which separates of a red color; $\frac{\mathrm{N}}{\mathrm{IO}}$ hydrochloric acid (say 7 cc.) is then added, followed, if necessary, by further additions of I cc. at a time until the red color of the aqueous layer is discharged; the excess of acid is then determined by $\frac{\mathrm{N}}{\mathrm{IO}}$ ammonia, the volume required being deducted from the volume of $\frac{\mathrm{N}}{10}$ hydrochloric acid, to ascertain the quantity of the latter required to neutralize the nicotine. Each cc. of $\frac{\mathrm{N}}{\mathrm{IO}}$ hydrochloric acid corresponds to $0.016 .2 \mathrm{gm}$. of nicotine, and the total quantity of the alkaloid is then ascertained by simple multiplication with the number of cc. of acid consumed. The product, multiplied by 20 , gives the percentage.

\section{LITERATURE} 265.

McCrae. Chem. Ztg., xxxi (I907), 45, and Pharm. Jour., March, I907,

Amé Pictet. Archiv. d. Pharm., Sept., I906, 375.

Pictet and Rotschy. Chem. Zeitschr., XLVI, p. I I8.

R. Kissling. Zeitschr. f. anal. Chem., xxI, No. I; and Proc. A. Ph. A., I882, 167 .

\section{ASSAY OF VERATRUM}

There are several species of veratrum and they contain a number of alkaloids, the nature of which is as yet imperfectly known. G. Bredemann shows that veratrum album contains four alkaloids, namely, protoveratrine $\left(\mathrm{C}_{32} \mathrm{H}_{51} \mathrm{NO}_{11}\right)$, jervine $\left(\mathrm{C}_{26} \mathrm{H}_{37} \mathrm{NO}_{3}\right)$, pseudojervine $\left(\mathrm{C}_{29} \mathrm{H}_{43} \mathrm{NO}_{7}\right)$, and rubijervine $\left(\mathrm{C}_{26} \mathrm{H}_{43} \mathrm{NO}_{2}\right)$. The same alkaloids are found in veratrum viride, but the proportion in which they exist differs in the different species. The most characteristic alkaloid is probably jervine, but in the present state of knowledge, this alkaloid cannot readily be separated. The therapeutic value of a sample of the drug cannot therefore be accurately determined by assay. A determination of the total alkaloids is the best that can be done at present.

Bredemann * proposes the following method: I 2 gms. of powdered drug are rotated with I $20 \mathrm{cc}$. of a mixture of equal parts of chloroform and ether, then ro cc. sodium hydroxid solution is added and the mixture shaken frequently during three hours; then sufficient 
water is added to cause coherence of the drug. The ether-chloroform solution, always more or less turbid, is decanted as completely as possible, shaken with magnesium oxid and three or four drops of water, and then the liquid poured through a dry filter and $100 \mathrm{cc}$. of the transparent filtrate corresponding to Io gms. drug collected. The chloroform-ether solution is extracted three times with $20 \mathrm{cc}$. of water containing acetic acid. The united acetic acid extractions are made alkaline with sodium hydroxid and extracted three times with a mixture of equal parts of chloroform and ether. The etherchloroform solution is driven off, the alkaloidal residue dried at $100^{\circ} \mathrm{C}$. and weighed. For the estimation of alkaloid in the tincture it is proposed that roo gms. tincture be concentrated to one-half its volume on a water-bath. Then I gm. paraffin and about $25 \mathrm{cc}$ water are added, and the liquid warmed until all alcohol has been expelled. To the warm liquid 2 gms. acetic acid are added and the liquid allowed to cool with frequent stirring. It is then filtered into a separator through a small wet filter. The paraffin and oil which remain on the filter are heated on a water-bath with $20 \mathrm{cc}$. water and I gm. acetic acid until the paraffin melts. The liquid is then allowed to cool, passed through the filter first used, and the dish and filter washed thoroughly with water. The united filtrates are made alkaline with sodium hydroxid and extracted with 20,10 , and 10 cc. chloroform.

Volumetric Method of Estimation. According to G. Bredemann the alkaloids of Veratrum album may be conveniently and accurately estimated by the volumetric method, employing for this purpose Keller's method, with some insignificant modifications. He has determined that I cc. of $\frac{\mathrm{N}}{100}$ hydrochloric acid requires for saturation:

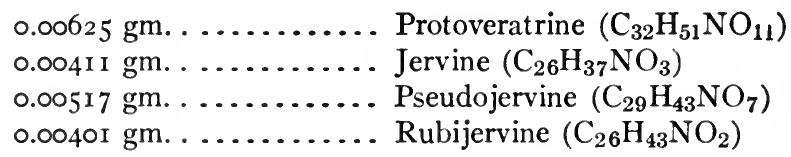

Furthermore, he has obtained experimental data which justify him in assuming the average molecular weight for the total alkaloids to be 424 , and that therefore $0.00424 \mathrm{gm}$. of the latter correspond to I cc. of the $\frac{\mathrm{N}}{100}$ acid. In the practical determinations applied to thirty-six specimens of veratrum rhizomes (both $V$. album and $V$. viride) he found variations of from 0.19928 to 0.93280 total alkaloid. In two cases he found the rootlets to have a higher alkaloid content than the rhizome, but to these the average molecukr weight cannot be applied, 
since they contain a larger proportion of protoveratrine, and therefore must be assumed to have a higher molecular weight.

\section{LITERATURE}

Wright and Luff. Ph. Jour. and Trans., May 3I, 1879 .

Salzberger. Archiv. d. Pharm. (I89I), 228, 462.

Kremel. Ph. Post. (1889), 227.

Bullock. A. J. Ph. (1879), 338 .

F. T. Gordon. Proc. Pa. Pharm. Assoc., I901, I25.

\section{ASSAY OF WILD CHERRY BARK}

(A. B. Stevens, Proceedings A. Ph. A., I896, 215). Io gms. of the ground bark are macerated in Ioo cc. of water for twenty-four hours, then distilled and the distillate containing the hydrocyanic acid is passed into a decinormal solution of potassium hydroxid.

The alkaline solution of potassium cyanid is then titrated with decinormal silver nitrate in the usual manner.

Dr. A. R. L. Dohme (Pharm. Runds., XIII, 260) distils the hydrocyanic acid by passing live steam into the flask containing the bark and water, instead of using direct flame; otherwise the process is the same as the above, except that he adds o.I gm. of $\mathrm{NaCl}$ to the distillate before titrating. This is deducted from the final result.

Another method consists in distilling by means of live steam and receiving the distillate in $\frac{\mathrm{N}}{\mathrm{IO}}$ silver nitrate solution. The distillation is known to be complete when, upon agitating the receiver, the distillate no longer produces a precipitate in the silver solution. The excess of silver nitrate is then estimated by Volhard's method. 


\section{CHAPTER LII}

\section{ASSAY OF GALENICAL PREPAR.ATIONS}

(I) J. U. Lloyd's Methods. I gm. of a solid extract which has been dissolved in 5 to $8 \mathrm{cc}$. of an alcoholic menstruum or a corresponding volume of the tincture evaporated to this bulk, or $5 \mathrm{cc}$. of the fluid extract, are mixed in a flat-bottomed porcelain mortar with $2 \mathrm{cc}$. of a solution of perchlorid of iron. To this is added sodium bicarbonate with constant trituration until a stiff magma results.* This magma is extracted by repeated trituration with chloroform, using first $20 \mathrm{cc}$. and then three portions of ro cc. each, decanting them severally by means of a guiding-rod, being careful that no suspended portions of the magma are drawn off. In order to make sure that all of the alkaloid has been extracted, add $5 \mathrm{cc}$. more of chloroform, draw it off, evaporate on a watch-glass, dissolve residue in dilute sulphuric acid, and test for alkaloids by Wagner's or Maver's reagent.

The mixed chloroformic extracts are collected and may be estimated volumetrically as follows:

Method $A$. To be used if the chloroformic extract is not colored.

The chloroformic solution is evaporated to dryness in a flask placed on a water-bath. To this residue is added an accurately measured excess of $\frac{\mathrm{N}}{25}$ sulphuric acid and the solution diluted with a little water, the indicator added and the excess of standard acid solution estimated by titrating with $\frac{\mathrm{N}}{50}$ potassium hydroxid V. S. The number of cc. of the $\frac{\mathrm{N}}{50}$ alkali $\dot{\mathrm{V}}$. S. used, divided by 2 and subtracted from the volume of $\frac{\mathrm{N}}{25}$ acirl $\mathrm{V} S \mathrm{~S}$. originally added, will give the number

* The ferric hydroxid which is produced in the above process serves to attract most of the tannates, gums, vegetable acids, and coloring matters, while the excess of sodium bicarbonate liberates the alkaloids, which are then dissolved by the chloroform. If the fluid extract is strongly alcoholic the chloroform will not separate easily, in which case the addition of a few cc. of water containing a very little glucose will cause a sharp separation. 
of cc. of the latter required for the alkaloid. This number, multiplied by the proper factor, will give the total alkaloid present in the fluid extract.

Example. The chloroformic residue obtained from $5 \mathrm{cc}$. of a fluid extract of hyoscyamus was dissolved in 12 cc. of $\frac{\mathrm{N}}{25}$ acid V. S., the solution titrated with $\frac{\mathrm{N}}{50}$ alkali V. S., $20.6 \mathrm{cc}$. of the latter were
used.

$20.6 \mathrm{cc}$. of $\frac{\mathrm{N}}{50} \mathrm{~V}$. S. is the equivalent of $10.3 \mathrm{cc}$. of $\frac{\mathrm{N}}{25} \mathrm{~V}$. S. $10.3 \mathrm{cc}$. subtracted from $\mathrm{I} 2 \mathrm{cc}$., the amount of $\frac{\mathrm{N}}{25}$ acid originally added, leaves I.7 cc., the quantity of $\frac{\mathrm{N}}{25}$ acid V.S. which was required for the alkaloid. This multiplied by the $\frac{\mathrm{N}}{25}$ factor for total alkaloids of hyoscyamus, o.orI475 gm., gives the quantity of alkaloids present in the $5 \mathrm{cc}$, which quantity multiplied by 20 gives the per cent:

I. 7 X0.0I $475=0.0194975 \mathrm{gm} . \times 20=0.3899$ per cent.

Method B. This method may be employed if the chloroformic extract is highly colored, the indicator fluorescin being used.

The residue from the evaporation of chloroform is dissolved in ro cc. of acid-free alcohol; then water is added to slight turbidity, followed by a measured excess of $\frac{\mathrm{N}}{25}$ acid V. S.; then the titration is completed with $\frac{\mathrm{N}}{50}$ alkali V.S. The first appearance of fluorescence marks the completion of the reaction. This is best observed by holding the flask over a dark surface and viewing by reflected light.

Method $C$. This is to be used in the case of highly colored extracts. It consists in removing the alkaloid in a pure state by shaking out in a separating funnel with immiscible solvents. The chloroformic extract is shaken out with several portions of acidulated water ( $\mathrm{I}: 50)$; this removes the alkaloid, leaving resins, fats, coloring matters, etc., in the chloroform. The acid alkaloidal solution is then treated with ether in a second separator, ammonia added to alkaline reaction, and the alkaloid thus liberated by ammonia dissolves in the ether, from which it is obtained by evaporation and estimated acidimetrically.

(2) Lyon's Method.* Fluid Extracts. Add ammonia to the fluid 
extract and shake out directly with an immiscible solvent; wash the alkaloid from this by means of acidulated water. Separate the solutions, add ammonia to the acid solution and shake out with the appropriate solvent. Finally, evaporate this alkaloidal solution on a waterbath and estimate the alkaloid in the residue alkalimetrically.

(3) Steiglitz's Method. Add ten to twenty times its volume of weak Prollius' mixture, shake together and let stand for some time. Pour off an aliquot part of the ethereal liquid, put in a separator and wash out with acidulated water. Separate the latter, add ammonia in slight excess and shake out with the appropriate solvent.

(4) F. A. Thompson's Method:* Into a capsule place about 5 gms. of oak sawdust, pour into this gradually Io cc. of the fluid extract, mix thoroughly, and dry at about $45^{\circ} \mathrm{C}$. (II $5^{\circ} \mathrm{F}$.), transfer to a flask and add 100 cc. of modified Prollius' mixture. Shake frequently during twenty minutes, pour off exactly $50 \mathrm{cc}$. of the ethereal liquid, and set aside in a warm place until the ether has nearly all evaporated. Then add $5 \mathrm{cc}$. of $\frac{\mathrm{N}}{5}$ sulphuric acid and $10 \mathrm{cc}$. of ether. Stir to redissolve in the ether all of the oily or waxy matter present and to insure solution of the alkaloids in the acid liquid. Evaporate completely the ether and any alcohol that may remain, and filter the acid aqueous .liquid through a very small dry filter into a bottle. The alkaloid is then liberated by treatment with an alkali and the appropriate solvent.

(5) J. Katz's Method (Arch. d. Pharm., I898, I, and Am. Drug., 1898, 28I). This method has the advantage of all other methods in that it enables one to rapidly and accurately estimate the alkaloids in a preparation without the necessity of applying heat for any purpose whatever during the process. The assay may be made in from one to three hours. $25 \mathrm{cc}$. of the tincture of an alcoholic strength of about 45 per cent are placed in a separatory funnel, $\mathbf{r}$ cc. of a 33 per cent solution of soda added, and the mixture shaken for five minutes with $50 \mathrm{cc}$. of ether, and set aside until the liquids have completely separated into two layers. The lower dark-colored aqueous layer is drawn off into a beaker. The ethereal layer which, besides the alkaloid, has taken up most of the alcohol and some coloring matter, is shaken up with $3 \mathrm{cc}$. of water, which after separating is drawn off and added to the first aqueous liquid. The ethereal liquid is then poured into a suitable flask, while the combined aqueous liquid is further treated with two portions of ether (25 cc. each), the ether to contain about io per cent of alcohol. These

* Proc. A. Ph. A., 1892, 446. 
ethereal extractions are washed each with $\mathrm{I} .5 \mathrm{cc}$. of water; the first extraction after washing may be added to the original ethereal solution, while the second is reserved and later on employed for washing the flask. The ethereal solution, which contains still some traces of aqueous fluid containing alkali, is dehydrated by treatment with 2 or 3 gms. of exsiccated calcium sulphaie and finally filtered into a glass-stoppered flask containing $50 \mathrm{cc}$. of water.

Titration by means of $\frac{\mathrm{N}}{\mathrm{IOO}}$ acid V. S. is employed, using alcoholic solution of iodeosin $(\mathrm{I}: 250)$ as indicator.

The method as above described is obviously applicable only to such alkaloids as are readily soluble in ether. If an estimation of alkaloids insoluble or only slightly soluble in ether, but soluble in chloroform, is to be made, the method is modified as follows:

$25 \mathrm{cc}$. of the tincture of 45 per cent alcoholic strength are vigorously shaken for five minutes with $30 \mathrm{cc}$. of a mixture of I part of chloroform and 2 parts of ether. The solution so obtained is washed with $3 \mathrm{cc}$. of a 30 per cent solution of sodium chlorid. This operation is repeated twice, using each time $\mathrm{I}_{5} \mathrm{cc}$. of the ether-chloroform mixture and washing with I.5 cc. of sodium-chlorid solution.

If the separation of the aqueous and ethereal liquids is not distinct an additional 2 or 3 gms. of sodium chlorid may be used. This prevents the emulsification, which if pure water were employed would occur.

If the tincture to be assayed contains more than 45 per cent of alcohol it is necessary to add water to reduce it to 40 or 50 per cent.

Tinctures containing chlorophyll or fat or fatty acids must first be deprived of these constituents, as these substances, possessing acid properties inferior to that of iodeosin, will act the part of an alkali toward it and thus be recorded as alkaloid.

To remove the chlorophyll and fatty acids, acidulate a mixture of equal parts of the tincture and water with a few drops of sulphuric acid, shake with talcum during several hours, and, after subsidence of the latter, filter. Of this filtrate 25 gms. (not cc., on account of the admixture of alcohol and water causing change of volume) are taken and the alkaloid estimated in the manner already described, after first removing, if necessary, the last traces of fat by a single shaking of the acid solution with petroleum ether.

For the assay of extracts I to I.5 gms. are dissolved in from 40 to $50 \mathrm{cc}$. of 45 per cent alcohol to make a solution containing less than 3 per cent extractive. For the assay of fluid extracts Io cc. are taken.

(6) C. Kippenberger's Method (Apoth. Ztg., I898, 664-674). The preparation to be examined, if strongly alcoholic, needs no 
preliminary treatment; if, however, it is a hydro-alcoholic or aqueous preparation-extracts and the like-it must preliminarily be dissolved in strong alcohol to which a certain percentage of hydrochloric acid has been added (tartaric acid in the case of aconite) and filtered off from the more or less abundant and generally smeary precipitate. An aliquot part of the filtrate is then evaporated on a water-bath, the residue dissolved in water, filtered, and the filtrate transferred to a beaker, in which the precipitation of the alkaloid as periodid is then effected by adding the prescribed quantity of the iodin solution* (usually $20 \mathrm{cc}$. for $\mathrm{I}-5 \mathrm{gms}$. of extract, according to the kind used). In most cases five to ten minutes are sufficient for complete deposition of the precipitate; but if it is desired that the precipitate shall become crystalline the mixture must be allowed to stand from twenty minutes to an hour. In either case the precipitate is collected on a small plaited filter and washed two or three times with water to which a few drops of the iodin solution have been added; or a little sodium chlorid may be dissolved in the wash water, which is particularly recommended in the case of atropine. The washed precipitate is now dissolved in acetone (pure) (of which from 15 to 20 cc. are required for $\mathrm{I}-5 \mathrm{gms}$. of extract employed), observing that it shall completely enter solution and that there shall be no waste. The acetone solution is then treated with alkali hydroxid solution in excess, then diluted hydrochloric acid in excess, the mixture diluted with water and shaken out with petroleum ether to remove the acetone, the last traces of which are removed by heating on a waterbath. The liquid is now supersaturated with alkali hydroxid and shaken out with chloroform or with a mixture of ether and chloroform. The chloroformic solution of pure alkaloid so obtained is cautiously evaporated and its amount determined either by direct weighing or by titration.

According to the preparation that is to be assayed, there must be some variation in the amount of solvents and precipitant. These are indicated by Kippenberger in the following examples:

Extract of Aconite Root. 7.5 gms. of the extract are dissolved in $75 \mathrm{cc}$. of water containing $3 \mathrm{gms}$. of tartaric acid and $50 \mathrm{cc}$. of the filtrate (=5 gms. of the extract) are mixed with $20 \mathrm{cc}$. of the iodin solution.

Tincture of Aconite Root. Ioo cc. of the tincture are evaporated to dryness with I gm. of tartaric acid, the residue dissolved in $60 \mathrm{cc}$.

* The iodin solution is made by dissolving $20 \mathrm{gms}$. of iodin and $60 \mathrm{gms}$. of potassium iodid in water to make $1000 \mathrm{cc}$. The diluted hydrochloric acid used is that of the German Pharmacopœia. 
of water containing I cc. of dilute hydrochloric acid, and $45 \mathrm{cc}$. of the filtrate $(=75 \mathrm{cc}$. of the tincture) are mixed with $20 \mathrm{cc}$. of the iodin solution.

Extract of Belladonna (either from leaves or root). 7.5 gms. of the extract are treated with $75 \mathrm{cc}$. of alcohol containing $2 \mathrm{cc}$. of diluted hydrochloric acid and filtered. $60 \mathrm{cc}$. of the filtrate are evaporated to dryness, the residue dissolved in $60 \mathrm{cc}$. of water containing I cc. of diluted hydrochloric acid, the solution filtered and $50 \mathrm{cc}$. of the filtrate ( $=5 \mathrm{gms}$ : of the extract) are mixed with $30 \mathrm{cc}$. of the iodin solution.

Tincture of Belladonna. Ioo cc. of the tincture are mixed with I cc. of diluted hydrochloric acid and evaporated to dryness, the residue dissolved in $50 \mathrm{cc}$. of water containing I cc. of diluted hydrochloric acid, and $40 \mathrm{cc}$. of the filtrate $(=80 \mathrm{cc}$. of the tincture $)$ are mixed with $20 \mathrm{cc}$. of the iodin solution.

Extract of Cinchona (Alcoholic). I.5 gms. of the extract are dissolved in $75 \mathrm{cc}$. of water containing $3 \mathrm{cc}$. of diluted hydrochloric acid, and to $50 \mathrm{cc}$. of the filtrate (= I gm. of the extract) 20 to 30 cc. of iodin solution are added. (The iodin solution for this assay is best made with only 30 gms. of potassium iodid per Iooo cc.).

Extract of Cinchona (Aqueous). I.5 gms. of the extract are treated with $80 \mathrm{cc}$. of alcohol containing $3 \mathrm{cc}$. of diluted hydrochloric acid, filtered, and the filtrate evaporated to dryness. The residue is dissolved in $75 \mathrm{cc}$. of water containing $2 \mathrm{cc}$. of diluted hydrochloric acid, and $50 \mathrm{cc}$. of the filtrate ( $=\mathrm{I} \mathrm{gm}$. of the extract) are precipitated with 20 to $30 \mathrm{cc}$. of the iodin solution, modified as under alcoholic extract of cinchona.

Tincture of Cinchona. $30 \mathrm{cc}$. of the tincture are evaporated to dryness, the residue dissolved in $60 \mathrm{cc}$. of water containing $3 \mathrm{cc}$. of diluted hydrochloric acid, the solution filtered and $50 \mathrm{cc}$. of the filtrate $(=25 \mathrm{cc}$. of the tincture) treated with $20 \mathrm{cc}$. of the iodin solution modified as under alcoholic extract of cinchona.

Extract of Hyoscyamus. I 2 gms. of the extract are treated with I20 cc. of alcohol containing $2.5 \mathrm{cc}$. of diluted hydrochloric acid and filtered, Ioo cc. of the filtrate evaporated to dryness, the residue dissolved in $60 \mathrm{cc}$. of water containing I cc. of diluted hydrochloric acid, filtered, and $45 \mathrm{cc}$. of the filtrate $(=7.5 \mathrm{gms}$. of extract $)$ mixed with $20 \mathrm{cc}$. of iodin solution.

Tincture of Hyoscyamus. $200 \mathrm{cc}$. of the tincture and $2 \mathrm{cc}$. of diluted hydrochloric acid are evaporated to dryness, the residue dissolved in $50 \mathrm{cc}$. of water, the filtrate rendered acid if necessary by the addition of a little diluted hydrochloric acid, and $40 \mathrm{cc}$. of it $(=\mathrm{r} 60 \mathrm{cc}$. of tincture $)$ are precipitated with 1o to $20 \mathrm{cc}$. of iodin solution. 
Extract of Nux. Vomica (Alcoholic). $\mathbf{1} .5 \mathrm{gms}$. of the extract are dissolved in $75 \mathrm{cc}$. of water containing $\mathrm{I} .5 \mathrm{cc}$. of diluted hydrochloric acid, and $50 \mathrm{cc}$. of the filtrate (=I $\mathrm{gm}$. of extract) are mixed with $20 \mathrm{cc}$. of the iodin solution.

Extract of Nux Vomica (Aqueous). 2 gms. of the extract are dissolved in $80 \mathrm{cc}$. of alcohol containing $2 \mathrm{cc}$. of diluted hydrochloric acid; $60 \mathrm{cc}$. of the filtrate are evaporated on a water-bath, the residue is dissolved in $75 \mathrm{cc}$. of water containing $\mathrm{I} .5 \mathrm{cc}$. of diluted hydrochloric acid, and $50 \mathrm{cc}$. of the filtrate from this (=I gm. of extract) are mixed with $20 \mathrm{cc}$. of iodin solution.

Tincture of Nux Vomica. $60 \cdot \mathrm{cc}$. of the tincture are evaporated on a water-bath to dryness, the residue dissolved in $60 \mathrm{cc}$. of water containing $2 \mathrm{cc}$. of diluted hydrochloric acid, and $50 \mathrm{cc}$. of the filtrate (=50 cc. of the tincture) are ntixed with $20 \mathrm{cc}$. of the iodin solution.

(7) Farr and Wright's Method for Tinctures as Modified by Seyler (Year-book of Pharmacy, I897):

(a) $50 \mathrm{cc}$. of the tincture are evaporated on a water-bath until all the alcohol is driven off, adding water from time to time, and finally adding enough water to make up the original volume.

(b) When the liquid has cooled off I cc. of semi-normal sulphuric acid is added and filtered through cotton into a separator. The dish and funnel arc rinsed with a little acidulated water and added to the contents of the separator, which is then shaken with 15 cc. of chloroform, drawn off and again shaken with two consecutive portions of $5 \mathrm{cc}$. each, of chloroform.

(c) The colored chloroformic solution is then washed with three successive portions of acidulated water $(20 \mathrm{cc}$. of water and $2 \mathrm{cc}$. semi-normal acid) to recover traces of alkaloid, and the washings added to the original acid liquid $(b)$.

(d) To the so purified aqueous liquid 2 cc. or more of ammoniawater B. P. are added and shaken out with consecutive portions of chloroform (of 10,5 , and $5 \mathrm{cc}$.) until this on evaporation gives no precipitate with Mayer's reagent.

(e) The chloroformic solution of crude alkaloid so obtained is now shaken out with three portions of acidulated water, as under $(c)$, the solution then made alkaline with ammonia-water and shaken out once more with chloroform, using 10,5 , and 5 cc., as under $(d)$.

The chloroformic solution is evaporated on a water-bath and the residue weighed and titrated as pure alkaloid.

For Fluid Extracts take to to $20 \mathrm{cc}$.

(8) Webster's Method.* In the following process the endeavor is made not orly to prevent the formation of emulsions (a most

*A. J. Ph., 1907, 301-307. 
objectionable feature of the shaking out process as usually carried out), but also to effect the complete elimination of ammonia:

In the shaking out process there are several drawbacks. The presence of fat, resin, gums, or other colloidal bodies, while they do not interfere with the solubility of the alkaloids and their salts, prevent, however (because of the increased viscosity of the liquids), the intimate contact necessary for their transference from one solvent to another, except by prolonged and vigorous agitation. Such treatment often results in the formation of an inseparable emulsion which interferes with the completion of the assay. In most assay processes the purified "ethereal" solution of the free alkaloid is, in the last stage, warmed to dissipate the solvent together with adherent ammonia, leaving the alkaloid in a suitable condition for weighing or titrating. The application of heat is liable to occasion loss from various causes: Reaction with chloroform, hydrolysis of the alkaloid, as in the case of cocaine, evaporation of coniine, spurting of strychnine, and more or less resinification of the alkaloids generally. The chief difficulty, however, is the elimination of ammonia, which adheres persistently to the residue. The elimination of the ammonia in the Webster process is effected by the addition of an excess of tartaric acid in the presence of absolute alcohol. Practically all of the ammonia will be precipitated, as well as nearly all of the albuminous and gummy matter. The filtrate can then be evaporated to a solid extract without injuring the alkaloids, which may be dissolved in acidified water, leaving behind the resins, chlorophyll and fat.

The process in detail is as follows: Add gradually $\mathrm{io} \mathrm{cc}$. of the fluid extract to $85 \mathrm{cc}$. of cold absolute alcohol in which $1.5 \mathrm{gms}$. of tartaric acid have been previously dissolved; add absolute alcohol to make roo cc. (in the cases of fluid extracts of gelsemium and veratrum where the menstruum is 95 per cent alcohol, 0.75 gms. of tartaric acid is added directly to $5 \mathrm{cc}$. of the fluid extract; the precipitate, if any, filtered off and washed with absolute alcohol, etc.). Shake well, then set aside for a few minutes. Filter, transfer $5^{\circ}$ cc. of the filtrate to a shallow porcelain basin of a diameter of six inches, and evaporate carefully on a water-bath, rotating the contents of the basin occasionally, and especially towards the end of the evaporation, so that the resulting extract may cover a large portion of the basin. When dry, cool the extract by floating the basin in cold water; add io cc. of half-normal sulphuric acid, and rotate the liquid about in the dish until the extract is dissolved or disintegrated; set aside for two minutes. Filter the liquid through a small, firmly packed pledget of cotton-wool previously moistened with water, into a large, pear-shaped separator. Rinse the evaporating dish with two successive 5 cc. portions of water, stirring to dis- 
integrate the insoluble substances, and pass the liquid through the filter. To the separator add $20 \mathrm{cc}$. of a mixture of $\mathrm{I} 6 \mathrm{cc}$. of chloroform and $4 \mathrm{cc}$. of ether; and $4 \mathrm{cc}$. or sufficient of a 20 per cent solution of $\mathrm{KOH}$, insert the stopper, and shake vigorously. When the fluids have separated, draw off the lower layer into a second separator containing $15 \mathrm{cc}$. of distilled water, and after agitation and separation as before, transfer the lower layer to a third separator also containing I 5 cc. of distilled water; agitate, and allow to separate. Pass the lower layer through a chloroform-moistened pledget of cotton-wool into a fourth separator of.about $150 \mathrm{cc}$. capacity. In the presence of a volatile alkaloid wash the cotton afterwards with $5 \mathrm{cc}$. of chloro. form; in all cases the stems of the separators should be washed with I cc. of chloroform after the solution has been run off and the washings added. Repeat the extraction till no more alkaloid is obtained, with two or more portions of the immiscible solvent which finally collect, after washing as before in separator No. 4. To the bulked ethereal extract add excess of $\frac{\mathrm{N}}{50}$ or $\frac{\mathrm{N}}{100}$ sulphuric acid, and shake thoroughly. After complete separation, reject the lower layer; add cochineal T. S. or iodeosin solution in water-saturated ether, and titrate back with $\frac{\mathrm{N}}{50}$ sodium hydroxid. .

For Solid Extracts. Dissolve 2 gms. of the extract in 10 cc. of 50 per cent alcohol, and treat as a fluid extract.

\section{ASSAYING OF PREPARATIONS OF ACONITE}

Assay of Fluid Extract of Aconite (U. S. P. VIII). Transfer Io cc. of fluid extract of aconite by means of a graduated pipette to a porcelain dish, and evaporate it carefully to dryness on a water-bath at a temperature not exceeding $60^{\circ} \mathrm{C}$. ( $140^{\circ} \mathrm{F}$ ). Add 5 cc. of tenth-normal sulphuric acid V. S. and ro cc. of distilled water. When the extract is dissolved, filter the liquid into a separator, washing the dish and filter, with about $40 \mathrm{cc}$. of distilled water; when this has passed through, add $25 \mathrm{cc}$. of ether and $2 \mathrm{cc}$. of ammoniawater to the separator, and agitate for one minute. Draw off the lower layer into a flask and filter the ether-solution into a beaker. Return the contents of the flask to the separator, add $15 \mathrm{cc}$. of ether, and agitate for one minute. Draw off the lower layer into the flask and filter the ether-solution into the beaker. Repeat, with two other portions of ro cc. each of ether. Evaporate the ether-solution to dryness, and dissolve the residue in $3 \mathrm{cc}$. of tenth-normal sulphuric acid V. S. diluted with $20 \mathrm{cc}$. of distilled water. Add to the solution 
5 drops of hematoxylin T. S., and then carefully run in fiftieth-normal potassium hydroxid V. S. until a violet color is produced, the transition stages being as follows: First yellow, then green, finally passing into violet. Divide the number of cc. of fiftieth-normal potassium hydroxid V. S. used by 5 , subtract this number from 3 (the $3 \mathrm{cc}$. of tenth-normal sulphuric acid V. S. taken), multiply the remainder by 0.064 , and this product by 10 , which will give the weight in grams of aconitine contained in one hundred cubic centimeters of the fluid extract of aconite.

Tincture of Aconite is assayed by evaporating roo cc. to dryness at a temperature not exceeding $60^{\circ} \mathrm{C}$. ( $\left(40^{\circ} \mathrm{F}\right.$.), and treating the resulting extract by the foregoing method, with the exception that the multiplication of the product by ro must be omitted. The result will represent the weight. in grams of aconitine contained in 100 cc. of the tincture.

\section{ASSAYING OF PREPARATIONS OF THE MYDRIATIC DRUGS}

Assay of Extract of Belladonna Leaves (U. S. P. VIII). Introduce 5 gms. of the extract of belladonna leaves into a small beaker and dissolve it in a mixture consisting of alcohol $5 \mathrm{cc}$., distilled water ro cc., ammonia-water $2 \mathrm{cc}$, , and chloroform $20 \mathrm{cc}$. When dissolved, transfer it to a separator, rinsing the beaker with a little alcohol and adding the rinsings to the separator. Insert the stopper securely and shake the separator for half a minute. Draw off the chloroformic layer into a second separator, and add to the first separator Io cc. more of chloroform. Shake it for half a minute, allow to separate, and again draw off the chloroformic layer into the second separator. Repeat this with ro cc. more of chloroform. To the united chloroformic liquids in the second separator, add 5 cc. of normal sulphuric acid V. S. and to cc. of distilled water, and shake it for half a minute. Draw off the chloroformic layer, after the liquids have separated, into the first separator, after cleaning it thoroughly, and the aqueous layer into a beaker, and repeat the process by adding to the first separator Io cc. of distilled water and I cc. of normal sulphuric acid V. S. Draw off the chloroformic layer, rejecting the same, and then run the acid aqueous layer into the beaker. Pass the combined acid aqueous solutions through a pledget of purified cotton into the first separator, after cleaning it thoroughly, rinsing the second separator, the beaker, and the funnel with about Io cc. of distilled water. To the first separator add I 5 cc. of chloroform, a small piece of red litmus paper, and enough ammonia-water to produce a distinctly alkaline reaction. Shake the separator for 
half a minute, and when the liquids have separated draw off the chloroformic layer into a beaker. Repeat this process with two portions of ro cc. each of chloroform, and evaporate the combined chloroformic liquids in the beaker to dryness on a water-bath containing warm water; dissolve the residue in $3 \mathrm{cc}$. of ether and allow the latter to evaporate completely. To the alkaloidal residue add $5 \mathrm{cc}$. of tenth-normal sulphuric acid V. S. and 5 drops of hæmatoxylin T. S. (or iodeosin T. S.), then titrate the excess of acid with fiftiethnormal potassium hydroxid V. S. Divide the number of cubic centimeters of fiftieth-normal potassium hydroxid V. S. used, by by 5 , subtract the quotient from 5 (the $5 \mathrm{cc}$. of tenth-normal sulphuric acid V. S. taken), and multiply the remainder by 0.0287 , and this product by 20 , to obtain the percentage of mydriatic alkaloids contained in the extract of belladonna leaves. The figure 0.0287 represents in weight the grams of mydriatic alkaloids (mainly atropine) required to neutralize I cc. of tenth-normal sulphuric acid V.S.

Assay of Fluid Extract of Belladonna Root (U. S. P., VIII). Transfer ro cc. of fluid extract of belladonna root by means of a graduated pipette to a separator, add $10 \mathrm{cc}$. of distilled water, $20 \mathrm{cc}$. of chloroform, and 2 cc. of ammonia-water. Shake the separator well for one minute, and draw off the lower chloroformic layer into a second separator. Repeat the extraction with two portions of ro cc. each of chloroform, and draw the chloroform solution into the second separator. To the latter add $8 \mathrm{cc}$. of normal sulphuric acid V. S. and $20 \mathrm{cc}$. of distilled water, shaking well for one minute. When perfectly separated draw off and reject the lower chloroformic layer, and filter the acid aqueous layer into a clean separator. Wash the separator and filter with Io cc. of distilled water, adding this to the clean separator. To the latter add $20 \mathrm{cc}$. of chloroform and $4 \mathrm{cc}$. of ammonia-water, and shake well for several minutes. Draw off the lower chloroformic layer into a beaker, and repeat the extraction with two portions of $\mathrm{I} 0 \mathrm{cc}$. each of chloroform, adding the chloroformic solution to the beaker. Allow the chloroform in the beaker to evaporate on a water-bath, containing warm water, until the residue is perfectly dry. To the alkaloidal residue add $5 \mathrm{cc}$. of tenth-normal sulphuric acid V, S., and when the residual alkaloids have all disssolved, titrate the solution with fiftieth-normal potassium hydroxid V. S., using 5 drops of hæmatoxylin or iodeosin T. S. as an indicator. Divide the number of cubic centimeters of fiftiethnormal potassium hydroxid V. S. used by 5 , subtract the quotient from 5 (the $5 \mathrm{cc}$. of tenth-normal sulphuric acid V. S. taken), and multiply the remainder by 0.0287 , and this product by 10 , to obtain the weight in grams of mydriatic alkaloids contained in one hundred cubic centimeters of the fluid extract of belladonna root. 
Assay of Tincture of Belladonna Leaves. Transfer roo cc. of tincture of belladonna leaves to an evaporating dish and evaporate it on a water-bath until it measures about ro cc. Add, if necessary, sufficient alcohol to dissolve any separated substance, and then assay the resulting liquid by the method given above, using the same details as there directed for Io cc. of fluid extract of belladonna root, with the exception that the multiplication of the product by ro be omitted. The result will represent the weight in grams of alkaloids contained in one hundred cubic centimeters of tincture of belladonna leaves.

The above assay of fluid extract of belladonna is a modification of Puckner's method (Ph. Rev., I898), which is as follows:

Measure ro cc. of the fluid extract into a separator and add $10 \mathrm{cc}$. of water, $20 \mathrm{cc}$. of chloroform, $2 \mathrm{cc}$. of a io per cent ammonia-water, and shake well. When the layers have separated, draw off the heavier, receiving it in a separator; complete the extraction of the alkaloid with two further portions of chloroform, io cc. each, adding this to the chloroform solution first drawn off; shake the chloroform solution of the alkaloid with $20 \mathrm{cc}$. of I per cent hydrochloric acid, and draw off the chloroform into a clean separator, using a few cc. of chloroform to rinse the stop-cock and outlet-tube of the first, and again shake the chloroform with I per cent hydrochloric acid, using only ro cc. this time.

Draw off and reject the chloroform and mix the acid extracts, add a few cc. of chloroform, draw off and reject the same. Now add $20 \mathrm{cc}$. of chloroform, 2 cc. of ammonia-water, and shake well. Draw off the clear chloroformic solution and complete the extraction with two further portions of chloroform, Io cc. each. Evaporate the chloroform and titrate the residue as follows:

Add to it $5 \mathrm{cc}$. of ether, about $5 \mathrm{cc}$. of cochineal solution, and gradually a slight excess of $\frac{\mathrm{N}}{20}$ acid. When complete solution has taken place, evaporate the ether and determine the excess of acid with $\mathrm{N}$ 20 of alkaloid. The advantage of this method is that there is no necessity for evaporating the fluid extract, the economy in material, and the purity of the alkaloid extracted.

Method of A. B. Lyons.* While the pharmacopœial process for the assay of fluid extracts of the mydriatic drugs is fairly satisfactory in the hands of skillful workers, it has, however, its drawbacks when applied to preparations which contain but a very small

* Ph. Rev., r908, XXVI, 22. 
quantity of alkaloid, as, for instance, preparations of hyoscyamus. The following is proposed as an alternative:

Put into a $200 \mathrm{cc}$. measuring flask $20 \mathrm{cc}$. of solution of lead subacetate (U. S. P.). Add 150 cc. of distilled water, mix and add with constant shaking $20 \mathrm{cc}$. of the fluid extract. Make up with distilled water to exactly $200 \mathrm{cc}$. Filter, using if necessary a double filter, and returning the first portion of the filtrate until it passes through quite clear. The filtrate should still contain some lead subacetate; it is not desirable that the excess should be very large, and it may therefore be a better way to use at first only i 5 cc. of the lead solution, then after adding the water and the fluid extract observing whether the mixture has any sweet taste, indicating that the lead is in excess. If not, more of the lead solution should be added, about I cc. at a time, until the sweet taste becomes perceptible. The measure should then be made up to $200 \mathrm{cc}$.

When the filter has practically finished dripping, add to the filtrate about a gram of granulated sodium phosphate, and stir until the salt is dissolved. If the mixture still has perceptible sweetness, add more of the phosphate, until this is in slight excess. Allow the mixture to stand at rest in a beaker a few minutes and test a few drops of the clear liquid that will shortly separate with a little sodium phosphate. If this produces any turbidity, add to the mixture more of the phosphate until this is surely in slight excess. All this takes but a few moments' time, although in the description it may seem a complicated operation.

Filter once more, when the filtrate will be quite clear and almost free from color. Transfer exactly Ioo cc. of the filtrate, representing Io cc. of the original fluid extract to a separator, add 25 cc. of chloform and about $2 \mathrm{cc}$. of water of ammonia. Shake carefully, using a rotary motion. Generally there is no serious danger of emulsification, but the possibility of such an occurrence must be always borne in mind. Nearly the whole of the alkaloid will pass into the chloroform in this first washing. Allow the chloroform to separate completely and draw it off. Repeat the extraction with two additional portions of chloroform, 20 and I5 cc. Evaporate the chloroform in a tared beaker, dry on a water-bath and weigh. Then determine the alkaloid in the usual way by titration.

Lyons employs the following as a routine method for such titrations:

The alkaloidal residue is dissolved in 2 cc. of alcohol. To the solution are added $2 \mathrm{cc}$. of decinormal sulphuric acid, $20 \mathrm{cc}$. of distilled water and five drops of cochineal indicator. Into a small flask are introduced at the same time $2 \mathrm{cc}$. of the same alcohol, $2 \mathrm{cc}$. of the decinormal acid, $20 \mathrm{cc}$. of the same distilled water, and five drops of the same indicator. Alkali (lime water or potassium hydroxid solution) 
is then added from a graduated pipette until the color changes. The amount of alkali consumed is read off, 2 cc. more of acid is added and the titration repeated. The two results will exactly correspond unless by any chance the alcohol or the distilled water used were not strictly neutral-a possibility always to be kept in mind in these delicate titrations. The second titration in any case gives us the exact strength of the alkali we are using. If it differs from the first, we may still go on with the determination, since we know the exact amount of the error due to imperfect neutrality of our solvents.

The next step is to titrate the alkaloidal solution, noting the exact amount of alkali required to neutralize the excess of acid. We have now all the data for our calculation. We subtract the amount of alkali consumed in neutralizing excess of acid in the alkaloidal solution from the amount consumed in the first blank experiment. The difference gives the alkalinity of the alkaloidal residue in units whose value is readily reduced to the "normal" standard. For example, suppose we found our alkaloidal residue to be the equivalent of $4.55 \mathrm{cc}$. of our "standard" alkali, and that we have found (in our second blank titration) that $2 \mathrm{cc}$. of $\frac{\mathrm{N}}{\mathrm{IO}}$ açid required for neutralization $7.45 \mathrm{cc}$. of our "standard" alkali (i.e., I cc. of decinormal acid corresponds with $3.725 \mathrm{cc}$. of the "standard" alkali). Then $4.55 \div 3.725=\mathrm{I} .22+\mathrm{cc}$. will be the quantity of decinormal alkali corresponding with the alkaloid present, and since each cc. of decinormal alkali corresponds with $0.0287 \mathrm{gm}$. of atropine, our residue contained $\mathrm{I} .22 \times 0.0287=0.035+\mathrm{gm}$. of alkaloid estimated as atropine.

The calculation reduced to the form of a "rule" is very simple. Subtract the result of the final titration expressed in cc. from the result of the first blank titration, multiply the remainder by 57.4 and divide the product by the result of the second blank titration. The quotient will be the quantity of atropine present, expressed in mgs. Fluid extract of belladonna root or of stramonium would be assayed in exactly the same way as the fluid extract of belladonna leaves. For the assay of tincture of belladonna or stramonium, take roo cc. of the tincture, evaporate to about 25 or $30 \mathrm{cc}$., treat with $10 \mathrm{cc}$. of solution of lead subacetate after making up to $85 \mathrm{cc}$., add distilled water to make exactly Ioo cc. and proceed as above described, using finally for the assay the equivalent of only $50 \mathrm{cc}$. of the tincture.

For the assay of fluid extract of hyoscyamus, use $25 \mathrm{cc}$. each of the lead solution and the fluic extract; make up to $250 \mathrm{cc}$. and take of the filtrate $\mathrm{I} 25$ or I50 cc. For the assay of tincture of hyoscyamus take at least $200 \mathrm{cc}$., evaporate to about $50 \mathrm{cc}$., dilute with water to I $75 \mathrm{cc}$., add $20 \mathrm{cc}$. of lead solution and make up to $200 \mathrm{cc}$. 
The Potassium-bismuth Iodid Method (Thoms*) is applied to preparations of the mydriatic drugs in the following manner: To 4 gms. of dry extract (extractum siccum) are added $50 \mathrm{cc} .90$ per cent alcohol, the mixture shaken frequently during three hours and filtered. Then $25 \mathrm{cc}$. of the filtrate, taken to represent 2 gms. extract, are heated on a water-bath until the alcohol has been driven off. The residue is taken up with $50 \mathrm{cc}$. water and to this added $10 \mathrm{cc}$. Io per cent sulphuric acid and $5 \mathrm{cc}$. potassium-bismuth iodid solution (prepared by pouring a solution of 80 gms. bismuth subnitrate in 200 gms. nitric acid (sp.gr. I.18), into a concentrated solution of $272 \mathrm{gms}$. potassium iodid in water and, after removal of the potassium nitrate crystals formed, diluting to I000 cc.). The precipitate is collected and with the filter placed in a cylinder and treated with $20 \mathrm{cc}$. I5 per cent sodium hydroxid solution and Io gms. coarsely powdered crystallized sodium carbonate. Next $50 \mathrm{cc}$. ether are added and the whole shaken frequently during three hours. Now about roo cc. water, $20 \mathrm{cc}$. ether, and five drops iodeosin solution are measured, into a stoppered flask and any red color, due to the alkalinity of the glass, destroyed by addition of a few drops of hundredth-normal hydrochloric acid. To this, $20 \mathrm{cc}$. of the ethereal alkaloid solution, representing I gm. of extract, are added, and its alkalinity determined with one-hundredth normal hydrochloric acid.

Assay of Belladonna Plaster (Rubber Base) (U. S. P. VIII). Into a suitable beaker containing $50 \mathrm{cc}$. of chloroform and $3 \mathrm{cc}$. of ammonia-water, introduce to gms. of the belladonna plaster cut into strips. Stir until the plaster is entirely removed from the cloth; then pour off the chloroform into another beaker, wash the cloth with $25 \mathrm{cc}$. of chloroform and I cc. of ammonia-water carefully, and add the washings to the chloroformic solution first obtained. If necessary, repeat the washing with $25 \mathrm{cc}$. of chloroform, and add this also to the chloroformic solution. Then dry the cloth at a low temperature; cool and weigh it, and subtract its weight from the original weight of the plaster. To the chloroformic solution, add four fifths of its volume of alcohol, stir gently, and allow the liquid to stand until all of the rubber has separated in a compact mass. Then pour off the supernatant liquid into a separator of $250 \mathrm{cc}$. capacity, and, having prepared a solution of sulphuric acid by diluting $40 \mathrm{cc}$. of normal sulphuric acid V. S. with $60 \mathrm{cc}$. of distilled water, add $20 \mathrm{cc}$. of the solution to the separator, and agitate for two minutes, rotating gently. Draw off the chloroformic solution into another separator, shake this with Io cc. of the sulphuric acid solution, and add the acid solution

*H. Thoms, Ber. d. Ph. Gesellsch., XV, 85; and Chem. Centralbl., I905, I, I34I. 
to that in the first separator. Repeat until the acid washings cease to give a reaction with mercuric potassium iodid T. S.; combine the acid liquids, and, having rendered this solution alkaline with ammoniawater, shake out the alkaloids with three successive portions of 25 , I5, and ro cc. of chloroform. Collect these in a flask, distil off all of the chloroform with the aid of a water-bath. To the alkaloidal residue add a slight excess of tenth-normal sulphuric acid V. S., noting the quantity used, and then add ten drops of chloroform and, after rotating, evaporate the latter by means of a water-bath. Then add five drops of hæmatoxylin T.S., and, rotating, titrate the excess of acid with fiftieth-normal potassium hydroxid V.S. Divide the number of cc. of fiftieth-normal potassium hydroxid V. S. used by 5 , subtract the quotient from the number of $\mathrm{cc}$. of tenth-normal sulphuric acid V. S. first added, and divide the difference by the number of grams of belladonna plaster separated from the cloth. Multiply the quotient by 0.0287 , and this product by 100 , which will give the percentage of mydriatic alkaloids in the belladonna plaster.

The Extracts of Hyoscyamus, Scopola, and Stramonium are assayed exactly as directed for extract of belladonna, except that in the case of hyoscyamus Io gms. are taken, and in the assay of scopola 2 gms. are sufficient for the assay.

The fluid extracts of these drugs are assayed in the manner directed for fluid extract of belladonna, except that $50 \mathrm{cc}$. of the fluid extract of hyoscyamus are taken, whereas Io cc. is taken of each of the others for the assay.

The tinctures of these drugs are likewise assayed as is the tincture of belladonna.

The German Pharmacopœia Method for the assay of extracts of belladonna and hyoscyamus is as follows: 2 gms. of the extract are dissolved in $5 \mathrm{gms}$. of water and $5 \mathrm{gms}$. of absolute alcohol. To this solution is added $50 \mathrm{gms}$. of ether and $20 \mathrm{gms}$. of chloroform, and, after briskly shaking, Io cc. of sodium carbonate solution $(\mathrm{I}: 3)$. The mixture is then allowed to stand for one hour with frequent shaking, after which $50 \mathrm{gms}$. of the clear chloroform-ether solution are filtered off through a dry well-covered filter, into a flask, and about half of it distilled off. The remaining chloroform-ether solution is then introduced into a separatory funnel. The flask is rinsed with three portions of ether of $5 \mathrm{cc}$. each, and the mixed solutions shaken out with $20 \mathrm{cc}$. of $\frac{\mathrm{N}}{\mathrm{I} 00}$ hydrochloric acid. After complete separation of the solutions (which may be facilitated by the addition of a small quantity of ether) the acid liquid is filtered through a small filter previously moistened with water, into a $200-\mathrm{cc}$. flask of white glass. The chloroform-ether solution is then washed out three times with 
water, using ro cc. each time, and the washings passed through the same filter. Sufficient water is then passed through the same filter to make the solution measure $100 \mathrm{cc}$. Ether is then added to make a layer $\mathrm{I} \mathrm{cm}$. in depth, five drops of iodeosin solution are now added and the mixture titrated with $\frac{\mathrm{N}}{100}$ potassium hydroxid solution, shaking after each addition of the alkali solution, until the lower layer assumes a pale red color.

\section{LITERATURE}

Karl Dieterich. Helfenberger Annalen, 1905, and $\mathrm{Ph}$. Centralh., (Ig06), XLVII, 9I6.

W. T. Hankey. Am. Drug., 1906, Xurx, 360.

G. Fromme. Geschäft's Bericht, Cæsar and Loretz, 1906, 24 .

W. Gadd and S. C. Gadd. Ph. Jour., Sept., 1905, 438.

F C. Bird. Ph. Jour., Aug., I900, I95

Farr and Wright. Ph. Jour., 1905, $546(\mathrm{xx})$.

Naylor and Chappel. Ph. Jour., 1906, 393 .

H. Thoms. Berichte d. Ph. Ges., xv, 85 .

H. Matthes and O. Ramstedt. Ph. Ztg., I906 (LI), I03r.

Assay of Fluid Extract of Cinchona (U. S. P. VIII). Transfer Io cc. of fluid extract of cinchona by means of a graduated pipette to an Erlenmeyer flask of $200 \mathrm{cc}$. capacity, and add a mixture of I00 cc. of ether, $25 \mathrm{cc}$. of chloroform, and Io cc. of ammonia-water. Insert the stopper securely, and shake the flask vigorously, at intervals, during ten minutes. Allow the liquids to separate, decant into a measuring cylinder exactly $66 \mathrm{cc}$. of the supernatant liquid (representing $5 \mathrm{cc}$. of the fluid extract), and transfer this to a separator, rinsing the cylinder with $5 \mathrm{cc}$. of ether and adding this to the separator. Add to the latter about ro cc. of normal sulphuric acid V. S., or enough to make the solution distinctly acid, and shake the separator vigorously for several minutes, and when the liquids have completely separated, draw off the lower layer into a second separator. To the first separator add $5 \mathrm{cc}$. more of normal sulphuric acid V.S., and $5 \mathrm{cc}$. of distilled water, shake it for several minutes, and when the liquids have separated, draw off the lower layer into the second separator. Now add $5 \mathrm{cc}$. of distilled water to the first separator, shake it, separate as before, and then draw off the lower aqueous layer into the second separator. To the second separator, add $25 \mathrm{cc}$. of ether, a small piece of red litmus paper, and then, gradually, ammonia-water, keeping the temperature of the liquids below $25^{\circ} \mathrm{C}$. $\left(77^{\circ} \mathrm{F}\right.$.), until the reaction is alkaline. Then shake, the separator vigorously for two minutes, and 
allow the liquids to stand for ten minutes at a temperature below $15^{\circ} \mathrm{C}$. $\left(59^{\circ} \mathrm{F}\right.$.). Draw off and reject the lower aqueous layer, and then transfer the ether layer into a tared beaker. Add $5 \mathrm{cc}$. more of ether to the separator, rinse carefully, and add the rinsings to the tared beaker, and entirely evaporate the ether at a moderate heat on a waterbath. Then dry the beaker in an air-bath at $120^{\circ} \mathrm{C}$. $\left(248^{\circ} \mathrm{F}\right.$.) for half an hour, cool, and weigh. Replace the beaker in the air-bath, and heat again at the same temperature for half an hour, cool, and weigh, repeating until the weight is constant. Multiply the weight by 20 to obtain the weight in grams of anhydrous ether-soluble alkaloids contained in 100 cc. of the fluid extract.

To make the assay volumetrically, dissolve the residue in 15 cc. of alcohol; dilute the solution with $40 \mathrm{cc}$. of water, add a few drops of hæmatoxylin solution, and then titrate with $\frac{\mathrm{N}}{\mathrm{IO}} \mathrm{HCl}$. Cochineal or rosolic acid may also be employed as indicators. The decinormal factor for succirubra alkaloid is $0.0304 \mathrm{gm}$.

The German Pharmacopoia employs the same method for the extracts of cinchona as for extract of belladonna with the difference that $\mathrm{N}$ $\frac{\mathrm{N}}{10}$ solutions are used, that the flask is rinsed with a mixture of chloroform (one part) and ether (three parts), instead of with ether alone, and that hæmatoxylin is used as indicator as follows: Of the roo cc. of acid solution, take $50 \mathrm{cc}$, add a freshly prepared solution of one granule of hæmatoxylin in I cc. of alcohol, and then titrate the mixture with standard $\mathrm{KOH}$ until the yellowish color upon shaking turns to bluish-violet.

Fluid Extract of Coca may be assayed by the method described for fluid extract of belladonna, U. S. P. VIII, except that ether is used as the solvent instead of chloroform. Benzin is also a very good solvent for the alkaloids of coca. See Assay of Coca Leaves.

Assay of Fluid Extract of Gelsemium. Method of Sayre and Havenhill.* The process is as follows: Take ${ }_{5}$ cc. of the fluid extract and evaporate at $60^{\circ} \mathrm{C}$. to a soft extract, or sufficiently to expel the alcohol. Add $5 \mathrm{cc}$. of normal sulphuric acid, which has been previously diluted with an equal volume of water, and allow the resulting mass to disintegrate. When thoroughly disintegrated transfer to a 15 -cc. graduated cylinder and complete the dilution to $15 \mathrm{cc}$. Mix thoroughly, and allow the precipitate to settle (the addition of a little purified talcum will sometimes be necessary), filter or decant off ro cc. into a separatory funnel and

* L. E. Sayre, Proc.A. Ph. A., 1907, 357. 
wash the acid solution with chloroform, using three portions (IO, 5, $5 \mathrm{cc}$.), wash the united chloroformic washings with about $5 \mathrm{cc}$. of slightly acidulated water. Unite the acid solutions; make the mixture alkaline with ammonium hydroxid, and shake out with chloroform, using three portions ( $15, \mathrm{ro}$, and $5 \mathrm{cc}$.). A fourth portion of ro cc. may sometimes be necessary to extract all the alkaloid before it ceases to give alkaloidal reaction with Mayer's reagent. Evaporate the chloroformic solution to constant weight, and weigh as crude chloroform-soluble gelsemium alkaloids.

A very satisfactory volumetric method is that described by $\mathrm{M}$. $\mathrm{H}$. Webster (A. J. Ph., 1907, 301-307). To 10 cc. of the fluid extract add I.50 gms. of tartaric acid; filter off the precipitate, washing it with absolute alcohol. Add sufficient absolute alcohol to make roo cc. Then proceed as directed under Webster's method (page 59I).

\section{ASSAYING OF PREPARATIONS OF HYDRASTIS}

Assay of Fluid Extract of Hydrastis. In the U.S. P. method, which is based upon that of W. A. Puckner,* the separation of the insoluble berberine hydroiodid from the hydrastine is effected by the employment of potassium iodid, and the hydrastine then estimated. Puckner's procedure differs, however, in avoiding the use of aliquot portions, and a more complete extraction of the alkaloid is aimed at. The method is as follows: Into a beaker containing water $25 \mathrm{cc}$. and potassium iodid T. S. $5 \mathrm{cc}$., fluid extract $5 \mathrm{cc}$, is measured. The mixture is stirred well and filtered through a $7 \mathrm{~cm}$. filter into a separator. The precipitate on the filter is washed with two portions, $5 \mathrm{cc}$. each, of a mixture consisting of water ig cc. and potassium iodid T.S. I cc. When the precipitate has drained, it is transferred with its filter back to the beaker and mixed with $5 \mathrm{cc}$. of the wash fluid above used. This is poured on a new filter, the filtrate passed into the separator and the beaker and precipitate washed with the remaining $5 \mathrm{cc}$. of the wash fluid. The liquid in the separator is made alkaline with ammonia-water, usually o.I cc. or three drops being sufficient, and extracted with three portions of ether, $20 \mathrm{cc}$. each; the aqueous solution being passed successively through three separators, each containing $20 \mathrm{cc}$. of ether. The ether is passed successively through a small pledget of cotton into a beaker, and finally the neck of the separator and stem of the funnel is washed with $5 \mathrm{cc}$. of ether. The ether is allowed to evaporate in a warm place, and the residue dried at $95^{\circ}$ to $98^{\circ}$ as long as further loss is noted.

* Pharm. Rev., XXVI, I33. 
Tincture of Hydrastis is assayed by evaporating roo cc. on a water-bath until it measures Io cc., adding a sufficient quantity of alcohol to redissolve any matter that may have been separated, and then assaying the resulting liquid as directed for fluid extract.

Heyl's Modification of Linde's Method.* 7.5 gms. of fluid extract are concentrated to a thick extract in an Erlenmeyer flask. The residue is dissolved in Io cc. water, and then Io gms. petroleum benzin and 50 gms. ether added, the flask stoppered, rotated and then 2.5 gms. Io per cent ammonia-water added. The mixture is shaken frequently during one hour and then transferred to a separator having a capacity of $250 \mathrm{cc}$. After separation has occurred, the aqueous fluid is drawn off. Benzin-ether solution is passed through a pledget of fat-free cotton into a dry Erlenmeyer flask and the flask stoppered. $50 \mathrm{gms}$. of this solution is weighed by difference into a separator and extracted with Io cc. of a mixture of one part hydrochloric acid and four parts. water, with two further portions of water $5 \mathrm{cc}$. each containing a few drops of dilute hydrochloric acid, and finally with $5 \mathrm{cc}$. water. To the acid extractions is added $50 \mathrm{gms}$. ether, 2.5 gms. ammonia-water, and the mixture shaken thoroughly and frequently. After one hour the fluid is transferred to a separator, the watery solution drawn off, and the ether filtered through a small plaited filter, the funnel being kept covered. 50 gms. of the filtrate are transferred into a tared vessel, the ether allowed to evaporate spontaneously and the residue dried at $105^{\circ}$. As a precaution, Heyl carried out all these operations as quickly as possible, although he has never observed the crystallization of hydrastine from ether solution reported by other investigators. This method is practically the same as that of the German Pharmacopoia.

The Rusting-Smeet's Method. $\dagger$ Io gms. of extract are mixed in a capacious tared vessel with $20 \mathrm{cc}$. water and the contents reduced by evaporation to from ro to II gms. Then I.5 cc. of 12.5 per cent hydrochloric acid are added and after cooling sufficient water to make 20 gms. Now $0.5 \mathrm{gm}$. of infusorial earth is added, the mixture well shaken, filtered and ro gms. transferred to a Ioo-cc. vial. To this $4 \mathrm{cc}$. of Io per cent ammonia-water and $25 \mathrm{cc}$. of ether are added, and after thorough shaking for a few minutes $25 \mathrm{cc}$. of petroleum ether, boiling at $50^{\circ}$ to $75^{\circ} \mathrm{C}$. After again agitating, $1.5 \mathrm{gm}$. of powdered tragacanth are added, the mixture shaken vigorously, $40 \mathrm{cc}$. of the clear liquid transferred to a tared flask, and the contents reduced to Io-II gms. The flask is stoppered and kept in a cool place for several hours. Then the liquid is carefully poured off, the crystals washed with a little petroleum ether, dried on a water-bath and weighed.

* Apoth. Ztg., I906 (XXI), 797.

$\dagger$ A. W. von der Haar, Apoth. Ztg., XXI, I05० (I906). 
ASSAYING OF PREPARATIONS OF IPECAC

Assay of Fluid Extract of Ipecac. The fluid extract and other galenical preparations of ipecac may be readily assayed by the Keller method, or, in fact, by any of the usual methods.

The following method may be used: $8 \mathrm{gms}$. of the fluid extract are diluted with $8 \mathrm{gms}$. of water in an ordinary vial, $32 \mathrm{gms}$. of chloroform and $48 \mathrm{gms}$. of ether are added and shaken up; $4 \mathrm{gms}$. of ammonia-water are now introduced and the mixture agitated frequently during half an hour. $50 \mathrm{gms}$. of the chloroform-ether solution (representing $5 \mathrm{gms}$. of the fluid extract) are separated, poured into a tared flask, and the solvent distilled or evaporated off; the varnish-like residue is twice treated with 5 to $10 \mathrm{cc}$. of ether and evaporated by forcing a current of air into the flask by means of a rubber bulb; the residue is then dried on a water-bath and weighed. For the titration the residue may be dissolved in a known quantity of decinormal hydrochloric acid; the solution may be assisted by gentle heat or the addition of a small quantity of alcohol; ten or twelve drops of Brazil-wood T.S. are then added and the excess of acid detcrmined by means of decinormal alkali, the latter being added until liquid is cardinal to purplish red in color. The quantity of alkali used is then subtracted from the quantity of decinormal acid first added. This gives the quantity of decinormal acid which was used to neutralize the alkaloids present. The decinormal factor, $0.025^{2} \mathrm{gm}$., is based upon the molecular weight of emetine (Kunz) (see Assay of Ipecac Root). This factor, multiplied by the number of cc. of $\frac{\mathrm{N}}{10}$ acid used gives the weight of emetine in 5 gms. of the fluid extract, and when this is multiplied by 20 the percentage is obtained.

The decinormal factor for "total " alkaloid is 0.0238 .

Method of U. S. P. VIII. Transfer ro cc. of fluid extract of ipecac by means of a graduated pipette to a porcelain evaporatingdish. Evaporate off the alcohol with the aid of a water-bath, and, when almost cool, add $5 \mathrm{cc}$. of normal sulphuric acid and stir the liquid at intervals for three minutes. Filter the liquid into a separator, rinse the dish, and wash the filter successively with ro cc. and $5 \mathrm{cc}$. of distilled water, and add these liquids to the separator. To the separator add $20 \mathrm{cc}$. of ether and a small piece of red litmus paper; render the liquid alkaline with ammonia-water and shake the separator for one minute. Draw off the aqueous layer into a beaker, and the ether layer into another beaker. Return the aqueous solution to the separator, add Io cc. more of ether, and shake the liquid, adding the ether solution to that already in the beaker, and returning 
the aqueous solution to the separator; repeat the extraction with Io cc. more of ether, and then add the ether layer to that already in the beaker. Allow the combined ether solutions to evaporate, either spontaneously or with the aid of a water-bath containing warm water, and then add Io cc. of tenth-normal sulphuric acid Stir the liquid carefully with a glass rod to facilitate the solution of the alkaloids, and when these have all dissolved, add five drops of hæmatoxylin T. S. From a graduated burette add sufficient fiftieth-normal potassium hydroxid to just cause the yellow color of the solution to turn purple. Divide the number of cc. of fiftieth-normal potassium V. S. used by 5, subtract the quotient from ro (the ro cc. of tenthnormal sulphuric acid taken), and multiply the remainder by 0.0238 , and this product by 1o, which will give the weight in grams of alkaloids contained in each one hundred cubic centimeters of fluid extract of ipecac.

See also

T. C. Bird. Ph. Jour., I900, Feb. 24, March 3I, and April $2 \mathrm{I}$.

Naylor and Chappel. Ph. Jour., I907 (IV), 393, 395.

\section{ASSAY OF NUX VOMICA PREPARATIONS}

Extract of Nux Vomica (Method of U. S. P., 1890). Extract of nux vomica dried at $100^{\circ} \mathrm{C}$., 2 gms.; alcohol; ammonia-water. sp.gr. 0.960; water, chloroform, decinormal sulphuric acid, centinormal potassium hydroxid, of each sufficient.

Put 2 gms. of the dried extract of nux vomica into a glass separator; add to it $20 \mathrm{cc}$. of a previously prepared mixture of 2 volumes of alcohol, I volume of ammonia-water, and I volume of water; shake the separator until the extract is dissolved. Then add $20 \mathrm{cc}$. of chloroform and agitate during five minutes. The chloroform dissolves the alkaloids which the ammonia liberated. Allow the chloroformic solution to separate, remove it as far as possible, pour a few cc. more of chloroform into the separator, and without shaking draw this off through the stop-cock to wash the outlet-tube. Repeat the extraction with two further portions of chloroform of $15 \mathrm{cc}$. each, washing the outlet-tube each time as just directed.

Collect all the chloroformic solutions in a wide beaker; expose the latter to a gentle heat on a water-bath until the chloroform and ammonia are completely dissipated. Add to the residue ro cc. of decinormal sulphuric acid measured accurately from a burette, stir gently, and then add $20 \mathrm{cc}$. of hot water. When solution has taken place add 2 cc. of Brazil-wood T. S. (The sulphuric acid combines with the alkaloids and forms sulphates of the alkaloids.) 
Now carefully run into this solution, centinormal potassium hydroxid until a permanent pinkish color is produced, showing a slight excess of the alkali. Divide the number of cc. of centinormal potassium hydroxid used by ro. Subtract the number found from Io (the ro cc. of $\frac{\mathrm{N}}{\text { IO }}$ acid first used), and the number of cc. of the $\frac{\mathrm{N}}{\mathrm{IO}}$ acid which went into combination with the alkaloids is found.

The two principal alkaloids of nux vomica are strychnine and brucine, and it is assumed that they are present in equa proportions; and thus the factor for total alkaloids is found by taking the mean of their respective molecular weight's:

$\begin{array}{llr}\text { Strychnine, } & 331.73 & \text { 2) } 723.04 \\ \text { Brucine } & \frac{391.31}{361.5^{2}}\end{array}$

361.52 gms. of the total alkaloids of nux vomica will neutralize I000 cc. of normal sulphuric acid. $36.15^{2}$ gms. will neutralize 1000 cc. of decinormal sulphuric acid.

Hence each cc. of decinormal sulphuric acid used in the above assay represents $0.03615^{2} \mathrm{gms}$. of an equal mixture of strychnine and brucine. And by multiplying the number of cc. used by this factor, the quantity of these alkaloids in the 2 gms. of extract taken is obtained, and this quantity multiplied by 50 will give the percentage.

The extract should contain 15 per cent of total alkaloids by the above assay.

Fhid Extract of Nux Vomica is evaporated to a solid extract and then assayed by the above process.

Tincture of Nux Vomica is assayed by evaporating $100 \mathrm{cc}$. to dryness, and the residue then tested by the above process. It should contain $0.3 \mathrm{gm}$. of alkaloids.

The German pharmacopoial method is identical with that described for extract of belladonna. Iodeosin is used as indicator and the total alkaloids determined.

Method of U. S. P. VIII. Introduce 2 gms. of the extract of nux vomica into a beaker, and dissolve it in $25 \mathrm{cc}$. of a mixture of $\mathrm{I} 6 \mathrm{cc}$. of ether, $5 \mathrm{cc}$. of chloroform, and $4 \mathrm{cc}$. of ammonia-water. When dissolved, transfer it to a separator, rinsing the beaker with a little chloroform, and adding the rinsings to the separator. Insert the stopper securely and shake the separator carefully for a few minutes. Draw off the aqueous layer into another separator, washing the ether-solution and separator with a little water, and adding this to the second sep- 
arator. Then shake out the aqueous liquid with two portions of $\mathrm{I}_{5}$ and Io cc., respectively, of chloroform, and add these to the first separator. If a few drops of the liquid left in the second separator still give a reaction with mercuric potassium iodid T. S. after acidulating, repeat the shaking-out with ro cc. more of chloroform. Now shake out the chloroformic solution in the first separator with three portions of 15, ro, and Io cc. of sulphuric acid solution (3 per cent), and collect the combined acid solutions in another separator. Introduce a small piece of red litmus paper, add enough ammonia-water to render the liquid alkaline, and extract the mixture with three portions, respectively, of I5, 10, and Io cc. of chloroform. Draw off the chloroformic solutions into a beaker, and evaporate the chloroform with the aid of a water-bath. Dissolve the alkaloidal residue in the beaker in $15 \mathrm{cc}$. of 3 per cent sulphuric acid solution by the aid of a water-bath, and allow the liquid to cool. To this solution add 3 cc. of a cooled mixture of equal volumes of nitric acid (sp.gr. I.40) and distilled water, and after rotating the liquid a few times, set it aside for exactly ten minutes, stirring it gently three times during this interval. Transfer the resulting red liquid to a separator containing 25 cc. of an aqueous solution of sodium hydroxid (I: IO), and wash the beaker three times with very small amounts of distilled water, and add the washings to the separator. If the liquid is not quite turbid, add 2 cc. more of the solution of sodium hydroxid. Now add $20 \mathrm{cc}$. of chloroform to the separator, and shake it well by a rotating motion for a few minutes, allow the liquids to separate, and draw off the chloroform through a small filter, wetted with chloroform, into a flask. Repeat this twice, using ro cc. of chloroform each time, and draw off both portions into the flask, using the same filter. Finally, wash the filter and funnel with $5 \mathrm{cc}$. of chloroform, and then evaporate all the chloroform by means of a waterbath, very carefully, to avoid decrepitation. To the alkaloidal residue, add ro cc. of tenth-normal sulphuric acid, 5 drops of iodeosin T. S., about $90 \mathrm{cc}$. of distilled water, and $20 \mathrm{cc}$. of ether. When all the alkaloid is dissolved, titrate the excess of acid with fiftiethnormal potassium hydroxid until the aqueous liquid just turns pink. Divide the number of cc. of fiftieth-normal potassium hydroxid used by 5 , subtract this number from ro (the Io cc. of tenth-normal sulphuric acid taken), multiply the remainder by 0.0332 , and this product by 50 , which will give the percentage of strychnine contained in the extract of nux vomica.

The fluid extract is assayed by evaporating ro cc. on a water-bath to dryness, and then treating as above described.

The tincture is assayed by evaporating roo cc. on a water-bath, and treating the resulting extract as directed above. 
See also

W. H. Lenton. Ph. Jour., 1905, 864.

Naylor and Chappel. Ph. Jour., 1907 (March), 393-395.

F. C. Bird. Ph. Jour., I900 (Sept.), 286.

H. Matthes and O. Ramstedt. Archiv. d. Ph., I907 (No. 2), II 2-132.

\section{ASSAY OF PREPARATIONS OF OPIUM}

Extract of Opium (Method of U. S. P., 1890). Extract of opium dried at $100^{\circ} \mathrm{C}$., 4 gms.; ammonia-water, $2.2 \mathrm{cc}$; alcohol, ether, water, of each a sufficient quantity.

Dissolve the extract of opium in $30 \mathrm{cc}$. of water, filter the solution through a small filter and wash the filter and residue with water until all soluble matters are extracted, collecting the washings separately. Evaporate in a tared porcelain capsule first the washings to a small volume, then add the first filtrate, and evaporate the whole to a weight of ro gms. Rotate the concentrated solution about in the capsule until the rings of extract are redissolved. Pour the liquid into a tared flask and rinse the capsule with a few drops of water at a time until the entire solution weighs 15 gms.

Then add $8.5 \mathrm{cc}$. of alcohol, shake well, add $20 \mathrm{cc}$. of ether, and shake again.

Now add the ammonia-water, stopper the flask with a sound cork, shake it thoroughly during ten minutes, and set it aside in a moderately cool place for at least six hours, or over night.

At the expiration of this time remove the stopper carefully and brush into the flask any crystals which may adhere to the cork. Place two rapidly acting, plainly folded filters, one within the other, in a small funnel, wet them well with ether and decant upon the inner one, the ethereal solution, as completely as possible.

Add Io cc. of ether to the contents of the flask, rotate, and again decant upon the filter; repeat this operation with another Io cc. of ether. Then pour the liquid in the bottle upon the filter in small portions at a time, in such a way as to transfer the greater portion of the crystals to the filter. When the liquid has passed through transfer the remaining crystals to the filter by rinsing the flask with several small portions of water, using not more than Io cc. in all.

Apply water to the crystals drop, by drop, until they are practically free from mother-liquor, and afterwards wash them with a saturated alcoholic solution of morphine, added drop by drop. When this has all passed through displace the remaining alcohol by ether, using about io cc. or more if necessary.

Dry to a constant weight at a temperature not exceeding $60^{\circ} \mathrm{C}$., and carefully transfer the crystals to a tared watch-glass and weigh 
them. The weight, multiplied by 25 , gives the percentage of crystallized morphine present in the extract.

Instead of drying and transferring the crystals to a watch-glass, as above directed, the filter containing them may be immersed in some boiling water in a beaker, and an excess of decinormal sulphuric acid added to dissolve the crystals (the quantity being noted); a few drops of methyl-orange are then added and the mixture titrated with decinormal potassium hydroxid. Deduct the quantity of the latter used from the quantity of decinormal acid first added, and the quantity of decinormal acid which combined with the morphine is found.

Iooo cc. of normal acid represents one molecular weight of the alkaloid.

- roo० cc. of decinormal acid represents one-tenth of a molecular weight of the alkaloid ( $30.092 \mathrm{gms}$.$) ; thus each cc. of \frac{\mathrm{N}}{\text { IO }}$ acid repre-
sents $0.0309^{2} \mathrm{gm}$. of crystallized morphine.

The number of cc. used, multiplied by this factor, gives the quantity of morphine present in the 4 gms. of extract taken.

This multiplied by 25 gives the per cent of crystallized morphine; it should contain 18 per cent.

Tincture of Opium (Laudanum) (Method of U. S. P., I89o). Tincture of opium roo cc., ammonia-water $3.5 \mathrm{cc}$., alcohol, ether, water, each a sufficient quantity. Evaporate the tincture to about $20 \mathrm{cc}$., add $40 \mathrm{cc}$. of water, mix thoroughly, and set the liquid aside for an hour, stirring occasionally and disintegrating the resinous flakes adhering to the capsule; then filter and wash the filter and residue with water, collecting the washings separately. Evaporate first the washings to a small volume, then add the first filtrate and evaporate to I4 gms. Pour the liquid into a tared flask, rinse the capsule, and add the rinsings until the entire solution weighs 20 gms. Then add I2.2 cc. of alcohol; shake well; add $25 \mathrm{cc}$. of ether; shake again. Now add the ammonia-water, cork well, shake for ten minutes, and set aside for at least six hours or overnight, so that the crystals may form.

At the expiration of this time decant the ethereal layer upon a double, plain, rapidly acting filter previously wet with ether; add Io cc. of ether to the contents of the flask, rotate, and again decant. Repeat this operation with another Io cc. of ether. Then pour the liquid in the bottle upon the filter, in small portions at a time, so as to transfer the greater portion of the crystals to the filter, and wash the remaining crystals on to the filter with the aid of a small quantity of water, using not more than ro cc. Then wash the crystals, first with a few drops of water, then with an alcoholic solution of morphine, and finally with ether to displace the alcohol. Dry the crystals to a constant weight and weigh on a tared watch-glass. 
If roo gms. of tincture have been operated upon, the weight of the crystals is at once the per cent of crystallized morphine. The yield should be 1.3 to 1.5 gms. of morphine from $100 \mathrm{cc}$. of tincture. After weighing, the crystals may be titrated as directed under Assay of Opium.

The gravimetric assay method of the U. S. P. VIII is an improvement upon the foregoing, and it or any of the methods described under Assay of Opium, may be used. The German pharmacopocial method is a very satisfactory one.

\section{LITERATURE-}

Mallinkrodt and Dunlap. J. A. C. S., 1905, 946.

T. E. Wetterstroem. Proc. A. Ph. A., 1906, 43I.

G. Bergstrom. Ph. Centralh., xLviI, I906, 632 .

G. Fromme. Report of Cæsar and Loretz, I906, 48.

Vanderkleed. Apoth., II, 534 .

Assay of Extract of Physostigma (U. S. P. VIII). Transfer I gm. of the extract of physostigma to a small porcelain dish, add $5 \mathrm{cc}$. of diluted alcohol, and digest for five minutes in a water-bath below boiling temperature; then add about 5 gms. of very clean, fine quartz sand, and evaporate to dryness on a water-bath, triturating thoroughly with a pestle to secure uniform admixture. When dry, carefully transfer the contents of the dish to an Erlenmeyer flask, add roo cc. of ether, and shake the flask. Then add ro cc. of an aqueous solution of sodium bicarbonate $(\mathrm{I}: 20)$, and shake the contents vigorously at intervals for one hour. Allow the mixture to stand, and, when settled, decant $50 \mathrm{cc}$. of the ether solution into $\mathrm{a}_{\mathrm{o}}$ separator, to which add a small piece of blue litmus paper, sufficient normal sulphuric acid to render the liquid acid, and ro $\mathrm{cc}$. of distilled water. Shake the separator well for one minute, and draw off the aqueous layer into another separator. Repeat the shaking-out process, using $2 \mathrm{cc}$. of normal sulphuric acid and $8 \mathrm{cc}$. of distilled water, and add the acid aqueous layer to the second separator; again repeat the extraction, using $\mathrm{I}$ cc. of normal sulphuric acid and $9^{\prime} \mathrm{cc}$. of distilled water, and add this to the second separator. To the combined acid liquids in the second separator, add $25 \mathrm{cc}$. of ether, a small piece of red litmus paper, and sufficient sodium bicarbonate solution $(\mathrm{r}: 20)$ to render it alkaline. Shake the separator for one minute, allow the liquids to separate, and draw off the ether into a beaker. Repeat the shaking-out process with $20 \mathrm{cc}$. and again with $15 \mathrm{cc}$. of ether added to the separator; shake each time for one minute, allow the liquids to separate, and draw of i the ether into the 
beaker. Carefully evaporate the ether from the combined solutions by means of a water-bath, and, when dry, dissolve the residue in $2 \mathrm{cc}$. of tenth-normal sulphuric acid; rinse the solution carefully into a 200-cc. flask with distilled water, add enough distilled water to bring the volume to about $90 \mathrm{cc}$., add $25 \mathrm{cc}$. of ether, and having shaken the flask, add five drops of iodeosin T. S., then titrate the excess of acid with fiftieth-normal potassium hydroxid, until, after shaking, the aqueous liquid just acquires a pink color. Divide the number of cc. of fiftieth-normal potassium hydroxid used by 5 , subtract the quotient from 2 (the 2 cc. of tenth-normal sulphuric acid taken), and multiply the remainder by 0.0273 , and this product by 200 ; the result will be the percentage of ether soluble alkaloids contained in the extract of physostigma.

Assay of Fluid Extract of Pilocarpus (U.S. P. VIII). Transfer Io cc. of fluid extract of pilocarpus by means of a graduated pipette to a porcelain dish containing a little clean sand, and evaporate it to dryness with the aid of a water-bath. Mix the sand uniformly with the extract and transfer the mixture to an Erlenmeyer flask of about Ioo cc. capacity, rinsing the dish with a mixture of $25 \mathrm{cc}$. of chloroform and $2.5 \mathrm{cc}$. of ammonia-water. Transfer the rinsings to the flask, cork it securely, and shake it well at intervals during one hour. Decant the liquid, transfer to a separator, wash the sand with several portions of chloroform, draw off and filter the chloroformic liquid into another separator. Then shake out the chloroform solution with I 5 cc. of normal sulphuric acid transferring the acid aqueous solution to another separator. Repeat the shaking-out with a mixture of $5 \mathrm{cc}$. of normal sulphuric acid and $5 \mathrm{cc}$. of distilled water, collecting the acid solution in the second separator. Again repeat the shaking-out with ro cc. of distilled water, and add the aqueous liquid to the second separator. Introduce into the second separator a small piece of red litmus paper, add enough ammonia-water to render the liquid alkaline, and shake out the liquid with $20 \mathrm{cc}$. of chloroform, drawing off the chloroformic solution into a beaker. Repeat the shaking-out with two portions of 15 and Io cc. each of chloroform, and add the chloroformic solutions to the beaker. Evaporate the chloroform by means of a water-bath, and dissolve the alkaloidal residue in 5 cc. of tenth-normal sulphuric acid. Add five drops of cochineal T. S. or iodeosin T. S., and titrate the excess of acid with fiftiethnormal potassium hydroxid. Divide the number of cc. of fiftiethnormal potassium hydroxid used by 5 , subtract the quotient from 8 (the $8 \mathrm{cc}$. of tenth-normal sulphuric acid taken), and multiply the remainder by 0.02 , and this product by Io, to obtain the weight in grams of alkaloids contained in Ioo cc. of the fluid extract of pilocarpus. 
Estimation of the Alkaloidal Strength of Scale Salts. 4 gms. of the scales are dissolved in $30 \mathrm{cc}$. of water in a capsule with the aid of gentle heat. The solution is cooled and transferred to a glass separator; an aqueous solution of $0.5 \mathrm{gm}$. of tartaric acid is then added, followed by an excess of solution of sodium hydroxid. The tartaric acid prevents the precipitation of $\mathrm{Fe}_{2}(\mathrm{OH})_{6}$, and the $\mathrm{NaOH}$ sets free the alkaloid. 'The alkaloid is then extracted by shaking up the mixture with successive portions of chloroform, I5 cc. each time. The chloroformic layers are separated each time and mixed and evaporated in a tared capsule on a water-bath, and the residue dried at $100^{\circ} \mathrm{C}$. $\left(212^{\circ} \mathrm{F}\right.$.), and weighed. Or the residue may be titrated by adding sufficient decinormal sulphuric or hydrochloric acid to dissolve the salts and still remain in excess, then titrating residually with decinormal $\mathrm{NaOH}$ or $\mathrm{KOH}$ to determine the excess of acid. 


\section{CHAPTER LIII}

\section{ASSAY OF PHENOL (CARBOLIC ACID)}

Preparation of Decinormal Bromin (Koppeschaar's Solution). 7.936 gms. in a liter.

$$
\begin{array}{ll}
\mathrm{KBr}=\mathrm{II} 8.22 & \mathrm{NaBr}=\mathrm{I} 02.24 \\
\mathrm{KBrO}_{3}=\mathrm{I} 65.86 & \mathrm{NaBrO}_{3}=\mathrm{I} 49.88
\end{array}
$$

This solution does not contain free bromin, but it contains two salts, a bromid and a bromate, which, when treated with hydrochloric acid, liberate a definite quantity of bromin.

It is made as follows: Dissolve $3 \mathrm{gms}$. of sodium bromate and 50 gms. of sodium bromid (or 3.2 gms. of potassium bromate and 50 gms. of potassium bromid) in sufficient water to makc $900 \mathrm{cc}$.

Transfer $20 \mathrm{cc}$. of this solution by means of a pipette into a bottle having a capacity of about $250 \mathrm{cc}$., provided with a glass stopper; add $75 \mathrm{cc}$. of water, then $5 \mathrm{cc}$. of pure hydrochloric acid, and immediately insert the stopper.

Shake the bottle a few times, then remove the stopper just sufficiently to quickly introduce $5 \mathrm{cc}$. of potassium iodid T. S., taking care that no bromin vapor escape, and immediately stopper the bottle.

Agitate the bottle thoroughly, remove the stopper, and rinse it and the neck of the bottle with a little water so that the washings flow into the bottle, then add from a burette decinormal sodium thiosulphate until the color of the free iodin is nearly all discharged, then add a few drops of starch T. S., and continue the titration with $\frac{\mathrm{N}}{\mathrm{IO}}$ thiosulphate until the blue color disappears.

Note the number of cc. of the $\frac{\mathrm{N}}{\mathrm{IO}}$ sodium thiosulphate thus used, and dilute the bromin solution so that equal volumes of it and the $\frac{\mathrm{N}}{\mathrm{IO}}$ sodium thiosulphate will exactly correspond to each other under the above-mentioned conditions. 
Example. Assuming that the $20 \mathrm{cc}$. of bromin solution required 25.2 cc. of the $\frac{\mathrm{N}}{\mathrm{IO}}$ thiosulphate to completely absorb the iodin, the bromin solution must be diluted in the proportion of 20 to 25.2; that is, each $20 \mathrm{cc}$. must be diluted to make $25.2 \mathrm{cc}$.

Thus if $85^{\circ} \mathrm{cc}$. are left, they must be diluted to make 1071 cc.; and the solution is decinormal.

A new trial should always be made after diluting, and the bromin solution should correspond, volume for volume, with the decinormal sodium thiosulphate.

The first step in the preparation of this solution is to dissolve the salts; then hydrochloric acid is added, which liberates a definite quantity of bromin, as the equation illustrates:

$$
5 \mathrm{NaBr}+\mathrm{NaBrO}_{3}+6 \mathrm{HCl}=6 \mathrm{NaCl}+{ }_{3} \mathrm{Br}_{2}+{ }_{3} \mathrm{H}_{2} \mathrm{O} .
$$

The stopper should be inserted into the bottle as soon as the hydrochloric acid has been added, in order that no bromin vapor escape, and the bottle rotated so as to mix the acid thoroughly with the liquid.

The next step is to determine the quantity of bromin which a definite volume of solution will liberate. The bromin solution should be of such strength that $1000 \mathrm{cc}$. of it will contain $7.936 \mathrm{gms}$. of available bromin. Bromin, like chlorin, liberates iodin from potassium iodid, and is estimated in the same manner.

One atomic weight of iodin is liberated by one atomic weight of bromin:

$$
\mathrm{Br}_{2}+2 \mathrm{KI}=2 \mathrm{KBr}+\mathrm{I}_{2}
$$

Thus by determining the quantity of iodin liberated the quantity of bromin is found.

The iodin is determined by the $\frac{\mathrm{N}}{\mathrm{IO}}$ sodium thiosulphate, one liter of which represents I 2.59 gms. of iodin, which is equivalent to 7.936 gms. of bromin, as is shown by the following equation:

$$
\begin{aligned}
& \left(\mathrm{Br}_{2}\right)=\mathrm{I}_{2}+2\left(\mathrm{Na}_{2} \mathrm{~S}_{2} \mathrm{O}_{3}+{ }_{5} \mathrm{H}_{2} \mathrm{O}\right)=2 \mathrm{NaI}+\mathrm{Na}_{2} \mathrm{~S}_{4} \mathrm{O}_{6}+10 \mathrm{H}_{2} \mathrm{O} \text {. } \\
& \text { 20) } \frac{58.72}{7.936} \text { gms. } \frac{20) 251.8}{12.59} \text { gms. } \frac{20)}{24.646} \text { gms. or } 1000 \text { cc. } \frac{N}{10} \text { V.S. }
\end{aligned}
$$

The Assay of Phenol. $1.556 \mathrm{gms}$. of the carbolic acid to be assayed is dissolved in sufficient water to make $1000 \mathrm{cc} .25 \mathrm{cc}$. of 
this solution, containing $0.0389 \mathrm{gm}$. of the acid, are transferred to a glass-stoppered bottle having a capacity of about $200 \mathrm{cc}$.

To this, $30 \mathrm{cc}$. of decinormal bromin, followed by $5 \mathrm{cc}$. of hydrochloric acid, are added, and the bottle immediately stoppered, and shaken repeatedly during half an hour.

Then the stopper is removed just sufficiently to introduce $5 \mathrm{cc}$. of a 20 per cent aqueous solution of potassium iodid, being careful that no bromin escape.

The bottle is then thoroughly shaken and the neck rinsed with a little water, the washings being allowed to flow into the bottle.

The solution is now ready for titration, and the decinormal sodium thiosulphate is delivered in from a burette, until the iodin is almost completely absorbed; then add a few drops of starch T. S., and continue the titration until the blue color is just discharged.

In the U. S. P. VIII it is directed to use $\mathrm{I}$ cc. of chloroform instead of starch. The precipitated tribromphenol interferes somewhat with the end-reaction when starch is used, and frequently with old phenol solutions * the precipitate possesses a bluish color which is not removed by an excess of sodium thiosulphate and which makes the end-reaction difficult. This difficulty is overcome by the use of a small quantity of chloroform which dissolves the tribromphenol and admits of a very sharp end-reaction.

When chloroform is used alone, the end-reaction is very clearly defined, and is known by a colorless aqueous solition and the chloroform being free from any tinge of pink, due to traces of iodin.

Note the number of cc. of $\frac{\mathrm{N}}{\mathrm{IO}}$ thiosulphate used; deduct this number from $30 \mathrm{cc}$. (the quantity of $\frac{\mathrm{N}}{\mathrm{IO}}$ bromin originally added), and the quantity of $\frac{\mathrm{N}}{\mathrm{IO}}$ bromin which went into combination with the phenol is obtained.

Each cc. of $\frac{\mathrm{N}}{10}$ bromin represents $0.001556 \mathrm{gm}$. of pure phenol.

Example. Assuming that $6 \mathrm{cc}$. of $\frac{\mathrm{N}}{\mathrm{IO}}$ sodium thiosulphate were required to discharge the color of the starch iodid, this deducted from $30 \mathrm{cc}$. leaves $24 \mathrm{cc}$., the quantity which combined with the phenol.

$$
\begin{aligned}
& 0.0015563 \times 24=0.037344 \mathrm{gm} . \\
& \frac{0.037344 \times 100}{0.039}=95.75 \text { of pure }
\end{aligned}
$$


The above method originated with Koppeschaar, and is the only volumetric method by which accurate results may be obtained.

It is based upon the fact that bromin reacts with phenol, producing an insoluble precipitate of tribromphenol.

The titration is not made directly; but the phenol solution is treated with an excess of standard bromin solution in the presence of some hydrochloric acid. The hydrochloric acid liberates the bromin, and the freed bromin then reacts with the phenol, as shown by the equations:

$$
\begin{aligned}
& 5 \mathrm{NaBr}+\mathrm{NaBrO}_{3}+6 \mathrm{HCl}=6 \mathrm{NaCl}+{ }_{3} \mathrm{H}_{2} \mathrm{O}+{ }_{3} \mathrm{Br}_{2} ; \\
& \mathrm{C}_{6} \mathrm{H}_{5} \mathrm{OH}+{ }_{3} \mathrm{Br}_{2}=\mathrm{C}_{6} \mathrm{H}_{2} \mathrm{Br}_{3} \mathrm{OH}+{ }_{3} \mathrm{HBr} \text {. } \\
& \text { 6) } \underline{93.34} \text { 6) } 476.16 \\
& \text { 10) } \lcm { 5 . 5 ^ { 6 } } \quad \longdiv { 1 0 ) 7 9 . 3 ^ { 6 } } \\
& \text { I. } 55^{6} \text { gms. } \frac{79 \cdot 3^{6}}{7 \cdot 93^{6}} \text { gms. or } 1000 \text { cc. } \frac{\mathrm{N}}{10} \text { bromin V.S. }
\end{aligned}
$$

Thus each cc. of the $\frac{\mathrm{N}}{\mathrm{IO}}$ bromin represents $0.001556 \mathrm{gm}$. of pure phenol.

The bromin solution which was added in excess, and the liberated bromin of which is not fixed by phenol, is then found by residual titration with $\frac{\mathrm{N}}{\mathrm{IO}}$ sodium thiosulphate after the addition of some potassium iodid.

The decinormal bromin solution and the decinormal sodium thiosulphate solution being equivalent, each cc. of the latter consumed represents one cc. of the former. Then by subtracting the number of cc. of the sodium thiosulphate solution used from the number of cc. of bromin solution originally added, the quantity of the latter which was actually consumed by the phenol present is found. This number, when multiplied by the factor for phenol, then gives the quantity of pure phenol present.

The hydrochloric acid used in the above estimation must contain no free chlorin. The potassium iodid must be free from iodate. The starch T. S. should not be added until most of the free iodin has been taken up, and the color of the solution has diminished to light yellow.

The carbolic acid should be diluted with water before titration, and should never be stronger than $0.1 \mathrm{gm}$. in $25 \mathrm{cc}$.

Mr. H. Beckurts reports that the precipitate obtained from phenol and bromin is not pure tribromphenol, but a mixture of tribromphenol $\left(\mathrm{C}_{6} \mathrm{H}_{2} \mathrm{Br}_{3} \mathrm{OH}\right)$ and tribromphenol bromid $\left(\mathrm{C}_{6} \mathrm{H}_{2} \mathrm{Br}_{3} \mathrm{OBr}\right)$.

Thus the results obtained by direct titration are often too high, since in the formation of tribromphenol only six atoms of bromin are 
required, while for the production of tribromphenol bromid eight atoms of bromin are taken up by one molecule of phenol.

The correct results obtained by Koppeschaar's method are attributable to the use of potassium iodid, which decomposes the tribromphenol bromid, liberating iodin, thus:

$$
\mathrm{C}_{6} \mathrm{H}_{2} \mathrm{Br}_{3} \mathrm{OBr}+2 \mathrm{KI}=\mathrm{C}_{6} \mathrm{H}_{2} \mathrm{Br}_{3} \mathrm{OK}+\mathrm{KBr}+\mathrm{I}_{2} \text {. }
$$

The free iodin is then estimated by residual titration, together with that liberated by the excess of bromin added.

Thus the nature of the original precipitate does not affect the final results.

S. J. Lloyd $*$ in a comprehensive series of experiments shows that the precipitate formed by the action of bromin water upon phenol, is at first pure white (tribromphenol), but on standing, the solution containing an excess of bromin, it gradually assumes a yellowish tint; the change is due to the formation of tribromphenol bromid. Thus the latter is not a primary product of the action of brominwater and phenol, but is formed by a gradual reaction between the precipitated tribromphenol and the excess of bromin.

This compound once formed is not quantitatively reconverted into tribromphenol by potassium iodid. Hence the less the quantity of tribromphenol bromid formed, the more accurate the analysis. The amount formed in a constant interval of time (say five minutes), increases with the excess of bromin and with the volume of the reacting mixture, and is diminished by adding acid or potassium bromid or by lowering the temperature. Hence, in order to avoid the formation of tribromphenol bromid when titrating phenol with bromin, the liquid must be strongly acid or must contain an excess of potassium bromid. The excess of bromin must not be too great, and the time during which the precipitate of tribromphenol is in contact with the excess of bromin must not be too long. Under these conditions only a mere trace of tribromphenol bromid is formed, and it is precisely when the quantity of that substance is small that it is more easily acted upon by HI, as shown in the above equation. In view of these facts, based upon his experiments, $\bar{\xi}$ loyd $\dagger$ suggests the following method:

Lloyd's Hypobromite Method. Solutions required. Fiftiethnormal thiosulphate and iodin; starch; hydrochloric acid (sp.gr. I.2); potassium iodid, I7 gms. in Ioo cc.; hyprobromite, prepared by dissolving $9 \mathrm{cc}$. of bromin in 2 liters of a solution containing 28 
gms. of caustic potash. The hypobromite must be compared with the thiosulphate by adding acid, and potassium iodid and titrating the iodin set tree.

The Analysis. Introduce the phenol solution into a glass-stoppered flask and add a volume of acid equal to about one third or one fourth of the combined volumes of the phenol solution and the hypobromite that will probably be added during the analysis.

Run in the hypobromite from a burette, shaking the flask, until the solution becomes permanently yellow. Then add an excess of the hypobromite (ro to 20 per cent of that used already), and shake well. Finally add an excess of potassium iodid, dilute with water, add ro cc. of chloroform, and determine the iodin with the standard thiosulphate. The object of diluting is to prevent the acid from acting on the potassium iodid or on the thiosulphate; if $10 \mathrm{cc}$. of water be added at this stage for every cc. of acid previously added, the solution will be sufficiently dilute. The use of chloroform may be dispensed with, if the mixture be allowed to stand five minutes before adding the potassium iodid; it is, however, better to use the chloroform-carbon disulphid is not satisfactory.

If these directions be adhered to, the phenol can be determined within I or 2 parts per thousand.

Dr. Waller's Method. Solutions Required. I. A standard solution of phenol containing Io gms. of pure phenol in I liter.

2. Diluted sulphuric acid of I 5 per cent or 20 per cent strength, saturated with alum. This is needed to facilitate the settling of the precipitate.

3. A solution of bromin in water.

The Estimation. Io gms. of the sample are introduced into a liter flask, and made up with water to I liter. This solution is filtered through a dry filter, and Io cc. of the clear filtrate taken for analysis. It is placed into an 8-oz. glass-stoppered bottle, and about $30 \mathrm{cc}$. of the acid-alum solution added. Into another bottle of the same kind ro cc. of the standard phenol solution is put, and to this also ,30 cc. of the acid-alum solution are added.

The bromin solution is now added from a burette to the bottle containing the standard phenol solution till no more precipitate forms, the bottle being stoppered and well shaken after each addition. The enid-reaction is further indicated by the appearance of a yellow color when a slight excess of bromin is reached. Near the end the precipitate forms slowly.

The other solution containing the sample under analy'sis is titrated in the same way. Then the calculation is made as follows:

The number of $\mathrm{cc}$. of bromin solution consumed by the sample 
is multiplied by זоo, and then divided by the number of cc. of bromin solution used by the standard phenol solution. The answer is the per cent of pure phenol contained in the sample analyzed.

The amount of water contained in a solution of carbolic acid may be determined by agitating the solution with an equal volume of chloroform in a graduated cylinder. After standing, the upper layer consists of the water contained in the mixture.

Crude or Impure Carbolic Acid.* Phenol in crude carbolic acid is estimated after separating the tarry matters. $20 \mathrm{cc}$. of the crude carbolic acid are placed in a beaker with $20 \mathrm{cc}$. of a strong solution of potassium hydroxid (sp.gr. about I.30). The mixture is well shaken and allowed to stand for half an hour; it is then diluted to $\frac{1}{4}$ liter with water. The tarry matters and other foreign impurities are thus set free, and may be removed by filtration, the filter and contents being washed with lukewarm water till the washings are no longer alkaline. The filtrate and washings are then slightly acidulated with hydrochloric acid, and made up to 3 liters with water.

The small quantity of tarry matters which is left in the filtrate does not interfere in the titration which follows. $50 \mathrm{cc}$. of this solution are now taken, and $\mathrm{I} 20 \mathrm{cc}$. of the decinormal bromin are added, followed by $5 \mathrm{cc}$. of hydrochloric acid, and the mixture shaken frequently during half an hour. Io cc. of potassium iodid T. S. are then added, shaken, allowed to rest (not longer than five minutes), and finally titrated with decinormal sodium thiosulphate, using starch T. S. as an indicator.

The number of cc. of the thiosulphate solution used are deducted from I 20 cc., the quantity of $\frac{\mathrm{N}}{\mathrm{IO}}$ bromin originally added, and the quantity of the latter which was actually taken up by the phenol is obtaned. This figure, when multiplied by the factor for phenol, c.0or 556 gm., gives the quantity of phenol present in the sample operated upon. It must be remembered that the $50 \mathrm{cc}$. of the diluted carbolic acid used in this assay represent $\frac{1}{3}$ of I cc. of the original sample.

Example. Let us assume that $80 \mathrm{cc}$. of decinormal sodium thiosulphate were required in the residual titration. Deducting this from $\mathrm{I} 20$ leaves $40 \mathrm{cc}$. of bromin which actually went into combination with the phenol; then $40 \times 0.001556=0.06224 \mathrm{gm}$. of phenol present in $0.33 \mathrm{cc}$. of the solution analyzed.

* Toth, Zeitschr. anal. Chem., XXV, I6o (1886). Stockmeier and Thurnauer, Chem. Zeit., r893, Ir9-r5I, recommend a sinilar method. 
Volumetric Determination with Potassium Permanganate. James. F. Tocher,* in search for a practical method for the volumetric determination of phenol in the presence of its homologues, gives the details of his studies and investigation which have led him to recommend potassium permanganate as suitable for this purpose. $\mathrm{He}$ finds that phenol, treated with potassium permanganate in the presence of normal or acid sodium carbonate, is oxidized to oxalic acid, while the hydrated oxids of manganese are deposited, and that in the absence of other oxidizable substances, as great, if not greater, accuracy can be attained, volumetrically, than by any of the halogen processes now commonly employed. The titration may be carried out as follows: I gm. phenol is dissolved in $1000 \mathrm{cc}$. of water, and of this solution ro cc. (=0.0I gm. phenol) taken for titration. About 3 to $4 \mathrm{gms}$. $\mathrm{NaHCO}_{3}$ is added, together with a little water; then 50 cc. decinormal permanganate are added, the liquid boiled for five minutes, and set aside to cool a little. Dilute $\mathrm{H}_{2} \mathrm{SO}_{4}$ is now added gradually, until the mixture is neutralized, and then to decided excess. The mixture is warmed to $60^{\circ} \mathrm{C}$., and decinormal solution of oxalic acid added, with stirring, until the color is discharged. If the phenol is pure, $29.78 \mathrm{cc}$. of the permanganate solution will have been consumed by $0.01 \mathrm{gm}$. of the substance taken.

The Estimation of Phenol in Pharmaceutical Products. Tablets, powders, and other pharmaceutical products may be assayed for phenol in the presence of substances which interfere with a direct estimation, by the method described below.

In a series of experiments Puckner and Clark $\dagger$ have found:

First. That phenol can be completely removed from a solution containing much potassium hydroxid by first saturating with carbon dioxid and then distilling with steam in a current of carbon dioxid. Under these conditions as much as $0.0150 \mathrm{gm}$. of phenol is obtained in the first $100 \mathrm{cc}$. of filtrate distillate.

Second. That sulphites, bromates, and nitrates do not affect the estimation by this method.

Third. That the U. S. P. method for the valuation of phenol is entirely satisfactory and also may be applied when the volume of the phenol solution is as great as $50 \mathrm{cc}$, and the amount of phenol present sufficient to absorb from io to 90 per cent of the bromin present. They have evolved the following method:

The substance containing the phenol was placed in a round. bottomed distilling flask and water sufficient to cover it was added. The flask was connected by means of a double perforated rubber

* Ph. Jour., March 25, 1901, 360, 36r.

† Proc. A. Ph. A., 1908; and A. J. Ph., LXXX, 484 (1908). 
stopper, with a Liebig condenser on the one hand, and on the other with a tin reservoir containing water. A current of carbon dioxid was then passed from a Kipp generator through the reservoir and distilling flask for fifteen minutes or more. (In the case of known mixtures of phenol and potassium hydroxid V. S., phenolphthalein was added and carbon dioxid passed until colorless, about five minutes being sufficient.) The water was then heated to boiling and the distillation continued, a brisk current of carbon dioxid * being passed through the apparatus continually until $250 \mathrm{cc} . \dagger$ of distillate was obtained. Of this distillate $50 \mathrm{cc}$. was taken and placed in a 250 -cc. glass-stoppered flask, $25^{\circ} \mathrm{cc}$. of standard bromin solution added, and the mixture acidulated with $5 \mathrm{cc}$. hydrochloric acid U. S. P.; the mixture was shaken frequently during one half hour, and then $5 \mathrm{cc}$. potassium iodid T. S. was quickly introduced and the mixture well shaken. The stopper and neck of the flask were rinsed with water, a small amount of chloroform added, and the iodin titrated with standard sodium thiosulphate V. S.

Meissinger and Vortmann have devised a method which is based upon the reaction between iodin and phenol in alkaline solution. This method is described on page $65^{\circ}$.

\section{LITERATURE}

Bader. Zeitschr. anal. Chem., xxxi, 58 (1392).

Meissinger and Vortmann. Ph. Ztg., xxix, 759.

Schaedler. Ph. Centrabl., XIII, 225.

Tocher. Ph. Jour., I901, 360.

Landolt. Berichte d. Chem. Ges., IV, 770 (I87I).

Degener. Zeitschr. prakt. Chem. N. F., XVII, 390 (I878).

Koppeschaar. Zeitschr. anal. Chem., xv, 233 (1876).

Chandelon. Bull. Soc. Chim., xxxviri, 69 (I882).

Telle. Chem. News, LxxxiII, 5I (I90I).

Waller. Chem. News, XLIII, I52 (I88I).

Seubert. Berichte d. Chem. Ges., XIV, I58I (I88I).

Beckurts. J. S. C. I., v, 546 (I886).

* Simple saturation with carbon dioxid will not liberate all the phenol, but a stream of the gas must be passed during the distillation; when in an experiment the supply of carbon dioxid was cut off as soon as the saturation was complete, and then the distillation continued, only 88.64 per cent of the phenol was recovered in one case, 90.48 per cent in another, and 86.68 per cent in a third.

$\dagger$ If $250 \mathrm{cc}$. of distillate is collected, as shown in an experiment with pure phenol, the first roo cc. of distillate in one case contained 96.48 per cent of the phenol taken and in another 97.22 per cent; with a mixture of phenol, opium, bismuth subnitrate, and aromatic powder, and containing 7.21 per cent phenol, the first roo cc. distillate contained 98.61 per cent of the phenol present. 
J. S. Lloyd. J. A. C. S., xxvir, 7 and r6 (rgo5).

Toth. Zeitschr. anal. Chem., xxv, r6o (r886).

Kleinert. Zeitschr. anal. Chem., XxiII, I (I884).

Fedeli. Berichte d. Chem. Ges., xxviII, 1060 (1895).

Giascosa. Zeitschr. physiol. Chem., vi, 43 (I878).

Neuberg. Zeitschr. physiol. Chem., XxVII, I23 (1899).

Kossler and Penny. Zeitschr. physiol. Chem., XviI, II 7 (I889).

Partheil. Apoth. Ztg. (I896).

Ditz and Cedivoda. Zeitschr. angew. Chem., r899, 873 and 897.

Stockmeier and Thurnauer. Chem. Zeit., I893, I 19 and 151.

Frehrichs. Centrabl., II (1896), 2 I 4 I.

Weinreb and Bondi. Monatsheft f. Chem., vi, 5 c6 (I885).

Werner. Bull Soc. Chim., XLIII, 373 (1885).

Kastle. Am Chem. Jour., Xxvir, 3I (1902).

Schryner. J. S. C. I. (1899), 553. 


\section{CHAPTER LIV}

\section{ESTIMATION OF GLYCERIN}

Glycerin (Glycerol) $\mathrm{C}_{3} \mathrm{H}_{5}(\mathrm{OH})_{3}=9 \mathrm{I} \cdot 37$. The estimation of glycerin, of fats, etc., may be made by the method of Benedikt and Zsigmondy. This method consists in saponifying the fat and oxidizing the resultant glycerin by permanganate in alkaline solution; thus oxalic acid, carbon dioxid, and water are formed. The excess of permanganate is then destroyed by sulphurous acid or a sulphite, the liquid filtered to separate the manganese dioxid, and the oxalic acid then precipitated by a soluble calcium salt in the presence of acetic acid, and the precipitated calcium oxalate then titrated with permanganate, or after ignition and conversion into carbonate titrated with standard acid solution in the usual way.

Aqueous solutions of glycerin may of course be assayed by this method very easily.

The reactions are as follows:

$$
\begin{aligned}
& \mathrm{C}_{3} \mathrm{H}_{5}(\mathrm{OH})_{3}+{ }_{2} \mathrm{KMnO}_{4}=\mathrm{K}_{2} \mathrm{C}_{2} \mathrm{O}_{4}+\mathrm{K}_{2} \mathrm{CO}_{3}+4 \mathrm{MnO}_{2}+{ }_{4} \mathrm{H}_{2} \mathrm{O} \text {; } \\
& 91.37 \\
& \text { (Potassium } \\
& \text { oxala te) } \\
& \begin{array}{r}
\text { oxalate } \\
\mathbf{1} 65.06
\end{array}
\end{aligned}
$$

then

$$
\underset{165.06}{\mathrm{~K}_{2} \mathrm{C}_{2} \mathrm{O}_{4}}+\mathrm{CaCl}_{2}=2 \mathrm{KCl}+\underset{\text { (Calcium oxalate) }}{\mathrm{CaC}_{27.14} \mathrm{O}_{4} ;}
$$

then

$$
\begin{aligned}
& { }_{5} \mathrm{CaC}_{2} \mathrm{O}_{4}+8 \mathrm{H}_{2} \mathrm{SO}_{4}+{ }_{2} \mathrm{KMnO}_{4}={ }_{5} \mathrm{CaSO}_{4} \\
& \text { roo) } 635.7 \\
& 6.357 \text { gms. } \quad \frac{100)}{3.1396} \text { gms. or I000 cc. } \frac{N}{10} \text { V.S. } \\
& +2 \mathrm{MnSO}_{4}+\mathrm{K}_{2} \mathrm{SO}_{4}+8 \mathrm{H}_{2} \mathrm{O}+\mathrm{roCO}_{2} \text {. }
\end{aligned}
$$

Thus $1000 \mathrm{cc} \cdot \frac{\mathrm{N}}{\mathrm{IO}}$ permanganate solution represents $6.357 \mathrm{gms}$. of calcium oxalate, which is equivalent to 8.25 gms. of potassium oxalate, which is equivalent to $4.568 \mathrm{gms}$. of glycerin. 
Thus each cc. of the permanganate solution of decinormal strength used up by the calcium oxalate represents $0.004568 \mathrm{gm}$. of glycerin.

If the precipitated calcium oxalate is ignited and converted into carbonate, and the carbonate then titrated with decinormal sulphuric or hydrochloric acid, the reactions are as follows:

$$
\begin{aligned}
& { }_{2} \mathrm{CaC}_{2} \mathrm{O}_{4}+\mathrm{O}_{2}={ }_{2} \mathrm{CaCO}_{3}+{ }_{2} \mathrm{CO}_{2} \text {; } \\
& \text { 4) } 254.28 \quad 4) \mathrm{r} 98.7 \\
& \frac{1 0 \longdiv { 6 3 . 5 7 }}{6.357 \text { gms. }} \quad \frac{\text { 10 }}{49.675} \\
& { }_{2} \mathrm{CaCO}_{3}+{ }_{2} \mathrm{H}_{2} \mathrm{SO}_{4}={ }_{2} \mathrm{CaSO}_{4}+{ }_{2} \mathrm{H}_{2} \mathrm{O}+{ }_{2} \mathrm{CO}_{2} \text {. } \\
& \frac{4) 198.7}{\text { I0) } 49.675} \frac{\text { 4) } 194.7}{\text { 10) } 48.675} \\
& 4.9675 \text { gms. } 4.8675 \text { gms. or rooocc. } \frac{\mathrm{N}}{\text { IO }} \text { V.S. }
\end{aligned}
$$

Thus each cc. of decinormal acid represents $0.0049675 \mathrm{gm}$. of $\mathrm{CaCO}_{3}$, or $0.006357 \mathrm{gm}$. of calcium oxalate, or $.004568 \mathrm{gm}$. of glycerin.

If Experimenting with Pure Glycerin, operate upon ro cc. of a 2 per cent solution. This is diluted with cold water to about 400 cc., about ro gms. of caustic potash are added to this, and then a saturated solution of potassium permanganate until the liquid is no longer green, but blue or blackish. An excess does not harm.

The liquid is then boiled for about one hour, and a strong solution of sodium sulphite is added to the boiling liquid until the violet or green color is destroyed; the liquid is then filtered while yet hot, to separate the precipitated manganese dioxid. When cool, it is acidified with acetic acid, and calcium chlorid added to precipitate the oxalic acid as calcium oxalate. When the deposition of calcium oxalate is complete it is separated by filtration, and titrated either with permanganate or after ignition with standard sulphuric acid.

The former method is preferable. For this purpose the filter is pierced, and the precipitate rinsed into a porcelain basin; about Io cc. of dilute sulphuric acid are then added through the funnel slowly, so that it comes into contact with and washes through any of the precipitate that may still cling to it.

The liquid is now diluted to about $200 \mathrm{cc}$., brought to $60^{\circ} \mathrm{C}$., and the decinormal permanganate run in from a burette, slowly, until a faint but distinct pink color appears and remains permanent after stirring; each cc. of the permanganate thus used represents 0.004568 $\mathrm{gm}$. of glycerin.

The Process for Estimating the Glycerin of Fats is as follows: io gms. of the fat or oil are placed in a strong small bottle together 
with 4 gms. of pure potassium hydroxid, dissolved in $25 \mathrm{cc}$. of water; the bottle is then closed with a solid rubber stopper and tied down firmly with wire; it is then placed in boiling water and heated, with occasional shaking, from six to ten hours, or until the fat or oil is completely saponified. The contents of the bottle are then poured into a beaker and diluted with hot water; this should give a clear solution.

A dilute acid is then added to separate the fatty acids, which are filtered out and the filtrate made up to a given volume.

This solution, which will usually contain 0.2 to $0.5 \mathrm{gm}$. of glycerin, according to its origin, is transferred to a porcelain basin, diluted with cold water to about $400 \mathrm{cc}$., and the glycerin estimated as described under the experiment with pure glycerin.

The Modified Form of Herbig and Mangold (Ulzer and Fraenkel) is as follows: 2 to 3 gms. of fat are saponified in pure methyl alcohol with potassium hydroxid. The alcohol is volatilized, the residual soap is dissolved in hot water, and is decomposed with dilute hydrochloric acid. It is then heated until the separated fatty acids form a clear layer. To the liquid fat some paraffin had better be added. It is cooled thoroughly, filtered off into a liter flask, and washed well. The solution is exactly neutralized with caustic potash and phenolphthalein. Io gms. more caustic potash are added, and as much 5 per cent potassium permanganate solution is added in the cold as would represent approximately one and a half times the theoretical amount. (For every part of glycerin 6.87 parts of potassium permanganate.)

The liquid will then no longer appear green, but blue or black. It is allowed to stand one half hour at ordinary temperature. Hydrogen dioxid, in not too great an excess, is added, until the supernatant liquid becomes colorless. It is diluted to the mark, is shaken briskly, and $500 \mathrm{cc}$. are filtered off through a dry filter. To decompose the hydrogen dioxid the liquid is boiled for half an hour, cooled to about $60^{\circ} \mathrm{C}$., and after addition of sulphuric acid the oxalic acid formed is titrated with permanganate.

In place of filtration, the filtrate, after acidifying with acetic acid, may be precipitated with calcium chlorid. When filtered it may be determined either gravimetrically by ignition to calcium oxid, or it may, after decomposition with sulphuric acid, be titrated with permanganate, as above.

The Acetin Method.* This method is a preferred one because of its simplicity and rapidity as compared with other methods. It depends on the phenomenon that glycerin on boiling with acetic

* Benedikt and Cantor, Monatsheft, IX, 52I. 
anhydrid is quantitatively transformed into triacetin. If the latter be then dissolved in water, and the free acetic acid be neutralized with sodium hydroxid, the dissolved triacetin can be saponified with sodium hydroxid and the excess of the latter titrated back.

The reactions occurring are expressed by the following equations:

$$
\begin{aligned}
& { }_{2} \mathrm{C}_{3} \mathrm{H}_{5}(\mathrm{OH})_{3}+{ }_{\text {Acetic anhydrid. }} \mathrm{C}_{4} \mathrm{H}_{6} \mathrm{O}_{3}= \\
& \mathrm{C}_{3} \mathrm{H}_{5}\left(\mathrm{C}_{2} \mathrm{H}_{3} \mathrm{O}_{2}\right)_{3}+{ }_{3} \mathrm{H}_{2} \mathrm{O} . \\
& \mathrm{C}_{3} \mathrm{H}_{5}\left(\mathrm{C}_{2} \mathrm{H}_{3} \mathrm{O}_{2}\right)_{3}+{ }_{3} \mathrm{NaOH} \mathrm{OH}={ }_{3} \mathrm{NaC}_{2} \mathrm{H}_{3} \mathrm{O}_{2}+\mathrm{C}_{3} \mathrm{H}_{5}(\mathrm{OH})_{3} .
\end{aligned}
$$

The necessary reagents are: (a) Normal hydrochloric acid.

(b) Dilute sodium hydroxid solution, not standardized, but containing about $20 \mathrm{gms}$. of sodium hydroxid to the liter.

(c) A stronger sodium hydroxid solution (about io per cent.) This should be preserved in a flask provided with a $25 \mathrm{cc}$. pipette.

(d) Phenolphthalein T. S.

The procedure. I to I.5 gms. of the sample are weighed out in a wide-necked, small, round-bottom flask of about roo cc. capacity; 7 to 8 gms. acetic anhydrid are added, with about 3 gms. dehydrated sodium acetate. The mass is boiled under a reflux for one to one and a half hours.

Triacetin is formed as per reaction (I). It is then allowed to cool somewhat and diluted with $50 \mathrm{cc}$. of water and the mixture likewise heated under a reflux (but not boiled), until upon agitation the triacetin is completely dissolved. The solution is then filtered into a large flask (500 cc.). Usually a flocculent white precipitate - remains on the filter. This is washed, the liquid cooled, phenolphthalein added, and the free acetic acid present exactly neutralized with dilute sodium hydroxid solution. The neutralization is known to be complete when the slightly yellowish color is changed to reddishyellow. It must not be pink, as this indicates an excess of the alkali, which excess will saponify some of the triacetin. The triacetin is now saponified by adding $25 \mathrm{cc}$. of the stronger sodium hydroxid solution and boiling for fifteen minutes under a reflux condenser. (See Reaction (2)). This is then titrated back with normal hydrochloric acid. A check titration is now made upon $25 \mathrm{cc}$. of the sodium hydroxid with normal hydrochloric acid, and from the difference the alkali used by the triacetin is found. This is then calculated into glycerin. Each cc. of the normal acid represents $0.03045 \mathrm{gm}$. of pure glycerin. The difference in the quantity of the standard acid used in the check titration, and in the actual analysis, multiplied by the above factor, gives the quantity of pure glycerin in the sample taken. 
In order to obtain accurate results, the steps should be conducted rapidly and continuously, and above all the free acetic acid should be neutralized with great care and an excess of alkali avoided.

The method gives results which, in the hands of careful workers, agree well with other methods.

O. Heller recommends the above method as being the best.

The Dichromate Method (Hehner)** This method depends upon oxidizing the glycerin with potassium dichromate in the presence of sulphuric acid. The quantity of standard dichromate used is then determined by means of a standard solution of ammonium ferrous sulphate.

One part of glycerin is completely oxidized (converted into $\mathrm{CO}_{2}$ ) by 7.486 parts of dichromate in the presence of sulphuric acid. The method is very easy and rapid, and is considered the best general method.

Solutions Required. Standard Potassium Dichromate Solution containing $74.86 \mathrm{gms}$. of the dichromate and $5^{\circ} \mathrm{cc}$. of concentrated sulphuric acid in a liter. One cc. of this solution should be equivalent to $0.0 \mathrm{Igm}$. of glycerin. The exact oxidizing power of this solution must be ascertained by titration against a solution containing a known quantity of iron wire, or pure ferrous ammonium sulphate.

Weaker Dichromate Solution, made by diluting the above so as to have a solution of one tenth the strength.

Standard Iron Solution. This should contain 240 gms. of ferrous ammonium sulphate and $50 \mathrm{cc}$. of concentrated sulphuric acid per liter. It is standardized against the dichromate solution, using potassium ferricyanid as the indicator.

The Process. With pure glycerin the oxidation by this method is absolutely quantitative. $0.2 \mathrm{gm}$. accurately weighed, are moderately diluted in a beaker. Io cc. of concentrated sulphuric acid are added, followed by 30 or $40 \mathrm{cc}$. of the stronger dichromate solution. The beaker is then covered with a watch-glass, placed in a water-bath and digested for two hours. The excess of dichromate is then determined by titration with the standard iron solution.

When great accuracy is required the weaker dichromate is useful for completing the titration. The operation should be conducted as near as possible at a temperature of $16^{\circ} \mathrm{C}$.

Crude glycerin must be first purified from chlorin or aldehyde as follows: I.5 gms. of the diluted sample is placed in a roo-cc. flask, heated with some moist silver oxid and let stand ten minutes; subacetate of lead solution is then added in slight excess and the whole 
diluted to roo cc. and filtered through a dry filter. $25 \mathrm{cc}$. of this are then digested with excess of dichromate and titrated as above described.

The estimation of glycerin in fats and oils is carried out as follows:

Saponify 3 gms. of fat with alcoholic $\mathrm{KOH}$ solution, do not drive off the alcohol, but dilute the soap solution to about $200 \mathrm{cc}$. and decompose with dilute sulphuric acid; filter off the insoluble acids, which may be estimated as usual. Vigorously boil the filtrate and washings, amounting together to $500 \mathrm{cc}$. in a covered beaker, down to one half. Then add sulphuric acid and standard dichromate as described.

The Iodic Acid Method (Chaumeil).* A. Chaumeil suggests that the difficulties of the dichromate method may be obviated by substituting iodic acid, which, besides being a more energetic oxidizing agent, has the advantage of not being destroyed by sulphuric acid, however concentrated the latter may be. The oxidation of glycerin, by iodic acid in the presence of sulphuric acid is complete, the reaction proceeding as follows:

$$
{ }_{5} \mathrm{C}_{3} \mathrm{H}_{5}(\mathrm{OH})_{3}+{ }_{7} \mathrm{I}_{2} \mathrm{O}_{5}={ }_{1} \mathrm{CO}_{2}+{ }_{2} \mathrm{OH} \mathrm{H}_{2} \mathrm{O}+{ }_{7} \mathrm{I}_{2},
$$

from which it is seen that five molecules of glycerin liberate seven molecules of iodin. In making the determination, a fragment of marble is placed in the distilling flask; the $\mathrm{CO}_{2}$ evolved maintains a slight pressure in the apparatus, and prevents absorption. The receiver contains potassium iodid solution, in which the iodin dissolves when it comes over. The iodin is readily determined by standard solution of sodium thiosulphate. Where the glycerin contains chlorids, the author does not eliminate the latter by a preliminary treatment with carbonate of silver, as has been recommended, but titrates the glycerin directly, and subtracts from the volume of the thiosulphate used that accounted for by the chlorids.

\section{ESTIMATION OF GLYCERIN IN FLUID EXTRACTS}

Take Io gms. of the fluid extract, evaporate it at a low temperature to 5 gms. It is important that a low temperature be employed in order that the alcohol, but not the glycerin, be volatilized.

Dissolve the residue in $50 \mathrm{cc}$. of water, and add solution of lead subacetate, drop by drop, until precipitation is complete. Allow the precipitate to subside, filter the liquid through a wet filter, wash the precipitate thoroughly with water, add to the filtrate and washings a few drops of dilute sulphuric acid, then ro gms. of solid potassium

* Bull. Soc. Chim., XXVII, I 2. 
hydroxid, and an excess of potassium permanganate solution. Bring the liquid to the boiling point and keep there for about one hour, then add sufficient of a strong solution of sodium sulphite to destroy the violet color due to the excess of permanganate.

Filter while still hot to separate the precipitated $\mathrm{MnO}_{2}$, and when cool acidify with acetic acid and add calcium chlorid solution to precipitate the oxalic acid as calcium oxalate.

When precipitation is complete filter and titrate (after the addition of sulphuric acid) with $\frac{\mathrm{N}}{\mathrm{IO}}$ potassium permanganate.

Each $\mathrm{cc} .=0.004568 \mathrm{gm}$. glycerin.

\section{LITERATURE}

C. Mangold. Zeitschr. angew. Chem., I89I, 400.

O. Hehner. J. S. C. I., VIII, 4 (I889).

Richardson and Jaffé. J. S. C. I., I898, 33 .

Benedikt and Cantor. Monatscheft, IX, 52 I.

E. Suhr. Archiv. f. Hygiene, XIV, 305.

Chaumeil. Bull. Soc. Chim., XxvII, I2, and Ph. Jour., April, I903, 490.

Lewkowitsch. Analyst, xxvin, I04, and Ph. Jour., April, I9०3, 558. 
ThE principal volumetric method for tannic acid depends upon the use of permanganate. The estimation of tannic acid by means of potassium permanganate is by no means as easily carried out as is the titration of ferrous salts with permanganate. Concordant results are obtained only when the titrations are carried out in exactly the same manner, and in accordance with definite directions. The tannic acid or tannins found in various parts of different plants (such as oak bark, nut-galls, cinchona, sumach, pine bark, tea, etc.) do not possess identical properties, and in fact, equal quantities of tannic acid from different sources will reduce different quantities of permanganate. Thus it is evident that the accurate determination of tannic acid is by no means a simple matter. If it were possible in every case to check the permanganate against the particular tannin to be estimated, this difficulty would be overcome. This is, however, rarely possible. Another difficulty to overcome is the fact that the various tanning materials contain besides tannin, other substances which have a reducing action upon permanganate. The method employed to overcome this difficuty is to titrate with permanganate and find the total reducing power of the mixed constituents, and then by means of gelatin, glue, or hide-powder remove the tannic acid and then again titrate with permanganate to determine the oxidizable matters other than tannin. The difference between the two titrations represents the tannin. Löwe (Zeitschr. anal. Chem., IV, 368) has shown that pectinous substances are frequently present in tanning material (especially oak bark) and that these must first be separated if accurate results are to be obtained, because these substances are precipitated by the substances which are used to precipitate tannin (particularly hide-powder). To separate these substances Löwe evaporates the liquid extract of, say, oak bark, with the addition of a drop of acetic acid, to dryness on a water-bath. The residue he then extracts with strong alcohol, which dissolves the tannin but not the pectinous substances. He then evaporates the alcoholic solution on a waterbath and takes up the dry residue with water. 
Löwenthal's method, which is described below, is accepted as the best volumetric method.

\section{ESTIMATION OF TANNIN IN BARKS, ETC. (LÖWENTHAL'S METHOD)}

The principle of this method depends upon the oxidation of the tannic acid, together with other easily oxidizable substances, by titrating with potassium permanganate.

The total amount of such substances is thus found, and expressed by a known volume of permanganate. The actual available tannin is then removed by gelatin or glue, ${ }^{*}$ and another titration made, to determine the amount of oxidizable matters other than tannin.

The difference between the amounts of permanganate solution used in the two titrations gives the amount of tannin present, which is available for tanning purposes, expressed in terms of permanganate.

Solutions Required. I. $\frac{N}{30}$ Potassium Permanganate (1.05 gm. per liter).

2. Indigo Solution. 6 gms. of pure precipitated indigo and $50 \mathrm{cc}$. of concentrated sulphuric acid are dissolved in sufficient water to make I liter.

3. Glue and Salt Solution. 25 gms. of good transparent glue are macerated in cold water, and then heated to dissolve; the solution is then made up to I liter and saturated with common salt. The solution should be filtered clear when used.

4. Acidified Solution of Common Salt. This is a saturated solution of common salt, containing in I liter $25 \mathrm{cc}$. of sulphuric acid.

The Analysis. 20 gms. of the bark or Io gms. of sumach are

* Neubauer (Zeitschr. anal. Chem. X, I) uses animal charcoal. Hammer (Zeitschr. prakt. Chem. LXXXI, I59) uses hide-powder (hide prepared for tanning and reduced to powder). Siemand (Zeitschr. anal. Chem. XXII, 595) recommends the employment of the glue-yielding tissue of bones or horn-cartilage, which are prepared as follows: Cut off the ends of hollow bones, remove the marrow and break the bones into large pieces. Digest these pieces for two days with a $\mathbf{5}$ per cent solution of sodium carbonate, then brush and wash them with water, leaving them in contact each time for several hours. Then break the bones into smaller pieces and treat with dilute hydrochloric acid, 8 liters of which contain $\mathbf{r}$ liter of commercial hydrochloric acid, until they become soft. Then wash with water to remove the acid and grind in a small mill whilst still moist. Remove the last traces of calcium salts and ferric oxid by digesting repeatedly with diluted hydrochloric acid ( $1: 20$ ) and thoroughly wash with water, press and dry. Horn-cartilage (the bony, vascular nucleus of cattle horn). This is freed from calcium salts in the manner described above. 
boiled with several portions of water until exhausted, and the solution when cold is made up to I liter.

Io cc. of this solution are diluted to rooo cc.; $25 \mathrm{cc}$. of the indigo solution are added, and the permanganate solution then run in, drop by drop, from a burette, stirring constantly, until the blue color changes to yellow, and the number of cc. of permanganate solution consumed noted.

$25 \mathrm{cc}$. of the indigo solution are now taken and diluted to rooo cc., titrated with permanganate, and the number of cc. again noted. By deducting this number from the number of cc. used in the first titration, the quantity of permanganate required by the tannin and the other oxidizable substances in the $10 \mathrm{cc}$. of solution taken is found.

The next step is to deprive a portion of the tannin solution of its tannin, and again titrate.

Ioo cc. of the tannin solution are treated with $50 \mathrm{cc}$. of the glue and salt solution, and, after stirring, roo cc. of the acidulated salt solution are added, the mixture stirred again, and set aside for several hours. The glue absorbs the tannin out of solution. The solution is then filtered. The filtrate should be perfectly clear.

Of this filtrate take $50 \mathrm{cc}$. (containing $20 \mathrm{cc}$. of the tannin solution), mix with $25 \mathrm{cc}$. of the indigo solution, and titrate with the permanganate solution as before, noting the number of cc. consumed.

Another $25 \mathrm{cc}$. of the indigo solution are now taken, diluted as in the other trial, and again titrated with permanganate. By deducting the number of cc. so obtained from the number required by the $50 \mathrm{cc}$. of filtrate, the quantity required by the oxidizable matter other than tannic acid in the $20 \mathrm{cc}$. of tannin solution is obtained. Therefore one half of this quantity, when deducted from the quantity of permanganate solution representing the total oxidizable matter in Io cc. of the tannin solution, gives the quantity of permanganate which was effected by the tannin above.

Duplicate titrations should always be made, and should agree within 0.1 or $0.2 \mathrm{cc}$. of the permanganate solution.

Thus far we have only the tannin value (expressed in terms of permanganate), of ro cc. of the original solution, representing $\frac{1}{100}$ of the material under examination.

The permanganate solution may be compared with a standard solution of the purest gallo-tannic acid obtainable, or with any tannin of known value, and thus a coefficient obtained.

According to the experiments of Neubauer, 63 gms. of pure crystallized oxalic acid (equivalent to $3 \mathrm{I} .4 \mathrm{gms}$. potassium permanganate) correspond to $4 \mathrm{I} .57 \mathrm{gms}$. of purified gallo-tannic acid (nutgall tannin). And Oser found that $63 \mathrm{gms}$. of oxalic acid correspond 
to 62.355 gms. of querci-tannic acid (oak-bark tannin). These coefficients are now largely used.

Based upon these figures each cc. of $\frac{\mathrm{N}}{30}$ permanganate solution represents .0013856 gm. of gallo-tannin, or .0020785 gm. of querci tannin. In most analyses, however, especially when the composition of the tannin is not exactly known, it is expressed as oxalic acid.

Notes. The Quantity of Material to be taken: This must be such as will make a solution which contains from 0.5 to $\mathrm{I}$ gm. of the tanning principle per liter. With this in view, the following quantities of the substances should be weighed off:

Pine bark. ................... Io to 5 gms.

Oak bark. ................... 9 to so gms.

Spanish chestnut wood............ 6 to $8 \mathrm{gms}$.

Valonia....................... 3 to 4 gmis.

Sumach. ................. 6 to $8 \mathrm{gms}$.

At a meeting of the A. O. A. C., November I6, I900, it was resolved that such a quantity of the material should be taken as will give about $0.8 \mathrm{gm}$. of total solids per roo $\mathrm{cc}$. of the solution. Then extract in a Soxhlet or other similar apparatus at steam heat, for non-starchy materials. For canaigre and substances containing like amounts of starch, use a temperature of $50^{\circ}$ to $55^{\circ} \mathrm{C}$. until near completion, finishing the extraction at steam heat. In the case of fluid extracts weigh such a quantity as will leave a residue of $0.8 \mathrm{gm}$. on evaporation of $100 \mathrm{cc}$. Dissolve in $800 \mathrm{cc}$. of water at a temperature of $80^{\circ} \mathrm{C}$., allow to stand twelve hours, and make up to I liter.

The Rate of Titration. The rate of speed at which the permanganate is added influences the results considerably. At least four minutes should be consumed in the titration. Towards the end of the titration, the permanganate should be added, drop by drop, in order to observe a sharp end-reaction.

The Indigo Solution. The concentration of the indigo solution is correct if $20 \mathrm{cc}$. of it require about an equal quantity of permanganate solution. If much more or less is required, the solution must be correspondingly diluted or strengthened. The color of the solution changes gradually from deep-blue to dark-green, then to light-green and then to yellowish-green. The last greenish tint disappears with the addition of the next drop of permanaganate solution. If this last change is not sharp, it indicates that the indigo solution is not pure enough; it probably contains some indigo-red, and cannot be used for accurate work. 
Estimation by Means of Egg Albumen. G. Fleury (Jour. Ph. Chim., I892, 499) proposes to use egg albumen for estimating tannin in wine and in the petals of red roses.

The hard-boiled egg-albumen is dried at a moderate temperature, and powdered. This is washed with dilute alcohol (Io per cent), very slightly acidulated with tartaric acid, to saturate the alkali. The albumen is again dried, and kept in a well-stoppered bottle.

The method of operation is as follows:

Albumen powder, equal to seven or eight times the quantity of tannin, which is supposed to be present, is added to the liquid in a flat dish. The dish is then set aside for forty-eight hours, stirring occasionally; the liquid must during this time be acid, not alkaline.

The end of the reaction is attained when the liquid ceases to give to give a color with ferric chlorid T. S.

The powder is then collected on a filter, washed with very dilute alcohol, and then dried at $100^{\circ} \mathrm{C}$. At the same time a sample of the original powder is dried and weighed, to determine the amount of water it contains.

The increase in weight of the albumen which was in contact with the tannin, minus the loss of weight of the albumen in the check experiment, gives the weight of the tannin present.

This method is not available for determining the tannin in nutgalls, because the absorption by the albumen is incomplete and too slow. In testing, it must be borne in mind that gallic acid is not absorbed by the albumen, and consequently still gives its reaction with ferric chlorid.

Estimation by Means of Hydrogen Dioxid. Thompson (Chem. Ztg., I902, 1085) gives the following method, in which tannin is determined by the quantity of oxygen which it absorbs when in alkaline solution.

The necessary nascent oxygen is obtained by the decomposition of hydrogen dioxid in concentrated alkaline solution upon the addition of chemically pure lead peroxid. The presence of the tannin does not in the least interfere with the complete decomposition of the hydrogen dioxid.

The tannin is freed of inorganic and pectinous substances by treatment with $9 \circ$ per cent alcohol, or purified 90 per cent methylalcohol.

The quantity of tannin is calculated out of the difference in the quantity of available oxygen from a definite quantity of hydrogen dioxid, and the quantity of available oxygen still present after treatment with a definite quantity of tannin. I gm. of C. P. anhydrous tannin absorbs (at $0^{\circ} \mathrm{C} .760 \mathrm{~mm}$. pressure) $20 \mathrm{cc}$. of oxygen. 
Estimation by the Aid of Safranin. L. Specht and F. Lorenz* recommend a method for the estimation of tannin which depends upon its precipitation with safranin as a tannin antimony lake and the reducibility of the uncombined safranin by means of hyposulphite. The tannin material is precipitated with tartar emetic and a known quantity of safranin, both in excess, and the excess of safranin is titrated with hyposulphite solution, the titer of which is adjusted to safranin. The amount of safranin consumed is determined by the difference, and the amount required for the tannin is ascertained by a blank experiment made with tannin of known purity. To avoid the interfering action of oxygen, the distilled water used throughout the process must be previously boiled, while the hyposulphite solution must be freshly prepared, as required. Ammonium hyposulphite, for the preparation of which specific directions are given, being found to possess the greatest stability.

The ammonium hyposulphite used in this process is made as follows: 50 gms. of pure zinc dust and $100 \mathrm{gms}$. of water are mixed together, and $600 \mathrm{cc}$. of solution of ammonium bisulphite $20^{\circ} \mathrm{B}$., neutralized with ammonia, is allowed to flow into this mixture, which must be refrigerated so that the temperature does not rise above $36^{\circ} \mathrm{F}$. The solution is clarified by subsidence, and $75 \mathrm{cc}$. of it are diluted with previously boiled and cooled water to $2000 \mathrm{cc}$. The -flocculent precipitate produced on dilution is allowed to subside, since filtration and consequent access of air must be avoided. The titer of this solution is then adjusted to safranin.

Estimation by Means of Silk. $\dagger$ Vignon recommends untwisted silk as superior to either gut-strings or powdered hide for the estimation of tannin in aqueous solutions. If an excess of silk (about $5 \mathrm{gms}$.) is immersed in a solution of $0.1 \mathrm{gm}$. of tannin in Ioo cc. during four or five hours at a temperature of $50^{\circ} \mathrm{C}$., the tannin will be completely absorbed, but none of the substances that usually are present, such as gallic acid, glucose, etc. The quantity of absorbed tannin may then be determined in several ways: by direct weighing; by the difference in the weight of extract obtained from equal weights of the solution before and after treatment, and by titration with permanganate before and after treatment with silk-r cc. of permanganate solution containing 3.I64 gms. $\mathrm{KMnO}_{4}$ per liter, corresponding to $0.004155 \mathrm{gm}$. tannin.

* Chem. Ztg., r9o0, No. I7.

† Ph. Ztg., Nov. 5, I898, 79I; from Jour. de Ph. et de Chim., I898, No. 8. 


\section{ESTMMATION OF TANNIN IN WINES *}

(a) Preparation of Reagents. (I) Oxalic Acid. Use tenthnormal solution; ' 10 cc. $=0.04157 \mathrm{gm}$. of tannin.

(2) Potassium Permanganate Solution. Dissolve I. $333 \mathrm{gms}$. of potassium permanganate in I liter of water and standardize the solution with the tenth-normal oxalic acid solution.

(3) Indigo Solution. Dissolve $6 \mathrm{gms}$. of sodium sulphindigotate in $500 \mathrm{cc}$. of water with the aid of heat; cool, add $50 \mathrm{cc}$. of concentrated sulphuric acid, make the solution up to I liter, and filter.

(4) Purified Boneblack. Extract finely pulverized boneblack with hydrochloric acid and wash with distilled water until the acid is entirely removed. The boneblack is kept covered with water.

(b) Determination. Dealcoholize roo cc, of the urine by evaporation and dilute with water to the original volume. Transfer ro cc. to a porcelain dish of about 2 liters capacity; add about a liter of water and exactly $20 \mathrm{cc}$. of indigo solution. Add tenth-normal potassium permanganate solution, a cc. at a time, until the blue color changes to green; then add a few drops at a time until the color becomes golden yellow. Designate the number of cc. of permanganate solution employed as $a$.

Treat Io cc. of the dealcoholized wine, prepared as above, with boneblack for fifteen minutes; filter and wash the boneblack thoroughly with water. Add a liter of water and $20 \mathrm{cc}$. of indigo solution and titrate with permanganate as above. Designate the number of cc. of permanganate employed above as $b$.

Then $a-b=c$, the number of $c c$. of permanganate solution required for the oxidation of the tannin and coloring matter in ro cc. of wine.

ESTIMATION OF TANNIN IN TEA $†$

\section{Proctor's Modification of Löwenthal's Method.}

\section{(a) Preparation of Reagents}

(I) Potassium permanganate. Make up a solution containing I.33 gms. per liter.

(2) Tenth-normal oxalic acid. Make up a solution containing 6.3 gms. per liter.

* Bulletin No. 107, Bureau of Chemistry, U. S. Dept. of Agriculture.

$\dagger$ Ibid. 
(3) Indigo carmine. Make up a solution containing $6 \mathrm{gms}$. of indigo carmine (free from indigo blue) and $50 \mathrm{cc}$. of concentrated sulphuric acid per liter.

(4) Gelatin solution. Prepare by soaking 25 gms. of gelatin for one hour in a saturated sodium chlorid solution, heat until the gelatin is dissolved, and make up to I liter after cooling.

(5) Mixture. Combine 975 cc. of saturated sodium chlorid solution and $25 \mathrm{cc}$. of concentrated sulphuric acid.

(6) Powdered kaolin.

\section{(b) Determination}

Obtain the value of the potassium perinanganate in terms of the oxalic acid. Boil 5 gms. of the tea for half an hour with $400 \mathrm{cc}$. of water; cool, transfer to a graduated 50o-cc. flask, and make up to the mark. To ro cc. of the infusion (filtered if not clear) add $25 \mathrm{cc}$. of the indigo carmine solution and about $750 \mathrm{cc}$. of water. Add from a burette the potassium permanganate solution, a little at a time while stirring, until the color becomes light green, then cautiously, drop by drop, until the color changes to bright yellow, or further, to a faint pink at the rim. The number of cc. of permanganate used furnishes the value $a$ of the formula given below.

Mix $100 \mathrm{cc}$. of the clear infusion of tea with $50 \mathrm{cc}$. of gelatin solution, roo cc. of salt acid solution, and ro gms. of kaolin, and shake several minutes in a corked flask. After settling decant through a filter. Mix $25 \mathrm{cc}$. of the filtrate (corresponding to Io cc. of the original infusion) with $25 \mathrm{cc}$. of the indigo solution and about $75^{\circ}$ cc. of water, and titrate with permanganate as before. The number of cc. of permanganate used gives the value $b ; a-b=c ; c$ equals the amount of permanganate required to oxidize the tannin. Assume that $0.04157 \mathrm{gm}$. of tannin (gallotannic acid) is equivalent to 0.063 gm. of oxalic acid.

\section{Tannin in Cloves and Allspice*}

Extract 2 gms. of material for twenty hours with absolute ether. Boil the residue for two hours with $300 \mathrm{cc}$. of water, cool, make up to $500 \mathrm{cc}$., and filter. Measure $25 \mathrm{cc}$. of this infusion into a flask of about $\mathrm{r} 200 \mathrm{cc}$. capacity, add $20 \mathrm{cc}$. of indigo solution and $750 \mathrm{cc}$. of distilled water, and proceed as directed under Estimation in Wines.

ro cc. of tenth-normal oxalic-acid solution are equivalent to $0.06232 \mathrm{gm}$. of quercitannic acid, or $0.008 \mathrm{gm}$. cf oxygen absorbed.

* Bulletin No. I07, Burean of Chemistry U. S. Dept. of Agriculture. 


\section{LITERATÜRE}

H. R. Procter. "Leather Industries Laboratory Book."

Hunt. Sutton's "Volumetric Analysis," 8th Ed., 365.

Kathreiner. Zeitschr. anal. Chem., XviI, II3.

A. H. Allen. "Commercial Analysis."

Fletcher and Allen. Chem. News, xxix, I69 (1874).

Dreaper, J S. C. I., XII, 4I 2.

Löwenthal. Jour. prakt. Chem., III, I50 (1860); and Zeitschr. anal. Chem., XVI, 33-20I; also xx, 9I.

Fr. Gaube. Zeitschr. anal. Chem., x, r.

Siemand. Ibid., XxıI, 595 .

Julius Löwe. Ibid., IV, 368 .

K. Hammer. Jour. prakt. Chem., LxxxI, 159.

Procter and Hewitt. Zeitschr. anal. Chem., XVIII, I $_{5}$.

Kramsky. Ibid., 1906, 776.

Specht and Lorenz. Ph. Ztg., Apr. 7, 1900, 266.

Vignon. Ibid., Nov. 5, 1898, 791.

Yocum. Proc. A. Ph. A., Am. Chem., Soc. Jan. 9, 1897.

Procter. J. S. C. I., XI, 329.

Eitner. Der Gerber., XIII, 245.

Trimble. Proc. Franklin Inst., Iv, 23.

Trimble and Peacock. A. J. Ph., xxirr, r6r.

Snyder, J. A. C. S., 1893. 


\section{CHAPTER LVI.}

\section{ESTIMATION OF FORMALDEHYDE *}

The Ammonia Method (Legler). This method is based upon the reaction between free ammonia and formaldehyde in which hexamethylene-tetramin is formed. It is for ordinary purposes sufficiently accurate.

It is this method which is recommended by Lederle $\dagger$ for use in the laboratory of the New York City Health Department, and by Prescott in the laboratory of the University of Michigan.

The assay is conducted as follows: 2 cc. of the solution are placed in a glass-stoppered bottle, the stopper of which is thickly coated with petrolatum, and $50 \mathrm{cc}$. of $\frac{\mathrm{N}}{2}$ ammonia solution added; let stand twelve hours, shaking occasionally. Then determine the excess of ammonia by titrating with $\frac{\mathrm{N}}{2}$ sulphuric acid solution, using rosolic acid or litmus as indicator. The excess of ammonia subtracted from the quantity added gives the quantity which combined with the formaldehyde, and thus the amount of the latter is ascertained.

The reaction is represented as follows:

$$
\begin{aligned}
& 6 \mathrm{CH}_{2} \mathrm{O}+{ }_{4} \mathrm{NH}_{3}=\left(\mathrm{CH}_{2}\right)_{6} \mathrm{~N}_{4}+6 \mathrm{H}_{2} \mathrm{O} \text {. } \\
& \text { 4) } \mathrm{r} 78.74 \quad 4) 67.72 \quad \text { Hexamethylenetetramin } \\
& \text { 2) } 4 4 . 6 8 \quad 2 \longdiv { 1 6 . 9 3 } \\
& \frac{44.68}{22.34} \text { gms. } \frac{163}{8.46} \text { gms. or rooo cc. } \frac{\mathrm{N}}{2} \text { V.S. } \\
& 0.02234 \mathrm{gm} \text {. } 0.00846 \mathrm{gm} \text {. or I cc. “ “ }
\end{aligned}
$$

Assuming that 22 cc. of $\frac{\mathrm{N}}{2}$ sulphuric acid were employed in the titration, 22 cc. of the $\frac{\mathrm{N}}{2}$ ammonia solution must have been in excess,

* Berichte d. Chem. Ges., XVI, I335, r883.

† Am. Drug., I897, 246. 
hence $28 \mathrm{cc}$. of the latter went into combination with the formaldehyde. Thus the $2 \mathrm{cc}$. of formaldehyde solution contained $28 \times 0.02234$ gm. $=0.6255 \mathrm{gm}$.

A. G. Craig* says that the chief difficulty in using the Legler method is the volatility of the ammonia. The difficulty is not so much the loss of strength, in the standard solution, but the loss during the determination. He proposes the following scheme by which this error is removed.

Prepare a normal solution of sulphuric acid. Make up an approximately normal solution of ammonia, the exact strength being immaterial. Procure several three-ounce prescription bottles with smooth sides and close-fitting soft rubber stoppers. Prepare a methyl-orange solution. Procure a boiler in which the bottles may be immersed to the neck without upsetting (a large beaker will do). Take as much of the sample as will contain $0.5 \mathrm{gm}$. of formaldehyde. Measure with the pipette, $25 \mathrm{cc}$. of the ammonia solution into each of the bottles, and to half of them add a sample of formaldehyde; stopper tightly. If the necks of the bottles are small, the stoppers need not be tied down. Place the bottles in the boiler, add cold water to the necks, and heat to boiling. Boil for one hour, and cool by running in cold water slowly, being careful not to allow the cold water to touch the hot bottles. Titrate with sulphuric acid and methyl-orange to the first indication of a color change. Take the difference between the readings for the blanks and those for the samples, as the ammonia consumed in normal cc. Of this difference, $\mathrm{I} \mathrm{cc.}=0.060 \mathrm{I} \mathrm{gm}$. of formaldehyde.

The Legler method is also liable to error because the compound formed is a weak base, and as such combines with acid, while at the same time it is liable to decompose into ammonia and formaldehyde and thus give an indefinite end-point when the residual ammonia is titrated with acid. Error is also liable to be introduced through the presence of carbonic acid in the ammonia-water, which, with the indicator rosolic acid, gives no sharp end-reaction.

The Ammonium Chlorid Method. In this method a solution of ammonium chlorid is used, from which ammonia is evolved by treatment with sodium hydroxid. The excess of alkali is then determined by titration with standard solution of sulphuric acid. This method, as devised by H. Schiff, $\uparrow$ and modifed by C. A. Male, $¥$ is as follows:

* T. A. C. S., XXIII, $6+2$ (190I).

† Chem. Ztg., XXVII, 14 (1903).

$\ddagger$ Pharm. Jour., June, 1905, 844. See also Carl E. Smith, A. J. Ph., LXX, 86, (1898). 
Introduce $2 \mathrm{gm}$. neutral ammonium chlorid, dissolved in $20 \mathrm{cc}$. of water, into a flask or bottle of about 200 cc. capacity, having wellfitting stopper. Dilute ro $\mathrm{cc}$. of the formaldehyde solution to roo cc. with water, and neutralize with sodium hydroxid solution, as the formaldehyde solution generally contains varying quantities of formic acid. Add $20 \mathrm{cc}$. of this neutralized solution to the ammonium chlorid solution, then $25 \mathrm{cc}$. of $\frac{\mathrm{N}}{\mathrm{I}} \mathrm{NaOH}$, and immediately stopper the flask, and leave for one hour. Afterwards determine the excess of alkali with $\frac{\mathrm{N}}{2} \mathrm{H}_{2} \mathrm{SO}_{4}$, using rosolic acid or litmus solution as indicator, both of which give a sharp end-reaction. The reaction and calculation is based upon the following equation:

$$
{ }_{2} \mathrm{NH}_{4} \mathrm{Cl}+{ }_{3} \mathrm{CH}_{2} \mathrm{O}+{ }_{2} \mathrm{KOH}=\mathrm{N}_{2}\left(\mathrm{CH}_{2}\right)_{3}+{ }_{2} \mathrm{KCl}+{ }_{5} \mathrm{H}_{2} \mathrm{O} \text {. }
$$

I cc. $\frac{\mathrm{N}}{\mathrm{I}} \mathrm{KOH}$ is equivalent to $0.045 \mathrm{gm}$. of formic aldehyde. This modified method is quite as simple, and gives results practically identical with that of Romijn.

Oxidation by Hydrogen Dioxid (Blank and Finkenbeiner)* This method depends upon the use of hydrogen dioxid for oxidizing formaldehyde into formic acid, in alkaline solution. The formic acid so produced neutralizes a portion of the alkali, and the excess of the latter is then determined by titration with standard acid. The method gives good results and can be very rapidly carried out. It is the method adopted in the U. S. P. VIII, in which it is described as follows:

Transfer $3 \mathrm{cc}$. of solution of formaldehyde to a well-stoppered Erlenmeyer flask, and weigh accurately. Add $50 \mathrm{cc}$. of normal sodium hydroxid V. S., and follow this immediately, but slowly, through a small funnel, with $50 \mathrm{cc}$. of solution of hydrogen dioxid, to which a drop of litmus T. S. has been added, and which has been neutralized with normal sodium hydroxid V. S. After the reaction has ceased and the foaming has subsided, rinse the funnel and sides of the vessel with distilled water, and, after allowing it to stand ten minutes, titrate back with normal sulphuric acid V. S., using litmus T. S. as indicator. Subtract the number of cc. of normal sulphuric acid V. S. consumed, from 50 (the number of cc. of normal sodium hydroxid V. S. employed), multiply the remainder by 2.979 , and divide the product by the weight of the solution taken; the quotient

* Berichte d. Chem. Ges., XXXI, 2979 (1898); and A. J. Ph., 1899, 486. 
represents the percentage, by weight, of absolute formaldehyde in the liquid.

In the original process, double normal sodium hydroxid was used. The reaction is as follows:*

$$
\mathrm{CH}_{2} \mathrm{O}+\mathrm{H}_{2} \mathrm{O}_{2}+\mathrm{NaOH}=\mathrm{NaOOCH}+2 \mathrm{H}_{2} \mathrm{O} \text {. }
$$

The Iodometric Method (Romijn). $\dagger$ This method, which is. considered the most rapid, most accurate, and most readily applied, depends upon the fact that iodin in the presence of an alkali acts as an indirect oxidizing agent, giving, when formaldehyde is present, the iodid of the base and formic acid:

$$
\mathrm{CH}_{2} \mathrm{O}+\mathrm{I}_{2}+2 \mathrm{NaOH}=2 \mathrm{NaI}+\mathrm{CHOOH}+\mathrm{H}_{2} \mathrm{O} \text {. }
$$

As modified by L. Reuter $\ddagger$ it is as follows:

$20 \mathrm{cc}$. of 35 to 40 per cent formaldehyde are introduced into a graduated flask and distilled water added to bring up the volume to $500 \mathrm{cc}$. Of this thoroughly mixed fluid, $5 \mathrm{cc}$. are introduced into a bottle capable of being perfectly closed. $30 \mathrm{cc}$. of normal sodium or potassium hydroxid are added, and then $\frac{\mathrm{N}}{5}$ iodin solution allowed to flow in from a burette with constant agitation, until the fluid remains of a bright yellow color (36-6o cc.). The shaking is then vigorously continued for one minute, when $40 \mathrm{cc}$. of normal sulphuric acid are added, and then the excess of iodin titrated with decinormal thiosulphate solution. Each cc. of $\frac{\mathrm{N}}{\mathrm{IO}}$ iodin solution consumed corresponds to $0.0015 \mathrm{gm}$. formaldehyde. A blank titration should always be made.

A solution containing as much as 5 per cent may be accurately estimated by this method, provided not more than 2 gms. be taken. The method is, however, not accurate in the presence of other aldehydes. F. O. Taylor, $\S$ commenting upon this method, says that it is quite satisfactory, but that the quantities of formaldehyde and reagents used are unnecessarily large and cumbersome and the method is hence modified by him as follows:

From a weighing bottle, consisting of a small Erlenmeyer flask, fitted with a perforated rubber stopper through which passes a dropper,

* See C. Allen Lyford, J. A. C. S., XXIX, I 227 (1907,) “The Action of Barium Peroxid and Hydrogen Peroxid upon Formaldehyde."

$\dagger$ Zeitschr. anal. Chem., XXXVI, 18-24 (1897); ibid., XXXIX, 60-63 (1900).

$\ddagger$ Ph. Rev., I903, 207.

$\S$ Bull. Ph., Aug., 1903, 323 . 
and containing about 25 or $30 \mathrm{cc}$. of the formaldehyde solution, weigh out accurately about 10 gms. of the solution into a stoppered $500 \mathrm{cc}$. flask and fill this to the mark with distilled water. For titration remove $5 \mathrm{cc}$. of this solution, corresponding to $0.0 \mathrm{Igm}$. of the weighed quantity of formaldehyde, and put into a 200-cc. Erlenmeyer flask. Into another flask put $5 \mathrm{cc}$. of water for a blank titration. To both now add $20 \mathrm{cc}$. of normal $\mathrm{NaOH}$ and then $20 \mathrm{cc}$. of an approximately $\frac{\mathrm{N}}{5}$ iodin solution, whose exact strength need not be known, and let stand for five or ten minutes for the entire completion of the reaction. Now add $25 \mathrm{cc}$. of normal $\mathrm{H}_{2} \mathrm{SO}_{4}$ and titrate the excess of iodin with $\frac{\mathrm{N}}{\mathrm{IO}}$ $\mathrm{Na}_{2} \mathrm{~S}_{2} \mathrm{O}_{3}$. The difference between the cc. of thiosulphate used on the assay and the cc. of the blank is the number of cc. of $\frac{\mathrm{N}}{\mathrm{IO}}$ iodin consumed by the formaldehyde. Each cc. of $\frac{\mathrm{N}}{\mathrm{IO}}$ iodin so used equals $0.00 \mathrm{I} 5$ gm. of $\mathrm{CH}_{2} \mathrm{O}$.

The Potassium Cyanid Method* This method is especially applicable to solutions containing small quantities of formaldehyde. It depends upon the fact that potassium cyanid and formaldehyde combine to form an addition product, in which one molecule of potassium cyanid combines with one molecule of formaldehyde, as shown by the following equation:<smiles></smiles>

In the estimation, the formaldehyde is mixed with a known quantity of potassium cyanid (in excess), the excess of the latter being determined by the use of standard silver nitrate solution, and thus the quantity of potassium cyanid, which combined with formaldehyde is found, and from this the quantity of formaldehyde is calculated.

The process is carried out as follows: $\dagger$ to cc. of $\frac{\mathrm{N}}{\mathrm{IO}}$ silver nitrate are treated with six drops of 50 per cent nitric acid in a 50-cc. flask. Io cc. of a solution of potassium cyanid (containing I gm. of $\mathrm{KCN}$ in $500 \mathrm{cc}$. of water) are then added and well shaken. An aliquot part

* Romijn, Zeitschr. anal. Chem., XXXVI, I8-24 (1897).

$\dagger$ Bernard H. Smith, J. A. C. S., XXV, I032 (1903). 

of the filtrate, say $25 \mathrm{cc}$., is then titrated by Volhard's method with $\frac{\mathrm{N}}{\text { IO }}$
ammonium sulphocyanate for excess of silver.

Another Io cc. of silver nitrate solution is then acidified with nitric acid and treated with ro cc. of the potassium cyanid solution to which has been added a measured quantity of dilute formaldehyde solution. The whole is made up to $50 \mathrm{cc}$. and then filtered. $25 \mathrm{cc}$. of the filtrate are titrated with $\frac{\mathrm{N}}{\mathrm{IO}}$ ammonium sulphocyanate for excess of silver as before.

The difference between these two results multiplied by 2 gives the amount of potassium cyanid which was used by the formaldehyde, in terms of $\frac{\mathrm{N}}{\mathrm{IO}}$ sulphocyanate.

The best results by this method are obtained, if the solution of formaldehyde is diluted to below I per cent. With I per cent solutions it is necessary to use $15 \mathrm{cc}$. of the silver solution. In estimating very dilute solutions, it is advisable to use a $200-\mathrm{cc}$. flask and to take Ioo cc. of the filtrate for the titration. It is possible by this method to determine with accuracy one part in Ioo,000.

A New Method for the Estimation of Free Formaldehyde has been devised by Clowes and Cullens,* depending upon the fact that when formaldehyde is mixed with phloroglucin and hydrochloric acid condensation occurs, resulting in the separation of phloroglucid according to the following equation:

$$
\mathrm{C}_{6} \mathrm{H}_{6} \mathrm{O}_{3}+\mathrm{HCOH}=\mathrm{C}_{7} \mathrm{H}_{6} \mathrm{O}_{3}+\mathrm{H}_{2} \mathrm{O} .
$$

The substance under examination is mixed with $5 \mathrm{cc}$. of water and a mixture of $15 \mathrm{cc}$. of hydrochloric acid with a specific gravity of I.I9, I5 cc. of water and a slight excess of phloroglucin and heated to a temperature of $70^{\circ}$ to $80^{\circ} \mathrm{C}$. on the water-bath for two hours. If the filtrate yeilds phloroglucid upon heating with concentrated sulphuric acid the hydrochloric acid has not been sufficient to cause decomposition of the methylene derivative. In this case a second sample of the substance is heated with $5 \mathrm{cc}$. of water and then with a mixture of 10 to $20 \mathrm{cc}$. of concentrated sulphuric acid, ro $\mathrm{cc}$. of water and a slight excess of phloroglucin.

Bernard H. Smith, $\dagger$ in reviewing the various methods, concludes that the Blank and Finkenbeiner method is very satisfactory for strong solutions. That the Legler method is fairly satisfactory, but gives

* Berichte d. Chem. Ges., XXXII, 2841.

† J. A. C. S, XXV, 1028 (1903). 
lower results. That the iodometric and the potassium cyanid methods give good results on dilute solutions. The latter method is best adapted to the estimation of very small quantities of formaldehyde.

\section{LITERATURE}

Legler. Berichte d. Chem. Ges., XvI, I333.

Lösekann. Ibid., XxII, 1565 .

Eschweiler. Ibid., I929.

Klar. Ph. Zeitg., XL, 6r r.

Smith. Analyst, XxI, I48 (I896).

G. Romijn. Zeitschr. anal. Chem., xxxvi, I8 (1897).

Lederle. Am. Drug., Lxx, 246 (I897).

Orchard. XxII, 4 (1897).

Grützner. Archiv. d. Ph., ccxxxiv, 634 .

Carl E. Smith. A. J. Ph., Lxx, 86, 432 (1898).

G. L. Taylor. A. J. Ph., Lxx, April, 1898.

Kebler. Ibid., 432 (1898).

Legler. Ph. Centralh., xxxix, 253 (1898).

Blank and Finkenbeiner. Berichte d. Chem. Ges., xxxi, 2979 (1898); ibid., XXXII, 2I4I (1899).

Kastle and Loevenhart. J. A. C. S., xxI, 262 (1899).

Clowes and Cullens. Berichte d. Chem. Ges., xxxIr, 284r (I899).

Brochet and Cambier. Compt. rend., cxx, 440.

Romijn. Zeitschr. anal. Chem., xxxix, 60 (I900).

Legler. Ph. Centralh., XuII, 65I (I90I).

Craig. J. A. C. S., xxIII, 638 (Igor).

Ganino. Zeitschr. anal. Chem., xL, 587 (rgor).

Vanini and Seiter. Ibid.

Ripper. Monatsheft, xxi, Iо79.

Haywood. Bull. No. 73, U. S. Dept. Agric., Igo2.

Pfaff. Chem. Zg., xxvi, 70I (Ig02).

F. O. Taylor. Bull. Ph., Aug., 1903, 323 .

Bernard H. Smith. J. A. C. S., Xxv, I028 (I903).

H. Schiff. Chem. Zg., xxviI, I4 (IgO3).

C. Wallnitz. Deutsche Gerber Zg., Nos. I-4; 6, 8, I2.

L. Reuter. Ph. Rev., Xxi, 207 (1903).

R. Clauser. Berichte d. Chem. Ges., xxxvi, Ior (1903).

Lemme. Chem. Zg., xxviII, 896 (1903).

Gleissner and Sayre. Drug. Cir., I92 (1904).

Clemens Kleber. Ph. Rev., XxII, 94 (1904).

C. A. Male. Ph. Jour., June, I905, 844 .

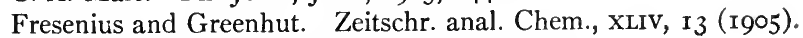

R. H. Williams. J. A. C. S., xxvIII, 596 (I905).

Haywood and Smith. Ibid., 1183. 


\section{CHAPTER LVII}

\section{ESTIMATION OF CHLOROFORM AND CHLORAL HYDRATE}

Chloroform, $\mathrm{CHCl}_{3}$. The volumetric estimation of chloroform is based upon the fact that when chloroform is.heated with an alkali hydroxid a formate and a chlorid of the alkali are formed. The reaction between the chloroform and the alkali takes place in definite proportions. An alcoholic solution of potassium hydroxid gives best results.

The process is carried out as follows: A weighed quantity of chloroform, which should be perfectly neutral in reaction, is introduced into a strong glass flask provided with a well-fitting glass stopper. To this is added an excess of normal alcoholic $\mathrm{KOH} \mathrm{V}$. S., the stopper securely tied down, and the flask warmed on a water-bath to $50^{\circ}$ or $60^{\circ} \mathrm{C}$. When reaction is complete the contents of the flask is cooled and then titrated with normal acid V. S. to find the excess of $\mathrm{KOH}$.

Each cc. of normal $\mathrm{KOH}$ represents $0.0296 \mathrm{I} \mathrm{gm}$. of chloroform.

$$
\begin{aligned}
& \mathrm{CHCl}_{3}+{ }_{4} \mathrm{KOH}={ }_{3} \mathrm{KCl}+\mathrm{HCOOK}+{ }_{2} \mathrm{H}_{2} \mathrm{O} \text {. } \\
& \frac{4) \mathrm{II} 8.45}{29.6 \mathrm{I}} \mathrm{gms} . \frac{4) 222.96}{55.74}=1000 \mathrm{cc} . \frac{\mathrm{N}}{10} \mathrm{~V} . \mathrm{S} . \\
& 0.0296 \mathrm{rgm} \text {. = I cc. " " }
\end{aligned}
$$

Instead of determining the excess of alkali as above described, a better method is that proposed by L. de Saint Martin.* In this process, the chloroform is heated with an excess of alcoholic potassium hydroxid in a sealed tube of glass. The tube is kept in boiling water for three hours, then cooled, opened, the alkali just neutralized with sulphuric acid, and then the chlorid determined with $\frac{\mathrm{N}}{10}$ silver nitrate solution, using chromate as indicator. W. A. Puckner $\dagger$ employs this method for the estimation of chloroform in chloroform-ether mixtures, such as are used in alkaloidal assaying. He uses instead of the sealed tube a two-ounce vial, stoppered with a sound cork 
firmly tied down. This vial is placed in boiling water in an upright position so that the contents do not come in contact with the cork, which would be acted upon by the alkali. The method is carried out as follows:

To Io cc. of an approximately normal alcoholic solution of potassium hydroxid, either free from chlorids or else of a known chlorid content, and contained in a vial, add a measured volume of the chloroform-ether mixture representing $0.05^{-0.2} \mathrm{gm}$. chloroform,* stopper with a sound cork, cover with cloth and tie this down firmly, mix the two liquids by rotation, then place the vial in boiling water in such a way that at no time the contents come in contact with the cork and retain the temperature for three hours. Remove the vial from the bath, let cool, add phenolphthalein and then sufficient sulphuric acid to exactly neutralize the liquid, then add two drops of potassium chromate T. S. and titrate with decinormal silver nitrate. Or if Volhard's method of estimation is preferred, add to the finished digestion Io cc. dilute nitric acid, an excess of decinormal silver nitrate, $5 \mathrm{cc}$. ferric ammonium sulphate T.S. and determine the excess of silver nitrate with decinormal potassium sulphocyanate. In either case I cc. of decinormal silver nitrate represents $0.003948 \mathrm{gm} . \mathrm{CHCl}_{3}$.

The method based upon the determination of the chlorid formed is to be preferred for accurate work to the alkalimetric method, for the reason that there is some decomposition of potassium formate with formation of potassium carbonate, which increases the alkalinity. An evident decomposition of the glass has the same effect, and hence the results by the alkalimetric method are low.

Chloral Hydrate $\left(\mathrm{C}_{2} \mathrm{HCl}_{3} \mathrm{O}+\mathrm{H}_{2} \mathrm{O}\right)$. When chloral hydrate is treated with an alkali it is decomposed and chloroform and an alkali formate are formed. The reaction must take place in the cold, or at least at the ordinary temperature, otherwise the alkali will attack and decompose the chloroform which is formed and hence the result would indicate too high a quantity of chloral hydrate.

The process is conducted as follows: A weighed quantity of chloral hydrate is dissolved in water, neutralized if it is acid, as is frequently the case, and then a measured excess of normal alkali is added. When a turbidity due to liberated chloroform is noted, swing until perfectly clear (one to two minutes). The excess is then determined by residual

* If the per cent of chloroform in the mixture is not even approximately known, I cc. may be digested with $25 \mathrm{cc}$. normal alcoholic potassium hydroxid solution for one hour, and the residual alkali determined with normal acid and phenolphthalein, when the cc. of normal alkali which disappeared during the digestion, multiplied by $0.0296 \mathrm{I}$, will give the amount of chloroform contained therein sufficiently close to judge the quantity to be taken for the actual determination. 
titration with normal hydrochloric acid, and thus the quantity of the alkali which reacted with the chloral hydrate is found, each cc. of which represents $0.16497 \mathrm{gm}$. of chloral hydrate.

The reaction is thus expressed:

$$
\begin{aligned}
& \mathrm{C}_{2} \mathrm{HCl}_{3}+\mathrm{H}_{2} \mathrm{O}+\mathrm{NaOH}=\mathrm{CHCl}_{3}+\mathrm{HCOONa}+\mathrm{H}_{2} \mathrm{O} \text {. } \\
& { }_{164.97} 40 \quad=1000 \mathrm{cc} . \frac{\mathrm{N}}{\mathrm{I}} \mathrm{V} . \mathrm{S} \text {. } \\
& 0.16497 \mathrm{gm} . \quad .040=\text { I cc. " “ " }
\end{aligned}
$$

The use of barium hydroxid is preferred by many to the sodium hydroxid.

Iodometric Method of Estimation. E. Rupp* recommends the following iodometric method for the estimation of chloral hydrate: To a mixture of $25 \mathrm{cc}$. of $\frac{\mathrm{N}}{\mathrm{rO}}$ iodin and $2.5 \mathrm{cc}$. $\frac{\mathrm{N}}{\mathrm{I}}$ potassium hydroxid in a glass-stoppered flask, ro cc. of chloral hydrate solution ( $\mathrm{I}: \mathrm{I} 00$ ) are added, the mixture shaken and allowed to stand five to ten minutes. It is then diluted with about $50 \mathrm{cc}$. of water, $5 \mathrm{cc}$. of hydrochloric acid added, and titrated with $\frac{\mathrm{N}}{\mathrm{ro}}$ thiosulphate, in the usual manner. The limit of $\frac{\mathrm{N}}{\mathrm{rO}}$ iodin consumed should be from Ir.75-rr.7 cc., as indicated by $13.25^{-1} 3.3$ cc. of $\frac{\mathrm{N}}{\text { ro }}$ thiosulphate consumed. I cc. $\frac{\mathrm{N}}{\text { 1O }} \mathrm{I}=0.008275$ gm. chloral hydrate.

* Arch. d. Ph., 241, No. 5 (July 31, 1903), 326-328. 


\section{CHAPTER LVIII}

\section{ASSAYING SURGICAL DRESSINGS}

THE assaying of surgical dressings, especially those most frequently employed, may be readily done by volumetric methods. Since the medicinal content of such dressings is usually very small, a sufficiently large quantity of the material must be taken for the assay.

The smallest quantity of medicinal substance is contained in the sublimate dressings, while salicylic, boric, and carbolic dressings contain larger proportions of the respective antiseptics, and as high as 30 per cent is contained in some iodoform dressings. Hence in assaying sublimate dressings a larger quantity of the material must be taken, while a comparatively small quantity is needed of iodoform dressing.

In taking a sample for analysis it is important that it be so selected from different parts of the package that it will fairly represent the average strength of the whole. S. W. Williams suggested that, as gauzes are sold by the yard, it is evident that the strength of the medication, even if expressed in terms per cent by weight, should have some definite relation to the measurement.

In the case of an expensive medication like iodoform it would seem far more equitable to give the strength in grains per square yard or grams per square meter. Thus the claims of the manufacturers might be compared on a common basis. This method would obviate the confusion attendant upon the allowance in the computation for water in the "moist dressings."

The weight of the water present in the so-called "moist dressings" is so varying a quantity that it must be excluded from the computation, in order to fairly compare the strength of moist dressings with the dry kind.

Carbolic Acid Dressing. If the dressing is of a low per cent, ro gms. are taken, if of a high per cent 5 gms. This is put into a liter flask, some water added and the whole warmed to and kept at about $80^{\circ} \mathrm{C}$. for some time, rotating the flask occasionally. Then allow to cool, dilute to the rooo-cc. mark, and filter. The paraffin, resin, or oil, etc., rises to the surface of the aqueous solution and is easily separated by filtration. The carbolic acid is then estimated 
by means of bromin solution, as described on page 615,25 or $50 \mathrm{cc}$. of the carbolic acid solution being taken.

Meissinger and Vortmann's Process (Pharm. Zeit. f. Russland, xxix, 759). Transfer $25 \mathrm{cc}$. of the carbolic acid solution prepared as above to a $150-\mathrm{cc}$. flask, and add $20 \mathrm{cc}$. of a I per cent solution sodium hydroxid (free from nitrite). Warm the mixture to $60^{\circ} \mathrm{C}$. and add $\frac{\mathrm{N}}{\mathrm{IO}}$ iodin solution from a burette, until the mixture in the flask assumes a permanent brownish-yellow color, and if much carbolic acid is present, deposits upon shaking, a red-colored precipitate. A lightrolored precipitate indicates deficiency of alkali. Cool the contents of the flask, acidulate with dilute sulphuric acid, and dilute to $150 \mathrm{cc}$. with distilled water and mix.

Remove ro cc. of this solution by means of a pipette, and titrate it with $\frac{\mathrm{N}}{\mathrm{IO}}$ sodium thiosulphate, using starch as indicator. The number of cc. thus found multiplied by 15 gives the cc. of $\frac{\mathrm{N}}{\mathrm{IO}}$ iodin solution which were added in excess to the carbolic acid solution. This deducted from the amount of iodin added, gives the number of $\mathrm{cc}$. of the latter which reacted with the carbolic acid.

One cc. of $\frac{\mathrm{N}}{10}$ iodin solution is equivalent to $0.001556 \mathrm{gm}$. of carbolic acid. Therefore, by multiplying this by the cc. of $\frac{\mathrm{N}}{\mathrm{IO}}$ iodin solution which reacted with the carbolic acid, the quantity of the latter in the $25 \mathrm{cc}$. of solution taken for analysis is ascertained.

If the carbolic acid solution is made from ro gms. of the dressing in a liter, $25 \mathrm{cc}$. of it represents 0.25 of the dressing. Hence to find the percentage by weight of the carbolic acid in the dressing, multiply the amount found by roo and divide by 0.25 .

Example. $25 \mathrm{cc}$. of the carbolic acid solution, representing $0.25 \mathrm{gm}$. of the dressing, were heated with $20 \mathrm{cc}$. of a I per cent solution of $\mathrm{NaOH}$, and $55 \mathrm{cc}$. of $\frac{\mathrm{N}}{\mathrm{IO}}$ iodin added, and the mixture made up to $\mathrm{I}_{50} \mathrm{cc}$. Io cc. of this titrated with $\frac{\mathrm{N}}{\mathrm{IO}}$ thiosulphate required $3.47 \mathrm{cc}$.

$$
3.47 \times 15=52.05 \mathrm{cc} \text {; }
$$

$$
55-52.05=2.95 \mathrm{cc} \text {; }
$$

$2.95 \times 0.001556=0.00459 \mathrm{gm}$.

then

$$
\frac{0.00459 \times 100}{0.25}=\mathrm{r} .83+\text { per cent. }
$$


In the above process the iodin reacts with the carbolic acid in proportion of one molecular weight of the latter and six atomic weights of iodin; the greater part of the iodin added is, however, taken up by the $\mathrm{NaOH}$ to form sodium iodid and iodate. Upon the addition of dilute sulphuric acid these two salts give up their iodin, but the iodin combined with the phenol is not liberated by the acid.

Salicylic Acid Dressings. 5 or Io gms. of the material, according to the claimed strength, are placed in a beaker or porcelain dish and heated with $500 \mathrm{cc}$. of distilled water. A few drops of phenolphthalein T. S. are then added and the solution titrated with $\frac{\mathrm{N}}{\mathrm{IO}}$ sodium hydroxid.

In case resinous or fatty matters are present they must be removed by filtration and the gauze or cotton thoroughly washed and pressed before the titration.

Each cc. of $\frac{\mathrm{N}}{\mathrm{IO}}$ alkali $=0.0137 \mathrm{gm}$. salicylic acid.

Benzoic acid in surgical dressings may be estimated in the same manner as the foregoing.

Each cc. $\frac{\mathrm{N}}{\mathrm{IO}}$ alkali $=0.0122 \mathrm{gm}$. benzoic acid.

Boric Acid Gauze. Beckurts and Danert (Apoth. Zeit.) give the following process for determining volumetrically the quantity of boric acid present in gauze: Cut 5 gms. of the gauze into fine shreds and shake with $400 \mathrm{cc}$. of a mixture of one part of glycerin and nineteen parts of water in a 500-cc. flask, adding later enough solvent to make up to $500 \mathrm{cc}$. Draw off $100 \mathrm{cc}$. of the clear fluid, and with the addition of phenolphthalein and some glycerin titrate with $\frac{\mathrm{N}}{\mathrm{IO}}$ sodium hydrate solution. The number of cc. of the solution required, when multiplied by 0.006 I 54 , gives the quantity of boric acid found in I gm. of the gauze; when multiplied by roo the percentage content is obtained. The quantity of glycerin added during titration is regulated by the appearance of alkalinity, for as soon as the solution shows an alkaline reaction glycerin is added; this is followed usually at first by disappearance of the red color until actual neutralization has taken place. If sufficient glycerin is added, the color reactions are rendered sharp.

Sublimate Dressings. In the estimation of mercuric chlorid in surgical dressings the available bichlorid only should be computed.

The mercury which is present in any other form but bichlorid must be excluded from consideration. It is to be remembered that corrosive 
sublimate reacts and forms with many organic substances, compounds which are insoluble or which have no germicidal power.

Moreover, sublimated dressings contain such minute proportions of the bichlorid that even traces of impurities, such as wood, gum, oil, fatty acids, etc., may be capable of destroying all of the bichlorid present. Even the cotton of which the dressing is made has a reducing influence upon the bichlorid.

Therefore, in assaying a sublimate dressing, the mercuric chlorid and not the mercury should be estimated. Hence the dressing should not be exhausted for the assay with acidulated water, which would tend to dissolve some of the bichlorid combined with the cellulose.

The available sublimate may best be extracted by means of hot sodium chlorid solution.

Denner's Process, Modified by Link and Vaswinkel. Macerate a weighed portion of the dressing, say 20 gms., in a 20-ounce glassstoppered bottle with $500 \mathrm{cc}$. of distilled water, shaking frequently for one hour. Decant an aliquot portion, say $250 \mathrm{cc}$., into an evaporating-dish. Add roo cc. of chlorin-water and a few drops of hydrochloric acid and evaporate to expel the chlorin and reduce to small bulk. Treat with sulphureted hydrogen, filter and wash the precipitate very thoroughly. Transfer with the filter-paper to a small glass-stoppered bottle; add I or 2 cc. of carbon disulphid and $50 \mathrm{cc}$. of $\frac{\mathrm{N}}{50}$ iodin solution. Stopper tightly and let stand, with frequent shaking, until the black precipitate of mercuric sulphid is dissolved. The carbon disulphid dissolves the separated sulphur and thus prevents it from interfering with the reaction. Open carefully to avoid loss by spurting, and add $50 \mathrm{cc}$. of $\frac{\mathrm{N}}{50}$ sodium thiosulphate and starchpaste indicator. Stopper the bottle, and shake vigorously until there remains no color indicative of free iodin. Open carefully again, and titrate the excess of thiosulphate with $\frac{\mathrm{N}}{50}$ iodin. This last result represents the amount of iodin bound by the mercuric sulphid, according to the equation:

$$
\mathrm{HgS}+2 \mathrm{KI}+\mathrm{I}_{2}=\left(\mathrm{HgI}_{2} \cdot 2 \mathrm{KI}\right)+\mathrm{S},
$$

and each cc. corresponds to $0.002688 \mathrm{gm}$. of mercury bichlorid in the $250 \mathrm{cc}$. of solution used for assay.

The Original Denner's Process * is as follows: A weighed quantity

* Ph. Centralh., XXIX. 207 (1888). 
of the dressing to be examined is exhausted by digestion with a known quantity of 0.7 per cent hot salt solution and then in a definite quantity of the filtered solution, faintly acidulated, the sublimated is precipitated with hydric sulphid. The mixture is then heated to boiling and the precipitated mercuric sulphid separated by filtration through cotton and thoroughly washed. The precipitate together with the cotton is then put into a glass-stoppered Erlenmeyer flask, and after the addition of several cc. of carbon disulphid, a measured excess of $\frac{\mathrm{N}}{\mathrm{IO}}$ iodin is added, and finally the excess of the latter found by titration with $\frac{\mathrm{N}}{10}$ sodium thiosulphate.

Beckurt's Process. Weigh about 20 gms. of the gauze or cotton to be assayed. Macerate in a liter cylinder with $250 \mathrm{cc}$. of warm distilled water containing $0.5 \mathrm{gm}$. of sodium chlorid. When cool dilute with distilled water to I liter, taking care that no air-bubbles are enmeshed in the material. Shake well and filter off $500 \mathrm{cc}$. (more accurately $493 \mathrm{cc}$., allowing for the volume of $20 \mathrm{gms}$. of cellulose) into a boiling flask with $0.2 \mathrm{gm}$. of ferrous sulphate in solution. Mix add excess of sodium hydroxid, and then dilute sulphuric acid in slight excess. The sublimate present is thus reduced to calomel, and may be estimated by adding from a burette $\frac{\mathrm{N}}{50}$ iodin until the color indicates a slight excess. Then titrate back the excess with $\frac{\mathrm{N}}{50}$ sodium thiosulphate and starch indicator. The difference between the $\frac{\mathrm{N}}{50}$ iodin and $\frac{\mathrm{N}}{50}$ thiosulphate used represents the iodin reacting with the mercury present (in the absence of other reducing agents), according to the equation

$$
\mathrm{H}_{\oint_{2}} \mathrm{Cl}_{2}+6 \mathrm{KI}+2 \mathrm{I}=2\left(\mathrm{HgI}_{2} \cdot 2 \mathrm{KI}\right)+2 \mathrm{KCl},
$$

and each cc. of $\frac{\mathrm{N}}{50}$ iodin so used corresponds to $0.0027688 \mathrm{gm}$. of sublimate in the $500 \mathrm{cc}$. of solution used for asșay.

Iodoform Dressings. Io gms. of the dressing are digested with alcohol until thoroughly exhausted. The alcoholic solution of iodoform is then placed upon a water-bath, acidulated with a few drops of nitric acid, and titrated with a $\frac{\mathrm{N}}{\mathrm{IO}}$ alcoholic solution of silver nitrate.

The end-point is known when a drop of the solution brought in contact with a drop of sodium chlorid solution produces a turbidity. 
It can be told approximately by the disappearance of the greenishyellow color of the solution. It becomes colorless when the reaction is complete. The reaction is as follows:

$$
\mathrm{CHI}_{3}+{ }_{3} \mathrm{AgNO}_{3}+\mathrm{H}_{2} \mathrm{O}={ }_{3} \mathrm{AgI}+{ }_{3} \mathrm{HNO}_{3}+\mathrm{CO} .
$$

Each cc. of the $\frac{\mathrm{N}}{10} \mathrm{AgNO}_{3}$ V. S. $=0.01302 \mathrm{gm}$. of iodoform.

The following process (Huss') may be applied to all dressings containing iodin, iodoform, iodol, sozoiodol, aristol, etc. It is based upon the fact that such compounds when heated with metallic zinc yield all their iodin with formation of zinc iodid.

Five grams of the dressing are placed in a dry test-tube, $20 \mathrm{gms}$. of zinc dust added, and shaken down, leaving a layer of the zinc $2 \mathrm{~cm}$. thick about the dressing. The whole is heated some time in a water-bath. The zinc is then washed out and transferred to a 500-cc. volume flask, which also bears a mark at $503 \mathrm{cc}$. The flask is filled with water to the $503-\mathrm{cc}$. mark (compensating for the volume of $20 \mathrm{gms}$. of zinc dust, sp.gr. 7.17). $250 \mathrm{cc}$. of the mixture is boiled with sodium carbonate, diluted to $500 \mathrm{cc}$., and $250 \mathrm{cc}$. filtered off. In this solution the iodin as sodium iodin is titrated with $\frac{\mathrm{N}}{\text { IO }}$ silver nitrate V. S., each cc. of which represents $0.01259 \mathrm{gm}$. iodin.

Commercial zinc dust usually contains zinc oxid; it is purified by treatment with dilute hydrochloric acid, washing with water till the chlorin reaction disappears, and subsequently with alcohol, and drying.

Other methods depend upon digesting the iodoform dressing with an alcoholic solution of sodium hydroxid, thus forming sodium iodid, which is estimated by titrating with $\frac{\mathrm{N}}{\mathrm{IO}} \mathrm{AgNO}_{3} \mathrm{~V}$. S.

Lehmann's Method. Martin Lehmann gave 'in the Berlin Pharmaceutische Zeitung (1900, No. I5) a method for the volumetric estimation of iodoform in surgical dressings, and in a later number of the same journal (No. 54) proposes some modifications which improve the process. The process, as modified, is carried out in the following manner: Io gms. of the dressing under examination, either gauze or cotton, are put into a glass-stoppered bottle, and $200 \mathrm{cc}$. of spirit of ether are poured on it. This is allowed to stand for twenty-four hours, being frequently shaken, at a temperature of from $20^{\circ}$ to $25^{\circ} \mathrm{C}$. The yellow solution of iodoform is gradually decomposed and in consequence changes from yellow to reddish brown from the liberation of free iodin. $20 \mathrm{cc}$. of this 
solution are placed in an Erlenmeyer flask having a capacity of $250 \mathrm{cc}$. A quantity of decinormal silver nitrate solution proportionate to the amount of iodin thought to be present is added, and after the addition of ten or fifteen drops of fuming nitric acid, the flask is warmed on a water-bath until the odor of both the ether and the nitrous acid have disappeared. After cooling and diluting with water the liquid is titrated with decinormal ammonium sulphocyanate solution, using I cc. of cold saturated ferric alum solution, until the color turns from white to a permanent light red. In examining gauze precisely one meter should be measured off and carefully weighed. The author gives the results of a series of examinations, which are of interest as demonstrating the fact that there is a constant loss in strength by keeping.

Styptic Cotton (C. E. Parker, Drug. Cir., I895, 23I). The iron in styptic cotton is determined by macerating 3 to $5 \mathrm{gms}$. in $50 \mathrm{cc}$. of water. The ferric salt is reduced by adding stannous chlorid in hydrochloric acid solution until the brown color disappears, then removing the excess of stannous chlorid by the addition of mercuric chlorid as long as a precipitate is produced, and finally titrating with $\frac{\mathrm{N}}{\mathrm{IO}}$ potassium dichromate solution until a drop tested on a white plate no longer gives a blue color with a drop of freshly prepared potassium ferricyanid solution, but only a bluish-gray color.

Each cc. of $\frac{\mathrm{N}}{\mathrm{IO}} \mathrm{K}_{2} \mathrm{Cr}_{2} \mathrm{O}_{7}=0.00555 \mathrm{gm}$. of metallic iron or $0.016 \mathrm{rO} 4$ gm. of ferric chlorid. 


\section{CHAPTER LIX}

\section{ESTIMATION OF COMPOUND ETHERS}

CoMpound ETHERs, also called esters, correspond in structure to the salts of the metals, in which the metal is replaced by a hydrocarbon radical.

All compound ethers when treated with a strong alkali give up their acids to the alkali, and set free the hydroxid of the hydrocarbon radical. Thus ethyl sulphate, $\left(\mathrm{C}_{2} \mathrm{H}_{5}\right)_{2} \mathrm{SO}_{4}$, when treated with ${ }_{2} \mathrm{KOH}$ reacts as follows:

$$
\left(\mathrm{C}_{2} \mathrm{H}_{5}\right)_{2} \mathrm{SO}_{4}+{ }_{2} \mathrm{KOH}={ }_{2} \mathrm{C}_{2} \mathrm{H}_{5} \mathrm{OH}+\mathrm{K}_{2} \mathrm{SO}_{4} \text {. }
$$

This decomposition is termed saponification and upon it is based a method for the estimation of compound ethers. In the process a measured quantity of normal alkali in decided excess is brought in contact with a weighed quantity of the compound ether, and when saponification is complete the excess of alkali is determined by retitration with normal acid solution and thus the quantity of normal alkali which went into combination with the ester is obtained, each cc. of which represents, of

$$
\begin{aligned}
& \text { Ethyl sulphate, }\left(\mathrm{C}_{2} \mathrm{H}_{5}\right)_{2} \mathrm{SO}_{4} \ldots \ldots \ldots \ldots \ldots . .076495 \mathrm{gm} \text {. } \\
& \text { Ethyl acetate, } \mathrm{C}_{2} \mathrm{H}_{5} \mathrm{C}_{2} \mathrm{H}_{3} \mathrm{O}_{2} \ldots \ldots \ldots \ldots . .0874 \mathrm{gm} \text {. } \\
& \text { Ethyl chlorid, } \mathrm{C}_{2} \mathrm{H}_{5} \mathrm{Cl} \ldots \ldots \ldots \ldots \ldots \ldots . . . . . .664 \mathrm{gm} \text {. } \\
& \text { Ethyl nitrite, } \mathrm{C}_{2} \mathrm{H}_{5} \mathrm{NO}_{2} \ldots \ldots \ldots \ldots \ldots . .0745 \mathrm{I} \text { gm. }
\end{aligned}
$$

In this process certain precautions must be taken. The decomposition does not occur immediately, but takes place slowly; it may be hastened by the application of heat, but since most esters are volatile, great care must be exercised so as not to dissipate it.

A quantity of the ether is weighed in a weighing-flask, which can be well closed with a tightly-fitting stopper. A measured excess of the alkali solution is then run in from a burette, the solution diluted with some water, for the decomposition is effected more readily in dilute than in concentrated solutions, the stopper inserted, and the flask placed upon a water-bath and heated to $50^{\circ}$ or $60^{\circ} \mathrm{C}$. It is 
kept at this temperature for two hours and frequently shaken. The flask and contents are then cooled and the stopper withdrawn. If the saponification is complete, the characteristic odor of the ether is no longer noticeable.*

The retitration with normal acid is now in order. If barium hydroxid was used for the saponification, normal oxalic acid solution is indicated.

In the case of esters containing volatile acids the direct method of analysis may be carried out as follows:

The ester is boiled with an excess of sulphuric acid until complete saponification is effected. The liberated volatile acid being distilled off in a current of steam and collected in a measured excess of standard alkali. The unneutralized alkali being then found by titration with standard acid, or if preferred, the saponification may be done with alkali, as in the foregoing method, and then an excess of sulphuric acid added and the liberated acid distilled into standard alkali solution.

\section{ESTIMATION OF SPIRIT OF NITROUS ETHER AND AMYL NITRITE}

(The Chlorate Method)

Spirit of Nitrous Ether. The following method for determining the amount of ethyl nitrite in spirit of nitrous ether is given in the Süddeutsche Apotheker-Zeitung, I897, 306. Io gms. of the spirit are treated with $20 \mathrm{gms}$. of a 5 per cent solution of potassium chlorate and 5 gms. of nitric acid (sp.gr. I.I53), and allowed to stand for an hour in a closed flask, shaking occasionally. The ethyl nitrite is thus oxidized to ethyl nitrate, and the potassium chlorate reduced to chlorid. The reaction is probably as follows:

$$
{ }_{3} \mathrm{C}_{2} \mathrm{H}_{5} \mathrm{NO}_{2}+\mathrm{KClO}_{3}=\mathrm{KCl}+{ }_{3} \mathrm{C}_{2} \mathrm{H}_{5} \mathrm{NO}_{3} .
$$

The chlorid so formed is then estimated by means of $\frac{\mathrm{N}}{\mathrm{IO}}$ silver nitrate solution.

To the above mixture $25 \mathrm{cc}$. of $\frac{\mathrm{N}}{10}$ silver nitrate solution are added, together with a few drops of a saturated solution of ammonio-ferric sulphate, and then the excess of silver nitrate solution determined by retitration with $\frac{\mathrm{N}}{\mathrm{IO}}$ ammonium sulphocyanate or potassium sulpho-

* It is usually better to boil the mixture in a flask provided with a reflux condenser. 
cyanate. By deducting the quantity of sulphocyanate solution used from the $25 \mathrm{cc}$. of $\frac{\mathrm{N}}{\mathrm{IO}}$ silver nitrate added, the quantity of the latter which combined with the potassium chlorid is found. This multiplied by 0.022353 gives the weight of ethyl nitrite in the ro gms. taken.

$$
\begin{aligned}
& { }_{3} \mathrm{C}_{2} \mathrm{H}_{5} \mathrm{NO}_{2}=\mathrm{KCl}=\mathrm{AgNO}_{3} \text {. }
\end{aligned}
$$

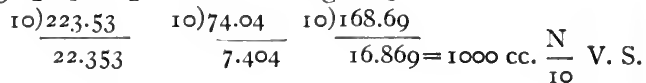

$$
\begin{aligned}
& 0.022353 \mathrm{gm} .=0.007404 \mathrm{gm} .=\mathrm{I} \text { cc. } " \text { " }
\end{aligned}
$$

Carl E. Smith (A. J. Ph., I898, 273) proposes the following modification of the above method: Into a I00-cc. flask or bottle of white glass, provided with a loosely-fitting stopper of glass, rubber, or cork, place successively ro cc. of distilled water, $5 \mathrm{cc}$. of a cold, aqueous, saturated solution of potassium chlorate, $5 \mathrm{cc}$. of the spirit to be tested, and $5 \mathrm{cc}$. of ro per cent nitric acid. Quickly insert the stopper and shake frequently during thirty minutes. Then add ro cc. of $\frac{\mathrm{N}}{\mathrm{IO}}$ silver nitrate, shake briskly for a moment, add ten drops of ferric ammonium sulphate solution, and titrate the excess of silver nitrate with $\frac{\mathrm{N}}{\mathrm{IO}}$ potassium sulphocyanate, without delay, so that the darkening of the precipitated silver chlorid may not obscure the end-reaction. This is reached, when, after momentary shaking, upon the addition of the last drop of solution, the appearing red color is not entirely dispersed, but leaves the liquid faintly reddish throughout. Assuming the spirit to contain 4 per cent by weight of ethyl nitrite, and to have a specific gravity of 0.84 , it would require $2.55 \mathrm{cc}$. $\frac{\mathrm{N}}{\mathrm{IO}}$ potassium sulphocyanate to precipitate the excess of silver in solution; and as each cc. of $\frac{\mathrm{N}}{10}$ silver nitrate consumed in precipitating the chlorid formed corresponds to $0.02235 \mathrm{gm}$. of ethyl nitrite, the calculation is as follows:

$$
\frac{(10-2.55) \times 0.02235 \times 100}{5 \times 0.84}=4.0 \text { per cent. }
$$

If it is desired to avoid calculation entirely, however, $2.7 \mathrm{cc}$. of spirit of nitrous ether and half the quantities of the reagents may be taken, in which case each cc. of $\frac{\mathrm{N}}{\mathrm{IO}}$ silver nitrate consumed indicates I per cent of ethyl nitrite. 
The valuation of amyl nitrite is made by first diluting the amyl nitrite with alcohol so as to make a 5 or 6 per cent dilution. Then, using just double the quantity of this (=Io cc.) and the reagents in the same doubled proportion, the process is carried out exactly as in the case of spirit of nitrous ether, assuming the alcoholic dilution to contain $6.037 \mathrm{gms}$ : of the sample in Ioo cc.; the ro cc. taken for assay contain $0.6037 \mathrm{gm}$. If in titrating the excess of silver, $5.45 \mathrm{cc}$. of $\frac{\mathrm{N}}{10}$ potassium sulphocyanate are required $(20-5.45)$ I $4.55 \mathrm{cc}$. of

$\frac{\mathrm{N}}{\mathrm{IO}}$ silver nitrate-each cc. equivalent to $0.03487 \mathrm{gm}$. of amyl nitrite -have been consumed in precipitating the chlorid formed in the reaction, and the calculation is as follows:

$\frac{\mathrm{I} 4.55 \times 0.03487 \times 100}{0.6037}=84.6$ per cent. 


\section{CHAPTER LX}

\section{URINE}

NORMAL URINE when fresh is clear and transparent. Its coior is yellowish, reddish, or colorless. It has a peculiar odor, a distinctly acid reaction, and its average specific gravity is from 1015 to 1028 .

On standing it generally gives a slight cloud of mucus, which slowly sinks to the bottom, and, after heavy exercise or a hearty meal of nitrogenous food, a sediment of urates.

If the urine be very dilute and the temperature is above the mean, decomposition rapidly takes place, and the urine becomes turbid, acquires an alkaline reaction, and develops a nauseous ammoniacal odor.

Reaction. The acid reaction of fresh urine is probably due to the presence of acid phosphate of sodium. If it has an alkaline reaction when first voided it is probably due to the conversion of urea into ammonium carbonate within the bladder; it is then generally turbid, and indicates an abnormal condition.

The reaction is best tested by dropping a small piece of a red and blue litmus-paper into it. If both are found red in a few minutes the reaction is acid, if both are blue it is alkaline, if both remain unchanged it is neutral.

Composition. The average composition of healthy urine is as follows:

\begin{tabular}{|c|c|c|}
\hline & Per Cent. & $\begin{array}{c}\text { Grains } \\
\text { per Diem. }\end{array}$ \\
\hline Water $\ldots \ldots \ldots \ldots \ldots \ldots \ldots \ldots, \ldots$ & 96.00 & 50 fl.ozs. \\
\hline Solids as tabulated below..... & 4.00 & $1000 \mathrm{grs}$. \\
\hline Urea ............... & 2.50 & $500 “$ \\
\hline Uric acid........... & 0.04 & $9.5 \mathrm{grs}$. \\
\hline Hippuric acid............ & 0.075 & $15.0^{\circ} \%$ \\
\hline Creatinine..$\ldots \ldots \ldots \ldots \ldots \ldots \ldots \ldots \ldots \ldots \ldots \ldots \ldots \ldots \ldots \ldots \ldots$ & 0.075 & $15.0 "$ \\
\hline Pigment, mucus, xanthin, and other extractives .... & 0.50 & $170.0 "$ \\
\hline Chlorids of potassium and sodium ............ & 0.50 & $170.0 “$ \\
\hline Sulphates of potassium and calcium ............ & 0.11 & $40.0^{\circ}$ \\
\hline Phosphates oi potassium and sodium ........... & 0.12 & $45.0^{\circ}$ \\
\hline Phosphates of magnesium and calcium .......... & 0.80 & $35.5 “$ \\
\hline
\end{tabular}


Beside these there have been found traces of indican, diastase, glucose, oxalic acid, lactic acid, carbolic acid, and unoxidized sulphur and phosphorus. (From "The Urine," Holland.)

The composition of urine is not constant: it is influenced by the amount of water and other fluids taken, by the temperature of the skin, by the emotions, the blood-pressure, the amount of work done, the time of day, age, sex, and medicine.

The Quantity passed in twenty-four hours varies considerably. The average quantity passed daily by a healthy adult is I400 to I600 cc.-about 50 fl.ozs. The quantity of total solids contained in this is, as seen in the table, about 60 gms., or rooo grains. About one half of these solids is composed of urea.

In making an analysis of urine the analyst looks for the presence of abnormal constituents, and determines the excess or deficiency of the normal constituents; and therefore, since the composition of urine is not the same at all hours of the day, it is important when accurate results are desired to examine a portion of the total quantity of the urine passed in twenty-four hours. If this cannot easily be obtained, or only a casual examination is to be made, the first urine passed in the morning may be used.

Specific Gravity. This varies from IOI5 to I028, according to the degree of dilution or concentration. But the pathological urine may vary from almost that of water to 1050. The urine of Bright's disease is, as a rule, of low specific gravity, and in diabetes of high specific gravity.

The specific gravity may be taken by any of the usual methods, but the urinometer (a special hydrometer, see Fig. 92) is generally used for this purpose. This instrument is usually graduated so that only the last two figures of the specific gravity appear upon the stem, and so as to read correctly at $60^{\circ} \mathrm{F}$. If the temperature is above $60^{\circ} \mathrm{F}$. it will be sufficiently accurate for ordinary clinical purposes to add one degree in specific gravity for every to degrees of temperature; that is, if it read ror 8 at $80^{\circ} \mathrm{F}$., it would read 1020 at $60^{\circ} \mathrm{F}$., or for every $\mathrm{I}^{\circ} \mathrm{F}$. above $60^{\circ}$ add $0.000 \mathrm{I}$ to the observed specific gravity. The urinometer is used as follows: Sufficient urine is placed in the upright jar or cylinder to float the urinometer, which is carefully introduced. When it has come to rest bring the eye on a level with the surface of the liquid in the jar, and take the reading at the lower edge of the meniscus formed by the upper surface of the urine.

The mark on the instrument which is cut by this line, and which can be distinctly seen, is taken as the correct reading.

If the urine be turbid this method cannot be employed. 
After taking the specific gravity, reaction, etc., set a portion of the urine aside in a conical glass so as to allow a deposit to form, which must be examined microscopically and chemically.

Total Solids. The total solids in urine may be roughly estimated as follows:

The last two figures of the specific gravity, when multiplied by the factor $2.33{ }^{*}$ will give the number of grams of solid matter in $1000 \mathrm{cc}$. of the urine.

From this it is easy to calculate the quantity of solids passed in twenty-four hours.

If, for example, $1500 \mathrm{cc}$. of urine were passed in twenty-four hours,
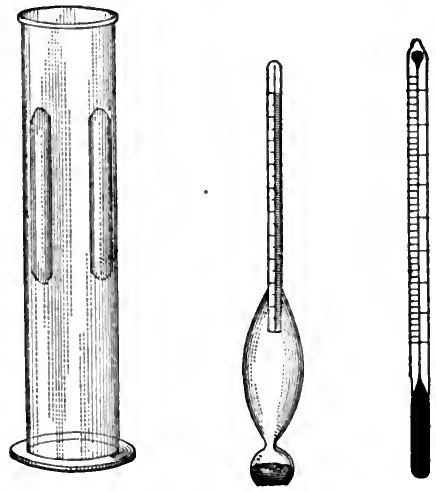

Fig. 92.

and the specific gravity of this was 1020, the total solids would be $20 \times 2.33=46.6 \mathrm{gms}$. in $1000 \mathrm{cc}$. In $1500 \mathrm{cc}$. there will be $\frac{46.6 \times 15}{10}$ $=69.9 \mathrm{gms}$. If it be desired to use the English measures, we may determine the total solids by multiplying the last two figures of the specific gravity by the number of fluid ounces of urine passed, for these last two figures represent approximately the grains of solid matter in a fluid ounce. Thus if 50 fluid ounces were passed and the specific gravity is 1020 , the total solids will be $50 \times 20=1000$ grs. in twentyfour hours.

* Neubauer, Zeitschr. anal. Chem., I, $166 . \quad$ J. H. Long (J. A. C. S., XXV, 257 and $87 \mathrm{I}$ ) discusses the relationship between the total solids and the specific gravity of urine, and as a result of his investigations establishes the factor 2.51. The specific gravity of the urine being taken at $25^{\circ} \mathrm{C}$. instead of $15^{\circ} \mathrm{C}$., as was done by Neubauer. 
A more exact method of determining the total solids is to evaporate Io cc. in a white porcelain dish and dry in a water-oven to a constant weight. The difference between the weight of the dish, and of the dish with the solids will be the weight of the solids in Io cc. of urine. Even by this method there is some loss through volatilization.

Chlorids. For the detection of chlorids a few drops of nitric acid are added to the urine in a test-tube, and then silver nitrate test solution. A white, curdy precipitate of silver chlorid forms, which should occupy not more than one fourth the volume of the urine taken. If it occupies more the chlorids are said to be increased; if it occupies less space than one fourth, the chlorids are diminished. It is always advisable to compare the specimen under examination with normal urine, subjected to the same test. In most cases such an approximate result is all that is required in a clinical examination.

The Volumetric Estimation. It is sometimes necessary to make a more accurate determination. For this purpose a decinormal solution of silver nitrate is used. Io cc. of the urine are diluted with about 50 cc. of water; a few drops of potassium chromate T. S. are added, and then the decinormal silver nitrate $\mathrm{V}$. S. run in from a burette until a permanent reddish color is produced. Note the number of cc. of the V. S. used, and multiply this number by the factor for chlorin, $0.003518 \mathrm{gm}$., or the factor for sodium chlorid, $0.005806 \mathrm{gm}$. This will give the quantity of chlorin or sodium chlorid in ro cc. of urine. This when multiplied by 10 gives the percentage. In highly colored urines this method is sometimes inapplicable, because the change of color is hidden by the color of the urine. In such cases Volhard's method (see page I22) may be employed.

Phosphates. Phosphoric acid exists in the urine combined with the alkalies and with the alkali earths. These phosphates are, therefore, generally distinguished by the terms alkali and earthy phosphates. By adding an alkali to normal urine the earthy phosphates (calcium and magnesium) are precipitated.

The earthy phosphates may be approximately estimated by adding a few drops of ammonia-water to the urine and observing the amount of turbidity produced after boiling. By comparing this with the amount obtained by the same treatment of normal urine the excess or deficiency is determined. The precipitate is $\mathrm{Ca}_{3}\left(\mathrm{PQ}_{4}\right)_{2}$ and $\mathrm{MgNH}_{4} \mathrm{PO}_{4}$.

The alkali phosphates may be detected in the filtrate from the earthy phosphates by the addition of a few drops of magnesium sulphate solution and some ammonium chlorid. The precipitate will be much more voluminous than that produced by the earthy phos- 
phates, and the excess or deficiency may be determined by comparison with normal urine. The precipitate has the composition $\mathrm{MgNH}_{4} \mathrm{PO}_{4}$.

The Quantitative Estimation of Phosphate is rarely required but may be made by the volumetric process with uranium nitrate (see also page 3 I4).

Total Phosphates. $50 \mathrm{cc}$. of the urine are poured into a beaker and $5 \mathrm{cc}$. of a saturated sodium acetate solution are added, together with a slight excess of acetic acid. The mixture is warmed over a water-bath to near boiling, and then titrated with the uranium solution, which is added, drop by drop, as long as a precipitate falls, or until a drop of the hot solution brought in contact, on a white porcelain plate, with a drop of freshly prepared potassium ferrocyanid solution, produces a brown color due to the formation of uranic ferrocyanid.

The quantity of uranic solution used is next read off and the phosphoric acid calculated.

Each cc. of uranic solution represents

$$
\begin{aligned}
& 0.005 \text { gm. of } \mathrm{P}_{2} \mathrm{O}_{5} ; \\
& 0.0069 \text { " " } \mathrm{H}_{3} \mathrm{PO}_{4} \text {. }
\end{aligned}
$$

A second and even a third titration should always be made, and all the above-named conditions should in every case be strictly adhered to.

The Earthy Phosphates are found by adding to $200 \mathrm{cc}$. of the urine a sufficient quantity of ammonia-water to render it strongly alkaline. This causes the earthy phosphates to precipitate. After the mixture has stood for twelve hours, the precipitate is collected on a filter and thoroughly washed with dilute ammonia-water $(\mathrm{I}: 3)$. A hole is then made in the bottom of the filter and the precipitate washed through it into a beaker and dissolved in the smallest possible quantity of hot acetic acid. To this solution are added $5 \mathrm{cc}$. of the sodium acetate solution and water sufficient to make $50 \mathrm{cc}$., and then treated as for total phosphates. The amount of earthy phosphates thus found subtracted from the total phosphates gives the alkali phosphates.

Sulphates. There are two kinds of sulphates in urine: the mineral sulphates $\left(\mathrm{K}_{2} \mathrm{SO}_{4}, \mathrm{Na}_{2} \mathrm{SO}_{4}\right.$, and $\left.\mathrm{MgSO}_{4}\right)$ and the ethereal sulphates. The mineral sulphates constitute about nine tenths of the total sulphates, the remaining one tenth is in the form of phenol, cresol, indoxyl, and skatoxyl sulphates of potassium. These latter are products of proteid putrefaction, absorbed from the intestinal canal. Their normal amount varies between 0.12 and $0.3 \mathrm{gm}$. Any increase is roughly indicative of intestinal indigestion.

About 30 grains, or 2 grams, of sulphates are daily discharged in the urine. 
Test. A few drops of hydrochloric acid are added to the in a test-tube to prevent the formation of barium phosphate. Barium chlorid T. S. is now added, which causes a white precipitate in the presence of sulphates.

Volumetric Estimation. This is done by the use of a standard solution of barium chlorid. (See also page 33I.)

The Gravimetric Method. Take roo cc. of urine, add $5 \mathrm{cc}$. $\mathrm{HCl}$ and heat to near boiling, then add barium chlorid T. S. in slight excess; place the beaker containing the mixture on a water-bath until the precipitate has subsided, decant the clear liquid carefully from the precipitate, add hot water, and when the precipitate has again settled decant again; continue this until the decanted liquid no longer gives a cloudiness with sulphuric acid. Then dry the precipitate and weigh carefully. This gives the quantity of $\mathrm{BaSO}_{4}$ which is precipitated out of the urine by barium chlorid.

207.7 parts of barium sulphate represent 98 parts of sulphuric acid. Therefore by multiplying the weight obtained by 98 and dividing by 207.7 the number of grams of sulphuric acid in the Ioo cc. of urine taken is obtained. From this we can easily calculate the quantity eliminated in twenty-four hours.

Estimation of Ethereal Sulphates Only. Take 200 cc. of urine, add an equal volume of barium chlorid solution, made alkaline with barium hydroxid, filter off the precipitate, and the clear filtrate will contain the ethereal sulphates. These are not precipitable by barium chlorid until they are decomposed by boiling with hydrochloric acid. Take $200 \mathrm{cc}$. of this filtrate (representing Ioo cc. of urine), add $20 \mathrm{cc}$. of hydorchloric acid and boil ten minutes. Filter, dry the precipitate, and weigh.

Total Acidity. Place $50 \mathrm{cc}$. of the urine in a beaker, add three or four drops of phenolphthalein, and then run into the beaker carefully from a burette decinormal sodium hydroxid V. S. until a faint permanent red color appears. The number of cc. of the decinormal alkali used, multiplied by 0.006255 , gives the acidity of $50 \mathrm{cc}$. of the urine expressed in grams of oxalic acid. From this the total acidity is determined by multiplying by the quantity of urine passed in twentyfour hours and dividing by 50 .

If the urine is highly colored the end-reaction is sometimes difficult to see. In such a case the color may be removed by shaking up a portion of the urine with coarsely powdered animal charcoal, then filtering. The urine is thus decolorized, and the pink color produced by the indicator at the completion of the reaction is easily seen.

Joulie's Method. The degree of acidity of the urine is a measure of the degree of the acidity of the blood. This acidity in both cases is due to acid phosphates, salts of $\mathrm{H}_{3} \mathrm{PO}_{4}$, that is, salts which, like 
$\mathrm{NaH}_{2} \mathrm{PO}_{4}, \mathrm{CaHPO}_{4}$, and $\mathrm{MgHPO}_{4}$, are still capable of taking up one or more atoms of base. It is therefore obvious that the real urinary acidity is phosphoric acid.

The specific gravity of the urine to be examined is taken at $15^{\circ} \mathrm{C}$. If the temperature differs from this figure a correction is made according to a printed table. The total acidity, that is to say, the acidity from all sources, is now calculated, and is expressed in terms of the amount present in a hundred parts of the excess of the urine over that of distilled water at the same temperature. In this way the errors inseparable from the varying amount of water in different samples of the same person's urine are altogether avoided. The degree of dilution of a specimen, always hitherto a matter of difficulty, may thus be disregarded. Thus, if the specimen in question shows a specific gravity of $\mathrm{r} .020$, the excess (which is called $E$ ) is 20 , because the density of the water at $15^{\circ} \mathrm{C}$. is known to be r.ooo. The total acidity is calculated by means of precipitation with a standard solution of sucrate of calcium (ro gms. of powdered chalk and 20 of sugar to a liter of distilled water). The figure thus obtained is called $A$. Thus we have $\frac{A}{E} \times 100=R A$ (i.e., the percentage of total acidity in. $E$ ). The normal standard for $R A$, as worked out by Joulie, is between 4 and 5 -as nearly as possible 4.5. But; as already seen, this total acidity is a matter of secondary importance. What we wish to ascertain is the amount which the acid phosphates contribute to this acidity; in other words, how much of this is serviceable phosphoric acidity, and how much of it is organic, fortuitous, and undesirable.

The next step is therefore to determine the amount of phosphoric acid, combined and uncombined, which is present in the given specimen. This is calculated by the classical method with nitrate of uranium and ferrocyanid of potassium, and is expressed in terms of the amount present in 100 parts of the excess of the urinary density over that of water at the same temperature. If we call the total phosphatic contents thus obtained $P$, we have the following formula $\frac{P}{E} \times 100=R P$ (i.e., the ratio of the phosphates; in other words, the percentage of total phosphatic contents in $E$ ). The normal standard for $R P$ as estimated by Joulie is between II and II.5, as nearly as possible I1.17. In order to find what we are in search of, namely, the amount which the phosphates contribute to the total acidity, it is necessary to divide $R P$ by $R A$, thus: $\frac{R P}{R A}=\frac{I I . I 7}{4.5}=2.45$. This represents the normal percentage of active phosphoric acid, that is, the amount which is capable of neutralizing an equal atomic weight 
of base, in $E$, which is the excess of urinary density over that of water.

When the readings show that both $R A$ and $R P$ are below the normal, the explanation is simple enough. It is that there is an insufficient quantity of phosphoric acid in the blood and the symptoms are therefore due, at least in part, to the deposition of earthy phosphates in certain organs and tissues.

Urea $\left(\mathrm{CO}\left(\mathrm{NH}_{2}\right)_{2}\right)$. This is the most important constituent of the urine, as it is the chief condition in which the nitrogen leaves the body. It may be detected by evaporating a few drops of urine on a glass slide, moistening with nitric acid, allowing it to crystallize, and examining the crystals of urea nitrate under a microscope of low power. As urea is generally looked upon as an index of the retrograde changes going on in the body, or of the eliminating power of the kidneys, its quantitative estimation is a matter of great importance.

The quantity of urea eliminated in twenty-four hours has been put as being 30 to 33 gms., or from 430 to 550 grains.

The Quantitative Estimation of Urea is effected by treating it with alkaline hypochlorites or hypobromites which decompose the urea into $\mathrm{CO}_{2}, \mathrm{~N}$, and $\mathrm{H}_{2} \mathrm{O}$. (See Part IV, Chapter LXV.)

S. R. Benedict and F. Gephart (I. A. C. S., xxx, I760 (I908)) recommend the following procedure for the accurate estimation of urea in urine: $5 \mathrm{cc}$. of urine, together with an equal volume of dilute hydrochloric acid (made by adding four volumes of distilled water to one of concentrated acid) are introduced into a rather wide test-tube. The mouth of the tube is covered with a cap of lead foil and is then placed in a small autoclave which is heated to a temperature of $150^{\circ}$ to $155^{\circ}$ (corresponding to a pressure of 6 kilograms per square centimeter). This temperature is maintained for an hour and an half. After the autoclave has cooled, the contents of the tube are washed into an 800-cc. Kjeldahl distillation flask, diluted to about $400 \mathrm{cc}$, treated with $20 \mathrm{cc}$. of ro per cent $\mathrm{NaOH}$ solution and distilled for about forty minutes into an excess of standard acid. The residue of acid is titrated, and the urea nitrogen calculated (after subtracting the previously determined ammonia nitrogen).

The process gives accurate results and compares favorably with the Kjeldahl process and with Folin's.

Uric Acid $\left(\mathrm{C}_{5} \mathrm{H}_{4} \mathrm{~N}_{4} \mathrm{O}_{3}\right)$ occurs in urine, sometimes in a free state, but oftener in combination with potassium, sodium, or ammonium, and occasionally with calcium and magnesium. These are called urates. It is detected microscopically, and varies in quantity from 0.4 to $0.8 \mathrm{gm}$. (6 to $\mathrm{I} 2 \mathrm{grs}$.) in twenty-four hours. The crystals are sometimes lerge enough to be seen by the naked eye. It deposits, 
upon standing, in the form of a brick-colored precipitate, commonly called brick-dust.

Qualitative Chemical Tests. The Murexid Test. A portion of the urine is evaporated to dryness in a porcelain dish upon a waterbath. The residue is then moistened with nitric acid, and after evaporating off the nitric acid the residue is moistened with ammonium hydroxid. If uric acid is present the residue assumes a beautiful purple-red color, due to the formation of murexid..

The Silver Carbonate Test. Make the urine alkaline with $\mathrm{Na}_{2} \mathrm{CO}_{3}$ or $\mathrm{K}_{2} \mathrm{CO}_{3}$, and moisten a filter-paper with the liquid. Now touch the moistened paper with a solution of $\mathrm{AgNO}_{3}$. In the presence of uric acid a distinct gray stain is produced.

Quantitative Estimation of Uric Acid. Acidulate a portion of the urine with $\mathrm{HCl}$, and set aside for twenty-four hours. The uric acid is thus set free, and, being insoluble, precipitates and adheres to the bottom and sides of the vessel. It is collected on a weighed filter, washed thoroughly, dried, and weighed. The heat used should not be over $100^{\circ} \mathrm{C}$. $\left(212^{\circ} \mathrm{F}\right)$. The weight of the filter and its contents, minus the weight of the filter alone, gives the weight of uric acid in the volume of urine taken. The quantity eliminated in twenty-four hours can then be calculated.

A volumetric method, depending upon the separation of uric acid by hydrochloric acid, and its subsequent titration with potassium permanganate is as follows, and is a fairly reliable and rapid process: $200 \mathrm{cc}$. of the urine are acidulated with a few drops of concentrated hydrochloric acid, and evaporated on a water-bath to about half the volume. It is then transferred to a stoppered flask, and $5 \mathrm{cc}$. of concentrated hydrochloric acid added, and the mixture violently shaken for a few minutes. It is then set aside for a half hour to settle, and decanted onto a filter of smooth, hard texture. The sediment in the flask is then washed with about $20 \mathrm{cc}$. of cold water and the water passed through the same filter. Then after washing the filter with a like quantity of cold water, a hole is made in its apex and any adhering precipitate washed into the original flask. To this is then added to cc. of to per cent potassium hydroxid solution and the mixture slightly warmed until a clear solution is obtained: $90 \mathrm{cc}$. of cold water and $20 \mathrm{cc}$. of dilute sulphuric acid are then added and the titration with $\frac{\mathrm{N}}{10}$ potassium permanganate carried out. Each cc. of $\frac{\mathrm{N}}{\mathrm{IO}}$ permanganate is equivalent to $0.0075 \mathrm{gm}$. of uric acid.

Bartley's Method.* This is based upon the precipitation of

* J. A. C. S., 1897, 649. 
uric acid by means of silver nitrate in the presence of magnesia mixture and a decided excess of ammonia. When precipitation is complete, the slightest trace of silver in solution is shown by a dark color produced in a drop of the clear solution by a soluble sulphid. The following description of the process is from Bartley's "Clinical Chemistry:"

"The solutions required are:

“I. A $\frac{\mathrm{I}}{50}$ normal solution of $\mathrm{AgNO}_{3}$, made by diluting one volume of a $\frac{\mathrm{N}}{\mathrm{rO}}$ solution with four volumes of distilled water.

"2. Magnesium mixture, made to contain about to gms. of crystallized $\mathrm{MgSO}_{4}, \mathrm{I} 2$ gms. of $\mathrm{NH}_{4} \mathrm{Cl}$, and I00 cc. of aqua ammonia (U. S. P.).

"3. A solution of ammonium sulphydrate, or potassium sulphid. This solution should be freshly made, and of such strength that its color is nearly that of the urine.

"When the urine contains a sediment of uric acid, or acid urates, it is to be put in solution by warming with a few drops of $\mathrm{NaOH}$ solution before beginning the process, and the excess of alkali neutralized with acetic acid. In very dark fever-urines it is best to dilute with an equal volume with water. The titration is performed in a hot solution to prevent the precipitation of the xanthin bases by silver nitrate.

"The process is conducted as follows: To $50 \mathrm{cc}$. of the clear urine add $5 \mathrm{cc}$. of the magnesium mixture and about ro cc. of ammonium hydroxid (sp.gr. 0.960), or enough to give a decided excess. Warm the solution on a water-bath, and add from a burette a $\frac{\mathrm{N}}{50}$ solution of silver nitrate. From time to time a drop is removed from the solution, by means of a dropper-pipette, with a bit of absorbent cotton wound tightly over the end, so as to make an efficient filter, and after removing the cotton filter, bring a drop of the solution in contact with a drop of the weak potassium sulphid solution on a white porcelain surface. Experiments with pure water showed that it required $\frac{1}{2} \mathrm{cc}$. of the silver solution in $50 \mathrm{cc}$. to give a marked reaction. This amount must therefore be deducted from the reading. The titration is continued until a dark ring or cloud is seen at the line of contact of the two drops, showing the presence of silver in the solution. Each cc. of silver solution corresponds to $0.00336 \mathrm{gm}$. of uric acid, and the number of cc. used (less $\frac{1}{2} \mathrm{cc}$. for each $50 \mathrm{cc}$. of urine), multiplied by this factor, gives the number of grams of uric acid in the urine taken. From this we may easily calculate the amount in roo cc. or that excreted in twenty-four hours. 
"As soon as the process is complete, the precipitate rapidly settles, and it is best to draw off a drop or two from this clear supernatant liquid and test it carefully again. We may also check our work by running in another drop of the silver solution, to see if it produces a cloud, or to see if the precipitation be complete. As there is no excess of silver in the hot liquid at any time, there can be no reduction of silver.

"If, after the titration is complete, the solution be cooled, it will usually be found that it will require from $\mathrm{I}$ to $3 \mathrm{cc}$. of the silver solution to again produce the end-reaction, because of the precipitation of the xanthin bases as silver compounds. The formula of the xanthin silver compound is $\mathrm{Ag}_{2} \mathrm{O} \cdot \mathrm{C}_{5} \mathrm{H}_{4} \mathrm{~N}_{4} \mathrm{O}_{2}$. The factor for the $\frac{\mathrm{N}}{50} \mathrm{AgNO}_{3}$ solution is 0.0015 -that is, if we calculate them all as xanthin, each cc. of silver solution used in the cold solution, more than is required by the hot solution, corresponds to the above amount of xanthin bases.

"By making two titrations, the one in the hot and the other in the cold urine, we may estimate both the uric acid and the xanthin bases, the latter by the difference in the results of the two titrations."

The Iodic Acid Method (Merck)** This method depends upon the fact that minute quantities of uric acid will react with iodic acid, in acidulated solutions, with liberation of iodin. This reaction is decidedly quantitative and if the liberated iodin is removed by chloroform and titrated with standard sodium thiosulphate solution, very good results are obtained. Every molecule of iodic acid $\left(\mathrm{HIO}_{3}\right)$ oxidizes five molecules of uric acid to diuric acid, with liberation of iodin (one atom), as per the equation:

$$
\underset{\text { Uric acid (1669.1) }}{\mathrm{IOC}_{5} \mathrm{H}_{4} \mathrm{~N}_{4} \mathrm{O}_{3}}+\underset{\text { Iodic acid (349.08) }}{\mathrm{HIO}_{3}}={ }_{5} \mathrm{C}_{10} \mathrm{~N}_{8} \mathrm{H}_{6} \mathrm{O}_{6}+6 \mathrm{H}_{2} \mathrm{O}+\underset{25 \mathrm{I} .8}{\mathrm{I}_{2}}
$$

As seen by this equation, $251.8 \mathrm{gms}$. of iodin are liberated from iodic acid by I669. I gms. of uric acid. Therefore 125.9 gms. of iodin are liberated by $834.55 \mathrm{gms}$. of uric acid. Each cc. of $\frac{\mathrm{N}}{100}$ sodium thiosulphate $(2.465 \mathrm{gms}$. per liter) equals $0.001259 \mathrm{gm}$. of iodin, and $0.008345 \mathrm{gm}$. of uric acid.

The Process. 50 to $100 \mathrm{cc}$. of urine are put into a glass-stoppered separatory burette $\dagger$ (if the urine is of high specific gravity it is diluted

* Ph. Zg., r 1905, 79r.

† This should be of 200 to $300 \mathrm{cc}$. capacity, and provided at its lower end with a 4 to $5 \mathrm{~cm}$. long neck of $0.5 \mathrm{~cm}$. caliber, and below the stop-cock a tip 0.5 to $1 \mathrm{~cm}$. in length. 
with an equal volume of water to prevent emulsification) acidulated with 5 to $9 \mathrm{cc}$. of 50 per cent tartaric or citric acid solution,* and then for every ro cc. of diluted urine, 0.4 to $0.7 \mathrm{cc} . \dagger$ of iodic acid solution ( $0.37 \mathrm{gm}$. per liter) are added, the mixture well shaken and let stand for one or two minutes.

The liberated iodin is then shaken out with three to five successive portions of chloroform, 2.5 to $3 \mathrm{cc}$. each.

The chloroform iodin solution is then collected and any iodic acid present washed out with water, the aqueous liquid being separated by means of a pipette.

After washing, the chloroform iodin solution is mixed with three to four times its volume of 50 per cent alcohol, in which a few crystals of potassium iodid are dissolved, and after the addition of starch solution titrated with $\frac{\mathrm{N}}{\mathrm{roO}}$ soldium thiosulphate.

If any albumen is present it must be removed by boiling. If iodid is present in the urine its quantity must be determined by testing with $\mathrm{NaNO}_{3}$ and acetic acid, or by means of hydrogen dioxid and sulphuric acid, and the difference between the quantity of iodin so found and the total iodin liberated gives the quantity which was liberated by, and hence represents, the uric acid in the urine. See also Gasometric Method, Part IV.

The Iodic Acid Method (Bouillet) $\$$ This differs from the foregoing in determining the amount of iodic acid consumed by the uric acid. It is carried out as follows: Precipitate the uric acid in the form of insoluble urate of barium by adding to $100 \mathrm{cc}$. of urine, previously neutralized with soda, chlorid of barium until no more precipitate is formed, then acidulate with $5 \mathrm{cc}$. of acetic acid I: roo let stand for fifteen or twenty minutes, filter, and wash the precipitate, which consists of urate, phosphate, and sulphate of barium. Transfer this precipitate into a porcelain crucible, using about 100 to $150 \mathrm{cc}$. of water for this purpose; add $20 \mathrm{cc}$. of $\mathrm{H}_{2} \mathrm{SO}_{4} \mathrm{I}$ : IO, so as to set free the uric acid, then boil. At this moment add ro cc. of the titrated solution of $\mathrm{I}_{2} \mathrm{O}_{5}$, and keep boiling gently until the whole of the iodin is liberated. Sometimes the liquid remains yellow, on account of the presence of the last traces of iodin, which are difficult to remove; this, however, can be easily effected by adding a little marble-the carbonic acid given off carries the iodin with it. After cooling, titrate the undecomposed $\mathrm{I}_{2} \mathrm{O}_{5}$; for this purpose, Io cc. of $\mathrm{HCl} \mathrm{I}$ : IO and $30 \mathrm{cc}$.

* These acids do not absorb iodin, even upon standing for days.

$\dagger$ It is best to make one or two trials, because the presence of tartaric or citric acid may cause liberation of $\mathrm{HCl}$ from the $\mathrm{NaCl}$.

$\ddagger$ Bull. Soc. Chim. (3), XXV, No. 5; and Chem. News, April 19, I901. 
of KI I : IO are successively added to the cold solution, and the iodin set at liherty is titrated with $\frac{\mathrm{N}}{10}$ hyposulphite. The difference between the original volume of the hyposulphite solution, $V$, and the present volume, $v$, gives the amount of iodic acid decomposed; this difference, multiplied by the factor 0.007 , gives the weight of uric acid. In a general way, the formula is $(V-v) \times 0.007=$ uric acid. The method is simple and rapid, and has proven extremely accurate.

\section{LITERATURE ON THE ESTIMATION OF URIC ACID AND UREA}

E. Riegler. Zeitschr. anal. Chem., r896, 3r.

Tunnicliffe and Rosenheim. Centrabl. f. Physiol., I897, xI, 434.

Rudisch and Boroschek. J. A. C. S., xxiv, 562 (I902).

Ludwig. Zeitschr. anal. Chem., XxI, I 48 (I882).

Salowski. Zeitschr. physiol. Chem., Xıv, 50, I890.

Folin and Shaffer. Ibid., XxxIr, 553, (I90I).

Haycraft. Zeitschr. anal. Chem., xxv, I69 (1886).

Hermann. Zeitschr. physiol. Chem., XII, 497.

Kruger. Ibid., XXI, 3 I I.

Czapek. Ibid., XII, 502 .

Folin. Ibid., XxxII, 504; XxxvI, 333; XxxvII, 548.

W. Braeutigam. Ph. Ztg., Nov. I3, I901.

B. Merck. Ibid., 1905, 79 I.

Neubauer and Vogel. "Urine Analysis."

Cammerer. J. Chem. Soc., LVI, Io40.

Hopkins and Groves. Chem. News, LXvi, ro7.

Dimmock and Branson. Ph. Jour., Jan. I2, 1907.

H. Bouillet. Bull. Soc. Chim. (3), xxv, No. 5; and Chem. News, April I9, I90I.

J. F. Tocher. Trans. Brit. Ph. Conf., 1902, 405-4I5.

A. H. Allen. Chem. News, Feb. 28, 1896.

Benedict and Gephart. J. A. C. S., xxx, 1760 (1908).

E. H. Bartley. Ibid., I897, 649.

Sellier. Ph. Ztg., Aug. 5, 1903, 627.

Dimmock and Branson. Trans. Brit. Ph. Conf., I903. 439.

Albumen. In all cases the urine should be clear before applying the tests for albumen. If not clear, it should be filtered.

(a) Boiling Test. About ro cc. of the clear urine are placed in a narrow test-tube, one drop of acetic or nitric acid is added, and the tube heated over a small flame in such a way that the upper portion of the liquid only will be heated. In the presence of albumen the urine will become turbid, more or less so in proportion to the amount of albumen present. 
If the acetic or nitric acid is not added before heating, a turbidity will be produced by the phosphates; this, however, will again disappear upon adding the acid.

(b) The Nitric Acid Test. About 2 cc. of pure nitric acid are placed in a test-tube, and the tube being inclined to one side, the urine is carefully run down the side of the tube so that it will float upon and not mix with the acid. An opaque-white zone will appear at the line of contact of the two liquids, if albumen is present.

A mixture of nitric acid one volume, and saturated solution of magnesium sulphate five volumes, is sometimes used instead of pure nitric acid in the above test, and is used in the same way.

(c) Ferrocyanid Test. A small portion of the urine is acidulated with acetic acid, and filtered if much of a precipitate forms. This acidulated urine is then floated on a solution of potassium ferroycanid. A white precipitate appears if albumen is present. This is a very delicate and reliable test; peptone, mucin, or alkaloids are not precipitated by it. This is known as Bödeker's Test.

(d) Picric Acid Test. A cold saturated solution of picric acid may be used in the same way as the nitric acid-by contact. A white zone appears at the line of contact. Alkaloids, mucin, peptones, and urates are, however, precipitated as well as albumen in this test, and the solution should be heated to redissolve these.

(e) Sodium Tungstate Test. The reagent is made by mixing equal parts of a cold saturated solution of sodium tungstate and citric acid solution. This is a very delicate test, and is applied in the same way as the nitric acid and the above. Peptones, alkaloids, mucin, and urates are also precipitated by this reagent, but these are redissolved upon boiling.

(f) Potassio-mercuric Iodid Test, or Tanret's Test. The reagent is prepared as follows: Mercuric chlorid, I.35 gms.; potassium iodid, 3.32 gms.; acetic acid, $20 \mathrm{cc}$; distilled water, $80 \mathrm{cc}$. The two salts are separately dissolved in water, and then the solutions mixed and the acetic acid added. This solution is also used by the contact method. It is very delicate, detecting one part of albumen in 20,000 parts of urine. It is necessary to heat in order to dissolve the alkaloids, mucin, and peptone, which are precipitated together with the albumen.

(g) Acidulated-brine Test. The reagent is made by adding one fluid ounce of hydrochloric acid to a pint of a saturated solution ot common salt and filtering.

It is used as follows: The solution is heated to boiling, and the urine added by the contact method. A white zone appears at the line of contact if albumen is present. Peptone, alkaloids, etc., are not precipitated by this reagent. 
The Quantitative Estimation of albumen is of great importance, but comparative tests are, as a rule, sufficient. An easy comparative test is to heat a given quantity of urine in a test-tube, add a few drops of nitric acid, and set aside for about twelve hours, and then note the volume occupied by the precipitated albumen. This is generally spoken of as volume per cent, and has no relation to actual percentage.

More accurate results are obtained with Esbach's Albuminometer. This is a graduated glass tube (Fig. 93). Fill the tube to $U$ with the urine, then to $R$ with the reagent. Close the tube with a rubber stopper, shake, and set aside for twenty-four hours. Then note the height of the precipitate, as indicated by the graduations. Each of the numbered divisions represents a gram of albumen in $1000 \mathrm{cc}$. of urine. The reading should be taken at the middle of the albuminous surface. The reagent: Picric acid, ro gms.; citric acid, 20 gms.; water, 1000 gms.

Blood. A small quantity of the urine is mixed in a test-tube with an equal volume of a mixture of freshly prepared tincture of guaiac and spirit of turpentine, which has been exposed to the air for some time. If blood-coloring matter is present the mixture assumes an indigo-blue color, the rapidity of formation of which depends upon the amount of blood-coloring matter present. Pus, saliva, and salts of iodin also give a blue color with this test; but it appears only after a considerable lapse of time, and it is seldom likely to mislead. Instead of the spirit of turpentine, peroxid of hydrogen may be used.

Fig. 93 .

Pus. The presence of pus is easily revealed by the microscope.

Urine containing pus is always turbid to the naked eye, and deposits a white or greenish-white sediment, which resembles urates or earthy phosphates. If heated the sediment does not disappear-difference from urates, neither is it dissolved by dilute acids-difference from earthy phosphates. It dissolves, however, in strongly alkaline solutions, giving a gelatinous, ropy liquid. Pus effervesces with hydrogen peroxid.

Sugar. (a) Bismuth Test. A few cc. of urine are placed in a test-tube, and an equal volume of sodium hydroxid solution and a little bismuth subnitrate; mix well, and boil for a few minutes. A black precipitate is produced if sugar is present.

If albumen is present it must be removed before applying the test, as it is decomposed by boiling with the alkali, forming a black sulphid of bismuth.

(b) Nylander's Test is a modification of the above. A solution is 
made of bișuth subnitrate 2 gms., Rochelle salt 4 gms., sodium hydroxid 8 gms., and distilled water roo cc.

Heat the urine to boiling, and add a few drops of this alkaline solution of bismuth, continuing the boiling. If sugar is present, the mixture turns black.

This is a very delicate test, but, as in the previous one, any albumen must be removed.

(c) Moore's Test. Add one part of liquor soda to two parts of urine, and boil. If sugar is present the urine will become blackish brown. Albumen must be removed before applying the test.

(d) Picric Acid Test. About 5 cc. of the urine are mixed with half as much of picric acid solution and about 2 cc. of liquor potassa, and boiled. A dark mahogany-red color is developed in the presence of sugar. Albumen will cause turbidity, but will not interfere with the test.

(e) Trommer's Test. 5 cc. of urine are mixed in a test-tube with one half of its volume of liquor soda, and one or two drops of a solution of $\mathrm{CuSO}_{4}$ (I:I0). In the presence of sugar a clear, deep-blue color is obtained. Heat the solution now almost, though not quite, to boiling. At first a greenish then a yellow turbidity forms, which rapidly changes to a reddish-yellow color, and precipitates red cuprous oxid. An excess of the copper solution should not be used.

(f) Haines' Test. The reagent used is a solution of copper sulphate in a mixture of equal parts of glycerin and water.

To about $5 \mathrm{cc}$. of urine add a few drops of this reagent, and then add sodium hydroxid solution until the liquid assumes a deep-blue color. The mixture is then gradually heated to boiling. If sugar is present the color changes to yellow, and finally brick-red.

(g) Indigo-carmine Test. The reagent is made by mixing one part of dried commercial extract of indigo with thirty parts of pure dry sodium carbonate.

The test: Add enough of this powder to $5 \mathrm{cc}$. of the urine to give it a transparent blue color, and heat to boiling. If sugar is present, the color changes to violet, cherry-red, and finally yellow. On gently agitating the tube the colors appear in the reversed order.

(h) Molisch's Test. Put I cc. of the urine in a test-tube, add 2 cc. of a saturated solution of alpha-naphthol, mix well, and then add an excess of sulphuric acid. A deep violet color is produced if sugar is present. On dilution with water a blue precipitate occurs.

Thymol or menthol may be used instead of naphthol. The color then produced is deep red.

Quantitative Estimation. This is generally effected by the use of Fehling's or Pavy's solution. The process is described on page $49^{2}$. 
By Fermentation. The Densimetric Method. This is performed by adding a small quantity of yeast to a certain volume of urine and setting aside for about twenty-four hours. As the sugar is decomposed the specific gravity of the urine becomes less. Therefore by taking the specific gravity of the urine before and after fermentation a fairly accurate estimation of sugar present may be made, provided the quantity be not less than 0.5 per cent. Each degree of the urinometer indicates 0.219 per cent of sugar. If the specific gravity of a sample of urine is found to be 1032 , and after subjecting it to fermentation it is 1022, the quantity of sugar present in the sample is ten times $0.219=2.19$ per cent.

Estimation of Sugar by Dr. Einhorn's Fermentation Saccharometer. Take I gm. of commercial compressed yeast (or $\frac{1}{1^{16}}$ of a cake

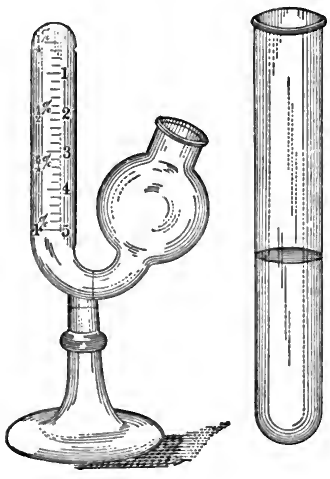

FIG. 94. of Fleischmann's yeast), shake thoroughly in the graduated test-tube with Io cc. of the urine to be examined. Then pour the mixture into the bulb of the saccharometer (Fig. 94). By inclining the apparatus the mixture will easily flow into the cylinder, thereby forcing out the air. Owing to the atmospheric pressure the fluid does not flow back, but remains there.

The apparatus is to be left undisturbed for twenty to twenty-four hours in a room of ordinary temperature.

If the urine contains sugar, the alcoholic fermentation begins in about twenty to thirty minutes. The evolved carbonic acid gas gathers at the top of the cylinder, forcing the fluid back into the bulb.

On the following day the upper part of the cylinder is filled with carbonic acid gas. The changed level of the fluid in the cylinder shows that the reaction has taken place, and indicates by the numbers -to which it corresponds - the approximate quantity of sugar present.

If the urine contains more than I per cent of sugar, then it must be diluted with water before being tested.

Diabetic urines of straw color, and a specific gravity of 1018-1022 may be diluted twice; of $1022-1028$, five times; 1028-1038, ten times.

The original (not diluted) urine contains in proportion to the dilution two, five, or ten times more sugar than the diluted urine.

In carrying out the fermentation test it is always recommendable to take, besides the urine to be tested, a normal one, and to make the same fermentation test with it.

The mixture of the normal urine with yeast will have on the follow- 
ing day only a small bubble on the top of the cylinder. That proves at once the efficacy and purity of the yeast.

If there is likewise in the suspected urine a small bubble on the top of the cylinder, then no sugar is present; but if there is a much larger gas volume, then we are sure that the urine contains sugar.

Tests for Bile. (a) Oliver's Test. Dissolve 2 gms. of fresh peptone (Savory and Moore's Pulverized), 0.25 gm. salicylic acid, and $2 \mathrm{cc}$. of 33 per cent acetic acid in water to make $200 \mathrm{cc}$. The solution should be rendered perfectly clear by filtration.

The urine should also be clarified by filtration, and diluted to a specific gravity of 1008. I cc. of this urine is added to $3 \mathrm{cc}$. of the above reagent. If biliary salts are present a distinct opalescence at once appears, which becomes more intense in about five minutes. This opalescence will be more or less distinct in proportion to the quantity of bile present.

(b) Gmelin's Test. 2 or 3 cc. of partially decomposed yellow nitric acid are placed in a test-tube, and an equal volume of the urine is cautiously poured on top. In the presence of bile pigments a play of colors, will appear, beginning with green, then passing through blue, violet, red, and yellow.

The nitric acid may be prepared for this test by adding a fragment of zinc to ordinary nitric acid.

(c) Pettenkofer's Test. Mix equal parts of urine and sulphuric acid, add one drop of simple syrup, and apply a gentle heat. The color will change from cherry-red to purple if biliary acids are present.

(d) Ultzmann's Test. 5 cc. of urine are mixed with $2 \mathrm{cc}$. of a strong solution of $\mathrm{KOH}(\mathrm{r}: 3)$ and then an excess of pure $\mathrm{HCl}$ added. The mixture will become emerald-green if biliary pigments are present.

(e) Tincture of Iodin Test. A few drops of iodin tincture are floated upon the surface of the urine. If biliary pigments are present, there will appear at the line of contact of the two liquids, after a few minutes, a beautiful emerald-green zone. 


\section{PART IV \\ A FEW GASOMETRIC ANALYSES}

\section{CHAPTER LXI}

\section{THE NITROMETER}

For general gas analysis, and for the rapid estimation of such substances as ethyl nitrite, hydrogen dioxid, urea, bleaching-powder, manganese dioxid, etc., an instrument called the nitrometer is used.

The apparatus in its simplest form is shown in Fig. 95. It consists

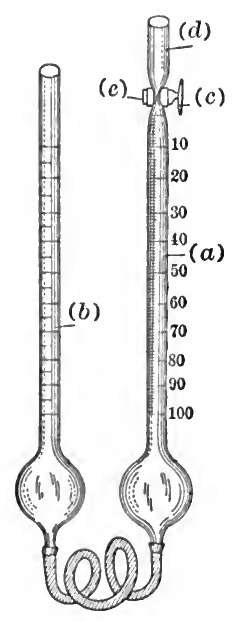

FIG. 95. of a measuring tube, $a$, of 50 or 100 cc. capacity, and graduated in tenths of a cc. This is connected by means of a stout rubber tube with an open "equilibrium tube $b$, also called "controltube," "pressure-tube," or "level-tube;" both tubes are preferably provided with a globular expansion near the lower end, and are held by suitable clamps upon a stand, in such a manner that either tube may be readily and quickly clamped at a higher or lower level. The measuring tube is fitted at the top with a stop-cock, $c$, and a graduated glass tube or cup, $d$. Some nitrometers are provided with a three-way stopcock, so arranged that according to the way it is turned, it will discharge the contents of the cup either into the measuring tube below, or out into the waste opening which is usually placed at $\boldsymbol{e}$, or it will discharge the contents of the measuring tube into the waste opening.

With this apparatus gases can be rapidly and accurately measured at definite temperature and pressure.

In measuring the gas the instrument is filled with some liquid in which the gas is insoluble-generally mercury. In many cases a saturated solution of salt may be used. 
Suppose we fill the instrument with mercury in such quantity that when the stop-cock is opened and the control-tube raised, the mercury will rise as far as the top, and about two inches in the controltube.

The top is now closed, the control-tube lowered, and a little carbonic acid gas admitted through $E$. The top is then again closed, and the instrument allowed to stand until its contents have acquired the temperature of the room. A centigrade thermometer suspended to the stand will then give the temperature of the gas.

The control-tube is now raised or lowered so as to make the level of the liquid in both tubes the same. This makes the pressure in the tube the same as the atmospheric pressure outside, and by referring to a barometer standing near this pressure is ascertained.

We now have a definite volume of the gas at a known temperature and pressure.

It now only remains to read off the volume of the gas, and correct it to the normal temperature and pressure by Charles' and Boyles' laws, respectively.

The normal temperature and pressure is $0^{\circ} \mathrm{C}$. and $760 \mathrm{~mm}$. pressure.

The weight of the gas in grams may then be calculated from its volume by multiplying the number of cc. at the normal temperature and pressure, by the weight of I cc. of the gas in grams.

This weight may be found as follows:

I000 cc. of hydrogen at normal temperature and pressure weigh $0.0896 \mathrm{gm}$. I cc. of $\mathrm{H}$ then weighs $0.0000896 \mathrm{gm}$.

One cc. of oxygen weighs sixteen times as much, and I cc. of nitrogen weighs fourteen times as much. Therefore, by multiplying the weight of $\mathrm{I}$ cc. of $\mathrm{H}$ by the atomic weight of an elementary gas, or half the molecular weight of a compound gas, the weight of I cc. of that gas is obtained.

According to the law of Charles, the volume of a gas under constant pressure varies directly with the absolute temperature.

All gases expand or contract by $\frac{1}{273}$ of their volume for each centigrade degree of temperature, increased or decreased.

We may regard a gas at $0^{\circ} \mathrm{C}$. as having passed through $273^{\circ} \mathrm{C}$. In other words, $273^{\circ}$ below zero must be regarded as the absolute zero, and $\circ^{\circ} \mathrm{C}$. as $273^{\circ}$ absolute temperature.

Thus the absolute temperature centigrade is the observed temperature $+273^{\circ}$.

Example. A given volume of oxygen gas at $15^{\circ} \mathrm{C}$. measures $20 \mathrm{cc}$. What will it measure at $0^{\circ} \mathrm{C}$. ?

$$
\frac{0^{\circ}+273^{\circ} \times 20}{15^{\circ}+273^{\circ}} \text { or } \frac{273^{\circ} \times 20}{288^{\circ}}=18.95 \mathrm{cc} . \text { Ans. }
$$


Boyle's Law. The volume of a confined gas is inversely proportional to the pressure brought to bear upon it. That is, the less the pressure the greater the volume, and vice versa.

Rule. Multiply the observed volume by the observed pressure, and divide by the normal pressure.

Example. A given volume of gas at $750 \mathrm{~mm}$. pressure measures $20 \mathrm{cc}$. What will it measure at $760 \mathrm{~mm}$. (the normal pressure)?

$$
\frac{750 \times 20 \mathrm{cc} .}{760}=19.73 \mathrm{cc} . \quad \text { Ans. }
$$

Now let us take an example in which both laws are involved.

A given volume of oxygen at $15^{\circ} \mathrm{C}$. subjected to a pressure of $75^{\circ} \mathrm{mm}$. measures $20 \mathrm{cc}$. What will it measure at the normal temperature and pressure?-i.e., $0^{\circ} \mathrm{C}$. and $760 \mathrm{~mm}$.

In the first example we find that $20 \mathrm{cc}$. of oxygen at $15^{\circ} \mathrm{C}$. will measure at $0^{\circ} \mathrm{C}$. $18.95 \mathrm{cc}$. Then

$$
\frac{750 \times 18.95 \mathrm{cc} .}{760}=18.70 \mathrm{cc} . \text { Ans. }
$$

Now to find the weight of this volume of oxygen we proceed as follows :

I cc. of $\mathrm{H}$ weighs 0.0000896 gm.;

I cc. of $\mathrm{O}$ weighs $\mathrm{I} 6 \times 0.000 c 8 \mathrm{y} 6=0.00 \mathrm{I} 4336 \mathrm{gm}$;

$18.70 \mathrm{cc}$. weigh $\mathrm{O}=18.70 \times 0.0014336 \mathrm{gm}$., or $0.02680832 \mathrm{gm}$.

The method of using the nitrometer for gasometric assay of various substances is illustrated in the assay of spirit of nitrous ether. (See page 683.)

The following tables, from the U. S. P. VIII, give the factors for corrections for temperature and barometric pressure. These factors may be used in order to obtain reasonably accurate results when the temperature and pressure are not nearly normal. The barometric correction is important at any locality more than $25^{\circ}$ meters above sea-level.

Example. Assuming that the volume of gas read off was $44.5 \mathrm{cc}$. at $32^{\circ} \mathrm{C}$. $\left(89.6^{\circ} \mathrm{F}\right.$.), and it is desired to ascertain the corresponding volume at $25^{\circ} \mathrm{C}$. $\left(77^{\circ} \mathrm{F}\right.$.), barometric pressure not being taken into consideration, then the $44.5 \mathrm{cc}$. must be reduced in the proportion of 
I to 0.977 (see temperature correction factors above), or 44.5 must be multiplied by 0.977 . The result will be $43.48(43.4765) \mathrm{cc}$. as the equivalent volume of gas at $25^{\circ} \mathrm{C}$. $\left(77^{\circ} \mathrm{F}\right.$.).

\section{FACTORS FOR TEMPERATURE CORRECTIONS}

(Normal Temperature, $25^{\circ} \mathrm{C}$.)

\begin{tabular}{|c|c|c|c|c|c|}
\hline Temperature. & Factor. & Temperature. & Factor. & Temperature. & Factor. \\
\hline $\mathrm{I} 5^{\circ} \mathrm{C}$. & I. 035 & $22^{\circ} \mathrm{C}$. & I.OIO & $29^{\circ} \mathrm{C}$. & 0.987 \\
\hline I $6^{\circ} \mathrm{C}$ & $I .031$ & $23^{\circ} \mathrm{C}$ & I . 007 & $30^{\circ} \mathrm{C}$. & 0.083 \\
\hline I $7^{\circ} \mathrm{C}$ & 1.028 & $24^{\circ} \mathrm{C}$. & I. .003 & $3 \mathrm{I}^{\circ} \mathrm{C}$. & 0.980 \\
\hline I $8^{\circ} \mathrm{C}$ & I. .024 & $25^{\circ} \mathrm{C}$ & I. . & $32^{\circ} \mathrm{C}$ & 0.977 \\
\hline $19^{\circ} \mathrm{C}$ & $\mathrm{I} .02 \mathrm{I}$ & $26^{\circ} \mathrm{C}$ & ○ 997 & $33^{\circ} \mathrm{C}$ & 0.974 \\
\hline $20^{\circ} \mathrm{C}$ & I. or 7 & $27^{\circ} \mathrm{C}$. & ○ 993 & $34^{\circ} \mathrm{C}$. & $0.97 \mathrm{I}$ \\
\hline $2 \mathrm{I}^{\circ} \mathrm{C}$ & I OI 4 & $28^{\circ} \mathrm{C}$ & 0.990 & $35^{\circ} \mathrm{C}$ & 0.968 \\
\hline
\end{tabular}

FACTORS FOR CORRECTION FOR BAROMETRIC PRESSURE

(Normal Barometer. $760 \mathrm{~mm}$.)

\begin{tabular}{|c|c|c|c|c|c|}
\hline \multicolumn{2}{|c|}{ Barometer Reading. } & \multirow{2}{*}{ Factor. } & \multicolumn{2}{|c|}{ Barometer Reading. } & \multirow{2}{*}{ Factor. } \\
\hline $\mathrm{Mm}$. & Inches. & & $\mathrm{Mm}$ & Inches. & \\
\hline 790 & 31.10 & 1.039 & 660 & 25.98 & 0.868 \\
\hline 780 & 30.71 & I.026 & $6_{50}$ & $25 \cdot 59$ & $0 . S_{55}$ \\
\hline 770 & $30.3 \mathrm{I}$ & I.OI3 & 640 & 25.20 & 0.842 \\
\hline 760 & 29.92 & I. .000 & 630 & 24.80 & 0.829 \\
\hline $75^{\circ}$ & 29.53 & 0.987 & 620 & $24 \cdot 4 \mathrm{I}$ & $0.8 \mathrm{r} 6$ \\
\hline 740 & 29 I3 & 0.974 & 6ro & 24.02 & 0.803 \\
\hline 730 & 28.74 & $0.96 \mathrm{I}$ & 600 & 23.62 & 0.789 \\
\hline 720 & 28.35 & 0.947 & 590 & 23.23 & 0.776 \\
\hline 710 & $27 \cdot 95$ & 0.934 & 580 & 22.83 & 0.76 .3 \\
\hline 700 & $27 \cdot 5^{6}$ & $\circ 92 \mathrm{I}$ & 570 & 22.44 & $0.75^{\circ}$ \\
\hline 690 & $27 \quad 17$ & 0.907 & 560 & 22.05 & $\circ 737$ \\
\hline 680 & 26.77 & 0.895 & $55^{\circ}$ & 21.65 & 0.724 \\
\hline 670 & $26.3^{8}$ & 0.882 & & & \\
\hline
\end{tabular}

Example. Assuming that the volume of gas read off was 43.48 $(43.4765)$ cc. at $590 \mathrm{~mm}$. barometric pressure, and it is desired to ascertain the corresponding volume at normal barometric pressure $(760 \mathrm{~mm}$.$) , temperatúre not being taken into consideration, then the$ $43.48 \mathrm{cc}$. must be reduced in the proportion of $\mathrm{I}$ to 0.776 (see barometric correction factors above), or 43.48 must be multiplied by 0.776 . The result will be $33.74 \mathrm{cc}$. as the equivalent volume of gas at normal barometric pressure. 


\section{ESTIMATION OF NITROGEN DIOXID}

$\mathrm{NO}=29.8 \mathrm{I}$; I liter at $\circ^{\circ} \mathrm{C}$. and $760 \mathrm{~mm} .=\mathrm{I} .3396 \mathrm{gms}$.

$$
\text { at } 25^{\circ} \mathrm{C} \text {. and } 760 \mathrm{~mm} .=\mathrm{r} .2272 \mathrm{gms} \text {. }
$$

One Cubic Centineter of Nitrogen Dioxid is the Equivalent of:

\begin{tabular}{|c|c|c|}
\hline & $\begin{array}{c}\text { At } 0^{\circ} \mathrm{C} \text {. and } \\
760 \mathrm{~mm} . \\
\text { Gram. }\end{array}$ & $\begin{array}{c}\text { At } 25^{\circ} \mathrm{C} \text {. and } \\
760 \mathrm{~mm} \text {. } \\
\text { Gram. }\end{array}$ \\
\hline 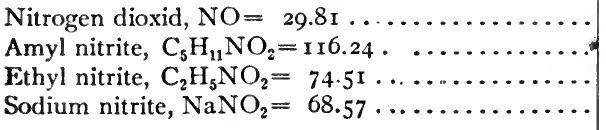 & $\begin{array}{l}0.0013336 \\
0.005^{2234} \\
0.0033482 \\
0.0030813\end{array}$ & $\begin{array}{l}0.0012272 \\
0.0047851 \\
0.0030673 \\
0.0028227\end{array}$ \\
\hline
\end{tabular}




\section{CHAPTER LXII}

\section{ASSAY OF NITRITES}

Spirit of Nitrous Ether. This is an alcoholic solution of ethyl nitrite $\left(\mathrm{C}_{2} \mathrm{H}_{5} \mathrm{NO}_{2}=74.5 \mathrm{I}\right)$, yielding when freshly prepared and tested in the nitrometer not less than Ir times its own volume of nitrogen dioxid $(\mathrm{NO}=29.8 \mathrm{r})$, U. S. P.

When nitrites are mixed with an excess of $\mathrm{KI}$ and acidulated with $\mathrm{H}_{2} \mathrm{SO}_{4}$, iodin is liberated, and all the nitrogen of the nitrite is evolved in the form of NO. as shown in the equation

$$
\begin{aligned}
& { }_{2} \mathrm{C}_{2} \mathrm{H}_{5} \mathrm{NO}_{2}+2 \mathrm{KI}+2 \mathrm{H}_{2} \mathrm{SO}_{4}={ }_{2} \mathrm{C}_{2} \mathrm{H}_{5} \mathrm{OH}+2 \mathrm{KHSO}_{4}+\mathrm{I}_{2}+2 \mathrm{NO} \text {. } \\
& \text { I } 49.02 \\
& 59.62
\end{aligned}
$$

The process is conducted as follows:

Open the stop-cock of the measuring tube, raise the control-tube, and pour into the latter a saturated solution of $\mathrm{NaCl}$ until the measuring tube, including the bore of the stop-cock, is completely filled. Then close the stop-cock and fix the control-tube at a lower level. Now introduce into the funnel at the top of the measuring tube a weighed quantity (about $4 \mathrm{gms}$.)* of spirit of nitrous ether; open the stop-cock, and allow the spirit to run into the nitrometer, being careful that no air enters at the same time. ro cc. of potassium iodid T. S. are now added in the same manner, and followed by ro cc. of normal sulphuric acid V. S. Effervescence takes place immediately, and after thirty to sixty minutes, when the volume of gas has become constant, the control-tube is lowered so as to make the level of the liquid in both tubes the same, and the volume of the gas in the graduated tube read off.

* It is convenient to take $5 \mathrm{cc}$. accurately measured, and calculate its weight by multiplying by the specific gravity. The U. S. P. directs to transfer $30 \mathrm{gms}$. of the spirit of nitrous ether, which has been previously shaken with $0.5 \mathrm{gm}$. of $\mathrm{KHCO}_{3}$ to a tared flask, and weigh it accurately. Add sufficient alcohol to bring the volume to exactly roo cc., and mix thoroughly. Introduce into the nitrometer exactly ro cc. of the alcoholic solution. 
This volume, multiplied by $0.0030673 \mathrm{gm}$. gives the weight of ethyl nitrite in the spirit taken for analysis. The product multiplied by Ioo, and then divided by the weight of the spirit taken, gives the per cent of pure ethyl nitrite present.

The temperature correction is one third of one per cent of the total percentage found, for each degree, additive, if the temperature is below $25^{\circ} \mathrm{C}$. The barometric correction is $\frac{4}{30}$ of one per cent for each millimeter, additive if above, subtractive if below 760 .

The volume of $\mathrm{NO}$ generated at the ordinary indoor temperature (assumed to be at or near $25^{\circ} \mathrm{C}$., $77^{\circ} \mathrm{F}$.) should not be less than $55 \mathrm{cc}$. if $5 \mathrm{cc}$. of the spirit are taken, corresponding to about 4 per cent of pure ethyl nitrite.

Sodium chlorid solution is used in the above assay, because, owing to its density, the spirit will float on top, and the gas evolved will not dissolve in it. At the same time the expense of using mercury is saved. It is important that no air be allowed to get into the measuring tube, because this would convert the NO into a higher oxid of nitrogen, which would dissolve in the salt solution, and thus vitiate the result.

It is required to correct the volume of gas evolved at higher temperatures, to its corresponding volume at $0^{\circ} \mathrm{C}$. The calculations involved are as explained below:

Example. 5 cc. of spirit of nitrous ether (sp.gr. 0.823) are treated in a nitrometer, and the $\mathrm{NO}$ evolved measures $55 \mathrm{cc}$.

The temperature at which the operation is conducted is $25^{\circ} \mathrm{C}$., and the atmospheric pressure normal.

What per cent of ethyl nitrite is present in the sample?

By consulting the equation given above, it will be seen that one molecular weight of $\mathrm{NO}=29.8 \mathrm{r}$ is evolved from one molecular weight of ethyl nitrite, $74.5 \mathrm{I}$.

Now reduce the volume of the gas liberated at $25^{\circ} \mathrm{C}$. to its corresponding volume at $0^{\circ} \mathrm{C}$. Thus

$$
273^{\circ}+25^{\circ}: 55:: 273^{\circ}+0^{\circ} . x . \quad x=50.4 \mathrm{cc} .
$$

Thus the gas evolved from $5 \mathrm{cc}$. of the spirit of nitrous ether, measured at $\circ^{\circ} \mathrm{C}$., is $50.4 \mathrm{cc}$.

The next step in the calculation is to find how much ethyl nitrite each cc. of the evolved $\mathrm{NO}$ represents. I liter of hydrogen at $0^{\circ} \mathrm{C}$. and normal pressure weighs $0.0896 \mathrm{gm}$.

By multiplying this weight by half the molecular weight of NO, the weight of $1000 \mathrm{cc}$. of the latter gas is obtained; this will be found to be r.3396. Now if r.3396 gms. of NO measures rooo cc., 29.8 I gnıs. will measure $22,328.24 \mathrm{cc}$.

$$
\text { I.3396: } 1000: \cdot 29.8 \mathrm{I}: x . \quad x=22,324.58 .
$$


Then if $22,324.58 \mathrm{cc}$, of $\mathrm{NO}$ are evolved by, and consequently represent, $74.5^{\mathrm{I}} \mathrm{gms}$. of ethyl nitrite, as the equation shows, I cc. of NO will represent $0.0033482 \mathrm{gm}$. of pure ethyl nitrite.

Now, since in the above example 50.4 cc. of gas were evolved at $0^{\circ} \mathrm{C}$., the $5 \mathrm{cc}$. of spirit of nitrous ether examined must contain

$$
50.4 \times 0.0033482 \mathrm{gm} .=0.16875 \mathrm{gm} \text {. }
$$

of pure ethyl nitrite.

In order to determine the percentage strength, the weight of the spirit taken must be known. This may be found by multiplying the measure by the specific gravity, $5 \mathrm{cc} . \times 0.823=4.115 \mathrm{gms}$. Then

$$
\text { 4.11 } 5 \text { gms.:0.16875 gms.: '100: } x . \quad x=4 \text { per cent. }
$$

Amyl Nitrite is a liquid containing about 80 per cent of amyl nitrite (principally iso-amyl nitrite), $\mathrm{C}_{5} \mathrm{H}_{11} \mathrm{NO}_{2}=\mathrm{I}_{1} 6.24$, together with variable quantities of undetermined compounds.

The U.S. P. assay is as follows: Transfer about $3 \mathrm{cc}$. of the amyl nitrite, which has been previously shaken with $0.5 \mathrm{gm}$. of potassium bicarbonate and carefully decanted, to a tared $100 \mathrm{cc}$. measuring flask, and weigh it accurately; add alcohol to bring the volume to exactly I00 cc. Io cc. of this alcoholic solution are introduced into the nitrometer as directed for spirit of nitrous ether; ro cc. of potassium iodid T. S. and to cc. of $\frac{\mathrm{N}}{\mathrm{I}} \mathrm{H}_{2} \mathrm{SO}_{4} \mathrm{~V}$. S. are then added, and the volume of NO generated, measured at the ordinary indoor temperature (assumed to be at or near $25^{\circ} \mathrm{C}$. or $77^{\circ} \mathrm{F}$.), should be about $40 \mathrm{cc}$. Each cc. at this temperature represents $0.004785 \mathrm{I}$ gm. of pure amyl nitrite, or about 2 per cent.

Sodium Nitrite $\left(\mathrm{NaNO}_{2}=68.57\right)$. This, like the other nitrites mentioned, when treated with potassium iodid and sulphuric acid, is decomposed, and NO is given off. The reaction is here illustrated:

$$
{ }_{2} \mathrm{NaNO}_{2}+{ }_{2} \mathrm{KI}+{ }_{2} \mathrm{H}_{2} \mathrm{SO}_{4}=\mathrm{K}_{2} \mathrm{SO}_{4}+\mathrm{Na}_{2} \mathrm{SO}_{4}+{ }_{2} \mathrm{H}_{2} \mathrm{O}+2 \mathrm{NO}+\mathrm{I}_{2} \text {. }
$$

A molecule of $\mathrm{NaNO}_{2}$ (68.57) evolves, when properly treated, one molecule of $\mathrm{NO}(29.8 \mathrm{I})$.

The assay process is as follows: Weigh out $0.15 \mathrm{gm}$. of $\mathrm{NaNO}_{2}$, dissolve it in about $5 \mathrm{cc}$. of water, and introduce the solution into a nitrometer. This is followed by a solution of $\mathrm{I} \mathrm{gm}$. of $\mathrm{KI}$ in $6 \mathrm{cc}$. of water and $\mathrm{I}_{5} \mathrm{cc}$. of $\frac{\mathrm{N}}{\mathrm{T}} \mathrm{H}_{2} \mathrm{SO}_{4}$. The gas which is liberated should 
measure not less than $50 \mathrm{cc}$. at $15^{\circ} \mathrm{C}$. $\left(59^{\circ} \mathrm{F}\right.$.) or $5 \mathrm{I} .7 \mathrm{cc}$. at $25^{\circ} \mathrm{C}$. $\left(77^{\circ} \mathrm{F}\right.$.), corresponding to not less than 97.6 per cent of the pure salt. Each cc. at $25^{\circ} \mathrm{C}$. represents $0.0028227 \mathrm{gm}$. and at $0^{\circ} \mathrm{C}$. $0.00308 \mathrm{r} 3$ gm. of pure $\mathrm{NaNO}_{2}$.

\section{ESTIMATION OF NITRIC ACID IN NITRATES}

This may also be effected by the use of the nitrometer.

When a nitrate is shaken up with an excess of sulphuric acid and mercury, the nitrate is decomposed and $\mathrm{NO}$ is evolved, as seen in the following equation:

$$
\begin{aligned}
& { }_{2} \mathrm{KNO}_{3}+{ }_{4} \mathrm{H}_{2} \mathrm{SO}_{4}+{ }_{3} \mathrm{Hg}={ }_{3} \mathrm{HgSO}_{4}+\mathrm{K}_{2} \mathrm{SO}_{4}+{ }_{2} \mathrm{NO}+{ }_{4} \mathrm{H}_{2} \mathrm{O} \text {. } \\
& \text { 2) } \frac{200.86}{100.43} \\
& \text { 2) } 59.62 \\
& 29.8 \mathbf{r}
\end{aligned}
$$

Thus each molecule of the nitrate radical $\mathrm{NO}_{3}$ gives off a molecule of NO.

Not more than $0.2 \mathrm{gm}$. of nitrate should be taken for analysis, since, if this quantity is exceeded, the volume of gas evolved will be greater than the instrument can conveniently hold. In this estimation the nitrometer is filled with mercury instead of brine; the nitrate is dissolved in $5 \mathrm{cc}$. of water, introduced into the nitrometer, and followed by excess of strong sulphuric acid. The instrument is well shaken for some time, and when action has ceased and the contents have cooled down to the temperature of the room, the level is adjusted and the volume of NO read off and calculated in the usual way. 


\section{CHAPTER LXIII}

\section{H Y D R O G N DIOXI D}

As stated in a previous chapter, hydrogen dioxid when acted upon by an acidulated solution of potassium permanganate, is decomposed and oxygen is evolved. One half of this oxygen comes from the dioxid and the other half from the permanganate.

Therefore if I cc. of the dioxid be treated in this way and $20 \mathrm{cc}$. of oxygen are evolved, the strength of the solution is ten volumes.

The nitrometer may be used for this estimation.

This instrument is charged with a concentrated solution of sodium sulphate (which in this case is better than brine), and I cc. of the dioxid introduced from the funnel, followed by excess of solution of permanganate acidulated with sulphuric acid.

This latter solution should be of such strength that when the reaction is completed, the solution should still have a purple color.

The reaction is thus illustrated.

$$
{ }_{5} \mathrm{H}_{2} \mathrm{O}_{2}+{ }_{3} \mathrm{H}_{2} \mathrm{SO}_{4}+{ }_{2} \mathrm{KMnO}_{4}=\mathrm{K}_{2} \mathrm{SO}_{4}+{ }_{2} \mathrm{MnSO}_{4}+8 \mathrm{H}_{2} \mathrm{O}+{ }_{5} \mathrm{O}_{2} \text {. }
$$

By the use of Squibb's Urea Apparatus (Fig. Ioo) the estimation may be easily and rapidly made.

Into the generating-bottle is put about $30 \mathrm{cc}$. of a strong, acidulated solution of potassium permanganate, and a small test-tube containing I cc. of $\mathrm{H}_{2} \mathrm{O}_{2}$ is carefully introduced. The two liquids must not be allowed to come in contact.

The larger flask is filled with water or, better, a solution of sodium sulphate; the connection is then made by means of the rubber tube, and the generating-bottle tipped over and agitated so that the liquids will mix and the reaction take place.

The liberated oxygen then passes into the larger bottle, displacing an equal volume of water, which is collected and measured. Half of this volume represents the volume strength of the $\mathrm{H}_{2} \mathrm{O}_{2}$.

An Improvised Nitrometer may be used. The author has found the following instrument convenient: 
To the bottom of an ordinary 50-cc burette is attached a suitable length of rubber tubing, to the other end of which is attached another burette or ungraduated tube, which serves as a control-tube.

Into the top of the burette is fitted a rubber stopper, through which passes a short glass tube, which is connected by means of a rubber tube to a generating bottle similar to that used with Squibb's Urea Apparatus. Into the control-tube is poured the solution of sodium sulphate, sufficient to fill the burette to the zero mark and have the surface of the liquid in both tubes on a level.

About $30 \mathrm{cc}$. of strong permanganate solution acidulated with sulphuric acid are now placed in the generating-bottle, and then the small test-tube or homeopathic vial, containing exactly I cc. of hydrogen dioxid, is placed in. The generating-bottle is then stoppered and agitated, the evolved gas passes over, and forces down the liquid in the burette; the control-tube is then lowered so as to bring the surfaces of the liquid in both tubes on a level.

The reading is then taken.

Each cc. of gas represents one half volume of oxygen evolved from the dioxid if I cc. of the latter is used. Each cc. of oxygen evolved from I cc. of the dioxid represents also $0.00 \mathrm{r} 688 \mathrm{gm}$. of absolute $\mathrm{H}_{2} \mathrm{O}_{2}$, or $0.0008 \mathrm{gm}$. of available oxygen.

Thus if from I cc. of the solution of hydrogen dioxid $20 \mathrm{cc}$. of gas are evolved, it is a so-called ten-volume solution, and contains $0.00 \mathrm{I} 688 \times 20=0.03376 \mathrm{gm}$. of absolute $\mathrm{H}_{2} \mathrm{O}_{2}$, or $0.0008 \times 20=0.016$ gm. of a vailable oxygen.

According to Naylor and Dyer (Trans. Brit. Ph. Conf., I90I, 339) the gasometric permanganate method is unreliable, because under the conditions of the test sulphuric acid added to the brine solution in the nitrometer naturally liberates a little hydrochloric acid, and this in the presence of permanganate becomes to some extent decomposed into chlorin. It is the uncertainty as to the extent to which the chlorin is absorbed by the water, which renders the accuracy of the method doubtful. The results of this method are uniformly too high, whether the gas, be collected over mercury, over saturated magnesium sulphate, or over brine, and in the latter case considerably higher. But when the dichromate V. S. is used (without acid), closely concordant results are obtained, whether the gas be collected over mercury, or the other solutions. The evolution of oxygen by the latter method is slower than when permanganate is used, but the oxygen obtained represents the volume available in the sample.

In the Hypochlorite.Method, the nitrometer is filled with a saturated solution of sodium chlorid. 2 cc. of the hydrogen dioxid are admitted into the measuring tube, the funnel tube filled with a little water, and this also let in, then $20 \mathrm{cc}$. of the chlorinated lime solution intro- 
duced. From this point the procedure is the same as in the gasometric permanganate method.

$$
\mathrm{Ca}(\mathrm{ClO})_{2}+{ }_{2} \mathrm{H}_{2} \mathrm{O}_{2}=\mathrm{O}_{4}+\mathrm{CaCl}_{2}+{ }_{2} \mathrm{H}_{2} \mathrm{O} \text {. }
$$

The presence of preservatives, except inorganic ones, gives low results.

The Hypobromite Method. W. M. Dehn (J. A. C. S., xxIx (9), I315) describes an accurate and rapid determination of hydrogen dioxid by means of sodium hypobromite, using a ureometer. The reaction involved is

$$
\mathrm{H}_{2} \mathrm{O}_{2}+\mathrm{NaBrO}=\mathrm{NaBr}+\mathrm{H}_{2} \mathrm{O}+\mathrm{O}_{2} .
$$

The apparatus is shown in Fig. 96. The following description of the method is by Dehn from the Journal of the American Chemical Society. "The stop-cock $E$ is opened and the stop-cocks $D$ and $F$ are closed; then the solution of sodium hypobromite* is poured in at the top of $C$ until it fills the tubes $A$ and $C$ to some point above the stop-cock $E$.

"The stop-cock $E$ is then closed and the stopcock $F$ is opened, so that the hypobromite in $C$ may run down to the constricted portion; the solution in $A$ is then sustained by atmospheric pressure. The stopcock $D$ (arranged so as to deliver only in the two directions of a right angle triangle) is turned from the position shown in the figure and is so controlled that $B$ may first be washed with a little of the hydrogen peroxid and then be filled with the same to a readable height on the scale. Upon turning $D$ so as to admit a regulated volume

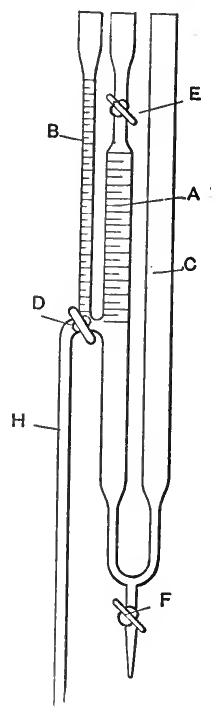

Fig. 96. of the hydrogen peroxid solution an immediate evaluation of oxygen results. After admitting most of the hypobromite held above $E$, and letting stand for a minute or two so as to drain properly, the columns of hypobromite in $A$ and $C$ are brought to the same level, the volume of oxygen is read off and its weight and that of the corresponding hydrogen dioxid are calculated by the usual formulas."

The author of this method also gives the following table, by means of which the cc. of oxygen, under various conditions of temperature

* This solution is prepared as directed under Estimation of Urea, except that it is finally diluted with an equal volume of water. 
and pressure, may be calculated into milligrams of hydrogen dioxid, and claims that by the use of this instrument, this hypobromite method and the table for calculating the assay of hydrogen dioxid, is not only rapid and accurate but the necessity of preparing and correcting standard solutions is avoided and the presence of the usual preservatives used in the dioxid solution may be ignored.

WEIGHT IN MILLIGRAMS OF $\mathrm{H}_{2} \mathrm{O}_{2}$ CORRESPONDING TO ONE CUBIC CENTIMETER OF MOIST OXYGEN

\begin{tabular}{|c|c|c|c|c|c|c|}
\hline$t / \mathrm{mm}$ & 728 & 732 & 736 & 740 & 744 & 748 \\
\hline $4^{\circ}$ & I. 2664 & I 2734 & I. 2802 & I. 2872 & I . 2942 & I . $301 \mathrm{II}$ \\
\hline 8 & I. 2463 & I . 253 I & I 2600 & I 2669 & I 2736 & I. 2805 \\
\hline 12 & I. $225 \mathrm{I}$ & I 2317 & I. .2387 & I. .2454 & I. 2522 & I . $25^{89}$ \\
\hline I6 & I 2044 & I. 2 I I I & I. 2178 & I. 2245 & I. 23 II & I. 2378 \\
\hline 20 & I. 18 I 7 & I. I 884 & I . I 948 & I. 2015 & I. 2080 & I. 2145 \\
\hline 24 & I 1583 & 1. 1649 & I. I 7 I9 & I. 1777 & I. . 843 & I. 1907 \\
\hline 28 & I. I 345 & I. I 4 I I & I. 1476 & I. I 538 & 1. 1603 & I. I 665 \\
\hline 32 & I. 1085 & I I I 49 & I I 213 & I. I 275 & I. I 338 & I. I 401 \\
\hline 36 & I . .0843 & I. 0905 & I. og67 & I. 1030 & I. 1093 & I. I I 55 \\
\hline 40 & I. .0605 & I. 0666 & I. .0725 & I. .0786 & I. .0849 & 1.0909 \\
\hline \multicolumn{2}{|c|}{${ }^{t} / \mathrm{mm}$} & 752 & 756 & 760 & 764 & 768 \\
\hline \multicolumn{2}{|c|}{$4^{\circ}$} & I. 3081 & I. $315^{I}$ & I. 3222 & I. 3290 & I. 3359 \\
\hline \multicolumn{2}{|c|}{8} & I. 2876 & I. 2944 & I. 3014 & I. $308 \mathrm{I}$ & I. 3150 \\
\hline \multicolumn{2}{|c|}{ I 2} & I. 2657 & I 2726 & 12823 & I. 2860 & I. 2928 \\
\hline \multicolumn{2}{|c|}{16} & I 2444 & I $25 \mathrm{I} 2$ & I. 2578 & I 2946 & I. $27 I_{3}$ \\
\hline \multicolumn{2}{|c|}{20} & I. 2213 & I 2279 & I 2345 & I. 2410 & I. 2475 \\
\hline \multicolumn{2}{|c|}{24} & I. I 972 & I. 2036 & I. 2100 & I. 2169 & I. 2230 \\
\hline \multicolumn{2}{|c|}{28} & I. I 731 & I. I 796 & I. I 857 & I. I 922 & I. I 986 \\
\hline \multicolumn{2}{|c|}{$3^{2}$} & I. I 465 & I. I 528 & I . I 589 & I. I 562 & I. I 7 I 5 \\
\hline \multicolumn{2}{|c|}{36} & I. I 2 I 4 & I. I 279 & I. I34I & I. 1402 & I. I 465 \\
\hline \multicolumn{2}{|c|}{40} & I . 097 I & I. 1033 & I. I094 & I. I I 55 & I. 1216 \\
\hline
\end{tabular}




\section{CHAPTER LXIV}

\section{ESTIMATION OF SOLUBLE CARBONATES BY THE USE OF THE} NITROMETER

THE nitrometer may be used for estimating ammonium carbonate in aromatic spirit of ammonia.

The nitrometer in this case must be charged with mercury, as the liberated $\mathrm{CO}_{2}$ is soluble in aqueous liquids.

A given volume of the spirit is introduced into the nitrometer followed by an excess of dilute $\mathrm{HCl}$, and the evolved gas then read off; and from its quantity the proportion of ammonium carbonate may be calculated by applying the equation

$$
\underset{{ }_{9} 6}{\left(\mathrm{NH}_{4}\right)_{2} \mathrm{CO}_{3}+{ }_{2} \mathrm{HCl}}=2 \mathrm{NH}_{4} \mathrm{Cl}+\mathrm{H}_{2} \mathrm{O}+\underset{*_{44}}{\mathrm{CO}_{2} .}
$$

The volume of gas liberated must first be reduced to its corresponding volume at $0^{\circ} \mathrm{C}$.

Each cc. of $\mathrm{CO}_{2}$ at $0^{\circ} \mathrm{C}$. weighs $0.001966 \mathrm{gm}$. Now if $44 \mathrm{gms}$. of $\mathrm{CO}_{2}$ represent $96 \mathrm{gms}$. of normal ammonium carbonate, how much ammonium carbonate does $0.001966 \mathrm{gm}$. of $\mathrm{CO}_{2}$ represent?

$$
\text { 44:96::0.001966: } x . \quad x=0.004289 \mathrm{gm} .
$$

Thus each cc. of $\mathrm{CO}_{2}$ at normal pressure and $\circ^{\circ} \mathrm{C}$. represents $0.004289 \mathrm{gm}$. of $\left(\mathrm{NH}_{4}\right)_{2} \mathrm{CO}_{3}$, approximately.

* The atomic weights are approximate. 


\section{CHAPTER LXV}

\section{ESTIMATION OF UREA AND URIC ACID IN URINE}

THE determination of urea is based upon the fact that when urea is decomposed by an alkaline hypochlorite or hypobromite, carbon dioxid and nitrogen are given off, as the equation shows:

$$
\mathrm{CO}\left(\mathrm{NH}_{2}\right)_{2}+{ }_{3} \mathrm{NaBrO}={ }_{3} \mathrm{NaBr}+\mathrm{CO}_{2}+\mathrm{N}_{2}+{ }_{2} \mathrm{H}_{2} \mathrm{O} \text {. }
$$

The liberated $\mathrm{N}$ may be measured, and from its quantity the quantity of urea calculated; the other products of the decomposition go into solution.

The hypobromite solution is prepared as follows: roo gms. $\mathrm{NaOH}$ are dissolved in $250 \mathrm{cc}$. of water, and when this solution has become cold $25 \mathrm{cc}$. of bromin are added, and the solution kept cold. This solution contains sodium hypobromite, bromate, and hydroxid. It readily undergoes decomposition, and should therefore always be freshly prepared when wanted for use. To $15 \mathrm{cc}$. of the $\mathrm{NaOH}$ solution add I cc. of bromin.

The solution of sodium hypochlorite is generally preferred to the hypobromite, because it is more stable, just as efficacious, and the disagreeable handling of bromin is obviated.

Various forms of apparatus have been devised for the quantitative estimation of urea.

The simplest of these is probably the one devised by Dr. Chas. A. Doremus. (See Fig. 97.)

The long arm of the ureometer is filled with the hypobromite solution, and then I cc. of the urine is introduced by the aid of the pipette. The pipette is introduced through the bulb as far as it will go in the bend, and the nipple is then gently but steadily compressed, being careful that no air is admitted. sided.

The volume of the liberated gas is read off after the froth has sub-

The ureometer indicates, according to its graduation, either milligrams of urea in I cc. or grains of urea per fluid ounce of urine. 
It also indicates by the signs,$+ \mathrm{N}$, and - whether the urea is present in an increased, normal, or decreased quantity.

Either Knop's or Squibb's solution, or Liquor Sodæ Chloratæ U. S. P. may be used in this instrument. Knop's solution is that described above. Squibb's solution contains potassium bromid as well as bromin. It is prepared by taking an equal weight of bromin and of potassium bromid, and adding eight times as many cc. of water as there were grams of bromin taken. For use mix equal volumes of this solution with the sodium hydroxid solution above described.

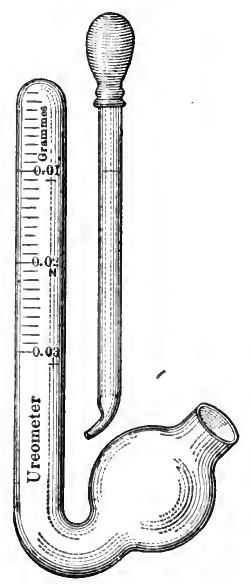

FIG. 97.

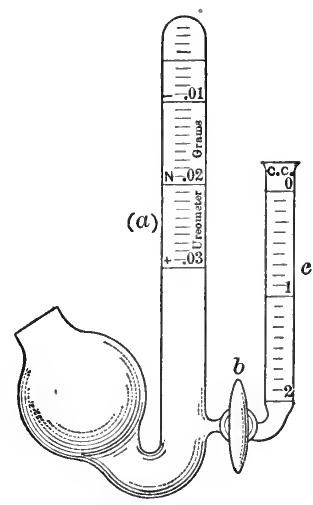

FIG. 98.

The Hinds-Doremus Ureometer. This apparatus, which is shown in Fig. 98, is capable of giving more exact results than the original apparatus, because the I cc. of urine can be delivered more accurately. It consists of a bulb with an upright tube $a$, graduated like the original, so that each of the smallest divisions represents $0.00 \mathrm{Igm}$. of urea in the urine used. The lower portion of this tube is in connection with a smaller tube $c$, graduated with a capacity of 2 cc.; between these tubes a glass stop-cock is situated. Closing the stopcock $b$, Knop's or Squibb's fluid (diluted one half) or liquor sodæ chloratæ U. S. P. are introduced into tube $a$ so as to completely fill it. The apparatus is then placed in an upright position and the smaller tube $c$ is filled to the zero mark with urine. The stop-cock is then turned slowly so as to admit gradually I cc. of the urine to tube $a$. After fifteen minutes the reading is taken. If the reading be 0.015 and the amount of urine taken was I cc., then multiplying by 100 gives $\mathrm{I} .5$ per cent of urea. 
Another Convenient Form of Apparatus is a tube closed at one end and graduated so that each division indicates a grain of urea in a fluid ounce of urine, when I cc. of urine is iaken for the estimation. (See Fig. 99.)

The process is conducted as follows: A 25 per cent solution

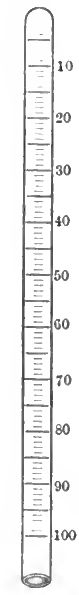
of $\mathrm{KBr}$ is introduced to the fifth division. The chlorinated soda solution is then added to the fifteenth or $t$ wentieth division. The tube is now inclined, and pure water carefully poured upon the liquid so that it will float on top; I cc. of urine is then added carefully, so that it will not mix with the reagents below, but remain in the water at the surface of the fluid. The open end of the tube is then quickly closed with the thumb, and the top firmly grasped in the right hand. The tube is then inverted, and the contents well mixed. The decomposition which takes place is usually ended in five minutes. As soon as the effervescence has ceased, the reading is taken at the surface of the liquid. The tube is now opened under water, when the column of fluid in the tube will fall; the reading is then again taken. The difference between the two readings gives the number of grains of urea in a fluid ounce FIG. 99. of the urine.

Squibb's Urea Apparatus (Fig. Ioo) is a very simple apparatus, and can be easily improvised in a drug store. It consists of two widemouthed bottles, the larger of which, $C$, capable of holding about $250 \mathrm{cc}$, is fitted with a rubber stopper, through which is passed a curved delivery-tube and a short straight tube, the latter connected by a piece of rubber tubing to the short glass tube in the rubber

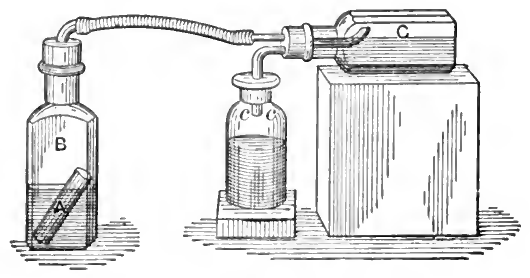

FIG. 100.

stopper of the smaller bottle or generating-bottle $B$. In the generatingbottle is a small test-tube $A$.

Into the test-tube $A$ is placed $5 \mathrm{cc}$. of urine, and into the smaller bottle $B$ is put $20 \mathrm{cc}$. of the hypobromite solution, or strong liquor sodæ chloratæ. The test-tube is then placed in the generating- 
bottle $B$, being careful that the urine and the reagent do not come in contact. The larger bottle $C$ is now filled with water and the two bottles connected by the rubber tube, the larger bottle being placed on its side upon a block, and when all connections are tight, the generating-bottle is shaken so that the urine will mix with the reagent.

Decomposition takes place, and the generated gas passes into the bottle $C$, displacing water, which is caught in a graduated cylinder or other measuring vessel. The volume of water displaced is equivalent to the volume of gas evolved.

Each cc. of nitrogen gas evolved at $0^{\circ} \mathrm{C}$. and normal pressure represents $0.0027 \mathrm{gm}$. of urea. Then by multiplying the number of cc. evolved by this number the quantity of urea in the $5 \mathrm{cc}$. of urine taken is ascertained.

The volume of gas obtained when the operation is conducted at ordinary temperatures should always be reduced to its corresponding volume at $0^{\circ} \mathrm{C}$. and $760 \mathrm{~mm}$.

The factor 0.0027 is found in the following manner:

$$
\begin{aligned}
& \text { rooo cc. of } \mathrm{H} \text { at } \circ^{\circ} \mathrm{C} \text {. weigh } 0.0896 \mathrm{gm} \text {.; } \\
& \text { rooo cc. of } \mathrm{N} \text { at } 0^{\circ} \mathrm{C} \text {. weigh } \mathrm{r} .248 \mathrm{gms} \text {. }
\end{aligned}
$$

By the equation it is seen that 59.65 gms. of urea evolve when decomposed 27.96 gms. of $\mathrm{N}$.

$$
\underset{59.65 \text { gms. }}{\mathrm{CO}\left(\mathrm{NH}_{2}\right)_{2}+{ }_{3} \mathrm{NaBrO}={ }_{3} \mathrm{NaBr}}+\underset{27.96 \text { gms. }}{\mathrm{CO}_{2}+\mathrm{N}_{2}+{ }_{2} \mathrm{H}_{2} \mathrm{O} .}
$$

Now we will find the volume occupied by 28 gms. of $\mathrm{N}$ at $\circ^{\circ} \mathrm{C}$.

$$
\text { I.248 gms. of } \mathrm{N}=\mathrm{I} 000 \mathrm{cc} \text {. }
$$

$$
\begin{array}{ccc}
\text { gms. } & \text { cc. } \quad \text { gms. } & \text { cc. } \\
\text { r. } 248 \cdot \text { r } & \text { 1000. }: 28: x . & x=23237
\end{array}
$$

Thus 59.65 gms. of urea evolve 23237 cc. of $\mathrm{N}$; I cc. of $\mathrm{N}$ thus represents $0.0027 \mathrm{gm}$. of urea.

By Means of Sellier's Urometer.* In order to obtain reliable results in urea determinations according to Sellier, it is necessary to operate upon the urine clarified by means of lead subacetate, and with freshly prepared bromin solution, in the presence of glucose.

* Ph. Ztg., 48, No. 62 (August 5, 1903), 627; from Chem. Centralbi., I9०3, II, No. 4 . 
The bromin solution is prepared according to Moreigne's formula: Io cc. of soda solution $36 \mathrm{~B}$., $70 \mathrm{cc}$. of water, and ro cc. of bromin. The

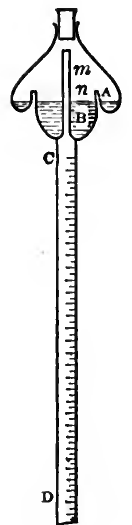

FIG. IOI. reaction is convenientlycarried out by the aid of the urometer (Fig. IoI). The bulb is divided by a central partition $m n$ into separate chambers $A$ and $B$, communicating with each other, and with the graduated tube $C D$, at $m$. In use, 2 cc. of the clarified urine and I cc. of 25 per cent glucose solution are allowed to flow along the wall into $A$, and I $_{5} \mathrm{cc}$. of the bromin solution in the same way into $B$. The stopper is inserted, the apparatus placed into water, until both have the same temperature, and water is then admitted into the tube up to the $0^{\circ}$ mark, by lifting the stopper, and adjusted to the level of the water of immersion. The reacting materials in $A$ and $B$ are then mixed, and the tube raised so that the interior and the outer level of the water shall be identical, observing also that the temperature has been readjusted, when the volume of nitrogen may be accurately read in cc. values.

Uric Acid. A. F. Dimmock and F. W. Branson* have devised a new method for the determination of uric acid in urine, which has been found to work easily. The urine (roo cc.) is warmed to about $40^{\circ} \mathrm{C}$., and then saturated with ammonium chlorid (3 $\mathrm{r}$ gms.), the whole being well shaken in a graduated flask until complete solution of the ammonium chlorid is effected and then left for from two to twelve hours (preferably the latter) for the complete precipitation and subsidence of the ammonium urate formed. The supernatant liquid is decanted, the residual ammonium urate collected on a small paper filter (about $5.5 \mathrm{~cm}$. in diameter) and carefully washed with a very dilute solution of ammonia, consisting of one part of liq. ammon. fort. in rooo parts of distilled water, until the filtrate yields only a slight precipitate with a 5 per cent solution of silver nitrate acidulated with 5 per cent of nitric acid-this indicating the absence of an appreciable amount of ammonium chlorid. The precipitate, with the filter-paper, is then placed into the generating-bottle shown in the accompanying illustration (Fig. I02) of the nitrometer, a tube containing $25 \mathrm{cc}$. of hypobromite solution (100 gms. sodium hydrate, $250 \mathrm{cc}$. water, and 22 cc. bromin) is lowered into the same bottle by means of a string, and, having immersed the latter in a vessel of water having the temperature of the room, the connection may, after two minutes or so, be made with the nitrometer; the reaction being established by tilting 
the generating-bottle so that the reagent may flow from the tube. The operation must, of course, be conducted with the usual precau-

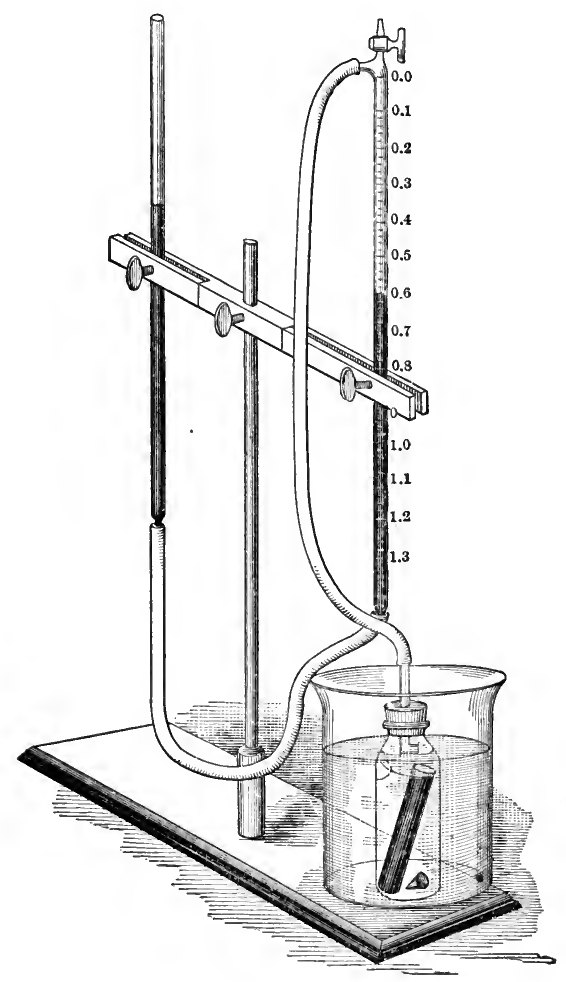

FIG. I02.

tions against leakage of apparatus, etc., which are explained in some detail. The method is available for urine containing from I in 1000 to $I$ in 10,000 uric acid. 



\section{INDEX}

PAGE

Abbreviations. ............................... ix

Acetate of lime, valuation of. . . . . . . . . . . . . . . . . 238

Acetates, metaliic, estimation of. ........................ 238

Acetic acid. . ....................................... 104

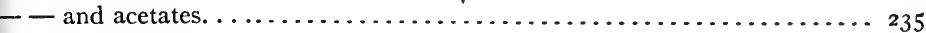

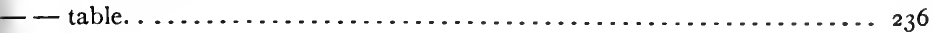

Acid, acetic....................................... 104

- and acetates. ................................. 235

Acid, arsenous, preparation of decinormal solution of............. 227

Acid, boric, estimation of. . . . . . . . . . . . . . . . . . . . 104, 240

- carbolic, assay of. . ............................... 6r 3

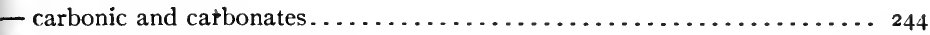

- carbolic, assay of, in dressings. . . . . . . . . . . . . . . . . . .649,651

- chloric, iodometric estimation of. ...................... 301

- chromic. . .................................. I 74

- estimation of, by digestion method................... 222

- estimation of, by distillation method .................. 2 I9

- citric . .................................. 105

- hydrobromic. ................................... I 20

- - assay of using chromate as indicator. . . . . . . . . . . . . . I I

- hydrochloric. ................................. 100, I 20

- half normal, V.S. . .......................... 70

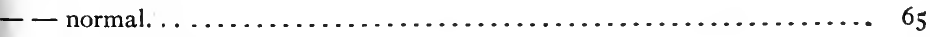

- - V.S. gravimetric standardization by means ot silver nitrate . . . . . 68

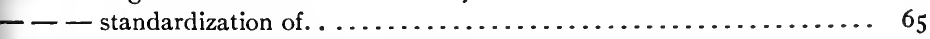

- - standardization by means of borax. .................. 66

- - - standardization by means of calc-spar................... 69

- - standardization by means of marble. ................ 70

— - standardization by means of silver nitrate volumetrically. . . .... 67

- - standardization of by means of specific gravity . . . . . . . . . . 66

- hydrocyanic assay, using chromate as indicator. . . . . . . . . . . . 127

- - use of Porrier Blue as indicator. . . . . . . . . . . . . . . . 127

- - using potassium iodid as indicator. . . . . . . . . . . . . . 28

- - estimation by Liebig's method. ....................... I 26

- hydriodic .................................... I 20

- - assay of by sulphocyanate. ........................ 123

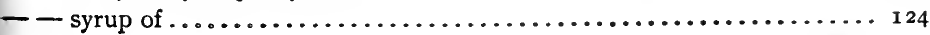


Acid, hydrosulphuric, estimation of by permanganate. $\ldots \ldots \ldots \ldots \ldots \ldots \ldots \ldots \ldots \ldots$

- - estimation of by means of iodin........................ 329

- hypophosphorous estimation. ........................ 167

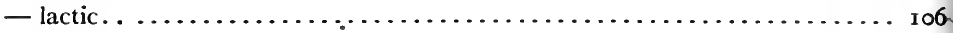

- nitric.............................................. 103

- - iodometric estimation of . . . . . . . . . .

- - nitrometer assay of. . ................................ 686

- nitrous, estimation of, in chamber acid. . ................... 303

- - estimation of by means of permanganate................ 65,302

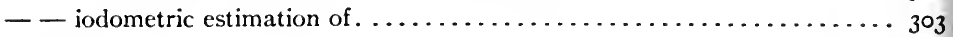

- oxalic. ......................................... 105

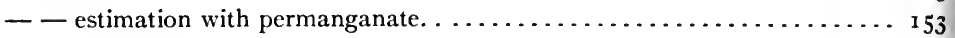

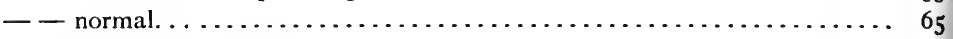

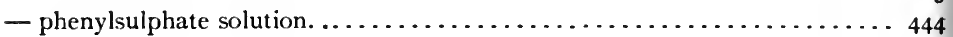

- phosphoric .................................. 10

- - estimation of, by Glückmann's method..................... 315

- - estimation of, by Pemberton's new method.................. 317

- - estimation of, by Pemberton's molybdic method. .............. $3^{16}$

- estimation by means of standard uranium. ................. $3^{12}$

- - Stolba's method . . ............................ 3 I I

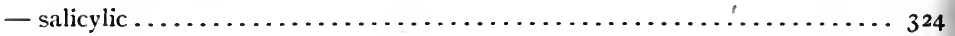

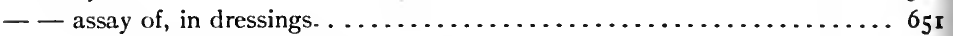

- - estimation of, by means of bromin.......................... 325

- - iodometric estimation of. . . . . . . . . . . . . . . . 324

- solution, preparation of standard.......................... 64

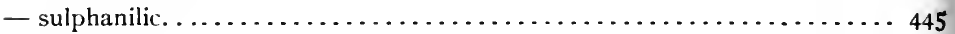

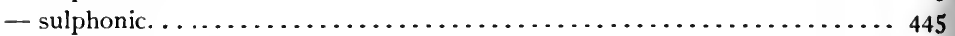

- sulphuric, estimation of. ........................ $33^{\mathbf{I}}$

- - V.S., gravimetric standardization by means of barium chlorid. ..... 73

- - iodometric standardization. . . ................. $7 \mathbf{I}$

- - - standardization by ammonium sulphate method........... 72

- - standardization by means of sodium carbonate............. 70

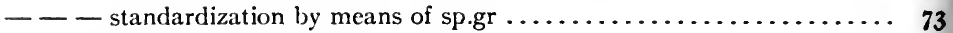

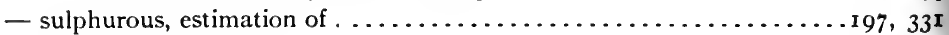

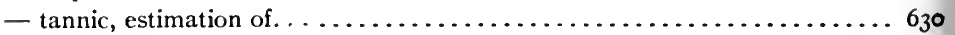

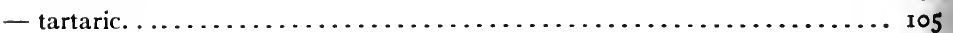

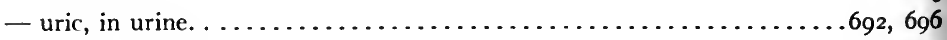

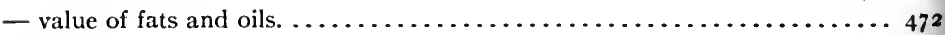

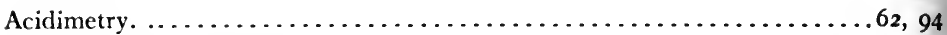

Acids, estimation of, in neutral salts. . . . . . . . . . . . . . 108

— estimation of, by neutralization....................... 94

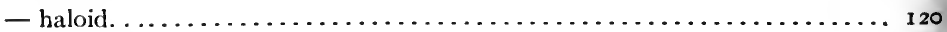

- inorganic. ..................................... 99

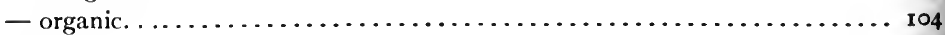

Aconite extract, assay of.............................. 588

- fluid extract, assay of..... $\quad \ldots \ldots \ldots \ldots \ldots \ldots \ldots \ldots \ldots \ldots \ldots \ldots \ldots \ldots \ldots \ldots \ldots$

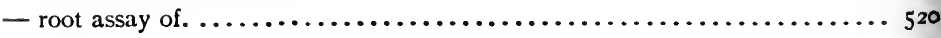


PAGE

Aconite tincture, assay of

Adams' method for fat in milk. ......................... 46 I

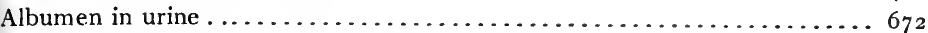

Albuminoid ammonia, estimation of in water.................. 44 I

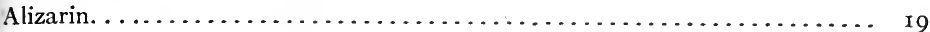

Alkali bicarbonate and carbonate mixed. . . . . . . . . . . . . . . 82

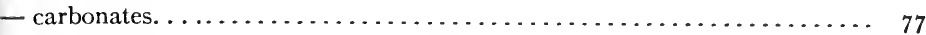

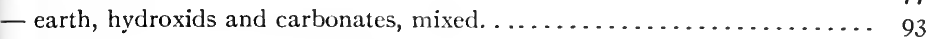

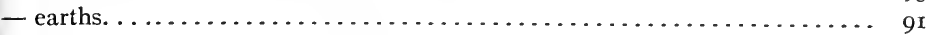

- hydroxid and carbonate mixed............................. $8 \mathrm{r}$

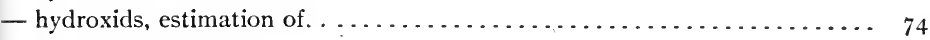

- metals, estimation of, in their salts. .................... 89

— standard solutions ................................ 96

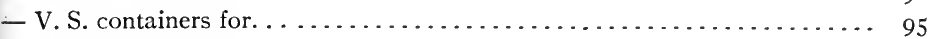

Alkalies, in presence of sulphites. . . . . . . . . . . . . . .

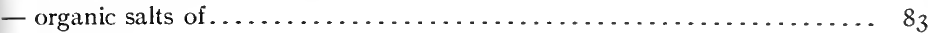

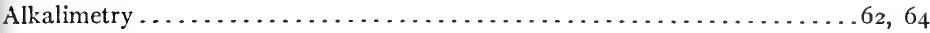

Alkaloidal assay by immiscible solvents...................... $5^{1} 2$

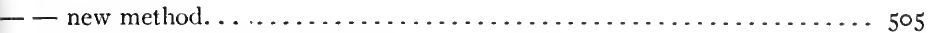

- salts, general method of procedure in estimating. .............. 507

- - solubility of in chloroform, etc. .......................... $5^{14}$

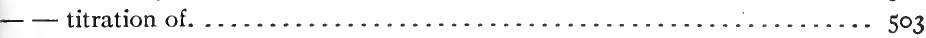

- strength of scale salts, estimation of. ..................... 6 2

Alkaloids, behavior of, with indicators. . . . . . . . . . . . . . . 503

- estimation of. . . . . . . . . . . . . . . . . . . . . . . 498

- estimation of by Gordon's modified alkalimetric method. . .......... 5 I0

- estimation of by Mayer's reagent. . . . . . . . . . . . . . . . . 505

- estimation of by Wagner's reagent. . ....................... 507

- extraction of from drugs.......................

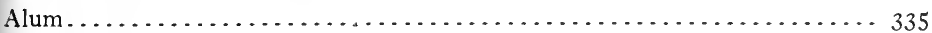

- estimation of by iodometric method. . . . . . . . . . . . . . . 337

- estimation by titration with barium hydroxid. . . . . . . . . . . . 336

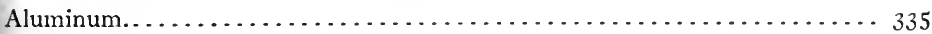

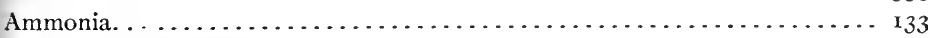

- albuminoid, in water............................

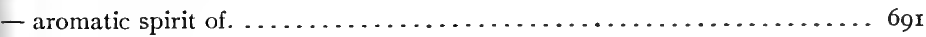

- estimation of. . . . . . . . . . . . . . . . . . . . . . . 338

- estimation of, in water.................................. 440

- water. . ....................................... 76

Ammoniated mercury, assay of . . . . . . . . . . . . . . . . .

Ammonium bromid. ............................... 6

- determination of the amount of chlorid present............. II7

- carbonate....................................... 79

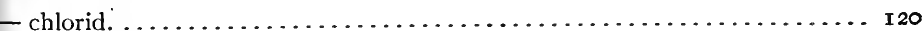

- - estimation of in ammonium bromid.................... II7

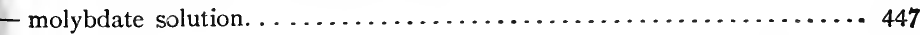

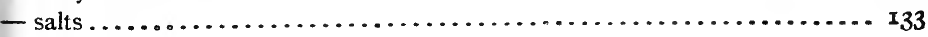


Ammonium salts, estimation of by ditillation

Ammonium salts, estimation of by distillation................... 338

- - estimation of by means of formaldehyde..................... 339

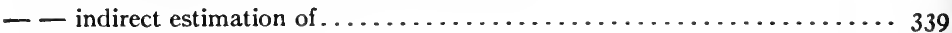

Amyl nitrite, nitrometer assay of . . . . . . . . . . . . . . . . . 685

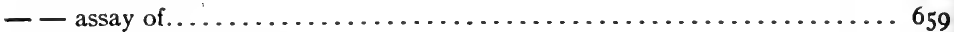

Analysis by neutralization. . . . . . . . . . . . . $\mathbf{6}_{2}$

- by oxidation and reduction. ......................... 137

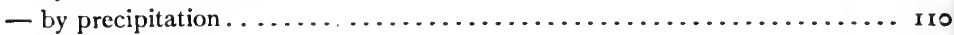

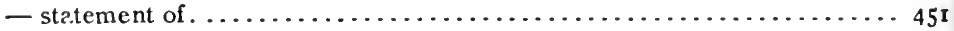

Anthracene Violet. .................................. 20

Antimonic acid and its salts................................ 344

Antimonous chlorid, estimation of. . . . . . . . .

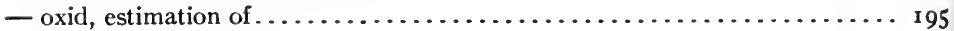

- sulphid, estimation of. . . . . . . . . . . . . . . . . . . 340

- - estimation of by ferric sulphate and permanganate............ 344

Antimony and potassium tartrate, estimation of. ............... 196

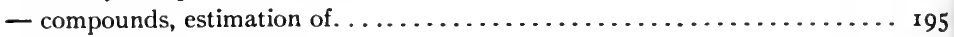

- estimation of by iodin. . . . . . . . . . . . . . . . . . . . 340

- estimation of by means of dichromate or permanganate........... 342

- estimation of by titration with standard bromate solution........... 34 I

- metallic, estimation of. . .............................. 340

Apparatus, on the use of. ........................... 44

- used in volumetric analyses. . . . . . . . . . . . . . . $3^{2}$

Aromatic spirit of ammonia. ......................... 6 r

Arsenates, estimation of by means of magnesia mixture. . . . . . . . . 348

Arsenic estimation of by dichromate. . . . . . . . . . . . . . . 346

- estimation of by means of iodate. ....................... 347

- estimation of in Paris green, Smith's method. . . . . . . . . . . . 350

- estimation of in small quantities. . . . . . . . . . . . . . . . . . 349

— in Paris green, estimation of Avery and Beans' method. . . . . . . . 35 I

- oxid, estimation of, after reduction....................... 348

- - estimation of by means of magnesia mixture.............. 348

- estimation of by means of uranium..................... 347

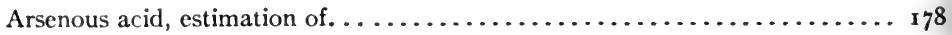

- - preparation of decinormal solution of . . . . . . . . . . .

- compounds, estimation of. ......................... 192

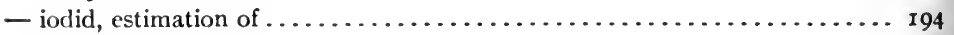

- oxid, estimation of . ............................. 192

- - estimation of by distillation with chromic and hydrochloric acids. . . . 348

Assaying drugs, general methods of. . . . . . . . . . . . . .

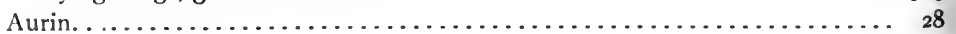

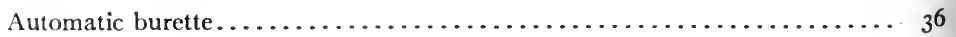

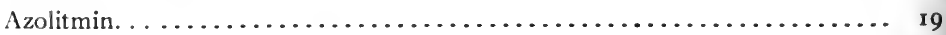

Babcock centrifugal method. . . . . . . . . . . . . . . . . 462

Barium compounds, estimation of. . . . . . . . . . . . . . . 353

- dioxid, estimation with permanganate................. 156, r6 
Barium, estimation of........................... $77^{8}$

- estimation of, by means of dichromate. . . . . . . . . . . . . 353

- estimation of, by indirect iodometric method. . . . . . . . . . . . . 354

- hydroxid solution, for estimation of starch.................. 484

- peroxid estimation, with permanganate $\ldots \ldots \ldots \ldots \ldots \ldots \ldots \ldots \ldots$ r $6 \ldots \ldots$

Baryta-water. . . . . . . . . . . . . . . . . . . . . . . . . . 484

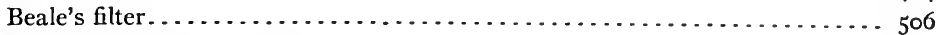

Belladonna extract, assay of. . . . . . . . . . . . . . . . . . . . 589, 593

— fluid extract, assay of. . . . . . . . . . . . . . . . . . . . . . . 594, 596

- leaves, assay of. . . . . . . . . . . . . . . . . . . . . . . 528

- plaster, assay of. . . . . . . . . . . . . . . . . . . . . . . . 598

- root, assay of................................. 527

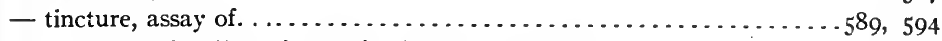

Bicarbonate of sodium, its use in titrations with iodin . . . . . . . . . . r 90

Bichlorid dressings, assay of. ............................ $65^{r}$

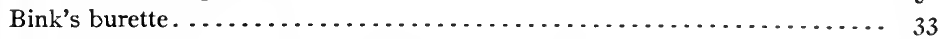

Bismuth, estimation of by precipitation as chromate............. 355

- estimation of by precipitation as molybdate. . . . . . . . . . . . 357

- estimation of by precipitation as oxalate. . . . . . . . . . . . . 355

- estimation of by precipitation as phosphate. . . . . . . . . . . $35^{6}$

- potassium iodid method for the assay of galenicals. . . . . . . . . . . 598

Bisulphite, sodium.............................. 201

Bitter almond water. . . . . . . . . . . . . . . . . . . . . . . r 28

Bleaching powder.................................... 2 10

— estimation of by means of arsenous acid. .................. 229

Blue, gentian. . . . . . . . . . . . . . . . . . . . . . . . . . 27

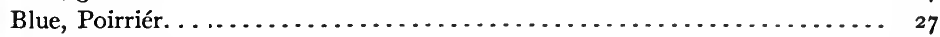

Borates. . .................................. $3^{2}$

Borax, estimation of. .............................. 240

Boric acid. . ....................................... 104

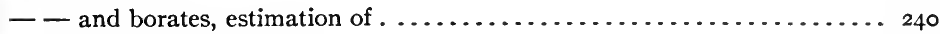

- detection of in milk. . . . . . . . . . . . . . . . . . . . . . 466

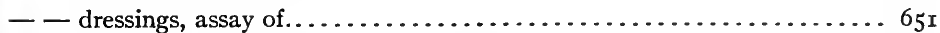

- estimation, with ferric salicylate indicator. . . . . . . . . . . . 24I

Bottle, for preserving stannous chlorid V. S.................. $23^{2}$

Boyle's law. ...................................... 680

Brazil wood T. S. . ................................ I9

Bromates, estimation of by digestion method. ................22, 223

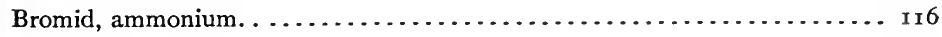

- lithium.................................... I 9

- sodium. .................................. I 19

- strontium. ................................. I 9

- potassium. .............................. I 9

- zinc. ................................ 19

Bromids, direct titration of, with chlorin water. . . . . . . . . . . $26_{5}$.

- estimation of. . .............................. I 5

- or chlorids, estimation of in presence of sulphocyanate. . . . . . . . . 268 
PAgE

Bromin absorption number. ....................... 478

- methods of estimation........................... 260

- solution, decinormal, preparation of .................... 6 1 $3_{3}$

- value of fats and oils........................... 478

- water. . . . . . . . . . . . . . . . . . . . . . . . . 213

Bunsen's distilling apparatus. ...................... 215

Bunsen valve. . . . . . . . . . . . . . . . . . . . . . . . . . 143

Burette, glass stop-cock ........................... 33

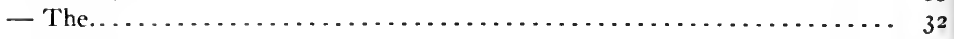

- Mohr's. ................................... $3^{2}$

- Weight. .................................... 59

- with blue striped background....................... 48

- with enameled sides. . . . . . . . . . . . . . . . . . . . . 48

Burettes, various forms of. . . . . . . . . . . . . . . . . 39

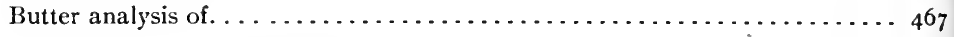

- estimation of volatile acids in. ........................ 467

Caffein, estimation of. ............................. 509

Calabar bean, assay of . . . . . . . . . . . . . . . . . . 576

Calchicum, assay of. . . . . . . . . . . . . . . . . . . . 542

Calcium chlorid, standard solution of. . . . . . . . . . . . . . . . 449

- estimation of, by precipitation as oxalate. . . . . . . . . . . . . 359

- hypophosphate, estimation. . . . . . . . . . . . . . . . . . . . I69

- salts, estimation as oxalate. . . . . . . . . . . . . . . . . 55

- estimation with permanganate. .................. r 70

Calculating analyses, methods of. . . . . . . . . . . . . . . . . 53

Calibration of instruments. . ....................... 49

Calx chlorinata. . . . . . . . . . . . . . . . . . . . . . . 210

Carbon monoxid in the air........................... 258

Carbolic acid, assay of. ............................ $66_{13}$

- - dressing, assay of. . . . . . . . . . . . . . . . . . . . . 649

Carbonates..................................... 132

- estimation of by the nitrometer. .................... $66_{91}$

- insoluble, estimation. . . . . . . . . . . . . . . . . . . . . . 245

- of the alkalies, estimation. . . . . . . . . . . . . . . . . . . 244

Carbonic acid and carbonates. . . . . . . . . . . . . . . . . . . . . 244

- gas. ................................ 133

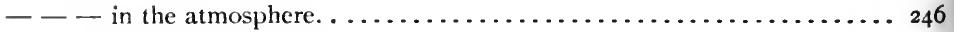

- - in insoluble carbonates........................... 245

- - in natural waters. . . . . . . . . . . . . . . . . . . $\ldots \ldots \ldots$

- in solutions in water. ............................ 246

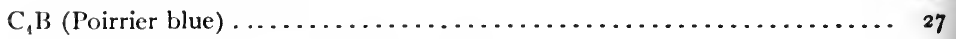

Chamber acid, estimation of nitrous acid in. ................. 303

Charles' law. .................................... 679

Cherry laurel water. ................................. 28

Chloral hydrate, estimation of. ........................ 647

Chlorates....................................... I33 


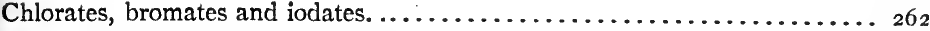

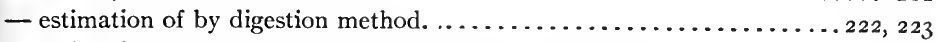

- estimation of by means of standard potassium iodate. . .......... 264

Chloric acid, iodometric estimation of . ...................... 301

Chlorid, ammonium. . ................................ I 20

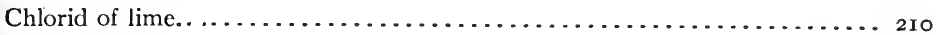

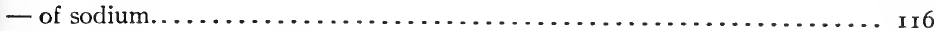

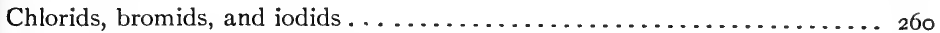

- - estimation of, in the presence of each other. . . . . . . . . . . .

- estimation of. ..................................... I 5

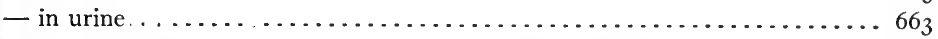

— or bromids, estimation of, in presence of sulphocyanate........... 268

Chlorinated lime........................................ 210

- - estimation of, by means of arsenous acid. ................ 229

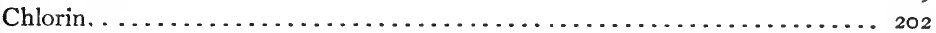

- methods of estimation............................... 260

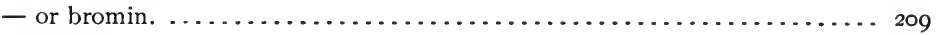

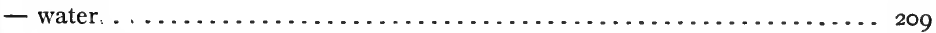

- estimation of by means of arsenous acid.................. 229

- estimation of iodids and bromids with. . . . . . . . . . . . . . 265

Chloroform, estimation of. . . . . . . . . . . . . . . . . . . 646

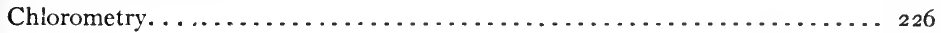

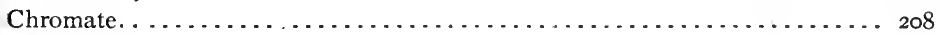

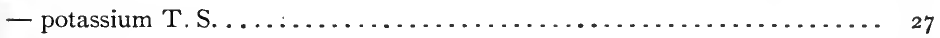

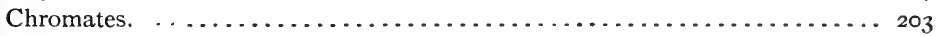

Chromic acid and chromates........................... I74

- - estimation of by distillation method.................. 2 I9

- - estimation of by digestion method ....................... 222

Chromophoric theory of indicators........................ ${ }_{4}$

Cinchona bark, assay of. ................................. 53 I

- extract, assay of. ....................................... 589

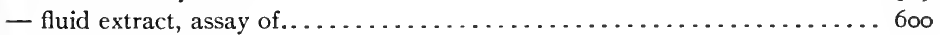

- tincture, assay of. . . ................................. 589

Citrates of the alkalies and alkali earths..................... 270

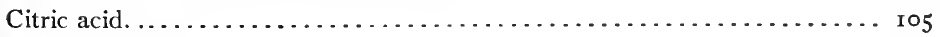

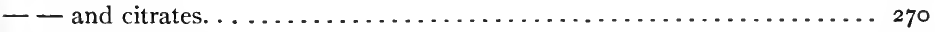

Cobaltic oxid, estimation of by distillation method. ............... 219

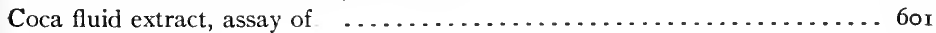

- leaves, assay of. ....................................... 537

Cochineal T.S. .................................... I9

Colostrum. . . . . . . . . . . . . . . . . . . . . . . . . . 458

Compound ethers, estimation of . . . . . . . . . . . .

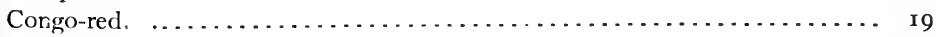

Conium, assay of. . . . . . . . . . . . . . . . 544

Copper, acidimetric estimation of, after precipitation with cuprous thiocyanate 372

- estimation of, as sulphid. . . . . . . . . . . . . . . . . . . . . .

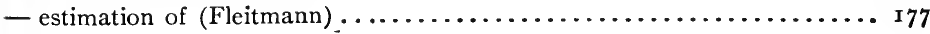


Copper, estimation of by means of ferrocyanid. . . . . . . . . . 375

- estimation of by means of potassium cyanid.................. 364

- estimation of by means of stannous chlorid. $\ldots \ldots \ldots \ldots \ldots \ldots \ldots \ldots \ldots \ldots \ldots \ldots$

- estimation of by means of thiocyanate. .................. $37 \mathbf{r}$

- estimation of by means of thiocyanate and permanganate. . . . . . . 373

- estimation of by precipitation as cuprous oxid. . . . . . . . . . . . 362

- estimation of by precipitation as metallic copper. . . . . . . . . . . 363

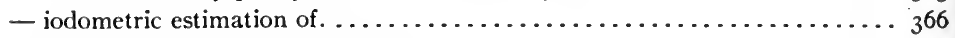

- ores, assay of by Dulin's process. . ...................... 366

- - assay of by Low's process............................ 369

- - assay of by Meade's process.......................... 374

- - assay of by Parr's process.............................. 375

- assay of by Steinbeck's process........................... 365

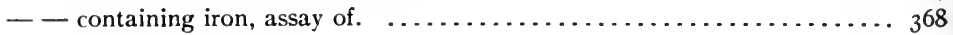

-

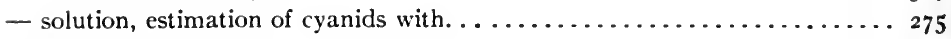

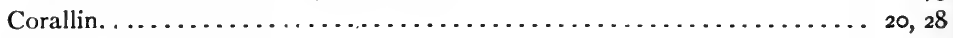

Cotton, styptic, assay of . . . . . . . . . . . . . . . . . . 655

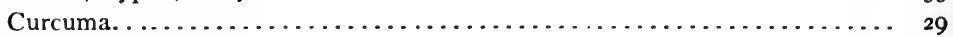

Cyanid, potassium............................... 29

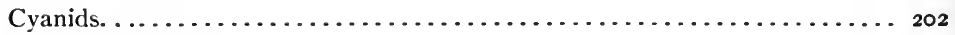

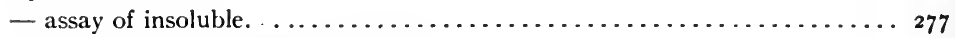

— estimation of by modified Kjeldahl process. . .............. 276

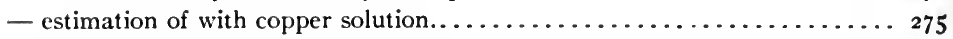

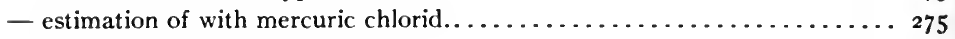

- titration of with iodin solution......................... 274

Cyanogen and its compounds. . . . . . . . . . . . . . 272

- estimation of. . . . . . . . . . . . . . 25

- estimation of by Liebig's method'. .................... 125

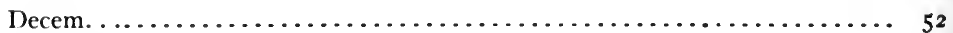

Decimillem. . . . . . . . . . . . . . . .

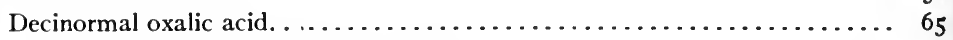

Diastase, use of in estimation of starch...................... 487

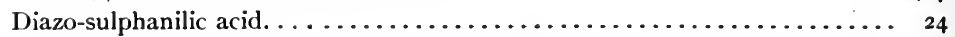

Dichromate, analysis by means of. .................... 178

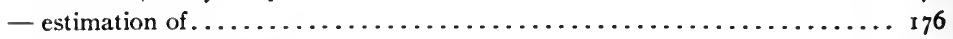

- method of titrating with. . . . . . . . . . . . . . . . . . 183

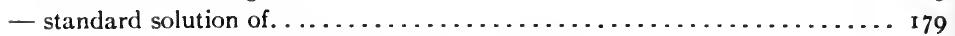

- use of, for standardizing sodium thiosulphate V. S. .............. 204

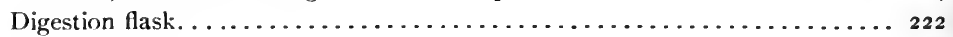

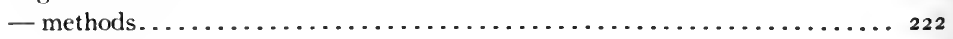

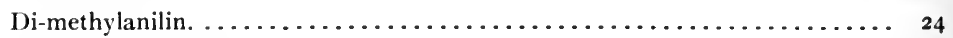

Dioxid of hydrogen. . . . . . . . . . . . . . . . . . . . 213

- assay of gasometrically................................... 687

Direct percentage estimations.......................... 53

Distillation apparatus for alkali iodids....................... 220 
Distillation methods. . . . . . . . . . . . . . . . . . $\ldots \ldots \ldots$

Dolomite, estimation of, with permanganate. ............... ${ }_{5} 6$

Dressings, surgical, assaying of............................ 649

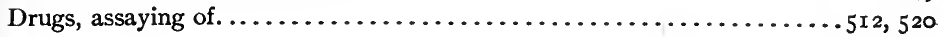

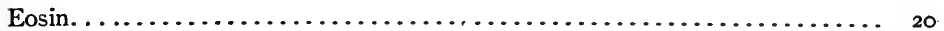

Eau de Javelle................................ 2 r $2_{2}$

Emetine (Kunz).................................. 499

Empirical solutions. ................................ 9 .

End-point, determination of when using Fehling's solution . . . . . . . . $49 \mathrm{r}$

- reactions, precision in determining in precipitation analyses.......... Ir $\mathrm{r}$

Erdman's float. . ............................... 47

Erythrosin B. ........................... 2 I

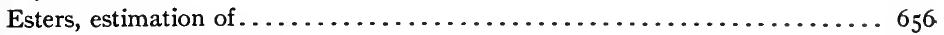

Ether, nitrous, estimation of. . . . . . . . . . . . . . . . . . . $66_{57}$

Ethers, compound, estimation of. . . . . . . . . . . . . . . . . . $6_{56}$

Expansion and contraction of fluids by changes of temperature........ 45

Extract of aconite root, assay of . . . . . . . . . . . . . . . . .

- of belladonna, assay of. . . . . . . . . . . . . . . . . . . . . 589, 593

- of cinchona, assay of . . . . . . . . . . . . . . . . . . . . . 589

- of hydrastis, assay of. . ........................... 603

- of hyoscyamus, assay of. . . . . . . . . . . . . . . . . . . . . 589

- of nux vomica, assay of.......................... 590,605

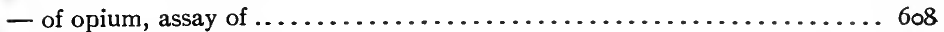

- of physostigma, assay of ......................... 6 ro

- assay of . . . . . . . . . . . . . . . . . . . . . . $584,587,592$.

Factor, how to find. . . . . . . . . . . . . . . . . . $5^{6}$

Factors for calculating analyses. . . . . . . . . . . . . . . . 55

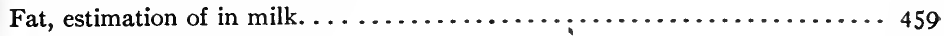

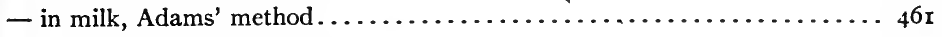

- Babcock centrifugal method . . . . . . . . . . . . . . . . . $44_{2}$

- - Werner-Schmid method. . ....................... 462

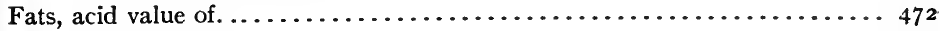

- and oils, bromin value of. . . . . . . . . . . . . . . . . . 478

- Hanus' iodin absorption number. . . . . . . . . . . . . . . . 476

- iodin absorption number. ....................... 474

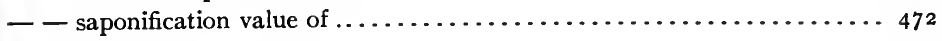

- volatile fatty acid value. ........................ 474

- Wijs' iodin absorption number. .................... 477

- oils and waxes, examination of. ... . . . . . . . . . . . .

Fatty acids, determination of, in soap. ................. 480

Fehling's solution. . . . . . . . . . . . . . . . . . . . . . . . . 489

Ferric alum indicator. . . . . . . . . . . . . . . . . . . . . .

— chlorid, estimation of, by U. S. P. method................. 225

- estimation with permanganate. .................... r64

- salts. 
PAGE

Ferric salts, estimation of............................ 178

- - estimation of, after reduction by various methods. ............. 225

- - estimation of by digestion method. . . . . .

- - estimation of by direct titration with sodium thiosulphate......... 380

- - estimation of by means of permanganate, after precipitation as ferrous

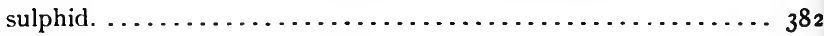

- - estimation of by means of permanganate, after reduction with stannous

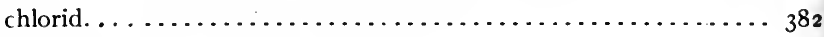

— - estimation of by means of stannous chlorid.............. 23 I

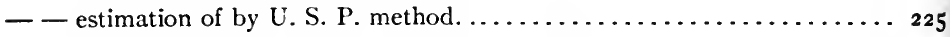

- - estimation with permanganate(after reduction) $\ldots \ldots \ldots \ldots \ldots \ldots \ldots \ldots \ldots \ldots$

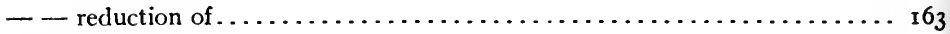

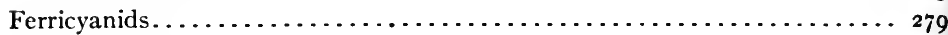

- potassium T. S. ................................. 27

- iodometric estimation of. . . . . . . . . . . . . . . . . . . . 280

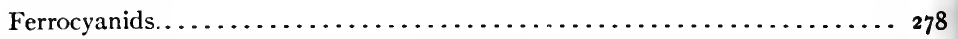

Ferrous carbonate, estimation of by dichromate.............. 182

- - estimation of, with permanganate V.S. . . . . . . . . . . . $\mathbf{r}_{52}$

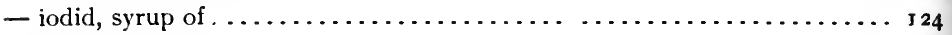

- salts, estimation of. ............................ 178

- - estimation of, by means of dichromate................. I8

- sulphate . ................................. $\mathbf{1}_{4}$

- - estimation of, with permanganate, V.S. . . .............. I50

Ferrum reductum, assay of, with permanganate V.S. . . . . . . . 152

Float, Erdman's and others. ........................... 47

Fluid extract of aconite, assay of. . . . . . . . . . 592

- - of belladonna, assay of............................ 596

- of cinchona, assay of. . .............................600

- - of coca, assay of. . . . . . . . . .

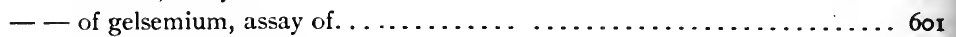

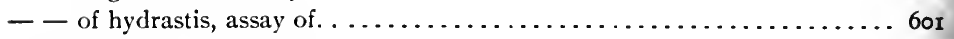

- of hyoscyanus, assay of. . ............................ 597

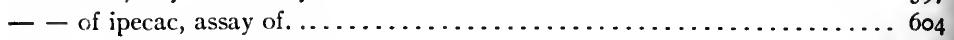

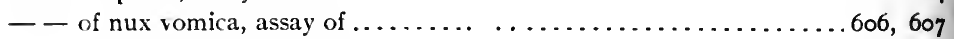

- - of pilocarpus, assay of. ............................ $6 \mathbf{r}_{\mathbf{1}}$

- - of stramonium, assay of. ......................... 597

Fluid extracts, assay of. . . . . . . . . .

Formaldehyde, estimation of. . ........................... $63^{6} 6$

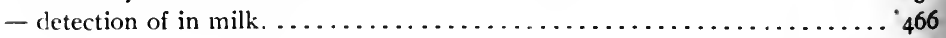

Fowler's solution, assay of. . .......................... 193

Free halogens, estimation of by means of arsenous acid. ....

Fresenius' distilling apparatus. ...................... 216

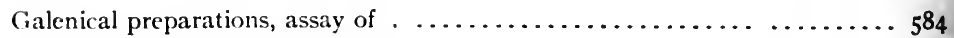

- - assay of, by Farr and Wright's method.................... 590

- - assay of, by Katz's method. . . . . . . . . . . . . . . . $5^{86}$

- assay of, by Kippenberger's method. . ................. $5^{87}$ 
Galenical preparations, assay of, by Lloyd's methods $\ldots \ldots \ldots \ldots \ldots \ldots \ldots \ldots$

- assay of, by Lyons' method. . . . . . . . . . . . . . . . . . $5^{8} 8_{5}$

- - assay of, by potassium-bismuth iodid method. . . . . . . . . . . . 598

- - assay of, by Steiglitz's method. . . . . . . . . . . . . . . . . . 56

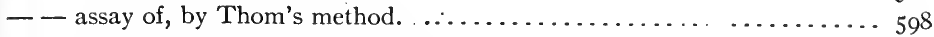

- - assay of, by Thompson's method. . . . . . . . . . . . . . . . . . . 586

- - assay of, by Webster's method...................... 590

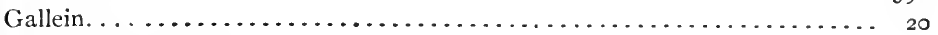

Gay-Lussac's burette .............................. 33

- degrees for expressing the value of chlorinated lime $\ldots \ldots \ldots \ldots$. . 2

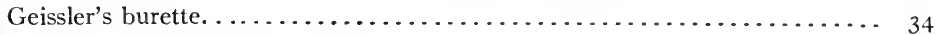

Gelsemium, fluid extract, assay of. . . . . . . . . . . . . . . . . . 6

General methods of assaying drugs. . . . . . . . . . . . . . . . 5

- principles. ............................... 4

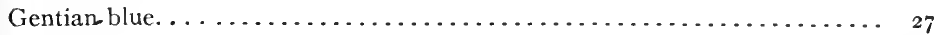

Gladding method for boric acid. . . . . . . . . . . . . . . . 242

Glass stop-cock burette. . . . . . . . . . . . . . . . . . . . . 33

Glue and salt solution, preparation of $\ldots \ldots \ldots \ldots \ldots \ldots \ldots \ldots \ldots \ldots \ldots \ldots$

Glycerin, determination of, in soap. . . . . . . . . . . . . . . 482

- estimation of. ................................... 623

- in fluid extracts, estimation of. ........................ 628

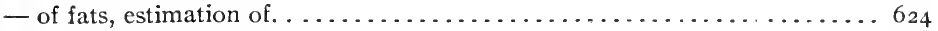

Glycerol, estimation of . . . . . . . . . . . . . . . . . . . . 623

Gold, estimation of . . . . . . . . . . . . . . . . . 378

Gordin's method. . . . . . . . . . . . . . . . . . . . . . . . 5 I 7

Gordin and Prescott's method......................... 5 r 8

Graduated cylinder. ............................. 43

Grain system of volumetric analysis. . . . . . . . . . . . . . .

Grouvelle's bleaching fluid. . . . . . . . . . . . . . . . . . . 2 I 2

Guide for the selection of indicators ........................ 30

Gunning method for nitrogen. ......................... 293

Haberland's method for the valuation of acetate of lime. . . . . . . . . 239

Halogens, estimation of, by means of arsenous acid $\ldots \ldots \ldots \ldots \ldots \ldots \ldots . \ldots 228$

Haloid acids. . ............................... I 20

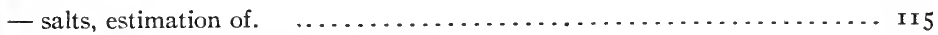

- estimation of, by Mohr's method . .................... I I5

- estimation of, with chromate indicator. . . . . . . . . . . . . I I5

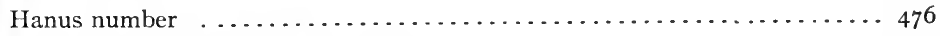

Hardness, determination of, in water. ....................... 448

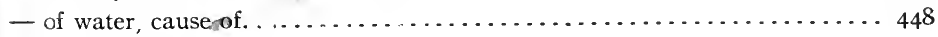

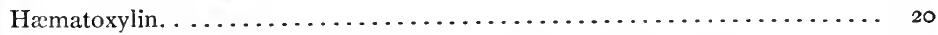

Hehner's method for the estimation of free mineral acids in vinegar...... 235

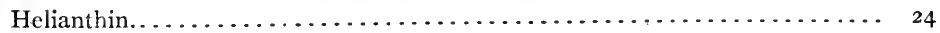

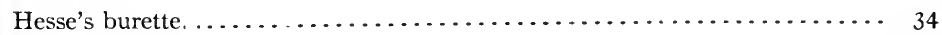

Hübl's number. .................................... 474

Hyde-powder. ................................. $62 \mathbf{1}$ 
PAGB

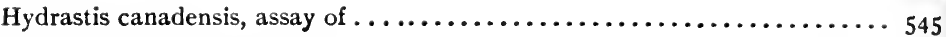

- extract, assay of. .............................. $60_{3}$

- fluid extract, assay of............................ $60_{2}$

- tincture, assay of. . . . . . . . . . ................... 603

Hydrazin, use of in estimation of iodates, bromates, and hypochlorites . . . . 265

Hydriodic acid. . ............................... r 20

- - assay of, by the sulphocyanate method ................. I 23

- syrup of................................... 124

Hydrobromic acid............................... I 20

- assay, using chromate as indicator. ................. I 2 I

Hydrochloric acid. ............................... I00, I 20

- - effect of its presence when titrating with permanganate.......... I 40

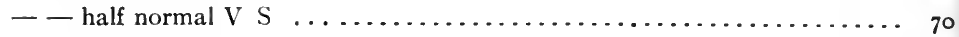

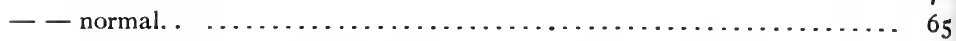

- - V S., gravimetric standardization by means of silver nitrate. . . . . . 68

- - - standardization by means of borax .................. 66

- - standardization by means of calc-spar .................. 68

- - standardization of, by means of specific gravity ............ 66

- - volumetric standardization by means of silver nitrate . . . . . . . 67

Hydrocyanic acid, assay, use of Poirrier blue as indicator . . . . . . . . . 127

- - using chromate as indicator. . . . . . . . . . . . . . . . 127

- - using potassium iodid as indicator. ................. I 28

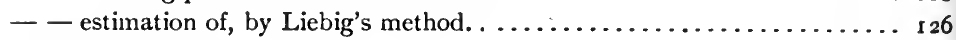

Hydrogen dioxid. . . . . . . . . . . . . . . . . . . . . . . . . 213, 309

- - assay, Kingzett's . . .......................... $2 \mathrm{r}_{3}$

- - assay of, gasometrically ........................ 687

- concentration by heating ........................ I 57

- estimation of volume strength. ...................... I 59

- estimation with permanganate. ..................... r 56 , I $5^{8}$

- iodometric assay of. . .......................... 213

- - reaction with permanganate. . . . . . . . . . . . . . . . . $5^{8}$

- _volume" strength. . ............................. I57

- peroxid. .................................. 309

- assay of gasometrically $\ldots \ldots \ldots \ldots \ldots \ldots \ldots \ldots \ldots \ldots \ldots \ldots \ldots \ldots \ldots$

- sulphid. . ................................... 202

- - estimation by means of copper sulphate. . . . . . . . . . . . . . 329

- estimation of, by means of arsenous acid. . . . . . . . . . . . . 330

- estimation of, by means of iodin...................... 329

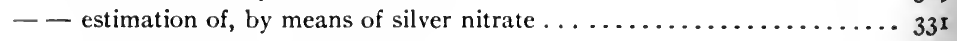

-- estimation of, by permanganate..................... 320

Hydroxylamin as a reducing agent in the estimation of chlorates, etc. . . ... 263

Hyoscyamus extract, assay of. . . . . . . . . . . . . . . . . . $5^{80}$

- fluid extract, assay of........................... 597

- leaves, assay of. . .............................. $5^{29}$

- tincture, assay of. . . . . . . . . . . . . . . . . . . . . . . . 589, 597

Hypophosphite calcium, estimation. . . . . . . . . . . . . . . . . I69

Hypophosphorous acid. . ......................... 103 
PAGE

Hypophosphorous acid, and hypophosphites, estimation with permanganate. . 167

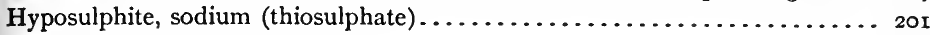

- solution, Schützenberger's................................ 308

Imperial gallon, system based upon. . . . . . . . .

Indicator, quantity to be used in a titration. ................... I8

- requirements of a good. ............................. r8

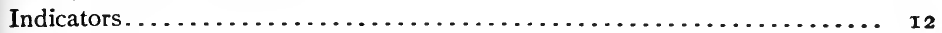

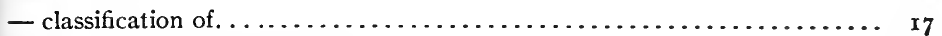

Indirect iodometric estimations. ............................. 208

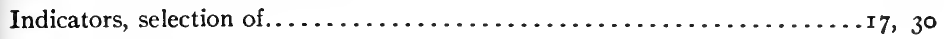

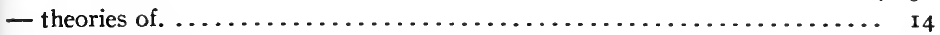

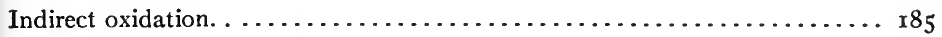

Indigo solution, preparation of.................................. $66_{3} 6$

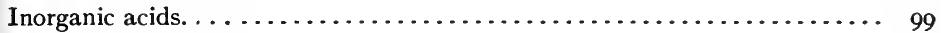

Instruments, calibration of.............................. 49

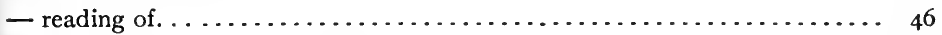

Interpretation of results, in water analysis. . . . . . . . . . . . . .

Iodate, potassium, titrations with standard solution of. ............. 263

Iodates, estimation of, by digestion method.................. 223

Iodeosin. . . . . . . . . . . . . . . . . . . . . . . . . .

Iodid, estimation of, by means of standard potassium iodate . . . . . . . 264

- potassium. .................................. I $_{7}$

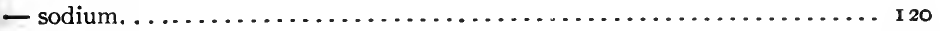

- strontium. ................................ 125

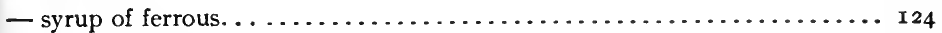

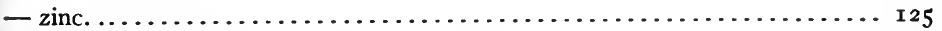

Iodids, direct titration of, with chlorin water $\ldots \ldots \ldots \ldots \ldots \ldots \ldots \ldots \ldots . \ldots \ldots 5$

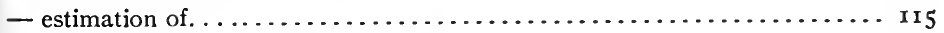

— estimation of, by distillation method ....................... 220

- estimation of, by means of standard potassium bi-iodate . . . . . . . . 265

- estimation of, by means of standard potassium dichromate .......... 265

- estimation of, by means of standard potassium permanganate . . . . . . 265

— estimation of, by mercuric chlorid V.S. . . . . . . . . . . . . . 134

Iodin absorption number of fats and oils. . . . . . . . . . . . . . .

- action of, as an indirect oxidizer .......................... 185

- empirical solution of. . ........................... 187

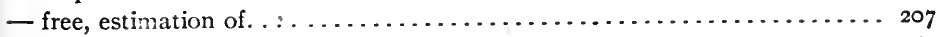

- - estimation of, by means of standard potassium iodate........... 264

- methods of estimation ..............................260

- purification of. . ............................. 186

- solution, estimation of cyanids with. . . . . . . . . . . . . . 274

- V.S., standardization by means of arsenous oxid.............. I88

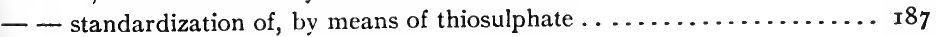

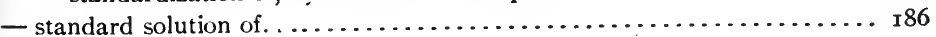

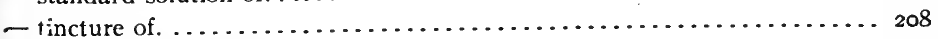

Iodized starch test paper. . ........................... 208 
Iodometric assay of hydrogen dioxid. ..................... $2 \mathrm{I}_{3}$

- estimations, indirect. . . . . . . . . . . . . . . . . . . . 208

- standardization of permanganate V S. .................... 46

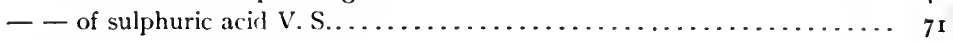

Iodoform, in dressings, assay of. . . . . . . . . . . . . . . $66_{53}$

Iodometry. . . . . . . . . . . . . . . . . . . 202

Ionization theory. ........................

Ipecac fluid extract, assay of. . . . . . . . . . . . . . . . . . . 604

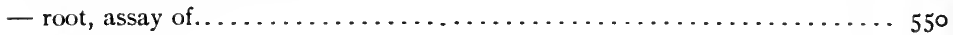

Iron, estimation of............................ 379

- estimation of, by means of stannous chlorid $\ldots \ldots \ldots \ldots \ldots \ldots \ldots \ldots \ldots$

- estimation of, in liquor ferri albuminata. . . . . . . . . . . . . . . $3^{85}$

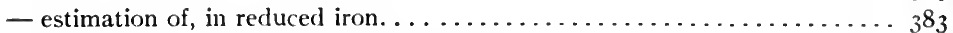

- estimation of, metallic, in ferrum reductum $\ldots \ldots \ldots \ldots \ldots \ldots \ldots \ldots \ldots$

- in styptic cotton, estimation of. ........................... 655

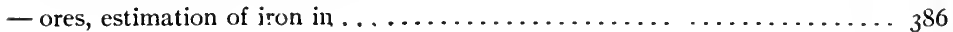

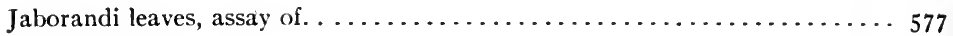

Javelle's water. . . . . . . . . . . . . . . . .

Jodlbauer's modification of Kjeldahl's method... . . . . . . . . . . . 290

Judgment, passing of, in water analyses. . . . . . . . . . .

Kebler's modification of the Keller method. . . . . . . . . . . . . . $5^{1} 7$

Keller's method. ............................... $55^{15}$

Kingzett's method for the assay of hydrogen dioxid. . . . . . . . . . . 213

Kjeldahl method, as adopted by the A. O. A. C. ............... 290

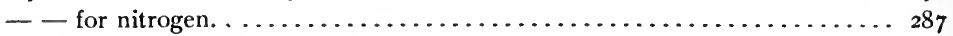

- process, estimation of cyanids with. . . . . . . . . . . . . . 276

Knapp's method for the estimation of sugar. ... . . . . . . . . . . 495

Knop's solution for urea estimation . . . . . . . . . . . . . . . . 693

Koppeschaar's solution. .......................... $6 \mathbf{1}_{3}$

Köttstorfer number. . . . . . . . . . . . . . . . . . . 472

Labarraque's solution. . . . . . . . . . . . . . . . . . . 2

Laborde's method for the estimation of mercuric salts . . . . . . . . . 233

Lacmoid. . . . . . . . . . . . . . . . .

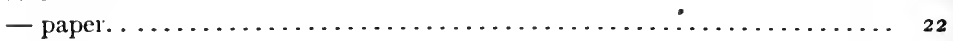

Lacmus. . ............................... 22

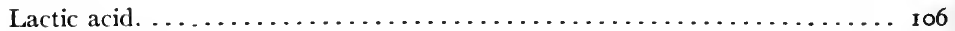

Laudanum, assay of. . . . . . . . . . . . . . . . . . . 609

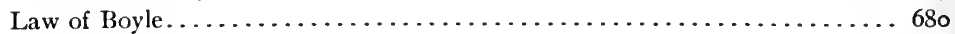

- of Charles. . . . . . . . . . . . . . . . . . . . . . . . . . 679

Lead, estimation of. . ........................ 178

— estimation of, by means of a standard sulphate solution. . . . . . . . 392

— estimation of by means of dichromate. . . . . . . . . . . . . 388

- estimation of, by precipitation as oxalate. . . . . . . . . . . . 393 
Lead, estimation of, by precipitation às chromate, and digestion with ferrous sulphate. .......................................... 390

- estimation of, by titration with dichromate, and ferrous sulphate ......... 390

- estimation of, iodometrically, after precipitation as chromate $\ldots \ldots \ldots \ldots . .392$

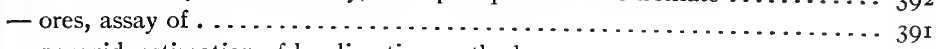

- peroxid, estimation of by digestion method.................. 222

- estimation of by distillation method..................

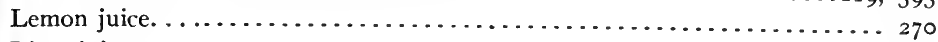

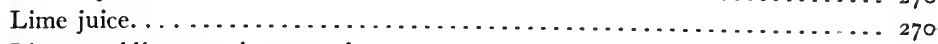

Liquor acidi arsenosi, assay of. . . . . . . . . . . . . . . . . . 193

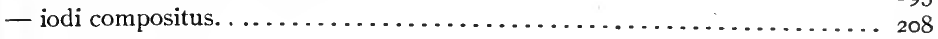

- potassii arsenitis, assay of. ........................... 193

- sodæ chlorinatæ............................. 212

Liter flasks..................................... 42

Litharge, assay of. . ................................... 389

Lithium bromid. ................................ I 19

- carbonate............................................ 79

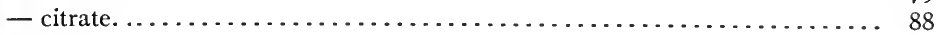

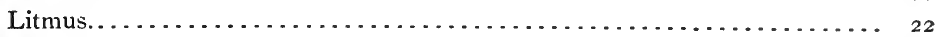

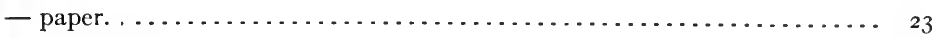

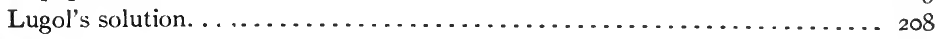

Luteol. ............................................... 24

Lyons' method. .................................... 516

Magnesia mixture. ................................ $3_{\text {I }}$

Magnesium, estimation of, as phosphate . . . . . . . . . . . . . 394

- estimation of by means of, sodium arsenate................ 395

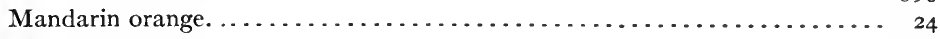

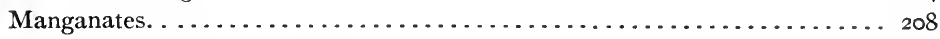

Manganese dioxid, estimation of........................... 400

- estimation of, by digestion method .................... 222

- estimation of, by distillation method ..................... 218

— estimation of, by means of arsenous acid ................ 230

- estimation with permanganate. .................... 7 I

- estimation of, by bismuthate method ..................... 404

' - estimation of, by direct titration with permanganate.............. $40 \mathrm{I}$

- estimation of, by distillation . . . . . . . . . . . . . . . . . . 399

- estimation of, by means of ferricyanid .................... 402

— estimation of, by Volhard's titration method . . . . . . . . . . . . $40 \mathrm{I}$

- ores, estimation of the available oxygen in.................. 397

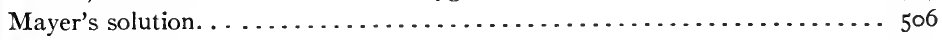

Meniscus. ........................................ 46

Mercuric chlorid, decinormal V. S............................. 409

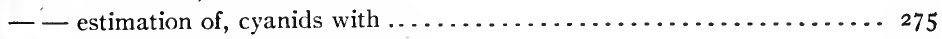

- estimation of, in colored tablets, etc...................... $4^{\mathrm{I}} 3$

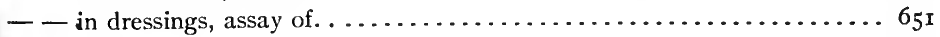

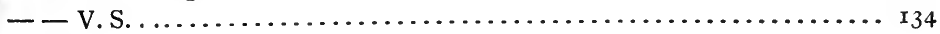


Mercuric cyanid, analysis of.

PAGE

- solution for the estimation of sugar........................ 495

— iodid solution for the estimation of sugar. .................. 496

- potassium iodid solution......................... 506

- salts, estimation of, by means of ferrous sulphate ............. 4ro

- estimation of, by means of stannous chlorid ............... 233

Mercurous compounds.................................. 202

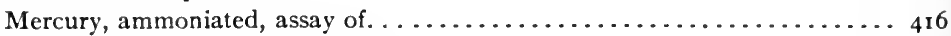

- estimation of, as mercurous chlorid ....................... 408

- estimation of, by direct titration with thiosulphate $\ldots \ldots \ldots \ldots \ldots \ldots \ldots$ 4 I I

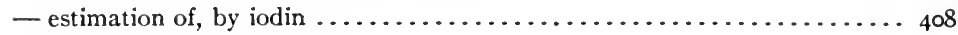

- estimation of, by means of dichromate . . . . . . . . . . . . 418

- estimation of, by means of potassium cyanid............... 4 I I

- estimation of, by potassium iodid ........................ 409

- estimation of, in its organic compounds .................. 4I5

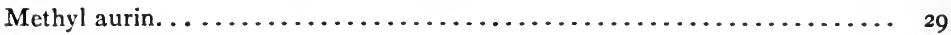

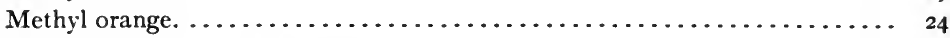

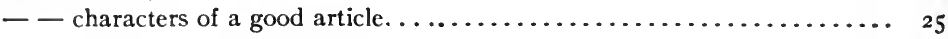

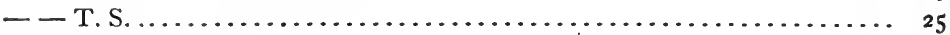

Milk, adulterations of. ...................................... 459

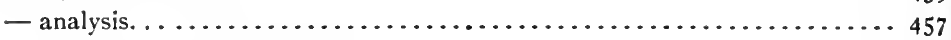

- calculation method for fat in. . ......................... $46_{3}$

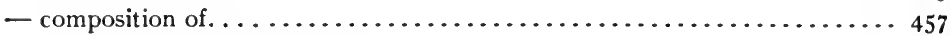

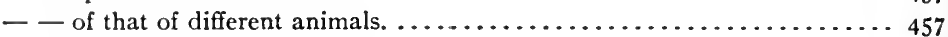

- detection of boric acid in. . . . . . . . . . . . . . . . . . 466

- of formaldehyde in................................. 466

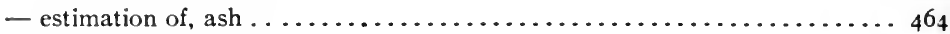

- estimation of, fat in ................................ 459

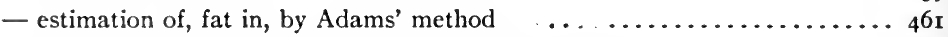

- estimation of, fat in. by Babcock centrifugal method............. 462

- estimation of, fat in, by Werner-Schmid method .............. 462

- estimation of, total proteids in . . . . . . . . . . . . . . . . . $44_{5}$

- estimation of, total solids and water in . .................. 459

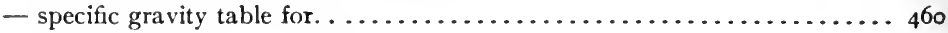

- sugar, estimation of, in milk ......................... 466

Molybdate ammonium solution.......................... 447

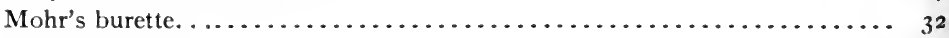

— distilling apparatus. ........................... 217

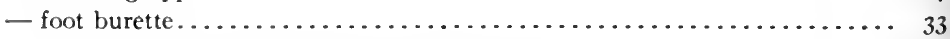

- method for estimating acetic acid in vinegar. ............... 237

- salt, standardization of permanganate V. S. with............... 147

Mydriatic drugs, assay of................................... 527

Naphthalamin hydrochlorate.................................... 445

Naphthylammonium chlorid. ................................... 445

Nessler's solution. ........................................... 440

Neutral salts, assay of, after conversion into chlorid. . . . . . . . 
PAGE

Neutralization analysis. . ........................... 62

Nitrate potassium, standard solution of. .................. 444

Nitrates. . . . . . . . . . . . . . . . . . . . . . . . . . . . . . 133, 294

- by modified Kjeldahl method............................. 293

— by the Schlössing method............................. 299

- by the Street-Ulsch method............................. 298

- by the Ulsch method .............................. 298

- by the zinc iron method. . ........................ 297

- estimation of. . ........................... 178

- estimation of, in water $\ldots \ldots \ldots \ldots \ldots \ldots \ldots \ldots \ldots \ldots \ldots \ldots \ldots \ldots \ldots \ldots \ldots$

- estimation (Pelouze method). ..................... I $7_{2}^{2}$

- nitrometer assay of.............................. 686

Nickel oxid, estimation of, by distillation method ............... 2 I9

Nitric acid ..................................... 103

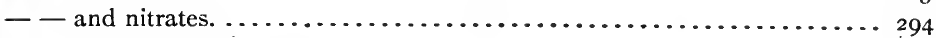

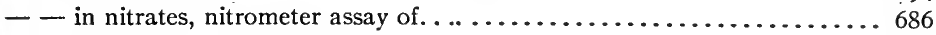

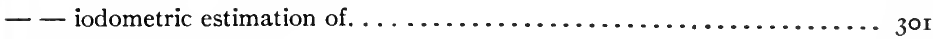

Nitrite of amyl, assay of. ............................ 659

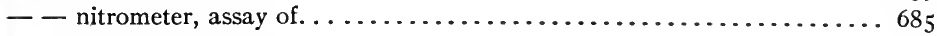

— of sodium, nitrometer assay of. ...................... $68_{5}$

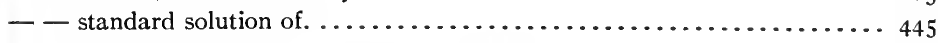

Nitrites, estimation of, by means of permanganate $\ldots \ldots \ldots \ldots \ldots \ldots \ldots 2$

- estimation of in water. .......................... 445

— estimation, U. S. P. method. . ...................... 166

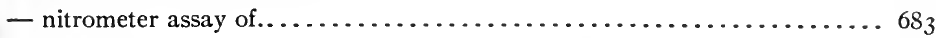

Nitrogen. .................................. I 33

- and its compounds. ................................ 283

— in nitrates, by modified Kjeldahl method... . . . . . . . . . . . . . . . 293

- in organic substances, Gunning method. .................... 293

- in organic substances, the Jodlbauer-Kjeldahl method. . . . . . . . . . 290

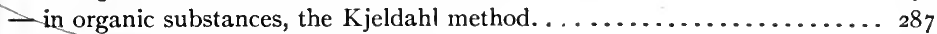

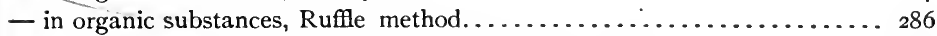

— in organic substances, Will and Varrentrapp's method. . . . . . . . ... 283

Nitrometer, improvised........................... 687

- The. ......................................... 678

Nitrous acid and nitrites, estimation with permanganate........... $16_{5}$

- estimation of, in chamber acid . . . . . . . . . . . . . . . . 303

- estimation of, by means of permanganaie ............... 302

- - iodometric estimation of. . ..................... 303

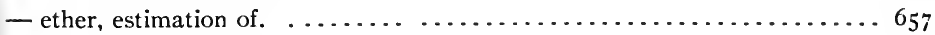

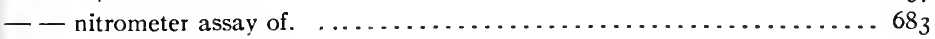

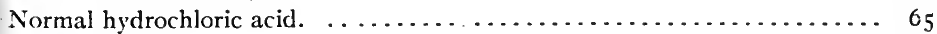

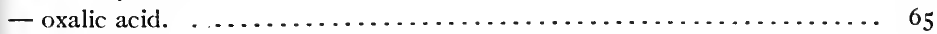

- solution. . ............................... 6

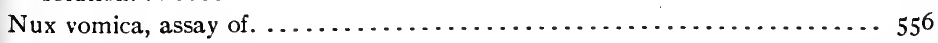

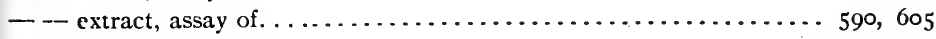

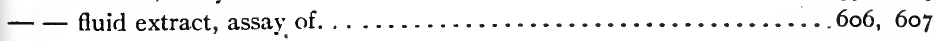

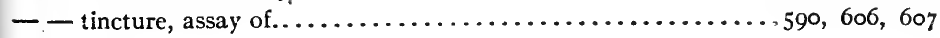




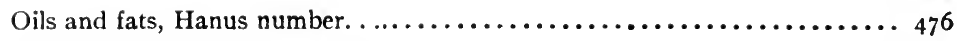

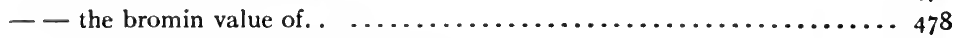

- Wijs', iodin absorption number. . ........................ 477

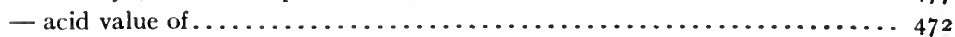

- fats and waxes, examination of. ........................ 472

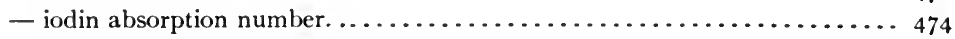

- saponification value of. ............................... 472

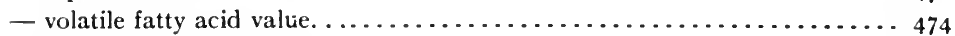

Oleomargarin, detection of. ........................ $47 \mathrm{I}$

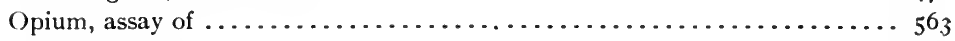

- extract, assay of. ....................................... 608

- tincture, assay of. . ................................ 609

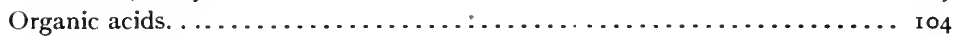

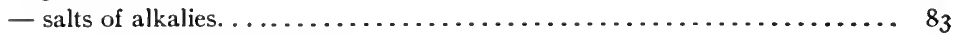

- - of alkalies and alkali earths. . . ..................... 133

Oxalates, estimation with permanganate.................... 5

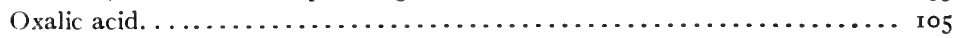

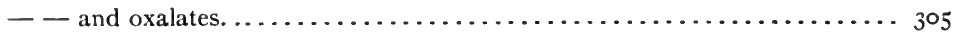

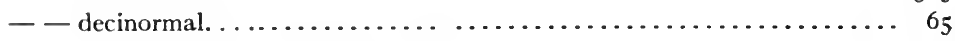

- estimation with permanganate. ....................... I 53

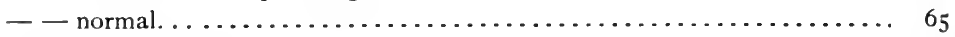

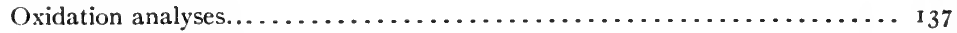

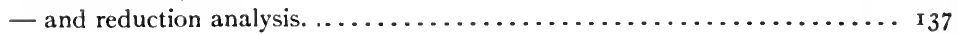

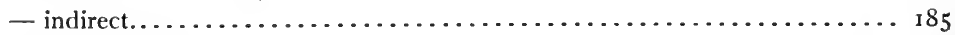

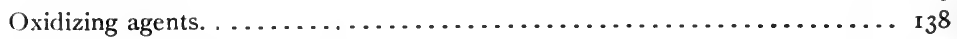

Oxy-chlor-diphenyl-quinoxalin ... ......................... 24

Oxygen and peroxids......................................... 307

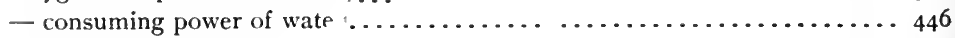

- dissolved in water....................................... 307

- dissolved in water, estimation of, by hyposulphite $\ldots \ldots \ldots \ldots \ldots \ldots \ldots . \ldots \ldots$

- dissolved in water, Schützenberger's method................... 308

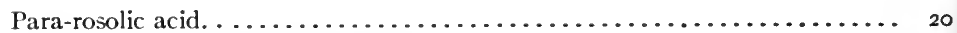

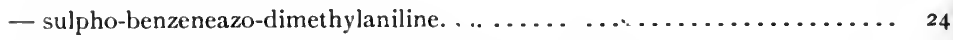

Paris green, estimation of arsenic in, by Smith's method............ $35^{\circ}$

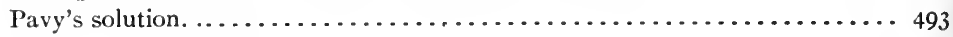

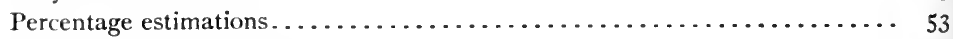

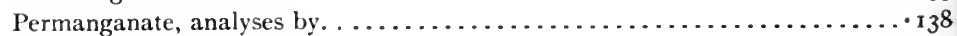

- direct titrations. . ......................... 14

- empirical solution, use of. . . . . . . . . . . . .

- indirect titration methods....................... I 4 I

- method for the estimation of sugar ........................ 495

- preparation of decinormal solution of. ................. 142

- residual titrations. . ......................... 167

- solution, standardization of, for use in estimating nitrous acid........ 302

- titration methods. . ............................. 4 I

- use of, for standardizing sodium thiosulphate................ 207 


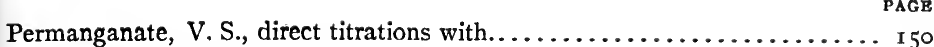

- - iodometric standardization of....................... 146

- - standardization by means of oxalic acid................... I 44

- standardization of, by means of iron .................. 142

- standardization of, by means of sodium oxalate .............. 148

— - standardization of, with ferrous ammonium sulphate . . . . . . . . . I47

- - standardization of, with hydrogen peroxid in the nitrometer ....... 148

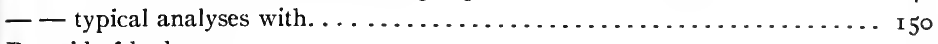

Peroxid of hydrogen. ........................................ 309

- of hydrogen, assay of, gasometrically....................... 687

- of lead, estimation of, by distillation . . . . . . . . . . . . . . 393

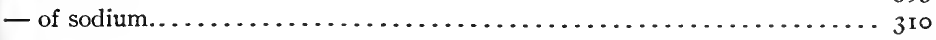

Peroxids. ......................................... 208

Personne's method for iodids.......................... 134

Persulphates, estimation of active oxygen in................... 310

Pettenkofer's method for carbonic acid gas in the atmosphere.......... 246

- - for estimating acetic acid in vinegar. . . . . . . . . . . . 237

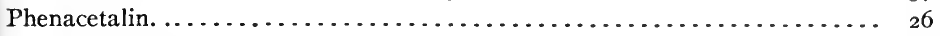

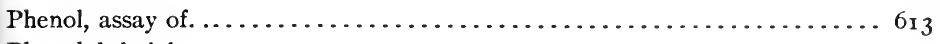

Phenolphthalein.................................... ${ }_{2} 6$

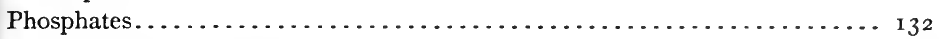

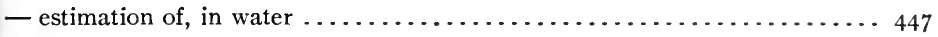

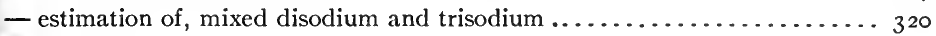

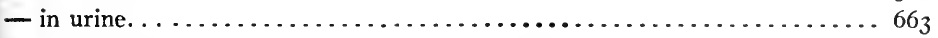

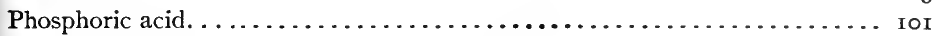

- - estimated by means of standard uranium................ 3 I 2

- estimation of, by Glückmann's method ................... $3^{1} 5$

- - estimation of, by Pemberton's molybdic method ............... $3^{1} 6$

- estimation of, by Pemberton's new method ................... 317

- Stolba's method................................ $3^{\text {I I }}$

Physostigma, assay of.. .................................... 575

- extract, assay of. .....................................

Pilocarpus fluid extract, assay of. . . . . . . . . . . . . . . .

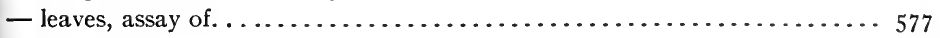

Pinch cocks. ......................................... 40

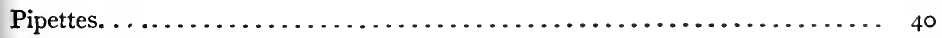

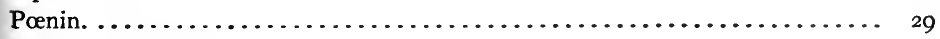

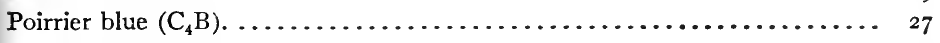

Poirer's orange III. . . . . . . . . . . . . 24

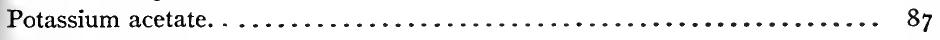

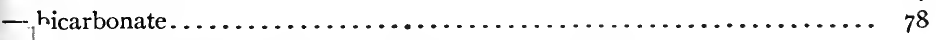

$\mathrm{f}_{2}$ odate, standard solution of, for estimation of iodids . ........... 265

-37 use of for standardizing sodium thiosulphate. ............... 206

$f^{26}$ nuth iodid method for assay of galenicals.................. 598

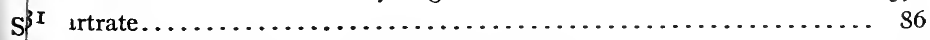

S 4 mate, estimation of, by the digestion method............... 224

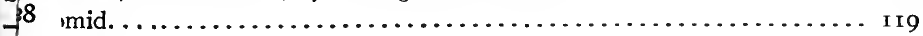


Potassium carbonate............................. 78

- chlorate, estimation of by the digestion method. . . . . . . . . . . 224

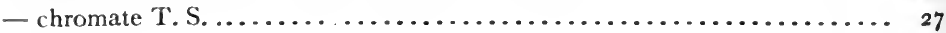

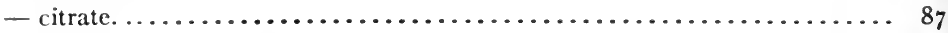

- cyanid. . . ................................. 1 29

- dichromate as an oxidizing agent. . . . . . . . . . . . . 8

- as a precipitating agent. ....................... 7

- estimation of. . ........................... ${ }_{7} 6$

- estimation of by distillation method.................. 219

- ferricyanid T. S. ............................. 27

- hydroxid. ...................................... 74

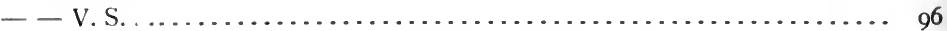

— - standardization of, by means of potassium bi-iodate . . . . . . . 98

— - - standardization by means of potassium bitartrate. . . . . . . . . 97

- jodate solution, titrations with. .................... $26_{3}$

- iodid. ................................ I 7

- - decinormal V. S............................. 409

- determination of the amount of chlorid present. ........... I 8

- permanganate, analyses by....................... 138

- - as an oxidizing agent. .......................... 8

- preparation of decinormal solution of. . . . . . . . . . . . . I 42

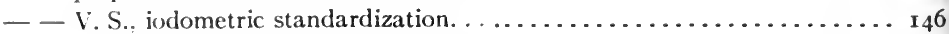

- - standardization by means of oxalic acid. . . . . . . . . . . . . . 144

- - standardization by means of sodium oxalate.............. I 48

- - standardization of, by means of iron $\ldots \ldots \ldots \ldots \ldots \ldots \ldots \ldots$ I42

- - standardization of, with Mohr's salt ................. I47

- - standardization with hydrogen peroxid in the nitrometer. . . . . . . I48

- sulphite. .................................. 200

- sulphocyanate V.S. .......................... II4

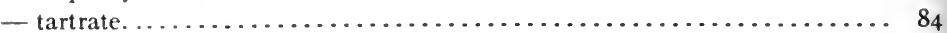

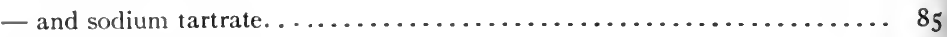

Precipitation analysis. . . . . . . . . . . . . . . . . . . . 110

- precision in determining end-reactions in ................ III

- - titration without indicator (Gay-Lussac). . . . . . . . . . . . ... I 20

- - with chromate indicator(Mohr's method) . . . . . . . . . . . . I 20

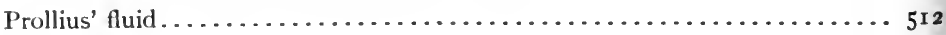

Puckner's method. . . . . . . . . . . . . . . . . . . . . . . . 5 I 6

Pyrogallo-phthalein. ............................... 20

Ramsay's bleaching fluid ........................... 212

Reading of graduated instruments. ..................... ${ }_{46}^{6}$

Reducing agents. . . . . . . . . . . . . . . . . . . . . . . . . . $3^{8} 8$,

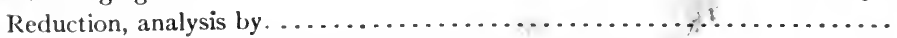

- methods, involving the use of standard arsenous acid solution. .........

- involving the use of stannous chlorid....................

Reichert number.

Resazurin. 


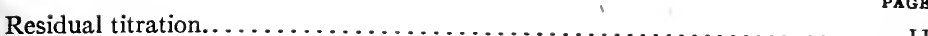

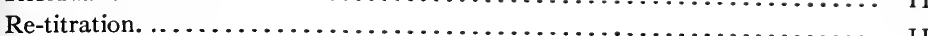

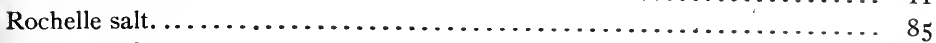

Rosolic acid. ..................................... 28

Sachsse's method for the estimation of sugar. . . . . . . . . . . . . 496

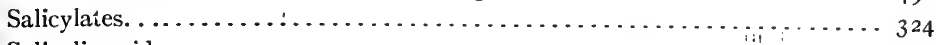

Salicylic acid. . . . . . . . . . . . . . . . . . . . . . . . 324

- - and salicylates, estimation by means of bromin. ............ 325

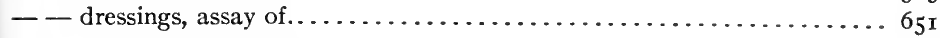

- iodometric estimation of. . . . . . . . . . . . . . . . . . 324

Saponification value of fats and oils. ................... 472

Standard acid solutions, preparation of. . . . . . . . . . . . . . 64

Saturation analysis, see neutralization analysis................ 62

Scale salts, estimation of alkaloidal strength of. . . . . . . . . . . . 6 r $2^{2}$

Schlössing's method for nitrates. . . . . . . . . . . . . . . . . . . . . 299

Scopola, assay of . . . . . . . . . . . . . . . . . . . . . 530

Selection of the indicator (Glaser) . . . . . . . . . . . . . .

- of the indicator, guide for the. ..................... 30

Separators.................................. $5^{13}$

Silver, estimation of. ........................... 4 I 7

- metallic, and alloys. . . . . . . . . . . . . . . . . . . . $3_{2}^{2}$

- nitrate, assay of, by means of sodium chlorid ............... 130

- - assay of, by means of sulphocyanate $\ldots \ldots \ldots \ldots \ldots \ldots \ldots \ldots \ldots \ldots$ I $3^{\mathrm{I}}$

- V. S., preparation of. ..................... II 2

- salts, assay of. . ............................ 130

Smith method for estimating boric acid. ................... 240

Soap, analy'sis of. . . . . . . . . . . . . . . . . . . . . . . . 480

- determination of fatty acids in. ................... 480

- of glycerin in. . . . . . . . . . . . . . . . . . . . . . . . 482

- standard solution of. . . . . . . . . . . . . . . . . . . . . 449

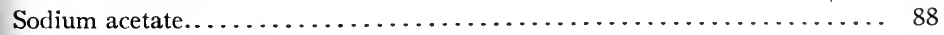

- and potassium tartrate. . . . . . . . . . . . . . . . . . . . 85

- benzoate................................ 88

- bicarbonate................................. 79

- its use in titrations with iodin. . . . . . . . . . . . . . . . .

- bisulphite. .............................. 20 r

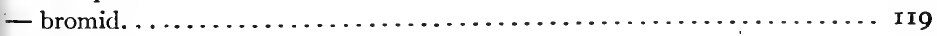

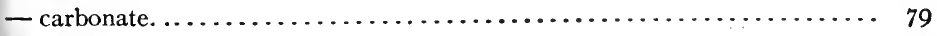

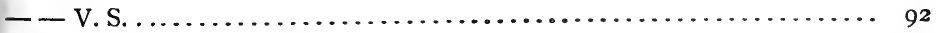

- chlorid. ................................ 1 6

- decinormal solution. . . . . . . . . . . . . . . . . . . . . 2

- preparation of pure.......................... II

- V.S. ................................ $\ldots \ldots$

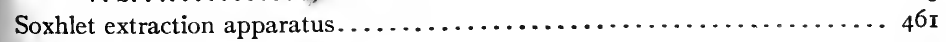

Sodium hydroxid. . . . . . . . . . . . . . . . . . . . . . . . 75

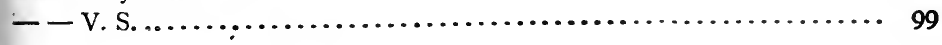




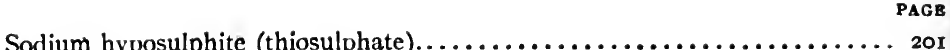

- iodid. ...................................... 20

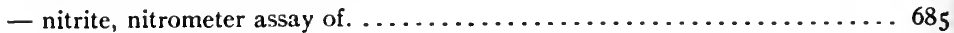

- salicylate. ....................................... 88

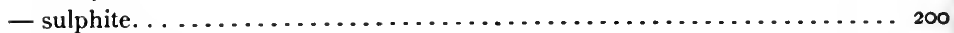

- thiosulphate......................... 20 r

- - decinormal solution. . . .......................... 203

- estimations involving use of. ........................ 202

Solution of chlorinated soda. . . . . . . . . . . . .

Soxhlet-Fehling method for sugar. ......................... 494

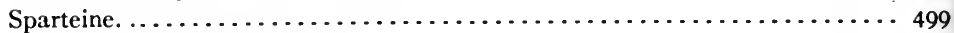

Spirit of nitrous ether, assay of. . ....................... 657

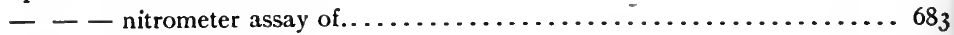

Spirits of ammonia. . . . . . . . . .

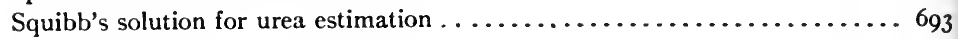

- urea apparatus. .................................... 694

Standard potassium iodate solution. . . . . . . . . . . . . . . . . . ${ }^{26} 3$

- silver nitrate V. S. . .............................. 12

- sodium chlorid V.S. ............................ 113

- solutions................................... 6

Standardization of decinormal sodium chlorid .................. I 3

- of hydrochloric acid V. S., by means of borax................ 66

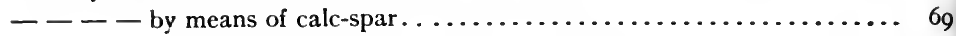

$-\ldots-$ by means of silver nitrate (gravimetrically)............... 67

$-\ldots,-$ by means of silver nitrate (volumetrically) ............. 68

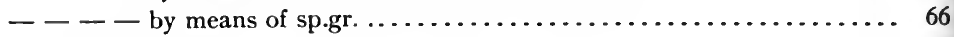

- of permanganate $V . S$. , by the iodometric method. .............. 146

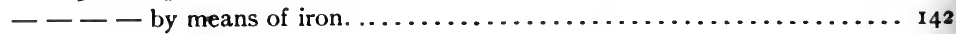

- - - by means of ferrous-ammonium sulphate............. 147

--- by means of oxalic acid........................ 144

-- by means of sodium oxalate. ....................... 148

--- with hydrogen peroxid, in the nitrometer............. 148

— of potassium hydroxid V.S., by means of potassium bi-iodate........ 97

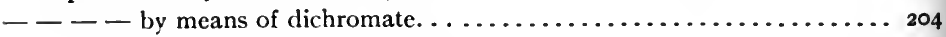

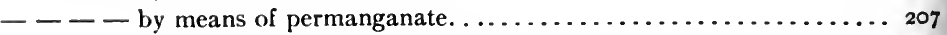

- - - by means of potassium bi-iodate. ..................... 206

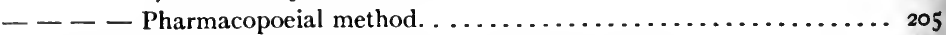

— of sulphuric acid V.S., by ammonium sulphate method. .......... $\boldsymbol{7}^{2}$

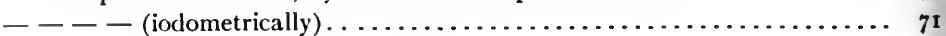

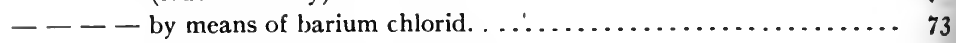

$--\longrightarrow$ by means of sodium carbonate...................... 70

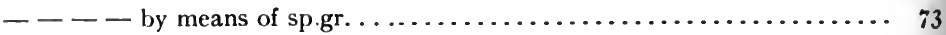

—of sulphuric acid by Wenig's method. ................... $\mathbf{7 2}^{2}$

- of thiosulphate V. S., by means of iodin V.S. . . . . . . . . . . 203

Standards, in sanitary water analyses. . . . . . . . . . . . . . . 455

Stannous chlorid, estimation of. . . . . . . .

- V. S., preparation of. ............................. $23^{2}$ 
PAGB

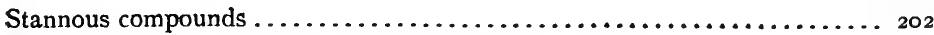

Starch, determination of, by means of barium hydroxid. ........... 484

- determination of, in cereals. . . . . . . . . . . . . . . . . . . . 484

- estimation of, after inversion by means of acid. ............... 486

- estimation of, after inversion by means of diastase............. 487

- iodized, test paper of. . ................................ 28

- solution. . ..................................... 29

- - application of.................................. 189

- - preparation of. ................................. 189

- - preservation of................................... 89

Stramonium fluid extract, assay of. . ....................... 597

- leaves, assay of. . ................................ 53 I

- tincture, assay of. . ............................ 597

Street-Ulsch method for nitrates. . ....................... 298

Strontium bromid. . .............................. I 9

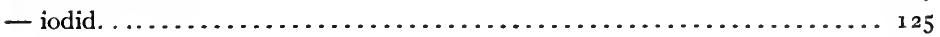

- salts, estimation of. .......................... 4 19

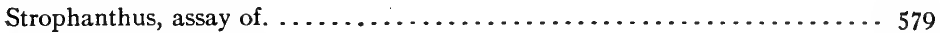

Styptic cotton, assay of. . . . . . . . . . . . . . . . . . . 655

Sublimate dressings, assay of.............................. 65 I

Sugar, estimation of, by Knapp's method. . .................. 495

— estimation of, by Pavy's solution. ........................ 493

- estimation of, by permanganate method.................... 495

- estimation of, by Sachsse's method. ....................... 496

- estimation of, by Soxhlet-Fehling method. . .................. 494

- in urin, estimation of. .................................. 492

Sugars, estimation of, by Fehling's solution. . .................. 489

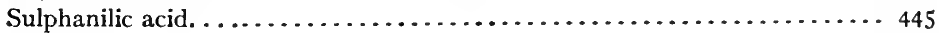

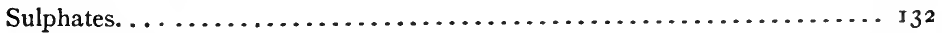

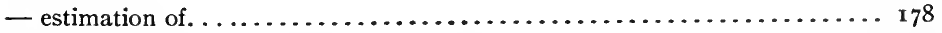

- estimation of, by means of barium chlorid................... 33 I

- estimation of, by precipitation as lead sulphate................. 333

- estimation of, with barium chlorid and potassium dichromate. ........ 333

- in urine. . ....................................... $66_{4}$

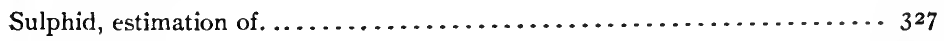

- hydrogen. .................................... 202

Sulphites. . . . . . . . . . . . . . . . . . . . . . . . . . 200

- estimation of. . .............................. 33I

Sulphocyanate method. ............................ 22

- V.S. . . .................................... II4

Sulphocyanates. ................................. $28 \mathbf{r}$

- estimation of, by means of cupric sulphate. . . . . . . . . . .

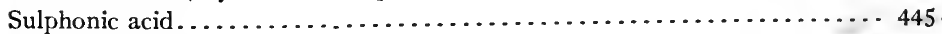

Sulphur and its compounds. . ............................... 326

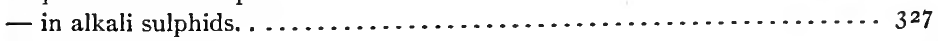

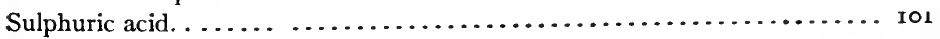

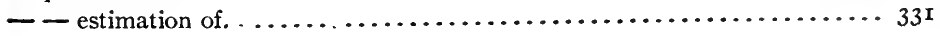


Sulphuric acid, normal V.S. ........................... 70 $^{2}$

- - V.S., standardization, by ammonium sulphate method ......... 72

- - gravimetric standardization, by means of barium chlorid. ....... 73

- - iodometric standardization................... it

- - standardization, by means of sp.gr. ................ 73

- - standardization, by means of sodium carbonate............ 70

Sulphurous acid and sulphites, estimation of. ............... 197

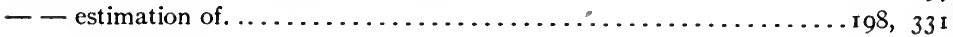

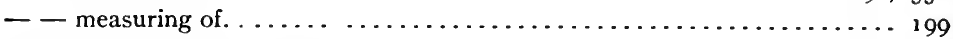

Surgical dressings, assaying of.............................. 649

Table of alkalies, alkali earths and acids, showing normal factors, etc. . . . 57

- of decinormal factors for alkaloids. . . . . . . . . . . . . . . . $55^{4} 4$

- of factors for correction for barometric pressure. ............. $68 \mathrm{I}$

- of factors for temperature corrections................... $68 \mathrm{r}$

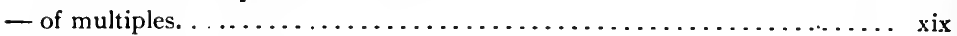

- of substances, estimated by means of standard iodin solution. . . . . . 201

- of substances, estimated by permanganate or dichromate.......... 185

- of substances, estimated by precipitation. ................. 135

- of the acids. .................................... 106

- of the alkali earth metals. . ......................... 94

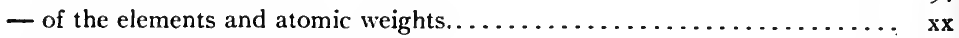

- of the organic salts of the alkalies. . .................... 89

- serving as a guide for the selection of a suitable indicator.......... 30

- showing amount of deviation in calibrated burętte.............. 50

- showing behavior of alkaloids, with indicators. . ............. 503

- showing color changes of indicators. . . . . . . . . . . . . 502

- showing iodin absorption by the three methods. . . . . . . . . . 477

- showing iodin factors of the alkaloids.................... 508

- showing quantity of substance to be taken in direct percentage estimations. 107

- showing relative expansion and contraction of water at different tempera-

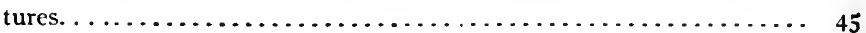

- showing U. S. P. requirement as to saponification number of fats and oils. 473

- showing volume of $0.00 \mathrm{gm}$. of carbon dioxid at various temperatures. 249

- showing weight of $\mathrm{H}_{2} \mathrm{O}_{2}$, corresponding to volume of oxygen.......6.60

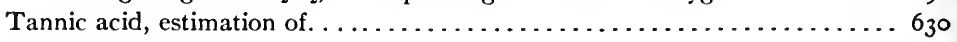

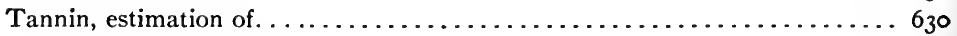

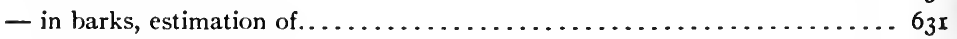

- in cloves and allspice, estimation of. . . . . . . . . . . . . . . 637

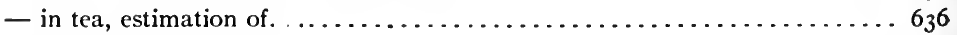

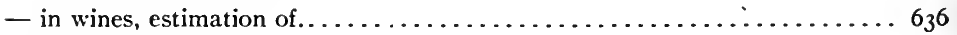

Tartaric acid.................................... 105

Tartar emetic, estimation of . . . .

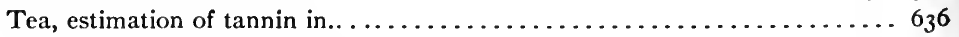

Tetra-bromo-resorcin-phthalein. . . . . . . . . . .

- iodo-fluorescein........................

Tetrazo-diphenyl-naphtionate $\ldots \ldots \ldots \ldots \ldots \ldots \ldots \ldots \ldots \ldots \ldots \ldots \ldots \ldots \ldots \ldots \ldots$ 
PAGE

The burette. $\ldots \ldots \ldots \ldots \ldots \ldots \ldots \ldots \ldots \ldots \ldots \ldots \ldots \ldots \ldots \ldots \ldots \ldots, 3^{2}$

Thiosulphate, sodium. . . . . . . . . . .

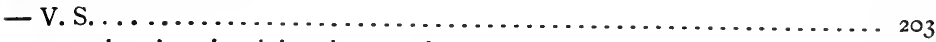

- estimations involving the use of. ......................... 202

- - standardization of, by means of dichromate................... 204

- - standardization of, by means of iodin V.S. .................. 203

- - standardization of, by means of permanganate................. 207

- - standardization of, by means of potassium bi-iodate. . . . . . . . . 206

Tin, estimation of (Löwenthal).............................. 177

- estimation of, by means of potassium dichromate............... 423

-- estimation of, by titration with iodin in acid solution. . . . . . . . 422

- estimation by titration with iodin in alkaline solution............ $42 \mathrm{I}$

- indirect titration by ferric chlorid and permanganate. . . . . . . . . 422

Tincture of aconite, assay of . . . . . . . . . . . . . . . . . 593

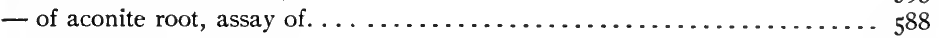

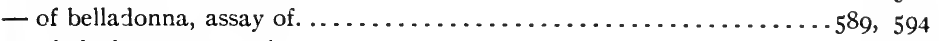

- of cinchona, assay of................................. 589

- of hydrastis, assay of. ................................ 603

— of hyoscyamus, assay of. ............................ 597

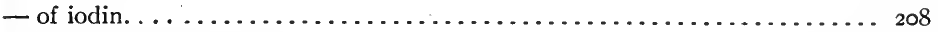

- of nux vomica, assay of................................ 606, 607

- of opium, assay of. . ...................................609

- of stramonium, assay of. . .......................... 597

Tinctures, assay of. . . . . . . . . . .

- containing chlorophyll, assay of......................... 587

Titer........................................ 6

Titration, residual. .......................... 1 I

Tobacco, assay of. . ........................................ 580

Total solids, estimation of, in urine. . . . . . . $66_{2}$

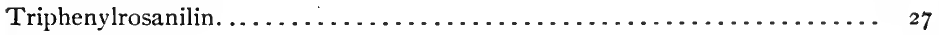

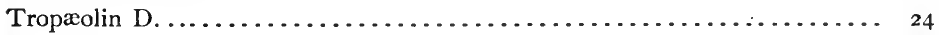

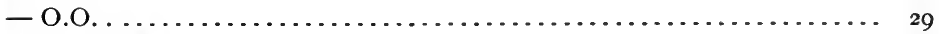

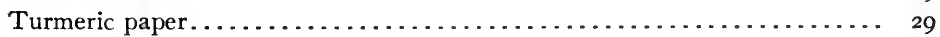

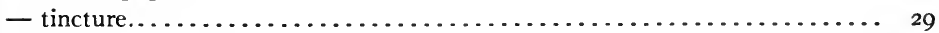

Type metal, assay of. ............................. 34 r

Ulsch method for nitrates............................. 298

Urea apparatus, Squibb's. .............................. 694

- estimation of ...................................... 692

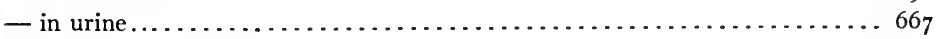

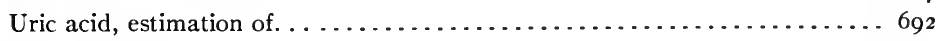

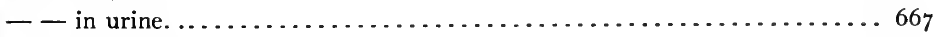

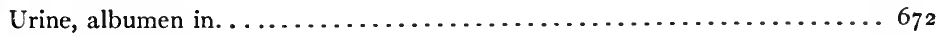

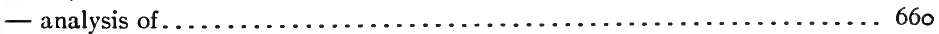

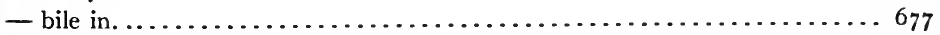

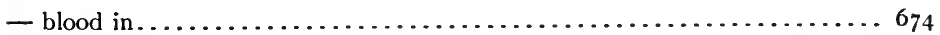

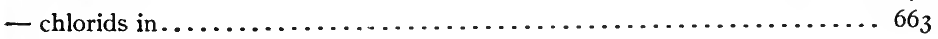


PAGE

Urine, composition of. ............................. 660

- estimation of sugar in. . . . . . . . . . . . . . . . . . . . 492

- ethereal sulphates in. . . . . . . . . . . . . . . . . . . . . $66_{5}$

- phosphates in. ...............................66 $66_{3}$

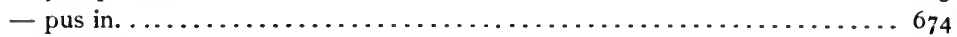

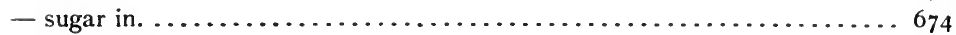

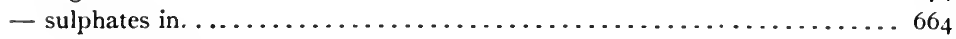

- total acidity of. ................................. $66_{5}$

- urea in....................................667,692

- uric acid in. ............................667,692,696

Ureometer, Doremus' ... . . . . . . . . . . . . . . . . . . . . . . . 692

- Hinds-Doremus. . . . . . . . . . . . . . . . . . . . . . . . . . . . 693

- Sellier's. . . . . . . . . . . . . . . . . . . . . . . . . . . . . . . . 695

Use of apparatus. ............................... 44

Veratrum, assay of. ................................ $55^{81}$

Vicarious methods. ............................. $5^{8}$

Vielhaber's method for cyanogen. . . . . . . . . . . . . . . . . . 127

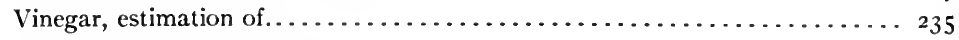

- estimation of acetic acid in. ........................... 237

- estimation of free mineral acids in. . . . . . . . . . . . . . . 235

Volatile fatty acid, value of oils........................ 474

Volhard's method. ............................. I 20, 122

- solution. . . .............................. I 14

Volumetric analysis by weight. ....................... 59

- - without a burette. . ............................ 59

- without weights and standard solutions. . . . . . . . . . . . . $5^{8}$

- or standard solutions. . . ............................ 6

Wagner's reagent, estimation of alkaloids with. .............. 507

Water, carbonic acid in, Lunge-Trillich method................. 255

- - in, Seyler method.............................. 255

- determination of hardness. . . . . . . . . . . . . . . . . . . . 448

- estimation of chlorin in. . . . . . . . . . . . . . . . . . . . . . 439

- estimation of free ammonia in.. ..................... 440

- estimation of nitrates in. . . . . . . . . . . . . . . . . . . . . 444

- estimation of nitrites in. . ........................ 445

- estimation of nitrogen in. ......................... 444

- interpretation of results of analyses. . . . . . . . . . . . . .

- organic and volatile matter. . . . . . . . . . . . . . . . . . . . . . 439

- oxygen-consuming power of . ....................... 446

- phosphates in. . .............................. 447

- sanitary analysis of. . . . . . . . . . . . . . . . . . . . . . 437

- total solids in. . . . . . . . . . . . . . . . . . . . . . . $43^{8}$

Waxes, fats and oils, examination of. . . . . . . . . . . . . 472

Weber's method for the valuation of acetate of lime. . . . . . . . . . . 238

Weighing flask. . ................................. 99 
PAGE

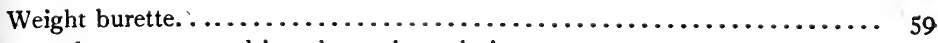

— and measures used in volumetric analysis. . . . . . . . . . . .

Wijs' iodin number. .................................... 477

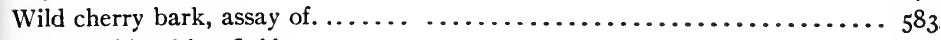

Wilson's bleaching fluid............................. 2 2

Wines, estimation of tannin in. . . . . . . . . . . . . . . . . . $6{ }_{3} 6$

Zinc bromid. ...................................... I 9

- estimation of, as arsenate. ............................... 435

- estimation of, as ferrocyanid.............................. 430

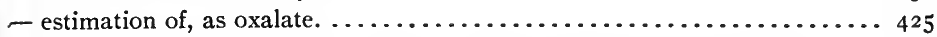

- estimation of, as sulphid, by means of ferric salt and permanganate. . . 428

- estimation of, as sulphid, using iron indicator. ................... 430

- estimation of, by precipitation as phosphate. .................. 437

- estimation of, by precipitation with standard sodium sulphid. ........ 427

- estimation of, indirect method.......................... 426

- estimation of, in the presence of manganese................... 433

- metallic estimation of. . . ............................ 202

- iodid. ..................................... I 25

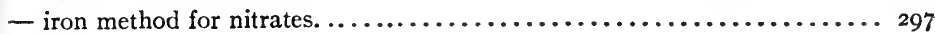





\title{
Short-Title Catalogue
}

OF THE

\section{PUBLICATIONS}

$\mathrm{OF}$

\section{JOHN WILEY \& SONS}

\author{
New York
}

LoNdon: CHAPMAN \& HALL, Limited

\section{ARRANGED UNDER SUBJECTS}

Descriptive circtlars sent on application. Books marked with an asterisk (*) are sold at net prices only. All books are bound in cloth unless otherwise stated.

\section{AGRICULTURE-HORTICULTURE-FORESTRY.}

Armsby's Principles of Animal Nutrition................... 8 vo, $\$ 400$ Budd and Hansen's American Horticultural Manual:

Part I. Propagation, Culture, and Improvement............ $12 \mathrm{mo}$, 150

Part II. Systematic Pomology........................ 150

Elliott's Engineering for Land Drainage............................ 150

Practical Farm Drainage. (Second Edition, Rewritten)........... 12 mo, 150

Graves's Forest Mensuration..............................8vo, 400

Green's Principles of American Forestry........................ 150

Grotenfelt's Principles of Modern Dairy Practice. (Woli.).............. 200

* Herrick's Denatured or Industrial Alcohol .................

Kemp and Waugh's Landscape Gardening. (New Edition, Rewritten. In Preparation).

* McKay and Larsen's Principles and Practice of Butter-making . . . . 8vo, 150 Maynard's Landscape Gardening as Applied to Home Decoration......12mo, 150 Sanderson's Insects Injurious to Staple Crops............... 150 Sanderson and Headlee's Insects Injurious to Garden Crops. (In Preparation).

* Schwarz's Longleaf Pine in Virgin Forests.................. 12mo, 125

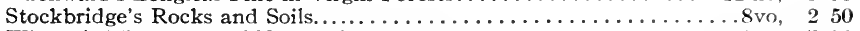

Winton's Microscopy of Vegetable Foods................... 7 vo, 50

Woll's Handbook for Farmers and Dairymen.................... 160

\section{ARCHITECTURE.}

Baldwin's Steam Heating for Buildings................. 250

Berg's Buildings and Structures of American Railroads..............

Birkmire's Architectural Iron and Steel.....................

Compound Riveted Girders as Applied in Buildings..............8vo, 200

Planning and Construction of American Theatres................8vo, 300

Planning and Construction of High Office Buildings............. 350

Skeleton Construction in Buildings....................... 300 
Briggs's Modern American School Buildings................8vo, Byrne's Inspection of Materials and Wormanship Employed in Construction.

Carpenter's Heating and Ventilating of Buildings............. 16mo

* Corthell's Allowable Pressure on Deep Foundations................ 12mo,

Freitag's Architectural Engineering.......................

Fireproofing of Steel Buildings....................8vo,

Gerhard's Guide to Sanitary Inspections. (Fourth Edition, Entirely Re-

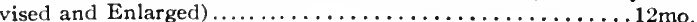

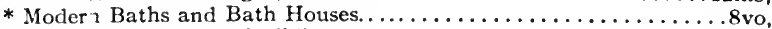

Sanitation of Public Buildings.......................

Theatre Fires and Panics............................

Johnson's Statics by Algebraic and Graphic Methods..............

Kellaway's How to Lay Out Suburban Home Grounds. . . . . . . . . . . . . 8vo,

Kidder's Architects' and Builders' Pocket-book..............

Merrill's Stones for Building and Decoration.................

Monckton's Stair-building........................4to,

Patton's Practical Treatise on Foundations................. 8vo,

Peabody's Naval Architecture..........................8vo,

Rice's Concrete-block Manufacture......................... 8vo,

Richey's Handbook for Superintendents of Construction ...... 16mo, mor.

Building Foreman's Pocket Book and Ready Reference. . 16mo, mor. * Building Mechanics' Ready Reference Series:

* Carpenters' and Woodworkers' Edition..........16mo, mor.

* Cement Workers' and Plasterers' Edition...........16mo, mor.

* Plumbers', Steam-Fitters', and Tinners' Edition. . .16mo, mor.

* Stone- and Brick-masons' Edition.............16mo, mor.

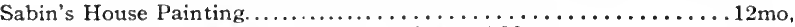

Siebert and Biggin's Modern Stone-cutting and Masonry...........8vo,

Snow's Principal Species of Wood.........................

Towne's Locks and Builders' Hardware................ 18mo, mor.

Wait's Engineering and Architectural Jurisprudence.............. 8vo,

Sheep,

Law of Contracts.................................... . . . . .

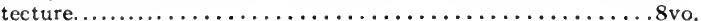

Sheep,

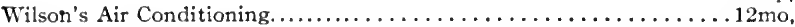

Worcester and Atkinson's Small Hospitals, Establishment and Maintenance, Suggestions for Hospital Architecture, with Plans for a Small Hospital.

\section{ARMY AND NAVY.}

Bernadou's Smokeless Powder, Nitro-cellulose, and the Theory of the Cellulose

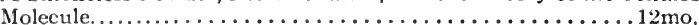

Chase's Art of Pattern Making........................ Serew Propellers and Marine Propulsion.................... 8vo,

* Cloke's Enlisted Specialists' Examiner...................... . 8vo, Gunner's Examiner.............................

Craig's Azimuth............................

Crehore and Squier's Polarizing Photo-chronograph..................

* Davis's Elements of Law..............................8vo,

* Treatise on the Military Law of United States. . . . . . . . . . . . . 8vo,

DeBrack's Cavalry Outpost Duties. (Carr.)................24mo, mor.

* Dudley's Military Law and the Procedure of Courts-martial. . .Large 12mo, Durand's Resistance and Propulsion of Ships...................

* Dyer's Handbook of Light Artillery............................ 12mo,

Eissler's Modern High Explosives......................... 8vo,

* Fiebeger's Text-book on Field Fortification.............. Large 12mo, Hamilton and Bond's The Gunner's Catechism.....................

* Hoff's Elementary Naval Tactics......................... 8vo,

Ingalls's Handbook of Problems in Direct Fire................8vo,

* Lissak's Ordnance and Gunnery.......................... 
* Ludlow's Logarithmic and Trigonometric Tables................8vo, \$1 00

* Lyons's Treatise on Electromagnetic Phenomena. Vols. I. and ii... . . . . 8vo, each, 6100

* Mahan's Permanent Fortifications. (Mercur.).............. 8vo. half mor. 750

Manual for Courts-martial.......................................... 150

* Mercur's Attack of Fortified Places........................................ 200

* Elements of the Art of War............................. 400

Nixon's Adjutants' Manual.................................... 100

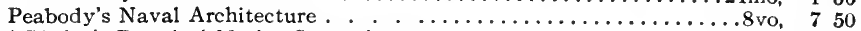

* Phelps's Practical Marine Surveying.................... 2 vo, 50

Putnam's Nautical Charts..............................

Rust's Ex-meridian Altitude. Azimuth and Star Finding Tables.......8vo, 500

Sharpe's Art of Subsisting Armies in War........................ mor, 150

* Tupes and Poole's Manual of Bayonet Exercises and Musketry Fencing. 24 mo, leather,

* Weaver's Military Explosives......................... 8vo,

Woodhull's Notes on Military Hygicne.....................

\section{ASSAYING.}

Betts's Lead.Refining by Electrolysis................... 8vo,

Fletcher's Practical Instructions in Quantitative Assaying with the Blowpipe. $16 \mathrm{mo}$, mor.

Furman and Pardoe's Manual of Practical Assaying. (Sixth Edition Revised and Enlarged $) \ldots \ldots \ldots \ldots \ldots \ldots \ldots \ldots \ldots \ldots$ vo,

Lodge's Notes on Assaying and Metallurgical Laboratory Experiments..8vo,

Low's Technical Methods of Ore Analysis.................... . . .

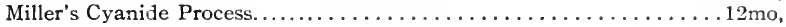

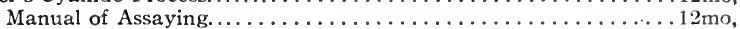

Minet's Production of Aluminum and its Industrial Use. (Waldo.)..12mo,

O'Driscoll's Notes on the Treatment of Gold Ores................. 8 vo,

Ricketts and Miller's Notes on Assaying...................... . . .

Robine and Lenglen's Cyanide Industry. (Le Clerc.)............. . 8vo,

Ulke's Modern Electrolytic Copper Refining................... 8vo,

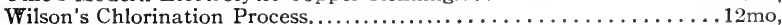
Cyanide Processes...............................

\section{ASTRONOMY.}

Comstock's Field Astronomy for Engineers..................8vo, 250

Craig's Azimuth....................................... 350

Crandall's Text-book on Geodesy and Least Squares..............8vo, 300

Doolittle's Treatise on Pracical Astronomy........................ 400

Hayford's Text-book of Geodetic Astronomy..................8vo, 300

Hosmer's Azimuth...................................... 100

Merriman's Elements of Precise Surveying and Geodesy..........8vo, 250

* Michie and Harlow's Practical Astronomy................. 8vo, 300

Rust's Ex-meridian Altitude, Azimuth and Star-Finding Tables...... 8vo, 500

*White's Elements of Theoretical and Descriptive Astronomy.......12mo, 200

\section{CHEMISTRY.}

* Abderhalden's Physiological Chemistry in Thirty Lectures. (Hall and

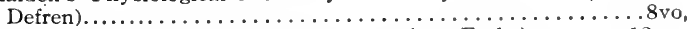
* Abegg's Theory of Electrolytic Dissociation. (von Ende.)........... Alexeyeff's General Principles of Organic Syntheses. (Matthews.)......8vo,

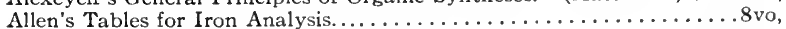
Armsby's Principles of Animal Nutrition.................... Arnold's Compendium of Chemistry. (Mandel.).................. $12 \mathrm{mo}$, Association of State and National Food and Dairy Departments, Hartford

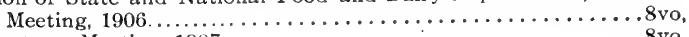
Jamestown Meeting, 1907 . 
Austen's Notes for Chemical Students.

Baskerville's Chemical Elements. (In Preparation).

Bernadou's Smokeless Powder.-Nitro-cellulose, and Theory of the Cellulose

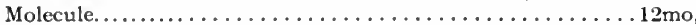

Biltz's Introduction to Inorganic Chemistry. (Hall and Phelan). (In Press). Laboratory Methods of Inorganic Chemistry. (Hall and Blanchard).

* Blanchard's Synthetic Inorganic Chemistry................. . . 12mo,

* Browning's Introduction to the Rarer Elements.................. . 8vo,

* Claassen's Bect-sugar Manufacture. (Hall and Rolfe.)............. 8vo, Classen's Quantitative Chemical Ana!ysis by Electrolysis. (Boltwood.).8vo,

Cohn's Indicators and Test-papers.........................

Tests and Reagents.......................... 8 vo,

* Danneel's Electrochemistry. (Merriam.).................... 12mo,

Dannerth's Methods of Textile Chemistry.................. 12mo,

Duhem's Thermodynamics and Chemistry. (Burgess.)............

Effront's Enzymes and their Applications. (Prescott.)..........8vo,

Eissler's Modern High Explosives......................... . . . . .

Erdmann's Introduction to Chemical Preparations. (Dunlap.)......12mo,

* Fischer's Physiology of Alimentation. . ................. Large 12mo,

Fletcher's Practical Instructions in Quantitative Assaying wlth the Blowpipe.

12 mo, mor.

Fowler's Sewage Works Analyses.......................... 12mo,

Fresenius's Manual of Qualitative Chemical Analysis. (Wells.)........8vo, 500 Manual of Qualitative Chemical Analysis. Part I. Descriptive. (Wells.) 8 vo, 300

Quantitative Chemical Analysis. (Cohn.) 2 vols...........8vo, 1250 When Sold Separately, Vol. I, \$6. Vol. II, \$8.

Fuertes's Water and Public Health..................... 150

Furman and Pardoe's Manual of Practical Assaying. (Sixth Edition,

Revised and Enlarged.)......................

* Getman's Exercises in Physical Chemistry.................... . . . .

Gill's Gas and Fuel Analysis for Engineers.................. 125

* Gooch and Browning's Outlines of Qualitative Chemical Analysis.

Grotenfelt's Principles of Modern Dairy Practice. (Woll.).........12mo, 200

Groth's Introduction to Chemical Crystallography (Marshall).......12mo, 125

Hammarsten's Text-book of Physiological Chemistry. (Mandel.)......8vo, 400

Hanausek's Microscopy of Technical Products. (Winton.)............8vo, 500

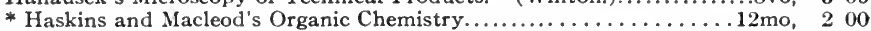

Hering's Ready Reference Tables (Conversion Factors)..........16mo, mor. 250

* Herrick's Denatured or Industrial Alcohol.................... 8vo, 400

Hinds's Inorganic Chemistry......................... 8vo, 300

* Laboratory Manual for Students............................... 100

* Holleman's Laboratory Manual of Organic Chemistry for Beginners.

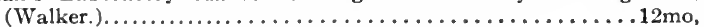

Text-book of Inorganic Chemistry. (Cooper.)................8vo,

Text-book of Organic Chemistry. (Walker and Mott.)............8vo,

* Holley's Lead and Zinc Pigments.................... Large 12mo,

Holley and Ladd's Analysis of Mixed Paints, Color Pigments, and Varnishes.

Large $12 \mathrm{mo}$,

Hopkins's Oil-chemists' Handbook. . . . . . . . . . . . . . . . . . . 8vo, Jackson's Directions for Laboratory Work in Physiological Chemistry. . 8vo, Johnson's Rapid Methods for the Chemical Analysis of Special Steels, Steel-

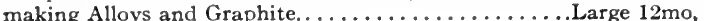

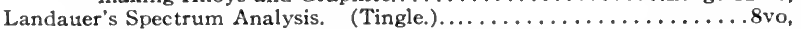

* Langworthy and Austen's Occurrence of Aluminum in Vegetable Products, Animal Products, and Natural Waters............. . . .

Lassar-Cohn's Application of Some General Reactions to Investigations in

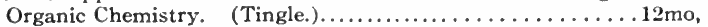

Leach's Inspection and Analysis of Food with Special Reference to State

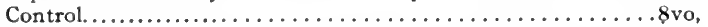

Löb's Electrochemistry of Organic Compounds. (Lorenz.)...........8vo,

Lodge's Notes on Assaying and Metallurgical Laboratory Experiments..8vo,

Low's Technical Method of Ore Analysis........................

Lunge's Techno-chemical Analysis. (Cohn.)....................

- McKay and Larsen's Principles and Practice of Butter-making.........8vo,

Maire's Modern Pigments and their Vehicles.................. 12mo, 
Mandel's Handbook for Bio-chemical Laboratory.

* Martin's Laboratory Guide to Qualitative Analysis with the Blowpipe

Mason's Examination of Water. (Chemical and Bacteriological) $12 \mathrm{mo}, 60$ Water-supply. (Considered Principally fromacteriological.).....12mo, 125

Principally from a Sanitary Standpoint.)

8 vo, 400

* Mathewson's First Principles of Chemical Theory...............8vo, 100

Matthews's Laboratory Manual of Dyeing and Textile Chemistry.......

Textile Fibres. 2d Edition, Rewritten..................8vo, 400

* Meyer's Determination of Radicles in Carbon Compounds. (Tingle.)

Third Edition................................... 125

Miller's Cyanide Process.......................... $100 \ldots \ldots \ldots \ldots$

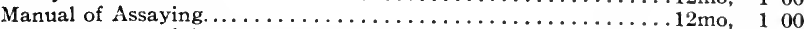

Minet's Production of Aluminum and its Industrial Use. (Waldo.).12mo, 250

Mixter's Elementary Text-book of Chemistry..................... 150

Morgan's Elements of Physical Chemistry...................... 300

Outline of the Theory of Solutions and its Results.............

* Physical Chemistry for Electrical Engineers.................... $12 \mathrm{mo}$, 50

Morse's Calculations used in Cane-sugar Factories...................... mor. 150

* Muir's History of Chemical Theories and Laws................... 4 vo, 400

Mulliken's General Method for the Identification of Pure Organic Compounds.

Vol. I. Compounds of Carbon with Hydrogen and Oxygen. Large 8vo, 500

Vol. II. Nitrogenous Compounds. (In Preparation).

Vol. III. The Commercial Dyestuffs. (In Press).

O'Driscoll's Notes on the Treatment of Gold Ores.................

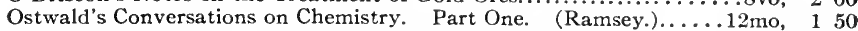

" " " " Part Two. (Turnbull.).....12mo, 200

Owen and Standage's Dyeing and Cleaning of Textile Fabrics.........12mo. 200

* Palmer's Practical Test Book of Chemistry...................... 100

* Pauli's Physical Chemistry in the Service of Medicine. (Fischer.).12mo, 125

Penfield's Tables of Minerals, Including the Use of Minerals and Statistics

of Domestic Production.........................

Pictet's Alkaloids and their Chemical Constitution (Biddle).....8vo, 00

Poole's Calorific Power of Fuels..........................

Prescott and Winslow's Elements of Water Bacteriology, with Special Refer-

ence to Sanitary Water Analysis.....................

Richards and Woodman's Air, Water, and Food from a Sanitary Stand-

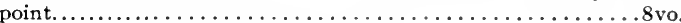

Ricketts and Miller's Notes on Assaying....................

Rideal's Disinfection and the Preservation of Food................. 8vo,

Sewage and the Bacterial Purification of Sewage............... 8 vo,

Rigg's Elementary Manual for the Chemical Laboratory............. . 8vo,

Robine and Lenglen's Cyanide Industry. (Le Clerc.)............ 8vo,

Ruddiman's Incompatibilities in Prescriptions................

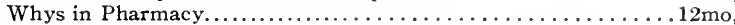

Ruer's Elements of Metallography. (Mathewson). (In Press.)

Sabin's Industrial and Artistic Technology of Paint and Varnish. .....8vo, 300

Salkowski's Physiological and Pathological Chemistry. (Orndorff.)....8vo, 250

Schimpf's Essentials of Volumetric Analysis....................

Manual of Volumetric Analysis. (Fifth Edition, Rewritten)....8vo, 500

* Qualitative Chemical Analysis......................... 125

Smith's Lecture Notes on Chemistry for Dental Students..........8vo, 250

Spencer's Handbook for Cane Sugar Manufacturers..................... mor. 300

Handbook for Chemists of Beet-sugar Houses............ $16 \mathrm{mo}$, mor. 300

Stockbridge's Rocks and Soils......................... 250

Stone's Practical Testing of Gas and Gas Meters.............. 8vo, 350

* Tillman's Descriptive General Chemistry......................... 300

* Elementary Lessons in Heat ......................... 150

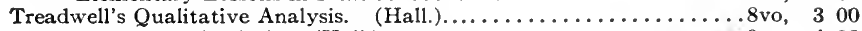

Quantitative Analysis. (Hall.)...................... 400

Turneaure and Russell's Public Water-supplies....................... 500

Van Deventer's Physical Chemistry for Beginners. (Boltwood.).....12mo, 150

Venable's Methods and Devices for Bacterial Treatment of Sewage.....8vo, 300

Ward and Whipple's Freshwater Biology. (In Press.)

Ware's Beet-sugar Manufacture and Refining. Vol. I.............8vo, 400 "s " " Vol. II.................. " 
Washington's Manual of the Chemical Analysis of Rocks............ 8vo, $\$ 200$

* Weaver's Military Explosives..........................8vo, 300

Wells's Laboratory Guide in Qualitative Chemical Analysis..........8vo, 150

Short Course in Inorganic Qualitative Chemical Analysis for Engineering

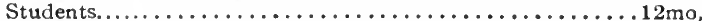

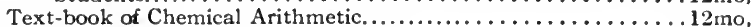

Whipple's Microscopy of Drinking-water.................... 8vo,

Wilson's Chlorination Process.....................................

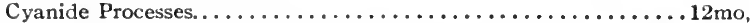

Winton's Microscopy of Vegetables Food.................... 8vo,

Zsigmondy's Colloids and the Ultramicroscope. (Alexander)...Large 12mo,

\title{
CIVIL ENGINEERING.
}

\section{BRIDGES AND ROOFS. HYDRAULICS. MATERIALS OF ENGINEER-}

\author{
ING. RAILWAY ENGINEERING.
}

Baker's Engineers' Surveying Instruments.................... Bixby's Graphical Computing Table.............Paper $\left.19 \frac{1}{2} \times 24\right\}$ inches. Breed and Hosmer's Principles and Practice of Surveying. Vol. I. Elemen-

tary Surveying...........................

Vol. II. Higher Surveying. ............................ . . . . .

* Burr's Ancient and Modern Engineering and the Isthmian Canal..... 8vo,

Comstock's Field Astronomy for Engineers...................... . .

* Corthell's Allowable Pressure on Deep Foundations ............ 12mo,

Crandall's Text-book on Geodesy and Least Squares............... . . .

Davis's Elevation and Stadia Tables.......................

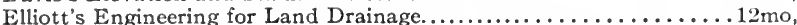

Practical Farm Drainage. (Second Edition Rewritten.).......12mo,

* Fiebeger's Treatise on Civil Engineering.................... 8vo,

Flemer's Photographic Methods and Instruments................ 8vo,

Folwell's Sewerage. (Designing and Maintenance.)............... 8vo,

Freitag's Architectural Engineering......................... 8 vo,

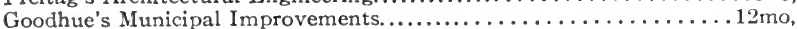

* Hauch and Rice's Tables of Quantities for Preliminary Estimates...12mo,

Hayford's Text-book of Geodetic Astronomy.................... . . . .

Hering's Ready Reference Tables (Conversion Factors)..........16mo, mor.

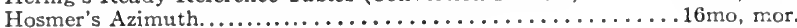

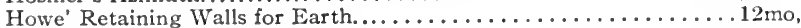

* Ives's Adjustments of the Engineer's Transit and Level. . . . . . . . 16mo, bds.

Johnson's (J. B.) Theory and Practice of Surveying. ........ Large 12mo, Johnson's (L. J.) Statics by Algebraic and Graphic Methods............ . 8vo, Kinnicutt, Winslow and Pratt's Purification of Sewage. (In Preparation).

* Mahan's Descriptive Geometry.............................

Merriman's Elements of Precise Surveying and Geodesy. . . . . . . . . . 8vo,

Merriman and Brooks's Handbook for Surveyors...........

Nugent's Plane Surveying........................... . . . .

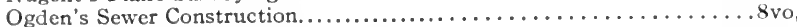

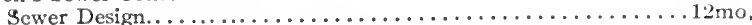

Parsons's Disposal of Municipal Refuse.................... . . . Patton's Treatise on Civil Engineering............... holf leather, Reed's Topographical Drawing and Sketching................. to, Rideal's Sewage and the Bacterial Purification of Sewage............ 8 vo, Riemer's Shaft-sinking under Difficult Conditions. (Corning and Peele.).8vo, Siebert and Biggin's Modern Stone-cutting and Masonry............ 8vo, Smith's Manual of Topographical Drawing. (McMillan.)..............

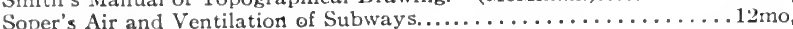
* Tracy's Exercises in Surveying. ..................... mo.

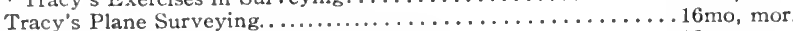
* Trautwine's Civit Engineer's Pocket-book...................... mor.

Venable's Garbage Crematories in America..................... 8vo, Methods and Devices for Bacterial Treatment of Sewage...........8vo, 
Wait's Engineering and Architectural Jurisprudence...............8vo, $\$ 600$

Sheep, 650

Law of Contracts................................. 300

Law of Operations Preliminary to Construction in Engineering and

Architecture................................ 500

Sheep, 550

Warren's Stereotomy-Problems in Stone-cutting.................

* Waterbury's Vest-Pocket Hand-book of Mathematics for Engineers.

$2 \frac{7}{8} \times 5 \frac{3}{8}$ inches, mor. 100

Webb's Problem's in the Use and Adjustment of Engineering Instruments. 16 mo, mor. 125

Wilson's Topographic Surveying....................

\section{BRIDGES AND ROOFS.}

Boller's Practical Treatise on the Construction of Iron Highway Bridges..8vo, 200

* Thames River Bridge........................................ paper, 500

Burr and Falk's Design and Construction of Metallic Bridges.........8vo, 500

Influence Lines for Bridge and Roof Computations...............8vo, 300

Du Bois's Mechanics of Engineering. Vol. II......................... 4to, 1000

Foster's Treatise on Wooden Trestle Bridges........................ 5 to, 50

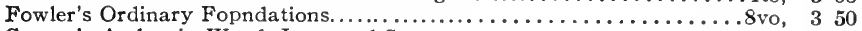

Greene's Arches in Wood, Iron, and Stone.......................... 250

Bridge Trusses..................................... 250

Roof Trusses.............................................. 125

Grimm's Secondary Stresses in Bridge Trusses.............................. 250

Heller's Stresses in Structures and the Accompanying Deformations..... . vo, 300

Howe's Design of Simple Roof-trusses in Wood and Steel.............8vo. 200

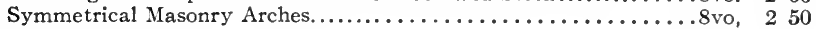

Treatise on Arches............................ 400

Johnson, Bryan and Turneaure's Theory and Practice in the Designing of

Modern Framed Structures................................. 4 to, 100

Merriman and Jacoby's Text-book on Roofs and B̈ridges:

Part I. Stresses in Simple Trusses..................... 250

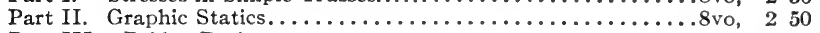

Part III. Bridge Design................................. 250

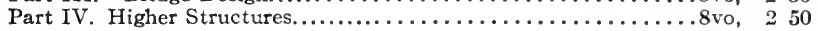

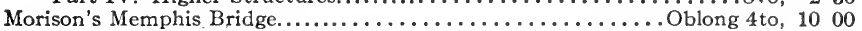

Sondericker's Graphic Statics, with Applications to Trusses, Beams, and

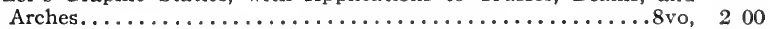

Waddell's De Pontibus, Pocket-book for Bridge Engineers.......16mo, mor. 200

* Specifications for Steel Bridges............................ 50

Waddell and Harringtoon's Bridge Engineering. (In Preparation.)

Wright's Designing of Draw-spans. Two parts in one volume..........8vo, 350

\section{HYDRAULICS.}

Barnes's Ice Formation............................... 8vo, 300

Bazin's Experiments upon the Contraction of the Liquid Vein Issuing from

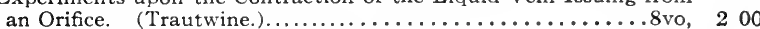

Bovey's Treatise on Hydraulics.......................... 500 Church's Diagrams of Mean Velocity of Water in Open Channels.

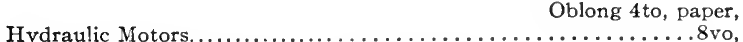
Coffin's Graphical Solution of Hydraulic Problems............16mo, mor.

Flather's Dynamometers, and the Measurement of Power.............. Folwell's Water-supply Engineering....................

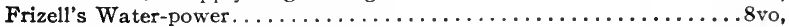

Fuertes's Water and Public Health...........................

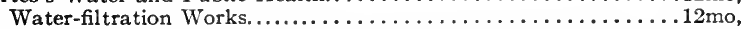

Ganguillet and Kutter's General Formula for the Uniform Flow of Water in Rivers and Other Channels. (Hering and Trautwine.)......8vo, 
Hazen's Clean Water and How to Get It.................... 12mo, \$1 50

Filtration of Public Water-supplies.................. 300

Hazelhurst's Towers and Tanks for Water-works................8vo, 250

Herschel's 115 Experiments on the Carrying Capacity of Large, Riveted, Metal

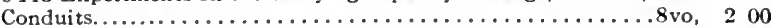

Hoyt and Grover's River Discharge....................

Hubbard and Kiersted's Water-works Management and Maintenance.

* Lyndon's Development and Electrical Distribution of Water Power.

Mason's Water-supply. (Considered Principally from a Sanitary Stand-

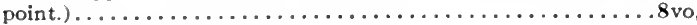

Merriman's Treatise on Hydraulics........................ 8 vo,

* Molitor's Hydraulics of Rivers, Weirs and Sluiccz............... . 8vo,

* Richards's Laboratory Notes on Industrial Water Analysis..... . . . 8vo,

Schuyler's Reservoirs for Irrigation, Water-power, and Domestic Watersupply. Second Edition, Revised and Enlarged....... Large 8vo,

* Thomas and Watt's Improvement of Rivers.................4 to,

Turneaure and Russell's Public Water-supplies..................8vo,

Wegmann's Design and Construction of Dams. 5th Ed., enlarged......4 to

Water-Supply of the City of New York from 1658 to $1895 \ldots \ldots \ldots \ldots 4$ to, 1000

Whipple's Value of Pure Water.............................. 12mo, 100

Williams and Hazen's Hydraulic Tables..................

Wilson's Irrigation Engineering......................... 4 vo, 400

Wood's Turbines........................................ 250

\section{MATERIALS OF ENGINEERING.}

Baker's Roads and Pavements......................... 8vo, 500

Treatise on Masonry Construction...................... 5 vo, 500

Black's United States Public Works.............................. 4 to, 500

Blanchard's Bituminous Roads. (In Press.)

Bleininger's Manufacture of Hydraulic Cement. (In Preparation.)

* Bovey's Strength of Materials and Theory of Structures.............8vo, $7 \mathbf{5 0}$

Burr's Elasticity and Resistance of the Materials of Engineering.......8vo, 750

Byrne's Highway Construction........................... 500 Inspection of the Materials and Workmanship Employed in Construction.

$16 \mathrm{mo}$,

Church's Mechanics of Engineering.

Du Bois's Mechanics of Engineering.

Vol. I. Kinematics, Statics, Kinetics.................. Small 4to,

Vol. II. The Stresses in Framed Structures, Strength of Materials and

Theory of Flexures............................................. 1000

* Eckel's Cements, Limes, and Plasters..................... 8vo, 600 Stone and Clay Products used in Engineering. (In Preparation.)

Fowler's Ordinary Foundations......................... 3 vo, 50

* Greene's Structural Mechanics...........................8vo, 250

* Holley's Lead and Zinc Pigments............................ 12mo, 300

Holley and Ladd's Analysis of Mixed Paints, Color Pigments and Varnishes. Large $12 \mathrm{mo}$

Johnson's (C. M.) Rapid Methods for the Chemical Analysis of Special Steels,

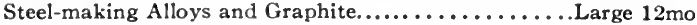

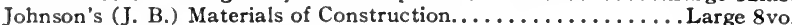

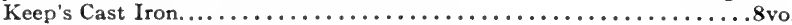
Lanza's Applied Mechanics................................ 8vo Maire's Modern Pigments and their Vehicles.................. 12mo, Martens's Handbook on Testing Materials. (Henning.) 2 vols.......8vo, Maurer's Technical Mechanics...........................8vo

Merrill's Stones for Building and Decoration..................8vo

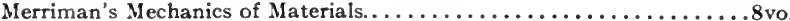

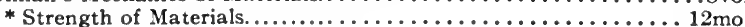

Metcalf's Steel. A Manual for Steel-users..................... 12mo

Morrison's Highway Engineering........................8vo

Patton's Practical Treatise on Foundations..................8vo,

Rice's Concrete Block Manufacture......................... 8vo, 
Richardson's Modern Asphalt Pavements.................. 8vo, \$3 00 Richey's Building Foreman's Pocket Book and Ready Reference.16mo,mor. 500

* Cement Workers' and Plasterers' Edition (Building Mechanics' Ready

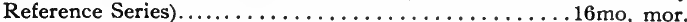

Handbook for Superintendents of Construction...........

* Stone and Brick Masons' Edition (Building Mechanics' Ready Reference Series $\ldots \ldots \ldots \ldots \ldots \ldots \ldots \ldots \ldots \ldots \ldots \ldots \ldots \ldots$ mor. m.

* Ries's Clays: Their Occurrence, Properties, and Uses................ 8vo,

* Ries and Leighton's History of the Clay-working Industry of the United

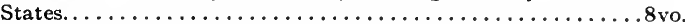

Sabin's Industrial and Artistic Technology of Paint and Varnish....... 8vo,

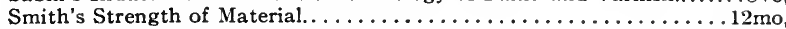
Snow's Principal Species of Wood.......................

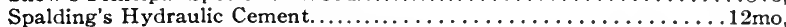

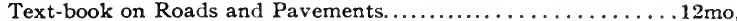

Taylor and Thompson's Treatise on Concrete, Plain and Reinforced....8vo, Thurston's Materials of Engineering. In Three Parts............... 8vo,

Part I. Non-metallic Materials of Engineering and Metallurgy... .8vo,

Part II. Iron and Steel..........................

Part III. A Treatise on Brasses, Bronzes, and Other Alloys and their

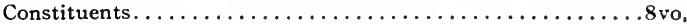

Tillson's Street Pavements and Paving Materials...............8vo, Turneaure and Maurer's Principles of Reinforced Concrete Construction. Second Edition, Revised and Enlarged............. 8vo, Waterbury's Cement Laboratory Manual..................... Wood's (De V.) Treatise on the Resistance of Materials, and an Appendix on the Preservation of Timber.......................

Wood's (M. P.) Rustless Coatings: Corrosion and Electrolysis of Iron and

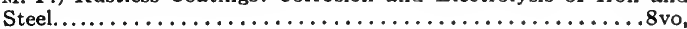

150 400 150 500 250 300 350 200 200 500

800 200 350

\section{RAILWAY ENGINEERING.}

Andrews's Handbook for Street Railway Engineers...... $3 \times 5$ inches, mor. Berg's Buildings and Structures of American Railroads.............4 to, Brooks's Handbook of Street Railroad Location............. Butts's Civil Engineer's Field-book.................... mo, mor.

Crandall's Railway and Other Earthwork Tables..................8vo,

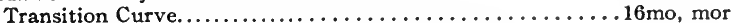

* Crockett's Methods for Earthwork Computaticns.............. . 8vo Dredge's History of the Pennsylvania Railroad. (1879) ................ Papet

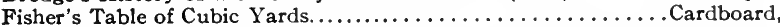
Godwin's Railroad Engineers' Field-book and Explorers' Guide. .16mo, mor. Hudson's Tables for Calculating the Cubic Contents of Excavations and Fm-

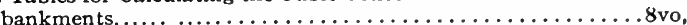

Ives and Hilts's Problems in Surveying, Railroad Surveying and Geodesy $16 \mathrm{mo}$, mor

Molitor and Beard's Manual for Resident Engineers..............16mo, Nagle's Field Manual for Railroad Engineers..............

* Orrock's Railroad Structures and Estimates................... 8vo, Philbrick's Field Manual for Engineers................. Raymond's Railroad Engineering. 3 volumes.

Vol. I. Railroad Field Geometry. (In Preparation.)

Vol. II Elements of Railroad Engineering. . . . . . . . . . . vo

Vol. III. Railroad Engineer's Field Book. (In Preparation.)

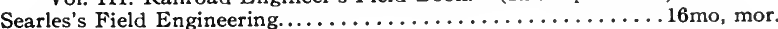

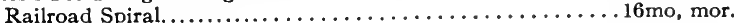

Taylor's Prismoidal Formulæ and Earthwork..................

* Trautwine's Field Practice of Laying Out Circular Curves for Railroads. $12 \mathrm{mo}$, mor.

* Method of Calculating the Cubic Contents of Excavations and Embankments by the Aid of Diagrams..................

Webb's Economics of Railroad Construction....................... $12 \mathrm{mo}$,

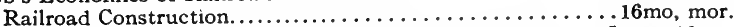
Wellington's Economic Theory of the Location of Railways.....Large 12mo, Wilson's Elements of Railroad-Track and Construction.............12mo,

125

500

150

250

150

150

: 50

j 00

25

250

100

150

100

300

300

300 


\section{DRAWING.}

Barr's Kinematics of Machinery............................. $\$ 250$

* Bartlett's Mechanical Drawing...................... 8vo, 300

* " " " Abridged Ed..................8vo, 150

Coolidge's Manual of Drawing.....................8vo, paper, 100

Coolidge and Freeman's Elements of General Drafting for Mechanical Engineers............................................................. 250

Durley's Kinematics of Machines............................ 400

Emch's Introduction to Projective Geometry and its Application.....8vo, 250

French and Ives' Stereotomy................................. 250

Hill's Text-book on Shades and Shadows, and Perspective............8vo, 200

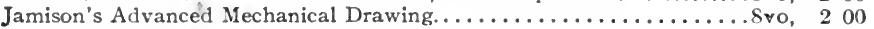

Elements of Mechanical Drawing...................... 250

Jones's Machine Design:

Part I. Kinematics of Machinery........................ 150

Part II. Form, Strength, and Proportions of Parts.............8vo, 300

Kimball and Barr's Machine Design. (In Press.)

MacCord's Elements of Descritpive Geometry............... 8vo, 300

Kinematics; or, Practical Mechanism................... 500

Alechanical Drawing................................. 400

Velocity Diagrams...................................... 150

McLeod's Descriptive Geometry.................................. 150

* Mahan's Descriptive Geometry and Stone-cutting..............8vo, 150

Industrial Drawing. (Thompson.)..................... 350

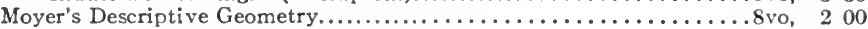

Reed's Topographical Drawing and Sketching.................4to, 500

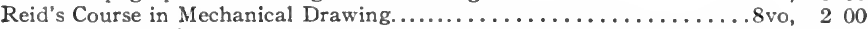

Text-book of Mechanical Drawing and Elementary Machine Design..8vo, 300

Robinson's Principles of Mechanism.......................... 300

Schwamb and Merrill's Elements of Mechanism...................8vo, 300

Smith (A. W.) and Marx's Machine Design...................... 8vo, 300

Smith's (R. S.) Manual of Topographical Drawing. (McMillan)........8vo, 250

* Titsworth's Elements of Mechanical Drawing..................... 8 8vo, 125

Warren's Drafting Instruments and Operations..............12mo, 125

Elements of Descriptive Geometry, Shadows, and Perspective.....8vo, 350

Elements of Machine Construction and Drawing..............8vo, 750

Elements of Plane and Solid Free-hand Geometrical Drawing....12mo, 100

General Problems of Shades and Shadows................8vo, 300

Manual of Elementary Problems in the Linear Perspective of Forms and

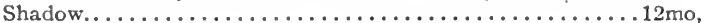

Manual of Elementary Projection Drawing..............

Plane Problems in Elementary Geometry....................

Problems, Theorems, and Examples in Descriptive Geometry......8vo,

Weisbach's Kinematics and Power of Transmission. (Hermann and

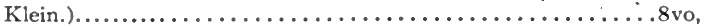

Wilson's (H. M.) Topographic Surveying....................

* Wilson's (V. T.) Descriptive Geometry...................... . . . . .

Free-hand Lettering. ..............................

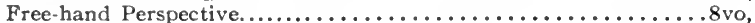

Woolf's Elementary Course in Descriptive Geometry.................... 8 vo,

\section{ELECTRICITY AND PHYSICS.}

* Abegg's Theory of Electrolytic Dissociation. (von Ende.).......12mo, 125

Andrews's Hand-book for Street Railway Engineering......3 35 inches, mor. 125

Anthony and Brackett's Text-book of Physics. (Magie.)....Large 12mo, 300

Anthony and Ball's Lecture-notes on the Theory of Electrical Measure-

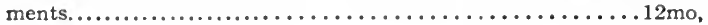

Benjamin's History of Electricity.....................

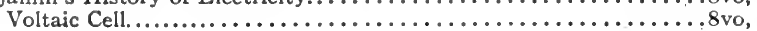


Betts's Lead Refining and Electrolysis.

$8 v o, \$ 400$

Classen's Quantitative Chemical Analysis by Electrolysis. (Boltwood.).8vo, 300

* Collins's Manual of Wireless Telegraphy and Telephony...........12mo, 150

* Mor. 200

Crehore and Squier's Polarizing Photo-chronograph............... 8vo, 300

* Danneel's Electrochemistry. (Merriam.)......................... 125

Dawson's "Engineering" and Electric Traction Pocket-book............. mor. 500

Dolezalek's Theory of the Lead Accumulator (Storage Battery). (von Ende.)

Duhem's Thermodynamics and Chemistry 12mo, 250

Flather's Dynamometers, and the

Getman's Introduction to Physical

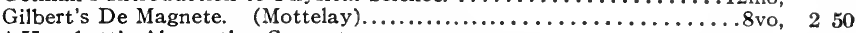

* Hanchett's Alternating Currents............................... I 00

Hering's Ready Reference Tables (Conversion Factors).......16mo, mor. 250

* Hobart and Ellis's High-speed Dynamo Electric Machinery......... 8vo, 600

Holman's Precision of Measurements...................... 200

Telescopic Mirror-scale Method, Adjustments, and Tests....Large 8vo, 75

* Karapetoff's Experimental Electrical Engineering.................8vo, 600

Kinzbrunner's Testing of Continuous-current Machines...........8vo, 200

Landauer's Spectrum Analysis. (Tingle.)...................

Le Chatelier's High-temperature Measurements. (Boudouard-Burgess.) $12 \mathrm{mo}, 300$

Löb's Electrochemistry of Organic Compounds. (Lorenz)............ 8vo, 300

* Lyndon's Development and Electrical Distribution of Water Power. .8vo, 300

* Lyons's Treatise on Electromagnetic Phenomena. Vols, I .and II. 8vo, each, 600

* Michie's Elements of Wave Motion Relating to Sound and Light....8vo, 400

Morgan's Outline of the Theory of Solution and its Results.........12mo, 100

* Physical Chemistry for Electrical Engineers............... $12 \mathrm{mo}, \quad 50$

* Norris's Introduction to the Study of Electrical Engineering.........8vo, 250

Norris and Dennıson's Course of Problems on the Electrical Characteristics of Circuits and Machines. (In Press.)

* Parshall and Hobart's Electric Machine Design.........4to, half mor, 1250 Reagan's Locomotives: Simple, Compound, and Electric. New Edition.

Large $12 \mathrm{mo}, 350$

* Rosenberg's Electrical Engineering. (Haldane Gee-Kinzbrunner.)..8vo, 200

Ryan, Norris, and Hoxie's Electrical Machinery. Vol. I.............8vo, 250

Schapper's Laboratory Guide for Students in Physical Chemistry.....12mo, 100

* Tillman's Elementary Lessons in Heat....................8vo, 150

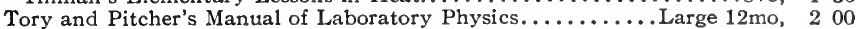

Ulke's Modern Electrolytic Copper Refining.................8vo, 300

\section{LAW.}

* Brennan's Hand-book of Useful Legal Information for Business Men.

$16 \mathrm{mo}$, mor.

* Davis's Elements of Law................................ 8vo,

* Treatise an the Military Law of United States.............

* Dudley's Military Law and the Procedure of Courts-martial. .Large 12mo, Manual for Courts-martial............................ mor. Wait's Engineering and Architectural Jurisprudence...............

Sheep,

Law of Contracts.........................................

Architecture..................................

\section{MATHEMATICS.}

Baker's Elliptic Functions............................

Briggs's Elements of Plane Analytic Geometry. (Bôcher).........12mo,

* Buchanan's Plane and Spherical Trigonometry................. 100 
Byerley's Harmonic Functions....................... 8vo, $\$ 100$

Chandler's Elements of the Infinitesimal Calculus..............12mo, 200

* Coffin's Vector Analysis. . . . . . . . . . . . . . . . . . . . . .

Compton's Manual of Logarithmic Computations...............

* Dickson's College Algebra........................................ 12mo, 150

* Introduction to the Theory of Algebraic Equations......................... 12mo, 125

Emch's Introduction to Projective Geometry and its Application..... 8vo, 250

Fiske's Functions of a Complex Variable..................8vo, 100

Halsted's Elementary Synthetic Geometry................ 8vo, 150

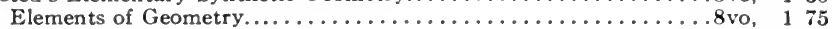

* Rational Geometry................................. 150

Hyde's Grassmann's Space Analysis. . . . . . . . . . . . . . . . . 8vo, 100

* Johnson's (J. B.) Three-place Logarithmic Tables: Vest-pocket size, paper, 15

* Mounted on heavy cardboard, $8 \times 10$ inches, 500

10 copies, 200

Johnson's (W. W.) Abridged Editions of Differential and Integral Calculus.

Large $12 \mathrm{mo}, 1$ vol. 250

Curve Tracing in Cartesian Co-ordinates................... 100

Differential Equations..................................... 100

Elementary Treatise on Differential Calculus................... 12mo, 150

Elementary Treatise on the Integral Calculus................. $12 \mathrm{mo}, 150$

* Theoretical Mechanics................................... 300

Theory of Errors and the Method of Least Squares........... $12 \mathrm{mo}$, 50

Treatise on Differential Calculus............................ $12 \mathrm{mo}, 300$

Treatise on the Integral Calculus.............................. $12 \mathrm{mo}, 300$

Treatise on Ordinary and Partial Differential Equations... Large 12mo, 350

Karapetoff's Engineering Applications of Higher Mathematics.

(In Preparation.)

Laplace's Philosophical Essay on Probabilities. (Truscott and Emory.) . 12mo,

* Ludlow and Bass's Elements of Trigonometry and Logarithmic and Other

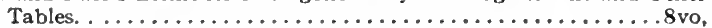

* Trigonometry and Tables published separately. ............Each,

* Ludlow's Logarithmic and Trigonometric Tables. . . . . . . . . . . . . 8vo,

Macfarlane's Vector Analysis and Quaternions. . . . . . . . . . . . . 8vo,

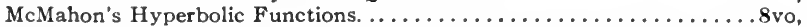

Manning's Irrational Numbers and their Representation by Sequences and

Series. . . . . . . . . . . . .

Mathematical Monographs. Edited by Mansfield Merriman and Robert

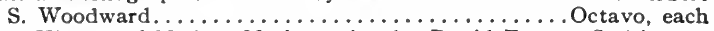

No. 1. History of Modern Mathematics, by David Eugene Smith.

No. 2. Synthetic Projective Geometry, by George Bruce Halsted.

No. 3. Determinants, by Laenas Gifford Weld. No. 4. Hyper-

bolic Functions, by James McMahon. No. 5. Harmonic Functions, by William E. Byerly. No. 6. Grassmann's Space Analysis, by Edward W. Hyde. No. 7. Probability and Theory of Errors, by Robert S. Woodward. No. 8. Vector Analysis and Quaternions, by Alexander Macfarlane. No. 9. Differential Equations, by William Woolsey Johnson. No. 10. The Solution of Equations, by Mansfield Merriman. No. 11. Functions of a Complex Variable, by Thomas S. Fiske.

Maurer's Technical Mechanics. ........................8vo, 400

Merriman's Method of Least Squares.....................8vo, 200

Solution of Equations. . ....................... 100

Rice and Johnson's Differential and Integral Calculus. 2 vols. in one.

Elementary Treatise on the Differential Calculus. ........ Large $12 \mathrm{mo}$,

Smith's History of Modern Mathematics. .................... 8vo

* Veblen and Lennes's Introduction to the Real Infinitesimal Analysis of One

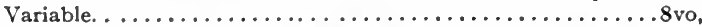

* Waterbury's Vest Pocket Hand-book of Mathematics for Engineers.

$2 \frac{7}{8} \times 5 \frac{3}{8}$ inches, mor. 100

Weld's Determinants. ................................. 100

Wood's Elements of Co-ordinate Geometry. ....................... 200

Woodward's Probability and Theory of Errors. . ................. 8vo, 100 


\section{MECHANICAL ENGINEERING.}

\section{MATERIALS OF ENGINEERING, STEAM-ENGINES AND BOILERS.}

Bacon's Forge Practice. .................................. $\$ 150$

Baldwin's Steam Heating for Buildings......................... 2 2 50

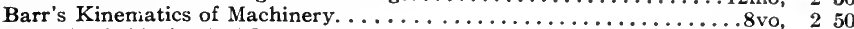

* Bartlett's Mechanical Drawing............................. 300

* " " " Abridged Ed.................

* Burr's Ancient and Modern Engineering and the Isthmian Canal. . . . 8vo, 350

Carpenter's Experimental Engineering. . . . . . . . . . . . . . . 6 8vo, 60

Heating and Ventilating Buildings................. 4 vo, 400

Clerk's Gas and Oil Engine. (New edition in press.)

Compton's First Lessons in Metal Working. .................. 12 mo, 150

Compton and De Groodt's Speed Lathe. ........................ 150

Coolidge's Manual of Drawing..................................... paper, 100

Coolidge and Freeman's Elements of Geenral Drafting for Mechanical En-

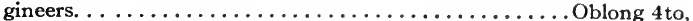

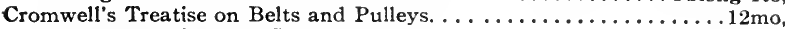

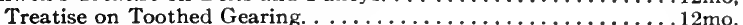

Dingey's Machinery Pattern Making.....................

Durley's Kinematics of Machines. . . . . . . . . . . . . . . . . . . 8vo,

Flanders's Gear-cutting Machinery. ..................... 12mo,

Flather's Dynamometers and the Measurement of Power...........12mo,

Rope Driving. . . . . . . . . . . . . . . . . . . . . .

Gill's Gas and Fuel Analysis for Engineers. . . . . . . . . . .

Goss's Locomotive Sparks. ........................... . . . . . .

Greene's Pumping Machinery. (In Preparation.)

Hering's Ready Reference Tables (Conversion Factors). . . . . . . 16mo, mor.

* Hobart and Ellis's High Speed Dynamo Electric Machinery. . . . . . . 8vo,

Hutton's Gas Engine. . . ........................8vo,

Jamison's Advanced Mechanical Drawing. ................. 8vo, Elements of Mechanical Drawing..................

Jones's Gas Engine. ............................ Machine Design:

Part I. Kinematics of Machinery.................. 150

Part II. Form, Strength, and Proportions of Parts...........8vo, 300

Kent's Mechanical Engineer's Pocket-Book..............6mo, mor. 500

Kerr's Power and Power Transmission. ........................ 200

Kimball and Barr's Machine Design. (In Press.)

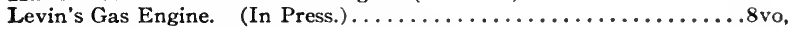

Leonard's Machine Shop Tools and Methods. ................8vo, 400

* Lorenz's Modern Refrigerating Machinery. (Pope, Haven, and Dean)..8vo, 400

MacCord's Kinematics; or, Practical Mechanism................8vo, 500

Mechanical Drawing......................... 400

Velocity Diagrams................................... 150

MacFarland's Standard Reduction Factors for Gases. . . ......... 8vo, 150

Mahan's Industrial Drawing. (Thompson.). ............... 3 vo, 30

Mehrtens's Gas Engine Theory and Design....................... 12mo, 250

Oberg's Handbook of Small Tools. ............................ 12mo, 300

* Parshall and Hobart's Electric Machine Design. Small 4to, half leather, 1250

Peele's Compressed Air Plant for Mines. . . . . . . . . . . . . . . 300

Poole's Calorific Power of Fuels. .....................8vo, 300

* Porter's Engineering Reminiscences, 1855 to $1882 \ldots \ldots \ldots \ldots \ldots \ldots \ldots$. $\ldots \ldots$ vo, 300

Reid's Course in Mechanical Drawing. ...................8vo, 200

Text-book of Mechanical Drawing and Elementary Machine Design.8vo, 300

Richards's Compressed Air................................. 150

Robinson's Principles of Mechanism.......................... 300

Schwamb and Merrill's Elements of Mechanism..............8vo, 300

Smith (A. W.) and Marx's Machine Design. . . . . . . . . . . . . 8vo, 300

Smith's (O.) Press-working of Metals..................... 300

Sorel's Carbureting and Combustion in Alcohol Engines. (Woodward and

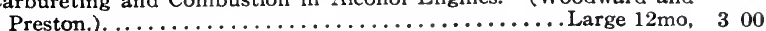

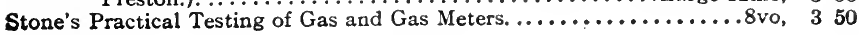


Thurston's Animal as a Machine and Prime Motor, and the Laws of Energetics.

$12 \mathrm{mo}, \$ 100$

Treatise on Friction and Lost Work in Machinery and Mill Work. . .8vo, 300

* Tillson's Complete Automobile Instructor. . . . . . . . . . . . 16mo, 150

* Titsworth's Elements of Mechanical Drawing.......................... 8vo, 125

Warren's Elements of Machine Construction and Drawing. ....... 8vo, 750

* Waterbury's Vest Pocket Hand-book of Mathematics for Engineers.

Weisbach's Kinematics and the Power of Transmission. (Herrmann-

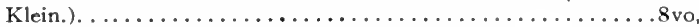

Machinery of Transmission and Governors. (Hermann-Klein.). .8vo,

Wood's Turbines..................................... 250

\section{MATERIALS OF ENGINEERING.}

* Bovey's Strength of Materials and Theory of Structures. . . . . . . .8vo, 750 Burr's Elasticity and Resistance of the Materials of Engineering. ......8vo, 750

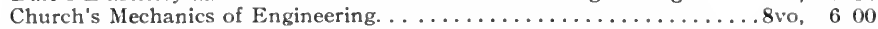

* Greene's Structural Mechanics. .......................... . . 250

* Holley's Lead and Zinc Pigments........................... 12 mo 300 Holley and Ladd's Analysis of Mixed Paints, Color Pigments, and Varnishes.

Large $12 \mathrm{mo}$,

Johnson's (C. M.) Rapid Methods for the Chemical Analysis of Special Steels, Steel-Making Alloys and Graphite........... Large $12 \mathrm{mo}$

Johnson's (J. B.) Materials of Construction. . ...................

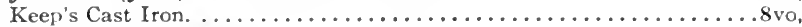

Lanza's Applied Mechanics. . . . . . . . . . . . . . . . . . . . . 8vo,

Maire's Modern Pigments and their Vehicles. ................. 12mo, Martens's Handbook on Testing Materials. (Henning.). ............8vo

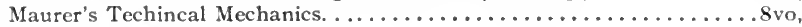

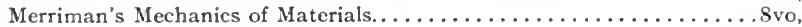

* Strength of Materials. . . . . . . . . . . . . . . .

Metcalf's Steel. A Manual for Steel-users..................... 12mo,

Sabin's Industrial and Artistic Technology of Paint and Varnish. . . . . 8vo, Smith's (A. W.) Materials of Machines. .................. 12mo,

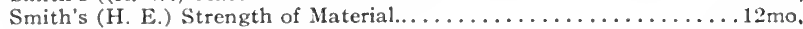

Thurston's Materials of Engineering................ vols., 8 vo,

Part I. Non-metallic Materials of Engineering, ............. 8vo,

Part II. Iron and Steel. . . . . . . . . . . . . . . . . . . . . .

Part III. A Treatise on Brasses, Bronzes, and Other Alloys and their

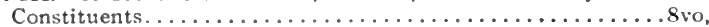

Wood's (De V.) Elements of Analytical Mechanics. .............. 8 vo,

Treatise on the Resistance of Materials and an Aprendix on the Preservation of Timber.........................

Wood's (M. P.) Rustless Coatings: Corrosion and Electrolysis of Iron and

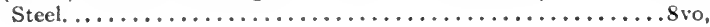

\section{STEAM-ENGINES AND BOILERS.}

Berry's Temperature-entropy Diagram................... 12 mo, Carnot's Reflections on the Motive Power of Heat. (Thurston.). . ..12mo,

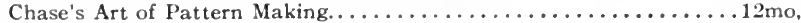
Creighton's Steam-engine and other Heat Motors. ............... Dawson's "Engineering" and Electric Traction Pocket-book. ...16mo, mor. Ford's Boiler Making for Boiler Makers. . . . . . . . . . . . . . 18mo,

* Gebhardt's Steam Power Plant Engineering. . . . . . . . . . . . . . 8vo,

Goss's Locomotive Performance......................... . . . . . Hemenway's Indicator Practice and Steam-engine Economy........12mo, Hutton's Heat and Heat-engines. . ....................... Mechanical Engineering of Power Plants. ............... 8vo,

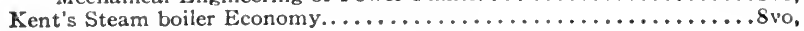


Kneass's Practice and Theory of the Injector. . . . . . . . . . . . 8vo, $\$ 150$

MacCord's Slide-valves. ............................

Meyer's Modern Locomotive Construction. .................. $10 . \ldots$ to, 10

Moyer's Steam Turbine. . . . . . . . . . $4 \ldots \ldots \ldots \ldots \ldots \ldots \ldots$ 8vo, 400

Peabody's Manual of the Steam-engine Indicator. . . . . . . . . 50

Tables of the Properties of Steam and Other Vapors and Temperature-

Entropy Table. ........................... 100

Thermodynamics of the Steam-engine and Other Heat-engines. .88 vo, 500

Valve-gears for Steam-engines.......................... 250

Peabody and Miller's Steam-boilers...................... 400

Pupin's Thermodynamics of Reversible Cycles in Gases and Saturated Vapors.

(Osterberg.)............................. 125

Reagan's Locomotives: Simple, Compound, and Electric. New Edition.

Large $12 \mathrm{mo}, 350$

Sinclair's Locomotive Engine Running and Management. . .........12mo, 200

Smart's Handbook of Engineering Laboratory Practice. .......... 12mo, 250

Snow's Steam-boiler Practice. . . . . . . . . . . . . . . . . . . . 300

Spangler's Notes on Thermodynamics. . . . . . . . . . . . . . . $12 \mathrm{mo}, 100$

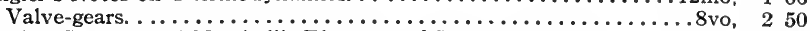

Spangler, Greene, and Marshall's Elements of Steam-engineering. ....8vo 300

Thomas's Steam-turbines. ........................... 400

Thurston's Handbook of Engine and Boiler Trials, and the Use of the Indi-

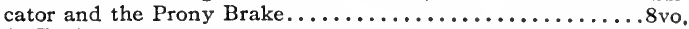

Manual of Steam-boilers; their Designs, Construction, and Operation 8vo, 500

Manual of the Steam-engine......................2vols., 8vo, 1000

Part I. History, Structure, and Theory............. 600

Part II. Design, Construction, and Operation............8vo, 600

Steam-boiler Explosions in Theory and in Practice............ 150

Wehrenfenning's Analysis and Softening of Boiler Feed-water. (Patterson).

Weisbach's Heat, Steam, and Steam-engines.

....................... 500

Wood's Thermodynamics, Heat Motors, and Refrigerating Machines. . .8vo, 400

\section{MECHANICS PURE AND APPLIED.}

Church's Mechanics of Engineering. ..................... 8vo, 600

Notes and Examples in Mechanics. .............................. 200

Dana's Text-book of Elementary Mechanics for Colleges and Schools .12mo, 150

Du Bois's Elementary Principles of Mechanics:

Vol.. I. Kinematics. ........................ 3 vo, 30

Vol. II. Statics............................8vo, 400

Mechanics of Engineering. $\mathrm{Vol}$. I.................................. 750

Vol. II. .................... Small 4 to, 1000

* Greene's Structural Mechanics. ........................... 250

James's Kinematics of a Point and the Rational Mechanics of a Particle.

Large $12 \mathrm{mo}, 200$

* Johnson's (W. W.) Theoretical Mechanics. ........................ 300

Lanza's Appiied Mechanics. . . . . . . . . . . . . . . . . . . . 750

* Martin's Text Book on Mechanics. Voi. I, Statics......................... 125

* Vol. II, Kinematics and Kinetics. $12 \mathrm{mo}, 150$

Maurer's Technical Mechanics.......................... 400

* Merriman's Elements of Mechanics. . . . . . . . . . . . 100

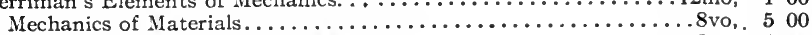

* Michie's Elements of Analytical Mechanics..................8vo. 400

Robinson's Principles of Mechanism. ................... 300

Sanborn's Mechanics Problems. ................................ 12mo, 150

Schwamb and Merrill's Elements of Mechanism.............. 300

Wood's Elements of Analytical Mechanics. ................... 300

Principles of Elementary Mechanics................... 125 


\section{MEDICAL.}

* Abderhalden's Physiological Chemistry in Thirty Lectures. (Hall and Defren. ....................................... \$5 00 von Behring's Suppression of Tuberculosis. (Bolduan.).........12mo, 100 Bolduan's Immune Sera. . . . . . . . . . . . . . . . . . . . . . 12mo, 150

Bordet's Studies in Immunity. (Gay). (In Press.)..............8vo, Daven port's Statistical Methods with Special Reference to Biological Varia-

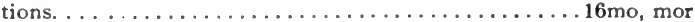

Ehrlich's Collected Studies on Immunity. (Bolduan.). . . . . . . . . . 8vo,

* Fischer's Physiology of Alimentation........................ 12mo, de Fursac's Manual of Psychiatry. (Rosanoff and Collins.).... Large 12mo, Hammarsten's Text-book on Physiological Chemistry. (Mandel.). ....8vo, Jackson's Directions for Laboratory Work in Physiological Chemistry . .8vo, Lassar-Cohn's Practical Urinary Analysis. (Lorenz.).............. 12mo, Mandel's Hand-book for the Bio-Chemical Laboratory............. 12mo,

* Pauli's Physical Chemistry in the Service of Medicine. (Fischer.)..12mo,

* Pozzi-Escot's Toxins and Venoms and their Antibodies. (Cohn.). 12mo, Rostoski's Serum Diagnosis. (Bolduan.)..................... 12mo,

Ruddiman's Incompatibilities in Prescriptions...............

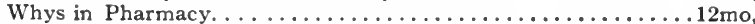

Salkowski's Physiological and Pathological Chemistry. (Orndorff.) ....8vo,

* Satterlee's Outlines of Human Embryology. . . . . . . . . . . . . . 12mo,

Smith's Lecture Notes on Chemistry for Dental Students.............8vo,

*Whipple's Tyhpoid Fever. ............................... 12mo,

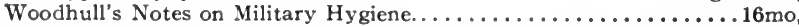

* Personal Hygiene. . . . . . . . . . . . . . . . . . . . . . . 12mo,

Worcester and Atkinson's Small Hospitals Establishment and Maintenance, and Suggestions for Hospital Architecture, with Plans for a Small Hospital. . . . . . . .........................12mo,

\section{METALLURGY.}

Betts's Lead Refining by Electrolysis. .........................8vo, Bolland's Encyclopedia of Founding and Dictionary of Foundry Terms used

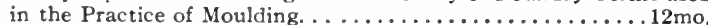

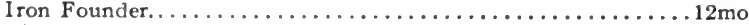

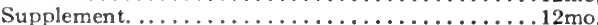

Douglas's Untechnical Addresses on Technical Subjects. . ........... 12mo,

Goesel's Minerals and Metals: A Reference Book..............16mo, mor.

* Iles's Lead-smelting. . . . . . . . . . . . . . . . . . . . . . . . . . 12mo,

Johnson's Rapid Methods for the Chemical Analysis of Special Steels,

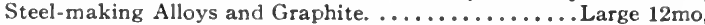

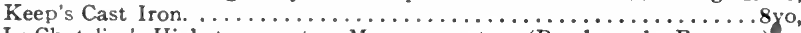

Le Chatelier's High-temperature Measurements. (Boudouard-Burgess.)

Metcalf's Steel. A Manual for Steel-users........................12mo

Minet's Production of Aluminum and its Industrial Use. (Waldo.). . 12mo,

Ruer's Elements of Metallography. (Mathewson).................8vo.

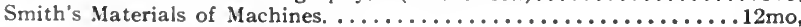

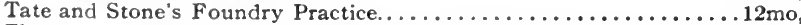

Thurston's Materials of Engineering. In Three Parts. ............. 8vo.

Part I. Non-metallic Materials of Engineering, see Civil Engineering, page 9.

Part II. Iron and Steel. ............................8vo

Part III. A Treatise on Brasses, Bronzes, and Other Alloys and their

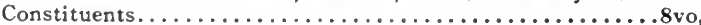

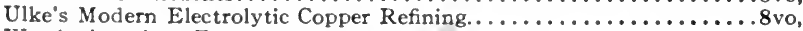
West's American Foundry Practice.......................12mo,

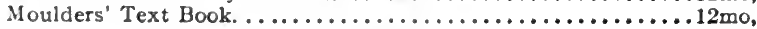




\section{MINERALOGY.}

Baskerville's Chemical Elements. (In Preparation.).

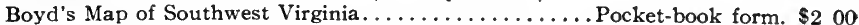

* Browning's Introduction to the Rarer Elements. . . . . . . . . . . 8vo, 150 Brush's Manual of Determinative Mineralogy. (Penfield.).........8vo, 400

Butler's Pocket Hand-book of Minerals. . . . . . . . . . . 300

Chester's Catalogue of Minerals....................

* Crane's Gold and Silver. ...................................... 125

Dana's First Appendix to Dana's New "System of Mineralogy". .Large 8vo, 100

Dana's Second Appendix to Dana's New "System of Mineralogy."

Manual of Mineralogy and Petrography.

.................... 200

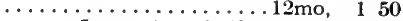

System of Mineralogy....................... 8 vo, half leather, 1250

Text-book of Mineralogy. ...................... 400

Douglas's Untechnical Addresses on Technical Subjects. . . . . . . . 12 mo, 100

Eakle's Mineral Tables............................ 8vo, 125

Eckel's Stone and Clay Products Used in Engineering. (In Preparation).

Goesel's Minerals and Metals: A Reference Book...........16mo, mor. 300

Groth's Introduction to Chemical Crystallography (Marshall)......12mo, 125

* Hayes's Handbook for Field Geologists................16mo, mor. 150

Iddings's Igneous Rocks. . . . . . . . . . . . . . . $5 \ldots \ldots \ldots$ vo, 00

Rock Minerals. . .........................8vo, 500

Johannsen's Determination of Rock-forming Minerals in Thin Sections. 8vo,

With Thumb Index 500

* Martin's Laboratory Guide to Qualitative Analysis with the Blow-

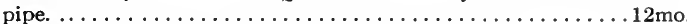

Merrill's Non-metallic Minerals: Their Occurrence and Uses. ... . . . . 8vo,

Stones for Building and Decoration. ....................

* Penfield's Notes on Determinative Mineralogy and Record of Mineral Tests. 8 vo, paper,

Tables of Minerals, Including the Use of Minerals and Statistics of

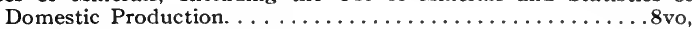

* Pirsson's Rocks and Rock Minerals...................... 12mo,

* Richards's Synopsis of Mineral Characters. . . . . . . . . . 12mo, mor.

* Ries's Clays: Their Occurrence, Properties and Uses......... . . . . 8vo,

* Ries and Leighton's History of the Clay-working Industry of the United

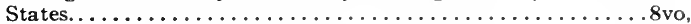

* Tillman's Text-book of Important Minerals and Rocks.......... . . 8vo, Washington's Manual of the Chemical Analysis of Rocks. . . . . . . . . . . 8vo,

\section{MINING.}

* Beard's Mine Gases and Explosions. .................. Large 12mo, 300

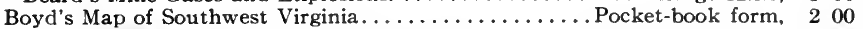

* Crane's Gold and Silver. ..........................8vo, 500

* Index of Mining Engineering Literature.............. 4 vo, 00

* 8vo, mor. 500

Douglas's Untechnical Addresses on Technical Subjects. . . . . . . . 12mo, 100

Eissler's Modern High Explosives.......................... 400

Goesel's Minerals and Metals: A Reference Book...........16mo, mor. 300

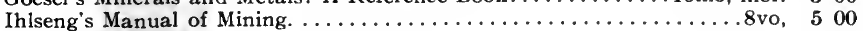

* Iles's Lead Smelting. ........................................ 250

Peele's Compressed Air Plant for Mines. . . . . . . . . . . . . . . 8vo, 300

Riemer's Shaft Sinking Under Difficult Conditions. (Corning and Peele).8vo, 300

* Weaver's Military Explosives. ............................. 300

Wilson's Hydriulic and Placer Mining. 2d edition, rewritten. . ...12mo, 250

Treatise on Practical and Theoretical Mine Ventilation.......12mo, 125 


\section{SANITARY SCIENCE.}

Association of State and National Food and Dairy Departments, Hartford

Meeting, 1906.................................. \$3 00

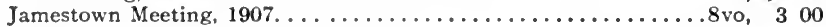

* Bashore's Outlines of Practical Sanitation......................... 125

Sanitation of a Country House. ........................ 100

Sanitation of Recreation Camps and Parks.............. $12 \mathrm{mo}, 00$

Folwell's Sewerage. (Designing, Construction, and Maintenance.)....8vo, 300 Water-supply Engineering. ......................... 400

Fowler's Sewage Works Analyses. . . . . . . . . . . . . . . . . 200

Fuertes's Water-filtration Works............................... 250

Water and Public Health. ................................ 150

Gerhard's Guide to Sanitary Inspections..................

* Modern Baths and Bath Houses....................... 8vo, 300

Sanitation of Public Buildings. ................... 150

Hazen's Clean Water and How to Get It............................ $12 \mathrm{mo}$, 150

Filtration of Public Water-supplies. ................. 8 vo, 300

Kinnicut, Winslow and Pratt's Purification of Sewage. (In Preparation.)

Leach's Inspection and Analysis of Food with Special Reference to State

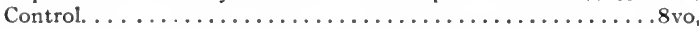

Mason's Examination of Water. (Chemical and Bacteriological).....12mo, Water-supply. (Considered principally from a Sanitary Standpoint).

* Merriman's Elements of Sanitary Enigneering. . . . . . . . . . . . . 8vo,

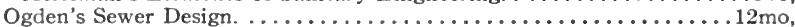

Parsons's Disposal of Municipal Refuse..................... 8vo, Prescott and Winslow's Elements of Water Bacteriology, with Special Refer-

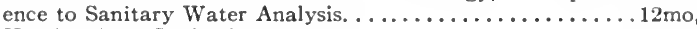

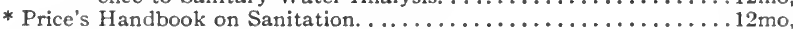

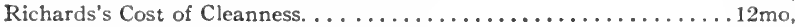
Cost of Food. A Study in Dietaries. . . . . . . . . . . . . . . 12mo,

Cost of Living as Modified by Sanitary Science. . . . . . . . . . 12mo,

Cost of Shelter. . . . . . . . . . . . . . . . . . . . . . . . . . .

* Richards and Williams's Dietary Computer........................

Richards and Woodman's Air, Water, and Food from a Sanitary Stand-

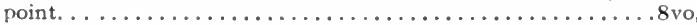

* Richey's Plumbers', Steam-fitters', and Tinners' Edition (Building Mechanics' Ready Reference Series)..............16mo, mor.

Rideal's Disinfection and the Preservation of Food. .............. 8vo,

Sewage and Bacterial Purification of Sewage. . . . . . . . . . . 8vo,

Soper's Air and Ventilation of Subways. . . . . . . . . . . . . . . 12mo,

Turneaure and Russell's Public Water-supplies................. . . . .

Venable's Garbage Crematories in America. . . . . . . . . . . . . . . . 8vo,

Method and Devices for Bacterial Treatment of Sewage. . ......8vo

Ward and Whipple's Freshwater Biology. (In Press.)

Whipple's Microscopy of Drinking-water. ...................8vo, 350

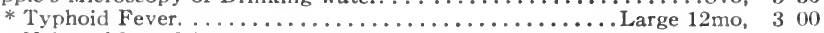

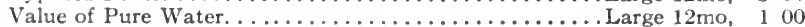

Winslow's Systematic Relationship of the Coccacex................ 12mo, 250

\section{MISCELLANEOUS.}

Emmons's Geological Guide-book of the Rocky Mountain Excursion of the International Congress of Geologists.....................

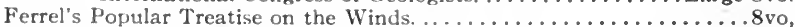
Fitzgerald's Boston Machinist. .......................... 18mo, Gannett's Statistical Abstract of the World. . . . . . . . . . . . . 24mo, Haines's Anerican Railway Nanagement. . . . . . . . . . . . . . 12mo,

* Hanusek's The Microscopy of Technical Products. (Winton)........ . . . . . . 
Jacobs's Betterment Briefs. A Collection of Published Papers on Or-

ganized Industrial Efficiency. ...................

Metcalfe's Cost of Manufactures, and the Administration of Workshops..8vo, 500

Putnam's Nautical Charts. ........................ 8vo, 200

Ricketts's History of Rensselaer Polytechnic Institute 1824-1894.

Large 12mo, 300

Rotherham's Emphasised New Testament. . . . . . . . . . . . Large 8vo, 200

Rust's Ex-Meridian Altitude, Azimuth and Star-finding Tables.......8vo, 500

Standage's Decoration of Wood, Glass, Metal, etc.............12mo, 200

Thome's Structural and Physiological Botany. (Bennett)................... 225

Westermaier's Compendium of General Botany. (Schneider). . . . . . 8vo, 200

Winslow's Elements of Applied Microscopy..................... 150

\section{HEBREW AND CHALDEE TEXT-BOOOKS.}

Gesenius's Hebrew and Chaldee Lexicon to the Old Testament Scriptures.

(Tregelles.)....................... Small 4to, half mor, 500

Green's Elementary Hebrew Grammar......................... 125 



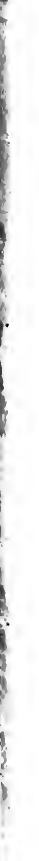





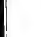



Physical \&

Applied Sci.

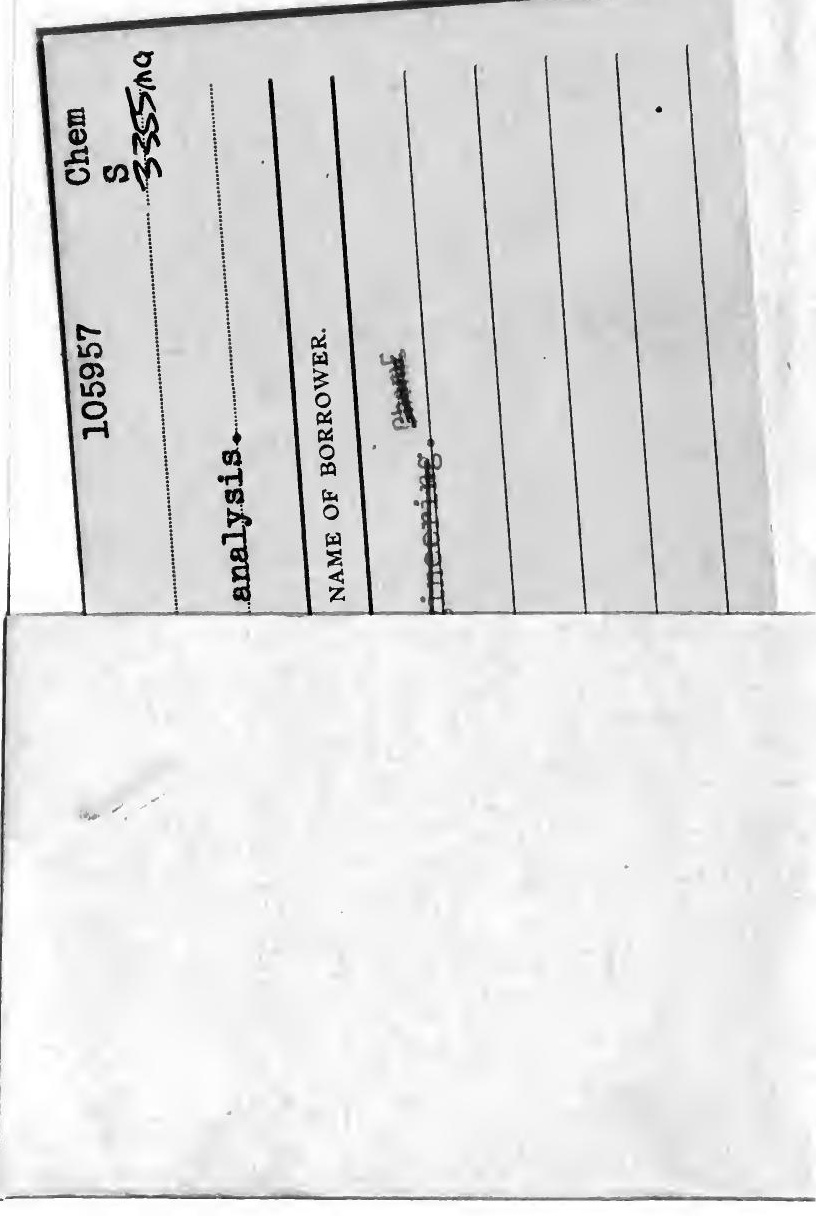


